\title{
Systems Analysis Programs for Hands-On Integrated Reliability Evaluations (SAPHIRE) Code Reference Manual
}

Parts $A$ and $B$

K. J. Kvarfordt

S. T. Wood

C. L. Smith

August 2008

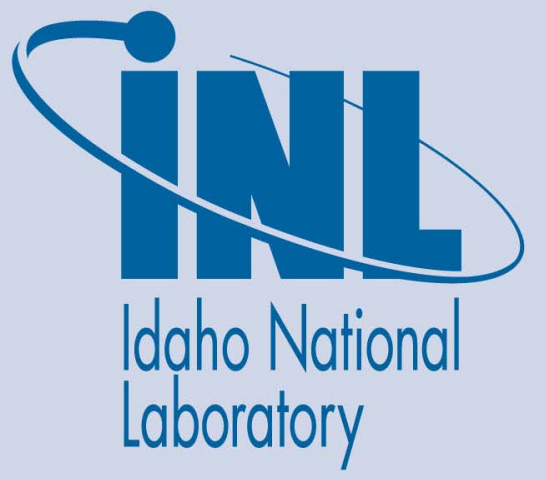

The INL is a U.S. Department of Energy National Laboratory operated by Battelle Energy Alliance 
INL/EXT-05-00644

Rev. 1

\section{Systems Analysis Programs for Hands-On Integrated Reliability Evaluations (SAPHIRE) Code Reference Manual}

Parts A and B

K. J. Kvarfordt

S. T. Wood

C. L. Smith

August 2008

Idaho National Laboratory
Idaho Falls, Idaho 83415

http://www.inl.gov

Prepared for the

Division of Risk Analysis

Office of Nuclear Regulatory Research

U.S. Nuclear Regulatory Commission

Washington D.C. 20555

Job Code $\mathrm{N} 6203$ 
INL/EXT-05-00644

Rev. 1

\title{
Systems Analysis Programs for Hands-On Integrated Reliability Evaluations (SAPHIRE) Code Reference Manual
}

\author{
Part A
}

\author{
K. J. Kvarfordt \\ S. T. Wood \\ C. L. Smith
}

August 2008

\section{Idaho National Laboratory \\ Idaho Falls, Idaho 83415}

http://www.inl.gov

Prepared for the

Division of Risk Analysis

Office of Nuclear Regulatory Research

U.S. Nuclear Regulatory Commission

Washington D.C. 20555

Job Code $\mathrm{N} 6203$ 


\title{
AVAILABILITY NOTICE
}

\author{
Availability of Reference Materials Cited in NRC Publications
}

Most documents cited in NRC publications will be available from one of the following sources:

1. The NRC Public Document Room, 11555 Rockville Pike, Rockville, MD 20852 (pdr@nrc.gov)

2. The Superintendent of Documents, U. S. Government Printing Office (GPO), Mail Stop SSOP, Washington, DC 20402-9328

3. The National Technical Information Service, Springfield, VA 22161

Although the listing that follows represents the majority of documents cited in NRC publications, it is not intended to be exhaustive.

Referenced documents available for inspection and copying for a fee from the NRC Public Document Room include NRC correspondence and internal NRC memoranda; NRC bulletins, circulars, information notices, inspection and investigative notices; licensee event reports; vendor reports and correspondence; Commission papers; and applicant and licensee documents and correspondence.

The following documents in the NUREG series are available for purchase from the GPO Sales Program: formal NRC staff and contractor reports, NRC-sponsored conference proceedings, international agreement reports, grant publications, and NRC booklets and brochures. Also available are regulatory guides, NRC regulations in the Code of Federal Regulations, and Nuclear Regulatory Commission Issuances.

Documents available from the National Technical Information Service include NUREG-series reports and technical reports prepared by other Federal agencies and reports prepared by the Atomic Energy Commission, forerunner agency to the Nuclear Regulatory Commission.

Documents available from public and special technical libraries include all open literature items, such as books, journal articles, and transactions. Federal Register notices, Federal and State legislation, and congressional reports can usually be obtained from these libraries.

Documents such as theses, dissertations, foreign reports and translations, and non-NRC conference proceedings are available for purchase from the organization sponsoring the publication cited.

Single copies of NRC draft reports are available free, to the extent of supply, upon written request to the Office of Administration, Distribution and Mail Services Section U. S. Nuclear Regulatory Commission, Washington, DC 20555-0001.

The public maintains copies of industry codes and standards used in a substantive manner in the NRC regulatory process at the NRC Library, Two White Flint North, 11545 Rockville Pike, Rockville, MD, 20852, for use. Codes and standards are usually copyrighted and may be purchased from the originating organization or, if they are American National Standards, from the American National Standards Institute, 1430 Broadway, New York, NY 10018. 


\section{DISCLAIMER NOTICE}

This report was prepared as an account of work sponsored by an agency of the United States Government. Neither the United States Government nor any agency thereof, or any of their employees, makes any warranty, expressed or implied, or assumes any legal liability of responsibility for any third party's use, or the results of such use, or any information, apparatus, product or process disclosed in this report, or represents that its use by such third party would not infringe privately owned rights. 


\section{PREVIOUS REPORTS}

Smith, C. L., et al., Testing, Verifying, and Validating SAPHIRE Versions 6.0 and 7.0, NUREG/CR6688, October 2000.

K. D. Russell, et al. Systems Analysis Programs for Hands-on Reliability Evaluations (SAPHIRE) Version 6.0 - System Overview Manual, NUREG/CR-6532, May 1999.

K. D. Russell et al., Integrated Reliability and Risk Analysis System (IRRAS) Version 5.0, Volume 2 Reference Manual, NUREG/CR-6116, EGG-2716, July 1994.

K. D. Russell et al., Verification and Validation $(V \& V)$, Volume 9 - Reference Manual, NUREG/CR6116, EGG-2716, July 1994.

K. D. Russell et al., Integrated Reliability and Risk Analysis System (IRRAS) Version 4.0, Volume 1 Reference Manual, NUREG/CR-5813, EGG-2664, January 1992.

K. D. Russell et al., Integrated Reliability and Risk Analysis System (IRRAS) Version 2.5 Reference Manual, NUREG/CR-5300, EGG-2613, March 1991.

K. D. Russell, M. B. Sattison, D. M. Rasmuson, Integrated Reliability and Risk Analysis System (IRRAS) - Version 2.0 User's Guide, NUREG/CR-5111, EGG-2535, manuscript completed March 1989, published June 1990.

K. D. Russell, D. M. Snider, M. B. Sattison, H. D. Stewart, S.D. Matthews, K. L. Wagner, Integrated Reliability and Risk Analysis System (IRRAS) User's Guide - Version 1.0 (DRAFT), NUREG/CR-4844, EGG-2495, June 1987. 


\section{ABSTRACT}

The Systems Analysis Programs for Hands-on Integrated Reliability Evaluations (SAPHIRE) is a software application developed for performing a complete probabilistic risk assessment (PRA) using a personal computer. SAPHIRE is funded by the U.S. Nuclear Regulatory Commission (NRC) and developed by the Idaho National Laboratory (INL). The INL's primary role in this project is that of software developer. However, the INL also plays an important role in technology transfer by interfacing and supporting SAPHIRE users comprised of a wide range of PRA practitioners from the NRC, national laboratories, the private sector, and foreign countries.

SAPHIRE can be used to model a complex system's response to initiating events, quantify associated damage outcome frequencies, and identify important contributors to this damage (Level 1 PRA) and to analyze containment performance during a severe accident and quantify radioactive releases (Level 2 PRA). It can be used for a PRA evaluating a variety of operating conditions, for example, for a nuclear reactor at full power, low power, or at shutdown conditions. Furthermore, SAPHIRE can be used to analyze both internal and external initiating events and has special features for transforming models built for internal event analysis to models for external event analysis. It can also be used in a limited manner to quantify risk in terms of release consequences to both the public and the environment (Level 3 PRA).

SAPHIRE includes a separate module called the Graphical Evaluation Module (GEM). GEM provides a highly specialized user interface with SAPHIRE that automates SAPHIRE process steps for evaluating operational events at commercial nuclear power plants. Using GEM, an analyst can estimate the risk associated with operational events in a very efficient and expeditious manner.

This reference guide will introduce the SAPHIRE Version 7.0 software. A brief discussion of the purpose and history of the software is included along with general information such as installation instructions, starting and stopping the program, and some pointers on how to get around inside the program. Next, database concepts and structure are discussed. Following that discussion are nine sections, one for each of the menu options on the SAPHIRE main menu, wherein the purpose and general capabilities for each option are furnished. Next, the capabilities and limitations of the software are provided. 


\section{FOREWORD}

The U.S. Nuclear Regulatory Commission has developed the Systems Analysis Programs for Hands-on Integrated Reliability Evaluations (SAPHIRE) software used to perform probabilistic risk assessments (PRAs) on a personal computer. SAPHIRE enables users to supply basic event data, create and solve fault and event trees, perform uncertainty analyses, and generate reports. In that way, analysts can perform PRAs for any complex system, facility, or process.

SAPHIRE can be used to model a plant's response to initiating events, quantify core damage frequencies, and identify important contributors to core damage (Level 1 PRA). The program can also be used to evaluate containment failure and release models for severe accident conditions, given that core damage has occurred (Level 2 PRA). In so doing, the analyst could build the PRA model assuming that the reactor is initially at full power, low power, or shutdown. In addition, SAPHIRE can be used to analyze both internal and external events, and it includes special features for transforming models built for internal event analysis to models for external event analysis. It can also be used in a limited manner to quantify the frequency of release consequences (Level 3 PRA). Because this software is a very detailed technical tool, users should be familiar with PRA concepts and methods used to perform such analyses.

SAPHIRE has evolved with advances in computer technology. The versions currently in use (6 and 7) run in the Microsoft Windows ${ }^{\circledR}$ environment. A user-friendly interface, Graphical Evaluation Module (GEM), streamlines and automates selected SAPHIRE inputs and processes for performing event assessments.

SAPHIRE has also evolved with users' needs, and Versions 6 and 7 include new features and capabilities for developing and using larger, more complex models. For example, Version 7 can solve up to 2 million sequences and includes enhancements for cut set slicing, event tree rule linkage, and reporting options.

This NUREG-series report comprises seven volumes, which address SAPHIRE/GEM Versions 6 and 7. Volume 1, "Overview/Summary," gives an overview of the functions available in SAPHIRE and presents general instructions for using the software. Volume 2, "Technical Reference," discusses the theoretical background behind the SAPHIRE functions. Volume 3, "SAPHIRE Users' Manual," provides installation instructions and a step-by-step approach to using the program's features. Volume 4, "SAPHIRE Tutorial Manual," provides an example of the overall process of constructing a PRA database. Volume 5, "GEM/GEMDATA Reference Manual," discusses the use of GEM. Volume 6, "SAPHIRE Quality Assurance (QA) Manual," discusses QA methods and tests. Lastly, Volume 7, "SAPHIRE Data Loading Manual," assists the user in entering PRA data into SAPHIRE using the built-in MAR-D ASCII-text file data transfer process.

Christiana H. Lui, Director

Division of Risk Analysis

Office of Nuclear Regulatory Research 


\section{CONTENTS}

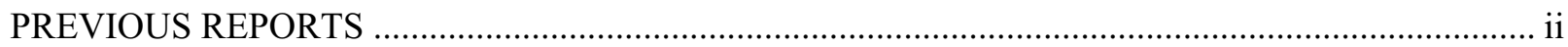

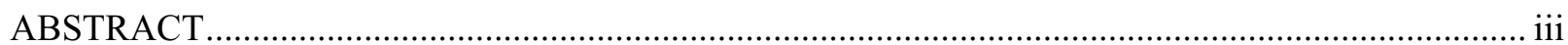

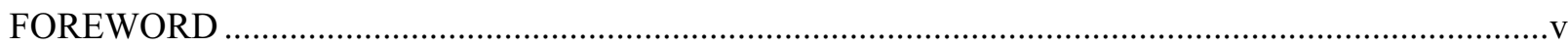

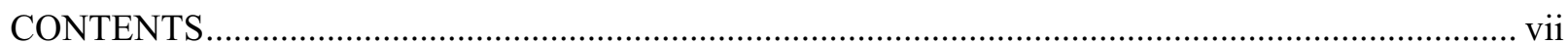

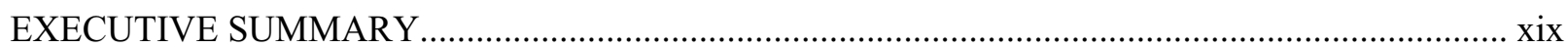

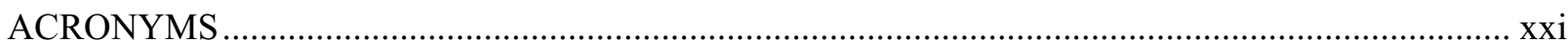

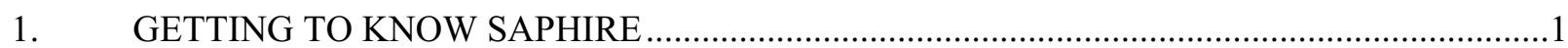

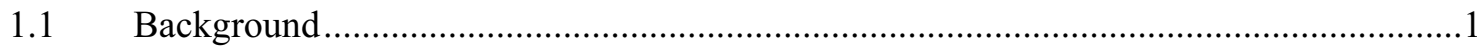

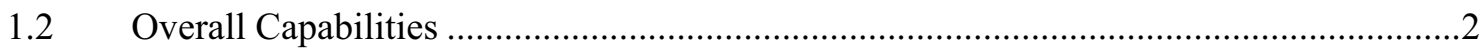

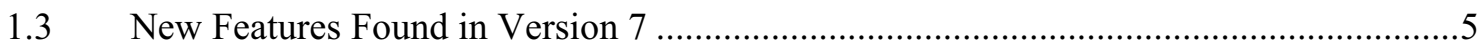

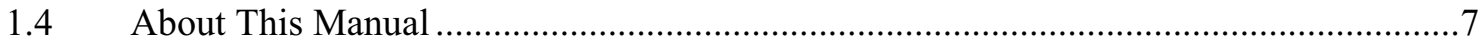

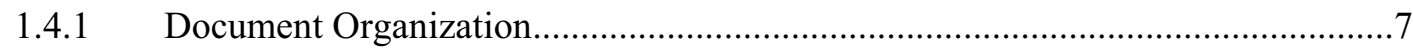

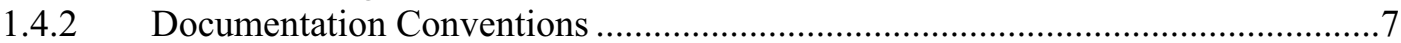

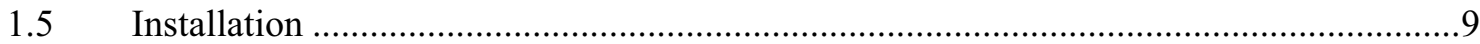

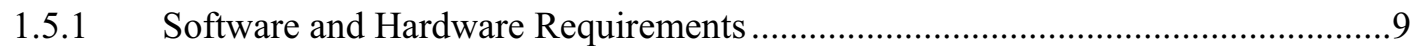

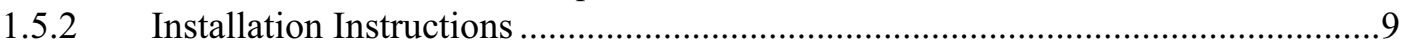

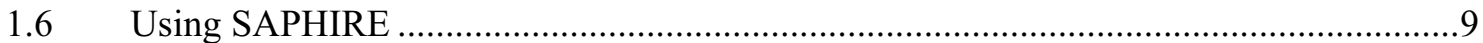

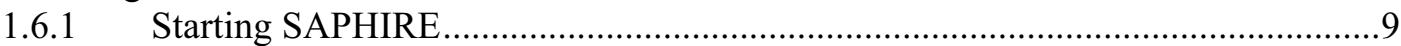

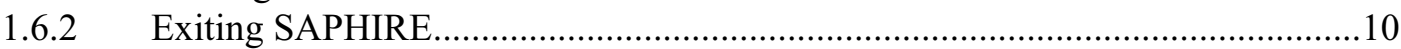

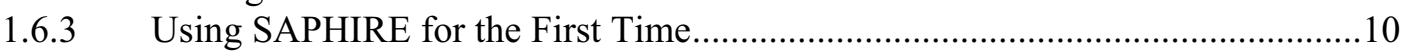

1.6.4 Converting from Previous SAPHIRE (IRRAS) Versions ...................................11

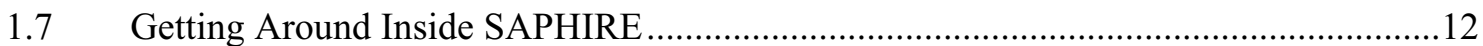

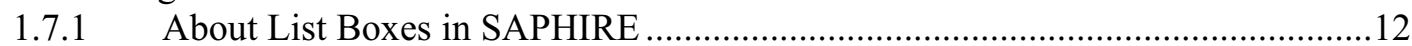

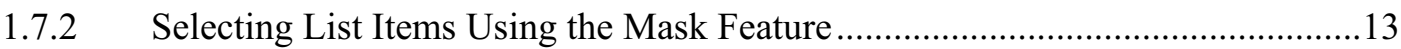

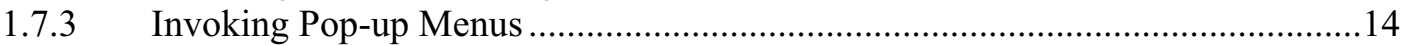

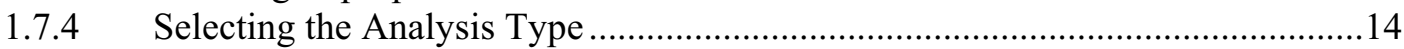

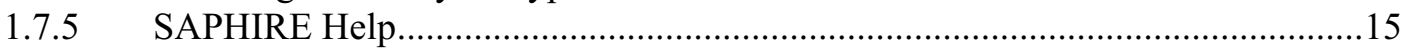

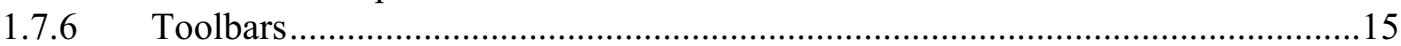

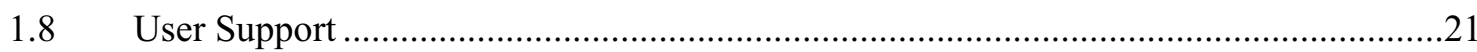

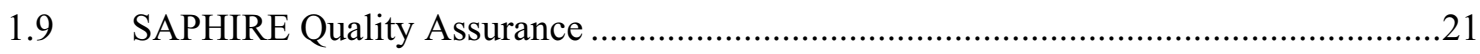

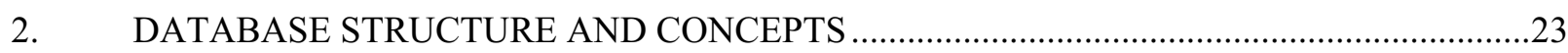

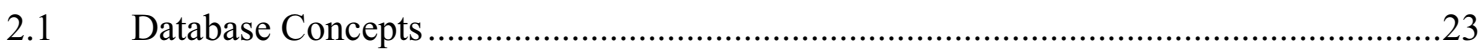

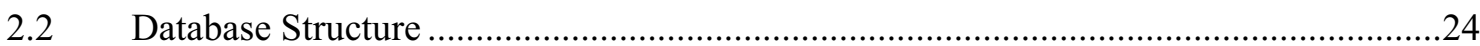

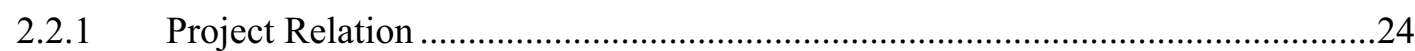

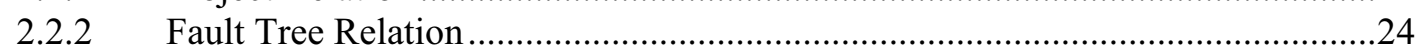




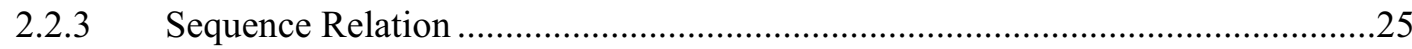

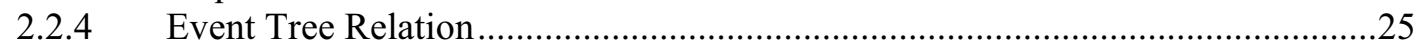

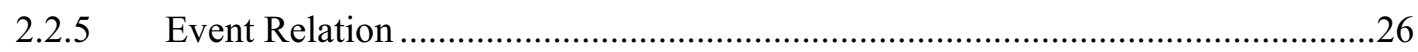

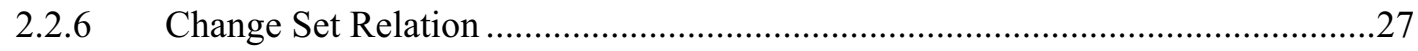

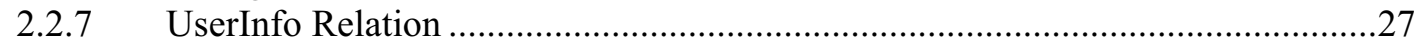

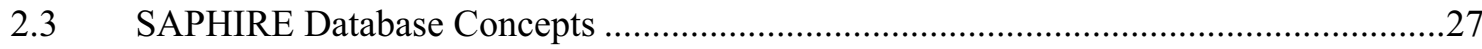

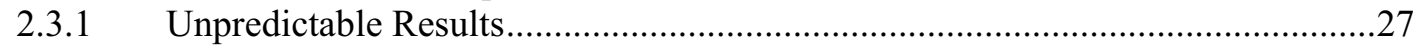

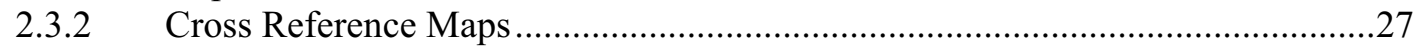

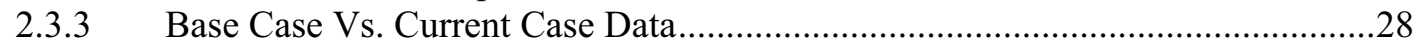

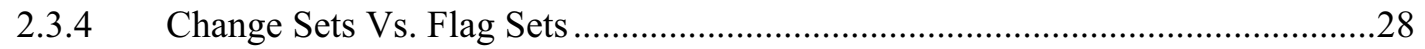

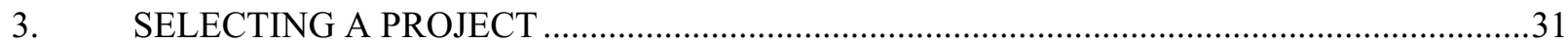

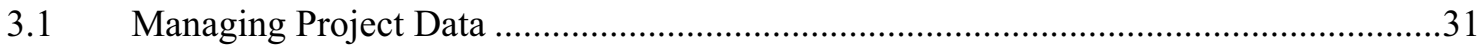

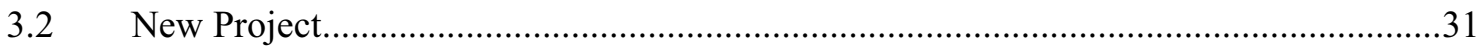

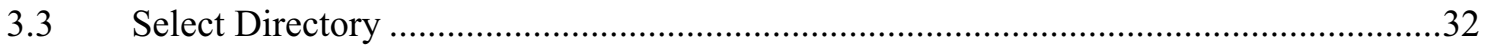

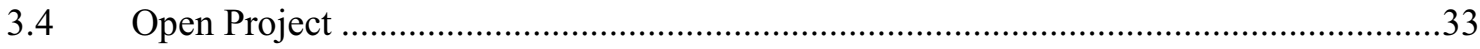

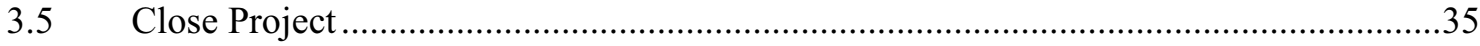

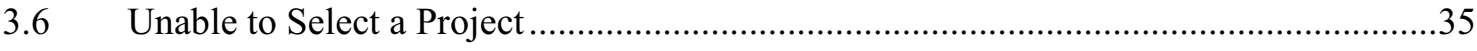

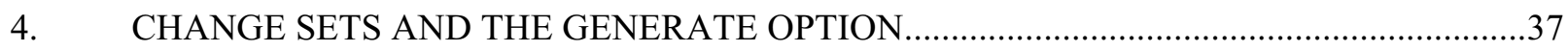

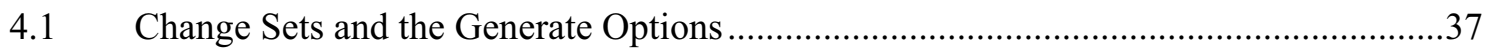

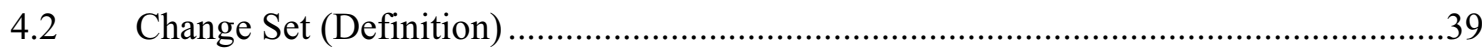

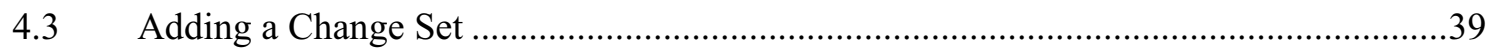

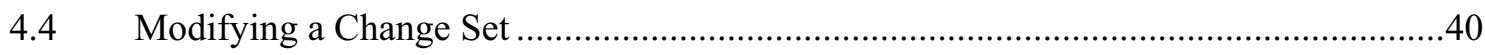

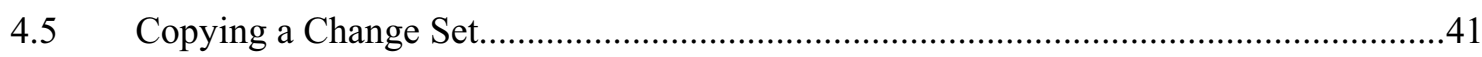

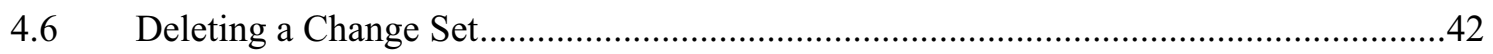

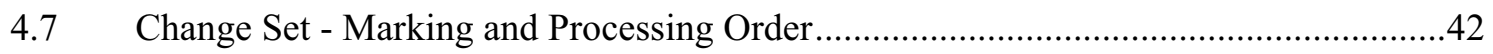

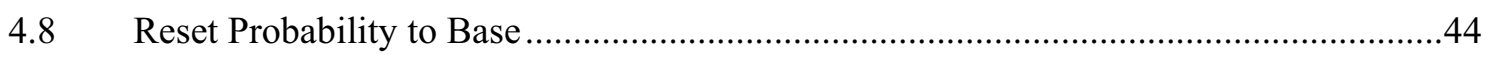

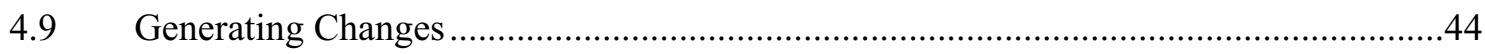

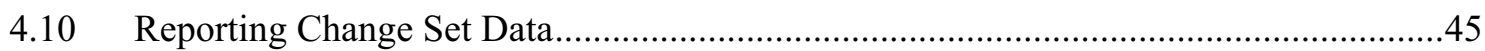

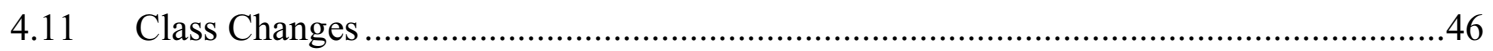

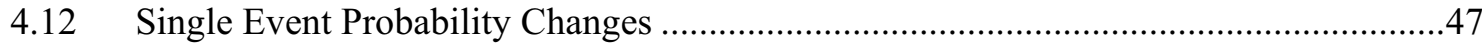

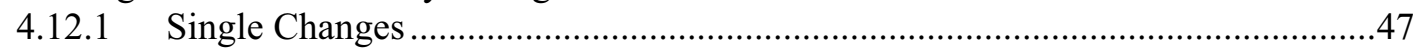

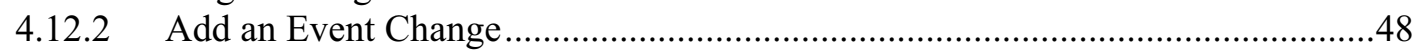




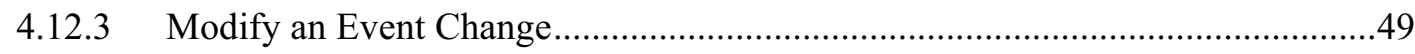

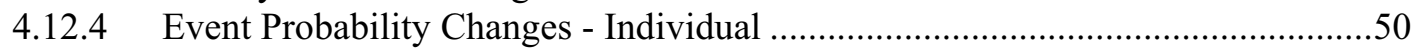

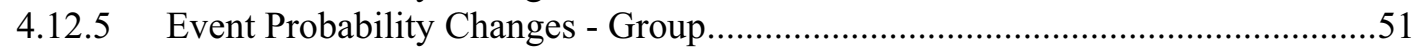

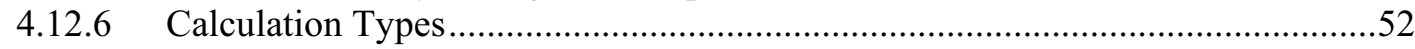

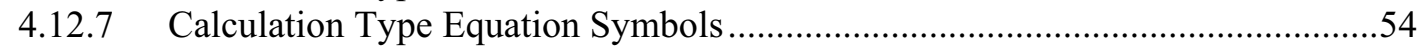

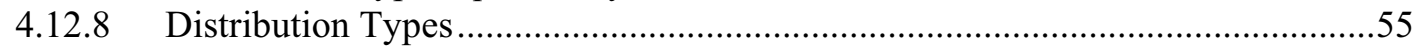

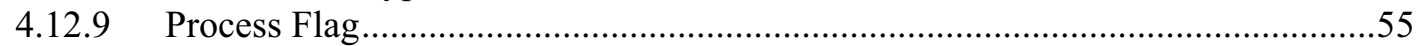

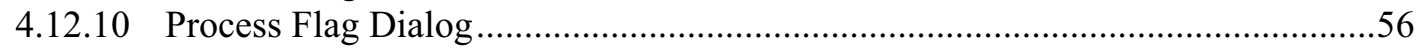

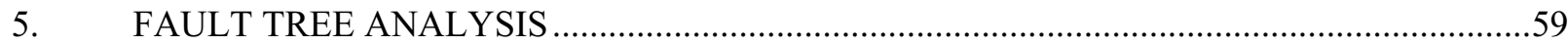

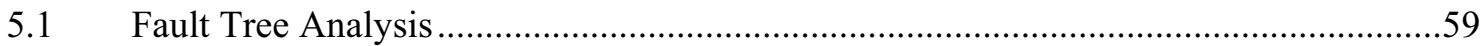

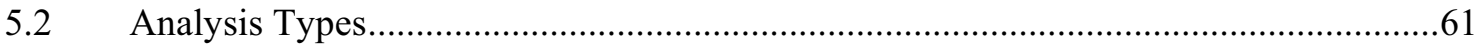

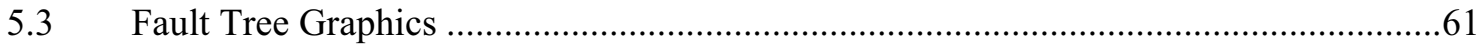

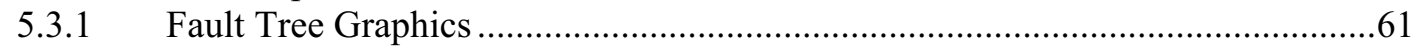

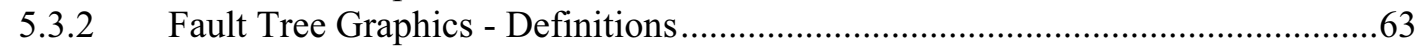

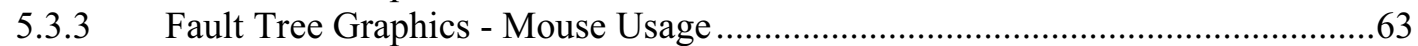

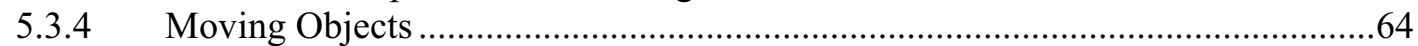

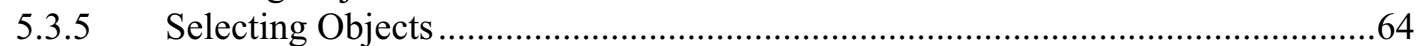

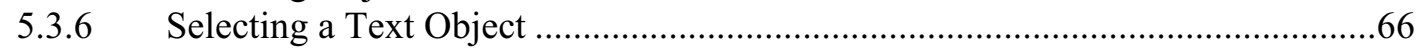

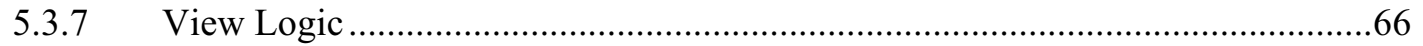

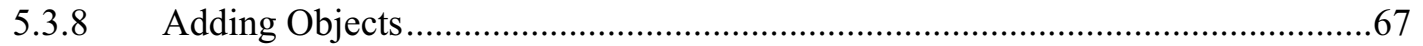

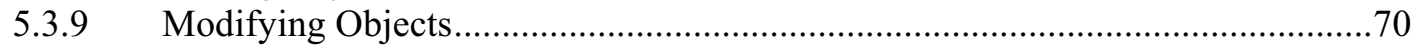

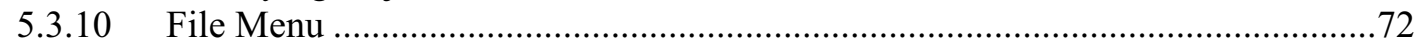

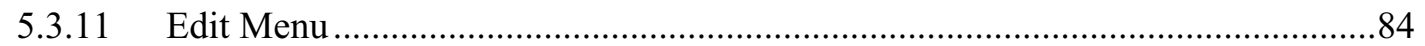

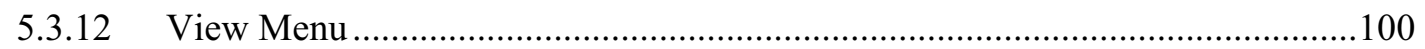

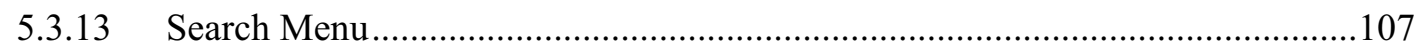

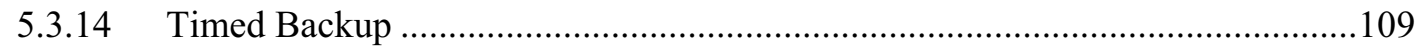

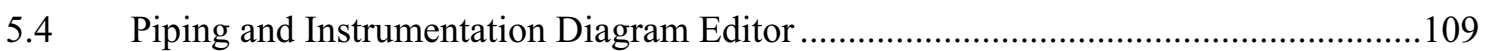

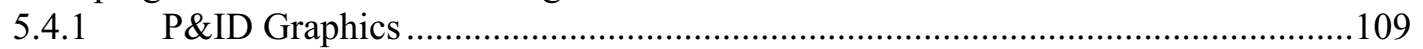

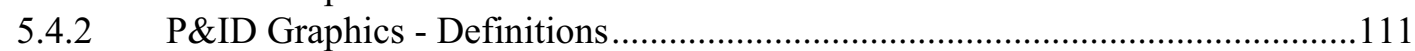

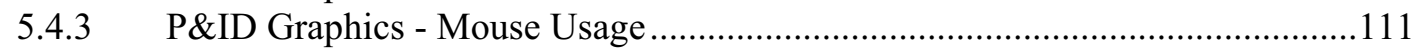

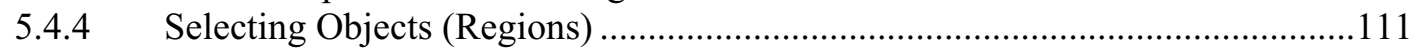

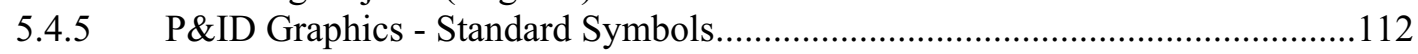

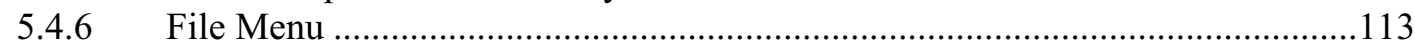

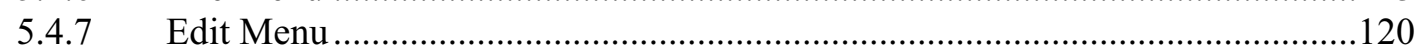

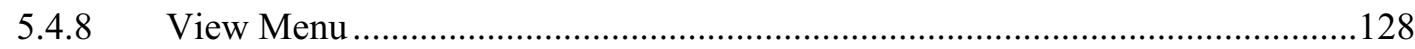

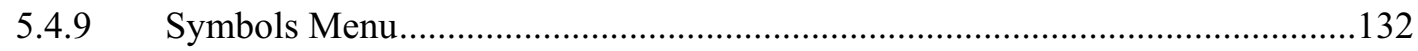

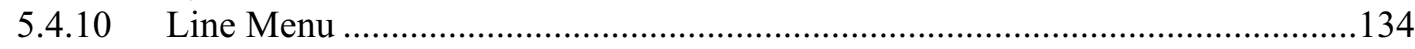

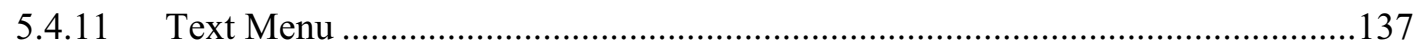

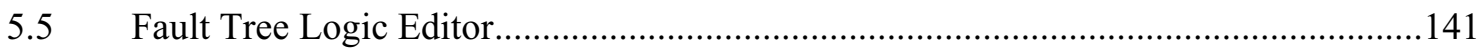

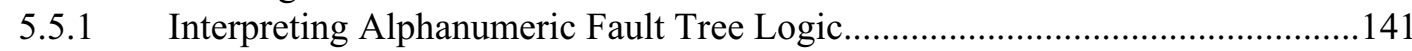

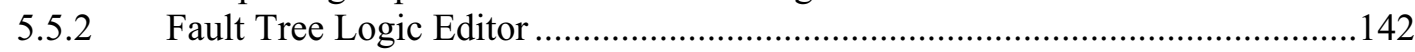

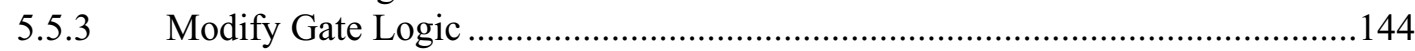

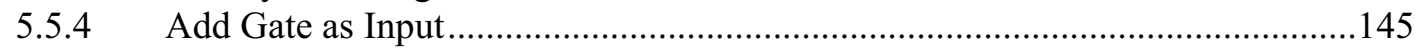

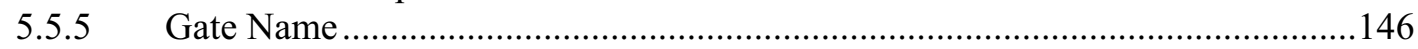

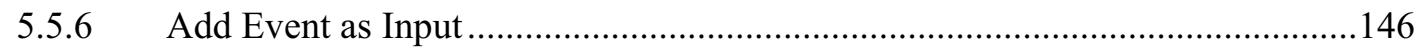

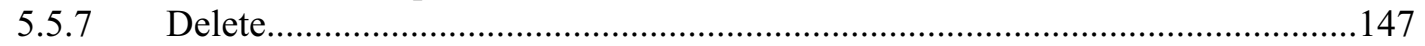




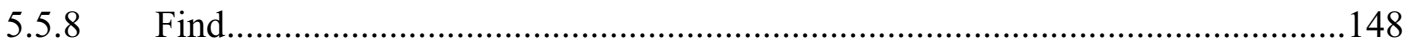

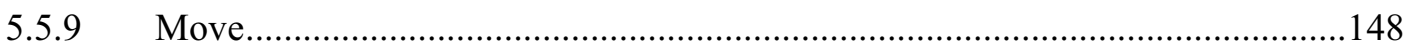

5.5.10 Follow Transfer .................................................................................. 149

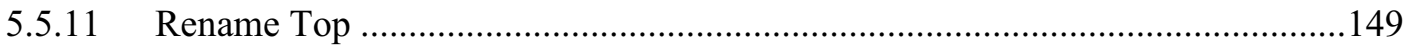

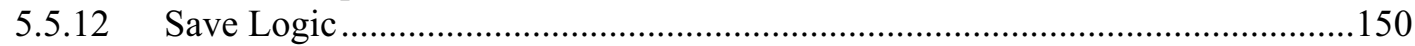

5.5.13 About Logic Dialogs ...............................................................................151

5.5.14 Tips on Using the Fault Tree Logic Editor.......................................................152

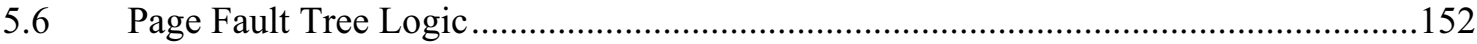

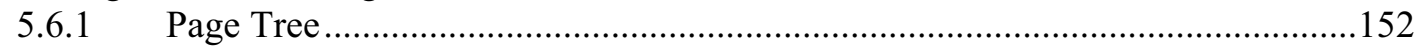

5.6.2 About Logic Dialogs ................................................................................154

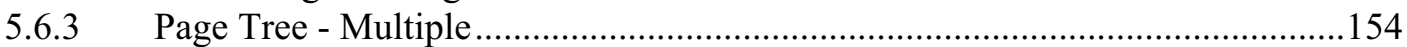

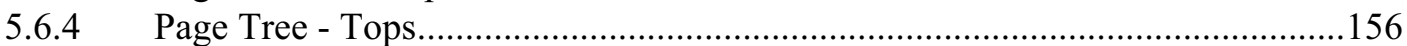

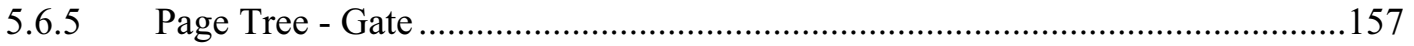

5.6.6 Page Tree - Find ……………………………………………………...158

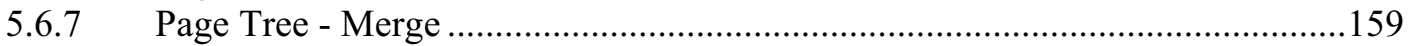

5.6.8 Page Tree - Auto Page .............................................................................. 160

5.6.9 Page Tree - Exit ...........................................................................................161

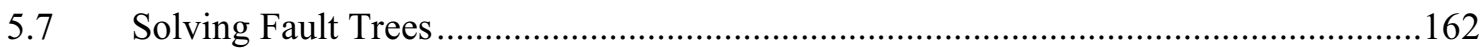

5.7.1 Solving Fault Trees ................................................................................162

5.7.2 Cut Set Generation Dialog...........................................................................165

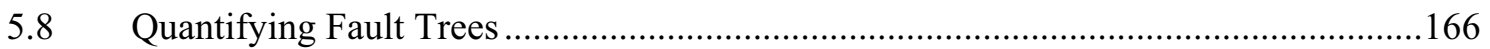

5.8.1 Quantifying Fault Trees....................................................................................166

5.8.2 Minimal Cut Set Upper Bound Approximation ……………………………….....167

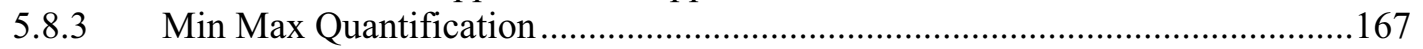

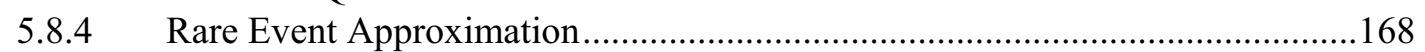

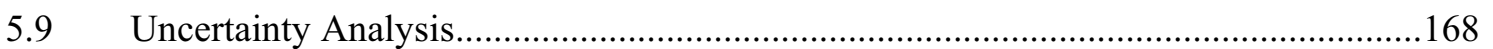

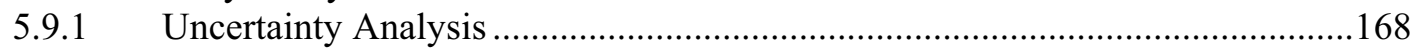

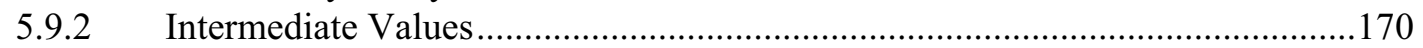

5.9.3 Monte Carlo Sampling (MCS) ....................................................................171

5.9.4 Latin Hypercube Sampling (LHS)..................................................................171

5.9.5 The Uncertainty Analysis Process ......................................................................172

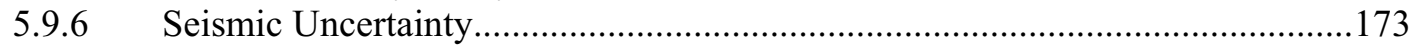

5.9.7 Uncertainty Distributions for Basic Events ......................................................174

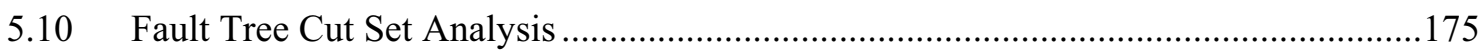

5.10.1 Fault Tree Cut Set Analysis...........................................................................175

5.10 .2 Updating Cut Sets.....................................................................................176

5.10.3 Cut Set Generation Truncation......................................................................177

5.10.4 Recovering Fault Tree Cut Sets....................................................................178

5.10.5 Editing Fault Tree Cut Sets ……………………………………………....... 186

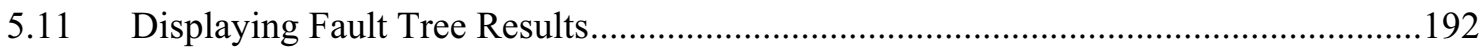

5.11.1 Displaying Fault Tree Results .......................................................................192

5.11.2 Displaying Cut Sets ..................................................................................... 193

5.11.3 Comparing Cut Sets................................................................................215

5.11.4 Displaying Importance Results....................................................................2219

5.11.5 Displaying Uncertainty................................................................................225 


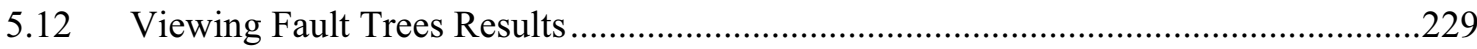

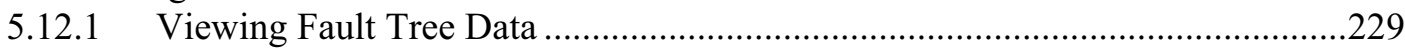

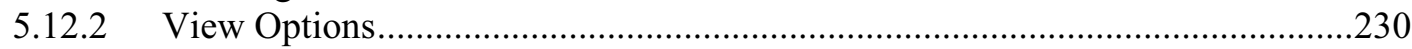

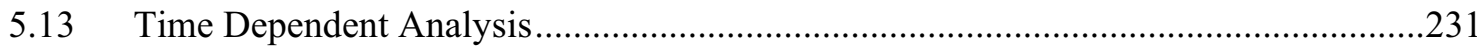

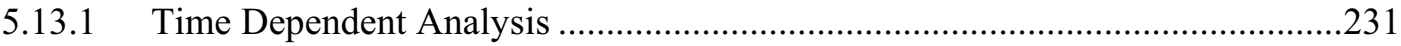

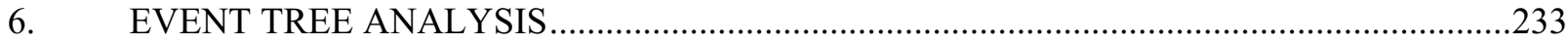

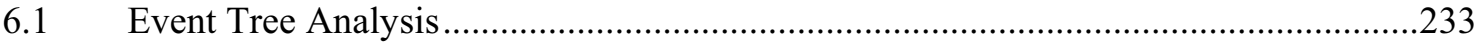

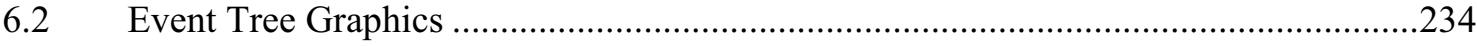

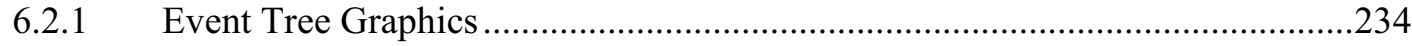

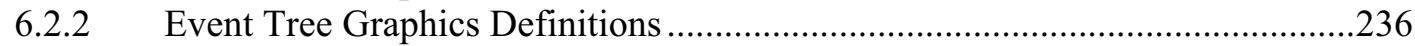

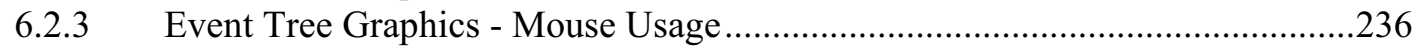

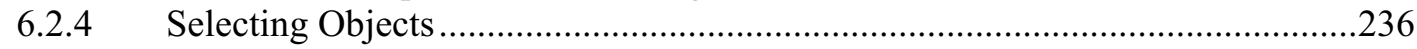

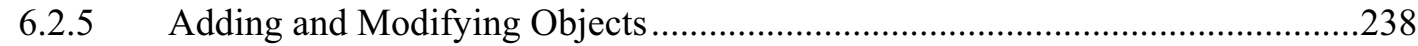

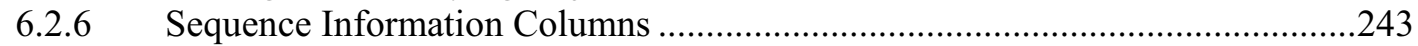

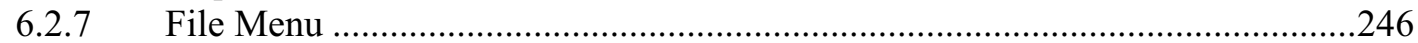

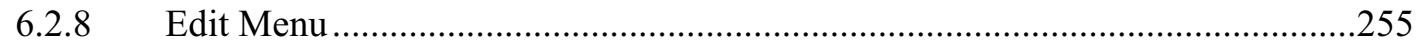

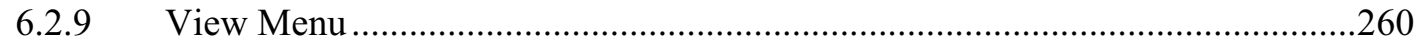

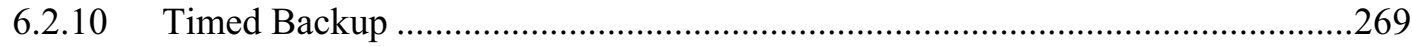

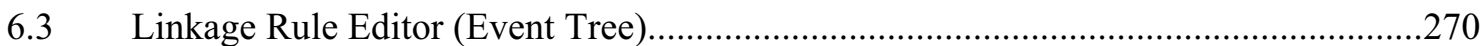

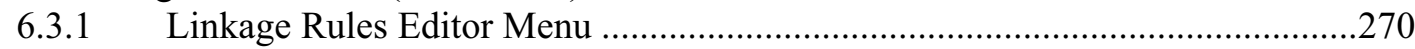

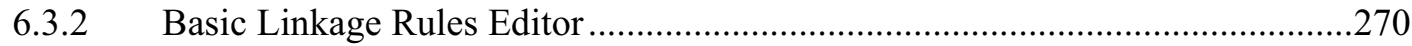

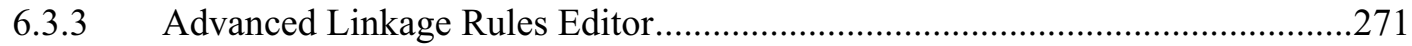

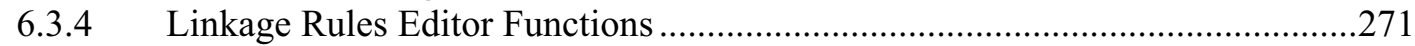

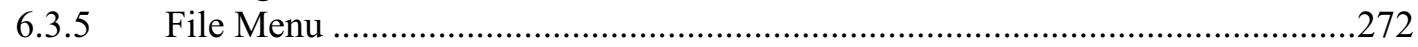

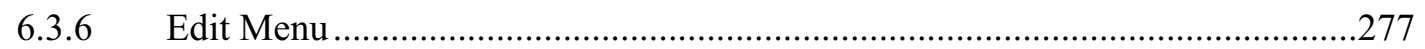

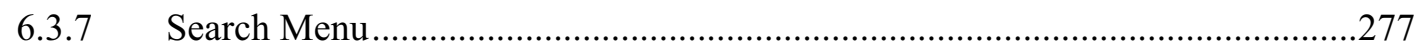

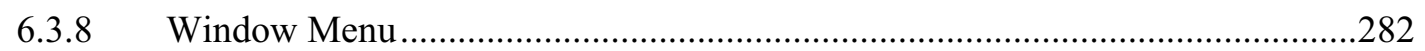

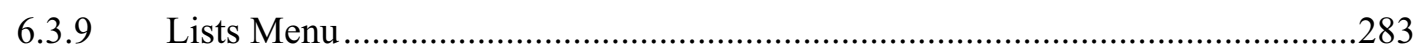

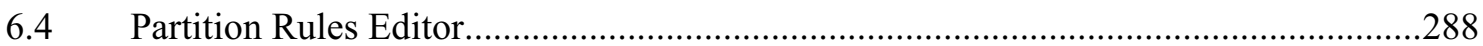

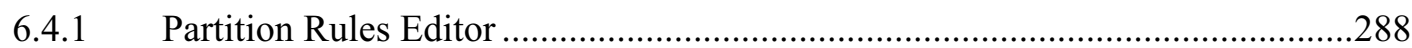

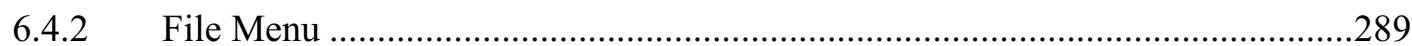

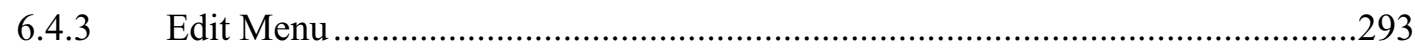

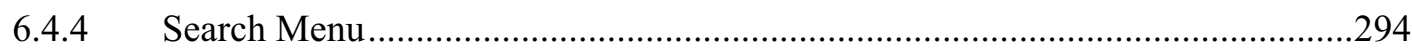

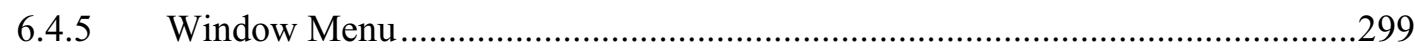

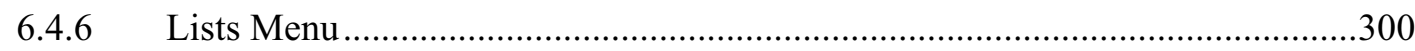

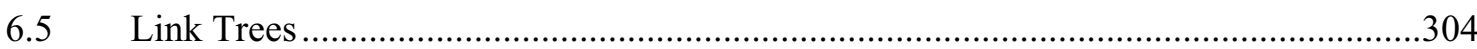

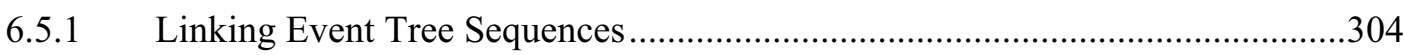

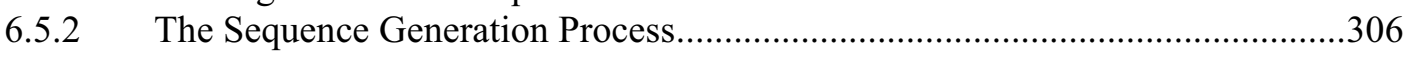

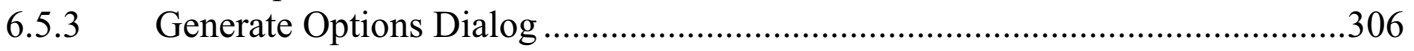

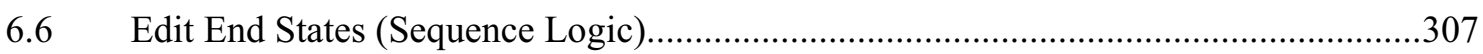

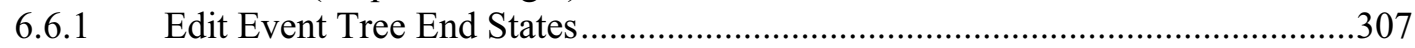

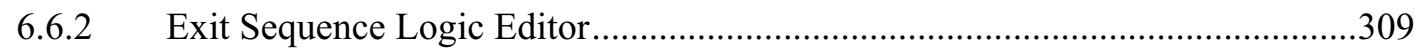

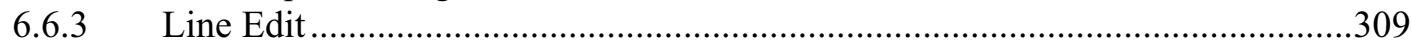

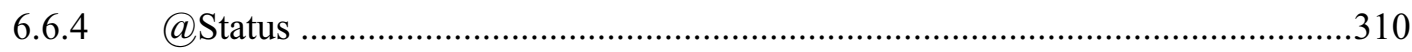




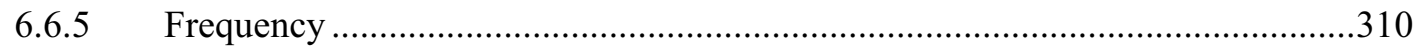

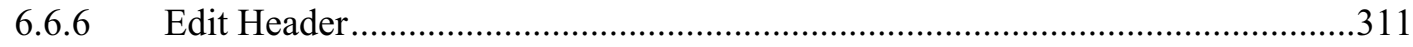

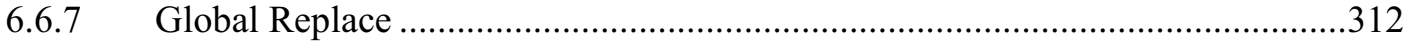

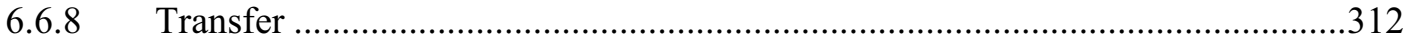

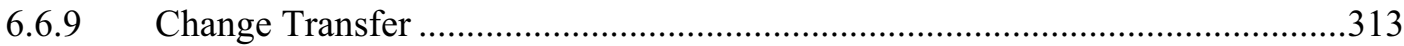

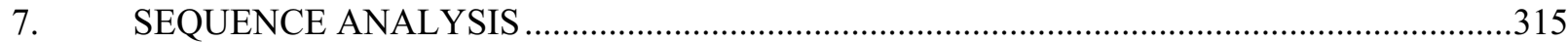

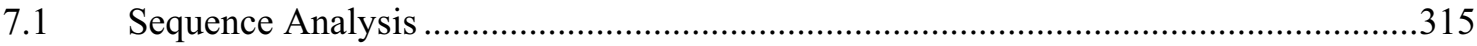

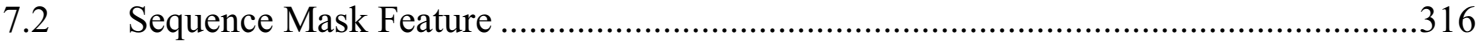

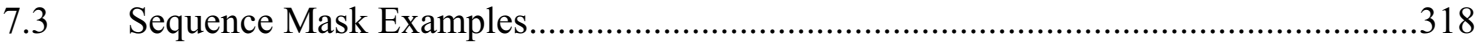

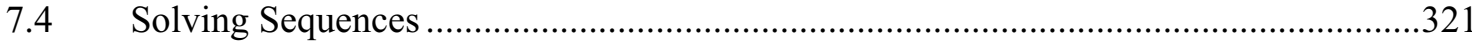

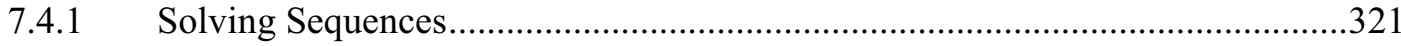

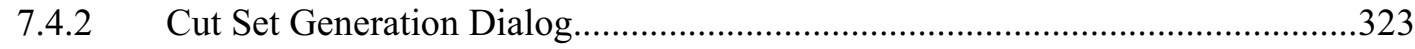

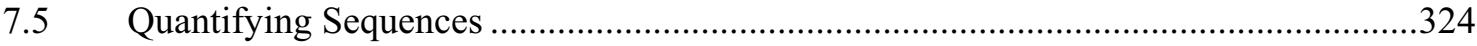

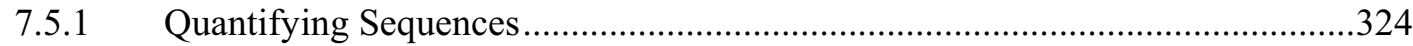

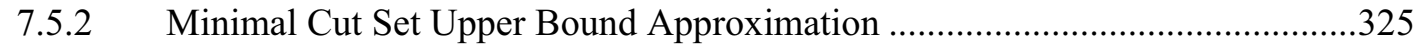

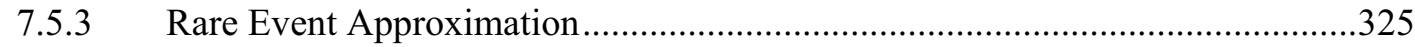

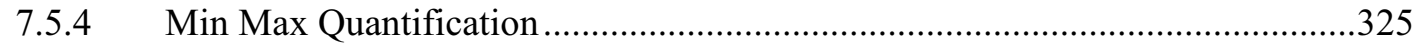

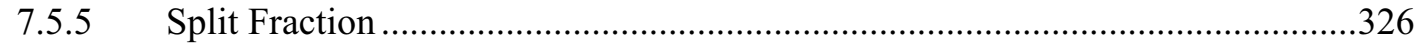

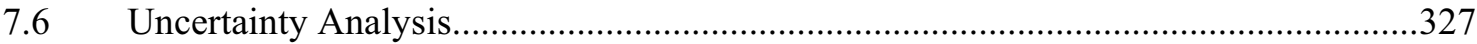

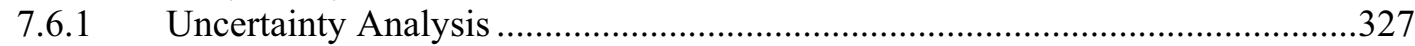

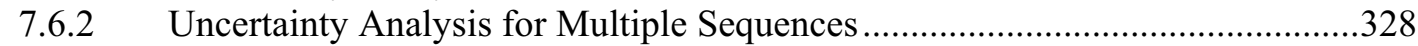

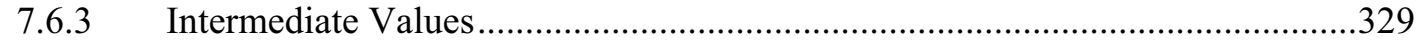

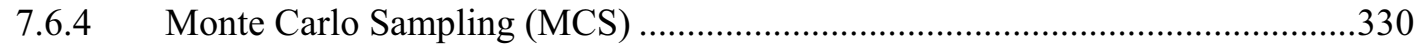

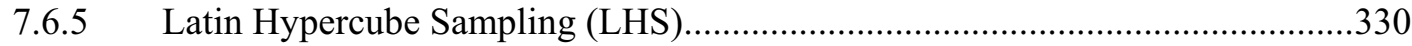

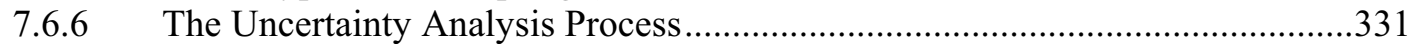

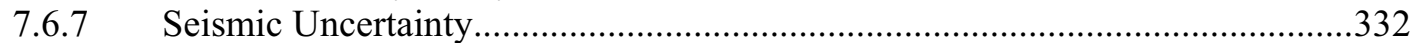

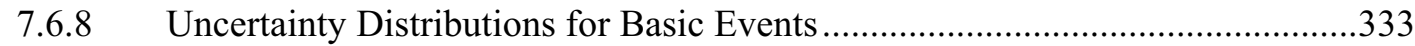

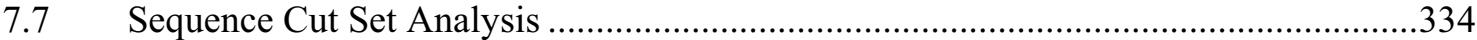

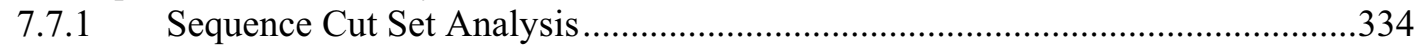

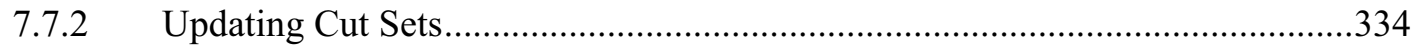

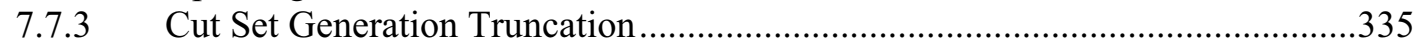

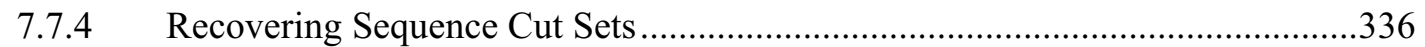

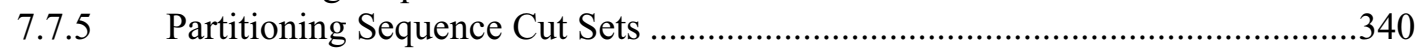

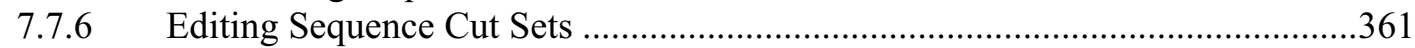

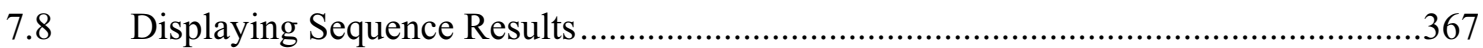

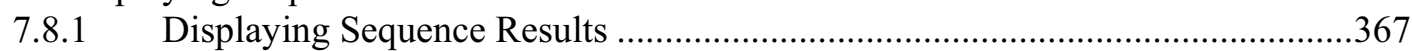

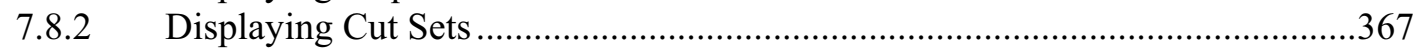

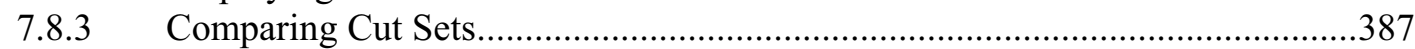

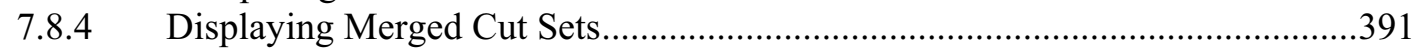

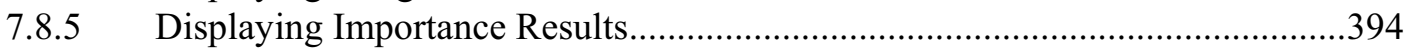

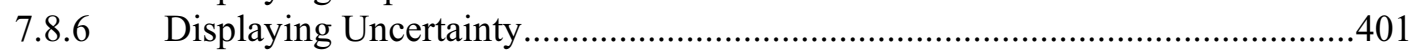

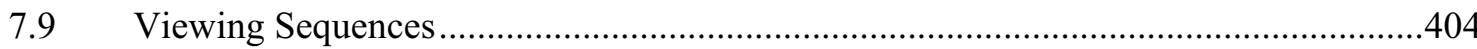




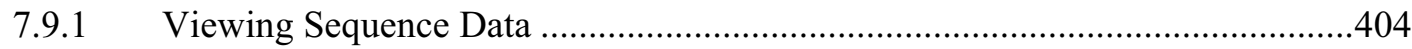

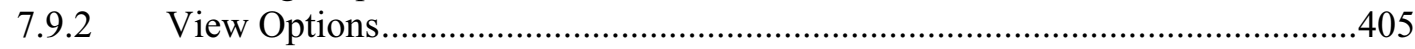

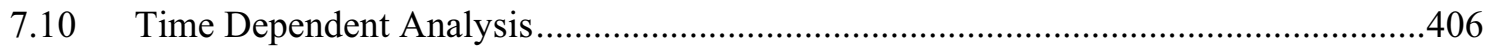

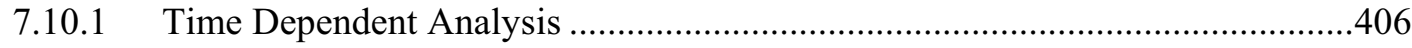

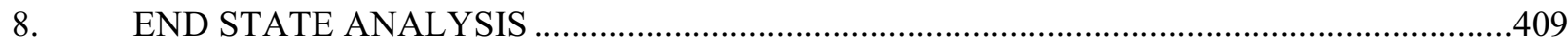

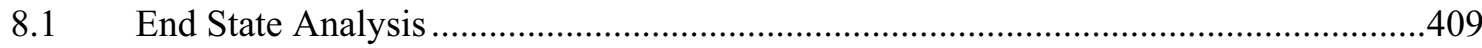

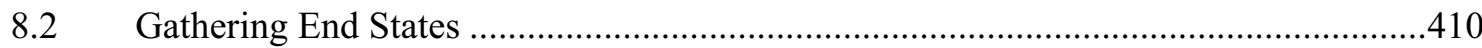

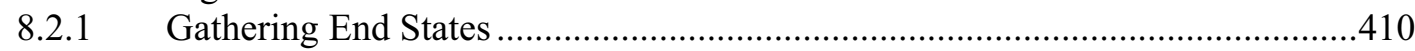

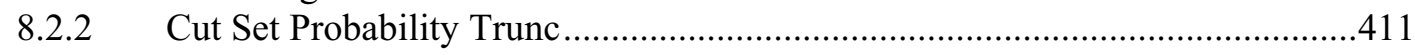

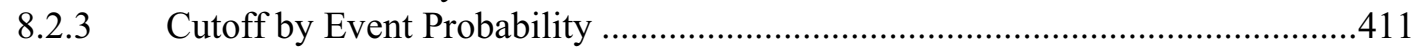

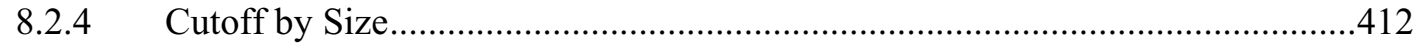

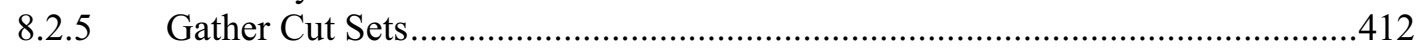

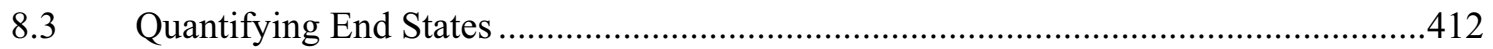

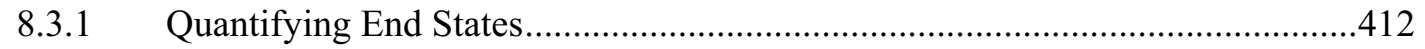

8.3.2 Minimal Cut Set Upper Bound Approximation .................................................

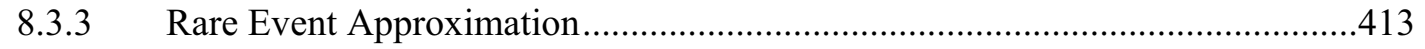

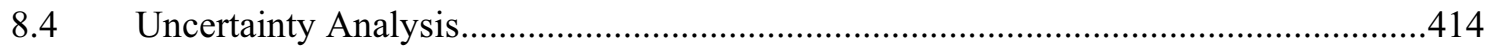

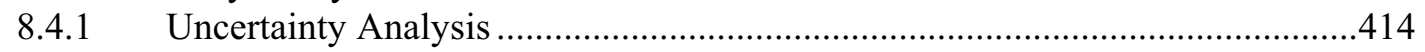

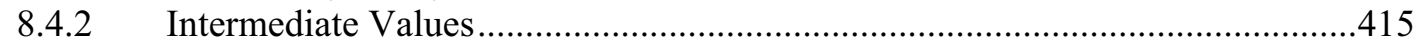

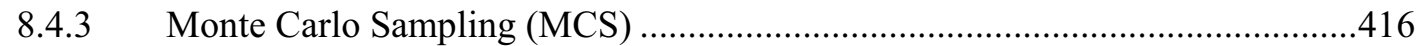

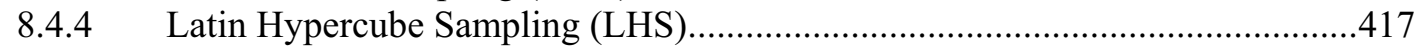

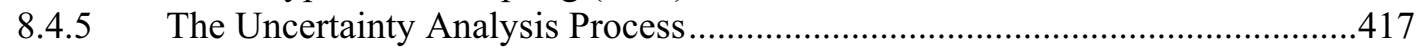

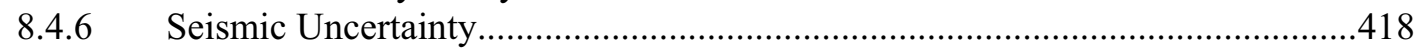

8.4.7 Uncertainty Distributions for Basic Events .....................................................419

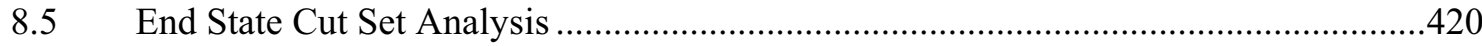

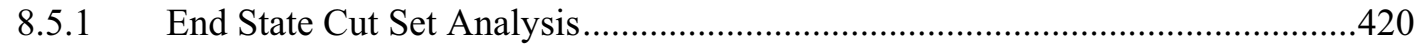

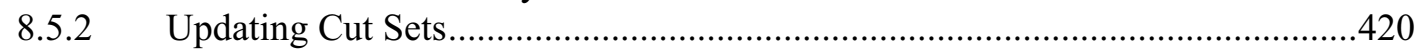

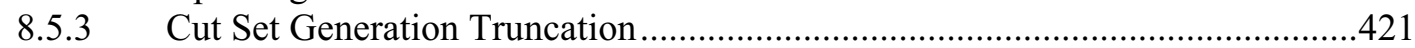

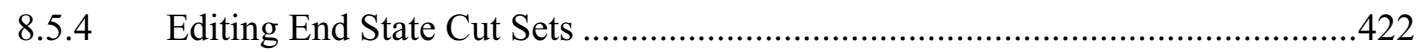

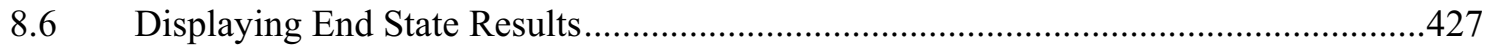

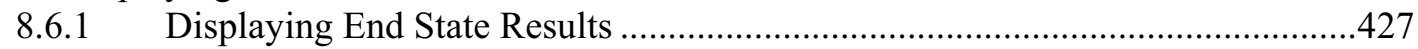

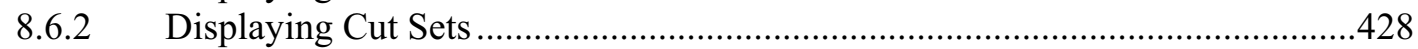

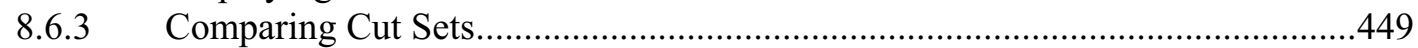

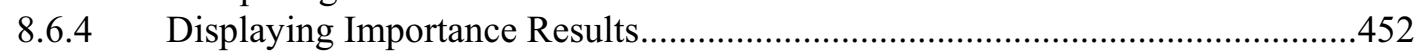

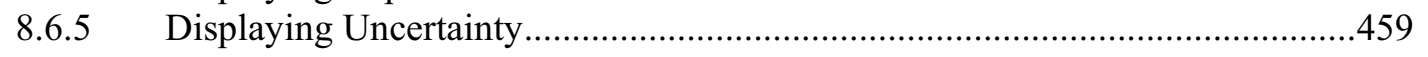

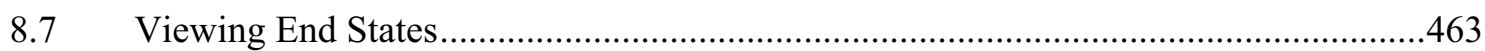

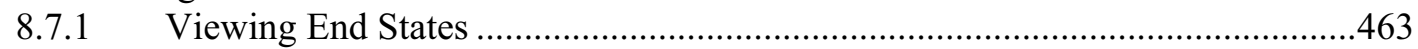

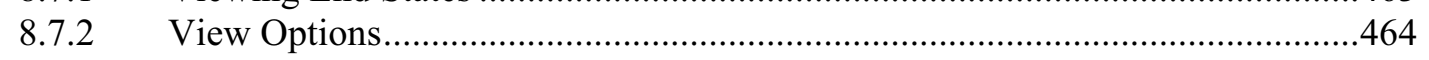

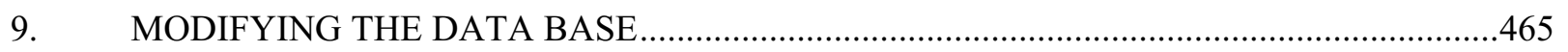

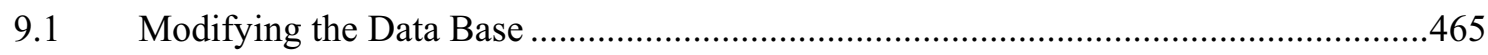

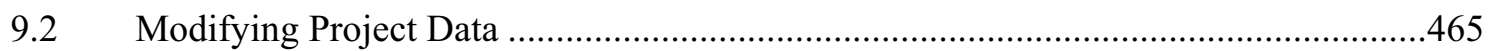




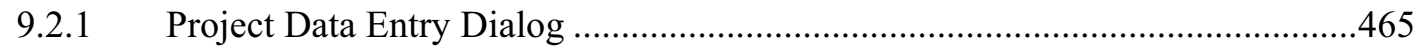

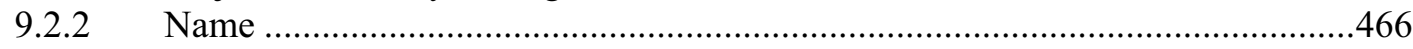

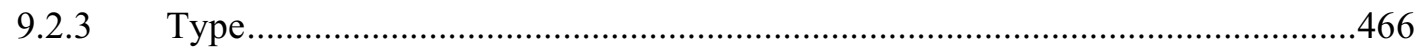

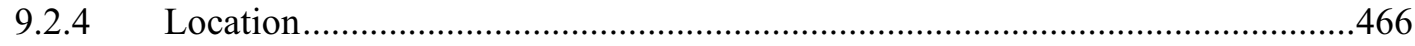

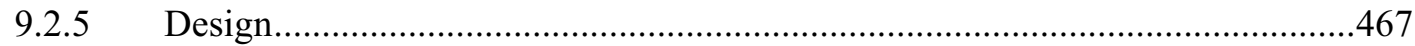

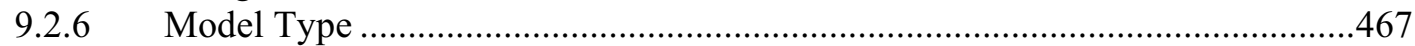

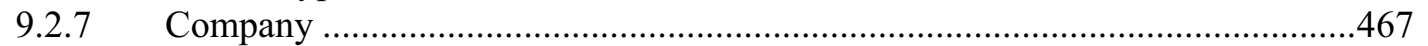

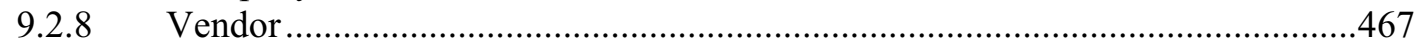

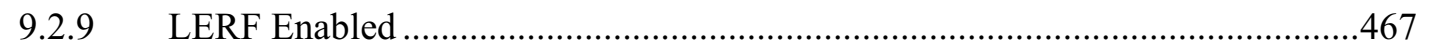

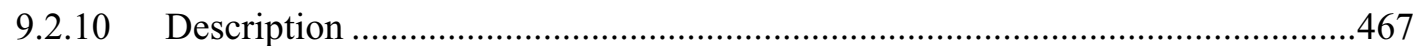

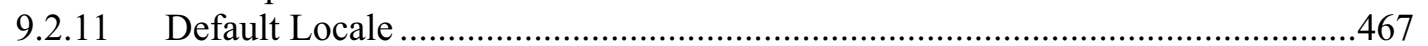

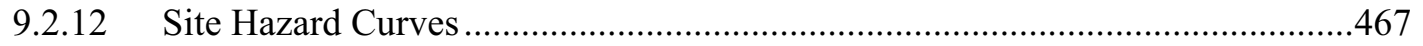

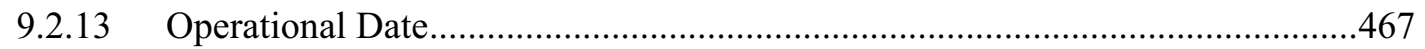

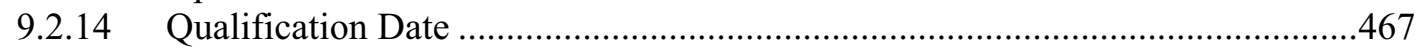

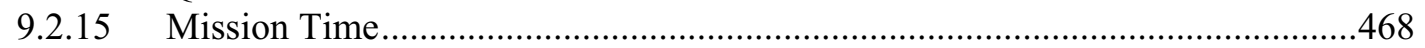

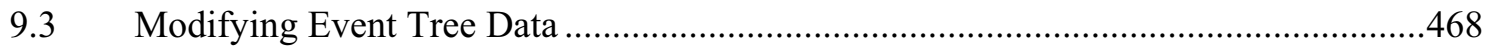

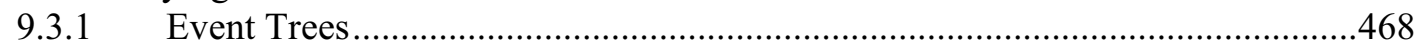

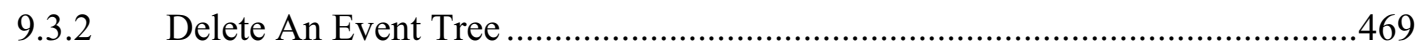

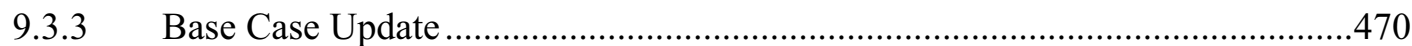

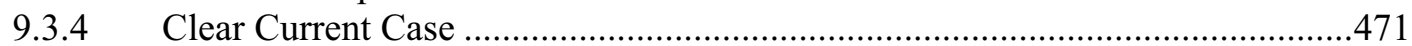

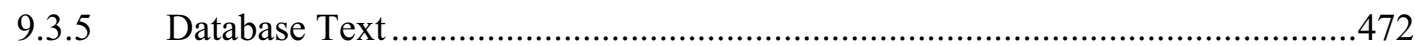

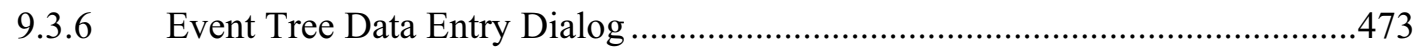

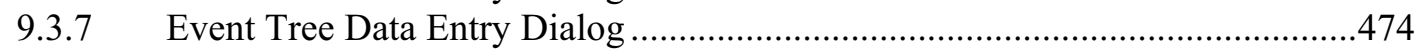

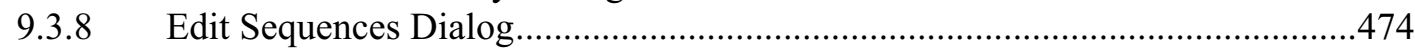

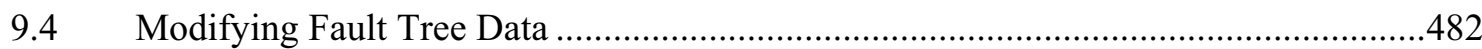

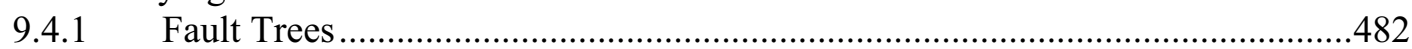

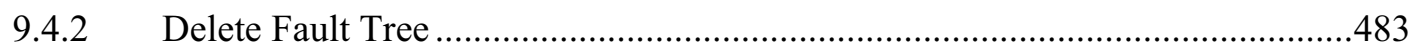

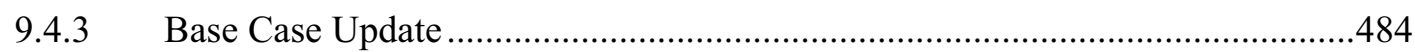

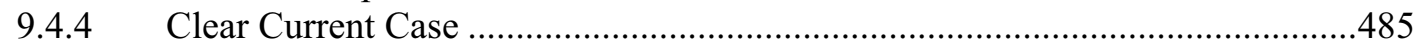

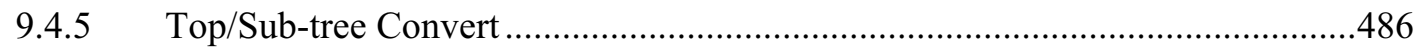

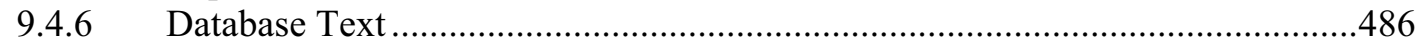

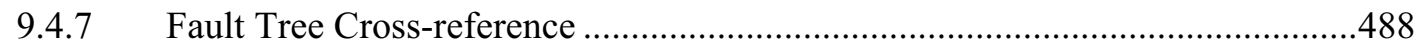

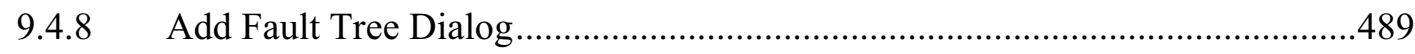

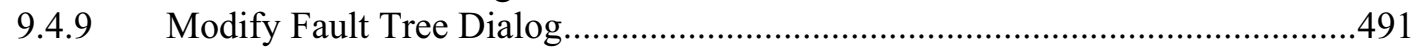

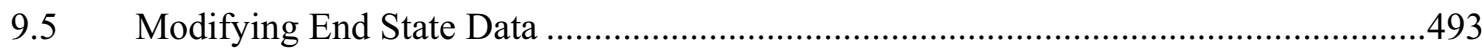

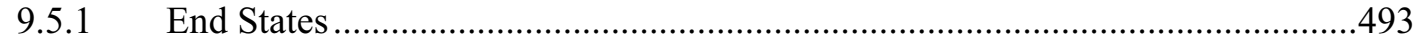

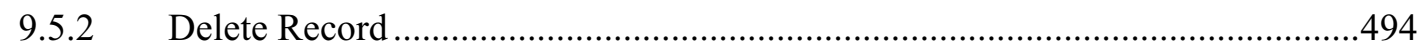

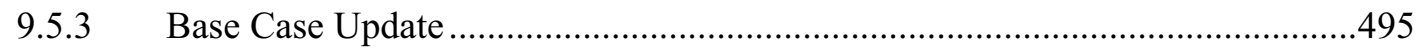

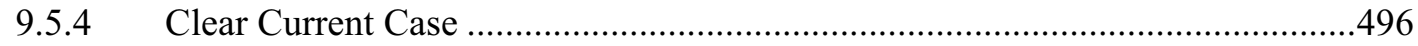

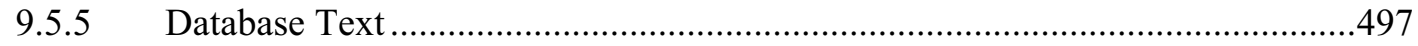

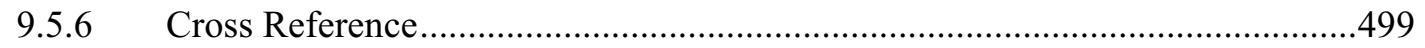

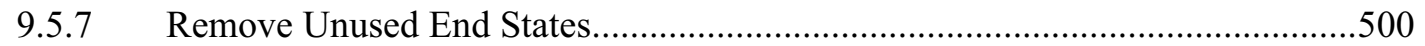

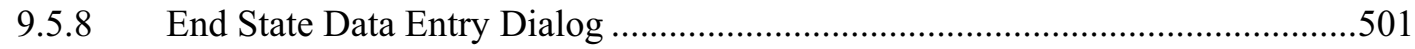

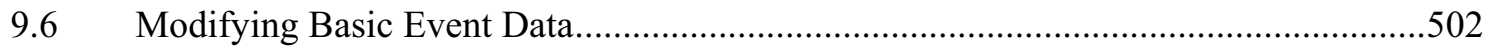

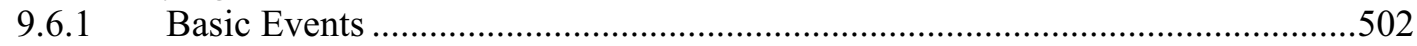

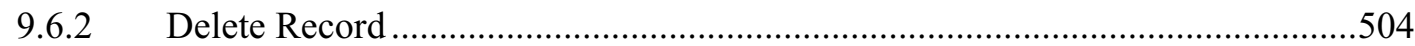

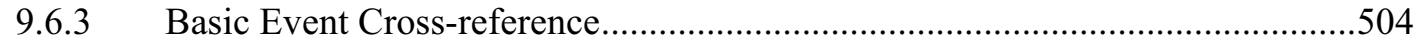




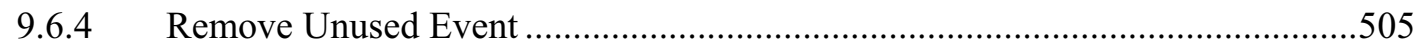

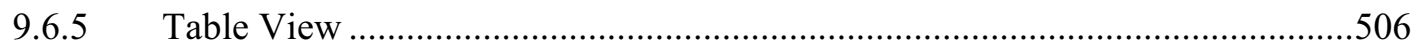

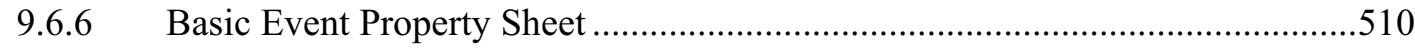

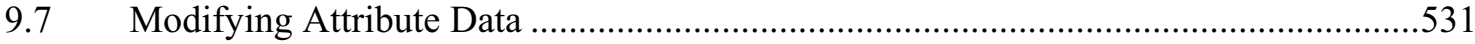

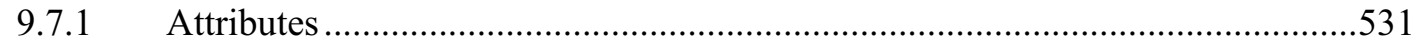

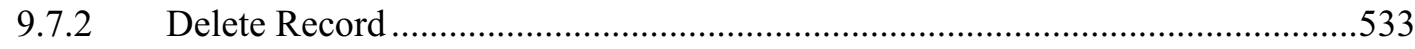

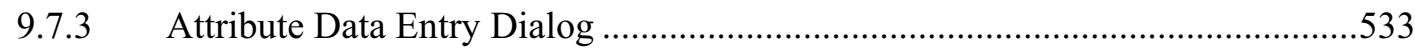

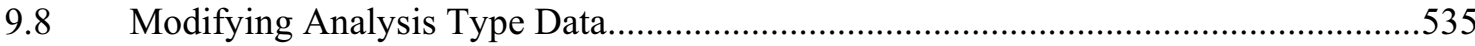

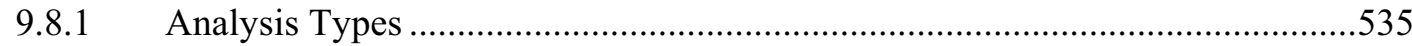

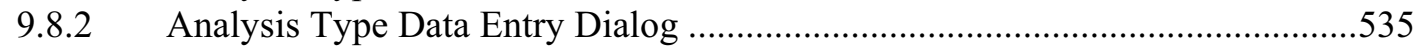

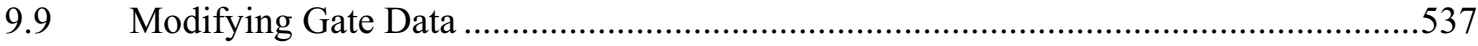

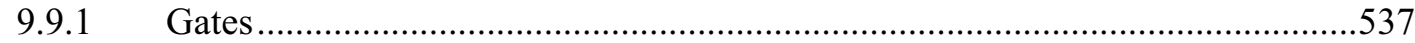

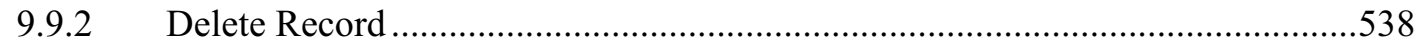

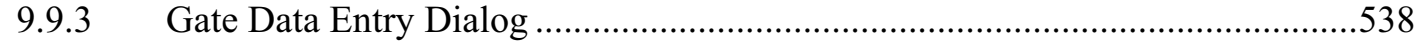

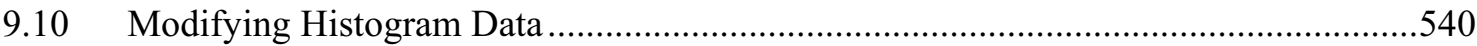

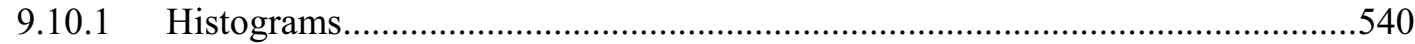

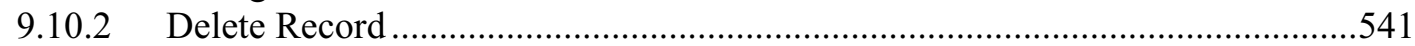

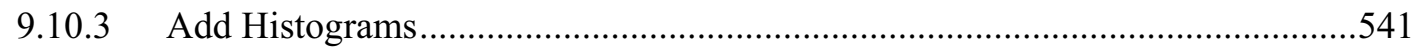

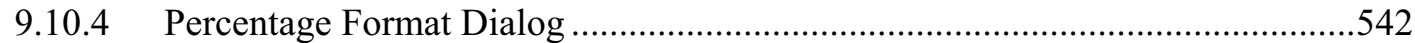

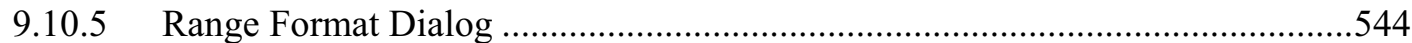

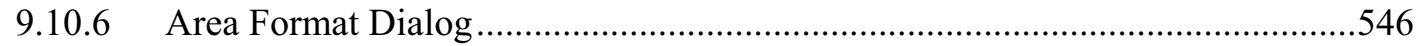

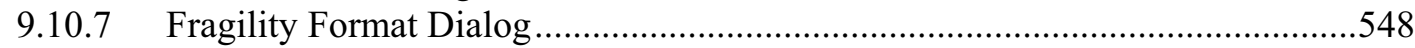

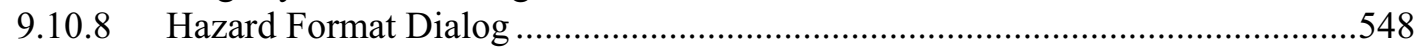

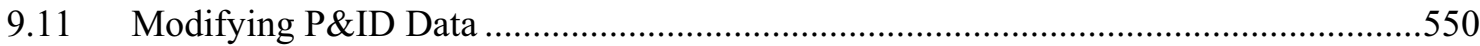

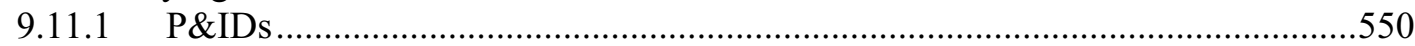

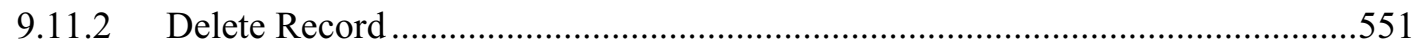

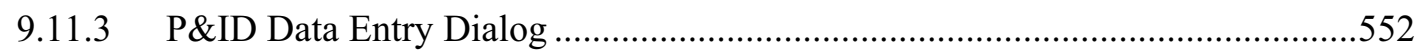

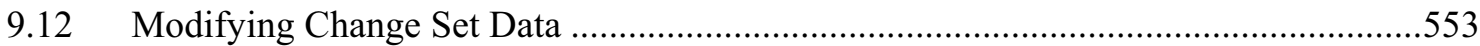

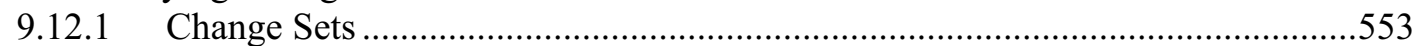

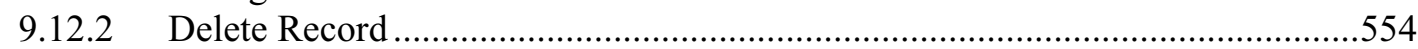

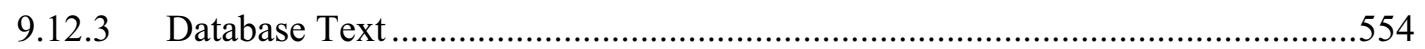

9.12.4 Change Set - Marking and Processing Order ....................................................556

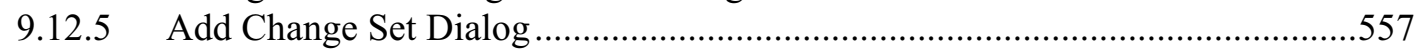

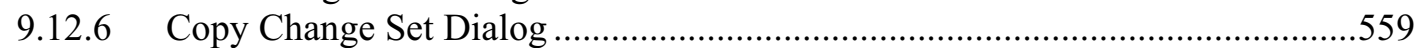

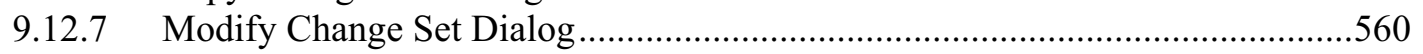

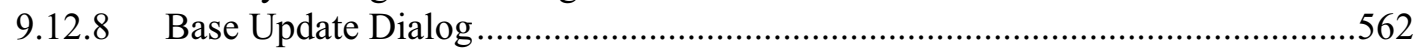

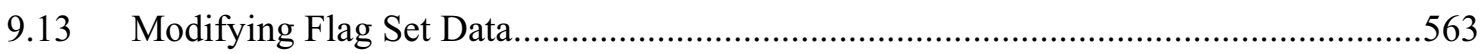

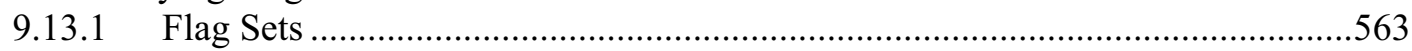

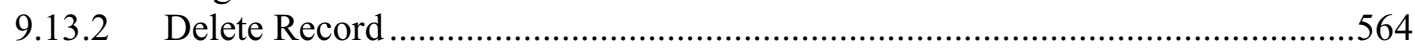

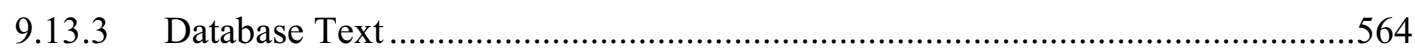

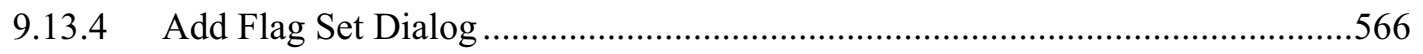

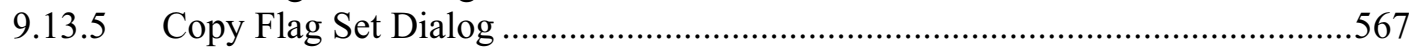

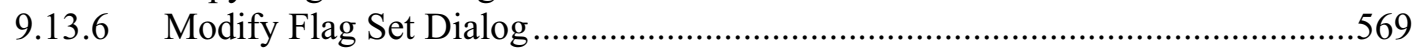

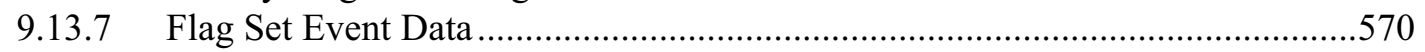




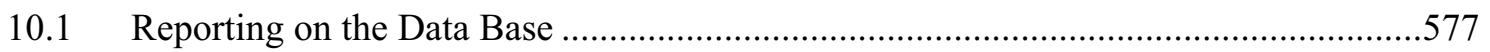

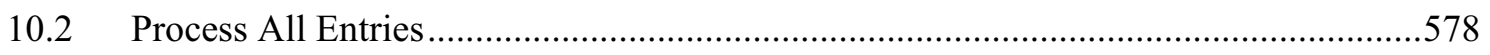

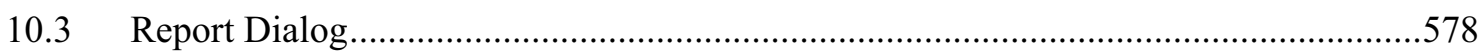

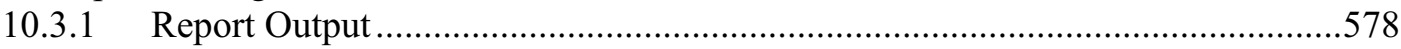

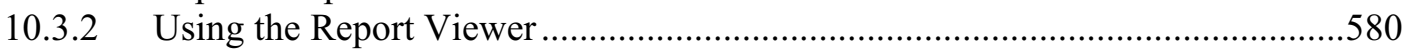

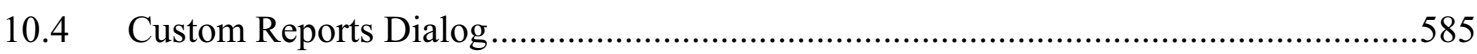

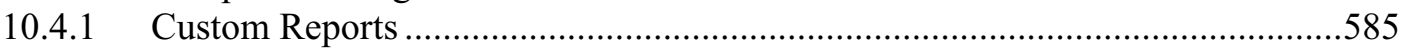

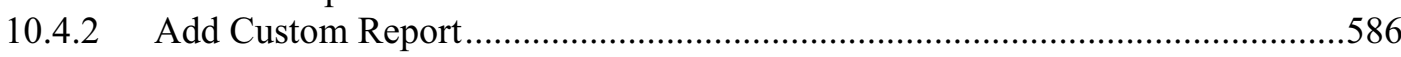

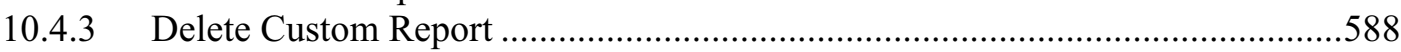

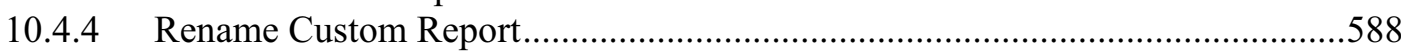

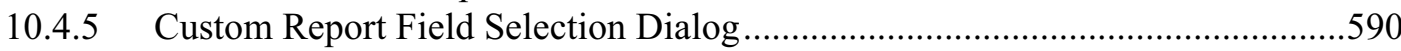

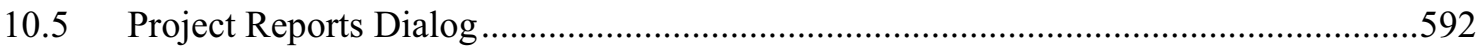

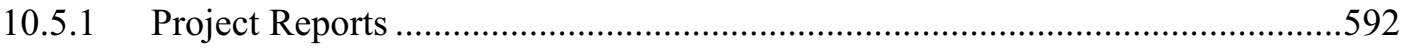

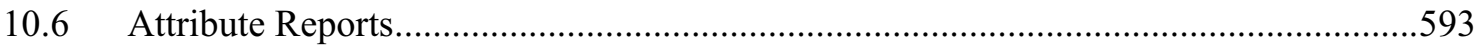

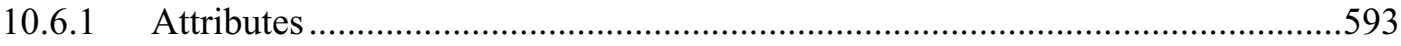

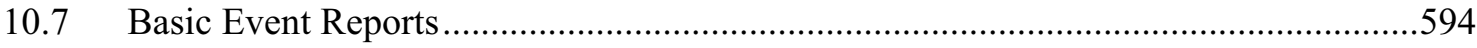

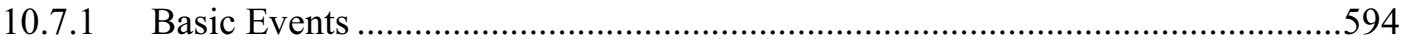

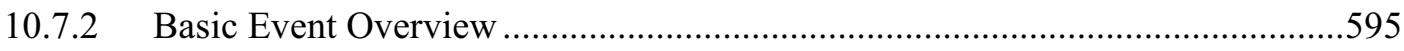

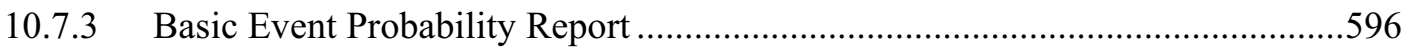

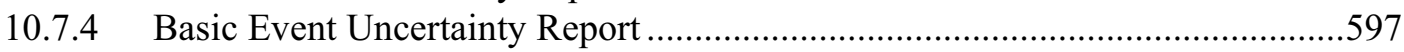

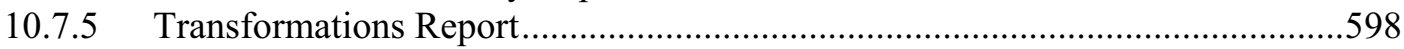

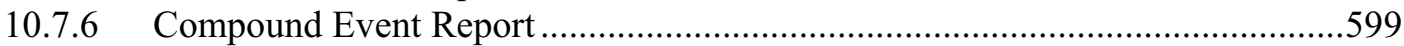

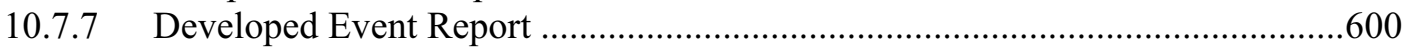

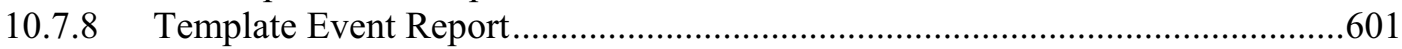

10.7.9 Basic Event Cross Reference Report................................................................602

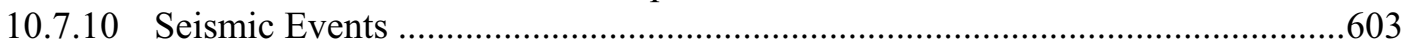

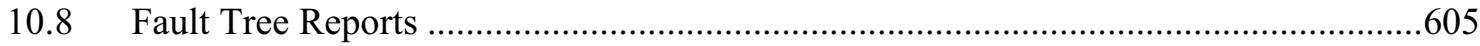

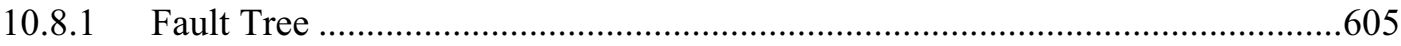

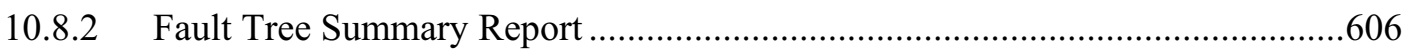

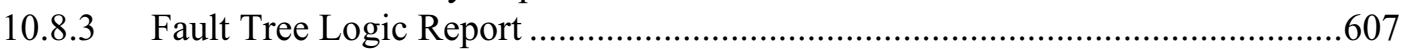

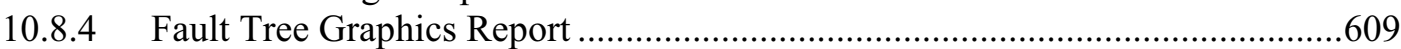

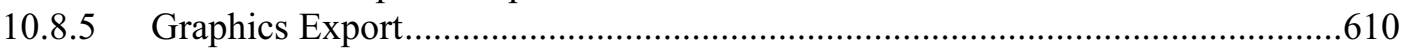

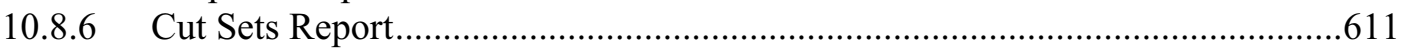

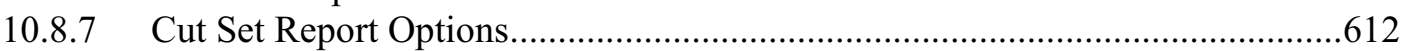

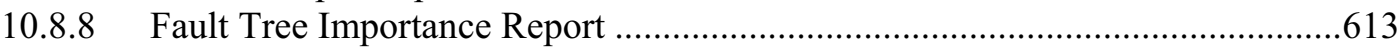

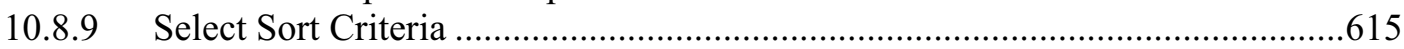

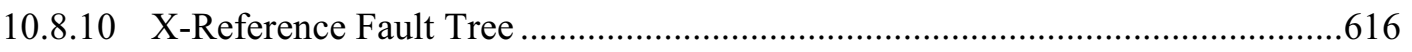

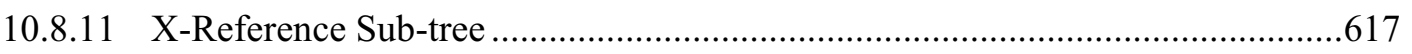

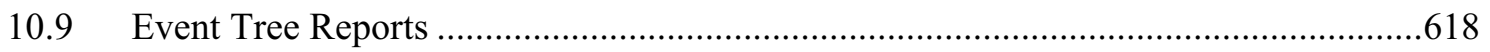

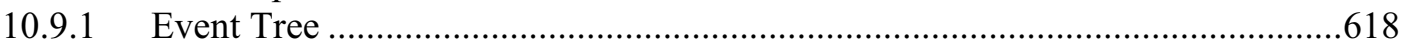

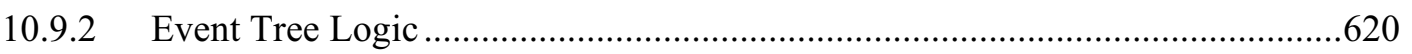

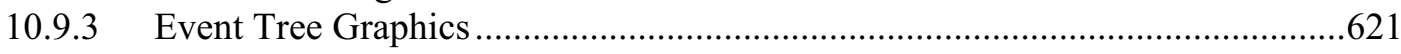




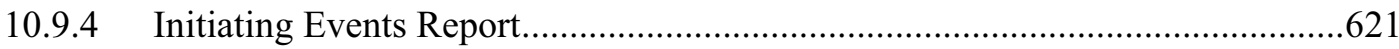

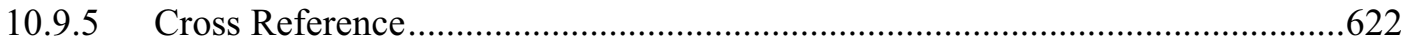

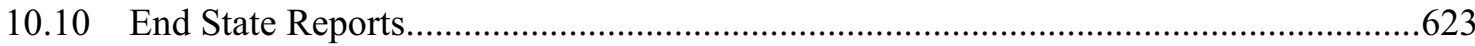

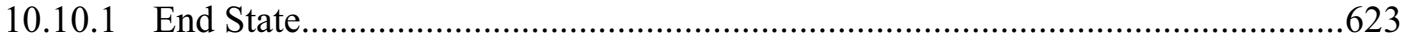

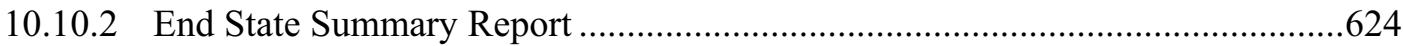

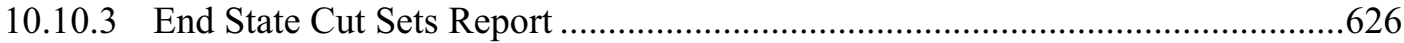

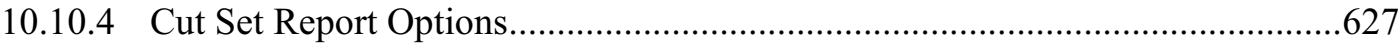

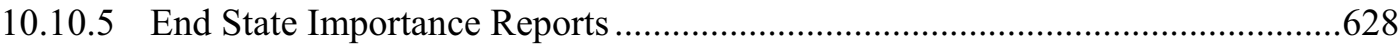

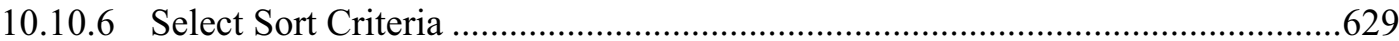

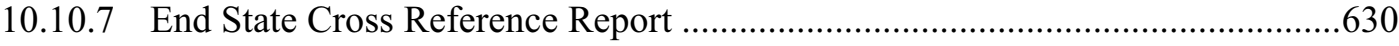

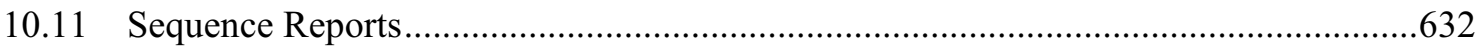

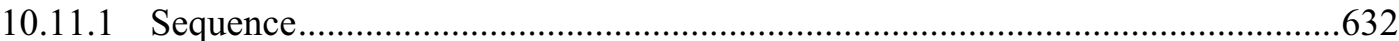

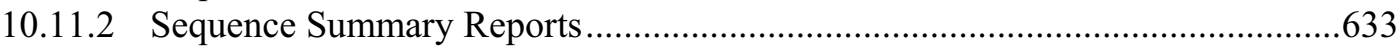

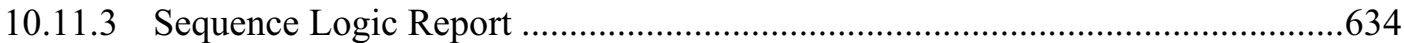

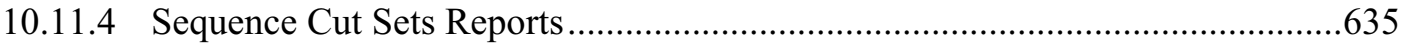

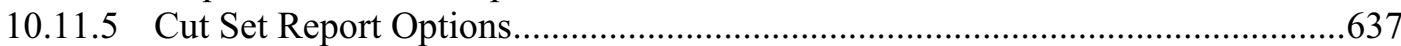

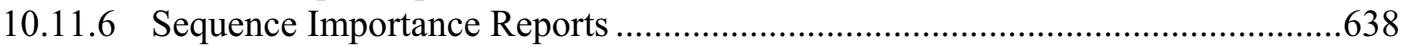

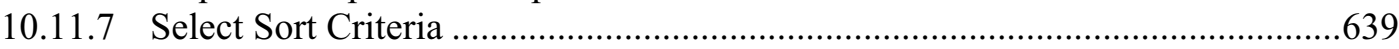

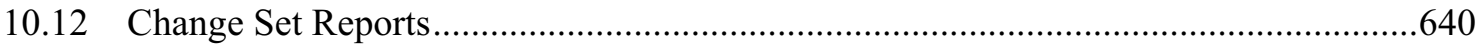

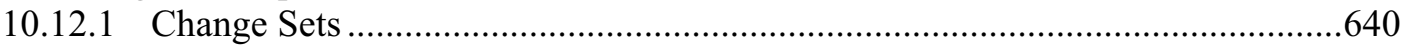

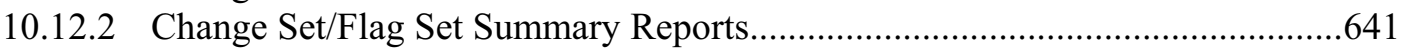

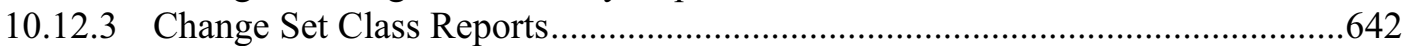

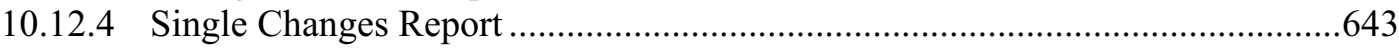

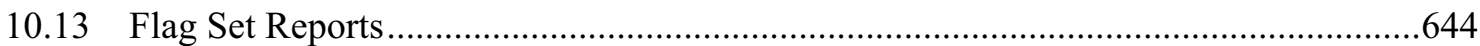

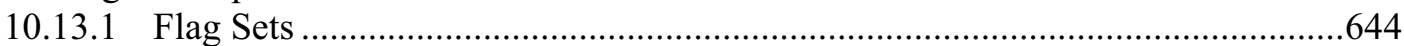

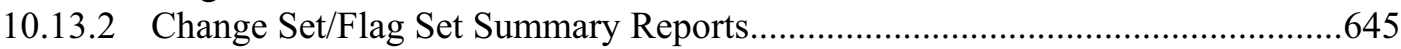

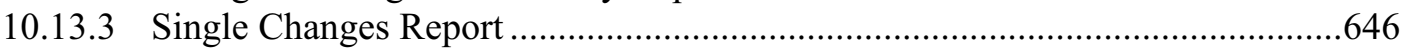

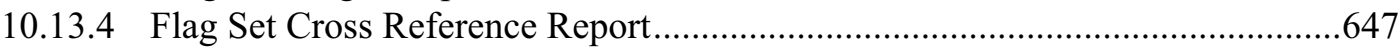

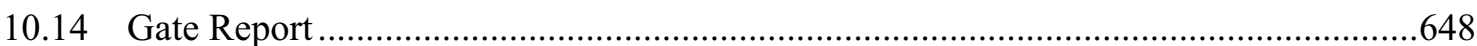

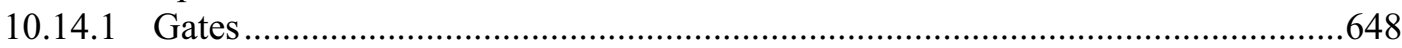

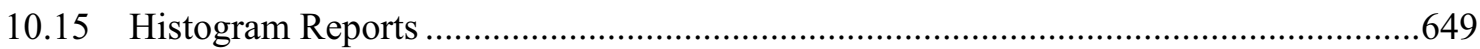

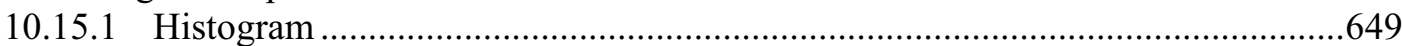

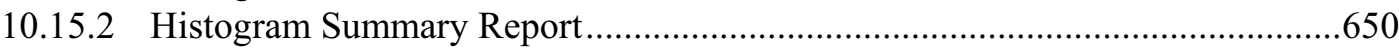

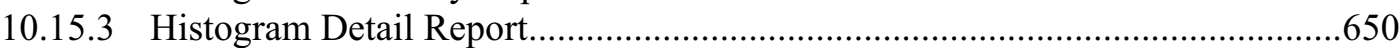

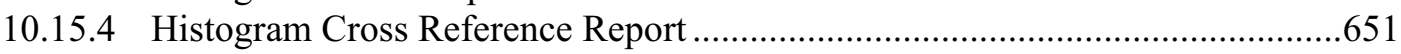

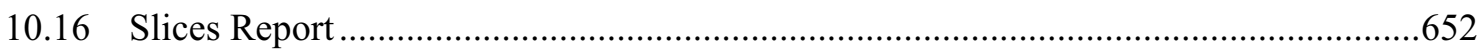

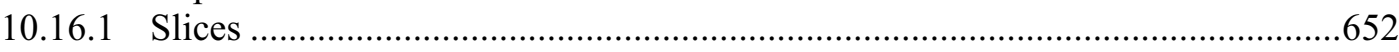

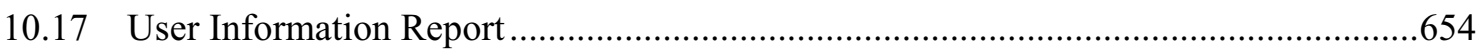

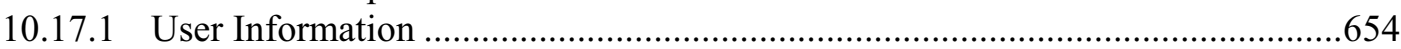


xviii 


\section{EXECUTIVE SUMMARY}

The U.S. Nuclear Regulatory Commission (NRC) has developed a powerful personal computer (PC) software application for performing probabilistic risk assessments (PRAs), called Systems Analysis Programs for Hands-on Integrated Reliability Evaluations (SAPHIRE). SAPHIRE capabilities for performing a PRA are summarized in this volume, and a more detailed description can be found in the individual sections of the documentation.

With the release of SAPHIRE versions 5 and 6, INL included a separate module called the Graphical Evaluation Module (GEM). GEM provides a highly specialized user interface with SAPHIRE, automating SAPHIRE process steps for evaluating operational events at commercial nuclear power plants. In particular, GEM implements many of the accident sequence precursor (ASP) program analysis methods. Using GEM, an analyst can estimate the risk associated with operational events very efficiently and expeditiously.

This SAPHIRE manual is designed to be an on-line reference guide to the program. Divided into two parts (A and B), this manual is a step-by-step approach to using the features of SAPHIRE. Introductions and installation instructions for the SAPHIRE are first presented, along with some information about the basic features of SAPHIRE. The next sections contain information about SAPHIRE data base concepts so that you can begin learning how SAPHIRE works. The sections following present a step-by-step approach to using the features of SAPHIRE. Later sections provide information about the capabilities and limitations of SAPHIRE, including some of the advanced features of SAPHIRE.

For readers who are familiar with previous versions of SAPHIRE, following are a list of some of the features that have been enhanced or are new to SAPHIRE version 7.

$\begin{array}{ll}\text { - } & \text { Improved Graphical Editing } \\ \text { - } & \text { Improved Fault Tree Logic Editing } \\ \text { - } & \text { Event Tree Linking Rules } \\ \text { - } & \text { Sequence Recovery Rules } \\ \text { - } & \text { Pault Tree Recovery Rules } \\ & \text { Partition Rules }\end{array}$

SAPHIRE automatically detects when the database schema for a project is different from the current version of the code. When SAPHIRE attempts to open a project created with an older version of the database schema, the Version Conflict dialog will appear. Once the data has been converted to version 7 , this data cannot be used with older versions of SAPHIRE (IRRAS, SARA, GEM, or FEP).

Users will find that SAPHIRE has evolved with advances in computers. Previously, it was run in the DOS operating system. It consisted of a suite of modules as described in the NUREG/CR-6116, "System Analysis Programs for Hands-on Integrated Reliability Evaluations (SAPHIRE) Version 5.0," series of volumes $1-8$. The current versions in use, 6 and 7, run under the Windows environment. Certain modules have been integrated in the SAPHIRE environment; however, the Graphical Evaluation Module (GEM) remains a separate interface with the SAPHIRE code. GEM is a user-friendly interface that streamlines and automates select SAPHIRE inputs and processes for performing events assessments.

SAPHIRE has also evolved with users' needs. New features and capabilities have been added in Versions 6 and 7 for developing and using larger, more complex models. For example, Version 7 can solve up to 2 
million sequences. In addition, enhancements have also been made in Version 7 for cut set slicing, event tree rule linkage rules, and reporting options.

To help assure the quality of new releases, SAPHIRE Versions 6 and 7 were used extensively with models created in earlier versions of SAPHIRE. The models were loaded into the current version of the software and results of the analyses were compared against SAPHIRE Version 5.0. Any discrepancies found were corrected in subsequent minor versions of the code. SAPHIRE has been validated in several ways. 


\section{ACRONYMS}

$\begin{array}{ll}\text { BPC } & \text { bound on the probability contribution } \\ \text { DOD } & \text { Department of Defense } \\ \text { DOE } & \text { Department of Energy } \\ \text { GEM } & \text { Graphical Evaluation Module } \\ \text { HEP } & \text { human error probability } \\ \text { HRA } & \text { human reliability analysis } \\ \text { INEEL } & \text { Idaho National Engineering and Environmental Laboratory } \\ \text { INL } & \text { Idaho National Laboratory } \\ \text { IRRAS } & \text { Integrated Reliability and Risk Analysis System } \\ \text { NRC } & \text { Nuclear Regulatory Commission } \\ \text { PC } & \text { personal computer } \\ \text { PRA } & \text { probabilistic risk analysis } \\ \text { PSF } & \text { performance shaping factor } \\ \text { RAW } & \text { risk achievement worth }\end{array}$

SAPHIRE Systems Analysis Programs for Hands-on Integrated Reliability Evaluations 


\section{Systems Analysis Programs for Hands-on Integrated Reliability Evaluations (SAPHIRE) Vol. 3 Code Reference Manual - Part A}

1. GETTING TO KNOW SAPHIRE

\subsection{Background}

The U.S. Nuclear Regulatory Commission (NRC) has developed a powerful personal computer (PC) software application for performing probabilistic risk assessments (PRAs), called Systems Analysis Programs for Hands-on Integrated Reliability Evaluations (SAPHIRE).

Using SAPHIRE on a PC, an analyst can perform a PRA for any complex system, facility, or process. Regarding nuclear power plants, SAPHIRE can be used to model a plant's response to initiating events, quantify associated core damage frequencies and identify important contributors to core damage (Level 1 PRA). It can also be used to evaluate containment failure and release models for severe accident conditions, given that core damage has occurred (Level 2 PRA). It can be used for a PRA assuming that the reactor is at full power, at low power, or at shutdown conditions. Furthermore, it can be used to analyze both internal and external initiating events, and it has special features for transforming models built for internal event analysis to models for external event analysis. It can also be used in a limited manner to quantify risk for release consequences to both the public and the environment (Level 3 PRA). For all of these models, SAPHIRE can evaluate the uncertainty inherent in the probabilistic models.

SAPHIRE development and maintenance has been undertaken by the Idaho National Laboratory (INL). The INL began development of a PRA software application on a PC in the mid 1980s when the enormous potential of PC applications started being recognized. The initial version, Integrated Risk and Reliability Analysis System (IRRAS), was released by the Idaho National Engineering Laboratory (now Idaho National Laboratory) in February 1987. IRRAS was an immediate success, because it clearly demonstrated the feasibility of performing reliability and risk assessments on a PC and because of its tremendous need (Russell 1987). Development of IRRAS continued over the following years. However, limitations to the state of the-art during those initial stages led to the development of several independent modules to complement IRRAS capabilities (Russell 1990; 1991; 1992; 1994). These modules were known as Models and Results Database (MAR-D), System Analysis and Risk Assessment (SARA), and Fault Tree, Event Tree, and Piping and Instrumentation Diagram (FEP).

IRRAS was developed primarily for performing a Level 1 PRA. It contained functions for creating event trees and fault trees, defining accident sequences and basic event failure data, solving system fault trees and accident sequence event trees, quantifying cut sets, performing sensitivity and uncertainty analyses, documenting the results, and generating reports.

MAR-D provided the means for loading and unloading PRA data from the IRRAS relational database. MAR-D used a simple ASCII data format. This format allowed interchange of data between PRAs performed with different types of software; data of PRAs performed by different codes could be converted into the data format appropriate for IRRAS, and vice-versa.

SARA provided the capability to access PRA data and results (descriptive facility information, failure data, event trees, fault trees, plant system model diagrams, and dominant accident sequences) stored in MAR-D. With SARA, a user could review and compare results of existing PRAs. It also provided the capability for performing limited sensitivity analyses. SARA was intended to provide easier access to PRA results to users that did not have the level of sophistication required to use IRRAS. 
FEP provided common access to the suite of graphical editors. The fault tree and event tree editors were accessible through FEP as well as through IRRAS, whereas the piping and instrumentation diagram (P\&ID) editor was only accessible through FEP. With these editors an analyst could construct from scratch as well as modify fault tree, event tree, and plant drawing graphical representations needed in a PRA.

Previous versions of SAPHIRE consisted of the suite of these modules. Taking advantage of the Windows 95 (or Windows NT) environment, all of these modules were integrated into SAPHIRE Version 6; more features were added; and the user interface was simplified.

With the release of SAPHIRE versions 5 and 6, INL included a separate module called the Graphical Evaluation Module (GEM). GEM provides a highly specialized user interface with SAPHIRE, automating SAPHIRE process steps for evaluating operational events at commercial nuclear power plants. In particular, GEM implements many of the accident sequence precursor (ASP) program analysis methods. Using GEM, an analyst can estimate the risk associated with operational events very efficiently and expeditiously.

\subsection{Overall Capabilities}

SAPHIRE capabilities for performing a PRA are summarized below. A more detailed description can be found in the individual sections of the documentation.

\section{Graphical fault tree construction}

Fault trees are constructed and modified with an interactive and flexible graphical editor; or with a fault tree logic editor similar to the hierarchical editor available in the Windows Explorer.

\section{Graphical event tree construction}

Event trees are constructed and modified with an interactive and extremely flexible graphical editor allowing the linkage of multiple event trees creating event trees of any size.

\section{Rule-based fault tree linking}

Accident sequences are generated using a linking module that uses a powerful set of user-defined rules to specify top event substitutions. These rules allow testing the current state of the solution process at each branch point.

\section{Fast cut set generation}

Extremely large fault trees and accident sequences are solved very quickly. SAPHIRE's solution engine is among the fastest in the industry.

\section{Conditional truncation}

Accident sequence cut sets can be generated using a "conditional truncation probability." 


\section{Fault tree flag sets}

"Flag sets" are included for pruning parts of fault trees to adjust their logic to different conditions. This saves generating multiple copies of a fault tree that are slightly different from one another.

\section{Failure data}

Failure data are handled through a failure data module that includes a "template." A template allows multiple basic events to refer back to a single "reference" basic event.

\section{Uncertainty analysis}

Both Monte Carlo and Latin Hypercube methods are available for performing uncertainty analysis. An event uncertainty "viewer" displays the cumulative distribution or probability density curve for individual basic events or groups of cut sets.

\section{Importance measures}

The traditional importance measures such as Fussell-Vesely, Risk Increase Ratio or Interval, Risk Reduction Ratio or Interval, and Birnbaum are available. However, because these measures are not suitable for all types of analyses, the NRC developed extended importance measures for passive components analysis which include cumulative Fussell-Vesely, Fussell-Vesely / Birnbaum, and group importance. These measures, including their uncertainty (via Monte Carlo methods) have been incorporated into SAPHIRE.

\section{Cut set editor and recovery analysis tools}

A rule-based editor is available for defining rules for modifying cut sets. These rules can be used to apply recovery on cut sets after an initial analysis has been performed. They can also be used to perform extensive changes to the cut set logic to incorporate new or different conditions.

\section{Versatile cut set slice and display}

A unique cut set "sort and slicer" is available to search and partition a large number of cut sets in fault trees, sequences, or end states.

\section{Cut set path tracing}

A "path tracer" is available to find the exact path through the logic that generated a particular cut set. As with other features contained within SAPHIRE, the speed of the "path tracer" is at the top of its class. This option will trace a cut set from an end state to its source in a fault tree.

\section{Cut set comparison}

A color-based cut set comparison utility is available for viewing changes between a "before and after" list of minimal cut sets.

\section{Cut set end state partitioning}

Cut sets can be partitioned into "end states" via a set of rules allowing each cut set to be assigned to a specific "end state." 


\section{End state analysis}

An "end state analysis module" provides the ability for gathering and analyzing accident sequence end states.

\section{Fire/Flood/Seismic analysis}

SAPHIRE provides a powerful set of tools for simplifying the process of performing external event analysis if an internal event PRA is available. Fault trees built for internal event analysis can be transformed into external event-specific fault trees automatically. Furthermore, locations can be clustered into zones, and location-specific event trees can be transformed into zone-specific fault trees.

\section{User-defined analysis types}

The user can define up to eight different analysis types for storing results. This is in addition to the eight fixed analysis types used by the software.

\section{Plug-in architecture}

A user-callable "plug-in" architecture allows interfacing to a customized library of probability calculation models. For example, included with SAPHIRE are plug-ins representing an alpha-factor common-cause, a multiple Greek letter common-cause, and a "supercomponent." Also, advanced plug-in modules are available to determine corrosion rates from flow-accelerated corrosion and its corresponding impact on the pipe failure probability.

\section{Report generation module}

SAPHIRE provides a powerful report generation module. The user can create extensive reports containing any type of information he/she wishes to include. Output capability includes WordPerfect, Word, Excel, and text formats. Reports can be automatically generated to fit the NRC's SPAR model report format.

\section{Utility functions}

SAPHIRE provides utility functions such as recovering a corrupted database, converting fault tree logic from alpha to graphics, and changing user defined constants.

\section{Graphical export to Windows metafiles}

With SAPHIRE a user can export the graphical fault trees and event trees to a Windows metafile. This allows importing the graphics into other Windows applications.

\section{International language support}

Enhancements to SAPHIRE allow for international text characters. For example, fault tree names can have a mixture of English and Russian. Consequently, logic gates or basic events with a name such as “Жзжібоњ TREE” are permissible. 


\section{Dual language support}

SAPHIRE has the capability to store two names, descriptions, and text fields for each entity in a project. The user can switch the display between the primary and alternate information easily. This allows a project to contain English and another language for each entity.

\section{On-line context sensitive help}

SAPHIRE contains a help manual with over seven hundred pages of useful information.

NOTE: This document assumes the reader is familiar with PRA methods and terminology. This manual serves as an overview for the SAPHIRE software. An on-line reference manual is available with installation of the software. The on-line manual does not instruct the reader in PRA methodology, but merely serves as a reference guide for the SAPHIRE software.

Information on recent changes and enhancements may be found on the SAPHIRE web site at saphire.inl.gov.

\subsection{New Features Found in Version 7}

For readers who are familiar with previous versions of SAPHIRE, following are a list of some of the features that have been enhanced or are new to SAPHIRE version 7.

Improved Graphical Editing

- Zoom 100\% - View and print fault trees at a uniform size.

- Undo - Roll back one or more changes to your fault tree or event tree diagram.

- Jump between event tree and fault tree diagrams - Now you can jump directly to the fault tree diagram that corresponds to a top in an event tree diagram.

- Fault tree graphics set up - Setting up a new fault tree diagram is now easier.

- Individual shape attributes - Now gates and events can be quickly be assigned unique color and font attributes, for easy visual differentiation.

Improved Fault Tree Logic Editing

- Edit and Add Fault trees, Gates, and Events - Add and edit items directly from the logic editor. Easier project creation - Setting up a new project space is now simpler.

Dual Language Support

- Assign events, fault trees, and other objects primary and alternate names and descriptions. Then, toggle the user interface to display either the primary or the alternate names. 
Event Table Viewer - Select only the event records and fields of interest, and view them at a glance, in the order of your choice.

Improved Reports

- The formatting of the reports has been updated. Output is now available in RTF, HTML, and ASCII. Additional reports are now available from the Reports menu, including change sets, flag sets, and various rule reports.

Improved Custom Reports - More complete information, including calculation summary results, is now available from this option.

Easier cross-reference reports - From the Modify option, see all references to an item in a single report.

- Fault Trees

- Basic Events

- End States

Slice Rules - Use a rule based approach to locating combinations of events in cut sets.

Advanced Rules - Continue to use SAPHIRE's original basic rules, or use a full scale programming language to develop linkage, recovery, and partition rules.

- $\quad$ Event Tree Linking Rules

- Sequence Recovery Rules

- Fault Tree Recovery Rules

- Partition Rules

Sensitivity Wizard - A convenient new interface for performing detailed sensitivity analysis.

Importance Measures Wizard - A convenient new interface for performing importance analysis, with added component grouping abilities.

Improved Load/Export capabilities - Load and/or extract groups of files at one time, to or from the folder of your choice.

- Extract All - Extract an entire project with one action.

- Load All - Load an entire project from any folder with one action.

- Extract All File Type - Extract a group of files that completely describe a particular type of object, with one action. 
- Load All/Group - Load a group of files from any folder with one action.

- Fault Tree Logic \& Related Information - Load the event and gate information directly referenced by a fault tree's logic. Maintain the paging information, too.

- Select a destination folder - Browse for file and/or folder names when you select an output destination.

Larger capacity - Now projects can contain up to 2,000,000 sequences.

Macro Script capabilities- Macro-scripts are written with keywords in a text editor and can be ran within SAPHIRE to automatically perform tedious and repetitious analysis options.

\subsection{About This Manual}

\subsubsection{Document Organization}

This manual is divided into two parts (A and B) and eight appendices. The first two sections provide an introduction to SAPHIRE, installation instructions and some information about the basic features of SAPHIRE. The third section contains information about SAPHIRE data base concepts so that you can begin learning how SAPHIRE works. The following sections present a step-by-step approach to using the features of SAPHIRE. Later sections provides information about the capabilities and limitations of SAPHIRE. The appendices cover some of the advanced features of SAPHIRE.

This manual is designed to be an on-line reference guide. Topics are generally limited to one full screen of information with links to related or more detailed information. At times, you may be able to click on a graphic figure for specific information about the area displayed. Unlike a traditional printed reference manual, you may "hop" about from link to link, therefore, there are no chapter or section numbers provided.

\subsubsection{Documentation Conventions}

Conventions used in this manual have been established to help you learn to use SAPHIRE quickly and easily.

- Dialog box names begin with uppercase letters and are italicized. For example, the Fault Trees List dialog box.

- $\quad$ Field names begin with uppercase letters and are italicized. For example, the Fault Tree Mask field.

- $\quad$ Key combinations joined with a plus sign indicate that you should press and hold the first key while you press the second key. Key combinations are printed in bold type. For example, $\mathbf{C t r l}+\mathbf{X}$.

- $\quad$ Menu names, sub-menu options, and button names are printed in bold type. When discussing a particular sequence of menu options, the nomenclature:

\section{Menu | Sub-menu Option | Button}

will be used to indicate the SAPHIRE menu options and any successive sub-menu options and/or buttons. For example, Modify | Project | Text refers to selecting the Modify option from the menu and then the Project sub-menu option, followed by the Text button. The menu option may be followed by a menu option found in a pop-up menu, such as, Fault Tree | View. This indicates 
that you should choose Fault Tree from the menu, select the desired fault tree(s), then right-click to invoke the pop-up menu and choose the View option.

- Topics will generally be arranged in the following format:

\section{Topic Title}

\section{PURPOSE}

This section will contain one to two paragraphs describing the primary functions(s) of this option.

\section{STEPS}

This section will list the actions needed to perform this function, often beginning with the menu bar selection.

A graphic image of the dialog box or menu options may be included.

由

Occasionally, an instruction to "Click on the graphic above for specific information" will appear here.

Field Name - This will contain one or two sentences briefly describing the purpose of the field andior data entry requirements. If more information is required, a link to another topic will be provided.

Button Name - This will contain one or two sentences briefly describing the action(s) performed as a result of choosing this button. If more information is required or another dialog box is launched, a link to another topic will be provided.

\section{SEE ALSO}

Other related topics (This will provide a direct link to related topics or more detailed information.

Links in topic text and on graphic images (also known as hot-spots) are indicated when the cursor changes to $\mathrm{fth}_{\mathrm{b}}$. Additionally, hot-spot text is displayed in green.

- $\quad$ Mouse manipulation instructions are for a standard two-button mouse as follows:

Click Right-click Double-click -
The left mouse button is pressed and released. The right mouse button is pressed and released. The left mouse button is pressed and released two times in rapid succession. 
Drag - $\quad$ The left mouse button is depressed while moving the mouse to a different screen position, then the mouse button is released.

\subsection{Installation}

\subsubsection{Software and Hardware Requirements}

- Windows 98 or greater

- $\quad$ Pentium class IBM-PC compatible with 2-button mouse

- $\quad 50 \mathrm{MB}$ free disk space (minimum for installation)

NOTE: The total disk space requirement is dependent on the number of projects you create and how large the projects are.

\subsubsection{Installation Instructions}

SAPHIRE can be installed from a variety of sources.

To install from SAPHIRE Web Site:

For SAPHIRE Users Group members, SAPHIRE can be installed from the Internet site, http://saphire.inl.gov. A password is required for access to this site.

1. Access the SAPHIRE web site at: http://saphire.inel.gov. Follow the instructions given on the web site to obtain the downloadable SAPHIRE program.

2. Once the executable is downloaded. Double click on the file or open the file from Windows Start Bar and click on Run.

3. The Setup program prompts you through the installation process. Follow the instructions on the screen.

To install from compact disk (CD):

1. Insert the $\mathrm{CD}$ in the disc drive.

2. From the Windows Start Bar, click Run.

3. Type d:ISETUP (replace $\mathrm{d}$ with the drive letter for your CD drive).

4. Choose OK.

5. The Setup program prompts you through the installation process. Follow the instructions on the screen.

\subsection{Using SAPHIRE}

\subsubsection{Starting SAPHIRE}

\section{PURPOSE}

Begin execution of the SAPHIRE code. 


\section{STEPS}

1. From the Windows Start Bar, click Programs.

2. Select SAPHIRE for Windows.

$$
\text { OR }
$$

3. From the Windows NT 3.51. Program manager or Windows NT 4.0/Windows 95 desktop, double click the SAPHIRE program icon.

4. Proceed by selecting a project with which to work.

\subsubsection{Exiting SAPHIRE}

\section{PURPOSE}

End this session of the SAPHIRE program.

\section{STEPS}

1. Select File from the menu.

2. Select the Exit sub-menu option. The SAPHIRE database will be closed and the program will be terminated.

\subsubsection{Using SAPHIRE for the First Time}

\section{PURPOSE}

When SAPHIRE for Windows is launched for the first time after installation, the constants dialog will be displayed. This dialog allows you to provide default values desired for various functions within SAPHIRE. Most of the fields have default values provided for you. This dialog will not appear on subsequent entries into SAPHIRE.

\section{STEPS}

1. The first time you run SAPHIRE for Windows after installation, the Define Constants dialog will appear.

2. Fill in and change the information as appropriate. 


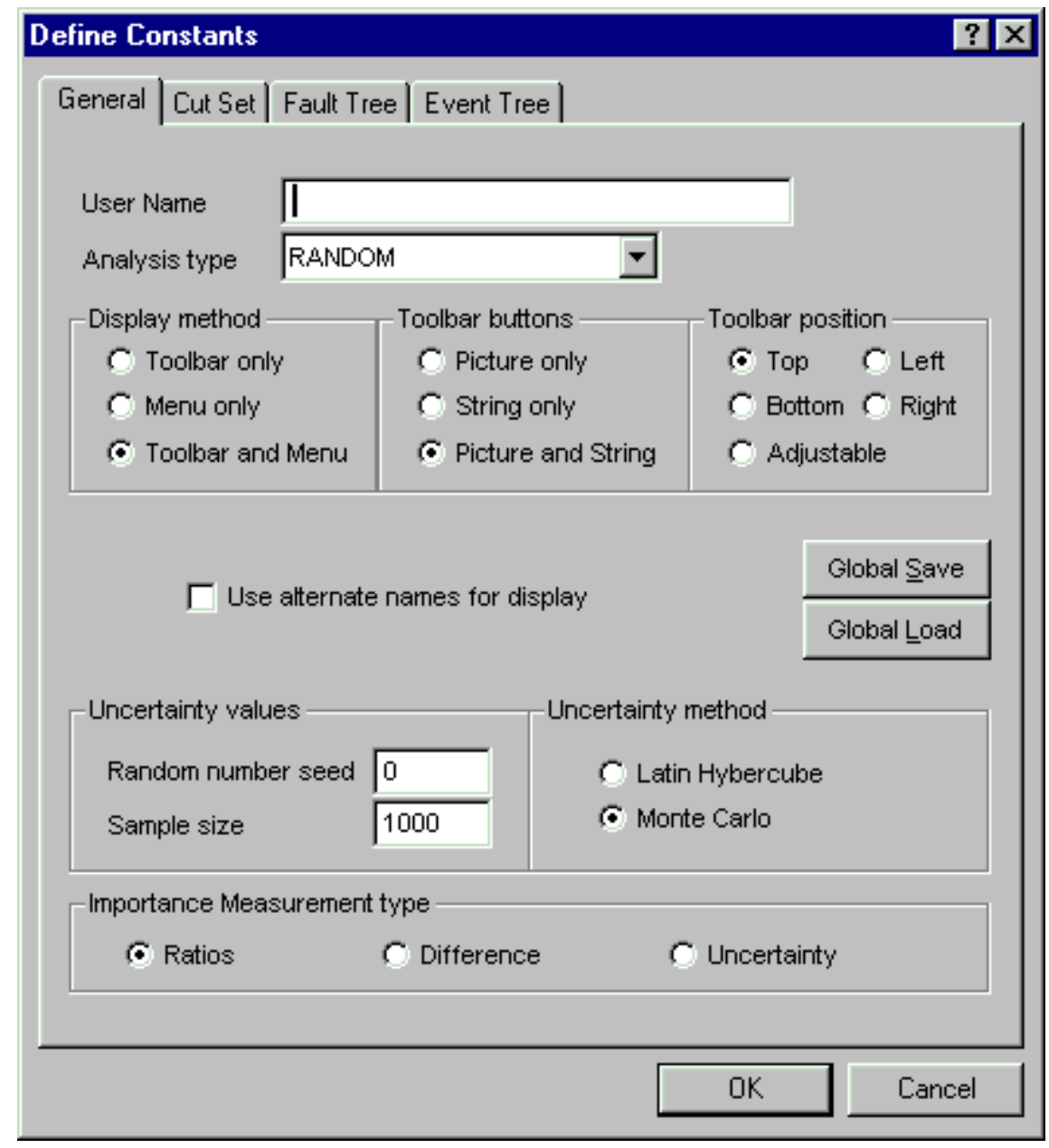

3. When you have finished, choose the OK button.

4. The SAPHIRE window will appear and you are now ready to proceed.

If you wish to change defaults, this dialog can be called up from the Utility | Define Constants option. To find out the definition for the various options and buttons, click on the question mark in the upper right corner of the dialog and then select the option in question.

\subsubsection{Converting from Previous SAPHIRE (IRRAS) Versions}

This section describes the procedure if you have been running a previous version of SAPHIRE (IRRAS, SARA, GEM, etc.).

SAPHIRE automatically detects when the database schema for a project is different from the current version of the code. When SAPHIRE attempts to open a project created with an older version of the database schema, the Version Conflict dialog will appear.

NOTE: Once the data has been converted to version 7 , this data cannot be used with older versions of SAPHIRE (IRRAS, SARA, GEM, or FEP). 


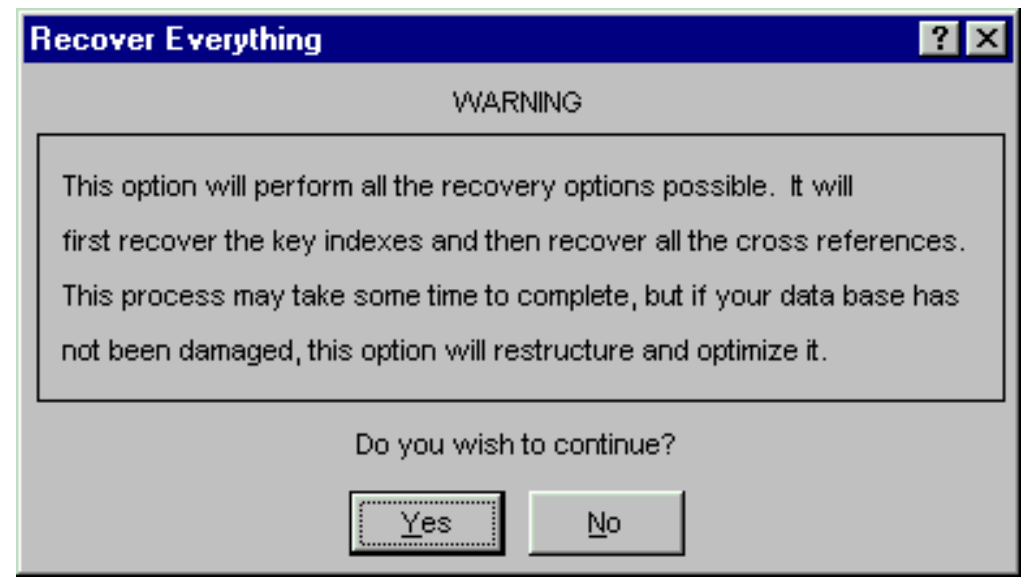

Yes - $\quad$ Convert the data for the current project to the current SAPHIRE version format. After conversion is complete, the SAPHIRE window will appear and you are now ready to proceed.

No - $\quad$ Do not convert the project data to the current SAPHIRE version format. The project will not be opened.

\subsection{Getting Around Inside SAPHIRE}

\subsubsection{About List Boxes in SAPHIRE}

Many dialogs in SAPHIRE contain list boxes. In some list boxes, multiple items can be selected for processing. The following discussion provides some hints on selecting list box items.

An item in a list box is "selected" if it is highlighted, that is, displayed in a color other than the other items in the list. The illustrations in this manual show selected list box items highlighted with a dark blue background and white foreground characters while the deselected items are displayed with a white background and black foreground characters. In the example here, the fault tree ECS is "selected." 


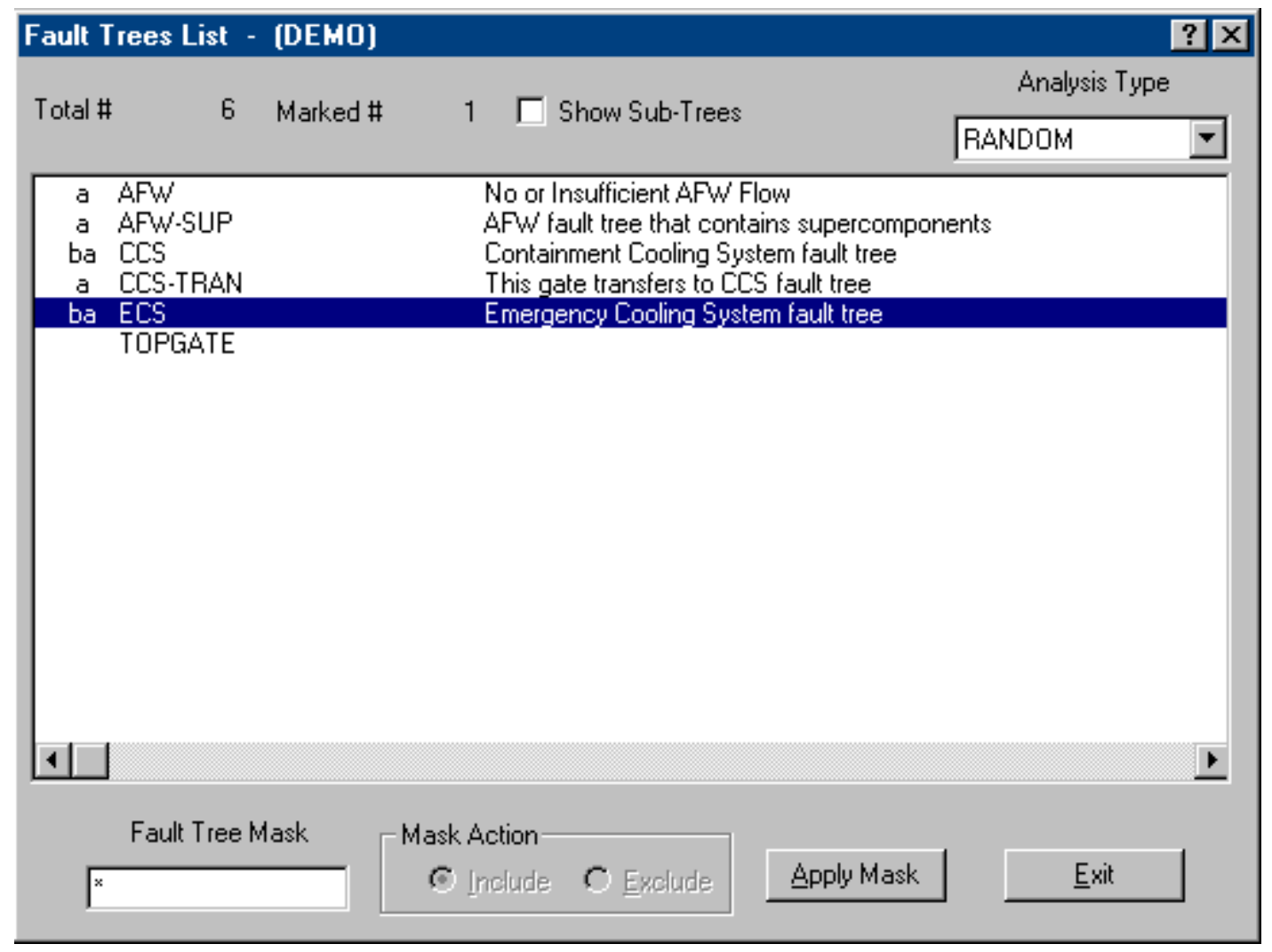

There are various ways to select items in a list:

- To select a single item, click on the desired item so that it is highlighted.

- To select multiple contiguous items, click on the first desired item in the list and drag up or down the list to the last desired item. Alternately, click the first desired item, then holding down the Shift key, click the last desired item.

- To select multiple non-contiguous items in the list, click several desired items while holding down the Ctrl (control) key.

- $\quad$ To select all items in the list, highlight the first item and press the Shift and End keys. Alternatively, pressing the $\mathbf{C t r l}$ and $\mathbf{A}$ keys selects all items.

\subsubsection{Selecting List Items Using the Mask Feature}

\section{PURPOSE}

Some dialogs in SAPHIRE with list boxes provide a "Mask" capability which allows you to select items from the list based on matched criteria. The example here shows the Fault Tree Mask entry field with the Include radio button selected.

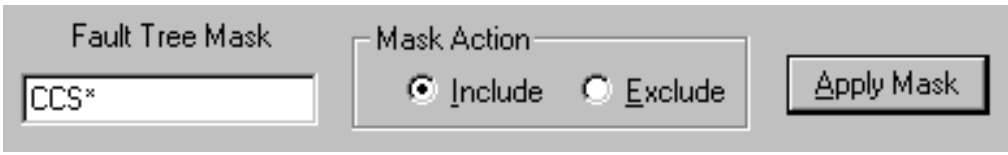


Generally, the mask is applied to the name of the item (e.g., Fault tree name or Event Tree name). The wildcard characters asterisk (*) and question mark (?) can be used in the mask. The asterisk represents one or more characters that a group has in common. The question mark represents a single character in that position of the string that a group has in common.

To use the mask capability, a dialog with a mask entry field must be active.

\section{STEPS}

1. With an appropriate dialog active, in the mask entry field type the common characters of the names you wish to match including any wildcard characters, if desired.

2. In the Mask Action section, choose either the Include or Exclude radio button, depending on whether you want these items included in the selection or excluded from it.

3. Choose the Apply Mask button. All list items with names matching your mask will be selected or deselected.

You are now ready to perform any of the available functions on the active dialog.

\subsubsection{Invoking Pop-up Menus}

Many dialogs in SAPHIRE have pop-up menus available. Once the dialog has been called up, invoke the pop-up menu by right-clicking. While holding down the right-hand side mouse button, select the desired menu option by highlighting it and then releasing the mouse button. Pop-up menus from any one dialog may vary, especially if the dialog contains a multiple-select list box. The options in a pop-up menu may be dependent upon whether or not, or how many, items are selected.

The examples below show the Fault Trees List dialog pop-up menus with one list item selected, more than one list item selected, and no list items selected:

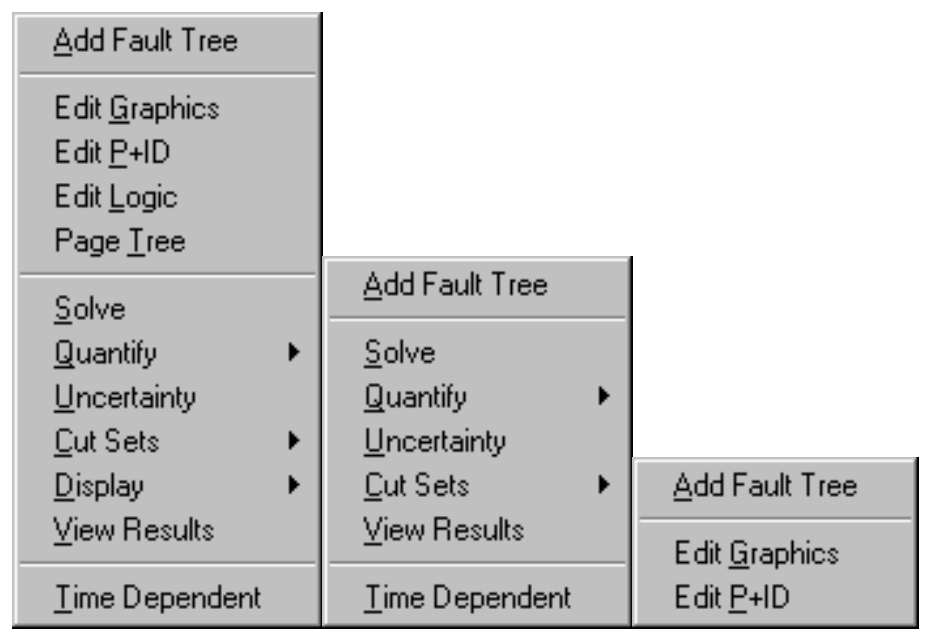

\subsubsection{Selecting the Analysis Type}

Many dialogs in SAPHIRE have an Analysis Type drop-down list. This drop-down list allows you to select from eight pre-defined and eight user-defined analysis types.

Select an analysis type by clicking on the down arrow on the right-hand side of the list and scrolling through the list until the desired analysis type is displayed. Then click on the desired analysis type. In the 
example here, Random is the current analysis type. The list has been scrolled about halfway down and the User 1 analysis type is currently highlighted.

\begin{tabular}{|l|}
\multicolumn{2}{c|}{ Analysis Type } \\
\hline RANDOM \\
\hline ASP_INITEVENT \\
\hline ASP_CONDITION \\
RESERVED \\
RESERVED4 \\
USERI \\
\hline USER2 \\
USER3 \\
\hline
\end{tabular}

By selecting one of the analysis types from the drop-down list, you indicate the type of analysis to be performed on the selected fault tree(s), sequence(s) or end state(s). See the section, Analysis Types, for more information.

\subsubsection{SAPHIRE Help}

Included with the SAPHIRE installation is an on-line hypertext help system containing a complete reference to SAPHIRE. The help system can be accessed via the Help option from the menu or through context-sensitive help on each dialog.

Context-sensitive help provides information about the selected control on the active dialog box. It is accessed by choosing the Help button (? $)$, located in the upper right-hand corner of the dialog.

In addition to the context-sensitive help, Tool Tips help is available on each dialog. To access Tool Tips, position the cursor over a control on the active dialog. A small box will appear containing a brief description about that control.

\subsubsection{Toolbars}

\subsubsection{SAPHIRE Toolbars}

The SAPHIRE toolbar provides single-click access to the common SAPHIRE functions.

Depending on what function is currently selected, the toolbar will change to reflect available options. When the current dialog contains a multiple-select list box, some of the toolbar options may be disabled, depending on the number of list items selected. During processing, such as cut set generation, only the Help button is available. 


\section{Main toolbar buttons}

\begin{tabular}{|c|c|c|c|}
\hline 5 & Exit the SAPHIRE program. & - 드를 & $\begin{array}{l}\text { Display the Sequences dialog . } \\
\text { Recalculate sequence values after event } \\
\text { failure data and/or cut sets have been } \\
\text { modified. }\end{array}$ \\
\hline & Create a new SAPHIRE project. & $\rightleftharpoons_{C-D}$ & $\begin{array}{l}\text { Display the End State List dialog. } \\
\text { Recalculate end state values after events } \\
\text { and/or cut sets have been modified. }\end{array}$ \\
\hline$\vec{ح}$ & Open an existing SAPHIRE project. & & $\begin{array}{l}\text { Activate the Modify Database toolbar. } \\
\text { Modify SAPHIRE database files (e.g., } \\
\text { project, event tree, basic event, etc.) }\end{array}$ \\
\hline & Generate change set data. & 茞曧 & $\begin{array}{l}\text { Display the Reports Menu dialog. } \\
\text { Generate reports containing information } \\
\text { about the current project. }\end{array}$ \\
\hline 뭄 & $\begin{array}{l}\text { Display the Fault Trees dialog. Build, } \\
\text { edit and analyze fault tree models }\end{array}$ & 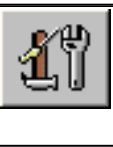 & $\begin{array}{l}\text { Activate the Utilities toolbar. Define } \\
\text { constants, recover the database, and } \\
\text { MAR D data exchange. }\end{array}$ \\
\hline$\vec{F}$ & $\begin{array}{l}\text { Display the Event Tree dialog. } \\
\text { Graphically build and edit event trees and } \\
\text { edit sequence logic }\end{array}$ & $\theta$ & Access the on-line hypertext help system. \\
\hline
\end{tabular}

The toolbar can be displayed or hidden by selecting the desired display method on the Define Constants dialog. Toolbar buttons can also be configured to display the bitmap (picture), text, or both.

\subsubsection{Fault Tree Toolbar}

The Fault Tree toolbar provides single-click access to the most common SAPHIRE fault tree options. This toolbar becomes active when the Fault Trees List dialog is displayed.

Depending the number of fault trees currently selected in the list, the toolbar will change to reflect available options. During processing, such as cut set generation, only the Help button is available.

\section{Fault tree toolbar buttons}

\begin{tabular}{|c|c|c|c|}
\hline$\angle m$ & $\begin{array}{l}\text { Close the Fault Trees List dialog and } \\
\text { return to the main toolbar. }\end{array}$ & 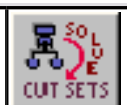 & $\begin{array}{l}\text { Generate cut sets for a fault tree based on } \\
\text { cut set generation cut off values. }\end{array}$ \\
\hline
\end{tabular}




\begin{tabular}{|c|c|c|c|}
\hline 旅 & $\begin{array}{l}\text { Add a new fault tree record to the } \\
\text { database. }\end{array}$ & $\begin{array}{l}P(\text { 옹 }) \\
=? ?\end{array}$ & $\begin{array}{l}\text { Calculate a new minimum cut set upper } \\
\text { bound for fault tree cut sets. }\end{array}$ \\
\hline 軍 & $\begin{array}{l}\text { Construct a new or modify the selected } \\
\text { graphical fault tree. }\end{array}$ & $\mathrm{K}_{\mathrm{LHS}}^{\mathrm{M}}$ & $\begin{array}{l}\text { Perform uncertainty analysis on a fault } \\
\text { tree. }\end{array}$ \\
\hline & $\begin{array}{l}\text { Construct a new or modify a graphical } \\
\text { P\&ID (Piping and Instrumentation } \\
\text { Diagram). }\end{array}$ & $C_{\text {SET }}^{C U T}$ & $\begin{array}{l}\text { Activate the Cut Set Options toolbar. } \\
\text { Perform various operations on cut sets: } \\
\text { Update, Prune, Recover, Edit. }\end{array}$ \\
\hline 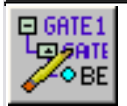 & $\begin{array}{l}\text { Modify fault tree logic in an } \\
\text { alphanumeric format. }\end{array}$ & \begin{tabular}{|l|l|l|l|} 
\\
\end{tabular} & $\begin{array}{l}\text { Activate the Display Options toolbar. } \\
\text { Display the results of fault tree analyses. }\end{array}$ \\
\hline 虽占 & Organize the fault tree logic into pages & 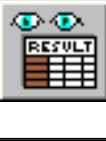 & $\begin{array}{l}\text { View fault tree analysis information and } \\
\text { compare base case and current case cut } \\
\text { set results. }\end{array}$ \\
\hline & & 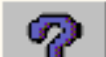 & Access the on-line hypertext help system \\
\hline
\end{tabular}

The toolbar can be displayed or hidden by selecting the desired display method on the Define Constants dialog. Toolbar buttons can also be configured to display the bitmap (picture), text, or both.

\subsubsection{Event Tree Toolbar}

The Event Tree toolbar provides single-click access to the most common SAPHIRE event tree options. This toolbar becomes active when the Event Tree List dialog is displayed

Depending the number of event trees currently selected in the list, the toolbar will change to reflect available options. During processing, such as sequence logic generation, only the Help button is available.

\section{Event tree toolbar buttons}

\begin{tabular}{|c|c|c|c|}
\hline & $\begin{array}{l}\text { Close the Event Tree List dialog and } \\
\text { return to the main toolbar. }\end{array}$ & 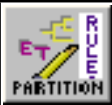 & Edit event tree partition rules. \\
\hline 角 & $\begin{array}{l}\text { Add a new event tree record to the } \\
\text { database. }\end{array}$ & 8 & $\begin{array}{l}\text { Link event tree sequences (i.e., generate } \\
\text { sequence logic). }\end{array}$ \\
\hline$-\sqrt{2}\left[\frac{1}{5}\right.$ & $\begin{array}{l}\text { Construct a new or modify the selected } \\
\text { graphical event tree. }\end{array}$ & t. & Edit event tree end states. \\
\hline & Edit event tree linkage rules. & 9 & Access the on-line hypertext help system. \\
\hline
\end{tabular}

The toolbar can be displayed or hidden by selecting the desired display method on the Define Constants dialog. Toolbar buttons can also be configured to display the bitmap (picture), text, or both.

\subsubsection{Sequence Toolbar}


The Sequence toolbar provides single-click access to the most common SAPHIRE sequence options. This toolbar becomes active when the Sequences dialog is displayed.

Depending on the number of sequences currently selected in the list, the toolbar will change to reflect available options. During processing, such as cut set generation, only the Help button is available.

\section{Sequence toolbar buttons}

\begin{tabular}{|c|c|c|c|}
\hline 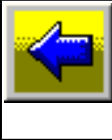 & $\begin{array}{l}\text { Close the Sequences dialog and return to } \\
\text { the main toolbar. }\end{array}$ & $\psi_{\text {SET }}^{\mathrm{CUT}}$ & $\begin{array}{l}\text { Activate the Cut Set Options toolbar. } \\
\text { Perform various operations on cut sets: } \\
\text { Update, Prune, Recover, Edit. }\end{array}$ \\
\hline 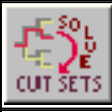 & $\begin{array}{l}\text { Generate cut sets for a sequence based on } \\
\text { cut set generation cut off values. }\end{array}$ & 慝 & $\begin{array}{l}\text { Activate the Display Options toolbar. } \\
\text { Display the results of sequence analyses. }\end{array}$ \\
\hline $\begin{array}{l}\mathrm{P}\left(\begin{array}{c}{ }^{\circ} \\
=\end{array}\right) \\
=? ?\end{array}$ & $\begin{array}{l}\text { Calculate a new minimum cut set upper } \\
\text { bound for sequence cut sets. }\end{array}$ & 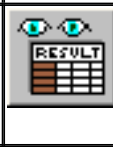 & $\begin{array}{l}\text { View sequence analysis information and } \\
\text { compare base case and current case cut } \\
\text { set results. }\end{array}$ \\
\hline $\mathrm{L}_{\mathrm{LHS}}^{\mathrm{MC}}$ & $\begin{array}{l}\text { Perform uncertainty analysis on selected } \\
\text { the sequence(s). }\end{array}$ & \% & Access the on-line hypertext help system. \\
\hline
\end{tabular}

The toolbar can be displayed or hidden by selecting the desired display method on the Define Constants dialog. Toolbar buttons can also be configured to display the bitmap (picture), text, or both.

\subsubsection{End State Toolbar}

The End State toolbar provides single-click access to the most common SAPHIRE end state options. This toolbar becomes active when the End State List dialog is displayed.

Depending on the number of end states currently selected in the list, the toolbar will change to reflect available options. During processing, such as cut set gathering, only the Help button is available.

\section{End State toolbar buttons}

\begin{tabular}{|c|c|c|c|}
\hline$/$ & $\begin{array}{l}\text { Close the End State List dialog and return } \\
\text { to the main toolbar. }\end{array}$ & EDIT// & Edit the current case end state cut sets. \\
\hline 2\% & $\begin{array}{l}\text { Gather cut sets for an end state based on } \\
\text { cut set generation cut off values. }\end{array}$ & EDIT/C & Edit the base case end state cut sets. \\
\hline $\begin{array}{l}\mathrm{P}(\mathrm{CD}) \\
=? ?\end{array}$ & $\begin{array}{l}\text { Calculate a new minimum cut set upper } \\
\text { bound for end state cut sets. }\end{array}$ & $\sqrt{17}$ & $\begin{array}{l}\text { Activate the Display Options toolbar. } \\
\text { Display the results of end state analyses. }\end{array}$ \\
\hline $\mathrm{L}_{\mathrm{LHS}}^{\mathrm{MC}}$ & $\begin{array}{l}\text { Perform uncertainty analysis on selected } \\
\text { the end state(s). }\end{array}$ & 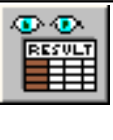 & $\begin{array}{l}\text { View end state analysis information and } \\
\text { compare base case and current case cut } \\
\text { set results. }\end{array}$ \\
\hline CUT & $\begin{array}{l}\text { Update the current case cut sets for a } \\
\text { selected fault tree. }\end{array}$ & 8 & Access the on-line hypertext help system. \\
\hline
\end{tabular}

The toolbar can be displayed or hidden by selecting the desired display method on the Define Constants dialog. Toolbar buttons can also be configured to display the bitmap (picture), text, or both.

\subsubsection{Cut Set Toolbar}


The Cut Set toolbar provides single-click access to the most common SAPHIRE Cut Set options. This toolbar becomes active when the either the Fault Trees List dialog or Sequences dialog is displayed and the Cut Set toolbar button is subsequently selected.

\section{Fault tree cut set toolbar buttons}

\begin{tabular}{|c|c|c|c|}
\hline 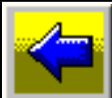 & Return to the Fault Tree toolbar. & 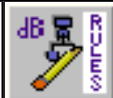 & Create and modify project recovery rules. \\
\hline 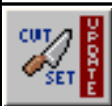 & $\begin{array}{l}\text { Update the current case cut sets for a } \\
\text { selected fault tree. }\end{array}$ & EDIT/P & Edit the current case fault tree cut sets. \\
\hline \begin{tabular}{|l|l|} 
员 \\
齐 \\
\end{tabular} & $\begin{array}{l}\text { Create and modify recovery rules for a } \\
\text { selected fault tree. }\end{array}$ & $\int_{\mathrm{BASE}}^{\mathrm{EDIT} / \mathrm{C}}$ & Edit the base case fault tree cut sets. \\
\hline $\begin{array}{l}\text { APPLY } \\
\text { 号 } \\
\text { 8. }\end{array}$ & $\begin{array}{l}\text { Apply all rules currently defined to the } \\
\text { selected fault tree(s). }\end{array}$ & P & Access the on-line hypertext help system. \\
\hline
\end{tabular}

\section{Sequence cut set toolbar buttons}

\begin{tabular}{|c|c|c|c|}
\hline & Return to the Sequence toolbar. & 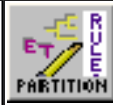 & $\begin{array}{l}\text { Define cut set partitioning rules for } \\
\text { individual event trees. }\end{array}$ \\
\hline$\overline{c \text { cur }}$ & $\begin{array}{l}\text { Update the current case cut sets for a } \\
\text { selected sequence. }\end{array}$ & 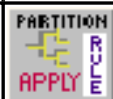 & $\begin{array}{l}\text { Apply the currently defined partition } \\
\text { rules to the selected sequence(s). }\end{array}$ \\
\hline & $\begin{array}{l}\text { Create and modify recovery rules for a } \\
\text { selected sequence. }\end{array}$ & 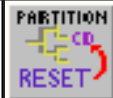 & $\begin{array}{l}\text { Clear the sequences created by the } \\
\text { partitioning rule. }\end{array}$ \\
\hline $\mathrm{E}_{\mathrm{T}}^{-1}$ & $\begin{array}{l}\text { Create and modify event tree recovery } \\
\text { rules. }\end{array}$ & EDIT/P & Edit the current case sequence cut sets. \\
\hline 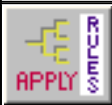 & $\begin{array}{l}\text { Apply all rules currently defined to the } \\
\text { selected sequences. }\end{array}$ & EDIT/ $/ 7$ & Edit the base case sequence cut sets. \\
\hline 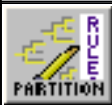 & $\begin{array}{l}\text { Define cut set partitioning rules for } \\
\text { individual sequences. }\end{array}$ & (9) & Access the on-line hypertext help system. \\
\hline
\end{tabular}

The toolbar can be displayed or hidden by selecting the desired display method on the Define Constants dialog. Toolbar buttons can also be configured to display the bitmap (picture), text, or both.

\subsubsection{Display Options Toolbar}

The Display Options toolbar provides single-click access to the most common SAPHIRE Display options. This toolbar becomes active when the either the Fault Trees List , or Sequences dialog is displayed and the Display Options toolbar button is subsequently selected.

\section{Display options toolbar buttons}

\begin{tabular}{|c|c|c|c|}
\hline & Return to the previous toolbar. & 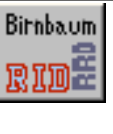 & $\begin{array}{l}\text { Difference - Birnbaum importance, Risk } \\
\text { Reduction Interval, and Risk Increase } \\
\text { Interval will be calculated. }\end{array}$ \\
\hline
\end{tabular}




\begin{tabular}{|c|c|c|c|}
\hline 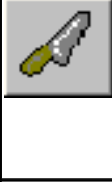 & $\begin{array}{l}\text { Display the cut sets, their percent of } \\
\text { contribution, the frequency, and the event } \\
\text { names that make up the cut sets. }\end{array}$ & $L_{I}$ & $\begin{array}{l}\text { Uncertainty Importance - Quantification } \\
\text { of the contribution of each individual } \\
\text { basic event's uncertainty to the total } \\
\text { uncertainty. }\end{array}$ \\
\hline ss 1 ss & $\begin{array}{l}\text { Display the base and current cut sets, } \\
\text { their percent of contribution, the } \\
\text { frequency, and the event names that } \\
\text { make up the cut sets. }\end{array}$ & $\underset{\mathrm{LHS}}{\mathrm{MC}}$ & Display uncertainty analysis results. \\
\hline $\begin{array}{l}\mathrm{F}-V_{\alpha} \\
\text { RIR }\end{array}$ & $\begin{array}{l}\text { Ratio - Fussell-Vesely importance, Risk } \\
\text { Reduction Ratio, and Risk Increase Ratio } \\
\text { will be calculated. }\end{array}$ & 9 & Access the on-line hypertext help system. \\
\hline
\end{tabular}

The toolbar can be displayed or hidden by selecting the desired display method on the Define Constants dialog. Toolbar buttons can also be configured to display the picture, text, or both.

\subsubsection{Modify Database Toolbar}

The Modify Database toolbar provides access to the SAPHIRE modify database functions.

\section{Modify Database toolbar buttons}

\begin{tabular}{|c|c|c|c|}
\hline & Return to the main toolbar. & 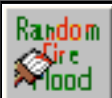 & Modify analysis type records. \\
\hline & Modify the project record. & 唐 & Modify gate records. \\
\hline $\overrightarrow{2}$ & $\begin{array}{l}\text { Add, modify, copy, or delete event tree } \\
\text { data records, or associated text. }\end{array}$ & 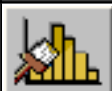 & Modify histogram records. \\
\hline 跎 & $\begin{array}{l}\text { Add, modify or delete fault tree data } \\
\text { records, or associated text. }\end{array}$ & & $\begin{array}{l}\text { Modify Piping and Instrumentation } \\
\text { Diagram (P\&ID) records. }\end{array}$ \\
\hline $\overrightarrow{20}$ & $\begin{array}{l}\text { Add, modify or delete the end state data } \\
\text { records, or associated text. }\end{array}$ & CHATG & Modify change set records. \\
\hline 店 & $\begin{array}{l}\text { Add, modify or delete the basic event } \\
\text { data records. }\end{array}$ & 189 & Modify flag set records. \\
\hline \begin{tabular}{|l} 
ATRRIEU TE \\
$\$ \$$
\end{tabular} & $\begin{array}{l}\text { Activate the Modify Attributes toolbar. } \\
\text { Edit records of the five attribute types in } \\
\text { the current project: Systems, Locations, } \\
\text { Failure Modes, Basic Event Types, and } \\
\text { Trains. }\end{array}$ & & Access the on-line hypertext help system. \\
\hline
\end{tabular}

\section{Modify Attributes toolbar buttons}

\begin{tabular}{|c|c|c|c|}
\hline & Return to the main toolbar. & 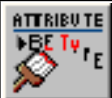 & Modify basic event type attribute records. \\
\hline 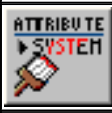 & Modify system attribute type records. & 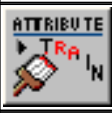 & Modify train attribute type records. \\
\hline 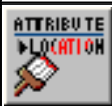 & Modify location attribute type records. & क & Access the on-line hypertext help system. \\
\hline
\end{tabular}




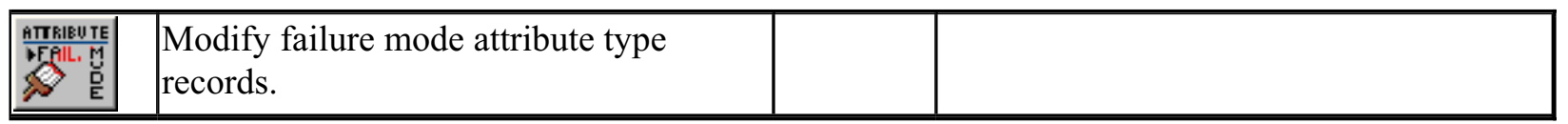

The toolbar can be displayed or hidden by selecting the desired display method on the Define Constants dialog. Toolbar buttons can also be configured to display the picture, text, or both.

\subsubsection{Utility Toolbar}

The Utilities toolbar provides access to the most common SAPHIRE utility functions.

\begin{tabular}{|c|c|c|c|}
\hline & Return to the main toolbar. & 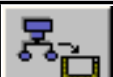 & Extract fault trees from the database. \\
\hline 占 & $\begin{array}{l}\text { Invoke the Define Constants dialog to } \\
\text { specify general information, cut set } \\
\text { option defaults, and default values for the } \\
\text { graphical editors. }\end{array}$ & 매교 & $\begin{array}{l}\text { Load fault tree graphic files into the } \\
\text { database. }\end{array}$ \\
\hline 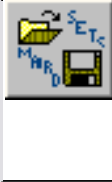 & $\begin{array}{l}\text { Load or extract data in the Models and } \\
\text { Results Data Base (MAR D) or Set } \\
\text { Equation Transformation System (SETS) } \\
\text { formats. }\end{array}$ & ${ }^{-5}$ & Extract event trees from the database. \\
\hline 且 & $\begin{array}{l}\text { Restructure the database and re index the } \\
\text { data }\end{array}$ & 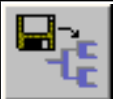 & $\begin{array}{l}\text { Load event tree graphic files into the } \\
\text { database. }\end{array}$ \\
\hline OR- 宮 & $\begin{array}{l}\text { Convert fault tree or sub-tree } \\
\text { alphanumeric logic to a graphical format. }\end{array}$ & P & Access the on-line hypertext help system. \\
\hline
\end{tabular}

The toolbar can be displayed or hidden by selecting the desired display method on the Define Constants dialog. Toolbar buttons can also be configured to display the picture), text, or both.

\subsection{User Support}

Training classes for NRC users, which provide instruction in the use of SAPHIRE, are supplied through the INEEL. Contact the NRC Program Manager for training information. Additional information about SAPHIRE training can be found on the SAPHIRE web site at: http://saphire.inel.gov. The SAPHIRE Users Group is designed to provide quality technical support for the SAPHIRE software along with regular updates and technical information.

\subsection{SAPHIRE Quality Assurance}

Software quality assurance comprises a variety of tasks. All of the methodologies applied to SAPHIRE to ensure software quality will not be covered in this document. This section will discuss the beta test activity performed prior to release of the current version of the software, the Validation and Verification $(\mathrm{V} \& \mathrm{~V})$ effort completed on SAPHIRE 5.0, and automated code testing techniques used to ensure quality software.

To help assure the quality of new releases, SAPHIRE Versions 6 and 7 were used extensively with models created in earlier versions of SAPHIRE. The models were loaded into the current version of the software and results of the analyses were compared against SAPHIRE Version 5.0. Any discrepancies 
found were corrected in subsequent minor versions of the code. SAPHIRE has been validated in several ways. The following summarize these validation processes.

\section{The Beta Version Process}

Each new SAPHIRE version is beta tested before its release. The beta testers are analysts experienced with PRA methods and terminology, and typically are familiar with earlier versions of SAPHIRE and IRRAS. The primary objective of the beta testing is to verify that the results produced by the new version are correct. The secondary objective is to ensure that the software is user-friendly and functional. Based on feedback from beta testers, programmatic errors and discrepancies are corrected, the user interface is improved, and new features are recommended.

\section{SAPHIRE V\&V}

A V\&V of SAPHIRE 5.0 in the traditional sense was performed in 1995 and documented in NUREG/CR6116, Volume 9, Systems Analysis Programs for Hands-on Integrated Reliability Evaluations (SAPHIRE) Version 5.0, Verification and Validation (V\&V) Manual, 1995.

\section{PRA Loading and Result Comparison}

Because INEEL is continually loading new PRAs into SAPHIRE, new SAPHIRE releases are tested extensively by comparing with PRA models and results of earlier versions and especially with PRAs loaded in SAPHIRE Version 5.0. About thirty PRAs have been (completely or partially) loaded in SAPHIRE. The loading process requires a detailed comparison of the models and results with the models and results of the original PRAs. Identified discrepancies are resolved through the interaction with the analysts who performed the PRA.

\section{Automated Code Testing}

Because of advances in the state-of-the-art of computer science, an automated testing process has been established during 1998. This automated testing tool allows the test engineers to develop and maintain scripts for selected critical functions of the software. Prior to each release, these scripts are run against the SAPHIRE software on a number of machines with varying configurations. The test results are automatically compared and any discrepancies are resolved. The automated testing process has been used for the Windows Versions 6 and 7 of SAPHIRE. 


\section{DATABASE STRUCTURE AND CONCEPTS}

\subsection{Database Concepts}

Before you can use SAPHIRE effectively, you need to have an understanding of the SAPHIRE 7.0 database structure and concepts. A relational database is used to manage inputs and outputs. SAPHIRE contains nine permanent relations, or tables, used to store and retrieve specific PRA information. For each relation, three files exist:

*.DAT - This file contains the actual data and information about which data constitutes a "key." The key is used for searching and locating information.

*.IDX - This file contains indices into the data for keyed fields.

*.BLK - This file contains variable length data (such as cut sets) that are accessible through pointers contained in the *.DAT file.

The SAPHIRE 7.0 database is divided into projects. Information for the project is divided into two separate parts, referred to as base case and current case. As delivered, SAPHIRE provides an initial project called DEMO. Access to any portion of the database is obtained through the appropriate project. Normally, a user will need to create a project for each PRA being performed. All the information in a project is stored together in a folder (or subdirectory).

NOTE: Only one project may be stored in an individual folder.

The following describes the steps a user would ordinarily take in order to perform a new PRA analysis using SAPHIRE. Certain information records are automatically added to the database depending on the functions performed by the user. Links are provided for detailed information about how the relations are used in each step:

1. Create a new project where the results of the PRA can be stored. Once the "Project" is created, the user is ready to add information to the database.

2. Adjust the program operating constants (via the Utility | Define Constants option).

3. Create the event trees for the Project.

4. Create the fault trees.

5. Enter the failure data for each basic event in the database.

6. Generate the sequence entries for each valid sequence in the event trees.

7. Perform analysis, varying failure data using change sets. 


\subsection{Database Structure}

\subsubsection{Project Relation}

A project is any logical grouping of fault trees and sequences with their associated basic events, cut sets, reliability data, and descriptions. Each project is contained within a separate folder.

Information about the project is stored in the Project relation. The Project relation contains general information about the PRA. Along with the project name, description, plant design, etc., it also contains cross reference information for relationships between basic events, fault tree, sequence, event tree, end state, change sets, and gate in the project. The cross reference maps are updated automatically each time one of these pieces of data is modified. The Project relation also contains the project sequence partition, and project sequence, fault tree, and end state recovery information along with the macro text.

\subsubsection{Fault Tree Relation}

The Fault Tree editor is used to create fault trees for the project. SAPHIRE creates an entry in the Fault Tree relation for each fault tree created. SAPHIRE requires that certain conventions be used for naming fault trees. To use a fault tree as a top event in an event tree, or reference it in a sequence, the name of the fault tree must be specified in a special manner. The name of the fault tree and the name of the top gate in the fault tree must be the same. Also, the name of the top event in the event tree and the fault tree name must be the same.

SAPHIRE adds all the basic event references in the fault tree to the Event relation. If a description is provided for a basic event, then this description is also added to the database. All other information for the basic event is left unchanged or set to its default values.

SAPHIRE also creates an entry in the Gate relation for each gate defined in the fault tree. The name, type, and description of each gate contained in the fault tree is stored in the database. This information can be edited. However, the changes are only reflected in the graphical fault trees if the user performs an "alpha to graphics" conversion on the fault trees that use the gate.

Finally, SAPHIRE creates an entry in the Event relation for each transfer gate defined in the fault tree. This is done to allow the user to specify whether a transfer gate is to be expanded or whether the basic event representing the transfer gate is to be used.

This relation maintains data for the following fault tree attributes:

- $\quad$ Fault tree name and description,

- $\quad$ Fault tree logic,

- Graphic representation,

- Minimal cut sets,

- Top event probability,

- Default flag set, 
- Default quantification method,

- Recovery rules,

- $\quad$ Importance measures, and

- Uncertainty analysis results.

\subsubsection{Sequence Relation}

The Sequence relation contains information about the sequences for an event tree. The user normally generates the sequence entries using the Link Trees option. When the user selects this option, SAPHIRE generates a sequence entry for each valid sequence in the event tree. Any sequences that may have been generated previously for this same event tree are automatically overwritten with the new valid sequence.

Each sequence contains an end state name that is created in the End State relation if it does not already exist. Changing the name of an end state in a sequence only changes the name for that specific sequence and does not change the information in the End State relation or the event tree for that sequence. When a sequence is solved, the cut sets generated are stored in the Sequence relation.

This relation maintains data for the following accident sequence attributes:

- $\quad$ Name and description,

- $\quad$ Logic (combination of successful and failed fault trees),

- $\quad$ Minimal cut sets,

- $\quad$ Sequence probability,

- Default flag set,

- Default quantification method,

- $\quad$ Sequence end state,

- $\quad$ Associated event tree,

- $\quad$ Partition and recovery rules,

- Importance measures, and

- Uncertainty analysis results.

\subsubsection{Event Tree Relation}

The Event Tree editor is used to generate the event trees for the project. When the user creates event trees using the Event Tree editor, SAPHIRE automatically adds certain information to the database. 
When the event tree is saved, an entry is created in the Event Tree relation that contains the graphical information associated with the event tree. If the event tree contains an initiating event as the first top event in the tree, then entries are automatically created in the Event Tree relation. Entries include the name of the event tree, the initiating event for the event tree, and other descriptive data.

Next, SAPHIRE creates an entry in the Fault Tree relation for each top event defined in the event tree. By default, SAPHIRE assumes that each top event is represented by a fault tree with the same name. At the time the fault tree entry is created, SAPHIRE also creates a basic event in the Event relation with the same name as the fault tree. This event is used when the fault tree logic is not expanded during fault tree analysis or when the logic has not yet been developed.

The Event Tree relation maintains data for the following event tree attributes:

- Event tree name and description,

- $\quad$ Event tree logic and graphic representation,

- Initiating event, and

- $\quad$ Event tree macro, recovery and partition rules

\subsubsection{Event Relation}

Once the fault trees are defined, the user will need to enter the failure data for each basic event in the database. This can be done with either the Modify option or with the Generate option and Base Case Update options.

If the Modify option is used to change the default failure data, then the user must be sure to use the Generate option to generate the current failure data to be used in the remainder of the analysis. Any time changes are made to the basic event failure data, the user MUST use the Generate option before the changes can be used. If it appears that changes made to basic event failure data are not being used properly by SAPHIRE, the user should check to ensure that the proper data were generated and saved.

The Event relation maintains data for the following basic event attributes:

- $\quad$ Basic event names and description,

- $\quad$ Event attributes,

- Transformation information,

- Uncertainty information,

- $\quad$ Failure data, and

- $\quad$ Process flag. 


\subsubsection{Change Set Relation}

The Change Set relation stores the name, description and event changes for change sets and flag sets created for this project. This information can be edited from the Modify option, or for change sets, from the Generate option.

This relation is maintained to track event changes relating to event class and event probability.

\subsubsection{UserInfo Relation}

The User Information relation contains basic operating constants for SAPHIRE. The user may change these constants to reflect the desired defaults to be used. These data are unrelated to trees and/or projects. Information stored includes cut set generation cut off values, event tree and fault tree graphical editor preferences, and menu and toolbar defaults.

\subsection{SAPHIRE Database Concepts}

\subsubsection{Unpredictable Results}

The information stored in the SAPHIRE database is buffered in and out of memory during processing. When the information is modified, SAPHIRE may not write the information out to the hard disk immediately. If the user does not exit SAPHIRE normally by using the File | Exit option from the main menu, then the information that was changed may be lost. The user should always exit SAPHIRE before turning off or rebooting the computer.

If a power failure or abnormal program abort occurs, the user should enter SAPHIRE, select the Utility option, and from that select the Recover Data Base option. The user should then rebuild everything in the database before continuing to use the program on this data. Failure to do this may cause unpredictable results.

If a database is generating results that the user cannot explain, the database project in question should be rebuilt and the results checked again. If the results still cannot be explained, then a bug may be causing the error and should be reported immediately.

\subsubsection{Cross Reference Maps}

As the user adds information to the SAPHIRE database, a cross reference map is maintained for each basic event, change set, sequence, fault tree, and event tree in the database. This map is updated automatically each time one of the specified pieces of information is added or changed.

When the user decides to delete a piece of information from the database, SAPHIRE checks these cross reference maps to ensure the piece of information being deleted is not used anywhere in the database. If it is used, SAPHIRE will not allow the information to be deleted until those records referencing it are changed or deleted. For instance, if a basic event is to be deleted, the user must ensure that the event is not used by a fault tree logic, fault tree cut sets (base or current), event tree logic, sequence logic, or sequence cut sets (base or current). In the Modify option, SAPHIRE will display a "--" character in front of any event which it determines is not used by anything in the database. 


\subsubsection{Base Case Vs. Current Case Data}

Base case and current case refer to two separate parts of a project data base. The base case data are stored in the data base files as a "permanent" record. The current case data, sometimes referred to as the alternate case, are used to perform an analysis (e.g., cut set generation and quantification).

All SAPHIRE calculations (e.g., cut set generation and sensitivity analysis) use the data stored in the current case. The current case is created by applying change sets to base case data (via the Generate option).

NOTE: The current case results are overwritten each time a specific analysis is performed. The base case results do not change each time an analysis is performed.

Current case data can equal the base case in order to reproduce the original study stored in the base case, however, only current case cut sets can be modified with the cut set editor. Changes made to cut sets with the cut set editor are implemented automatically. A cut set update is necessary if changes are made that create non-minimal cut sets.

Base case data and results are changed by updating the base case. Updating the base case transfers the current case data or results into the base case.

\subsubsection{Change Sets Vs. Flag Sets}

Change sets are a user-defined set of changes that will be applied to the base case data when event data is transferred to the current case. There is no limit to the number of change sets that can be added to the data base. A change set can contain one class change and unlimited individual probability changes, also referred to as "single" changes.

Class changes use a basic event attribute to search for a class of basic events to which the defined change applies:

- $\quad$ The search criteria are defined first

- The change to be applied is then defined

Probability, or single changes only modify a user-identified basic event

- $\quad$ The desired basic event is selected

- The changes to the basic event are then defined

Multiple change sets can be defined and applied singly or in combination to create different sensitivity studies. When multiple change sets are used in combination, the order of "marking" a change set is important. (Change sets are marked by double-clicking the line containing the change set.)

- The first selected change set will be the first one that is applied

- $\quad$ Later changes will overwrite earlier ones if there is any overlap

- Within a change set, individual probability changes will overwrite a class change 
EXAMPLE: Two change sets are developed. The first is named "A" and sets all valves to failed. The second is named " $\mathrm{B}$ " and sets all pumps to failed. The possible scenarios are

\begin{tabular}{|l|l|}
\hline Change set(s) that are marked & Sensitivity case \\
\hline None & Original base case analysis \\
\hline A & Valves failed \\
\hline B & Pumps failed \\
\hline A and B & Both valves and pumps failed \\
\hline
\end{tabular}

Flag sets are a special type of change set used to indicate modifications to particular events on a sequence-by-sequence basis. They are different from change sets in that they can only contain individually-specified types of changes. No "Class Changes" are allowed in a flag set.

Flag Sets can only contain either house flag changes to the calculation type or process type changes. You can not make changes to the probability of failure (e.g., change the probability from $2 \mathrm{E}-3$ to $1 \mathrm{E}-1$ ) for events in a Flag Set. Consequently, the allowable changes that can be made in a Flag Set are:

\begin{tabular}{|l|l|}
\hline Type of change & Allowable values \\
\hline Calculation type & T (TRUE) \\
& F (FALSE) \\
& I (IGNORE) \\
\hline \hline Process Flag & X \\
& Y \\
& W \\
\hline
\end{tabular}




\section{SELECTING A PROJECT}

\subsection{Managing Project Data}

\section{PURPOSE}

A project is a group of models, such as those for a single plant, unit, or facility. When SAPHIRE is invoked, the most recent project used is automatically opened. From the File menu option, the ability to create a new project, open an existing project, or close the current project is available.

\section{STEPS}

1. From the SAPHIRE menu select File.

2. The drop-down menu with available items will be displayed.

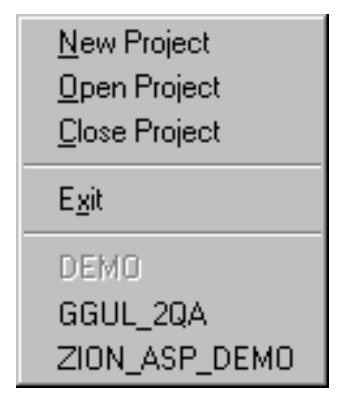

A list of recently used projects is displayed on the File menu. By selecting one of the projects, the current project is automatically closed and the selected project will be opened.

New Project - Closes the current project and creates a new one.

Open Project - Closes the current project and opens an existing one.

Close Project - Closes the current project.

Exit - Closes the current project and terminates the SAPHIRE program.

\subsection{New Project}

\section{PURPOSE}

This option provides the means of creating a new project. The new project you are creating must reside in its own folder (i.e., a single folder cannot contain more than ".SRA" file).

\section{STEPS}

1. From the menu select File.

2. Choose the New Project submenu option. The New Project dialog will be displayed. 


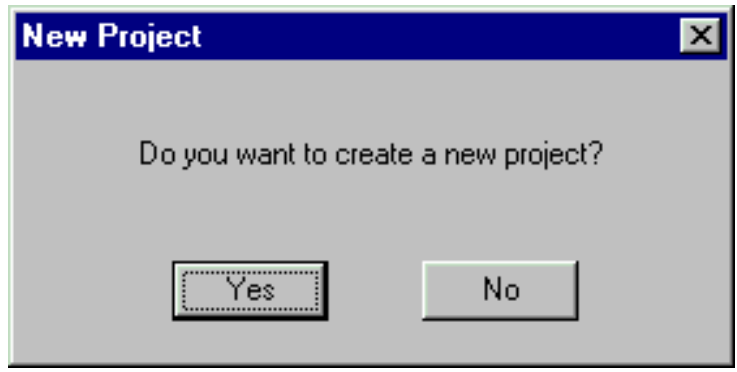

3. Choose the Yes button. The New Project-Name Project dialog will appear.

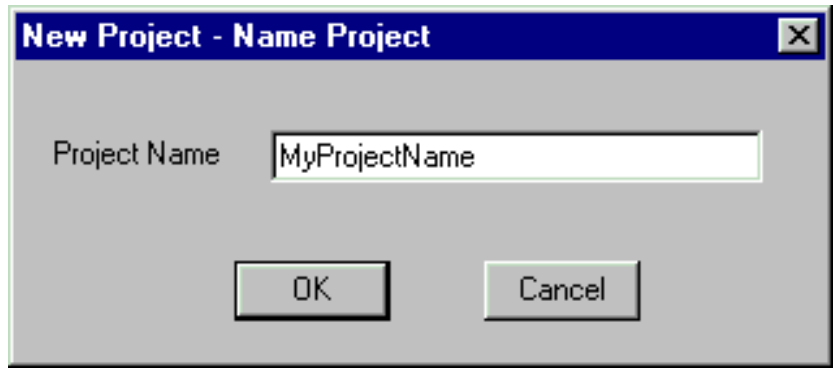

4. Enter the name of the project in the Project name field.

5. Choose the OK button. The New Project - Directory Info dialog will appear.

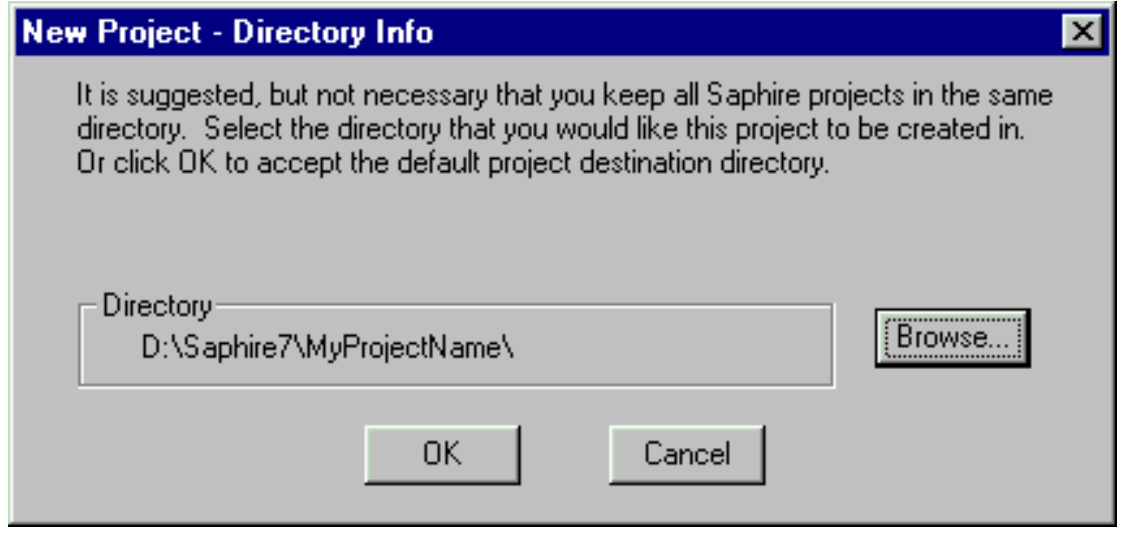

To accept the default directory, choose the OK button. You may select a different directory by choosing the browse button. When you choose OK, the directory will be created, and the new project will become SAPHIRE's current project.

Directory - $\quad$ The currently selected directory in which to place the new project.

Browse - $\quad$ Open the Select Directory dialog to choose a directory.

Open - $\quad$ Create the project and close the New Project dialog.

Cancel - $\quad$ Close the New Project dialog without creating a project.

\subsection{Select Directory}

\section{PURPOSE}

This option allows you to select, and optionally create, a directory. 


\section{STEPS}

1. From the menu select File.

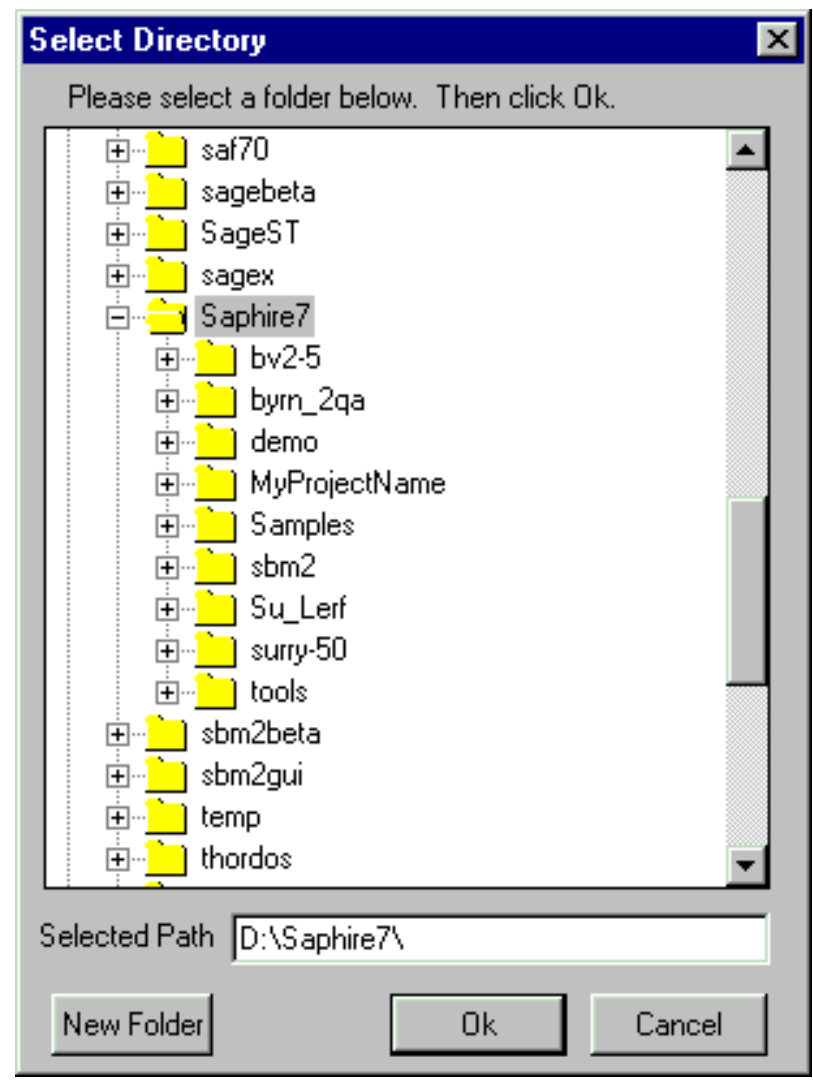

Lists the available folders in a tree view, so you can see how the folders fit into your system hierarchy. Click $₫$ to expand a folder. Click $\square$ to collapse an expanded folder.

Selected Path - Contains the name of the currently selected directory, or, you may type in the name of an existing directory.

New Folder - Allows you to enter the name of a new directory. The new directory will be added beneath the currently selected folder in the tree view.

Ok - $\quad$ Select the currently highlighted directory.

Cancel - $\quad$ Close the Select directory dialog without selecting a directory.

\subsection{Open Project}

\section{PURPOSE}

This option allows you to select (open) the project data set you wish to work with and provides the capability of copying raw (MAR-D) data files or SAPHIRE database files into a specific project. When the project is successfully selected, the name is shown on the title bar of the SAPHIRE window.

\section{STEPS}

1. From the menu select File. 
2. Choose the Open Project sub-menu option. The Open Project dialog will be displayed.

3. Choose the folder that contains the desired project.

4. Double-click on the project ".SRA" file or "FAM.DAT" file.

OR

5. Highlight the desired project and choose the Open button.

The selected project will now be the current project and its name will appear in the title bar of the SAPHIRE window.

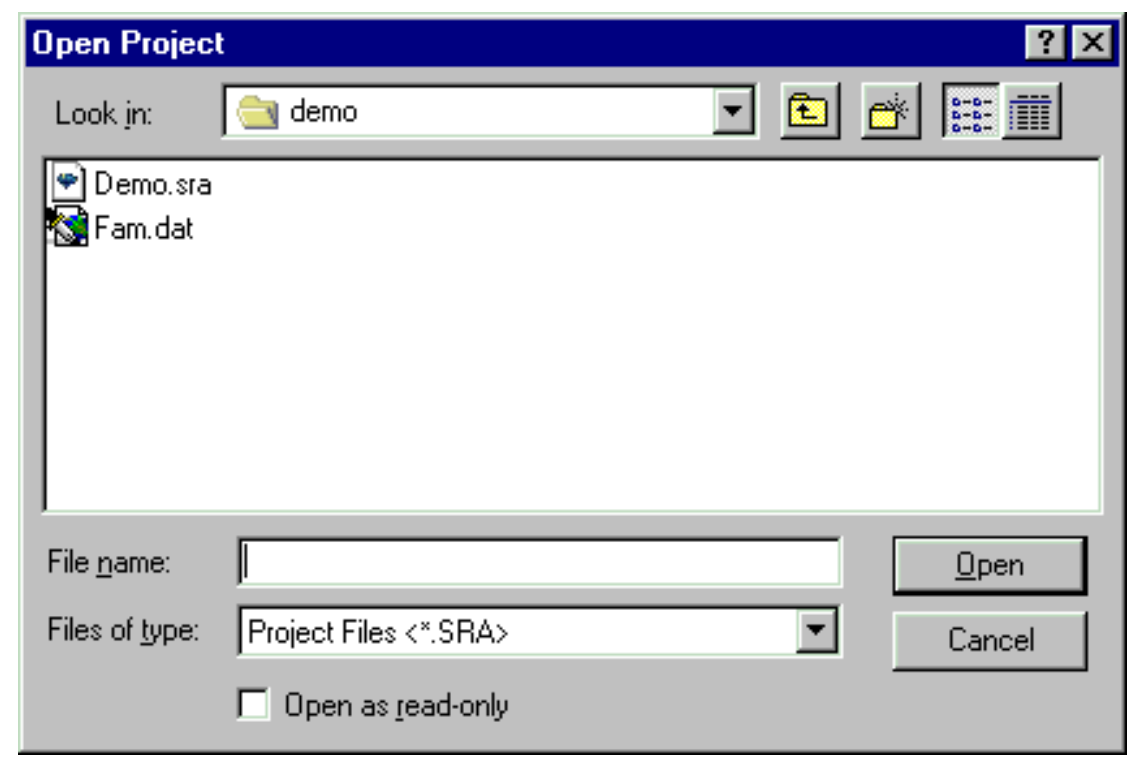

Look in - $\quad$ Lists the available folders and files. The box directly below it shows the contents of the current folder. The down-arrow to the right of this field shows how the folder fits into your system hierarchy.

Moves up one level in the system hierarchy.

Creates a new folder at the current level.

Displays the icons and names of the contents of the current folder.

Displays the icons, names, size, type, date and time modified, and archive flags of the contents of the current folder.

File Name - Contains the name of the project.

Files of Type - Lists the types of files to display. This is useful for narrowing the list of files displayed to only those you are interested in. In this case, only ".SRA" files are listed.

Open - $\quad$ Open the highlighted project and close the Open Project dialog.

Cancel - $\quad$ Close the Open Project dialog without opening a project.

Double-click - Open the project and close the Open Project dialog.

The current directory is the current project unless you select another project. SAPHIRE retains the last project you selected when you exited the program so when you enter SAPHIRE again the last project selected is the current project. 
If the selected project was created with an older version of SAPHIRE (IRRAS), the Version Conflict dialog will be displayed.

\subsection{Close Project}

\section{PURPOSE}

This option provides the means of closing the current project.

\section{STEPS}

1. From the menu select File.

2. Choose the Close Project submenu option.

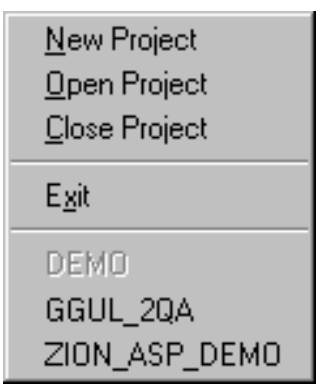

The current project database files will be closed. No project name will appear in the title bar of the SAPHIRE window. SAPHIRE menu options are no longer available, with the exception of File and Help, until a project is opened or created.

\subsection{Unable to Select a Project}

If for any reason the project cannot be selected, the message, "Unable to select desired project" appears, the previously selected project will be retained, and you will be given another chance to select a project.

If the highlighted project's data version does not match the current software version, the Version Conflict dialog appears, and you will be asked if you want to rebuild the data. 


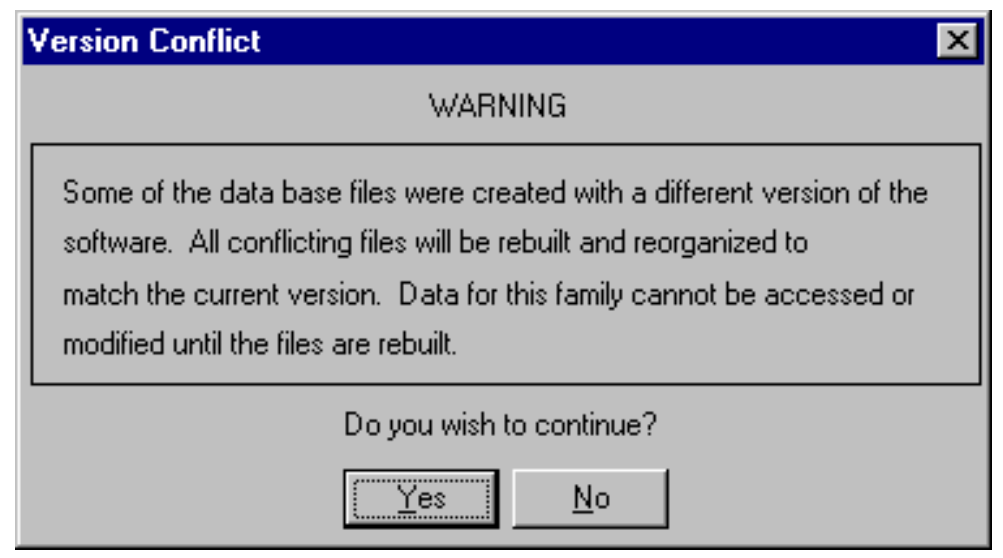

Yes - $\quad$ Rebuild the project data. This button must be chosen in order to selected the project. After the rebuild process is complete, the project is selected as the current project.

No - Do not rebuild the project's data. The project will not be selected, the former selected project will be retained, and the message, "Unable to select desired project" appears in the message bar. 


\section{CHANGE SETS AND THE GENERATE OPTION}

\subsection{Change Sets and the Generate Options}

\section{PURPOSE}

The Generate option is used to create a working copy of user event data for use in a particular analysis. This data can then be modified using change sets or used to update the base case data. SAPHIRE always uses current case data for analysis.

The event data can be manipulated, via change sets, to examine the changes in the probabilities of plant accidents and accident sequence failures based on basic events. A change set is a set of sensitivity analysis data modifications to be applied to basic events during fault tree or sequence analysis. Change sets modify the current case basic event data. A change set consists of probability and/or class changes for a group of basic events.

Within a change set, the user can add events to the change set, modify selected event probabilities, or reset those event probabilities to the base case values. The probability of events occurring can be recalculated. The user can construct change sets to be applied to the basic events for later propagation through existing cut sets. In addition, the user can perform a "base case update" that takes all current basic event data and uses it to overwrite the base case data. Note that the original base case data are overwritten in the process.

If the base case data is changed and the Generate option is not performed, the data that is used for the analysis will not reflect the changes. But, if the user attempts to perform an analysis after making a data change, SAPHIRE will prompt the user to generate data changes.

\section{STEPS}

1. From the SAPHIRE menu select Generate.

2. The Generate dialog will be displayed. 


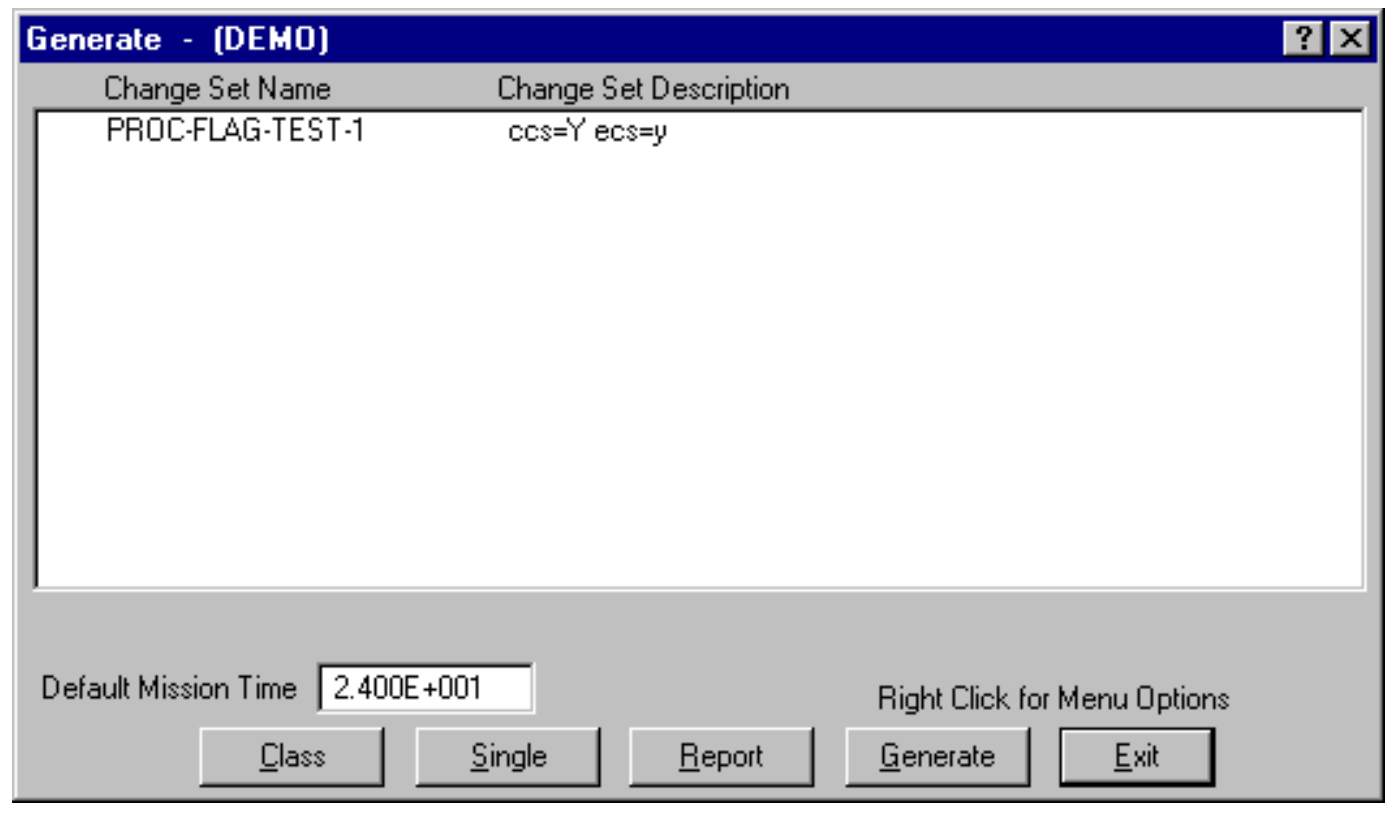

Pop-up Menu Options - Perform operations on the change set relation.

Add - $\quad$ Add a new change set record to the current project database. When complete, the message, "Record added," is displayed in the message bar of the SAPHIRE window.

Modify - $\quad$ Edit the selected change set record. When complete, the message, "Record modified," is displayed in the message bar of the SAPHIRE window.

Delete - $\quad$ Delete the selected change set record(s).

Copy - $\quad$ Create a new change set record by copying an existing one.

Text Edit - $\quad$ View and edit the descriptive text associated with the selected change set.

Mark/Unmark - Set the order of change set processing. Change sets can also be marked by double-clicking on the desired change set in the list.

\section{Button Options - Perform operations on the change set data.}

Class - $\quad$ Add a class probability change to a group of events.

Single - Create and reset individual event probability changes.

Report - Create reports that reflect the event modifications that currently exist within the database.

Generate - Generate new current case event data using the temporary modifications made to the marked change sets.

Exit - $\quad$ Close the Generate dialog. 


\subsection{Change Set (Definition)}

A change set is a set of sensitivity data modifications to be applied to basic events during fault tree or sequence analysis. Change sets modify the current case basic event data. A change set consists of probability and/or class changes for a group of basic events.

Within a change set, you may add events to the change set, modify selected event probabilities, or reset those event probabilities to the base case values. In addition, you may perform a base case update that takes all current basic event data and makes it the new base case data. The original base case data are overwritten in this process.

\subsection{Adding a Change Set}

\section{PURPOSE}

This option allows you to create a new change set record. Enter the new change set's name, description and date.

\section{STEPS}

1. From the SAPHIRE menu select Modify $\mid$ Change Sets. The Change Sets dialog is displayed. OR

2. From the SAPHIRE menu select Generate. The Generate dialog is displayed.

3. Right-click to invoke the pop-up menu and choose Add. The Add Change Set dialog is displayed.

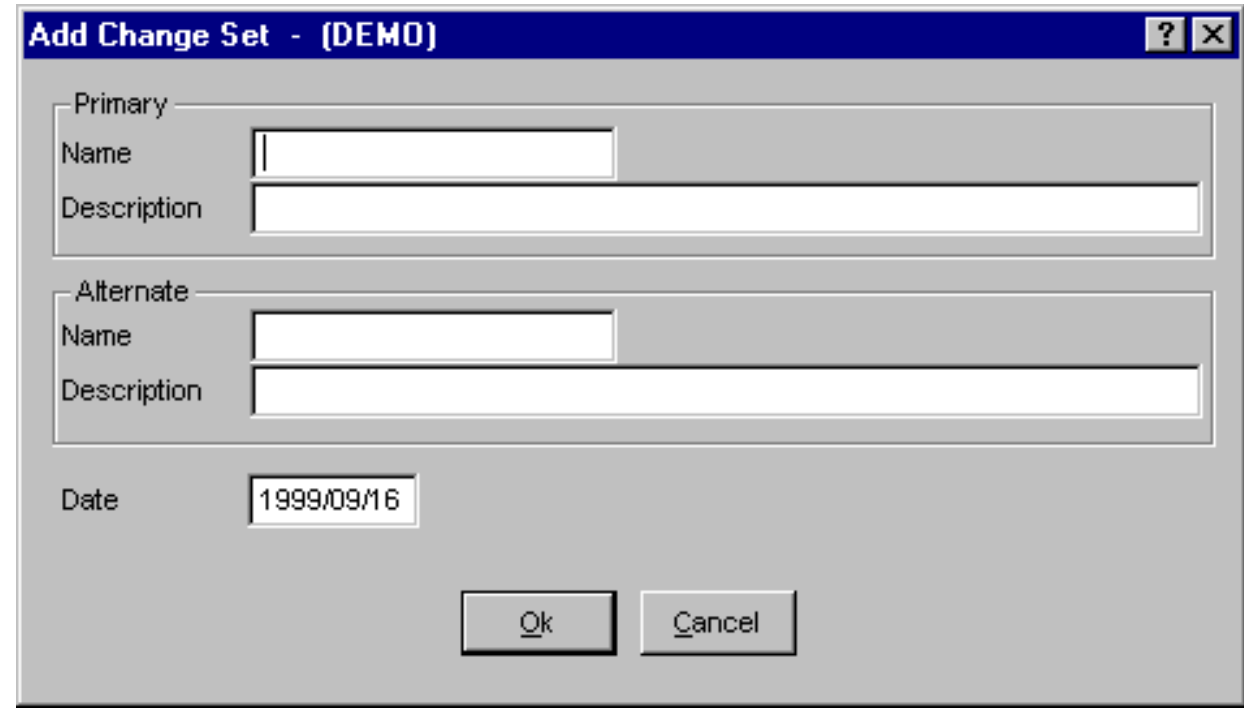

Ok - Save the new change set, and close the dialog. If the add operation is successful, the message, "Record added," is displayed in the message bar. If a change set record already exists with the same name, the message, "Duplicate record name - not added," is displayed in the message bar and the Add Change Set dialog remains open.

Cancel - Close the dialog without saving. A new change set record is not added to the database. 
By default the set type is a Change Set and the Read-only attribute is not selected. These fields are not available on the Add Change Set dialog. If you wish to alter the set type or read-only attribute, you must Modify the change set.

\subsection{Modifying a Change Set}

\section{PURPOSE}

This option allows you to modify change set record data. In addition to editing the change set name, description, and date, you can also edit the set type and read-only attribute.

\section{STEPS}

1. From the SAPHIRE menu select Modify | Change Sets. The Change Sets dialog is displayed. $O R$

2. From the SAPHIRE menu select Generate. The Generate dialog is displayed.

3. Highlight the change set you wish to edit.

4. Right-click to invoke the pop-up menu and choose Modify. The Modify Change Set dialog is displayed.

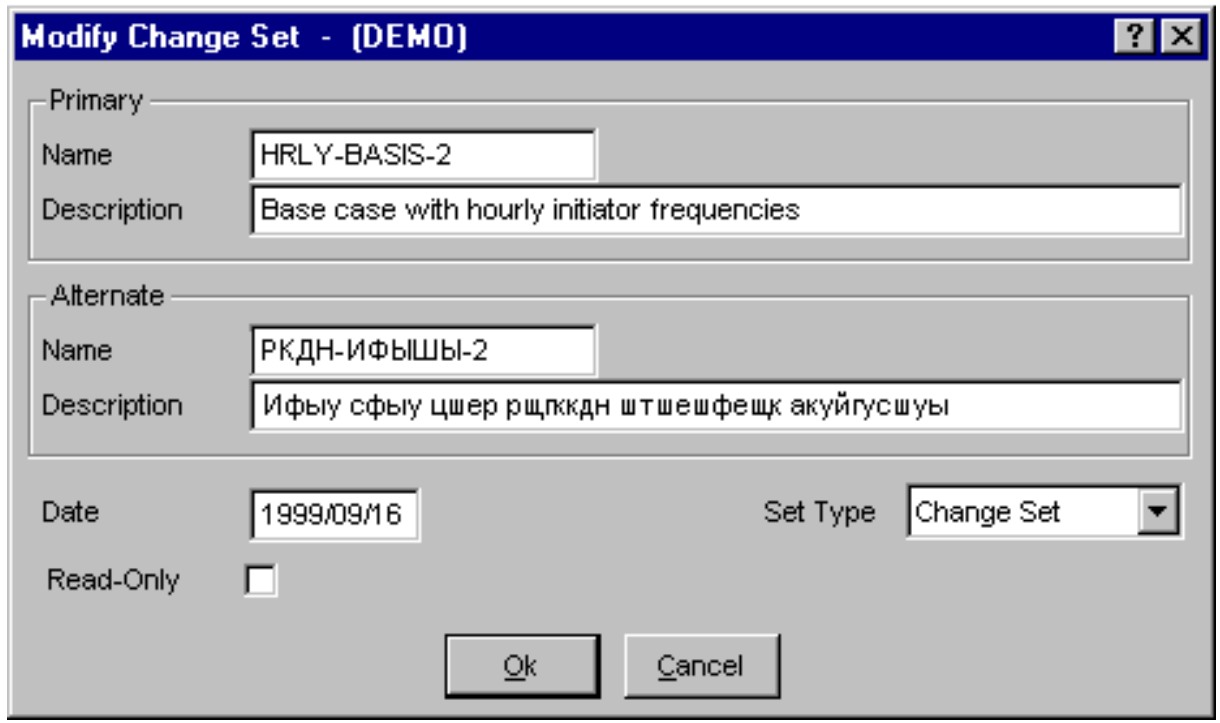

Ok - Save the modified change set and close the dialog. If the modify operation is successful, the message, "Record modified," is displayed in the message bar. If a change set record already exists with the same name, the message, "Revised name is a duplicate - not modified," is displayed in the message bar and the Modify Change Set dialog remains open.

Cancel - Close the dialog without saving the modifications. 


\subsection{Copying a Change Set}

\section{PURPOSE}

This option allows you to create a new change set record based on the highlighted one. The original change set name is displayed in the Copy From: field. You MUST enter a unique name for the new change set. Along with the source change set's name, description, and date, probability and class changes are also duplicated.

\section{STEPS}

1. From the SAPHIRE menu select Modify | Change Sets. The Change Sets dialog is displayed. OR

2. From the SAPHIRE menu select Generate. The Generate dialog is displayed.

3. Highlight the change set you wish to copy.

4. Right-click to invoke the pop-up menu and choose Copy. The Copy Change Set dialog is displayed.

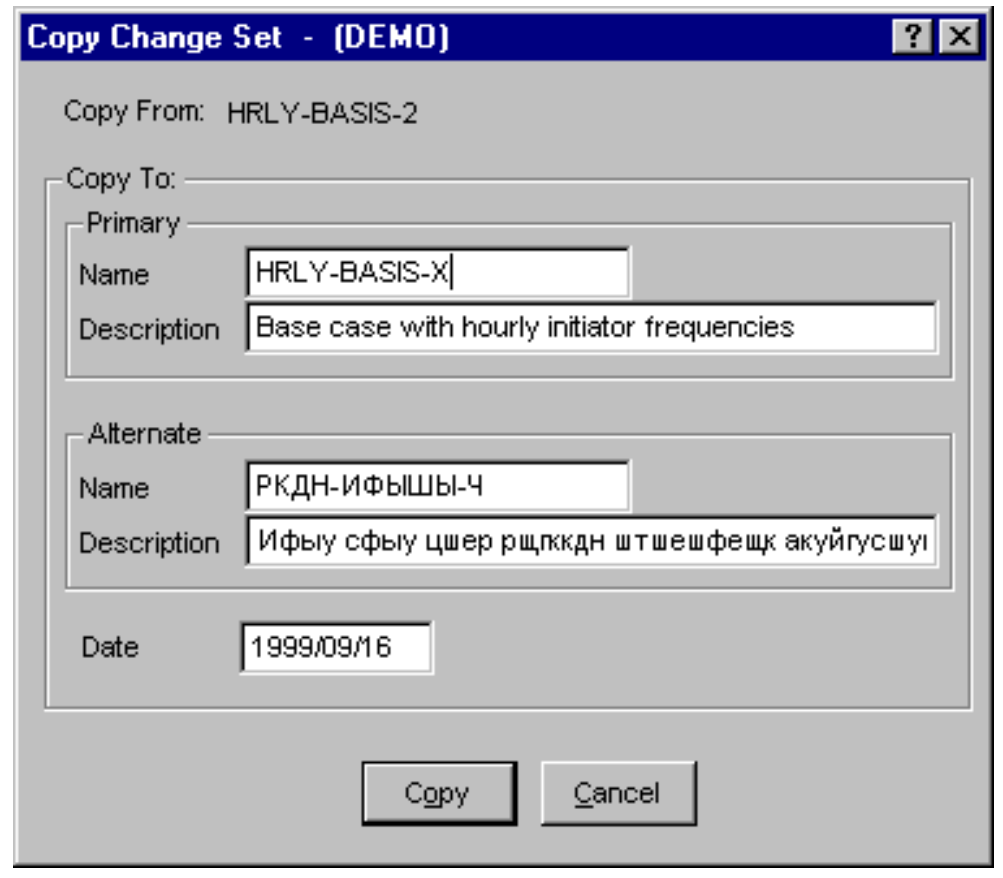

Copy - Save the newly created change set and close the dialog. If the copy operation is successful, the message, "Record added," is displayed in the message bar. If a change set record already exists with the same name, the message, "Duplicate record name - not added," is displayed in the message bar and the Copy Change Set dialog remains open.

Cancel - Close the dialog without saving the change set. A new change set record is not added to the database. 
The set type and read-only flag of the source (original) change set will be duplicated in the destination (new) change set along with the basic event data changes. If you wish to alter the set type or read-only attribute, you must Modify the change set record. If you wish to alter the basic event data changes, you must choose the Class or Single buttons from the Generate dialog.

\subsection{Deleting a Change Set}

\section{PURPOSE}

This option allows you to remove the highlighted change set record from the database.

\section{STEPS}

1. From the SAPHIRE menu select Modify | Change Sets. The Change Sets dialog is displayed. $O R$

2. From the SAPHIRE menu select Generate. The Generate dialog is displayed.

3. Highlight the change set you wish to delete.

4. Right-click to invoke the pop-up menu and choose Delete.

5. A message box will be displayed as shown below.

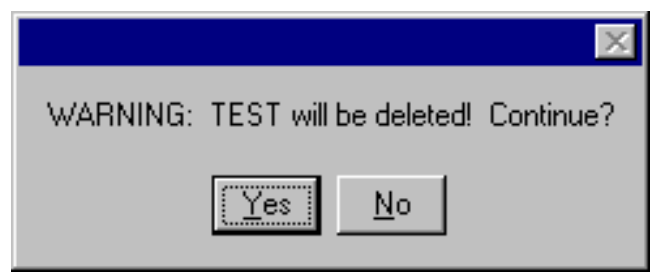

Yes - Perform the delete operation. The selected record will be removed from the database. The message, "Record deleted" is displayed in the message bar.

No - Cancel the delete operation. The selected record will NOT be removed from the database.

\subsection{Change Set - Marking and Processing Order}

\section{PURPOSE}

Change sets are "marked" to determine which change sets will be used to generate current case data. Order of marking determines which event changes take precedence. This option acts in the manner of a toggle.

\section{Marking Change Sets:}

\section{STEPS}

1. From the SAPHIRE menu select Modify | Change Sets. The Change Sets dialog is displayed. $O R$

2. From the SAPHIRE menu select Generate. The Generate dialog is displayed.

3. Double-click on the desired change set to mark or unmark it. 
If the selected change set was not previously marked, a numeric value (1 - 99) will appear to the left of the change set name, indicating the order of marking. The change sets in the list are rearranged by marked order, then alphabetically by type: first by condition assessment, then initiating event assessment type, finally by ordinary change set type.

If the change set was previously marked, it is unmarked (i.e., the numeric value is removed) and it is repositioned in the list.

If no change sets are marked when you select the Generate option, then the current case data will be initialized to the base case data.

\section{Change Set Processing:}

During change set processing (i.e., generating event data or updating base case values), three criteria are evaluated: probability changes, class changes, and the order of marked change sets.

If an event in a marked change set has both a class change and a probability change associated with it, the probability change takes precedence over the class change if any discrepancies arise..

If more than one change set is marked, then the probability and class changes in the change sets marked with the highest number ( 2 is higher than 1, 3 is higher than 2 ) will take precedence over any changes from lower numbered change sets. For example, suppose that Event A has a probability change associated with it in Change Set 1, and a class change associated with it in Change Set 2, then the class change from Change Set 2 would overwrite the probability change from Change Set 1.

The following example illustrates the outcome of processing three marked change sets with four events:

- $\quad$ Event A has both a probability change and a class change associated with it in Change Set 2.

- $\quad$ Event B has a probability change in Change Set 1, and a class change in Change Set 3.

- $\quad$ Event $\mathrm{C}$ has only a class change in Change Set 1.

- $\quad$ Event D has no probability changes or class changes associated with it in any of the three marked change sets.

The outcome of the Generate operation on these three marked change sets would be as follows:

- $\quad$ The current case data for event A would be set to the values associated with the probability change in Change Set 2. (Probability changes take precedence over class changes when they occur for the same event within the same change set.)

- $\quad$ The current case data for event B would be set equal to the values associated with the class change in Change Set 3. (The probability or class change in higher numbered change sets take precedence over changes in lower numbered change sets.)

- $\quad$ The current case data for event $\mathrm{C}$ would be set to the values associated with the class change in Change Set 1.

- $\quad$ The current case data for event $\mathrm{D}$ would be set to the base case values for event $\mathrm{D}$. (There were no probability changes or class changes made to event $\mathrm{D}$ in any of the marked change sets.) 


\subsection{Reset Probability to Base}

\section{PURPOSE}

This option allows you to reset the probability changes (not class changes) for selected events back to the base case values. You may reset probability changes for a single event, a group of events, or all events.

\section{STEPS}

1. Choose Generate from the menu. The Generate dialog will be displayed.

2. Highlight the desired change set.

3. Choose the Single button. The Selected Events dialog will be displayed.

4. Highlight the basic events you wish to modify.

5. Right-click to invoke the pop-up menu and choose Reset.

6. A message box will be displayed as shown below.

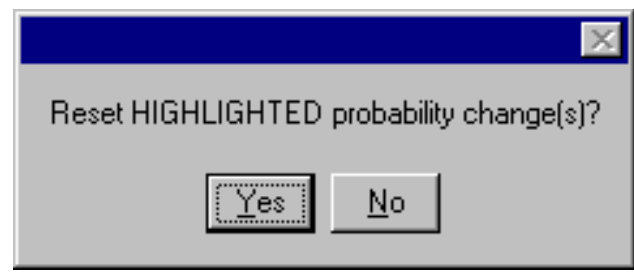

Yes - Reset the probability back to the base case value.

No - Terminate the reset operation.

\subsection{Generating Changes}

\section{PURPOSE}

This option applies the event data modifications specified by the marked change sets to the current case basic event data. This option must be executed before any data analysis if you wish the change set modifications to be reflected in the analysis results. The Generate option creates new current event data, which is used when a user wishes to perform an analysis.

If an event in the change set has both a class change and a probability change associated with it, the probability change takes precedence over the class change when generating new current case event data values. If the event does not have an associated probability change or class change, then the current case event data values will be set equal to the base case data for that event.

\section{STEPS}

1. Choose Generate from the menu.

2. From the Generate dialog, mark the desired change set(s).

3. Change the Mission Time, if desired.

4. Choose the Generate button. 
"New event data has been generated with changes" is displayed when the changes are successfully generated.

\subsection{Reporting Change Set Data}

\section{PURPOSE}

Reports can be created that reflect the event modifications that currently exist within the basic event change data file. There are three report types: Unaffected Events, Affected Events, and All Events. Each of the report types may be sorted by event name, ascending probability, or change sets. In addition, a report may be routed to the Report Viewer, a printer, or a disk file.

\section{STEPS}

1. Choose Generate from the menu. The Generate dialog will be displayed.

2. Highlight the desired change set(s).

3. Choose the Report button.

If changes have been made to the selected change sets, but the Generate operation has not been performed, a warning dialog will be displayed. Otherwise, the Event Change Report dialog will be displayed.

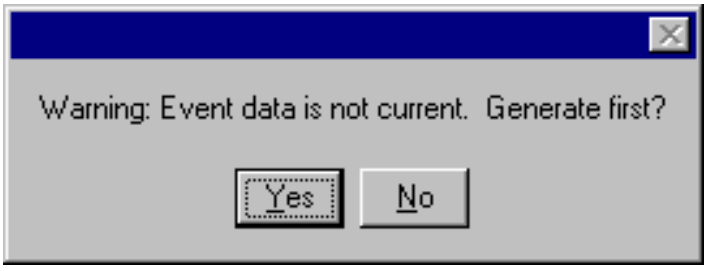

Yes - Automatically perform the generate operation before displaying the Event Change Report dialog.

No - Display the Event Change Report dialog without first generating the changes. The report will present the basic event change data file as it currently exists. However, this file does not reflect the change set modifications made after the last generate operation. 


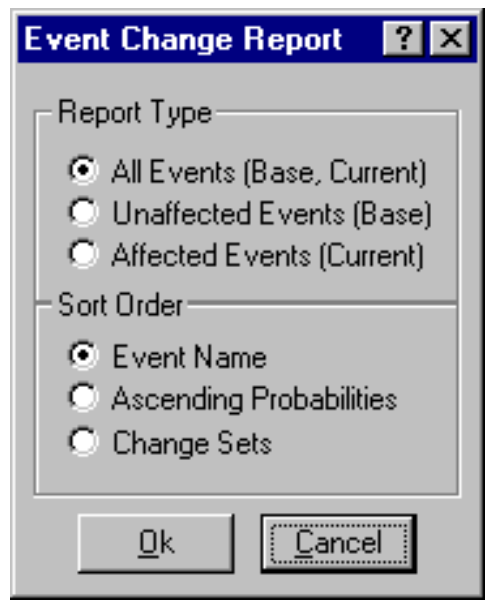

Report Type options:

All Events (Base, Current) -

Unaffected Events (Base) -

Affected Events (Current) -

Sort Order options:

Event Name -

Ascending Probabilities -

Change Sets -
Generate a report which includes all basic events in the database.

Generate a report with the events that are not included in any of the change sets for which current event data has been generated.

Generate a report with the events whose current case value has been changed from the base case. These events are included in one or more change sets that have been marked and the basic event data generated.

Show the events included by the Report Type selection ordered by event name.

Show the events included by the Report Type selection in ascending order by the current probability value.

Show the events included by the Report Type selection by marked change set order.

The report generated by this option shows the basic event name, and current values for calculation type, lambda value, tau value, mission time, probability value, change set name, and type of change applied.

Ok - Generate the report based on the selected options. The Report Viewer will display the report.

Cancel - Close the Event Change Report dialog without creating a report.

\subsection{Class Changes}

\section{PURPOSE}

This option allows you to change event data parameters for a specified grouping of events in a change set.

\section{STEPS}

1. Choose Generate from the menu. 
2. From the Generate dialog, highlight the desired change set and choose the Class button.

3. The Edit Event Class dialog will be displayed.

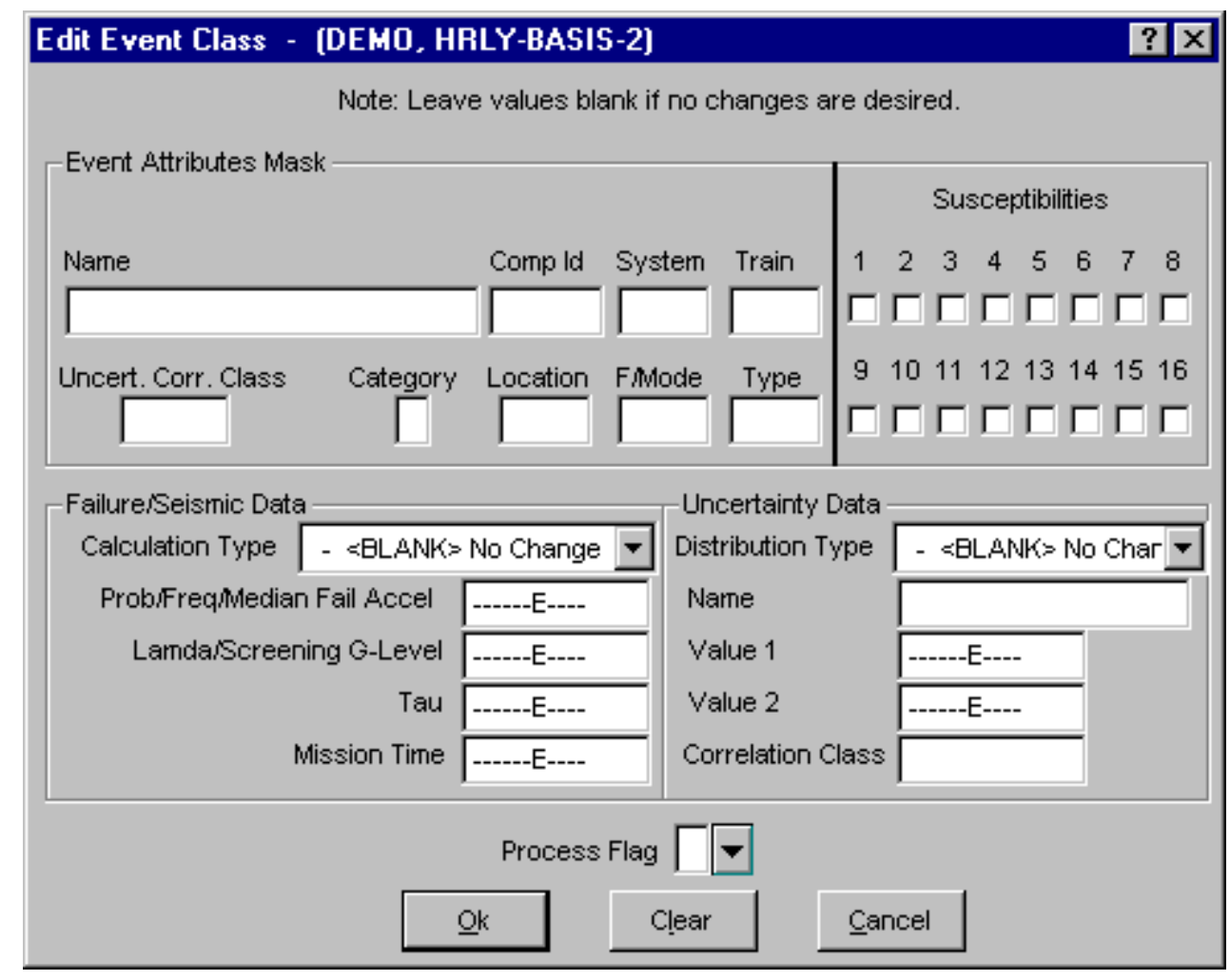

The event class is defined by entering some search criteria in the mask fields. The more of these fields that are filled in, the finer the class definition becomes.

Ok - $\quad$ Save the class mask and close the Edit Event Class dialog.

Clear - Clear the fields and close the Edit Event Class dialog.

Cancel - Close the Edit Event Class dialog without saving the mask.

It is important to remember that probability changes have a higher priority than class changes. When both are applied to an event, the probability change will be used during an analysis.

\subsection{Single Event Probability Changes}

\subsubsection{Single Changes}

\section{PURPOSE}

This function allows the user the flexibility to experiment with setting individual basic event failure, uncertainty, and seismic fragility data. These data values may be set for a single event or for a selected group of events. This current data can then be applied to the specified event(s) for use during the analysis. The single change set type also provides a reset option to set data values back to the base case values. 


\section{STEPS}

1. Choose Generate from the menu. The Generate dialog will be displayed.

2. Highlight the desired change set and choose the Single button.

3. The Selected Events dialog will be displayed.

This dialog lists the event name and description of each event included in the change set. In addition, the dialog shows the event usage and change set flags. The usage column (i.e., SLF) indicates whether the event has been marked or not used and whether the event is used in the base case, current, or both. The event usage flag is supplied for sequence cut sets ("S"), fault tree logic ("L"), or fault tree cut sets ("F"). The change set flag indicates if the event has been marked for a probability (single) change ("P"), a class change ("C") or both ("c"). The change set name is shown in the title bar of this dialog.

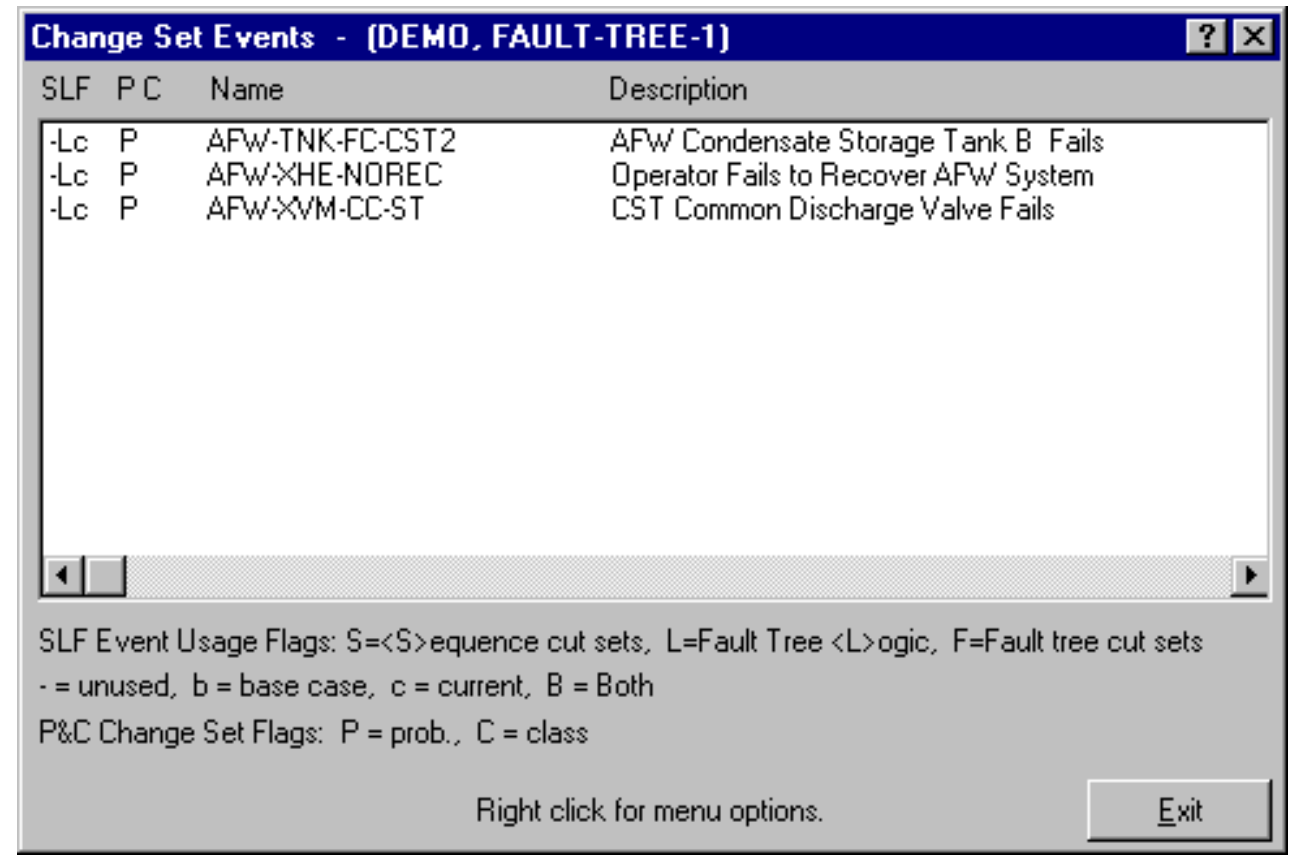

Add - Add basic event(s) to this change set and change their uncertainty, failure data, and/or seismic fragility values.

Modify - Modify uncertainty, failure data, and/or seismic fragility values of basic event(s) in this change set.

Reset - Reset changes for selected event(s) back to the base case values.

\subsubsection{Add an Event Change}

\section{PURPOSE}

This option allows you to add a basic event change to the currently selected change set. 


\section{STEPS}

1. Choose Generate from the menu. The Generate dialog will be displayed.

2. Highlight the desired change set and choose the Single button. The Selected Events dialog will be displayed.

3. Right-click to invoke the pop-up menu and choose the Add option.

4. The Select Event dialog will be displayed.

\begin{tabular}{|c|c|c|}
\hline Select Change Event & - [Right click for options] & $x$ \\
\hline $\begin{array}{l}\langle\text { FALSE }\rangle \\
\langle\text { INIT }\rangle \\
\langle\text { PASS }\rangle \\
\langle\text { TRUE }\rangle \\
\text { AFW } \\
\text { AFW-CST-TANK } \\
\text { AFW-MDP-A-F } \\
\text { AFW-MDP-B-F } \\
\text { AFW-MDP-CF-AB } \\
\text { AFW-MDP-FC-1A } \\
\text { AFW-MDP-FC-1B } \\
\text { AFW-PMP-CF-ALL } \\
\text { AFW-SUP } \\
\text { AFW-TDP-C-F }\end{array}$ & $\begin{array}{l}\text { System Generated Success Event } \\
\text { System Generated Initiating Event } \\
\text { System Generated Ignore Event } \\
\text { System Generated Failure Event } \\
\text { No or Insufficient AFW Flow } \\
\text { CST system fails } \\
\text { MDP train-A supercomponent } \\
\text { MDP train-B supercomponent } \\
\text { Common Cause Failure of AFW Motor Driven Pumps } \\
\text { AFW Motor Driven Pump 1A. Fails } \\
\text { AFW Motor Driven Pump 1B Fails } \\
\text { Common Cause Failure of AFW' Pumps (All Types] } \\
\text { AFW system fails } \\
\text { TDP train-A supercomponent }\end{array}$ & 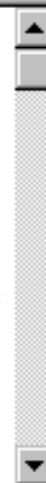 \\
\hline 4 & & \\
\hline
\end{tabular}

5. Highlight the event(s) to be added to the change set.

6. Right-click to invoke the pop-up menu and choose the Add option.

If only a single event has been selected, then the Event Probability Changes dialog for single event changes will be displayed. If a group of events were selected, then the Event Probability Changes dialog for a group of events will be displayed.

The probability changes made are reflected on the Selected Events dialog by showing a "P" to the left of the event name. Probability changes have a higher priority than class changes. When both are applied to an event, the probability change will be used during fault tree/sequence analysis. This is indicated by flagging the affected events with a "P" and "c".

\subsubsection{Modify an Event Change}

\section{PURPOSE}

This option allows you to modify probability changes for the selected basic event(s) in the current change set.

\section{STEPS}

1. Choose Generate from the menu. The Generate dialog will be displayed.

2. Highlight the desired change set and choose the Single button.

3. The Selected Events dialog will be displayed.

4. Highlight the desired basic event(s).

5. Right-click to invoke the pop-up menu and choose Modify. 
If only a single event has been selected, then the Event Probability Changes dialog for single event changes will be displayed. If a group of events were selected, then the Event Probability Changes dialog for a group of events will be displayed.

The probability changes made are reflected on the Selected Events dialog by showing a "P" to the left of the event name. Probability changes have a higher priority than class changes. When both are applied to an event, the probability change will be used during fault tree/sequence analysis. This is indicated by flagging the affected events with a "P" and "c".

\subsubsection{Event Probability Changes - Individual}

\section{PURPOSE}

This function allows you to temporarily change the current uncertainty, failure data, and seismic fragility values for the selected basic event. This current data can then be applied to the specified event for use during event tree analysis.

\section{STEPS}

1. Choose Generate from the menu. The Generate dialog will be displayed.

2. Highlight the desired change set.

3. Choose the Single button. The Selected Events dialog will be displayed.

4. Highlight the basic event you wish to modify.

5. Right-click to invoke the pop-up menu and choose Add or Modify.

6. The Event Probability Changes dialog for individual event changes will be displayed. 


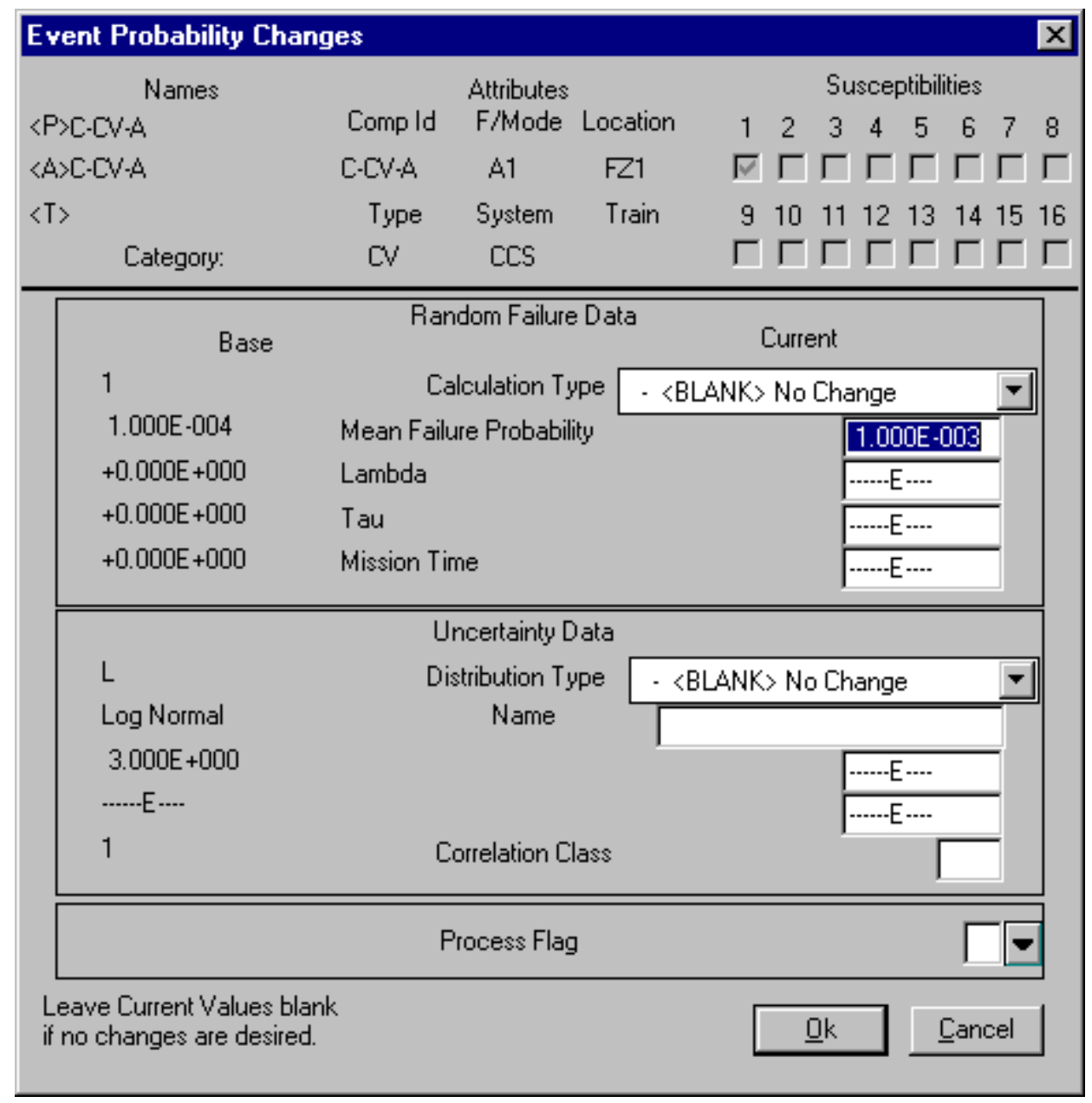

The dialog is divided into five data areas: Event Attributes, Random Failure Data, Uncertainty Data, Process Flag, and Seismic Fragility.

The Event Attributes data section is for information only; no changes may be made to the data fields in this area.

The Failure Data and Uncertainty Data areas display both the base case and current data values. You may change only the current data values.

Ok - $\quad$ Save the basic event probability changes and close the Event Probability Changes dialog.

Cancel - Close the Event Probability Changes dialog without saving the changes.

\subsubsection{Event Probability Changes - Group}

\section{PURPOSE}

This function allows you to temporarily change the current uncertainty, failure data, and seismic fragility for a group of selected basic events. This current data can then be applied to the specified events for use during event tree analysis. 


\section{STEPS}

1. Choose Generate from the menu. The Generate dialog will be displayed.

2. Highlight the desired change set.

3. Choose the Single button. The Selected Events dialog will be displayed.

4. Highlight the basic events (two or more) you wish to modify.

5. Right-click to invoke the pop-up menu and choose Add or Modify.

6. The Event Probability Changes dialog for group event changes will be displayed.

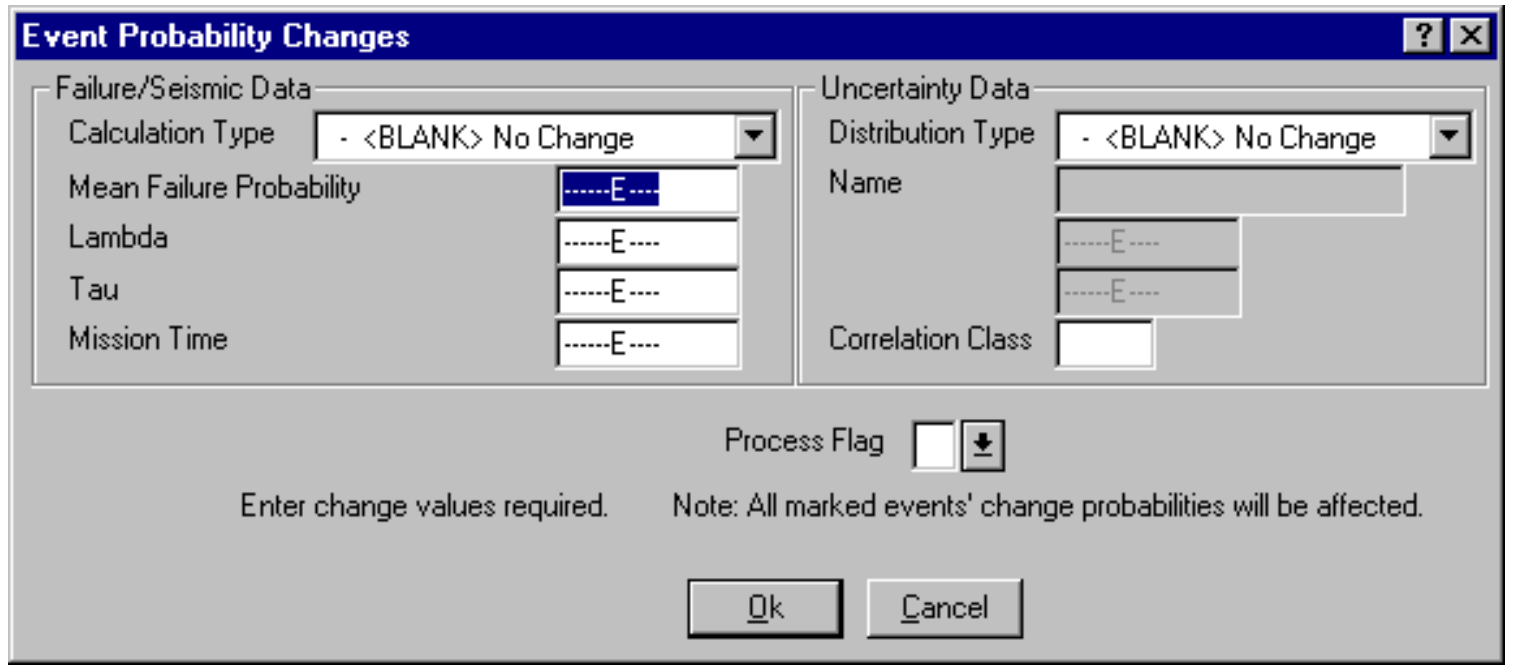

This dialog is divided into three areas: Failure Data, Uncertainty Data, and Process Flag..

The changes entered on this dialog will be applied to all selected events.
Ok -
Save the basic event probability changes and close the Event Probability Changes dialog.
Cancel - $\quad$ Close the Event Probability Changes dialog without saving the changes.

\subsubsection{Calculation Types}

In the Failure Data section the calculation type is a numerical reference to the calculation method to be used. Choose the desired calculation type from the drop-down list. An equation for each calculation type follows. See the symbol table for more details.

$1 \quad \mathrm{P}=\mathrm{p}$. 
The value specified in the probability field is directly used as the basic event failure probability or initiating event frequency

$3 \quad \mathrm{P}=1-\operatorname{Exp}\left(-\mathrm{L} * \mathrm{t}_{\mathrm{m}}\right)$.

This calculation type is the full equation for the failure probability of an operating component without repair in a non-demand failure mode.

$5 \mathrm{P}=([\mathrm{L} * \mathrm{~T}] /[1+\{\mathrm{L} * \mathrm{~T}\}]) *\left(1 \mathrm{EXP}\left[(\mathrm{L}+1 / \mathrm{T}) * \mathrm{t}_{\mathrm{m}}\right]\right)$.

This calculation type is the full equation for the failure probability of an operating component with consideration given to the ability to repair the component.

$7 \quad \mathrm{P}=1+(\operatorname{EXP}[\mathrm{L} * \mathrm{~T}] 1) /(\mathrm{L} * \mathrm{~T})$.

This calculation type is the full equation for the failure probability of a standby component in a non-demand failure mode with consideration given to periodic testing.

$8 \quad \mathrm{P}=\mathrm{bp}+\mathrm{p}$.

This calculation type is available only in change sets. It indicates the value given in the change set probability field is to be added to the base case probability value.

$9 \quad \mathrm{P}=\mathrm{bp} * \mathrm{p}$.

This calculation type is available only in change sets. It indicates the value given in the change set probability field is to be multiplied with the base case probability value.

$\mathrm{T} \quad \mathrm{P}=1.0$ (House event - failed).

This calculation type indicates that the basic event is to be treated as a house event that is always failed. A house event never appears in the minimal cut sets. The model is modified to reflect the logic, given that the indicated basic event is always failed. To do this for an event that is guaranteed to occur (failure probability $=1.0$ ), the event is removed from the logic where it appears as an input to an AND gate. If the basic event is input to an OR gate, the entire gate and its inputs are removed from the logic. The resulting minimal cut sets show the failure combinations that must occur for top event or sequence failure given that the indicated basic event is always failed.

F $\quad \mathrm{P}=0.0$ (house event - successful).

This calculation type indicates that the basic event is to be treated as a house event that is never failed. A house event never appears in the minimal cut sets. The model is modified to reflect the logic given that the indicated basic event is never failed. To do this for an event that is guaranteed successful, the basic event is removed from the logic where it appears as an input to an OR gate. If the basic event is input to an AND gate, the entire gate and its inputs are removed from the logic. The resulting minimal cut sets show the failure combinations that must occur for top event or sequence failure given that the indicated basic event can never fail.

I $\quad \mathrm{P}=0.0$ (ignore event).

This calculation type indicates that the basic event is to be treated as if it did not exist in the logic for the fault tree. Before the tree is solved, the logic is edited to remove all references to the specified event from the fault tree.

$\mathrm{S} \quad \mathrm{P}=0.0$ (find a fault tree with the same name and use its current mincut upper bound as the probability)

This calculation type indicates that the basic event is to replace its matching fault tree. If no matching fault tree exists, the probability will be set to 0.0 .

E $\quad \mathrm{P}=0.0$ (find an end state tree with the same name and use its current mincut upper bound as the probability)

This calculation type indicates that the basic event is to use its matching end state value. If no matching end state exists, the probability will be set to 0.0. This type is useful in level two analysis. 
$\mathrm{G} \quad \mathrm{P}=\mathrm{II}[\ln (\mathrm{g} / \mathrm{a}) / \mathrm{Br}]$.

This calculation type indicates that the basic event is to be treated as a seismic event. The probability value for screening will be calculated using the ground acceleration, failure acceleration, and $\mathrm{Br}$ entered by the user.

$\mathrm{H} \quad \mathrm{P}=\mathrm{II}[\ln (\mathrm{g} / \mathrm{a}) / \mathrm{Br}]$.

This calculation type indicates that the basic event is to be treated as a seismic event. The probability for screening will be calculated from the failure acceleration, $\mathrm{Br}$, and ground acceleration. The ground acceleration will be the highest g-level specified in the medium project hazard curve.

$\mathrm{V} \quad \mathrm{P}=\mathrm{N} / \mathrm{A}$ (not applicable)

The event is a "value" event and will not appear directly in fault tree or sequence logic. The value specified in the Value field is not necessarily a probability or frequency, but is rather an input value to a compound event.

B $\quad \mathrm{P}=\mathrm{bp} /$

This option is only available for use in a change set. It indicates that the base case value is to be used. This is useful when multiple change sets are marked and it is desired to override a previous change.

C $\quad \mathrm{P}=\mathrm{F}(\mathrm{x})$.

The event is a compound event whose probability/frequency value is defined as a function of other input values, formulas, and/or events (value events and regular basic events). This option is not currently available in change sets, since compound event data cannot be added via a change set (but it can be removed via a change set).

$\mathrm{X} \quad \mathrm{P}=\mathrm{F}(\mathrm{x})$

This calculation type indicates that the event is a human action and will use built-in worksheets to calculate the human error probability (HEP). For each event, human performance shaping factors can be applied in the worksheet to adjust the HEP probability. Performance shaping factors include: stress/stressors, available time, complexity, experience/training, procedures, ergonomics, fitness for duty, and work processes.

\subsubsection{Calculation Type Equation Symbols}

The following symbols are used in the equations for calculating failure probability:

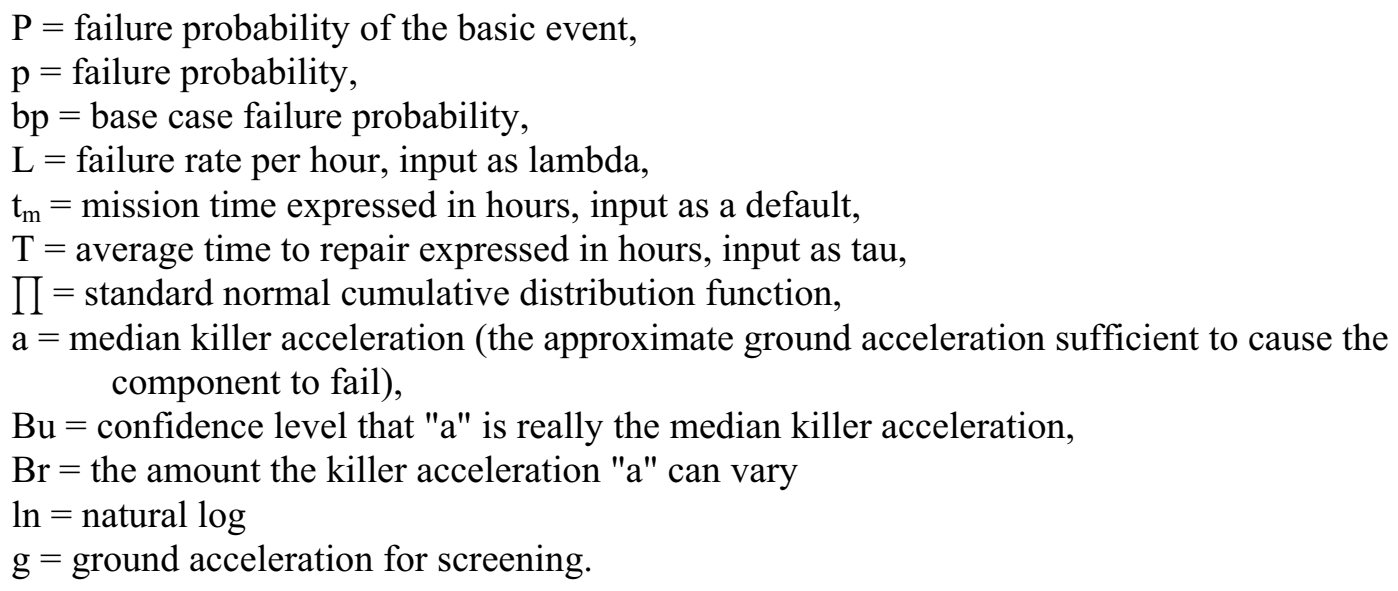




\subsubsection{Distribution Types}

For the Uncertainty Data section, there are nine predefined distribution types available. The predefined distribution types are:

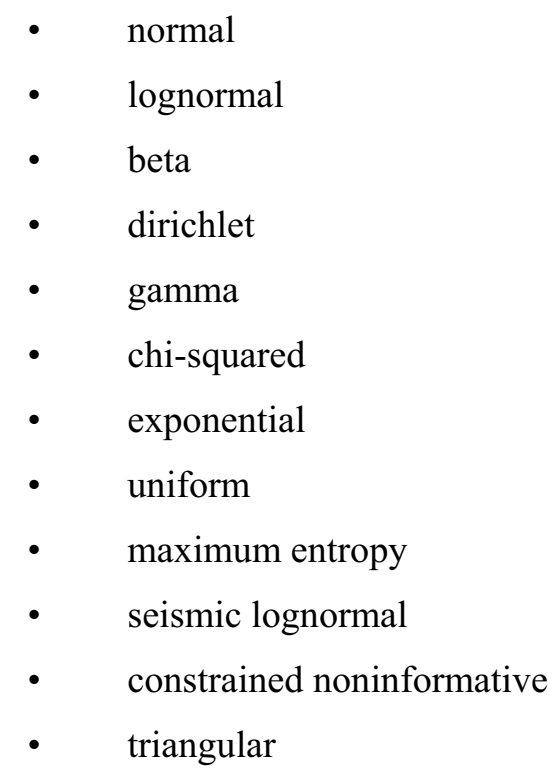

In addition to these predefined distribution types, user-defined histograms may be used. The default distribution type is no distribution. Choose the desired distribution type from the drop-down list.

\subsubsection{Process Flag}

The Process Flag is a one-character field that specifies if certain processes should take special note of the selected event. Select one of the following values: 


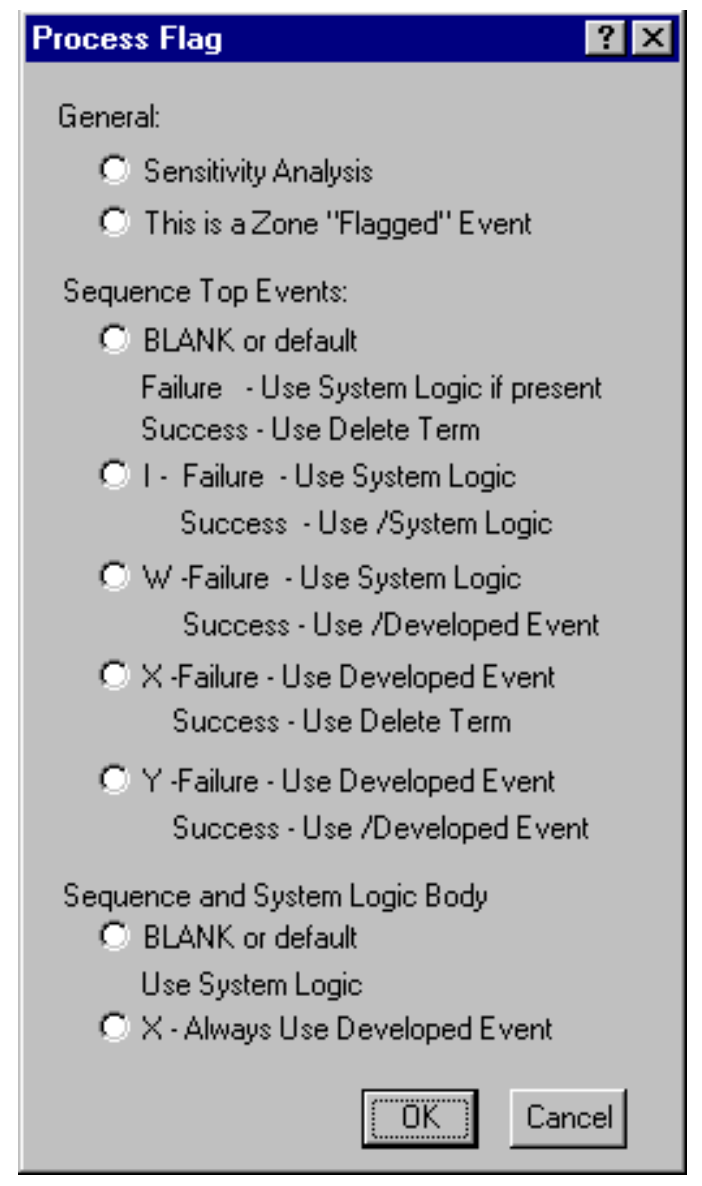

\subsubsection{Process Flag Dialog}

\subsubsection{Sensitivity Analysis}

If an event is marked for sensitivity analysis, SAPHIRE will map a results frequency or probability plot. A sensitivity analysis allows you to see how sensitive the frequency or probability is in relation to an event.

\subsubsection{Zoned Flagged Event}

A zone flagged event is an event that has been marked as representing a zone (i.e., location or area). An example of a zone is a fire zone or a flood zone. When SAPHIRE encounters a zone flagged event, it performs a transformation. A transformation is an event or set of events that replace a zone flagged event.

\subsubsection{Process Flag ' $I$ '}

Use fault tree logic (if top event fails), use the complement of the fault tree logic (if top event succeeds). That is, if the top event is a failure, SAPHIRE will expand the fault tree and solve; if the event succeeds, SAPHIRE will complement the fault tree logic and solve it. An "I" causes SAPHIRE to treat the transfer as independent. Logic below this transfer is expanded for failure references, and for success references the complement of the logic is used. 


\subsubsection{Process Flag ' $W$ '}

Use fault tree logic (if top event fails), use complement of the developed event (if top event succeeds). That is, if the event fails SAPHIRE will expand the fault tree and solve; if the event succeeds, SAPHIRE will use the complement of the developed event for the fault tree.

\subsubsection{Process Flag ' $X^{\prime}$}

Use developed event (if event fails), use cut set matching to eliminate cut sets (if event succeeds). That is, an "X" tells SAPHIRE that the top event is to be used for failure references, but success references are to be treated the same as if the flag was blank.

\subsubsection{Process Flag ' $Y^{\prime}$}

Use developed event (if event fails), use complement of developed event (if event succeeds). That is, a "Y" indicates that a transfer is to be replaced with its basic event for failed references and the complement of the event is to be used for success references.

\subsubsection{Process Flag ' $X$ '}

Always use developed event. That is, do not expand the fault tree or transfer. Instead, simply use the event probability.

\subsubsection{Blank or Default}

When the Process Flag field is blank, the transfer logic associated with this event is expanded for failure references. For success references, the transfer is also expanded; however, any "impossible" cut sets (i.e., those both failing and succeeding) are removed from the resulting failure cut sets using cut set matching (also known as the "delete term" process). 


\section{FAULT TREE ANALYSIS}

\subsection{Fault Tree Analysis}

\section{PURPOSE}

A fault tree is a model of a system that is suitable for probabilistic analysis. The model is usually tailored for the study of the system's failure.

This option allows you to build, edit and analyze fault tree models. You are provided with the means to recalculate fault tree values after events and/or cut sets have been modified. The Fault Trees List shows the fault trees in the current project.

\section{KEY TO FLAGS}

Located to the right of each fault tree is a set of flags that indicate the status of the fault tree:

C - $\quad$ both current and base case cut sets exist for the selected analysis type - only base case cut sets exist for the selected analysis type

c - $\quad$ only current case cut sets exist for the selected analysis type

G - both logic and graphics exist for the fault tree

L - only logic exists for the fault tree

g - only graphics exist for the fault tree

\section{STEPS}

1. From the SAPHIRE menu select Fault Tree.

2. The Fault Trees List dialog will be displayed. 


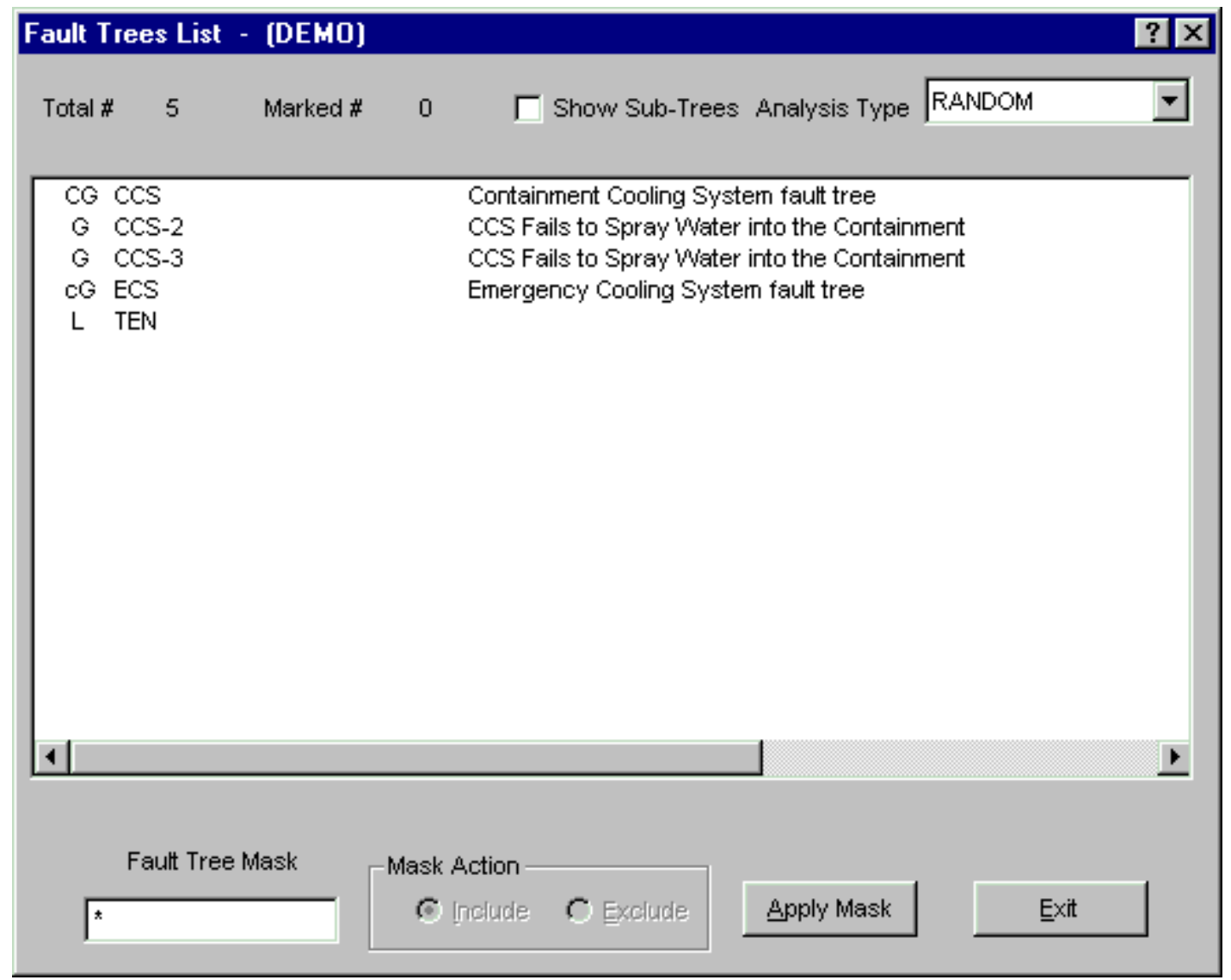

From this dialog, you can select fault trees using the mouse or the Mask feature.

Analysis type -

Show Sub-Trees -

Pop-up Menu Options - The following functions may be accessed from the pop-up menu,

Add Fault Tree Edit Graphics -

Edit P\&ID -

Edit Logic -

Page Tree -

Solve -

Quantify -

Uncertainty depending on whether or how many fault trees are highlighted.

Select the type of analysis from the drop-down list.

When checked, include sub-trees in the list of fault trees. Only logical and graphical pop-up options are available when this option is selected

Add a new fault tree record to the current project database.

Construct and modify fault tree diagrams using the graphical Fault Tree

Editor.

Construct and modify piping and instrumentation diagrams using the Piping \& Instrumentation Diagram Editor.

Modify the logic of a fault or sub-tree in an alphanumeric format.

Organize the fault tree logic into pages.

Generate cut sets for a fault tree based on cut set generation cut off values.

Calculate a new minimum cut set upper bound for fault tree cut sets using current data values (using event data changes and current case cut sets).

Perform uncertainty analysis on a fault tree using either the Monte Carlo or Latin Hypercube simulation technique. 
Cut Sets -

Display -

View Results -

Time Dependent -
Perform various operations on cut sets: Update, Prune, Recover, Edit. Display the results of fault tree analyses in various report forms. View fault tree analysis information and compare base case and current case minimal cut set upper bound results.

Perform time dependent fault tree analysis.

\subsection{Analysis Types}

SAPHIRE allows the user to store multiple analysis types in the same project. For instance, the user can perform a normal random analysis and then using the same database, perform a fire or flood analysis. Each analysis uses the same fault trees and failure data, but stores the results in a different location in the database. In SAPHIRE, there are eight reserved analysis types and eight user definable types.

The first six reserved types are "Random," "Fire," "Flood," "Seismic," "Event Assessment," and "Condition Assessment." The remaining two reserved types are undefined. When the user selects a different analysis type, the cut sets and other results generated for that analysis type are stored in a separate location. This ability allows the user to track all the results from a comprehensive analysis in the same database.

The default analysis type is "Random." This default value can be changed in the Utility | Define Constants | Analysis option.

Associated with the analysis type is the ability to perform event transformations. This ability is useful in doing vital area or location analysis. With this ability, the user is able to define transformations for a basic event. When this event is used in a fault tree, the user can then choose to transform this event according to their definitions. The analysis type corresponds to the basic event susceptibility type. If an event is "susceptible" to "Fire," then the user marks the event flag to indicate this. When a fault tree associated with this event is loaded, it will automatically be transformed if the current analysis type is one this event is "susceptible" to.

\subsection{Fault Tree Graphics}

\subsubsection{Fault Tree Graphics}

\section{PURPOSE}

The Fault Tree Editor provides the means to construct a fault tree diagram. A fault tree diagram is a graphical representation or picture of the fault tree model built from fault tree objects.

A fault tree is a model of a system that is suitable for probabilistic analysis. The model is usually tailored for the study of the system's failure. The fault tree logic structure can consist of any combination of the logic symbols that do not result in a logical loop. Fault tree logic can be thought of as a group or set equations that describe the possible failure combinations of the system.

\section{STEPS}

1. From the SAPHIRE menu select Fault Tree. The Fault Trees List dialog will be displayed. 
2. Highlight the desired fault tree. (Skip this step if creating a new fault tree.)

3. Right-click to invoke the pop-up menu and select the Edit Graphics option.

4. The Fault Tree Editor window will be displayed. The name of the current fault tree will be displayed in the title bar.

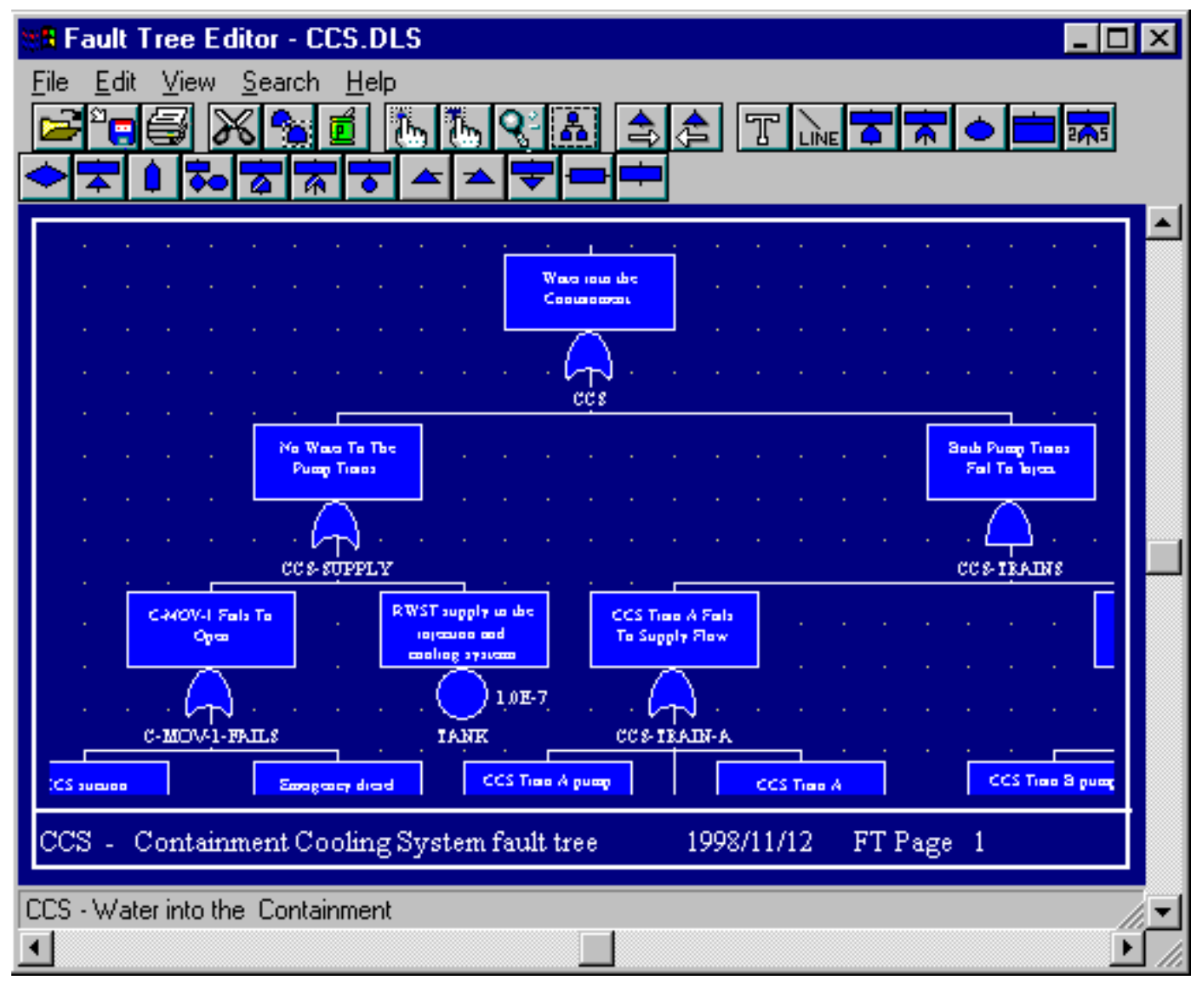

The Fault Tree Editor allows you to build the model using graphical symbols for gates, basic events, and connecting lines. These objects are tied to the SAPHIRE database. Various editing functions such as cut, paste, and copy are provided along with file functions such as save and print.

Options are available from the menu and/or the toolbar.

\section{Menu Options}

File - $\quad$ File manipulation functions such as open, save, print, etc.

Edit - Editing functions such as copy and paste along with attribute setting.

View - Control of visual display such as zoom, refresh, etc.

Search - Search and cursor positioning operations (e.g., Find and Find and Replace).

Help - SAPHIRE on-line help.

\section{Toolbar Buttons}

Open a fault tree graphics file from disk.

AND gate object 

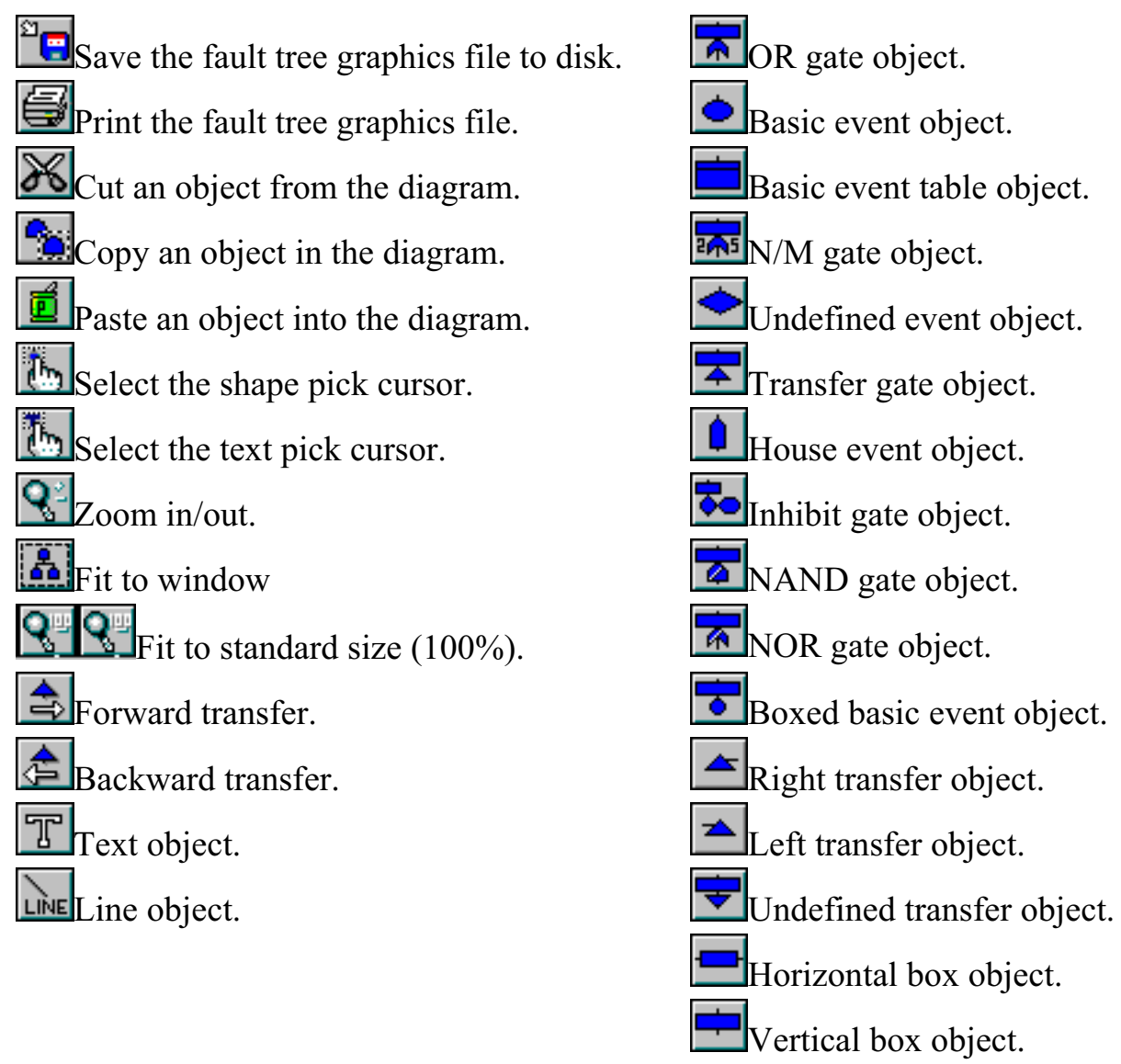

\subsubsection{Fault Tree Graphics - Definitions}

\section{PURPOSE}

The following definitions are used with reference to the SAPHIRE graphical Fault Tree Editor.
Objects -
The building blocks of a fault tree diagram. They include shapes, lines that connect shapes, and descriptive text. Each object has attributes that may include color, size, and name.
Pick Box - An invisible rectangle which surrounds every object. It defines the area of the object where the mouse cursor must be positioned in order to select the object.
Rubber band rectangle -A rectangle displayed on the screen showing the area currently selected during a drag function. This is used when selecting more than one object.

\subsubsection{Fault Tree Graphics - Mouse Usage}

All mouse actions defined in the SAPHIRE graphical Fault Tree Editor are for a standard right handed two-button mouse.

Click - $\quad$ The left mouse button is pressed and released.

Right-click - The right mouse button is pressed and released. 
Double-click - The left mouse button is pressed and released two times in rapid succession. Drag - $\quad$ The left mouse button is pressed and held down while moving the mouse.

End-drag - $\quad$ The left mouse button that was being held down during the $d r a g$ is released.

\subsubsection{Moving Objects}

\section{PURPOSE}

Move selected object(s) to a different location in the diagram.

\section{STEPS}

1. Select the desired object.

2. Drag the mouse to the new position.

3. End-drag.

While dragging the mouse, the Move cursor 5 ?

\subsubsection{Selecting Objects}

\section{PURPOSE}

Prior to manipulating a shape object (e.g., moving, copying, etc.) in the fault tree diagram, it must be selected. This topic describes how to select object(s).

\section{STEPS}

\section{To select a single object}

1. Choose the Pick button from the toolbar.

2. Move the cursor ( $\left(\mathrm{r}^{\mathrm{b}} \mathrm{b}\right)$ on top of the object you wish to select.

3. Click the mouse.

If the mouse is clicked while inside an object's pick box, that object is selected. A dashed line will appear surrounding the selected object. 


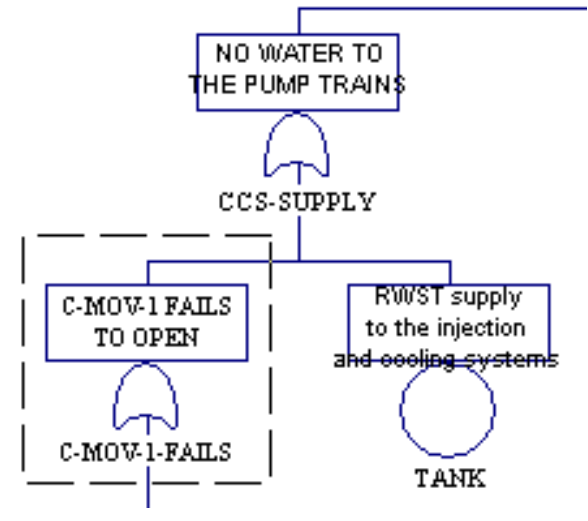

In the example here, the OR gate, C-MOV-1-FAILS, is selected.

\section{STEPS}

\section{To select a group of objects}

1. Choose the Pick button from the toolbar.

2. Starting outside of any pick box, drag the mouse until the rubber band rectangle encloses all the objects you wish to manipulate.

If, when you begin dragging the mouse, an object's pick box appears, you were inside that object's pick box. Find an area without any visible objects to begin the mouse drag operation.

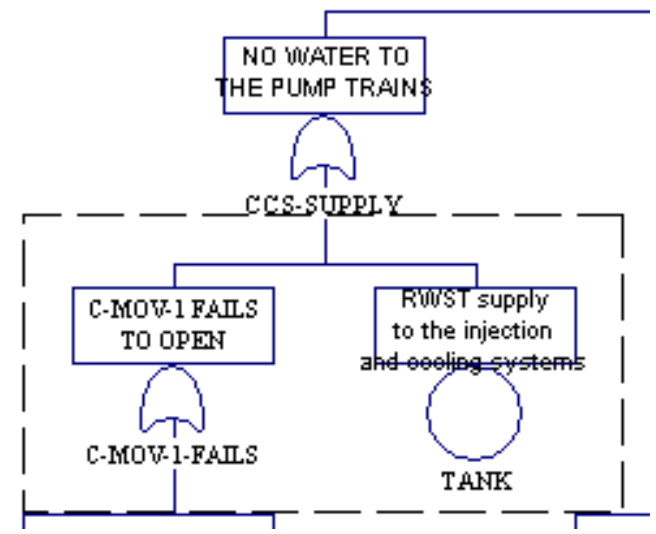

In the example here, the inputs to the CCS-SUPPLY gate, are selected.

NOTE: Because some object's pick boxes may overlap, there is an order of selection. Shapes get first priority, followed by lines, and then text. If objects of the same type overlap, then the object that was added first will be selected. Because text has the lowest priority when selecting, there is a special Text cursor that allows you to pick text that is superimposed on a shape or line. If desired text is not close to another object, you can select it by following the steps described above for a generic object. If the desired text is on top of or close to a shape or line, you should use the Text Pick cursor. 


\subsubsection{Selecting a Text Object}

\section{PURPOSE}

Prior to manipulating a text object (e.g., moving, copying, etc.) in the fault tree diagram, it must be selected. This option describes how to select a text object.

Because some object's pick boxes may overlap, there is an order of selection. Shapes get first priority, followed by lines, and then text. If objects of the same type overlap, then the object that was added first will be selected.

Because text has the lowest priority when selecting, there is a special text cursor that allows you to pick text that is superimposed on a shape or line. If desired text is not close to another object, you can select it by using the Pick cursor. If the desired text is on top of or close to a shape or line, you should follow the steps below.

\section{STEPS}

1. Choose the

2. Move the cursor

3. Click the mouse.

If the mouse is clicked while inside a text object's pick box, that text object is selected.

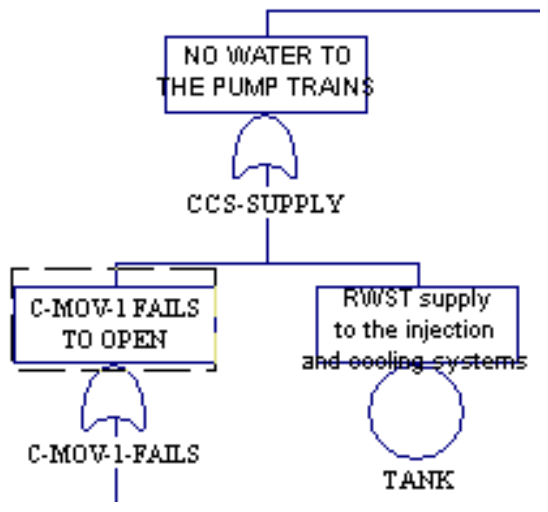

In the example here, the text associated with the OR gate, C-MOV-1-FAILS, is selected.

\subsubsection{View Logic}

\section{PURPOSE}

This option allows you to view the current diagram logic represented in the form of a hierarchical tree, where each gate can be expanded or condensed by clicking on the gate. The logic can only be viewed, not edited, from this dialog. See the topic, "About Logic Dialogs," for a description of the icons presented on this dialog. 


\section{STEPS}

1. Right click anywhere in the Fault Tree Graphical Editor.

2. Select View Logic menu option.

3. The Fault Tree Logic dialog will be displayed

\section{Edit Fault Tree Logic - [DEMO, CCS]}

$\square$ CCS OR CCS Fails to Spray Water into the Containment

-1 CCS-SUPPLY OR No Water To The Pump Trains

tank BE 1.000E-007 RWST supply to the injection and cooling systems

$\checkmark$ C-MOV-1-FAILS OR C-MOV-1 Fails To Open

c-mov-1 BE 1.000E-003 CCS suction isolation valve

$\bigcirc \mathrm{dg}-b \mathrm{BE} \quad 2.000 \mathrm{E}-002$ Emergency diesel generator $\mathrm{B}$

$\checkmark$ CCS-TRAINS AND Both Pump Trains Fail To Inject

ces-train-a BE $1.000 \mathrm{E}+000$

$\checkmark$ CCS-TRAIN-B OR CCS Train B Fails To Supply Flow

c-cv-b BE 1.000E-004 CCS Train B pump discharge check valve

c-mov-b BE 5.000E-003 CCS Train B pump discharge isolation valve

$\bigcirc$ c-pump-b BE 3.000E-003 CCS Train B motor-driven pump

$\bigcirc \mathrm{dg}-\mathrm{b}$ BE 2.000E-002 Emergency diesel generator $\mathrm{B}$

Exit - Close the Fault Tree Logic dialog.

\subsubsection{Adding Objects}

\subsubsection{Adding An Object}

\section{PURPOSE}

Add a shape object (e.g., AND gate, basic event, etc.) to the diagram. 


\section{STEPS}

1. Choose the desired object button (OR gate, AND gate, basic event, etc.) from the toolbar. The cursor will change to the associated object cursor.

2. Position the cursor at the desired location.

3. Click the mouse. The shape indicated by the cursor will be added.

Below is an example of each shape cursor available in the graphical Fault Tree Editor:
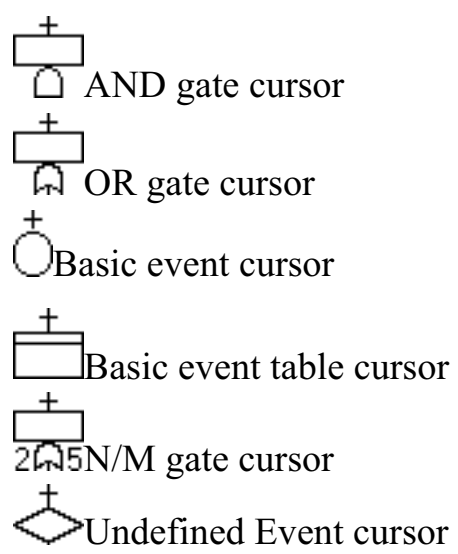

$\stackrel{+}{\triangle}$ Transfer gate cursor



十

OInhibit gate cursor

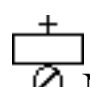

$\square$ NOT AND (NAND) gate cursor

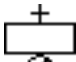

Tit NOT OR (NOR) gate cursor

$+$

Boxed basic event cursor

$+\triangle$ Left transfer cursor

$\triangle+$ Right transfer cursor

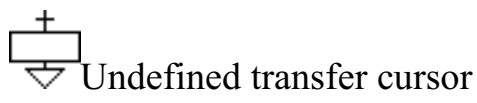

$+\square$ Horizontal Box

+ Vertical Box

\subsubsection{Adding Text}

\section{PURPOSE}

Adds text to the diagram.

\section{STEPS}

1. Choose the Text object button from the toolbar. The cursor will change to the Text cursor + 맘.

2. Position the Text cursor at the desired location.

3. Click the mouse. The Text Attributes dialog will be displayed. 


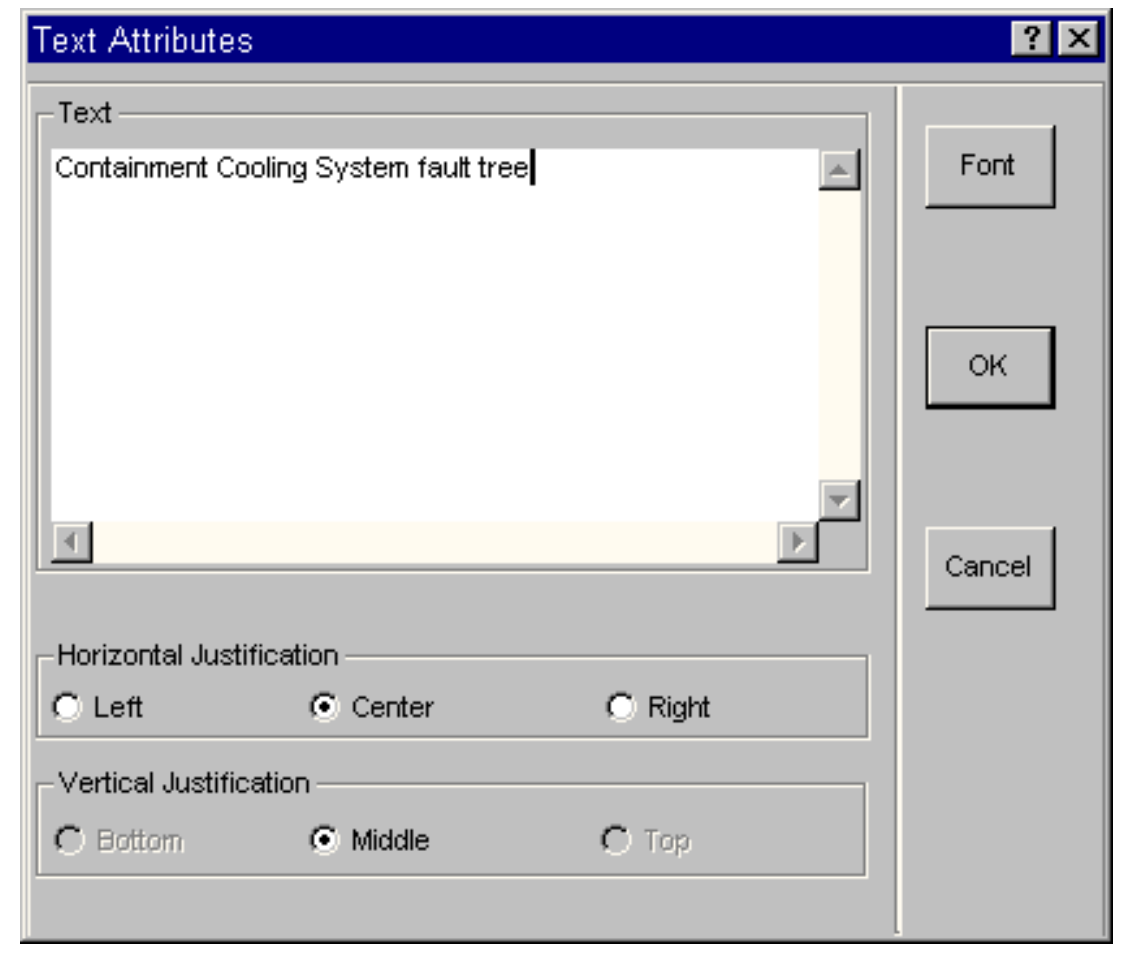

Text - $\quad$ Descriptive text for a shape or explanatory text for the entire fault tree. Maximum Justification of 600 alphanumeric upper- and lowercase characters.

Horizontal - The horizontal alignment of the text.

Vertical - The vertical alignment of the text.

Text Font - $\quad$ The font for the text.

OK - $\quad$ Close the Text Attributes dialog and add the input text to the diagram.

Cancel - $\quad$ Close the Text Attributes dialog without adding the text to the diagram.

\subsubsection{Adding An N/M Gate}

\section{PURPOSE}

Adds an N/M gate to the diagram.

\section{STEPS}

1. Choose the N/M gate object button $2 \mathbf{2}^{5}$ from the toolbar. The cursor will change to the N/M gate cursor $\stackrel{+}{2.725}$.

2. Position the N/M gate cursor at the desired location.

3. Click the mouse. The Define N/M Inputs dialog will be displayed. 


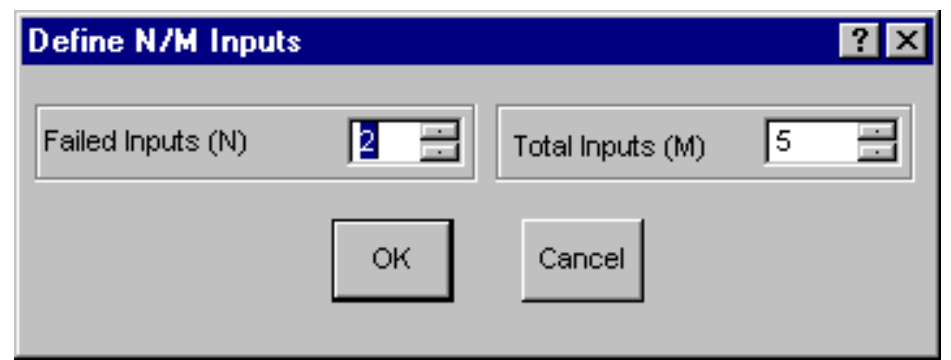

Failed Inputs (N) - $\quad$ Must be less than the Total Inputs. Choose the value by using the buddycontrol or enter the value.

Total Inputs (M) - $\quad$ Must be greater than 2 and less than 100. Choose the value by using the buddy-control or enter the value.

OK - $\quad$ Close the Define N/M Inputs dialog and add the N/M Gate to the diagram.

Cancel - $\quad$ Close the Define N/M Inputs dialog without adding the N/M Gate to diagram.

\subsubsection{Adding Connecting Lines}

\section{PURPOSE}

Adds connecting lines to the diagram. Connecting lines are used to logically link shape objects in the diagram.

\section{STEPS}

1. Choose the Line object button 네트 from the toolbar. The cursor will change to the Line cursor + .

2. Drag the mouse from one endpoint and to the other endpoint. (Hold the left mouse button down.)

3. Release the left mouse button (End drag) and the line will be drawn.

\subsubsection{Modifying Objects}

\subsubsection{Modifying Objects}

\section{PURPOSE}

This option allows you to modify the attributes of the selected shape object in the diagram.

\section{STEPS}

1. Select the object using the Pick cursor rhy $^{\text {th }}$. 
2. Right-click to invoke the pop-up menu and choose Edit.

$$
O R
$$

3. Choose Edit | Attributes from the menu.

4. The Shape Definition dialog will be displayed.

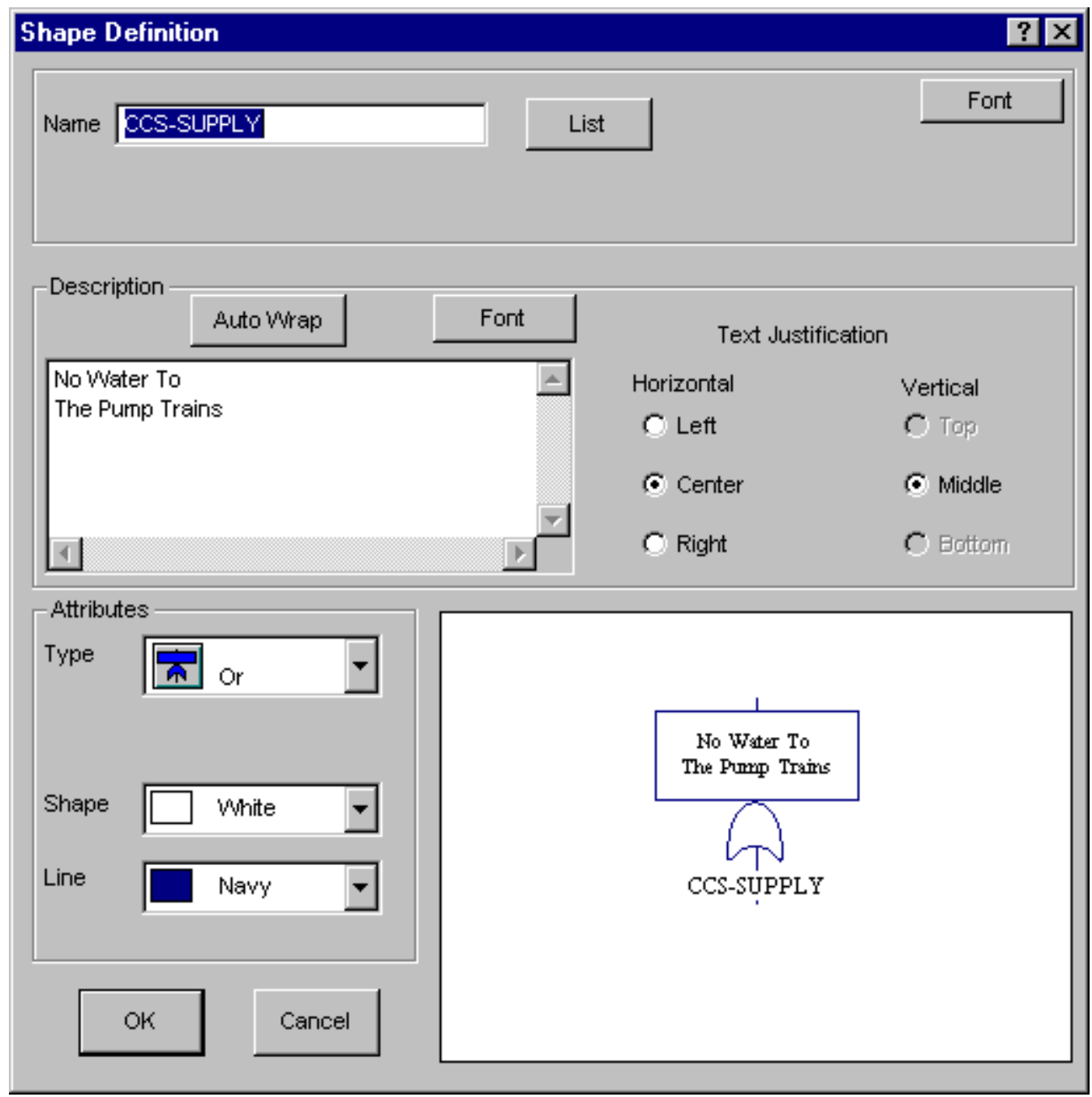

Name -

List -

Font -

Description -
The name of the selected shape. Enter up to 24 alphanumeric characters. (Embedded spaces are not allowed). The name entered here will be used in the Name field in the SAPHIRE database.

The available basic events and gates in the database.

The font attributes (such as typeface, size, color, etc.) used to display the shape name.

Brief description of the shape. Maximum length is 600 characters. The first 60 characters of the text entered here will be used in the Description field in the SAPHIRE database. 
Auto Wrap -

Font -

Text Justification -

Attributes

Type -

Shape -

Line -

Cancel -

OK -
Choose this button to fit the text inside the shape's text region.

The font attributes used to display the shape description.

The horizontal and vertical alignment of the shape description.

Change the shape's type by selecting from the drop-down list.

The fill color of the shape.

The outline color of the shape.

\subsubsection{File Menu}

\subsubsection{File}

These options provide file manipulation functions.

New -
Open -
Save -
Save As -
Export As -
Merge -
Forward Transfer -
Backward Transfer -
Print -
Exit -

New -

Create a new file in the current window. Open a file into the current window. Save the file in the current window to disk. Save the file in the current window with a new file name. Convert the file to RTF, EMF, or WMF format. Graft another file into the current window.

Follow a transfer gate.

Display a previously viewed transfer. Print the file in the current window. Terminate the Fault Tree Editor. If changes have been made you will be queried if you want to save the file.

\subsubsection{New}

\section{PURPOSE}

This option will allow you to clear the window and start a new diagram.

\section{STEPS}

1. From the Fault Tree Editor menu select File $\mid$ New.

OR

2. Select and right click on a fault tree that does not yet contain graphics from the SAPHIRE Fault Tree List, and choose Edit Graphics from the pop-up menu. 
3. If you have not saved the current diagram you will be prompted by a Caution dialog to do so.

4. The Enter top gate information dialog will appear, and you can begin building a new diagram.

Note: SAPHIRE requires the fault tree name and the top gate name to be the same. The name field and list button are disabled when the name of the fault tree is known. When a new diagram is started via the Fault Tree Editor File|New menu option, the name of the fault tree is not known. It is up to the user to save the diagram with the same name as the top gate.

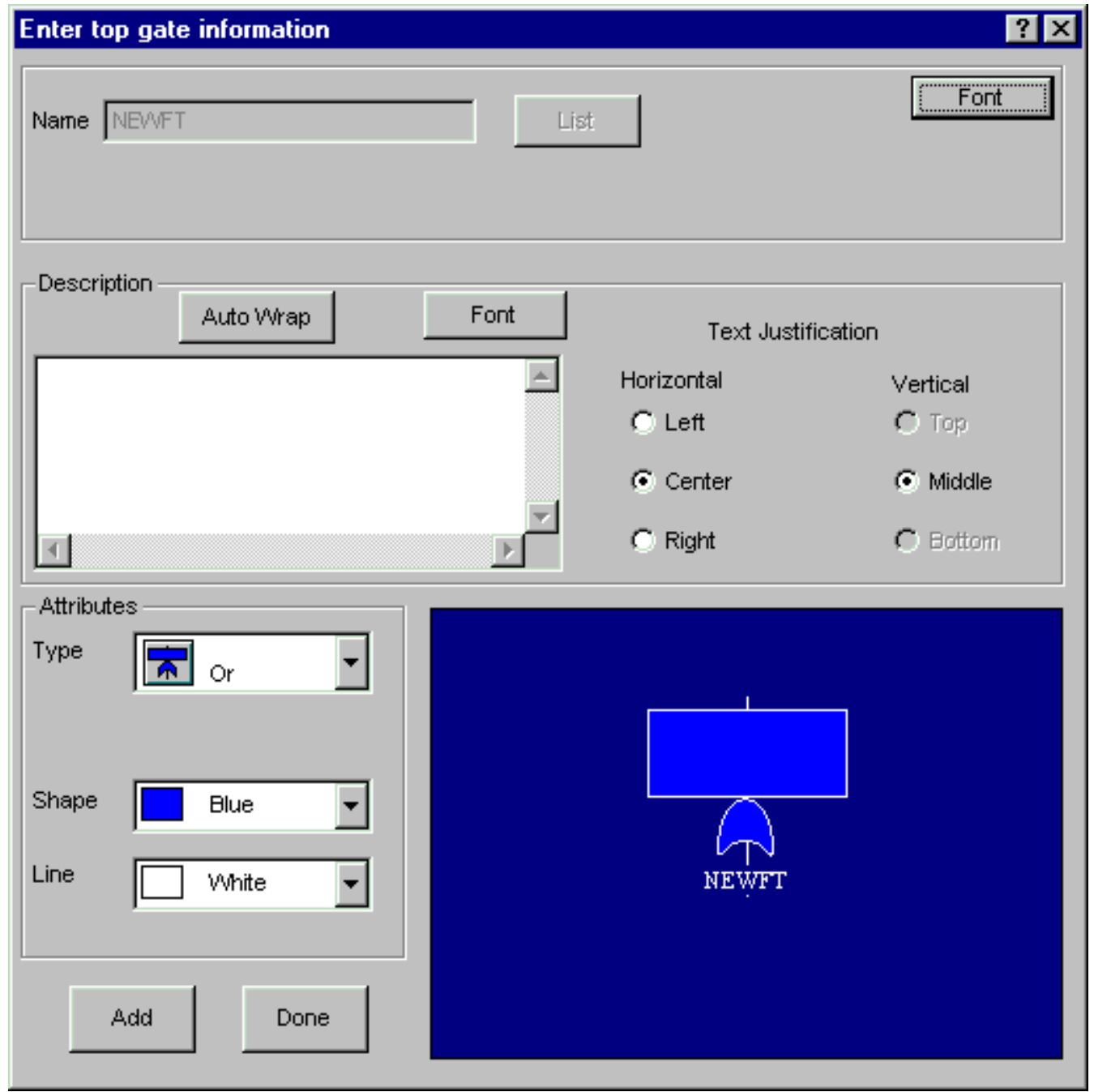

Add - Add the top gate to the information, and proceed to the Enter input to top gate dialog.

Done - Add the top gate to the diagram, close the Enter Top Gate Information dialog, and begin free form editing.

See Modifying Objects for more details on the rest of the information available from this dialog.

\subsubsection{Enter input to top gate}




\section{PURPOSE}

This option will add shapes as inputs to the top gate of the diagram. It initially appears after the Add button is selected from the Enter Top Gate Information dialog. It then appears repeatedly until the Done button is selected.

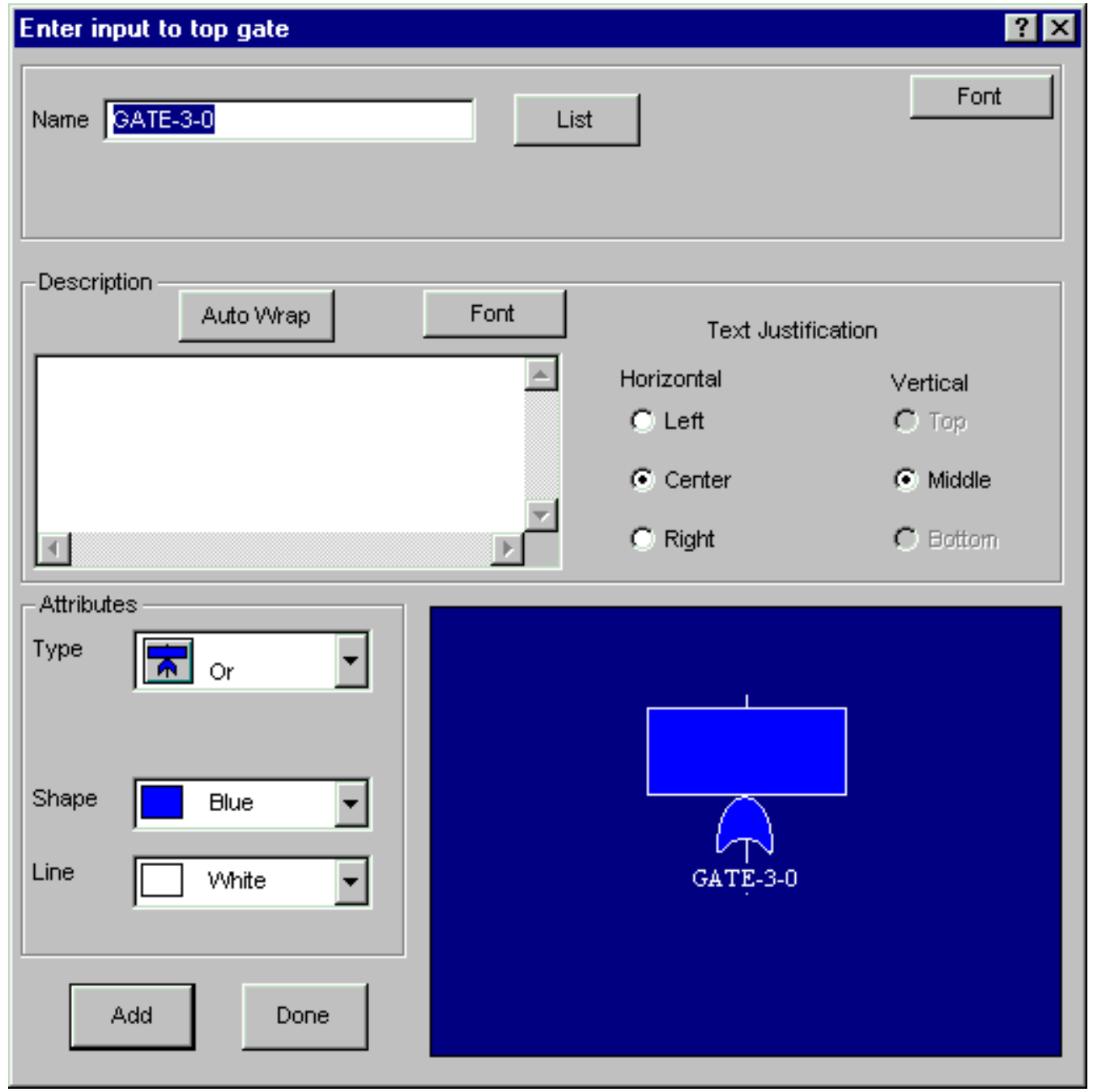

Add - Attach the input to the top gate, and begin editing a new input to the top gate.

Done - Attach the input to the top gate, close the Enter input to top gate dialog, and begin free form editing.

See Modifying Objects for more details on the rest of the information available from this dialog.

\subsubsection{Open}

\section{PURPOSE}

This option will allow you to select an existing diagram (i.e., fault tree graphics file) to view or edit. 


\section{STEPS}

1. From the Fault Tree Editor menu select File | Open.

OR

2. Choose the Open Diagram button on the toolbar.

3. The Open Fault Tree common dialog will be displayed listing all the fault tree diagrams (.DLS files) in the current project directory

4. Select the desired diagram and choose Open,

OR

5. Double-click on the diagram name.

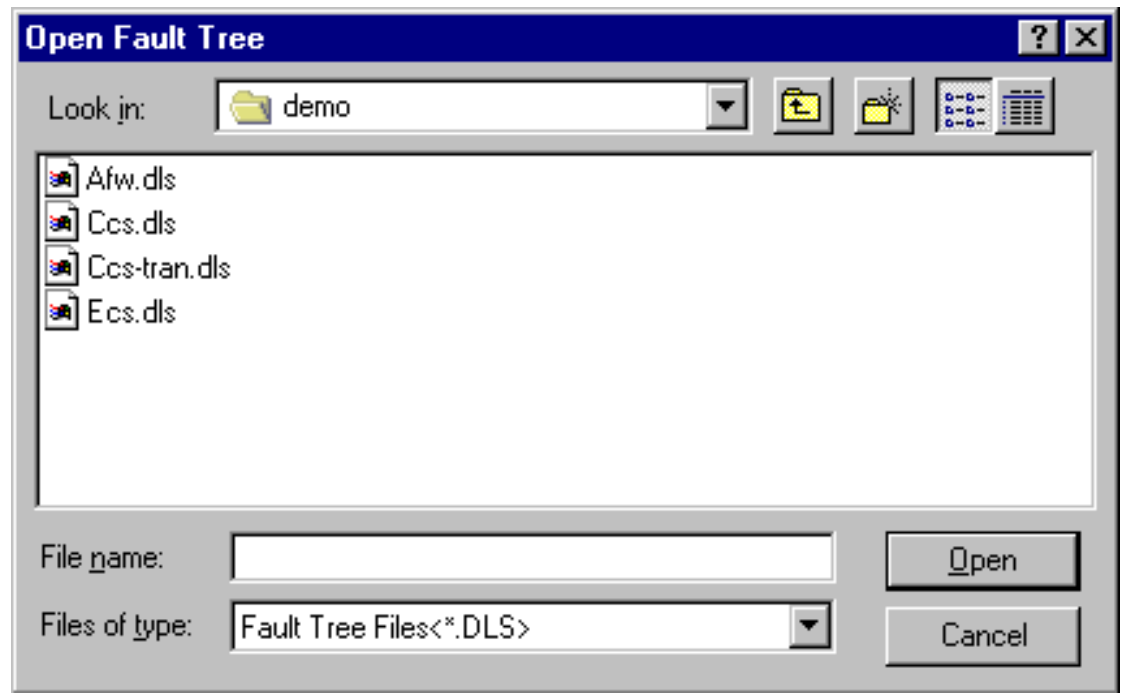

Look in - $\quad$ Lists the available folders and files. The box directly below it shows the contents of the current folder. The down-arrow to the right of this field shows how the folder fits into your system hierarchy.

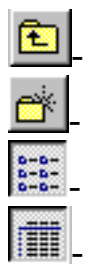

Moves up one level in the system hierarchy.

Creates a new folder at the current level.

Displays the icons and names of the contents of the current folder.

Displays the icons, names, size, type, date and time modified, and archive flags of the contents of the current folder.

File Name - Contains the name of the file in which the diagram will be saved.

Files of Type - Lists the types of files to display. This is useful for narrowing the list of files displayed to only those you are interested in. In this case, only ".DLS" files are listed.

Open - $\quad$ Open the highlighted diagram file and close the Open Fault Tree dialog.

Cancel Close the Open Fault Tree dialog without selecting a different diagram.

Double-click - Open the diagram file and close the Open Fault Tree dialog.

If you have selected a diagram to open and you have not saved the current one you will be prompted by a Caution dialog to do so. 


\subsubsection{Saving Changes}

\section{PURPOSE}

This option will save any changes made in the current fault tree diagram to the file presently open. If this is a new diagram, then you will be allowed to name the file. The logical representation of the diagram will also be saved in the SAPHIRE database.

\section{STEPS}

1. From the Fault Tree Editor menu select File | Save.

OR

2. Choose the Save Diagram button

3. If this is not a new diagram, the data is saved.

OR

4. If this is a new diagram, see Saving Changes to a Different File.

\subsubsection{Saving Changes to Different File}

\section{PURPOSE}

This option will save any changes made in the current fault tree diagram to a file you select. The logical representation of the diagram will also be saved in the SAPHIRE database.

\section{STEPS}

1. From the Fault Tree Editor menu select File.

2. Choose Save as from the menu

3. The Save Fault Tree common dialog will be displayed listing all the fault tree diagrams (.DLS files) in the current project directory.

4. Enter the name of the file in the File Name field, OR

5. Select the desired diagram file and choose Save, OR

6. Double-click on the diagram name. 


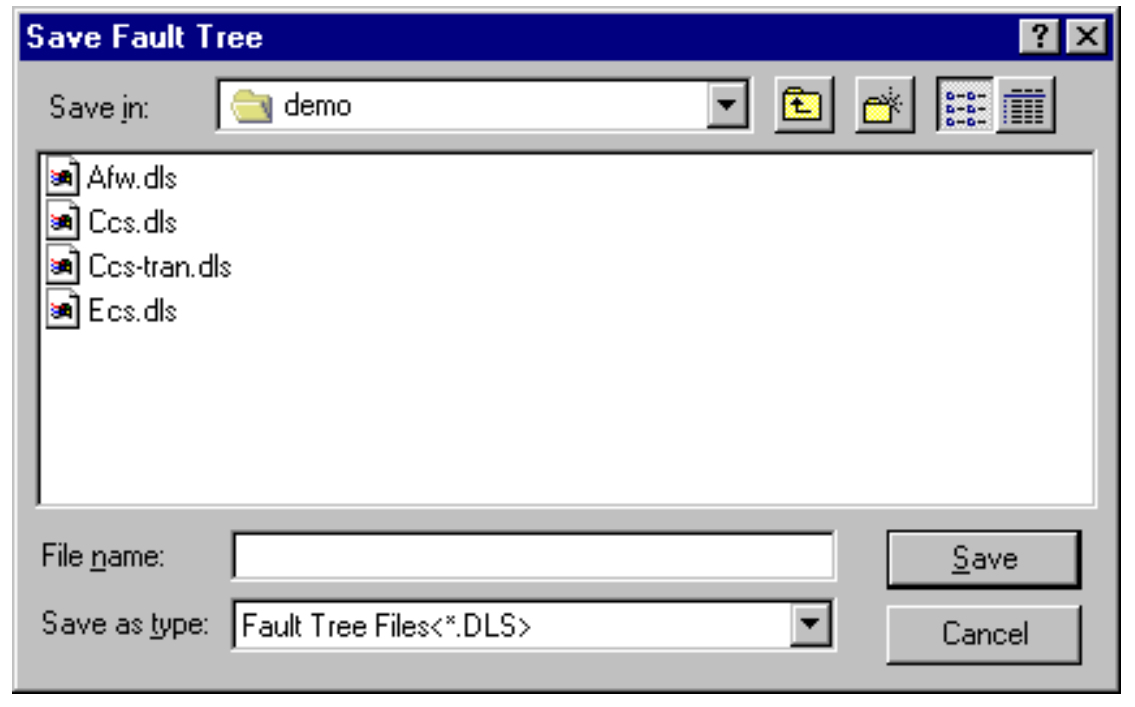

Save in - $\quad$ Lists the available folders and files. The box directly below it shows the contents of the current folder. The down-arrow to the right of this field shows how the folder fits into your system hierarchy.

Moves up one level in the system hierarchy.

Creates a new folder at the current level.

Displays the icons and names of the contents of the current folder.

Displays the icons, names, size, type, date and time modified, and archive flags of the contents of the current folder.

File Name - Contains the name of the file in which the diagram will be saved.

Files of Type - Lists the types of files to display. This is useful for narrowing the list of files displayed to only those you are interested in. In this case, only .DLS files are listed.

Save - $\quad$ Save the current diagram to the file in the File name field and close the Save Fault Tree dialog. If an existing file was selected, a warning dialog will be displayed.

Double-click - Save the current diagram to the file in the File name field and close the Save Fault Tree dialog.

Cancel - $\quad$ Close the Save Fault Tree dialog without saving the diagram.

\subsubsection{Save Fault Tree Warning Dialog}

This dialog is displayed when you choose to save the current diagram to an already existing file.

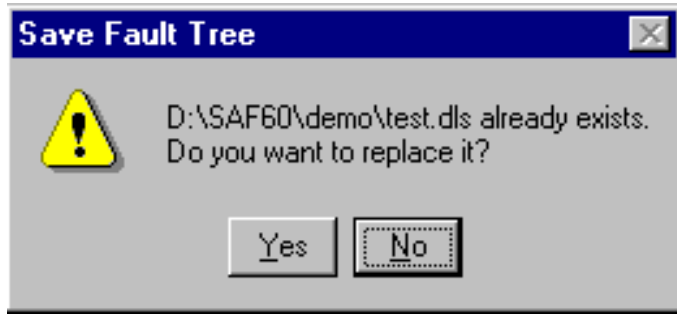


Save the current diagram to the selected file. The diagram in the existing file will be overwritten.

No - $\quad$ Do not save the current diagram to the select file.

\subsubsection{Warning: File name and top gate name are different}

When saving a fault tree diagram, this dialog is displayed when name of the fault tree file and the name of the top gate do not match. To correctly interpret fault tree logic, SAPHIRE requires the two names to be the same.

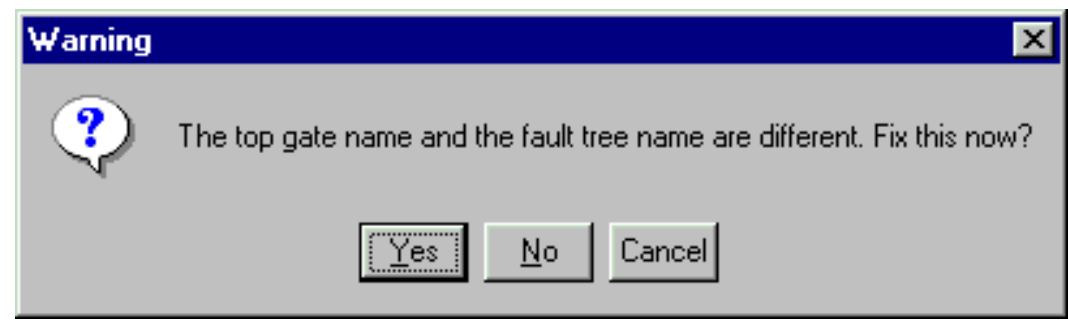

Yes - $\quad$ Let SAPHIRE rename the top gate to match the file name.

No - $\quad$ Save the diagram anyway, but leave the file name and top gate names alone. SAPHIRE will not be able to use the logic from this diagram until the problem has been corrected.

Cancel - $\quad$ Cancel the save, and return to editing the diagram. Use this option if you prefer to fix the problem yourself.

\subsubsection{Export As}

\section{PURPOSE}

This option will convert the diagram to a file in either Enhanced Metafile (EMF), Windows Metafile (WMF) format, or Rich Text Format (RTF). This option is available from the Fault Tree Graphic Editor and the Event Tree Graphic Editor.

\section{STEPS}

1. From the Fault Tree Editor menu select File.

2. Choose Export as from the menu

3. The Export Fault Tree As common dialog will be displayed listing all the EMF or WMF files in the current project directory.

4. Enter the name of the file in the File Name field,

$O R$

5. Select the desired file and choose Save,

OR

6. Double-click on the file name. 


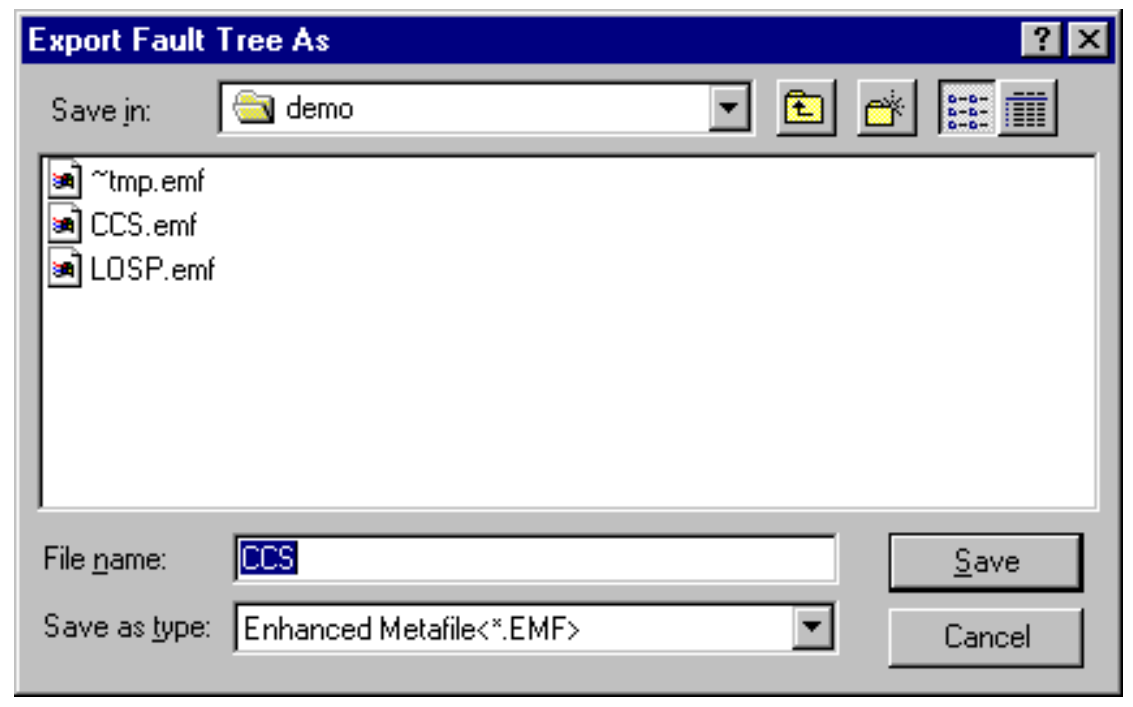

Save in - $\quad$ Lists the available folders and files. The box directly below it shows the contents of the current folder. The down-arrow to the right of this field shows how the folder fits into your system hierarchy.

Moves up one level in the system hierarchy.

Creates a new folder at the current level.

Displays the icons and names of the contents of the current folder.

Displays the icons, names, size, type, date and time modified, and archive flags of the contents of the current folder.

File name - Contains the name of the export file.

Save as type - $\quad$ Lists the types of files to display. This is useful for narrowing the list of files displayed to only those you are interested in. In this case, .RTF, .EMF or WMF files are listed.

Save - $\quad$ Save the current file in the File name field and close the Export Fault Tree As dialog. If an existing file was selected, a warning dialog will be displayed.

Double-click - Export the current diagram to the file in the File name field and close the Export Fault Tree As dialog.

Cancel - $\quad$ Close the Export Fault Tree As dialog without saving the diagram.

\subsubsection{Merge Diagram}

\section{PURPOSE}

This option will integrate an existing fault tree diagram into the current fault tree diagram. 


\section{STEPS}

1. From the Fault Tree Editor menu select File.

2. Choose Merge from the menu.

3. The Merge Fault Tree dialog will be displayed listing all the fault tree diagrams (.DLS files) in the current project directory.

4. Select the desired diagram and choose Open,

OR

5. Double-click on the diagram name.

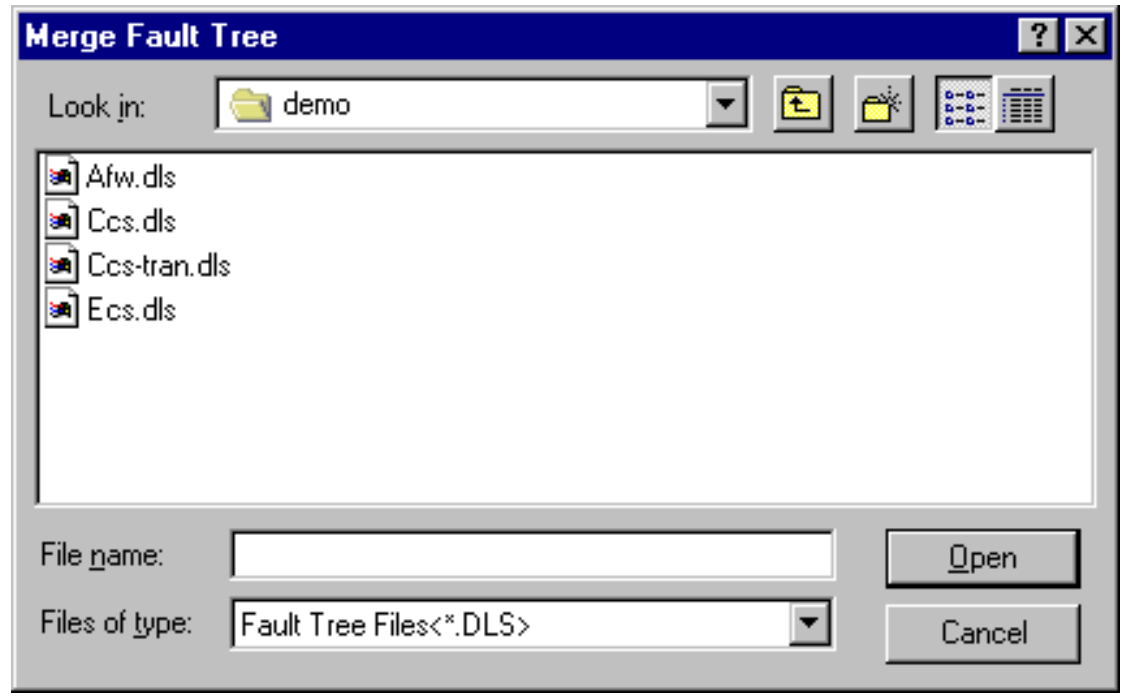

Open - $\quad$ Change the cursor to the Merge cursor and close the Merge Fault Tree dialog. Continue following the steps below.

Double-click - Change the cursor to the Merge cursor and close the Merge Fault Tree dialog. Continue following the steps below.

Cancel - $\quad$ Close the Merge Fault Tree dialog without merging in a diagram.

6. Position the Merge cursor $\overline{\overline{\mathrm{V}}}$ over the area where you want to graft in the diagram.

7. Click the mouse. The selected diagram will be grafted in.

OR

8. To cancel the merge operation, right-click the mouse and the cursor will change to the Pick cursor the

\subsubsection{Forward Transfer}

\section{PURPOSE}

This option will display the diagram corresponding to the selected transfer gate in the current fault tree diagram. Any changes made to the current fault tree diagram can be saved before viewing the transfer diagram. 


\section{STEPS}

1. Select the transfer gate you wish to follow.

2. From the Fault Tree Editor menu select File | Forward Transfer.

OR

3. Choose the Forward Transfer button $\Rightarrow 0$ on the toolbar.

4. If changes were made to the current diagram, the Caution dialog will be displayed.

5. If the diagram corresponding to the transfer gate exists, that will become the current diagram.

6. If no corresponding diagram exists, the Status dialog will be displayed.

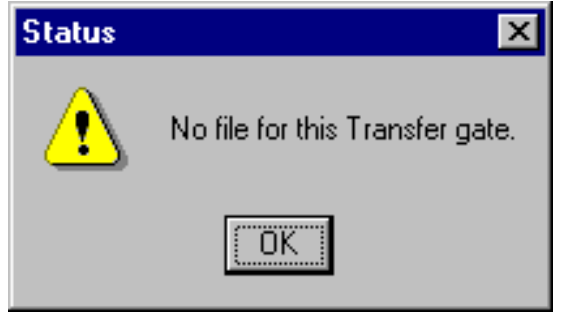

OK - Close the Status dialog.

\subsubsection{Backward Transfer}

\section{PURPOSE}

This option will list all the diagrams previously viewed in the current chain of transfers, including the current diagram. Select the fault tree diagram you wish to edit. Any changes made to the current fault tree diagram can be saved before viewing the transfer diagram.

\section{STEPS}

1. From the Fault Tree Editor menu select File | Backward Transfer.

OR

2. Choose the Backward Transfer button 순 on the toolbar.

3. The Select Transfer dialog will be displayed.

4. Highlight a diagram in the list and choose $\mathbf{O K}$. 


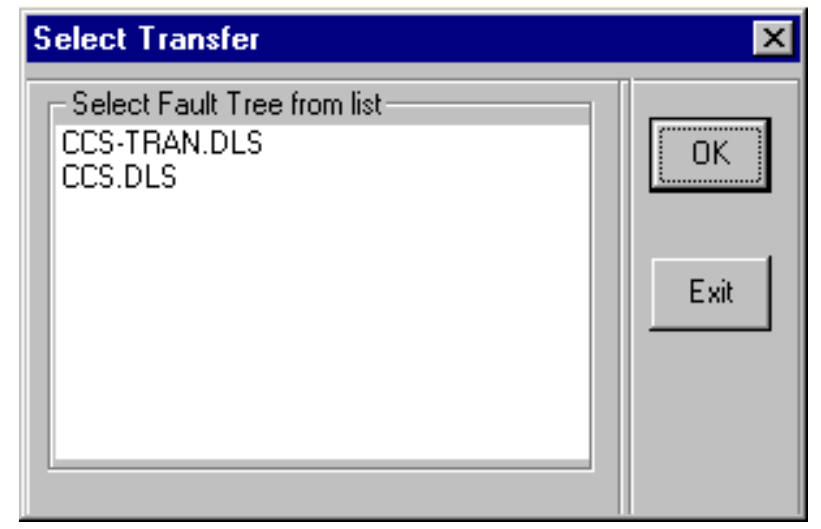

OK - Close the Select Transfer dialog and load the selected fault tree diagram. If you Exit - $\quad$ Close the Select Transfer dialog without loading a new diagram.

\subsubsection{Print}

\section{PURPOSE}

This option will print the current diagram to the selected printer. You are provided the opportunity to set additional options, such as page range, number of copies, etc., before printing the diagram.

\section{STEPS}

1. From the Fault Tree Editor or Event Tree Editor or P\&ID Editor menu select File | Print. OR

2. When in the Fault Tree Editor, choose the Print button 與 on the toolbar.

3. The Print common dialog will be displayed. 


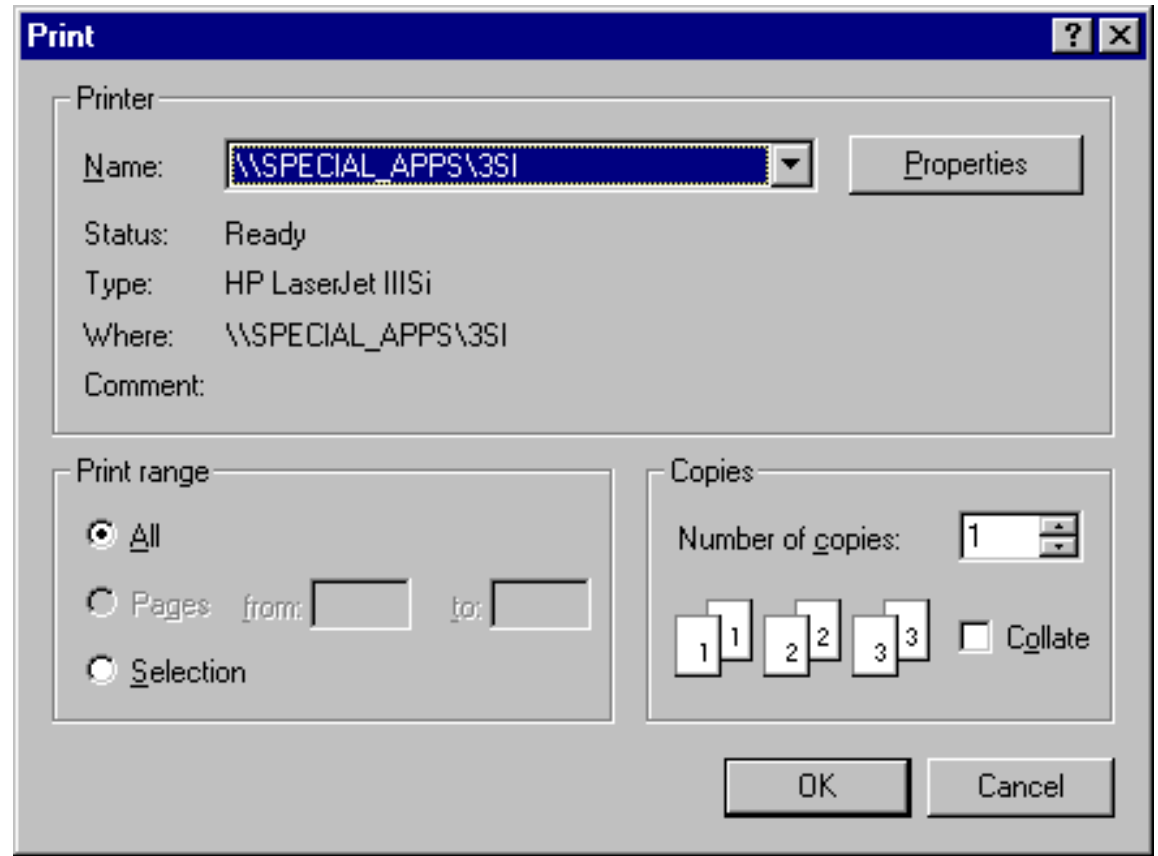

Name - $\quad$ List the printers that are set up for this computer.

Properties - $\quad$ Set-up options for the selected printer. Available options depend on the features of the printer.

Status - $\quad$ Condition of the selected printer.

Type - Kind of selected printer.

Where - Location of the selected printer.

Comment - $\quad$ Miscellaneous information about the selected printer.

Print Range - Print the entire document, specified range, or highlighted selection.

Copies - $\quad$ Enter the number of copies you wish to print.

Collate - $\quad$ For more than one copy, specify whether you want the copies collated.

OK - $\quad$ Close the Print Dialog and print the current diagram to the selected output device.

Cancel - $\quad$ Close the Print Dialog without printing the diagram.

\subsubsection{Caution: Save Changes To Current Drawing}

\section{PURPOSE}

This dialog will appear any time you are leaving the current diagram, which has not been saved. You leave the current diagram by exiting, following a transfer, opening another diagram, or creating a new diagram.

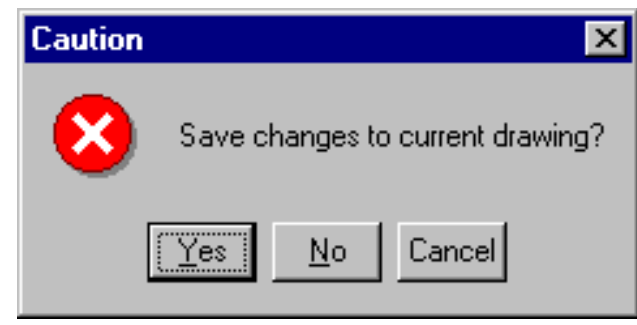


Yes -

No -

Cancel -
The current diagram will be saved to disk, and the next operation (Exit or change diagram) will be performed.

The current diagram will not be saved to disk, and the next operation (Exit or change diagram) will be performed.

The current diagram will not be saved to disk and the next operation (Exit or change diagram) will not be performed.

\subsubsection{Edit Menu}

\subsubsection{Edit}

These options provide editing and text formatting functions.

Cut - $\quad$ Remove the selected object(s) from the current window.

Copy - $\quad$ Copy the selected object(s) from the current window.

Paste - $\quad$ Copy the contents of the temporary file into the current window at the cursor position.

Delete - $\quad$ Permanently remove the selected object(s) from the current window.

Attributes - Set the attributes of the selected object(s)

\subsubsection{Undo}

\section{PURPOSE}

This feature allows you to undo previous actions. SAPHIRE maintains a list of actions taken by the user in a diagram editing session. When the list contains items, Undo will roll back the most recently completed action. Successive undo commands will roll back the next most recent action.

Note: The rollback list is cleared whenever the diagram is saved. (This applies only to user performed saves, not to timed backups.)

\section{STEPS}

1. From the Fault Tree Editor menu or Event Tree Editor menu select Edit $\mid$ Undo.

2. The most recent action will be undone.

\subsubsection{Cutting Objects}

\section{PURPOSE}

This option removes objects from the diagram and saves them in the clipboard.

\section{STEPS}

1. Select the object(s) you wish to cut. 
2. Press the $\mathbf{C t r l}+\mathbf{X}$ key combination.

OR

3. Choose the Cut button $\$ 8$ on the toolbar.

OR

4. From the Fault Tree Editor or Event Tree Editor menu select Edit | Cut.

OR

5. Right-click to invoke the pop-up menu and select Cut.

The selected object(s) will be removed from the diagram and placed in a temporary file as objects, as well as in the clipboard as a bitmap. This bitmap can be pasted (imported) into any document that can copy bitmaps from the clipboard.

\subsubsection{Copying Objects}

\section{PURPOSE}

This option copies selected objects from the diagram and places them in the clipboard.

\section{STEPS}

1. Select the object(s) you wish to copy.

2. Press the $\mathbf{C t r}+\mathbf{C}$ key combination.

$O R$

3. Choose the Copy button 3 (FTE) or ${ }^{-5}$ (ETE) on the toolbar.

OR

4. $\quad$ From the Fault Tree Editor or Event Tree Editor menu select Edit | Copy.

OR

5. Right-click to invoke the pop-up menu and select Copy.

The selected object(s) will be copied from the diagram and placed in a temporary file as objects, as well as in the clipboard as a bitmap. This bitmap can be pasted (imported) into any document that can copy bitmaps from the clipboard.

\subsubsection{Pasting Objects}

\section{PURPOSE}

This option pastes (imports) objects into the current diagram.

\section{STEPS}

1. Press the $\mathbf{C t r l + V}$ key combination.

$$
\text { OR }
$$

2. Choose the Paste button on the toolbar.

OR

3. From the Fault Tree Editor or Event Tree Editor menu select Edit | Paste.

OR 
4. $\quad$ Right-click to invoke the pop-up menu and select Paste.

5. The cursor is changed to the paste cursor ${ }^{+} \stackrel{\text { 焉. }}{.}$

6. Move the cursor to the location in the diagram where you wish to paste the object(s).

7. Click the mouse.

The selected object(s) will be copied the diagram centered, about the cursor.

\subsubsection{Deleting Objects}

\section{PURPOSE}

This option permanently removes the selected object(s) from the diagram. They are NOT put in the clipboard.

\section{STEPS}

1. Select the object(s) you wish to delete.

2. Press the Delete key. $O R$

3. From the Fault Tree Editor or Event Tree Editor menu select Edit | Delete.

The object(s) will be permanently deleted.

\subsubsection{Edit Database}

\section{PURPOSE}

This option allows you to modify the event record or gate record associated with the selected shape.

\section{STEPS}

1. Select the desired event or gate using the Pick cursor ${ }^{\text {r thy }}$

2. Right-click to invoke the pop-up menu.

3. Choose Edit Database from the pop-up menu.

4. The Modify Event or Modify Gate dialog will be displayed, depending on which shape type you selected.

\subsubsection{Temporary File}

\section{DEFINITION}

A holding place for objects that have been cut or copied. It is created when the graphical Fault Tree Editor is started and is available during the editing session. Objects in the holding file can be pasted into the current diagram. As long as the editing session is not ended and no new objects are cut or copied, the contents of the temporary file can be pasted.

\subsubsection{Modifying Attributes}




\subsection{Modifying Attributes}

The attributes of any of the objects in a fault tree diagram can be modified, individually or as a group. Depending on the type of object selected, different attributes can be changed.

\section{STEPS}

1. Select the object whose attributes you wish to modify.

2. Right-click to invoke the pop-up menu and choose Edit $O R$

3. Select Edit from the Fault Tree Editor menu and choose Attributes.

See the following for specific details:

Modifying Objects (Shapes)

Modifying Inhibit Gates

Modifying N/M Gates

Modifying Lines

Modifying Text

Modifying Groups of Objects

\subsection{Modifying Objects}

\section{PURPOSE}

This option allows you to modify the attributes of the selected shape object in the diagram.

\section{STEPS}

1. Select the object using the Pick cursor rht $^{\text {th }}$.

2. Right-click to invoke the pop-up menu and choose Edit.

$O R$

3. Choose Edit $\mid$ Attributes from the menu.

4. The Shape Definition dialog will be displayed. 


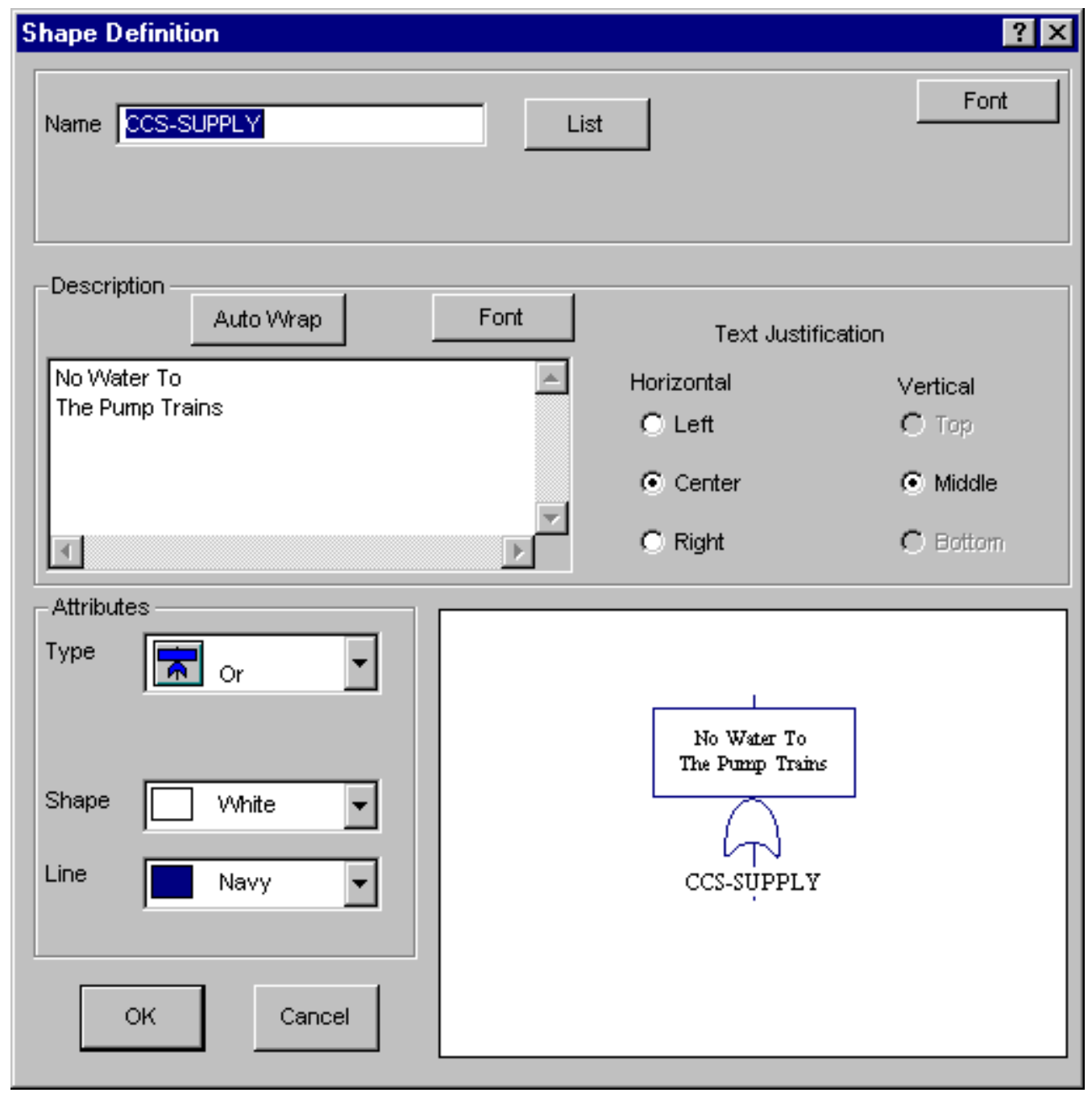

Name -

List -

Font -

Description -

Auto Wrap -

Font -

Text Justification - The horizontal and vertical alignment of the shape description.
The name of the selected shape. Enter up to 24 alphanumeric characters. (Embedded spaces are not allowed). The name entered here will be used in the Name field in the SAPHIRE database.

The available basic events and gates in the database.

The font attributes (such as typeface, size, color, etc.) used to display the shape name.

Brief description of the shape. Maximum length is 600 characters. The first 60 characters of the text entered here will be used in the Description field in the SAPHIRE database.

Choose this button to fit the text inside the shape's text region.

The font attributes used to display the shape description. 


\section{Attributes}

Type - $\quad$ Change the shape's type by selecting from the drop-down list.

Shape - $\quad$ The fill color of the shape.

Line - $\quad$ The outline color of the shape.

Cancel -

Close the Shape Definition dialog without applying the changes.

OK -

Close the Shape Definition dialog and apply any changes.

\subsection{Listing Event/Gates}

\section{PURPOSE}

This option allows you to select an event or gate name when modifying the attributes of the selected gate.

\section{STEPS}

1. Select the desired N/M gate using the Pick cursor thy

2. Right-click to invoke the pop-up menu and choose Edit.

$O R$

3. Choose Edit | Attributes from the menu.

4. The Shape Definition dialog will be displayed.

5. $\quad$ Choose the List button. The Find Event/Gate dialog will be displayed. 


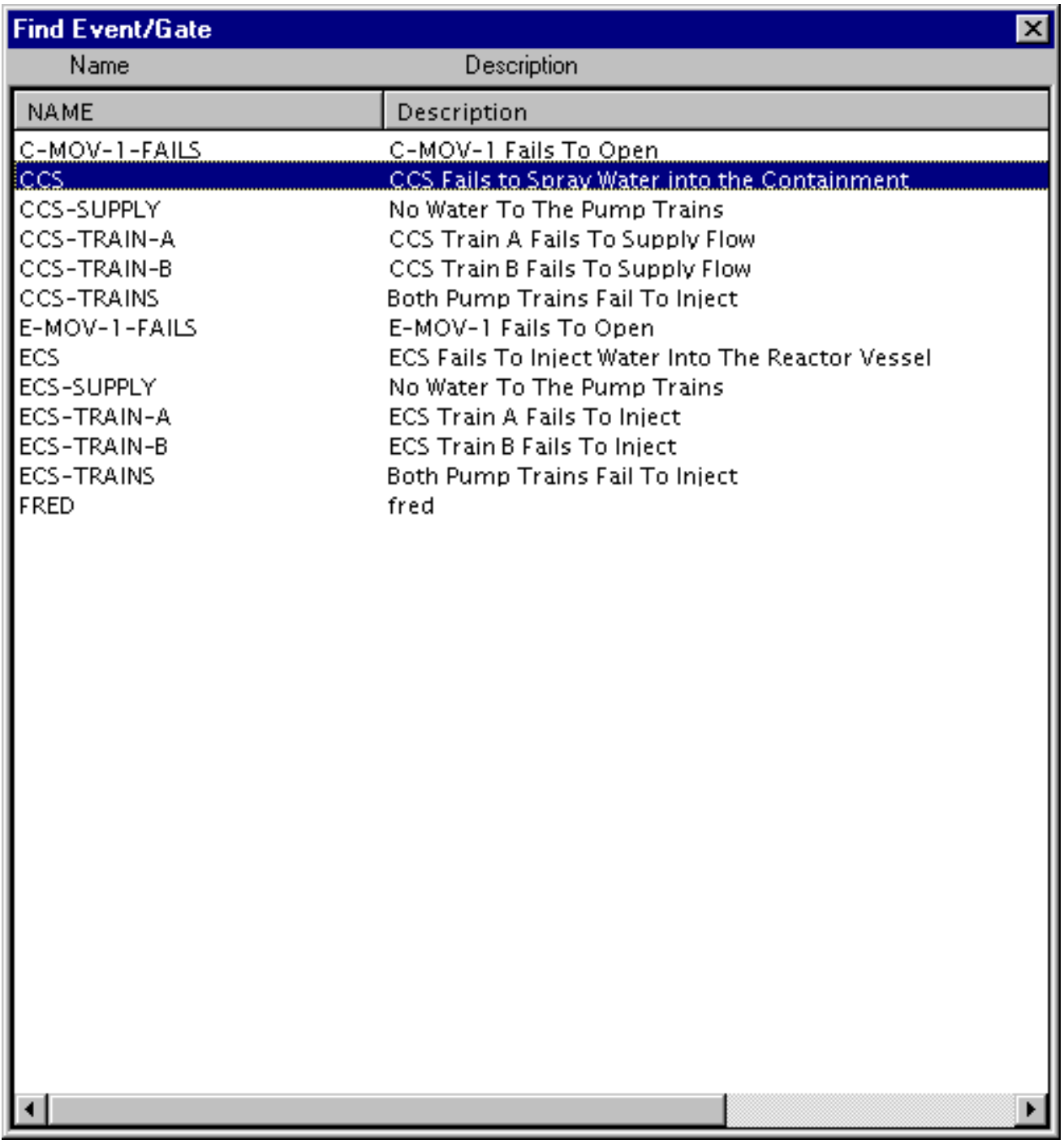

Double-click - Selects the event or name in the list and closes the Find Event/Gate dialog.

\subsection{Modifying Inhibit Gates}

\section{PURPOSE}

This option allows you to modify the attributes of the selected inhibit gate.

\section{STEPS}

1. Select the desired inhibit gate using the Pick cursor $\mathrm{rth}_{\mathrm{t}}$.

2. Right-click to invoke up the pop-up menu and choose Edit.

$O R$

3. Choose Edit $\mid$ Attributes form the menu.

4. The Shape Definition dialog will be displayed. 


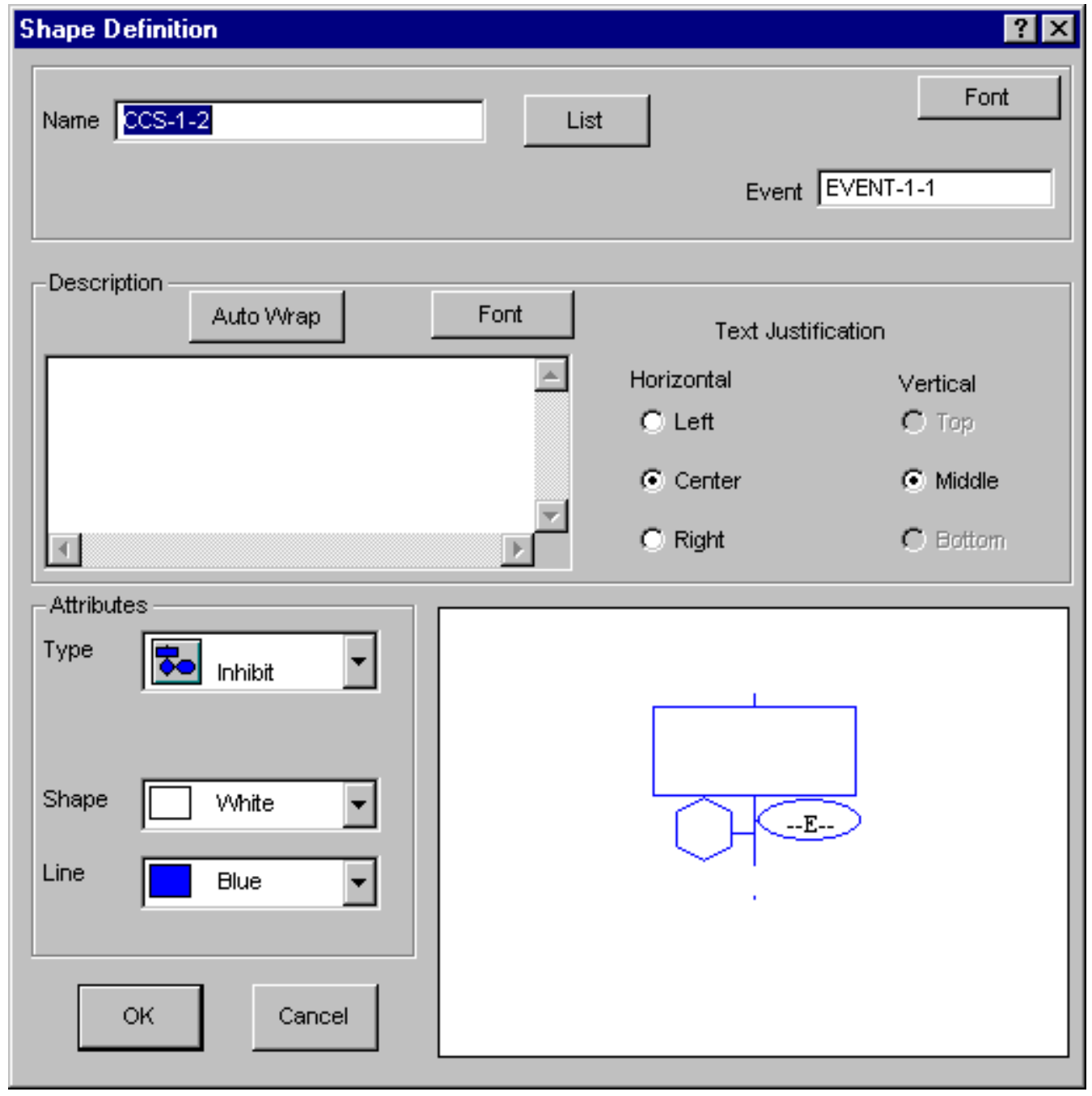

Name -

List -

Font -

Event -

Description -

\section{Auto Wrap -}

Font -

Text Justification -
The name of the gate. Enter up to 24 alphanumeric characters. (Embedded spaces are not allowed). The name entered here will be used in the Name field of the Gate relation in the SAPHIRE data base.

The available basic events and gates in the database.

The font attributes (such as typeface, size, color, etc.) used to display the shape name.

The name of the inhibiting basic event. Enter up to 24 alphanumeric characters. (Embedded spaces are not allowed). The name entered here will be used in the Name field of the Event relation in the SAPHIRE data base. 


\section{Attributes}

Type - $\quad$ Change the shape's type by selecting from the drop-down list.

Shape - $\quad$ The fill color of the shape.

Line - $\quad$ The outline color of the shape.

Cancel -

Close the Shape Definition dialog without applying the changes.

OK -

Close the Shape Definition dialog and apply any changes.

\subsection{Modifying N/M Gates}

\section{PURPOSE}

This option allows you to modify the attributes of the selected N/M gate.

\section{STEPS}

1. Select the desired N/M gate using the Pick cursor

2. Right-click to invoke the pop-up menu and choose Edit

OR

3. Choose Edit | Attributes from the menu.

4. The Shape Definition dialog will be displayed. 


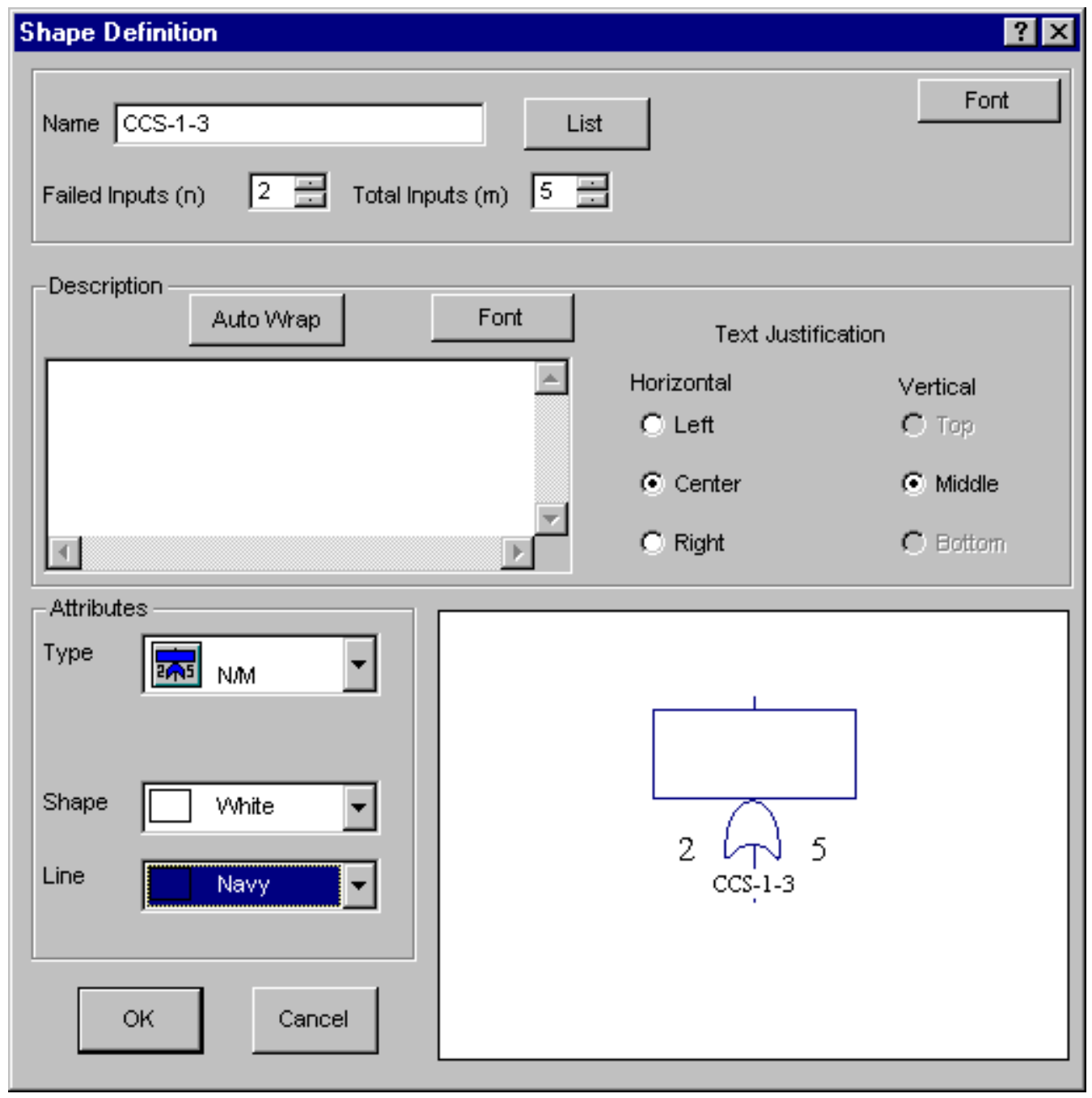

Name -

List -

Font -

Failed Inputs (n) -

Total Inputs (m) -

Description -

\section{Auto Wrap - \\ Font - \\ Text Justification - \\ Attributes}

Type - $\quad$ Change the shape's type by selecting from the drop-down list.
The name of the gate. Enter up to 24 alphanumeric characters. (Embedded spaces are not allowed). The name entered here will be used in the Name field of the Gate relation in the SAPHIRE data base. The available basic events and gates in the database.

The font attributes (such as typeface, size, color, etc.) used to display the shape name.

Must be less than the Total Inputs. Choose the value by using the buddycontrol or enter the value.

Must be greater than 2 and less than 100. Choose the value by using the buddy-control or enter the value

Brief description of the gate. Maximum length is 600 characters. The first 60 characters of the text entered here will be used in the Description field of the Gate relation in the SAPHIRE data base. Choose this button to fit the text inside the shape's text region. The font attributes used to display the shape description.

The horizontal and vertical alignment of the shape description. 

Shape -
Line -
The fill color of the shape.
The outline color of the shape.

Cancel -

Close the Shape Definition dialog without applying the changes.

OK -

Close the Shape Definition dialog and apply any changes.

\subsection{Modifying Lines}

\section{PURPOSE}

This option allows you to modify the attributes of the selected line in the diagram.

\section{STEPS}

1. Select the desired line using the Pick cursor oftry.

2. Right-click to invoke the pop-up menu and choose Edit.

$O R$

3. Choose Edit | Attributes from the menu.

4. The Line Attributes dialog will be displayed.

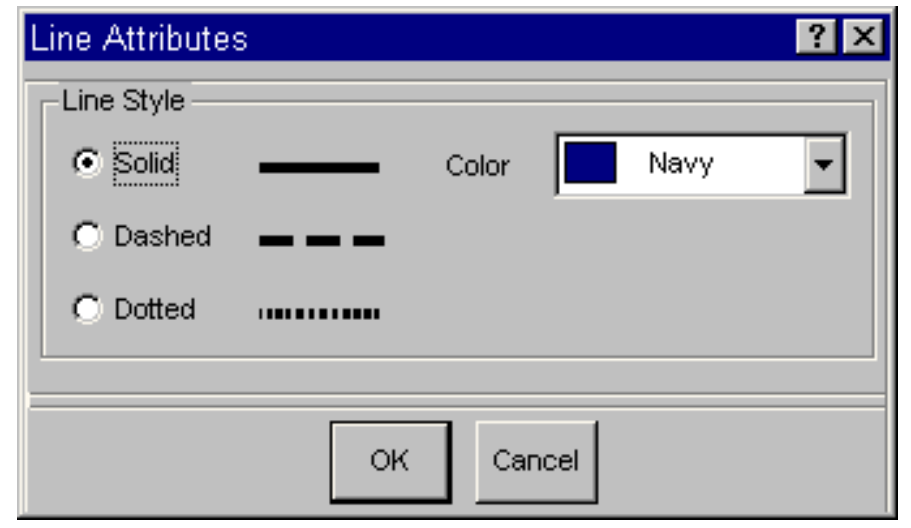

Line Style - $\quad$ Indicates the line's type (solid, dashed, or dotted). Choose the desired radio button

Color - $\quad$ Indicates the line's color. Select from the drop-down list.

Cancel - $\quad$ Close the Line Attributes dialog, without applying the changes.

OK - $\quad$ Close the Line Attributes dialog and apply any changes.

\subsection{Modifying Text}

\section{PURPOSE}

This option allows you to modify the attributes of the selected text in the diagram.

\section{STEPS}

1. Select the desired text using the Text Pick cursor 
2. Right-click to invoke the pop-up menu and select Edit.

$O R$

3. Choose Edit | Attributes from the menu.

4. The Text Attributes dialog will be displayed.

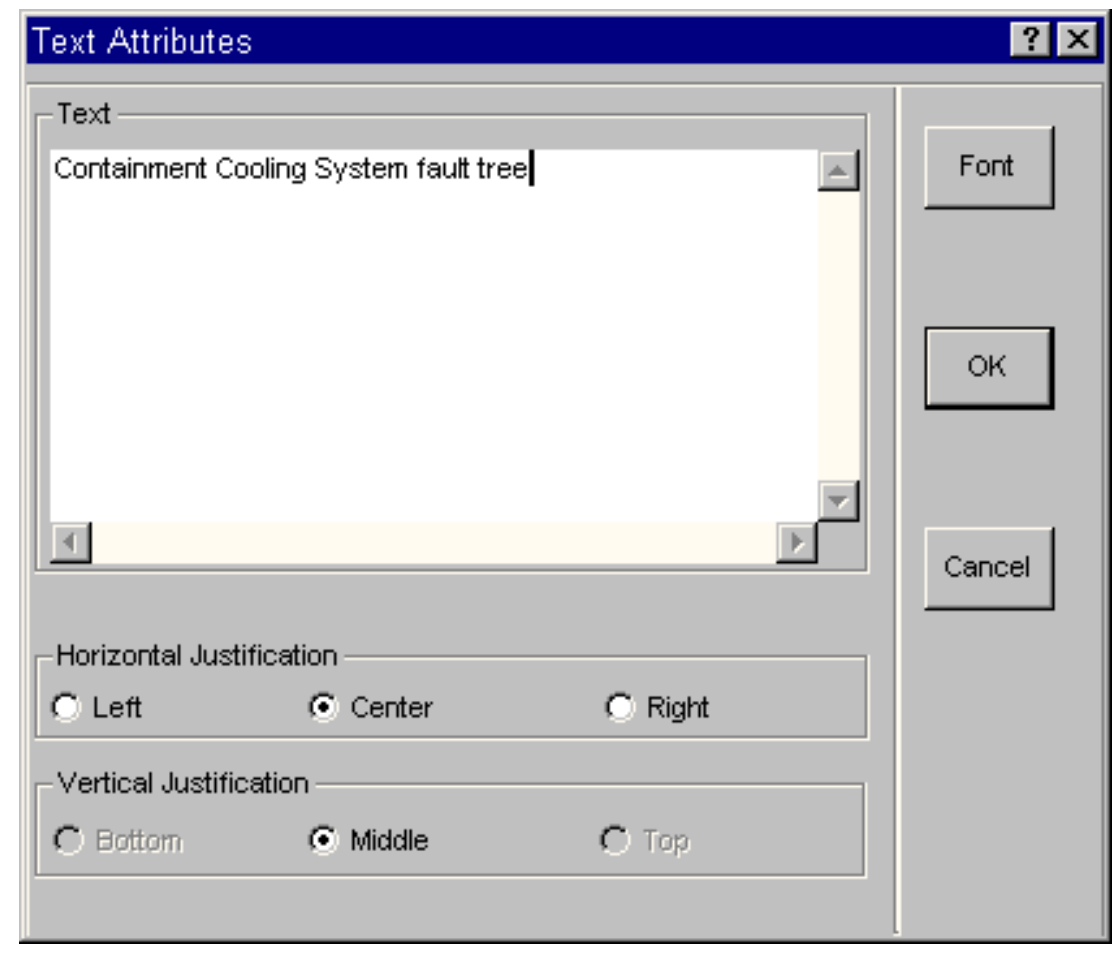

Text -

Horizontal Justification Vertical Justification -

Font -

Cancel -

OK -
Descriptive text for a shape or explanatory text for the entire fault tree. Maximum of 600 alphanumeric upper- and lowercase characters.

The horizontal alignment of the text.

The vertical alignment of the text.

The font attributes for the text.

Close the Text Attributes dialog without applying the changes.

Close the Text Attributes dialog and apply any changes.

\subsection{Modifying Groups Of Objects}

\section{PURPOSE}

This option allows you to modify the attributes of any selected objects in the diagram. This dialog appears when multiple objects are selected simultaneously.

\section{STEPS}

1. Select the desired objects to be changed using the Pick cursor

2. Right-click to invoke the pop-up menu and select Edit. 


\section{OR}

3. Choose Edit | Attributes from the menu.

4. The Attributes dialog will be displayed.

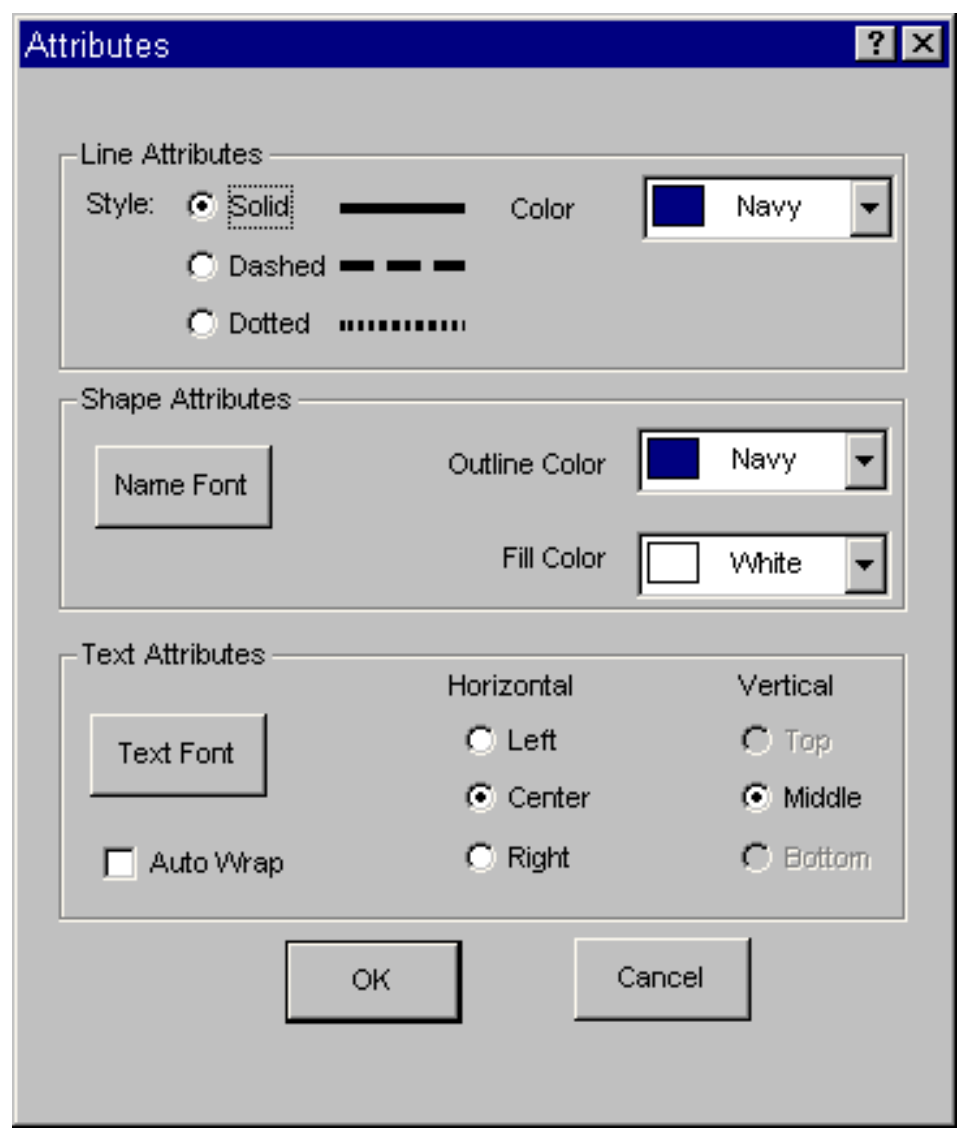

\section{Line Attributes}

Style -

Color -

The line style for all selected lines (solid, dashed, or dotted).

The color for all selected connecting lines. Select from the drop-down list.

\section{Shape Attributes}

Name Font -

The font attributes for all the selected shape names.

Outline Color - The exterior color for all selected shapes. Select from the drop-down list. Fill Color The interior color for all selected shapes. Select from the drop-down list.

\section{Text Attributes}

Text Font - $\quad$ The font attributes for all the selected text objects.

Horizontal - $\quad$ The horizontal alignment for all selected text.

Vertical - $\quad$ The vertical alignment for all selected text.

OK-

Close the Attributes dialog and apply any changes. 


\subsection{Font Selection}

\section{PURPOSE}

This option allows you to change attributes for a text object font or shape name font. This option is accessible from either the Preferences dialog or any of the shape attributes dialogs in the Fault Tree Editor and from any of the "edit" dialogs in the Event Tree Editor.

\section{STEPS}

1. In the Editor window, select the desired object(s) whose font you want to change.

2. Choose Edit $\mid$ Attributes.

$O R$

3. Choose View | Preferences. (Objects do not need to be selected before invoking this option)

4. On dialog, choose the Font button. The Font dialog will be displayed.

5. Select the desired font from the list and choose or change the style, size, color, etc.

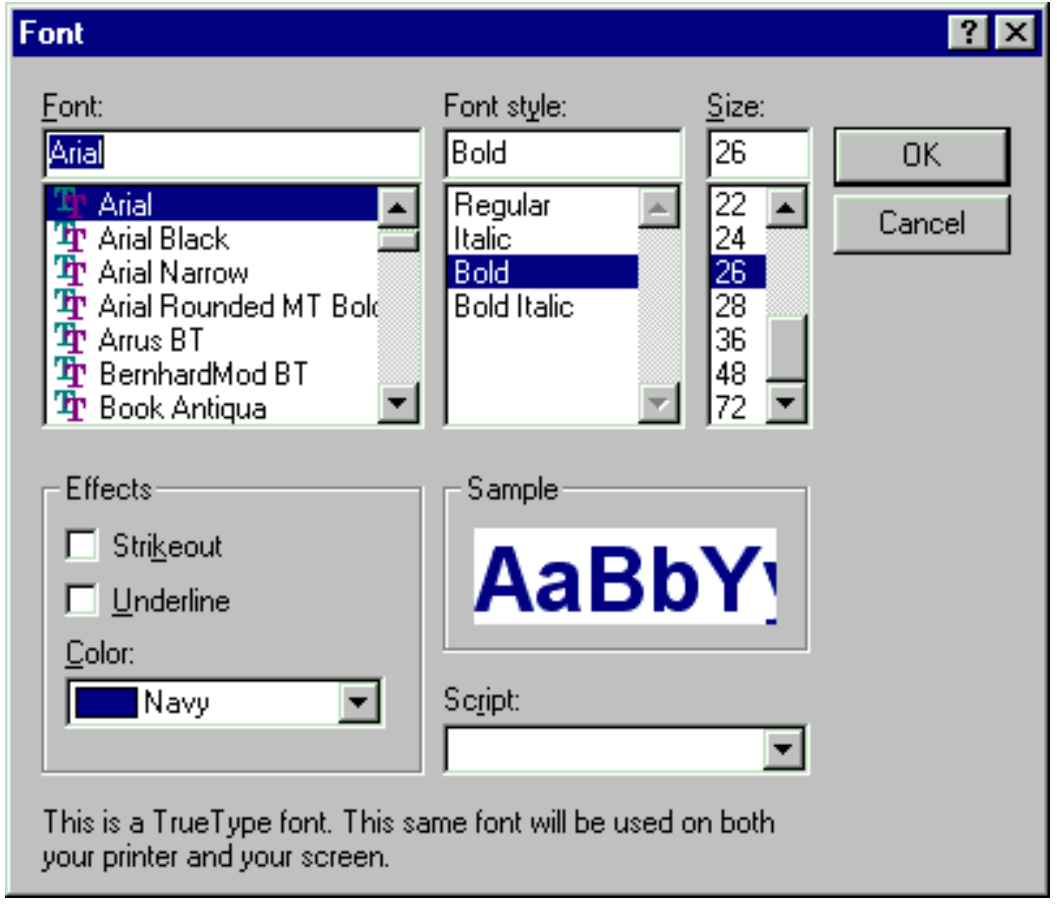

Font - $\quad$ List of available typeface names.

Font Style - $\quad$ Bold, italic, bold italic, or regular styles.

Size - $\quad$ Point size of the selected typeface.

Effects - $\quad$ Underline, strikeout text color.

Sample - $\quad$ Example of the appearance of the highlighted font.

Script - Character set. 
OK - $\quad$ Close the Select Font dialog and change the font. The font change will not be Cancel - $\quad$ Close the Select Font dialog without changing the font.

NOTE: The series of steps shown here, illustrate how to access the dialogs from the Fault Tree Editor menu. The dialogs can also be accessed via the pop-up menu.

\subsection{Line Style}

\section{PURPOSE}

This option allows you to change the type of line being drawn. This option is accessible from either the Preferences dialog or the Line Attributes dialog.

\section{STEPS}

1. In the Fault Tree Editor window, select the desired line(s) whose style you want to change.

2. Choose Edit $\mid$ Attributes.

$O R$

3. Choose View | Preferences. (A line does not need to be selected before invoking this option.)

When the dialog is initially displayed, the style radio button and color are selected for the current default (Preferences dialog) or the currently selected line(s) (Attributes dialog).

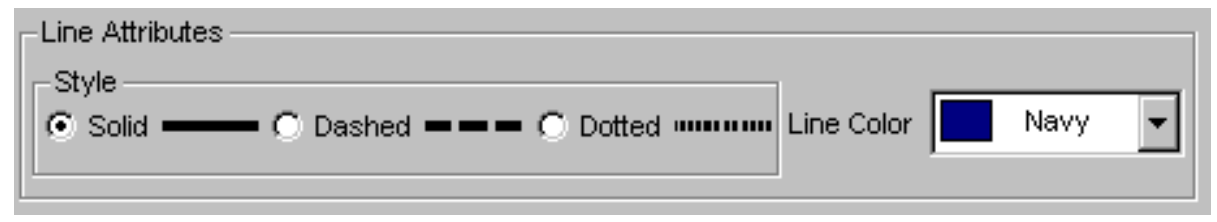

\section{Line Style}

Choose the radio button to the left of the desired style. The possible line styles are solid, dashed, and dotted.

\section{Line Color}

Choose the line color from the drop-down list.

The line will be redrawn using the new style and color if the Ok button is chosen.

NOTE: The series of steps shown here illustrate how to access the dialogs from the Fault Tree Editor menu. The dialogs can also be accessed via the pop-up menu.

\subsection{Horizontal Justification}

\section{PURPOSE}

Allows you to change the horizontal justification of text. This option is accessible from the Preferences dialog, the Text Attributes dialog, or the Attributes dialog. 
Each text object has a pick box that surrounds it called the text box. You can align text on the left or right sides of the text box or center text between the sides of the text box.

\section{STEPS}

In the Fault Tree Editor window, select the desired text object(s) whose justification you want to change.

1. Choose Edit | Attributes.

$O R$

2. Choose View | Preferences. (A text object does not need to be selected before invoking this option.)

3. In the Horizontal Justification section, choose the radio button to the left of the desired style.

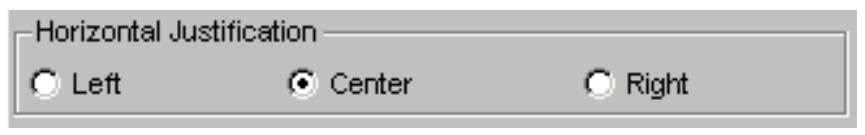

Left - Align the text along the left side of the text box.

Center - $\quad$ Align the text so it is centered between the two sides of the text box.

Right - $\quad$ Align the text along the right side of the text box.

NOTE: The series of steps shown here illustrate how to access the dialogs from the Fault Tree Editor menu. The dialogs can also be accessed via the pop-up menu.

\subsection{Vertical Justification}

\section{PURPOSE}

Allows you to change the vertical justification of text. This option is accessible from the Preferences dialog, the Text Attributes dialog, or the Attributes dialog.

Each text object has a pick box that surrounds it, called the text box. You can align text along the top or bottom of the text box or slightly above the bottom (base) of the text box.

\section{STEPS}

1. In the Fault Tree Editor window, select the desired text object(s) whose justification you want to change.

2. Choose Edit | Attributes.

OR

3. Choose View | Preferences. (A text object does not need to be selected before invoking this option.)

4. In the Vertical Justification section, choose the radio button to the left of the desired style. 


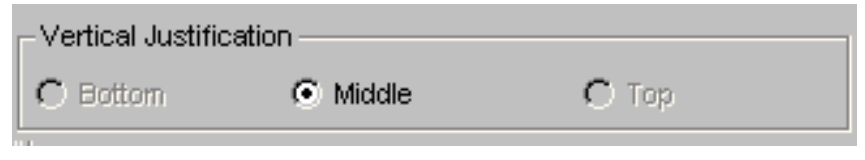
Bottom -
Align the text at the bottom of the text box.
Middle -
Align the text in the middle of the text box.
Top -
Align the text at the top of the text box.

NOTE: The series of steps shown here, illustrate how to access the dialogs from the Fault Tree Editor menu. The dialogs can also be accessed via the pop-up menu.

\subsubsection{View Menu}

\subsubsection{View}

These menu options allow you to change the portion of the current diagram in the viewing window. For simple diagrams with a small number of gates, this is generally not necessary. For larger, more complex diagrams that do not fit on one screen, you may need to view different portions of the diagram. You can move the view window up, down, left, and right using the scroll bars or menu commands. You can change the size of the viewing window by zooming in or out, adjust the diagram to fit inside the viewing window, and restore the view window to its original size.

Page Up - $\quad$ Move the viewing window up about one-half page (i.e., screen) above the current location.

Page Down - $\quad$ Move the viewing window down about one-half page (i.e., screen) below the current location.

Page Left - $\quad$ Move the viewing window left about one-half page (i.e., screen) left of the current location.

Page Right - $\quad$ Move the viewing window right about one-half page (i.e., screen) right of the current location.

Zoom - $\quad$ Enlarge or shrink a portion of the diagram.

Normal - $\quad$ Restore the view window to its original size.

Fit - $\quad$ Adjust the diagram to fit inside the view window.

Zoom Selected - Enlarge the selected portion of the diagram.

Preferences - Change the fault tree graphical editor defaults.

Refresh - $\quad$ Redraw the viewing window.

\subsubsection{Using Scroll Bars To Move The View Window}

\section{PURPOSE}

This option allows you to change the portion of the diagram in the view window. 


\section{STEPS}

To move the viewing window to the left slightly:

1. Click on the left arrow in the bottom scroll bar.

2. The viewing window will move a little to the left.

To move the viewing window to the right slightly:

1. Click on the right arrow in the bottom scroll bar.

2. The viewing window will move a little to the right.

To move the viewing window up slightly:

1. Click on the up arrow in the scroll bar on the right hand side.

2. The viewing window will move up a little.

To move the viewing window down slightly:

1. Click on the down arrow in the scroll bar on the right hand side.

2. The viewing window will move down a little.

To move the viewing window to the left about one-eighth page:

1. Click on bottom scroll bar between the left arrow and the thumb.

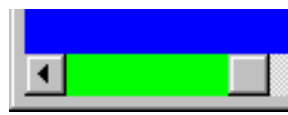
The area to click in is highlighted in green in this example.

2. The viewing window will move to the left.

To move the viewing window to the right about one-eighth page:

1. Click on bottom scroll bar between the right arrow and the thumb.

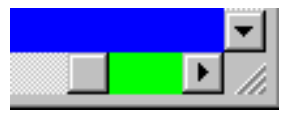
The area to click in is highlighted in green in this example.

2. The viewing window will move to the right. 
To move the viewing window up about one-eighth page:

1. Click on bottom scroll bar between the top arrow and the thumb. highlighted in green in this example

2. The viewing window will move up.

To move the viewing window down about one-eighth page:

1. Click on right scroll bar between the bottom arrow and the thumb. The area to click in is highlighted in green in this example.

2. The viewing window will move down.

\subsubsection{Zooming In/Out}

\section{PURPOSE}

This option allows you to enlarge or shrink the diagram in the viewing window to change the portion of the diagram that can be seen. It has the effect of magnifying or reducing the diagram size.

\section{STEPS}

1. From the Fault Tree Editor or Event Tree Editor menu select View $\mid$ Zoom.

OR

2. Select the Zoom button (FTE) or $_{\text {(ETE) from the toolbar. }}$

OR

3. Press the $\mathbf{C t r l}+\mathbf{Z}$ key combination.

4. The cursor is changed to the Zoom cursor

5. Move the cursor to the desired location.

To zoom in or magnify the diagram:

1. Click the mouse. The diagram will be magnified by $40 \%$. 


\section{To zoom out or reduce the diagram:}

1. Right-click the mouse. The diagram will be reduced by $40 \%$.

\subsubsection{Restore the View Window}

\section{PURPOSE}

This option allows you to restore the diagram position in the viewing window to the same relative position as when the diagram file was initially opened. Changes to the objects (i.e., their position, attributes, etc.) are not restored.

\section{STEPS}

1. From the Fault Tree Editor or Event Tree Editor menu select View | Normal.

$O R$

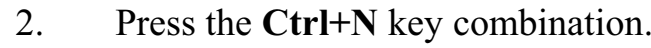

3. The initial position of the diagram is redisplayed.

\subsubsection{Fit the View Window}

\section{PURPOSE}

This option allows you to adjust the entire diagram to fit inside the viewing window. If the diagram is relatively large, the size of the shapes and text will be reduced so that the entire fault tree can be viewed without scrolling or paging the window. If the diagram is relatively small, the size of the shapes and text will be enlarged so that the diagram fills the window.

\section{STEPS}

1. From the Fault Tree Editor menu select View | Fit or from the Event Tree Editor menu select View | Fit to Screen.

$O R$

2. Select the Fit to Window button (FTE) from the toolbar.

OR

3. Press the $\mathbf{C t r}+\mathbf{F}$ key combination.

4. The diagram will be enlarged or reduced to fit inside the viewing window.

\subsubsection{Zoom 100\%}

\section{PURPOSE}

This option allows you to resize the diagram to a standard size defined as $100 \%$. The shapes included in all diagrams set to be viewed at $100 \%$ will be the same size. Likewise, all text (of matching point size) across diagrams will have the same magnification.

Use this option across diagrams to achieve a uniform magnification. To print a group of diagrams from the Reports menu at this size, diagrams must have been previously saved with this option set.

\section{STEPS}

1. From the Fault Tree Editor menu select View | Zoom 100\% . 
OR

2. Select the Standard Size button $\mathrm{Q}$ from the toolbar.

$O R$

3. Press the Ctrl+1 key combination.

The diagram will be magnified to the standard $100 \%$ size.

\subsubsection{Zoom Selected}

\section{PURPOSE}

This option allows you to enlarge the diagram in the viewing window to change the portion of the diagram that can be seen. It has the effect of magnifying the diagram size.

\section{STEPS}

1. Using the pick cursor, outline the area to be enlarged.

2. From the Fault Tree Editor menu select View $\mid$ Zoom Selected.

3. The diagram will magnified to display the selected area.

\subsubsection{Modifying Preferences}

\section{PURPOSE}

This option allows you to modify the preferences for the graphical Fault Tree Editor. The changes made here will be reflected in the default values in the Utility | Define Constants | Fault Tree option.

\section{STEPS}

1. Right-click to invoke the pop-up menu.

$O R$

2. Select View from the menu

3. Select Preferences from the menu. The Preferences dialog will be displayed. 


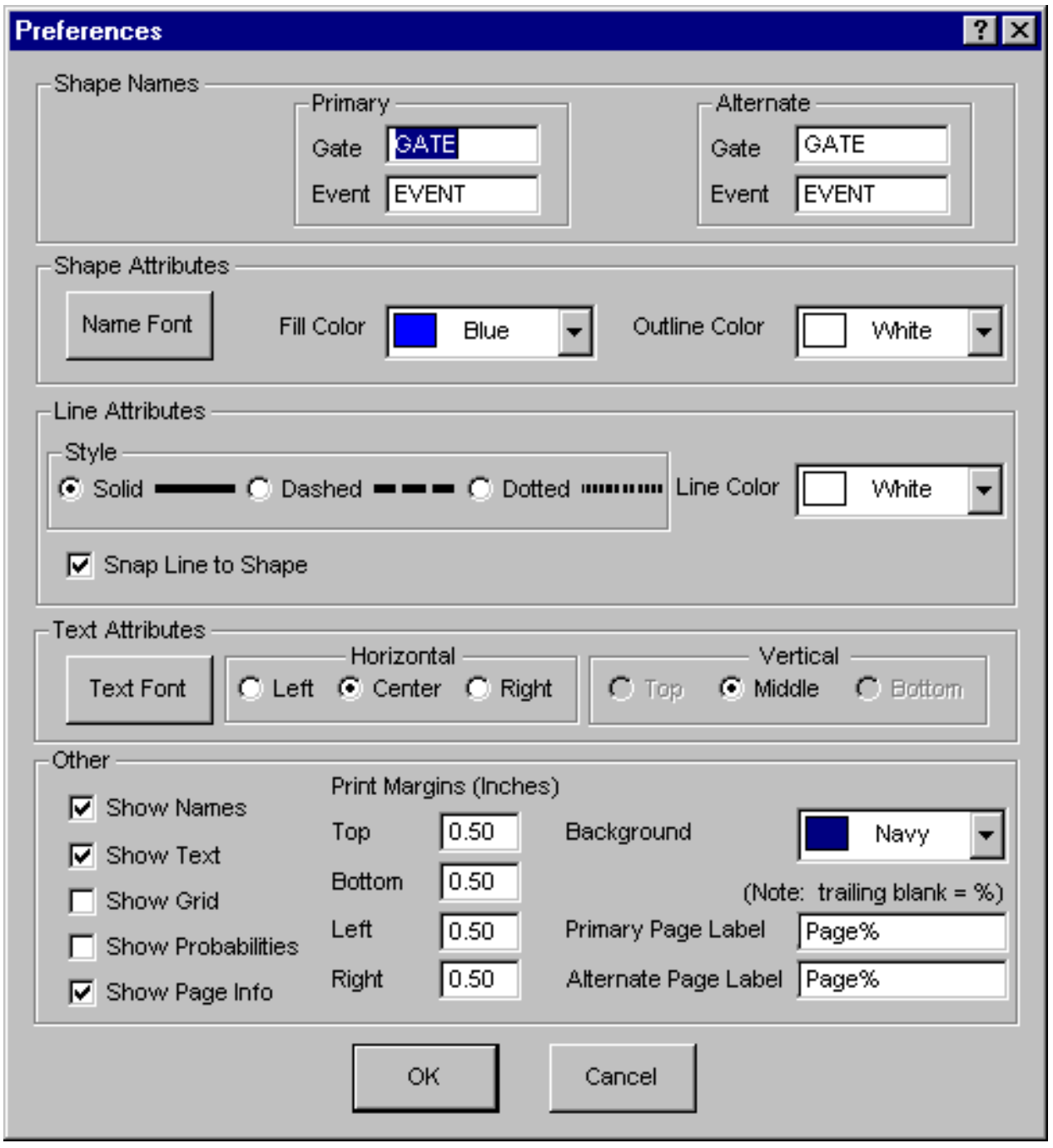

\section{Shape Names -}

Primary

Gate Name Event Name -

Alternate

Gate Name -

Event Name -

Shape Attributes -

Name Font -

Fill Color -

Outline Color -
All shapes created following these changes will use the prefixes entered here.

Default prefix for the primary gate name.

Default prefix for the primary event name.
Default prefix for the alternate gate name.

Default prefix for the alternate event name.

All shapes created following these changes will use the attributes selected here.

The shape name font attributes.

The interior color for shapes. Select from the drop-down list.

The exterior color for shapes. Select from the drop-down list. 
Line Attributes -

Style -

Color -

Snap Line to Shape -

Text Attributes -

Text Font -

Horizontal -

Vertical -

Other

\begin{abstract}
Show names -
Show Text -

Show Grid -
\end{abstract}

Show Probabilities -

Show Page Info -

Print Margins -

Background -

Primary Page Label -

Alternate Page Label -
All lines created following these changes will use the attributes selected here.

The line style (solid, dashed, or dotted).

The color for connecting lines. Select from the drop-down list.

If checked, lines drawn from any part of one shape to any part of another will be adjusted to connect the two shapes. If not checked, lines will be placed exactly where dropped.

All text objects created following these changes will use the attributes selected here.

The font attributes for text objects.

The horizontal alignment for text objects.

The vertical alignment for text objects.

If checked, the names for all shapes will be displayed.

If checked, all descriptive text will be displayed.

If checked, a background grid will be displayed to help align shapes and lines.

If checked, the probabilities of the basic events will be displayed.

If checked, turns on the display of page information at the

bottom of the diagram.

Set the top, bottom, left, and right margins, in inches, for printing. Enter the values in real numbers.

Indicates the background color in the editor window. Select from the drop-down list.

The label entered is inserted in front of the page number. This label is used when primary names are displayed and printed.

The label entered is inserted in front of the page number. This label is used when alternate names are displayed and printed. The alternate name display feature is set in the Define Constants option.

OK - Close the Preferences dialog and apply any changes.

Cancel - Close the Preferences dialog without applying the changes.

\subsubsection{Default Shape Name}

\section{PURPOSE}

Define the usage of the default gate and event names in the Preferences dialog.

When a new gate is added to the diagram, the entry in the Gate field is used as a prefix for every new gate's name. This default gate name prefix is combined with a counter number to form a gate name. When a new event is added, the entry in the Event field is used as a prefix for every new event's name. This default event name prefix is combined with a counter number to form an event name. The counter number will be incremented by one every time a gate or event is added. The counter number is initialized to 0 every time you start the graphical Fault Tree Editor. 
For example, suppose that the default gate prefix is GATE and the default event prefix is EVENT. Also suppose that shapes for the fault tree are created in the following order: gate, gate, event, event, gate. The name of the first gate is, by default, GATE0. The name of the next gate is GATE1. The first event added is named EVENT2. The next event added is EVENT3. The last shape in this series is a gate. Its name is GATE4.

\section{STEPS}

1. In the Fault Tree Editor window, right-click to invoke the pop-up menu.

$O R$

2. Choose View from the menu.

3. Select Preferences from the menu. The Preferences dialog will be displayed.

4. In the Shape Names section, enter the desired prefix in the Gate or Event field.

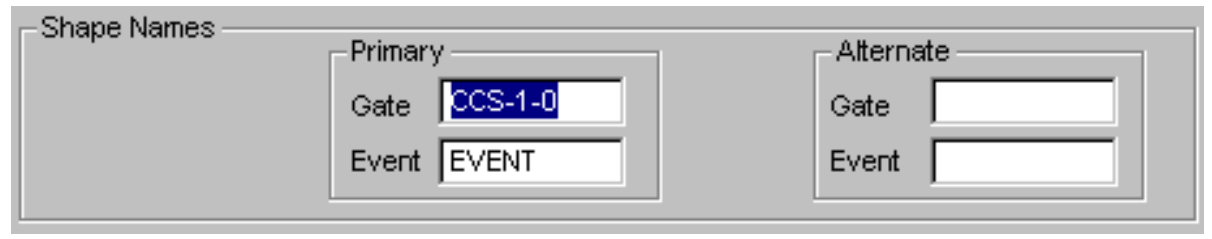

\subsubsection{Search Menu}

\subsubsection{Search}

These options provide search and cursor positioning operations.

Find and Replace - $\quad$ Find a word and replace it with another word.

Find Name - $\quad$ Find a particular shape or verify that a certain shape exists in the diagram.

\subsubsection{Global Find and Replace Text}

\section{PURPOSE}

This option allows you to find a word (i.e., sequence of alphanumeric characters) and replace it with another word. All descriptive text and shape names will be searched and the replaced if found. The search operation is case-sensitive.

\section{STEPS}

1. From the Fault Tree Editor menu select Search.

2. Select Find and Replace from the menu.

3. The Global Find and Replace Text dialog will be displayed 


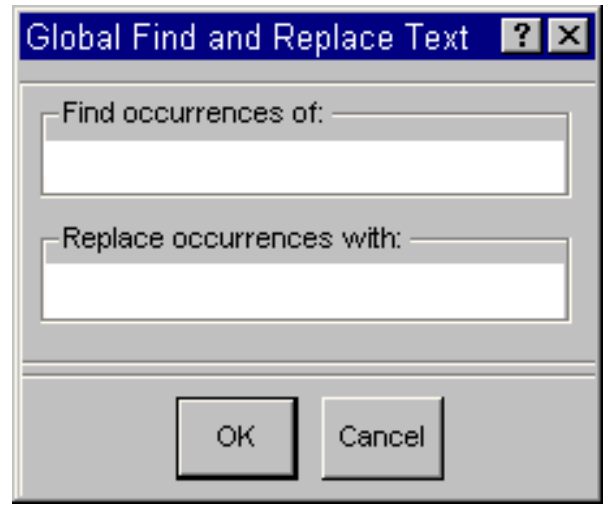

Find occurrences of -

Enter the word or phrase you wish to replace

Replace occurrences with Enter the replacement word or phrase.

OK -

Search for the exact occurrence of the "find" word or phrase and substitute it with the exact replacement word or phrase. The

Cancel Global Find and Replace Text dialog will be closed. Close the Global Find and Replace Text dialog without performing the search-and-replace operation.

\subsubsection{Find Name}

\section{PURPOSE}

This option allows you to find a particular shape or verify that a certain shape exists in the diagram. The search operation is case-sensitive.

\section{STEPS}

1. From the Fault Tree Editor menu select Search.

2. Select Find Name from the menu.

3. The Find Shapes dialog will be displayed.

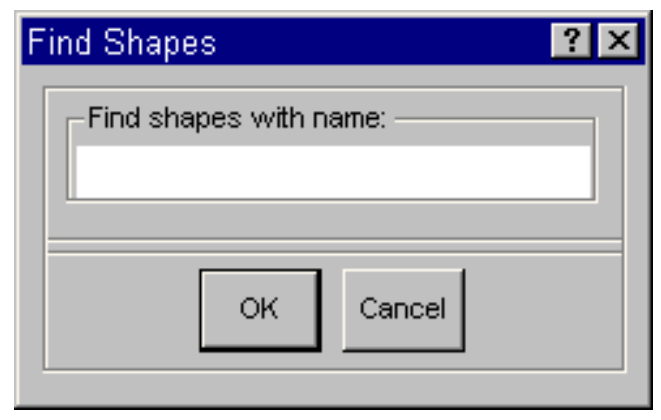

Find shapes with name - $\quad$ Enter the shape name search criteria (i.e., the name of the shape to perform the search on). 
OK -

Cancel -
Search for the shape whose name exactly matches the entered name and close the Find Shapes dialog. The shape matching the search criteria will be highlighted, if found.

Close the Find Shapes dialog without performing the search operation.

\subsubsection{Timed Backup}

\subsubsection{Restore Backup File}

While editing a fault tree or event tree diagram, SAPHIRE will periodically make a backup of the file. If the editing session terminates abnormally, the next time the fault tree diagram is edited, the Restore Backup File dialog will be displayed.

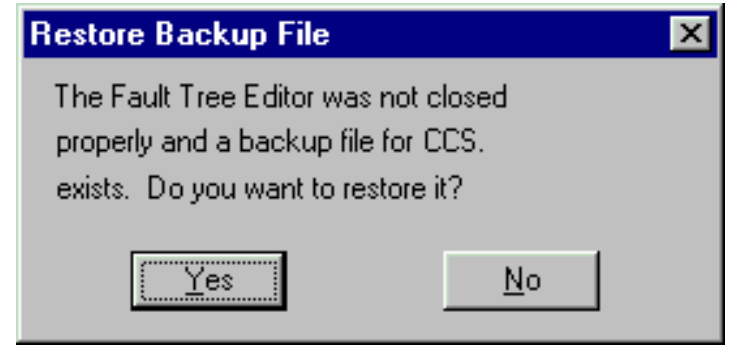

Yes - $\quad$ Edit the backup version of the diagram. This option will restore most of the changes made from the aborted editing session.

No - $\quad$ Discard the backup version of the diagram. Edit the diagram as it existed prior to the aborted editing session.

\subsection{Piping and Instrumentation Diagram Editor}

\subsubsection{P\&ID Graphics}

\section{PURPOSE}

This graphical editor provides the ability to construct piping and instrumentation flow diagrams using the Piping \& Instrumentation Diagram Editor. These diagrams are used only for graphical reference and are intended to represent the physical layout of a system.

\section{STEPS}

1. From the SAPHIRE menu select Fault Tree. The Fault Trees List dialog will be displayed.

2. Highlight the desired fault tree. (Skip this step if creating a new P\&ID.)

3. Right-click to invoke the pop-up menu and select Edit P+ID.

4. The PID Editor window will be displayed. 


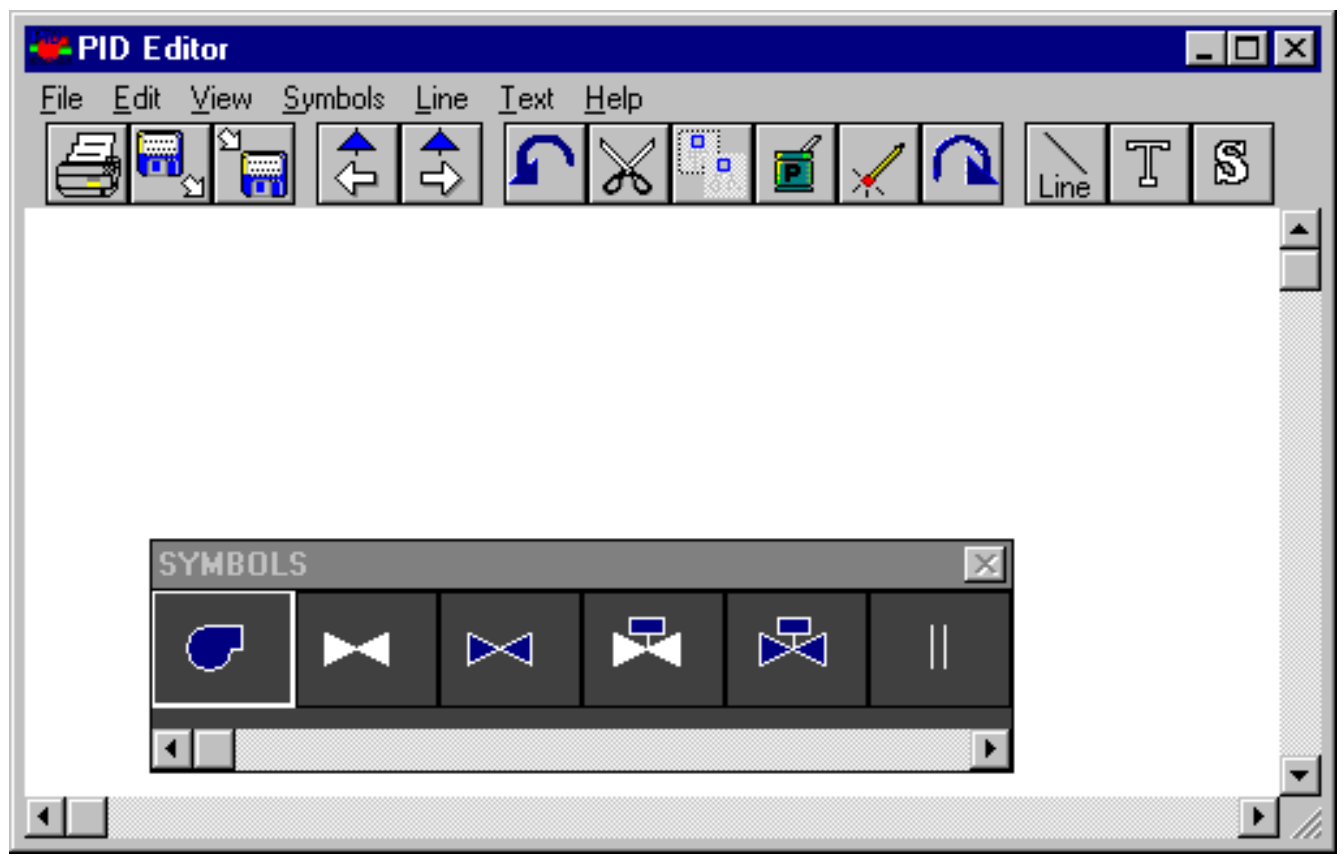

Options are available from the menu and/or the too bar.

\section{Menu Options}

File - $\quad$ File manipulation functions such as open, save, print, etc.

Edit - Editing functions such as copy and paste along with attribute setting.

View - Control of visual display such as zoom, refresh, etc.

Symbols - Modify symbol attributes such as display of symbol table and color.

Line - Modify line attributes such as style and color.

Text - $\quad$ Modify text attributes such as color, size, alignment, etc.

Help - SAPHIRE on-line help.

\section{Tool Bar Options}

\section{Print the current diagram}

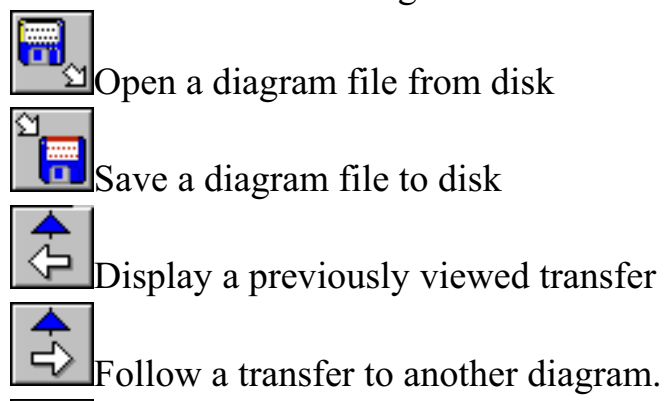

\section{Not yet available.}

$\$$

\$6 Cut an object from the diagram

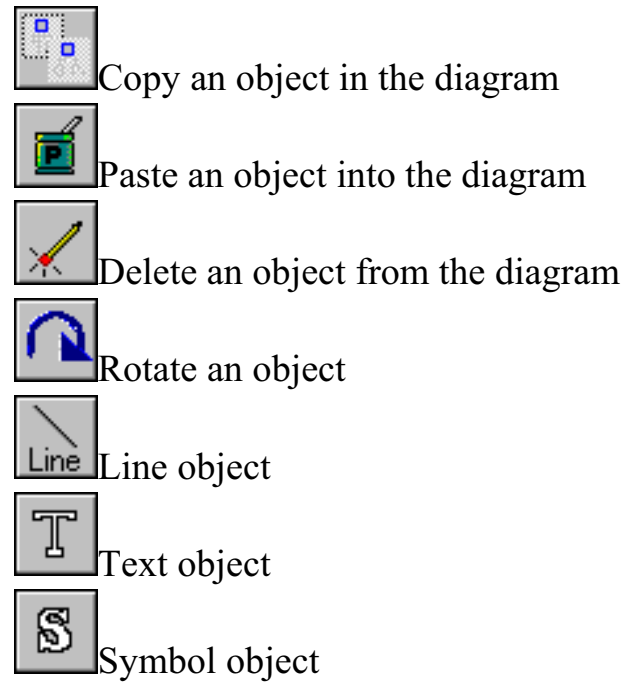




\subsubsection{P\&ID Graphics - Definitions}

The following definitions are used with reference to the SAPHIRE graphical P\&ID Editor.

Object -

Pick Box -

Rubber band rectangle -

Symbol -

Line -
The building blocks of a piping and instrumentation flow diagram. They include symbols, lines that connect symbols, and descriptive text. Each object has attributes which may include color, size, and name.

An invisible rectangle which surrounds every object. It defines the area of the object where the mouse cursor must be positioned in order to select the object.

A rectangle displayed on the screen showing the area currently selected during a drag function, sometimes referred to as a "region". This is used when selecting more than one object. The region enclosed by the rubber band rectangle can be redefined by positioning the mouse over one of the sides or corners of the rubber band rectangle. When one of the resize cursors (e.g.,

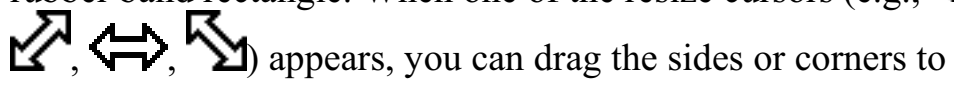
adjust the size of the rectangle.

A graphical representation of a component (e.g., pump, valve, etc.).

A special symbol used to link other symbols together, such as a pipe connecting two valves.

\subsubsection{P\&ID Graphics - Mouse Usage}

All mouse actions defined in the SAPHIRE graphical $P \& I D$ Editor are for a standard two-button mouse.

Click - $\quad$ The left mouse button is pressed and released.

Right-click - The right mouse button is pressed and released.

Double-click - The left mouse button is pressed and released two times in rapid succession.

Drag - $\quad$ The left mouse button is pressed and held down while moving the mouse.

\subsubsection{Selecting Objects (Regions)}

\section{PURPOSE}

Prior to changing the attributes of objects (e.g., color, size, etc.) in the diagram, they must be selected. This option describes how to select objects. 


\section{STEPS}

1. Position the standard cursor over an area beside the desired object(s).

2. Drag the mouse to form a rubber band rectangle that completely surrounds the object(s).

3. Release the mouse button. The objects inside the rubber band rectangle are now the currently selected objects (i.e., are inside the selected region).

4. Editing functions can now be performed on these objects.

It is important to note that only those items that are completely within the boundaries of the rubber band rectangle will be "selected", except the lines. The rectangle only need to intersect with the lines in order for them to be "selected".

The rubber band rectangle can be redefined by repeating steps 2 and 3 or by using one of the resize cursors.

\subsubsection{P\&ID Graphics - Standard Symbols}

The following are examples of the symbols in the standard P\&ID Graphics standard Symbol Table.
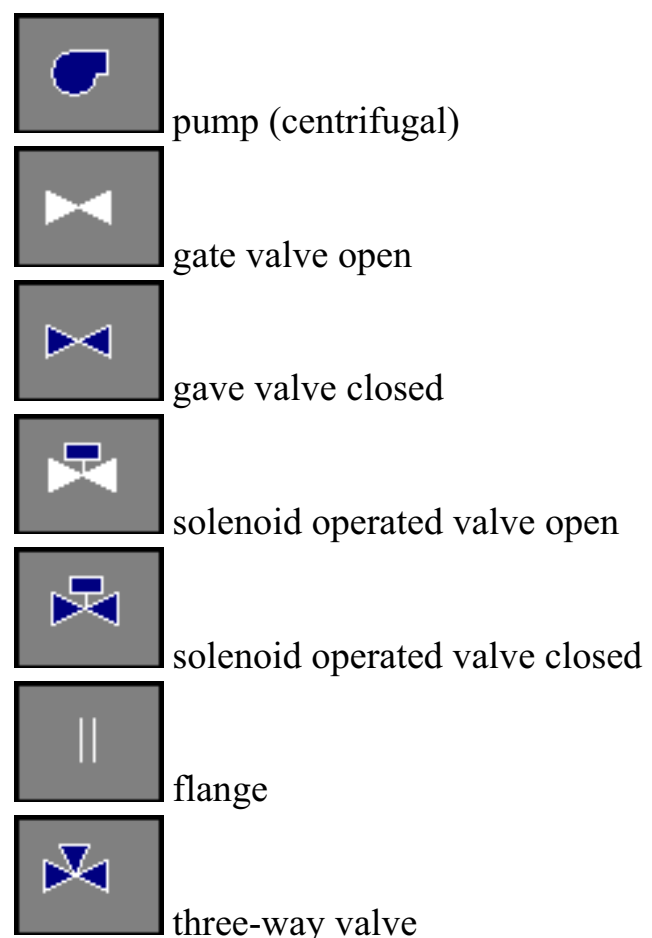

flange

three-way valve

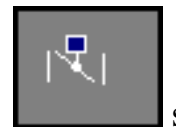

solenoid operated butterfly valve

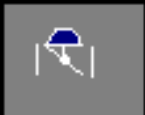

diaphragm operated butterfly valve

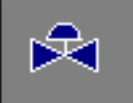

diaphragm operated valve closed

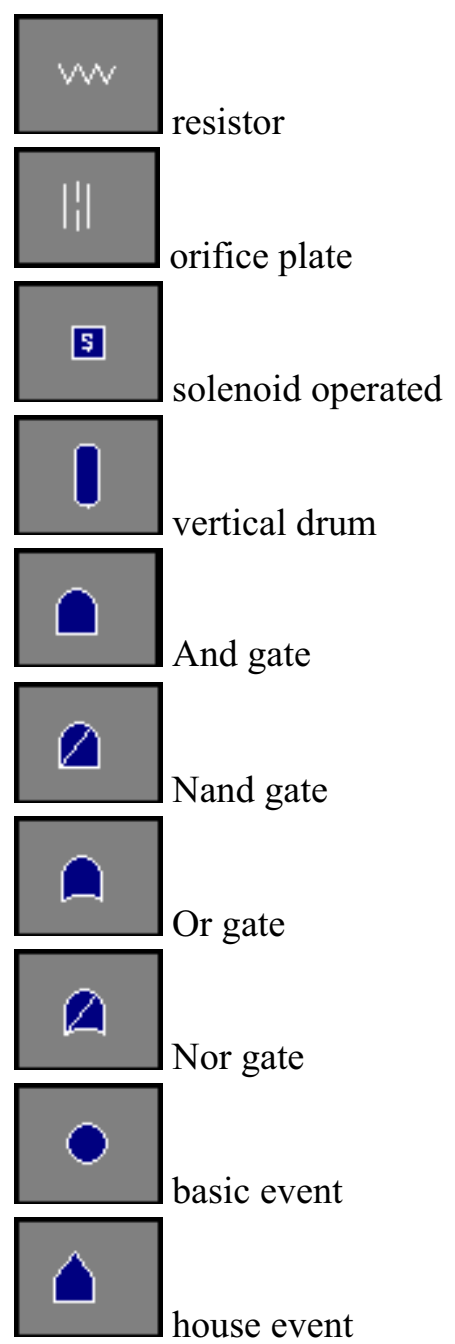



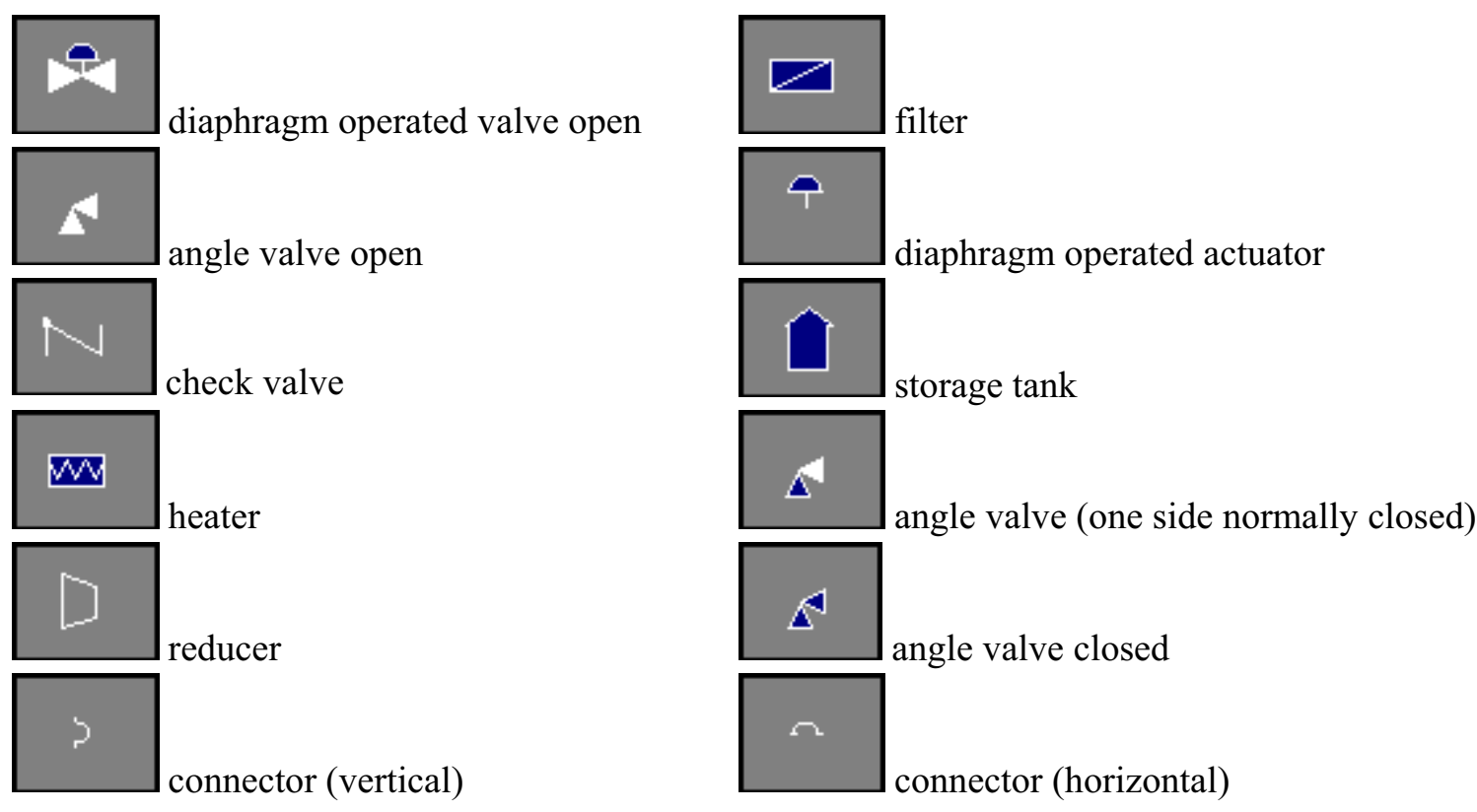

\subsubsection{File Menu}

\subsubsection{File}

These options provide file (diagram) manipulation functions in the P\&ID Editor.

New -
Open -
Save -
Save As -
Print -
Transfer Out -
Transfer In -
New Transfer -
Load New Symbol Table -
Defaults -
Exit -

Create a new file in the editing window. Open a file in the editing window Save changes in the diagram to disk Save the file in the editing window with a new file name. Print the file in the editing window. Display a previously viewed transfer. Follow a transfer to another diagram. Add a transfer symbol to the diagram. Use a different symbol table.

Change the P\&ID graphical editor preferences.

Terminate the P\&ID Editor. If changes have been made you will be queried if you want to save the file.

\subsubsection{New}

\section{PURPOSE}

This option will allow you to clear the window and begin creating a new diagram.

\section{STEPS}

1. From the $P \& I D$ Editor menu select File $\mid$ New.

2. The contents of the window will be cleared and you can begin building a new diagram. 
If you have not saved the current diagram you will be prompted by a Message dialog to do so.

\subsubsection{Open}

\section{PURPOSE}

This option will allow you to select an existing diagram (i.e., P\&ID graphics file) to view or edit.

\section{STEPS}

1. From the P\&ID Editor menu select File |Open.

OR

2. Choose the Open button

3. The Open common dialog will be displayed listing all the P\&ID diagrams (.PID files) in the current project directory.

\subsubsection{Open Dialog}

\section{PURPOSE}

This dialog is displayed with opening an existing diagram or adding a transfer to an existing diagram.

\section{STEPS}

1. Select the desired diagram and choose Open,

OR

2. Double-click on the diagram name.

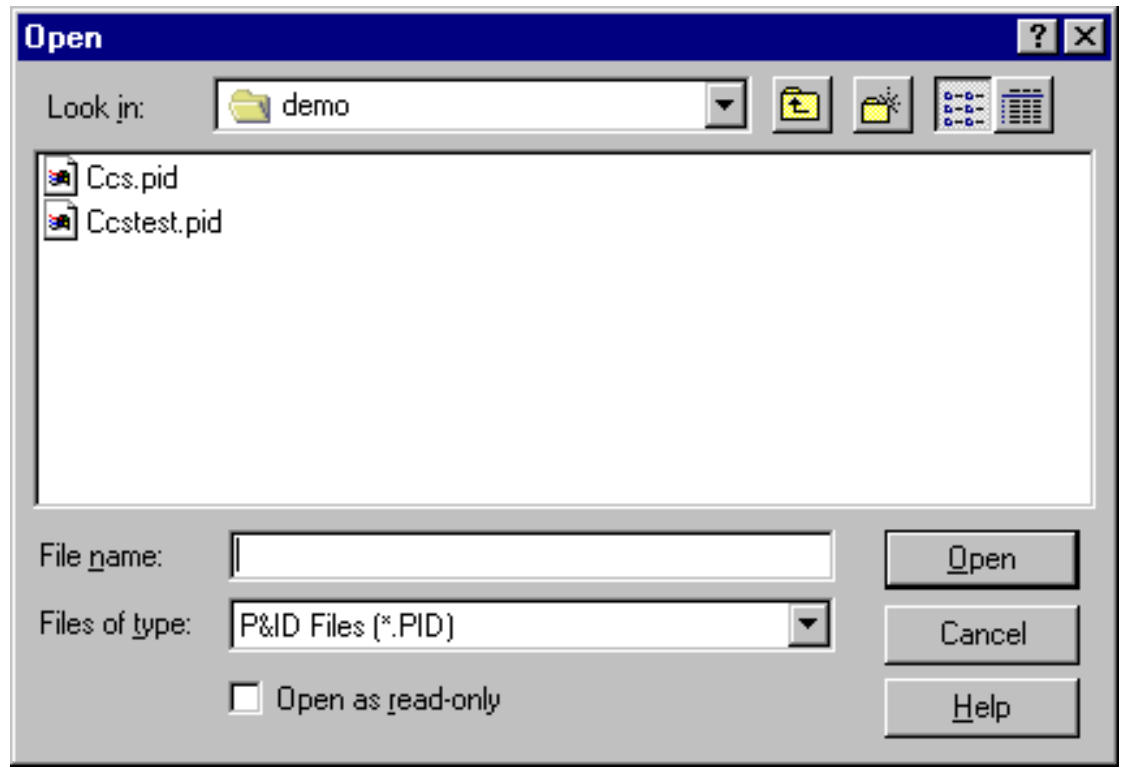


Look in - $\quad$ Lists the available folders and files. The box directly below it shows the contents of the current folder. The down-arrow to the right of this field shows how the folder fits into your system hierarchy.

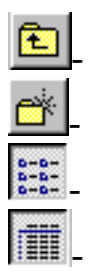

Moves up one level in the system hierarchy.

Creates a new folder at the current level.

Displays the icons and names of the contents of the current folder.

Displays the icons, names, size, type, date and time modified, and archive flags of the contents of the current folder.

File Name - Contains the name of the file in which the diagram will be saved.

Files of Type - Lists the types of files to display. This is useful for narrowing the list of files displayed to only those you are interested in. In this case, only ".PID” files are listed.

Open - $\quad$ Open the highlighted diagram file and close the Open dialog.

Cancel - $\quad$ Close the Open dialog without selecting a diagram.

Double-click - Open the diagram file and close the Open dialog.

If you have selected a diagram to open and you have not saved the current one you will be prompted by a Message dialog to do so.

\subsubsection{Message : Current Drawing Not Saved}

\section{PURPOSE}

This dialog will appear any time you are leaving the current diagram which has not been saved. You leave the current diagram by exiting, following a transfer, opening another diagram, or creating a new diagram.

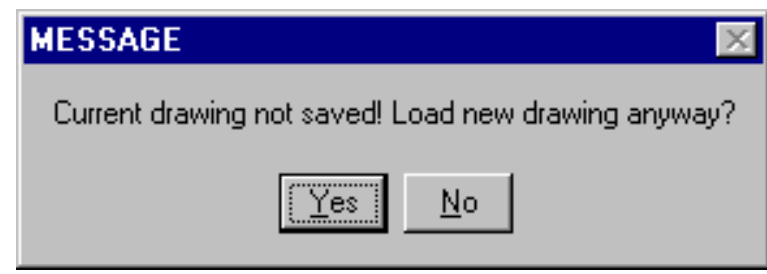

Yes -

The current diagram will be saved to disk, and the next operation (Exit or change diagram) will be performed.

No The current diagram will not be saved to disk and the next operation (Exit or change diagram) will not be performed.

\subsubsection{Save}

\section{PURPOSE}

This option will save any changes made in the current P\&ID diagram to the file presently open. If this is a new diagram, then you will be allowed to name the file.

\section{STEPS}

1. From the P\&ID Editor menu select File | Save. 
OR

2. Choose the Save button on the toolbar.

3. If this is not a new diagram, the data is saved.

OR

4. If this is a new diagram, see Save As .

\subsubsection{Save As}

\section{PURPOSE}

This option will save any changes made in the current P\&ID diagram to a file you select.

\section{STEPS}

1. From the $P \& I D$ Editor menu select File.

2. Choose Save as from the menu

3. The Save common dialog will be displayed listing all the P\&ID diagrams (.PID files) in the current project directory.

4. Enter the name of the file in the File Name field,

$O R$

5. Select the desired diagram file and choose Save,

$O R$

6. Double-click on the diagram name.

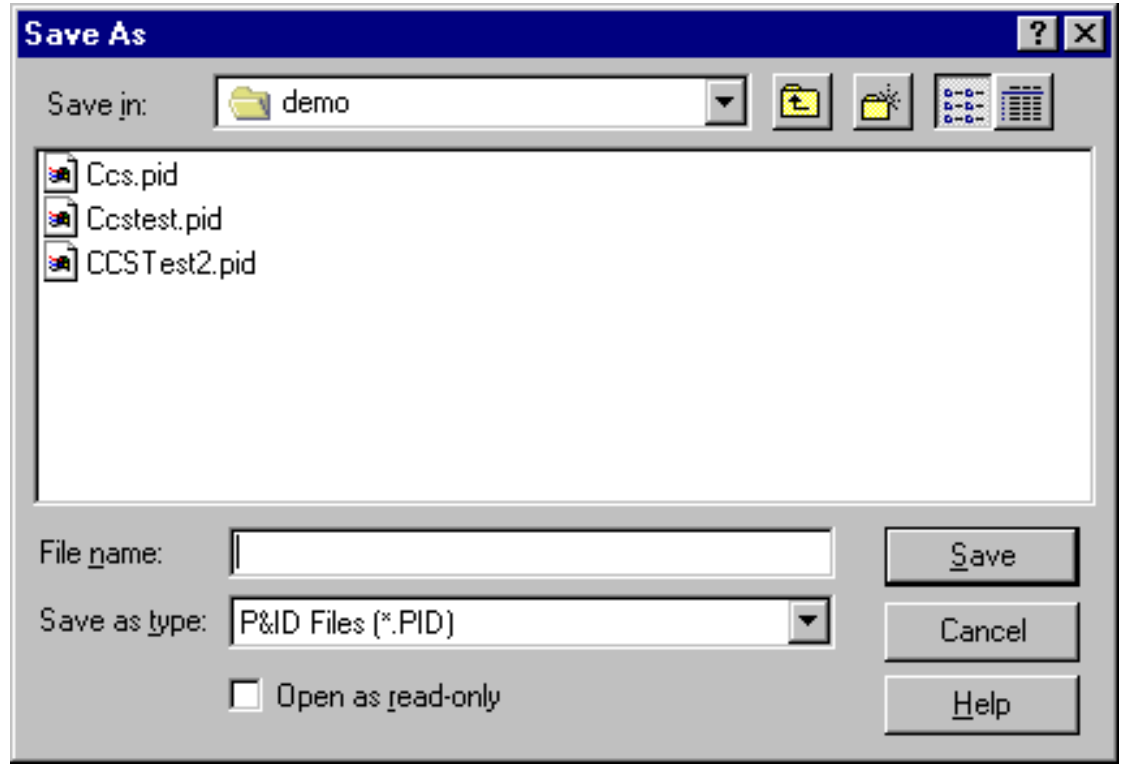

Save in - $\quad$ Lists the available folders and files. The box directly below it shows the contents of the current folder. The down-arrow to the right of this field shows how the folder fits into your system hierarchy.

Moves up one level in the system hierarchy. 
Creates a new folder at the current level.

Displays the icons and names of the contents of the current folder.

Displays the icons, names, size, type, date and time modified, and archive flags of the contents of the current folder.

File Name - Contains the name of the file in which the diagram will be saved.

Files of Type - Lists the types of files to display. This is useful for narrowing the list of files displayed to only those you are interested in. In this case, only .PID files are listed.

Save - $\quad$ Save the current diagram to the file in the File name field and close the Save dialog. If an existing file was selected, a warning dialog will be displayed.

Double-click - Save the current diagram to the file in the File name field and close the Save dialog.

Cancel - $\quad$ Close the Save dialog without saving the diagram.

\subsubsection{Message : File Already Exists}

This dialog is displayed when you choose to save the current diagram to an already existing file.

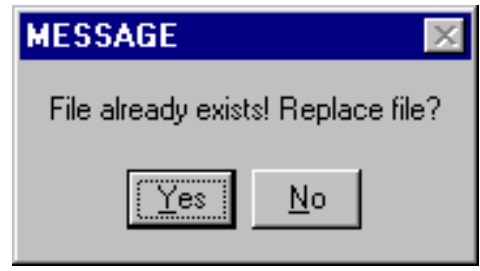

Yes - $\quad$ Save the current diagram to the selected file. The diagram in the existing file will be overwritten.

No - $\quad$ Do not save the current diagram to the select file.

\subsubsection{Print}

\section{PURPOSE}

This option will print the current diagram to the selected printer. You are provided the opportunity to set additional options, such as page range, number of copies, etc., before printing the diagram.

\section{STEPS}

1. From the Fault Tree Editor or Event Tree Editor or P\&ID Editor menu select File | Print. OR

2. When in the Fault Tree Editor, choose the Print button on the toolbar.

3. The Print common dialog will be displayed. 


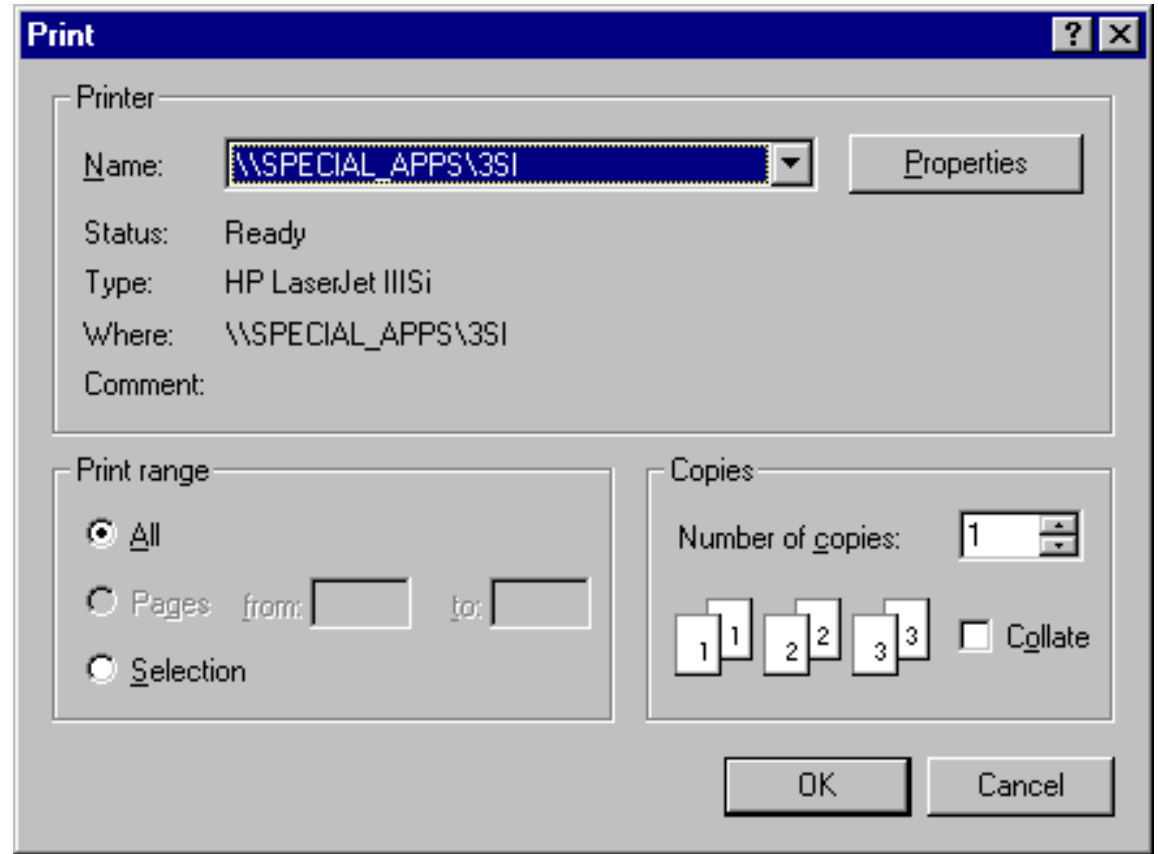

Name - $\quad$ List the printers that are set up for this computer.

Properties - $\quad$ Set-up options for the selected printer. Available options depend on the features of the printer.

Status - $\quad$ Condition of the selected printer.

Type - Kind of selected printer.

Where - Location of the selected printer.

Comment - $\quad$ Miscellaneous information about the selected printer.

Print Range - Print the entire document, specified range, or highlighted selection.

Copies Enter the number of copies you wish to print.

Collate - $\quad$ For more than one copy, specify whether you want the copies collated.

OK - $\quad$ Close the Print Dialog and print the current diagram to the selected output device.

Cancel - $\quad$ Close the Print Dialog without printing the diagram.

\subsubsection{Transfer Out}

This option is not yet available.

\section{PURPOSE}

This option will list all the diagrams previously viewed in the current chain of transfers, including the current diagram. Select the diagram you wish to edit. Any changes made to the current diagram can be saved before viewing the transfer diagram.

\section{STEPS}

1. $\quad$ From the P\&ID Editor menu select File | Transfer Out.

$O R$ 


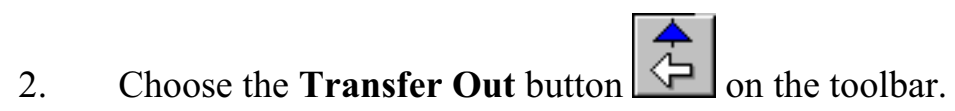

\subsubsection{Transfer In}

This option is not yet available.

\section{PURPOSE}

This option will display the diagram corresponding to the selected transfer in the current diagram. Any changes made to the current diagram can be saved before viewing the transfer diagram.

\section{STEPS}

1. From the $P \& I D$ Editor menu select File | Transfer In.

OR

2. Choose the Transfer In button $\stackrel{\Delta}{\Rightarrow}$ on the toolbar.

\subsubsection{New Transfer}

\section{PURPOSE}

This option allows you to add a transfer symbol to the current diagram. The transfer symbol is used to represent a corresponding P\&ID file.

\section{STEPS}

1. From the P\&ID Editor menu select File.

2. Choose New Transfer from the menu.

3. The cursor is changed to the Transfer cursor

4. Position the cursor over the desired transfer location and click the mouse.

5. The Open common dialog will be displayed listing all the P\&ID files (.PID files) in the current project directory.

6. Select the desired P\&ID file and choose $\mathbf{O K}$.

7. The transfer symbol ccs will be placed in the diagram. The symbol name will be the name of the corresponding file.

\subsubsection{Load New Symbol Table}

This option is not yet available. 


\section{PURPOSE}

This option allows you to use a different symbol table with this diagram.

\subsubsection{Defaults}

\section{PURPOSE}

This option allows you to modify the preferred defaults for the graphical P\&ID Editor.

\section{STEPS}

1. From the P\&ID Editor menu select File.

2. Choose Defaults from the menu.

3. The Default Settings dialog will be displayed.

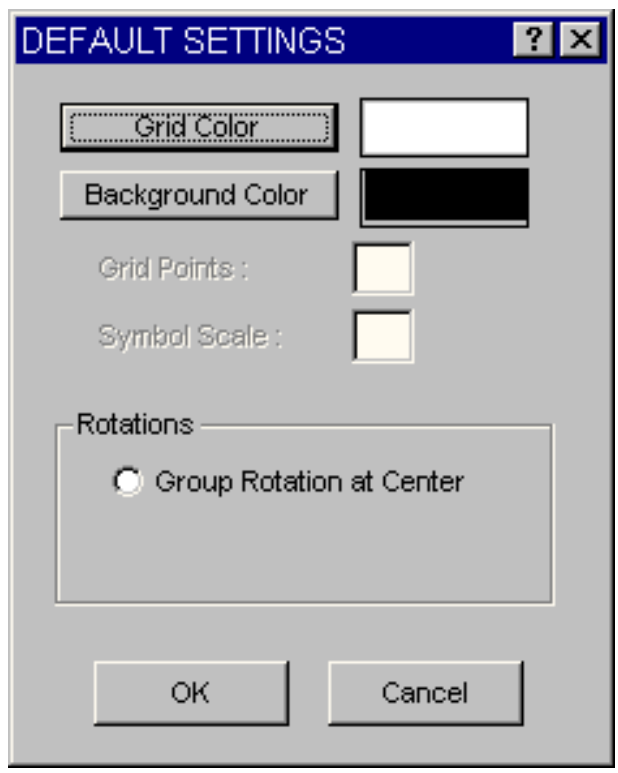

Grid Color -

The color of the grid.

Background Color - The background color for the window.

Grid Points This option is not yet available.

Symbol Scale This option is not yet available.

Rotations This option is not yet available.

OK Apply the changes and close the Default Settings dialog.

Cancel Close the Default Settings dialog without applying the changes.

\subsubsection{Edit Menu}

\subsubsection{Edit}

These options provide editing and text formatting functions in the P\&ID Editor.

Undo - Undo the previous editing operation. 
Cut - Cut selected objects from the diagram.

Copy - Copy selected objects in the diagram.

Paste - Copy the clipboard object into the diagram at the cursor position.

Delete - Remove selected objects from the diagram.

Move - Move selected objects in the diagram.

Rotate - Turn selected objects in the diagram.

Scale - Change the size of selected objects in the diagram.

Flip - Invert selected objects in the diagram.

Symbol Fill Color - Change the symbol interior color.

Symbol Name - Change the name of selected symbols in the diagram.

Symbol Name Font - Change the font for symbol name display.

Line Color - Change the color of selected lines.

Text Attributes - Change the font, color, or alignment of the selected text objects.

\subsubsection{Undo}

This option is not yet available.

\subsubsection{Cut}

This option is not yet available.

\subsubsection{Copy}

\section{PURPOSE}

This option copies selected objects in the diagram and allows you to place the copies in another region of the diagram.

\section{STEPS}

1. Select the region in the diagram to be copied.

2. From the P\&ID Editor menu select Edit | Copy.

OR

3. Choose the Copy button 10 on the toolbar.

4. Position the cursor in the area of the diagram where you want the copied symbols to be placed.

Click the mouse.

\subsubsection{Paste}

This option is not yet available.

\subsubsection{Delete}

\section{PURPOSE}

This option deletes selected object(s) from the diagram. 


\section{STEPS}

1. Select the object(s) in the diagram to be deleted. It is important to note that only those objects that are completely within the boundaries of the rubber band rectangle will be deleted, except for lines. To delete lines, the rectangle only needs to intersect with the lines.

2. From the $P \& I D$ Editor menu select Edit | Delete.

$O R$

3. Choose the Delete button on the toolbar.

4. The Delete Region dialog will be displayed.

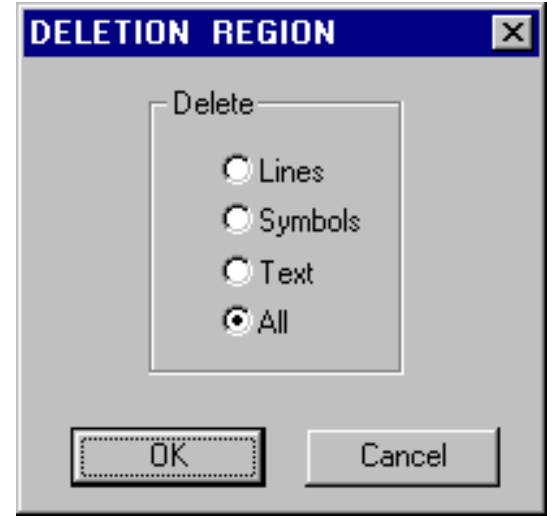

\section{Delete}

Lines - Delete all lines within the selected region.

Symbols - Delete all symbols within the selected region.

Text - Delete all text within the selected region.

All - $\quad$ Delete all objects within the selected region.

OK - $\quad$ Perform the delete operation based on the selected radio button and close the Delete Region dialog.

Cancel - $\quad$ Close the Delete Region dialog without deleting the selected objects.

\subsubsection{Move}

This option is not yet available.

\section{PURPOSE}

This option allows you to move the selected objects to a new location in the diagram.

\subsubsection{Rotate}

\section{PURPOSE}

This option rotates or turns selected object(s) in the diagram. 


\section{STEPS}

1. Select the object(s) in the diagram to be rotated.

2. From the $P \& I D$ Editor menu select Edit | Rotate.

OR

3. Choose the Rotate button on the button bar.

4. The Rotation dialog will be displayed.

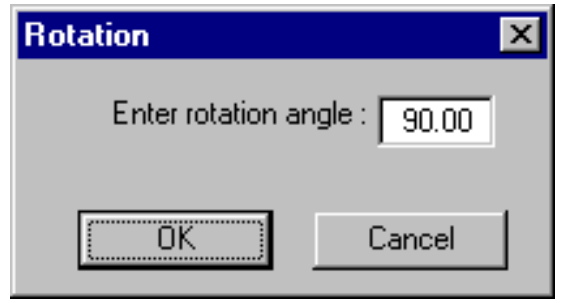

Enter Rotation Angle - The angle, in degrees (0 to 360), to turn the object(s).

OK -

Rotate the selected object(s) the indicated number of degrees and close

Cancel the Rotation dialog. Close the Rotation dialog without rotating the object(s).

\subsubsection{Scale}

This option is not yet available.

\section{PURPOSE}

This option allows you to change the size of objects in the diagram.

\subsubsection{Flip}

This option is not yet available.

\section{PURPOSE}

This option allows you to invert a symbol. For example, a given symbol may be defined in a particular drawing as having one input and one output. The input is on the left side of the symbol and the output on the right. This defines the flow or direction of the symbol as left to right. However, the logic or drawing may dictate that the flow is going in a right to left direction. Connecting the symbols inappropriately may yield incorrect logic in the diagram. Therefore, the symbol may be flipped to invert the direction of flow through the symbols.

\subsubsection{Symbol Fill Color}

This option is not yet available.

\subsubsection{Symbol Name}




\section{PURPOSE}

This option allows you to change the name of the selected symbol(s).

\section{STEPS}

1. Select the object(s) in the diagram to be renamed.

2. From the P\&ID Editor menu select Edit.

3. Choose Symbol Name from the menu.

4. The Symbol Attributes dialog will be displayed.

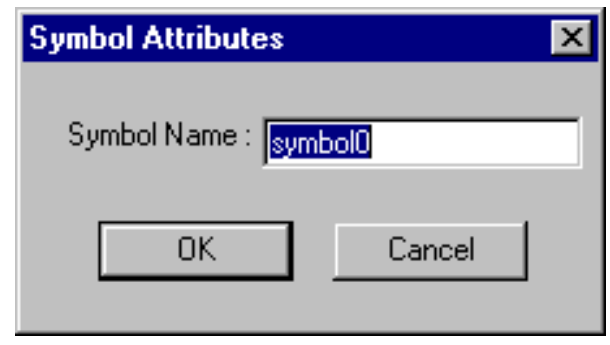

Symbol Name - The current name of the symbol is displayed in this field. Enter the new name by typing over of inserting new text.

OK - $\quad$ Apply the change and close the Symbol Attributes dialog. If more than one object has been selected, the Symbol Attributes dialog will be displayed for each selected object.

Cancel - $\quad$ Close the Symbol Attributes dialog without changing the name.

\subsubsection{Symbol Name Font}

This option is not yet available.

\subsubsection{Modify Line Color}

\section{PURPOSE}

This option allows you to change the color of the selected line(s).

\section{STEPS}

1. Select the desired line(s) in the diagram.

2. From the $P \& I D$ Editor menu select Edit.

3. Choose Line Color from the menu.

4. The Choose a Color dialog will be displayed.

\subsubsection{Choose a Color Dialog}




\section{PURPOSE}

This option allows you to change the color of selected objects and of objects created after this change.

Select the desired color by clicking on it.

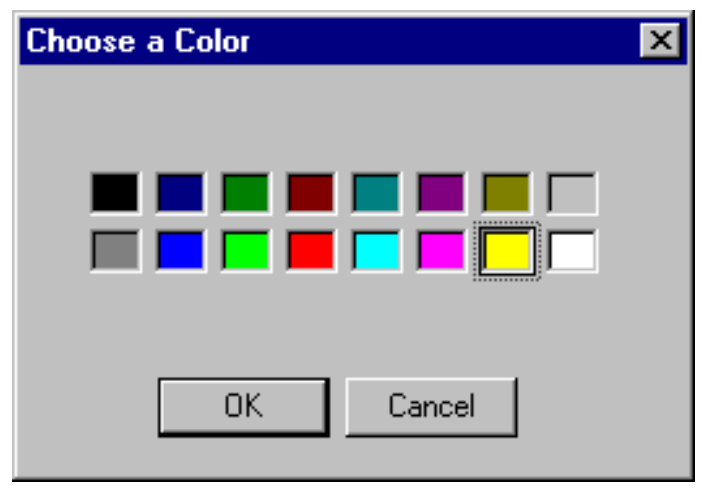

In the example above the color, yellow, is currently selected.

OK - Change the color of the selected object(s) and close the Choose a Color dialog. Objects that are created after this change will be created with this color.

Cancel - Close the Choose a Color dialog without applying the color change.

\subsubsection{Text Attributes}

\section{PURPOSE}

This option allows you to change the font, color, or alignment of the selected text object(s).

\section{STEPS}

1. Select the desired line(s) in the diagram.

2. From the P\&ID Editor menu select Edit.

3. Choose Text Attributes from the menu.

4. The Text Style dialog will be displayed.

If you choose the $\mathbf{O K}$ button on this dialog, the changes will be applied to the selected text. This change will apply to all new text objects.

\subsubsection{Text Style Dialog}




\section{PURPOSE}

This option allows you to change the font, color, or alignment of the text object(s).

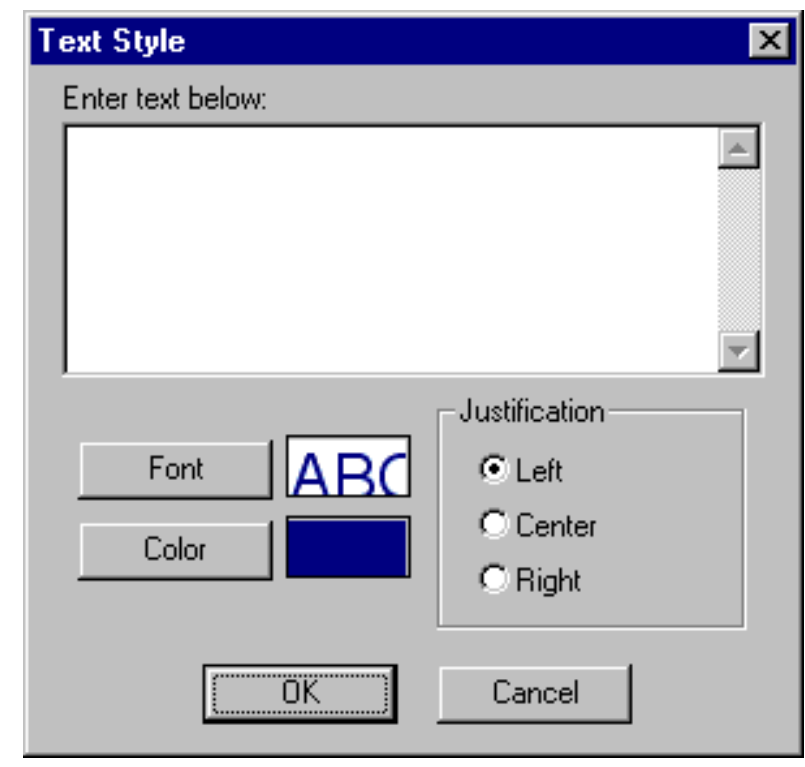

Enter text below: - The alphanumeric characters that make up the text object.

Font Change the font, font style, or size used to display the text.

Color Change the color used to display the text.

\section{Justification}

Left - Align the text to the left

Center - $\quad$ Align the text in the center

Right - $\quad$ Align the text to the right

OK -

Save the changes and close the Text Style dialog.

Cancel -

Close the Text Style dialog without saving the changes.

\subsubsection{Font/Color Dialog}

\section{PURPOSE}

This option allows you to change the font and color of the selected text object(s) and all text created after this change. 


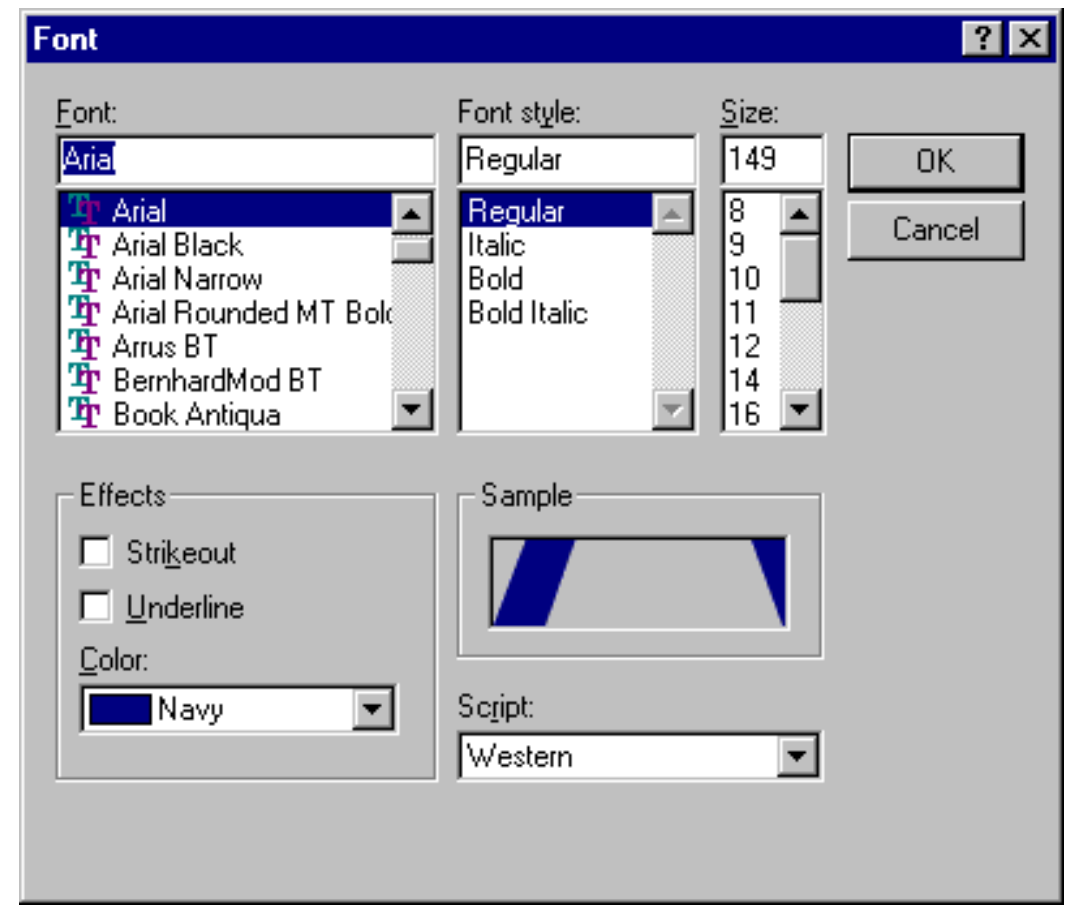

Font - $\quad$ The currently selected font and list of available fonts. Choose a new font from the drop-down list.

Font Style - $\quad$ The currently selected font style (e.g., bold, italic, etc.) and list of available styles. Choose a new style from the drop-down list.

Size - $\quad$ The currently selected size and list of available sizes. Choose from the dropdown list or enter any positive numeric value.

\section{Effects}

Strikeout - $\quad$ Display the text in strikeout mod. (Shown with a horizontal line through

Underline - $\quad$ Display the text with an underline.

Color - $\quad$ The currently selected color. Choose a new color from the drop-down list.

Sample - $\quad$ Displays the current color of the text.

Script - $\quad$ Select the script from the drop-down list.

OK - $\quad$ Save the changes and close the Font dialog.

Cancel - $\quad$ Close the Font dialog without saving the changes. 


\subsubsection{View Menu}

\subsubsection{View}

These menu options allow you to change the portion of the current diagram in the P\&ID Editor viewing window. You can move the view window up, down, left, and right using the scroll bars or menu commands. You can change the size of the viewing window by zooming in or out, adjust the diagram to the previous zoom window, and restore the view window to its original size.

Zoom In - "Enlarge" the objects in the viewing window.

Zoom Out - "Shrink" the objects in the viewing window.

Zoom Restore - Restore the viewing window to its original size

Zoom Previous - Restore viewing window to the previous position.

Grid - $\quad$ Display the background grid.

Snap - $\quad$ Align objects to the grid.

Show Names - Display the symbol names in the diagram.

Show Text - Display text objects in the diagram.

\subsubsection{Zoom In}

\section{PURPOSE}

This option allows you to "enlarge" the objects in the viewing window. It has the effect of reducing the total amount of the diagram included in the viewing window.

\section{STEPS}

1. From the $P \& I D$ Editor menu select View.

2. Choose Zoom In from the menu.

3. The cursor will be changed to the Zoom cursor $\mathbb{R}^{\mathbb{Z}}$.

4. Outline the region of the diagram to be enlarged.

\subsubsection{Zoom Out}

\section{PURPOSE}

This option allows you to "shrink" the objects in the viewing window. It has the effect of increasing the total amount of the diagram included in the viewing window.

\section{STEPS}

1. From the P\&ID Editor menu select View.

2. Choose Zoom Out from the menu.

\subsubsection{Zoom Restore}




\section{PURPOSE}

This option allows you to restore the diagram position in the viewing window to the same relative position as when the diagram file was initially opened. Changes to the objects (i.e., their position, attributes, etc.) are not restored.

\section{STEPS}

1. From the $P \& I D$ Editor menu select View.

2. Choose Zoom Restore from the menu.

3. The initial position of the diagram will be redisplayed.

\subsubsection{Zoom Previous}

\section{PURPOSE}

This option allows you to restore the diagram position in the viewing window to the position just prior to the most recent zoom action. Changes to the objects (i.e., their position, attributes, etc.) are not restored.

\section{STEPS}

1. From the $P \& I D$ Editor menu select View.

2. Choose Zoom Previous from the menu.

3. The previous position of the diagram will be redisplayed.

\subsubsection{Grid}

\section{PURPOSE}

This option displays a background grid to help align symbols, text, and lines. If selected, a check mark to the left of this menu item will be displayed and the grid will appear in the editing window. If deselected, there will not be a check mark next to the menu item and the background grid will not be displayed. This option acts as a toggle.

\section{STEPS}

1. From the $P \& I D$ Editor menu select View.

2. Choose Grid from the menu. The background grid will be displayed.

The next time the View menu is invoked, a check mark will appear to the left of this item. 


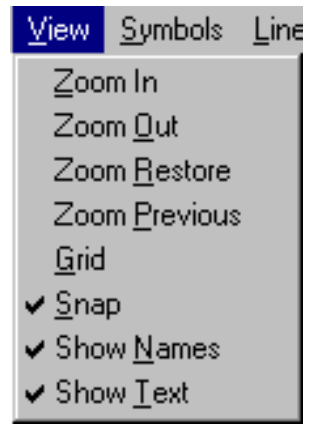

In the example of the View menu shown here, the Grid option is not currently selected.

\subsubsection{Snap}

\section{PURPOSE}

This option aligns symbols, text, and lines to the grid as they are created. If selected, a check mark to the left of this menu item will be displayed and the snap option will be activated. If deselected, there will not be a check mark next to the menu item and snap option will be deactivated. This option acts as a toggle.

\section{STEPS}

1. From the $P \& I D$ Editor menu select View.

2. Choose Snap from the menu.

Until this option is deactivated, objects created or in the diagram will be aligned with the grid. The next time the View menu is invoked, a check mark will appear to the left of this item.

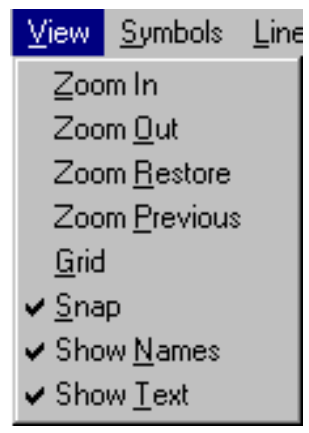

In the example of the View menu shown here, the Snap option is currently selected.

\subsubsection{Show Names}

\section{PURPOSE}

This option allows you to display the symbol names. If selected, a check mark to the left of this menu item will be displayed and the symbol names will appear in the editing window. If deselected, there will not be a check mark next to the menu item and the symbol names will not be displayed. This option acts as a toggle. 


\section{STEPS}

1. From the $P \& I D$ Editor menu select View.

2. Choose Show Names from the menu. The symbol names will be displayed.

The next time the View menu is invoked, a check mark will appear to the left of this item.

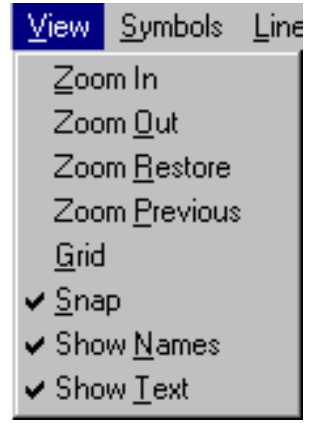

In the example of the View menu shown here, the Show Names option is currently selected.

\subsubsection{Show Text}

\section{PURPOSE}

This option allows you to display text objects in the diagram. If selected, a check mark to the left of this menu item will be displayed and the text objects will appear in the editing window. If deselected, there will not be a check mark next to the menu item and the text objects will not be displayed. This option acts as a toggle.

\section{STEPS}

1. From the $P \& I D$ Editor menu select View.

2. Choose Show Text from the menu. The text objects will be displayed.

The next time the View menu is invoked, a check mark will appear to the left of this item.

\begin{tabular}{|l|}
\hline View Symbols Line \\
\hline Zoom In \\
Zoom Qut \\
Zoom Reestore \\
Zoom Previous \\
Grid \\
$\checkmark$ Snap \\
$\checkmark$ Show Names \\
$\checkmark$ Show Iext \\
\hline
\end{tabular}

In the example of the View menu shown here, the Text option is currently selected. 


\subsubsection{Symbols Menu}

\subsubsection{Symbols}

These menu options allow you to modify symbol attributes in the P\&ID Editor.

Symbol Table - Display the Symbol Table.

Color - $\quad$ Set the default symbol fill color.

Draw - $\quad$ Add a symbol to the diagram.

\subsubsection{Symbol Table}

\section{PURPOSE}

This option allows you to display the symbol table. If selected, a check mark to the left of this menu item will be displayed and the symbol table will appear in the editing window. If deselected, there will not be a check mark next to the menu item and the symbol table will not be displayed. This option acts as a toggle.

The symbol table is used to select a symbol and place it in the diagram.

\section{STEPS}

1. From the P\&ID Editor menu select Symbols.

2. Choose Symbol Table from the menu. The Symbol Table will be displayed.

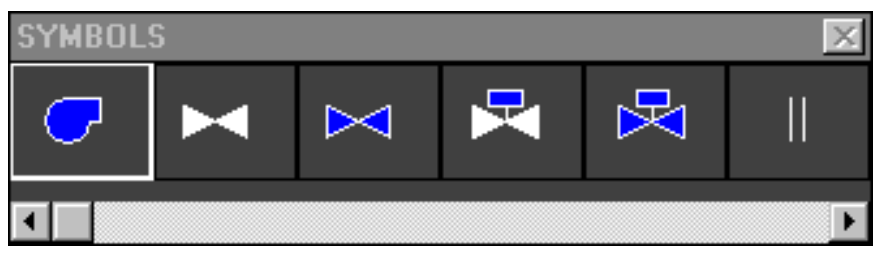

As shown, this table contains a graphic representation of each symbol in the current symbol file. The symbol table is capable of displaying six symbols at one time. If there are more symbols contained within the current symbol file, the horizontal scroll bar will be available.

The next time the Symbols menu is invoked, a check mark will appear to the left of this item.

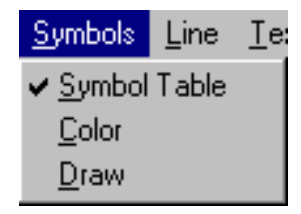

In the example of the Symbols menu shown here, the Symbol Table option is currently selected.

\subsubsection{Symbol Color}




\section{PURPOSE}

This option allows you to set the fill (interior) color of the symbol(s) that will be created following this selection.

\section{STEPS}

1. From the P\&ID Editor menu select Symbols.

2. Choose Color from the menu. The Choose a Color dialog will be displayed.

\subsubsection{Choose a Color Dialog}

\section{PURPOSE}

This option allows you to change the color of selected objects and of objects created after this change.

Select the desired color by clicking on it.

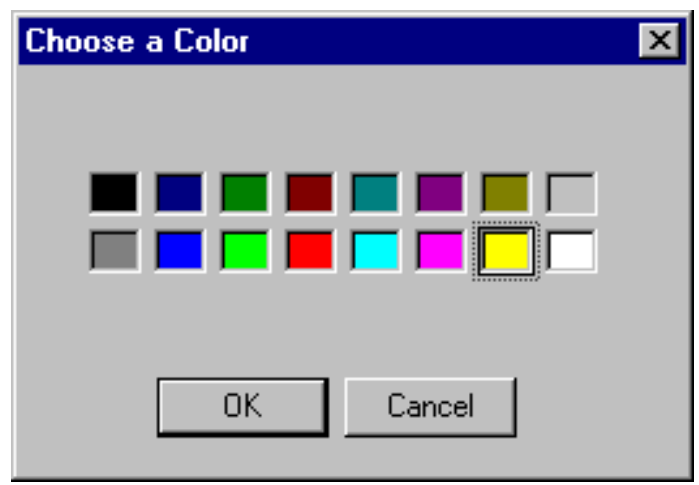

In the example above the color, yellow, is currently selected.

OK - Change the color of the selected object(s) and close the Choose a Color dialog. Objects that are created after this change will be created with this color.

Cancel - Close the Choose a Color dialog without applying the color change.

\subsubsection{Draw Symbol}

\section{PURPOSE}

This option allows you to add a new symbol to the diagram.

\section{STEPS}

1. Select the desired symbol from the Symbol Table, if not currently selected. 
2. From the $P \& I D$ Editor menu select Symbols | Draw.

OR

3. Choose the Symbol button 0 on the toolbar.

4. The cursor will change to the Symbol cursor ${ }^{6}$.

5. Place the cursor over the desired position in the diagram.

6. Click the mouse. The symbol will be placed in the diagram.

The cursor will remain the Symbol cursor. Repeat steps 3 and 4 as many times as desired. Right-click to change the cursor to the standard cursor.

\subsubsection{Line Menu}

\subsubsection{Line}

These menu options allow you to modify line attributes in the $P \& I D$ Editor.

Style - $\quad$ Set the default line style and color.

Orthogonal - Set the default line style to orthogonal. (Draw lines at right angles.)

Color - $\quad$ Set the default line color.

Draw - Add a line to the diagram.

\subsubsection{Line Style}

\section{PURPOSE}

This option allows you to set the style of the line(s) that will be created following this selection.

\section{STEPS}

1. From the P\&ID Editor menu select Line.

2. Choose Style from the menu.

3. The Select Line Style dialog will be displayed. 


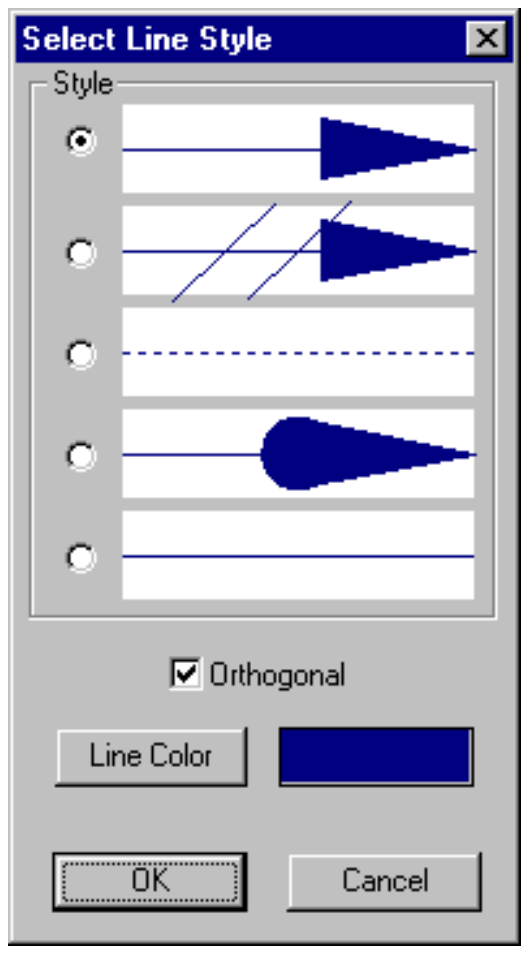

Style - $\quad$ Select one of the five desired line styles:

Orthogonal - Draw lines at right angles.

Line Color - $\quad$ Select the color of lines that will be drawn following this selection.

OK -

Save the changes and close the Select Line Style dialog.

Cancel - $\quad$ Close the Select Line Style dialog without saving the changes.

\subsubsection{Orthogonal}

\section{PURPOSE}

This option allows you to set the line style so that they will be drawn with right angles between connected symbols that are not directly horizontally or vertically aligned. If selected, a check mark to the left of this menu item will be displayed. If deselected, there will not be a check mark next to the menu item. This option acts as a toggle

\section{STEPS}

1. From the $P \& I D$ Editor menu select Line.

2. Choose Orthogonal from the menu.

\begin{tabular}{|l|}
\hline Line Iext Helf \\
\hline Style \\
$\checkmark$ Qrthogonal \\
Color \\
Draw \\
\hline
\end{tabular}


In the example of the Line menu shown here, the Orthogonal option is currently selected.

NOTE: This option can also be set on the Select Line Style dialog.

\subsubsection{Line Color}

\section{PURPOSE}

This option allows you to set the color of the line(s) and symbol outline(s) that will be created following this selection.

\section{STEPS}

1. From the $P \& I D$ Editor menu select Line.

2. Choose Color from the menu.

3. The Choose a Color dialog will be displayed.

\subsubsection{Choose a Color Dialog}

\section{PURPOSE}

This option allows you to change the color of selected objects and of objects created after this change.

Select the desired color by clicking on it.

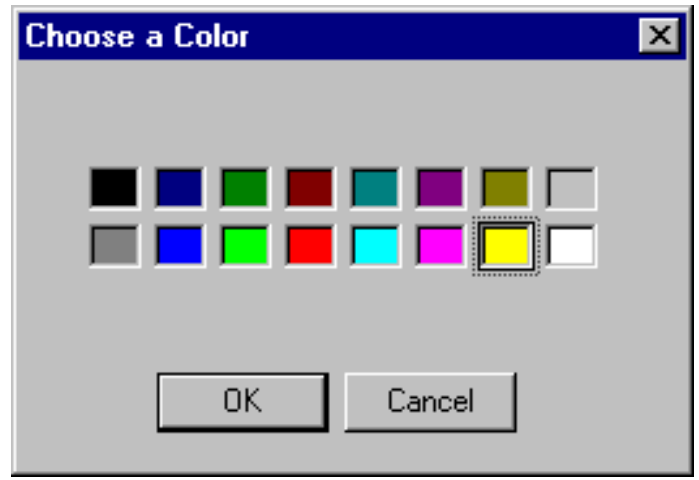

In the example above the color, yellow, is currently selected.

OK - Change the color of the selected object(s) and close the Choose a Color dialog. Objects that are created after this change will be created with this color.

Cancel - Close the Choose a Color dialog without applying the color change.

\subsubsection{Draw Line}

\section{PURPOSE}

This option allows you to add a new line to the diagram. 


\section{STEPS}

1. From the $P \& I D$ Editor menu select Line | Draw.

OR

2. Choose the Line Line button on the toolbar.

The cursor will change to the Line cursor 1 .

3. Place the cursor over the desired position for an endpoint for the line in the diagram and click the mouse.

4. Move the cursor to the next endpoint position and click the mouse.

The cursor will remain the Line cursor. Repeat step 5 as many times as desired. Right-click to change the cursor to the standard cursor.

\subsubsection{Text Menu}

\subsubsection{Text}

These menu options allow you to modify text attributes in the $P \& I D$ Editor.

Font - $\quad$ Set the text font.

Draw - $\quad$ Add a text object to the diagram

Left Justify - $\quad$ Set default text alignment to the left of the text box.

Center - $\quad$ Set default text alignment to the center of the text box.

Right Justify - Set default text alignment to the right of the text box.

\subsubsection{Font}

\section{PURPOSE}

This option allows you to set the font and color of the text object(s) and symbol name(s) that will be created following this selection.

\section{STEPS}

1. From the P\&ID Editor menu select Text.

2. Choose Font from the menu. The Font dialog will be displayed.

\subsubsection{Font/Color Dialog}

\section{PURPOSE}

This option allows you to change the font and color of the selected text object(s) and all text created after this change. 


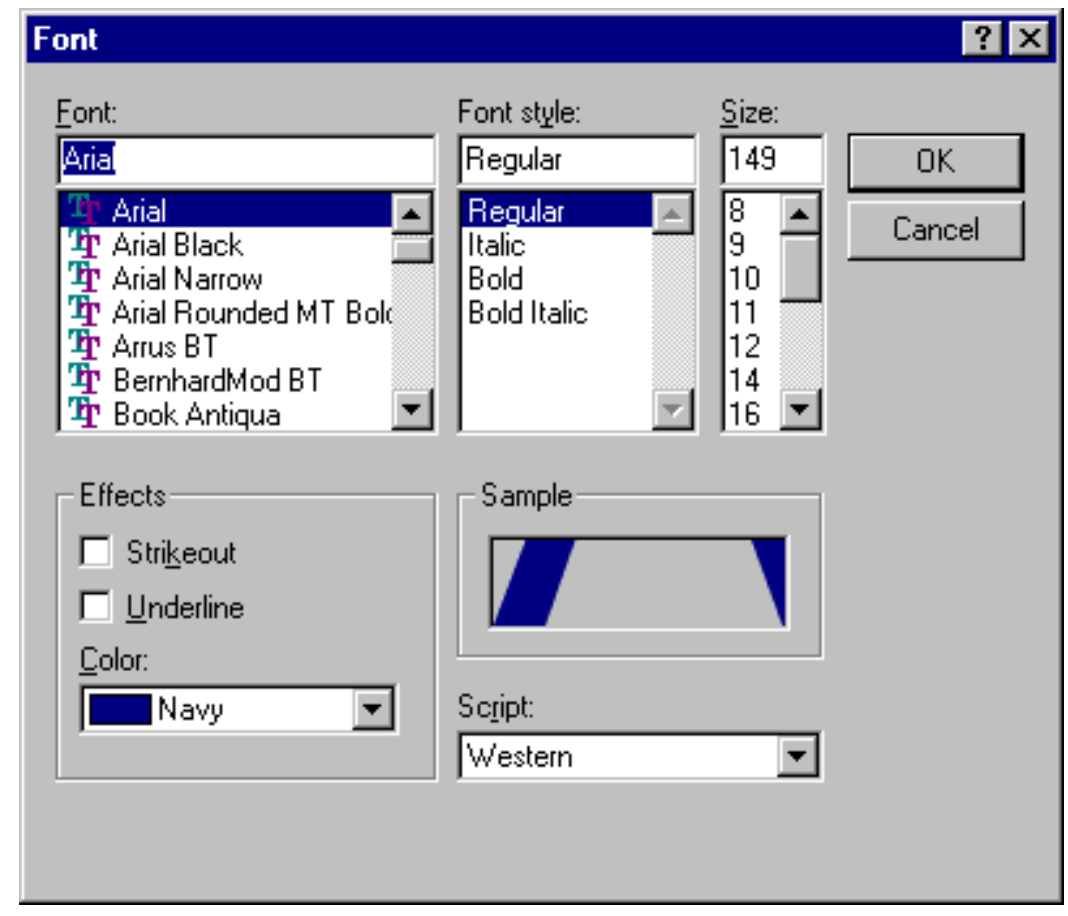

Font - $\quad$ The currently selected font and list of available fonts. Choose a new font from the drop-down list.

Font Style - $\quad$ The currently selected font style (e.g., bold, italic, etc.) and list of available styles. Choose a new style from the drop-down list.

Size - $\quad$ The currently selected size and list of available sizes. Choose from the dropdown list or enter any positive numeric value.

Effects

Strikeout - Display the text in strikeout mod. (Shown with a horizontal line through the characters.)

Underline - $\quad$ Display the text with an underline.

Color - $\quad$ The currently selected color. Choose a new color from the drop-down list.

Sample - $\quad$ Displays the current color of the text.

Script - $\quad$ Select the script from the drop-down list.

OK - $\quad$ Save the changes and close the Font dialog.

Cancel - $\quad$ Close the Font dialog without saving the changes.

\subsubsection{Draw Text}

\section{PURPOSE}

This option allows you to add a new text object to the diagram. 


\section{STEPS}

1. From the $P \& I D$ Editor menu select Text $\mid$ Draw.

OR

2. Choose the Text object button $\stackrel{\square}{\square}$ on the toolbar.

3. The Text Style dialog will be displayed.

4. Enter the desired text and set the desired font and color. Choose $\mathbf{O K}$.

5. The cursor will change to the Text cursor $\llbracket$.

6. Place the cursor over the desired position in the diagram.

7. Click the mouse. The text object will be placed in the diagram.

The cursor will change to the standard cursor when step 7 is complete.

To cancel the operation after step 6, right-click and the cursor will change to the standard cursor.

\subsubsection{Text Style Dialog}

\section{PURPOSE}

This option allows you to change the font, color, or alignment of the text object(s).

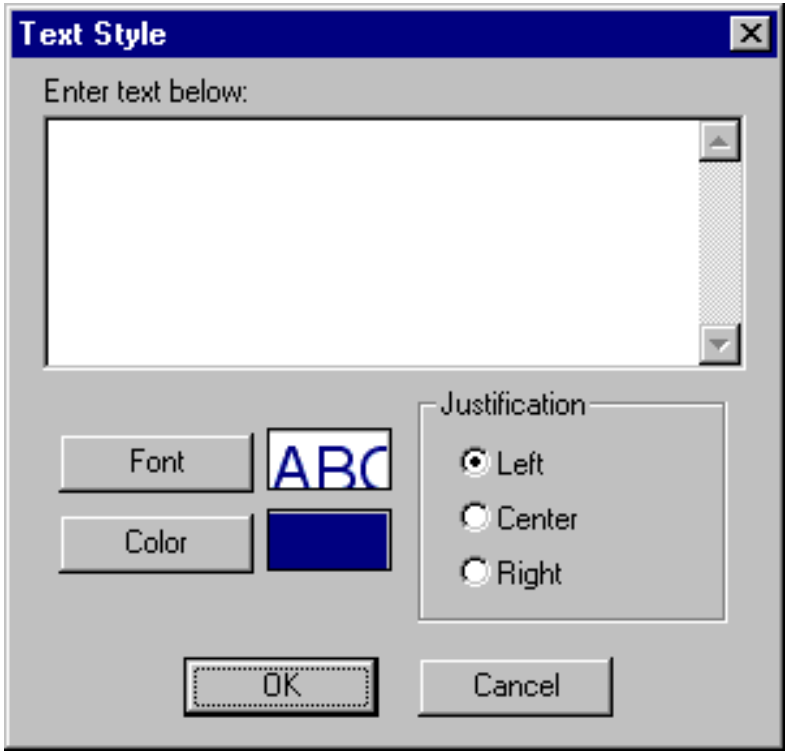

Enter text below: - The alphanumeric characters that make up the text object.

Font - $\quad$ Change the font, font style, or size used to display the text.

Color - $\quad$ Change the color used to display the text. 


\section{Justification}

Left - Align the text to the left

Center - $\quad$ Align the text in the center

Right - $\quad$ Align the text to the right

OK -

Save the changes and close the Text Style dialog.

Cancel -

Close the Text Style dialog without saving the changes.

\subsubsection{Left Justify}

\section{PURPOSE}

This option allows you to set the text alignment so that text objects created after this change will be aligned along the left side of the text object box.

\section{STEPS}

1. From the $P \& I D$ Editor menu select Text.

2. Choose Left Justify from the menu. A check mark to the left of this menu item will be displayed the next time the Text menu is invoked. The prior text alignment option will be deselected.

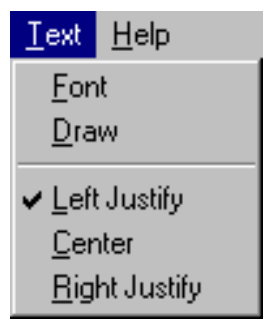

In the example of the Text menu shown here, the Left Justify option is currently selected.

\subsubsection{Center}

\section{PURPOSE}

This option allows you to set the text alignment so that text objects created after this change will be centered inside the text object box.

\section{STEPS}

1. From the $P \& I D$ Editor menu select Text.

2. Choose Center from the menu. A check mark to the left of this menu item will be displayed the next time the Text menu is invoked. The prior text alignment option will be deselected. 


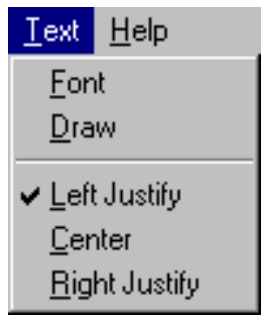

In the example of the Text menu shown here, the Center option is NOT currently selected.

\subsubsection{Right Justify}

\section{PURPOSE}

This option allows you to set the text alignment so that text objects created after this change will be aligned along the right side of the text object box.

\section{STEPS}

1. From the $P \& I D$ Editor menu select Text.

2. Choose Right Justify from the menu. A check mark to the left of this menu item will be displayed the next time the Text menu is invoked. The prior text alignment option will be deselected.

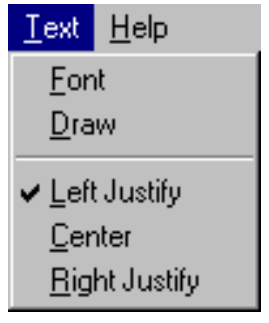

In the example of the Text menu shown here, the Right Justify option is NOT currently selected.

\subsection{Fault Tree Logic Editor}

\subsubsection{Interpreting Alphanumeric Fault Tree Logic \\ PURPOSE}

This example illustrates how the CCS graphical fault tree is presented in an alphanumeric format. 


\section{Containment Cooling Fault Tree}
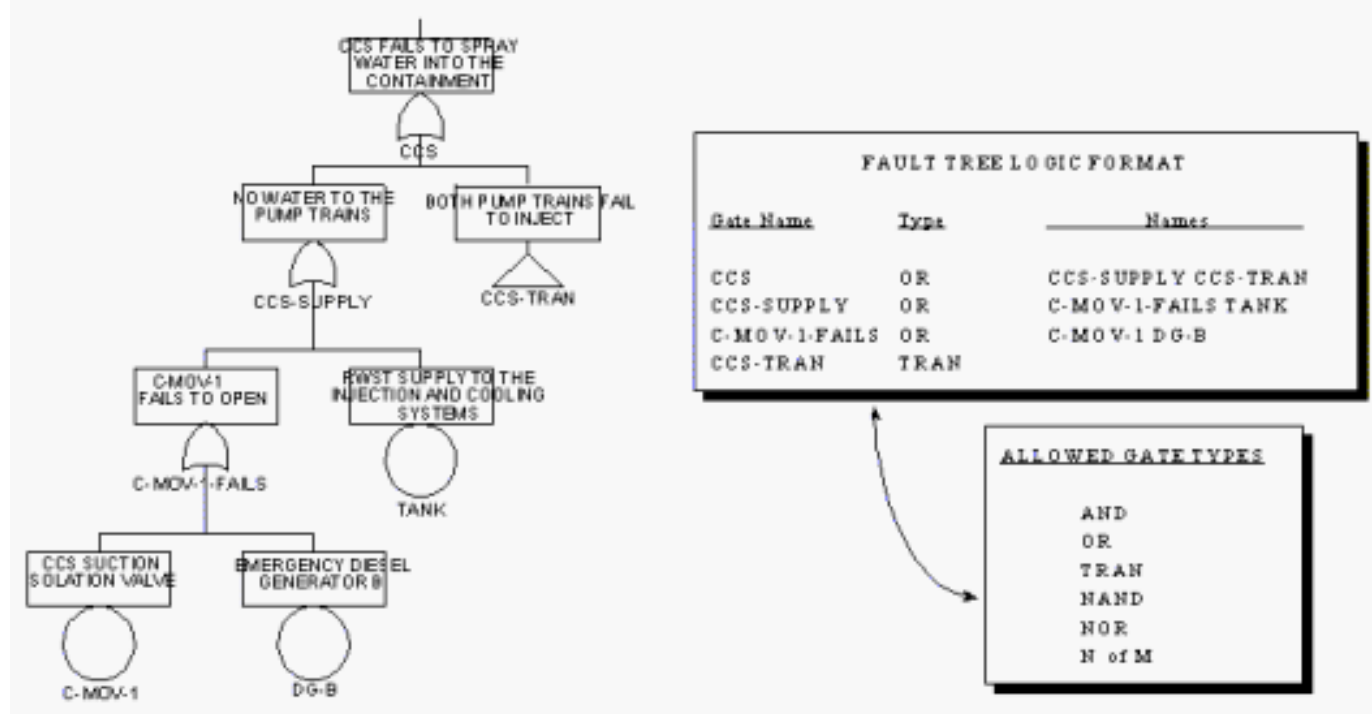

\subsubsection{Fault Tree Logic Editor}

\section{PURPOSE}

This option provides a means for editing the fault tree logic in an alphanumeric format. All gates, types, and inputs contained in the selected fault tree or sub-tree will be displayed. The fault tree logic editor is a convenient tool for quickly editing fault tree logic and entering gate and basic event data.

\section{STEPS}

1. From the SAPHIRE menu select Fault Tree. The Fault Trees List dialog will be displayed.

2. Highlight the desired fault tree.

3. Right-click to invoke the pop-up menu and select the Edit Logic option.

4. The Edit Fault Tree Logic dialog will be displayed. 


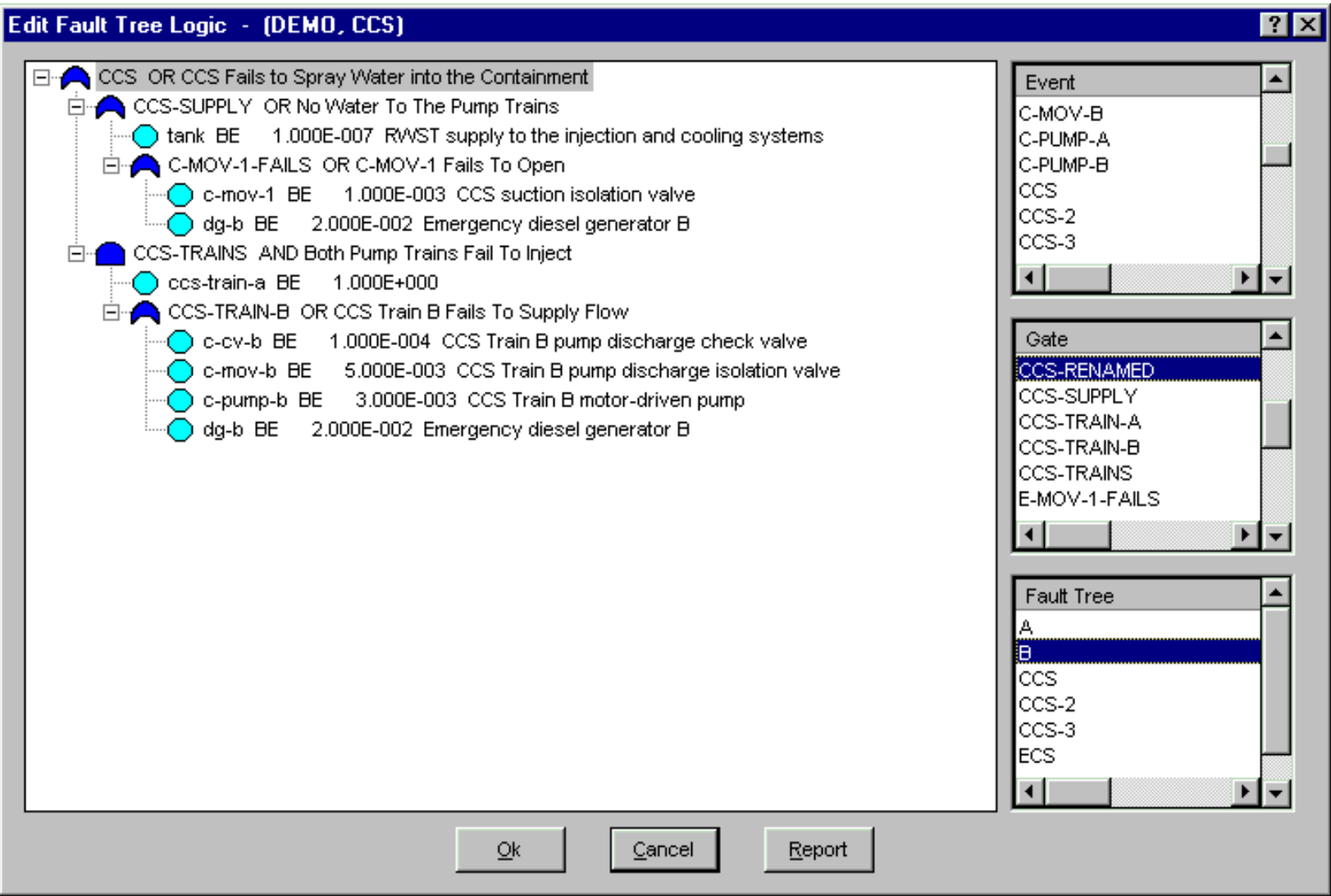

As shown here, the logic is represented in the form of a hierarchical tree, where each gate can be expanded or condensed by clicking on the gate. See the topic, "About Logic Dialogs ," for a description of the icons presented on this dialog.

\section{Logic Tree View Pop-up Menu Options}

Modify - Edit the selected basic event or gate logic .

\section{Insert}

Gate - Add a new gate to the database and insert it into the logic.

Event - Add a new basic event to the database and insert it into the logic.

Top Gate - Add a new or existing gate to the logic at the top level.

Delete - Remove the gate or basic event from the logic.

Find - Locate a specific basic event or gate in the logic.

Move- $\quad$ Move the selected gate or basic event to another location in the logic.

Follow Transfer - Follow the selected transfer. Available only when a transfer gate is selected.

Transfer Back - Follow the transfer back to the original tree. Available only after a transfer has been followed. 
Rename Top - Change the name of the top gate. Available only when the top gate is selected.

\section{Event Pop-up Menu Options}

Add - $\quad$ Add a new event to the database.

Modify - Edit the selected basic event.

Select - Insert the selected basic event in the list to the selected point in the logic tree view.

\section{Gate Pop-up Menu Options}

Add - $\quad$ Add a new gate to the database.

Modify - Edit the selected gate.

Select - I nsert the selected gate in the list to the selected point in the logic tree view.

\section{Fault Tree Pop-up Menu Options}

Add - $\quad$ Add a new fault tree to the database.

Modify - Edit the selected fault tree .

Select - Insert the selected fault tree as a transfer.

Edit - Load and edit the selected fault tree logic. If changes have been made to the current tree, you will be prompted to save those changes before switching to the

\section{Button Options} selected fault tree.

Report - Generate a report displaying the logic.

Ok - Save the changes made to the logic and close the Edit Fault Tree Logic dialog.

Cancel - Close the Edit Fault Tree Logic dialog without saving changes to the logic.

\subsubsection{Modify Gate Logic}

\section{PURPOSE}

This option allows you to edit the inputs to and/or the type of the selected gate.

\section{STEPS}

1. From the SAPHIRE menu select Fault Tree. The Fault Trees List dialog will be displayed.

2. Highlight the desired fault tree.

3. Right-click to invoke the pop-up menu and select the Edit Logic option.

4. From the Edit Fault Tree Logic dialog, select the gate in the tree to be modified.

5. Right-click to invoke the pop-up menu and select the Modify option.

6. The Edit dialog will be displayed showing the gate name, type and description along with its inputs. 


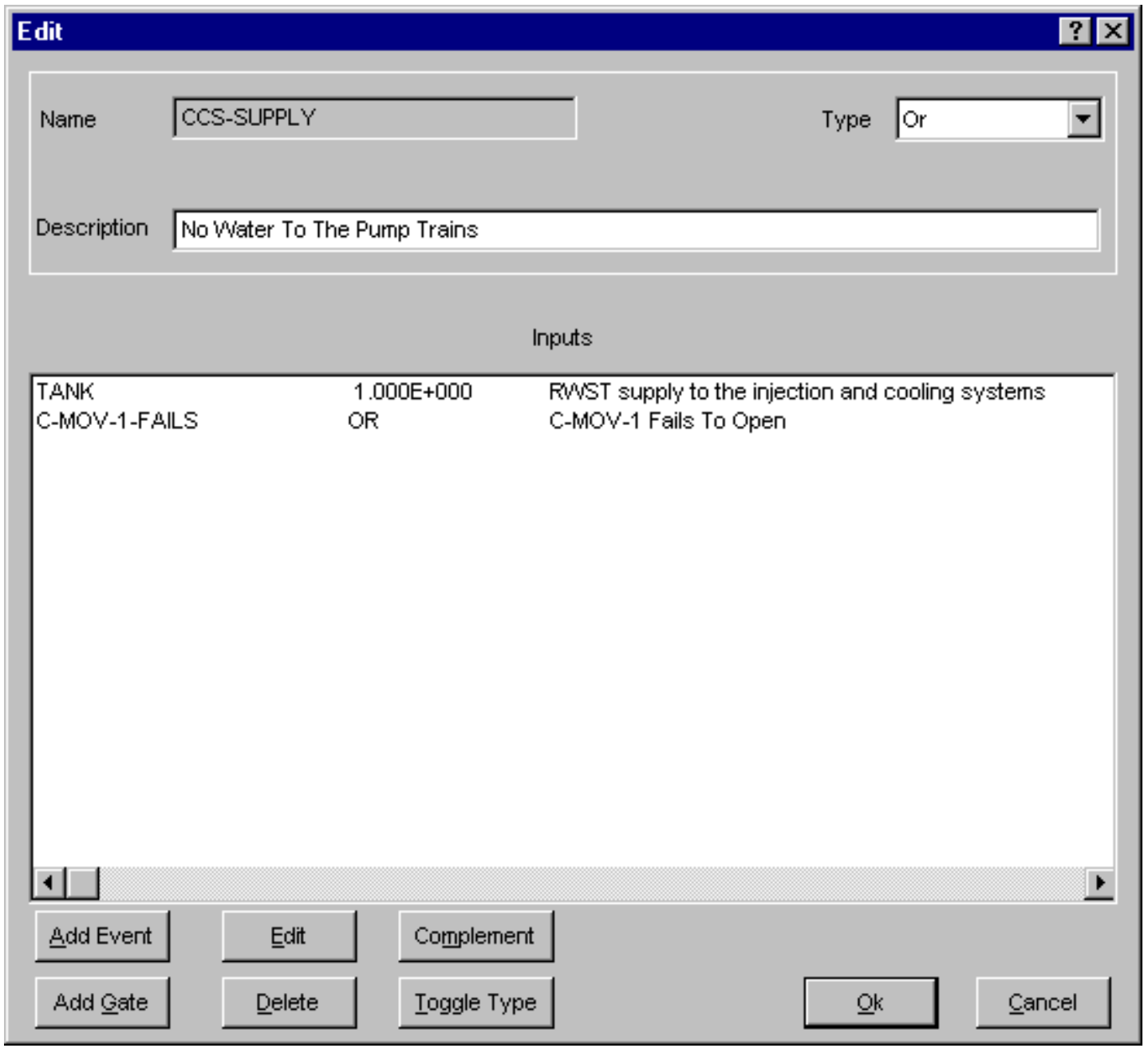

\section{Gate Options}

Change the gate type by choosing from the Type drop-down list.

\section{Input Options}

Add Event - $\quad$ Add a new event as an input to the current gate.

Add Gate - $\quad$ Add a new gate as an input to the current gate.

Edit -

Delete -

Modify the gate or event currently highlighted in the Inputs list.

Complement -

Remove the highlighted event or gate from the Inputs list.

Toggle Type -

Change the highlighted event or gate to its complement.

Ok -

Change the highlighted gate to an event, or event to a gate.

Cancel -

Close the Edit dialog and save the changes.

Close the Edit dialog without saving the changes.

\subsubsection{Add Gate as Input}

\section{PURPOSE}

This option allows you to add a gate as an input to the currently selected gate. The new gate will be displayed in the Inputs list and the hierarchical tree. 


\section{STEPS}

1. From the SAPHIRE menu select Fault Tree. The Fault Trees List dialog will be displayed.

2. Highlight the desired fault tree.

3. Right-click to invoke the pop-up menu and select the Edit Logic option. The Edit Fault Tree Logic dialog will be displayed.

4. Right-click to invoke the pop-up menu and select the Insert Top option. OR

5. Highlight the gate to be modified.

6. Right-click to invoke the pop-up menu and select the Modify option.

7. The Edit dialog will be displayed showing the gate name, type and description along with its inputs.

8. Choose the Add Gate button.

9. The Gate dialog will be displayed.

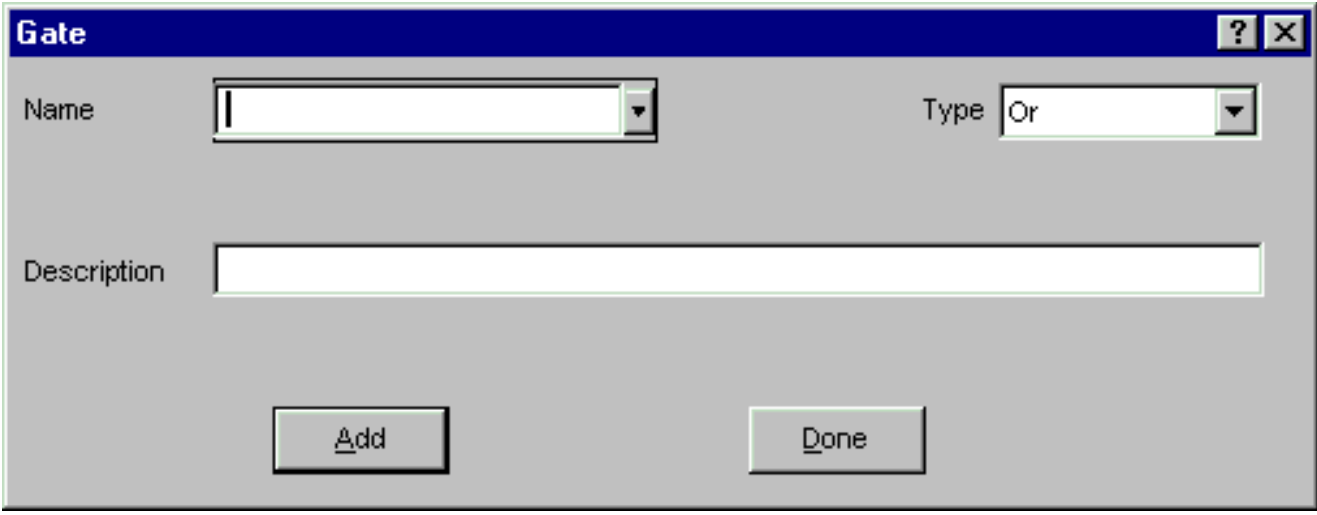

Add - $\quad$ Add the gate as an input to the current gate. The dialog will remain open so you may continue to add gates.

Done - Close the Gate dialog without adding the gate as an input to the current gate.

\subsubsection{Gate Name}

This is a required field. Choose from the drop-down list or enter a new name. A maximum of 24 uppercase, alphanumeric characters may be entered. Embedded blanks are not allowed. The name should be descriptive so it can be readily identified.

\subsubsection{Add Event as Input}

\section{PURPOSE}

This option allows you to add the basic events used as inputs to a gate. 


\section{STEPS}

1. From the SAPHIRE menu select Fault Tree. The Fault Trees List dialog will be displayed.

2. Highlight the desired fault tree.

3. Right-click to invoke the pop-up menu and select the Edit Logic option.

4. From the Edit Fault Tree Logic dialog, select a gate.

5. Right-click to invoke the pop-up menu and select the Modify option. The Modify Gate Logic dialog will be displayed.

6. Select the Add Event button.

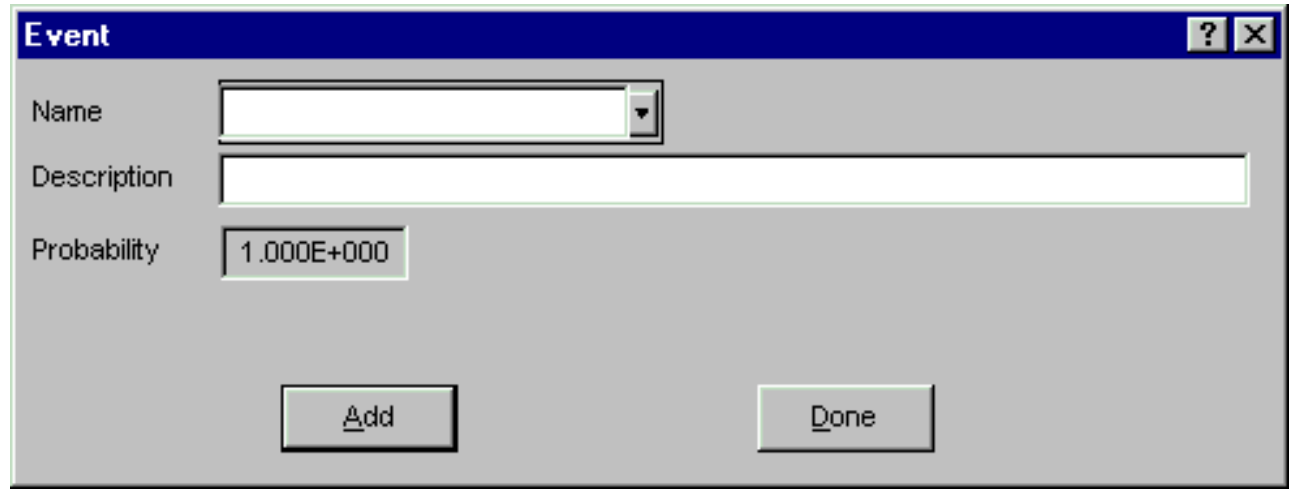

Select the desired event from the drop-down list. The associated description and probability will appear, but cannot be modified. Or, enter a new event name and description.

Add - Add the basic event as an input to the current gate. The dialog will remain open so you may continue to add events.

Done - Close the Event dialog.

\subsubsection{Delete}

\section{PURPOSE}

This option allows you to remove any gate or event from the current fault tree logic.

\section{STEPS}

1. From the SAPHIRE menu select Fault Tree. The Fault Trees List dialog will be displayed.

2. Highlight the desired fault tree.

3. Right-click to invoke the pop-up menu and select the Edit Logic option.

4. From the Edit Fault Tree Logic dialog, select the gate in the tree to be modified.

5. Right-click to invoke the pop-up menu and select the Modify option.

6. The Edit dialog will be displayed showing the gate name, type and description along with its inputs.

7. Select the desired event or gate in the Inputs list.

8. Choose the Delete button. 
No confirmation of the delete operation is provided. The event or gate is removed immediately from the Inputs list. The event or gate will NOT be deleted from the database - only the logic.

\subsubsection{Find}

\section{PURPOSE}

This option is used to locate a specific event or gate. If the located event or gate is an input to a condensed gate, that gate will be expanded.

\section{STEPS}

1. From the SAPHIRE menu select Fault Tree. The Fault Trees List dialog will be displayed.

2. Highlight the desired fault tree.

3. Right-click to invoke the pop-up menu and select the Edit Logic option. The Edit Fault Tree Logic dialog will be displayed.

4. Right-click to invoke the pop-up menu and select the Find option. The Find dialog will be displayed.

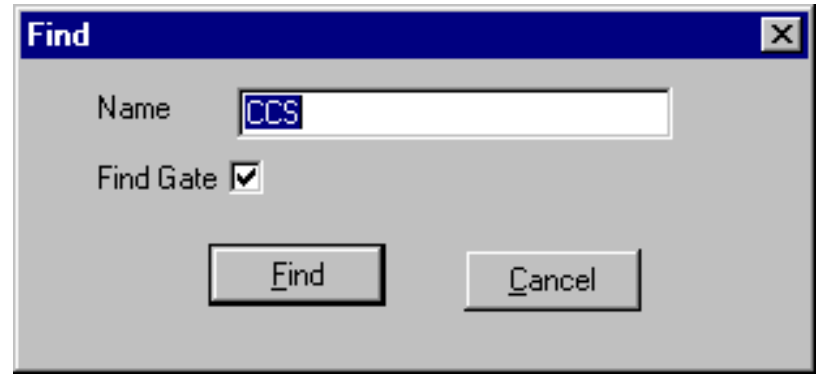

Name - $\quad$ Enter the name of the event or gate to be located.

Find Gate - $\quad$ Select this check box if the search is for a gate. Deselect this check box if the search is for an event.

Find - $\quad$ Execute the "find" operation based on the current criteria.

Cancel - $\quad$ Close the Find dialog without executing the find operation.

\subsubsection{Move}

\section{PURPOSE}

This option allows you to move a gate from one position in the fault tree logic to another. 


\section{STEPS}

1. From the SAPHIRE menu select Fault Tree. The Fault Trees List dialog will be displayed.

2. Highlight the desired fault tree.

3. Right-click to invoke the pop-up menu and select the Edit Logic option. The Edit Fault Tree Logic dialog will be displayed.

4. Highlight the input (gate or event) to be moved.

5. Right-click to invoke the pop-up menu and select the Move option. The message, "Select gate to attach to" will be displayed in the message bar.

6. Select the gate as instructed. The input will be moved to the new location.

\subsubsection{Follow Transfer}

\section{PURPOSE}

This option can be used only on transfer gates. This option displays the logic of the fault tree specified by the transfer gate.

\section{STEPS}

1. From the SAPHIRE menu select Fault Tree. The Fault Trees List dialog will be displayed.

2. Highlight the desired fault tree.

3. Right-click to invoke the pop-up menu and select the Edit Logic option. The Edit Fault Tree Logic dialog will be displayed.

4. Selected the desired transfer gate.

5. Right-click to invoke the pop-up menu and select the Follow Transfer option.

The Edit Fault Tree Logic dialog will be displayed containing the logic of the specified fault tree. The name of the fault tree will be displayed in the title bar. The regular Edit Fault Tree Logic options are available. Notice that the Transfer Back pop-up menu option is now available. Selecting this option will return you to the previous logic display.

\subsubsection{Rename Top}

\section{PURPOSE}

This option allows you to move a gate from one position in the fault tree logic to another.

\section{STEPS}

1. From the SAPHIRE menu select Fault Tree. The Fault Trees List dialog will be displayed.

2. Highlight the desired fault tree.

3. Right-click to invoke the pop-up menu and select the Edit Logic option. The Edit Fault Tree Logic dialog will be displayed.

5. Highlight the top gate.

6. Right-click to invoke the pop-up menu and select the Rename Top option. The Rename Top Gate dialog will be displayed. 
Ok - Change the name of the top gate and close the Rename Top Gate dialog.

Cancel - Close the Rename Top Gate dialog without changing the top gate name.

\subsubsection{Save Logic}

\section{PURPOSE}

This option allows you to save the logic, and optionally, create graphics to match the logic.

\section{STEPS}

1. From the SAPHIRE menu select Fault Tree. The Fault Trees List dialog will be displayed.

2. Highlight the desired fault tree.

3. Right-click to invoke the pop-up menu and select the Edit Logic option. The Edit Fault Tree Logic dialog will be displayed.

4. Make the desired changes.

5. Select Ok.

6. The Save Graphics dialog will be displayed.

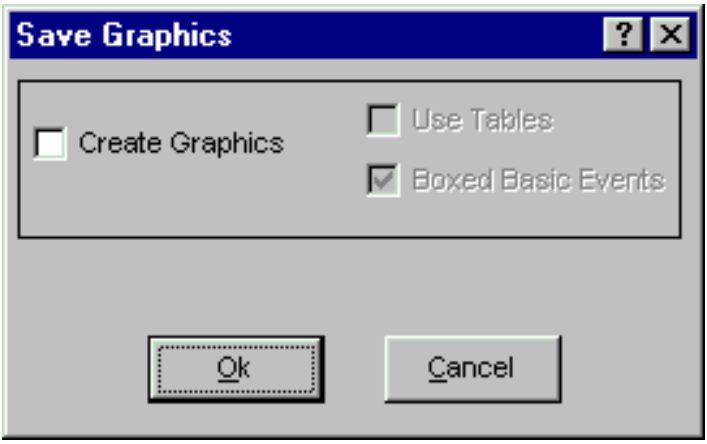




\section{Create Graphics -}

Use Tables -

Boxed Basic Events -

Ok -

Cancel -
Select this check box to automatically convert the alphanumeric logic changes to the graphical format. This will create corresponding .DLS for each new fault tree or sub-tree.

For each logic gate with many basic events as inputs, create a table of basic events, using the Primary name field. SAPHIRE processes the table of basic events as if they were separate symbols. Enabled only when Create Graphics is checked.

Create each basic event with a box for descriptive text. The text from the description field of the associated event will be placed in the box. This does not influence the logic of the fault tree, but adds clarity to the model for those using and reviewing it. Enabled only when Create Graphics is checked.

Save the logic, create the graphics (if selected), and exit.

Close the Save Graphics dialog without saving the logic but do not close the Edit Fault Tree Logic dialog.

\subsubsection{About Logic Dialogs}

The gate names and gate types are displayed in uppercase characters. The event names are displayed in lowercase characters. The cut set events, indicated only in the Path Search option on the Selected Cut Set Events dialog, are enclosed in brackets $(\diamond)$.

Presentation of the logic in dialogs and reports generated by SAPHIRE is limited to 255 characters in width. If the representation of the logic requires expansion past this limit, it will be truncated for display and reporting. The actual logic will remain in tact.

\section{The following graphics are used for easy identification of gate types and their status:}

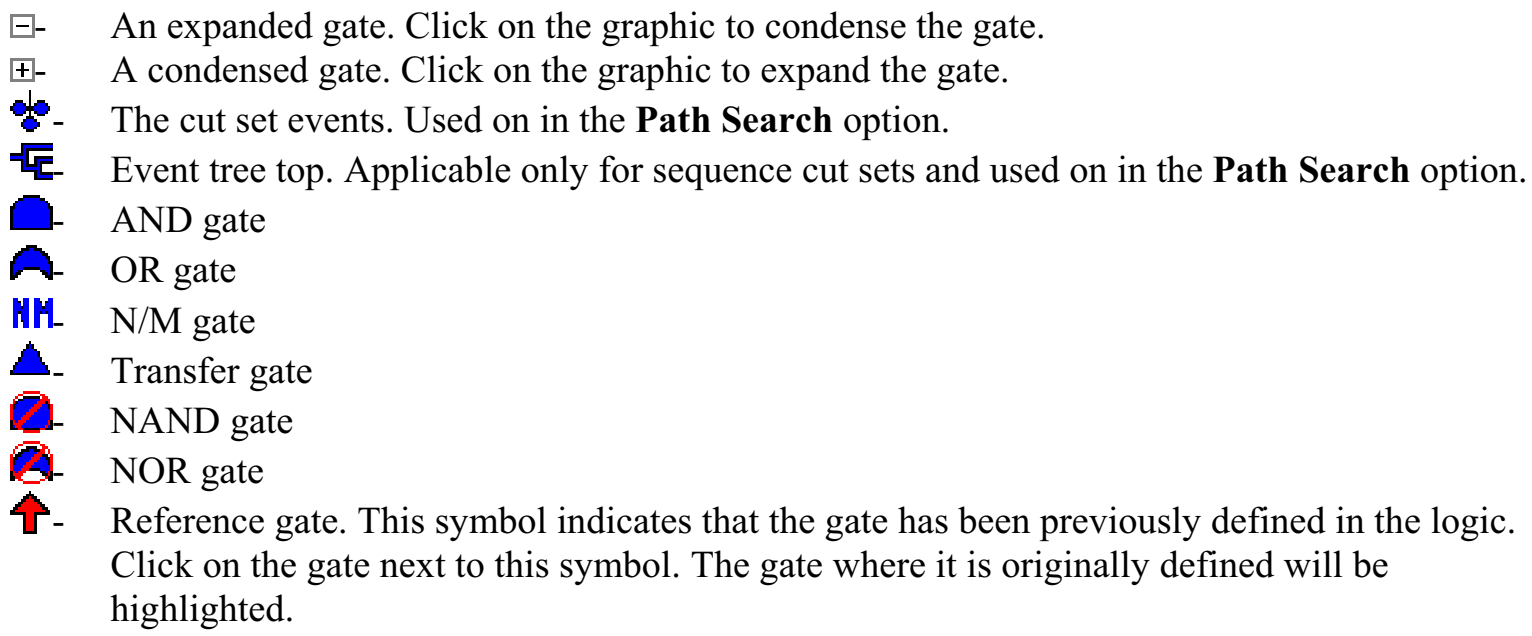




\subsubsection{Tips on Using the Fault Tree Logic Editor}

\section{PURPOSE}

Following are some hints on using the fault tree alphanumeric Logic Editor.

Tips on changing gate or event names:

When you change the name of a gate, the gate name will be changed everywhere it occurs.

When you change the name of a basic event as an input to a gate, it is changed everywhere in the current logic. If you highlight an event and choose the Modify option from the pop-up menu, it is changed at that location.

The fault tree Logic Editor does not allow the edited fault tree to be saved with a new fault tree name. The top gate in the fault tree must match the fault tree name.

After changes are made to the fault tree logic using the Logic Editor, select the Create Graphics check box to update the fault tree graphical file. It is not necessary to update the graphical file in order to perform analyses with the updated logic; however, to keep the fault tree logic and the fault tree graphic identical, you must perform this conversion.

\subsection{Page Fault Tree Logic}

\subsubsection{Page Tree}

\section{PURPOSE}

This option provides a variety of functions: (1) automatically divide a large fault tree logic file into a set of equivalent logic files with each file fitting more conveniently on a page (Auto Page); (2) break out a sub-tree at a user-specified gate (Gate); (3) output gates with multiple references into a separate sub-tree (Multiple); or (4) output multiple top gates into separate fault trees (Tops).

The fault tree shown below will be used to illustrate how the various Page Tree functions work. Examples described in the various Page Tree functions refer to this basic fault tree file, called TOPGATE.FTL.

Note that TOPGATE.FTL has a couple of interesting features that will be explored with the examples. First, this logic file has two top events, TOPGATE and TOPGATE2. Second, there are four gates that are referenced more than once: GATE6, GATE10, GATE11, and GATE12.

\section{STEPS}

1. From the SAPHIRE menu select Fault Tree. The Fault Trees List dialog will be displayed.

2. Highlight the desired fault tree.

3. Right-click to invoke the pop-up menu and choose Page Tree.

4. The Page Fault Tree Logic dialog will be displayed.

See the topic, "About Logic Dialogs ," for a description of the icons presented on this dialog. 


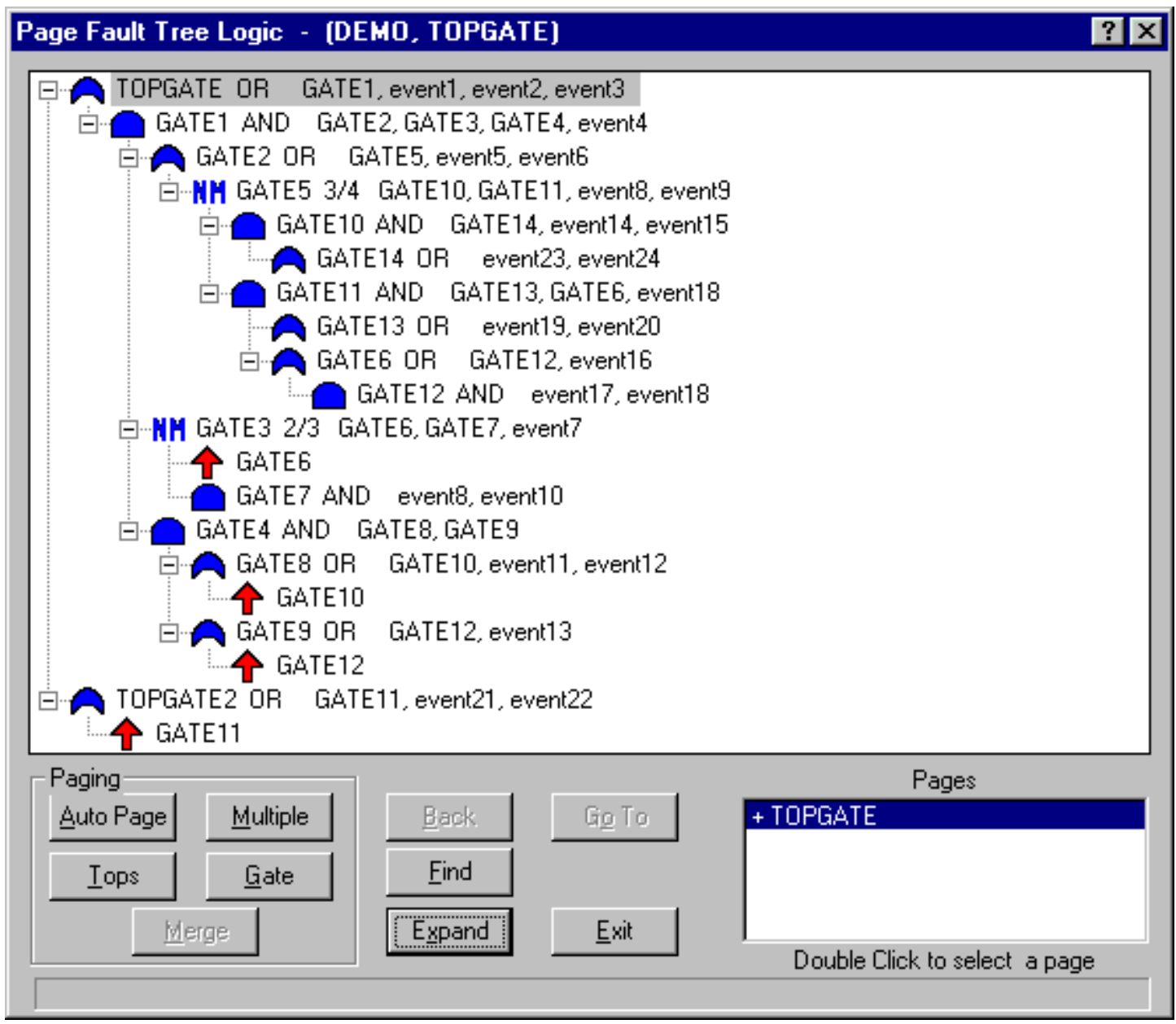

\section{Paging Options}

Auto Page -

Multiple -

Tops -

Gate -

Merge -
Automatically break the fault tree into easily viewable segments

Break out gates with multiple references (i.e., gates that appear in more than one location within the fault tree) and output the logic into separate sub-trees. Enabled only when multiply-referenced gates are detected.

Automatically separate the logic file into different fault trees by the top events. Enabled only when multiple tops in the logic are detected.

Create a separate fault tree logic file with the specified gate as the top event.

Merge the logic represented by a transfer gate into the current fault tree file. This will occur only if the gate is not referenced more than once within the fault tree. Only transfer gates created during the current paging session can be merged. (Sub trees that existed prior to the current paging session cannot be merged from here. To merge a saved sub tree, see Fault Tree Logic Options .) 
Back - $\quad$ Reload the previous fault tree file. This option is enabled only after executing the Go To option.

Find - $\quad$ Locate a specified gate.

Expand - $\quad$ Expand all gates in the current fault tree file.

Go To - $\quad$ Follow a transfer to another fault tree file.

Exit - $\quad$ Close the Page Fault Tree Logic dialog. If changes were made to the fault tree logic, you will be prompted to save the changes before exiting.

Pages - $\quad$ List of existing pages. Double-click on a page in the list to make it the current page.

\subsubsection{About Logic Dialogs}

The gate names and gate types are displayed in uppercase characters. The event names are displayed in lowercase characters. The cut set events, indicated only in the Path Search option on the Selected Cut Set Events dialog, are enclosed in brackets $(<)$.

Presentation of the logic in dialogs and reports generated by SAPHIRE is limited to 255 characters in width. If the representation of the logic requires expansion past this limit, it will be truncated for display and reporting. The actual logic will remain in tact.

\section{The following graphics are used for easy identification of gate types and their status:}

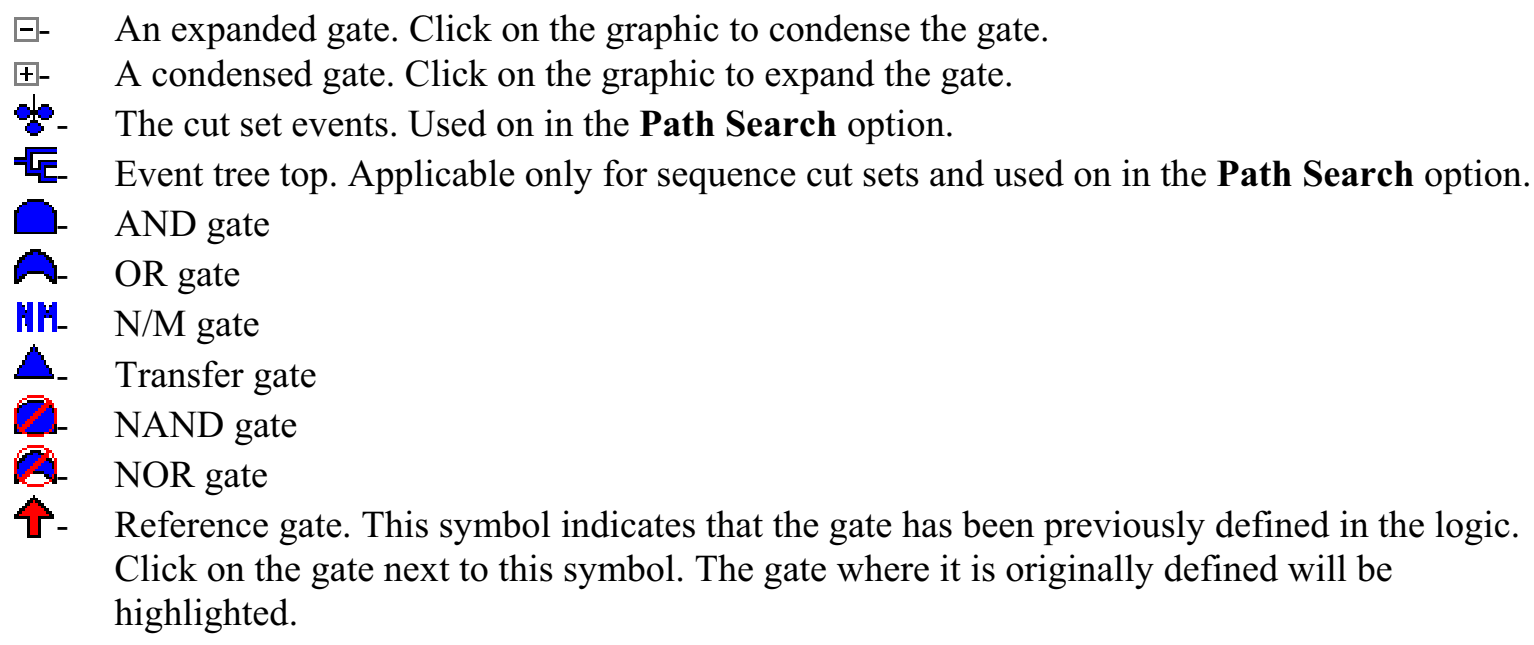

\subsubsection{Page Tree - Multiple}

\section{PURPOSE}

This option breaks out gates with multiple references (i.e., gates that appear in more than one location within the fault tree) and outputs sub-tree logic files that meet the established screening criteria. This has the effect of breaking the fault tree logic file into smaller sections that will reduce the amount of repeated logic. When this process is executed, transfer gates are inserted as appropriate so that the final linked logic is equivalent to the original logic. 
Screening criteria have been established to prevent the logic file from being broken into a large number of trivial fault trees. A gate will be broken out as a sub-tree only if:

It is referenced more than once in the logic file, and

It has in its input at least one logic gate that is referenced only once.

A gate with multiple references will not be broken out as a sub-tree if its inputs consist only of basic events, transfer gates, tables of events, other gates with multiple references, or any combination of these inputs.

This option is enabled only when gates with multiple references are detected in the fault tree logic file.

\section{STEPS}

1. From the SAPHIRE menu select Fault Tree. The Fault Trees List dialog will be displayed.

2. Highlight the desired fault tree.

3. Right-click to invoke the pop-up menu and choose Page Tree.

4. $\quad$ The Page Fault Tree Logic dialog will be displayed.

5. Choose the Multiple button.

\section{EXAMPLE}

Using the example fault tree, the execution of the Multiple function results in four logic files:

TOPGATE.FTL, TOPGATE2.FTL, GATE10.FTL and GATE11.FTL. Notice that the original fault tree logic file included two top events and four gates with multiple references. The two top event logic files have been split off because the Tops function is automatically executed during this process. Two other logic files were created because only GATE10 and GATE11 met the screening criteria. GATE6 does not meet the screening criteria because it has as one of its inputs a gate with multiple references (GATE12). GATE12 only has basic events as inputs and therefore, does not meet the screening criteria either.

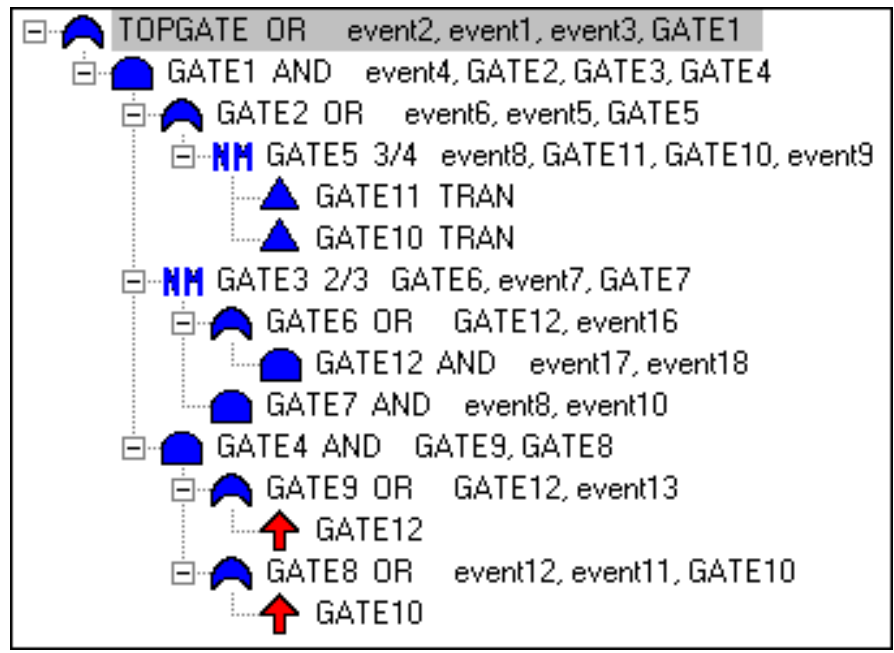

TOPGATE.FTL after the Multiple command. 
$\square$ TOPGATE2 OR event21, event22, GATE11

A GATE11 TRAN

TOPGATE2.FTL after the Multiple command.

$\square$ GATE10 AND GATE14, event14, event15

A GATE14 OR event23, event24

GATE10.FTL after the Multiple command.

GATE11 AND GATE13, event18, GATE6
GATE13 OR event19, event20
GATE6 OR GATE12, event16
GATE12 AND event17, event18

GATE11.FTL after the Multiple command.

\subsubsection{Page Tree - Tops}

\section{PURPOSE}

This option automatically separates the logic file into different fault trees by the top events. This option is enabled only when multiple tops in the logic are detected.

\section{STEPS}

1. From the SAPHIRE menu select Fault Tree. The Fault Trees List dialog will be displayed.

2. Highlight the desired fault tree.

3. Right-click to invoke the pop-up menu and choose Page Tree.

4. The Page Fault Tree Logic dialog will be displayed.

5. Choose the Tops button.

\section{EXAMPLE}

Notice that the example fault tree logic file included two top events. Execution of the Tops function results in four logic files: TOPGATE.FTL, TOPGATE2.FTL, GATE10.FTL and GATE11.FTL. The logic files GATE10.FTL and GATE11.FTL have been split off because the Multiple function is automatically executed during this process. The resulting top event logic files are each separate and complete. Further processing on these files can now be performed. 


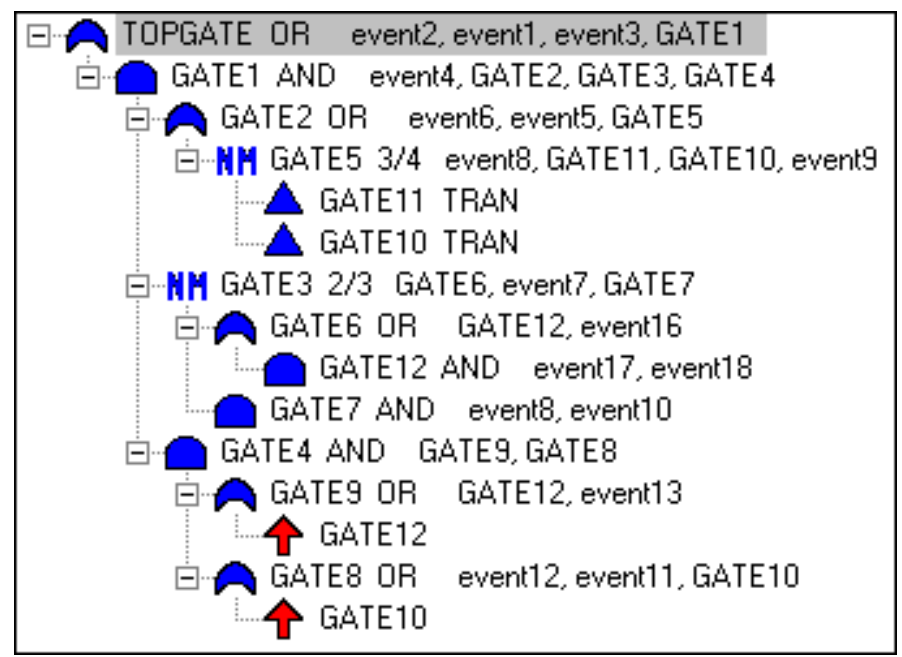

TOPGATE.FTL after the Tops command.

๑ A TOPGATE2 OR event21, event22, GATE11

A GATE11 TRAN

TOPGATE.FTL after the Tops command.

\subsubsection{Page Tree - Gate}

\section{PURPOSE}

This option creates a separate fault tree logic file with the specified gate as the top event. Transfer gates will be inserted as appropriate so that the final linked logic is equivalent to the original logic.

\section{STEPS}

1. From the SAPHIRE menu select Fault Tree. The Fault Tree List dialog will be displayed.

2. Highlight the desired fault tree.

3. Right-click to invoke the pop-up menu and choose Page Tree. The Page Fault Tree Logic dialog will be displayed.

4. Highlight the desired gate.

5. Choose the Gate button.

\section{EXAMPLE}

Using the example fault tree, GATE11 was selected. A new fault tree logic file has been created with GATE11 as the top event. Notice the new TOPGATE.FTL file now contains a transfer to GATE11. 


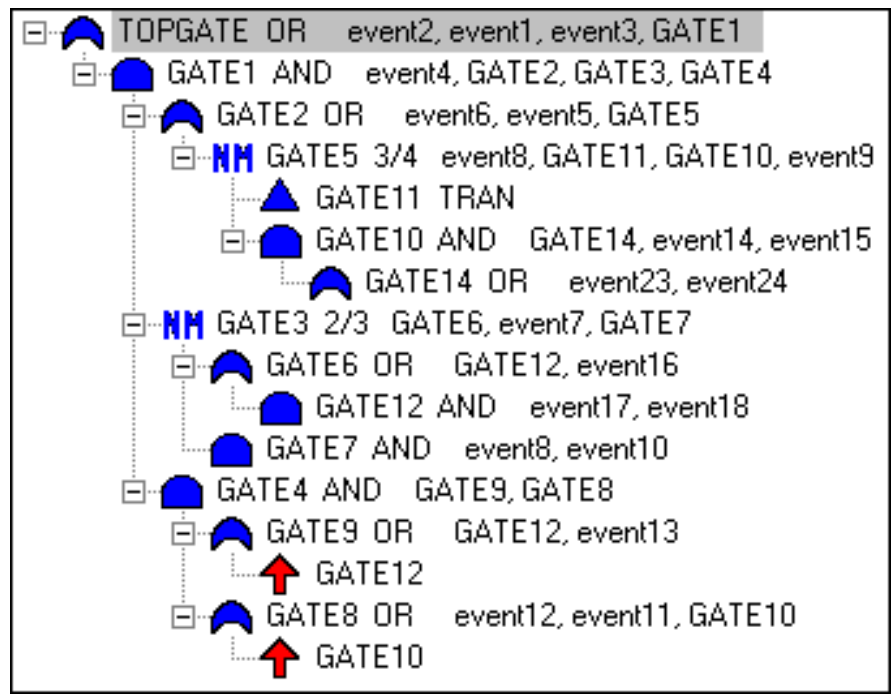

TOPGATE.FTL after the Gate command.

GATE11 AND GATE13, event18, GATE6
GATE13 OR event19, event20
GATE6 OR GATE12, event16
GATE12 AND event17, event18

GATE11.FTL after the Gate command.

\subsubsection{Page Tree - Find}

\section{PURPOSE}

This option locates the specified gate.

\section{STEPS}

1. From the SAPHIRE menu select Fault Tree. The Fault Trees List dialog will be displayed.

2. Highlight the desired fault tree.

3. Right-click to invoke the pop-up menu and choose Page Tree.

4. $\quad$ The Page Fault Tree Logic dialog will be displayed.

5. Choose the Find button.

6. The Find Gate dialog will be displayed.

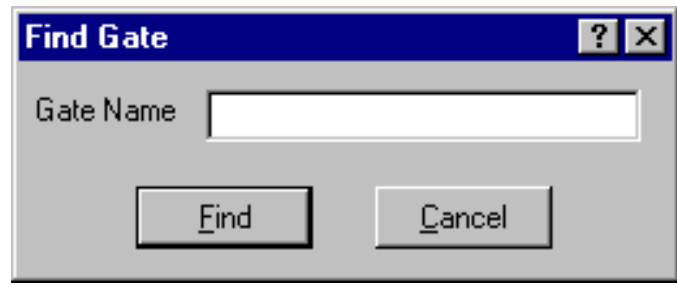

Gate Name - $\quad$ The name of the gate to be located. 
Find - $\quad$ Perform the find operation and close the Find Gate dialog. If the operation is successful, the located gate will be highlighted.

Cancel - $\quad$ Close the Find Gate dialog without performing the find operation.

\subsubsection{Page Tree - Merge}

\section{PURPOSE}

This option incorporates the logic represented by a transfer gate into the current fault tree file. This process will occur only if the gate is not referenced more than once within the fault tree. If an attempt is made to merge a transfer gate that is referenced more than once, the message, "Transfer used in other places, cannot merge" will be displayed in the message bar of the Page Fault Tree Logic dialog.

This option is enabled only when transfer gates are detected in the fault tree logic file.

\section{STEPS}

1. From the SAPHIRE menu select Fault Tree. The Fault Trees List dialog will be displayed.

2. Highlight the desired fault tree.

3. Right-click to invoke the pop-up menu and choose Page Tree. The Page Fault Tree Logic dialog will be displayed.

4. Highlight the desired transfer gate.

5. Choose the Merge button.

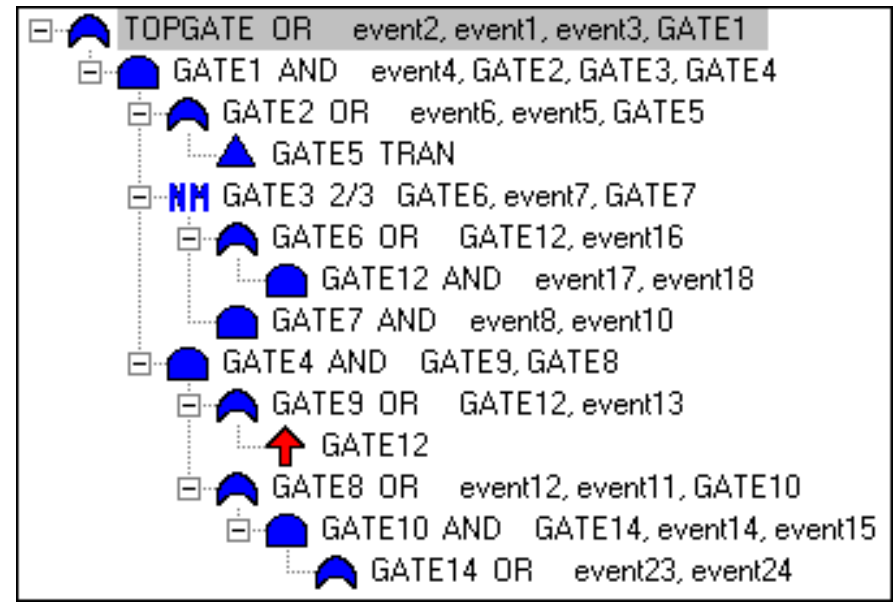

\section{EXAMPLE}

Using the example TOPGATE.FTL file above (created by using the Gate option on GATE5 in the basic example), notice that GATE5 is a transfer gate. After performing the Merge function on transfer GATE5, the result is a single TOPGATE.FTL file. 


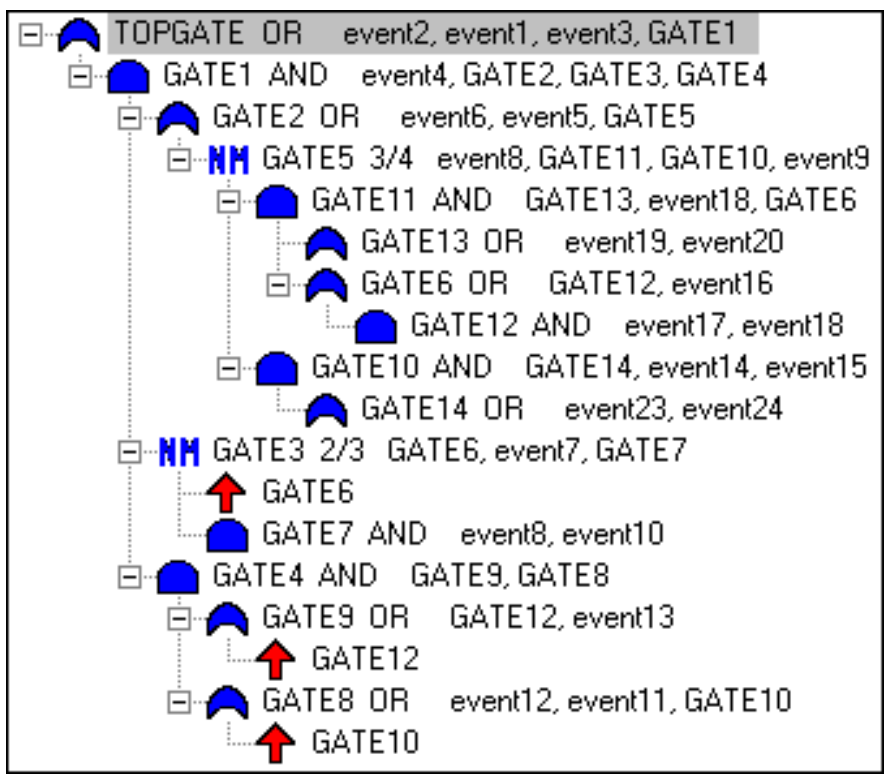

TOPGATE.FTL after the Merge command.

\subsubsection{Page Tree - Auto Page}

\section{PURPOSE}

This option automatically breaks the fault tree into easily viewable segments. When this process is executed, the Multiple and Tops operations are performed automatically. Transfer gates will be inserted as appropriate so that the final linked logic is equivalent to the original logic. Generally, the process will result in pages containing trees no more than four levels deep and five inputs wide.

\section{STEPS}

1. From the SAPHIRE menu select Fault Tree. The Fault Trees List dialog will be displayed.

2. Highlight the desired fault tree.

3. Right-click to invoke the pop-up menu and choose Page Tree. The Page Fault Tree Logic dialog will be displayed.

4. Highlight the desired gate.

5. Choose the Auto Page button.

\section{EXAMPLE}

Using the example fault tree, the execution of the Auto Page function results in six logic files:

TOPGATE.FTL, TOPGATE2.FTL, GATE2.FTL, GATE4.FTL, GATE10.FTL, and GATE11.FTL. Notice that the original fault tree logic file included two top events and four gates with multiple references. Remember that the Auto Page function automatically performs the Multiple and Tops functions. TOPGATE2 was recognized as a separate top event and was split off as a separate fault tree. Performing the Multiple function resulted in two more FTL files: GATE10 and GATE11 which met the screening criteria. Although GATE6 and GATE12 are also referenced more than once, GATE6 has as one of its inputs a gate with multiple references (GATE12) and GATE12 only has basic events as inputs and therefore, neither meet the screening criteria. GATE2 and GATE4 FTL files were split off for viewing convenience. 


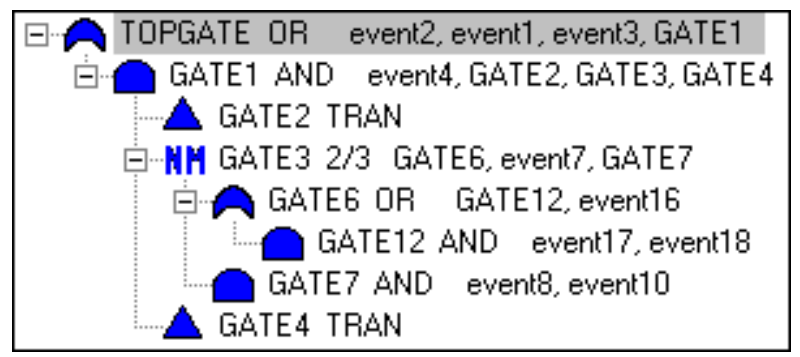

TOPGATE.FTL after the Auto Page command.

$\square$ A TOPGATE2 OR event21, event22, GATE11

$\triangle$ GATE11 TRAN

TOPGATE2.FTL after the Auto Page command.

$\square$ A GATE2 OR event6, event5, GATE5

D HM GATE5 3/4 event8, GATE11, GATE10, event9

A GATE11 TRAN

$\triangle$ GATE10 TRAN

GATE2.FTL after the Auto Page command.

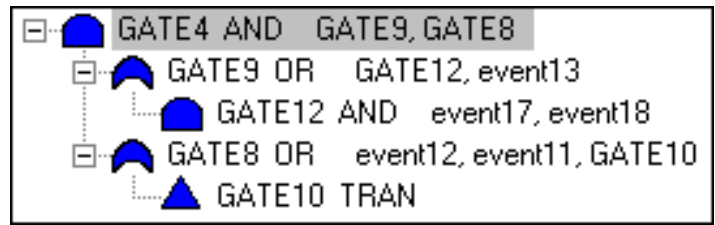

GATE4.FTL after the Auto Page command.

\section{$\square$ GATE10 AND GATE14, event14, event15}

A GATE14 OR event23, event24

GATE10.FTL after the Auto Page command.

GDATE11 AND GATE13, event18, GATE6
GATE13 OR event19, event20
GATE6 OR GATE12, event16
GATE12 AND event17, event18

GATE11.FTL after the Auto Page command.

\subsubsection{Page Tree - Exit}

\section{PURPOSE}

This option closes the Page Fault Tree Logic dialog. If changes were made to the fault tree logic, you will be prompted to save the changes before exiting by the Exit Pager dialog. 


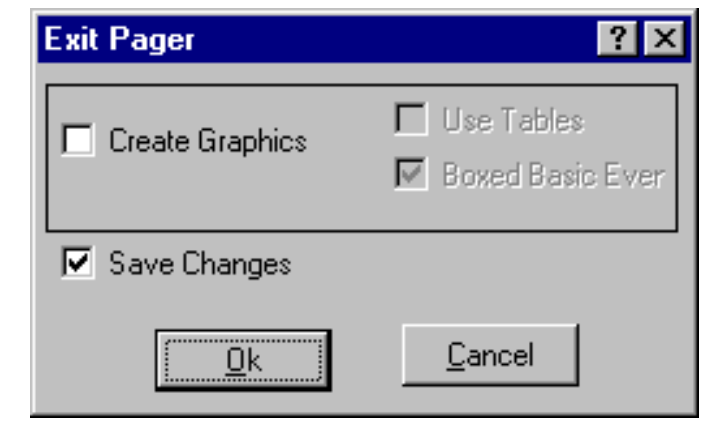

\section{Create Graphics -}

Select this check box to automatically convert the alphanumeric logic changes to the graphical format. This will create corresponding .DLS for each new fault tree or sub-tree.

\footnotetext{
Use Tables -

For each logic gate with many basic events as inputs, create a table of basic events, using the Primary name field. SAPHIRE processes the table of basic events as if they were separate symbols.
Boxed Basic Events -
Create each basic events with a box for descriptive text. The text from the description field of the associated event will be placed in the box. This does not influence the logic of the fault tree, but adds clarity to the model for those using and reviewing it.
Save Changes - $\quad$ Save the changes made to the fault tree logic file. If this check box is NOT selected, and the Ok button is chosen, the Page Fault Tree Logic dialog will be closed and the changes will not be saved.
Ok - $\quad$ Close the Page Fault Tree Logic dialog. Changes will be saved and graphic files created if the appropriate check boxes are selected.
Cancel - $\quad$ Close the Exit Pager dialog but do not close the Page Fault Tree Logic dialog.

\subsection{Solving Fault Trees}

\subsubsection{Solving Fault Trees}

\section{PURPOSE}

This option generates the cut sets for selected fault tree(s) based on cut set generation cutoff values. You are given the ability to generate cut sets for a selected fault tree, a group of fault trees, or all fault trees 
within the current project. Various options are provided to allow cut sets to be generated in various ways. You are given the opportunity to specify several cut set generation cutoff values that will be used to determine if a cut set is to be retained or discarded from the selected fault tree(s).

\section{STEPS}

1. From the SAPHIRE menu select Fault Tree.

2. The Fault Trees List dialog will be displayed.

3. Highlight the desired fault tree(s).

4. Right-click to invoke the pop-up menu and choose Solve.

Whether you are generating cut sets for a single fault tree, a group of fault trees, or for all fault trees the Cut Set Generation dialog will be displayed.

The dialog below is displayed when the Analysis Type is "Random".

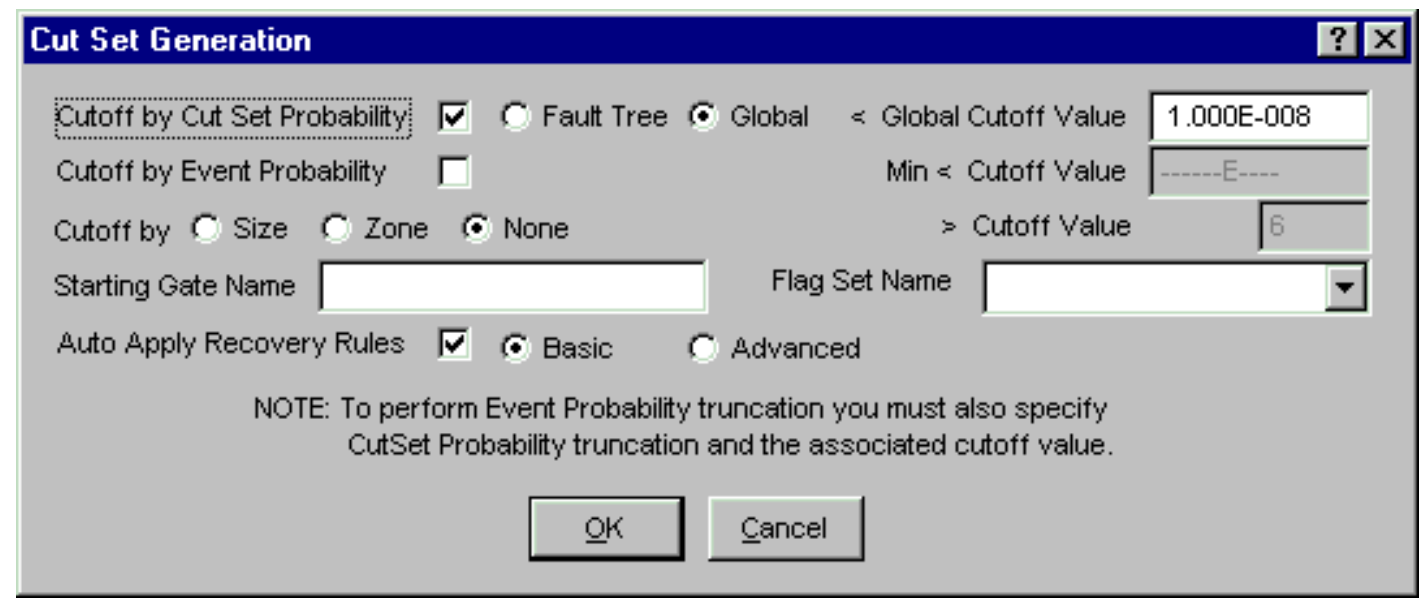

The dialog below is displayed when any other Analysis Type is selected. 


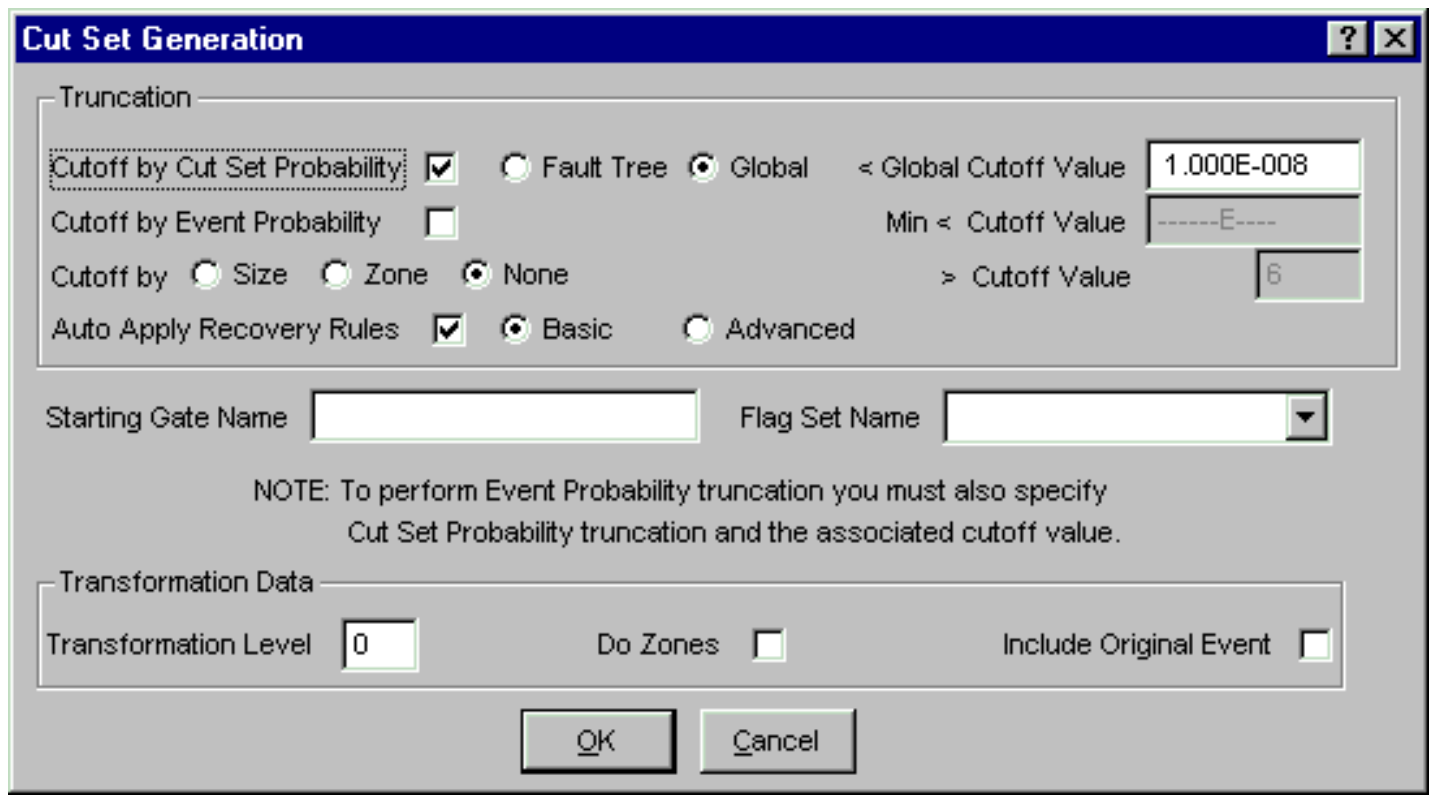

You may change any of the data fields on this dialog. The default values that appear on this dialog may be reset to new values by selecting Utility from the SAPHIRE menu and then invoking the Define Constants option.

Cutoff by Cut Set Probability - Used for determining if the cut set should be retained or discarded based on the value in the < Global Cutoff Value field.

Cutoff by Event Probability - Used for determining if the cut set should be retained or discarded based on the value in the Min < Cutoff Value field.

Cutoff by Size Used for determining if the cut set should be retained or discarded based on the value in the > Cutoff Value field or zoned flagged events.

\author{
Starting Gate Name - \\ Flag Set Name - \\ Auto Apply Recovery Rules - \\ Basic - \\ Advanced -
}

Transformation Level -

\section{Do Zones -}

\section{Include Original Event -}

Used to specify the top gate of the tree.

Used to specify the flag set to be used during processing.

Used for automatically applying recovery rules while solving.

Apply rules created using the basic rule syntax

Apply rules created using the advanced rule syntax.

An integer $0-255$ which indicates the default level of substitution for the transformations to be performed.

If checked, perform zone transformation during cut set generation.

Used for determining if the original event should be retained or discarded during processing.

During processing, the Cut Set Generation Results dialog is displayed and updated as the calculations proceed. Upon completion of the cut set generation, the summary results are displayed in this dialog. Note that the displayed results reflect the values prior to the application of recover rules, if that option was selected. Choose the OK button to close the dialog, or View Results button to review or print the results.

The only limit on the number of cut sets that can be stored for a given fault tree is the available hard drive space. When processing is complete, the Fault Trees List dialog is updated to show the letter "c" in front of the fault tree(s) with current case cut sets. 


\subsubsection{Cut Set Generation Dialog}

\section{Cutoff by Cut Set Probability}

If you select this check box and choose the:

$\begin{array}{ll}\text { Global radio button - } & \begin{array}{l}\text { Only those cut sets whose product for all of its event } \\ \text { probabilities is greater than or equal to the value in the }<\text { Global } \\ \text { Cutoff Value field will be kept. }\end{array} \\ \text { Fault Tree radio button - } & \begin{array}{l}\text { Only those cut sets whose product for all of its event } \\ \text { probabilities is greater than or equal to the fault tree's cutoff } \\ \text { value will be kept. This value is entered in the Modify | Fault } \\ \text { Trees option. }\end{array}\end{array}$

All other cut sets will be removed.

If you uncheck this box, then the probability for the cut set will be irrelevant for determining if the cut set should be retained or discarded.

\subsubsection{Cutoff by Event Probability}

If you select this check box, then you must also choose the Cutoff by Cut Set Probability check box. This option will check all cut sets that are below the probability cutoff (Min $<$ Cutoff Value field) and remove them only if they contain an event whose probability is below this value.

When you select his option, SAPHIRE will also perform the quantification.

\subsubsection{Cutoff by Size}

Size - If you choose this radio button, then only the cut sets whose number of events is less than or equal to the value specified in the $>$ Cutoff Value field will be kept in the cut sets for that fault tree. All other cut sets will be removed.

Zone - If you choose this radio button, then only zone flagged events will be checked.

None - If you choose this radio button, then the number of events in a cut set will be irrelevant for determining if the cut set should be retained or discarded.

\subsubsection{Starting Gate Name}

If a gate is specified in this field, then this gate will be the gate assumed to be the top gate of the tree. If a name is not specified, the top gate will be determined by finding the gate that is not referenced by any other gate. This top gate should be named the same as the fault tree or sub-tree name.

\subsubsection{Flag Set Name}

Select the desired flag set, if any, from the drop-down list. If the field is left blank, the fault tree-specific flag set, if any, will be used. If a flag set is specified, that flag set will be used during processing. A predefined flag set option, NONE, is available. If you choose this option, no flag sets are used.

\subsubsection{Include Original Event}

If checked, the original event will be included in the logic along with the transformation event(s) during cut set generation. Otherwise, the original event will be replaced by the transformation event(s). 


\subsection{Quantifying Fault Trees}

\subsubsection{Quantifying Fault Trees}

\section{PURPOSE}

The quantification process will re-quantify the cut sets using current data values (event change sets and current case cut sets). Quantification can be applied to a selected fault tree, for a group of fault trees, or for all of the fault trees within the current project. The new value is saved with the current case cut sets for the selected fault tree.

\section{STEPS}

1. From the SAPHIRE menu select Fault Tree. The Fault Trees List dialog will be displayed.

2. Highlight the desired fault tree(s) and right-click to invoke the pop-up menu.

3. Choose Quantify, then one of the submenu options.

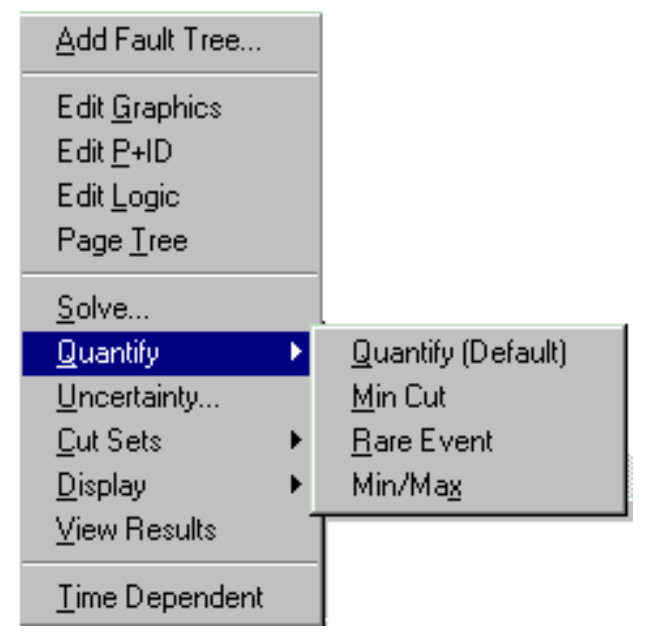

Quantify (Default) - $\quad$ Re-quantify using the quantification method for the specified fault tree.

Min Cut This is selected in the Modify | Fault Trees option.

Rare Event Re-quantify using the Minimal Cut Set Upper Bound Approximation. Re-quantify by adding together the probabilities for the cut sets of a top event.

$\operatorname{Min} / \operatorname{Max}-$ Re-quantify using the "exact" calculation for the union of cut sets via the inclusion-exclusion rule.

If the selected quantification method differs from the default method of the fault tree, a warning box with the message, "The chosen quantification method was different from the Fault Tree default" is displayed. Choose the Ok button to continue processing.

During processing, the Quantification Results dialog is displayed. If an error occurs, the message, "Error quantifying cut sets" will be displayed in the message bar. If a single fault tree was selected, upon 
completion of the quantification process, the results are displayed in the Quantification Results dialog. Choose Ok to close the dialog.

\subsubsection{Minimal Cut Set Upper Bound Approximation}

This calculation approximates the probability of the union of the minimal cut sets for the fault trees. The equation for the minimal cut set upper bound is

$$
S=1-\prod_{i=1}^{m}\left(1-C_{i}\right)
$$

where

$\mathrm{S}=$ minimal cut set upper bound for the fault tree unavailability,

$\mathrm{C}_{\mathrm{i}}=$ probability of the $\mathrm{i}=$ th cut set, and

$\mathrm{m}=$ the number of cut sets.

Example: If the cut sets for a fault tree are $\mathrm{X}=\mathrm{A} \cup \mathrm{B} \cup \mathrm{C}$ (i.e., the union of three events, $\mathrm{A}$, $\mathrm{B}$, and $\mathrm{C}$ ); then the cut sets can be written as $\mathrm{X}=\mathrm{A}+\mathrm{B}+\mathrm{C}$ with the plus symbol indicating union. The fault tree unavailability computed from the minimal cut set upper bound approximation is then $X=1-(1-A)(1-$ B)(1 - C).

\section{SEE TECHNICAL REFERENCE}

\section{Minimal Cut Set Upper Bound}

\subsubsection{Min Max Quantification}

The Min-Max quantification option quantifies the current case cut sets using the "exact" probability quantification algorithm. This algorithm can be extremely time consuming if the number of cut sets is large. The user should be careful when selecting this option.

When you choose this option, the dialog shown below will be displayed. You are required to enter the number of passes. The number passes indicates the number of intermediate terms SAPHIRE will calculate when determining the minimum and maximum quantification values. Type a number from 1 to 10 to indicate the number of passes you want SAPHIRE to make. The higher the number, the more the calculation speed decreases and the accuracy increases. Choose the Ok button to proceed.

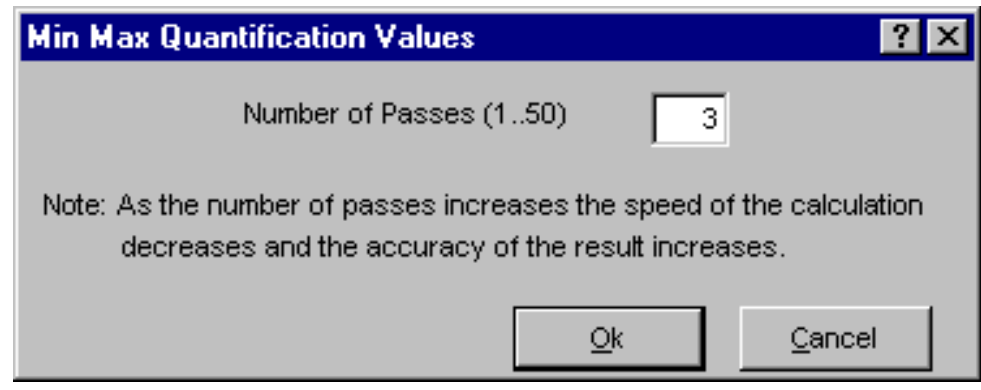


SAPHIRE will perform the specified number of intermediate terms and when complete will show the results on the Quantification Results dialog. The results of the Min/Max Quantification option are displayed only on this dialog.

\section{How this option works:}

To quantify the union of events, the first pass consists of adding the events, the second pass consists of subtracting pairs of events, the third pass consists of adding "triples", and so on.

For a simple example, assume that a fault tree has only three cut sets which are the union of $\mathrm{A}, \mathrm{B}$, and $\mathrm{C}$ which can be expressed as $\mathrm{A} \cup \mathrm{B} \cup \mathrm{C}$. In this example, each cut set consists of only one term; however, the approach is not limited to one term per cut set. For 3 passes, the solution is shown below.

$$
\mathrm{X}=(\mathrm{A}+\mathrm{B}+\mathrm{C})-(\mathrm{A} * \mathrm{~B}+\mathrm{A} * \mathrm{C}+\mathrm{B} * \mathrm{C})+(\mathrm{A} * \mathrm{~B} * \mathrm{C})
$$

The Min/Max algorithm applies the Boolean idempotent law $(\mathrm{A} * \mathrm{~A}=\mathrm{A})$ to reduce identical terms during the multiplication of cut sets.

The table below shows the Min/Max equation and results, with $\mathrm{A}=\mathrm{B}=\mathrm{C}=0.8$. The minimal cut set upper bound approximation would be $1-(1-0.8)^{3}=0.992$. (Note that the Min Max calculation will be the same as the minimal cut set upper bound when the cut sets do not contain common events.)

\begin{tabular}{|c|c|c|}
\hline $\begin{array}{c}\# \\
\text { Passes }\end{array}$ & Min/Max Equation & Min/Max Probability \\
\hline 1 & $\mathrm{~A}+\mathrm{B}+\mathrm{C}$ & 2.4 \\
\hline 2 & $\mathrm{~A}+\mathrm{B}+\mathrm{C}-(\mathrm{A} * \mathrm{~B}+\mathrm{A} * \mathrm{C}+\mathrm{B} * \mathrm{C})$ & $2.4-1.92=0.48$ \\
\hline 3 & $\mathrm{~A}+\mathrm{B}+\mathrm{C}-(\mathrm{A} * \mathrm{~B}+\mathrm{A} * \mathrm{C}+\mathrm{B} * \mathrm{C})+(\mathrm{A} * \mathrm{~B} * \mathrm{C})$ & $2.4-1.92+0.512=0.992$ \\
\hline 4 & $\mathrm{~A}+\mathrm{B}+\mathrm{C}-(\mathrm{A} * \mathrm{~B}+\mathrm{A} * \mathrm{C}+\mathrm{B} * \mathrm{C})+(\mathrm{A} * \mathrm{~B} * \mathrm{C})$ & $2.4-1.92+0.512=0.992$ \\
\hline
\end{tabular}

\subsubsection{Rare Event Approximation}

The rare event approximation approach adds together the probabilities for the cut sets of a top event. This approximation is a good method when the cut set probabilities are small.

\subsection{Uncertainty Analysis}

\subsubsection{Uncertainty Analysis}

\section{PURPOSE}

This option can estimate the variability (due to the uncertainties in the basic event probabilities) of either a fault tree top event probability or an event tree sequence frequency. To do this, SAPHIRE provides two sampling techniques: Monte Carlo simulation and Latin Hypercube simulation. In addition, you may perform a seismic analysis to integrate the seismic fragility curve with the site hazard curve using the uncertainty analysis option. 
In a traditional uncertainty analysis, SAPHIRE samples the user-specified distributions for each basic event in a group of cut sets, then quantifies these cut sets using the sample values. For a seismic uncertainty, however, SAPHIRE performs the analysis at each G-level in the site hazard curve for the current project. The seismic events are sampled at each new G-level and the quantification performed.

You are given the option of performing an uncertainty analysis on the current case cut sets for either a single, for a group, or for all of the fault trees (or sequences or end states) within the current project.

\section{STEPS}

1. From the SAPHIRE menu select Fault Tree/Sequence/End State.

2. The Fault Trees List/Sequences/End State List dialog will be displayed.

3. Highlight the desired fault tree(s)/sequence(s)/end state(s) and right-click to invoke the pop-up menu.

4. Choose Uncertainty.

5. The Uncertainty Calculation Values dialog will be displayed.

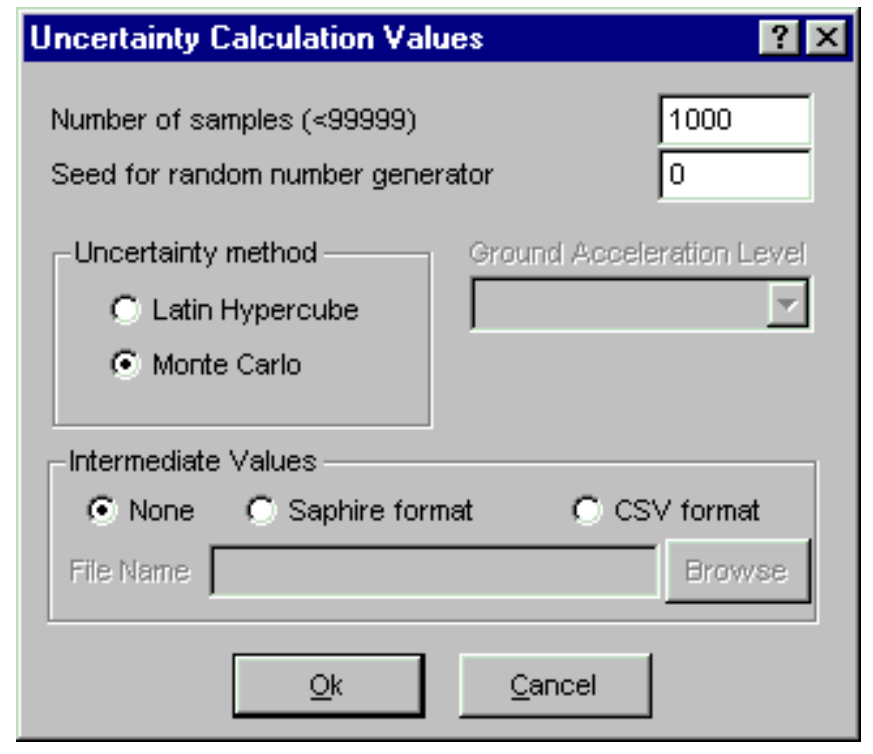

You may change any of the data fields on this dialog. The default values that appear on this dialog may be reset to new values by selecting the Utility $\mid$ Define Constants option.

Number of samples $(<99999)$ -

Seed for random number generator -
A default value is provided for the number of samples to use in simulation. You may use this value or enter another value. The number must be less than 99,999 .

A default seed is provided for the random seed. You may use this value or enter a new value for the seed. To obtain a random seed from the system clock, you must enter a zero in this field.

Select the appropriate radio button for the desired uncertainty sampling technique. 
Ground Acceleration Level -

Intermediate Values -

None -

SAPHIRE format -

CSV format -

File Name -

Browse -
If seismic uncertainty is selected, a ground level acceleration must be provided. This indicates the acceleration rate at which the component will always fail. The user can select to process all G-levels combined, all G-levels separately, or a specified G-level only.

Select this check box if you wish for the results and intermediate samples to be written to an ASCII file. Select this check box if you wish for the results and intermediate samples to be written to an ASCII file, formatted in the traditional SAPHIRE format.

Select this check box if you wish for the results and intermediate samples to be written to an ASCII file, formatted as a comma delimited file, for easy import into a spreadsheet program.

Specifies the name of the ASCII file where the intermediate values will be stored.

Opens the Choose File dialog, to select a directory and file name to store the intermediate results.

\subsubsection{Intermediate Values}

\section{PURPOSE}

This option allows you to output the results and intermediate samples to the ASCII file specified in the File Name field.

\section{STEPS}

1. From the SAPHIRE menu select Fault Tree/Sequences/End State. The Fault Trees List/Sequences/End State List dialog will be displayed.

2. Highlight the desired fault tree(s)/sequence(s)/end state(s) and right-click to invoke the pop-up menu.

3. Choose Uncertainty. The Uncertainty Calculation Values dialog will be displayed.

4. Check the Output Values check box and enter a name in the File Name field.

The example below describes the layout of a SAPHIRE formatted ASCII file. The CSV (comma delimited file) layout is similar, but is comma delimited to be easily imported into a spreadsheet program.

Format for ASCII file containing intermediate samples: 


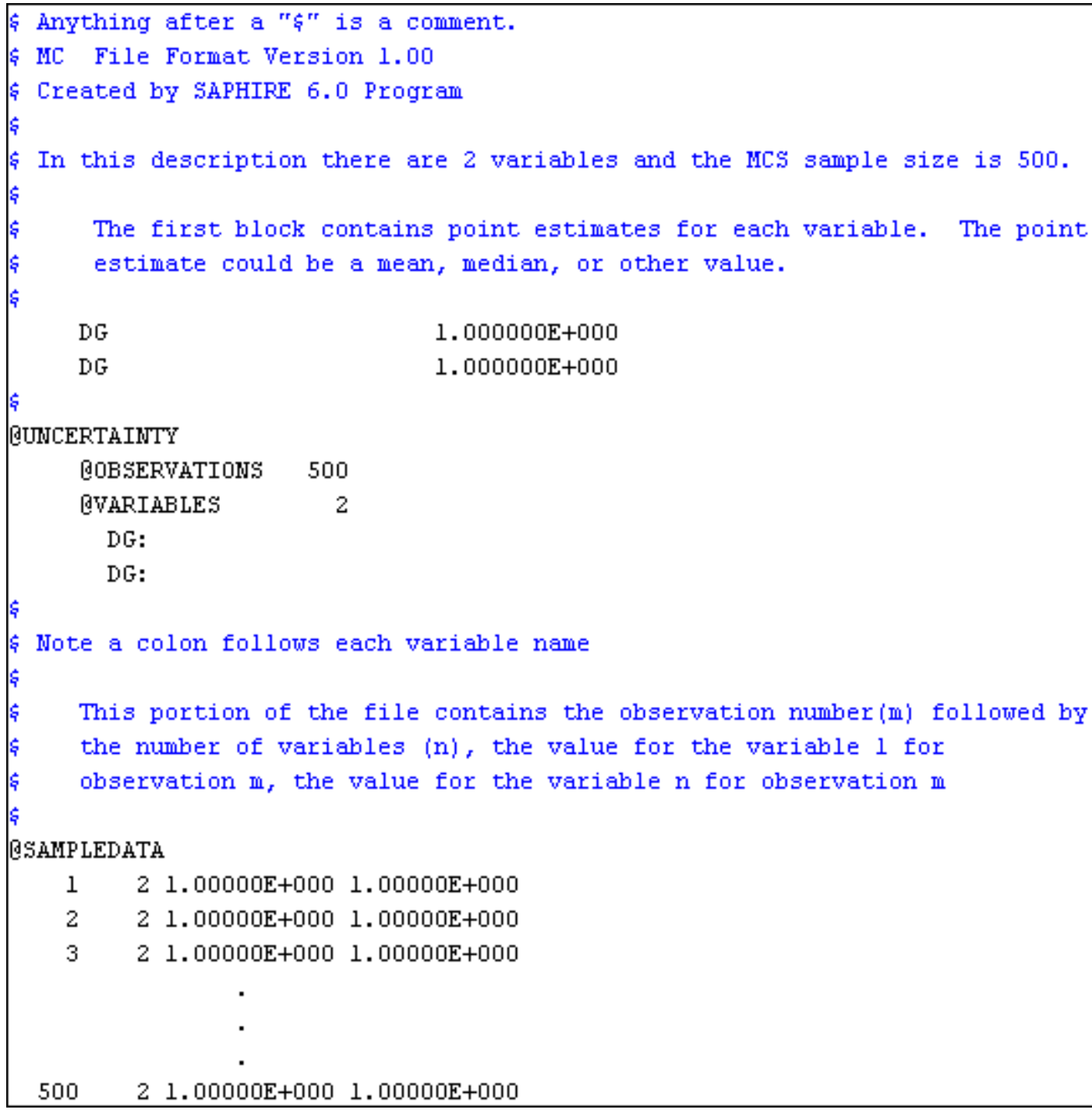

\subsubsection{Monte Carlo Sampling (MCS)}

Simple MCS is a fundamental uncertainty sampling approach. To perform the sampling, SAPHIRE makes repeated quantifications of the fault tree/sequence/end state cutsets using samples from the basic event uncertainty distributions. This type of sampling requires more samples than LHS for the same degree of accuracy.

When using this sampling technique, if the number of samples entered is less than ten, then the number of samples will be increased to ten before the uncertainty analysis process will continue. Any number of samples greater than or equal to ten will be allowed, but a number of at least 1000 is probably a better value for improving the reliability of the Monte Carlo results.

\subsubsection{Latin Hypercube Sampling (LHS)}

LHS is a stratified sampling technique where the random variable distributions are divided into equal probability intervals. A probability is randomly selected from within each interval for each basic event. 
Generally, LHS will require fewer samples than simple MCS for similar accuracy. However, due to the stratification method, it may take longer to generate a value than for a MCS.

When using this technique, if the number of samples entered is less than twice the total number of unique events in the fault tree/sequence/end state, then the number of samples will be increased to two times the total number of unique events before the analysis will continue. The LHS technique gives its best results if the number of samples is at least twice the total number of unique events.

\subsubsection{The Uncertainty Analysis Process}

The following is a description of the uncertainty analysis process performed by SAPHIRE after values have been entered in the Uncertainty Calculation Values dialog.

Once the number of samples has been accepted and a seed obtained from the system clock (if necessary), checks will be run to ensure the events with the same correlation classes have consistent failure data, uncertainty data, and distribution types. If any events with inconsistencies exist, an error message will be displayed and the uncertainty analysis process will be terminated so that the inconsistent values may be corrected.

If an error occurs during the uncertainty analysis process, the process is terminated and a message box providing information about the specific error is displayed.

If all of the events successfully pass the correlation class checks, then the distribution parameters for the events will be checked to ensure that they are valid. If any of the parameters are invalid, error messages will be displayed and the process will be terminated so the distribution parameters may be corrected.

After both of these checks have been passed, a point estimate will be calculated for the selected fault tree (or sequence or end state). At this point the samples for each event will be generated using the selected sampling technique, either the Monte Carlo Sampling technique or the Latin Hypercube Sampling technique. The uncertainty analysis function provides you with 11 different distribution types for both sampling techniques. The distribution types include normal, lognormal, beta, gamma, chi-squared, exponential, uniform, Dirichlet, constrained non-informative, maximum entropy, and the user-defined histograms.

During processing, the Uncertainty Results dialog will be displayed and updated as the samples are generated. When the requested number of samples have been generated, statistical information will be calculated using the generated samples. A sample mean, median, and standard deviation will be calculated for the selected fault tree (or sequence or end state). Coefficients of skewness and kurtosis, and quantile values will also be calculated. This data will be saved in the database for the selected fault tree (or sequence or end state).

Upon completion of these calculations, the following values will be displayed on the Uncertainty Results dialog for viewing: the name, random seed used, the number of samples generated in this process, the total number of events and cut sets being processed, the point estimate, the mean, the median, the 5th and 95th percentile values, the minimum and maximum generated sample values, the standard deviation, the skewness and kurtosis, and the time involved to perform the analysis. 


\begin{tabular}{|c|c|c|}
\hline Uncertainty Results & \multicolumn{2}{|r|}{$\mathrm{x}$} \\
\hline \multicolumn{3}{|l|}{ Name } \\
\hline Random Seed 41877 & Events & 10 \\
\hline Sample Size $\quad 1000$ & Cut Sets & 15 \\
\hline Point estimate & \multicolumn{2}{|c|}{$2.120 \mathrm{E}-002$} \\
\hline Mean Value & \multicolumn{2}{|c|}{$2.047 \mathrm{E}-002$} \\
\hline 5th Percentile Value & \multicolumn{2}{|c|}{$1.524 \mathrm{E}-003$} \\
\hline Median Value & \multicolumn{2}{|c|}{$8.788 \mathrm{E}-003$} \\
\hline 95th Percentile Value & \multicolumn{2}{|c|}{$6.852 E-002$} \\
\hline Minimum Sample Value & \multicolumn{2}{|c|}{$2.150 \mathrm{E}-004$} \\
\hline Maximum Sample Value & \multicolumn{2}{|c|}{ 6.591E-001 } \\
\hline Standard Deviation & \multicolumn{2}{|c|}{$4.138 \mathrm{E}-002$} \\
\hline Skewness & \multicolumn{2}{|c|}{$7.312 \mathrm{E}+000$} \\
\hline Kurtosis & \multicolumn{2}{|c|}{$8.155 E+001$} \\
\hline Elapsed Time & \multicolumn{2}{|c|}{ 00:00:00.690 } \\
\hline \multicolumn{3}{|c|}{ Cancel } \\
\hline
\end{tabular}

If only one fault tree (or sequence or end state) was selected (highlighted) for the uncertainty analysis process, then you will need to close the Uncertainty Results dialog. However, if more than one is being processed, the Uncertainty Results dialog will be displayed for each, and when all of the selected fault trees (or sequences or end states) have been processed, the Uncertainty Results dialog will automatically be closed.

\subsubsection{Seismic Uncertainty}

\section{PURPOSE}

A seismic analysis integrates the seismic fragility curve with the site hazard curve using the uncertainty analysis option. If the user has chosen to perform a seismic analysis, then a G-level was also selected. This option allows the user to specify how SAPHIRE performs the analysis.

\section{STEPS}

1. From the SAPHIRE menu select Fault Tree/Sequence/End State. The Fault Trees List/Sequences/End State List dialog will be displayed.

2. Select the "SEISMIC" value from the Analysis Type drop-down list.

3. Highlight the desired fault tree(s)/sequence(s)/end state(s) and right-click to invoke the pop-up menu.

4. Choose Uncertainty. The Uncertainty Calculation Values dialog will be displayed.

5. Provide appropriate values for the data entry fields on this dialog and choose one of the Ground Acceleration Level values.

The G-levels are described here: 
ALL COMBINED - SAPHIRE performs an uncertainty analysis on all G-levels defined in the site hazard curve for this project. The combined results are then stored in the database.

ALL SEPARATE - SAPHIRE performs an uncertainty analysis on all G-levels, but does the analysis and stores the results separately for each G-level.

Specific G-level - $\quad$ SAPHIRE only performs the analysis for that specified G-level.

\subsubsection{Uncertainty Distributions for Basic Events}

Within the SAPHIRE code, eleven types of uncertainty distributions are supported. The table below lists the different distributions, their identifier within SAPHIRE, and the uncertainty parameter that is needed by the code.

Along with the uncertainty parameter, most distributions require a second parameter. SAPHIRE requires that the mean value be specified for all distributions. The mean value is put in the database as a mean failure probability or a mean failure rate, depending on which calculation type is used.

To demonstrate how to specify an uncertainty distribution in SAPHIRE, assume that a basic event has a mean failure probability of 0.5 and the standard deviation is 0.2 . The uncertainty distribution type is unknown. Assuming that the event is distributed with a uniform distribution, we can calculate the upper end point to be 0.846 from the equations (for a uniform distribution)

Mean $=(1 / 2)(b+a)$

Variance $=(1 / 12)(b-a)^{2}$,

where $a$ is the lower end point and $b$ is the upper end point. Consequently, to enter this distribution in SAPHIRE, the mean value of 0.5 would be entered, a uniform distribution would be chosen (type U), and the upper end point of 0.846 would be specified.

Alternatively, if we assume that the event is lognormally distributed, we would need to calculate the error factor $(\mathrm{EF})$. Knowing the mean $\left(\mu_{\mathrm{ln}}\right)$ and standard deviation $\left(\sigma_{\ln }\right)$, we can calculate the EF from the equation

$$
E F=e^{1.645 \sqrt{\ln \left(1+\left(\sigma_{\ln } / \mu_{\ln }\right)^{2}\right)}} .
$$

From this equation, the EF is calculated to be 1.88. To enter this distribution in SAPHIRE, the mean value of 0.5 would be entered, a lognormal distribution would be chosen (type L), and the EF of 1.88 would be specified.

\section{Basic event uncertainty distribution types supported by SAPHIRE.}

$\begin{array}{lll}\text { Distribution } & \text { Identifier } & \text { Uncertainty Parameter } \\ \text { none } & \text { blank } & \text { none } \\ \text { lognormal } & \text { L } & \text { error factor } \\ \text { normal } & \text { N } & \text { standard deviation } \\ \text { beta } & \text { B } & \text { b in Beta }(a, b)\end{array}$




\begin{tabular}{lll} 
gamma & $\mathrm{G}$ & $\mathrm{r}$ in $\Gamma(\mathrm{r})$ \\
chi-squared & $\mathrm{C}$ & degrees of freedom \\
exponential & $\mathrm{E}$ & none \\
uniform & $\mathrm{U}$ & upper end point \\
histograms & $\mathrm{H}$ & histogram number (i.e., identifier) \\
maximum entropy & $\mathrm{M}$ & lower and upper end point \\
constrained noninformative & $\mathrm{O}$ & none \\
Seismic & $\mathrm{S}$ & Beta $\mathrm{r}$, Beta $\mathrm{u}$ \\
\hline
\end{tabular}

If the event was assumed to be normally distributed, we would simply put the mean value in the probability data field and the standard deviation in the uncertainty parameter data field. To enter this distribution, the mean value of 0.5 would be entered, a normal distribution would be chosen (type N), and the standard deviation of 0.2 would be specified.

To review the process of specifying an uncertainty distribution in SAPHIRE, the required steps are to:

1. Enter the mean value for the basic event.

2. Choose the distribution type (from those shown in the "Identifier" column in the table above).

3. Specify the appropriate uncertainty distribution parameter (shown in the "Uncertainty Parameter" column in the table above).

Additional information on the uncertainty distributions and their parameters can be found in the SAPHIRE Technical Reference Manual.

\subsection{Fault Tree Cut Set Analysis}

\subsubsection{Fault Tree Cut Set Analysis}

\section{PURPOSE}

This option provides the ability to perform a variety of analysis functions on the fault tree cut sets.

\section{STEPS}

1. From the SAPHIRE menu select Fault Tree. The Fault Trees List dialog will be displayed.

2. Highlight the desired fault tree(s) and right-click to invoke the pop-up menu.

3. Choose Cut Sets, then one of the sub-menu options. 


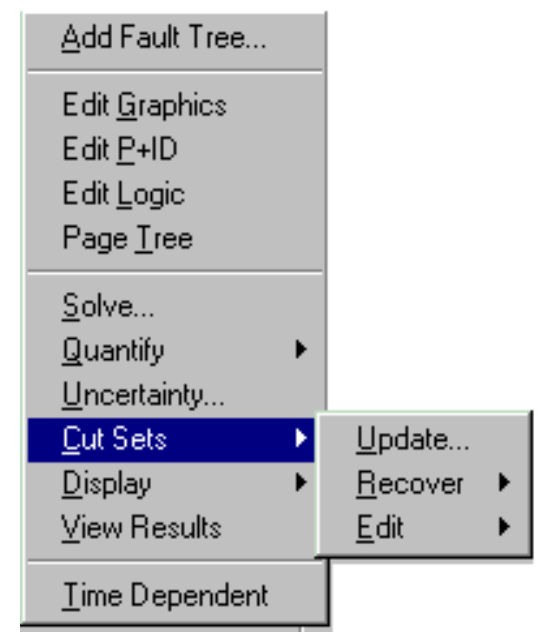

Update - $\quad$ Update the current cut sets for a selected fault tree based on cut set generation cutoff values.

Prune - $\quad$ Eliminate cut sets from a selected fault tree that contains events that conflict in some way with one another.

Recover - $\quad$ Modify cut sets by adding recovery actions to them.

Edit - $\quad$ Modify the base case/current fault tree cut sets.

\subsubsection{Updating Cut Sets}

\section{PURPOSE}

This option will update the current case cut sets for a selected fault tree (or sequence or end state) based on cut set generation cutoff values. You are given the opportunity to specify several cut set generation cutoff values that will be used to determine if a cut set is to be retained or discarded. You are also given the choice of using either the base case cut sets or the current case cut sets as the starting set of cut sets to be updated. The updated version of the cut sets will be saved as the new current case cut sets.

Current case cut sets can be updated for a selected fault tree (or sequence or end state), a group of fault trees (or sequences or end states), or all of the fault trees (or sequences or end states) within the current project

\section{STEPS}

1. From the SAPHIRE menu select Fault Tree/Sequence/End State. The Fault Trees List/Sequences/End State List dialog will be displayed.

2. Highlight the desired fault tree(s)/sequence(s)/end state(s) and right-click to invoke the pop-up menu.

3. Choose Cut Sets, then the Update sub-menu option.

4. The Cut Set Generation Truncation dialog will be displayed. 


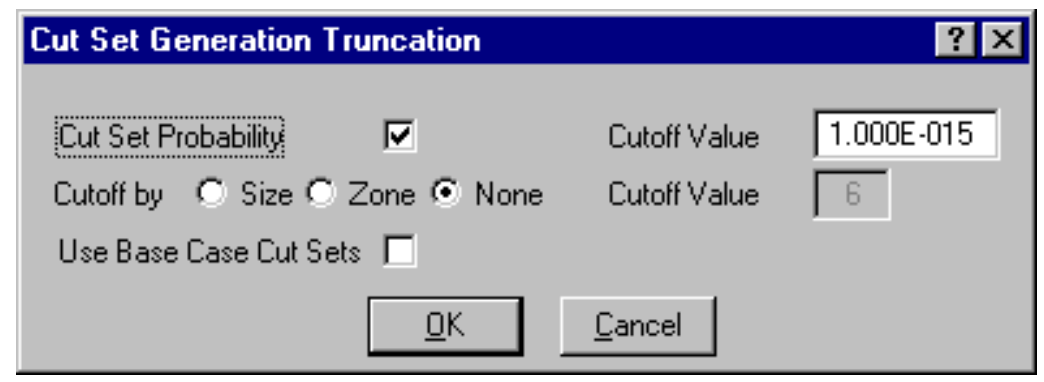

You may change any of the data fields on this dialog. The default values that appear on this dialog may be reset to new values by selecting Utility on the SAPHIRE menu and then invoking the Define Constants option.

Cut Set Probability - Used for determining if the cut set should be retained or discarded based on the value in the Cutoff Value field.

Cutoff by Size - $\quad$ Used for determining if the cut set should be retained or discarded based on zoned flagged events or the value in the Cutoff Value field.

Use Base Case Cut Sets -Used to specify base case cut sets or current case cut sets.

During processing, the Cut Set Generation Results dialog is displayed and updated as the calculations proceed. If a single fault tree (or sequence or end state) was selected, upon completion of the cut set generation, the results are displayed in this dialog. Choose the Ok button to close the dialog.

Once the cut sets are updated, they are automatically quantified.

\subsubsection{Cut Set Generation Truncation}

\subsubsection{Cut Set Probability}

If you select this check box, then only the cut sets whose product for all of its event probabilities is greater than or equal to the value in the Cutoff Value field will be kept. All other cut sets will be removed from current case cut sets for that fault tree.

If you uncheck this box, then the probability for the cut set will not be relevant for determining if the cut set should be retained or discarded.

\subsubsection{Cutoff by Size}

Size - If you choose this radio button, then only the cut sets whose number of events is less than or equal to the value specified in the $>$ Cutoff Value field will be kept in the cut sets for that fault tree. All other cut sets will be removed. 
Zone - If you choose this radio button, then only zone flagged events will be checked.

None - If you choose this radio button, then the number of events in a cut set will be irrelevant for determining if the cut set should be retained or discarded.

\subsubsection{Use Base Case Cut Sets}

If you select this check box, then base case cut sets will be used as the cut sets to be updated and then stored in the current case cut sets. However, if unchecked, the current cut sets will be used as the cut sets to be updated and then resaved in the current case cut sets.

\subsubsection{Recovering Fault Tree Cut Sets}

\subsubsection{Recovering Fault Tree Cut Sets}

\section{PURPOSE}

The "recovery" option allows the user to post-process cut sets by modifying them according to a predefined set of rules. The user constructs recovery rules to be applied to each cut set which then define the action to be taken. The rules are constructed using the same editor that is used for event tree rules definition. This editor is a macro-based editor, which means that the user can define macros (or variables) that can be used as the search criteria tests.

\section{STEPS}

1. From the SAPHIRE menu select Fault Tree. The Fault Trees List dialog will be displayed.

2. Highlight the desired fault tree(s) and right-click to invoke the pop-up menu.

3. Choose Cut Sets | Recover.

4. Select one of the sub-menu options

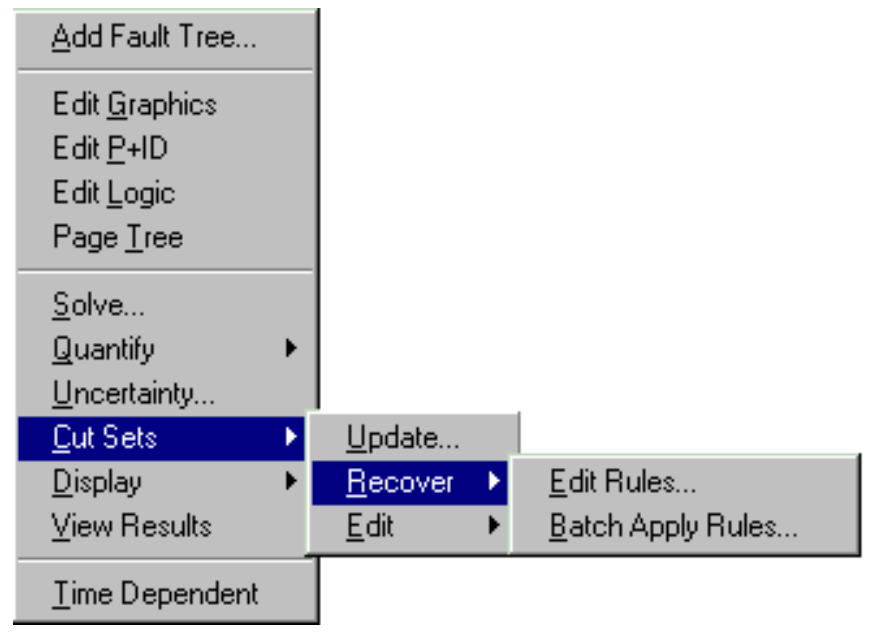

Edit Rules - Create and modify recovery rules for a highlighted fault tree.

Batch Apply - Apply all rules currently defined to the highlighted fault trees. 


\subsubsection{Batch Apply}

\section{PURPOSE}

This option allows you to apply all rules currently defined to the highlighted or marked fault trees.

The application of recovery rules can potentially require numerous comparisons of rules to cut sets, resulting in a long analysis time. Consequently, when the rules are applied in SAPHIRE for the entire project, the analyst is presented with the option to apply rules to cut sets down to a specified truncation level. By setting a truncation value in the Probability cutoff value field, only those cut sets with a "high" probability will have rules applied.

\section{STEPS}

1. From the SAPHIRE menu select Fault Tree. The Fault Trees List dialog will be displayed.

2. Highlight the desired fault tree(s) and right-click to invoke the pop-up menu.

3. Choose Cut Sets | Recover.

4. Select the Batch Apply submenu option.

5. The Fault Tree Recovery dialog will be displayed.

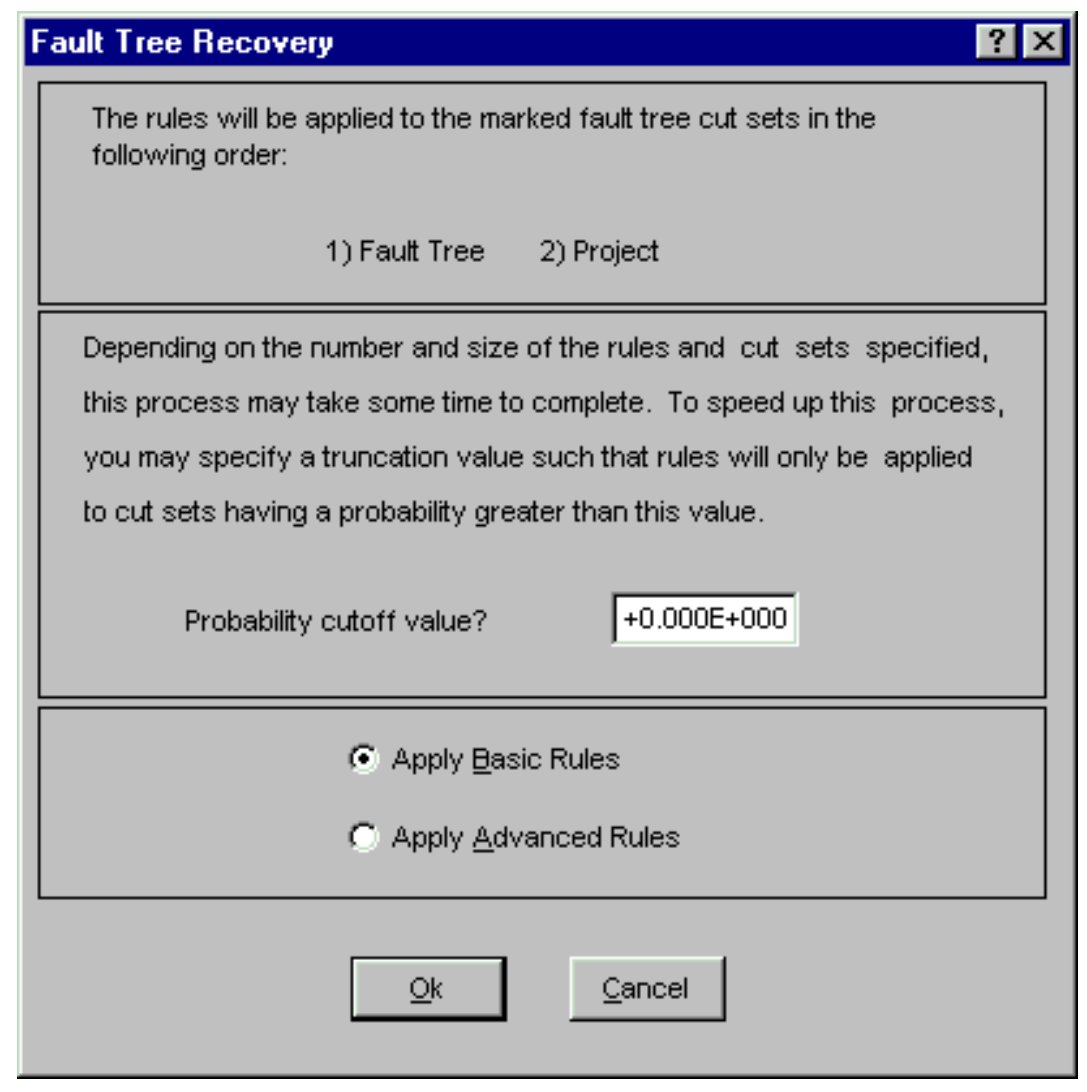

Apply Basic Rules -

Recover the selected fault trees using rules created using Basic Rules . 
Apply Advanced Rules -

Probability cutoff value -

Ok -

Cancel -
Recover the selected fault trees using rules created using Advanced Rules .

The rules are applied only to those cut sets having a probability greater than this value. A value of zero will force the rules to be applied to all cut sets.

Apply the selected rules using the specified cutoff value.

Close the Fault Tree Recovery dialog without applying rules. 


\subsubsection{Fault Tree Recovery Rule Options}

\section{PURPOSE}

The "recover" option allows the user to post-process cut sets by modifying them according to a predefined set of rules. The user constructs recovery rules to be applied to each cut set which then define the action to be taken. The rules are constructed using the same editor that is used for event tree rules definition. This editor is a macro-based editor, which means that the user can define macros (or variables) that can be used as the search criteria tests.

The rules may be developed for a particular fault tree or all fault trees, depending on the Rule Level selected.

\section{STEPS}

1. From the SAPHIRE menu select Fault Tree. The Fault Trees List dialog will be displayed.

2. Highlight the desired fault tree(s) and right-click to invoke the pop-up menu.

3. Choose Cut Sets | Recover | Edit Rules.

4. The Edit Recovery Rules dialog will be displayed.

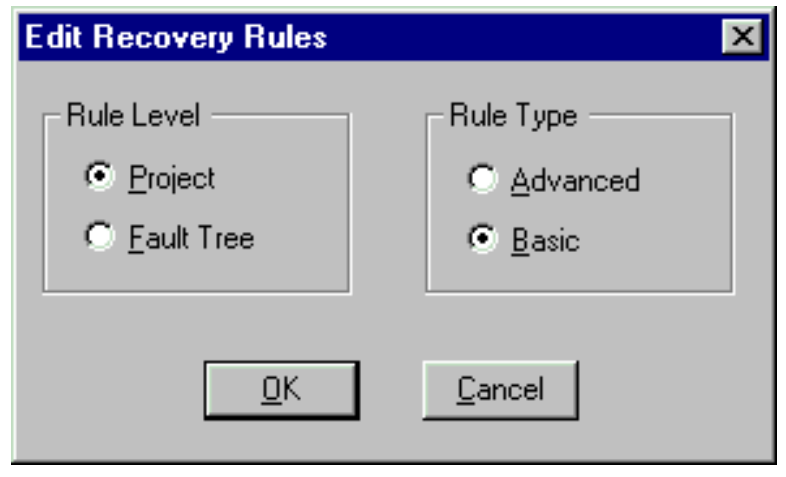

\section{Rule Level}

Project -

Fault Tree -
Edit rules whose scope you wish to apply to all or multiple fault trees across the project.

Edit rules whose scope you wish to apply only to the highlighted fault tree.

\section{Rule Type}

Advanced Basic -

OK -

Cancel -
Edit rules using the advanced recovery rules language.

Edit rules using the basic recovery rules language.

Create and modify fault tree recovery rules.

Close the Edit Recovery Rules dialog without editing any rules. 


\subsubsection{Basic Recovery Rules Editor}

\section{PURPOSE}

This is a free format line editor that provides you with an if-then-else logic structure. There is no limit to the number of rules the user may input to the editor. Each rule is processed in sequential order.

Basic rules can have different logic than the Advanced Rules and are saved separately.

\section{SEE}

Recovery Rules Editor Functions

\subsubsection{Advanced Recovery Rules Editor}

\section{PURPOSE}

This editor functions much the same as the Basic Rules Editor. However, the Modula-2 programming language is used to allow more robust programming capabilities. Using the Advanced Editor, users can define variables that can be assigned values based on specified criteria during run-time. Procedures can also be implemented which allows use of modular programming techniques.

When the advanced rules are compiled, a special dynamic link library (DLL) is created and saved in a special folder.

When this option is invoked the first time (before any advanced rules for the selected project/ fault tree exist), you will be given the option to convert a copy of the basic rules, if any, into advanced rule format. At that time, applying the basic or advanced rules should produce identical results. However, advanced rules are saved separately, and can be modified to have different logic than the Basic Rules.

\section{SEE}

Recovery Rules Editor Functions

Applying Recovery Rules

\subsubsection{Edit Rules}

\subsection{Recovery Rules Editor}

\section{PURPOSE}

This option allows you to create or modify rules that affect existing cut sets in a "post-processing" fashion. The rule-based editor is available for both fault tree and sequence cut sets. The Recovery Rules editor provides a means to develop logic rules that allow for probabilistic risk assessment techniques such as automated inclusion of recovery events, inclusion of common-cause failure cut sets, or elimination of mutually-exclusive events (e.g., restricted or impossible combinations of events).

The rules are entered in a free-form text editor within SAPHIRE. The same editor is used to create both basic and advanced recovery rules. The following discussions use the basic rule syntax, except where 
noted. Although the syntax/keywords differ between basic and advanced rules, the objectives and application of either kind of rule are the same.

Recovery rules follow a format similar to the structure that is found in traditional programming languages (e.g., BASIC or PASCAL). As such, the ability exists to define "macros" and "if...then" type of structures. After creating or editing rules, SAPHIRE compiles the rules to check their validity. Alternatively, rules can be exported from SAPHIRE or entered in any word processor or text editor (that can output ASCII files) and then loaded directly into the SAPHIRE database.

The recovery rules can be applied to cut sets for a particular sequence, a single event tree, or all sequences in the project. The rules can also be applied to cut sets for a particular fault tree or all fault trees in the project.

\section{STEPS}

1. From the SAPHIRE menu select Fault Tree/Sequence. The Fault Trees List/Sequences dialog will be displayed.

2. Highlight the desired fault tree(s)/sequence(s) and right-click to invoke the pop-up menu.

3. Choose Cut Sets | Recover | Edit Rules. The Edit Recovery Rules dialog will appear.

4. $\quad$ Select the desired Rule Level and Rule Type, and press OK.

If rules have been previously defined they will be displayed in the edit window.

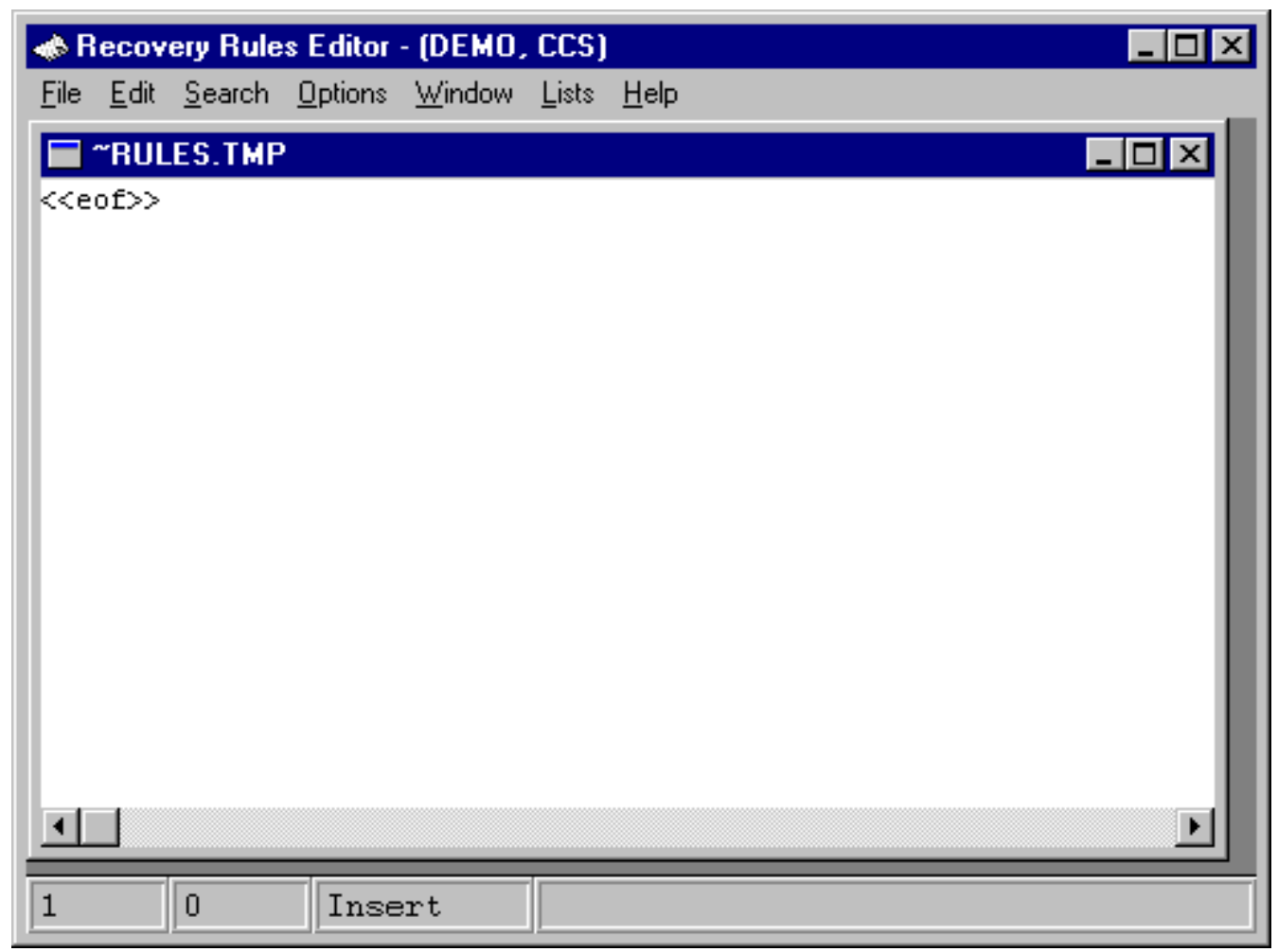

Type the rules in the editor. Note that rules are not case sensitive.

File - $\quad$ File functions such as open, save, print, preferences, etc. 
Edit -

Search -

Window -

Lists -
Editing functions such as copy and paste along with text format operations. Search and cursor positioning operations such as find, replace, goto, etc. Window management functions such as cascade, tile horizontally, etc. Display lists of initiators, fault trees, etc. for inserting into the rules.

\subsection{Editor File Functions}

These options provide file management functions.

\begin{tabular}{|c|c|}
\hline Open - & $\begin{array}{l}\text { Open a file into a new window. This feature is not usually used while editing } \\
\text { SAPHIRE rules. }\end{array}$ \\
\hline New - & $\begin{array}{l}\text { Create a new file in a new window. This feature is not usually used while editing } \\
\text { SAPHIRE rules. }\end{array}$ \\
\hline Close - & $\begin{array}{l}\text { Close the edit current window. If changes have been made you will be prompted } \\
\text { to save the file. }\end{array}$ \\
\hline Quit - & Close the current edit window without saving. \\
\hline ave - & Save the file in the current window to disk. \\
\hline Save As - & Save the file in the current window with a new file name. \\
\hline Save All - & Save all the files in all edit windows currently open. \\
\hline Save Block - & $\begin{array}{l}\text { Save the currently highlighted text into a new file. The Save As dialog will be } \\
\text { invoked. }\end{array}$ \\
\hline Import File - & $\begin{array}{l}\text { Insert the contents of a file into the current edit window at the current cursor } \\
\text { position. The Edit external file dialog will be invoked. }\end{array}$ \\
\hline Print - & Print the file in the current edit window. \\
\hline Page setup - & Set printer page layout options, .such as pages per sheet and line numbers. \\
\hline $\begin{array}{l}\text { Preferences - } \\
\text { Exit - }\end{array}$ & $\begin{array}{l}\text { Select editing options such as tab width, undo levels, etc. } \\
\text { Terminate the Editor session. }\end{array}$ \\
\hline
\end{tabular}

\subsection{Editor Edit Functions}

These options provide editing and text formatting functions.
Undo -
Undo the previous editing operation.
Cut -
Copy -
Paste -
Delete -
Shift left indent - Shift right indent - Shift left space - Shift right space - Delete to end of line -
Delete line - Capitalize word - Downcase word -
Remove the highlighted text from the current window and place it in the clipboard.
Copy the highlighted text from the current window and place it in the clipboard.
Copy the clipboard text into the current window at the cursor position. Remove the highlighted text from the current window without placing it in the clipboard.
Shift the text to the left by the Shift Size specified under Options. Shift the text to the right by the Shift Size specified under Options. Shift the text to the left by a single space.
Shift the text to the right by a single space.
Delete to the end of the current line beginning after the current cursor position.
Delete the line on which the cursor is currently positioned.
Convert the entire word under the current cursor position to upper case. Convert the entire word under the current cursor position to lower case.

\subsection{Editor Search Functions}


These options provide search and cursor positioning operations.

Find -

Replace -

Find Procedure Find/Replace again Find Altered lines Find line number Match symbol -

Goto next error Goto previous error Goto Marker -
Search the text in the current window for the specified string. Search the text in the current window for a string and replace it with another string.

Search the text in the current window for the specified procedure.

Repeat the previous search operation.

Find the lines of text that have changed since the last save operation.

Go to the specified text line number in the current window.

Find the matching symbol pair for the character under the cursor "(), \{\} , [], (**)"

Position the cursor at the next compilation error.

Position the cursor at the previous compilation error.

Position the cursor at the previously saved file position. Up to four marker positions can be retrieved.

Set Marker -

\subsection{Editor Window Functions}

These options are used for managing the open edit windows within the application window, such as the Linkage Rules Editor or the Event Tree Logic Editor window. Along with the options listed below and segregated by a separation bar, the names of each open file is listed in the menu.

Cascade windows -

Tile windows vertically -

Arrange Icons -
Resize and reposition the non-minimized windows in an overlapping fashion so that the title of each window is displayed in a cascade arrangement. The current edit window will be on top.

Resize and reposition the non-minimized windows in a horizontally tiled arrangement.

Resize and reposition the non-minimized windows in a vertically tiled arrangement.

Arrange the minimized window icons at the bottom of the application window.

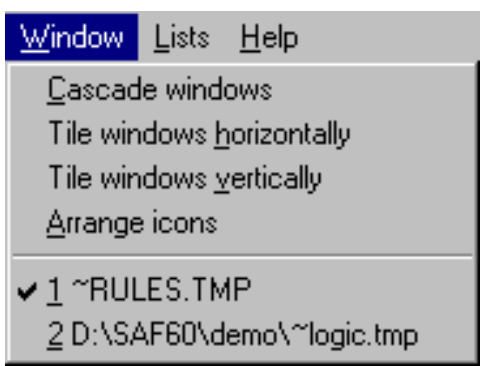

In the example above, the files " $\sim$ RULES.TMP" and " $\sim$ LOGIC.TMP" are open. $\sim$ RULES.TMP is the active window, as indicated by the check mark on the menu.

\subsection{Recovery Rules Lists}

Selecting from the lists allows you to insert or replace items in the editor. 
Macros -

Events -

Initiators -

Recovery Events -
Allows you to insert a macro into the editor.

Select basic events used to replace others instead of typing in the basic event names.

Select initiators used to replace others instead of typing in the initiator names.

Select recovery events used to replace others instead of typing in the event names.

\subsection{MACRO}

(Basic Rule syntax)

A macro is a user-definable keyword that specifies a search criteria. The macro name must be all uppercase, must be 24 characters or less, and must not include any of the restricted characters (e.g., a space, *, ?, \,/). The macro line can wrap around to more than one line, but must end with a semicolon.

MACRO-NAME = SEARCH-CRITERIA;

if MACRO-NAME “and optional other search criteria” then

perform some action on each cut set...;

endif

| Macros are only applicable in the particular rule they are entered into.

\subsubsection{Editing Fault Tree Cut Sets}

\subsubsection{Editing Cut Sets}

\section{PURPOSE}

The Cut Set Editor provides you with the means to edit the fault tree (or sequence or end state) cut sets. SAPHIRE provides space for two sets of cut sets in the database: base case and current case. Whenever SAPHIRE generates cut sets, they are stored in the current case location. The user may save a set of cut sets to the base case location by performing a Base Case Update. The user can choose to edit either base case or current cut sets; however, the results of the editing are always stored in the current case location.

Using the cut set editor, you can insert new cut sets, delete cut sets, or add or modify basic events in the cut sets. Any event name entered during cut set editing may be preceded by a "/" to indicate that it is to be treated as a complemented event. The probability of a complemented event is one minus the failure probability.

\section{STEPS}

1. From the SAPHIRE menu select Fault Tree/Sequence/End State. The Fault Trees List/Sequences/End State List dialog will be displayed. 
2. Highlight the desired record(s) and right-click to invoke the pop-up menu.

3. Choose Cut Sets, then the Edit sub-menu option.

4. Choose either Current or Base from the sub-menu.

5. The Cut Set Editor dialog will be displayed.

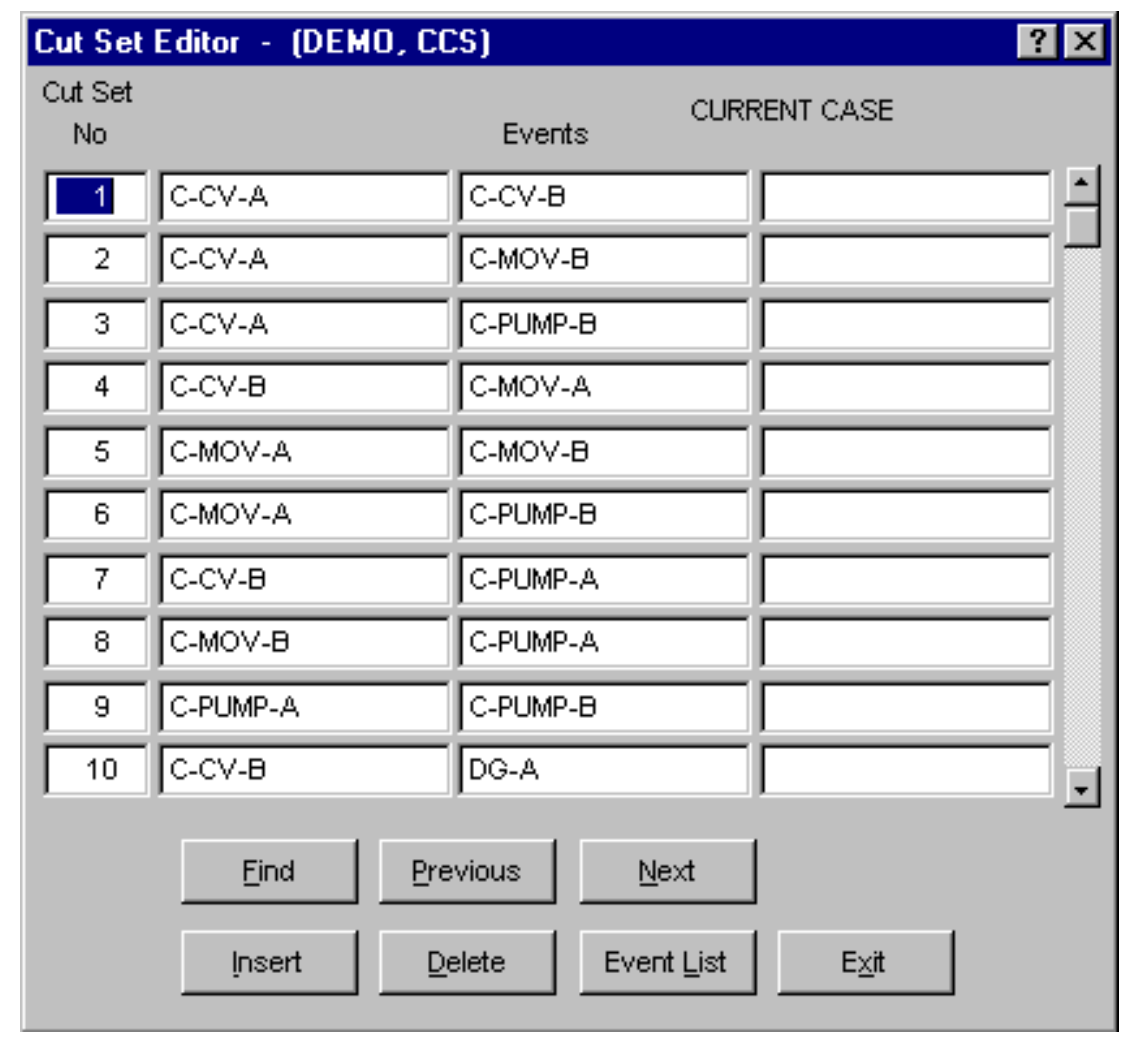

Find - $\quad$ Locate the cut set(s) containing the marked basic event(s).

Previous - $\quad$ Locate the previous cut set containing the event(s) matching the criteria of most recent "Find" operation.

Next - $\quad$ Locate the next cut set containing the event(s) matching the criteria of most recent "Find" operation.

Insert - $\quad$ Add a new cut set or basic event.

Delete - $\quad$ Delete an existing cut set or basic event.

Event List - $\quad$ List the basic events in the data base.

Exit - $\quad$ Close the Cut Set Editor dialog. The Cut Set Editor message box is displayed with three options:

Yes - $\quad$ Close the dialog and save changes to the cut sets.

No - Close the dialog without saving changes to the cut sets.

Cancel - Do not close the dialog. Changes are not saved.

\subsubsection{Find}

\section{PURPOSE}

This option locates the cut set(s) containing the selected criteria. 


\section{STEPS}

1. From the SAPHIRE menu select Fault Tree/Sequence/End State. The Fault Trees List/Sequences/End State List dialog will be displayed.

2. Highlight the desired record(s) and right-click to invoke the pop-up menu.

3. Choose Cut Sets, then the Edit sub-menu option.

4. Choose either Current or Base from the sub-menu.

5. The Cut Set Editor dialog will be displayed.

6. Choose the Find button.

7. The Event List dialog will be displayed.

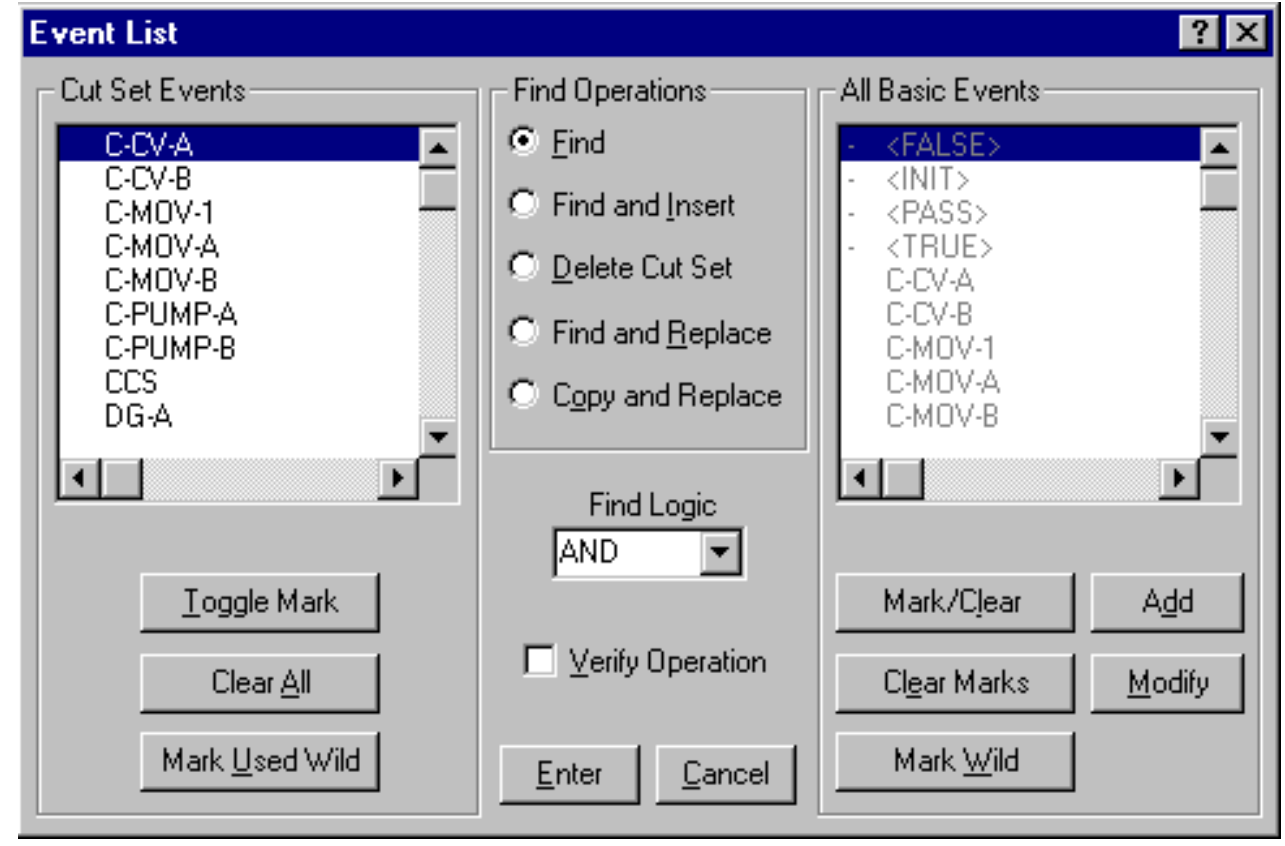

The Event List dialog consists of four sections. The functions that apply to each section are discussed briefly:

\section{Cut Set Events}

Basic Event List - $\quad$ Contains all basic events used in the cut sets being edited.

Toggle Mark - $\quad$ Marks or unmarks selected events in the Cut Set Events list. An asterisk

$\left(^{*}\right)$ next to the event name indicates that it is currently marked. A slash

(/) indicates that the event is marked as a complimented event. 
Clear All -

Mark Used Wild -

Find Operations

Find -

Find and Insert -

Delete Cut Set -

Find and Replace -

Copy and Replace -
Removes all marks from the events in the Cut Set Events list.

Marks events in the Cut Set Events list based on matching criteria. The Event Class Mask dialog is displayed.

Locate the cut set(s) containing the marked event(s) in the Cut Set Events list using the selected Find Logic.

Locate the cut sets(s) containing the marked event(s) in the Cut Set Events list using the selected Find Logic and insert the marked event(s) from the All Basic Events list. The new events are inserted into the cut set after the "find" event(s).

Remove the cut set(s) containing the marked event(s) in the Cut Set Events list using the selected Find Logic.

Locate the cut sets(s) containing the marked event(s) in the Cut Set Events list using the selected Find Logic and replace them with the marked event(s) from the All Basic Events list.

Copy the cut sets(s) containing the marked event(s) in the Cut Set Events list using the selected Find Logic and replace the event(s) with the marked event(s) from the All Basic Events list. This creates a new cut set for each cut sets matching the "find" criteria.

All Basic Events - This section is enabled for use with the Find and Insert, Find and Replace, and Copy and Replace functions:

Basic Event List - $\quad$ Contains all basic events in the database.

Mark/Clear -

Marks or unmarks selected events in the All Basic Events list.

Clear Marks -

Remove all marks from the events in the All Basic Events list.

Mark Wild -

Marks events in the All Basic Events list based on matching criteria. The Event Class Mask dialog is displayed.

Add -

Add a new basic event to the database. This new event will appear in the All Basic Events list.

Modify - $\quad$ Modify the selected event in the All Basic Events list.

\section{Other options}

Find Logic drop-down list -
Allows selection between the logical AND and OR operations. The selected logical operation is applied to the events in the Cut Set Events. 


\section{Verify Option -}

Enter -

Cancel -
If this check box is selected, you are prompted to confirm the operation for each successful find. If this check box is deselected, the operation will be performed immediately.

Perform the "find" operation using the currently selected criteria.

Do not perform the "find" operation and close the Event List dialog.

\subsubsection{Insert}

\section{PURPOSE}

This option allows you to insert a new cut set or basic event. If the I-bar is in a Cut Set No cell (i.e., the first column of cells on the Cut Set Editor dialog), then a row for a cut set will be added at the end of the cut set list. If the I-bar is in one of the Events cells, and the last event row for the current cut set (i.e., all three cells in the current cut set row) is filled, then a row for the current cut set will be added.

\section{STEPS}

1. From the SAPHIRE menu select Fault Tree/Sequence/End State. The Fault Trees List/Sequences/End State List dialog will be displayed.

2. Highlight the desired record(s) and right-click to invoke the pop-up menu.

3. Choose Cut Sets, then the Edit sub-menu option.

4. Choose either Current or Base from the sub-menu.

5. The Cut Set Editor dialog will be displayed.

6. Place the cursor in the desired cell and choose the Insert button.

\subsubsection{Delete}

\section{PURPOSE}

This option allows you to delete an existing cut set or basic event. If the I-bar is in a Cut Set No cell (i.e., the first column of cells on the Cut Set Editor dialog), then the entire cut set will be deleted. If the I-bar is in one of the Events cells, then the event in that cell will be removed from the cut set.

\section{STEPS}

1. From the SAPHIRE menu select Fault Tree/Sequence/End State. The Fault Trees List/Sequences/End State List dialog will be displayed.

2. Highlight the desired record(s) and right-click to invoke the pop-up menu.

3. Choose Cut Sets, then the Edit sub-menu option.

4. Choose either Current or Base from the sub-menu.

5. The Cut Set Editor dialog will be displayed. 
6. Place the cursor in the desired cell and choose the Delete button.

If you are deleting a cut set, you will be prompted:

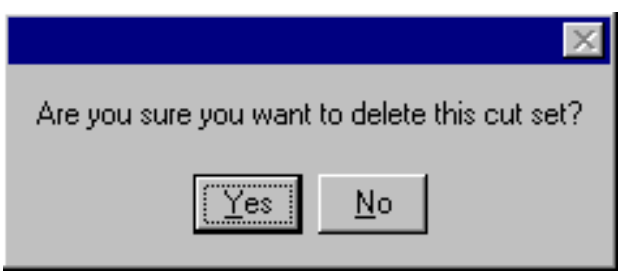

Yes - Continue the delete operation.

No - Cancel the delete operation.

\subsubsection{Event List}

\section{PURPOSE}

This option lists all the basic events in the database. From this dialog you can add a new event to the database, modify an existing event's data, or select the event to be included in the current cut set. The cursor must be positioned in one of the Events cells in order to activate this option. The selected event will replace an existing event in the current cell.

\section{STEPS}

1. From the SAPHIRE menu select Fault Tree/Sequence/End State. The Fault Trees List/Sequences/End State List dialog will be displayed.

2. Highlight the desired record(s) and right-click to invoke the pop-up menu.

3. Choose Cut Sets, then the Edit sub-menu option.

4. Choose either Current or Base from the sub-menu.

5. The Cut Set Editor dialog will be displayed.

6. Place the cursor in the desired Event cell.

7. Choose the Event List button. The Event List dialog will be displayed. 


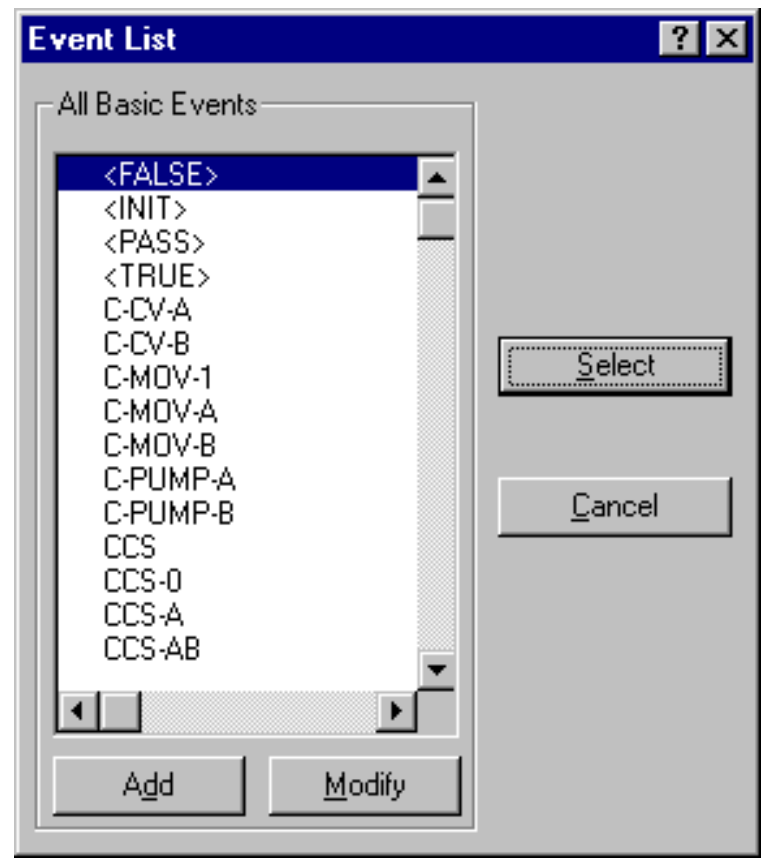

Add - $\quad$ Add basic event to the data base. The Basic Event data entry dialog will be displayed.

Modify - Modify the data of the highlighted basic event. The Basic Event data entry dialog will be displayed.

Select - Include the highlighted basic event in the cut set. The selected event will be placed in the cell currently occupied by the cursor.

Cancel - Close the Event List dialog and do not change the current cell.

\subsection{Displaying Fault Tree Results}

\subsubsection{Displaying Fault Tree Results}

\section{PURPOSE}

This option displays the results of fault tree analysis functions. You can display the case cut sets and uncertainty analysis results, and perform importance measure analyses.

\section{STEPS}

1. From the SAPHIRE menu select Fault Tree. The Fault Trees List dialog will be displayed.

2. Highlight the desired fault tree and right-click to invoke the pop-up menu.

3. Choose Display, then the desired sub-menu option. 


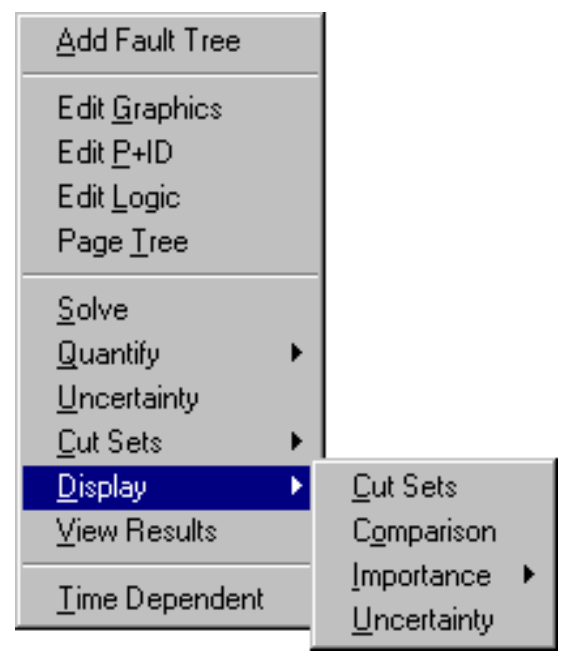

Cut Sets - $\quad$ Display the fault tree's cut sets, their percent of contribution to the fault tree, the frequency, and the event names that make up the cut sets.

Comparison - Display the fault tree's base and current cut sets, their percent of contribution to the fault tree, the frequency, and the event names that make up the cut sets.

Importance - Perform and display "reliability-worth" information about the basic events in the cut sets.

Uncertainty - Display uncertainty analysis results.

\subsubsection{Displaying Cut Sets}

\subsubsection{Displaying Cut Sets}

\section{PURPOSE}

This option displays the current case cut sets. Three tabbed pages provide different views of the cut sets. The first tab, labeled Full List, contains the complete list of cut sets for the selected fault tree (or sequences or end states). This page always contains the complete set of cut sets found for the selected fault tree/sequences/end state.

When the dialog is invoked, the fault tree (or sequence or end state) cut sets are shown. Displayed with each cut set is its associated percent of contribution to the minimal cut set upper bound, its probability (or, perhaps, frequency), and the event names that make up the cut sets. Also displayed are the minimal cut set upper bound for the total, the number of cut sets that make up the total, the current slice minimal cut set upper bound, the percentage that the slice contributes to the total, and the number of cut sets in the slice.

The Included In List and Excluded From List tabs will not contain data until a slice has been defined, via one of the Slice By options.

The second tab, labeled Included In Slice, contains those cut sets specified (qualified) by the user via either the Remove and/or Slice By options. The third tab, labeled Excluded From Slice, contains the inverse of the second tab; it contains those cut sets not included in the qualified cut set list. The summary information at the top of each page shows the summary information for just the cut sets shown on the current page, and also in relation to the full list of cut sets. 


\section{STEPS}

1. From the SAPHIRE menu select Fault Tree/Sequence/End State. The Fault Trees List/Sequences/End State List dialog will be displayed.

2. Highlight the desired list item(s) and right-click to invoke the pop-up menu.

3. Choose Display, then the Cut Sets sub-menu option.

4. The Selected Cut Sets dialog will be displayed.

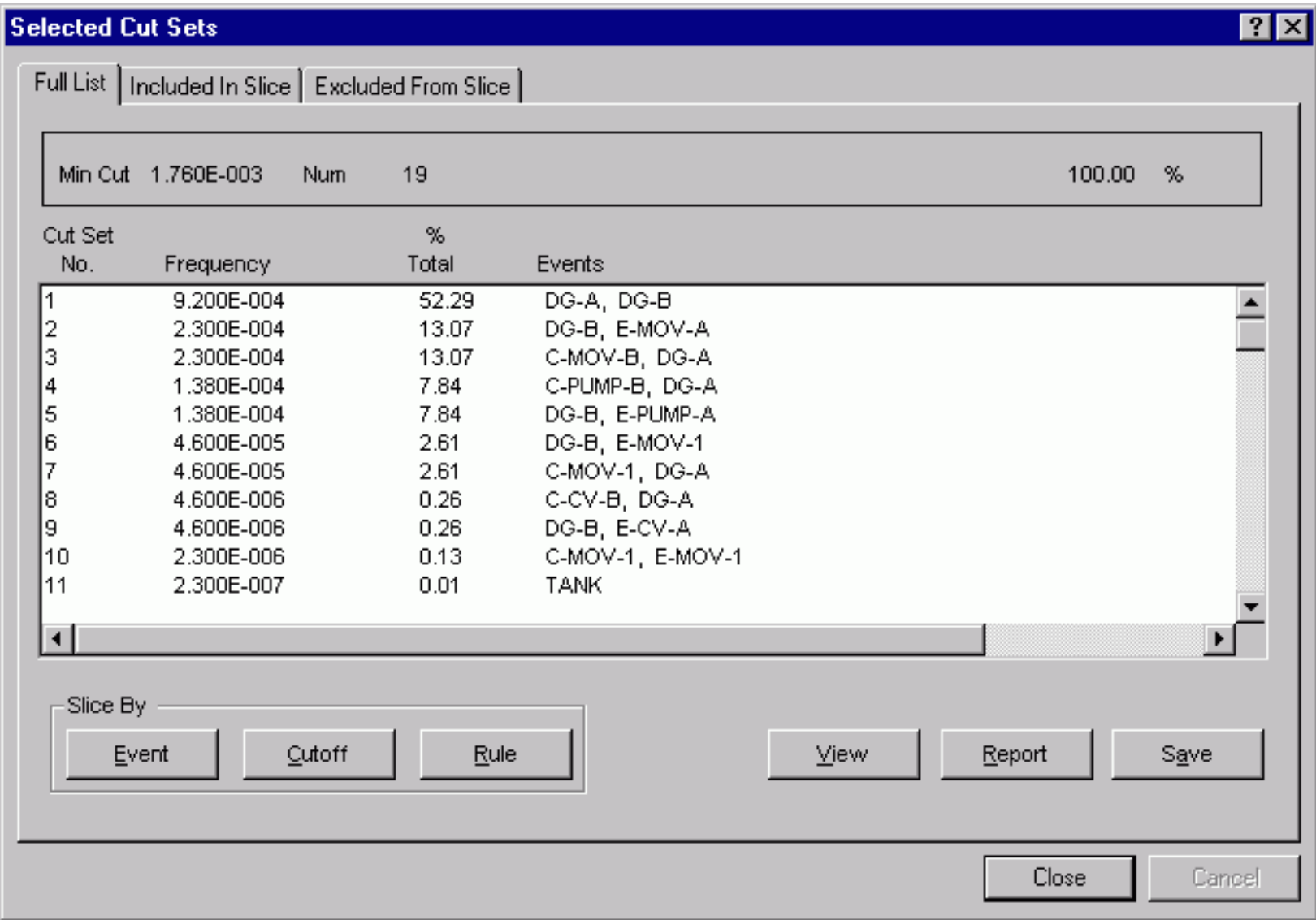

Tabbed pages

Full List -

Included in Slice -

Display the complete (unsliced) list of cut sets.

Excluded From Slice - Display all currently unqualified cut sets.

Pop-up menu (only) functions

Path -

Remove -

Reset Explicit -
Display the logic that generated the highlighted cut set.

Remove the highlighted cut set(s) from the current list, and place them into the Excluded From Slice list.

Restore the cut set(s) that were deleted, via the Remove option, to the current list. 

Slice By
Event -
Define the event criteria upon which cut sets will be qualified.
Cutoff -
Define the cut off criteria upon which cut sets will be qualified.
Rule -
Define the rule-based criteria upon which cut sets will be qualified.
View -
Display information about the selected cut set including basic event data.
Report - Generate a report of the cut sets that are displayed in the currently selected tab.
Save -
Copy the cut sets from the currently selected tab to an end state.
Close - Close the Selected Cut Sets dialog.

\subsubsection{Path Search}

\section{PURPOSE}

This option displays the representation of the logic that generated the selected cut set. The representation is in the form of a hierarchical tree, where each gate can be expanded or condensed by clicking on the gate. Only the failure path is traced here.

\section{STEPS}

1. From the SAPHIRE menu select Fault Tree/Sequence/End State. The Fault Tree List/Sequences/End State List dialog will be displayed.

2. Highlight the desired list item and right-click to invoke the pop-up menu.

3. Choose Display | Cut Sets. The Selected Cut Sets dialog will be displayed.

4. Highlight the desired cut set and right-click to invoke the pop-up menu.

5. Choose the Path option.

6. The Selected Cut Set Events dialog will be displayed.

See the topic, "About Logic Dialogs ," for a description of the icons presented on this dialog. 


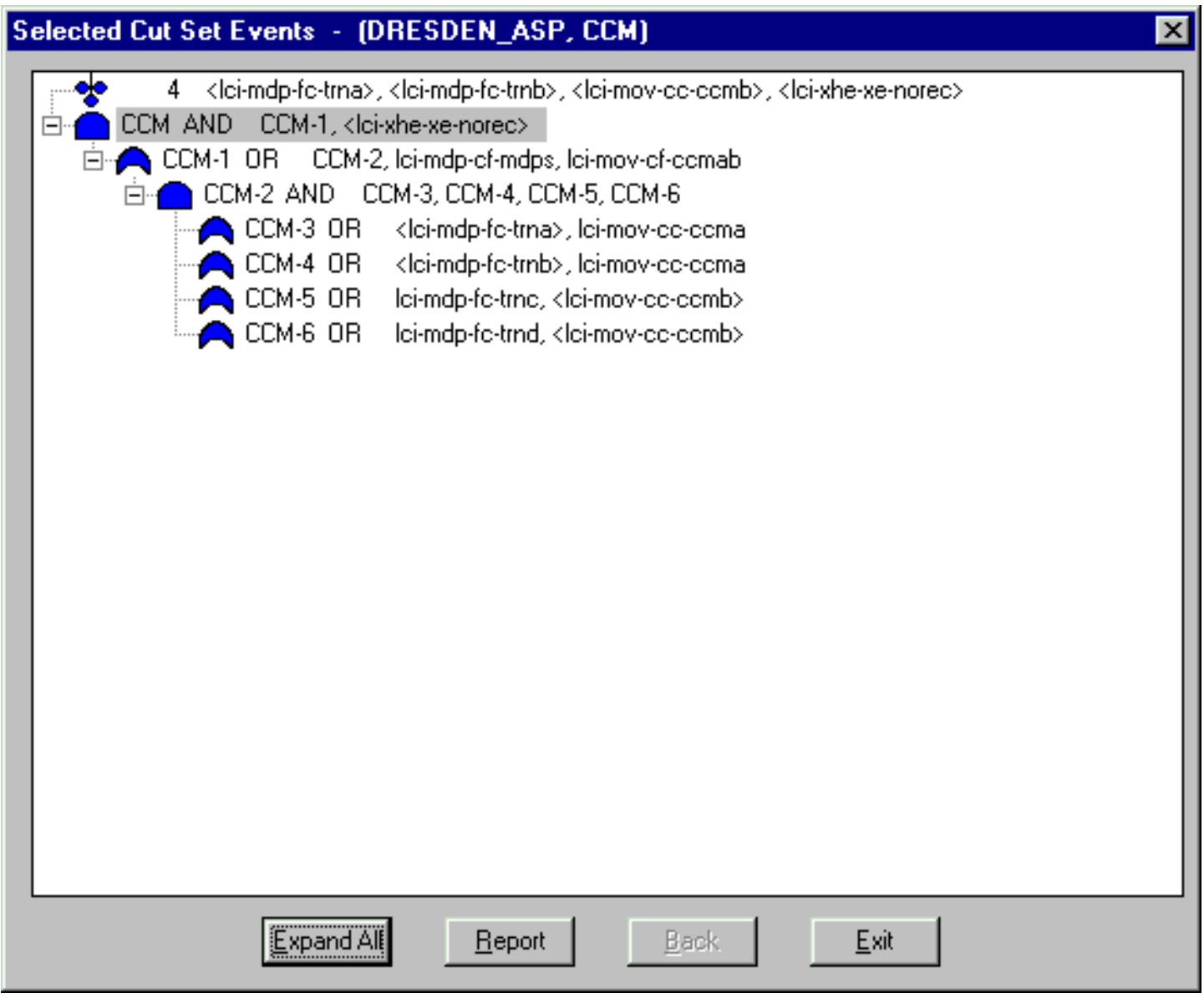

Expand All - Expand all gates.

Report - $\quad$ Generate a report representing the expanded hierarchical tree. The output destination for the report must be specified.

Back - $\quad$ Return to the referenced gate. Active only after a referenced gate has been traced to the location where it was originally defined (i.e., the reference gate icon has been clicked).

Exit - $\quad$ Close the Selected Cut Set Events dialog.

\subsubsection{About Logic Dialogs}

The gate names and gate types are displayed in uppercase characters. The event names are displayed in lowercase characters. The cut set events, indicated only in the Path Search option on the Selected Cut Set Events dialog, are enclosed in brackets $(<>)$.

Presentation of the logic in dialogs and reports generated by SAPHIRE is limited to 255 characters in width. If the representation of the logic requires expansion past this limit, it will be truncated for display and reporting. The actual logic will remain in tact.

The following graphics are used for easy identification of gate types and their status:

$\square-\quad$ An expanded gate. Click on the graphic to condense the gate. 


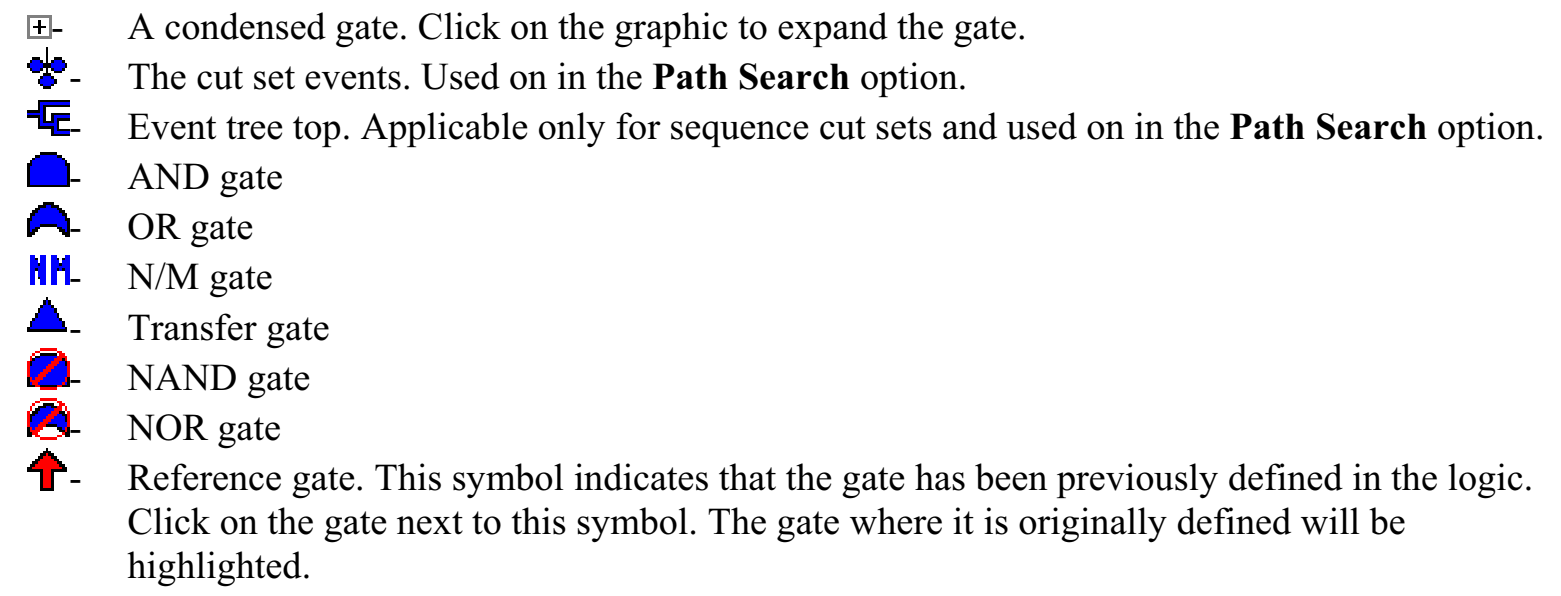

\subsubsection{Save}

\section{PURPOSE}

Save the results of the existing cut set slice (currently displayed on the Selected Cut Sets dialog) to an end state.

\section{STEPS}

1. From the SAPHIRE menu select Fault Tree/Sequence/End State. The Fault Tree List/Sequences/End State List dialog will be displayed.

2. Highlight the desired list item and right-click to invoke the pop-up menu.

3. Choose Display | Cut Sets. The Selected Cut Sets dialog will be displayed.

4. Slice, or qualify, the cut sets as desired. Select the tab containing the cut set list you want to save.

5. Choose the Save button. The Save To An End State dialog will be displayed.

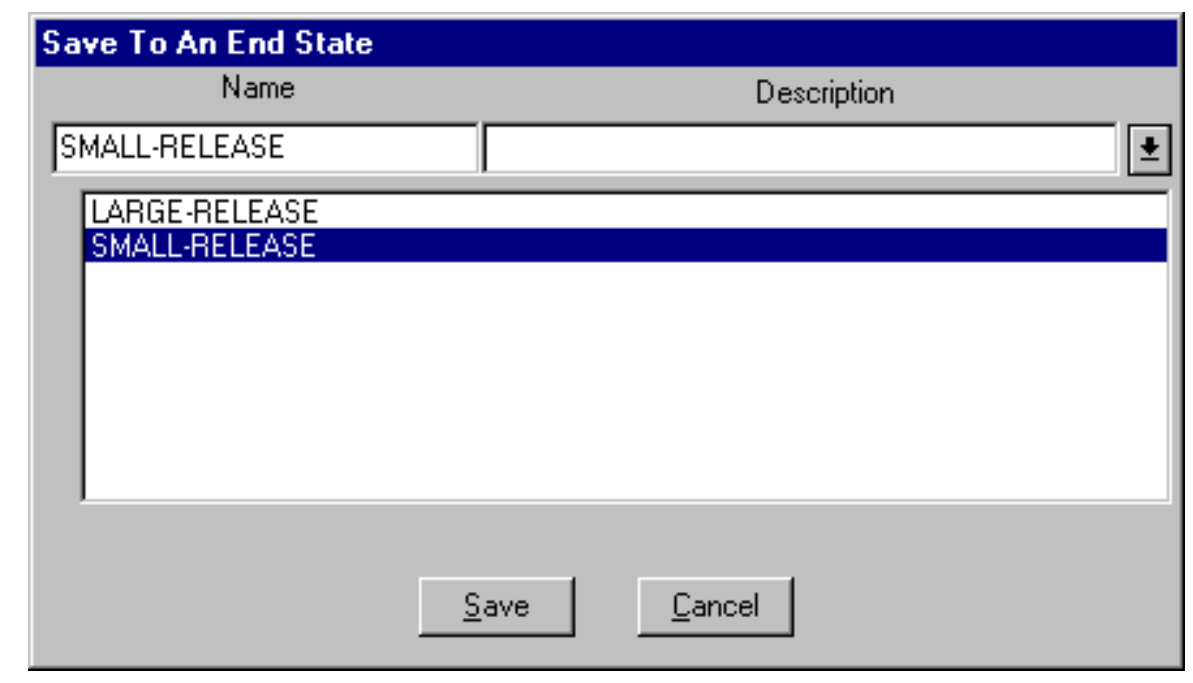

In the example here, the down arrow to the right of the Description field was selected to display the list of existing end states. 
Name - $\quad$ Enter the name of the new end state. Up to 24 uppercase, alphanumeric characters are allowed.

Description - Enter the description of the new end state. Up to 60 upper- and lowercase, alphanumeric characters area allowed

Save - $\quad$ Perform the save operation. A new end state record will be saved in the SAPHIRE data base with the name and description as entered here, and current slice for its cut sets. Selecting an existing end state will cause the existing cut sets for that end state to be overwritten.

Cancel - $\quad$ Close the Save To An End State dialog without saving the cut sets or creating a new end state record.

\subsubsection{Slice Options}

\subsection{Slice By Cutoff}

\section{PURPOSE}

This option provides the ability to divide the original cut set list into two subsets (slices) - qualified and unqualified cut sets. This is accomplished by defining a cut off value to determine whether or not a cut set is qualified. This qualification criteria can be used to help the analyst determine the affect of specified cut sets on the minimal cut set upper bound.

\section{STEPS}

1. From the SAPHIRE menu select Fault Tree/Sequence/End State. The Fault Tree List/Sequences/End State List dialog will be displayed.

2. Highlight the desired list item and right-click to invoke the pop-up menu.

3. Choose Display | Cut Sets. The Selected Cut Sets dialog will be displayed.

4. Choose the Slice By Cutoff button.

5. The Slice By Value dialog will be displayed.

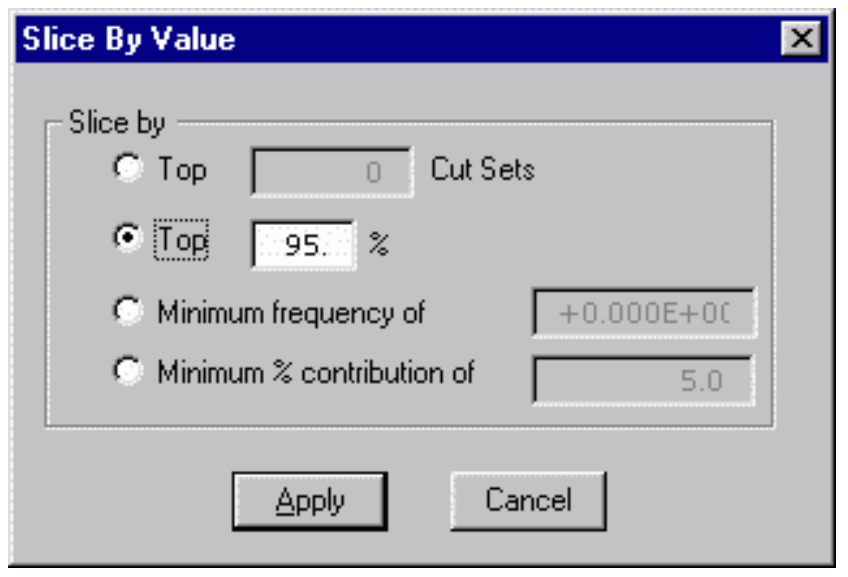

Select one of four types of cutoff value expressions: 
Top X\% -

Minimum frequency of $\mathbf{X}-$
Display only the cut sets which contribute to the top $\mathrm{X} \%$ of the min cut upper bound.

Display only the cut sets which contribute at least a value of $\mathrm{X}$ to the min cut upper bound.

Minimum \% contribution of $\mathbf{X}$ - Display only the cut sets which contribute at least a $\mathrm{X} \%$ to the min cut upper bound.

Apply - Cut sets in the current slice will be evaluated. Those cut sets meeting the qualification criteria will be retained in the current slice of qualified cut sets. Other cut sets not meeting the qualification criteria will be placed in the unqualified list.

Cancel - Close the Slice By Value dialog without applying the qualification criteria.

\subsection{Slice By Event}

\subsection{Slice By Event}

\section{PURPOSE}

This option provides the ability to divide the original cut set list into two subsets (slices) - qualified and unqualified cut sets. This is accomplished by defining a set of events used to evaluate whether or not a cut set is qualified. This qualification criteria can be used to help the analyst determine the affect of specified events on the minimal cut set upper bound.

\section{STEPS}

1. From the SAPHIRE menu select Fault Tree/Sequence/End State. The Fault Tree List/Sequences/End State List dialog will be displayed.

2. Highlight the desired list item and right-click to invoke the pop-up menu.

3. Choose Display | Cut Sets. The Selected Cut Sets dialog will be displayed.

4. Choose the Slice By Event button.

5. The Event List dialog will be displayed. 


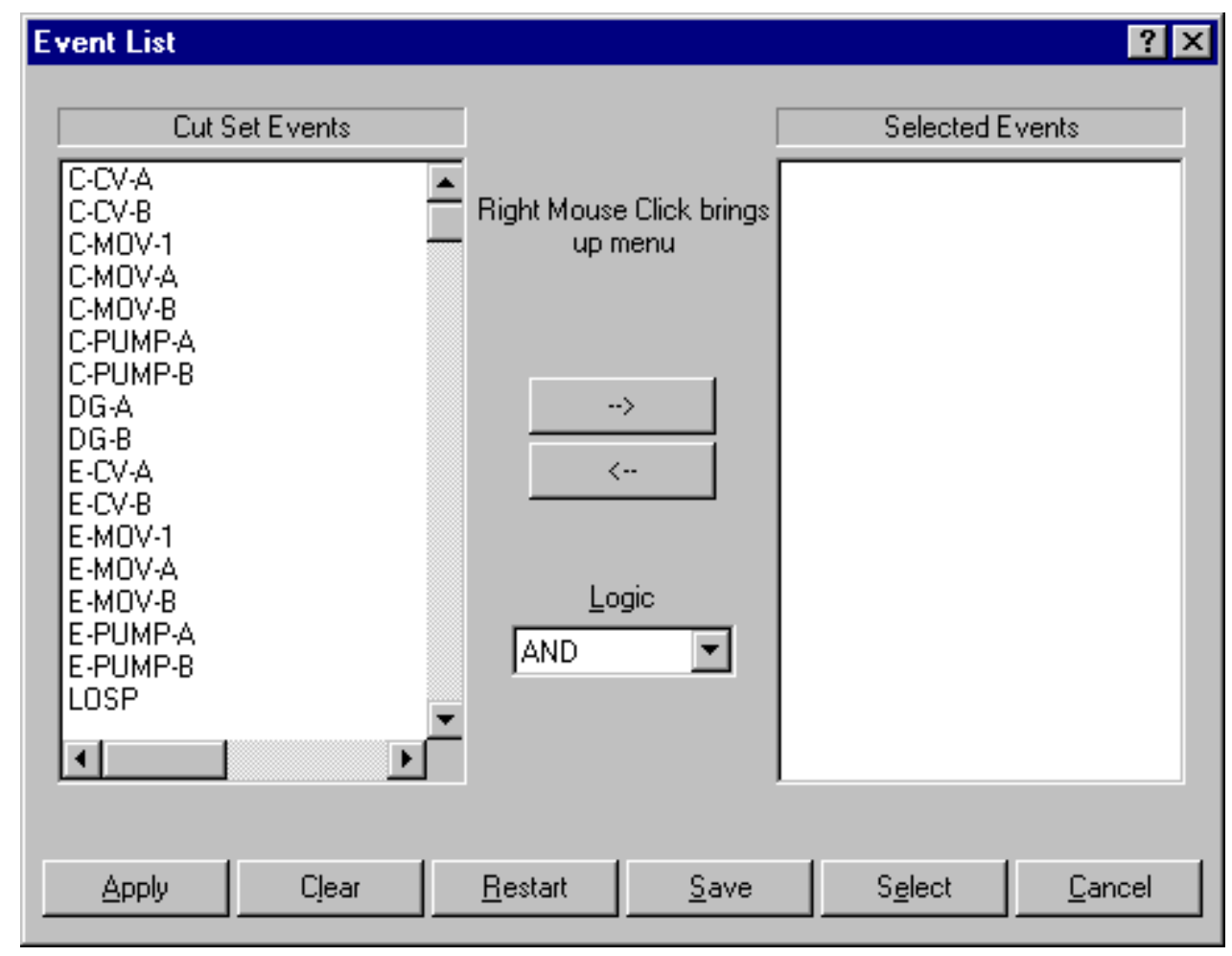

The Event List dialog is divided into three sections:

Cut Set Events list - Contains all the basic events included in the cut set(s) for the currently selected fault tree, sequence(s) or end state(s).

Selected Events list - Contains all basic events that define the qualification criteria. This list is initially empty.

Logic drop-down list - Allows selection between the logical "AND" and "OR" operations. The selected logical operation is applied to the events in the Selected Events list to determine which cut sets are qualified.

The various functions available from this dialog are accessed three different ways. When an event in the Cut Set Events list is highlighted, the following options are available from the pop-up menu. The highlighted event(s) will be added to the Selected Events list.

Wild Card Mark -

Add Event -

Add Failure Event Add Success Event Add NOT Event In -

Apply Selected -
Use the event class mask to highlight events in the Cut Set Events list.

Cut sets containing this event, in any state (either failed or success), will be qualified.

Cut sets containing the failure $(+)$ of this event will be qualified. Cut sets containing the success (/) of this event will be qualified. Cut sets that do not contain $(\sim)$ this event, in any state (either failed or success), will be qualified.

Evaluate the qualification criteria. This is the same process as if the Apply button was chosen. 
When an event in the Selected Events list is highlighted, the following options are available from the pop-up menu. The qualification criteria state will be changed for the highlighted event(s):
Remove Event -
Delete this event from the Selected Events list. This event will no
Event (either / or + ) - longer be used in the qualification criteria.
Failed Event - The state of
is irrelevant.
Success Event - Change the state of the highlighted event to failed $(+)$.
Event NOT In - Change the state of the highlighted event to successful (/). The highlighted event are is not contained $(\sim)$ in qualified cut sets.
Apply Selected -
Evaluate the qualification criteria. This is the same process as if the Apply button was chosen.

The following functions are performed by choosing the appropriate button:
Apply -
Cut sets in the current slice will be evaluated. Those cut sets meeting the qualification criteria will be retained in the current slice of qualified cut sets. Other cut sets not meeting the qualification criteria will be placed in the unqualified list.
Clear -
Remove all events from the Selected Events list (i.e., clear the qualification criteria).
Restart -
Restore the original cut sets and clear the qualification criteria. The Event List dialog will be closed and the Included In List and Excluded From List pages will be cleared.
Save -
Save the selected events in a stored slice record.
Select -
Choose a saved slice record and place the events in the Selected Events list.
Cancel -
Close the Event List dialog without applying the qualification criteria.

\subsection{Wild Card Mark}

\section{PURPOSE}

This option uses the event class mask to highlight events in the Cut Set Events list. This selection is used with either the Cut Set Editor or the Cut Set | Slice option.

\section{STEPS}

1. From the SAPHIRE menu select Fault Tree/Sequence/End State. The Fault Tree List/Sequences/End State List dialog will be displayed.

2. Highlight the desired list item and right-click to invoke the pop-up menu. 
To access from the Slice\#1

1. Choose Display | Cut Sets. The Selected Cut Sets dialog will be displayed.

2. Choose the Slice button. The Event List dialog will be displayed.

3. Right-click to invoke the pop-up menu and choose the Wild Card Mark option.

To access from the Cut Set Editor\#1

1. Choose Cut Sets | Edit | Current or Base. The Cut Set Editor dialog will be displayed.

2. Choose the Find button. The Event List dialog will be displayed.

3. Choose the Mark Used Wild button. The Event Class Mask dialog will be displayed.

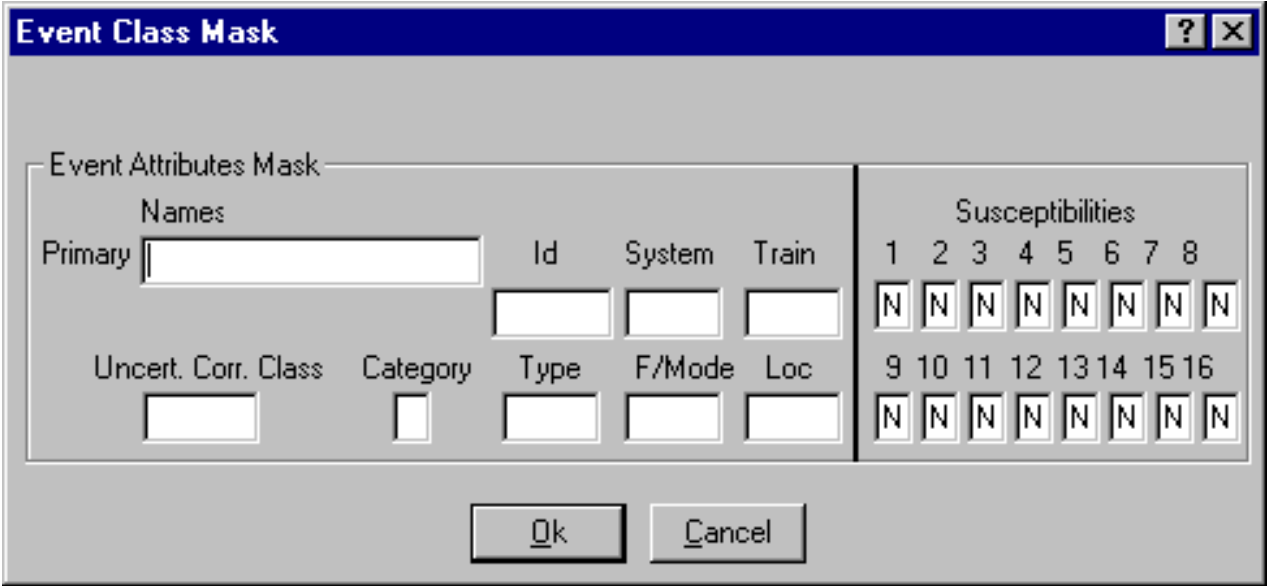

Primary -

Id -

System -

Train -

Uncert. Corr. Class -

Category -

Type -

F/Mode -

Location -

Susceptibilities -
Select all basic events with matching primary names (maximum 24 characters). Wildcard characters may be used.

Select all basic events with matching component identifiers.

Select all basic event with matching systems.

Select all basic event with matching trains

Select all basic event with matching uncertainty correlation classes.

Select all basic event with matching categories.

Select all basic event with matching types.

Select all basic event with matching failure modes.

Select all basic event with matching locations.

Select all basic event with matching susceptibility attributes set.

\subsection{Save Slice}

\section{PURPOSE}

This option allows you to save the basic events currently displayed in the Selected Events list in a stored slice record or a set. 


\section{STEPS}

1. From the SAPHIRE menu select Fault Tree/Sequence/End State. The Fault Tree

List/Sequences/End State List dialog will be displayed.

2. Highlight the desired list item and right-click to invoke the pop-up menu.

3. Choose Display | Cut Sets. The Selected Cut Sets dialog will be displayed.

4. Choose the Slice button. The Event List dialog will be displayed.

5. Select the desired events to be saved in the slice.

6. Choose the Save button. The Save Slice dialog will be displayed.

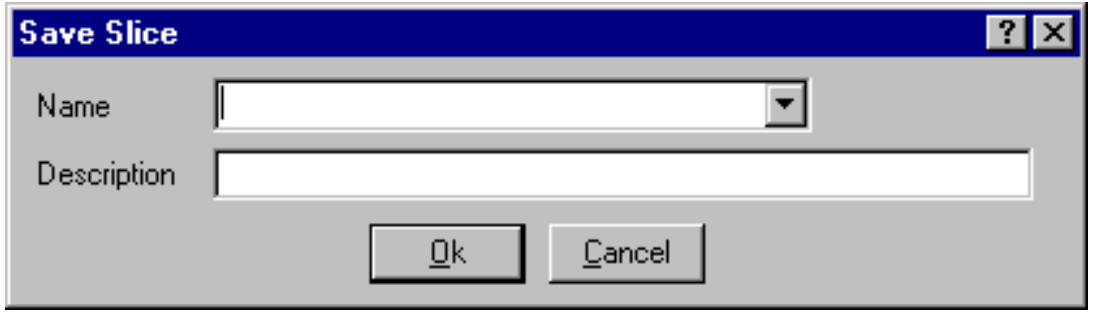

Ok - $\quad$ Save the new slice record and close the dialog.

Cancel - Close the dialog without saving.

\subsection{Slice Name}

This is a required field. A maximum of 24 uppercase, alphanumeric characters may be entered, or an existing slice may be overwritten by selecting it from the drop-down list. When entering a new name, embedded blanks are not allowed. The name should be descriptive so the slice can be readily identified.

\subsection{Select Slice}

\section{PURPOSE}

Choose a saved slice record and place the events in the Selected Events list. When a slice record is loaded, the basic events saved in the slice replace all basic events currently displayed in the Selected Events list. If a basic event that was saved in a slice does not appear in the Cut Set Events list, it will not be displayed in the Selected Events list, although it will remain in the slice record.

\section{STEPS}

1. From the SAPHIRE menu select Fault Tree/Sequence/End State. The Fault Tree List/Sequences/End State List dialog will be displayed.

2. Highlight the desired list item and right-click to invoke the pop-up menu.

3. Choose Display | Cut Sets. The Selected Cut Sets dialog will be displayed.

4. Choose the Slice button. The Event List dialog will be displayed.

5. Choose the Select button. The Select Slice dialog will be displayed. 


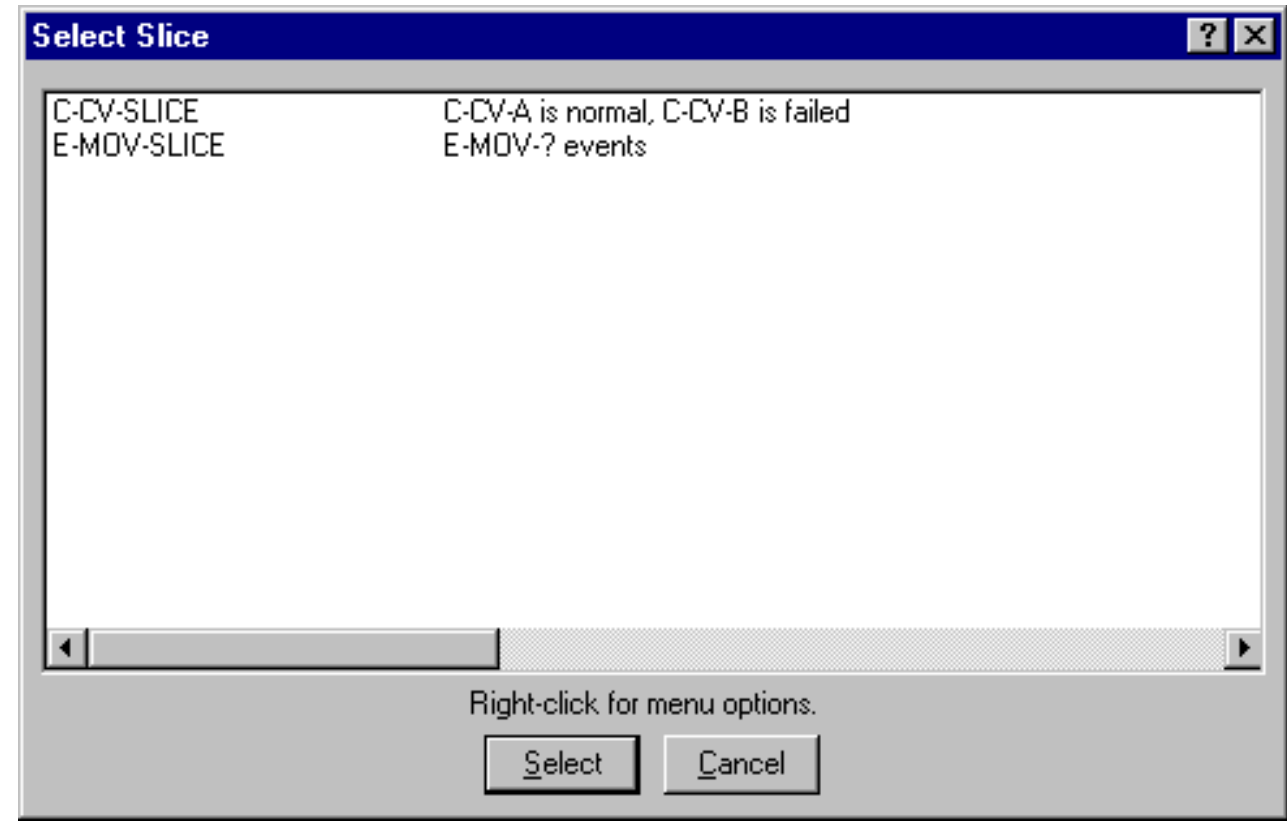

\section{Pop-up Menu Options}

Modify - Change the slice record name and/or description. See Save Slice .

Delete - Delete the slice record from the database.

Exit - $\quad$ Close the Select Slice dialog.

\section{Button Options}

Select - Choose the highlighted slice record and load the saved events.

Cancel - Close the Select Slice dialog.

\subsection{Slice By Rule}

\subsection{Slice By Rule}

\section{PURPOSE}

This option provides the ability to divide the original cut set list into two subsets (slices) - qualified and unqualified cut sets. This is accomplished by defining one or more rules to evaluate whether or not a cut set is qualified. The rules provide the ability to qualify cut sets using logical expressions, including mixed logic (combining "ands" and "ors") if desired. The rules are saved in the project for repeated use.

When the dialog is invoked, a list of slice rules is displayed. The highlighted rule is previewed in the bottom text area. To add/modify/delete a slice rule name and description, right click and select the appropriate menu option. To edit a rule, choose the Edit Rule button. To apply a rule to the current cut sets, choose the Apply button.

To clear the qualified and unqualified lists, choose the Reset option from the Slice By Event option, or, apply a different slice option to override the current slice.

\section{STEPS}

1. From the SAPHIRE menu select Fault Tree/Sequence/End State. The Fault Tree List/Sequences/End State List dialog will be displayed. 
2. Highlight the desired list item and right-click to invoke the pop-up menu.

3. Choose Display | Cut Sets. The Selected Cut Sets dialog will be displayed.

4. Choose the Slice By Rule button. The Slice Rules dialog will be displayed.

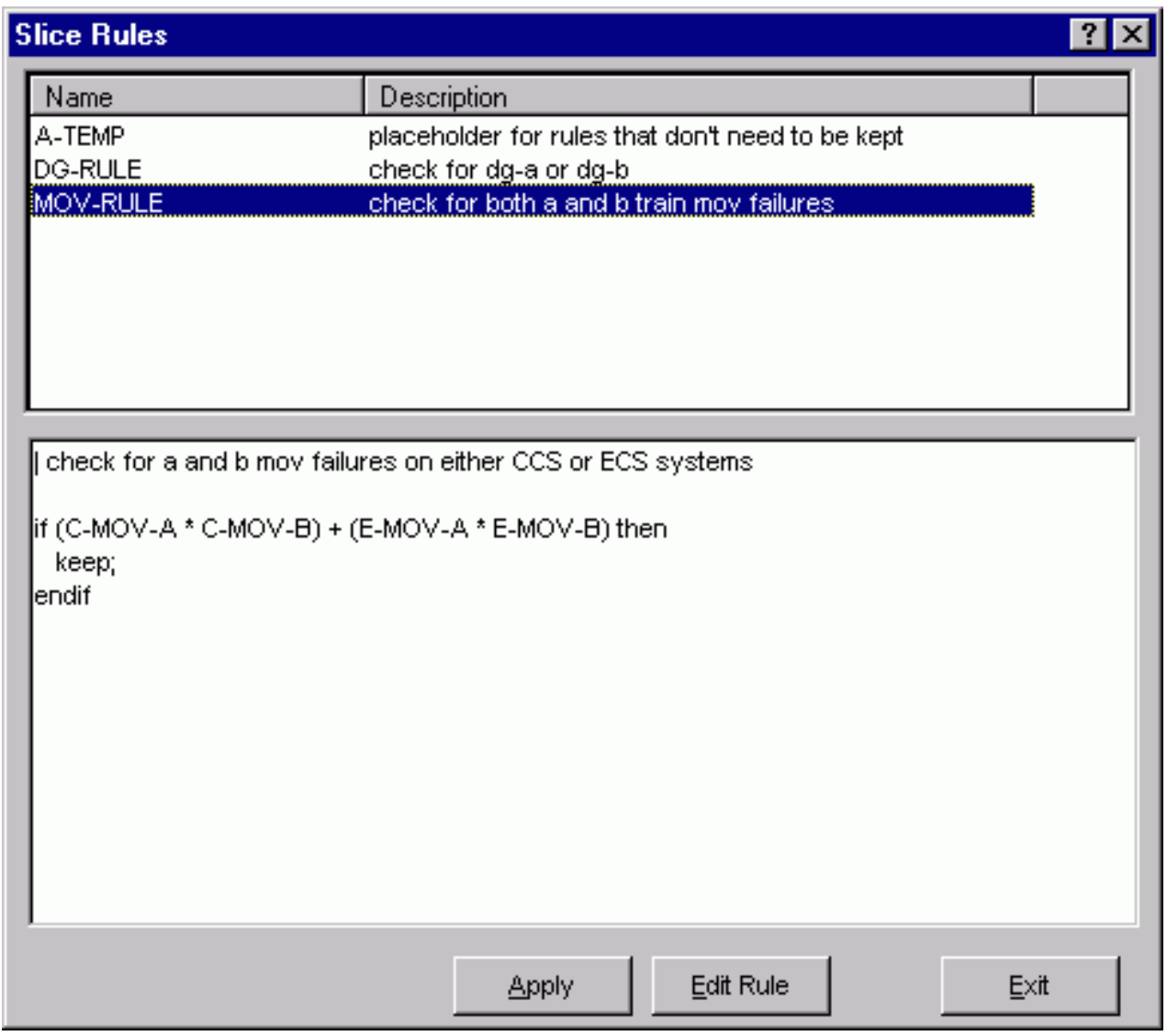

\section{Pop-up Menu Options}

Add - Create a new, empty rule.

Copy - Make a copy of an existing rule. You must supply a unique name before saving the rule.

Modify - Modify the name and/or description of the selected rule.

Delete - Remove the rule from the project.

Apply - The full list of current cut sets will be evaluated. Those cut sets meeting the qualification criteria will be retained in the current slice of qualified cut sets. Other cut sets not meeting the qualification criteria will be placed in the unqualified list.

Edit Rules-Edit the selected rule logic.

Exit - $\quad$ Close the Slice Rules dialog without applying a rule. 


\subsection{Slice Rules Editor}

\section{PURPOSE}

This option allows you to create or modify rules that divide cut sets into two subsets - qualified and unqualified. The Slice Rules editor provides a means to develop logic rules that locate and separate cut sets containing desired event combinations.

The slice rules are entered in a free-form text editor within SAPHIRE. The editor options and rule syntax are similar to that of SAPHIRE's recovery rules. However, unlike to recovery rules, slice rules are not tied to the fault tree, sequence(s) or end state(s) whose cut sets are currently displayed. (A slice rule is available to be applied to any type of displayed cut sets.)

Slice rules follow a format similar to the structure that is found in traditional programming languages (e.g., BASIC or PASCAL). As such, the ability exists to define "macros" and "if...then" type of structures. After creating or editing rules, SAPHIRE compiles the rules to check their validity.

Note: An alternative method of entering rules into the project is to export them from SAPHIRE and create or edit them in any word processor or text editor (that can output ASCII files) and then loaded directly into the SAPHIRE database.

\section{STEPS}

1. From the SAPHIRE menu select Fault Tree/Sequence/End State. The Fault Trees List/Sequences/End States dialog will be displayed.

2. Highlight the desired fault tree(s)/sequence(s)/end state(s) and right-click to invoke the pop-up menu.

3. Choose Display | Cut Sets. The Selected Cut Sets dialog will appear.

4. Choose the Slice By Rule button. The Slice Rules dialog will appear.

5. If necessary, add a new rule by right clicking and choosing the Add menu option.

6. Highlight the desired slice rule and choose Edit Rules. If rules have been previously defined they will be displayed in the edit window. 


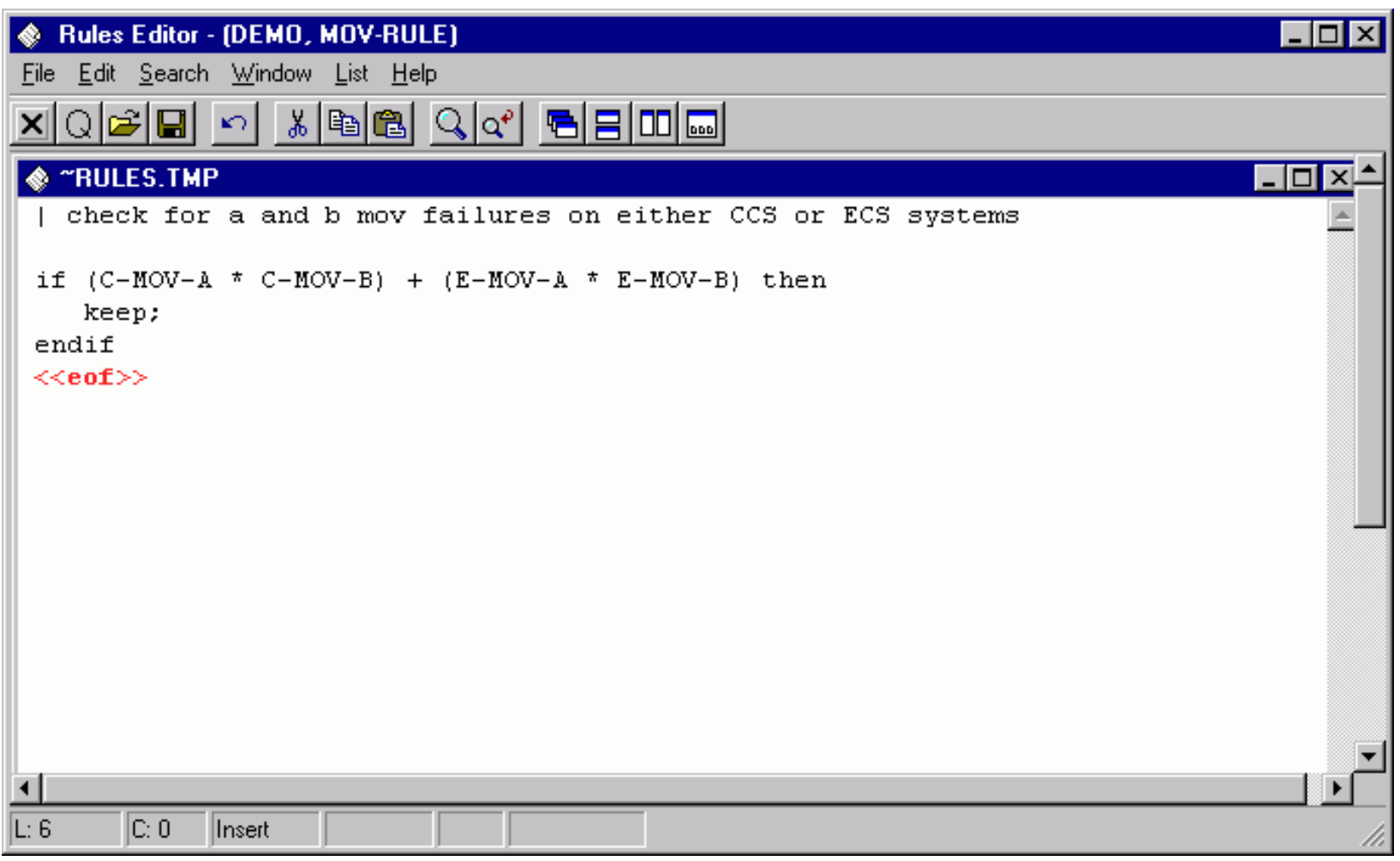

Type the rules in the editor. Note that the rules are not case sensitive.

File - $\quad$ File functions such as open, save, print, preferences, etc.

Edit - $\quad$ Editing functions such as copy and paste along with text format operations.

Search - $\quad$ Search and cursor positioning operations such as find, replace, goto, etc.

Window - Window management functions such as cascade, tile horizontally, etc.

Lists - $\quad$ Display lists of macros and events for inserting into the rules. Three different event lists are available: initiators, a complete list of events, and only those events available in the currently displayed cut sets.

\subsection{Editor File Functions}

These options provide file management functions.

Open - $\quad$ Open a file into a new window. This feature is not usually used while editing SAPHIRE rules.

New - $\quad$ Create a new file in a new window. This feature is not usually used while editing SAPHIRE rules.

Close - $\quad$ Close the edit current window. If changes have been made you will be prompted to save the file.

Quit - $\quad$ Close the current edit window without saving.

Save - $\quad$ Save the file in the current window to disk.

Save As - $\quad$ Save the file in the current window with a new file name. 
Save All - $\quad$ Save all the files in all edit windows currently open.

Save Block - $\quad$ Save the currently highlighted text into a new file. The Save As dialog will be invoked.

Import File - Insert the contents of a file into the current edit window at the current cursor position. The Edit external file dialog will be invoked.

Print - $\quad$ Print the file in the current edit window.

Page setup - $\quad$ Set printer page layout options, such as pages per sheet and line numbers.

Preferences - Select editing options such as tab width, undo levels, etc.

Exit -

Terminate the Editor session.

\subsection{Editor Edit Functions}

These options provide editing and text formatting functions.
Undo -
Undo the previous editing operation.
Cut -
Remove the highlighted text from the current window and place it in the clipboard.
Copy -
Copy the highlighted text from the current window and place it in the clipboard.
Paste -
Delete -
Copy the clipboard text into the current window at the cursor position.
Remove the highlighted text from the current window without placing it
Shift left indent - in the clipboard.
Shift right indent -
Shift the text to the left by the Shift Size specified under Options.
Shift left space -
Shift the text to the right by the Shift Size specified under Options.
Shift right space -
Shift the text to the left by a single space.
Delete to end of line -
Shift the text to the right by a single space.
Delete to the end of the current line beginning after the current cursor position.
Delete line -
Delete the line on which the cursor is currently positioned.
Capitalize word -
Convert the entire word under the current cursor position to upper case.
Downcase word -
Convert the entire word under the current cursor position to lower case.

\subsection{Editor Search Functions}

These options provide search and cursor positioning operations.

Find -

Replace -

Find Procedure -

Find/Replace again -

Find Altered lines -

Find line number -

Match symbol -

Goto next error -

Goto previous error -

Goto Marker -

Set Marker -

5.11.2.5.3.6
Search the text in the current window for the specified string.

Search the text in the current window for a string and replace it with another string.

Search the text in the current window for the specified procedure.

Repeat the previous search operation.

Find the lines of text that have changed since the last save operation.

Go to the specified text line number in the current window.

Find the matching symbol pair for the character under the cursor "( $),\{\}$, [], (**)"

Position the cursor at the next compilation error.

Position the cursor at the previous compilation error.

Position the cursor at the previously saved file position. Up to four marker positions can be retrieved.

Save the current file position for later retrieval.

Editor Window Functions 
These options are used for managing the open edit windows within the application window, such as the Linkage Rules Editor or the Event Tree Logic Editor window. Along with the options listed below and segregated by a separation bar, the names of each open file is listed in the menu.

Cascade windows -

Tile windows horizontally -

Tile windows vertically -

Arrange Icons -
Resize and reposition the non-minimized windows in an overlapping fashion so that the title of each window is displayed in a cascade arrangement. The current edit window will be on top.

Resize and reposition the non-minimized windows in a horizontally tiled arrangement.

Resize and reposition the non-minimized windows in a vertically tiled arrangement.

Arrange the minimized window icons at the bottom of the application window.

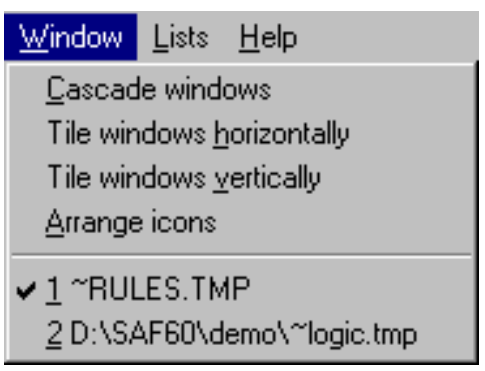

In the example above, the files " $\sim$ RULES.TMP" and " $\sim$ LOGIC.TMP" are open. $\sim$ RULES.TMP is the active window, as indicated by the check mark on the menu.

\subsection{Slice Rules Lists}

Selecting from the lists allows you to insert or replace items in the editor.

Macros - $\quad$ Allows you to insert a macro into the editor.

Events - $\quad$ Select any basic event instead of typing in the basic event name.

Cut Set Events - Select from a list of only those events found in the currently displayed cut sets, instead of typing in the event name.

Initiators - $\quad$ Select an initiating event instead of typing in the initiator name.

\subsection{MACRO}

(Basic Rule syntax)

A macro is a user-definable keyword that specifies a search criteria. The macro name must be all uppercase, must be 24 characters or less, and must not include any of the restricted characters (e.g., a space, $*, ?, \backslash, /)$. The macro line can wrap around to more than one line, but must end with a semicolon.

MACRO-NAME $=$ SEARCH-CRITERIA;

if MACRO-NAME “and optional other search criteria" then 
perform some action on each cut set...;

endif

| Macros are only applicable in the particular rule they are entered into.

\subsubsection{Importance}

\subsection{Sets Importance}

\section{PURPOSE}

This option displays the Fussell-Vesely importance of the selected set(s) of cut sets including interactions among the events. The selected sets are previously saved slices.

\section{STEPS}

1. From the SAPHIRE menu select Fault Tree/Sequence/End State. The Fault Tree List/Sequences/End State List dialog will be displayed.

2. Highlight the desired list item and right-click to invoke the pop-up menu.

3. Choose Display | Cut Sets. The Selected Cut Sets dialog will be displayed.

4. Choose the Sets button. The Select Set(s) for Set Importance dialog will be displayed.

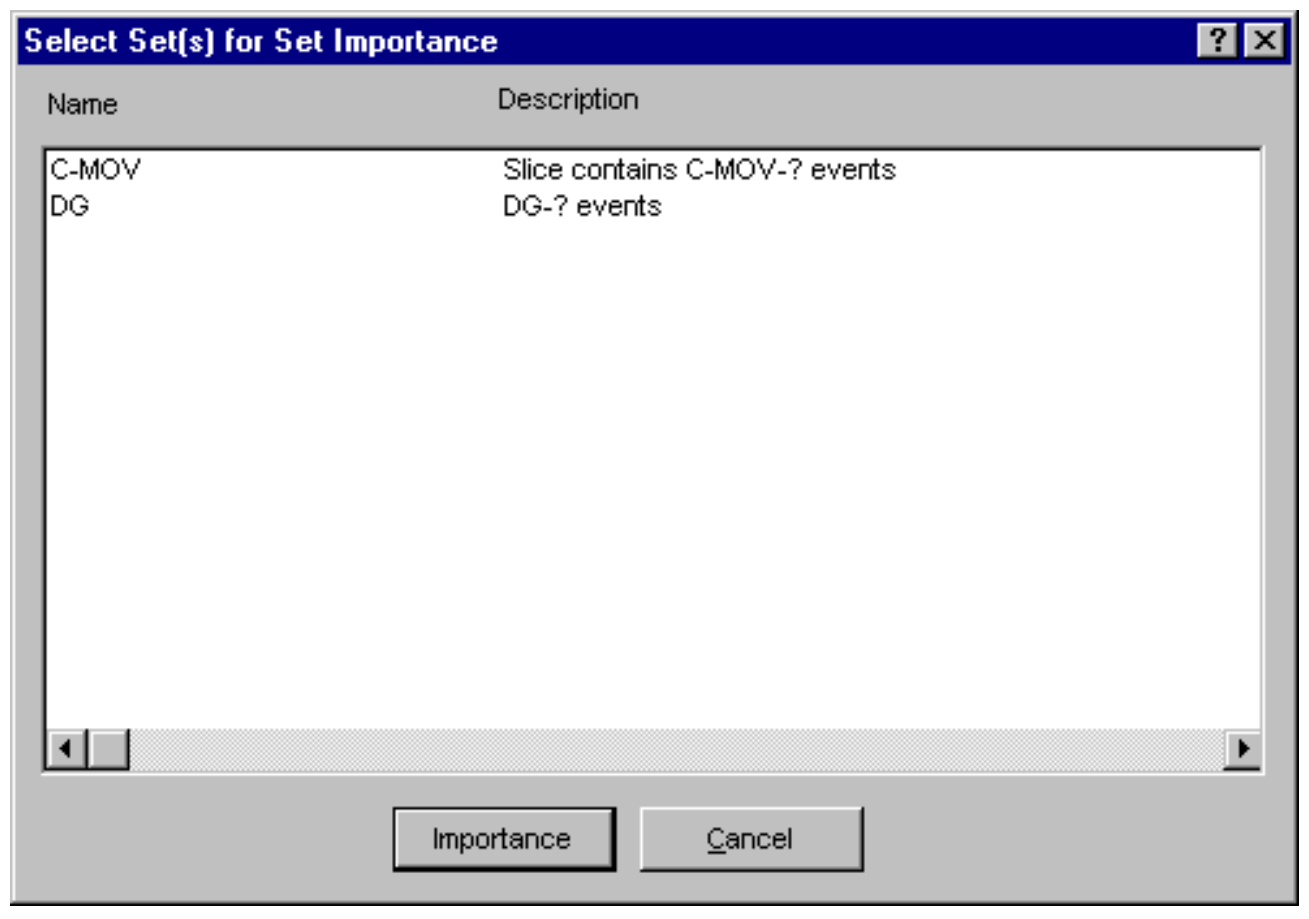

Select the desired set(s) and choose the Importance button.

The Sets Importance dialog will be displayed containing the Fussell-Vesely importance, normalized Fussell-Vesely, name of the set, and its description, for each selected set. 


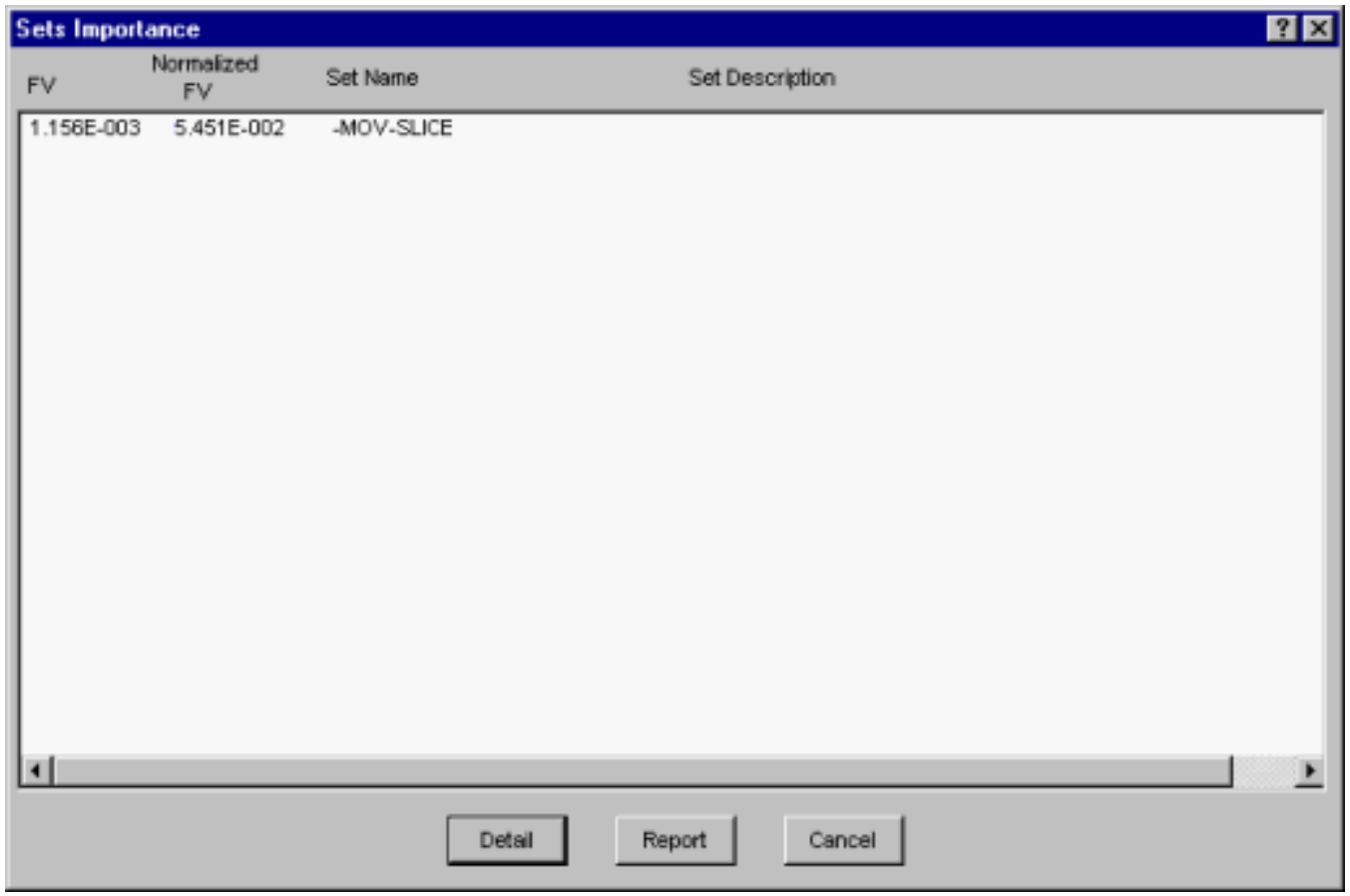

Detail - Displays the Cut Set Importance dialog after selecting one of the sets. Choose the Top or Bottom buttons for Sets importance details .

Report - Generates a report containing the Fussell-Vesely importance, normalized Fussell-Vesely, set name, and description of the selected set.

Cancel - Closes the Sets Importance dialog.

\subsubsection{View Option}

\subsection{View Cut Set}

\section{PURPOSE}

This option displays the basic events of the selected cut set with their failure probabilities and descriptions. At the bottom of the Selected Cut Set Events dialog, information about this cut set is displayed: percent of contribution to the total, the cut set frequency, and the number of events that make up this cut set.

\section{STEPS}

1. From the SAPHIRE menu select Fault Tree/Sequence/End State. The Fault Tree List/Sequences/End State List dialog will be displayed.

2. Highlight the desired list item and right-click to invoke the pop-up menu. 
3. Choose Display | Cut Sets. The Selected Cut Sets dialog will be displayed.

$O R$

4. Choose Display | Comparison. The Base \& Current Case Cut Sets dialog will be displayed.

5. Highlight the desired cut set.

6. Choose the View button. The Selected Cut Set Events dialog will be displayed.

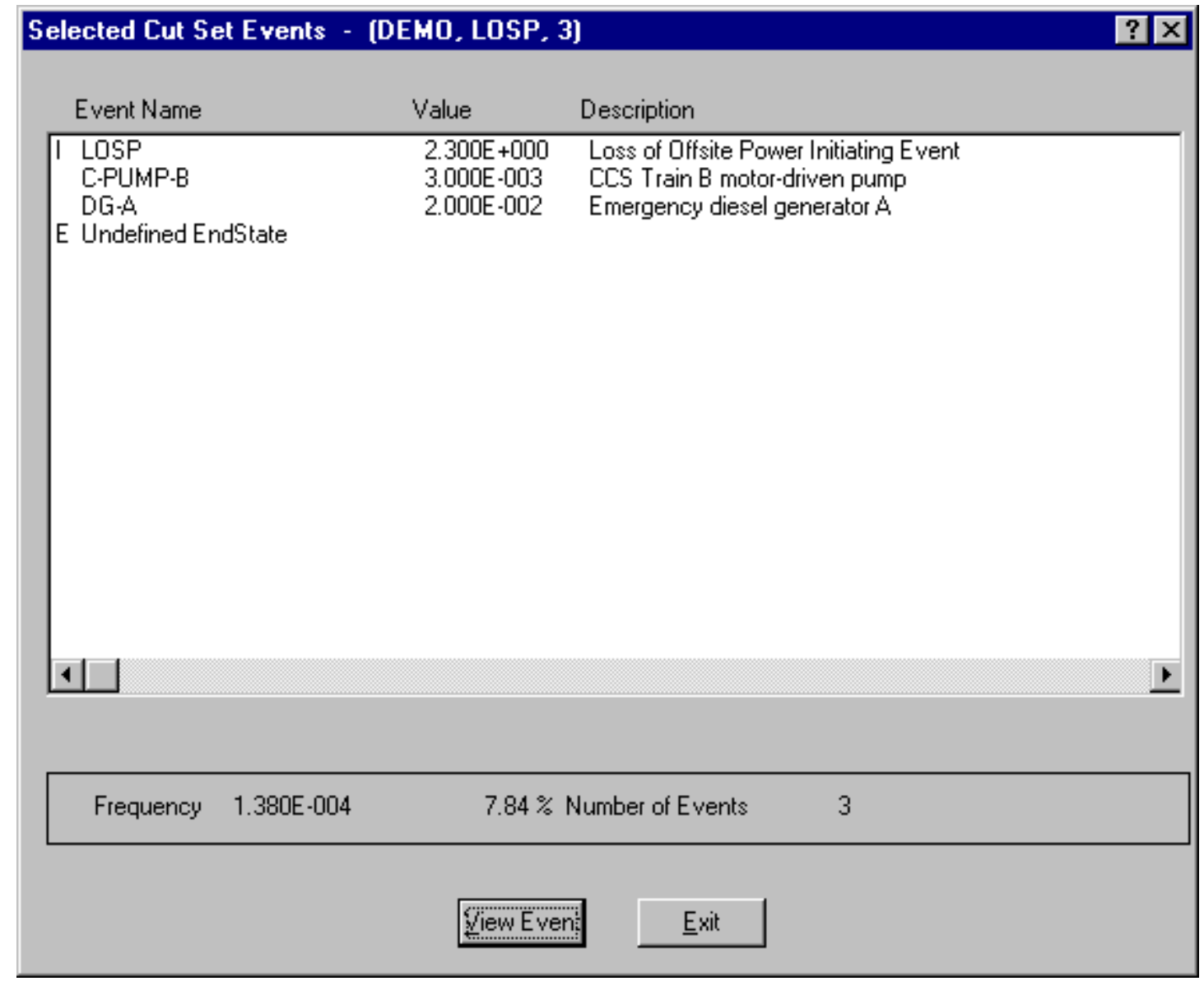

When viewing fault tree cut sets, the end state, if any, is designated with the letter "E" to the left of the Event Name column. It will be the last item listed.

When viewing sequence cut sets, the initiating event is designated with the letter "I" to the left of the Event Name column. It will be the first item listed. The end state will be designated with the letter "E" to the left of the Event Name column and will be the last item listed.

When viewing end state cut sets, the initiating event is designated with the letter "I" to the left of the Event Name column. It will be the first item listed. The originating sequence is designated by the letter "S" to the left of the Event Name column and will be the last item listed.

View Event - Display individual basic event information.

\subsection{View Event}




\section{PURPOSE}

This option provides the following detailed information about the basic events that make up a cut set: event names and description, event attributes, random failure data, uncertainty data, susceptibility flags, and transformation data.

\section{STEPS}

1. From the SAPHIRE menu select Fault Tree/Sequence/End State. The Fault Tree List/Sequences/End State List dialog will be displayed.

2. Highlight the desired list item and right-click to invoke the pop-up menu.

3. Choose Display | Cut Sets. The Selected Cut Sets dialog will be displayed. OR

4. Choose Display | Comparison. The Base \& Current Case Cut Sets dialog will be displayed.

5. Highlight the desired cut set.

6. Choose the View Cut Set button. The Selected Cut Set Events dialog will be displayed.

7. Highlight the desired basic event.

8. Choose the View Event button. The View Event dialog will be displayed. 


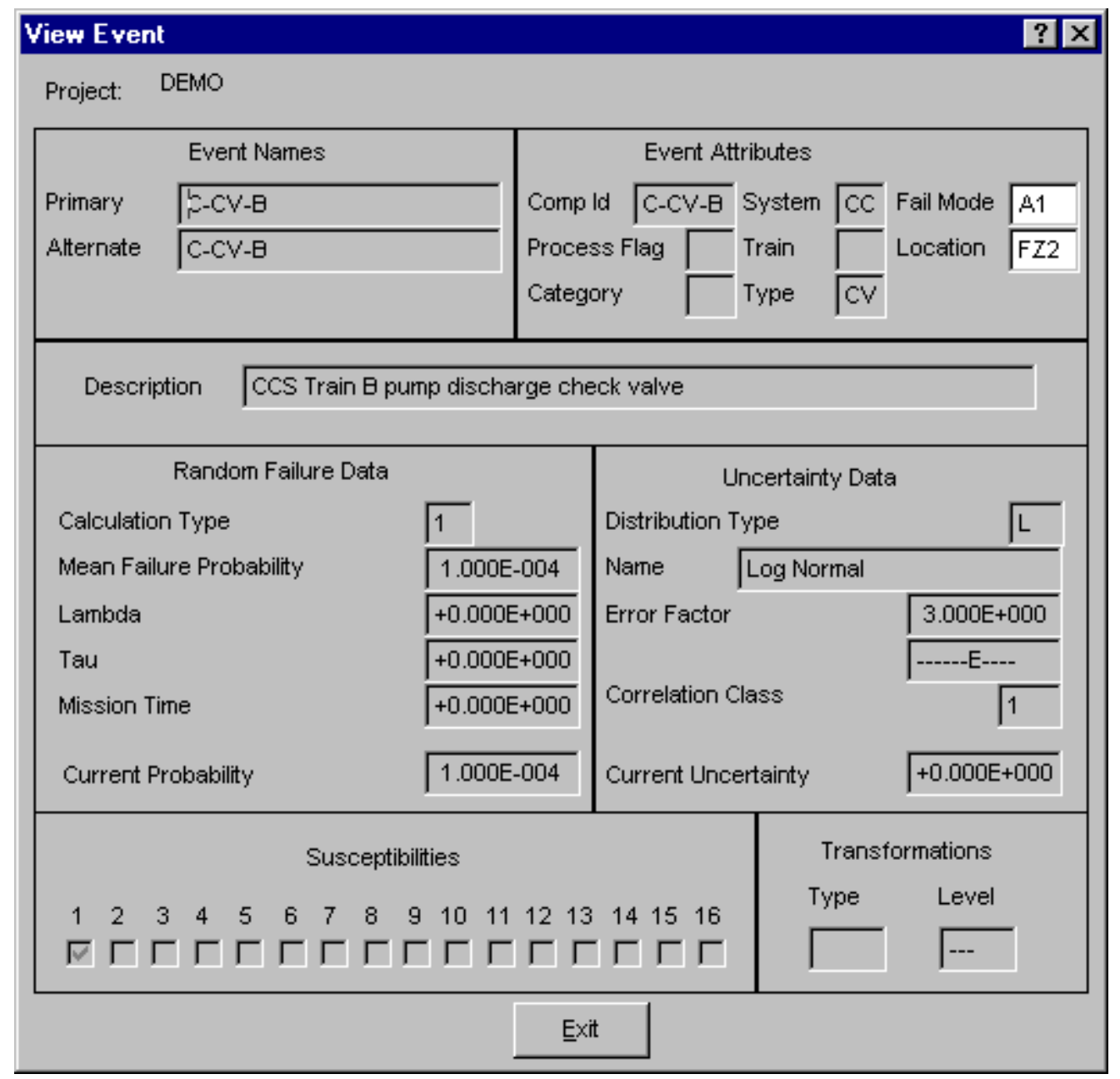

Information on this dialog is for display only and cannot be modified.

\subsubsection{Report Dialog}

\subsection{Cut Set Report}

\section{PURPOSE}

This option allows you to generate a report of the data that is currently displayed on the dialog. The report may be displayed in the Report Viewer, sent to a printer, or saved to a file for later processing.

\section{STEPS}

1. From the SAPHIRE menu select Fault Tree/Sequence/End State. The Fault Tree List/Sequences/End State List dialog will be displayed.

2. Highlight the desired list item and right-click to invoke the pop-up menu.

3. Choose Display | Cut Sets. The Selected Cut Sets dialog will be displayed.

4. Choose the Report button. The Report dialog will be displayed.

5. Select the desired report options and choose $\mathbf{O K}$. The Report Viewer will display the report. 


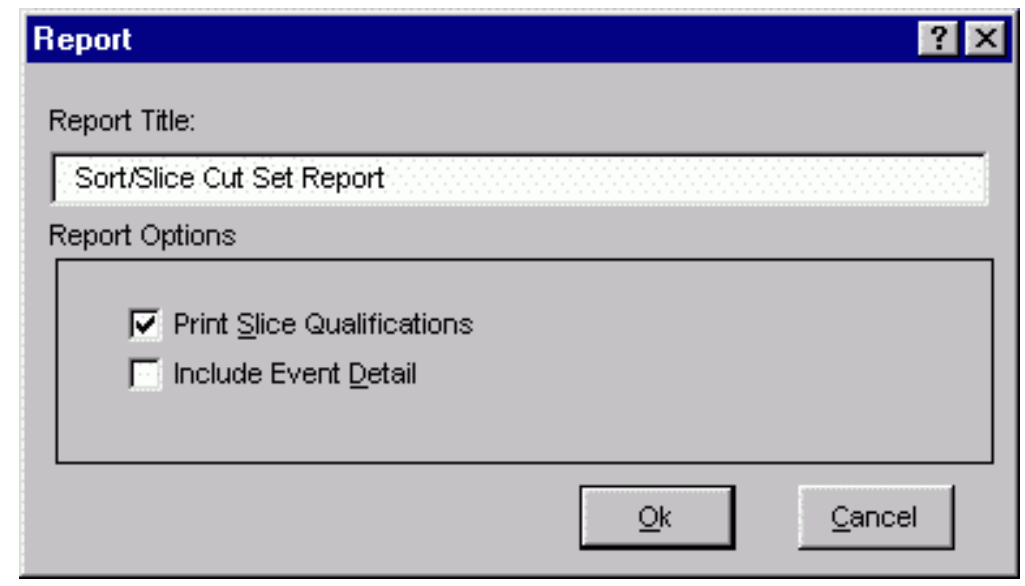

Report Title -

Print Slice Qualifications -

Include Event Detail -
A default report title is provided. Edit this value to customize the title. When checked, the criteria used to qualify the cut sets is included in the report.

When checked, the report will report one event per line, and including event probability and description information. When this option is not checked, the events will appear as a comma delimited list, with no supplementary event information included.

\subsubsection{Comparing Cut Sets}

\subsubsection{Cut Set Comparison}

\section{PURPOSE}

This option displays both the base case and current case cut sets for the selected fault tree (or sequences or end states). The source (either base or current) of the cut sets is indicated both by color and a symbol. The color red and the "-" symbol in the first column indicate a base case cut set. The color black and a blank (" ") in the first column indicate that the cut set is both a current case and base case cut set. The color green and the "+" symbol in the first column indicate a current case cut set.

\section{STEPS}

1. From the SAPHIRE menu select Fault Tree/Sequence/End State. The Fault Trees List/Sequences/End State List dialog will be displayed.

2. Highlight the desired list item(s) and right-click to invoke the pop-up menu.

3. Choose Display, then the Comparison sub-menu option. The Base \& Current Case Cut Sets dialog will be displayed. 


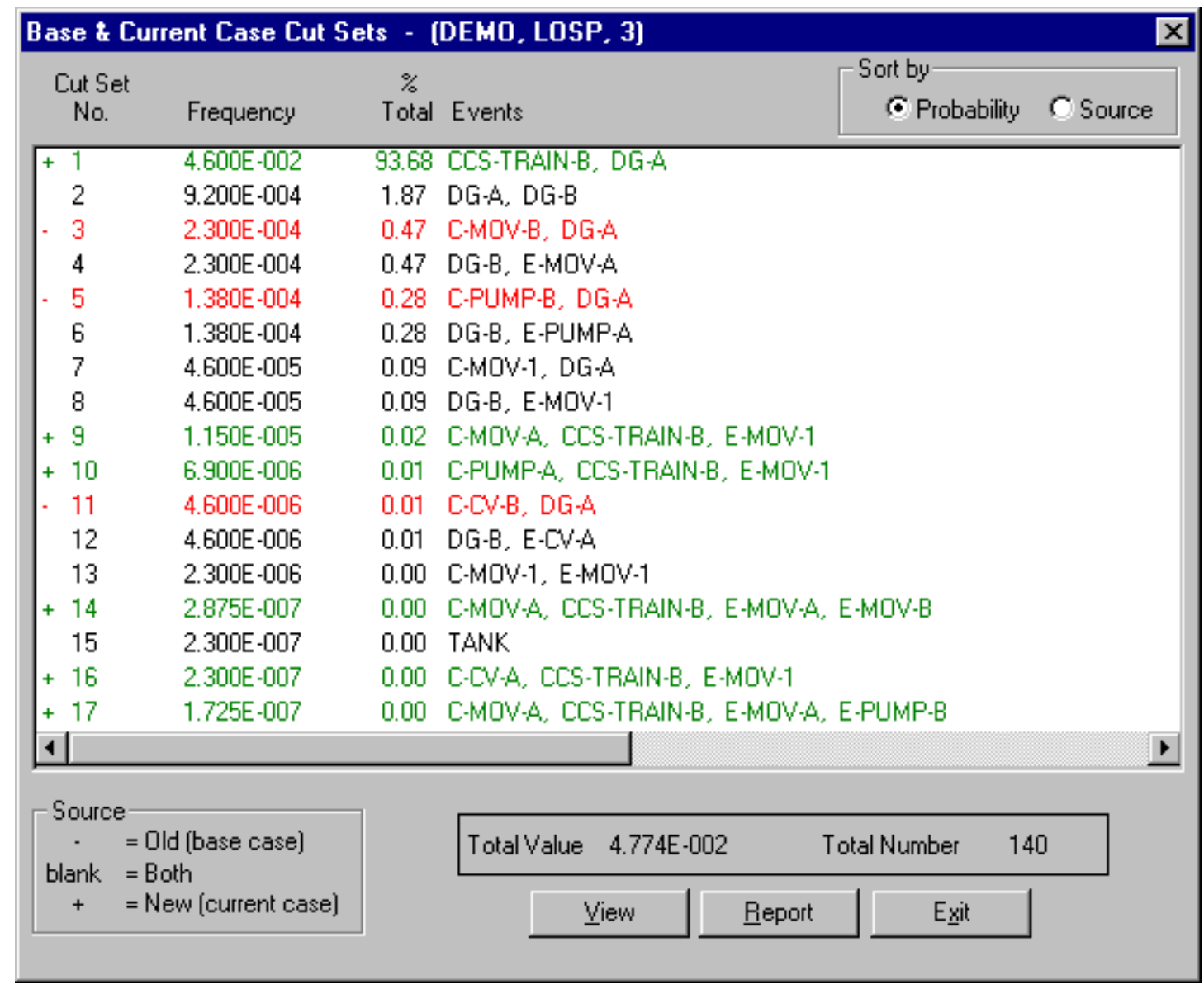

\section{Sort by}

Probability - List the cut sets by frequency in descending order.

Source - $\quad$ List the cut sets by source, listing first base case cut sets, then cut sets

View -

Report -

Exit existing in both, followed by current case cut sets. Display information about the selected cut set including basic event data. Generate a report of the data that is displayed on the dialog. Close the Base \& Current Case Cut Sets dialog.

\subsubsection{View Cut Set}

\section{PURPOSE}

This option displays the basic events of the selected cut set with their failure probabilities and descriptions. At the bottom of the Selected Cut Set Events dialog, information about this cut set is displayed: percent of contribution to the total, the cut set frequency, and the number of events that make up this cut set. 


\section{STEPS}

1. From the SAPHIRE menu select Fault Tree/Sequence/End State. The Fault Tree List/Sequences/End State List dialog will be displayed.

2. Highlight the desired list item and right-click to invoke the pop-up menu.

3. Choose Display | Cut Sets. The Selected Cut Sets dialog will be displayed. OR

4. Choose Display | Comparison. The Base \& Current Case Cut Sets dialog will be displayed.

5. Highlight the desired cut set.

6. Choose the View button. The Selected Cut Set Events dialog will be displayed.

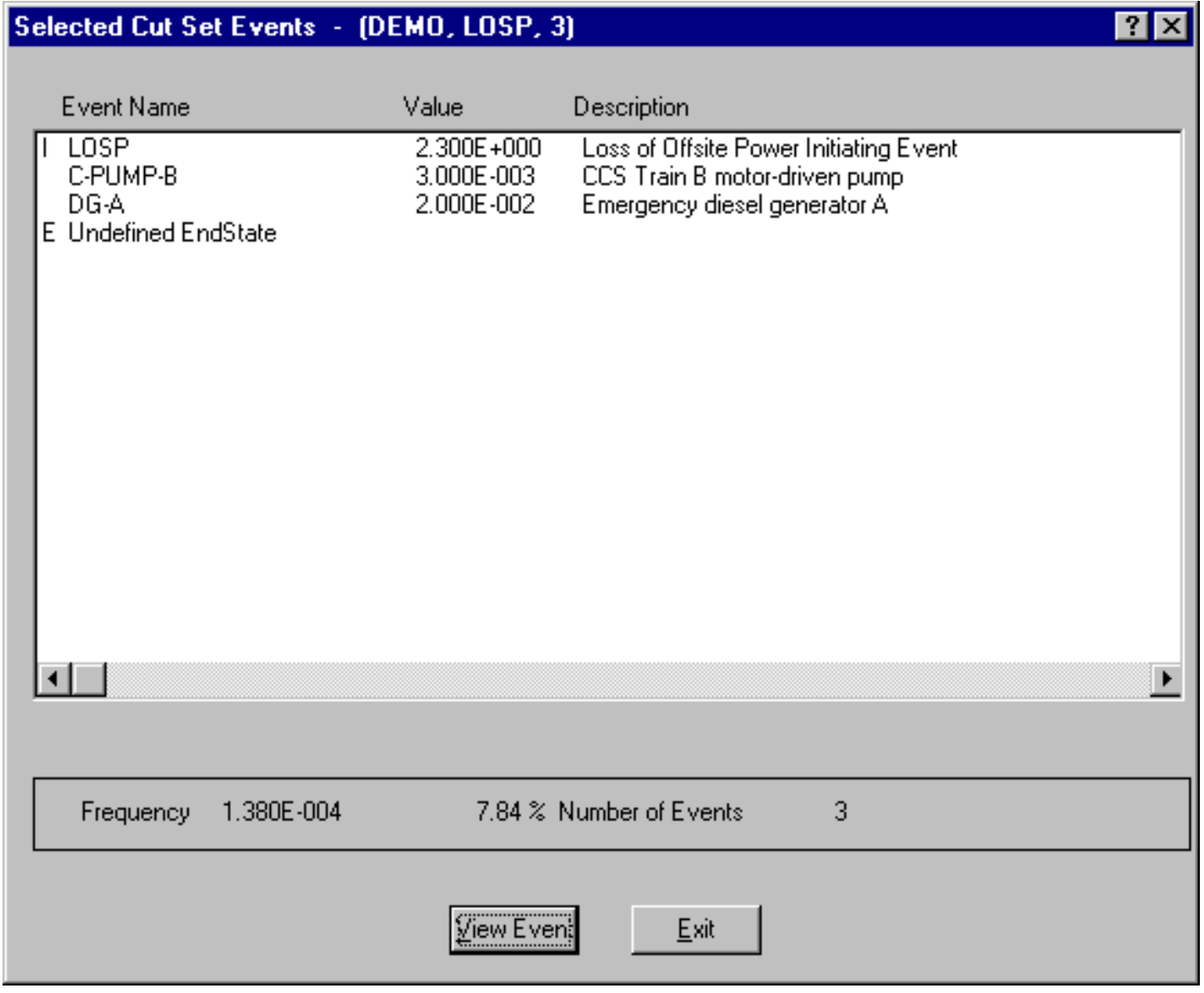

When viewing fault tree cut sets, the end state, if any, is designated with the letter "E" to the left of the Event Name column. It will be the last item listed.

When viewing sequence cut sets, the initiating event is designated with the letter "I" to the left of the Event Name column. It will be the first item listed. The end state will be designated with the letter "E" to the left of the Event Name column and will be the last item listed. 
When viewing end state cut sets, the initiating event is designated with the letter "I" to the left of the Event Name column. It will be the first item listed. The originating sequence is designated by the letter "S" to the left of the Event Name column and will be the last item listed.

View Event - Display individual basic event information.

\subsubsection{Cut Set Report}

\section{PURPOSE}

This option allows you to generate a report of the data that is currently displayed on the dialog. The report may be displayed in the Report Viewer, sent to a printer, or saved to a file for later processing.

\section{STEPS}

1. From the SAPHIRE menu select Fault Tree/Sequence/End State. The Fault Tree List/Sequences/End State List dialog will be displayed.

2. Highlight the desired list item and right-click to invoke the pop-up menu.

3. Choose Display | Cut Sets. The Selected Cut Sets dialog will be displayed.

4. Choose the Report button. The Report dialog will be displayed.

5. Select the desired report options and choose OK. The Report Viewer will display the report.

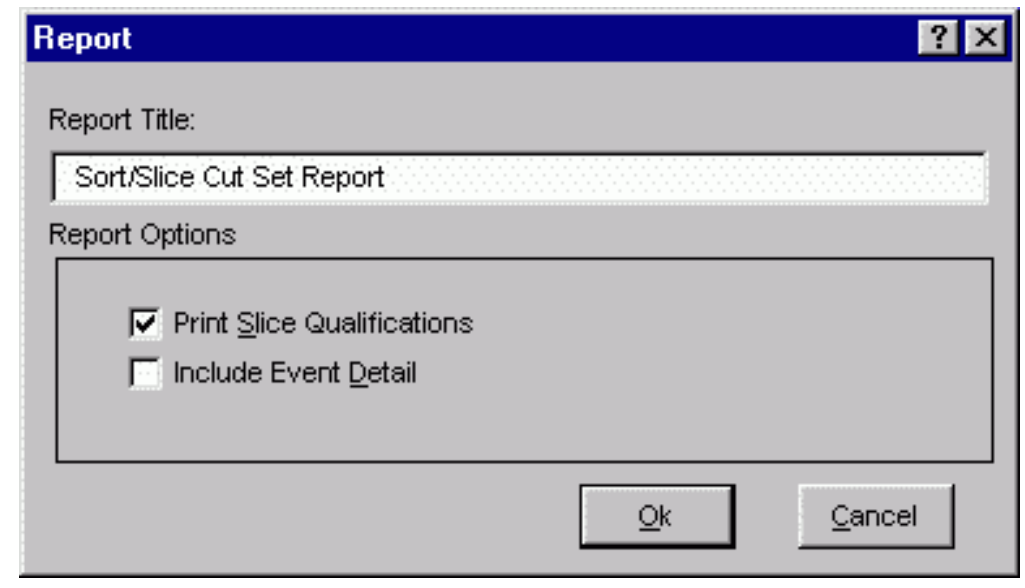

\section{Report Title - \\ Print Slice Qualifications -}

Include Event Detail -
A default report title is provided. Edit this value to customize the title. When checked, the criteria used to qualify the cut sets is included in the report.

When checked, the report will report one event per line, and including event probability and description information. When this option is not checked, the events will appear as a comma delimited list, with no supplementary event information included. 


\subsubsection{Displaying Importance Results}

\subsubsection{Importance Measures}

\section{PURPOSE}

Importance measures provide "reliability-worth" information about basic events appearing in the cut sets for a fault tree or accident sequence. The "importance" for a basic event is essentially the event's contribution to the overall top event probability (for fault trees) or sequence frequency (for event trees). The measure of contribution can take on many forms depending on what concern the analyst has for a particular basic event. Consequently, SAPHIRE calculates seven different basic event importance measures. These are the Fussell-Vesely importance, risk reduction ratio, risk increase ratio, Birnbaum (or first derivative) importance, risk reduction difference, risk increase difference, and the uncertainty importance.

The ratio importance measures are dimensionless and consider only relative changes. The difference definitions account for the actual risk levels that exist and are more appropriate when actual risk levels are of concern, such as comparisons or prioritizations across different plants. For purely relative evaluations, such as prioritizations within a plant, the ratios sometimes give more graphic results.

These importance measures are calculated for each basic event for the respective fault tree or accident sequence. Once the results have been calculated, they can be displayed and then partitioned as a subset of the original cut sets.

\section{STEPS}

1. From the SAPHIRE menu select Fault Tree/Sequence/End State. The Fault Tree List/Sequences/End State List dialog will be displayed.

2. Highlight the desired list item and right-click to invoke the pop-up menu.

3. Choose Display, then the Importance sub-menu option.

4. Choose from one of the following sub-menu options: 


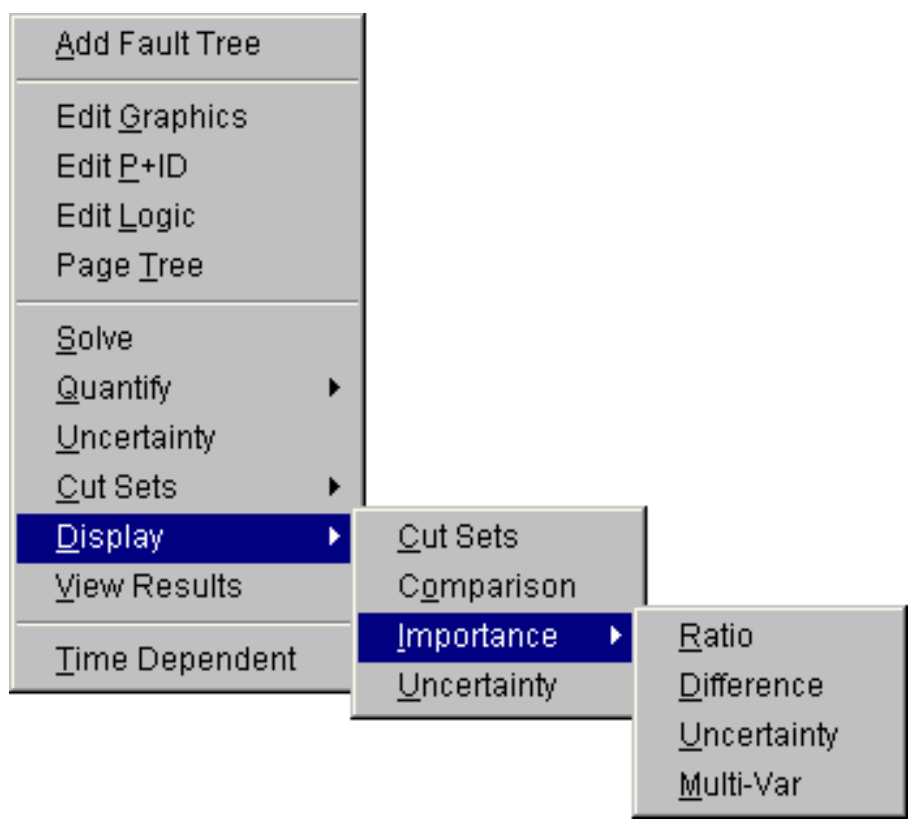

Ratio - $\quad$ Fussell-Vesely importance, Risk Reduction Ratio, and Risk Increase Ratio will be calculated and displayed.

Difference - $\quad$ Birnbaum importance, Risk Reduction Interval, and Risk Increase Interval will be calculated and displayed.

Uncertainty - Quantification of the contribution of each individual basic event's uncertainty to the total output uncertainty.

Multi-Var - Importance for a group of events will be calculated and displayed.

\subsubsection{Fussell-Vesely Importance (FV)}

The FV measure is an indication of the percentage of the minimal cut set upper bound contributed by the cut sets containing the basic event. The equation for FV importance is

$$
\mathbf{F V}=\mathbf{F}(\mathbf{i}) / \mathbf{F}(\mathbf{x})
$$

where

$\mathrm{F}(\mathrm{i})$ is the minimal cut set upper bound for the group of cut sets containing the event $\mathrm{F}(\mathrm{x})$ is the original minimal cut set upper bound

Prior to SAPHIRE version 7.27, the FV equation used and approximate expression

$$
\mathbf{F V}=\mathbf{1}-\mathbf{F}(\mathbf{0}) / \mathbf{F}(\mathbf{x})
$$

where

$\mathrm{F}(0)$ is the minimal cut set upper bound with the event probability set equal to 0.0 . $\mathrm{F}(\mathrm{x})$ is the original minimal cut set upper bound.

\subsubsection{Birnbaum Importance (B)}

This indicates the sensitivity of the minimal cut set upper bound with respect to a change in the basic event probability. 


$$
\mathbf{B}=\mathbf{F}(\mathbf{1})-\mathbf{F}(\mathbf{0})
$$

where

$\mathrm{F}(1)$ is the minimal cut set upper bound with the event probability set equal to 1.0 .

$\mathrm{F}(0)$ is the minimal cut set upper bound with the event probability set equal to 0.0 .

\subsubsection{Risk Reduction Ratio (RRR) or Risk Reduction Interval (RRI)}

These are an indication of how much the minimal cut set upper bound would decrease if the basic event was reduced to a probability or 0.0 (typically if the corresponding component never failed).

$$
\begin{aligned}
& R R R=F(x) / F(0) \\
& R R I=F(x)-F(0)
\end{aligned}
$$

Note the similarity between RRI and FV; the relative importance ranking of basic events will be the same for the two importance measures.

\subsubsection{Risk Increase Ratio (RIR) or Risk Increase Interval (RII)}

These are an indication of how much the minimal cut set upper bound would increase if the basic event was increased to 1.0 (typically if the corresponding component always failed). Note: If the event probability is close to 1.0, this importance measure may yield a small RIR or RII.

$$
\begin{aligned}
& \text { RIR }=\mathbf{F}(\mathbf{1}) / F(x) \\
& \text { RII }=\mathbf{F}(\mathbf{1})-\mathbf{F}(\mathbf{x})
\end{aligned}
$$

where

$\mathrm{F}(\mathrm{x})$ is the original minimal cut set upper bound

$\mathrm{F}(1)$ is the minimal cut set upper bound with the event probability set equal to 1.0 .

\subsubsection{Uncertainty Importance}

The uncertainty in each input parameter, as expressed through its probability distribution, contributes to the uncertainty in the output parameter of interest (e.g., core damage frequency). The uncertainty importance measure in SAPHIRE attempts to quantify the contribution of each individual basic event $=\mathrm{S}$ uncertainty to this total output uncertainty. The measure used in SAPHIRE is based on a Taylor series expansion of the variance of the output of interest. The equation used by SAPHIRE is

$$
\operatorname{Var}(R) \approx \sum_{i=1}^{n}\left(\frac{\partial R}{\partial p_{i}}\right)^{2} \sigma_{i}^{2}
$$

where $\mathrm{R}$ is the output of interest, $\mathrm{p}_{\mathrm{i}}$ is the probability of the ith basic event, and $\sigma_{\mathrm{i}}^{2}$ is the variance of the uncertainty distribution for the ith event. This approximation, which hinges upon the basic events being mutually statistically independent, says that the variance of the output is approximately the sum of $n$ separate contributions, one from each basic event. The magnitude of each contribution (each contribution is positive) measures how much of the output variance is contributed by each basic event. Because it is 
more convenient, SAPHIRE uses the square root of each individual contribution as the uncertainty importance:

$$
I_{u n c}=\frac{\partial R}{\partial p_{i}} \sigma_{i}
$$

where $\sigma_{\mathrm{i}}$ is the standard deviation of the uncertainty distribution of the ith basic event. Note that the partial derivative in the above equation for the uncertainty importance is, by definition, the Birnbaum importance of that event. Therefore, no new calculations are needed; the uncertainty importance is the Birnbaum importance multiplied by the standard deviation of the input probability distribution.

\subsubsection{Multi-Var Importance}

This option allows the user to calculate an importance for a group of events.

For instance, the user can select all motor operated valves in a set of cut sets and get a combined importance measure for these valves together. All of the valves are treated as a single event in the importance calculations and the result is displayed with the name "Multi-variable."

\subsubsection{Importance Measures Dialog}

\subsection{Importance Measures Results}

\section{PURPOSE}

This option displays the results of the importance measure analysis.

\section{STEPS}

1. From the SAPHIRE menu select Fault Tree/Sequence/End State. The Fault Tree List/Sequences/End State List dialog will be displayed.

2. Highlight the desired list item and right-click to invoke the pop-up menu.

3. Choose Display, then the Importance submenu option.

4. Choose from one of the submenu options: Ratio, Difference, Uncertainty, Mult-Var. The Importance Measures dialog will be displayed.

The results shown here are Fussell-Vesely importance measures. If the analysis type is seismic, the program will prompt you to select a ground acceleration level defined in the project hazard curve. 


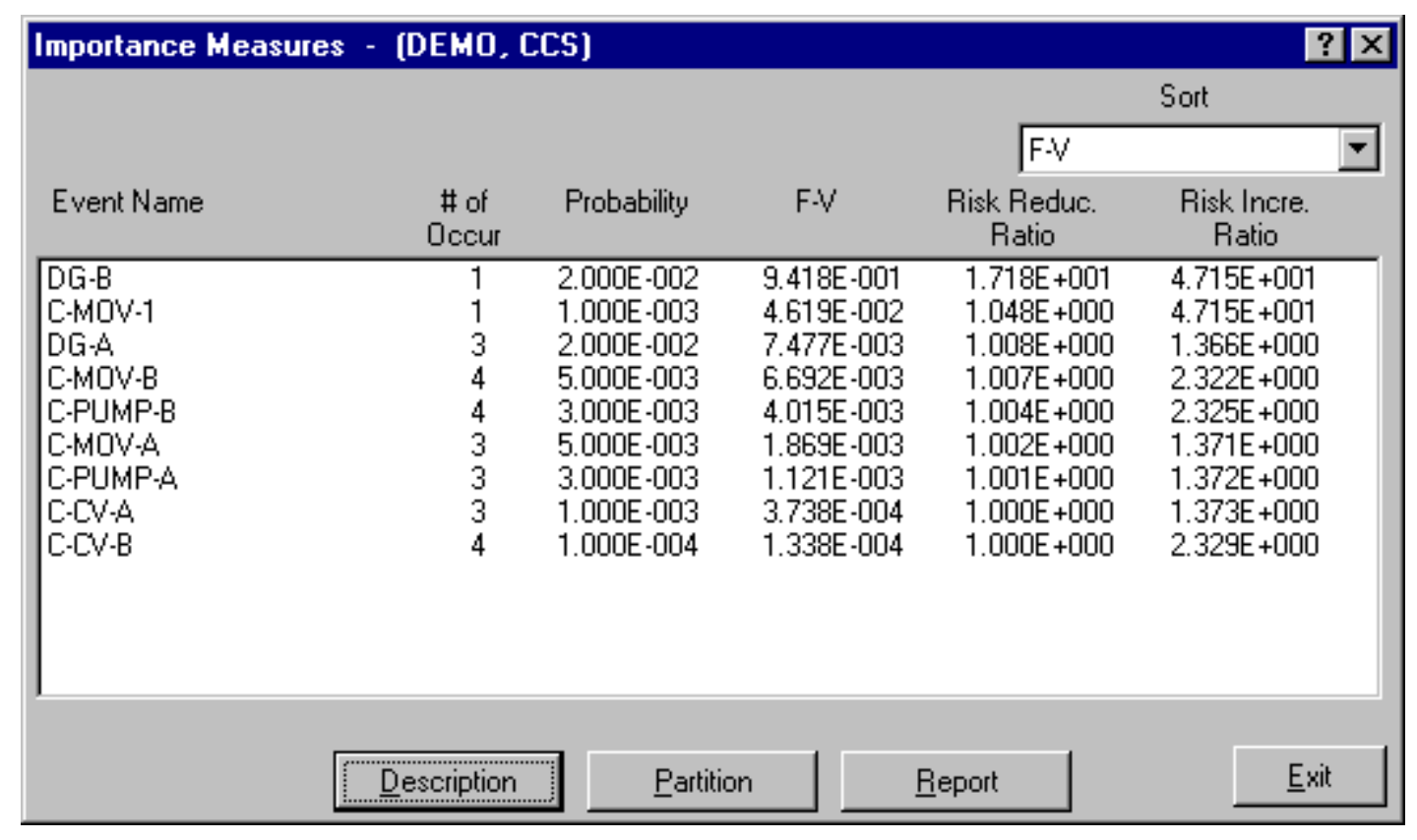

Sort -

Select from the drop-down list to sort results by Occurrence, Probability, F-V / Birnbaum / Uncertainty, Reduction, or Increase. By default, when the importance data are first displayed it is sorted, high to low, by F-V / Birnbaum / Uncertainty importance value.

Description - Display the full description for the highlighted event in the message bar of the SAPHIRE main window.

Partition - $\quad$ Limit which events are displayed/reported.

Report - $\quad$ Generate a report of the data that is displayed on the dialog.

Exit - $\quad$ Close the Importance Measures dialog.

\subsection{Importance Results Partition}

\section{PURPOSE}

This option allows you to redefine a fault tree as a subset of the original cut sets. This is accomplished by defining a set of events to be used to determine whether a cut set belongs to a partition.

\section{STEPS}

1. From the SAPHIRE menu select Fault Tree/Sequence/End State. The Fault Tree List/Sequences/End State List dialog will be displayed.

2. Highlight the desired list item and right-click to invoke the pop-up menu.

3. Choose Display | Importance.

4. Choose from one of the submenu options: Ratio, Difference, Uncertainty, Mult-Var. The Importance Measures dialog will be displayed.

5. Choose the Partition button. The Partition dialog will be displayed. 


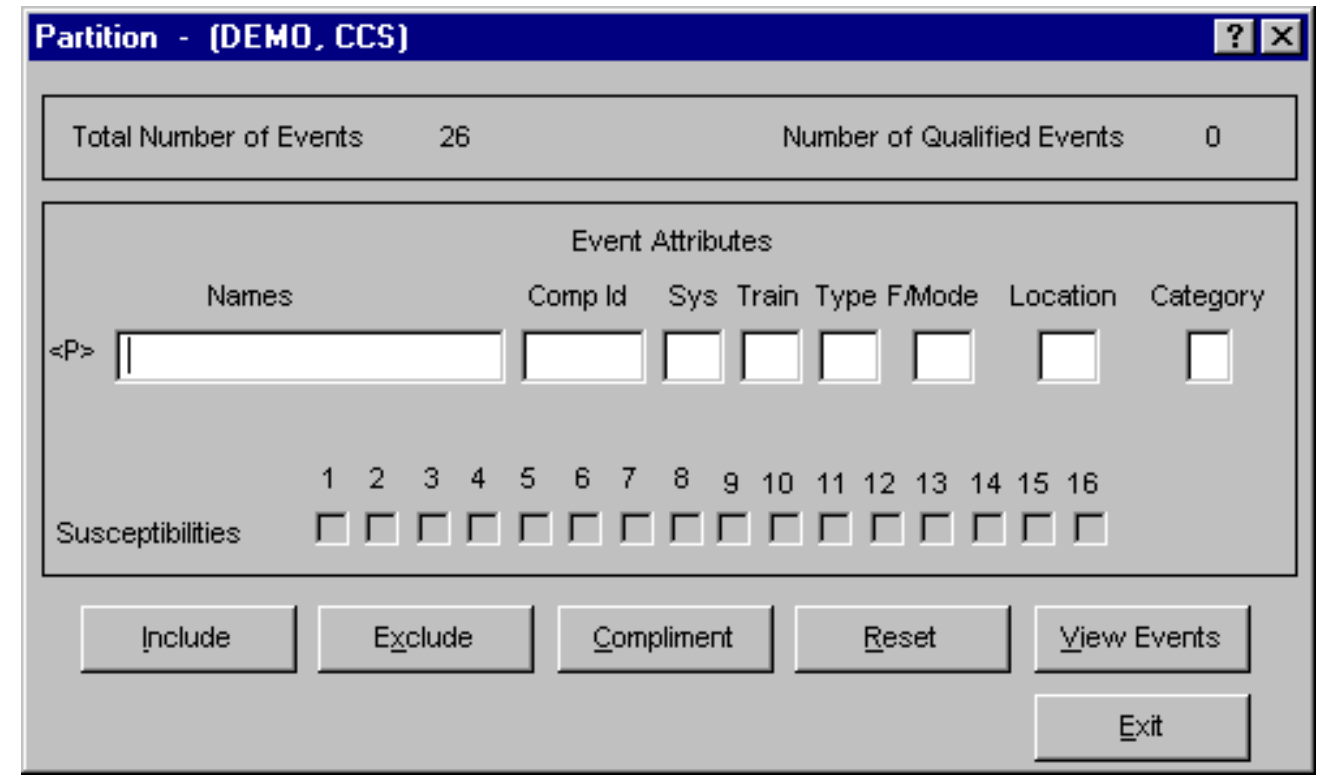

Include - $\quad$ Fill in the entry fields that are to be used to qualify the events that may be used in the new partition. Choose the Include button. The events in the cut set are qualified and when complete, will update the Number of Qualified Events field that appears in the upper right of the Partition dialog.

Exclude - $\quad$ Fill in the entry fields that are to be used to remove events from the list of qualified events. Choose the Exclude button. The events in the cut set are qualified and when complete, will update the Number of Qualified Events field that appears in the upper right of the Partition dialog.

Compliment - Cause all currently qualified events to be disqualified, and all unqualified events to become the set of qualified events.

Reset - $\quad$ Set all events in the database to be qualified. This removes all partitioning from the current cut sets.

View Events - Display the list of all events in the project. Mark those events that are to be considered qualified.

Exit - $\quad$ Close the Partition dialog. Only those cut sets that are made up of qualified events are displayed on the Importance Measures dialog.

\subsection{View Events}

\section{PURPOSE}

This option allows you to display the list of all events in the project and mark those events that are to be used to determine whether a cut set belongs to a partition. 


\section{STEPS}

1. From the SAPHIRE menu select Fault Tree/Sequence/End State. The Fault Tree List/Sequences/End State List dialog will be displayed.

2. Highlight the desired list item and right-click to invoke the pop-up menu.

3. Choose Display | Importance.

4. Choose from one of the sub-menu options: Ratio, Difference, Uncertainty, Mult-Var.

5. The Importance Measures dialog will be displayed.

6. Choose the Partition button. The Partition dialog will be displayed.

7. Choose the View Events button. The Events dialog will be displayed.

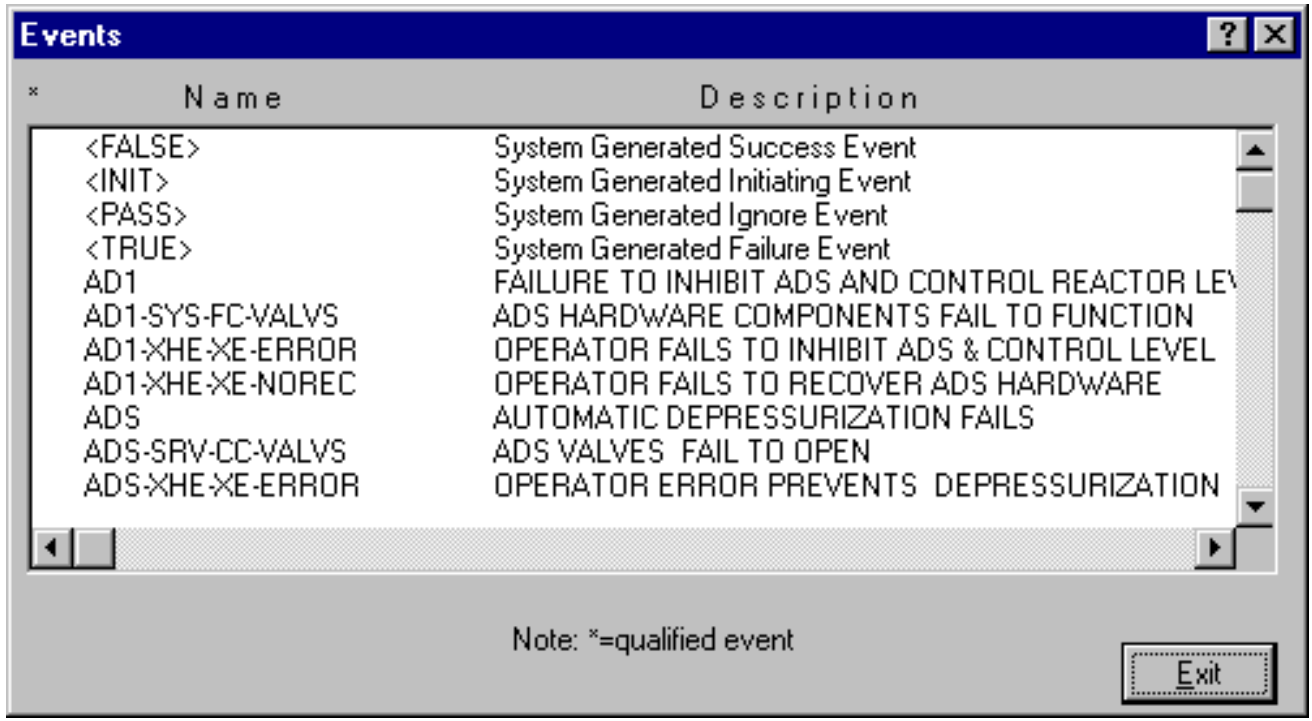

Double-click - Mark the selected event. An asterisk (*) will appear to the left of the event name. Exit Close the Events dialog. Choose the Include or Exclude button from the Partition dialog..

\subsubsection{Displaying Uncertainty}

\subsubsection{Displaying Uncertainty Results}

\section{PURPOSE}

This option displays the results of the uncertainty analysis previously performed. The results displayed are the distribution and probability bounds for both base and current results. These values were calculated using either the Latin Hypercube or the Monte Carlo simulation technique. 


\section{STEPS}

1. From the SAPHIRE menu select Fault Tree/Sequence/End State. The Fault Tree List/Sequences/End State List dialog will be displayed.

2. Highlight the desired list item and right-click to invoke the pop-up menu.

3. Choose Display, then the Uncertainty sub-menu option. The Fault Tree/Sequence/End State Uncertainty dialog will be displayed.

If the analysis type is seismic, you will be prompted to select a ground acceleration level defined in the project hazard curve.

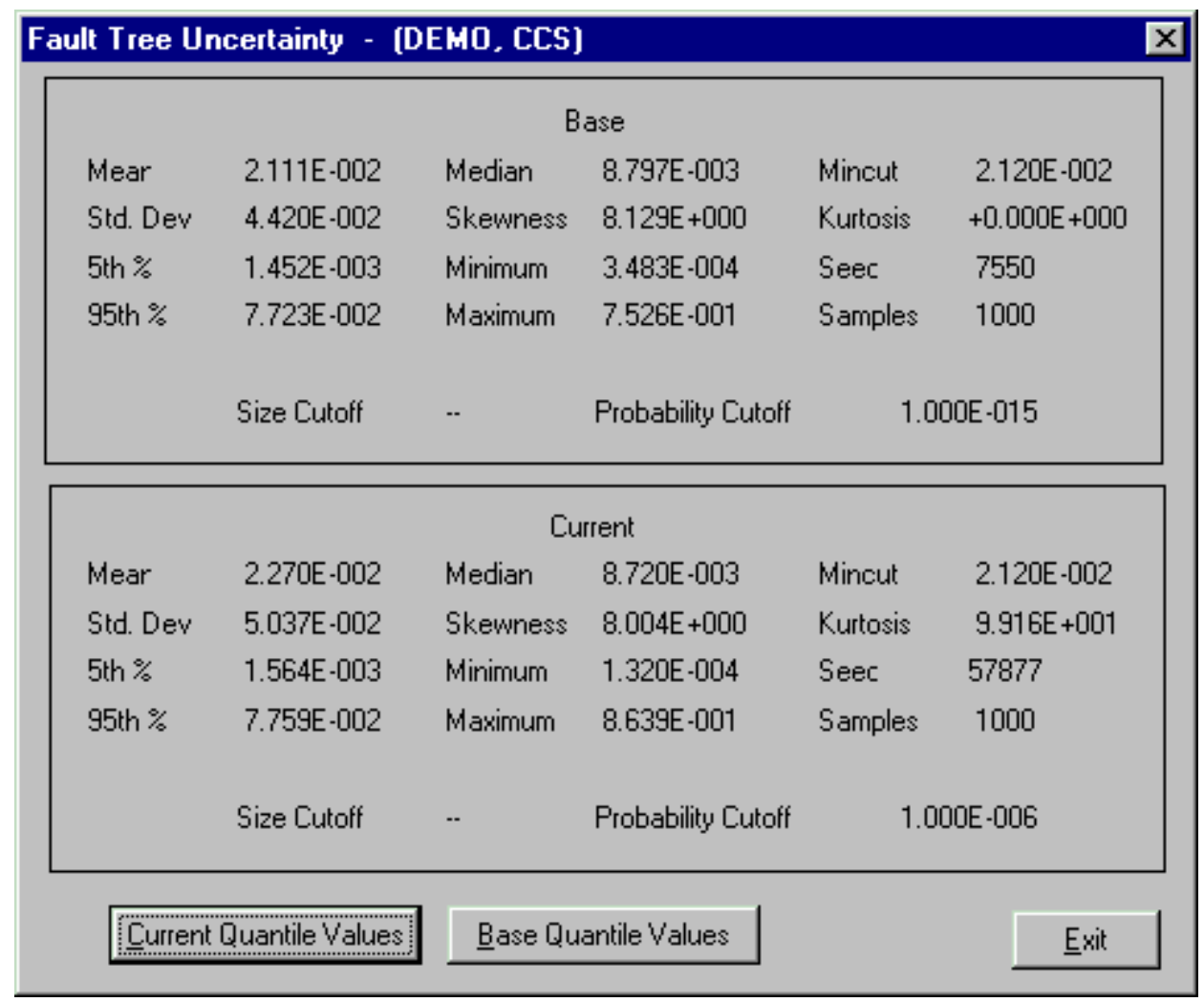

Current Quantile Values - View the quantile values associated with the current case data. Base Quantile ValuesExit View the quantile values associated with the base case data. dialog.

Close the Fault Tree/Sequence/End State Uncertainty

\subsubsection{Uncertainty Quantile Values}

\section{PURPOSE}

Display the uncertainty quantile (or probability) values associated with either the current or base case data. These quantile values can be used to construct a cumulative distribution plot of the analysis uncertainty results. 


\section{STEPS}

1. From the SAPHIRE menu select Fault Tree/Sequence/End State. The Fault Tree List/Sequences/End State List dialog will be displayed.

2. Highlight the desired fault tree and right-click to invoke the pop-up menu.

3. Choose Display, then the Uncertainty sub-menu option. The Fault Tree/Sequence/End State Uncertainty dialog will be displayed.

4. Choose the Current Quantile Values or Base Quantile Values button.

\begin{tabular}{|c|c|c|c|c|c|}
\hline \multicolumn{3}{|c|}{ Uncertainty Quantile Values - [DEMO, CCS] } & \multicolumn{3}{|c|}{$? \times$} \\
\hline $\begin{array}{l}\text { Distribution } \\
\text { Quantile Level } \\
\text { (in per cent) }\end{array}$ & $\begin{array}{c}95 \% \text { Confidence } \\
\text { Interval On } \\
\text { Quantile Level in } \\
\%(+i-)\end{array}$ & Quantile Value & \multicolumn{3}{|c|}{$\begin{array}{l}95 \% \text { Confidence } \\
\text { Interval on Quantile }\end{array}$} \\
\hline 0.5 & 0.5 & $6.2185 E-004$ & $3.6160 \mathrm{E}-004$ & $8.1985 E-004$ & $\bar{\Delta}$ \\
\hline 1.0 & 0.7 & 8.1985E-004 & 5.3501E-004 & $1.0688 \mathrm{E}-003$ & \\
\hline 2.5 & 1.0 & 1.1657E-003 & 1.0634E-003 & 1.3215E-003 & - \\
\hline 5.0 & 1.4 & 1.6235E-003 & $1.3281 \mathrm{E}-003$ & $1.7995 \mathrm{E}-003$ & \\
\hline 10.0 & 1.9 & 2.2045E-003 & $2.0049 E-003$ & $2.4574 \mathrm{E}-003$ & \\
\hline 20.0 & 2.5 & 3.3830E-003 & 3.0875E-003 & 3.6982E-003 & \\
\hline 25.0 & 2.7 & $4.0422 \mathrm{E}-003$ & $3.6952 \mathrm{E}-003$ & 4.3167E-003 & \\
\hline 30.0 & 2.9 & $4.5621 \mathrm{E}-003$ & $4.2275 \mathrm{E}-003$ & $5.0094 \mathrm{E}-003$ & \\
\hline 40.0 & 3.1 & $6.2820 \mathrm{E}-003$ & 5.6327E-003 & 6.8446E-003 & \\
\hline 50.0 & 3.1 & 8.8435E-003 & $7.9240 E-003$ & $9.5918 \mathrm{E}-003$ & \\
\hline 60.0 & 3.1 & $1.1394 \mathrm{E}-002$ & 1.0605E-002 & $1.2502 \mathrm{E}-002$ & \\
\hline \multirow[t]{2}{*}{70.0} & 2.9 & 1.6375E-002 & $1.4758 \mathrm{E}-002$ & $1.8275 \mathrm{E}-002$ & $\nabla$ \\
\hline & Plot & Report & Exit & & \\
\hline
\end{tabular}

Distribution Quantile Level (in percent) - The probability level, which ranges from $0.5 \%$ to $99.5 \%$.

95\% Confidence Interval On Quantile Level in \% (+/-) - The 95\% confidence level on the quantile level (e.g., 5\%, 50\%, 90\%) expressed in terms of the percent of the quantile level. Note that this is the confidence on the quantile level, not the quantile value.

Quantile Value - The value obtained from the uncertainty analysis at the i'th \% quantile (or probability) level. The units on the value varies depending on the type of analysis (e.g., failure probability, core damage frequency, etc.).

95\% Lower Bound Confidence Level On Quantile Value - The lower bound confidence level on the quantile value.

95\% Upper Bound Confidence Level On Quantile Value - The upper bound confidence level on the quantile value.

Plot - Show a graphical representation of the uncertainty distribution defined by the uncertainty quantile values.

Report - Generate a report containing the quantile values as displayed here.

Exit - Close the Uncertainty Quantile Values dialog.

\subsubsection{Plot Uncertainty Quantile Values}




\section{PURPOSE}

Show a graphical representation of the distribution defined by the uncertainty quantile (or probability) values associated with either the current or base case data.

\section{STEPS}

1. From the SAPHIRE menu select Fault Tree/Sequence/End State. The Fault Tree List/Sequences/End State List dialog will be displayed.

2. Highlight the desired fault tree and right-click to invoke the pop-up menu.

3. Choose Display, then the Uncertainty sub-menu option. The Fault Tree/Sequence/End State Uncertainty dialog will be displayed.

4. Choose the Current Quantile Values or Base Quantile Values button.

5. Choose the Plot button. The Uncertainty plot dialog will be displayed.

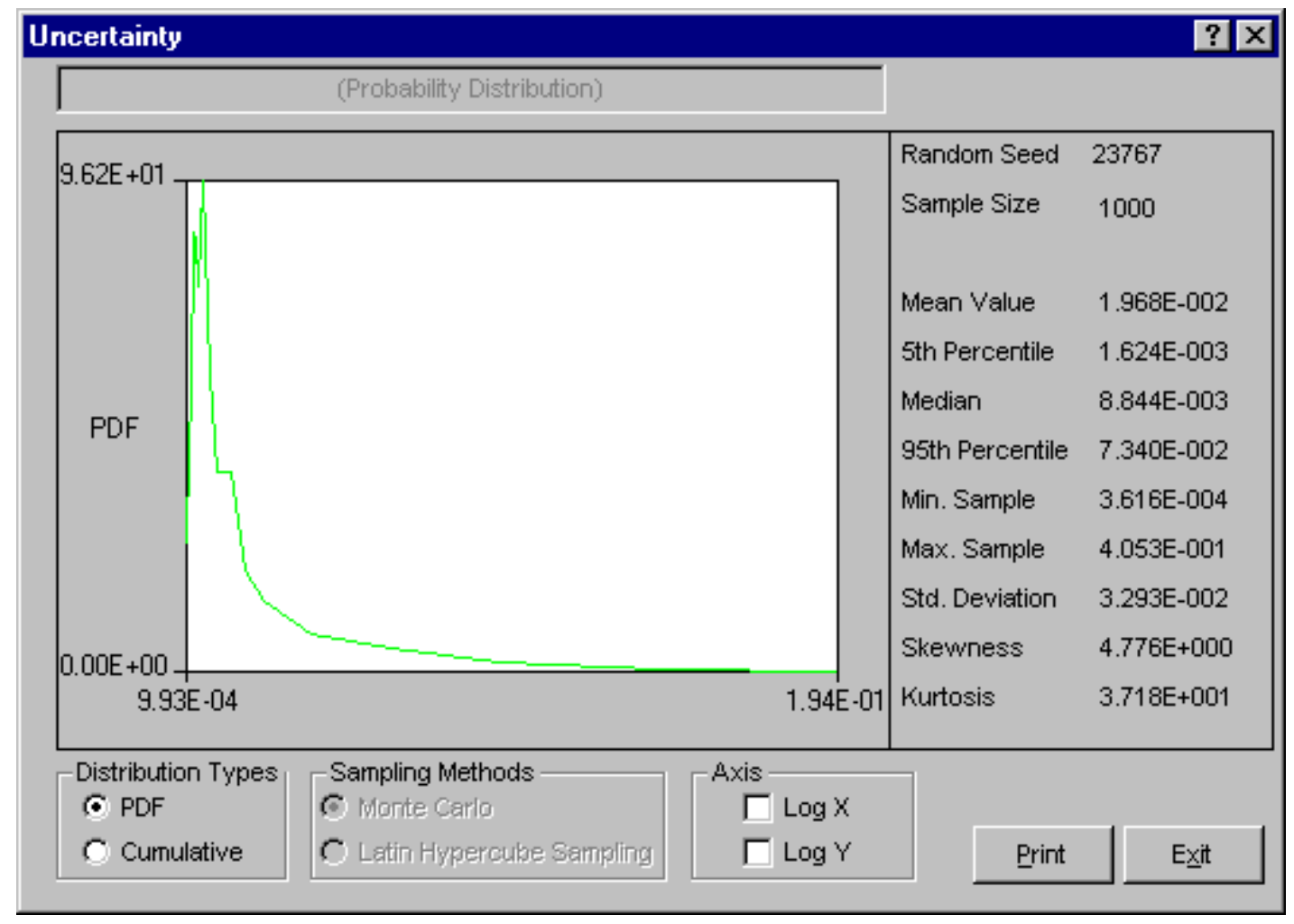

Print - Print the plot as displayed. The Print common dialog will be launched where you can select the desired printer.

Exit - Close the Uncertainty plot dialog.

\subsubsection{Distribution Types}


Select the type of graph to be displayed:

PDF - $\quad$ Default) - Displays a graph showing the Probability Density Function.

Cumulative - Displays a graph showing the Cumulative Density Function.

\subsubsection{Sampling Methods}

The selected radio button indicates the sampling method used for the uncertainty calculation. Note the sampling method used cannot be changed here. In order to view results using a different sampling method, the uncertainty analysis must be re-run using the desired sampling method.

Monte Carlo - $\quad$ Displays a graph that is based on Monte Carlo Sampling of the defined uncertainty distribution.

Latin Hypercube Sampling - Displays a graph that is based on Latin Hypercube Sampling of the defined uncertainty distribution.

\subsubsection{Axis}

$\log$ X - Produces a graph where the Log base 10 of the X-Values are used instead of just the XValues.

Log Y - Produces a graph where the Log base 10 of the Y-Values are used instead of just the YValues.

\subsection{Viewing Fault Trees Results}

\subsubsection{Viewing Fault Tree Data}

\section{PURPOSE}

The data displayed from this option originates from the fault tree analyses previously performed. One or more fault trees can be viewed using this option.

\section{STEPS}

1. From the SAPHIRE menu select Fault Tree. The Fault Tree List dialog will be displayed.

2. Highlight the desired fault tree(s) and right-click to invoke the pop-up menu.

3. Choose View Results from the menu. The Fault Tree Review dialog will be displayed. 


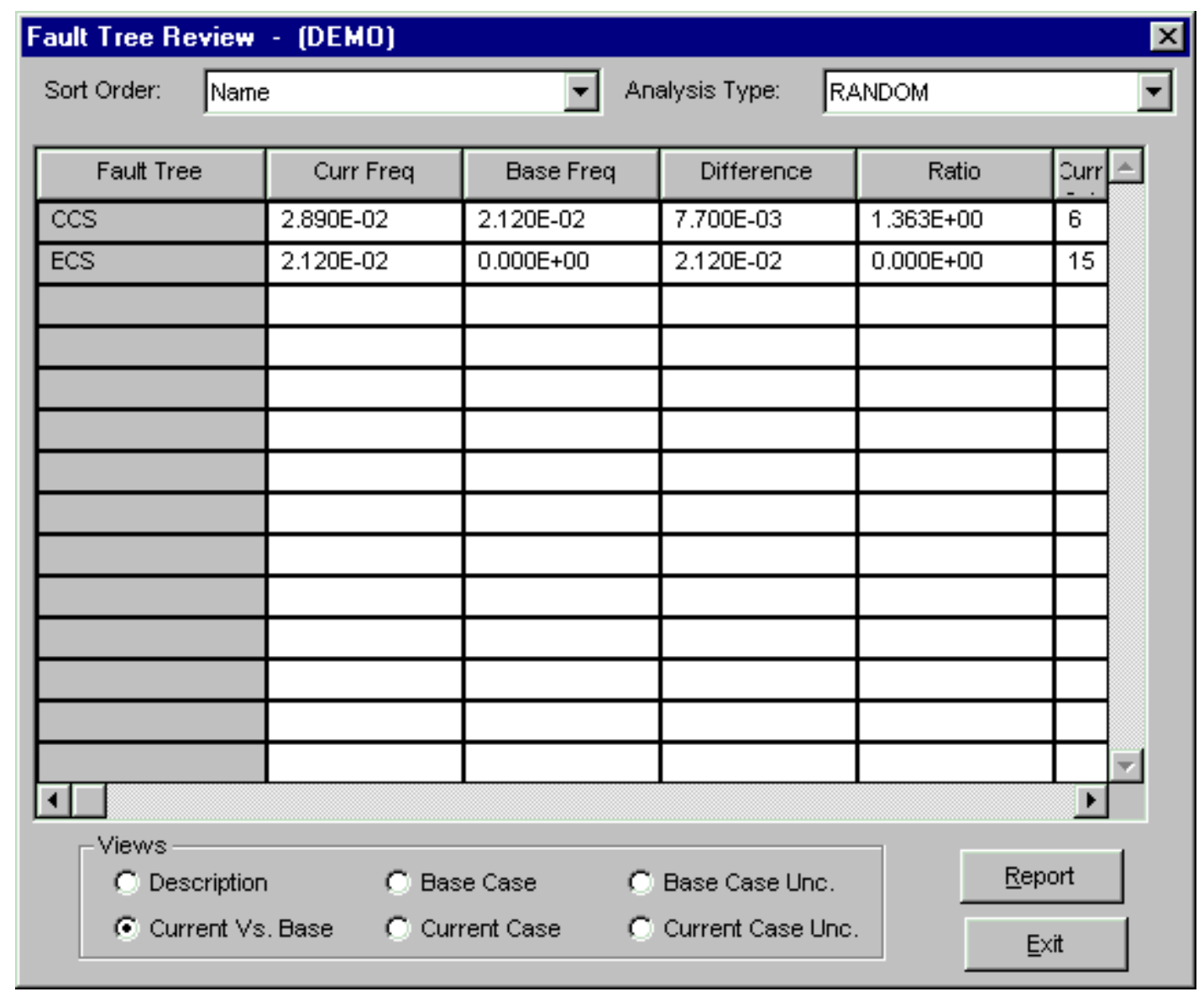

The selected fault trees are displayed in this dialog. The Fault Tree column contains the primary names of the fault trees. Other columns' titles and data vary based on the current "View".

Sort Order - Select from the drop-down list to sort results by Name, Base case min cut frequency, Current case min cut frequency, Delta min cut (base-current), Base case mean frequency, Current mean frequency, Delta mean frequency.

Report - $\quad$ Produces a report of the data currently displayed. The output can go to the Report Viewer, a printer, or a file.

Exit -

Close the Fault Tree Review dialog.

\subsubsection{View Options}

Description -

Current Vs. Base -

Base Case -
Includes the full description. (60 character maximum) Includes the current case cut set probability, base case cut set probability, the probability difference (current - base), ratio, current case cut set count, base case cut set count, the cut set count difference (current - base).

Includes base case values for cut set probability, mean, $5^{\text {th }}$ percentile, median, $95^{\text {th }}$ percentile, probability truncation, and size truncation used when solving. 
Current Case -

Base Case Uncertainty -

Current Case Uncertainty -
Includes current case values for cut set probability, mean, $5^{\text {th }}$ percentile, median, $95^{\text {th }}$ percentile, probability truncation, and size truncation used when solving.

Includes base case values for cut set probability, mean, $5^{\text {th }}$ percentile, median, $95^{\text {th }}$ percentile, minimum, maximum, standard deviation, skewness, kurtosis, sample size, and random number seed.

Includes current case values for cut set probability, mean, $5^{\text {th }}$ percentile, median, $95^{\text {th }}$ percentile, minimum, maximum, standard deviation, skewness, kurtosis, sample size, and random number seed.

\subsection{Time Dependent Analysis}

\subsubsection{Time Dependent Analysis}

\section{PURPOSE}

The time dependent analysis function allows the user to calculate a time profile of the selected fault tree or sequence cut sets. The user defines a start and stop "mission" time and a delta time or number of samples. From this data, SAPHIRE calculates a probability for each event in the cut sets using the start mission time. The cut sets are quantified and a min cut upper bound is determined. The mission time is then incremented by a delta and new event probabilities and a new min cut upper bound are calculated. Events that do not have mission time as part of their calculation type are not changed. This process continues until the mission time equals the stop time, at which point the results of the distribution are displayed. This allows the user to calculate a distribution of results for events that are dependent on time.

\section{STEPS}

1. From the SAPHIRE menu select Fault Tree/Sequence. The Fault Tree List/Sequences dialog will be displayed.

2. Highlight the desired list item(s) and right-click to invoke the pop-up menu.

3. Choose Time Dependent from the menu. The Time Dependent Calculation Values dialog will be displayed.

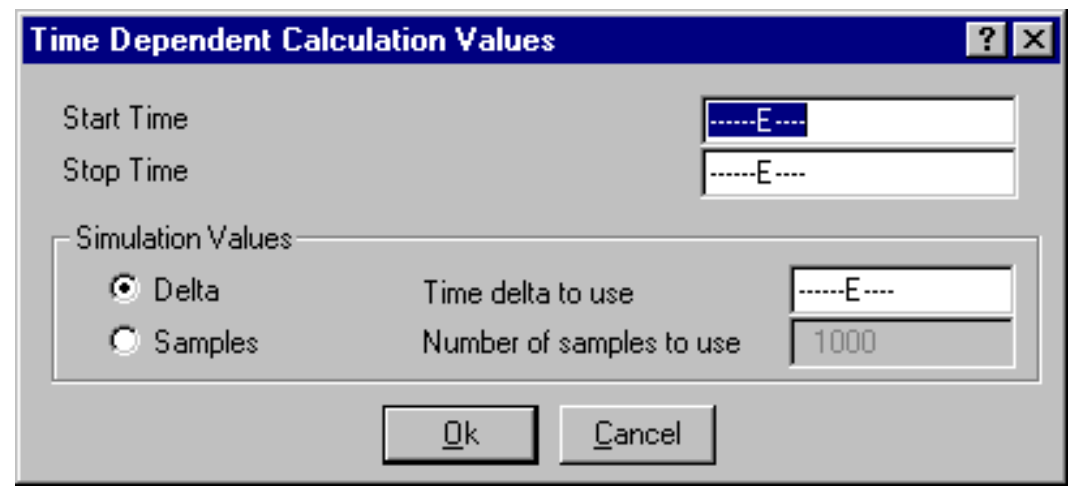


Start Time - The time, in hours, to begin the analysis.

Stop Time - $\quad$ The time, in hours, to end the analysis.

\section{Simulation Values}

Delta -

Time delta to use -

Sample -
If this radio button is selected, use the delta indicated in the Time delta to use field to determine the number of samples used in the analysis. The smaller the delta, the greater the number of samples.

The incremental change, in hours, for which the analysis will be performed beginning at the Start Time and terminating at the Stop Time.

If this radio button is selected, use the Number of samples to use field to determine the time delta used in the analysis. The greater the number of samples, the smaller the delta.

Number of samples to use - The total number of samples used for this analysis. The number of samples will determine the time delta to be used.

Ok - $\quad$ Begin the time analysis.

Cancel - $\quad$ Close the Time Dependent Calculation Values dialog without calculating the time profile. 


\section{EVENT TREE ANALYSIS}

\subsection{Event Tree Analysis}

\section{PURPOSE}

An event tree is a model of relationships of failed or successful fault tree tops.

This option allows you to graphically build and edit event trees and edit sequence logic. The ability to define linkage and partition rules is also provided. The Event Tree List shows the event trees in the current project.

\section{KEY TO FLAGS}

Located to the right of each event tree is a set of flags that indicate the status of the event tree: A " + " in the first column indicates that event tree linkage rules exist.

A "+" in the second column indicates event tree level partition rules exist.

A "-" in either column indicates that the respective rules exist but are not compiled.

An "s" indicates that an event tree is a sub-tree (a tree that is transferred to), rather than a front-line event tree.

\section{STEPS}

1. From the SAPHIRE menu select Event Tree. The Event Tree List dialog will be displayed.

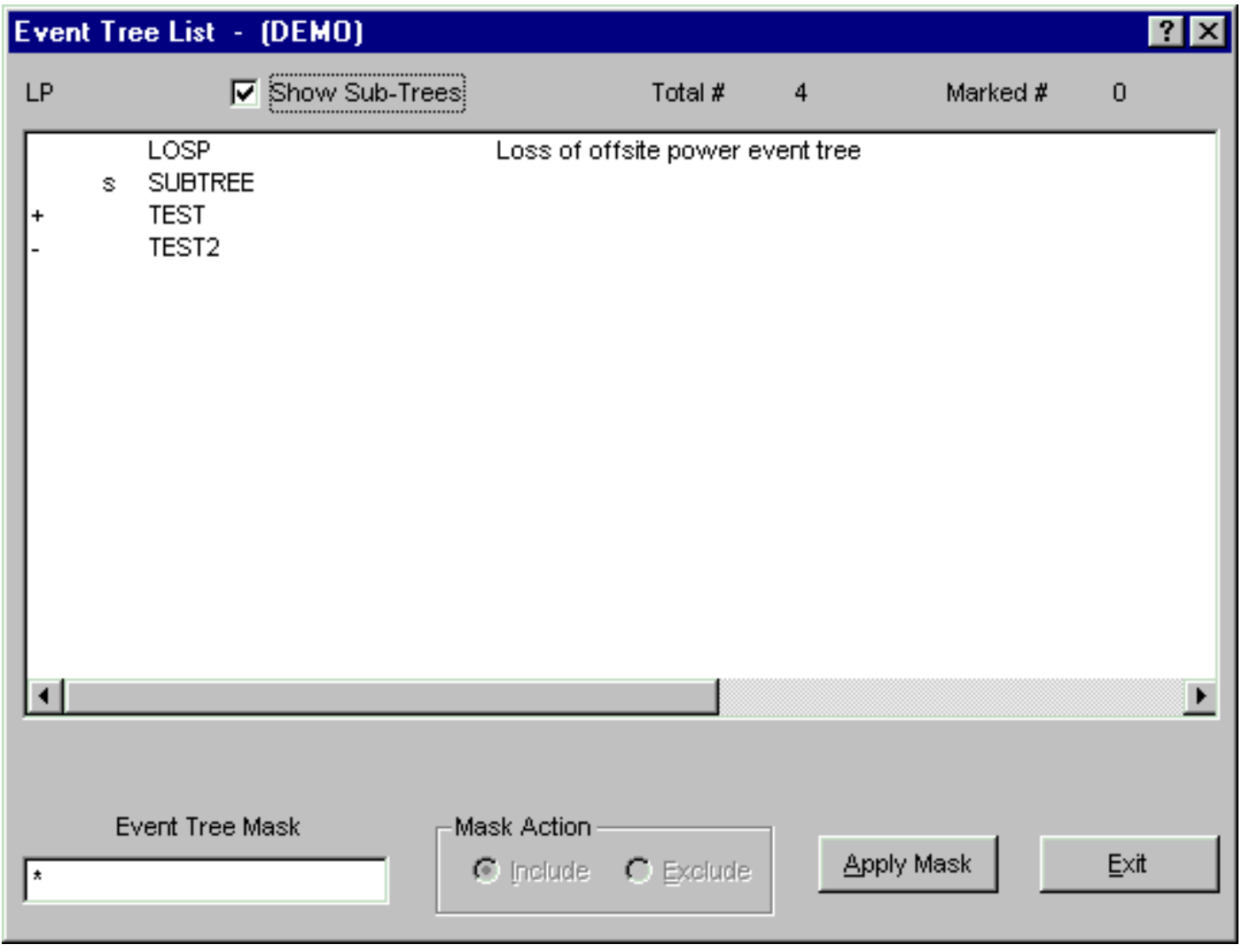


From this dialog, you can select event trees using the mouse or the Mask feature. The following functions may be accessed from the pop-up menu:

Show Sub-Trees - When checked, include sub-trees in the list of event trees.

Pop-up Menu Options - The following functions may be accessed from the pop-up menu.

Add Event Tree - $\quad$ Add a new event tree record to the current project database.

Edit Graphics - $\quad$ Build and edit event tree diagrams.

Edit Rules - $\quad$ Define linkage rules.

Edit Partition Rules - Define the end states to be associated with each cut set in a sequence.

Link Trees - Generate sequence logic.

Edit End States - $\quad$ Edit event tree sequences after an event tree has been created.

\subsection{Event Tree Graphics}

\subsubsection{Event Tree Graphics}

\section{PURPOSE}

This option allows you to construct or edit an event tree diagram from the graphical Event Tree Editor. An event tree is a model of relationships of failed or successful fault trees or tops. An event tree diagram is a graphical representation or picture of that model.

You may start building the diagram from scratch or from an existing file to generate or modify logic. The initiating event, basic events, sequences, and end states are tied to the SAPHIRE database. They are automatically added to the database as the event tree is being constructed. Various editing functions such as cut, paste, and copy along with file functions such as save and print are provided.

\section{STEPS}

1. From the SAPHIRE menu select Event Tree. The Event Tree List dialog will be displayed.

To create a new diagram:

Do not highlight an event tree

To edit an existing file:

Highlight the desired event tree in the list

2. Right-click to invoke the pop-up menu and choose Edit Graphics. The Event Tree Editor will be displayed. The name of the current event tree will be displayed in the title bar. 


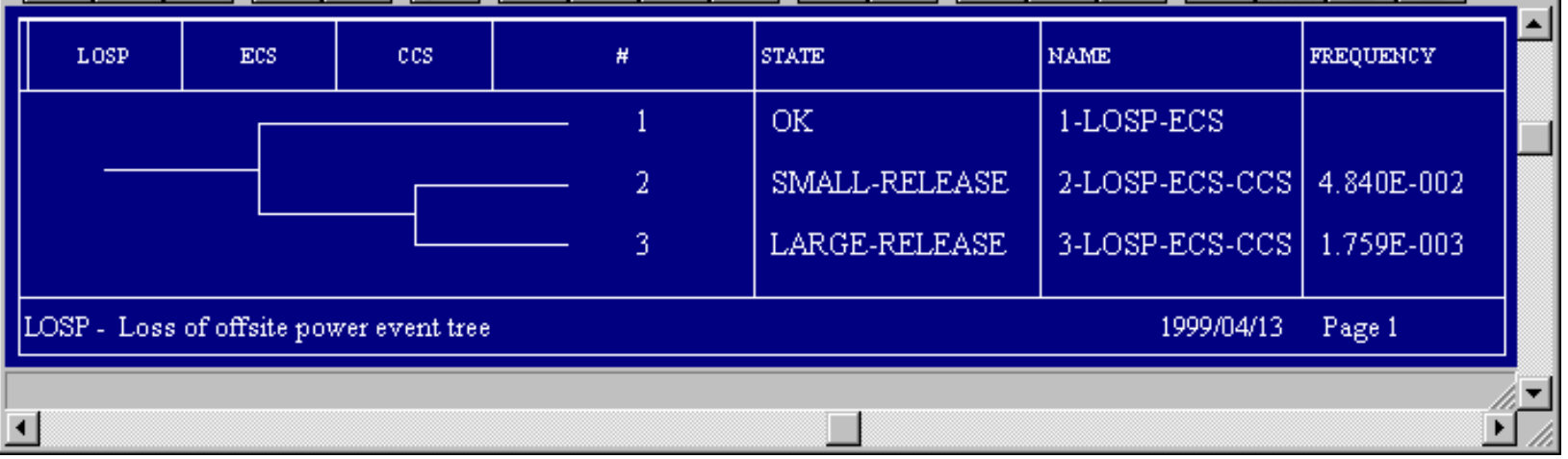

Note that an up branch indicates success of the top event while a down branch indicates a failure of the top branch.

Options are available from the menu and /or the toolbar.

\section{Menu Options}

File - $\quad$ File manipulation functions such as open, save, print, etc.

Edit - $\quad$ Editing functions such as copy and paste along with attribute setting.

View - Control of visual display parameters such as zoom, refresh, etc.

Help - SAPHIRE on-line help.

\section{Toolbar Buttons}

\begin{tabular}{|c|c|c|c|}
\hline 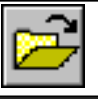 & $\begin{array}{l}\text { Open an event tree graphics file } \\
\text { from disk. }\end{array}$ & 尛 & Erase \\
\hline 兴 & $\begin{array}{l}\text { Save the event tree graphics file to } \\
\text { disk. }\end{array}$ & $\bar{\square}$ & Create a text object. \\
\hline 国 & Print the event tree graphics file. & TOP & Add a Top \\
\hline 泾-C & Backward transfer & 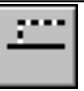 & Add branch above \\
\hline 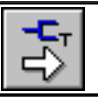 & Forward transfer & $\overline{\vdots . . .}$ & Add branch below \\
\hline -3닫 & Page tree & 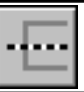 & Pass \\
\hline 20 & Cut & $-\mathrm{Ct}$ & Zoom \\
\hline$t_{-}^{-5}$ & Copy & - & Fit to window \\
\hline 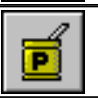 & Paste & 10 & Select the pick cursor \\
\hline & & 藏 & Select the text pick cursor \\
\hline
\end{tabular}




\subsubsection{Event Tree Graphics Definitions}

\section{PURPOSE}

The following definitions are used with reference to the SAPHIRE graphical Event Tree Editor.

Object - $\quad$ The building blocks of an event tree diagram. They include tops or fault trees, sequence branches or paths (lines that indicate the failure and success of the tops), end states, and descriptive text. Each object has attributes that may include color, size, and height.

Pick Box - $\quad$ An invisible rectangle that surrounds each sequence branch, top and end state. It defines the area of the object where the mouse cursor must be to select the object.

Sequence Path - A sequence is a path through an event tree. Each sequence will have a unique number and can have a name, end state, and two other user-defined fields. The unique number will start at 1 and increment for every sequence that is added to the event tree. A sequence with only an initiator and one top will have two sequences: the top sequence will be sequence number 1 , and the bottom sequence will be sequence number 2 .

Branch Point - Directly beneath each top in the diagram is its branch point. A sequence is a line that connects one or more branch points. At these branch points, the sequence path line can continue as one line to the right (pass-through), or branch into two or more lines.

Branch - $\quad$ A path that begins at a branch point and continues to the right is a branch. All subsequent branches in a tree are included as a part of the branch.

Pass Through - A sequence path line that passes through a branch point without branching is a pass-through. This indicates that the corresponding top's success or failure has no impact on the sequence.

\subsubsection{Event Tree Graphics - Mouse Usage}

All mouse actions are defined in the SAPHIRE graphical Event Tree Editor are for a standard right handed two-button mouse.

Click - $\quad$ The left mouse button is pressed and released.

Right-click - The right mouse button is pressed and released.

Double-click - The left mouse button is pressed and released two times in rapid succession.

\subsubsection{Selecting Objects}

\subsubsection{Selecting Objects}




\section{PURPOSE}

Prior to manipulating (e.g., moving, copying, etc.) an object in an event tree diagram, that object or group of objects must be selected. By choosing the Pick button you can select a branch, top, or end state .

\subsubsection{Selecting a Branch}

\section{PURPOSE}

This option describes how to select a branch.

\section{STEPS}

1. Invoke the Pick cursor $\mathrm{rth}_{\mathrm{J}}$ by choosing the Pick button from the button bar.

2. Move the cursor on top of the branch you wish to select.

3. Click the mouse.

4. The branch will be redisplayed in the highlight color. The highlight color is defined as the inverse of the line color.

\subsubsection{Selecting an End State}

\section{PURPOSE}

This option describes how to select an end state.

\section{STEPS}

1. Invoke the Pick cursor ${ }^{n}$ by choosing the Pick button from the button bar.

2. Move the cursor on top of the end state you wish to select.

3. Click the mouse.

4. The end state will be surrounded by a pick box.

\subsubsection{Selecting a Top or Fault Tree}

\section{PURPOSE}

This option describes how to select a Top or Fault Tree.

\section{STEPS}

1. Invoke the Pick cursor $\mathrm{r}_{7}$ by choosing the Pick button from the button bar.

2. Move the cursor on over the Top you wish to select.

3. Click the mouse. 
4. The Top will be surrounded by a pick box.

\subsubsection{Selecting Text}

\section{PURPOSE}

This option describes how to select a Text object.

Because text is a free-floating object, its pick box can overlap those of Tops, Branches, or End States. Therefore, there is an order of selection. Because text has the lowest priority when selecting, there is a special text cursor that allows you to pick text that is superimposed on another object. If the desired text is not close to another object, you can select it by using the Pick cursor the If the desired text is on top of or close to another object, you should follow the steps below.

\section{STEPS}

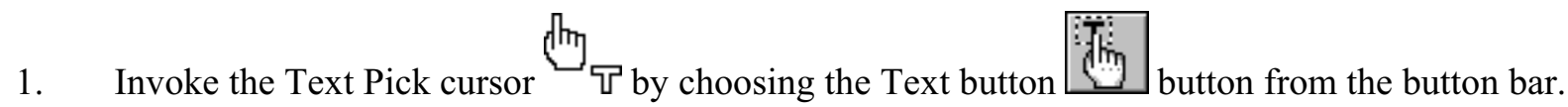

2. Move the cursor over the text you wish to select.

3. Click the mouse.

4. The text will be surrounded by a pick box.

\subsubsection{Adding and Modifying Objects}

\subsubsection{Add Top}

\section{PURPOSE}

This option allows you to add a new top or fault tree to the current diagram.

\section{STEPS}

1. Choose the Top object button $\mathrm{TOP}$ from the button bar. The cursor will change to the Top cursor TOF.

2. Move the cursor to the top in the header that will follow the top you are adding. To add a top after the rightmost top, move the cursor to the right of the last top.

3. Click the mouse. The Edit Event dialog will be displayed

If you choose the $\mathbf{O K}$ button on the Edit Event dialog, the new top will be inserted into the diagram with all sequence paths passing through it.

\subsubsection{Modify Top}

\section{PURPOSE}

This option allows you to change the currently selected top or fault tree or its attributes. 


\section{STEPS}

1. Select the top using the Pick ${ }^{\text {rth }}$ cursor.

2. Right-click. The Edit Event dialog will be displayed.

\subsubsection{Add Text}

\section{PURPOSE}

This option allows you to add text to the diagram. Text can be "free-form" or attached to a branch, depending on the method selected.

\section{STEPS}

1. Choose the Text object button from the button bar. The cursor will change to the Text cursor $[$.

2. Position the text cursor at the desired location.

3. Click the mouse. The Edit Text dialog will be displayed.

Note: The position of text added in this way is independent from the rest of the diagram. When the diagram is resized, the text may appear to move (actually, the text stays in place; the rest of the diagram moves). To add text to a relative position within the diagram, use the following steps.

\section{STEPS}

1. Select the branch where you wish to attach text.

2. Right-click to invoke the pop-up menu and select Edit Text.

3. The Edit Text dialog will be displayed.

Text added or modified in this way will be "attached" to the designated branch and will reposition itself to remain next to the branch whenever the branch moves.

\subsubsection{Modify Text}

\section{PURPOSE}

This option allows you to change existing text in the diagram. 


\section{STEPS}

1. Select the desired text using the Text Pick cursor

2. Right-click. The Edit Text dialog will be displayed.

$\mathrm{OR}$, if the text is attached to a branch, you may do the following:

3. Select the branch containing the text you wish to edit.

4. Right-click to invoke the pop-up menu and select Edit Text. The Edit Text dialog will be displayed.

TIP: If the Edit Text dialog does not contain any text, then the text is not attached to the branch. In that case, use the first method to edit the text. If you would like to attach the text to the branch, first cut the text from the Edit Text dialog to the clipboard, then use the second method to bring up another Edit Text dialog, and finally, copy the text from the clipboard.

\subsubsection{Edit Text Dialog}

\section{PURPOSE}

This option allows you to set the attributes of a new text object or change the attributes of the currently selected text object. This dialog is displayed any time text is added or modified.

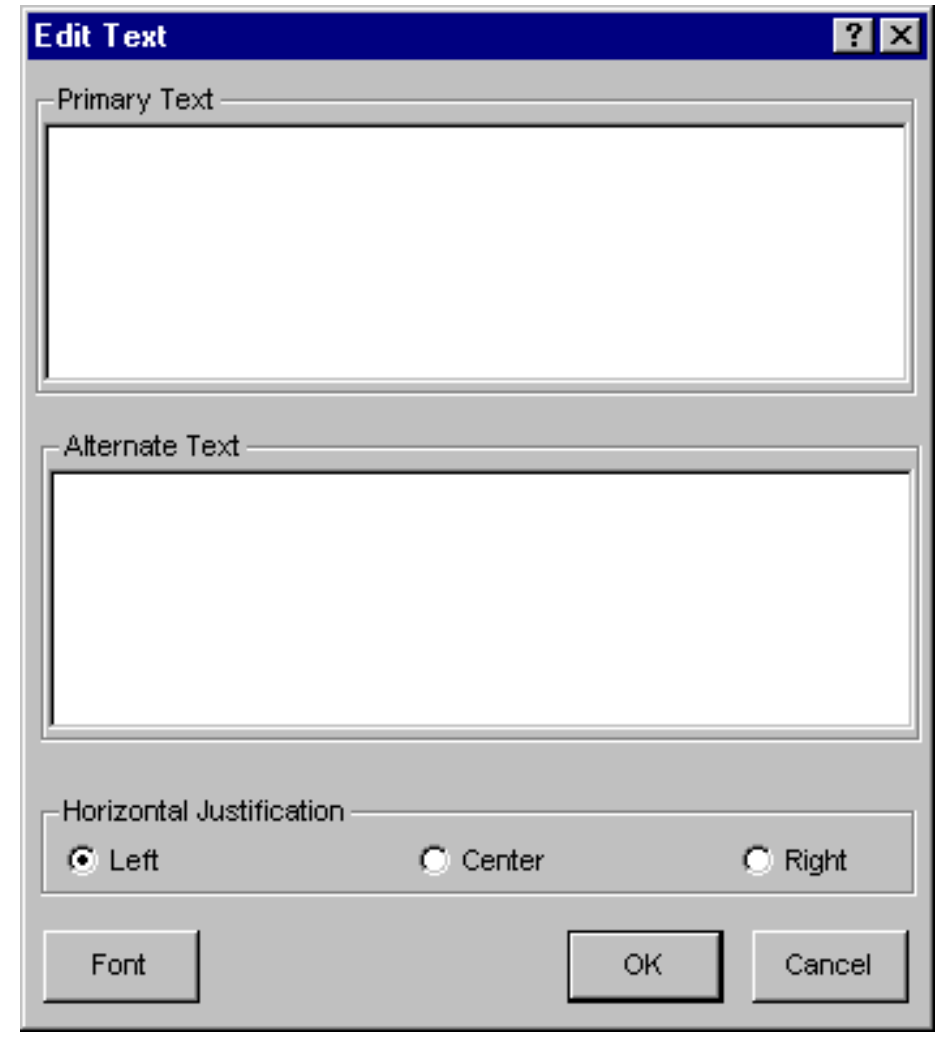


Primary Text -

Alternate Text -

Horizontal Justification -

Font -

OK -

Cancel -
Descriptive text for the event tree. Maximum of 600

alphanumeric, upper- and lowercase characters.

Descriptive text for the event tree. Maximum of 600 alphanumeric, upper- and lowercase characters. This feature allows text to be displayed and reported using a different locale. For this text to be displayed on reports instead of the Primary text, choose Use alternate names for display in the Define Constants option.

Set the horizontal alignment of the text.

Choose the font with which to display the text.

Close the Edit Text dialog and add or modify the text in the diagram.

Close the Edit Text dialog without adding or modifying the text in the diagram.

\subsubsection{Edit Event}

\subsection{Edit Event Dialog}

\section{PURPOSE}

This option allows you to set the attributes of a new event or change the attributes of the currently selected event. This dialog is displayed any time a top is added or modified . 


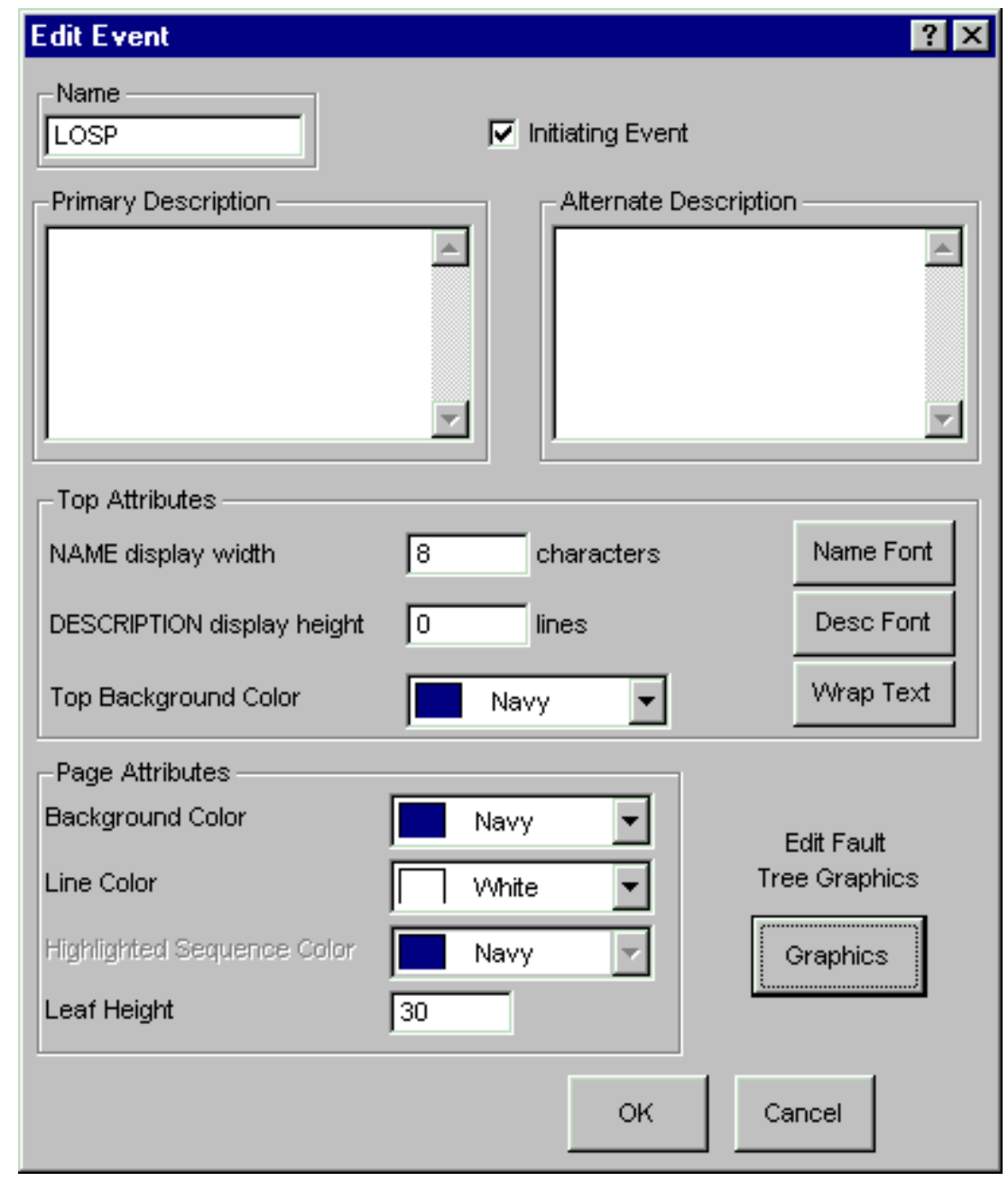

OK - Apply the addition of or modifications to the top and close the Edit Event dialog.

Cancel - Close the Edit Event dialog without applying the addition of or modifications to the top.

\subsection{Name}

The name of the top. Maximum of 24 alphanumeric characters.

\subsection{Description}

Brief description of the top. Maximum of 80 alphanumeric, upper- and lowercase characters.

\subsection{Initiating Event}

Select this check box if the top is an initiating event. (Only enabled on the first or leftmost top.)

\subsection{Wrap Text}

Choose this button to automatically fit the text inside the description box, using the specified Description Display Height 


\subsection{Edit Fault Tree Graphic}

Jump to the Fault Tree Graphic Editor to edit the fault tree graphic that corresponds to the event tree's selected top. The Event Tree Graphic Editor remains open, but is inaccessible until the fault tree editor is closed.

\subsection{Highlighted Sequence Color}

Select the color to be used for the highlighted sequence from the drop-down list. (Not yet available.)

\subsubsection{Sequence Information Columns}

\subsubsection{Modify Sequence Column Location}

\section{PURPOSE}

Change the location of a sequence information column in the diagram.

\section{STEPS}

1. Choose the Pick button

2. Position the Pick cursor $\mathrm{rth}_{\mathrm{b}}$ over the line to the left of the desired column.

3. The cursor will change to the Header cursor $\Leftrightarrow$.

4. Drag the mouse to the desired location. The cursor will change to the Move Column cursor

5. Release the mouse and the column will be moved to the new location.

\subsubsection{Modify Sequence Information}

\subsection{Modify Sequence Information}

\section{PURPOSE}

This option allows you to change the data in the sequence information columns in the diagram.

Each sequence path has some additional information that can help define it. This information includes the name of the sequence, the end state of this sequence, its frequency, and a user-defined field. If the sequence continues through another event tree then the end state is the name of the transfer tree. You have an option to display this additional information and change its location.

\section{STEPS}

1. Select the desired sequence using the Pick thrt

2. Right-click. The Edit Sequence dialog will be displayed. 


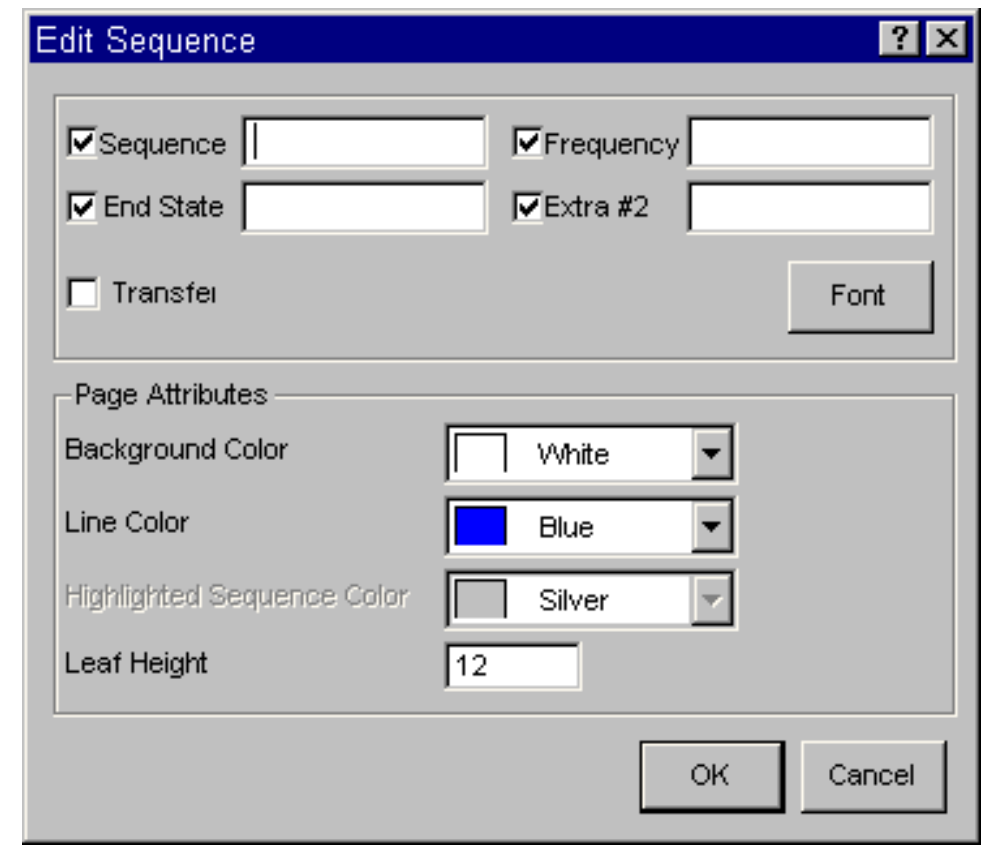

OK - Close the Edit Sequence dialog and apply the changes the diagram.

Cancel - Close the Edit Sequence dialog without applying the changes to the diagram.

\subsection{Sequence}

The name of the sequence. Maximum of 24 alphanumeric characters. With both the check box to the left of this field selected and the View | Show Sequence Names option selected, the name in this field will be displayed.

\subsection{End State}

The name of the sequence end state. Maximum of 24 alphanumeric characters. With both the check box to the left of this field selected and the View | Show End State Names option selected, the name in this field will be displayed.

\subsection{Frequency}

Extra information column \#1 for the sequence. Can contain the frequency for the sequence. Maximum 24 alphanumeric characters. With both the check box to the left of this field selected and the View | Show Frequency option selected, the data in this field will be displayed.

\subsection{Extra \#2}

Extra information column \#2 for the sequence. Maximum 24 alphanumeric characters. With both the check box to the left of this field selected and the View | Show Extra-\#2 option selected, the data in this field will be displayed.

\subsection{Transfer}


Check this box if the sequence continues in another event tree. The end state field contains the name of the transfer event tree.

\subsubsection{Modify Sequence Column Header}

\subsection{Modify Sequence Column Header}

\section{PURPOSE}

Change the sequence information column headers in the diagram.

\section{STEPS}

2. Position the Pick cursor over the sequence column header area. (Ensure that a sequence is not currently selected or the Edit Sequence dialog will be displayed instead.)

3. Right-click. The Edit Sequence Header dialog will be displayed.

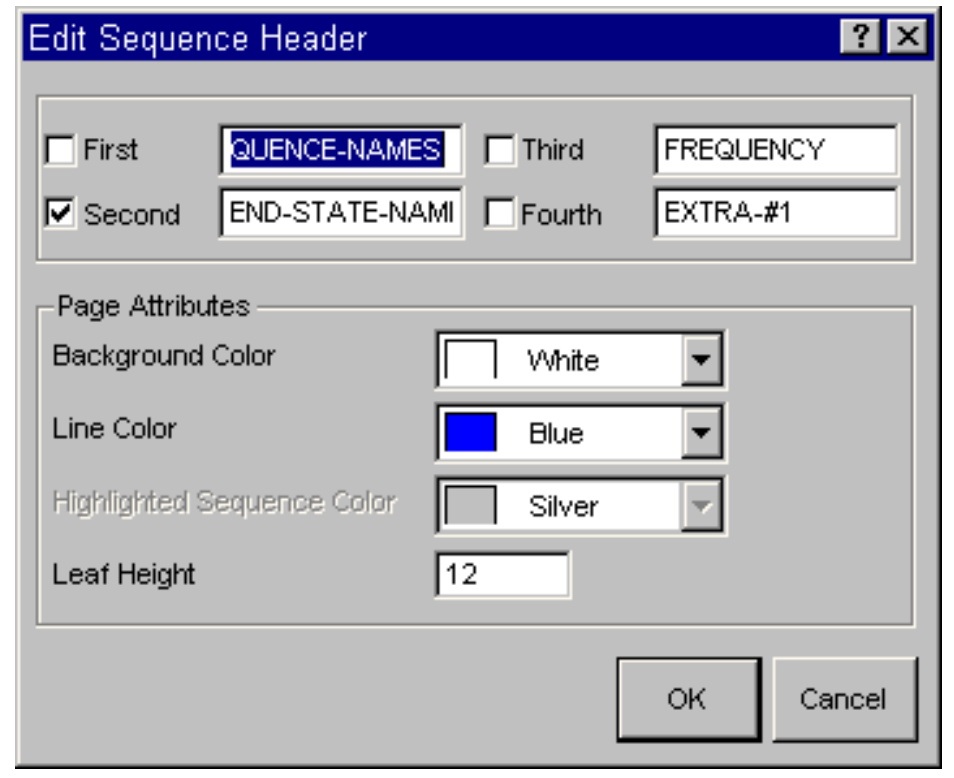

OK - Close the Edit Sequence Header dialog and apply the changes the diagram.

Cancel - Close the Edit Sequence Header dialog without applying the changes to the diagram.

\subsection{First}

The sequence column header. Maximum 24 alphanumeric characters. The data in this column will always contain the sequence names. Select the check box to the left of this field to display this column.

\subsection{Second}


The sequence end state column header. Maximum 24 alphanumeric characters. The data in this column will always contain the end state or transfer tree names. Select the check box to the left of this field to display this column.

\subsection{Third}

The header for the third sequence column. Maximum 24 alphanumeric characters. This column can contain whatever information you wish, however, options exist elsewhere in the program that will put the frequency of the sequence in this column. Select the check box to the left of this field to display this column.

\subsection{Fourth}

The header for extra information for the sequence. Maximum 24 alphanumeric characters. This column can contain whatever information you wish. Select the check box to the left of this field to display this column.

\subsubsection{File Menu}

\subsubsection{File}

These options provide file manipulation functions.

$\begin{array}{ll}\text { New - } & \text { Create a new file in the current window. } \\ \text { Open - } & \text { Open a file into the current window. } \\ \text { Save - } & \text { Save the file in the current window to disk. } \\ \text { Save As - } & \text { Save the file in the current window with a new file name. } \\ \text { Export As - } & \text { Save the file in RTF, EMF, or WMF format. } \\ \text { Page Tree - } & \text { Copy the highlighted event tree branch to a new or existing diagram. } \\ \text { Forward Transfer - } & \text { Follow a transfer to another event tree. } \\ \text { Backward Transfer - } & \text { Display a previously viewed transfer. } \\ \text { Print - } & \begin{array}{l}\text { Print the file in the current window. } \\ \text { Exit - }\end{array} \\ & \text { Terminate the Event Tree Editor. If changes have been made you will be } \\ & \text { queried if you want to save the file. }\end{array}$

\subsubsection{New}

\section{PURPOSE}

This option will allow you to clear the window and begin building a new diagram.

\section{STEPS}

1. From the Event Tree Editor menu select File | New. The Initiating Event or Top Name dialog will be displayed. 


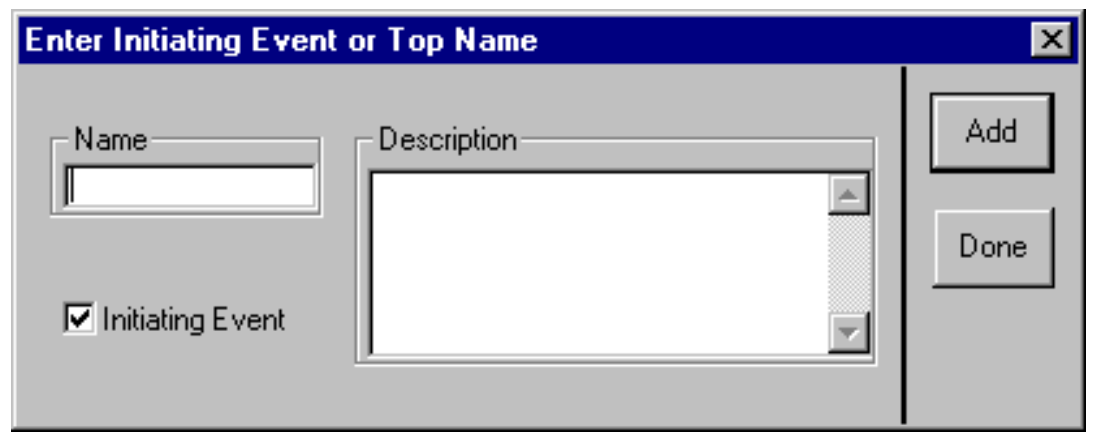

Name -

Description Initiating Event -

Add -

Done -
The name of the top (basic event).

The description the top (basic event).

Select this check box if the top is an initiating event.

Add the top to the top list. The Event Name dialog will be displayed. After entering at least two basic events, the Event Name dialog will be closed, the contents of the window will be initialized with the tops entered.

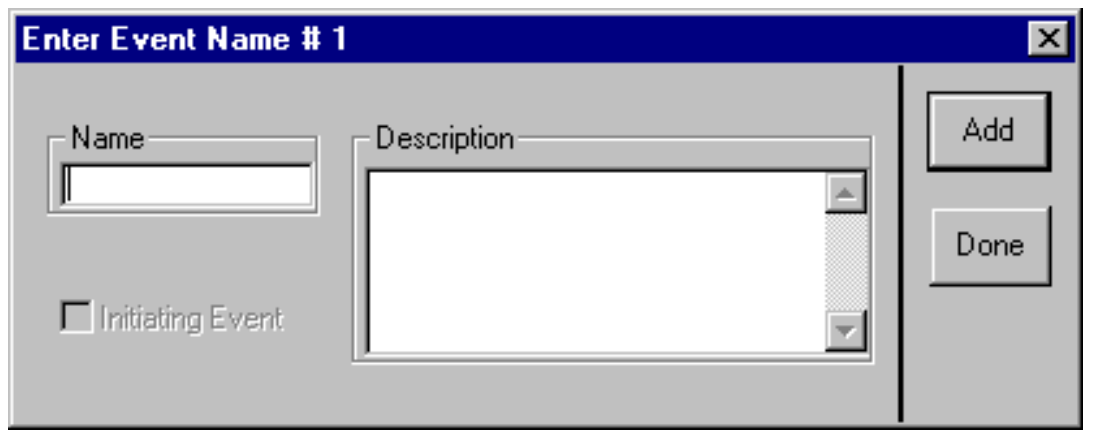

Enter data for each top to be included in the diagram, choosing Add after each entry. After adding the last top, choose the Done button.

If you have not saved the current diagram you will be prompted by a Caution dialog to do so.

\subsubsection{Open}

\section{PURPOSE}

This option will allow you to select an existing diagram (i.e., event tree graphics file) to view or edit.

\section{STEPS}

1. From the Event Tree Editor menu select File | Open.

$$
\text { OR }
$$

2. Choose the Open Diagram button on the button bar. 
3. The Open Event Tree common dialog will be displayed listing all the event tree diagrams (.EGT files) in the current project directory

4. Select the desired diagram and choose Open,

$O R$

5. Double-click on the diagram name.

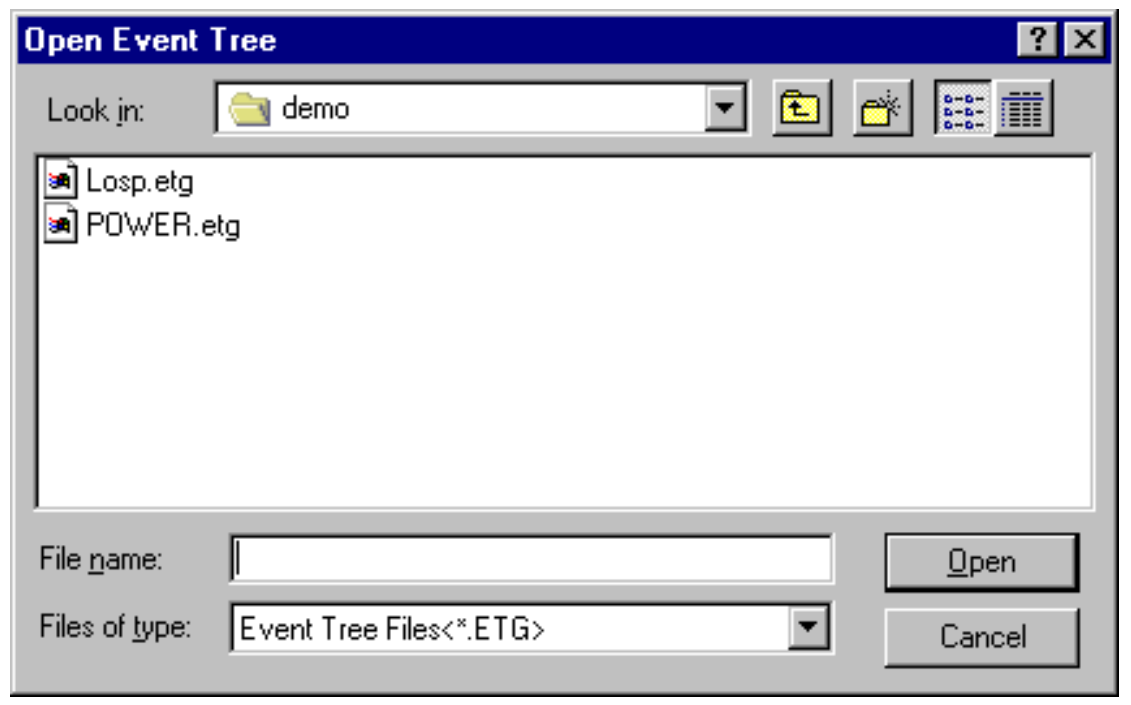

Look in - $\quad$ Lists the available folders and files. The box directly below it shows the contents of the current folder. The down-arrow to the right of this field shows how the folder fits into your system hierarchy.

Moves up one level in the system hierarchy.

Creates a new folder at the current level.

Displays the icons and names of the contents of the current folder.

Displays the icons, names, size, type, date and time modified, and archive flags of the contents of the current folder.

File Name - Contains the name of the file in which the diagram will be saved.

Files of Type - Lists the types of files to display. This is useful for narrowing the list of files displayed to only those you are interested in. In this case, only ".ETG" files are listed.

Open - $\quad$ Open the highlighted diagram file and close the Open Event Tree dialog.

Cancel Close the Open Event Tree dialog without selecting a different diagram.

Double-click - Open the diagram file and close the Open Event Tree dialog.

If you have selected a diagram to open and you have not saved the current one you will be prompted by a Caution dialog to do so.

\subsubsection{Save}




\section{PURPOSE}

This option will save any changes made in the current event tree diagram to the file presently open. If this is a new diagram, then you will be allowed to name the file. The logical representation of the diagram will also be saved in the SAPHIRE database.

\section{STEPS}

1. From the Event Tree Editor menu select File | Save.

$O R$

2. Click the Save Diagram button

3. If this is not a new diagram, the data is saved.

OR

4. If this is a new diagram, see Saving Changes to a Different File .

\subsubsection{Saving Changes to a Different File}

\section{PURPOSE}

This option will save any changes made in the current event tree diagram to a file you select. The logical representation of the diagram will also be saved in the SAPHIRE database.

\section{STEPS}

1. From the Event Tree Editor menu select File.

2. Choose Save as from the menu

3. The Save Event Tree common dialog will be displayed listing all the event tree diagrams (.ETG files) in the current project directory.

4. Enter the name of the file in the File Name field, OR

5. Select the desired diagram file and choose Save, $O R$

6. Double-click on the diagram name. 


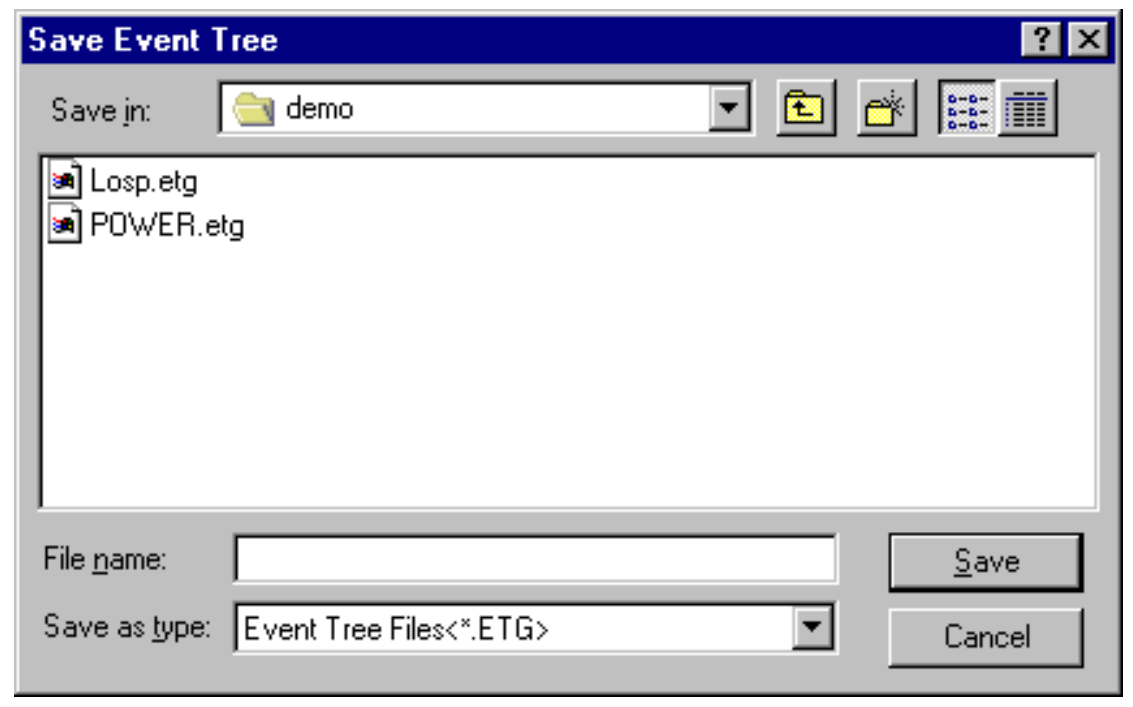

Save in - $\quad$ Lists the available folders and files. The box directly below it shows the contents of the current folder. The down-arrow to the right of this field shows how the folder fits into your system hierarchy.

Moves up one level in the system hierarchy.

Creates a new folder at the current level.

Displays the icons and names of the contents of the current folder.

Displays the icons, names, size, type, date and time modified, and archive flags of the contents of the current folder.

File Name - Contains the name of the file in which the diagram will be saved.

Files of Type - Lists the types of files to display. This is useful for narrowing the list of files displayed to only those you are interested in. In this case, only .ETG files are listed.

Save - $\quad$ Save the current diagram to the file in the File name field and close the Save Event Tree dialog. If an existing file was selected, a warning dialog will be displayed.

Double-click - Save the current diagram to the file in the File name field and close the Save Event Tree dialog.

Cancel - $\quad$ Close the Save Event Tree dialog without saving the diagram.

\subsubsection{Export As}

\section{PURPOSE}

This option will convert the event tree diagram to a file in Enhanced Metafile (EMF), Windows Metafile (WMF) format, or Rich Text Format (RTF). 


\section{STEPS}

1. From the Event Tree Editor menu select File.

2. Choose Export as from the menu

3. The Export Event Tree As common dialog will be displayed listing all the EMF, WMF, OR RTF files in the current project directory.

4. Enter the name of the file in the File Name field,

$O R$

5. Select the desired file and choose Save,

OR

6. Double-click on the file name.

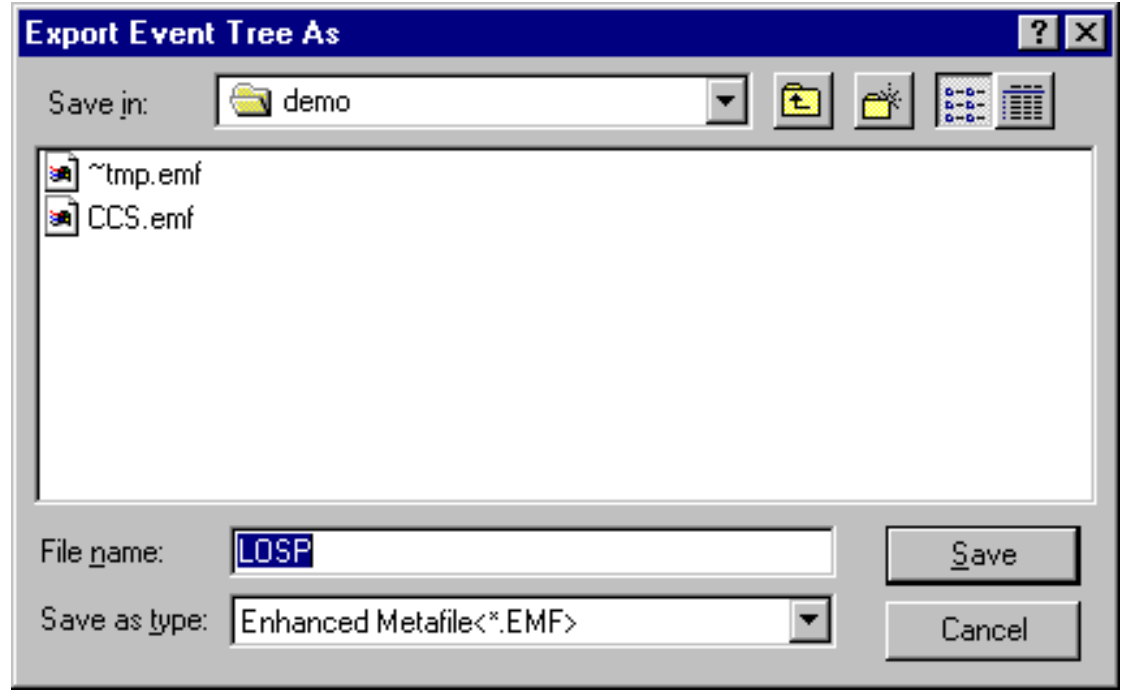

Save in - $\quad$ Lists the available folders and files. The box directly below it shows the contents of the current folder. The down-arrow to the right of this field shows how the folder fits into your system hierarchy.

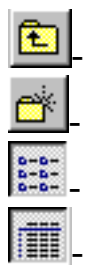

Moves up one level in the system hierarchy.

Creates a new folder at the current level.

Displays the icons and names of the contents of the current folder.

Displays the icons, names, size, type, date and time modified, and archive flags of the contents of the current folder.

File name - Contains the name of the export file.

Save as type - $\quad$ Lists the types of files to display. This is useful for narrowing the list of files displayed to only those you are interested in. In this case, only .EMF, .WMF, or .RTF files are listed.

Save - $\quad$ Save the current file in the File name field and close the Export Event Tree As dialog. If an existing file was selected, a warning dialog will be displayed.

Double-click - Export the current diagram to the file in the File name field and close the Export Event Tree As dialog.

Cancel - $\quad$ Close the Export Event Tree As dialog without saving the diagram. 


\subsubsection{Page Tree}

\section{PURPOSE}

This option will allow you to copy the highlighted event tree branch to a new or existing diagram. The highlighted branch will be replaced in the current diagram with a straight pass-through of all the subsequent branch points and will transfer to the diagram you selected.

\section{STEPS}

1. Select the branch to be paged.

2. From the Event Tree Editor menu select File.

3. Choose Page Tree from the menu. The Page Event Tree dialog will be displayed.

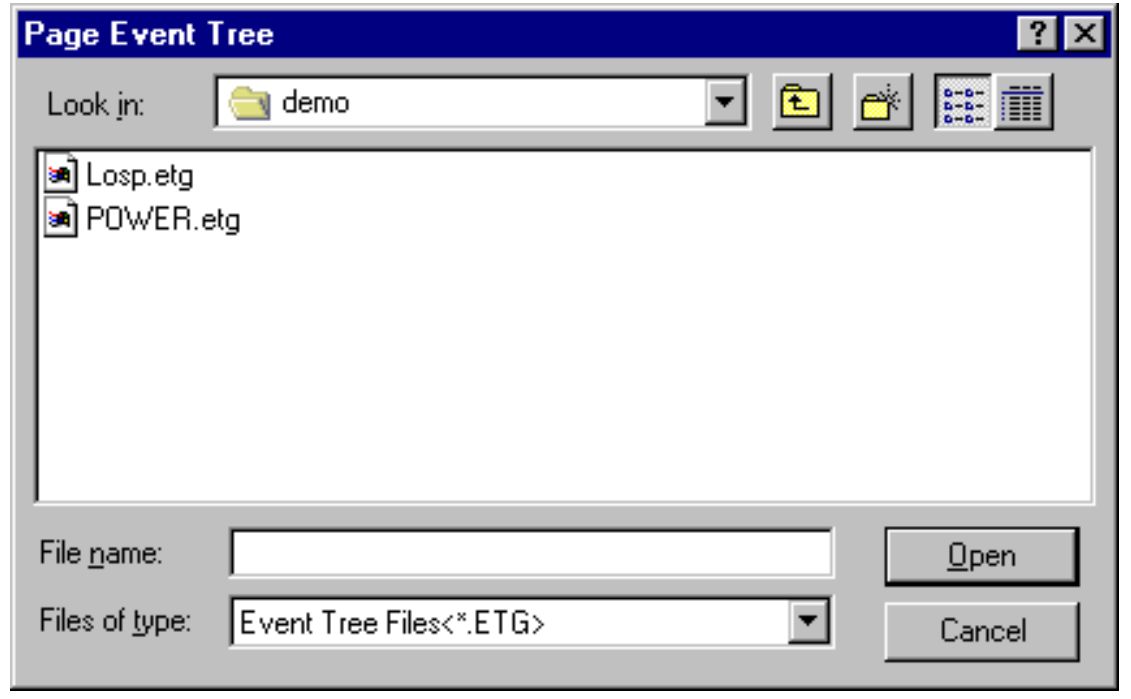

Look in - $\quad$ Lists the available folders and files. The box directly below it shows the contents of the current folder. The down-arrow to the right of this field shows how the folder fits into your system hierarchy.

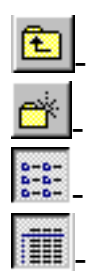

File Name Files of Type -

Moves up one level in the system hierarchy.

Creates a new folder at the current level.

Displays the icons and names of the contents of the current folder.

Displays the icons, names, size, type, date and time modified, and archive flags of the contents of the current folder.

Contains the name of the file in which the branch will be saved.

Lists the types of files to display. This is useful for narrowing the list of files displayed to only those you are interested in. In this case, only .ETG files are listed.

Open - $\quad$ Save the current branch to the file in the File name field and close the Page Event Tree dialog. If an existing file was selected, a warning dialog will be displayed.

Double-click - Save the current branch to the file in the File name field and close the Page Event Tree dialog. 
Cancel - $\quad$ Close the Page Event Tree dialog without saving the branch to a different file.

\subsubsection{Forward Transfer}

\section{PURPOSE}

This option will display the diagram corresponding to the selected transfer in the current event tree diagram. Any changes made to the current event tree diagram can be saved before viewing the transfer diagram.

\section{STEPS}

1. Select the transfer leaf you wish to follow.

2. From the Event Tree Editor menu select File | Forward Transfer.

$$
\text { OR }
$$

3. Choose the Forward Transfer button $\stackrel{-c_{T}}{{ }^{-}}$on the button bar.

4. If changes were made to the current diagram, the Caution dialog will be displayed.

5. If the diagram corresponding to the transfer branch exists, that will become the current diagram. OR

6. If no corresponding diagram exists, the Status dialog shown below will be displayed.

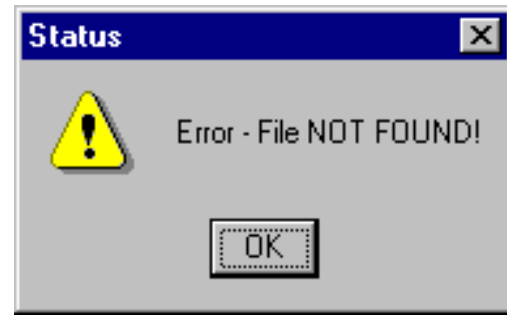

$$
O R
$$

7. If the selected branch is not a transfer, the Status dialog shown below will be displayed.

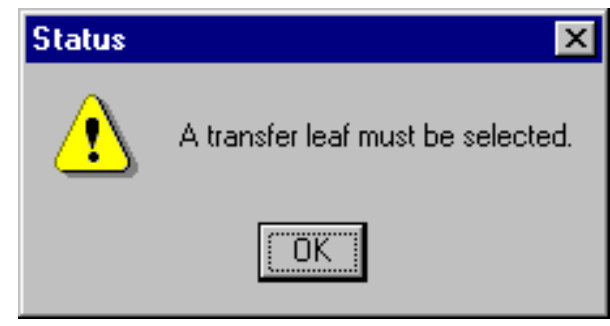

OK - Close the Status dialog.

\subsubsection{Backward Transfer}




\section{PURPOSE}

This option will list all the diagrams previously viewed in the current chain of transfers, including the current diagram. Select the event tree diagram you wish to edit. Any changes made to the current event tree diagram can be saved before viewing the transfer diagram.

\section{STEPS}

1. From the Event Tree Editor menu select File | Backward.

OR

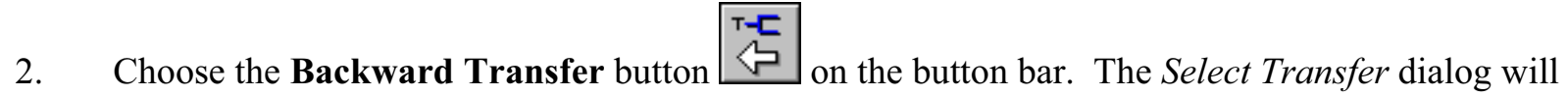
be displayed.

3. Highlight a diagram in the list and choose $\mathbf{O K}$.

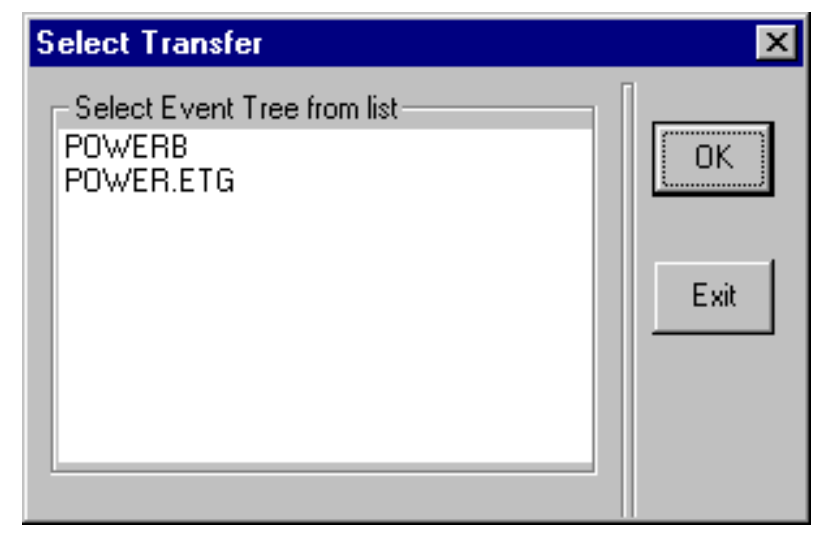

OK - Close the Select Transfer dialog and load the selected fault tree diagram. If you have made any changes to the current diagram, the Caution dialog will appear.

Exit - Close the Select Transfer dialog without loading a new diagram.

\subsubsection{Print}

\section{PURPOSE}

This option will print the current diagram to the selected printer. You are provided the opportunity to set additional options, such as page range, number of copies, etc., before printing the diagram.

\section{STEPS}

1. From the Fault Tree Editor or Event Tree Editor or P\&ID Editor menu select File | Print. OR

2. When in the Fault Tree Editor, choose the Print button 且 on the toolbar. The Print common dialog will be displayed. 


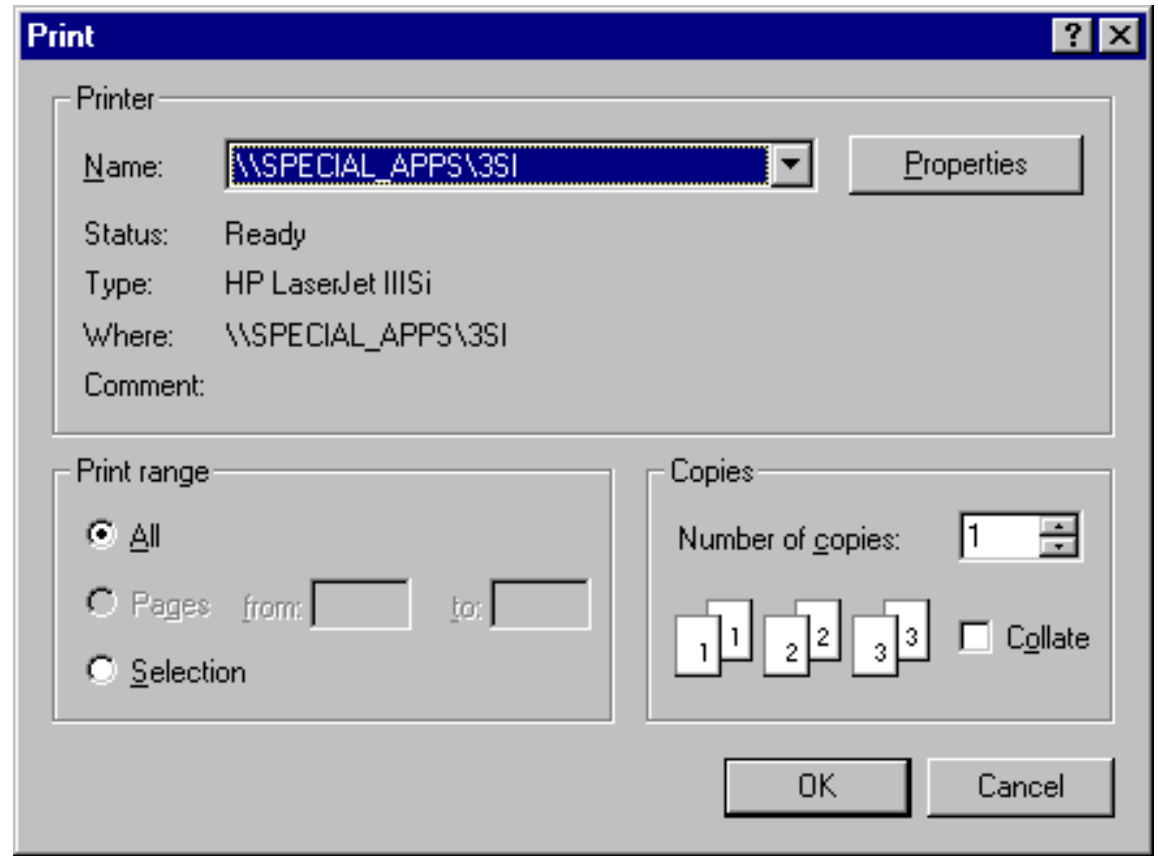

Name - $\quad$ List the printers that are set up for this computer.

Properties - $\quad$ Set-up options for the selected printer. Available options depend on the features of the printer.

Status - $\quad$ Condition of the selected printer.

Type - Kind of selected printer.

Where - Location of the selected printer.

Comment - Miscellaneous information about the selected printer.

Print Range - Print the entire document, specified range, or highlighted selection.

Copies - $\quad$ Enter the number of copies you wish to print.

Collate - $\quad$ For more than one copy, specify whether you want the copies collated.

OK - $\quad$ Close the Print Dialog and print the current diagram to the selected output device.

Cancel - $\quad$ Close the Print Dialog without printing the diagram.

\subsubsection{Edit Menu}

\subsubsection{Edit}

These options provide editing and text formatting functions.

Cut -

Copy -

Paste -

Delete -

Add Branch Above Add Branch Below -

Pass -

Attributes -
Remove the selected object(s) from the current window. Copy the selected object(s) from the current window.

Copy the contents of the temporary file into the current window at the cursor position.

Permanently remove the selected object(s) from the current window.

Add a new branch above the currently selected branch.

Add a new branch below the currently selected branch.

Replace the selected branch with a straight Pass-Through.

This menu item is for information only, indicating the action to perform

(right-click) in order to invoke the appropriate attributes dialog. 


\subsubsection{Undo}

\section{PURPOSE}

This feature allows you to undo previous actions. SAPHIRE maintains a list of actions taken by the user in a diagram editing session. When the list contains items, Undo will roll back the most recently completed action. Successive undo commands will roll back the next most recent action.

Note: The rollback list is cleared whenever the diagram is saved. (This applies only to user performed saves, not to timed backups.)

\section{STEPS}

1. $\quad$ From the Fault Tree Editor menu or Event Tree Editor menu select Edit $\mid$ Undo.

The most recent action will be undone.

\subsubsection{Cutting Objects}

\section{PURPOSE}

This option removes objects from the diagram and saves them in the clipboard.

\section{STEPS}

1. Select the object(s) you wish to cut.

2. Press the $\mathbf{C t r l}+\mathbf{X}$ key combination.

$$
\text { OR }
$$

3. Choose the Cut button on the toolbar.

$$
\text { OR }
$$

4. From the Fault Tree Editor or Event Tree Editor menu select Edit | Cut. OR

5. Right-click to invoke the pop-up menu and select Cut.

The selected object(s) will be removed from the diagram and placed in a temporary file as objects, as well as in the clipboard as a bitmap. This bitmap can be pasted (imported) into any document that can copy bitmaps from the clipboard.

\subsubsection{Copying Objects}

\section{PURPOSE}

This option copies selected objects from the diagram and places them in the clipboard.

\section{STEPS}

1. Select the object(s) you wish to copy. 
2. Press the $\mathbf{C t r l}+\mathbf{C}$ key combination.

$O R$

3. Choose the Copy button 3 (FTE) or ${ }^{5}-\mathrm{ETE}$ ) on the toolbar.

OR

4. From the Fault Tree Editor or Event Tree Editor menu select Edit | Copy.

OR

5. Right-click to invoke the pop-up menu and select Copy.

The selected object(s) will be copied from the diagram and placed in a temporary file as objects, as well as in the clipboard as a bitmap. This bitmap can be pasted (imported) into any document that can copy bitmaps from the clipboard.

\subsubsection{Pasting Objects}

\section{PURPOSE}

This option pastes (imports) objects into the current diagram.

\section{STEPS}

1. Press the $\mathbf{C t r l + V}$ key combination.

$O R$

2. Choose the Paste button 迎

$$
\text { OR }
$$

3. From the Fault Tree Editor or Event Tree Editor menu select Edit | Paste.

OR

4. Right-click to invoke the pop-up menu and select Paste. The cursor is changed to the paste cursor ${ }^{+}$晋.

5. Move the cursor to the location in the diagram where you wish to paste the object(s).

6. Click the mouse.

The selected object(s) will be copied the diagram centered, about the cursor.

\subsubsection{Deleting Objects}

\section{PURPOSE}

This option permanently removes the selected object(s) from the diagram. They are NOT put in the clipboard.

\section{STEPS}

1. Select the object(s) you wish to delete.

2. Press the Delete key. 
OR

3. From the Fault Tree Editor or Event Tree Editor menu select Edit | Delete.

The object(s) will be permanently deleted.

\subsubsection{Add Branch Above}

\section{PURPOSE}

Add a new branch above the currently selected branch.

\section{STEPS}

1. Select the branch you wish to add a branch above.

2. From the Event Tree Editor menu select Edit | Add Branch Above.

OR

3. Choose the Add Branch Above button $\square$ on the button bar.

$O R$

4. Right-click to invoke the pop-up menu and select Add Branch Above.

A new branch will appear in the event tree above the selected branch. This branch will pass-through all the tops to the right of the top being branched. The selected branch will remain highlighted.

\subsubsection{Add Branch Below}

\section{PURPOSE}

Add a new branch below the currently selected branch

\section{STEPS}

1. Select the branch you wish to add a branch below.

2. $\quad$ From the Event Tree Editor menu select Edit | Add Branch Below.

OR

3. Choose the Add Branch Below button $\bar{\square}$ on the button bar.

$O R$

4. Right-click to invoke the pop-up menu and select Add Branch Above.

A new branch will appear in the event tree below the selected branch. This branch will pass-through all the tops to the right of the top being branched. The selected branch will remain highlighted.

\subsubsection{Pass}

\section{PURPOSE}

Replace the selected branch with a straight Pass-Through. 


\section{STEPS}

1. Select the branch you wish to change.

2. $\quad$ From the Event Tree Editor menu select Edit | Pass.

OR

3. Choose the Pass button on the button bar.

OR

4. Right-click to invoke the pop-up menu and select Pass.

The selected branch will be changed into a pass-through. This branch will pass-through all the tops to the right of the top being branched. The new pass-through branch will be highlighted.

\subsubsection{Attributes}

\section{PURPOSE}

This dialog is used to set or change the attributes of the current graphical event tree. Changing these attributes does not affect the Preferences.

\section{STEPS}

1. Ensure that no object (e.g., branch, top, event, sequence information column) is selected.

2. Right-click on the Event Tree Editor window. The Attributes dialog will be displayed.

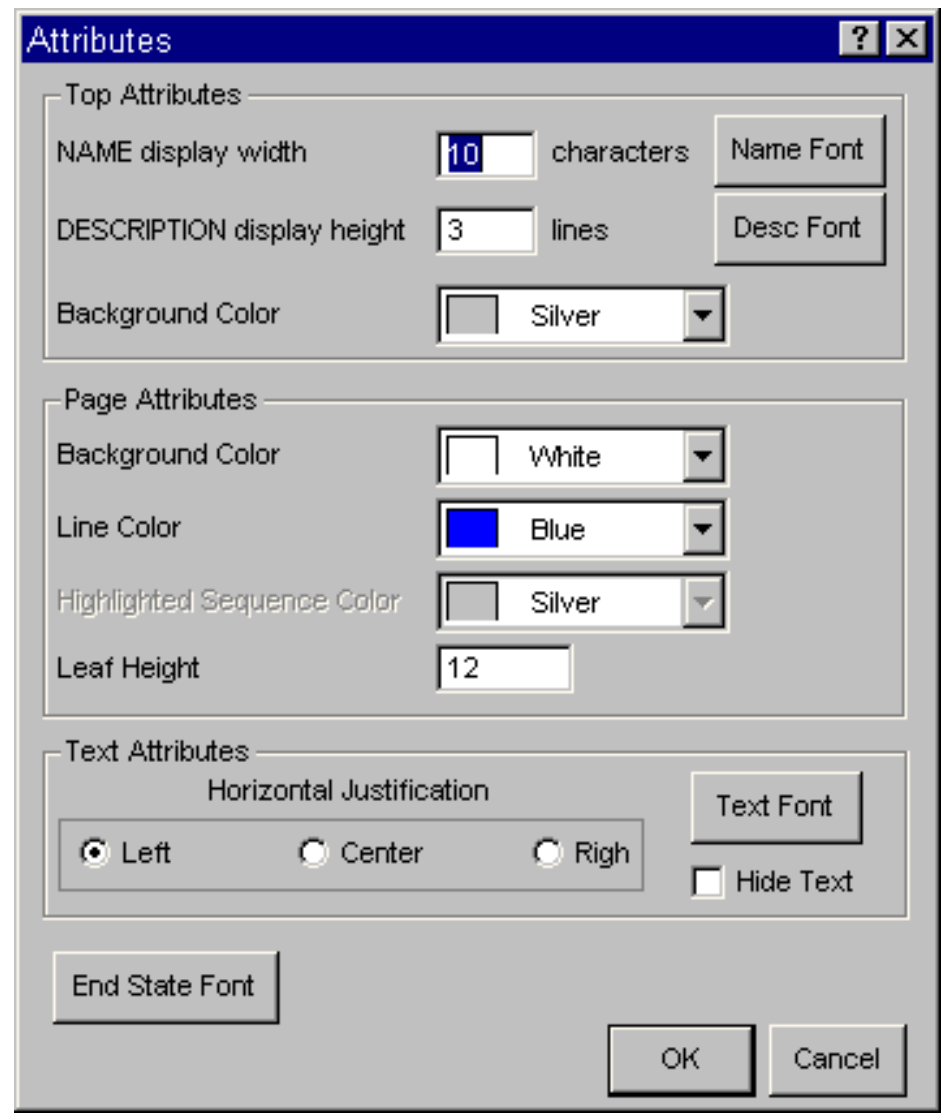


OK - Apply the changes and close the Attributes dialog.

Cancel - Close the Attributes dialog without applying the changes.

\subsubsection{View Menu}

\subsubsection{View}

These menu options allow you to change the portion of the current diagram in the viewing window. For simple diagrams with a small number of branches, this is generally not necessary. For larger, more complex diagrams that do not fit on one screen, you may need to view different portions of the diagram. You can move the view window up, down, left, and right using the scroll bars or menu commands. You can change the size of the viewing window by zooming in or out, adjust the diagram to fit inside the viewing window, and restore the view window to its original size.

\begin{tabular}{|c|c|}
\hline Show Sequence Names - & Display the sequence names column. \\
\hline Show End State Names - & Display the end state names column. \\
\hline Show Frequency - & Display the frequency (extra information \#1) column. \\
\hline Show Extra-\#2. & Display the extra information $\# 2$ column. \\
\hline Show Grid - & Display the background grid. \\
\hline Preferences - & Change the event tree graphical editor defaults. \\
\hline Refresh - & Redraw the viewing window. \\
\hline Zoom - & Enlarge or shrink a portion of the diagram. \\
\hline View Normal - & Restore the view window to its original size. \\
\hline Fit to Screen - & Adjust the diagram to fit inside the view window. \\
\hline Page Up - & $\begin{array}{l}\text { Move the viewing window up about one-half page (i.e., screen) } \\
\text { above the current location. }\end{array}$ \\
\hline Page Down - & $\begin{array}{l}\text { Move the viewing window down about one-half page (i.e., } \\
\text { screen) below the current location. }\end{array}$ \\
\hline Page Left - & $\begin{array}{l}\text { Move the viewing window left about one-half page (i.e., screen) } \\
\text { left of the current location. }\end{array}$ \\
\hline Page Right - & $\begin{array}{l}\text { Move the viewing window right about one-half page (i.e., } \\
\text { screen) right of the current location. }\end{array}$ \\
\hline
\end{tabular}

\subsubsection{Show Sequence Names}

\section{PURPOSE}

This option allows you to display the sequence names column. If selected, a check mark to the left of this menu item will be displayed and the column will appear in the editor window. If deselected, there will not be a check mark next to the menu item and the sequence name column will not be displayed. This option acts as a toggle.

This column will always contain the sequence names. By default, the sequence names column is the first column following the event tree branches and is therefore, referred to as the "first" column. The physical location of this column can be changed.

\section{STEPS}

1. From the Event Tree Editor menu select View. 
2. Choose Show Sequence Names from the menu.

\begin{tabular}{|c|c|}
\hline \multicolumn{2}{|l|}{ View Help } \\
\hline $\begin{aligned} & \text { Show Sequer } \\
& \text { Show End St: } \\
& \text { Show Frequer } \\
& \text { Show Extra-\# }\end{aligned}$ & \\
\hline \multicolumn{2}{|l|}{$\checkmark$ Show Grid } \\
\hline \multicolumn{2}{|l|}{ Preferences... } \\
\hline Refresh & F5 \\
\hline Zoom & Ctrl $+Z$ \\
\hline View Normal & $\mathrm{Ctrl}+\mathrm{V}$ \\
\hline Fit to Screen & HOME \\
\hline Page Up & $P g U_{p}$ \\
\hline Page Down & $\mathrm{PgDn}$ \\
\hline Page Left & Ctrl+Left \\
\hline Page Right & Ctrl+Right \\
\hline
\end{tabular}

In the example of the View menu shown here, the Show Sequence Names option is NOT currently selected.

This toggle can also be set in the Edit Sequence dialog or the Edit Sequence Header dialog.

\subsubsection{Show End State Names}

\section{PURPOSE}

This option allows you to display the end state names column. If selected, a check mark to the left of this menu item will be displayed and the column will appear in the editor window. If deselected, there will not be a check mark next to the menu item and the end state name column will not be displayed. This option acts as a toggle.

This column will always contain the sequence end state or transfer tree names. If the sequence continues through another event tree then the end state is the name of the transfer tree. By default, the end state names column is the second column following the event tree branches and is therefore, referred to as the "second" column. The physical location of this column can be changed.

\section{STEPS}

1. From the Event Tree Editor menu select View.

2. Choose Show End State Names from the menu. 


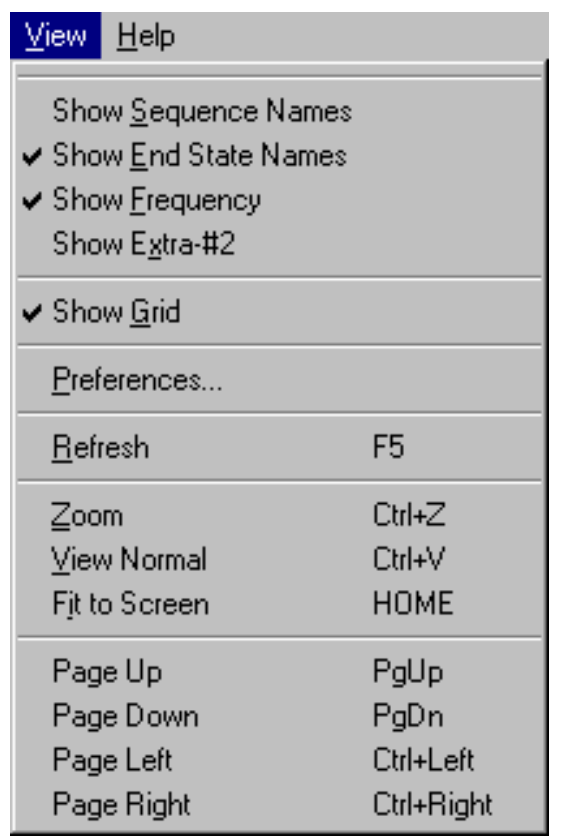

In the example of the View menu shown here, the Show End State Names option is currently selected.

This toggle can also be set in the Edit Sequence dialog or the Edit Sequence Header dialog.

\subsubsection{Show Frequency}

\section{PURPOSE}

This option allows you to display the extra information \#1 column. If selected, a check mark to the left of this menu item will be displayed and the column will appear in the editor window. If deselected, there will not be a check mark next to the menu item and the column will not be displayed. This option acts as a toggle.

This column can be used for whatever information you wish, however, options exist elsewhere in the program that will put the frequency of this sequence in this column. By default, this is the third column following the event tree branches and is therefore, referred to as the "third" column. The physical location of this column can be changed.

\section{STEPS}

1. From the Event Tree Editor menu select View.

2. Choose Show Frequency from the menu. 


\begin{tabular}{|c|c|}
\hline View & \\
\hline $\begin{aligned} & \text { Show Sequer } \\
& \checkmark \text { Show End St } \\
& \text { Show Frequer } \\
& \text { Show Extra-\# }\end{aligned}$ & \\
\hline$\checkmark$ Show Grid & \\
\hline Preferences... & \\
\hline Refresh & F5 \\
\hline Zoom & Ctrl +2 \\
\hline View Normal & Ctrl+V \\
\hline Fit to Screen & HOME \\
\hline Page Up & Pgup \\
\hline Page Down & $\mathrm{PgDn}$ \\
\hline Page Left & Ctrl+Left \\
\hline Page Right & Ctrl+Right \\
\hline
\end{tabular}

In the example of the View menu shown here, the Show Frequency option is currently selected.

This toggle can also be set in the Edit Sequence dialog or the Edit Sequence Header dialog.

\subsubsection{Show Extra-\#2}

\section{PURPOSE}

This option allows you to display the extra information \#2 column. If selected, a check mark to the left of this menu item will be displayed and the column will appear in the editor window. If deselected, there will not be a check mark next to the menu item and the column will not be displayed. This option acts as a toggle.

This column can be used for whatever information you wish. By default, this column is the fourth column following the event tree branches and is therefore, referred to as the "fourth" column. The physical location of this column can be changed .

\section{STEPS}

1. From the Event Tree Editor menu select View.

2. Choose Show Extra-\#2 from the menu. 


\begin{tabular}{|c|c|}
\hline View & \\
\hline $\begin{aligned} & \text { Show Sequer } \\
& \checkmark \text { Show End St } \\
& \text { Show Frequer } \\
& \text { Show Extra-\# }\end{aligned}$ & \\
\hline$\checkmark$ Show Grid & \\
\hline Preferences... & \\
\hline Refresh & F5 \\
\hline Zoom & Ctrl +2 \\
\hline View Normal & Ctrl+V \\
\hline Fit to Screen & HOME \\
\hline Page Up & Pgup \\
\hline Page Down & $\mathrm{PgDn}$ \\
\hline Page Left & Ctrl+Left \\
\hline Page Right & Ctrl+Right \\
\hline
\end{tabular}

In the example of the View menu shown here, the Show Extra-\#2 option is NOT currently selected.

This toggle can also be set in the Edit Sequence dialog or the Edit Sequence Header dialog.

\subsubsection{Show Grid}

\section{PURPOSE}

This option allows you to display a background grid to help align branches. If selected, a check mark to the left of this menu item will be displayed and the grid will appear on the background of the editor window. If deselected, there will not be a check mark next to the menu item and the background grid will not be displayed. This option acts as a toggle.

\section{STEPS}

1. From the Event Tree Editor menu select View.

2. Choose Show Grid from the menu. 


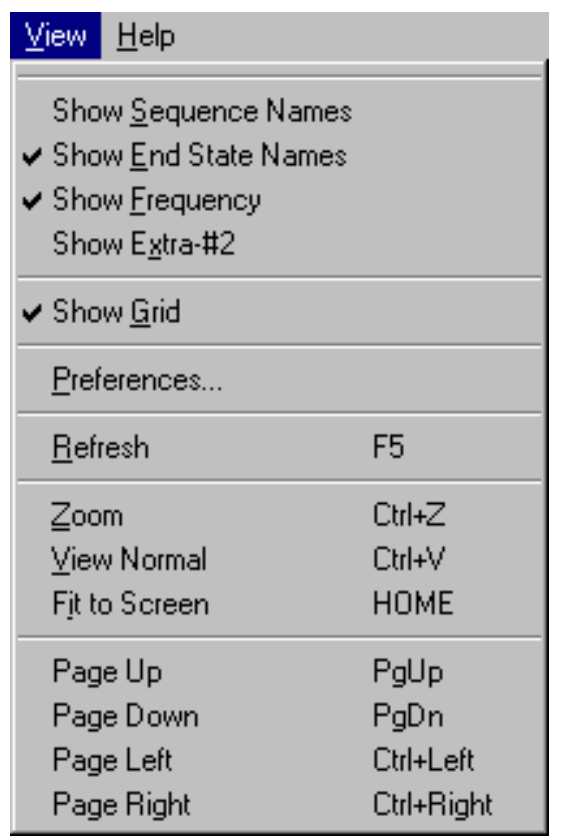

In the example of the View menu shown here, the Show Grid option is currently selected.

\subsubsection{Preferences}

\section{PURPOSE}

This option allows you to modify the preferred defaults for the graphical Event Tree Editor. The changes made here will be reflected in the default values in the Utility | Define Constants | Event Tree option. These changes affect only new graphical event trees and does not affect the attributes of existing ones.

\section{STEPS}

1. From the Event Tree Editor menu select View.

2. Choose Preferences from the menu.

3. The Preferences dialog will be displayed. 


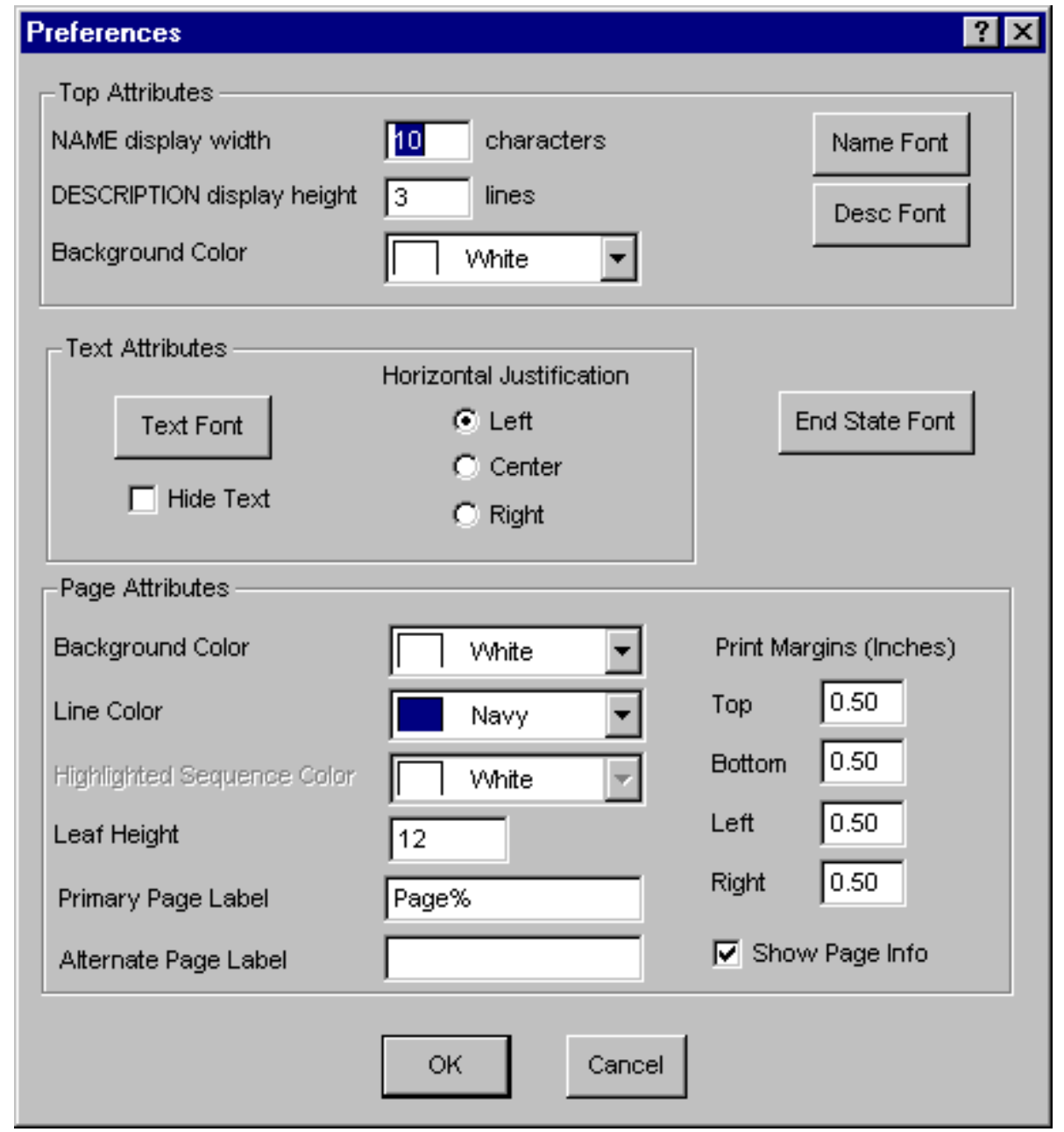

OK - $\quad$ Save the changes and close the Preferences dialog.

Cancel - Close the Preferences dialog without saving the changes.

\subsubsection{Zooming In/Out}

\section{PURPOSE}

This option allows you to enlarge or shrink the diagram in the viewing window to change the portion of the diagram that can be seen. It has the effect of magnifying or reducing the diagram size.

\section{STEPS}

1. From the Fault Tree Editor or Event Tree Editor menu select View $\mid$ Zoom.

$$
\text { OR }
$$

2. Select the Zoom button 8 (FTE) or (ETE) from the toolbar.

$$
O R
$$

3. Press the $\mathbf{C t r}+\mathbf{Z}$ key combination. The cursor is changed to the Zoom cursor

4. Move the cursor to the desired location. 
To zoom in or magnify the diagram:

1. Click the mouse. The diagram will be magnified by $40 \%$.

To zoom out or reduce the diagram:

1. Right-click the mouse. The diagram will be reduced by $40 \%$.

\subsubsection{Using Scroll Bars To Move The View Window}

\section{PURPOSE}

This option allows you to change the portion of the diagram in the view window.

\section{STEPS}

To move the viewing window to the left slightly:

1. Click on the left arrow in the bottom scroll bar.

The viewing window will move a little to the left.

To move the viewing window to the right slightly:

1. Click on the right arrow in the bottom scroll bar.

The viewing window will move a little to the right.

To move the viewing window up slightly:

1. Click on the up arrow in the scroll bar on the right hand side.

The viewing window will move up a little.

To move the viewing window down slightly:

1. Click on the down arrow in the scroll bar on the right hand side.

The viewing window will move down a little. 
To move the viewing window to the left about one-eighth page:

1. Click on bottom scroll bar between the left arrow and the thumb.

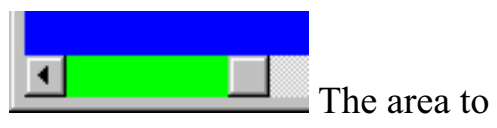
click in is highlighted in green in this example.

The viewing window will move to the left.

To move the viewing window to the right about one-eighth page:

1. Click on bottom scroll bar between the right arrow and the thumb.

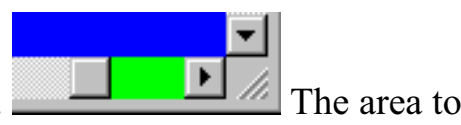
click in is highlighted in green in this example.

The viewing window will move to the right.

To move the viewing window up about one-eighth page:

1. Click on bottom scroll bar between the top arrow and the thumb. The area to click in is highlighted in green in this example

The viewing window will move up.

To move the viewing window down about one-eighth page:

1. Click on right scroll bar between the bottom arrow and the thumb. highlighted in green in this example.

The viewing window will move down.

\subsubsection{Restore the View Window}

\section{PURPOSE}

This option allows you to restore the diagram position in the viewing window to the same relative position as when the diagram file was initially opened. Changes to the objects (i.e., their position, attributes, etc.) are not restored. 


\title{
STEPS
}

1. From the Fault Tree Editor or Event Tree Editor menu select View | Normal.

$O R$

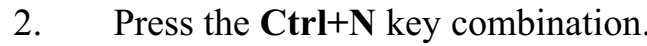

The initial position of the diagram is redisplayed.

\subsubsection{Fit the View Window}

\section{PURPOSE}

This option allows you to adjust the entire diagram to fit inside the viewing window. If the diagram is relatively large, the size of the shapes and text will be reduced so that the entire fault tree can be viewed without scrolling or paging the window. If the diagram is relatively small, the size of the shapes and text will be enlarged so that the diagram fills the window.

\section{STEPS}

1. From the Fault Tree Editor menu select View | Fit or from the Event Tree Editor menu select View | Fit to Screen.

\author{
OR
}

2. Select the Fit to Window button (FTE) from the toolbar.

OR

3. Press the $\mathbf{C t r l + F}$ key combination.

The diagram will be enlarged or reduced to fit inside the viewing window.

\subsubsection{Timed Backup}

\subsubsection{Restore Backup File}

While editing a fault tree or event tree diagram, SAPHIRE will periodically make a backup of the file. If the editing session terminates abnormally, the next time the fault tree diagram is edited, the Restore Backup File dialog will be displayed.

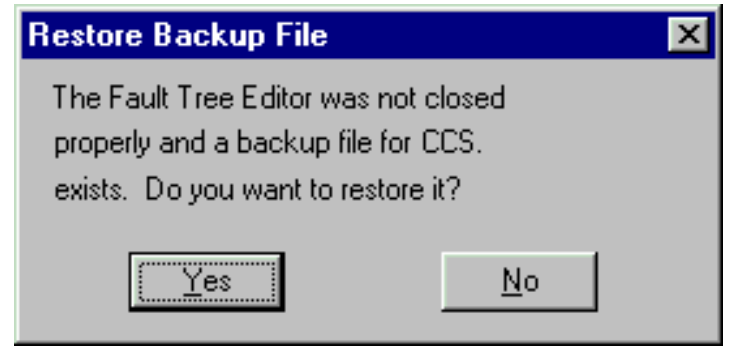

Yes - Edit the backup version of the diagram. This option will restore most of the changes made from the aborted editing session.

No - Discard the backup version of the diagram. Edit the diagram as it existed prior to the aborted editing session. 


\subsection{Linkage Rule Editor (Event Tree)}

\subsubsection{Linkage Rules Editor Menu}

\section{PURPOSE}

This option allows you to specify linkage rules for an event tree. A linkage rule is a special case, an exception, or substitution to the normal sequence generation. Typically, these rules are used to replace default fault trees (i.e., top events) with substituted fault trees (or "split-fractions") based on logical conditions that are specified in the rules. During sequence logic generation the logic for a sequence is created as specified in the event tree logic, and then the linkage rules are applied.

For example, event tree A contains a sequence named SEQ-1. According to the strict logic of the event tree, the fault trees that make up SEQ-1's logic are SYS-1, SYS-2, SYS-3, and SYS-4. A linkage rule would enable you to replace all occurrences of SYS-4 in any sequence in the event tree with SYS-5. Alternatively, you may only want to replace SYS-4 with SYS-5 if and only if SYS-2 and SYS-3 are also present in the sequence logic.

\section{STEPS}

1. From the SAPHIRE menu select Event Tree. The Event Tree List dialog will be displayed.

2. Highlight the desired event tree in the list.

3. Right-click to invoke the pop-up menu and choose Edit Rules.

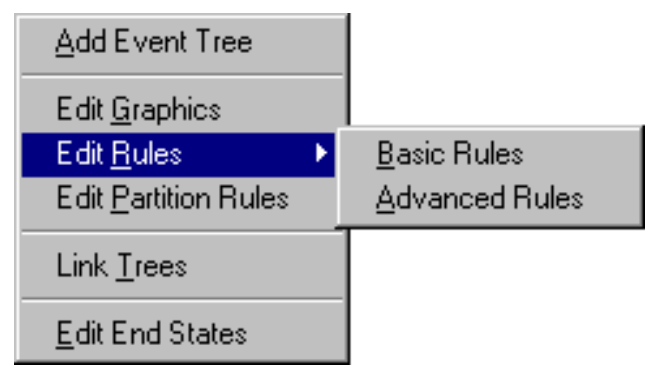

4. Choose either Basic Rules or Advanced Rules. The Linkage Rules Editor will be invoked.

\subsubsection{Basic Linkage Rules Editor}

\section{PURPOSE}

This is a free format line editor that provides you with an if-then-else logic structure. By default the editor assumes that a name is the name of a fault tree, if one exists. If a fault tree by the name does not exist then the editor assumes that the name is the name of a macro. If the user desires to change the default assumptions, then they can "cast" the names to something else by enclosing the name in parenthesis with a cast name preceding it. There is no limit to the number of rules the user may input to the editor. Each rule is processed in sequential order. 
Basic rules can have different logic than the Advanced Rules and are saved separately.

\section{SEE}

Linkage Rules Editor Functions

\subsubsection{Advanced Linkage Rules Editor}

\section{PURPOSE}

This editor functions much the same as the Basic Rules Editor. However, the Modula-2 programming language is used to allow more robust programming capabilities. Using the Advanced Editor, users can define variables which can be assigned values based on specified criteria during run-time. Procedures can also be implemented which allows use of modular programming techniques.

When the advanced rules are compiled, a special dynamic link library (DLL) is created and saved in a special folder.

Advanced rules can have different logic than the Basic Rules and are saved separately.

\section{SEE}

Linkage Rules Editor Functions

\subsubsection{Linkage Rules Editor Functions}

\section{PURPOSE}

The editor contains file management functions such as open, save, save as, print, import, etc. Editing functions such as cut, copy, paste, delete, undo, etc., are provided. Text format operations such as indent, capitalization, etc. are supplied. Search functions such as find, replace, set marker, goto marker, etc., are provided. Preference attributes such as undo levels, select font, shift size, show altered lines, etc., can be set. Also, window functions such as tile, cascade, and arrange icons can be set. Finally, list options that provide the ability to insert database items such as basic events, initiators, fault trees, flags, etc., are provided. 


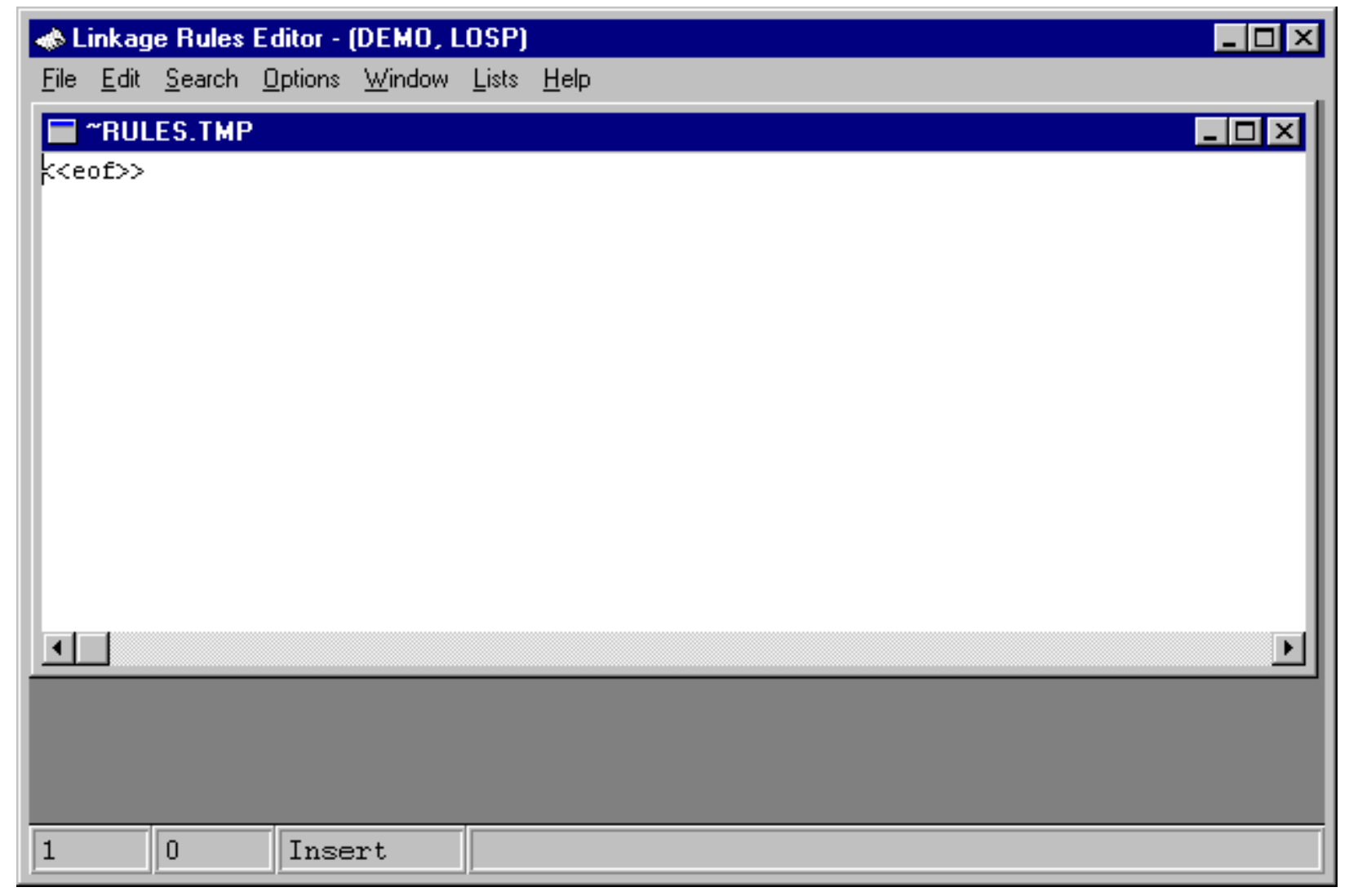

File -

File functions such as open, save, print, preferences, etc.

Edit Editing functions such as copy and paste along with text format operations.

Search - Search and cursor positioning operations such as find, replace, goto, etc.

Window - Window management functions such as cascade, tile horizontally, etc.

Lists - Display lists of initiators, fault trees, etc. for inserting into the rules.

\subsubsection{File Menu}

\subsubsection{Editor File Functions}

These options provide file management functions.

Open - $\quad$ Open a file into a new window. This feature is not usually used while editing SAPHIRE rules.

New - $\quad$ Create a new file in a new window. This feature is not usually used while editing SAPHIRE rules.

Close - $\quad$ Close the edit current window. If changes have been made you will be prompted to save the file.

Quit - $\quad$ Close the current edit window without saving.

Save - $\quad$ Save the file in the current window to disk.

Save As - $\quad$ Save the file in the current window with a new file name.

Save All - $\quad$ Save all the files in all edit windows currently open.

Save Block - Save the currently highlighted text into a new file. The Save As dialog will be invoked. 
Import File - Insert the contents of a file into the current edit window at the current cursor position. The Edit external file dialog will be invoked.

Print - $\quad$ Print the file in the current edit window.

Page setup - $\quad$ Set printer page layout options, .such as pages per sheet and line numbers.

Preferences - $\quad$ Select editing options such as tab width, undo levels, etc.

Exit -

Terminate the Editor session.

\subsubsection{Open}

\section{PURPOSE}

This option allows you to open an existing file into a new editing window within the Linkage Rules Editor or the Event Tree Logic Editor.

\section{STEPS}

1. From the Linkage Rules Editor or the Event Tree Logic Editor menu select File.

2. Select the Open menu option. The Edit external file dialog will be displayed listing all of the files in the current project directory.

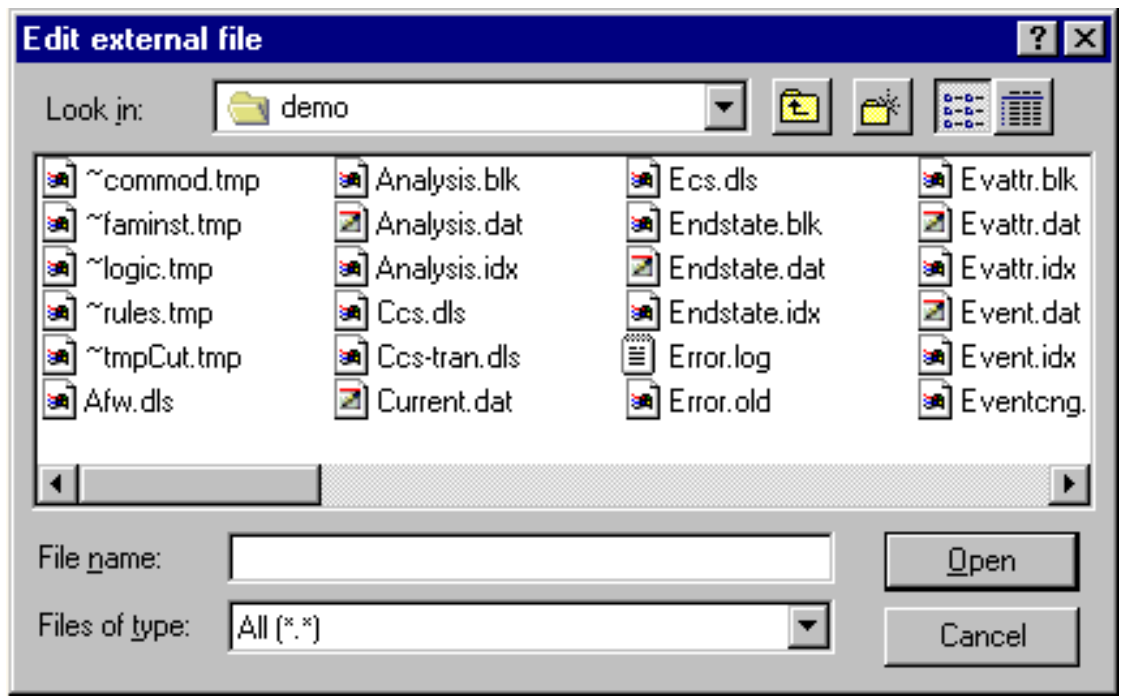

Look in - $\quad$ Lists the available folders and files. The box directly below it shows the contents of the current folder. The down-arrow to the right of this field shows how the folder fits into your system hierarchy.

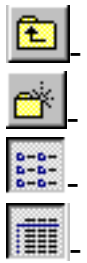

Moves up one level in the system hierarchy.

Creates a new folder at the current level.

Displays the icons and names of the contents of the current folder.

Displays the icons, names, size, type, date and time modified, and archive flags of the contents of the current folder.

File Name - Contains the name of the file in which the diagram will be saved. 
Files of Type - Lists the types of files to display. This is useful for narrowing the list of files displayed to only those you are interested in.

Open - $\quad$ Open the selected file in a new window and close the Edit external file dialog.

Cancel - $\quad$ Close the Edit external file dialog without opening another file.

Double-click - Open the selected file in a new window and close the Edit external file dialog.

\subsubsection{Save As}

\section{PURPOSE}

This option allows you to save the file in the current editing window with a new file name.

\section{STEPS}

1. From the Linkage Rules Editor or the Event Tree Logic Editor menu select File.

2. Select the Save As menu option. The Save As dialog will be displayed.

\begin{tabular}{|c|c|c|c|c|c|}
\hline \multicolumn{6}{|l|}{ Save As } \\
\hline Save in: & 9 dem & & $\nabla$ t & ن: & $\mid \begin{array}{l}0=0- \\
\vdots:-2: \\
0=0-\end{array}$ \\
\hline \multicolumn{2}{|c|}{ \%commod.tmp } & Afw.dls & 3] Current.dat & & Error.old \\
\hline \multicolumn{2}{|c|}{ itaminst.tmp } & Analysis.blk & Ecs.ds & & Evattr.blk \\
\hline \multicolumn{2}{|l|}{$\sim \operatorname{logic} . \operatorname{tmp}$} & 3] Analysis.dat & Endstate.blk & & ㄱ.] Evattr.dat \\
\hline \multicolumn{2}{|l|}{ 〜rules.BAK } & Analysis.idx & [] Endstate.dat & & Evattr.idx \\
\hline \multicolumn{2}{|l|}{$\sim$ rules.tmp } & Cos.dls & Endstate.idx & & (]) Event.dat \\
\hline \multicolumn{2}{|c|}{ 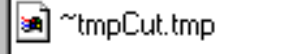 } & Cos-tran.dls & Eis Eror.log & & Event.idx \\
\hline \multicolumn{2}{|l|}{1} & & & & 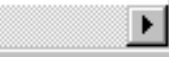 \\
\hline \multirow{2}{*}{$\begin{array}{l}\text { File name: } \\
\text { Save as type: }\end{array}$} & \multicolumn{3}{|c|}{ 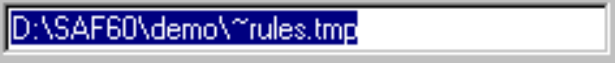 } & & Save \\
\hline & All $\left[{ }^{*} .{ }^{*}\right]$ & & $\nabla$ & & Cancel \\
\hline
\end{tabular}

Save in -

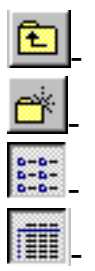

File Name -
Lists the available folders and files. The box directly below it shows the contents of the current folder. The down-arrow to the right of this field shows how the folder fits into your system hierarchy.

Moves up one level in the system hierarchy.

Creates a new folder at the current level.

Displays the icons and names of the contents of the current folder.

Displays the icons, names, size, type, date and time modified, and archive flags of the contents of the current folder.

Contains the name of the file to be saved. 
Files of Type -

Save -

Double-click -

Cancel -
Lists the types of files to display. This is useful for narrowing the list of files displayed to only those you are interested in.

Save the data in the current editing window to the file in the File name field and close the Save Fault Tree dialog. If an existing file was selected, a warning dialog will be displayed.

Save the current diagram to the file in the File name field and close the Save As dialog.

Close the Save As dialog without performing the save operation.

\subsubsection{Print}

\section{PURPOSE}

This option allows you to print the file in the current edit window to the default (Windows System) printer.

\section{STEPS}

1. From the Linkage Rules Editor or the Event Tree Logic Editor menu select File.

2. Select the Print menu option. The Print dialog will be displayed.

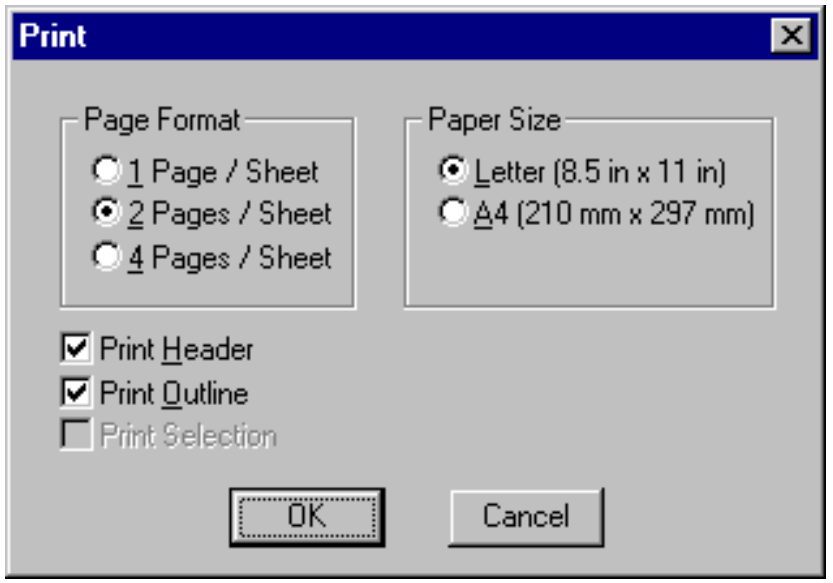

Page Format - Select the desired format option. A "page" of data is approximately 66 lines, including header information and outlines.

1 Page / Sheet - Prints one "page" of data on a single sheet of paper. The page is printed using the Portrait orientation (i.e., the short edge of the paper is horizontal).

2 Pages / Sheet - Prints two "pages" of data on a single sheet. The page is printed using the Landscape orientation (i.e., the long edge of the paper is horizontal).

4 Pages/ Sheet - Print four "pages" of data on a single sheet. The page is printed using the Landscape orientation.

Paper Size -

Print Header -
Select the size of the paper to print on.

Include header information in the print-out. Header information includes the current date, the file name, and the page number. These items are printed across the top of the page, followed by a line, separating it from the data. 
Print Outline - Outline each "page" in a rectangle. This is especially useful when using the 2 Pages / Sheet or 4 Pages / Sheet options.

Print Selection - Print the currently highlighted data.

OK - $\quad$ Print the data to the Windows System Printer using the selected options and close the Print dialog.

Cancel - $\quad$ Close the Print dialog without printing the data.

\subsubsection{Exit}

\section{PURPOSE}

This option allows you to terminate the Editor session. The rules are automatically compiled upon exiting the Editor. If there is a compilation error, the Editor will be reopened with the current file and a message will be displayed in the editor's message bar.

\section{STEPS}

1. From the Linkage Rules Editor or the Event Tree Logic Editor menu select File.

2. Select the Exit menu option.

If changes have been made to the data, you will be prompted to save the file.

\subsubsection{Save This File}

\section{PURPOSE}

This dialog will appear any time you are closing the current editing window or exiting the Editor and changes to the file have not been saved (i.e., written to disk).

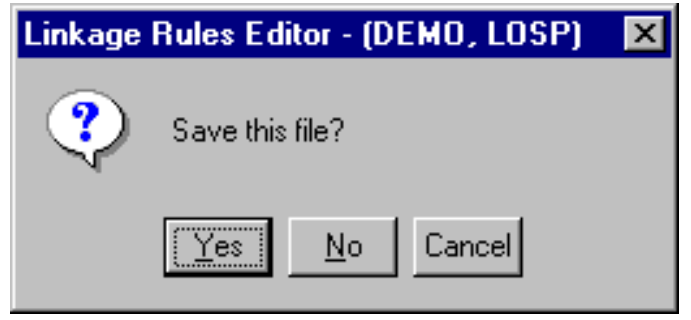

Yes - $\quad$ Save the changes made to the file in the current editing window and close the current editing window.

No - Do not save the changes made to the file in the current editing window and close the current editing window.

Cancel - Do not save the changes made to the file in the current editing window and do not close the current editing window. 


\subsubsection{Edit Menu}

\subsubsection{Editor Edit Functions}

These options provide editing and text formatting functions.
Undo -
Undo the previous editing operation.
Cut -
Remove the highlighted text from the current window and place it in the
Copy - clipboard.

\section{Paste - \\ Delete -}
Shift left indent - Copy the highlighted text from the current window and place it in the clipboard.
Shift right indent -
Shift left space -
Shift right space -
Delete to end of line -
Delete line -
Capitalize word -
Downcase word -
Link edit Windows -
Copy the clipboard text into the current window at the cursor position. Remove the highlighted text from the current window without placing it in the clipboard.
Shift the text to the left by the Shift Size specified under Options.
Shift the text to the right by the Shift Size specified under Options.
Shift the text to the left by a single space.
Shift the text to the right by a single space.
Delete to the end of the current line beginning after the current cursor position.
Delete the line on which the cursor is currently positioned.
Convert the entire word under the current cursor position to upper case. Convert the entire word under the current cursor position to lower case.

\subsubsection{Search Menu}

\subsubsection{Editor Search Functions}

These options provide search and cursor positioning operations.

Find -

Replace -

Find Procedure Find/Replace again Find Altered lines Find line number Match symbol -

Goto next error Goto previous error Goto Marker -

Set Marker -
Search the text in the current window for the specified string. Search the text in the current window for a string and replace it with another string.

Search the text in the current window for the specified procedure.

Repeat the previous search operation.

Find the lines of text that have changed since the last save operation.

Go to the specified text line number in the current window.

Find the matching symbol pair for the character under the cursor "( $),\{\}$, [], $(* *)$ "'

Position the cursor at the next compilation error.

Position the cursor at the previous compilation error.

Position the cursor at the previously saved file position. Up to four marker positions can be retrieved.

Save the current file position for later retrieval.

\subsubsection{Find}




\section{PURPOSE}

This option allows you to search the text in the current window for the specified string. If the search criteria are met, the first string matching the criteria is highlighted.

\section{STEPS}

1. From the Linkage Rules Editor or the Event Tree Logic Editor menu select Search.

2. Select the Find menu option. The Find dialog will be displayed.

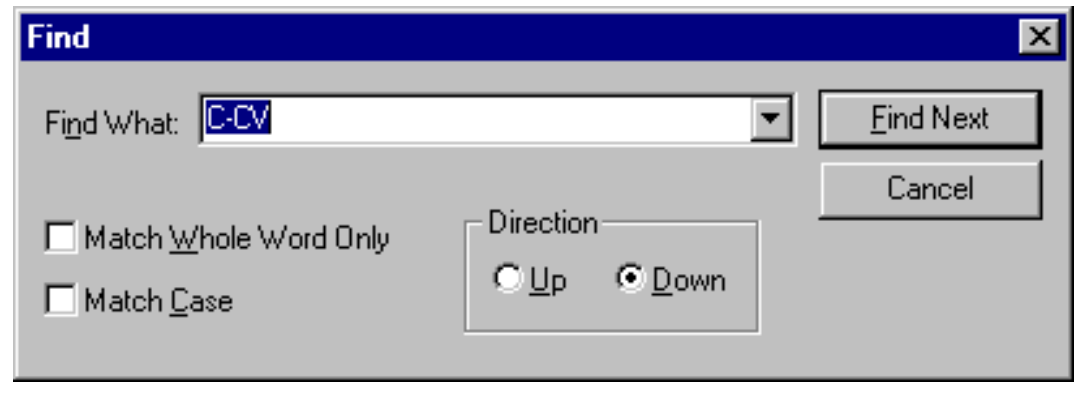

Find What -

Match Whole Word Only -

Match Case -

Direction

Up -

Down -

Find Next -

Cancel -
The string of alphanumeric characters upon which the search is based (i.e., the "search string"). Type the text in the field or select from the drop-down list.

If this check box is selected, only the series of alphanumeric characters matching the search string and delimited by a space, comma, dash, period, etc. are located.

If this check box is selected, only the series of alphanumeric characters matching the upper- and lowercase characters as entered in the Find What field are located (i.e., the search will be case-sensitive).

Search the text from the current cursor position to the beginning or top of the text.

Search the text from the current cursor position to the end or bottom of the text.

Perform the search operation using the selected criteria. If the search is not successful, an information dialog will be displayed. If the search criteria are met, the Find dialog, shown below, will become active and the current Find dialog will be closed.

Close the Find dialog without performing the search operation.

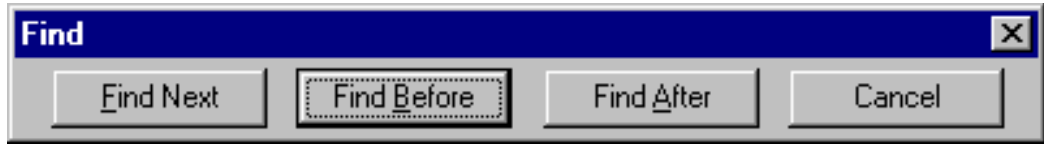


Find Next -

Find Before -

Find After -

Cancel -

\subsubsection{Replace}

\section{PURPOSE}

This option allows you to search the text in the current window for the specified string and replace it with another string. If the search criteria are met, the first string matching the criteria is highlighted.

\section{STEPS}

1. From the Linkage Rules Editor or the Event Tree Logic Editor menu select Search.

2. Select the Replace menu option. The Replace dialog will be displayed.

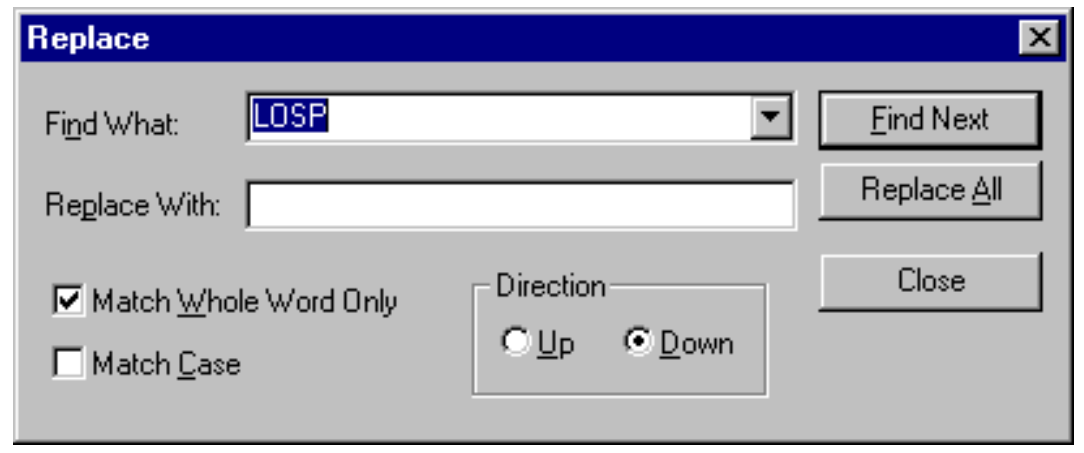

Find What -

Replace With -

Match Whole Word Only -

Match Case -
The string of alphanumeric characters upon which the search is based (i.e., the "search string"). Type the text in the field or select from the drop-down list.

The string of alphanumeric characters that will be substituted for the search string. Type the text in the field.

If this check box is selected, only the series of alphanumeric characters matching the search string and delimited by a space, comma, dash, period, etc. are located.

If this check box is selected, only the series of alphanumeric characters matching the upper- and lowercase characters as entered in the Find What field are located (i.e., the search will be case-sensitive). 


\section{Direction}

Up -

Down -

Find Next -

Replace All -

Close -
Search the text from the current cursor position to the beginning or top of the text.

Search the text from the current cursor position to the end or bottom of the text.

Perform the search operation using the selected criteria. If the search is not successful, an information dialog will be displayed. If the search criteria are met, the Find/Replace again dialog will become active and the current Replace dialog will be closed..

Find all occurrences matching the search criteria and automatically substitute with the "replace string."

Close the Replace dialog without performing the search and replace operation.

\subsubsection{Find Procedure}

\section{PURPOSE}

This option allows you to search the text in the current window for the specified procedure.

\section{STEPS}

1. From the Linkage Rules Editor, the Recovery Rules Editor, or the Partition Rules Editor menu select Search.

2. Select the Find Procedure menu option. The Procedure to search for dialog will be displayed.

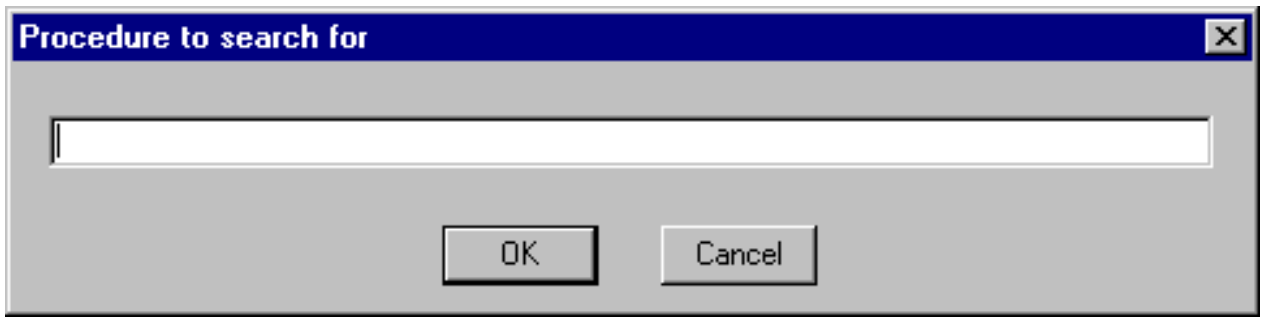

OK - Locate the procedure matching the current search criteria.

Cancel - Close the Procedure to search for dialog without performing the search operation.

\subsubsection{Find/Replace again}

\section{PURPOSE}

This option allows you to search the text in the current window based on the previously defined search string and replace it with the previously defined replace string. If the search criteria are met, the first string matching the criteria is highlighted. 


\section{STEPS}

1. From the Linkage Rules Editor or the Event Tree Logic Editor menu select Search.

2. Select the Find/Replace again menu option. The Replace dialog will be displayed.

\begin{tabular}{|c|c|c|c|}
\hline Replace & & & \\
\hline Find Next & Replace & Replace All & Close \\
\hline
\end{tabular}

Find Next - Locate the next string matching the current search criteria. A substitution will not be made.

Replace - $\quad$ Substitute the replace string for the located search string and continue searching.

Replace All - Substitute the replace string for all occurrences matching the search criteria, beginning at the current cursor position.

Close - $\quad$ Close the Replace dialog without performing the search and replace operation.

NOTE: This dialog can also be invoked from the Search | Replace | Find Next menu options.

\subsubsection{Find line number}

\section{PURPOSE}

Position the cursor on the specified line number in the text in the current window.

\section{STEPS}

1. From the Linkage Rules Editor or the Event Tree Logic Editor menu select Search.

2. Select the Find line number menu option.

3. The Enter line number dialog will be displayed.

4. Type the desired line number in the field.

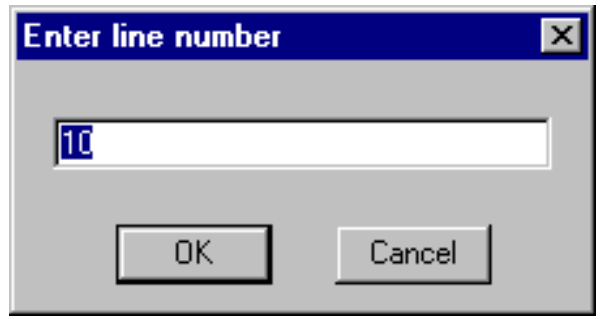

OK - Position the cursor at the beginning of the desired line and close the dialog.

Cancel - Close the dialog without repositioning the cursor.

\subsubsection{Goto Marker}




\section{PURPOSE}

Position the cursor at the previously saved file position. Up to four marker positions can be retrieved for each editing window.

\section{STEPS}

1. From the Linkage Rules Editor or the Event Tree Logic Editor menu select Search.

2. Select the Goto Marker menu option.

3. Choose one of the four marker positions available from the submenu.

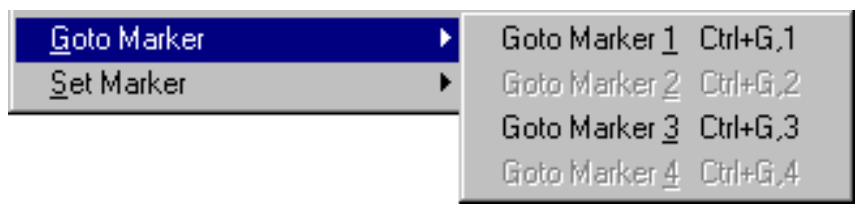

In this example, two markers have been set at \#1 and \#3. After choosing one of the available markers, the cursor is positioned at the beginning of the line.

The marker positions are saved only during the current editing session.

\subsubsection{Set Marker}

\section{PURPOSE}

Save the current file position for later retrieval. Up to four marker positions can be saved for each editing window.

\section{STEPS}

1. Position the cursor on the desired line of the text in the edit window.

2. From the Linkage Rules Editor or the Event Tree Logic Editor menu select Search.

3. Select the Set Marker menu option.

4. Choose one of the four marker positions available from the submenu.

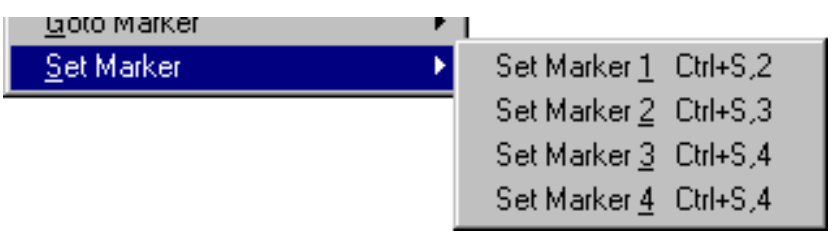

The marker positions are saved only during the current editing session.

\subsubsection{Window Menu}

\subsubsection{Editor Window Functions}


These options are used for managing the open edit windows within the application window, such as the Linkage Rules Editor or the Event Tree Logic Editor window. Along with the options listed below and segregated by a separation bar, the names of each open file is listed in the menu.

Cascade windows -

Tile windows horizontally -

Tile windows vertically -

Arrange Icons -
Resize and reposition the non-minimized windows in an overlapping fashion so that the title of each window is displayed in a cascade arrangement. The current edit window will be on top.

Resize and reposition the non-minimized windows in a horizontally tiled arrangement.

Resize and reposition the non-minimized windows in a vertically tiled arrangement.

Arrange the minimized window icons at the bottom of the application window.

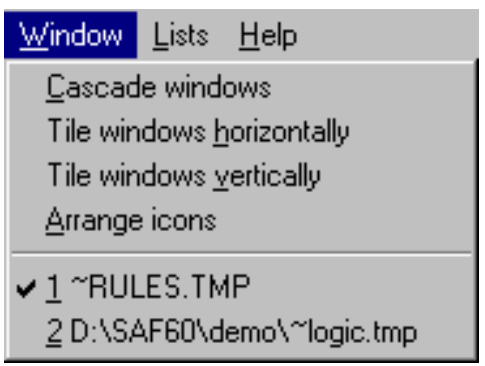

In the example above, the files " $\sim$ RULES.TMP" and " $\sim$ LOGIC.TMP" are open. $\sim$ RULES.TMP is the active window, as indicated by the check mark on the menu.

\subsubsection{Lists Menu}

\subsubsection{Linkage Rules Lists}

Selecting from the lists allows you to insert items in the editor. Any or all of the lists can be open simultaneously.

Macros - $\quad$ Insert a macro name into the text.

Events - $\quad$ Insert a basic event name into the text.

Initiators - Insert an initiating event name into the text.

Fault Trees - Insert a fault tree name into the text.

End States - $\quad$ Insert an end state name into the text.

Event Trees - Insert an event tree name into the text.

Flags - $\quad$ Insert a flag set name into the text.

\subsubsection{Macros}

\section{PURPOSE}

This option allows you to insert a macro into the text of the current edit window. 


\section{STEPS}

1. Place the cursor at the desired location in the text.

2. From the Linkage Rules Editor or the Event Tree Logic Editor menu select Lists.

3. Select the Macros menu option. The Macro List dialog will be displayed.

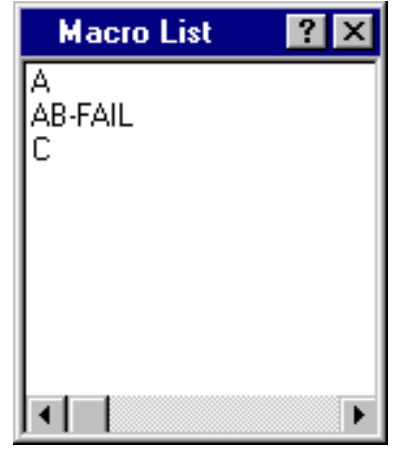

4. Double-click on the desired macro name in the list. The selected name will be entered at the current cursor location.

The Macro List dialog will remain open in the application window during the current session unless explicitly closed.

\subsubsection{Events}

\section{PURPOSE}

This option allows you to insert a basic event name into the text of the current edit window.

\section{STEPS}

1. Place the cursor at the desired location in the text.

2. From the Linkage Rules Editor or the Event Tree Logic Editor menu select Lists.

3. Select the Events menu option. The Event List dialog will be displayed containing all of the basic events in the current family's database.

\begin{tabular}{|c|c|}
\hline Event List & ? $\mathrm{x}$ \\
\hline $\begin{array}{l}\langle\text { FALSE }\rangle \\
\langle\text { NII }\rangle\end{array}$ & \\
\hline 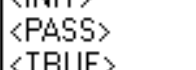 & - \\
\hline $\begin{array}{l}\text { RTRUE } \\
\text { C-CV-A } \\
\text { C-CV-B }\end{array}$ & \\
\hline C-MOV-1 & $\mp$ \\
\hline 111 & 11 \\
\hline
\end{tabular}


4. Double-click on the desired event name in the list. The selected name will be entered at the current cursor location.

The Event List dialog will remain open in the application window during the current session unless explicitly closed.

\subsubsection{Initiators}

\section{PURPOSE}

This option allows you to insert an initiating basic event name into the text of the current edit window.

\section{STEPS}

1. Place the cursor at the desired location in the text.

2. From the Linkage Rules Editor or the Event Tree Logic Editor menu select Lists.

3. Select the Initiators menu option. The Initiator List dialog will be displayed containing all of the basic events whose category attribute is set to "I" in the current family's database.

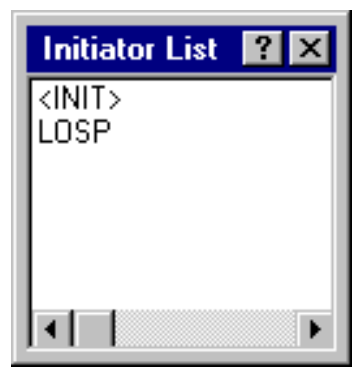

4. Double-click on the desired initiating event name in the list. The selected name will be entered at the current cursor location.

The Initiator List dialog will remain open in the application window during the current session unless explicitly closed.

\subsubsection{Systems}

\section{PURPOSE}

This option allows you to insert a fault tree name into the text of the current edit window.

\section{STEPS}

1. Place the cursor at the desired location in the text.

2. From the Linkage Rules Editor or the Event Tree Logic Editor menu select Lists. 
3. Select the Systems menu option. The System List dialog will be displayed containing all of the fault trees in the current family's database.

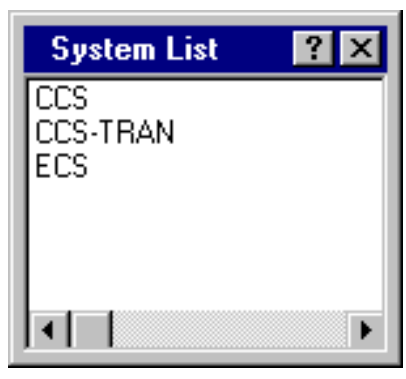

4. Double-click on the desired fault tree name in the list. The selected name will be entered at the current cursor location.

The System List dialog will remain open in the application window during the current session unless explicitly closed.

\subsubsection{End States}

\section{PURPOSE}

This option allows you to insert an end state name into the text of the current edit window.

\section{STEPS}

1. Place the cursor at the desired location in the text.

2. From the Linkage Rules Editor or the Event Tree Logic Editor menu select Lists.

3. Select the End States menu option. The Endstate List dialog will be displayed containing all of the end states in the current family's database.

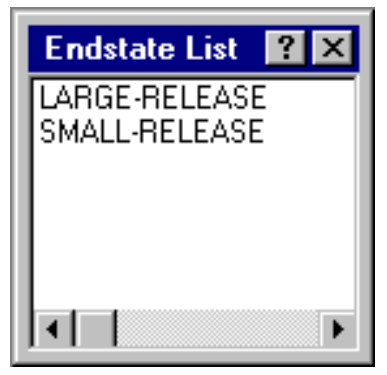

4. Double-click on the desired end state name in the list. The selected name will be entered at the current cursor location. 
The Endstate List dialog will remain open in the application window during the current session unless explicitly closed.

\subsubsection{Event Trees}

\section{PURPOSE}

This option allows you to insert an event tree name into the text of the current edit window.

\section{STEPS}

1. Place the cursor at the desired location in the text.

2. From the Linkage Rules Editor or the Event Tree Logic Editor menu select Lists.

3. Select the Event Trees menu option. The Event Tree List dialog will be displayed containing all of the event trees in the current family's database.

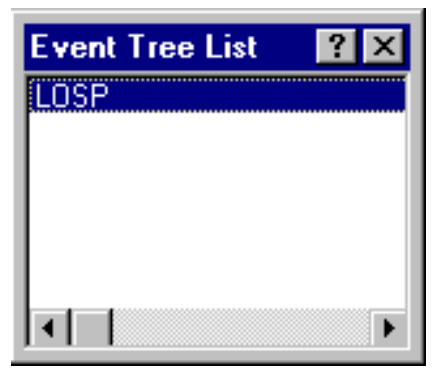

4. Double-click on the desired event tree name in the list. The selected name will be entered at the current cursor location.

The Event Tree List dialog will remain open in the application window during the current session unless explicitly closed.

\subsubsection{Flags}

\section{PURPOSE}

This option allows you to insert a flag set name into the text of the current edit window.

\section{STEPS}

1. Place the cursor at the desired location in the text.

2. From the Linkage Rules Editor or the Event Tree Logic Editor menu select Lists.

3. Select the Flags menu option. The Flag List dialog will be displayed containing all of the flag sets in the current family's database. 


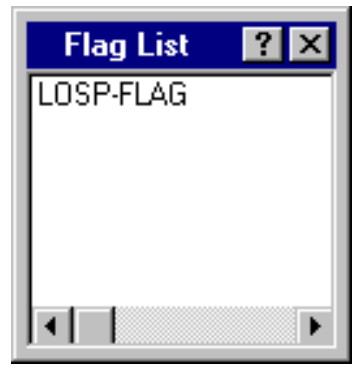

4. Double-click on the desired flag set name in the list. The selected name will be entered at the current cursor location.

The Flag List dialog will remain open in the application window during the current session unless explicitly closed.

\subsection{Partition Rules Editor}

\subsubsection{Partition Rules Editor}

\section{PURPOSE}

This editor allows you to define a set of rules that map the cut sets for a sequence to an end state. This is a free format, macro-based line editor that provides you with an if-then-else logic structure. If rules have already been defined they will be displayed in the edit window.

By default, the editor assumes that a name is the name of a basic event, if one exists in the database. If a basic event by the name does not exist then the editor assumes that the name is the name of a macro. If you want to change the default assumptions, then you can "cast" the names to something else by enclosing the name in parenthesis with a cast name preceding it.

There is no limit to the number of rules you may input to the editor. Each rule is processed in sequential order.

\section{STEPS}

1. From the SAPHIRE menu select Sequence. The Sequences dialog will be displayed.

2. Highlight the desired sequence(s) and right-click to invoke the pop-up menu.

3. Choose Cut Sets | Partition | Edit Rules. The Edit Recovery Rules dialog will appear.

4. $\quad$ Select the desired Rule Level and Rule Type, and press OK.

If rules have been previously defined they will be displayed in the edit window. 


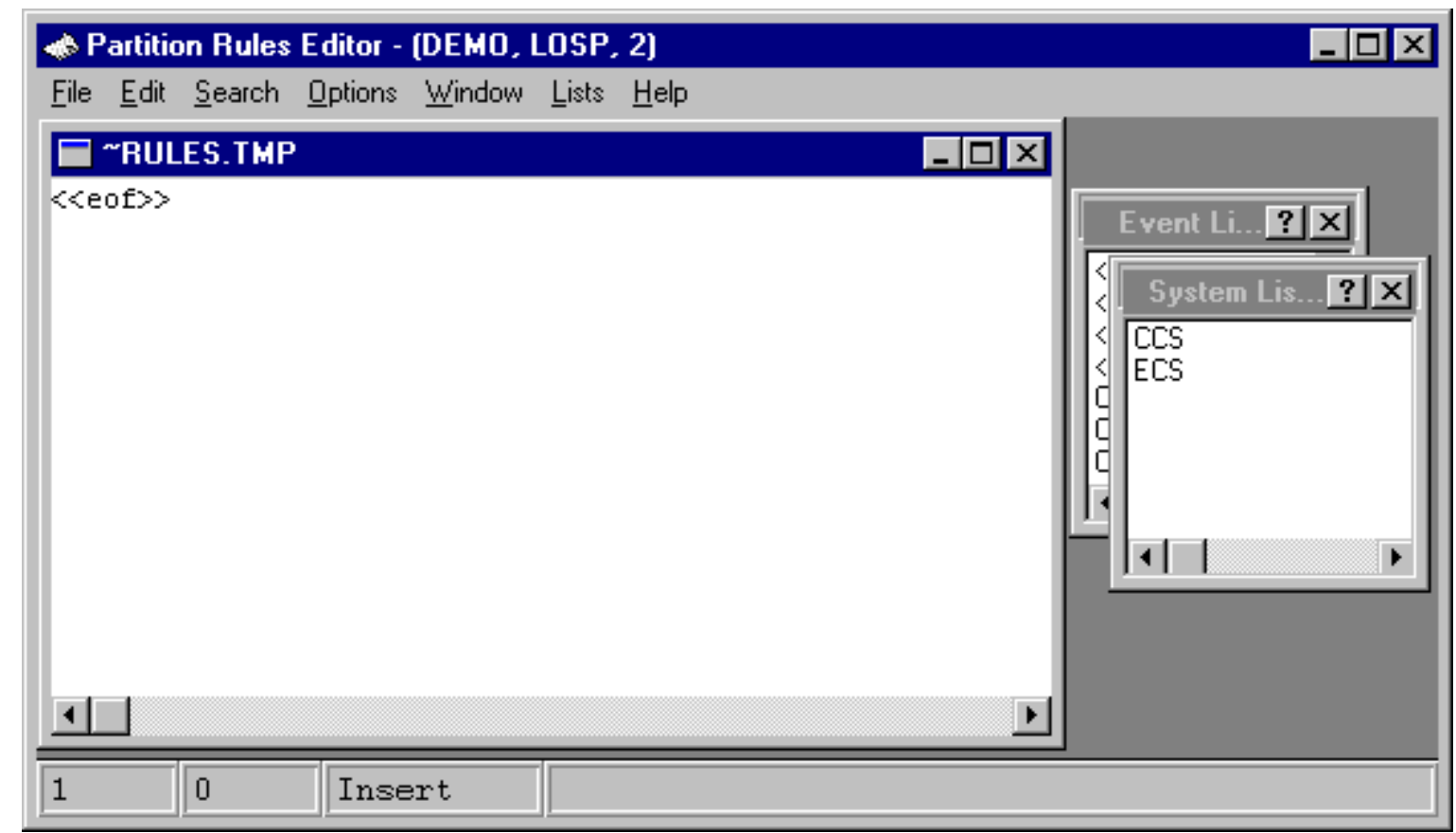

File File functions such as open, save, print, preferences, etc.

Edit Editing functions such as copy and paste along with text format operations.

Search Search and cursor positioning operations such as find, replace, goto, etc.

Window - Window management functions such as cascade, tile horizontally, etc.

Lists - $\quad$ Display lists of initiators, fault trees, etc. for inserting into the rules.

\subsubsection{File Menu}

\subsubsection{Editor File Functions}

These options provide file management functions.

Open - $\quad$ Open a file into a new window. This feature is not usually used while editing SAPHIRE rules.

New -

Create a new file in a new window. This feature is not usually used while editing SAPHIRE rules.

Close - $\quad$ Close the edit current window. If changes have been made you will be prompted to save the file.

Quit - Close the current edit window without saving.

Save - $\quad$ Save the file in the current window to disk.

Save As - $\quad$ Save the file in the current window with a new file name.

Save All - $\quad$ Save all the files in all edit windows currently open.

Save Block - $\quad$ Save the currently highlighted text into a new file. The Save As dialog will be invoked.

Import File - Insert the contents of a file into the current edit window at the current cursor position. The Edit external file dialog will be invoked.

Print - $\quad$ Print the file in the current edit window.

Page setup - $\quad$ Set printer page layout options, such as pages per sheet and line numbers.

Preferences - $\quad$ Select editing options such as tab width, undo levels, etc. 
Exit -

Terminate the Editor session.

\subsubsection{Open}

\section{PURPOSE}

This option allows you to open an existing file into a new editing window within the Linkage Rules Editor or the Event Tree Logic Editor.

\section{STEPS}

1. From the Linkage Rules Editor or the Event Tree Logic Editor menu select File.

2. Select the Open menu option.

3. The Edit external file dialog will be displayed listing all of the files in the current project directory.

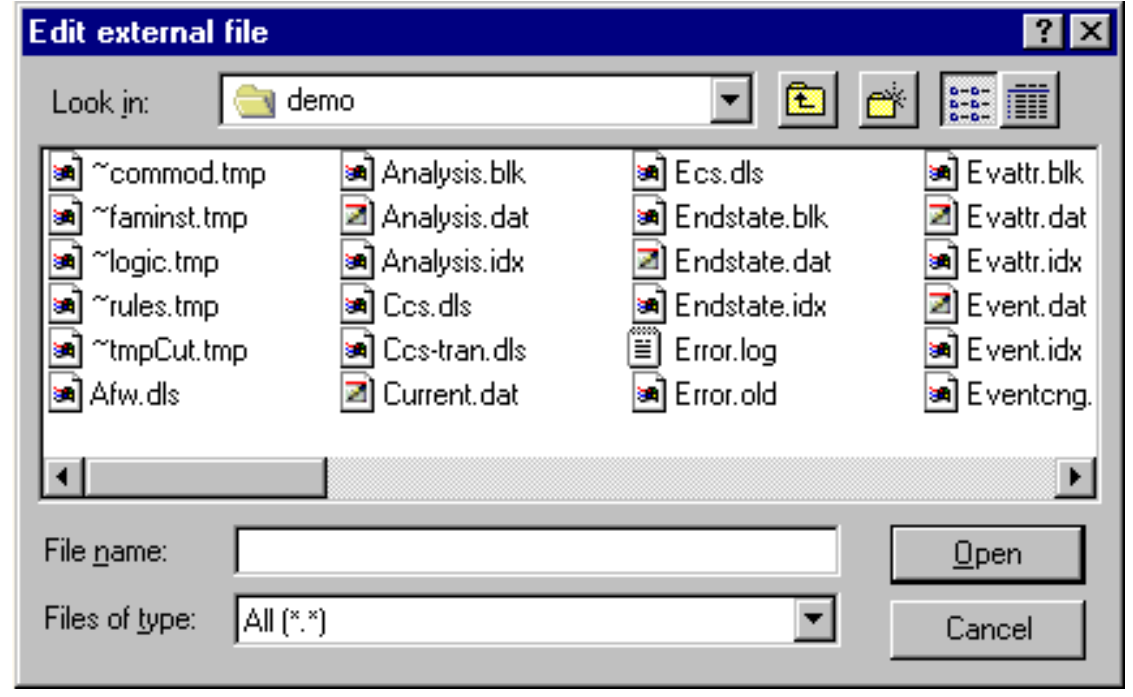

Look in - Lists the available folders and files. The box directly below it shows the contents of the current folder. The down-arrow to the right of this field shows how the folder fits into your system hierarchy.

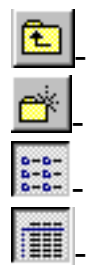

Moves up one level in the system hierarchy.

Creates a new folder at the current level.

Displays the icons and names of the contents of the current folder.

Displays the icons, names, size, type, date and time modified, and archive flags of the contents of the current folder.

File Name - Contains the name of the file in which the diagram will be saved.

Files of Type - Lists the types of files to display. This is useful for narrowing the list of files displayed to only those you are interested in.

Open -

Cancel Open the selected file in a new window and close the Edit external file dialog. Close the Edit external file dialog without opening another file. 
Double-click - Open the selected file in a new window and close the Edit external file dialog.

\subsubsection{Save As}

\section{PURPOSE}

This option allows you to save the file in the current editing window with a new file name.

\section{STEPS}

1. From the Linkage Rules Editor or the Event Tree Logic Editor menu select File.

2. Select the Save As menu option. The Save As dialog will be displayed.

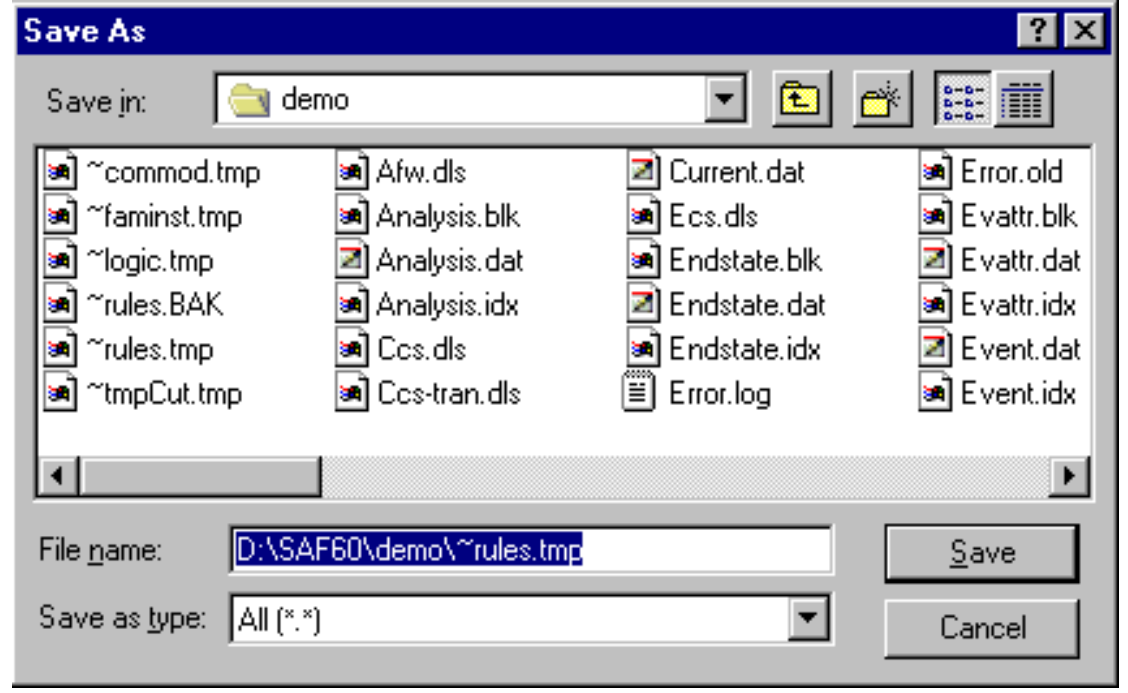

Save in - $\quad$ Lists the available folders and files. The box directly below it shows the contents of the current folder. The down-arrow to the right of this field shows how the folder fits into your system hierarchy.

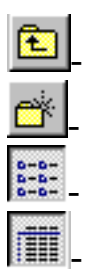

File Name Files of Type -

Save -

Double-click -

Cancel -
Moves up one level in the system hierarchy.

Creates a new folder at the current level.

Displays the icons and names of the contents of the current folder.

Displays the icons, names, size, type, date and time modified, and archive flags of the contents of the current folder.

Contains the name of the file to be saved.

Lists the types of files to display. This is useful for narrowing the list of files displayed to only those you are interested in.

Save the data in the current editing window to the file in the File name field and close the Save Fault Tree dialog. If an existing file was selected, a warning dialog will be displayed.

Save the current diagram to the file in the File name field and close the Save As dialog.

Close the Save As dialog without performing the save operation.

\subsubsection{Print}




\section{PURPOSE}

This option allows you to print the file in the current edit window to the default (Windows System) printer.

\section{STEPS}

1. From the Linkage Rules Editor or the Event Tree Logic Editor menu select File.

2. Select the Print menu option. The Print dialog will be displayed.

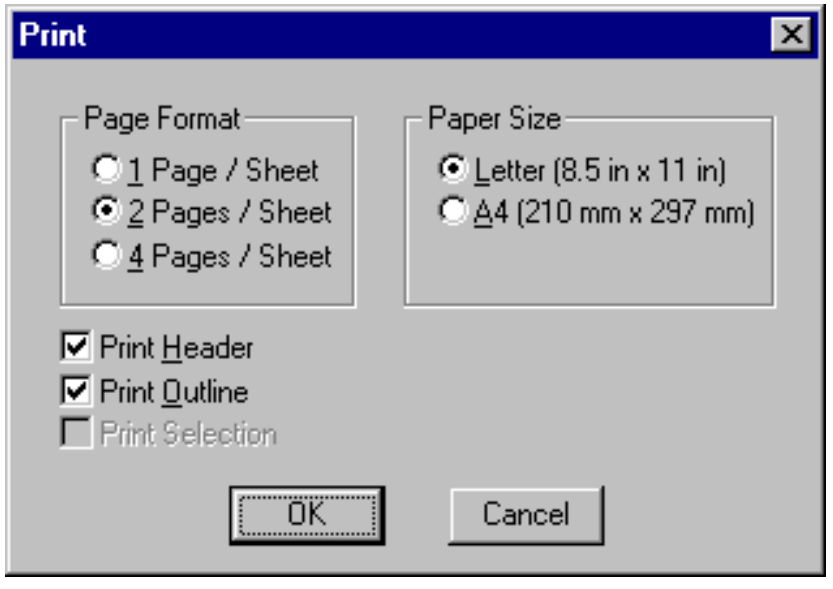

Page Format - Select the desired format option. A "page" of data is approximately 66 lines, including header information and outlines.

1 Page / Sheet - Prints one "page" of data on a single sheet of paper. The page is printed using the Portrait orientation (i.e., the short edge of the paper is horizontal).

2 Pages / Sheet - Prints two "pages" of data on a single sheet. The page is printed using the Landscape orientation (i.e., the long edge of the paper is horizontal).

4 Pages/ Sheet - Print four "pages" of data on a single sheet. The page is printed using the Landscape orientation.

Paper Size - $\quad$ Select the size of the paper to print on.

Print Header - Include header information in the print-out. Header information includes the current date, the file name, and the page number. These items are printed across the top of the page, followed by a line, separating it from the data.

Print Outline - Outline each "page" in a rectangle. This is especially useful when using the 2 Pages / Sheet or 4 Pages / Sheet options.

Print Selection - Print the currently highlighted data.

OK -

Cancel -
Print the data to the Windows System Printer using the selected options and close the Print dialog.

\subsubsection{Exit}




\section{PURPOSE}

This option allows you to terminate the Editor session. The rules are automatically compiled upon exiting the Editor. If there is a compilation error, the Editor will be reopened with the current file and a message will be displayed in the editor's message bar.

\section{STEPS}

1. From the Linkage Rules Editor or the Event Tree Logic Editor menu select File.

2. Select the Exit menu option.

If changes have been made to the data, you will be prompted to save the file.

\subsubsection{Save This File}

\section{PURPOSE}

This dialog will appear any time you are closing the current editing window or exiting the Editor and changes to the file have not been saved (i.e., written to disk).

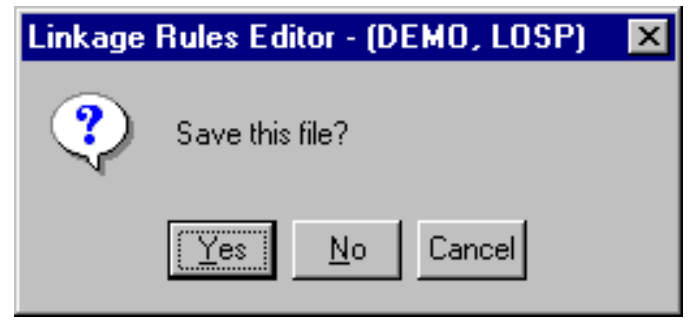

Yes - $\quad$ Save the changes made to the file in the current editing window and close the current editing window.

No - Do not save the changes made to the file in the current editing window and close the current editing window.

Cancel - Do not save the changes made to the file in the current editing window and do not close the current editing window.

\subsubsection{Edit Menu}

\subsubsection{Editor Edit Functions}

These options provide editing and text formatting functions.
Undo -
Undo the previous editing operation.
Cut -
Copy -
Paste -
Delete -
Shift left indent -
Remove the highlighted text from the current window and place it in the clipboard.
Copy the highlighted text from the current window and place it in the clipboard.
Copy the clipboard text into the current window at the cursor position. Remove the highlighted text from the current window without placing it in the clipboard.
Shift the text to the left by the Shift Size specified under Options. 
Shift right indent -

Shift left space -

Shift right space -

Delete to end of line -

Delete line -

Capitalize word -

Downcase word -

Link edit Windows -
Shift the text to the right by the Shift Size specified under Options.

Shift the text to the left by a single space.

Shift the text to the right by a single space.

Delete to the end of the current line beginning after the current cursor position.

Delete the line on which the cursor is currently positioned.

Convert the entire word under the current cursor position to upper case.

Convert the entire word under the current cursor position to lower case.

\subsubsection{Search Menu}

\subsubsection{Editor Search Functions}

These options provide search and cursor positioning operations.

Find -

Replace -

Find Procedure Find/Replace again Find Altered lines Find line number Match symbol -

Goto next error -

Goto previous error -

Goto Marker -

Set Marker -
Search the text in the current window for the specified string.

Search the text in the current window for a string and replace it with another string.

Search the text in the current window for the specified procedure.

Repeat the previous search operation.

Find the lines of text that have changed since the last save operation.

Go to the specified text line number in the current window.

Find the matching symbol pair for the character under the cursor "( ), \{\} , [], (**)"

Position the cursor at the next compilation error.

Position the cursor at the previous compilation error.

Position the cursor at the previously saved file position. Up to four marker positions can be retrieved.

Save the current file position for later retrieval.

\subsubsection{Find}

\section{PURPOSE}

This option allows you to search the text in the current window for the specified string. If the search criteria are met, the first string matching the criteria is highlighted.

\section{STEPS}

1. From the Linkage Rules Editor or the Event Tree Logic Editor menu select Search.

2. Select the Find menu option. The Find dialog will be displayed. 


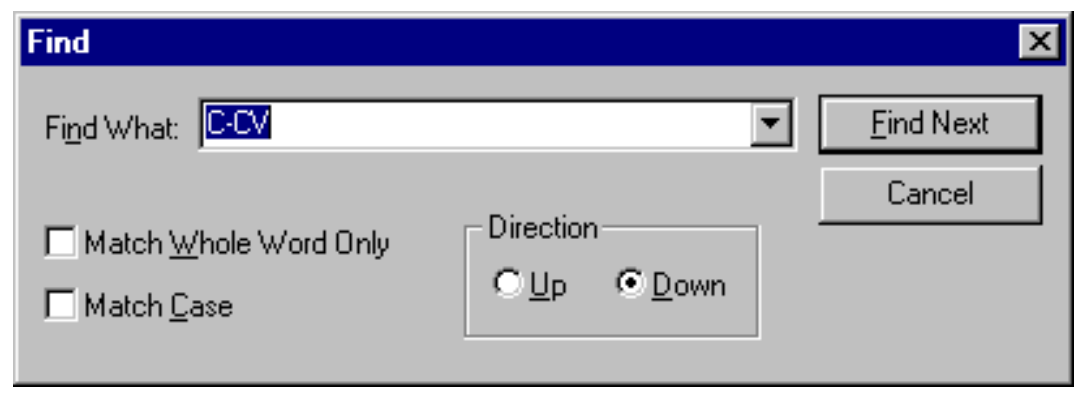

Find What -

Match Whole Word Only -

Match Case -

Direction

Up -

Down -

Find Next -

Cancel -
The string of alphanumeric characters upon which the search is based (i.e., the "search string"). Type the text in the field or select from the drop-down list.

If this check box is selected, only the series of alphanumeric characters matching the search string and delimited by a space, comma, dash, period, etc. are located.

If this check box is selected, only the series of alphanumeric characters matching the upper- and lowercase characters as entered in the Find What field are located (i.e., the search will be case-sensitive).

Search the text from the current cursor position to the beginning or top of the text.

Search the text from the current cursor position to the end or bottom of the text.

Perform the search operation using the selected criteria. If the search is not successful, an information dialog will be displayed. If the search criteria are met, the Find dialog, shown below, will become active and the current Find dialog will be closed..

Close the Find dialog without performing the search operation.

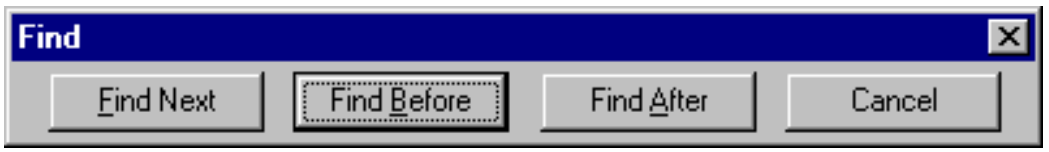

Find Next - $\quad$ Locate the next string matching the current search criteria.

Find Before - Locate the previous string matching the current search criteria.

Find After - $\quad$ Locate the next string matching the current search criteria.

Cancel - $\quad$ Close the Find dialog without performing the search operation.

\subsubsection{Replace}




\section{PURPOSE}

This option allows you to search the text in the current window for the specified string and replace it with another string. If the search criteria are met, the first string matching the criteria is highlighted.

\section{STEPS}

1. From the Linkage Rules Editor or the Event Tree Logic Editor menu select Search.

2. Select the Replace menu option. The Replace dialog will be displayed.

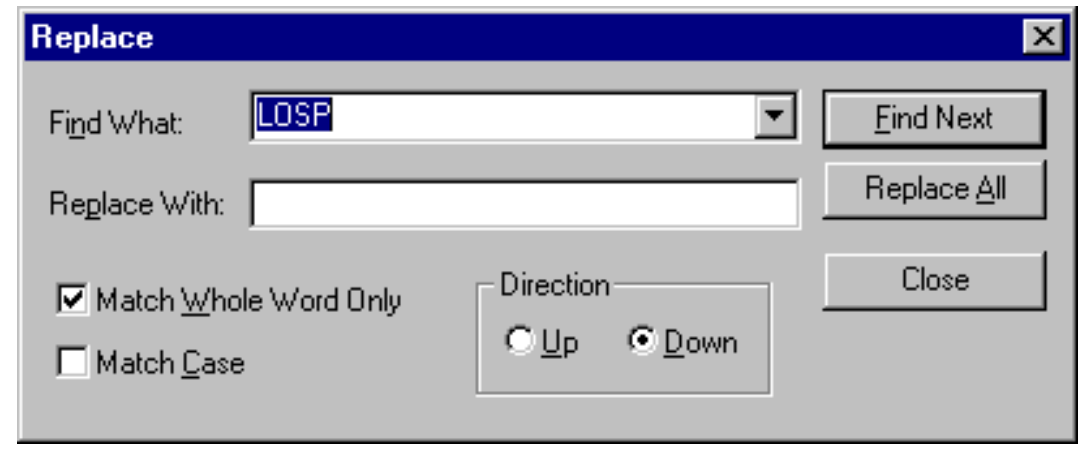

Find What -

Replace With -

Match Whole Word Only -

Match Case -

Direction

Up -

Down -

Find Next -

Replace All -
The string of alphanumeric characters upon which the search is based (i.e., the "search string"). Type the text in the field or select from the drop-down list.

The string of alphanumeric characters that will be substituted for the search string. Type the text in the field.

If this check box is selected, only the series of alphanumeric characters matching the search string and delimited by a space, comma, dash, period, etc. are located.

If this check box is selected, only the series of alphanumeric characters matching the upper- and lowercase characters as entered in the Find What field are located (i.e., the search will be case-sensitive).

Search the text from the current cursor position to the beginning or top of the text.

Search the text from the current cursor position to the end or bottom of the text.

Perform the search operation using the selected criteria. If the search is not successful, an information dialog will be displayed. If the search criteria are met, the Find/Replace again dialog will become active and the current Replace dialog will be closed.

Find all occurrences matching the search criteria and automatically substitute with the "replace string." 
Close -

\subsubsection{Find/Replace again}

\section{PURPOSE}

This option allows you to search the text in the current window based on the previously defined search string and replace it with the previously defined replace string. If the search criteria are met, the first string matching the criteria is highlighted.

\section{STEPS}

1. From the Linkage Rules Editor or the Event Tree Logic Editor menu select Search.

2. Select the Find/Replace again menu option. The Replace dialog will be displayed.

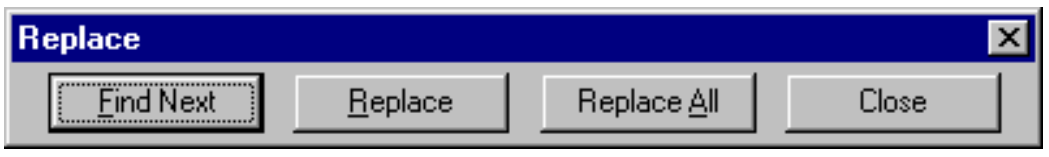

Find Next - $\quad$ Locate the next string matching the current search criteria. A substitution will not be made.

Replace - $\quad$ Substitute the replace string for the located search string and continue searching.

Replace All - Substitute the replace string for all occurrences matching the search criteria, beginning at the current cursor position.

Close - $\quad$ Close the Replace dialog without performing the search and replace operation.

NOTE: This dialog can also be invoked from the Search | Replace | Find Next menu options.

\subsubsection{Find Procedure}

\section{PURPOSE}

This option allows you to search the text in the current window for the specified procedure.

\section{STEPS}

1. From the Linkage Rules Editor, the Recovery Rules Editor, or the Partition Rules Editor menu select Search.

2. Select the Find Procedure menu option. The Procedure to search for dialog will be displayed.

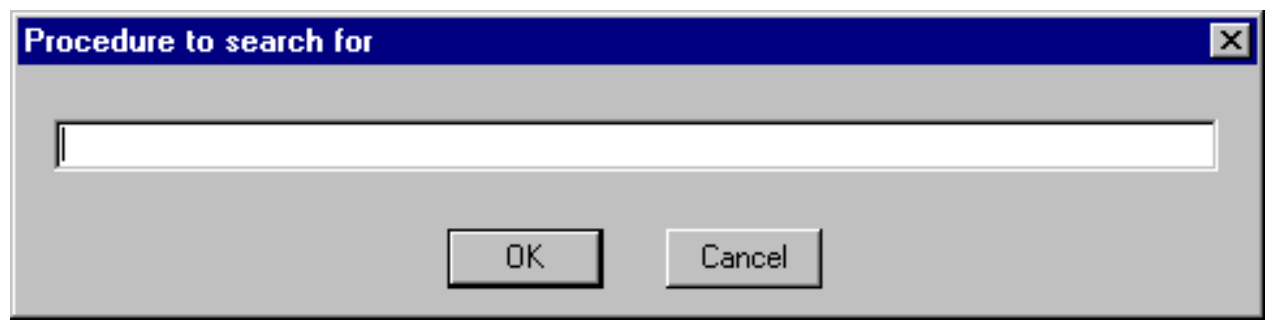

OK - Locate the procedure matching the current search criteria.

Cancel - Close the Procedure to search for dialog without performing the search operation. 


\subsubsection{Find line number}

\section{PURPOSE}

Position the cursor on the specified line number in the text in the current window.

\section{STEPS}

1. From the Linkage Rules Editor or the Event Tree Logic Editor menu select Search.

2. Select the Find line number menu option.

3. The Enter line number dialog will be displayed.

4. Type the desired line number in the field.

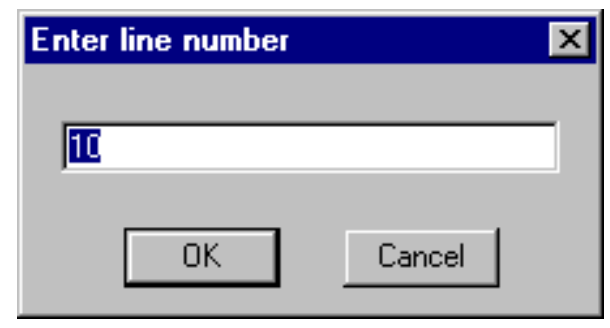

OK - Position the cursor at the beginning of the desired line and close the dialog.

Cancel - Close the dialog without repositioning the cursor.

\subsubsection{Goto Marker}

\section{PURPOSE}

Position the cursor at the previously saved file position. Up to four marker positions can be retrieved for each editing window.

STEPS

1. From the Linkage Rules Editor or the Event Tree Logic Editor menu select Search.

2. Select the Goto Marker menu option.

3. Choose one of the four marker positions available from the submenu.

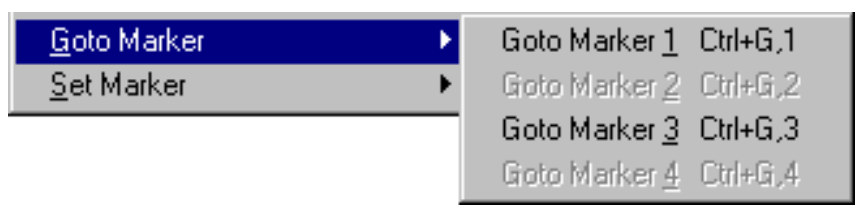

In this example, two markers have been set at \#1 and \#3. After choosing one of the available markers, the cursor is positioned at the beginning of the line.

The marker positions are saved only during the current editing session. 


\subsubsection{Set Marker}

\section{PURPOSE}

Save the current file position for later retrieval. Up to four marker positions can be saved for each editing window.

\section{STEPS}

1. Position the cursor on the desired line of the text in the edit window.

2. From the Linkage Rules Editor or the Event Tree Logic Editor menu select Search.

3. Select the Set Marker menu option.

4. Choose one of the four marker positions available from the submenu.

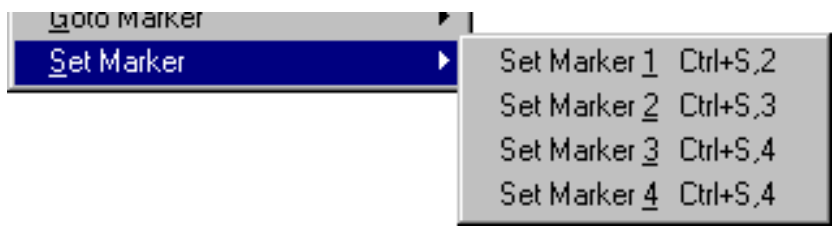

The marker positions are saved only during the current editing session.

\subsubsection{Window Menu}

\subsubsection{Editor Window Functions}

These options are used for managing the open edit windows within the application window, such as the Linkage Rules Editor or the Event Tree Logic Editor window. Along with the options listed below and segregated by a separation bar, the names of each open file is listed in the menu.

Cascade windows -

Tile windows horizontally -

Tile windows vertically -

Arrange Icons -
Resize and reposition the non-minimized windows in an overlapping fashion so that the title of each window is displayed in a cascade arrangement. The current edit window will be on top.

Resize and reposition the non-minimized windows in a horizontally tiled arrangement.

Resize and reposition the non-minimized windows in a vertically tiled arrangement.

Arrange the minimized window icons at the bottom of the application window.

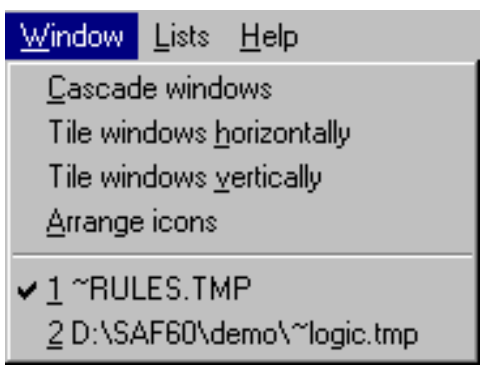


In the example above, the files " $\sim$ RULES.TMP" and " $\sim$ LOGIC.TMP" are open. $\sim$ RULES.TMP is the active window, as indicated by the check mark on the menu.

\subsubsection{Lists Menu}

\subsubsection{Partition Rules Lists}

Selecting from the lists allows you to insert or replace items in the editor.

Macros - $\quad$ Allows you to insert a macro into the editor.

Events - $\quad$ Select basic events used to replace others instead of typing in the basic event names.

Initiators - $\quad$ Select initiators used to replace others instead of typing in the initiator names.

Fault Trees - $\quad$ Select fault trees as top events and fault trees used to replace others instead of typing in the fault trees names.

End States - $\quad$ Select end states used to replace others instead of typing in the end state names.

Event Trees - Select event trees used to replace others instead of typing in the event tree names.

\subsubsection{Macros}

\section{PURPOSE}

This option allows you to insert a macro into the text of the current edit window.

\section{STEPS}

1. Place the cursor at the desired location in the text.

2. From the Linkage Rules Editor or the Event Tree Logic Editor menu select Lists.

3. Select the Macros menu option. The Macro List dialog will be displayed.

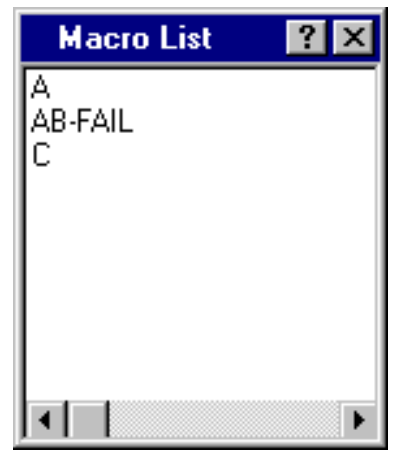

4. Double-click on the desired macro name in the list. The selected name will be entered at the current cursor location.

The Macro List dialog will remain open in the application window during the current session unless explicitly closed.

\subsubsection{Events}




\section{PURPOSE}

This option allows you to insert a basic event name into the text of the current edit window.

\section{STEPS}

1. Place the cursor at the desired location in the text.

2. From the Linkage Rules Editor or the Event Tree Logic Editor menu select Lists.

3. Select the Events menu option. The Event List dialog will be displayed containing all of the basic events in the current family's database.

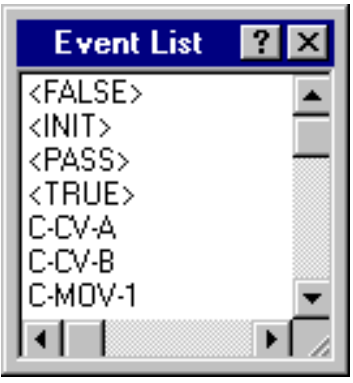

4. Double-click on the desired event name in the list. The selected name will be entered at the current cursor location.

The Event List dialog will remain open in the application window during the current session unless explicitly closed. 


\subsubsection{Initiators}

\section{PURPOSE}

This option allows you to insert an initiating basic event name into the text of the current edit window.

\section{STEPS}

1. Place the cursor at the desired location in the text.

2. From the Linkage Rules Editor or the Event Tree Logic Editor menu select Lists.

3. Select the Initiators menu option. The Initiator List dialog will be displayed containing all of the basic events whose category attribute is set to "I" in the current family's database.

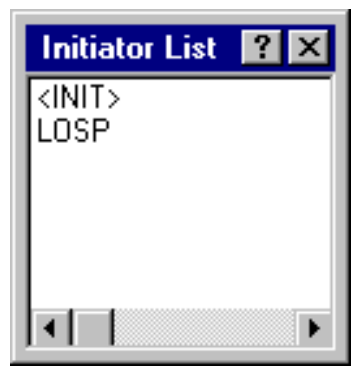

4. Double-click on the desired initiating event name in the list. The selected name will be entered at the current cursor location.

The Initiator List dialog will remain open in the application window during the current session unless explicitly closed.

\subsubsection{Systems}

\section{PURPOSE}

This option allows you to insert a fault tree name into the text of the current edit window.

\section{STEPS}

1. Place the cursor at the desired location in the text.

2. From the Linkage Rules Editor or the Event Tree Logic Editor menu select Lists.

3. Select the Systems menu option. The System List dialog will be displayed containing all of the fault trees in the current family's database. 


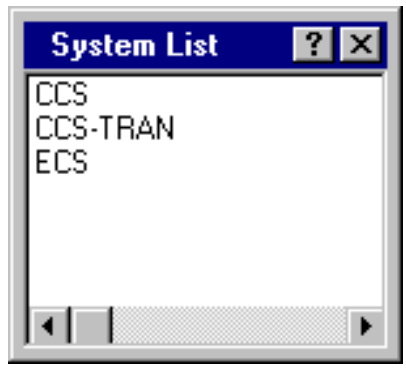

4. Double-click on the desired fault tree name in the list. The selected name will be entered at the current cursor location.

The System List dialog will remain open in the application window during the current session unless explicitly closed.

\subsubsection{End States}

\section{PURPOSE}

This option allows you to insert an end state name into the text of the current edit window.

\section{STEPS}

1. Place the cursor at the desired location in the text.

2. From the Linkage Rules Editor or the Event Tree Logic Editor menu select Lists.

3. Select the End States menu option. The Endstate List dialog will be displayed containing all of the end states in the current family's database.

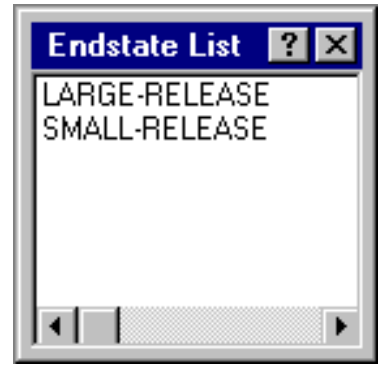

4. Double-click on the desired end state name in the list. The selected name will be entered at the current cursor location.

The Endstate List dialog will remain open in the application window during the current session unless explicitly closed. 


\subsubsection{Event Trees}

\section{PURPOSE}

This option allows you to insert an event tree name into the text of the current edit window.

\section{STEPS}

1. Place the cursor at the desired location in the text.

2. From the Linkage Rules Editor or the Event Tree Logic Editor menu select Lists.

3. Select the Event Trees menu option. The Event Tree List dialog will be displayed containing all of the event trees in the current family's database.

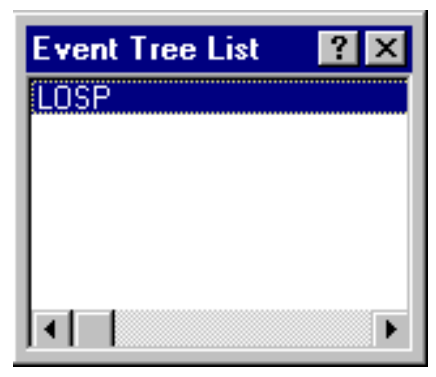

4. Double-click on the desired event tree name in the list. The selected name will be entered at the current cursor location.

The Event Tree List dialog will remain open in the application window during the current session unless explicitly closed.

\subsection{Link Trees}

\subsubsection{Linking Event Tree Sequences}

\section{PURPOSE}

This option allows you to generate sequence logic using the event tree graphical files. During sequence generation the sequence logic is created as specified in the event tree logic, and then the linkage rules are applied.

\section{STEPS}

1. From the SAPHIRE menu select Event Tree. The Event Tree List dialog will be displayed.

2. Highlight the desired event tree(s) in the list.

3. Right-click to invoke the pop-up menu and choose Link Trees. The Sequence Logic Generate dialog will be displayed. 


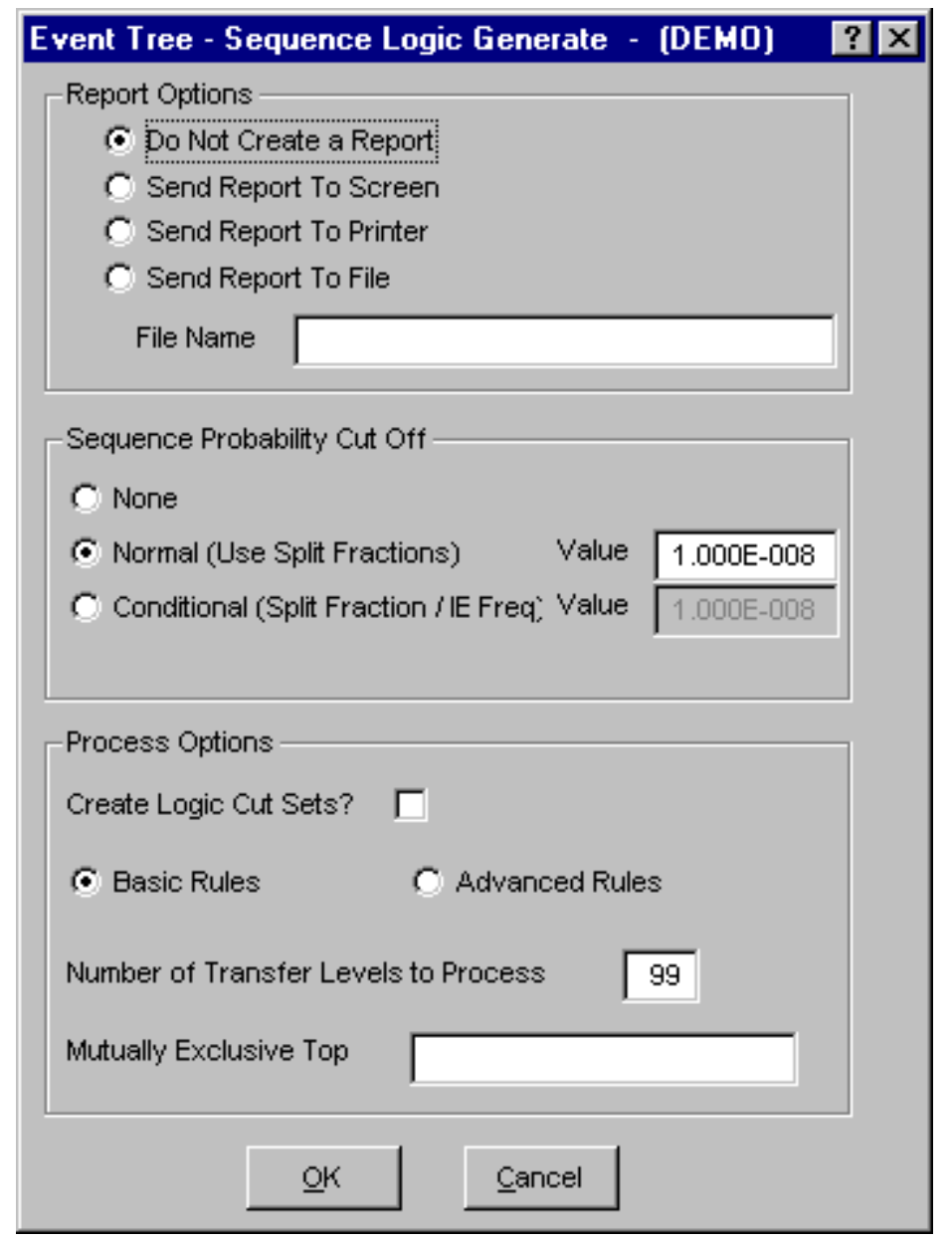

\section{Report Options -}

\section{Sequence Probability Cutoff-}

\section{Create Logic Cut Sets? -}

Rules

Basic Rules -

Advanced Rules -

Number of Transfer Levels to Process -

Mutually Exclusive Top -

OK -

\section{Cancel -}

The Sequence Generation report contains the generating details of the sequence.

Select the type of probability cutoff to be performed, if any.

Generate a sequence cut set when the sequence logic is generated.

Use event tree linkage rules written using the basic if-then-else syntax.

Use event tree linkage rules written using the Modula-2 programming language.

Set the level of sub-tree tops contained in the sequences.

Specify a top to add to the sequence logic.

Generate sequences based on the selected options.

Terminate the process without generating the sequences. 


\subsubsection{The Sequence Generation Process}

After sequence generation begins, a number of messages will be displayed in the message bar of the SAPHIRE window. One message displays the number of sequences being added or deleted. During this process, any new sequences are added and any sequences of the event tree that are no longer valid are removed. Another series of messages lists the name of the sequence being processed along with a running total of the number of good (i.e., valid) sequences processed and the total number of sequences processed. When complete, a final total of the number of sequences saved, the number of valid sequences encountered, and the number of sequences processed is displayed. If you choose to create a report, the name of the file containing the generating details of the sequence is displayed.

Invalid sequences can result in several ways.

1. The logic of an event tree may be such as to create sequences that have tops in them that are both failed and successful. These are invalid and are deleted.

2. The user may have modified the event tree logic, reducing the number of sequences for the tree. Sequences that existed previously are invalid, also.

3. Sequences that have an end state value of "OK," "SUCCESS" or begin with an "@" character are never generated by SAPHIRE.

\subsubsection{Generate Options Dialog}

\subsubsection{Sequence Generation Report Options}

The Sequence Generation report contains the listing of the sequences' names, the fault trees that make up the sequences, any substitutions of fault trees (based on the linkage rules), and any transfers to other event trees.
Do Not Create a Report - Send Report To Screen - Send Report To Printer - Send Report To File -

No report is generated, but the sequences are generated. Sends the report to the Report Viewer. Sends the report to the Windows default printer. Sends the report to the specified file name. A default name is provided, which can be changed by simply typing over the default name.

\subsubsection{Sequence Probability Cut Off}

Select the type of probability cutoff to be performed, if any. If the tops are independent, then the sequence logic can be truncated on probability. Each top is treated as a basic event with its probability assumed to be the value of its split-fraction. As the sequence logic is being created and each top is appended to the logic, the probability for the sequence can be checked to ensure that its probability is greater than the truncation value specified in the Cut Off Value field.

None - $\quad$ Do not discard sequences when generating logic.

Normal - $\quad$ Use the split fraction values as indicated above. 
Conditional - Use the split-fraction values, but without considering the initiating event as part of the sequence probability (i.e., the initiating event probability $=1.0$ ). This is initiating event value is used only during truncation, but not during quantification.

Cut Off Value - Enter the sequence probability truncation value. If either the Normal or Conditional radio button is selected, each sequence whose probability is less than the truncation probability is discarded and the logic is not followed.

\subsubsection{Create Logic Cut Sets?}

If you select this check box, sequence "cut sets" will be generated when the sequence logic is generated. Each top, whether failed or successful, is treated as a basic event and placed in a single "cut set" for the generated sequence. This technique is used to reproduce the "large event tree" methodology.

\subsubsection{Basic Rules}

Use the basic linkage rules for sequence logic generation. These rules were created using the Basic Linkage Rules Editor and can be different from those rules created using the Advanced Linkage Rules Editor.

\subsubsection{Advanced Rules}

Use the advanced linkage rules for sequence logic generation. These rules were created using the Advanced Linkage Rules Editor and can be different from those rules created using the Basic Linkage Rules Editor.

\subsubsection{Number of Transfer Levels to Process}

A level refers to a transfer to a sub-tree. The default value of 99 will generate sequences whose logic will contain all tops for all the sub-trees. If the level is set to less than 99 , the generated sequences will contain only tops from sub-trees less than the specified level.

\subsubsection{Mutually Exclusive Top}

Optionally specify a top to add to the sequence logic. This top will be displayed in the logic as a complemented fault tree and will be treated accordingly when the sequence is solved. The purpose is to provide a method of removing illegal combinations of events.

Note that this option has been superceded by the incorporation of recovery rules.

\subsection{Edit End States (Sequence Logic)}

\subsubsection{Edit Event Tree End States}

\section{PURPOSE}

This option allows you to view or modify event tree sequence information after an event tree has been created. 


\section{STEPS}

1. From the SAPHIRE menu select Event Tree. The Event Tree List dialog will be displayed.

2. Highlight the desired event tree in the list.

3. Right-click to invoke the pop-up menu and choose Edit End States.

4. The Sequence Editor dialog will be displayed showing all the sequence names and end states defined for the selected event tree.

NOTE: Any changes made to sequence and end state names using this option, will automatically be updated throughout the database, including the event tree graphical (.ETG) file (i.e., the corresponding drawing will be updated).

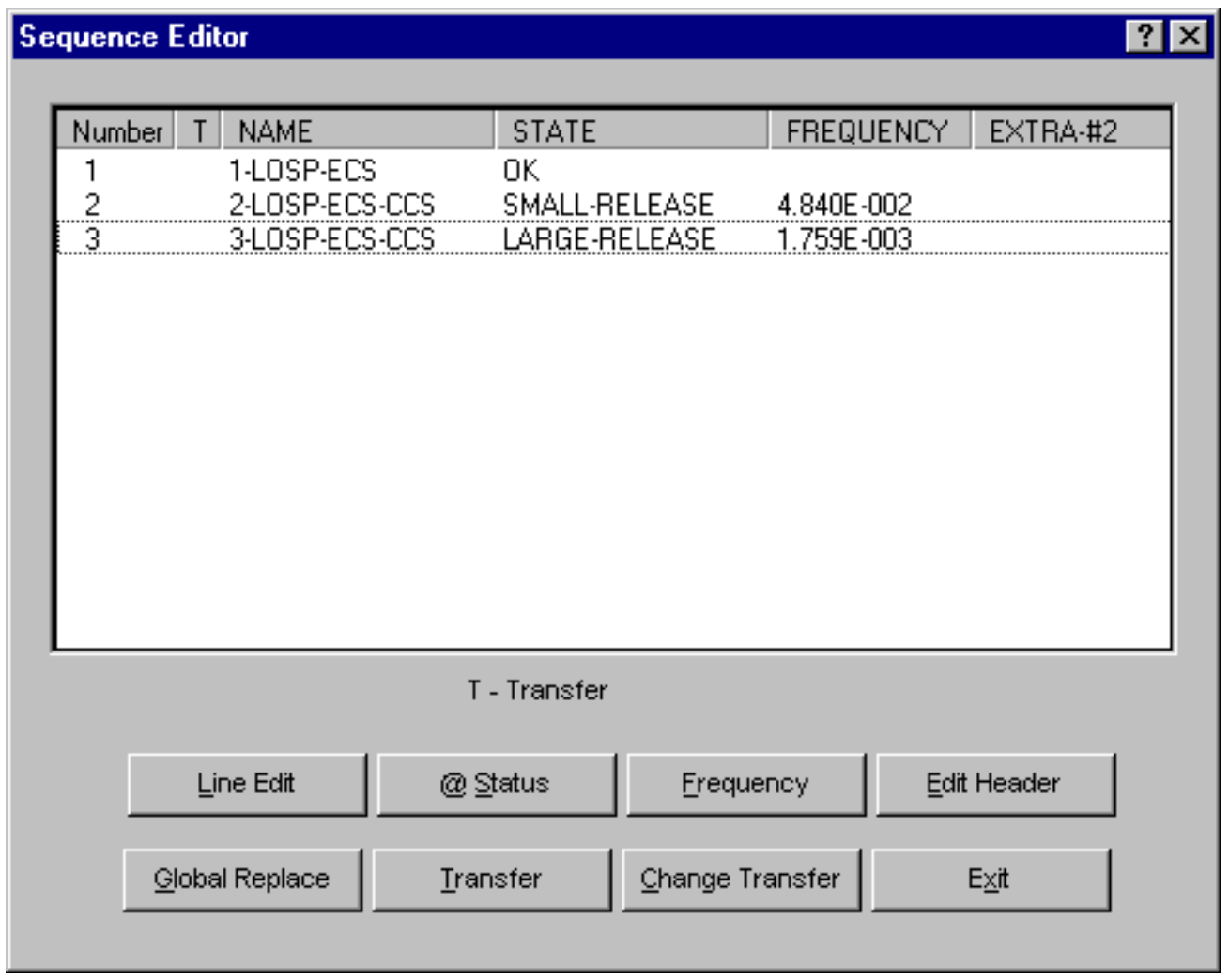

\section{Line Edit - \\ @Status -}

Frequency -

Edit Header -

Global Replace -

Transfer -

Change Transfer -

Exit -
Edit the data displayed one line at a time.

Toggle the current state of a sequence (i.e., sequence will be generated or ignored).

Display the frequencies or min cut upper bound values associated with the selected sequence.

Change the headers that appear across the top of the columns.

Replace a specified string at every occurrence with another string

Follow the transfer of the selected sequence to sub-trees.

Turn on or off transfer points.

Terminate the sequence logic editor. 


\subsubsection{Exit Sequence Logic Editor}

\section{PURPOSE}

This option closes the Sequence Editor dialog and allows you to save any changes made.

\section{STEPS}

1. From the SAPHIRE menu select Event Tree. The Event Tree List dialog will be displayed.

2. Highlight the desired event tree in the list.

3. Right-click to invoke the pop-up menu and choose Edit End States. The Sequence Editor dialog will be displayed.

4. Make desired changes to the logic.

5. Choose the Exit button. You will be prompted to save the changes before exiting.

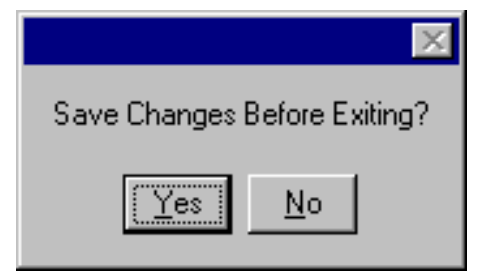

Yes - Save the changes to the sequence logic. The corresponding event tree graphical (.ETG) file will be updated with the changes at this time.

No - Do not save the changes to the sequence logic.

NOTE: You will not be prompted with this dialog if no changes were made to the sequence logic.

\subsubsection{Line Edit}

\section{PURPOSE}

This option allows you to edit the data displayed one line at a time, including sequence names, sequence end state names, sequence frequencies.

\section{STEPS}

1. From the SAPHIRE menu select Event Tree. The Event Tree List dialog will be displayed.

2. Highlight the desired event tree in the list.

3. Right-click to invoke the pop-up menu and choose Edit End States. The Sequence Editor dialog will be displayed.

4. Highlight the desired sequence and choose the Line Edit button.

5. The Line Edit dialog will be displayed with the sequence information. 


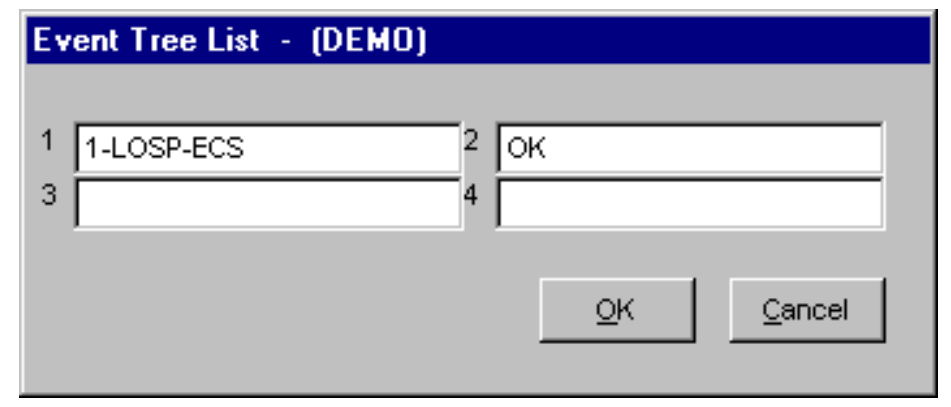

You may change any of the fields displayed by simply typing over the existing data. . These changes made are not saved until you exit the Sequence Editor dialog. At that time, you will be asked whether or not you wish to save all changes made before exiting.

OK - Exit and show the changes made.

Cancel - Exit without showing the changes made. These changes will not be saved when you exit the Sequence Editor dialog.

\subsection{4@Status}

\section{PURPOSE}

This option allows you to toggle the current state of a sequence (i.e., sequence will be generated or ignored). An "@" means that the corresponding sequence will not be generated; it will be ignored. (Remember that "successful" sequences are not generated when OK or SUCCESS is present in the End State column, or when the @Status has been assigned.)

\section{STEPS}

1. From the SAPHIRE menu select Event Tree. The Event Tree List dialog will be displayed.

2. Highlight the desired event tree in the list.

3. Right-click to invoke the pop-up menu and choose Edit End States. The Sequence Editor dialog will be displayed.

4. Highlight the desired the sequence whose status is to be changed and choose the @Status button.

Depending on the current status, an "@" will appear or disappear in front of the end state name.

\subsubsection{Frequency}

\section{PURPOSE}

This option will display the frequencies or minimal cut set upper bound values associated with the selected sequence. The values displayed are the results of the last time the sequences were solved. Frequency values are NOT recalculated using this option. 


\section{STEPS}

1. From the SAPHIRE menu select Event Tree. The Event Tree List dialog will be displayed.

2. Highlight the desired event tree in the list.

3. Right-click to invoke the pop-up menu and choose Edit End States. The Sequence Editor dialog will be displayed.

4. Highlight the desired sequence(s) and choose the Frequency button.

The EXTRA-\#1 column will be renamed to FREQUENCY and the corresponding frequencies will be displayed. You may now invoke the Line Edit command to modify frequencies, if desired.

\subsubsection{Edit Header}

\section{PURPOSE}

This option allows you to change the headers that appear across the top of the columns (note: EXTRA-\#2 is a user-defined field).

\section{STEPS}

1. From the SAPHIRE menu select Event Tree. The Event Tree List dialog will be displayed.

2. Highlight the desired event tree in the list.

3. Right-click to invoke the pop-up menu and choose Edit End States. The Sequence Editor dialog will be displayed.

4. Choose the Edit Header button. The Header Edit dialog will be displayed.

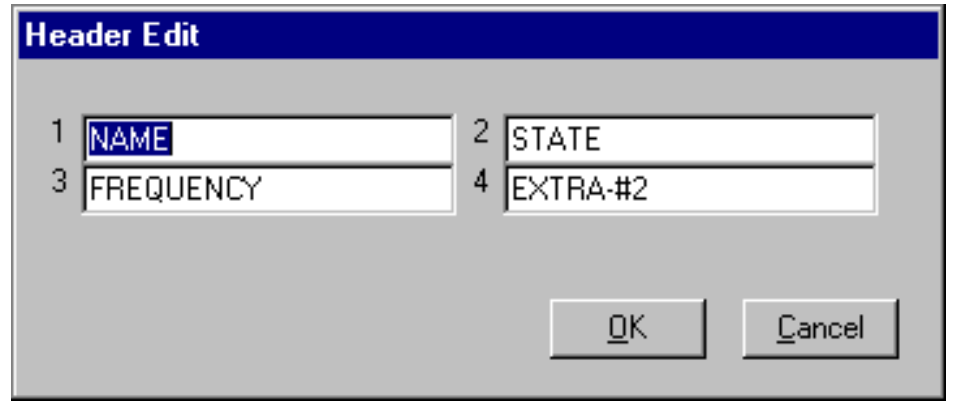

To make changes, simply type over the existing header. These changes made are not saved until you exit the Sequence Editor dialog. At that time, you will be asked whether or not you wish to save all changes made before exiting.

OK - Exit and show the changes made.

Cancel - Exit without showing the changes made. These changes will not be saved when you exit the Sequence Editor dialog. 


\subsubsection{Global Replace}

\section{PURPOSE}

This option allows you to replace a specified string at every occurrence with another string.

\section{STEPS}

1. From the SAPHIRE menu select Event Tree. The Event Tree List dialog will be displayed.

2. Highlight the desired event tree in the list.

3. Right-click to invoke the pop-up menu and choose Edit End States. The Sequence Editor dialog will be displayed.

4. Choose the Global Replace button. The Event Tree Search Replace dialog will be displayed.

5. Enter the string for which to search in the Search for field.

6. Enter the replacement string in the Replace with field.

7. Select the column $(1,2,3$, or 4$)$ on which to perform the search.

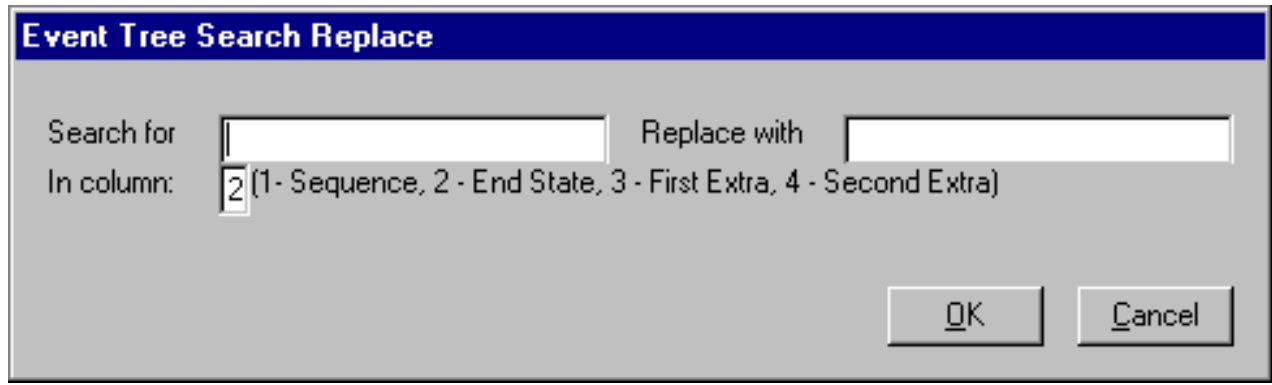

If the search string is found, all occurrences of the search string will be replaced by the string specified in the Replace with field. If the string is not found, the message, "No such string found" will be displayed in message bar of the SAPHIRE window and the Event Tree Search Replace dialog will be closed.

\subsubsection{Transfer}

\section{PURPOSE}

This option will allow you specify event tree transfers (to sub-trees), control the status of event tree transfers, and transfer to sub-trees within the editor.

\section{STEPS}

1. From the SAPHIRE menu select Event Tree. The Event Tree List dialog will be displayed.

2. Highlight the desired event tree in the list.

3. Right-click to invoke the pop-up menu and choose Edit End States. The Sequence Editor dialog will be displayed.

4. Highlight the desired sequence and choose the Transfer button. 
If changes were made to the current event tree, you will be asked if you wish to save those changes before following the transfer.

The Sequence Editor dialog will be displayed with the name of the transfer tree appearing the Event Tree box.

After desired changes are made to this event tree, choosing the Exit button closes this dialog. The Sequence Editor dialog will be redisplayed with the previous event tree sequences listed.

\subsubsection{Change Transfer}

\section{PURPOSE}

This option works as a toggle switch to turn on and off transfer points. If the sequence was marked as a transfer to an event tree sub-tree, this option will turn off the transfer. If the sequence was not marked as a transfer, it turns on the transfer. (The sub-tree name is then entered in the End State column.) A "T" appears in the column in front of the sequence names.

\section{STEPS}

1. From the SAPHIRE menu select Event Tree. The Event Tree List dialog will be displayed.

2. Highlight the desired event tree in the list.

3. Right-click to invoke the pop-up menu and choose Edit End States. The Sequence Editor dialog will be displayed.

4. Highlight the desired sequence and choose the Change Transfer button. 


\section{SEQUENCE ANALYSIS}

\subsection{Sequence Analysis}

\section{PURPOSE}

When developing a risk assessment code, accident sequence analysis must also be considered. Accident sequences are defined in SAPHIRE by developing event trees. Once an event tree has been developed, the sequence logic from the event tree can be generated. The sequence logic is the list of systems that succeed or fail during this accident sequence. These system failures and successes are top events of fault trees. This logic is used by SAPHIRE to generate the cut sets for the sequence.

This option provides the means to recalculate sequence values after event failure data and/or cut sets have been modified. The Sequences dialog shows all sequences, ordered by event tree name and sequence name, in the current project.

\section{KEY TO FLAGS}

Located to the right of each sequence is a set of flags that indicate the status of the sequence:

b - base case cut sets exist for the selected analysis type

c - current case cut sets exist for the selected analysis type

\section{STEPS}

1. From the SAPHIRE menu select Sequence. The Sequences dialog will be displayed. 


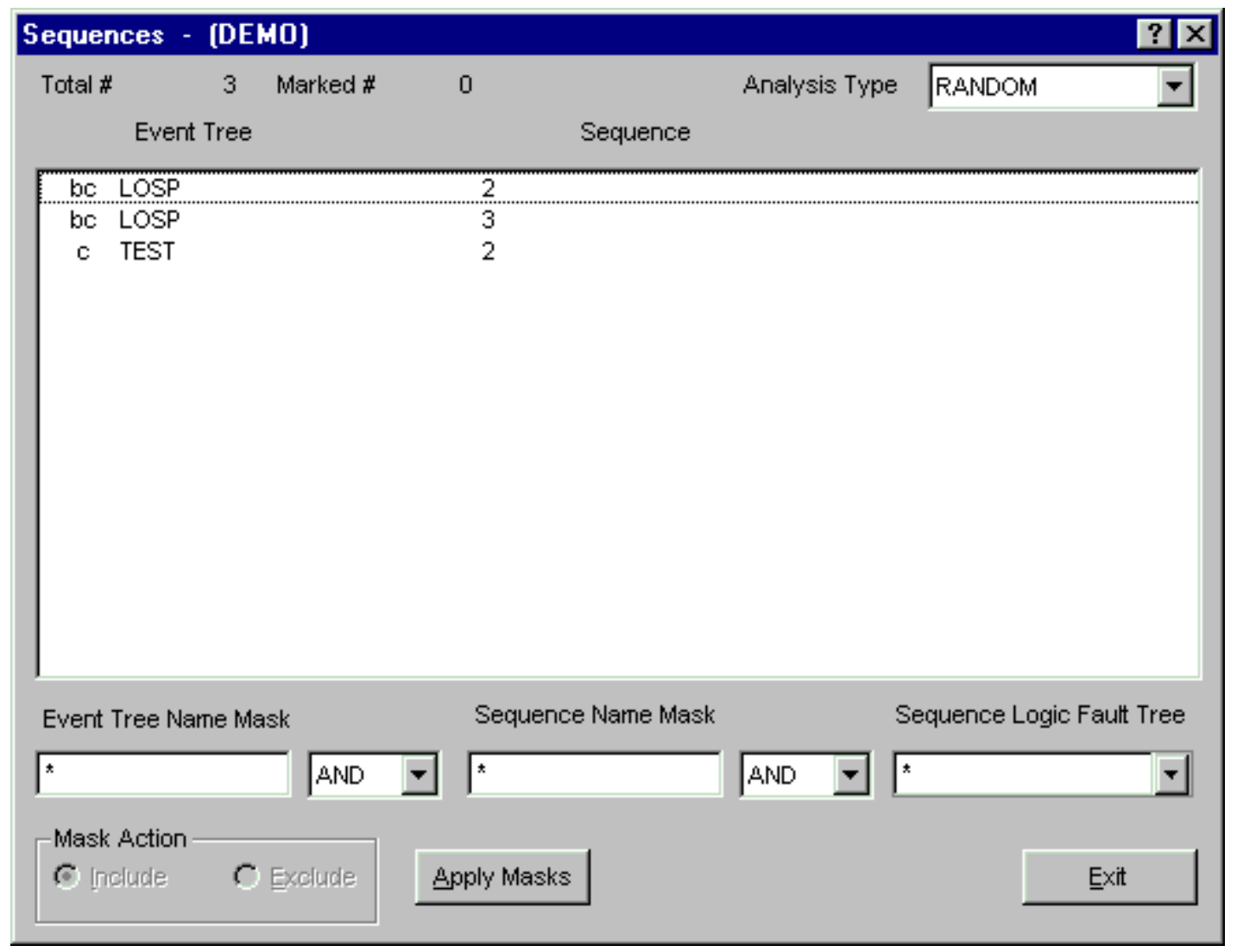

From this dialog, you can select sequences using the mouse or the Mask feature. The analysis type can be selected from the drop-down list. Additionally, the following functions may be accessed from the pop-up menu:

Solve -

Quantify -

Uncertainty -

Cut Sets -

Display -

View -

Time Dependent -
Generate cut sets for a sequence based on cut set generation cut off values.

Calculate a new minimum cut set upper bound for sequence cut sets using current data values (using event data changes and current case cut sets).

Perform uncertainty analysis on a sequence using either the Monte Carlo or Latin Hypercube simulation technique.

Perform various operations on sequence cut sets: Update, Prune, Recover, Edit.

Display the results of sequence analyses in various report forms. View sequence analysis information and compare base case and current case minimal cut set upper bound results.

Perform time dependent sequence analysis.

\subsection{Sequence Mask Feature}

\section{PURPOSE}

This feature facilities selection of event tree sequences in the list by matching criteria in the three mask fields in combination with the selected logic operations. 
The wildcard characters asterisk $\left(^{*}\right)$ and question mark (?) can be used in any of the mask fields. The asterisk represents one or more characters that a group has in common. The question mark represents a single character in that position of the string that a group has in common.

\section{STEPS}

1. Specify the event tree name criterion, if any.

2. Choose the logic operator from the drop-down list.

3. Specify the sequence name criterion, if any.

4. Choose the logic operator from the drop-down list.

5. Specify the fault tree name criterion, if any.

6. Choose the desired mask action.

7. Choose the Apply Masks button. All sequences matching the specified qualification criteria will be selected or deselected, depending on the current mask action.

8. Right-click to invoke the pop-up menu and select the desired operation, or repeat steps 1 through 7 to select/deselect additional sequences based on another set of qualification criteria.

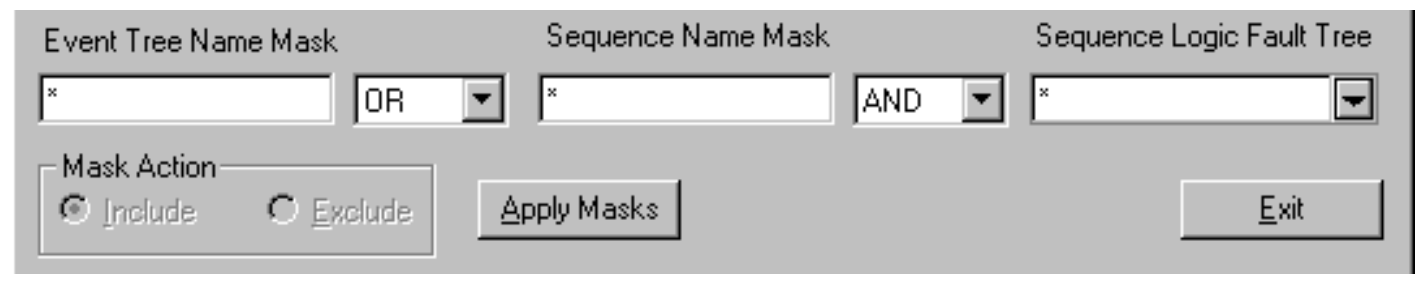

The graphic here shows the mask fields as they appear when the Sequences dialog is initially invoked.

Event Tree Name Mask - $\quad$ Used to specify the desired event tree(s) by name. An asterisk will allow all event trees to be considered.

Sequence Name Mask -

Sequence Logic Fault Tree -

Logic Operators -
Used to specify the desired sequence(s) by name. An asterisk will allow all sequences to be considered.

Used to specify the desired fault tree(s) that are used in the sequence logic by fault tree name. A specific fault tree can be selected from the drop-down list by clicking on the down arrow to the right of this field. All fault trees in the current project will be listed.

Two logic operator drop-down lists are available, each providing two options: AND and OR. The selected logical operation for the drop-down list on the left is applied to the event tree name and sequence name criteria. The selected logical operation for the drop-down list on the right is applied to the sequence name and sequence logic fault tree mask criteria. 
AND -

OR -

Mask Action

Include -

Exclude -

Apply Masks -
Choosing this option tells SAPHIRE that both sets of qualification criteria in the applicable mask fields must be met. Choosing this option tells SAPHIRE that either set of qualification criteria in the applicable mask fields may be met.

The sequences that match the qualification criteria will be selected.

The sequences that match the qualification criteria will be deselected.

Search for the sequences that match the qualification criteria and apply the selected mask action.

\subsection{Sequence Mask Examples}

\section{PURPOSE}

Provide examples for using the sequence mask feature. The BYRON_ASP project is used in these examples.

\section{Example 1 STEPS}

To select all sequences in the TRANS event tree:

1. Enter "TRANS" in the Event Tree Name Mask field.

2. Choose the AND logic operation (default value).

3. Enter "*” in the Sequence Name Mask field (default value).

4. Choose the AND logic operation (default value).

5. Enter “*” in the Sequence Logic Fault Tree field (default value).

6. Choose the Include radio button.

7. Choose Apply Masks.

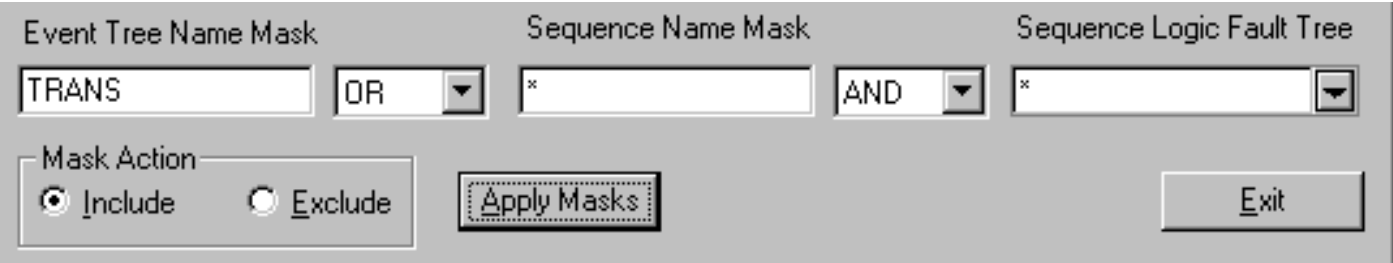

All 13 of the TRANS event tree sequences will be selected. Note the Marked \# indicator at the top of the Sequences dialog. 


\section{Example 2 STEPS}

To deselect all sequences:

1. Enter an asterisk in each of the mask fields.

2. Choose Exclude.

3. Choose Apply Masks.

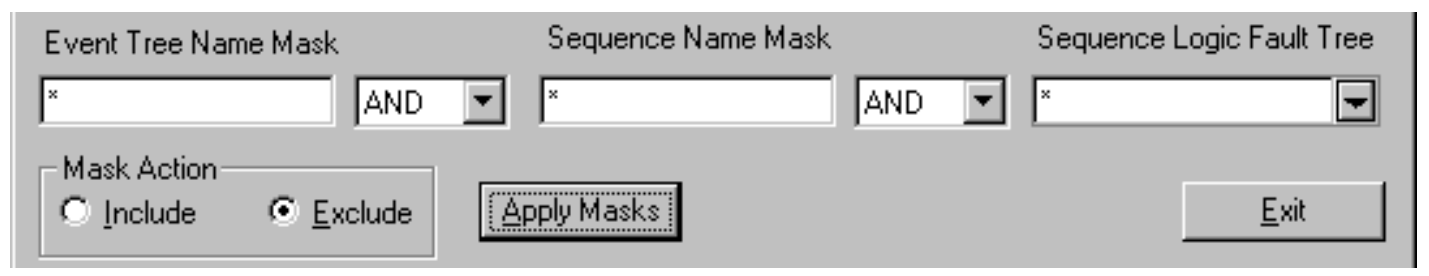

\section{Example 3 STEPS}

To select all sequences that include the RHR fault tree in their logic:

1. Leave all fields with default values, unless otherwise specified. (“*” in the Event Tree Name Mask and Sequence Name Mask fields, AND logical operators selected.)

2. Enter "RHR" in the Sequence Logic Fault Tree field (or select it from the drop-down list).

3. Choose the Include radio button.

4. Choose Apply Masks.

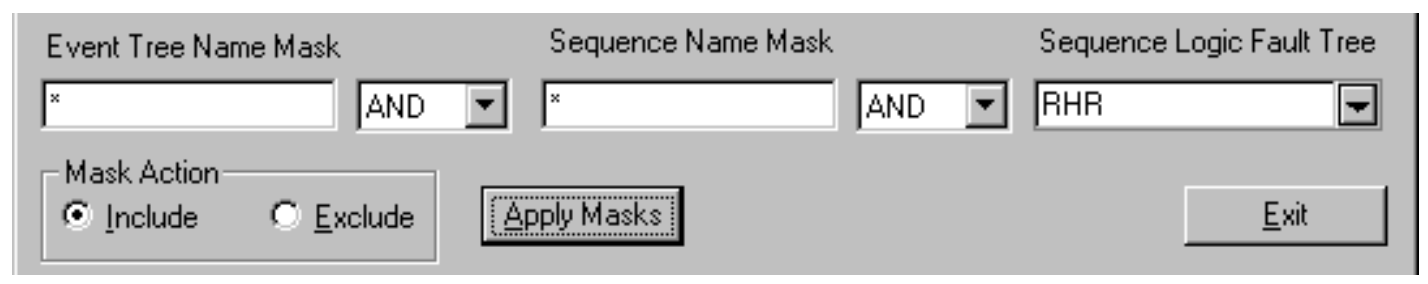

11 sequences will be selected - Three LOOP, two SGTR, three SLOCA, and three TRANS. If the Example 2 steps had been skipped, 21 sequences would have been selected - the 13 TRANS in addition to the three LOOP, two SGTR, and three SLOCA.

\section{Example 4 STEPS}

To select all sequences in the TRANS event tree along with all sequences that include the RHR fault tree in their logic (examples 1 and 3 without performing two separate mask operations):

1. Deselect all sequences (Example 2). 
2. Enter "TRANS" in the Event Tree Name Mask field.

3. Choose the AND logic operation.

4. Enter “*” in the Sequence Name Mask field.

5. Choose the OR logic operation.

6. Enter "RHR" in the Sequence Logic Fault Tree field.

7. Choose the Include radio button.

8. Choose Apply Masks.

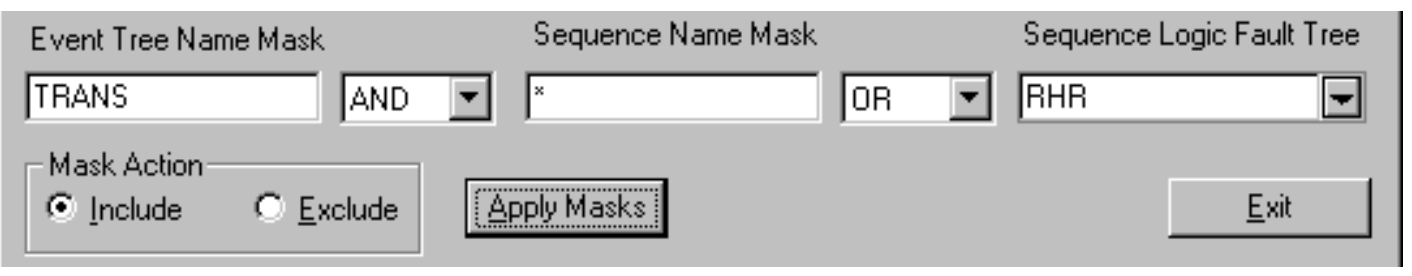

The 21 sequences, discussed above will be selected.

\section{Example 5 STEPS}

To select only those sequences in the TRANS event tree that include the RHR fault tree in their logic:

1. Deselect all sequences (Example 2).

2. Enter "TRANS" in the Event Tree Name Mask field.

3. Choose the AND logic operation.

4. Enter “*” in the Sequence Name Mask field.

5. Choose the AND logic operation.

6. Enter "RHR" in the Sequence Logic Fault Tree field.

7. Choose the Include radio button.

8. Choose Apply Masks.

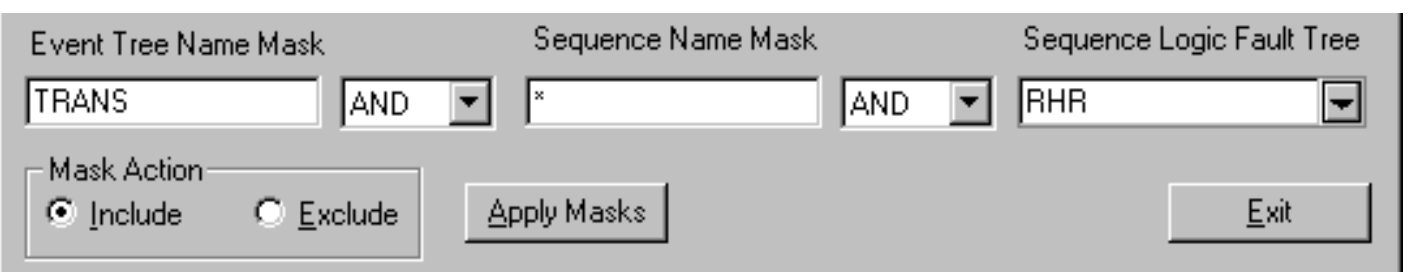

Three sequences will be selected. Note: The only difference between Example 4 and Example 5 is the logical operator selection in STEP 5. 


\subsection{Solving Sequences}

\subsubsection{Solving Sequences}

\section{PURPOSE}

This option allows you to generate the cut sets for selected sequence(s) based on cut set generation cutoff values. You are given the opportunity to specify several cut set generation cutoff values that will be used to determine if a cut set is to be retained or discarded from the selected sequence(s). You are given the ability to generate cut sets for a selected sequence, a group of sequences, or all sequences within the current project.

\section{STEPS}

1. From the SAPHIRE menu select Sequence. The Sequences dialog will be displayed.

2. Highlight the desired sequence(s) and right-click to invoke the pop-up menu.

3. Choose Solve.

Whether you are generating cut sets for a single sequence, a group of sequences, or for all sequences the Cut Set Generation dialog will be displayed.

The dialog below is displayed when the Analysis Type is "Random".

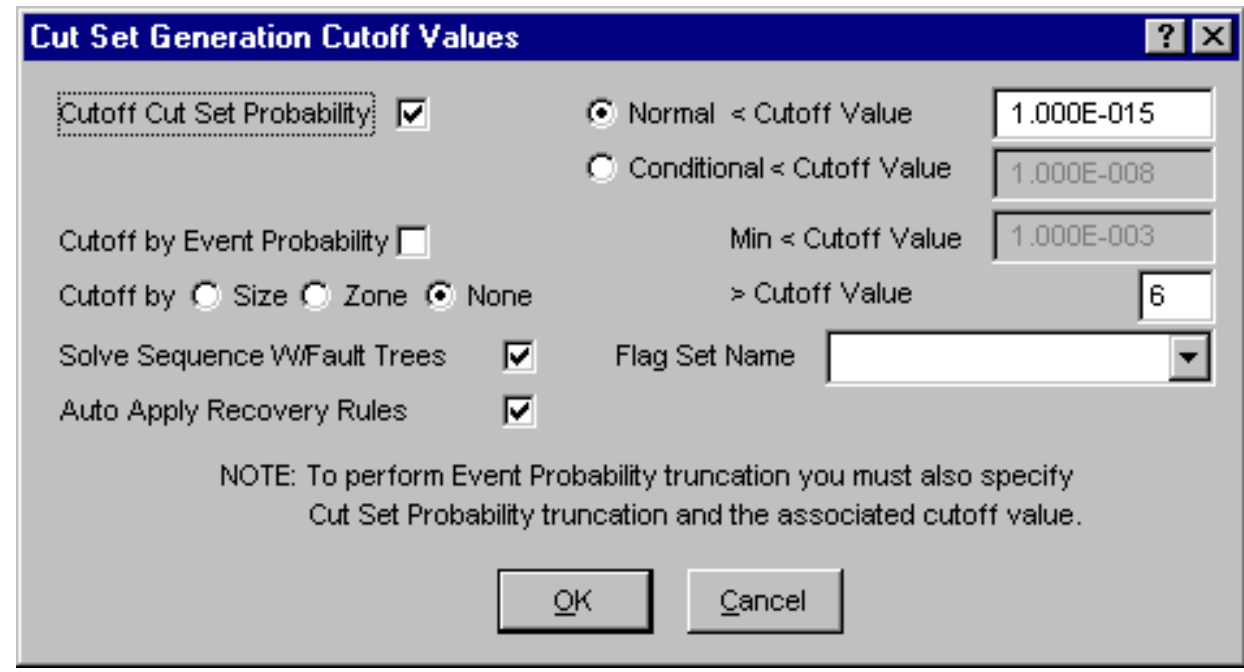

The dialog below is displayed when any other Analysis Type is selected. 


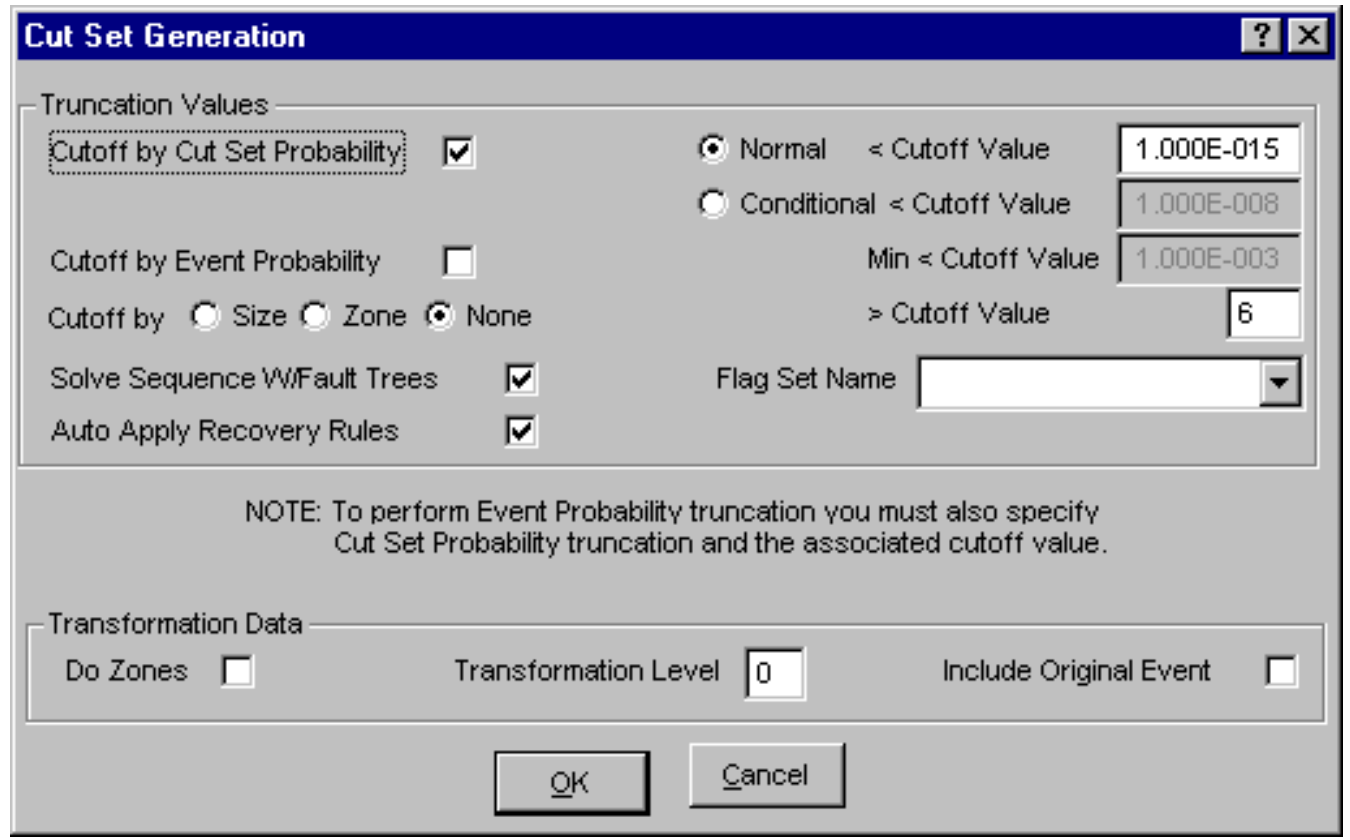

You may change any of the data fields on this dialog. The default values that appear on this dialog may be reset to new values by selecting Utility from the SAPHIRE menu and then invoking the Define Constants option.

Cutoff by Cut Set Probability - Used for determining if the cut set should be retained or discarded based on the value in the $<$ Cutoff Value field.

Cutoff by Event Probability - Used for determining if the cut set should be retained or discarded based on the value in the Min < Cutoff Value field.

Cutoff by Size Used for determining if the cut set should be retained or discarded based on the value in the $>$ Cutoff Value field or zoned flagged events.

Solve Sequence W/Fault Trees - Use the fault trees to solve the tree; otherwise the fault tree cut sets are used.

Flag Set Name Used to specify the flag set to be used during processing.

Auto Apply Recovery Rules Basic Advanced -

Do Zones Used for automatically applying recovery rules while solving. Apply rules created using the basic rule syntax Apply rules created using the advanced rule syntax. If checked, perform zone transformation during cut set generation.

Transformation Level An integer $0-255$ which indicates the default level of substitution for the transformations to be performed.

Include Original Event - $\quad$ Used for determining if the original event should be retained or discarded during processing.

During processing, the Cut Set Generation Results dialog is displayed and updated as the calculations proceed. Upon completion of the cut set generation, the summary results are displayed in this dialog. Note that the displayed results reflect the values prior to the application of recover rules, if that option was selected. Choose the $\mathbf{O K}$ button to close the dialog, or View Results button to review or print the results. 
The only limit on the number of cut sets that can be stored for a given fault tree is the available hard drive space. When processing is complete, the Sequences List dialog is updated to show the letter "c" in front of the sequence(s) with current case cut sets.

\subsubsection{Cut Set Generation Dialog}

\subsubsection{Cutoff By Cut Set Probability}

If you select this check box and choose the:

Normal radio button - Only those cut sets whose product for all of its event probabilities is greater than or equal to the value in the $<$ Cutoff Value field will be kept.

Conditional radio button - Only those cut sets whose product for all of its event probabilities, excluding the initiating event probability, is greater than or equal to the value in the $<$ Cutoff Value field will be kept.

All other cut sets will be removed.

If you deselect this check box, then the probability for the cut set will be irrelevant for determining if the cut set should be retained or discarded.

\subsubsection{Cutoff by Event Probability}

If you select this check box, then you must also choose the Cutoff by Cut Set Probability check box. This option will check all cut sets that are below the probability cutoff (Min $<$ Cutoff Value field) and remove them only if they contain an event whose probability is below this value.

When you select his option, SAPHIRE will also perform the quantification.

\subsubsection{Cutoff by Size}

Size - If you choose this radio button, then only the cut sets whose number of events is less than or equal to the value specified in the $>$ Cutoff Value field will be kept in the cut sets for that fault tree. All other cut sets will be removed.

Zone - If you choose this radio button, then only zone flagged events will be checked.

None - If you choose this radio button, then the number of events in a cut set will be irrelevant for determining if the cut set should be retained or discarded.

\subsubsection{Solve Sequence W/Fault Trees}

If you check this box, then fault tree logic will be used. If the box is unchecked, previously generated fault tree cut sets will be used.

\subsubsection{Flag Set Name}


This option allows the user to specify changes to the flags for each sequence without having to regenerate the failure data each time. The fault tree logic is pruned before it is solved, dependent on these flags.

Select the desired flag set, if any, from the drop-down list. If this field is left blank, then SAPHIRE checks to see if there is a default setting for this sequence. The default can be set using the Modify |

Event Trees | Sequences menu options. If there is a default setting, then SAPHIRE uses it; otherwise, SAPHIRE uses no flags.

If you specify a flag set, that flag set will be used during processing. A pre-defined flag set option, NONE, is available. If you choose this option, no flag sets are used.

\subsection{Quantifying Sequences}

\subsubsection{Quantifying Sequences}

\section{PURPOSE}

The quantification process will re-quantify the cut sets using current data values (event change sets and current case cut sets) for a selected sequence, for a group of sequences, or for all of the sequences within the current project. The new value is saved with the current case cut sets for the selected sequence.

\section{STEPS}

1. From the SAPHIRE menu select Sequence. The Sequences dialog will be displayed.

2. Highlight the desired sequence(s) and right-click to invoke the pop-up menu.

3. Choose Quantify, then one of the sub-menu options.

\begin{tabular}{|l|l|}
\hline Solve & \\
\hline$\underline{\text { Quantify }}$ & Quantify (Default) \\
\hline$\underline{\text { Uncertainty }}$ & Min Cut \\
Cout Sets & Rare Event \\
Display & Min/Max \\
View & $\underline{\text { Split Fraction }}$ \\
\hline Iime Dependent & \\
\hline
\end{tabular}

Quantify (Default) - $\quad$ Re-quantify using the quantification method for the specified sequence.

Min Cut This is selected in the Modify | Event Trees | Sequences option.

Rare Event -

$\operatorname{Min} / \operatorname{Max}-$

Split Fraction -
Re-quantify using the Minimal Cut Set Upper Bound Approximation. Re-quantify by adding together the probabilities for the cut sets of a top event.

Re-quantify using the "exact" calculation for the union of cut sets via the inclusion-exclusion rule.

Quantify the cut sets using the min cut upper bound values that have been calculated for each successful or failed fault tree in this sequence. 
If the selected quantification method differs from the sequence(s) default, a warning box with the message, "The chosen quantification method was different from the Sequence default" is displayed.

Choose the Ok button to continue processing.

During processing, the Quantification Results dialog is displayed. If an error occurs, the message, "Error quantifying cut sets" will be displayed in the message bar.

If a single sequence was selected, upon completion of the quantification process, the results are displayed in the Quantification Results dialog. Choose Ok to close the dialog.

\subsubsection{Minimal Cut Set Upper Bound Approximation}

This calculation approximates the probability of the union of the minimal cut sets for the fault trees. The equation for the minimal cut set upper bound is

$$
S=1-\prod_{i=1}^{m}\left(1-C_{i}\right)
$$

where

$$
\begin{aligned}
& \mathrm{S}=\text { minimal cut set upper bound for the fault tree unavailability, } \\
& \mathrm{C}_{\mathrm{i}}=\text { probability of the } \mathrm{i}=\text { th cut set, and } \\
& \mathrm{m}=\text { the number of cut sets. }
\end{aligned}
$$

Example: If the cut sets for a fault tree are $\mathrm{X}=\mathrm{A} \cup \mathrm{B} \cup \mathrm{C}$ (i.e., the union of three events, $\mathrm{A}$, $\mathrm{B}$, and $\mathrm{C}$ ); then the cut sets can be written as $\mathrm{X}=\mathrm{A}+\mathrm{B}+\mathrm{C}$ with the plus symbol indicating union. The fault tree unavailability computed from the minimal cut set upper bound approximation is then $X=1-(1-A)(1$ B)(1 - C).

\section{SEE TECHNICAL REFERENCE}

\section{Minimal Cut Set Upper Bound}

\subsubsection{Rare Event Approximation}

The rare event approximation approach adds together the probabilities for the cut sets of a top event. This approximation is a good method when the cut set probabilities are small.

\section{SEE TECHNICAL REFERENCE}

Rare Event

\subsubsection{Min Max Quantification}

The Min-Max quantification option quantifies the current case cut sets using the "exact" probability quantification algorithm. This algorithm can be extremely time consuming if the number of cut sets is large. The user should be careful when selecting this option.

When you choose this option, the dialog shown below will be displayed. You are required to enter the number of passes. The number passes indicates the number of intermediate terms SAPHIRE will calculate when determining the minimum and maximum quantification values. Type a number from 1 to 10 to 
indicate the number of passes you want SAPHIRE to make. The higher the number, the more the calculation speed decreases and the accuracy increases. Choose the Ok button to proceed.

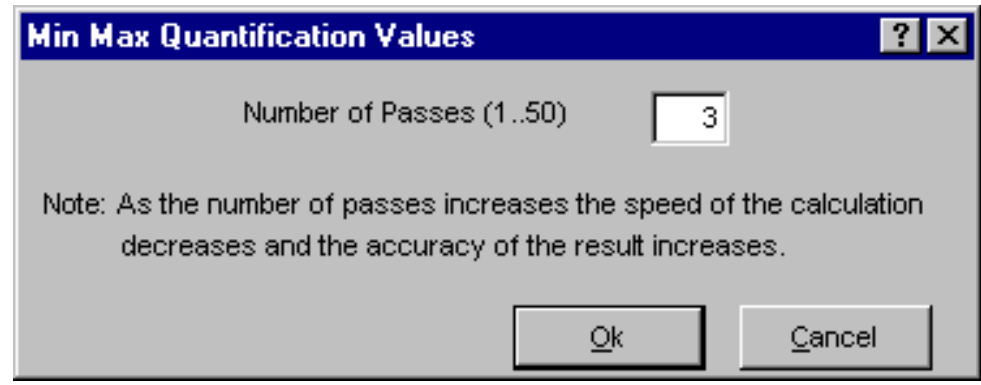

SAPHIRE will perform the specified number of intermediate terms and when complete will show the results on the Quantification Results dialog. The results of the Min/Max Quantification option are displayed only on this dialog.

\section{How this option works:}

To quantify the union of events, the first pass consists of adding the events, the second pass consists of subtracting pairs of events, the third pass consists of adding "triples", and so on.

For a simple example, assume that a fault tree has only three cut sets which are the union of $\mathrm{A}, \mathrm{B}$, and $\mathrm{C}$ which can be expressed as $\mathrm{A} \cup \mathrm{B} \cup \mathrm{C}$. In this example, each cut set consists of only one term; however, the approach is not limited to one term per cut set. For 3 passes, the solution is shown below.

$$
\mathrm{X}=(\mathrm{A}+\mathrm{B}+\mathrm{C})-(\mathrm{A} * \mathrm{~B}+\mathrm{A} * \mathrm{C}+\mathrm{B} * \mathrm{C})+(\mathrm{A} * \mathrm{~B} * \mathrm{C})
$$

The Min/Max algorithm applies the Boolean idempotent law $(\mathrm{A} * \mathrm{~A}=\mathrm{A})$ to reduce identical terms during the multiplication of cut sets.

The table below shows the Min/Max equation and results, with $\mathrm{A}=\mathrm{B}=\mathrm{C}=0.8$. The minimal cut set upper bound approximation would be $1-(1-0.8)^{3}=0.992$. (Note that the Min Max calculation will be the same as the minimal cut set upper bound when the cut sets do not contain common events.)

\begin{tabular}{|c|c|c|}
\hline $\begin{array}{c}\# \\
\text { Passes }\end{array}$ & Min/Max Equation & Min/Max value \\
\hline 1 & $\mathrm{~A}+\mathrm{B}+\mathrm{C}$ & 2.4 \\
\hline 2 & $\mathrm{~A}+\mathrm{B}+\mathrm{C}-(\mathrm{A} * \mathrm{~B}+\mathrm{A} * \mathrm{C}+\mathrm{B} * \mathrm{C})$ & $2.4-1.92=0.48$ \\
\hline \hline 3 & $\mathrm{~A}+\mathrm{B}+\mathrm{C}-(\mathrm{A} * \mathrm{~B}+\mathrm{A} * \mathrm{C}+\mathrm{B} * \mathrm{C})+\left(\mathrm{A} * \mathrm{~B}^{*} \mathrm{C}\right)$ & $2.4-1.92+0.512=0.992$ \\
\hline \hline 4 & $\mathrm{~A}+\mathrm{B}+\mathrm{C}-(\mathrm{A} * \mathrm{~B}+\mathrm{A} * \mathrm{C}+\mathrm{B} * \mathrm{C})+\left(\mathrm{A} * \mathrm{~B}^{*} \mathrm{C}\right)$ & $2.4-1.92+0.512=0.992$ \\
\hline
\end{tabular}

\subsubsection{Split Fraction}

This option allows you to quantify the sequence cut sets using the min cut upper bound values that have been calculated for each successful or failed fault tree which make up this sequence. This option does not generate cut sets and is usually used to give you a quick approximation of the actual result. The actual results may be obtained by generating cut sets and quantifying these cut sets. 


\subsection{Uncertainty Analysis}

\subsubsection{Uncertainty Analysis}

\section{PURPOSE}

This option can estimate the variability (due to the uncertainties in the basic event probabilities) of either a fault tree top event probability or an event tree sequence frequency. To do this, SAPHIRE provides two sampling techniques: Monte Carlo simulation and Latin Hypercube simulation. In addition, you may perform a seismic analysis to integrate the seismic fragility curve with the site hazard curve using the uncertainty analysis option.

In a traditional uncertainty analysis, SAPHIRE samples the user-specified distributions for each basic event in a group of cut sets, then quantifies these cut sets using the sample values. For a seismic uncertainty, however, SAPHIRE performs the analysis at each G-level in the site hazard curve for the current project. The seismic events are sampled at each new G-level and the quantification performed.

You are given the option of performing an uncertainty analysis on the current case cut sets for either a single, for a group, or for all of the fault trees (or sequences or end states) within the current project.

\section{STEPS}

1. From the SAPHIRE menu select Fault Tree/Sequence/End State. The Fault Trees List/Sequences/End State List dialog will be displayed.

2. Highlight the desired fault tree(s)/sequence(s)/end state(s) and right-click to invoke the pop-up menu.

3. Choose Uncertainty. The Uncertainty Calculation Values dialog will be displayed.

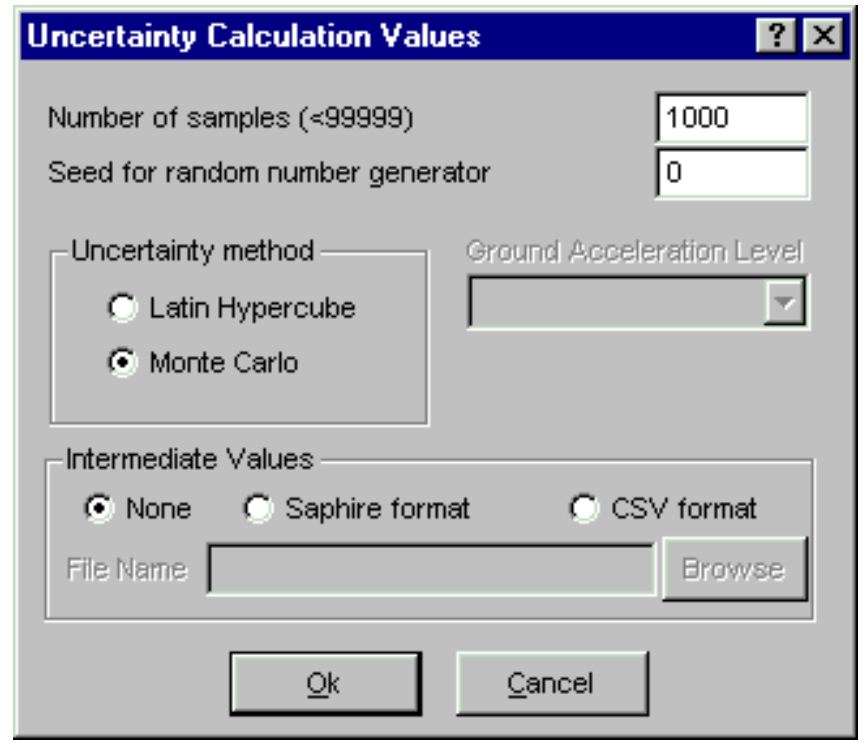


You may change any of the data fields on this dialog. The default values that appear on this dialog may be reset to new values by selecting the Utility $\mid$ Define Constants option.

Number of samples $(<99999)$ -

Seed for random number generator -

Uncertainty method -

Ground Acceleration Level -

Intermediate Values None -

SAPHIRE format -

CSV format -

File Name -

Browse -
A default value is provided for the number of samples to use in simulation. You may use this value or enter another value. The number must be less than 99,999.

A default seed is provided for the random seed. You may use this value or enter a new value for the seed. To obtain a random seed from the system clock, you must enter a zero in this field.

Select the appropriate radio button for the desired uncertainty sampling technique.

If seismic uncertainty is selected, a ground level acceleration must be provided. This indicates the acceleration rate at which the component will always fail. The user can select to process all G-levels combined, all G-levels separately, or a specified G-level only.

Select this check box if you wish for the results and intermediate samples to be written to an ASCII file. Select this check box if you wish for the results and intermediate samples to be written to an ASCII file, formatted in the traditional SAPHIRE format.

Select this check box if you wish for the results and intermediate samples to be written to an ASCII file, formatted as a comma delimited file, for easy import into a spreadsheet program.

Specifies the name of the ASCII file where the intermediate values will be stored.

Opens the Choose File dialog, to select a directory and file name to store the intermediate results.

\subsubsection{Uncertainty Analysis for Multiple Sequences}

When multiple sequences are selected for processing, you may combine results using the group or project option. All options are the same as the dialog for uncertainty analysis for single sequences, with the addition of the Uncertainty type group. 


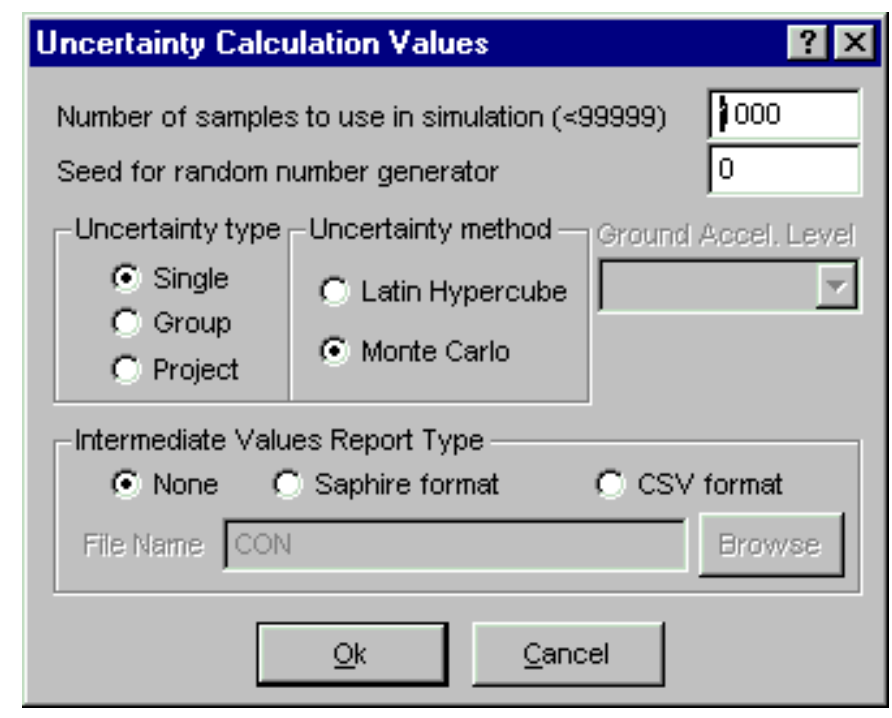

Single - Obtain uncertainty analysis results for each marked sequence separately. This data will be saved in the database for the selected sequence.

Group - Obtain uncertainty analysis results for marked sequences as a group. Group uncertainty results are not saved in the database. These results are not available from the Display option.

Project - Obtain uncertainty analysis results for the sequences in the project. This data will be saved in the database for the current project.

\subsubsection{Intermediate Values}

\section{PURPOSE}

This option allows you to output the results and intermediate samples to the ASCII file specified in the File Name field.

\section{STEPS}

1. From the SAPHIRE menu select Fault Tree/Sequences/End State. The Fault Trees List/Sequences/End State List dialog will be displayed.

2. Highlight the desired fault tree(s)/sequence(s)/end state(s) and right-click to invoke the pop-up menu.

3. Choose Uncertainty. The Uncertainty Calculation Values dialog will be displayed.

4. Check the Output Values check box and enter a name in the File Name field.

The example below describes the layout of a SAPHIRE formatted ASCII file. The CSV (comma delimited file) layout is similar, but is comma delimited to be easily imported into a spreadsheet program.

Format for ASCII file containing intermediate samples: 


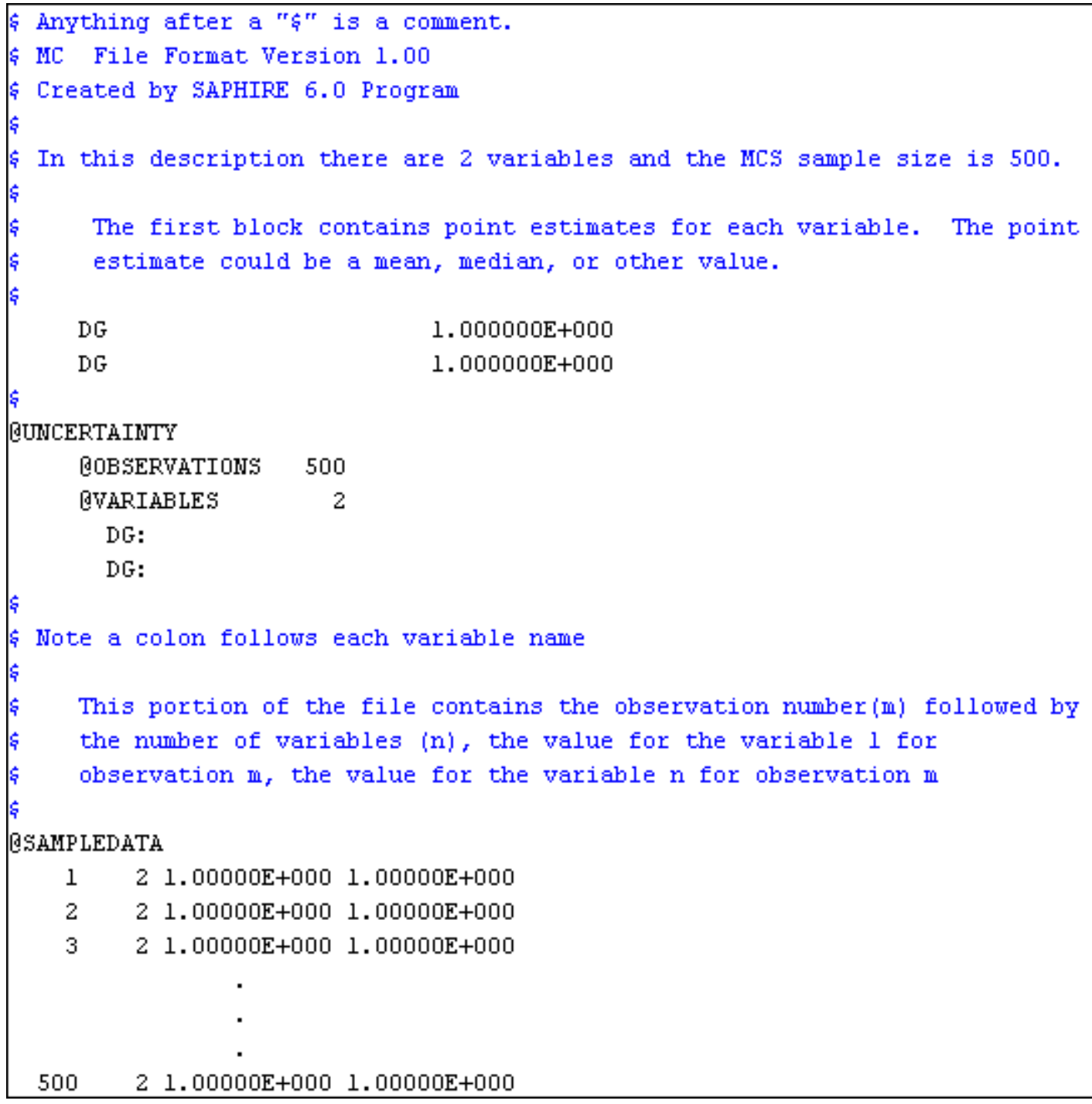

\subsubsection{Monte Carlo Sampling (MCS)}

Simple MCS is a fundamental uncertainty sampling approach. To perform the sampling, SAPHIRE makes repeated quantifications of the fault tree/sequence/end state cutsets using samples from the basic event uncertainty distributions. This type of sampling requires more samples than LHS for the same degree of accuracy.

When using this sampling technique, if the number of samples entered is less than ten, then the number of samples will be increased to ten before the uncertainty analysis process will continue. Any number of samples greater than or equal to ten will be allowed, but a number of at least 1000 is probably a better value for improving the reliability of the Monte Carlo results.

\subsubsection{Latin Hypercube Sampling (LHS)}

LHS is a stratified sampling technique where the random variable distributions are divided into equal probability intervals. A probability is randomly selected from within each interval for each basic event. 
Generally, LHS will require fewer samples than simple MCS for similar accuracy. However, due to the stratification method, it may take longer to generate a value than for a MCS.

When using this technique, if the number of samples entered is less than twice the total number of unique events in the fault tree/sequence/end state, then the number of samples will be increased to two times the total number of unique events before the analysis will continue. The LHS technique gives its best results if the number of samples is at least twice the total number of unique events.

\subsubsection{The Uncertainty Analysis Process}

The following is a description of the uncertainty analysis process performed by SAPHIRE after values have been entered in the Uncertainty Calculation Values dialog.

Once the number of samples has been accepted and a seed obtained from the system clock (if necessary), checks will be run to ensure the events with the same correlation classes have consistent failure data, uncertainty data, and distribution types. If any events with inconsistencies exist, an error message will be displayed and the uncertainty analysis process will be terminated so that the inconsistent values may be corrected.

If an error occurs during the uncertainty analysis process, the process is terminated and a message box providing information about the specific error is displayed.

If all of the events successfully pass the correlation class checks, then the distribution parameters for the events will be checked to ensure that they are valid. If any of the parameters are invalid, error messages will be displayed and the process will be terminated so the distribution parameters may be corrected.

After both of these checks have been passed, a point estimate will be calculated for the selected fault tree (or sequence or end state). At this point the samples for each event will be generated using the selected sampling technique, either the Monte Carlo Sampling technique or the Latin Hypercube Sampling technique. The uncertainty analysis function provides you with 11 different distribution types for both sampling techniques. The distribution types include normal, lognormal, beta, gamma, chi-squared, exponential, uniform, Dirichlet, constrained non-informative, maximum entropy, and the user-defined histograms.

During processing, the Uncertainty Results dialog will be displayed and updated as the samples are generated. When the requested number of samples have been generated, statistical information will be calculated using the generated samples. A sample mean, median, and standard deviation will be calculated for the selected fault tree (or sequence or end state). Coefficients of skewness and kurtosis, and quantile values will also be calculated. This data will be saved in the database for the selected fault tree (or sequence or end state).

Upon completion of these calculations, the following values will be displayed on the Uncertainty Results dialog for viewing: the name, random seed used, the number of samples generated in this process, the total number of events and cut sets being processed, the point estimate, the mean, the median, the 5th and 95th percentile values, the minimum and maximum generated sample values, the standard deviation, the skewness and kurtosis, and the time involved to perform the analysis. 


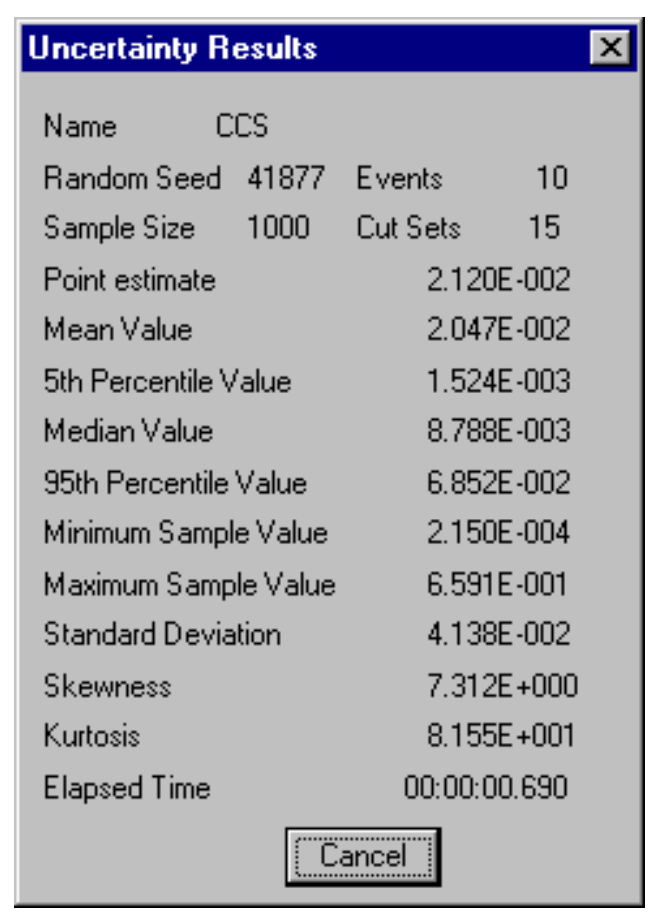

If only one fault tree (or sequence or end state) was selected (highlighted) for the uncertainty analysis process, then you will need to close the Uncertainty Results dialog. However, if more than one is being processed, the Uncertainty Results dialog will be displayed for each, and when all of the selected fault trees (or sequences or end states) have been processed, the Uncertainty Results dialog will automatically be closed.

\subsubsection{Seismic Uncertainty}

\section{PURPOSE}

A seismic analysis integrates the seismic fragility curve with the site hazard curve using the uncertainty analysis option. If the user has chosen to perform a seismic analysis, then a G-level was also selected. This option allows the user to specify how SAPHIRE performs the analysis.

\section{STEPS}

1. From the SAPHIRE menu select Fault Tree/Sequence/End State. The Fault Trees List/Sequences/End State List dialog will be displayed.

2. Select the "SEISMIC" value from the Analysis Type drop-down list.

3. Highlight the desired fault tree(s)/sequence(s)/end state(s) and right-click to invoke the pop-up menu.

4. Choose Uncertainty. The Uncertainty Calculation Values dialog will be displayed.

5. Provide appropriate values for the data entry fields on this dialog and choose one of the Ground Acceleration Level values.

The G-levels are described here:

ALL COMBINED - SAPHIRE performs an uncertainty analysis on all G-levels defined in the site hazard curve for this project. The combined results are then stored in the database. 
ALL SEPARATE - SAPHIRE performs an uncertainty analysis on all G-levels, but does the analysis and stores the results separately for each G-level.

Specific G-level - $\quad$ SAPHIRE only performs the analysis for that specified G-level.

\subsubsection{Uncertainty Distributions for Basic Events}

Within the SAPHIRE code, eleven types of uncertainty distributions are supported. The table below lists the different distributions, their identifier within SAPHIRE, and the uncertainty parameter that is needed by the code.

Along with the uncertainty parameter, most distributions require a second parameter. SAPHIRE requires that the mean value be specified for all distributions. The mean value is put in the database as a mean failure probability or a mean failure rate, depending on which calculation type is used.

Basic event uncertainty distribution types supported by SAPHIRE.

\begin{tabular}{lll} 
Distribution & Identifier & Uncertainty Parameter \\
none & blank & None \\
lognormal & $\mathrm{L}$ & error factor \\
normal & $\mathrm{N}$ & standard deviation \\
beta & $\mathrm{B}$ & $\mathrm{b}$ in Beta $(\mathrm{a}, \mathrm{b})$ \\
gamma & $\mathrm{G}$ & $\mathrm{r}$ in $\Gamma(\mathrm{r})$ \\
chi-squared & $\mathrm{C}$ & degrees of freedom \\
exponential & $\mathrm{E}$ & None \\
uniform & $\mathrm{U}$ & upper end point \\
histograms & $\mathrm{H}$ & histogram number (i.e., identifier) \\
maximum entropy & $\mathrm{M}$ & lower and upper end point \\
constrained noninformative & $\mathrm{O}$ & None \\
Seismic & $\mathrm{S}$ & Beta $r$, Beta u \\
\hline
\end{tabular}

If the event was assumed to be normally distributed, we would simply put the mean value in the probability data field and the standard deviation in the uncertainty parameter data field. To enter this distribution, the mean value of 0.5 would be entered, a normal distribution would be chosen (type N), and the standard deviation of 0.2 would be specified.

To review the process of specifying an uncertainty distribution in SAPHIRE, the required steps are to:

1. Enter the mean value for the basic event.

2. Choose the distribution type (from those shown in the "Identifier" column in the table above).

3. Specify the appropriate uncertainty distribution parameter (shown in the "Uncertainty Parameter" column in the table above).

Additional information on the uncertainty distributions and their parameters can be found in the SAPHIRE Technical Reference Manual. 


\subsection{Sequence Cut Set Analysis}

\subsubsection{Sequence Cut Set Analysis}

\section{PURPOSE}

This option provides the ability to perform a variety of analysis functions on the sequence cut sets.

\section{STEPS}

1. From the SAPHIRE menu select Sequence. The Sequences dialog will be displayed.

2. Highlight the desired sequence(s) and right-click to invoke the pop-up menu.

3. Choose Cut Sets, then one of the sub-menu options.

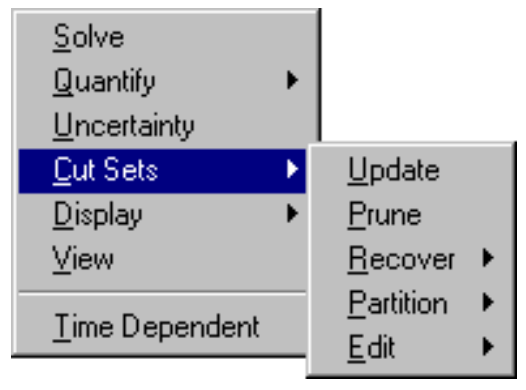

Update - Update the current cut sets for selected sequence(s) based on cut set generation cutoff values.

Prune - Eliminate cut sets from selected sequence(s) that contains events that conflict in some way with one another.

Recover -Modify cut sets by adding recovery actions to them.

Partition-Define the end states to be associated with each cut set in a sequence.

Edit - Modify the base case/current sequence cut sets.

\subsubsection{Updating Cut Sets}

\section{PURPOSE}

This option will update the current case cut sets for a selected fault tree (or sequence or end state) based on cut set generation cutoff values. You are given the opportunity to specify several cut set generation cutoff values that will be used to determine if a cut set is to be retained or discarded. You are also given the choice of using either the base case cut sets or the current case cut sets as the starting set of cut sets to be updated. The updated version of the cut sets will be saved as the new current case cut sets.

Current case cut sets can be updated for a selected fault tree (or sequence or end state), a group of fault trees (or sequences or end states), or all of the fault trees (or sequences or end states) within the current project.

\section{STEPS}

1. From the SAPHIRE menu select Fault Tree/Sequence/End State. The Fault Trees List/Sequences/End State List dialog will be displayed. 
2. Highlight the desired fault tree(s)/sequence(s)/end state(s) and right-click to invoke the pop-up menu.

3. Choose Cut Sets, then the Update sub-menu option.

4. The Cut Set Generation Truncation dialog will be displayed.

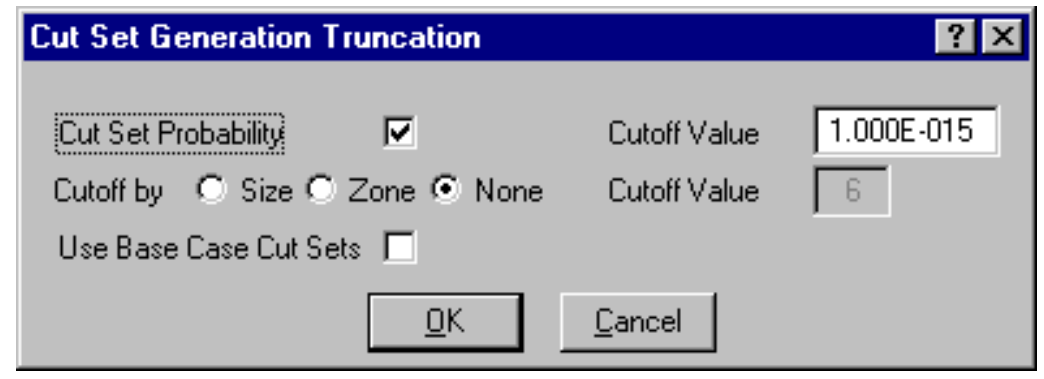

You may change any of the data fields on this dialog. The default values that appear on this dialog may be reset to new values by selecting Utility on the SAPHIRE menu and then invoking the Define Constants option.

Cut Set Probability - Used for determining if the cut set should be retained or discarded based on the value in the Cutoff Value field.

Cutoff by Size - $\quad$ Used for determining if the cut set should be retained or discarded based on zoned flagged events or the value in the Cutoff Value field.

Use Base Case Cut Sets -Used to specify base case cut sets or current case cut sets.

During processing, the Cut Set Generation Results dialog is displayed and updated as the calculations proceed. If a single fault tree (or sequence or end state) was selected, upon completion of the cut set generation, the results are displayed in this dialog. Choose the Ok button to close the dialog.

Once the cut sets are updated, they are automatically quantified.

\subsubsection{Cut Set Generation Truncation}

\subsubsection{Cut Set Probability}

If you select this check box, then only the cut sets whose product for all of its event probabilities is greater than or equal to the value in the Cutoff Value field will be kept. All other cut sets will be removed from current case cut sets for that fault tree.

If you uncheck this box, then the probability for the cut set will not be relevant for determining if the cut set should be retained or discarded.

\subsubsection{Cutoff by Size}

Size - If you choose this radio button, then only the cut sets whose number of events is less than or equal to the value specified in the $>$ Cutoff Value field will be kept in the cut sets for that fault tree. All other cut sets will be removed.

Zone - If you choose this radio button, then only zone flagged events will be checked.

None - If you choose this radio button, then the number of events in a cut set will be irrelevant for determining if the cut set should be retained or discarded. 


\subsubsection{Use Base Case Cut Sets}

If you select this check box, then base case cut sets will be used as the cut sets to be updated and then stored in the current case cut sets. However, if unchecked, the current cut sets will be used as the cut sets to be updated and then resaved in the current case cut sets.

\subsubsection{Recovering Sequence Cut Sets}

\subsubsection{Recovering Sequence Cut Sets}

\section{PURPOSE}

The "recovery" option allows the user to post-process cut sets by modifying them according to a predefined set of rules. You can recover a selected event or events based on sequence, applied, or derived rules. The user constructs recovery rules to be applied to each cut set which then define the action to be taken. The rules are constructed using the same editor that is used for event tree rules definition. This editor is a macro-based editor, which means that the user can define macros (or variables) that can be used as the search criteria tests.

\section{STEPS}

1. From the SAPHIRE menu select Sequence. The Sequences dialog will be displayed.

2. Highlight the desired sequence(s) and right-click to invoke the pop-up menu.

3. Choose Cut Sets | Recover.

4. Select one of the sub-menu options

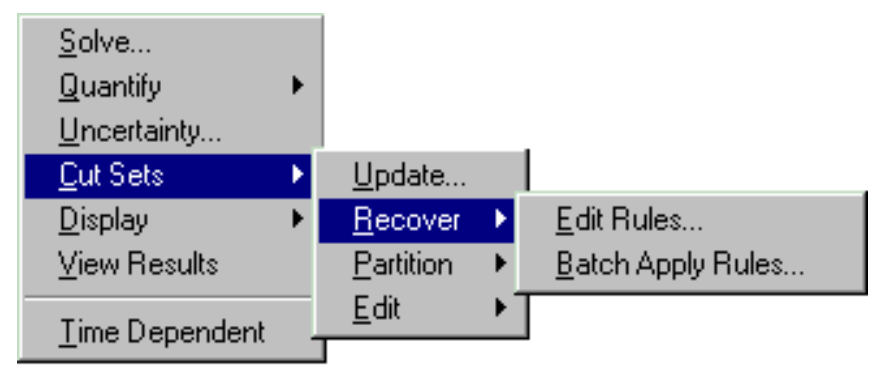

Edit Rules - Create and modify recovery rules for a highlighted sequence.

Apply Batch - Apply all rules currently defined to the highlighted sequences.

\subsubsection{Recovery Rules Editor}

\section{PURPOSE}

This option allows you to create or modify rules that affect existing cut sets in a "post-processing" fashion. The rule-based editor is available for both fault tree and sequence cut sets. The Recovery Rules editor provides a means to develop logic rules that allow for probabilistic risk assessment techniques such as automated inclusion of recovery events, inclusion of common-cause failure cut sets, or elimination of mutually-exclusive events (e.g., restricted or impossible combinations of events). 
The rules are entered in a free-form text editor within SAPHIRE. The same editor is used to create both basic and advanced recovery rules. The following discussions use the basic rule syntax, except where noted. Although the syntax/keywords differ between basic and advanced rules, the objectives and application of either kind of rule are the same.

Recovery rules follow a format similar to the structure that is found in traditional programming languages (e.g., BASIC or PASCAL). As such, the ability exists to define "macros" and "if...then" type of structures. After creating or editing rules, SAPHIRE compiles the rules to check their validity. Alternatively, rules can be exported from SAPHIRE or entered in any word processor or text editor (that can output ASCII files) and then loaded directly into the SAPHIRE database.

The recovery rules can be applied to cut sets for a particular sequence, a single event tree, or all sequences in the project. The rules can also be applied to cut sets for a particular fault tree or all fault trees in the project.

\section{STEPS}

1. From the SAPHIRE menu select Fault Tree/Sequence. The Fault Trees List/Sequences dialog will be displayed.

2. Highlight the desired fault tree(s)/sequence(s) and right-click to invoke the pop-up menu.

3. Choose Cut Sets | Recover | Edit Rules. The Edit Recovery Rules dialog will appear.

4. Select the desired Rule Level and Rule Type, and press OK.

If rules have been previously defined they will be displayed in the edit window.

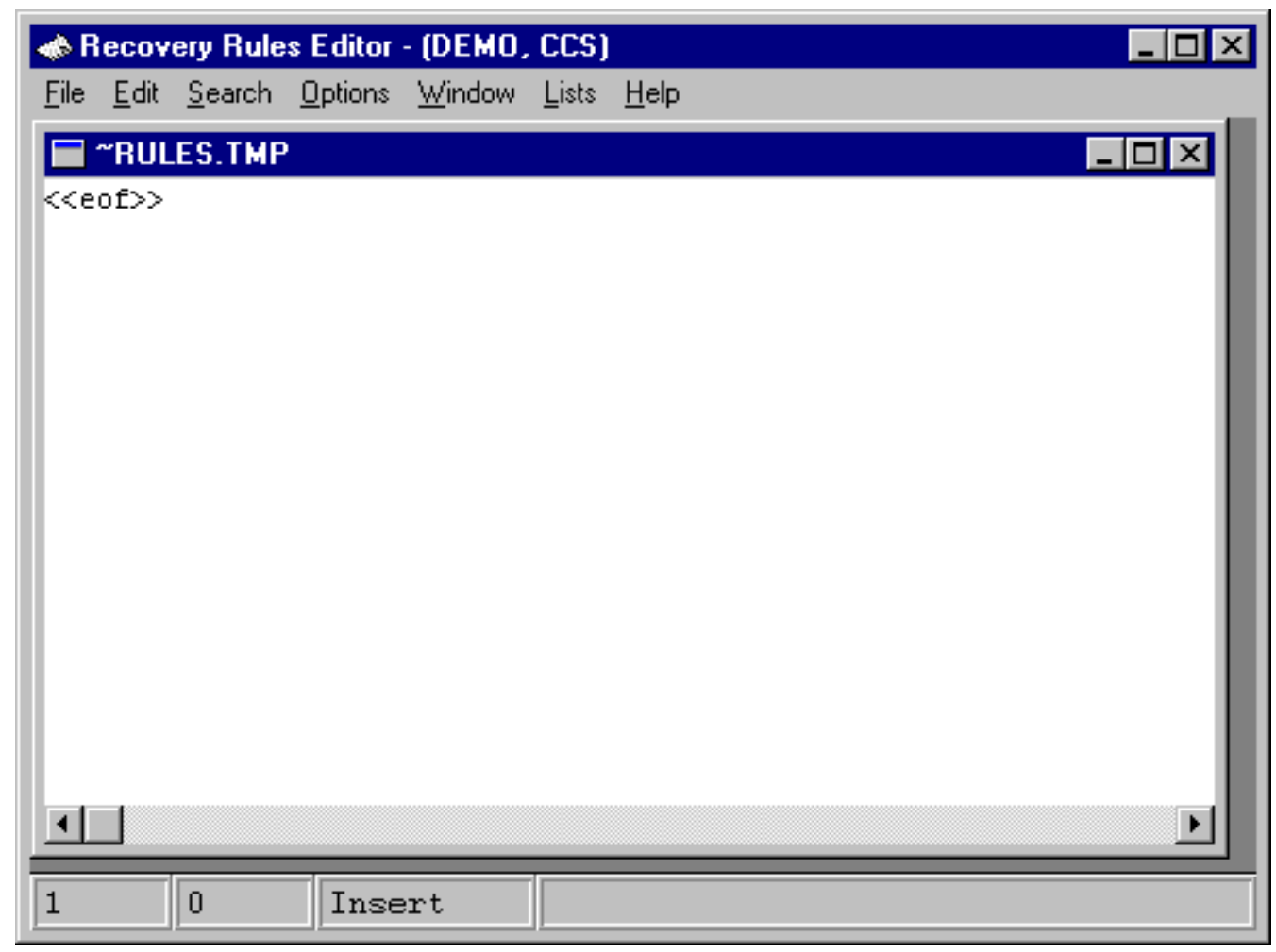

Type the rules in the editor. Note that rules are not case sensitive. 
File -

Edit -

Search -

Window -

Lists -
File functions such as open, save, print, preferences, etc.

Editing functions such as copy and paste along with text format operations. Search and cursor positioning operations such as find, replace, goto, etc. Window management functions such as cascade, tile horizontally, etc. Display lists of initiators, fault trees, etc. for inserting into the rules.

\section{PURPOSE}

This option allows you to apply all rules currently defined to the highlighted sequences.

The application of recovery rules can potentially require numerous comparisons of rules to cut sets, resulting in a long analysis time. Consequently, when the rules are applied in SAPHIRE for the entire project, the analyst is presented with the option to apply rules to cut sets down to a specified truncation level. By setting a truncation value in the Probability Cutoff Value field, only those cut sets with a "high" probability will have rules applied.

\section{STEPS}

1. From the SAPHIRE menu select Sequence. The Sequences dialog will be displayed.

2. Highlight the desired sequence(s) and right-click to invoke the pop-up menu.

3. Choose Cut Sets | Recover.

4. Select the Apply Batch sub-menu option. The Sequence Recovery dialog will be displayed.

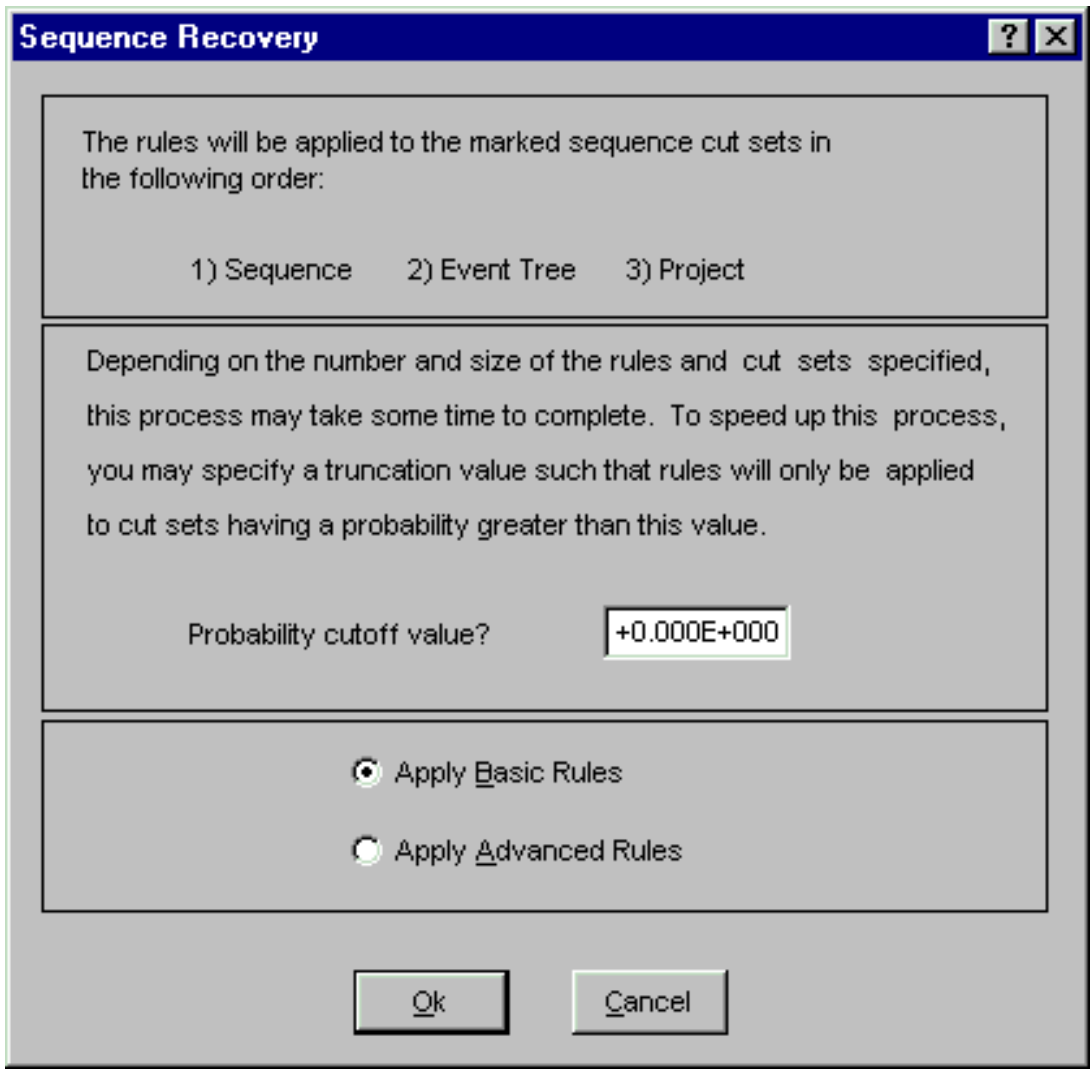


Apply Basic Rules -

Apply Advanced Rules -

Probability cutoff value -

Ok -

Cancel -
Recover the selected fault trees using rules created using Basic Rules.

Recover the selected fault trees using rules created using Advanced Rules.

The rules are applied only to those cut sets having a probability greater than this value. A value of zero will force the rules to be applied to all cut sets.

Apply the selected rules using the specified cutoff value.

Close the Sequence Recovery dialog without applying rules.

\subsubsection{Sequence Recovery Options}

\section{PURPOSE}

The "recovery" option allows the user to post-process cut sets by modifying them according to a predefined set of rules. The user constructs recovery rules to be applied to each cut set which then define the action to be taken. The rules are constructed using the same editor that is used for event tree rules definition. This editor is a macro-based editor, which means that the user can define macros (or variables) that can be used as the search criteria tests.

The rules may be developed for a particular fault tree or all fault trees, depending on the Rule Level selected.

\section{STEPS}

1. From the SAPHIRE menu select Sequence. The Sequences List dialog will be displayed.

2. Highlight the desired sequence(s) and right-click to invoke the pop-up menu.

3. Choose Cut Sets | Recover | Edit Rules. The Edit Recovery Rules dialog will be displayed.

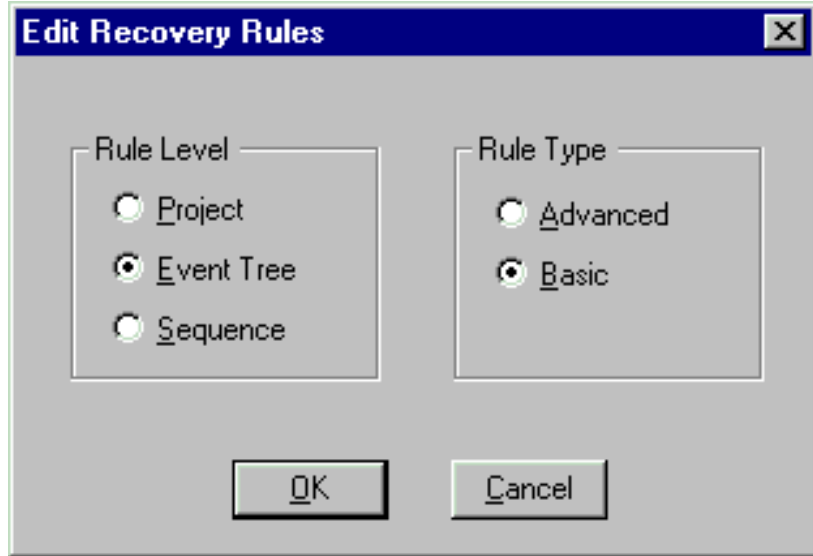

Rule Level

Project - $\quad$ Edit rules whose scope you wish to apply to all or multiple fault trees across the project. 
Event Tree - $\quad$ Edit rules whose scope you wish to apply only to the sequences of the highlighted event tree.

Sequence - $\quad$ Edit rules whose scope you wish to apply only to the sequences of the highlighted sequence. (Not generally recommended, because these rules may be deleted during future event tree linking.)

\section{Rule Type}

Advanced Basic -

Edit rules using the advanced recovery rules language.

Edit rules using the basic recovery rules language.

OK -

Create and modify sequence recovery rules.

Cancel -

\subsubsection{Basic Recovery Rule Editor}

\section{PURPOSE}

This is a free format line editor that provides you with an if-then-else logic structure. There is no limit to the number of rules the user may input to the editor. Each rule is processed in sequential order.

Basic rules can have different logic than the Advanced Rules and are saved separately.

\subsubsection{Advanced Recovery Rule Editor}

\section{PURPOSE}

This editor functions much the same as the Basic Rules Editor. However, the Modula-2 programming language is used to allow more robust programming capabilities. Using the Advanced Editor, users can define variables that can be assigned values based on specified criteria during run-time. Procedures can also be implemented which allows use of modular programming techniques.

When the advanced rules are compiled, a special dynamic link library (DLL) is created and saved in a special folder.

When this option is invoked the first time (before any advanced rules for the selected project/event tree/sequence exist), you will be given the option to convert a copy of the basic rules, if any, into advanced rule format. At that time, applying the basic or advanced rules should produce identical results. However, advanced rules are saved separately, and can be modified to have different logic than the Basic Rules.

\subsubsection{Partitioning Sequence Cut Sets}

\subsubsection{Partitioning Sequence Cut Sets}




\section{PURPOSE}

This option allows you to define the end states to be associated with each cut set in an accident sequence. You can define a set of rules that map the cut sets for a sequence to a particular end state. The rules are defined using the Partition Rules Editor.

\section{STEPS}

1. From the SAPHIRE menu select Sequence. The Sequences dialog will be displayed.

2. Highlight the desired sequence(s) and right-click to invoke the pop-up menu.

3. Choose Cut Sets | Partition.

4. Select one of the sub-menu options

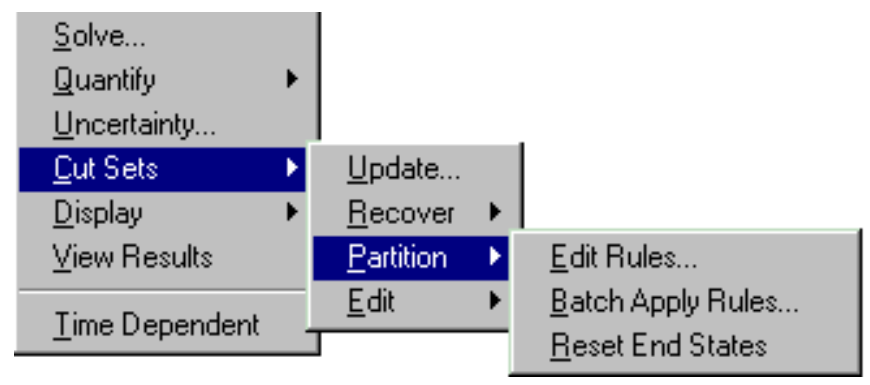

Edit Rules Apply Batch Reset End States -
Define cut set partitioning rules for individual sequences. Apply the currently defined partition rules to the selected sequence(s). Clear the sequences created by the partitioning rule.

Once the rules have been defined, they need to be applied . Rules are applied sequentially; that is, project rules, followed by event tree rules, and finally sequence rules. If rules conflict, the latest applied rule will take precedence over earlier ones. After you have entered the partitioning rules and applied them, you must then "gather" cut sets for the various end states that you have defined.

\subsubsection{Apply Batch}

\section{PURPOSE}

This option actually applies the partition rules to the selected sequence cut sets. Rules are sequentially applied; that is, project rules, followed by event tree rules, and finally sequence rules. If rules conflict, the latest applied rule will take precedence over earlier ones.

\section{STEPS}

1. From the SAPHIRE menu select Sequence.

2. The Sequences dialog will be displayed.

3. Highlight the desired sequence(s) and right-click to invoke the pop-up menu.

4. Choose Cut Sets | Partition. 
5. Select the Apply Batch sub-menu option. The Partition Sequence Cut Sets dialog will be displayed.

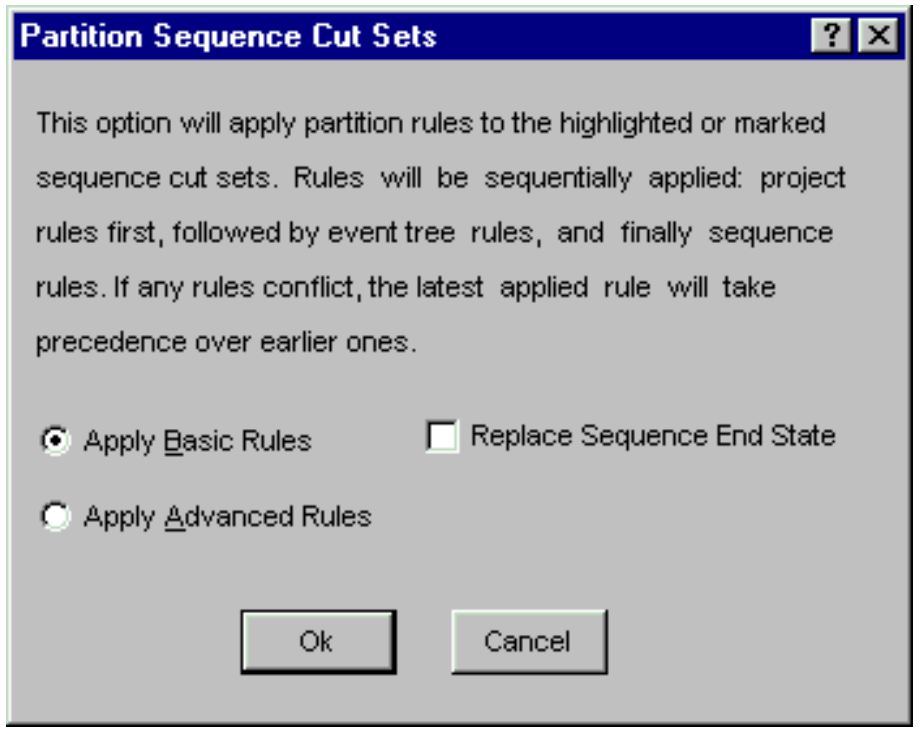

Apply Basic Rules -

Apply Advanced Rules -

Replace Sequence End State -

Ok -

Cancel -
Recover the selected fault trees using rules created using Basic Rules.

Recover the selected fault trees using rules created using Advanced Rules.

Replace the end state at the sequence level, rather than at the cut set level.

Apply the rules to the selected sequence cut sets. Close the Partition Sequence Cut Sets dialog without applying rules.

\subsubsection{Reset End States}

\section{PURPOSE}

This option clears the sequence end states created by the partitioning rules. By performing this function, the sequence end state cross-reference map is set to blank. However, cut sets will still appear in the end states until the end state is removed, or gathered again. By clearing the cross-reference map, the end state can be removed, if desired, from the end states listed by using the Modify | End States option.

\section{STEPS}

1. From the SAPHIRE menu select Sequence. The Sequences dialog will be displayed.

2. Highlight the desired sequence(s) and right-click to invoke the pop-up menu.

\section{Choose Cut Sets | Partition.}


4. Select the Reset End States sub-menu option. The message, "Reset complete," will be displayed in the status bar of the SAPHIRE window.

\subsubsection{Partition Rule Edit Options}

\section{PURPOSE}

The "partition" option allows the user to post-process cut sets by assigning an end state to each cut set according to a pre-defined set of rules. The user constructs partition rules to be applied to each cut set which then define the end state to be assigned. The rules are constructed using the same editor that is used for event tree rules definition, and recovery rule definition. This editor is a macro-based editor, which means that the user can define macros (or variables) that can be used as the search criteria tests.

The rules may be developed for a particular sequence, all sequences belonging to an event tree, or for all sequences, depending on the Rule Level selected.

\section{STEPS}

1. From the SAPHIRE menu select Sequence. The Sequences List dialog will be displayed.

2. Highlight the desired sequence(s) and right-click to invoke the pop-up menu.

3. Choose Cut Sets | Partition | Edit Rules.

4. The Edit Partition Rules dialog will be displayed.

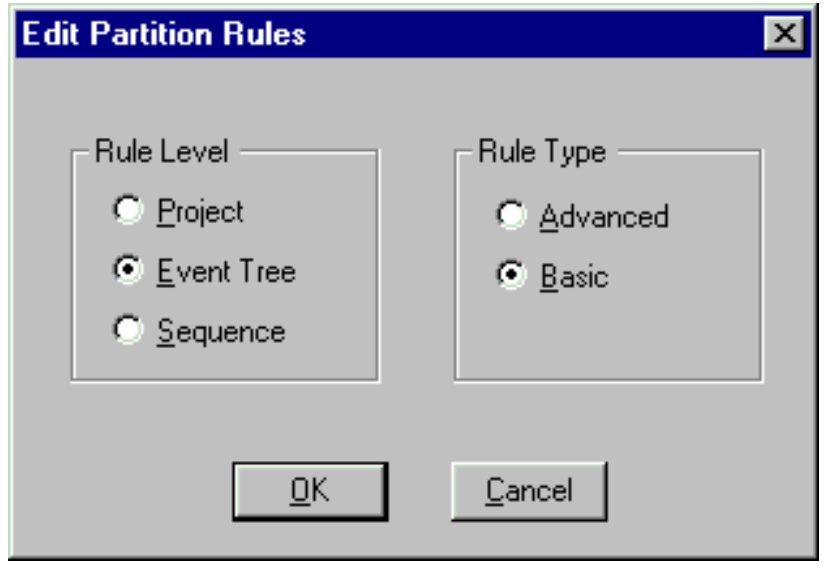

Rule Level

Project -

Event Tree -

Sequence -
Edit rules whose scope you wish to apply to all or multiple event tree sequences across the project.

Edit rules whose scope you wish to apply only to the sequences of the highlighted event tree.

Edit rules whose scope you wish to apply only to the sequences of the highlighted sequence. (Not generally recommended, because these rules may be deleted during future event tree linking.) 


\section{Rule Type}

Advanced - $\quad$ Edit rules using the advanced recovery rules language.

Basic Edit rules using the basic recovery rules language.

OK -

Cancel -
Create and modify sequence recovery rules.

Close the Edit Partition Rules dialog without editing any rules.

\subsubsection{Basic Partition Rule Editor}

\section{PURPOSE}

This is a free format line editor that provides you with an if-then-else logic structure. There is no limit to the number of rules the user may input to the editor. Each rule is processed in sequential order.

Basic rules can have different logic than the Advanced Rules and are saved separately.

\subsubsection{Advanced Partition Rule Editor}

\section{PURPOSE}

This editor functions much the same as the Basic Rules Editor. However, the Modula-2 programming language is used to allow more robust programming capabilities. Using the Advanced Editor, users can define variables that can be assigned values based on specified criteria during run-time. Procedures can also be implemented which allows use of modular programming techniques.

When the advanced rules are compiled, a special dynamic link library (DLL) is created and saved in a special folder.

When this option is invoked the first time (before any advanced rules for the selected project/event tree/sequence or fault tree exist), you will be given the option to convert a copy of the basic rules, if any, into advanced rule format. At that time, applying the basic or advanced rules should produce identical results. However, advanced rules are saved separately, and can be modified to have different logic than the Basic Rules.

\subsubsection{Partition Rules Editor}

\subsection{Partition Rules Editor}

\section{PURPOSE}

This editor allows you to define a set of rules that map the cut sets for a sequence to an end state. This is a free format, macro-based line editor that provides you with an if-then-else logic structure. If rules have already been defined they will be displayed in the edit window.

By default, the editor assumes that a name is the name of a basic event, if one exists in the database. If a basic event by the name does not exist then the editor assumes that the name is the name of a macro. If you want to change the default assumptions, then you can "cast" the names to something else by enclosing the name in parenthesis with a cast name preceding it.

There is no limit to the number of rules you may input to the editor. Each rule is processed in sequential order. 


\section{STEPS}

1. From the SAPHIRE menu select Sequence. The Sequences dialog will be displayed.

2. Highlight the desired sequence(s) and right-click to invoke the pop-up menu.

3. Choose Cut Sets | Partition | Edit Rules. The Edit Recovery Rules dialog will appear.

4. $\quad$ Select the desired Rule Level and Rule Type, and press $\mathbf{O K}$.

If rules have been previously defined they will be displayed in the edit window.

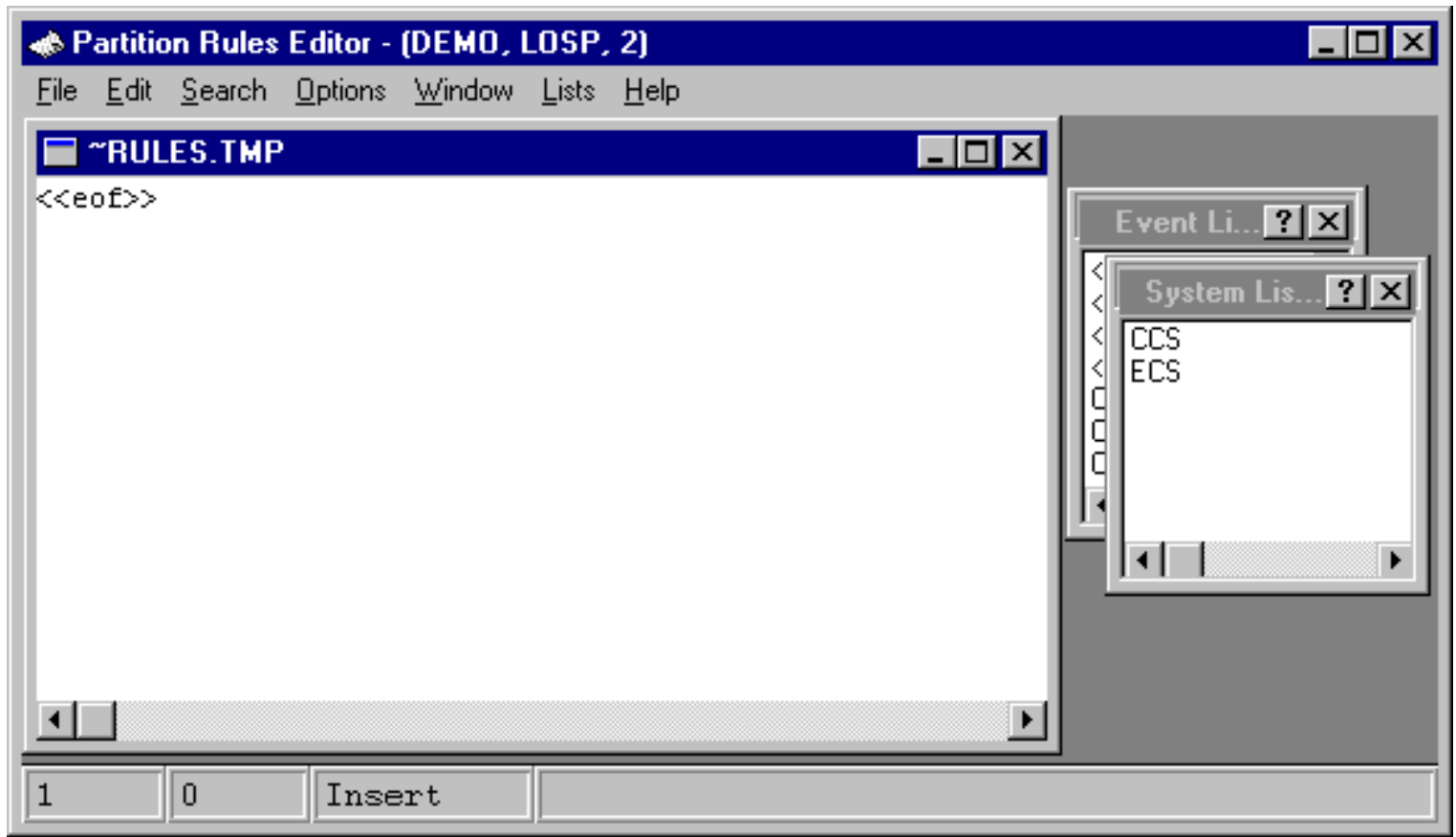

File - $\quad$ File functions such as open, save, print, preferences, etc.

Edit - $\quad$ Editing functions such as copy and paste along with text format operations.

Search - Search and cursor positioning operations such as find, replace, goto, etc.

Window - Window management functions such as cascade, tile horizontally, etc.

Lists - $\quad$ Display lists of initiators, fault trees, etc. for inserting into the rules.

\subsection{File Menu}

\subsection{Editor File Functions}

These options provide file management functions.

Open - $\quad$ Open a file into a new window. This feature is not usually used while editing SAPHIRE rules.

New - $\quad$ Create a new file in a new window. This feature is not usually used while editing SAPHIRE rules.

Close - $\quad$ Close the edit current window. If changes have been made you will be prompted to save the file.

Quit - $\quad$ Close the current edit window without saving.

Save - $\quad$ Save the file in the current window to disk. 
Save As -

Save All -

Save Block -

Import File -

Print -

Page setup -

Preferences -

Exit -
Save the file in the current window with a new file name.

Save all the files in all edit windows currently open.

Save the currently highlighted text into a new file. The Save As dialog will be invoked.

Insert the contents of a file into the current edit window at the current cursor position. The Edit external file dialog will be invoked.

Print the file in the current edit window.

Set printer page layout options, .such as pages per sheet and line numbers.

Select editing options such as tab width, undo levels, etc.

Terminate the Editor session.

\subsection{Open}

\section{PURPOSE}

This option allows you to open an existing file into a new editing window within the Linkage Rules Editor or the Event Tree Logic Editor.

\section{STEPS}

1. From the Linkage Rules Editor or the Event Tree Logic Editor menu select File.

2. Select the Open menu option.

The Edit external file dialog will be displayed listing all of the files in the current project directory.

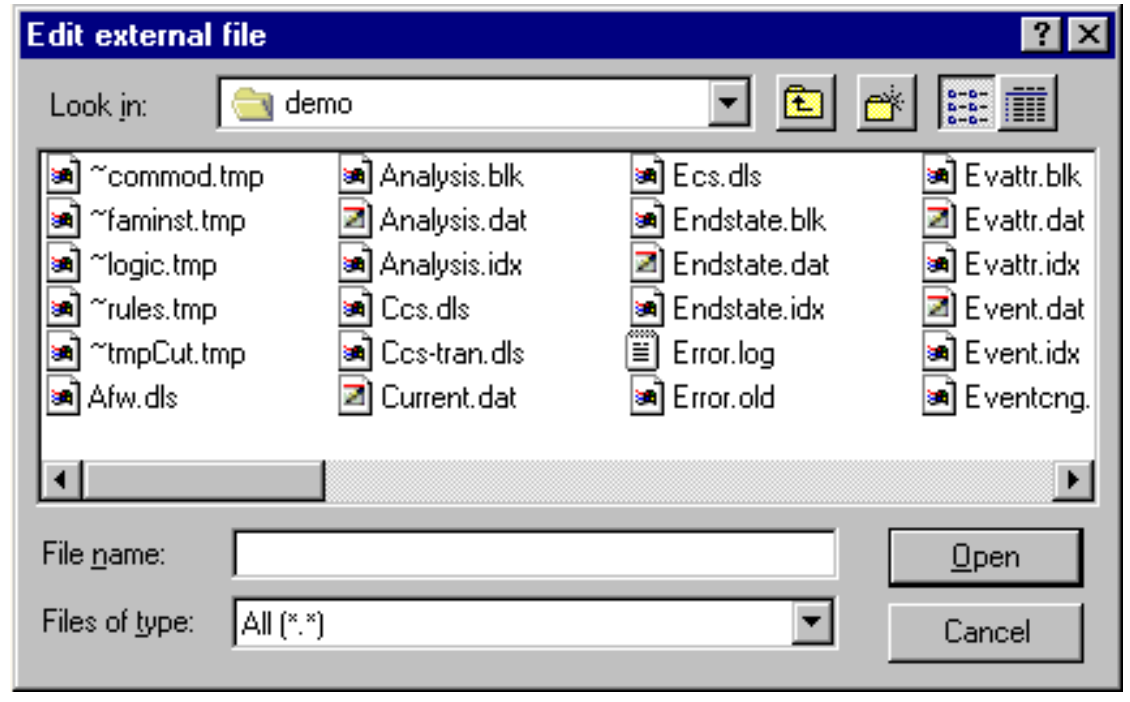

Look in - $\quad$ Lists the available folders and files. The box directly below it shows the contents of the current folder. The down-arrow to the right of this field shows how the folder fits into your system hierarchy.

Moves up one level in the system hierarchy.

Creates a new folder at the current level.

Displays the icons and names of the contents of the current folder. 
位烡

File Name -

Files of Type -

Open -

Cancel -

Double-click -
Displays the icons, names, size, type, date and time modified, and archive flags of the contents of the current folder.

Contains the name of the file in which the diagram will be saved.

Lists the types of files to display. This is useful for narrowing the list of files displayed to only those you are interested in.

Open the selected file in a new window and close the Edit external file dialog. Close the Edit external file dialog without opening another file.

Open the selected file in a new window and close the Edit external file dialog. 


\subsection{Save As}

\section{PURPOSE}

This option allows you to save the file in the current editing window with a new file name.

\section{STEPS}

1. From the Linkage Rules Editor or the Event Tree Logic Editor menu select File.

2. Select the Save As menu option. The Save As dialog will be displayed.

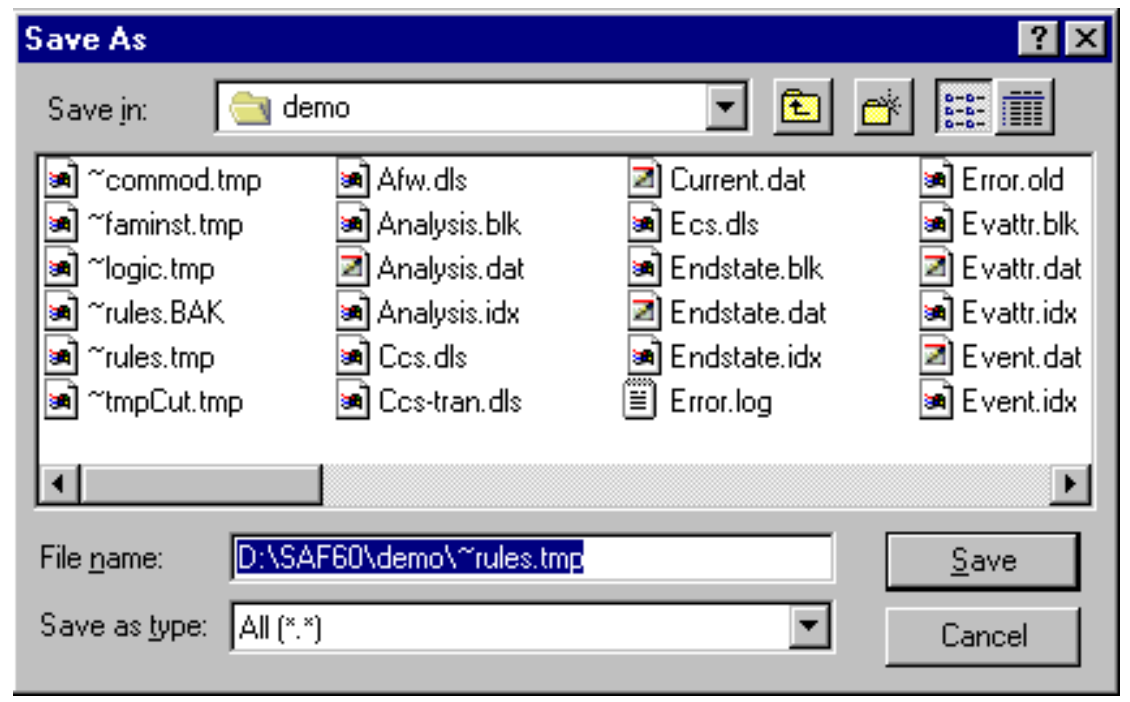

Save in - $\quad$ Lists the available folders and files. The box directly below it shows the contents of the current folder. The down-arrow to the right of this field shows how the folder fits into your system hierarchy.

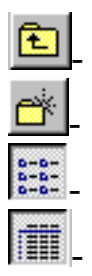

File Name Files of Type -

Save -

Double-click -

Cancel -
Moves up one level in the system hierarchy.

Creates a new folder at the current level.

Displays the icons and names of the contents of the current folder.

Displays the icons, names, size, type, date and time modified, and archive flags of the contents of the current folder.

Contains the name of the file to be saved.

Lists the types of files to display. This is useful for narrowing the list of files displayed to only those you are interested in.

Save the data in the current editing window to the file in the File name field and close the Save Fault Tree dialog. If an existing file was selected, a warning dialog will be displayed.

Save the current diagram to the file in the File name field and close the Save As dialog.

Close the Save As dialog without performing the save operation.

\subsection{Print}




\section{PURPOSE}

This option allows you to print the file in the current edit window to the default (Windows System) printer.

\section{STEPS}

1. From the Linkage Rules Editor or the Event Tree Logic Editor menu select File.

2. Select the Print menu option. The Print dialog will be displayed.

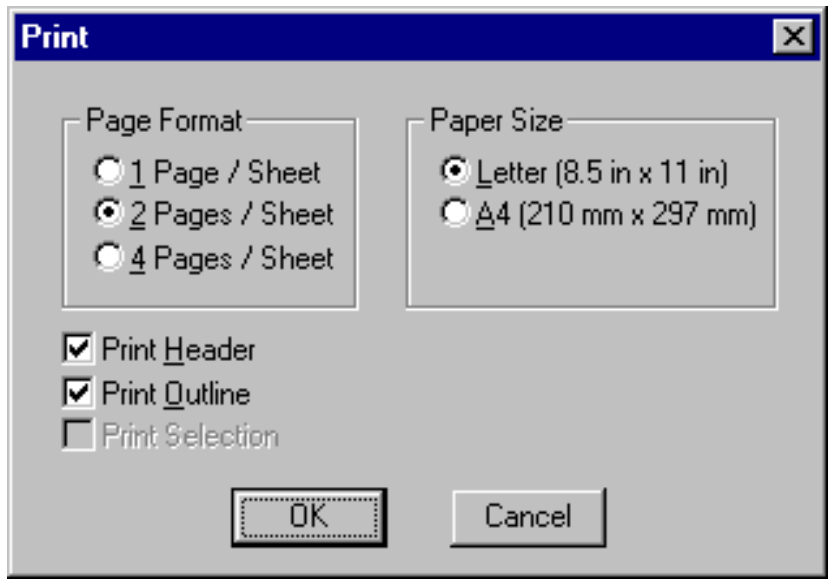

Page Format - Select the desired format option. A "page" of data is approximately 66 lines, including header information and outlines.

1 Page / Sheet - Prints one "page" of data on a single sheet of paper. The page is printed using the Portrait orientation (i.e., the short edge of the paper is horizontal).

2 Pages / Sheet - Prints two "pages" of data on a single sheet. The page is printed using the Landscape orientation (i.e., the long edge of the paper is horizontal).

4 Pages/ Sheet - Print four "pages" of data on a single sheet. The page is printed using the Landscape orientation.

Paper Size - $\quad$ Select the size of the paper to print on.

Print Header - Include header information in the print-out. Header information includes the current date, the file name, and the page number. These items are printed across the top of the page, followed by a line, separating it from the data.

Print Outline - Outline each "page" in a rectangle. This is especially useful when using the 2 Pages / Sheet or 4 Pages / Sheet options.

Print Selection - Print the currently highlighted data.

OK -

Cancel -
Print the data to the Windows System Printer using the selected options and close the Print dialog.

Close the Print dialog without printing the data. 


\subsection{Exit}

\section{PURPOSE}

This option allows you to terminate the Editor session. The rules are automatically compiled upon exiting the Editor. If there is a compilation error, the Editor will be reopened with the current file and a message will be displayed in the editor's message bar.

\section{STEPS}

1. From the Linkage Rules Editor or the Event Tree Logic Editor menu select File.

2. Select the Exit menu option.

If changes have been made to the data, you will be prompted to save the file.

\subsection{Save This File}

\section{PURPOSE}

This dialog will appear any time you are closing the current editing window or exiting the Editor and changes to the file have not been saved (i.e., written to disk).

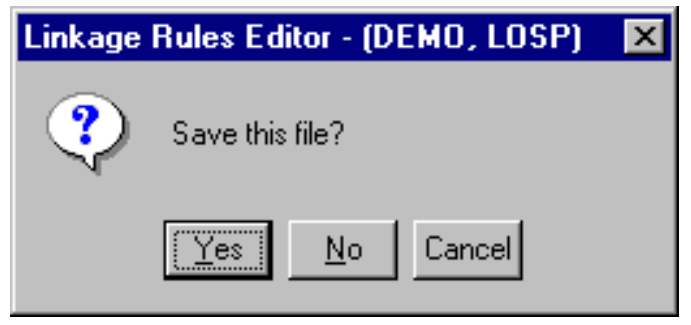

Yes - $\quad$ Save the changes made to the file in the current editing window and close the current editing window.

No - Do not save the changes made to the file in the current editing window and close the current editing window.

Cancel - Do not save the changes made to the file in the current editing window and do not close the current editing window.

\subsection{Edit Menu}

\subsection{Editor Edit Functions}

These options provide editing and text formatting functions.
Undo -
Undo the previous editing operation.
Cut -
Remove the highlighted text from the current window and place it in the
Copy - clipboard.
Paste -
Delete - Copy the highlighted text from the current window and place it in the clipboard.
Copy the clipboard text into the current window at the cursor position. Remove the highlighted text from the current window without placing it in the clipboard. 
Shift left indent -

Shift right indent -

Shift left space -

Shift right space -

Delete to end of line -

Delete line -

Capitalize word -

Downcase word -
Shift the text to the left by the Shift Size specified under Options. Shift the text to the right by the Shift Size specified under Options. Shift the text to the left by a single space.

Shift the text to the right by a single space.

Delete to the end of the current line beginning after the current cursor position.

Delete the line on which the cursor is currently positioned.

Convert the entire word under the current cursor position to upper case.

Convert the entire word under the current cursor position to lower case.

\subsection{Search Menu}

\subsection{Editor Search Functions}

These options provide search and cursor positioning operations.

Find -

Replace -

Find Procedure Find/Replace again Find Altered lines Find line number Match symbol -

Goto next error Goto previous error Goto Marker -

Set Marker -
Search the text in the current window for the specified string.

Search the text in the current window for a string and replace it with another string.

Search the text in the current window for the specified procedure. Repeat the previous search operation. Find the lines of text that have changed since the last save operation. Go to the specified text line number in the current window.

Find the matching symbol pair for the character under the cursor " ()$,\{\}$, [], (**)"

Position the cursor at the next compilation error.

Position the cursor at the previous compilation error.

Position the cursor at the previously saved file position. Up to four marker positions can be retrieved.

Save the current file position for later retrieval.

\subsection{Find}

\section{PURPOSE}

This option allows you to search the text in the current window for the specified string. If the search criteria are met, the first string matching the criteria is highlighted.

\section{STEPS}

1. From the Linkage Rules Editor or the Event Tree Logic Editor menu select Search.

2. Select the Find menu option. The Find dialog will be displayed. 


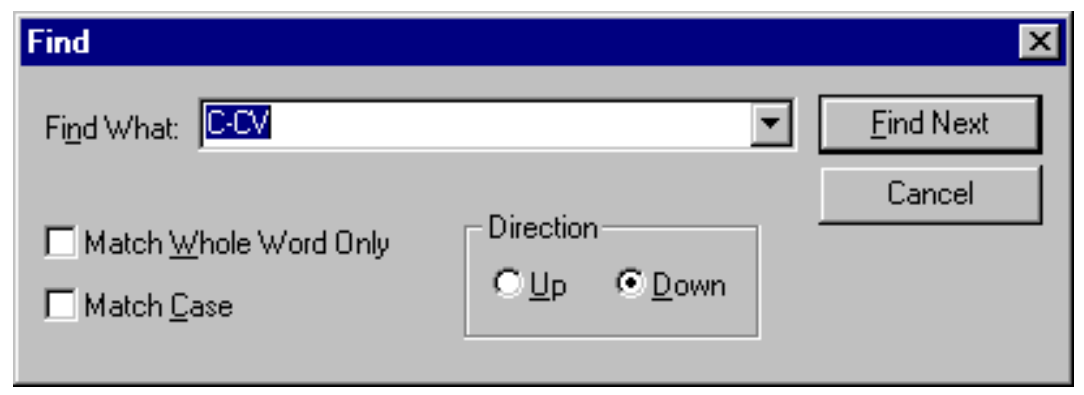

Find What -

Match Whole Word Only -

Match Case -

Direction

Up -

Down -

Find Next -

Cancel -
The string of alphanumeric characters upon which the search is based (i.e., the "search string"). Type the text in the field or select from the drop-down list.

If this check box is selected, only the series of alphanumeric characters matching the search string and delimited by a space, comma, dash, period, etc. are located.

If this check box is selected, only the series of alphanumeric characters matching the upper- and lowercase characters as entered in the Find What field are located (i.e., the search will be case-sensitive).

Search the text from the current cursor position to the beginning or top of the text.

Search the text from the current cursor position to the end or bottom of the text.

Perform the search operation using the selected criteria. If the search is not successful, an information dialog will be displayed. If the search criteria are met, the Find dialog, shown below, will become active and the current Find dialog will be closed..

Close the Find dialog without performing the search operation.

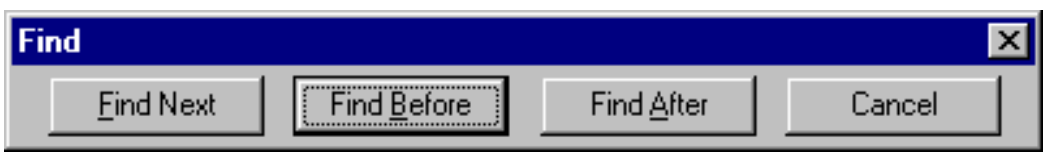

Find Next -

Find Before -

Find After -

Cancel -
Locate the next string matching the current search criteria.

Locate the previous string matching the current search criteria. Locate the next string matching the current search criteria. Close the Find dialog without performing the search operation.

\subsection{Replace}

\section{PURPOSE}

This option allows you to search the text in the current window for the specified string and replace it with another string. If the search criteria are met, the first string matching the criteria is highlighted. 


\section{STEPS}

1. From the Linkage Rules Editor or the Event Tree Logic Editor menu select Search.

2. Select the Replace menu option. The Replace dialog will be displayed.

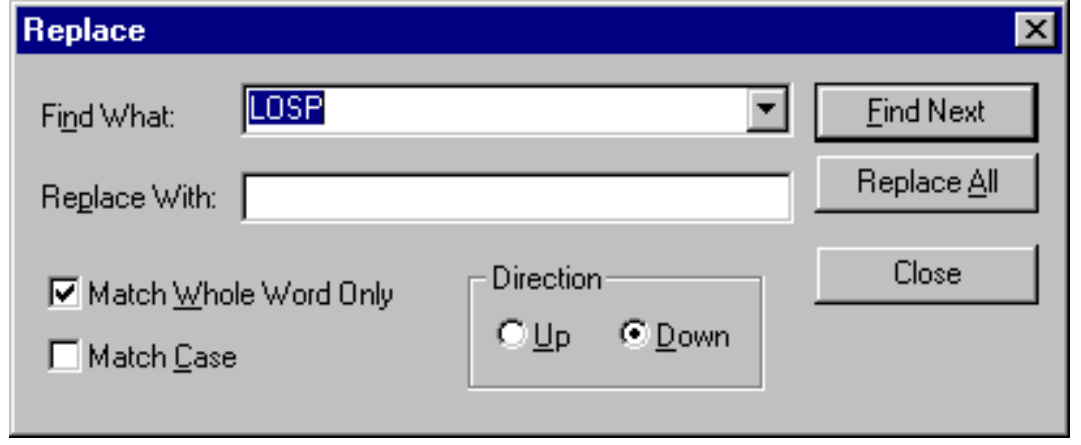

Find What -

Replace With -

Match Whole Word Only -

Match Case -

Direction

Up -

Down -

Find Next -

Replace All -

Close -
The string of alphanumeric characters upon which the search is based (i.e., the "search string"). Type the text in the field or select from the drop-down list.

The string of alphanumeric characters that will be substituted for the search string. Type the text in the field.

If this check box is selected, only the series of alphanumeric characters matching the search string and delimited by a space, comma, dash, period, etc. are located.

If this check box is selected, only the series of alphanumeric characters matching the upper- and lowercase characters as entered in the Find What field are located (i.e., the search will be case-sensitive).

Search the text from the current cursor position to the beginning or top of the text.

Search the text from the current cursor position to the end or bottom of the text.

Perform the search operation using the selected criteria. If the search is not successful, an information dialog will be displayed. If the search criteria are met, the Find/Replace again dialog will become active and the current Replace dialog will be closed..

Find all occurrences matching the search criteria and automatically substitute with the "replace string."

Close the Replace dialog without performing the search and replace operation.

\subsection{Find Procedure}




\section{PURPOSE}

This option allows you to search the text in the current window for the specified procedure.

\section{STEPS}

1. From the Linkage Rules Editor, the Recovery Rules Editor, or the Partition Rules Editor menu select Search.

2. Select the Find Procedure menu option. The Procedure to search for dialog will be displayed.

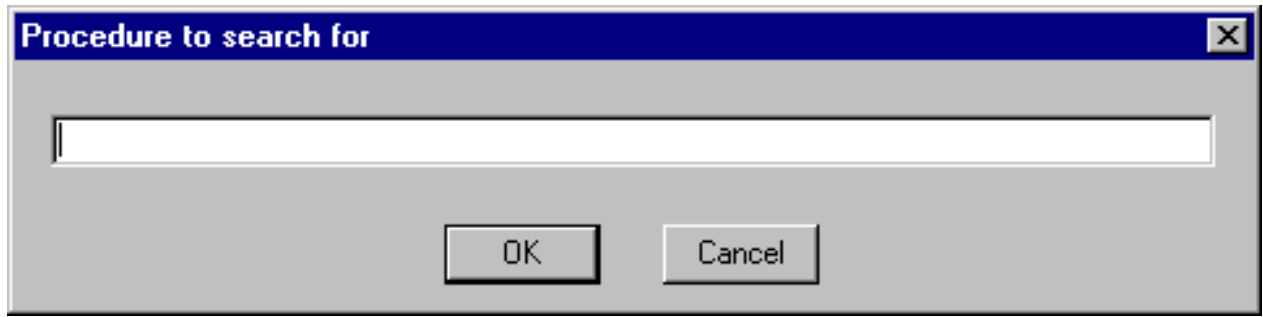

OK - Locate the procedure matching the current search criteria.

Cancel - Close the Procedure to search for dialog without performing the search operation.

\subsection{Find/Replace again}

\section{PURPOSE}

This option allows you to search the text in the current window based on the previously defined search string and replace it with the previously defined replace string. If the search criteria are met, the first string matching the criteria is highlighted.

\section{STEPS}

1. From the Linkage Rules Editor or the Event Tree Logic Editor menu select Search.

2. Select the Find/Replace again menu option. The Replace dialog will be displayed.

\begin{tabular}{l|l|l|l|}
\hline Replace & \multicolumn{1}{|c|}{ Close } \\
\hline Find Next & Replace & Replace All \\
\hline
\end{tabular}

Find Next - $\quad$ Locate the next string matching the current search criteria. A substitution will not be made.

Replace - $\quad$ Substitute the replace string for the located search string and continue searching.

Replace All - Substitute the replace string for all occurrences matching the search criteria, beginning at the current cursor position.

Close - $\quad$ Close the Replace dialog without performing the search and replace operation.

NOTE: This dialog can also be invoked from the Search | Replace | Find Next menu options.

\subsection{Find Procedure}




\section{PURPOSE}

This option allows you to search the text in the current window for the specified procedure.

\section{STEPS}

1. From the Linkage Rules Editor, the Recovery Rules Editor, or the Partition Rules Editor menu select Search.

2. Select the Find Procedure menu option. The Procedure to search for dialog will be displayed.

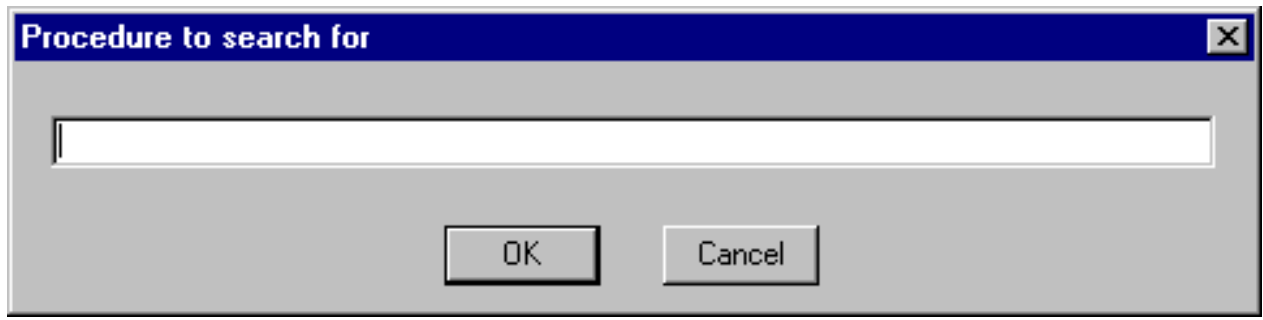

OK - Locate the procedure matching the current search criteria.

Cancel - Close the Procedure to search for dialog without performing the search operation.

\subsection{Find line number}

\section{PURPOSE}

Position the cursor on the specified line number in the text in the current window.

\section{STEPS}

1. From the Linkage Rules Editor or the Event Tree Logic Editor menu select Search.

2. Select the Find line number menu option.

3. The Enter line number dialog will be displayed.

4. Type the desired line number in the field.

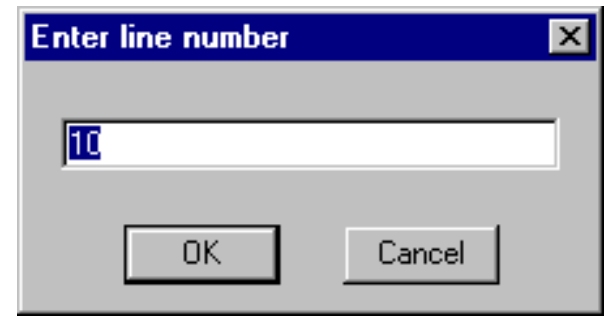

OK - Position the cursor at the beginning of the desired line and close the dialog.

Cancel - Close the dialog without repositioning the cursor.

\subsection{Goto Marker}




\section{PURPOSE}

Position the cursor at the previously saved file position. Up to four marker positions can be retrieved for each editing window.

\section{STEPS}

1. From the Linkage Rules Editor or the Event Tree Logic Editor menu select Search.

2. Select the Goto Marker menu option.

3. Choose one of the four marker positions available from the submenu.

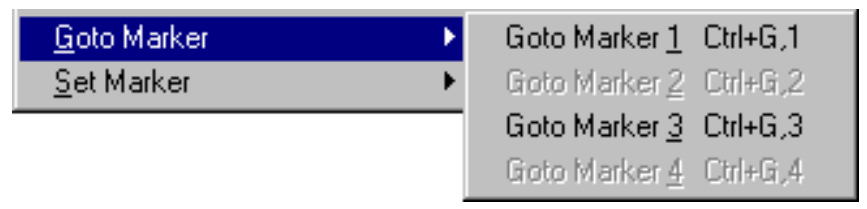

In this example, two markers have been set at \#1 and \#3. After choosing one of the available markers, the cursor is positioned at the beginning of the line.

The marker positions are saved only during the current editing session.

\subsection{Set Marker}

\section{PURPOSE}

Save the current file position for later retrieval. Up to four marker positions can be saved for each editing window.

\section{STEPS}

1. Position the cursor on the desired line of the text in the edit window.

2. From the Linkage Rules Editor or the Event Tree Logic Editor menu select Search.

3. Select the Set Marker menu option.

4. Choose one of the four marker positions available from the submenu.

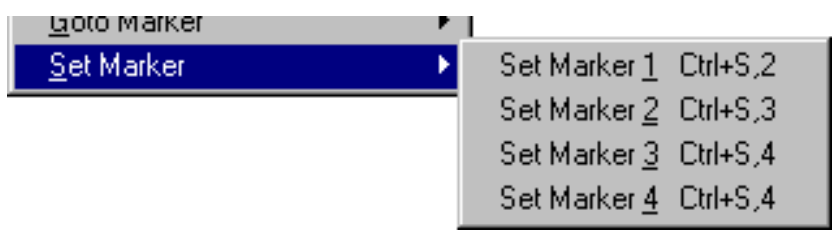

The marker positions are saved only during the current editing session.

\subsection{Window Menu}

\subsection{Editor Window Functions}


These options are used for managing the open edit windows within the application window, such as the Linkage Rules Editor or the Event Tree Logic Editor window. Along with the options listed below and segregated by a separation bar, the names of each open file is listed in the menu.

Cascade windows -

Tile windows horizontally -

Tile windows vertically -

Arrange Icons -
Resize and reposition the non-minimized windows in an overlapping fashion so that the title of each window is displayed in a cascade arrangement. The current edit window will be on top.

Resize and reposition the non-minimized windows in a horizontally tiled arrangement.

Resize and reposition the non-minimized windows in a vertically tiled arrangement.

Arrange the minimized window icons at the bottom of the application window.

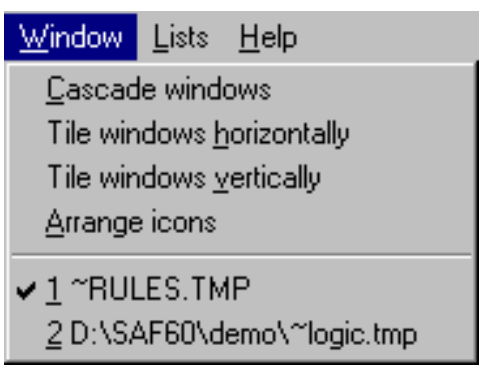

In the example above, the files " $\sim$ RULES.TMP" and " $\sim$ LOGIC.TMP" are open. $\sim$ RULES.TMP is the active window, as indicated by the check mark on the menu.

\subsection{Lists Menu}

\subsection{Partition Rules Lists}

Selecting from the lists allows you to insert or replace items in the editor.

Macros - $\quad$ Allows you to insert a macro into the editor.

Events - $\quad$ Select basic events used to replace others instead of typing in the basic event names.

Initiators - $\quad$ Select initiators used to replace others instead of typing in the initiator names.

Fault Trees - $\quad$ Select fault trees as top events and fault trees used to replace others instead of typing in the fault trees names.

End States - $\quad$ Select end states used to replace others instead of typing in the end state names.

Event Trees - Select event trees used to replace others instead of typing in the event tree names.

\subsection{Macros}

\section{PURPOSE}

This option allows you to insert a macro into the text of the current edit window. 


\section{STEPS}

1. Place the cursor at the desired location in the text.

2. From the Linkage Rules Editor or the Event Tree Logic Editor menu select Lists.

3. Select the Macros menu option. The Macro List dialog will be displayed.

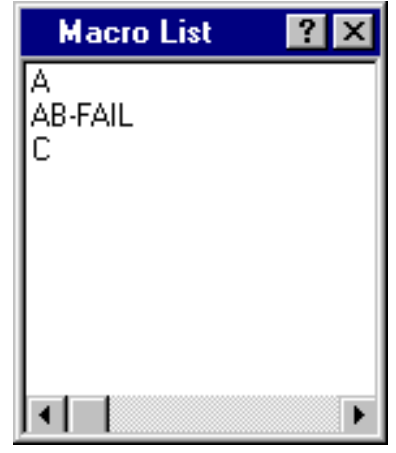

Double-click on the desired macro name in the list. The selected name will be entered at the current cursor location.

The Macro List dialog will remain open in the application window during the current session unless explicitly closed.

\subsection{Events}

\section{PURPOSE}

This option allows you to insert a basic event name into the text of the current edit window.

\section{STEPS}

1. Place the cursor at the desired location in the text.

2. From the Linkage Rules Editor or the Event Tree Logic Editor menu select Lists.

3. Select the Events menu option. The Event List dialog will be displayed containing all of the basic events in the current family's database.

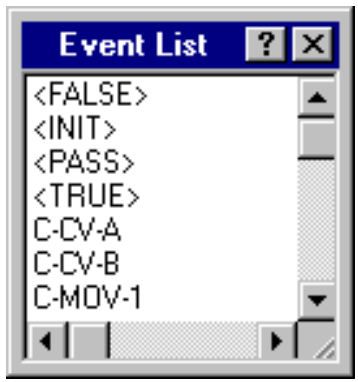

4. Double-click on the desired event name in the list. The selected name will be entered at the current cursor location. 
The Event List dialog will remain open in the application window during the current session unless explicitly closed.

\subsection{Initiators}

\section{PURPOSE}

This option allows you to insert an initiating basic event name into the text of the current edit window.

\section{STEPS}

1. Place the cursor at the desired location in the text.

2. From the Linkage Rules Editor or the Event Tree Logic Editor menu select Lists.

3. Select the Initiators menu option. The Initiator List dialog will be displayed containing all of the basic events whose category attribute is set to "I" in the current family's database.

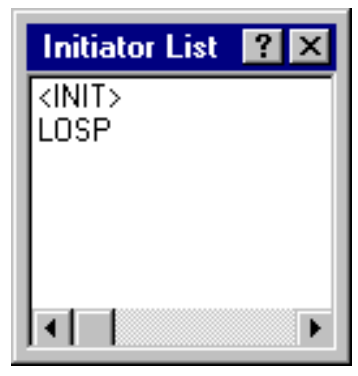

4. Double-click on the desired initiating event name in the list. The selected name will be entered at the current cursor location.

The Initiator List dialog will remain open in the application window during the current session unless explicitly closed.

\subsection{Systems}

\section{PURPOSE}

This option allows you to insert a fault tree name into the text of the current edit window.

\section{STEPS}

1. Place the cursor at the desired location in the text.

2. From the Linkage Rules Editor or the Event Tree Logic Editor menu select Lists.

3. Select the Systems menu option. The System List dialog will be displayed containing all of the fault trees in the current family's database. 


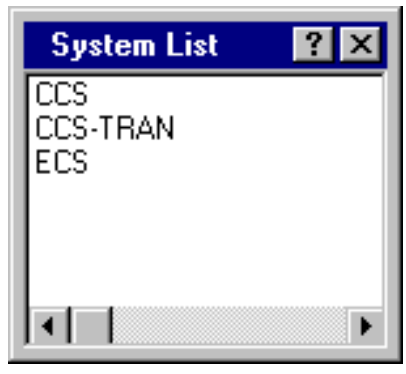

4. Double-click on the desired fault tree name in the list. The selected name will be entered at the current cursor location.

The System List dialog will remain open in the application window during the current session unless explicitly closed.

\subsection{End States}

\section{PURPOSE}

This option allows you to insert an end state name into the text of the current edit window.

\section{STEPS}

1. Place the cursor at the desired location in the text.

2. From the Linkage Rules Editor or the Event Tree Logic Editor menu select Lists.

3. Select the End States menu option. The Endstate List dialog will be displayed containing all of the end states in the current family's database.

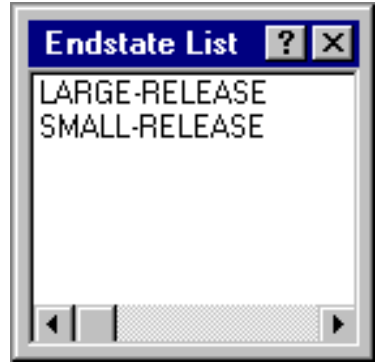

4. Double-click on the desired end state name in the list. The selected name will be entered at the current cursor location.

The Endstate List dialog will remain open in the application window during the current session unless explicitly closed.

\subsection{Event Trees}




\section{PURPOSE}

This option allows you to insert an event tree name into the text of the current edit window.

\section{STEPS}

1. Place the cursor at the desired location in the text.

2. From the Linkage Rules Editor or the Event Tree Logic Editor menu select Lists.

3. Select the Event Trees menu option. The Event Tree List dialog will be displayed containing all of the event trees in the current family's database.

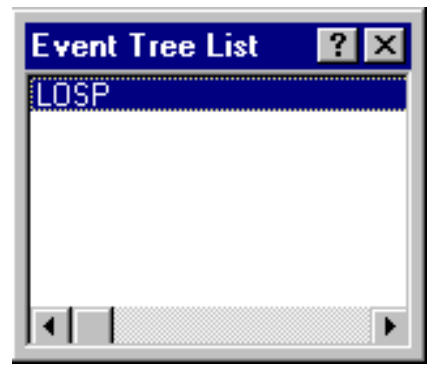

4. Double-click on the desired event tree name in the list. The selected name will be entered at the current cursor location.

The Event Tree List dialog will remain open in the application window during the current session unless explicitly closed.

\subsubsection{Editing Sequence Cut Sets}

\subsubsection{Editing Cut Sets}

\section{PURPOSE}

The Cut Set Editor provides you with the means to edit the fault tree (or sequence or end state) cut sets. SAPHIRE provides space for two sets of cut sets in the database: base case and current case. Whenever SAPHIRE generates cut sets, they are stored in the current case location. The user may save a set of cut sets to the base case location by performing a Base Case Update. The user can choose to edit either base case or current cut sets; however, the results of the editing are always stored in the current case location.

Using the cut set editor, you can insert new cut sets, delete cut sets, or add or modify basic events in the cut sets. Any event name entered during cut set editing may be preceded by a "/" to indicate that it is to be treated as a complemented event. The probability of a complemented event is one minus the failure probability. 


\section{STEPS}

1. From the SAPHIRE menu select Fault Tree/Sequence/End State. The Fault Trees List/Sequences/End State List dialog will be displayed.

2. Highlight the desired record(s) and right-click to invoke the pop-up menu.

3. Choose Cut Sets, then the Edit sub-menu option.

4. Choose either Current or Base from the sub-menu. The Cut Set Editor dialog will be displayed.

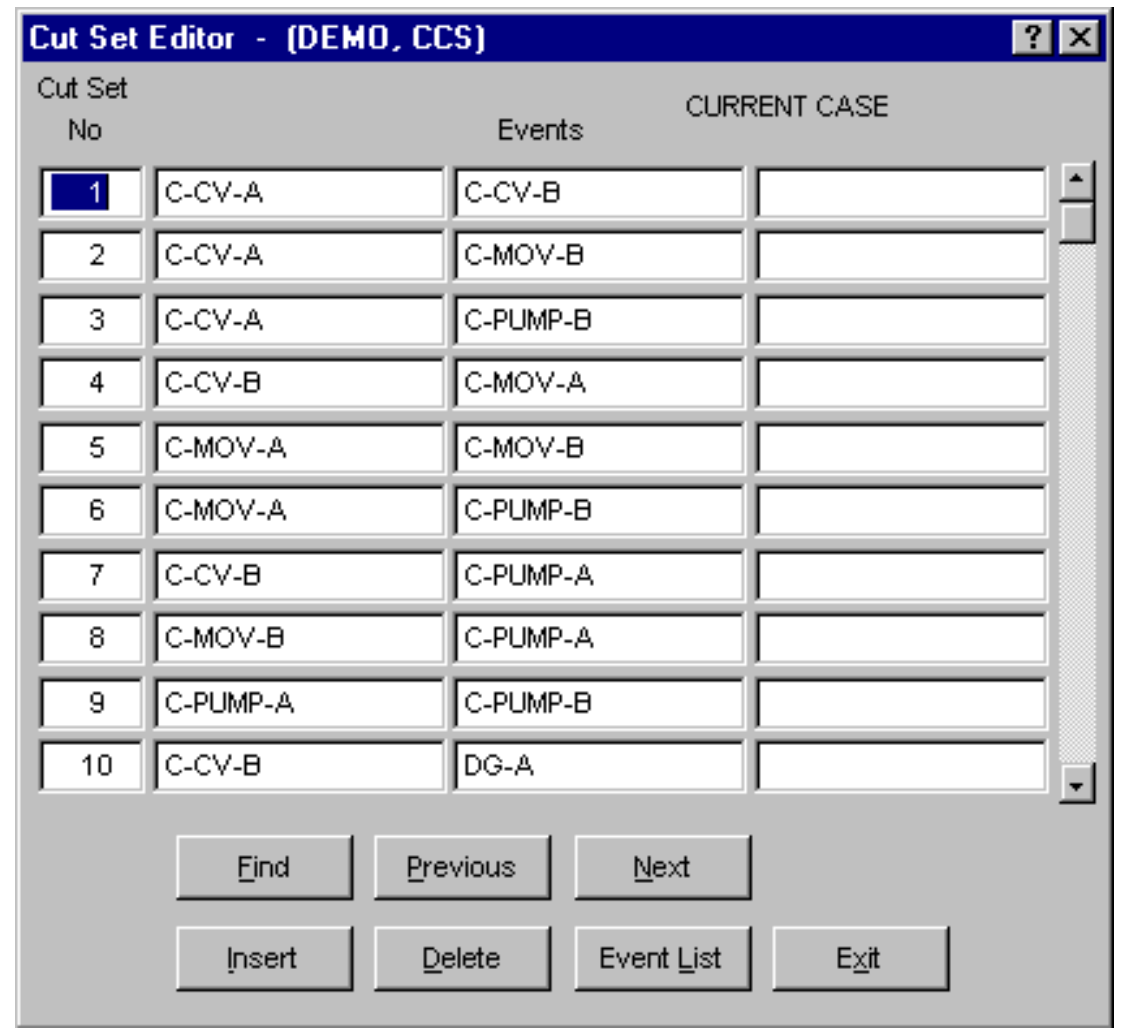

Find - $\quad$ Locate the cut set(s) containing the marked basic event(s).

Previous - $\quad$ Locate the previous cut set containing the event(s) matching the criteria of most recent "Find" operation.

Next - $\quad$ Locate the next cut set containing the event(s) matching the criteria of most recent "Find" operation.

Insert - $\quad$ Add a new cut set or basic event.

Delete - Delete an existing cut set or basic event.

Event List - List the basic events in the data base.

Exit - $\quad$ Close the Cut Set Editor dialog. The Cut Set Editor message box is displayed with three options:

Yes - Close the dialog and save changes to the cut sets.

No - Close the dialog without saving changes to the cut sets.

Cancel - Do not close the dialog. Changes are not saved. 


\subsubsection{Find}

\section{PURPOSE}

This option locates the cut set(s) containing the selected criteria.

\section{STEPS}

1. From the SAPHIRE menu select Fault Tree/Sequence/End State. The Fault Trees List/Sequences/End State List dialog will be displayed.

2 Highlight the desired record(s) and right-click to invoke the pop-up menu.

3. Choose Cut Sets, then the Edit sub-menu option.

4. Choose either Current or Base from the sub-menu. The Cut Set Editor dialog will be displayed.

5. Choose the Find button. The Event List dialog will be displayed.

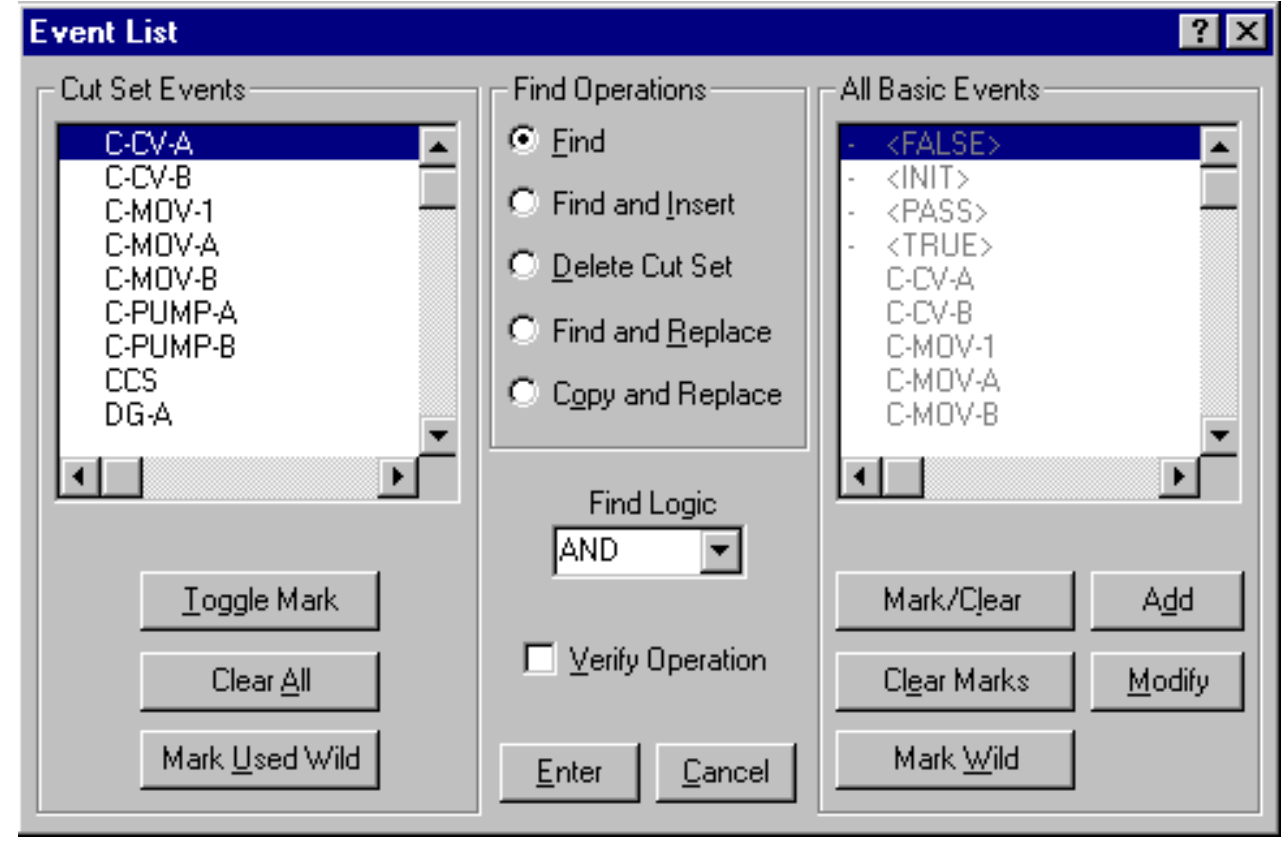

The Event List dialog consists of four sections. The functions that apply to each section are discussed briefly:

\section{Cut Set Events}

Basic Event List - Contains all basic events used in the cut sets being edited. 
Toggle Mark -

Clear All Mark Used Wild -

Find Operations

Find -

Find and Insert -

Delete Cut Set -

Find and Replace -

Copy and Replace -
Marks or unmarks selected events in the Cut Set Events list. An asterisk

(*) next to the event name indicates that it is currently marked. A slash

(/) indicates that the event is marked as a complimented event.

Removes all marks from the events in the Cut Set Events list. Marks events in the Cut Set Events list based on matching criteria. The Event Class Mask dialog is displayed.

Locate the cut set(s) containing the marked event(s) in the Cut Set Events list using the selected Find Logic.

Locate the cut sets(s) containing the marked event(s) in the Cut Set Events list using the selected Find Logic and insert the marked event(s) from the All Basic Events list. The new events are inserted into the cut set after the "find" event(s).

Remove the cut set(s) containing the marked event(s) in the Cut Set Events list using the selected Find Logic.

Locate the cut sets(s) containing the marked event(s) in the Cut Set Events list using the selected Find Logic and replace them with the marked event(s) from the All Basic Events list.

Copy the cut sets(s) containing the marked event(s) in the Cut Set Events list using the selected Find Logic and replace the event(s) with the marked event(s) from the All Basic Events list. This creates a new cut set for each cut sets matching the "find" criteria.

All Basic Events - This section is enabled for use with the Find and Insert, Find and Replace, and Copy and Replace functions:

Basic Event List Mark/Clear Clear Marks Mark Wild -

Add -

Modify -
Contains all basic events in the database.

Marks or unmarks selected events in the All Basic Events list. Remove all marks from the events in the All Basic Events list. Marks events in the All Basic Events list based on matching criteria. The Event Class Mask dialog is displayed.

Add a new basic event to the database. This new event will appear in the All Basic Events list.

Modify the selected event in the All Basic Events list.

Other options:

Find Logic drop-down list - Allows selection between the logical AND and OR operations. The selected logical operation is applied to the events in the Cut Set Events.

Verify Option -

Enter -

Cancel -
If this check box is selected, you are prompted to confirm the operation for each successful find. If this check box is deselected, the operation will be performed immediately.

Perform the "find" operation using the currently selected criteria. Do not perform the "find" operation and close the Event List dialog.

\subsubsection{Insert}




\section{PURPOSE}

This option allows you to insert a new cut set or basic event. If the I-bar is in a Cut Set No cell (i.e., the first column of cells on the Cut Set Editor dialog), then a row for a cut set will be added at the end of the cut set list. If the I-bar is in one of the Events cells, and the last event row for the current cut set (i.e., all three cells in the current cut set row) is filled, then a row for the current cut set will be added.

\section{STEPS}

1. From the SAPHIRE menu select Fault Tree/Sequence/End State. The Fault Trees List/Sequences/End State List dialog will be displayed.

2. Highlight the desired record(s) and right-click to invoke the pop-up menu.

3. Choose Cut Sets, then the Edit sub-menu option.

4. Choose either Current or Base from the sub-menu. The Cut Set Editor dialog will be displayed.

5. Place the cursor in the desired cell and choose the Insert button.

\subsubsection{Delete}

\section{PURPOSE}

This option allows you to delete an existing cut set or basic event. If the I-bar is in a Cut Set No cell (i.e., the first column of cells on the Cut Set Editor dialog), then the entire cut set will be deleted. If the I-bar is in one of the Events cells, then the event in that cell will be removed from the cut set.

\section{STEPS}

1. From the SAPHIRE menu select Fault Tree/Sequence/End State. The Fault Trees List/Sequences/End State List dialog will be displayed.

2. Highlight the desired record(s) and right-click to invoke the pop-up menu.

3. Choose Cut Sets, then the Edit sub-menu option.

4. Choose either Current or Base from the sub-menu. The Cut Set Editor dialog will be displayed.

5. Place the cursor in the desired cell and choose the Delete button.

If you are deleting a cut set, you will be prompted:

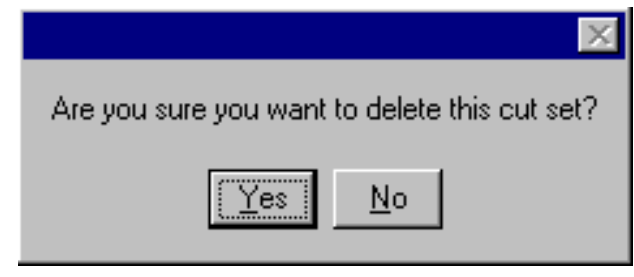

Yes - Continue the delete operation.

No - Cancel the delete operation.

\subsubsection{Event List}




\section{PURPOSE}

This option lists all the basic events in the database. From this dialog you can add a new event to the database, modify an existing event's data, or select the event to be included in the current cut set. The cursor must be positioned in one of the Events cells in order to activate this option. The selected event will replace an existing event in the current cell.

\section{STEPS}

1. From the SAPHIRE menu select Fault Tree/Sequence/End State. The Fault Trees List/Sequences/End State List dialog will be displayed.

2. Highlight the desired record(s) and right-click to invoke the pop-up menu.

3. Choose Cut Sets, then the Edit sub-menu option.

4. Choose either Current or Base from the sub-menu. The Cut Set Editor dialog will be displayed.

5. Place the cursor in the desired Event cell.

6. $\quad$ Choose the Event List button. The Event List dialog will be displayed.

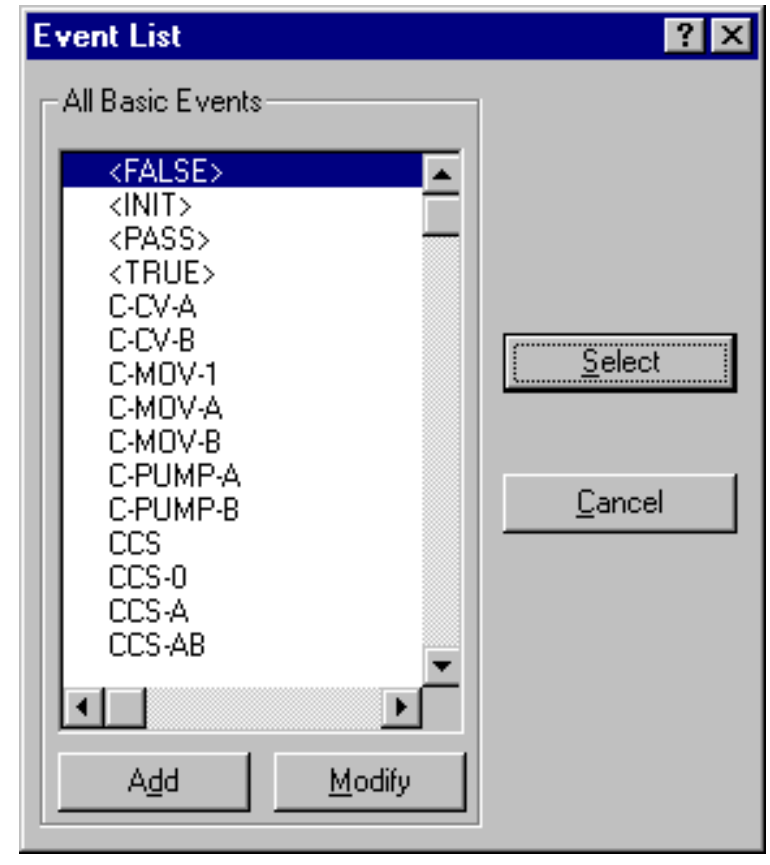

Add - $\quad$ Add basic event to the data base. The Basic Event data entry dialog will be displayed.

Modify - $\quad$ Modify the data of the highlighted basic event. The Basic Event data entry dialog will be displayed.

Select - $\quad$ Include the highlighted basic event in the cut set. The selected event will be placed in the cell currently occupied by the cursor.

Cancel - $\quad$ Close the Event List dialog and do not change the current cell. 


\subsection{Displaying Sequence Results}

\subsubsection{Displaying Sequence Results}

\section{PURPOSE}

This option displays the results of sequence analysis functions. You can view the current case cut sets and uncertainty analysis results, and perform importance measure analysis.

\section{STEPS}

1. From the SAPHIRE menu select Sequence. The Sequences dialog will be displayed.

2. Highlight the desired sequence and right-click to invoke the pop-up menu.

3. Choose Display, then the desired sub-menu option.

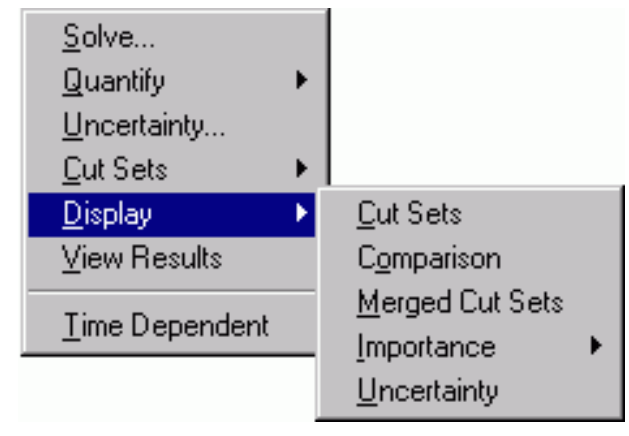

Cut Sets - $\quad$ Display the sequences' cut sets, their percent of contribution to the

Comparison - Display the sequences' base and current cut sets, their percent of contribution to the sequence, the frequency, and the event names that make up the cut sets.

Merged Cut Sets - $\quad$ Display cut sets from a level 2 sequence merged with its corresponding Perform and display "reliability-worth" information about the basic events in the cut sets.

Uncertainty - $\quad$ Display uncertainty analysis results.

\subsubsection{Displaying Cut Sets}

\subsubsection{Displaying Cut Sets}

\section{PURPOSE}

This option displays the current case cut sets. Three tabbed pages provide different views of the cut sets. The first tab, labeled Full List, contains the complete list of cut sets for the selected fault tree (or sequences or end states). This page always contains the complete set of cut sets found for the selected fault tree/sequences/end state. 
When the dialog is invoked, the fault tree (or sequence or end state) cut sets are shown. Displayed with each cut set is its associated percent of contribution to the minimal cut set upper bound, its probability (or, perhaps, frequency), and the event names that make up the cut sets. Also displayed are the minimal cut set upper bound for the total, the number of cut sets that make up the total, the current slice minimal cut set upper bound, the percentage that the slice contributes to the total, and the number of cut sets in the slice.

The Included In List and Excluded From List tabs will not contain data until a slice has been defined, via one of the Slice By options.

The second tab, labeled Included In Slice, contains those cut sets specified (qualified) by the user via either the Remove and/or Slice By options. The third tab, labeled Excluded From Slice, contains the inverse of the second tab; it contains those cut sets not included in the qualified cut set list. The summary information at the top of each page shows the summary information for just the cut sets shown on the current page, and also in relation to the full list of cut sets.

\section{STEPS}

1. From the SAPHIRE menu select Fault Tree/Sequence/End State. The Fault Trees List/Sequences/End State List dialog will be displayed.

2. Highlight the desired list item(s) and right-click to invoke the pop-up menu.

3. Choose Display, then the Cut Sets sub-menu option. The Selected Cut Sets dialog will be displayed.

Full List | Included In Slice | Excluded From Slice |

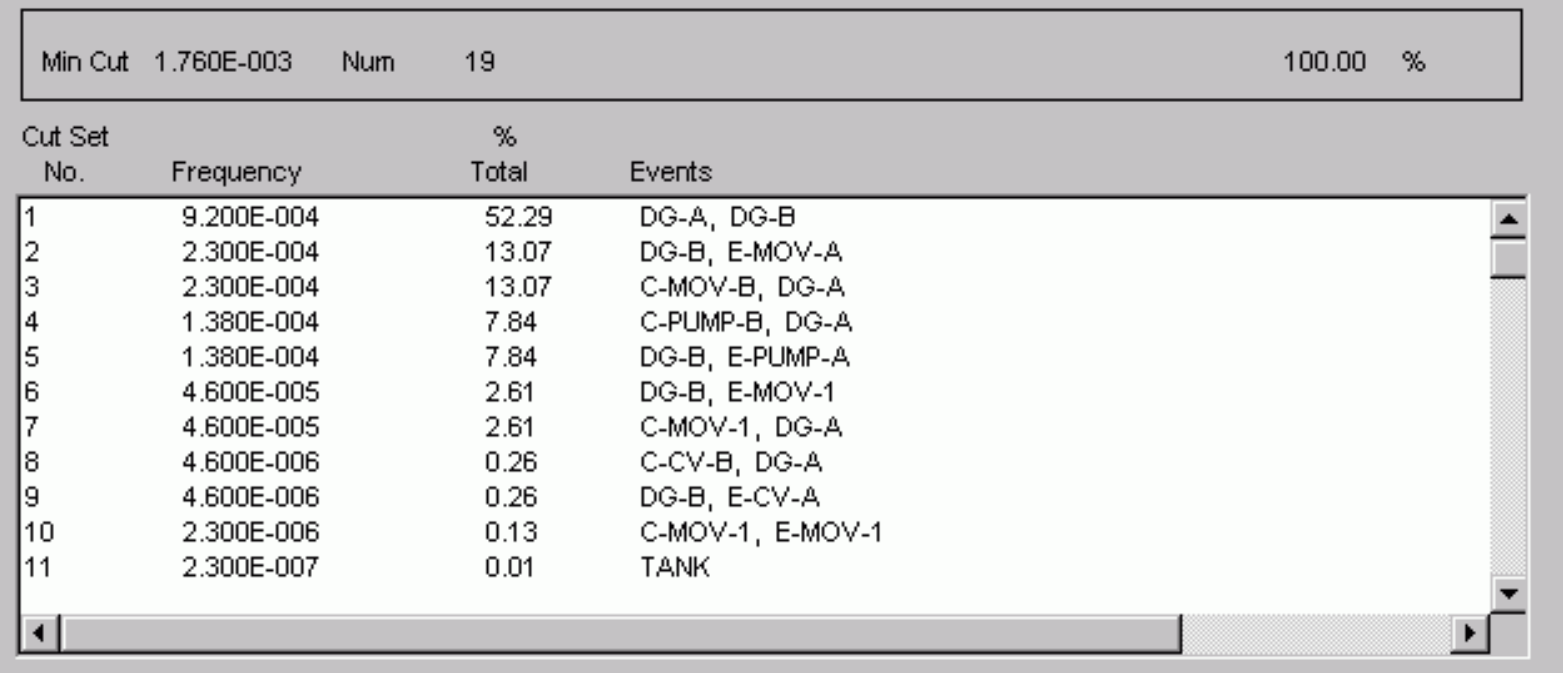

Slice By
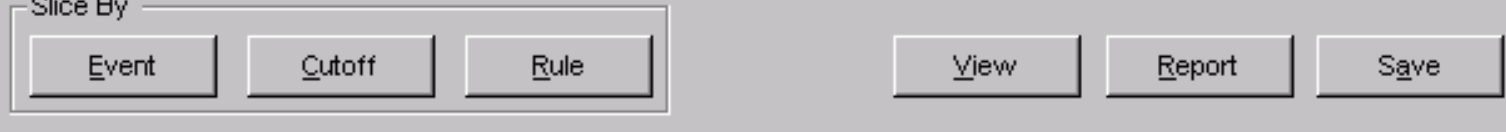
Tabbed pages:

Full List -

Display the complete (unsliced) list of cut sets.

Included in Slice - Display all currently qualified cut sets.

Excluded From Slice - Display all currently unqualified cut sets.

Pop-up menu (only) functions:

Path -

Remove -

Reset Explicit -
Display the logic that generated the highlighted cut set.

Remove the highlighted cut set(s) from the current list, and place them into the Excluded From Slice list.

Restore the cut set(s) that were deleted, via the Remove option, to the current list.

Slice By

Event -

Define the event criteria upon which cut sets will be qualified.

Cutoff -

Define the cut off criteria upon which cut sets will be qualified.

Rule -

Define the rule-based criteria upon which cut sets will be qualified.

View -

Display information about the selected cut set including basic event data.

Report -

Generate a report of the cut sets that are displayed in the currently selected tab.

Save -

Copy the cut sets from the currently selected tab to an end state.

Close Close the Selected Cut Sets dialog.

\subsubsection{Path Search}

\section{PURPOSE}

This option displays the representation of the logic that generated the selected cut set. The representation is in the form of a hierarchical tree, where each gate can be expanded or condensed by clicking on the gate. Only the failure path is traced here.

\section{STEPS}

1. From the SAPHIRE menu select Fault Tree/Sequence/End State. The Fault Tree List/Sequences/End State List dialog will be displayed.

2. Highlight the desired list item and right-click to invoke the pop-up menu.

3. Choose Display | Cut Sets. The Selected Cut Sets dialog will be displayed.

4. Highlight the desired cut set and right-click to invoke the pop-up menu.

5. Choose the Path option. The Selected Cut Set Events dialog will be displayed.

See the topic, "About Logic Dialogs," for a description of the icons presented on this dialog. 


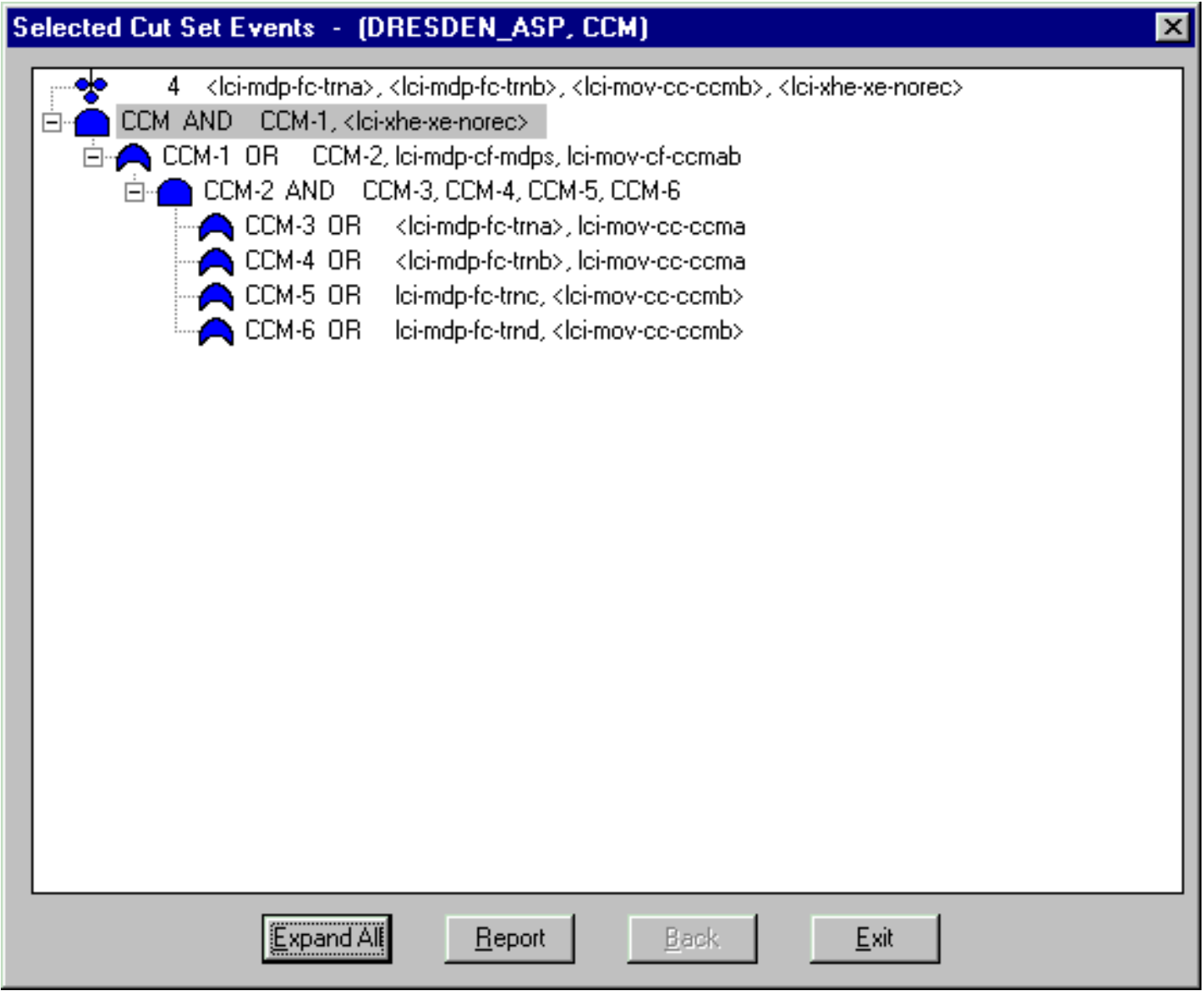

Expand All - Expand all gates.

Report - $\quad$ Generate a report representing the expanded hierarchical tree. The output destination for the report must be specified.

Back - $\quad$ Return to the referenced gate. Active only after a referenced gate has been traced to the location where it was originally defined (i.e., the reference gate icon has been clicked).

Exit - $\quad$ Close the Selected Cut Set Events dialog.

\subsubsection{Slice Options}

\subsection{Slicing Cut Sets}

\section{PURPOSE}

The Selected Cut Sets dialog displays the current case cut sets for the selected fault tree/sequences/end state. The cut sets can be viewed as a whole, or "sliced" into two lists, according to a user defined set of criteria. The three tabbed pages on the dialog present different views of the cut sets:

The first tab, labeled Full List, contains the complete list of cut sets for the selected fault tree (or sequences or end states). This page always contains the complete set of cut sets found for the selected fault tree/sequences/end state. 
The second tab, labeled Included In Slice, contains those cut sets specified (qualified) by the user via either the Remove and/or Slice By options. This list will be empty until the user qualifies the data.

The third tab, labeled Excluded From Slice, contains the inverse of the second tab; it contains those cut sets not included in the qualified cut set list. This list will also be empty until the user qualifies the data.

A user slices (qualifies) cut sets according to one of several methods: Slice By Event, Slice By Cutoff, and/or Slice By Rule.

Note - To clear the qualification criteria (i.e., clear the Included in List and Excluded From List), choose the Slice By Event | Restart option.

\section{STEPS}

1. From the SAPHIRE menu select Fault Tree/Sequence/End State. The Fault Tree List/Sequences/End State List dialog will be displayed.

2. Highlight the desired list item and right-click to invoke the pop-up menu.

3. Choose Display | Cut Sets. The Selected Cut Sets dialog will be displayed.

4. Choose one of the Slice By buttons. Define the qualification criteria using the selected method.

\subsection{Slice By Event}

\subsection{Slice By Event}

\section{PURPOSE}

This option provides the ability to divide the original cut set list into two subsets (slices) - qualified and unqualified cut sets. This is accomplished by defining a set of events used to evaluate whether or not a cut set is qualified. This qualification criteria can be used to help the analyst determine the affect of specified events on the minimal cut set upper bound.

\section{STEPS}

1. From the SAPHIRE menu select Fault Tree/Sequence/End State. The Fault Tree List/Sequences/End State List dialog will be displayed.

2. Highlight the desired list item and right-click to invoke the pop-up menu.

3. Choose Display | Cut Sets. The Selected Cut Sets dialog will be displayed.

4. Choose the Slice By Event button. The Event List dialog will be displayed. 


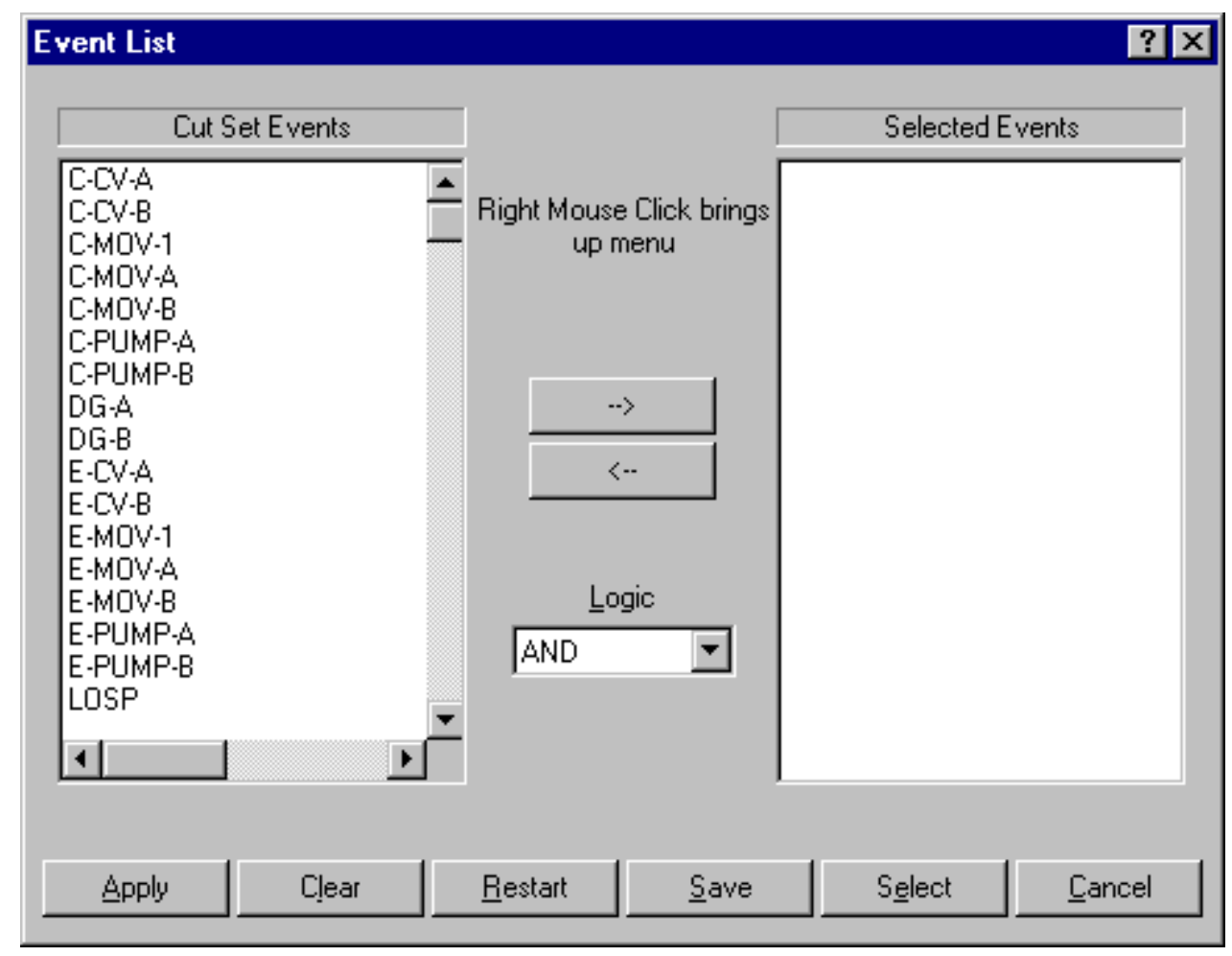

The Event List dialog is divided into three sections:

Cut Set Events list - Contains all the basic events included in the cut set(s) for the currently selected fault tree, sequence(s) or end state(s).

Selected Events list - Contains all basic events that define the qualification criteria. This list is initially empty.

Logic drop-down list - Allows selection between the logical "AND" and "OR" operations. The selected logical operation is applied to the events in the Selected Events list to determine which cut sets are qualified.

The various functions available from this dialog are accessed three different ways. When an event in the Cut Set Events list is highlighted, the following options are available from the pop-up menu. The highlighted event(s) will be added to the Selected Events list.

Wild Card Mark -

Add Event -

Add Failure Event Add Success Event Add NOT Event In -

Apply Selected -
Use the event class mask to highlight events in the Cut Set Events list.

Cut sets containing this event, in any state (either failed or success), will be qualified.

Cut sets containing the failure $(+)$ of this event will be qualified. Cut sets containing the success (/) of this event will be qualified. Cut sets that do not contain $(\sim)$ this event, in any state (either failed or success), will be qualified.

Evaluate the qualification criteria. This is the same process as if the Apply button was chosen. 
When an event in the Selected Events list is highlighted, the following options are available from the pop-up menu. The qualification criteria state will be changed for the highlighted event(s):
Remove Event -
Delete this event from the Selected Events list. This event will no
Event (either / or + ) - longer be used in the qualification criteria.
Failed Event - The state of
is irrelevant.
Success Event - Change the state of the highlighted event to failed $(+)$.
Event NOT In - Change the state of the highlighted event to successful (/). The highlighted event are is not contained $(\sim)$ in qualified cut sets.
Apply Selected - $\quad$ Evaluate the qualification criteria. This is the same process as if the Apply button was chosen.

The following functions are performed by choosing the appropriate button:
Apply - Cut sets in the current slice will be evaluated. Those cut sets meeting the qualification criteria will be retained in the current slice of qualified cut sets. Other cut sets not meeting the qualification criteria will be placed in the unqualified list.
Clear - $\quad$ Remove all events from the Selected Events list (i.e., clear the qualification criteria).
Restart - Restore the original cut sets and clear the qualification criteria. The Event List dialog will be closed and the Included In List and Excluded From List pages will be cleared.
Save - $\quad$ Save the selected events in a stored slice record.
Select - Choose a saved slice record and place the events in the Selected Events list.
Cancel - Close the Event List dialog without applying the qualification criteria.

\subsection{Wild Card Mark}

\section{PURPOSE}

This option uses the event class mask to highlight events in the Cut Set Events list. This selection is used with either the Cut Set Editor or the Cut Set | Slice option.

\section{STEPS}

1. From the SAPHIRE menu select Fault Tree/Sequence/End State. The Fault Tree List/Sequences/End State List dialog will be displayed.

2. Highlight the desired list item and right-click to invoke the pop-up menu.

To access from the Slice\#1

1. Choose Display | Cut Sets. The Selected Cut Sets dialog will be displayed.

2. Choose the Slice button. The Event List dialog will be displayed.

3. Right-click to invoke the pop-up menu and choose the Wild Card Mark option.

To access from the Cut Set Editor\#1

1. Choose Cut Sets | Edit | Current or Base. The Cut Set Editor dialog will be displayed. 
2. Choose the Find button. The Event List dialog will be displayed.

3. Choose the Mark Used Wild button.

4. The Event Class Mask dialog will be displayed.

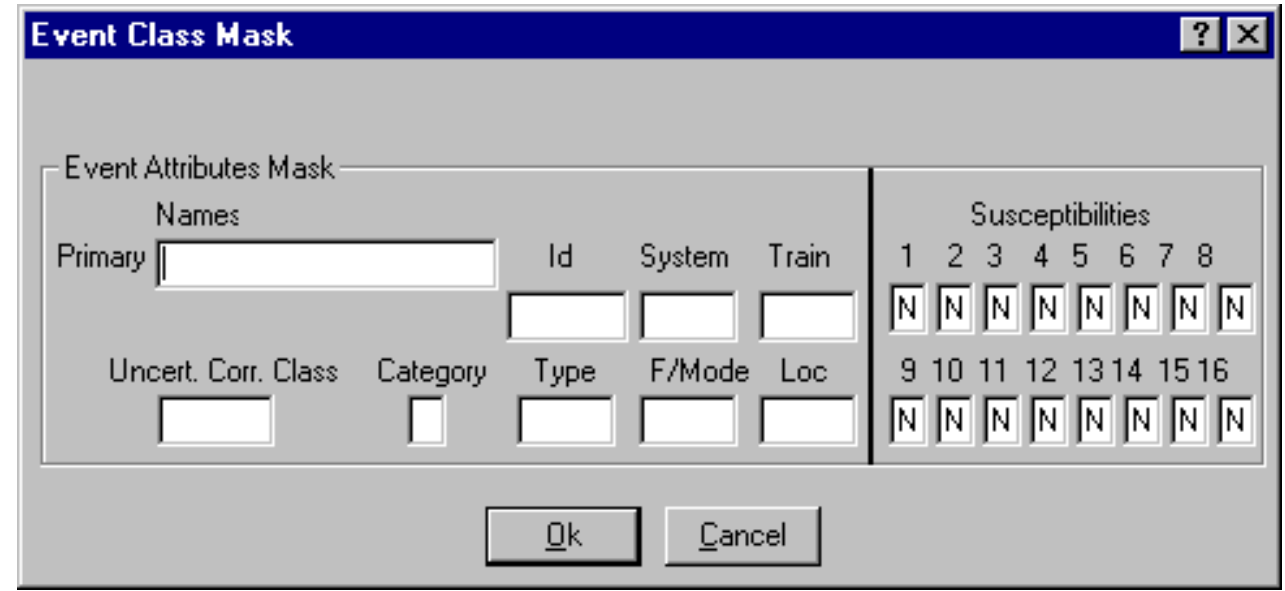

Primary -

Id -

System -

Train -

Uncert. Corr. Class -

Category -

Type -

F/Mode -

Location -

Susceptibilities -
Select all basic events with matching primary names (maximum 24 characters). Wildcard characters may be used.

Select all basic events with matching component identifiers.

Select all basic event with matching systems.

Select all basic event with matching trains

Select all basic event with matching uncertainty correlation classes.

Select all basic event with matching categories.

Select all basic event with matching types.

Select all basic event with matching failure modes.

Select all basic event with matching locations.

Select all basic event with matching susceptibility attributes set.

\subsection{Save Slice}

\section{PURPOSE}

This option allows you to save the basic events currently displayed in the Selected Events list in a stored slice record or a set.

\section{STEPS}

1. From the SAPHIRE menu select Fault Tree/Sequence/End State. The Fault Tree List/Sequences/End State List dialog will be displayed.

2. Highlight the desired list item and right-click to invoke the pop-up menu.

3. Choose Display | Cut Sets. The Selected Cut Sets dialog will be displayed. 
4. Choose the Slice button. The Event List dialog will be displayed.

5. Select the desired events to be saved in the slice.

6. Choose the Save button. The Save Slice dialog will be displayed.

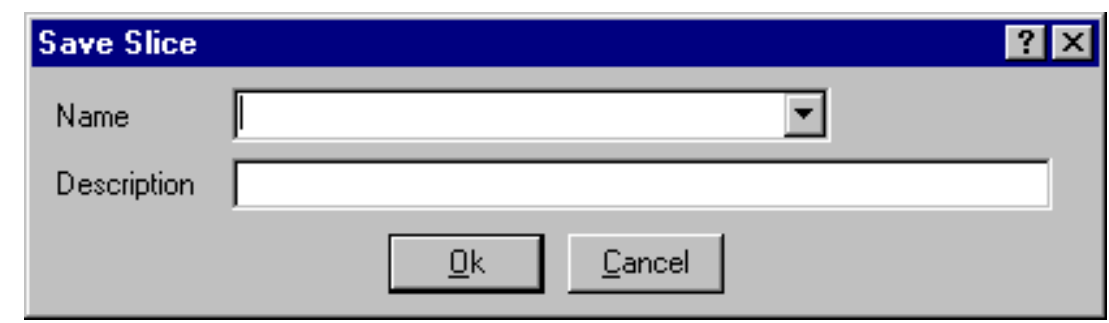

Ok - Save the new slice record and close the dialog.

Cancel - Close the dialog without saving.

\subsection{Select Slice}

\section{PURPOSE}

Choose a saved slice record and place the events in the Selected Events list. When a slice record is loaded, the basic events saved in the slice replace all basic events currently displayed in the Selected Events list. If a basic event that was saved in a slice does not appear in the Cut Set Events list, it will not be displayed in the Selected Events list, although it will remain in the slice record.

\section{STEPS}

1. From the SAPHIRE menu select Fault Tree/Sequence/End State. The Fault Tree List/Sequences/End State List dialog will be displayed.

2. Highlight the desired list item and right-click to invoke the pop-up menu.

3. Choose Display | Cut Sets. The Selected Cut Sets dialog will be displayed.

4. Choose the Slice button. The Event List dialog will be displayed.

5. Choose the Select button. The Select Slice dialog will be displayed. 


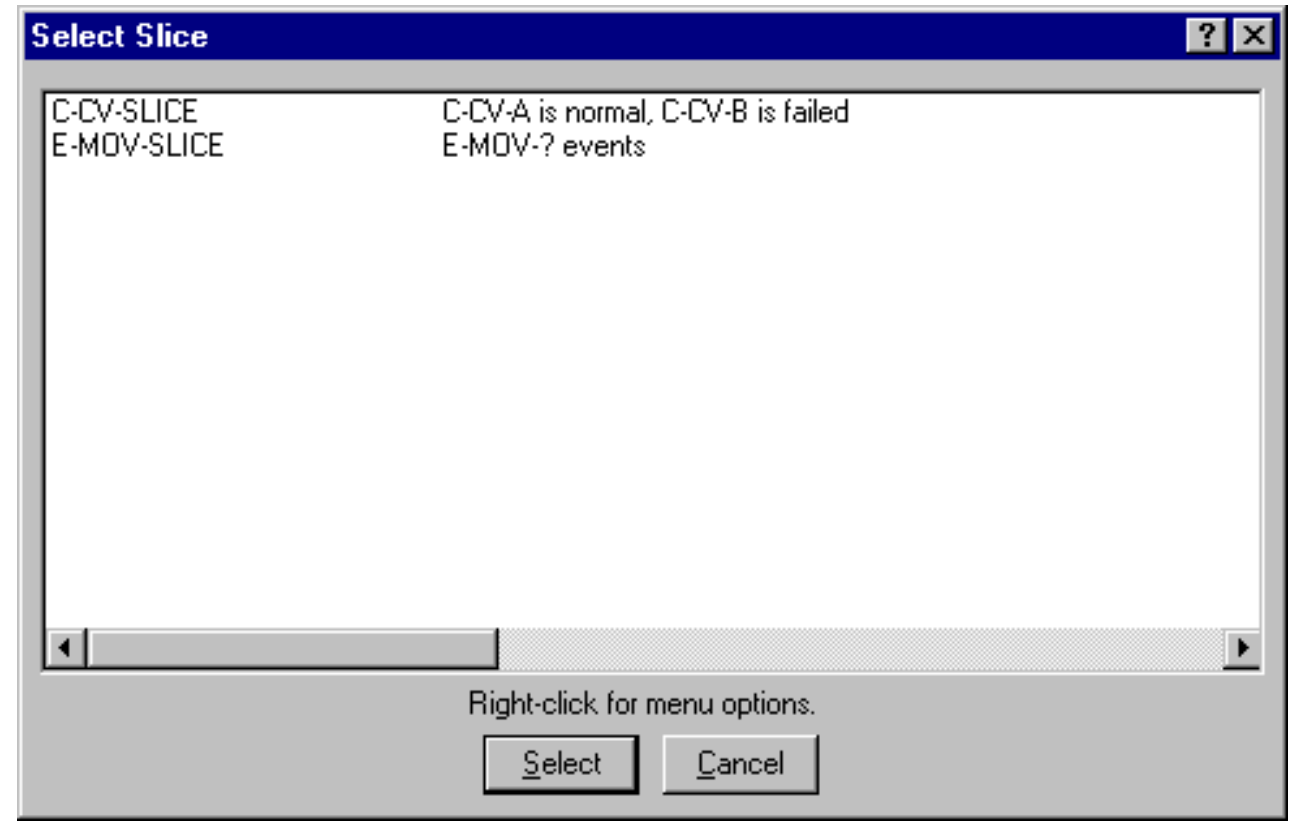

\section{Pop-up Menu Options}

Modify - Change the slice record name and/or description. See Save Slice .

Delete - Delete the slice record from the database.

Exit - Close the Select Slice dialog.

\section{Button Options}

Select - Choose the highlighted slice record and load the saved events.

Cancel - Close the Select Slice dialog.

\subsection{Slice By Cutoff}

\section{PURPOSE}

This option provides the ability to divide the original cut set list into two subsets (slices) - qualified and unqualified cut sets. This is accomplished by defining a cut off value to determine whether or not a cut set is qualified. This qualification criteria can be used to help the analyst determine the affect of specified cut sets on the minimal cut set upper bound.

\section{STEPS}

1. From the SAPHIRE menu select Fault Tree/Sequence/End State. The Fault Tree List/Sequences/End State List dialog will be displayed.

2. Highlight the desired list item and right-click to invoke the pop-up menu.

3. Choose Display | Cut Sets. The Selected Cut Sets dialog will be displayed.

4. $\quad$ Choose the Slice By Cutoff button. The Slice By Value dialog will be displayed. 


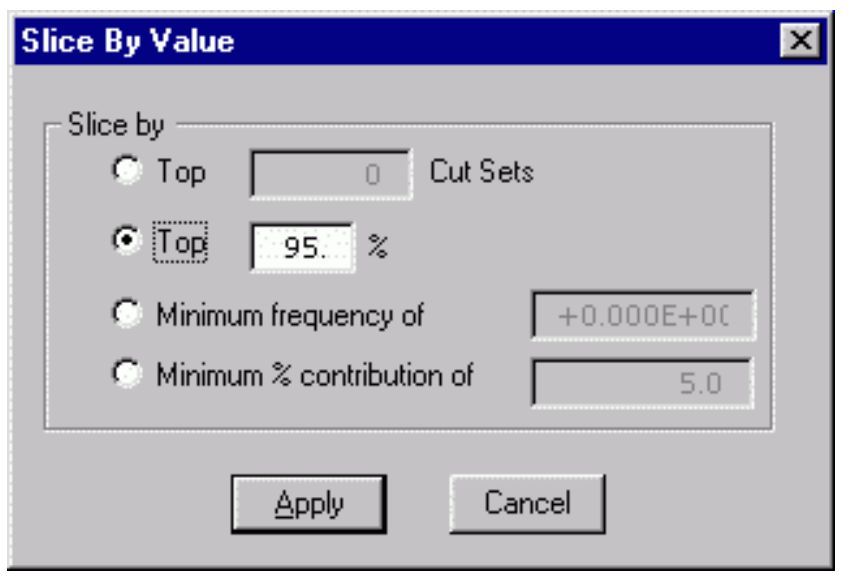

Select one of four types of cutoff value expressions:

Top X Cut Sets -

Top X\% -

Minimum frequency of $\mathbf{X}-$
Display only the top X cut sets in the qualified list.

Display only the cut sets which contribute to the top X\% of the min cut upper bound.

Display only the cut sets which contribute at least a value of $\mathrm{X}$ to the min cut upper bound.

Minimum \% contribution of $\mathbf{X}$ - Display only the cut sets which contribute at least a X\% to the min cut upper bound.

Apply -

Cancel -
Cut sets in the current slice will be evaluated. Those cut sets meeting the qualification criteria will be retained in the current slice of qualified cut sets. Other cut sets not meeting the qualification criteria will be placed in the unqualified list.

Close the Slice By Value dialog without applying the qualification criteria.

\subsection{Slice By Rule}

\subsection{Slice By Rule}

\section{PURPOSE}

This option provides the ability to divide the original cut set list into two subsets (slices) - qualified and unqualified cut sets. This is accomplished by defining one or more rules to evaluate whether or not a cut set is qualified. The rules provide the ability to qualify cut sets using logical expressions, including mixed logic (combining "ands" and "ors") if desired. The rules are saved in the project for repeated use. 
When the dialog is invoked, a list of slice rules is displayed. The highlighted rule is previewed in the bottom text area. To add/modify/delete a slice rule name and description, right click and select the appropriate menu option. To edit a rule, choose the Edit Rule button. To apply a rule to the current cut sets, choose the Apply button.

To clear the qualified and unqualified lists, choose the Reset option from the Slice By Event option, or, apply a different slice option to override the current slice.

\section{STEPS}

1. From the SAPHIRE menu select Fault Tree/Sequence/End State. The Fault Tree List/Sequences/End State List dialog will be displayed.

2. Highlight the desired list item and right-click to invoke the pop-up menu.

3. Choose Display | Cut Sets. The Selected Cut Sets dialog will be displayed.

4. Choose the Slice By Rule button. The Slice Rules dialog will be displayed.

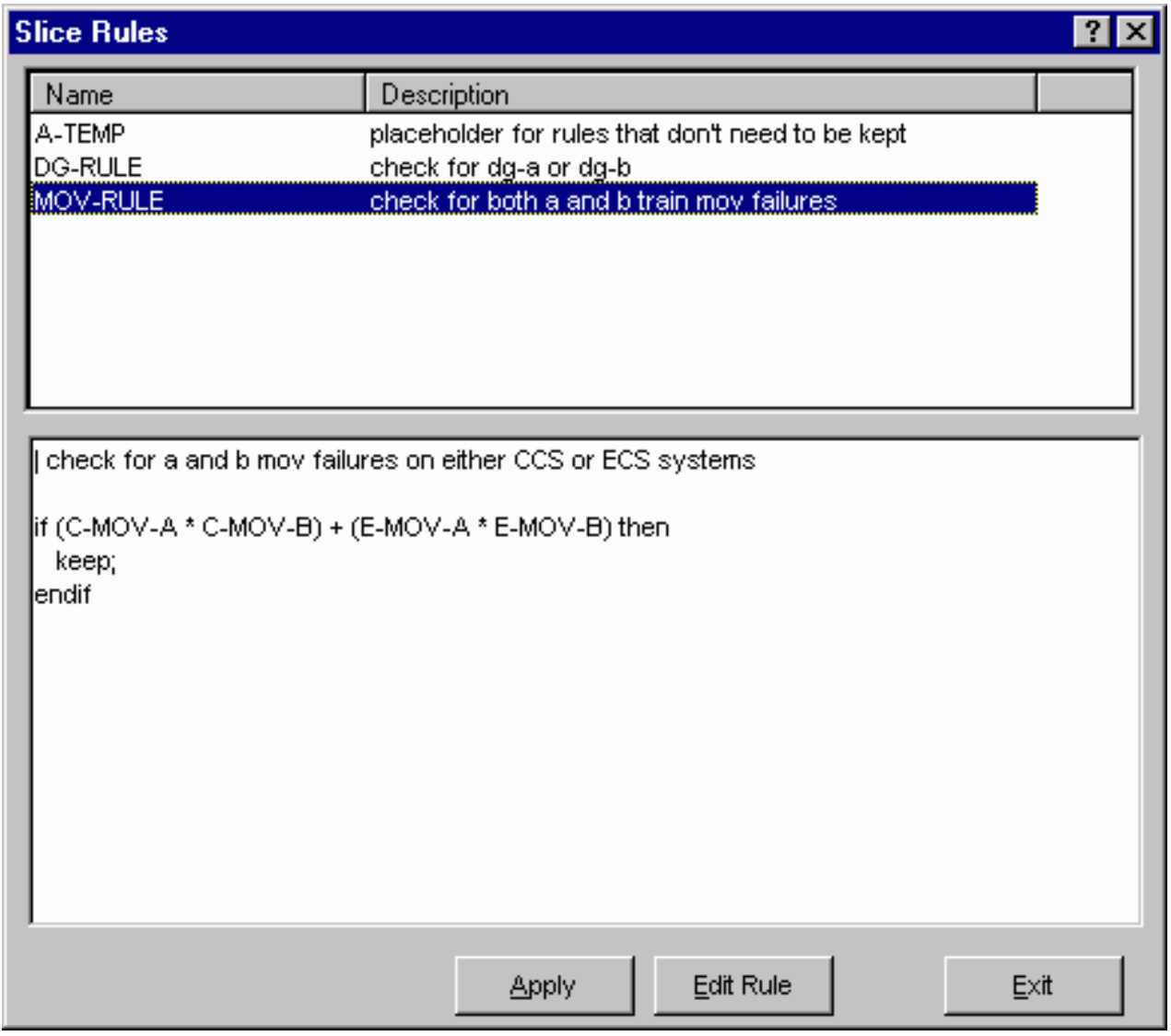




\section{Pop-up Menu Options}

Add - $\quad$ Create a new, empty rule.

Copy - Make a copy of an existing rule. You must supply a unique name before saving the rule.

Modify - Modify the name and/or description of the selected rule.

Delete - Remove the rule from the project.

Apply - The full list of current cut sets will be evaluated. Those cut sets meeting the qualification criteria will be retained in the current slice of qualified cut sets. Other cut sets not meeting the qualification criteria will be placed in the unqualified list.

Edit Rules-Edit the selected rule logic.

Exit - $\quad$ Close the Slice Rules dialog without applying a rule.

\subsection{Slice Rules Editor}

\section{PURPOSE}

This option allows you to create or modify rules that divide cut sets into two subsets - qualified and unqualified. The Slice Rules editor provides a means to develop logic rules that locate and separate cut sets containing desired event combinations.

The slice rules are entered in a free-form text editor within SAPHIRE. The editor options and rule syntax are similar to that of SAPHIRE's recovery rules. However, unlike to recovery rules, slice rules are not tied to the fault tree, sequence(s) or end state(s) whose cut sets are currently displayed. (A slice rule is available to be applied to any type of displayed cut sets.)

Slice rules follow a format similar to the structure that is found in traditional programming languages (e.g., BASIC or PASCAL). As such, the ability exists to define "macros" and "if...then" type of structures. After creating or editing rules, SAPHIRE compiles the rules to check their validity.

Note: An alternative method of entering rules into the project is to export them from SAPHIRE and create or edit them in any word processor or text editor (that can output ASCII files) and then loaded directly into the SAPHIRE database.

\section{STEPS}

1. From the SAPHIRE menu select Fault Tree/Sequence/End State. The Fault Trees List/Sequences/End States dialog will be displayed.

2. Highlight the desired fault tree(s)/sequence(s)/end state(s) and right-click to invoke the pop-up menu.

3. Choose Display | Cut Sets. The Selected Cut Sets dialog will appear.

4. Choose the Slice By Rule button. The Slice Rules dialog will appear.

5. If necessary, add a new rule by right clicking and choosing the Add menu option.

6. Highlight the desired slice rule and choose Edit Rules. If rules have been previously defined they will be displayed in the edit window. 


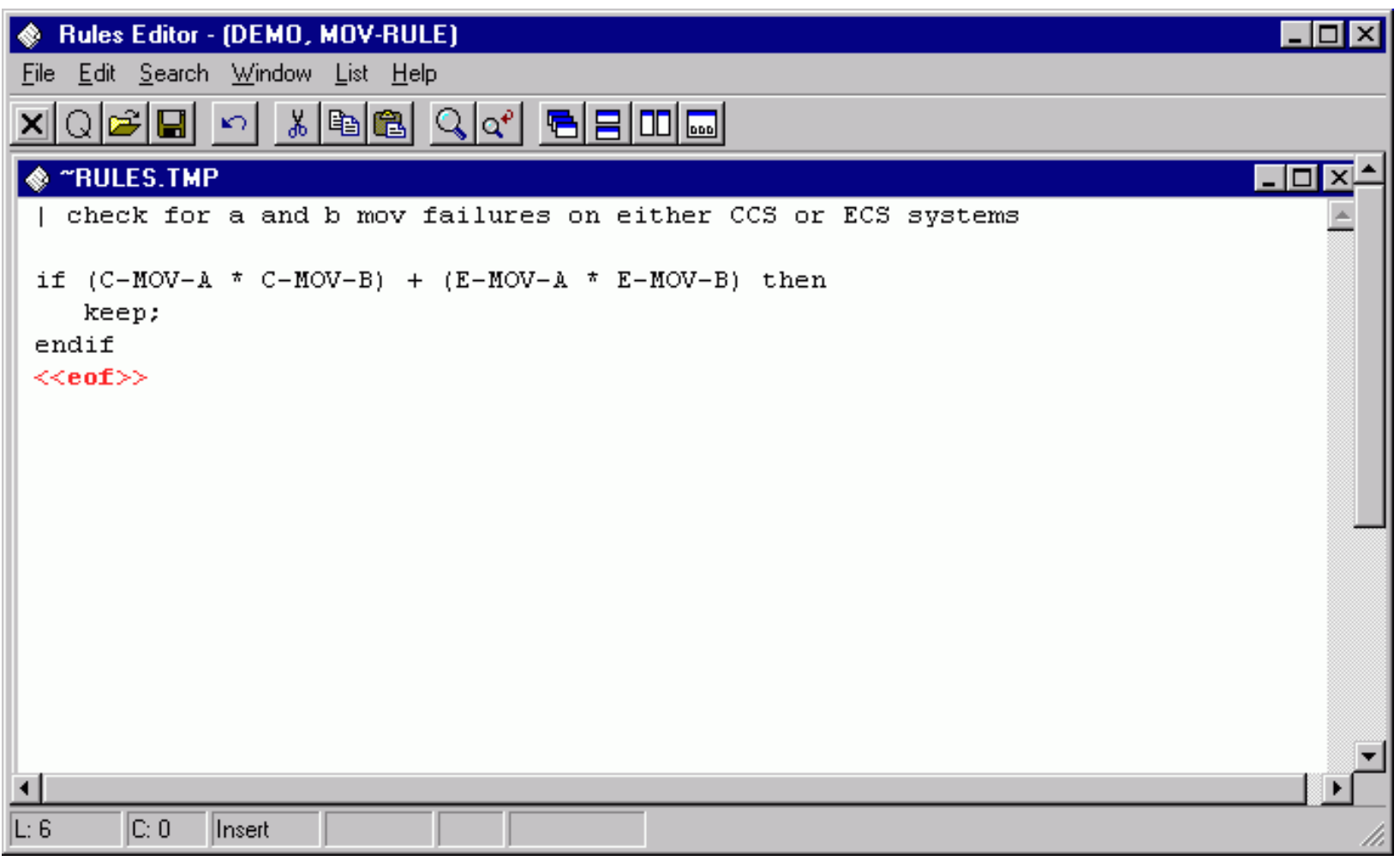

Type the rules in the editor. Note that the rules are not case sensitive.

File -

File functions such as open, save, print, preferences, etc.

Edit -

Search -

Editing functions such as copy and paste along with text format operations.

Window -

Search and cursor positioning operations such as find, replace, goto, etc.

Lists -

Window management functions such as cascade, tile horizontally, etc.

Display lists of macros and events for inserting into the rules. Three different

event lists are available: initiators, a complete list of events, and only those events available in the currently displayed cut sets.

\subsection{Editor File Functions}

These options provide file management functions.

Open - $\quad$ Open a file into a new window. This feature is not usually used while editing SAPHIRE rules.

New - $\quad$ Create a new file in a new window. This feature is not usually used while editing SAPHIRE rules.

Close - $\quad$ Close the edit current window. If changes have been made you will be prompted to save the file.

Quit - $\quad$ Close the current edit window without saving.

Save - $\quad$ Save the file in the current window to disk.

Save As - $\quad$ Save the file in the current window with a new file name.

Save All - $\quad$ Save all the files in all edit windows currently open.

Save Block - Save the currently highlighted text into a new file. The Save As dialog will be invoked. 
Import File - Insert the contents of a file into the current edit window at the current cursor position. The Edit external file dialog will be invoked.

Print - $\quad$ Print the file in the current edit window.

Page setup - $\quad$ Set printer page layout options, .such as pages per sheet and line numbers.

Preferences - $\quad$ Select editing options such as tab width, undo levels, etc.

Exit -

Terminate the Editor session.

\subsection{Editor Edit Functions}

These options provide editing and text formatting functions.
Undo -
Undo the previous editing operation.
Cut -
Remove the highlighted text from the current window and place it in the clipboard.
Copy -
Copy the highlighted text from the current window and place it in the
Paste - clipboard.
Delete -
Copy the clipboard text into the current window at the cursor position.
Shift left indent - in the clipboard.
Shift right indent -
Shift left space -
Shift right space -
Delete to end of line -
Delete line -
Capitalize word -
Downcase word -
Shift the text to the left by the Shift Size specified under Options.
Shift the text to the right by the Shift Size specified under Options.
Shift the text to the left by a single space.
Shift the text to the right by a single space.
Delete to the end of the current line beginning after the current cursor position.
Delete the line on which the cursor is currently positioned.
Convert the entire word under the current cursor position to upper case.
Convert the entire word under the current cursor position to lower case.

\subsection{Editor Search Functions}

These options provide search and cursor positioning operations.

Find -

Replace -

Find Procedure Find/Replace again Find Altered lines Find line number Match symbol -

Goto next error -

Goto previous error Goto Marker -

Set Marker -
Search the text in the current window for the specified string.

Search the text in the current window for a string and replace it with another string.

Search the text in the current window for the specified procedure. Repeat the previous search operation.

Find the lines of text that have changed since the last save operation.

Go to the specified text line number in the current window.

Find the matching symbol pair for the character under the cursor " ()$,\{\}$, [], (**)"

Position the cursor at the next compilation error.

Position the cursor at the previous compilation error.

Position the cursor at the previously saved file position. Up to four marker positions can be retrieved.

Save the current file position for later retrieval.

\subsection{6}

Editor Window Functions 
These options are used for managing the open edit windows within the application window, such as the Linkage Rules Editor or the Event Tree Logic Editor window. Along with the options listed below and segregated by a separation bar, the names of each open file is listed in the menu.

Cascade windows -

Tile windows horizontally -

Tile windows vertically -

Arrange Icons -
Resize and reposition the non-minimized windows in an overlapping fashion so that the title of each window is displayed in a cascade arrangement. The current edit window will be on top.

Resize and reposition the non-minimized windows in a horizontally tiled arrangement.

Resize and reposition the non-minimized windows in a vertically tiled arrangement.

Arrange the minimized window icons at the bottom of the application window.

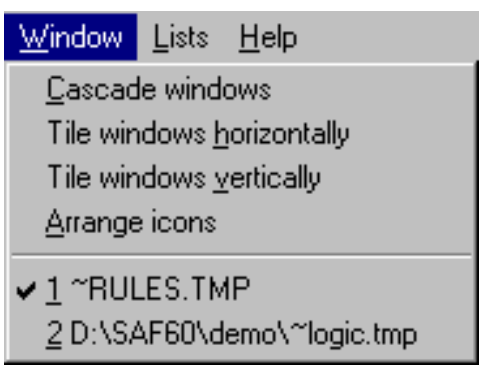

In the example above, the files " $\sim$ RULES.TMP" and " $\sim$ LOGIC.TMP" are open. $\sim$ RULES.TMP is the active window, as indicated by the check mark on the menu.

\subsection{Slice Rules Lists}

Selecting from the lists allows you to insert or replace items in the editor.

Macros - $\quad$ Allows you to insert a macro into the editor.

Events - $\quad$ Select any basic event instead of typing in the basic event name.

Cut Set Events - Select from a list of only those events found in the currently displayed cut sets, instead of typing in the event name.

Initiators - $\quad$ Select an initiating event instead of typing in the initiator name.

\subsection{MACRO}

(Basic Rule syntax)

A macro is a user-definable keyword that specifies a search criteria. The macro name must be all uppercase, must be 24 characters or less, and must not include any of the restricted characters (e.g., a space, *, ?, \,/). The macro line can wrap around to more than one line, but must end with a semicolon.

MACRO-NAME = SEARCH-CRITERIA;

if MACRO-NAME “and optional other search criteria” then 
perform some action on each cut set...;

endif

| Macros are only applicable in the particular rule they are entered into.

\subsubsection{View Option}

\subsection{View Cut Set}

\section{PURPOSE}

This option displays the basic events of the selected cut set with their failure probabilities and descriptions. At the bottom of the Selected Cut Set Events dialog, information about this cut set is displayed: percent of contribution to the total, the cut set frequency, and the number of events that make up this cut set.

\section{STEPS}

1. From the SAPHIRE menu select Fault Tree/Sequence/End State. The Fault Tree List/Sequences/End State List dialog will be displayed.

2. Highlight the desired list item and right-click to invoke the pop-up menu.

3. Choose Display | Cut Sets. The Selected Cut Sets dialog will be displayed. OR

4. Choose Display | Comparison. The Base \& Current Case Cut Sets dialog will be displayed.

5. Highlight the desired cut set.

6. Choose the View button. The Selected Cut Set Events dialog will be displayed. 


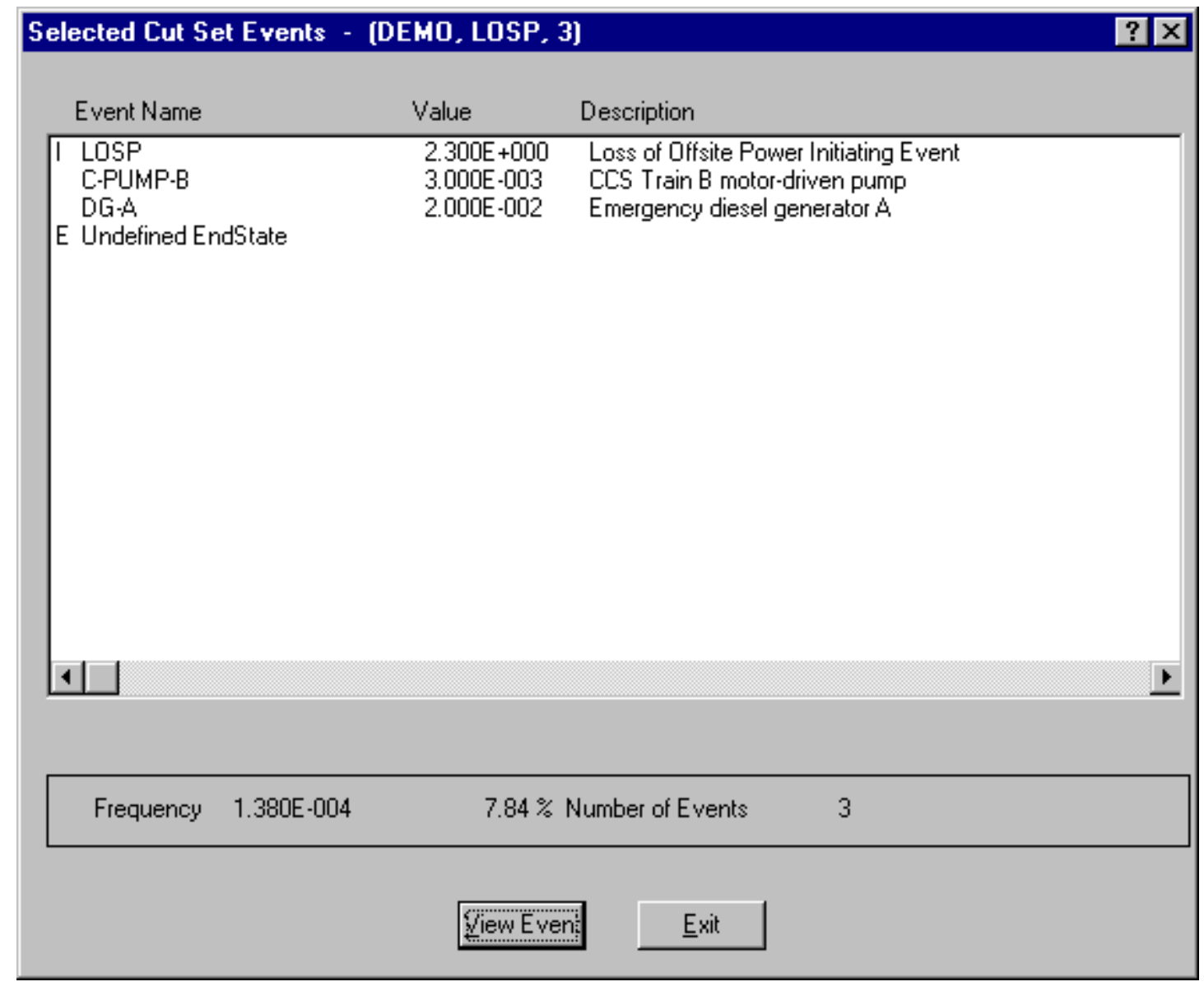

When viewing fault tree cut sets, the end state, if any, is designated with the letter " $E$ " to the left of the Event Name column. It will be the last item listed.

When viewing sequence cut sets, the initiating event is designated with the letter "I" to the left of the Event Name column. It will be the first item listed. The end state will be designated with the letter "E" to the left of the Event Name column and will be the last item listed.

When viewing end state cut sets, the initiating event is designated with the letter "I" to the left of the Event Name column. It will be the first item listed. The originating sequence is designated by the letter "S" to the left of the Event Name column and will be the last item listed.

View Event - Display individual basic event information.

\subsection{View Event}

\section{PURPOSE}

This option provides the following detailed information about the basic events that make up a cut set: event names and description, event attributes, random failure data, uncertainty data, susceptibility flags, and transformation data. 


\section{STEPS}

1. From the SAPHIRE menu select Fault Tree/Sequence/End State. The Fault Tree List/Sequences/End State List dialog will be displayed.

2. Highlight the desired list item and right-click to invoke the pop-up menu.

3. Choose Display | Cut Sets. The Selected Cut Sets dialog will be displayed. OR

4. Choose Display | Comparison. The Base \& Current Case Cut Sets dialog will be displayed.

5. Highlight the desired cut set.

6. Choose the View Cut Set button. The Selected Cut Set Events dialog will be displayed.

7. Highlight the desired basic event.

8. Choose the View Event button. The View Event dialog will be displayed.

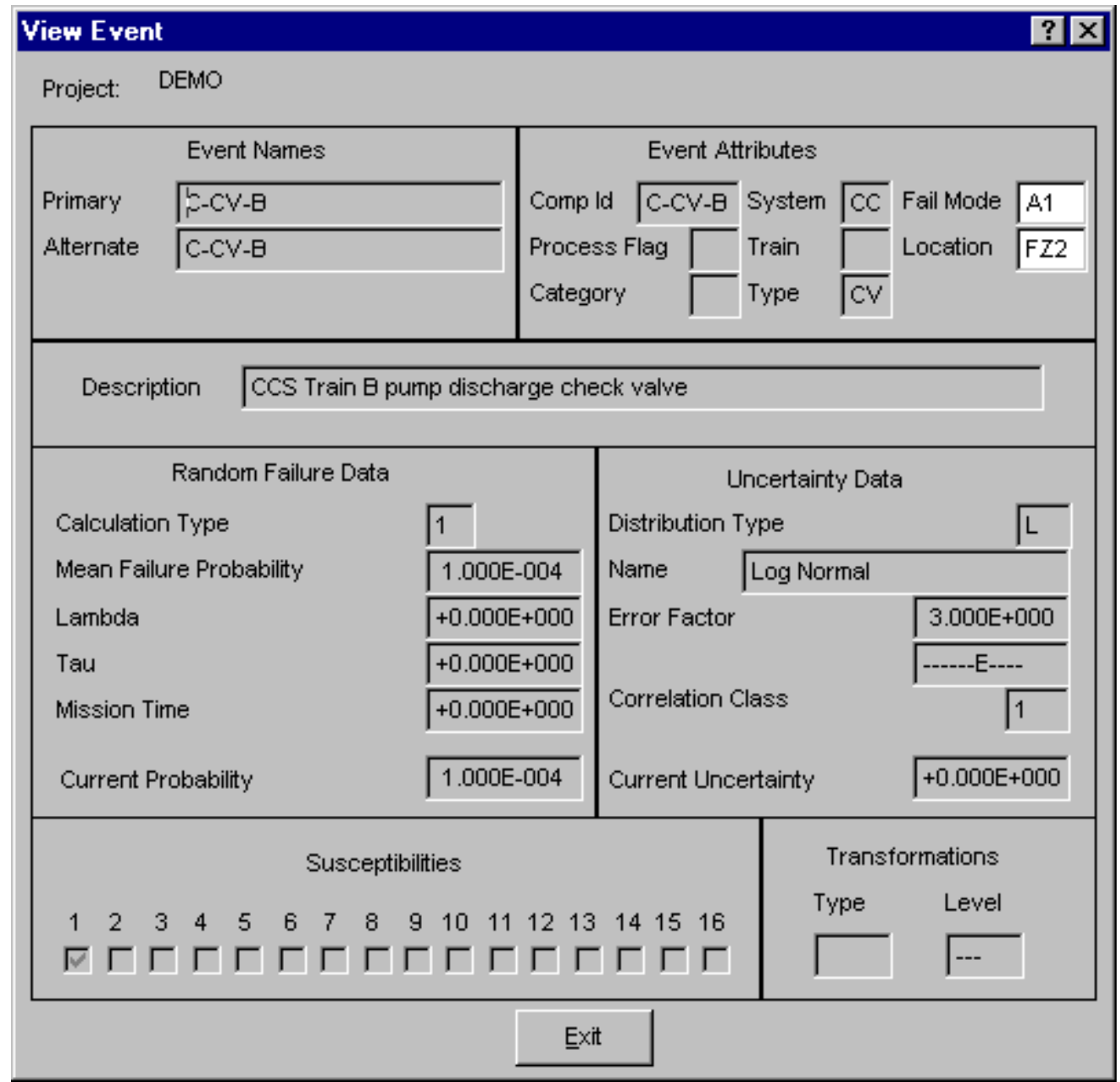

Information on this dialog is for display only and cannot be modified. 


\subsubsection{Cut Set Report}

\section{PURPOSE}

This option allows you to generate a report of the data that is currently displayed on the dialog. The report may be displayed in the Report Viewer, sent to a printer, or saved to a file for later processing.

\section{STEPS}

1. From the SAPHIRE menu select Fault Tree/Sequence/End State. The Fault Tree List/Sequences/End State List dialog will be displayed.

2. Highlight the desired list item and right-click to invoke the pop-up menu.

3. Choose Display | Cut Sets. The Selected Cut Sets dialog will be displayed.

4. Choose the Report button. The Report dialog will be displayed.

5. Select the desired report options and choose OK. The Report Viewer will display the report.

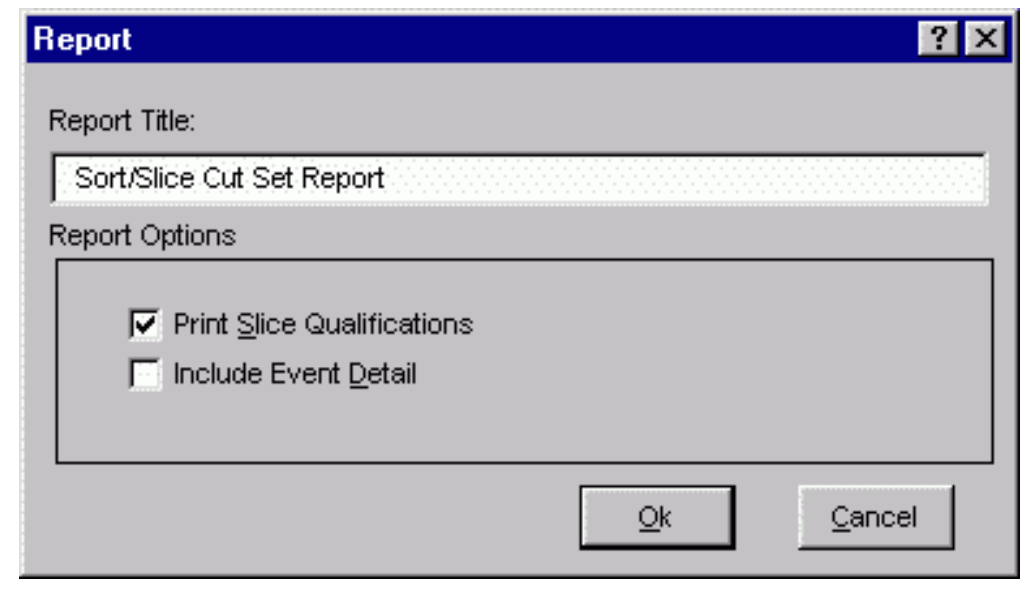

\section{Report Title - \\ Print Slice Qualifications -}

Include Event Detail -
A default report title is provided. Edit this value to customize the title. When checked, the criteria used to qualify the cut sets is included in the report.

When checked, the report will report one event per line, and including event probability and description information. When this option is not checked, the events will appear as a comma delimited list, with no supplementary event information included.

\subsubsection{Save}

\section{PURPOSE}

Save the results of the existing cut set slice (currently displayed on the Selected Cut Sets dialog) to an end state. 


\section{STEPS}

1. From the SAPHIRE menu select Fault Tree/Sequence/End State. The Fault Tree List/Sequences/End State List dialog will be displayed.

2. Highlight the desired list item and right-click to invoke the pop-up menu.

3. Choose Display | Cut Sets. The Selected Cut Sets dialog will be displayed.

4. Slice, or qualify, the cut sets as desired. Select the tab containing the cut set list you want to save.

5. Choose the Save button. The Save To An End State dialog will be displayed.

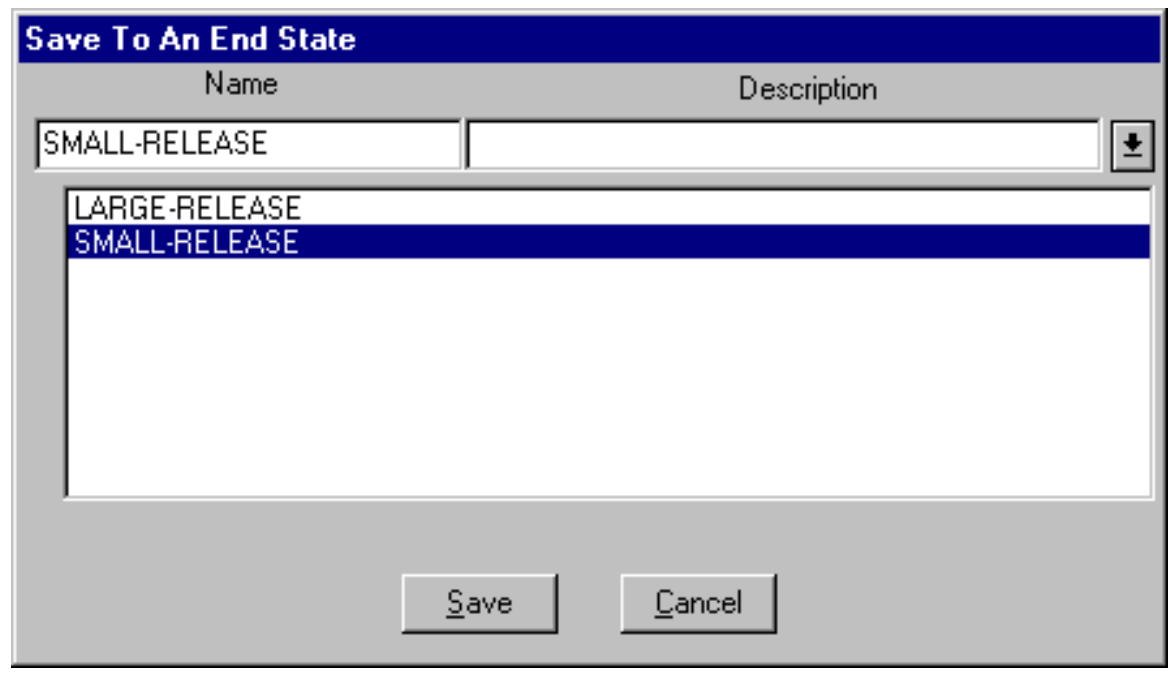

In the example here, the down arrow to the right of the Description field was selected to display the list of existing end states.
Name -
Enter the name of the new end state. Up to 24 uppercase, alphanumeric characters are allowed.
Description - Enter the description of the new end state. Up to 60 upper- and lowercase, alphanumeric characters area allowed
Save - $\quad$ Perform the save operation. A new end state record will be saved in the SAPHIRE data base with the name and description as entered here, and current slice for its cut sets. Selecting an existing end state will cause the existing cut sets for that end state to be overwritten.
Cancel - $\quad$ Close the Save To An End State dialog without saving the cut sets or creating a new end state record.

\subsubsection{Comparing Cut Sets}

\subsubsection{Cut Set Comparison}

\section{PURPOSE}

This option displays both the base case and current case cut sets for the selected fault tree (or sequences or end states). The source (either base or current) of the cut sets is indicated both by color and a symbol. The color red and the "-" symbol in the first column indicate a base case cut set. The color black and a 
blank (" ") in the first column indicate that the cut set is both a current case and base case cut set. The color green and the "+" symbol in the first column indicate a current case cut set.

\section{STEPS}

1. From the SAPHIRE menu select Fault Tree/Sequence/End State. The Fault Trees List/Sequences/End State List dialog will be displayed.

2. Highlight the desired list item(s) and right-click to invoke the pop-up menu.

3. Choose Display, then the Comparison sub-menu option. The Base \& Current Case Cut Sets dialog will be displayed.

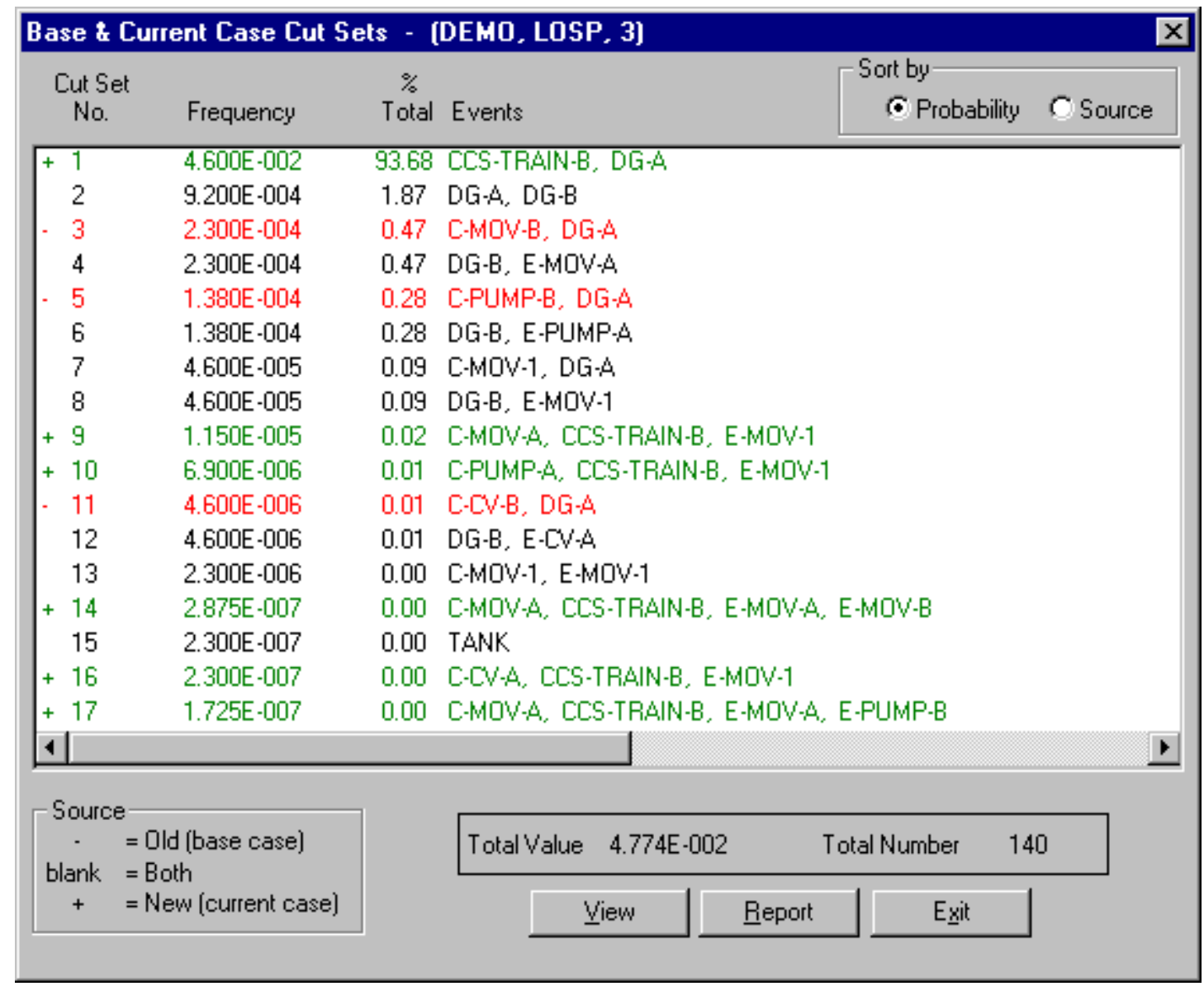

\section{Sort by}

Probability - List the cut sets by frequency in descending order.

Source -

View -

Report -

Exit -
List the cut sets by source, listing first base case cut sets, then cut sets existing in both, followed by current case cut sets.

Display information about the selected cut set including basic event data. Generate a report of the data that is displayed on the dialog. Close the Base \& Current Case Cut Sets dialog.

\subsubsection{View Cut Set}




\section{PURPOSE}

This option displays the basic events of the selected cut set with their failure probabilities and descriptions. At the bottom of the Selected Cut Set Events dialog, information about this cut set is displayed: percent of contribution to the total, the cut set frequency, and the number of events that make up this cut set.

\section{STEPS}

1. From the SAPHIRE menu select Fault Tree/Sequence/End State. The Fault Tree List/Sequences/End State List dialog will be displayed.

2. Highlight the desired list item and right-click to invoke the pop-up menu.

3. Choose Display | Cut Sets. The Selected Cut Sets dialog will be displayed. OR

4. Choose Display | Comparison. The Base \& Current Case Cut Sets dialog will be displayed.

5. Highlight the desired cut set.

6. Choose the View button. The Selected Cut Set Events dialog will be displayed.

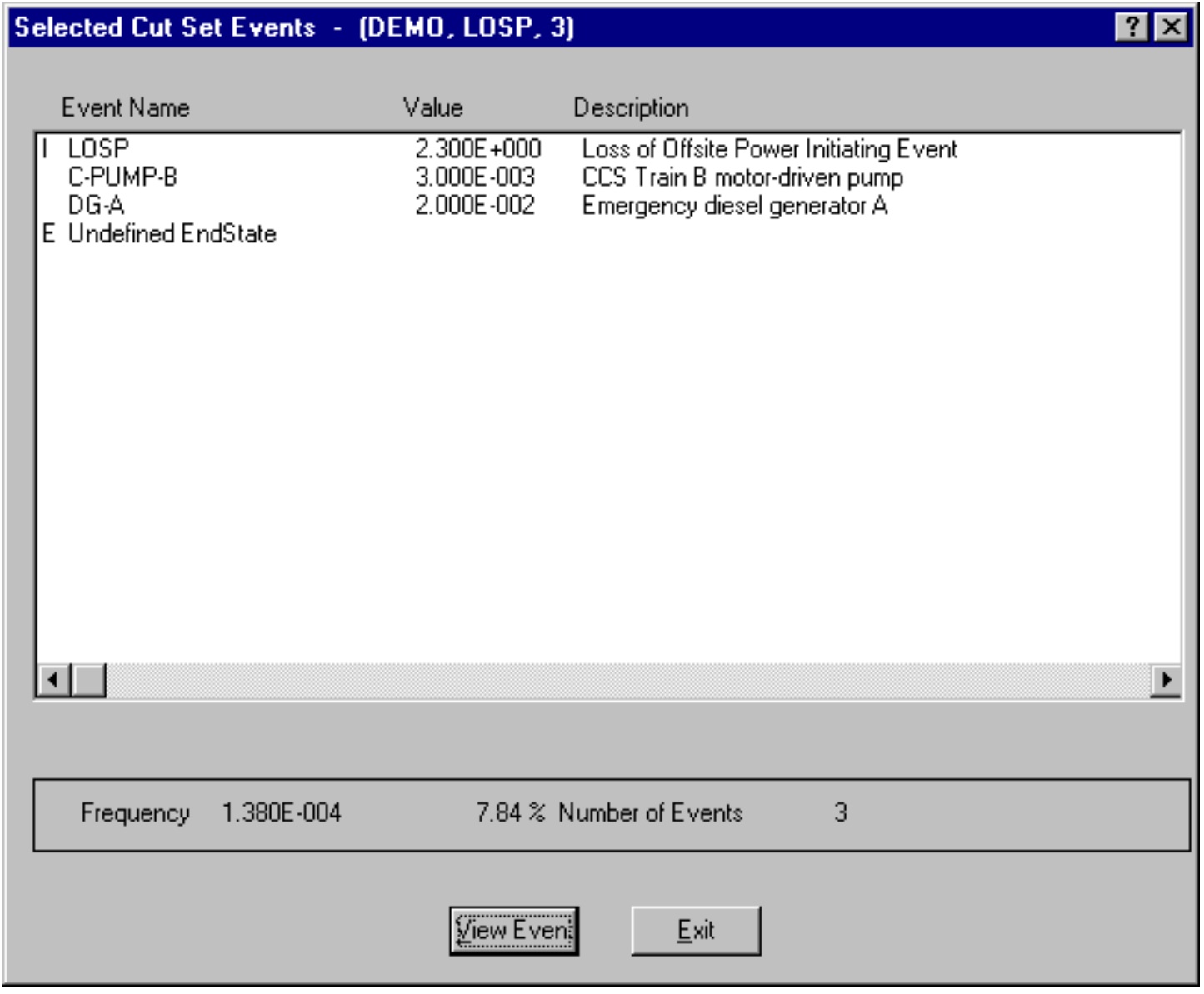

When viewing fault tree cut sets, the end state, if any, is designated with the letter "E" to the left of the Event Name column. It will be the last item listed. 
When viewing sequence cut sets, the initiating event is designated with the letter "I" to the left of the Event Name column. It will be the first item listed. The end state will be designated with the letter "E" to the left of the Event Name column and will be the last item listed.

When viewing end state cut sets, the initiating event is designated with the letter "I" to the left of the Event Name column. It will be the first item listed. The originating sequence is designated by the letter "S" to the left of the Event Name column and will be the last item listed.

View Event - Display individual basic event information.

\subsubsection{Cut Set Report}

\section{PURPOSE}

This option allows you to generate a report of the data that is currently displayed on the dialog. The report may be displayed in the Report Viewer, sent to a printer, or saved to a file for later processing.

\section{STEPS}

1. From the SAPHIRE menu select Fault Tree/Sequence/End State. The Fault Tree List/Sequences/End State List dialog will be displayed.

2. Highlight the desired list item and right-click to invoke the pop-up menu.

3. Choose Display | Cut Sets. The Selected Cut Sets dialog will be displayed.

4. Choose the Report button. The Report dialog will be displayed.

5. Select the desired report options and choose $\mathbf{O K}$. The Report Viewer will display the report.

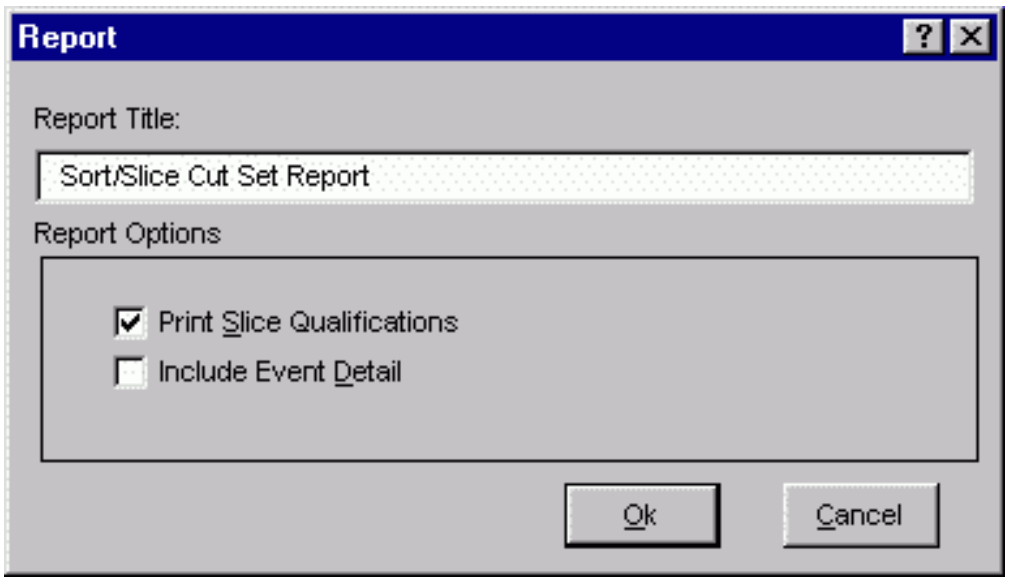

Report Title Print Slice Qualifications -
A default report title is provided. Edit this value to customize the title. When checked, the criteria used to qualify the cut sets is included in the report. 
When checked, the report will report one event per line, and including event probability and description information. When this option is not checked, the events will appear as a comma delimited list, with no supplementary event information included.

\subsubsection{Displaying Merged Cut Sets}

\subsubsection{Merged Cut Sets}

\section{PURPOSE}

This option is provided for (and is meaningful only to) projects that perform level two analysis. It provides a mechanism for viewing level two sequence cut sets merged (combined) with the corresponding level one sequence cut sets.

This option will only appear as a pop-up menu option when a single sequence is highlighted. Results will be available only for valid level two sequences. A valid level two sequence is defined as a sequence that 1) contains (level two) cut sets, and 2) has an initiator of the same name as an end state which contains level 1 cut sets.

The cut sets from the level two sequence will be multiplied with the cut sets from the corresponding level one end state to produce the merged cut sets. The combined cut sets will be minimized prior to display.

\section{STEPS}

1. From the SAPHIRE menu select Sequence. The Sequences list dialog will be displayed.

2. Highlight the desired level 2 sequence and right-click to invoke the pop-up menu.

3. Choose Display, then the Merged Cut Sets sub-menu option. The Merge Cut Sets Options dialog will be displayed.

4. Select the desired Merge options, and choose OK.

If valid cut sets for both level two and level one are located, the Selected Cut Sets dialog will be displayed, containing the merged cut set results.

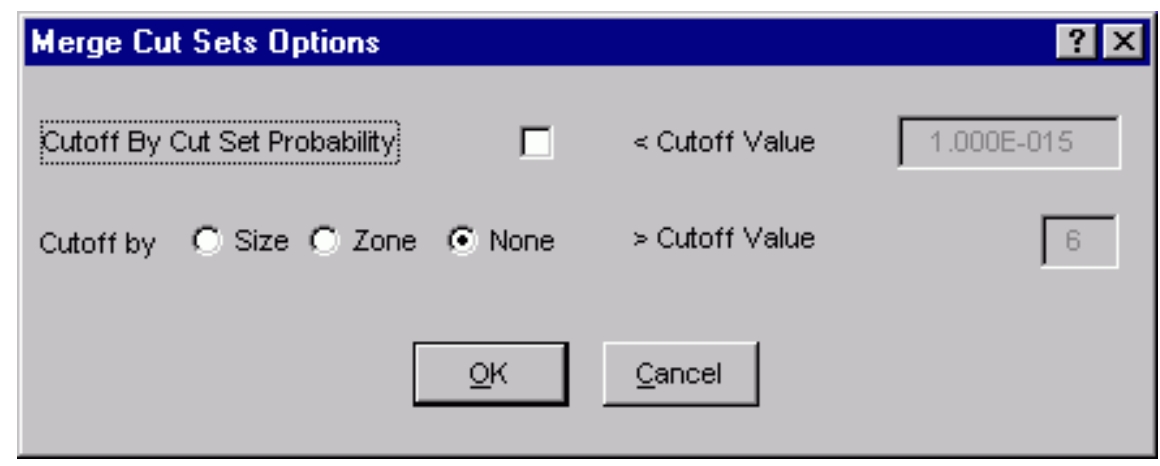

\subsubsection{Merge Cut Set Options}




\section{PURPOSE}

This option allows you to specify the cut set probability and size cutoff values desired when a cut set merge is performed.

\section{STEPS}

1. From the SAPHIRE menu select Sequence. The Sequences list dialog will be displayed.

2. Highlight the desired level 2 sequence and right-click to invoke the pop-up menu.

3. Choose Display, then the Merged Cut Sets sub-menu option. The Merge Cut Sets Options dialog will be displayed.

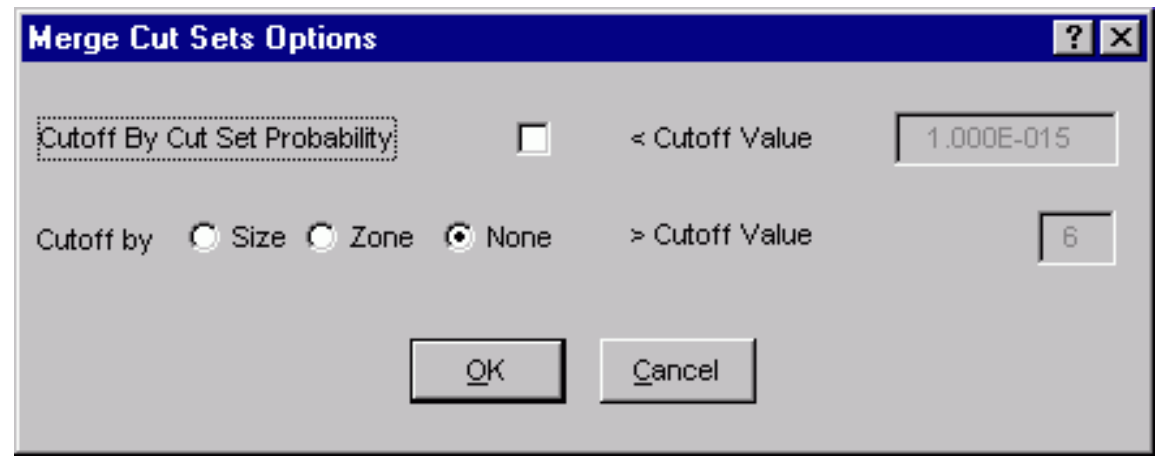

You may change any of the data fields on this dialog. The default values that appear on this dialog may be reset to new values by selecting Utility from the SAPHIRE menu and then invoking the

Define Constants option.

Cutoff by Cut Set Probability - Used for determining if the cut set should be retained or discarded based on the value in the $<$ Cutoff Value field.

Cutoff by Used for determining if the cut set should be retained or discarded based on the value in the $>$ Cutoff Value field or zoned flagged events.

\subsubsection{Merge Cut Sets Example}

\section{PURPOSE}

This topic illustrates the merge cut sets process. Cut sets from level one end states are multiplied with the level two sequences. When common events cause non-minimal cut sets, the cut sets are automatically reduced to their minimal terms.

The following examples assume a level two project contains the following items:

Suppose the level two project contained two level one sequences "L1-SEQ-1A" and "L1SEQ-1B", both of which have "PDS-X" as an end state, and "INIT-1" as an initiator. The level two event tree would then also be called "PDS-X", having an initiating event also called "PDS-X".

Assume the level two event tree has a single sequence called "L2-SEQ-2X". 


\section{Example 1}

Suppose sequence L1-SEQ-1A contains a single cut set, EV-1A * EV-1B (with initiator INIT-1).

Suppose sequence L1-SEQ-1B contains a single cut set, EV-1C (with initiator INIT-1).

Then the cut sets for end state PDS-X would be $\mathrm{EV}-1 \mathrm{~A} * \mathrm{EV}-1 \mathrm{~B}+$ $\mathrm{EV}-1 \mathrm{C}$

Suppose the level two sequence L2-SEQ-2A contained two cut sets, EV-2X + EV-2Y (with initiator PDS-X).

Then the merged cut sets for L2-SEQ-1 would be (all with initiator INIT-1).

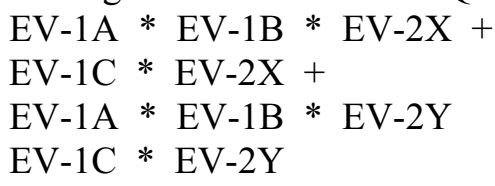

\section{Example 2}

Suppose sequence L1-SEQ-1A contains a single cut set, EV-1A * EV-1B (with initiator INIT-1).

Suppose sequence L1-SEQ-1B contains a single cut set, EV-M (with initiator INIT-1).

Then the cut sets for end state PDS-X would be

$\mathrm{EV}-1 \mathrm{~A} * \mathrm{EV}-1 \mathrm{~B}+$

EV-M

Suppose the level two sequence L2-SEQ-2A contained two cut sets, EV-2X + EV-M (with initiator PDS-X).

Then the merged cut sets for L2-SEQ-1 would be (all with initiator INIT-1).

$\mathrm{EV}-1 \mathrm{~A} * \mathrm{EV}-1 \mathrm{~B} * \mathrm{EV}-2 \mathrm{X}+$

EV-M

Because the level one and level two cut sets share a common event, EV-M, the multiplied cut sets become non-minimal; so SAPHIRE automatically reduces the terms prior to display.

\subsubsection{Level Two Analysis}

\section{PURPOSE}

This topic briefly describes the level two analysis process. 


\section{STEPS}

1. Create a level one event tree as usual.

2. Assign plant damage end states either manually or via partition rules using the general format

if ...some criteria... then

globalPartition="PDS-name1"

transfer = "Level-2-Event-Tree-Name1"

else

$\cdots$

endif

3. If partition rules were created to assign end states, apply the partition rules. During the partition process, an end state, event tree, and initiating event, all sharing the same name, will each be created, if necessary, for each end state defined with the globalPartition statement. The partition rule will automatically create event tree logic consisting of the initiating event of the same name, and a single "Pass" sequence that transfers to the level two event tree specified by the transfer statement. The initiating event will have a Calc Type of "E", which means it will use the current min cut upper bound of the end state as its frequency. If end states were assigned manually, the corresponding event tree and initiating event must also be created manually.

4. Solve the level one sequences.

5. Gather the plant damage end states to collect the level one cut sets.

6. Generate current event data to assure that the level two initiator reflects the plant damage state min cut upper bound.

7. Create the logic for the level two transfer tree; link, and solve the level two sequences.

8. View the level one and level two cut sets merged, if desired.

\subsubsection{Displaying Importance Results}

\subsubsection{Importance Measures}

\section{PURPOSE}

Importance measures provide "reliability-worth" information about basic events appearing in the cut sets for a fault tree or accident sequence. The "importance" for a basic event is essentially the event's contribution to the overall top event probability (for fault trees) or sequence frequency (for event trees). The measure of contribution can take on many forms depending on what concern the analyst has for a particular basic event. Consequently, SAPHIRE calculates seven different basic event importance measures. These are the Fussell-Vesely importance, risk reduction ratio, risk increase ratio, Birnbaum (or first derivative) importance, risk reduction difference, risk increase difference, and the uncertainty importance. 
The ratio importance measures are dimensionless and consider only relative changes. The difference definitions account for the actual risk levels that exist and are more appropriate when actual risk levels are of concern, such as comparisons or prioritizations across different plants. For purely relative evaluations, such as prioritizations within a plant, the ratios sometimes give more graphic results.

These importance measures are calculated for each basic event for the respective fault tree or accident sequence. Once the results have been calculated, they can be displayed and then partitioned as a subset of the original cut sets.

\section{STEPS}

1. From the SAPHIRE menu select Fault Tree/Sequence/End State. The Fault Tree List/Sequences/End State List dialog will be displayed.

2. Highlight the desired list item and right-click to invoke the pop-up menu.

3. Choose Display, then the Importance sub-menu option.

4. Choose from one of the following sub-menu options:

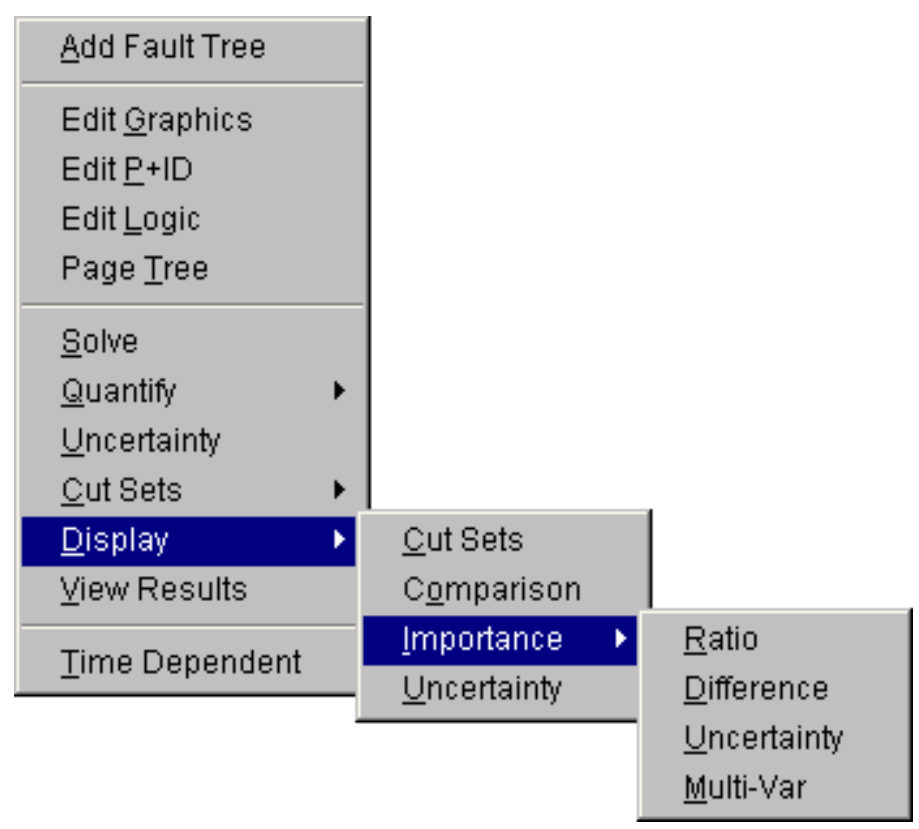

Ratio - $\quad$ Fussell-Vesely importance, Risk Reduction Ratio, and Risk Increase Ratio will be calculated and displayed.

Difference - $\quad$ Birnbaum importance, Risk Reduction Interval, and Risk Increase Interval will be calculated and displayed. 
Uncertainty - Quantification of the contribution of each individual basic event's uncertainty to the total output uncertainty.

Multi-Var - Importance for a group of events will be calculated and displayed.

\subsubsection{Fussell-Vesely Importance (FV)}

The FV measure is an indication of the percentage of the minimal cut set upper bound contributed by the cut sets containing the basic event. The equation for FV importance is

$$
\mathbf{F V}=\mathbf{F}(\mathbf{i}) / \mathbf{F}(\mathbf{x})
$$

where

F(i) is the minimal cut set upper bound for the group of cut sets containing the event $\mathrm{F}(\mathrm{x})$ is the original minimal cut set upper bound

Prior to SAPHIRE version 7.27, the FV equation used and approximate expression

$$
\mathbf{F V}=1-\mathbf{F}(\mathbf{0}) / \mathbf{F}(\mathbf{x})
$$

where

$\mathrm{F}(0)$ is the minimal cut set upper bound with the event probability set equal to 0.0 .

$\mathrm{F}(\mathrm{x})$ is the original minimal cut set upper bound.

\subsubsection{Birnbaum Importance (B)}

This indicates the sensitivity of the minimal cut set upper bound with respect to a change in the basic event probability.

$$
\mathbf{B}=\mathbf{F}(\mathbf{1})-\mathbf{F}(\mathbf{0})
$$

where

$\mathrm{F}(1)$ is the minimal cut set upper bound with the event probability set equal to 1.0. $\mathrm{F}(0)$ is the minimal cut set upper bound with the event probability set equal to 0.0 .

\subsubsection{Risk Reduction Ratio (RRR) or Risk Reduction Interval (RRI)}

These are an indication of how much the minimal cut set upper bound would decrease if the basic event was reduced to a probability or 0.0 (typically if the corresponding component never failed).

$$
\begin{aligned}
& \text { RRR }=F(x) / F(0) \\
& \text { RRI }=\mathbf{F}(\mathbf{x})-\mathbf{F}(0)
\end{aligned}
$$

Note the similarity between RRI and FV; the relative importance ranking of basic events will be the same for the two importance measures.

\subsubsection{Risk Increase Ratio (RIR) or Risk Increase Interval (RII)}

These are an indication of how much the minimal cut set upper bound would increase if the basic event was increased to 1.0 (typically if the corresponding component always failed). Note: If the event probability is close to 1.0, this importance measure may yield a small RIR or RII. 


$$
\begin{aligned}
& \text { RIR }=F(1) / F(x) \\
& \text { RII = F(1) - F(x) }
\end{aligned}
$$

where

$\mathrm{F}(\mathrm{x})$ is the original minimal cut set upper bound

$\mathrm{F}(1)$ is the minimal cut set upper bound with the event probability set equal to 1.0 .

\subsubsection{Uncertainty Importance}

The uncertainty in each input parameter, as expressed through its probability distribution, contributes to the uncertainty in the output parameter of interest (e.g., core damage frequency). The uncertainty importance measure in SAPHIRE attempts to quantify the contribution of each individual basic event $=\mathrm{S}$ uncertainty to this total output uncertainty. The measure used in SAPHIRE is based on a Taylor series expansion of the variance of the output of interest. The equation used by SAPHIRE is

$$
\operatorname{Var}(R) \approx \sum_{i=1}^{n}\left(\frac{\partial R}{\partial p_{i}}\right)^{2} \sigma_{i}^{2}
$$

where $\mathrm{R}$ is the output of interest, $\mathrm{p}_{\mathrm{i}}$ is the probability of the ith basic event, and $\sigma_{i}^{2}$ is the variance of the uncertainty distribution for the ith event. This approximation, which hinges upon the basic events being mutually statistically independent, says that the variance of the output is approximately the sum of $n$ separate contributions, one from each basic event. The magnitude of each contribution (each contribution is positive) measures how much of the output variance is contributed by each basic event. Because it is more convenient, SAPHIRE uses the square root of each individual contribution as the uncertainty importance:

$$
I_{u n c}=\frac{\partial R}{\partial p_{i}} \sigma_{i}
$$

where $\sigma_{i}$ is the standard deviation of the uncertainty distribution of the ith basic event. Note that the partial derivative in the above equation for the uncertainty importance is, by definition, the Birnbaum importance of that event. Therefore, no new calculations are needed; the uncertainty importance is the Birnbaum importance multiplied by the standard deviation of the input probability distribution.

\subsubsection{Multi-Var Importance}

This option allows the user to calculate an importance for a group of events.

For instance, the user can select all motor operated valves in a set of cut sets and get a combined importance measure for these valves together. All of the valves are treated as a single event in the importance calculations and the result is displayed with the name "Multi-variable." 


\subsubsection{Importance Measures Dialog}

\subsection{Importance Measures Results}

\section{PURPOSE}

This option displays the results of the importance measure analysis.

\section{STEPS}

1. From the SAPHIRE menu select Fault Tree/Sequence/End State. The Fault Tree List/Sequences/End State List dialog will be displayed.

2. Highlight the desired list item and right-click to invoke the pop-up menu.

3. Choose Display, then the Importance submenu option.

4. Choose from one of the submenu options: Ratio, Difference, Uncertainty, Mult-Var.

5. The Importance Measures dialog will be displayed.

The results shown here are Fussell-Vesely importance measures. If the analysis type is seismic, the program will prompt you to select a ground acceleration level defined in the project hazard curve.

\begin{tabular}{|c|c|c|c|c|c|c|}
\hline \multicolumn{6}{|c|}{ Importance Measures - [DEHO, CCS] } & $x$ \\
\hline \multirow[b]{3}{*}{ Event Name } & \multirow[b]{3}{*}{$\begin{array}{l}\# \text { of } \\
\text { Dccur }\end{array}$} & \multirow[b]{3}{*}{ Probability } & \multirow[b]{3}{*}{$F \cdot V$} & \multicolumn{3}{|c|}{ Sort } \\
\hline & & & & \multicolumn{2}{|l|}{$F \cdot V$} & $\nabla$ \\
\hline & & & & $\begin{array}{c}\text { Risk Reduc. } \\
\text { Ratio }\end{array}$ & $\begin{array}{c}\text { Risk Incre. } \\
\text { Ratio }\end{array}$ & \\
\hline \multirow[t]{2}{*}{$\begin{array}{l}\text { DG-B } \\
\text { C-MOV-1 } \\
\text { DG-A } \\
\text { C-MOV-B } \\
\text { C-PUMP-B } \\
\text { C-MOV-A } \\
\text { C-PUMP-A } \\
\text { C-CV-A } \\
\text { C-CV-B }\end{array}$} & $\begin{array}{l}1 \\
1 \\
3 \\
4 \\
4 \\
3 \\
3 \\
3 \\
4\end{array}$ & $\begin{array}{l}2.000 \mathrm{E}-002 \\
1.000 \mathrm{E}-003 \\
2.000 \mathrm{E}-002 \\
5.000 \mathrm{E}-003 \\
3.000 \mathrm{E}-003 \\
5.000 \mathrm{E}-003 \\
3.000 \mathrm{E}-003 \\
1.000 \mathrm{E}-003 \\
1.000 \mathrm{E}-004\end{array}$ & $\begin{array}{l}9.418 \mathrm{E}-001 \\
4.619 \mathrm{E}-002 \\
7.477 \mathrm{E}-003 \\
6.692 \mathrm{E}-003 \\
4.015 \mathrm{E}-003 \\
1.869 \mathrm{E}-003 \\
1.121 \mathrm{E}-003 \\
3.738 \mathrm{E}-004 \\
1.338 \mathrm{E}-004\end{array}$ & $\begin{array}{l}1.718 \mathrm{E}+001 \\
1.048 \mathrm{E}+0000 \\
1.008 \mathrm{E}+000 \\
1.007 \mathrm{E}+000 \\
1.004 \mathrm{E}+000 \\
1.002 \mathrm{E}+000 \\
1.001 \mathrm{E}+000 \\
1.000 \mathrm{E}+000 \\
1.000 \mathrm{E}+000\end{array}$ & \multicolumn{2}{|l|}{$\begin{array}{l}4.715 \mathrm{E}+001 \\
4.715 \mathrm{E}+001 \\
1.366 \mathrm{E}+000 \\
2.322 \mathrm{E}+000 \\
2.325 \mathrm{E}+000 \\
1.371 \mathrm{E}+000 \\
1.372 \mathrm{E}+000 \\
1.373 \mathrm{E}+000 \\
2.329 \mathrm{E}+000\end{array}$} \\
\hline & Description & \multicolumn{2}{|c|}{ Partition } & Report & \multicolumn{2}{|c|}{ Exit } \\
\hline
\end{tabular}

Sort -

Select from the drop-down list to sort results by Occurrence, Probability, F-V / Birnbaum / Uncertainty, Reduction, or Increase. By default, when the importance data are first displayed it is sorted, high to low, by F-V / Birnbaum / Uncertainty importance value.

Description - $\quad$ Display the full description for the highlighted event in the message bar of the SAPHIRE main window.

Partition - $\quad$ Limit which events are displayed/reported. 
Report - $\quad$ Generate a report of the data that is displayed on the dialog.

Exit -

Close the Importance Measures dialog.

\subsection{Importance Results Partition}

\section{PURPOSE}

This option allows you to redefine a fault tree as a subset of the original cut sets. This is accomplished by defining a set of events to be used to determine whether a cut set belongs to a partition.

\section{STEPS}

1. From the SAPHIRE menu select Fault Tree/Sequence/End State. The Fault Tree List/Sequences/End State List dialog will be displayed.

2. Highlight the desired list item and right-click to invoke the pop-up menu.

3. Choose Display | Importance.

4. Choose from one of the submenu options: Ratio, Difference, Uncertainty, Mult-Var. The Importance Measures dialog will be displayed.

5. Choose the Partition button. The Partition dialog will be displayed.

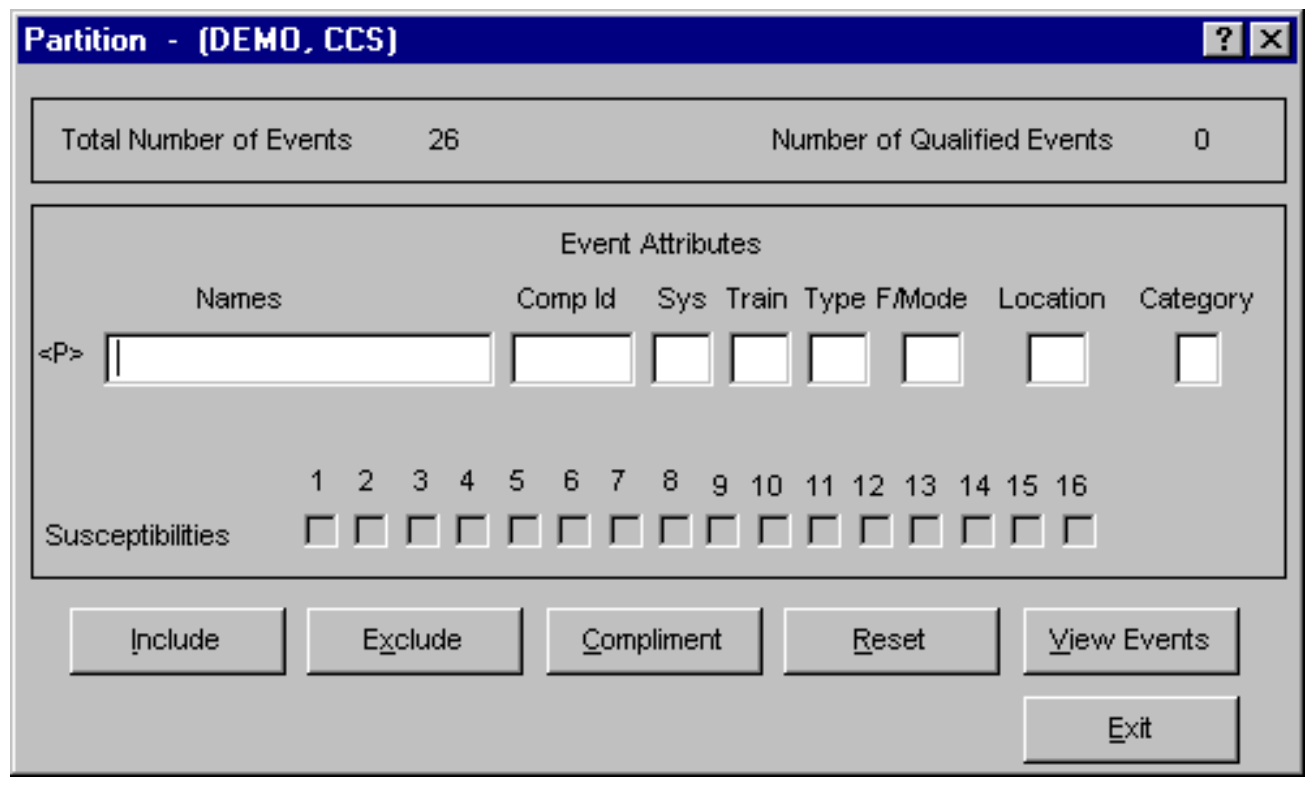

Include - $\quad$ Fill in the entry fields that are to be used to qualify the events that may be used in the new partition. Choose the Include button. The events in the cut set are qualified and when complete, will update the Number of Qualified Events field that appears in the upper right of the Partition dialog.

Exclude - $\quad$ Fill in the entry fields that are to be used to remove events from the list of qualified events. Choose the Exclude button. The events in the cut set are qualified and when complete, will update the Number of Qualified Events field that appears in the upper right of the Partition dialog.

Compliment - Cause all currently qualified events to be disqualified, and all unqualified events to become the set of qualified events. 
Reset - $\quad$ Set all events in the database to be qualified. This removes all partitioning from the current cut sets.

View Events - Display the list of all events in the project. Mark those events that are to be considered qualified.

Exit - $\quad$ Close the Partition dialog. Only those cut sets that are made up of qualified events are displayed on the Importance Measures dialog.

\subsection{View Events}

\section{PURPOSE}

This option allows you to display the list of all events in the project and mark those events that are to be used to determine whether a cut set belongs to a partition.

\section{STEPS}

1. From the SAPHIRE menu select Fault Tree/Sequence/End State. The Fault Tree List/Sequences/End State List dialog will be displayed.

2. Highlight the desired list item and right-click to invoke the pop-up menu.

3. Choose Display | Importance.

4. Choose from one of the sub-menu options: Ratio, Difference, Uncertainty, Mult-Var.

5. The Importance Measures dialog will be displayed.

6. Choose the Partition button. The Partition dialog will be displayed.

7. Choose the View Events button. The Events dialog will be displayed.

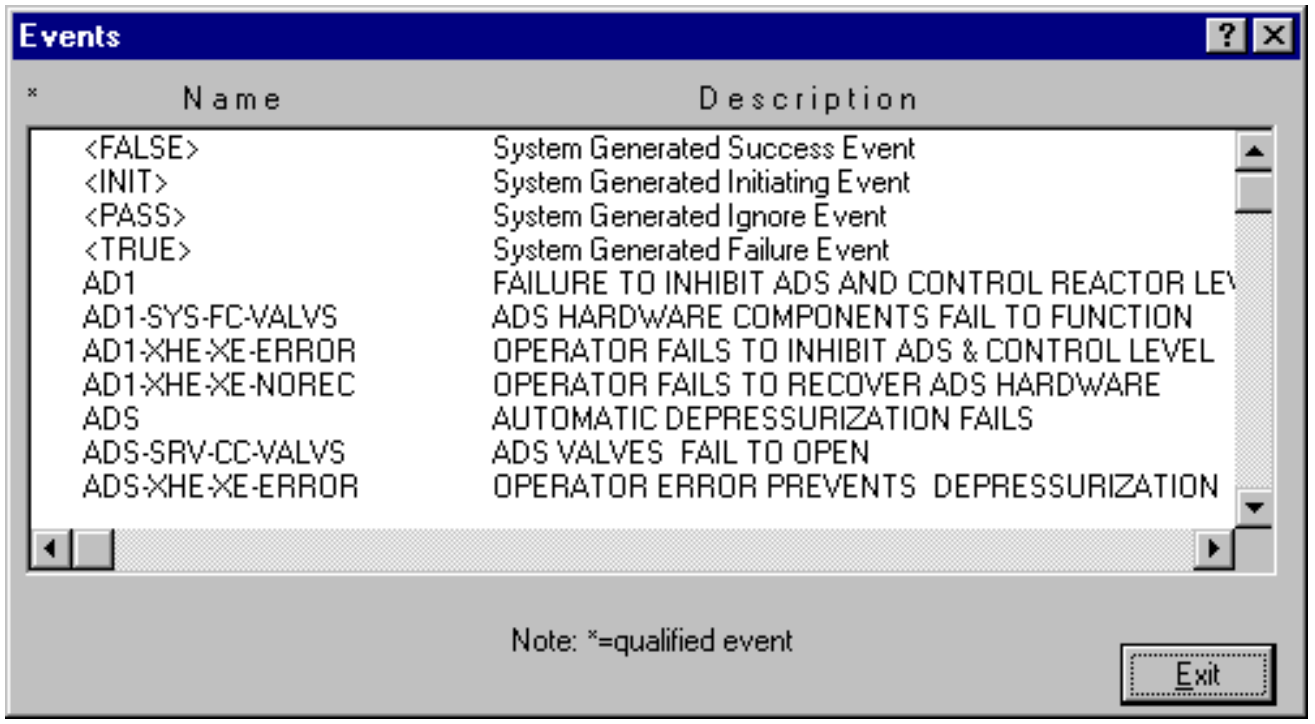

Double-click - Mark the selected event. An asterisk (*) will appear to the left of the event name. Exit Close the Events dialog. Choose the Include or Exclude button from the Partition dialog. 


\subsubsection{Displaying Uncertainty}

\subsubsection{Displaying Uncertainty Results}

\section{PURPOSE}

This option displays the results of the uncertainty analysis previously performed. The results displayed are the distribution and probability bounds for both base and current results. These values were calculated using either the Latin Hypercube or the Monte Carlo simulation technique.

\section{STEPS}

1. From the SAPHIRE menu select Fault Tree/Sequence/End State. The Fault Tree List/Sequences/End State List dialog will be displayed.

2. Highlight the desired list item and right-click to invoke the pop-up menu.

3. Choose Display, then the Uncertainty sub-menu option. The Fault Tree/Sequence/End State Uncertainty dialog will be displayed.

If the analysis type is seismic, you will be prompted to select a ground acceleration level defined in the project hazard curve.

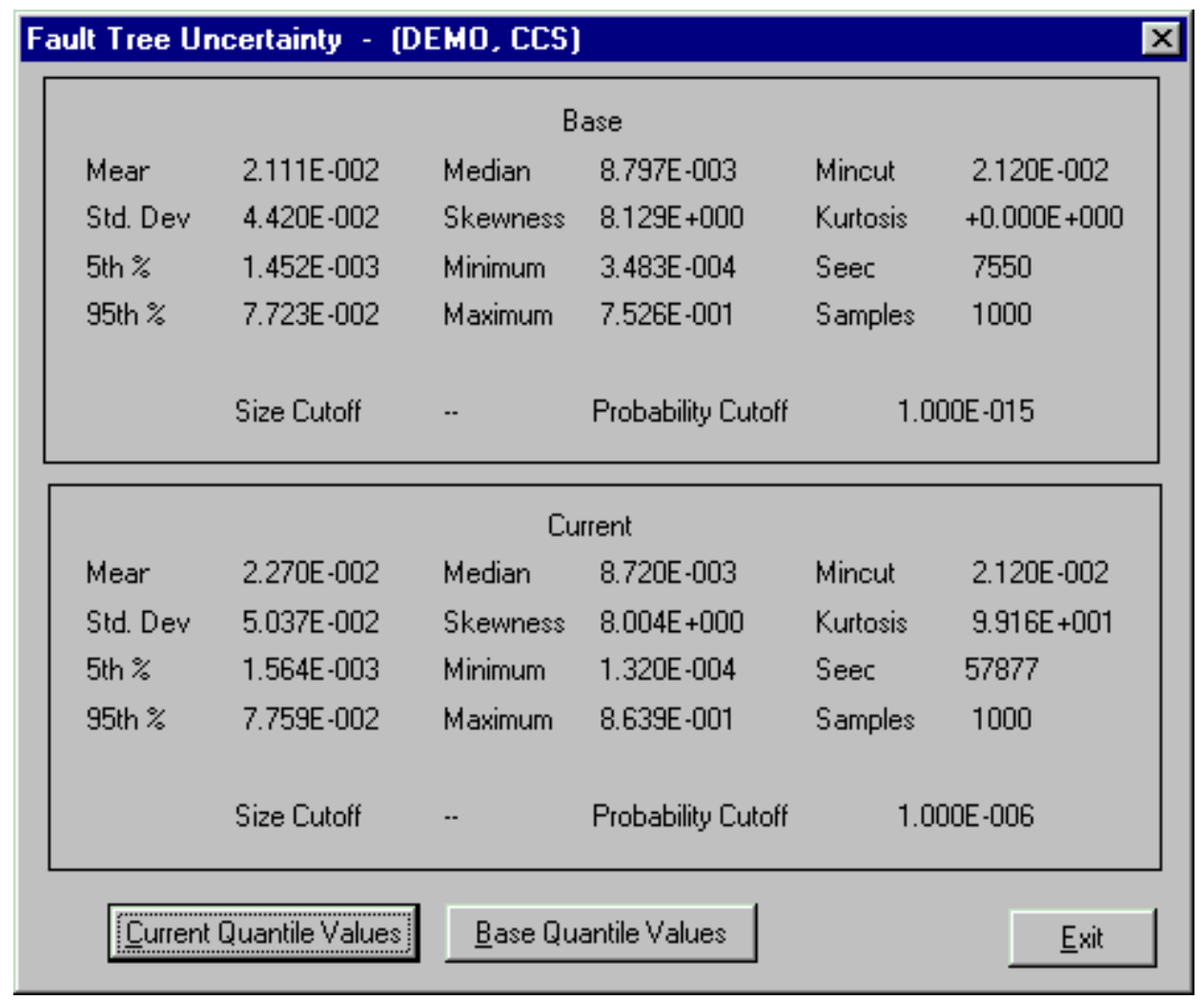

Current Quantile Values Base Quantile ValuesExit dialog.
View the quantile values associated with the current case data. View the quantile values associated with the base case data. Close the Fault Tree/Sequence/End State Uncertainty 


\subsubsection{Uncertainty Quantile Values}

\section{PURPOSE}

Display the uncertainty quantile (or probability) values associated with either the current or base case data. These quantile values can be used to construct a cumulative distribution plot of the analysis uncertainty results.

\section{STEPS}

1. From the SAPHIRE menu select Fault Tree/Sequence/End State. The Fault Tree List/Sequences/End State List dialog will be displayed.

2. Highlight the desired fault tree and right-click to invoke the pop-up menu.

3. Choose Display, then the Uncertainty sub-menu option. The Fault Tree/Sequence/End State Uncertainty dialog will be displayed.

4. Choose the Current Quantile Values or Base Quantile Values button.

\begin{tabular}{|c|c|c|c|c|c|}
\hline \multicolumn{3}{|c|}{ Uncertainty Quantile Values - [DEMO, CCS] } & \multicolumn{3}{|c|}{$? \sqrt{x}$} \\
\hline $\begin{array}{l}\text { Distribution } \\
\text { Quantile Level } \\
\text { (in per cent) }\end{array}$ & $\begin{array}{c}95 \% \text { Confidence } \\
\text { Interval On } \\
\text { Quantile Level in } \\
\%(+i-)\end{array}$ & Quantile Value & \multicolumn{3}{|c|}{$\begin{array}{l}95 \% \text { Confidence } \\
\text { Interval on Quantile }\end{array}$} \\
\hline 05 & 05 & $62185 \mathrm{~F}-004$ & $36160 F_{-004}$ & $81985 F-004$ & 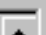 \\
\hline $\begin{array}{l}0.5 \\
1.0\end{array}$ & 0.7 & $8.1985 \mathrm{E}-004$ & $5.3501 \mathrm{E}-004$ & $\begin{array}{l}0.1905 \mathrm{E}-004 \\
1.0688 \mathrm{E}-003\end{array}$ & $\boldsymbol{\Delta}$ \\
\hline 2.5 & 1.0 & 1.1657E-003 & $1.0634 \mathrm{E}-003$ & $1.3215 \mathrm{E}-003$ & 我 \\
\hline 5.0 & 1.4 & 1.6235E-003 & 1.3281E-003 & 1.7995E-003 & \\
\hline 10.0 & 1.9 & $2.2045 \mathrm{E}-003$ & $2.0049 \mathrm{E}-003$ & $2.4574 \mathrm{E}-003$ & \\
\hline 20.0 & 2.5 & 3.3830E-003 & 3.0875E-003 & $3.6982 E-003$ & \\
\hline 25.0 & 2.7 & $4.0422 \mathrm{E}-003$ & $3.6952 \mathrm{E}-003$ & 4.3167E-003 & \\
\hline 30.0 & 2.9 & $4.5621 \mathrm{E}-003$ & $4.2275 \mathrm{E}-003$ & 5.0094E-003 & \\
\hline 40.0 & 3.1 & $6.2820 \mathrm{E}-003$ & 5.6327E-003 & $6.8446 \mathrm{E}-003$ & \\
\hline 50.0 & 3.1 & $8.8435 E-003$ & $7.9240 \mathrm{E}-003$ & $9.5918 \mathrm{E}-003$ & \\
\hline 60.0 & 3.1 & $1.1394 \mathrm{E}-002$ & 1.0605E-002 & 1.2502E-002 & \\
\hline \multirow[t]{2}{*}{70.0} & 2.9 & 1.6375E-002 & 1.4758E-002 & 1.8275E-002 & $\nabla$ \\
\hline & Plot & Report & Exit & & \\
\hline
\end{tabular}

Distribution Quantile Level (in percent) - The probability level, which ranges from $0.5 \%$ to 99.5\%.

95\% Confidence Interval On Quantile Level in \% (+/-) - The 95\% confidence level on the quantile level (e.g., $5 \%, 50 \%, 90 \%$ ) expressed in terms of the percent of the quantile level. Note that this is the confidence on the quantile level, not the quantile value.

Quantile Value - The value obtained from the uncertainty analysis at the i'th \% quantile (or probability) level. The units on the value varies depending on the type of analysis (e.g., failure probability, core damage frequency, etc.).

95\% Lower Bound Confidence Level On Quantile Value - The lower bound confidence level on the quantile value.

95\% Upper Bound Confidence Level On Quantile Value - The upper bound confidence level on the quantile value. 
Plot - Show a graphical representation of the uncertainty distribution defined by the uncertainty quantile values.

Report - Generate a report containing the quantile values as displayed here.

Exit - Close the Uncertainty Quantile Values dialog.

\subsubsection{Plot Uncertainty Quantile Values}

\section{PURPOSE}

Show a graphical representation of the distribution defined by the uncertainty quantile (or probability) values associated with either the current or base case data.

\section{STEPS}

1. From the SAPHIRE menu select Fault Tree/Sequence/End State. The Fault Tree List/Sequences/End State List dialog will be displayed.

2. Highlight the desired fault tree and right-click to invoke the pop-up menu.

3. Choose Display, then the Uncertainty sub-menu option. The Fault Tree/Sequence/End State Uncertainty dialog will be displayed.

4. Choose the Current Quantile Values or Base Quantile Values button.

5. Choose the Plot button. The Uncertainty plot dialog will be displayed.

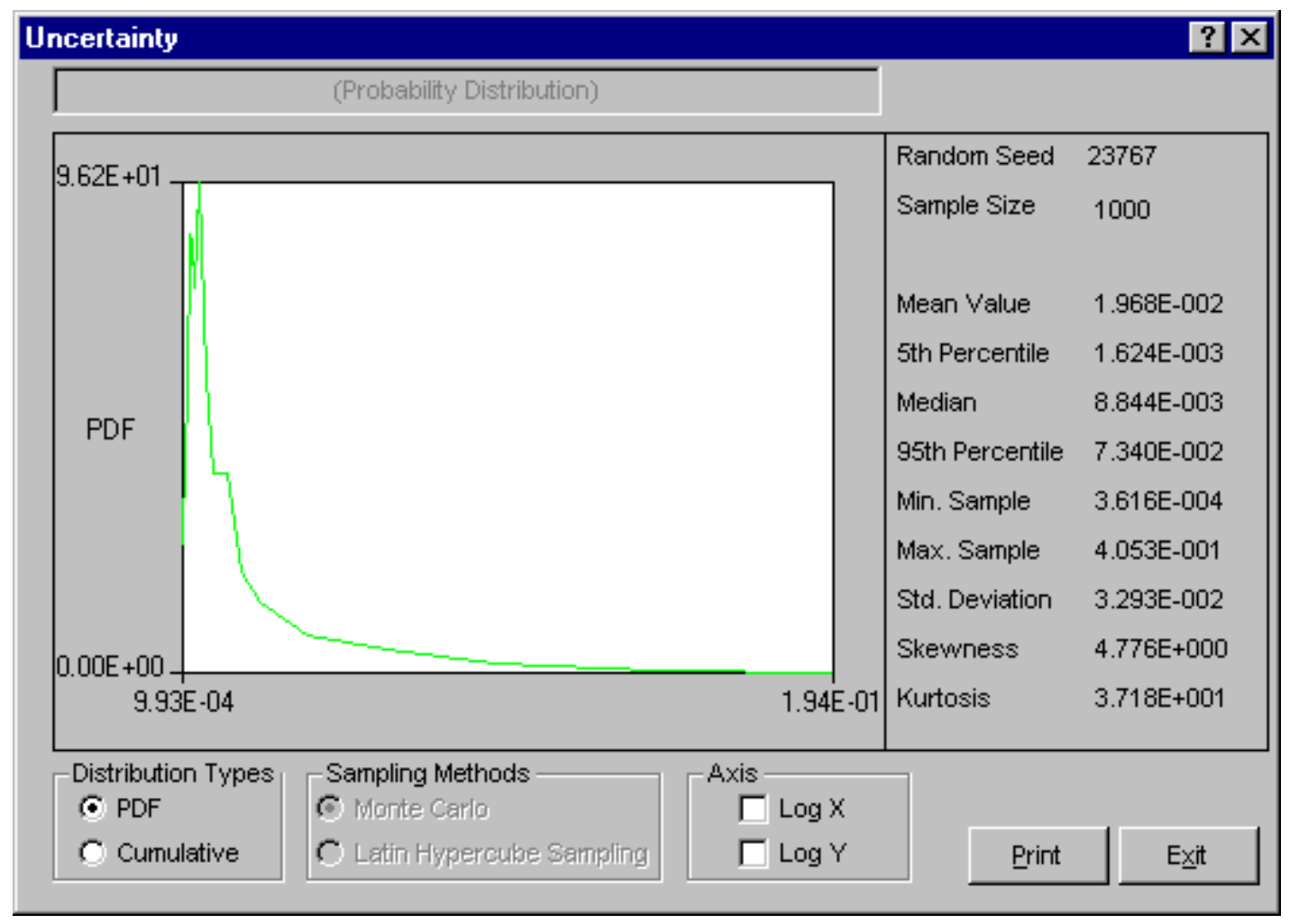

Print - Print the plot as displayed. The Print common dialog will be launched where you can select the desired printer.

Exit - Close the Uncertainty plot dialog. 


\subsubsection{Distribution Types}

Select the type of graph to be displayed:

PDF - $\quad$ (Default) - Displays a graph showing the Probability Density Function.

Cumulative - Displays a graph showing the Cumulative Density Function.

\subsubsection{Sampling Methods}

The selected radio button indicates the sampling method used for the uncertainty calculation. Note the sampling method used cannot be changed here. In order to view results using a different sampling method, the uncertainty analysis must be re-run using the desired sampling method.

Monte Carlo - $\quad$ Displays a graph that is based on Monte Carlo Sampling of the defined uncertainty distribution.

Latin Hypercube Sampling - Displays a graph that is based on Latin Hypercube Sampling of the defined uncertainty distribution.

7.8.6.6 Axis

$\log \mathbf{X}$ - Produces a graph where the Log base 10 of the X-Values are used instead of just the XValues.

$\log$ Y - Produces a graph where the Log base 10 of the Y-Values are used instead of just the YValues.

\subsection{Viewing Sequences}

\subsubsection{Viewing Sequence Data}

\section{PURPOSE}

This option presents the sequence analyses in various report forms. One or more sequences can be viewed using this option.

\section{STEPS}

1. From the SAPHIRE menu select Sequence. The Sequences dialog will be displayed.

2. Highlight the desired sequence(s) and right-click to invoke the pop-up menu.

3. Choose View Results from the menu. The Sequence Review dialog will be displayed. 


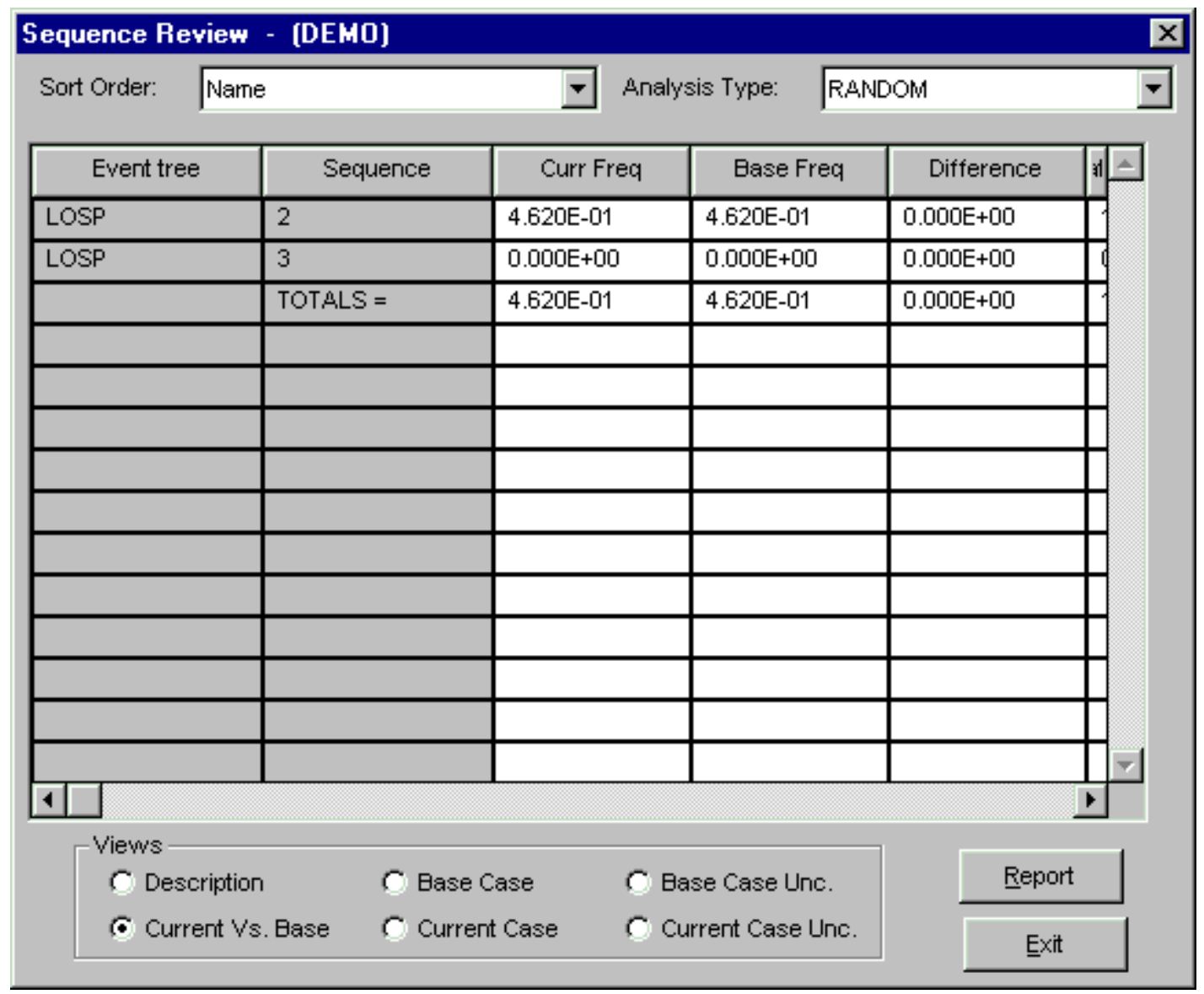

The selected sequences are displayed in this dialog. The Sequence column contains the sequences' primary names. Other columns' titles and data vary depending on the selected "View".

The last row in the grid show the summation totals of applicable columns.

Report - Produce a report of the data currently displayed.

Exit - $\quad$ Close the Sequence Review dialog.

\subsubsection{View Options}

Description -

Current Vs. Base -

Base Case -
Includes the full description. (60 character maximum)

Includes the current case cut set probability, base case cut set probability, the probability difference (current - base), ratio, current case cut set count, base case cut set count, the cut set count difference (current - base).

Includes base case values for cut set probability, mean, $5^{\text {th }}$ percentile, median, $95^{\text {th }}$ percentile, probability truncation, and size truncation used when solving. 
Current Case -

Base Case Uncertainty -

Current Case Uncertainty -
Includes current case values for cut set probability, mean, $5^{\text {th }}$ percentile, median, $95^{\text {th }}$ percentile, probability truncation, and size truncation used when solving.

Includes base case values for cut set probability, mean, $5^{\text {th }}$ percentile, median, $95^{\text {th }}$ percentile, minimum, maximum, standard deviation, skewness, kurtosis, sample size, and random number seed.

Includes current case values for cut set probability, mean, $5^{\text {th }}$ percentile, median, $95^{\text {th }}$ percentile, minimum, maximum, standard deviation, skewness, kurtosis, sample size, and random number seed.

\subsection{Time Dependent Analysis}

\subsubsection{Time Dependent Analysis}

\section{PURPOSE}

The time dependent analysis function allows the user to calculate a time profile of the selected fault tree or sequence cut sets. The user defines a start and stop "mission" time and a delta time or number of samples. From this data, SAPHIRE calculates a probability for each event in the cut sets using the start mission time. The cut sets are quantified and a min cut upper bound is determined. The mission time is then incremented by a delta and new event probabilities and a new min cut upper bound are calculated. Events that do not have mission time as part of their calculation type are not changed. This process continues until the mission time equals the stop time, at which point the results of the distribution are displayed. This allows the user to calculate a distribution of results for events that are dependent on time.

\section{STEPS}

1. From the SAPHIRE menu select Fault Tree/Sequence. The Fault Tree List/Sequences dialog will be displayed.

2. Highlight the desired list item(s) and right-click to invoke the pop-up menu.

3. Choose Time Dependent from the menu. The Time Dependent Calculation Values dialog will be displayed.

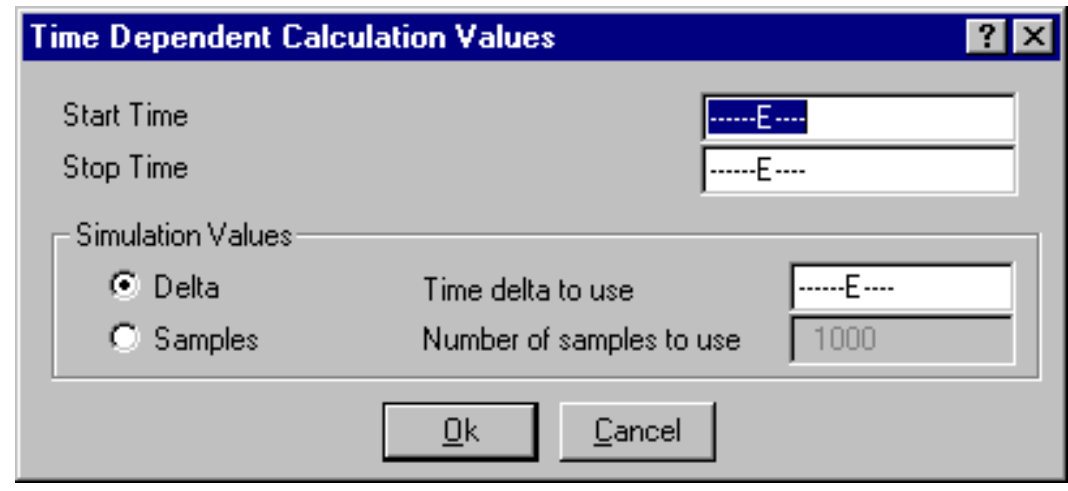


Start Time - The time, in hours, to begin the analysis.

Stop Time - $\quad$ The time, in hours, to end the analysis.

\section{Simulation Values}

Delta -

Time delta to use -

Sample -

Number of samples to use -

Ok -

Cancel -
If this radio button is selected, use the delta indicated in the Time delta to use field to determine the number of samples used in the analysis. The smaller the delta, the greater the number of samples.

The incremental change, in hours, for which the analysis will be performed beginning at the Start Time and terminating at the Stop Time.

If this radio button is selected, use the Number of samples to use field to determine the time delta used in the analysis. The greater the number of samples, the smaller the delta.

The total number of samples used for this analysis. The number of samples will determine the time delta to be used.

Begin the time analysis.

Close the Time Dependent Calculation Values dialog without calculating the time profile. 


\section{END STATE ANALYSIS}

\subsection{End State Analysis}

\section{PURPOSE}

This option provides the means to recalculate end state values after events and/or cut sets have been modified. The End State List dialog shows all end states defined for the current project, ordered by end state name.

\section{KEY TO FLAGS}

Located to the right of each end state is a set of flags that indicate the status of the end state:

b - base case cut sets exist for the selected analysis type

c - current case cut sets exist for the selected analysis type

\section{STEPS}

1. From the SAPHIRE menu select End State. The End State List dialog will be displayed.

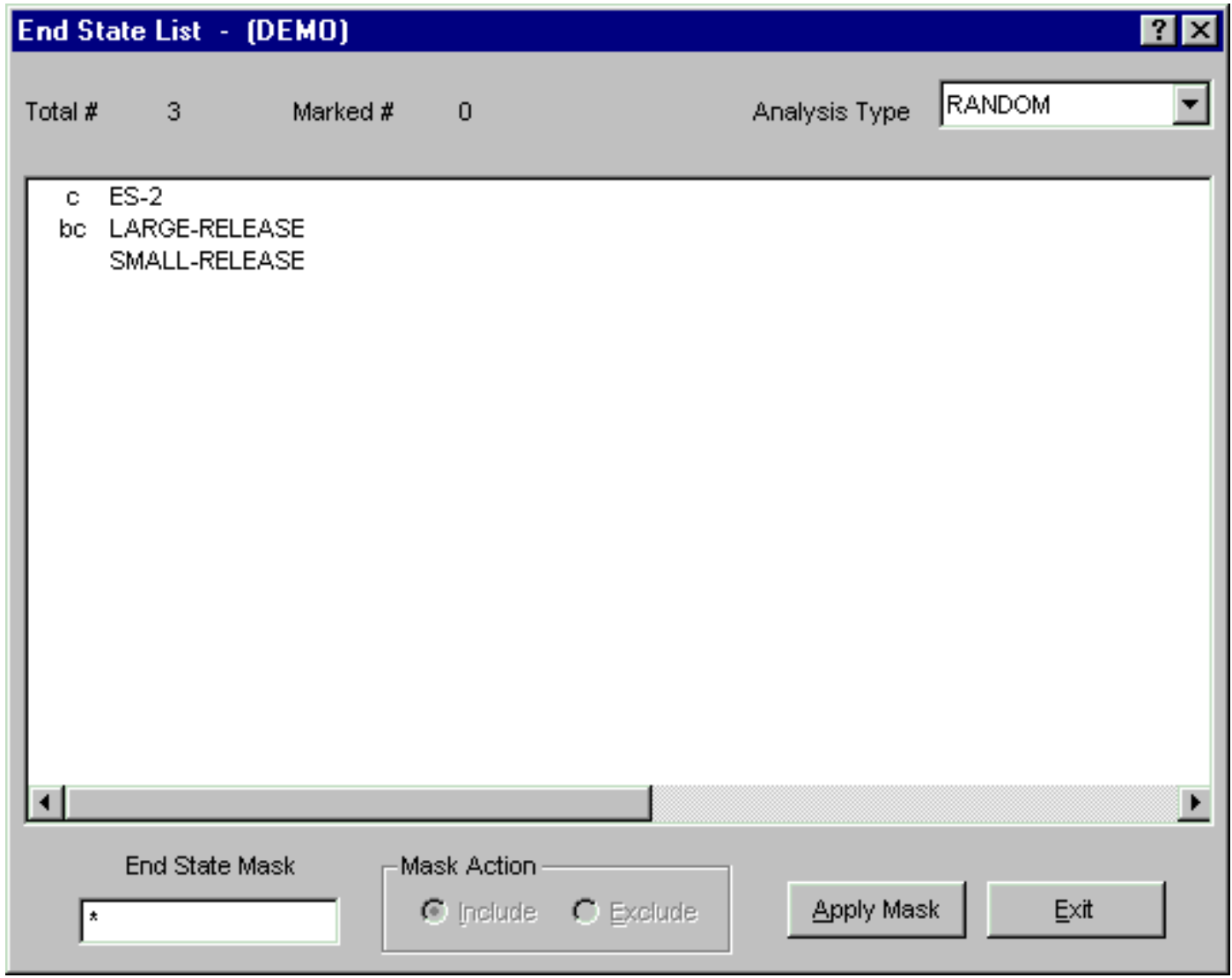


From this dialog, you can select end states using the mouse or the Mask feature. The analysis type can be selected from the drop-down list. Additionally, the following functions may be accessed from the pop-up menu:

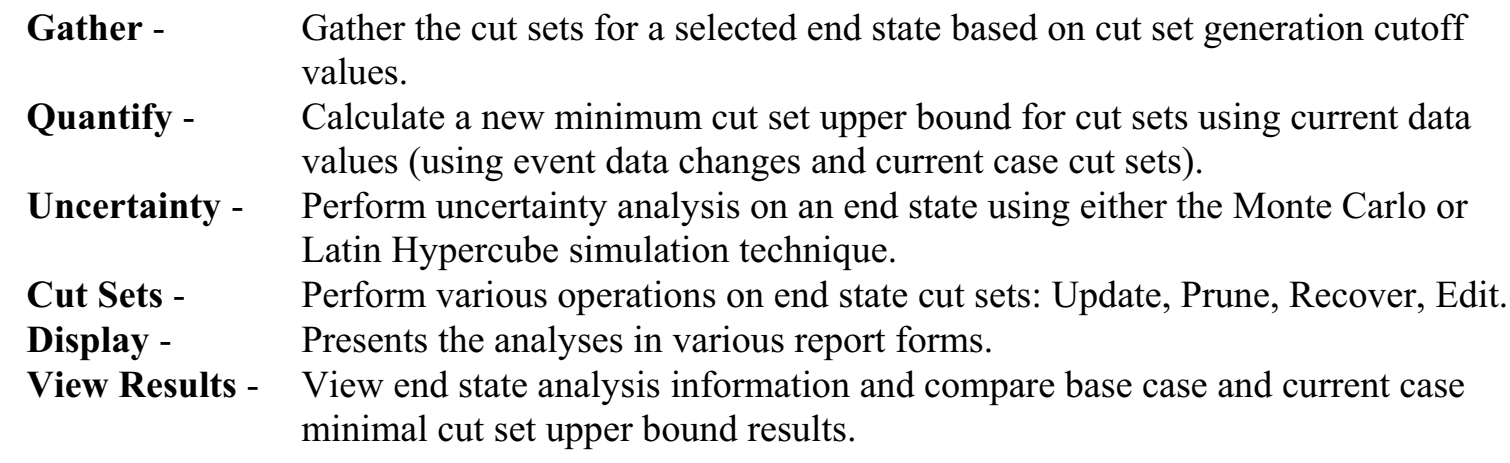

\subsection{Gathering End States}

\subsubsection{Gathering End States}

\section{PURPOSE}

This option allows you to gather the cut sets for a selected end state based on cut set generation cutoff values. You are given the opportunity to specify several cut set generation cutoff values that will be used to determine if a cut set is to be retained or discarded from the selected end state.

You are given the ability to generate cut sets for a selected end state, a group of end states, or all end states within the current project.

\section{STEPS}

1. From the SAPHIRE menu select End State. The End State List dialog will be displayed.

2. Highlight the desired end state(s) and right-click to invoke the pop-up menu.

3. Choose Gather.

Whether you are gathering cut sets for a single end state, a group of end states, or for all end states, the Cut Set Generation dialog will be displayed. 


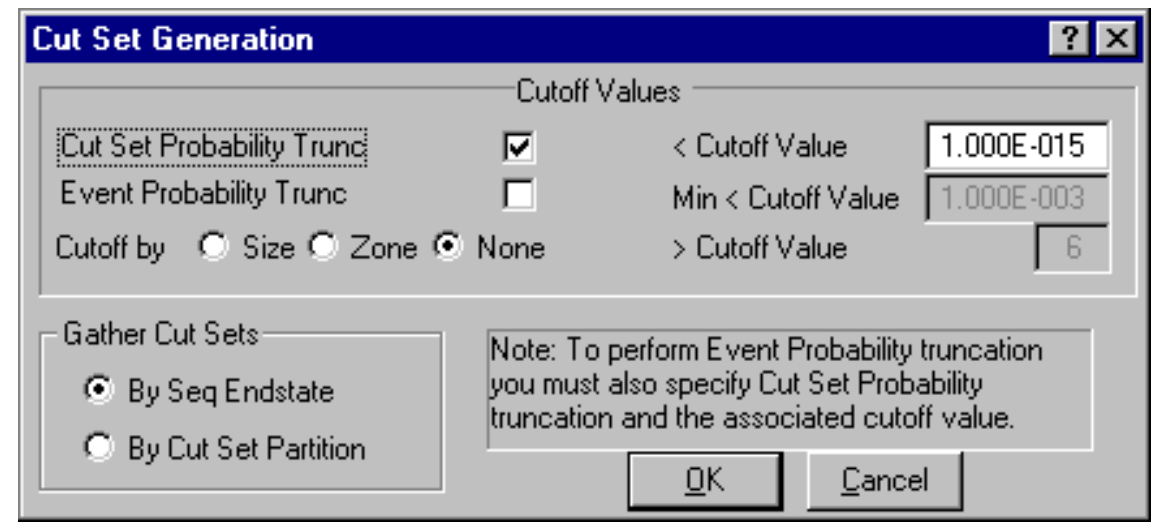

You may change any of the data fields on this dialog. The default values that appear on this dialog may be reset to new values by selecting Utility from the SAPHIRE menu and then invoking the Define Constants option.

Cutoff by Cut Set Probability - Used for determining if the cut set should be retained or discarded based on the value in the < Cutoff Value field.

Cutoff by Event Probability - Used for determining if the cut set should be retained or discarded based on the value in the Min < Cutoff Value field.

Cutoff by Size Used for determining if the cut set should be retained or discarded based on the value in the > Cutoff Value field or zoned flagged events.

Gather Cut Sets Used for determining the method for gathering cut sets.

During processing, the Cut Set Generation Results dialog is displayed and updated as the calculations proceed. Upon completion of the cut set generation, the summary results are displayed in this dialog. Choose the OK button to close the dialog, or View Results button to review or print the results.

The only limit on the number of cut sets that can be stored for a given fault tree is the available hard drive space. When processing is complete, the End State List dialog is updated to show the letter "c" in front of the end state(s) with current case cut sets.

\subsubsection{Cut Set Probability Trunc}

If you select this check box, then the only cut sets whose product for all of its event probabilities is greater than or equal to the value in the Cutoff Value field will be kept. All other cut sets will be removed.

If you deselect this check box, then the probability for the cut set will be irrelevant for determining if the cut set should be retained or discarded.

\subsubsection{Cutoff by Event Probability}

If you select this check box, then you must also choose the Cutoff by Cut Set Probability check box. This option will check all cut sets that are below the probability cutoff (Min < Cutoff Value field) and remove them only if they contain an event whose probability is below this value.

When you select his option, SAPHIRE will also perform the quantification. 


\subsubsection{Cutoff by Size}

Size - If you choose this radio button, then only the cut sets whose number of events is less than or equal to the value specified in the $>$ Cutoff Value field will be kept in the cut sets for that fault tree. All other cut sets will be removed.

Zone -If you choose this radio button, then only zone flagged events will be checked.

None- If you choose this radio button, then the number of events in a cut set will be irrelevant for determining if the cut set should be retained or discarded.

\subsubsection{Gather Cut Sets}

This option allows you to select which method to use for gathering cut sets.

By Seq Endstate - $\quad$ Gather cut sets by the end state assigned to each sequence (via the event tree graphic editor).

By Cut Set Partition - The end state of each cut set will be used for gathering. (via end state partition rules).

\subsection{Quantifying End States}

\subsubsection{Quantifying End States}

\section{PURPOSE}

The quantification process will calculate a new minimum cut set upper bound for the end state cut sets using the current data values (event change sets and current case cut sets). The new value is saved with the current case cut sets for the selected end state.

\section{STEPS}

1. From the SAPHIRE menu select End State. The End State List dialog will be displayed.

2. Highlight the desired end state(s) and right-click to invoke the pop-up menu.

3. Choose Quantify, then one of the sub-menu options.

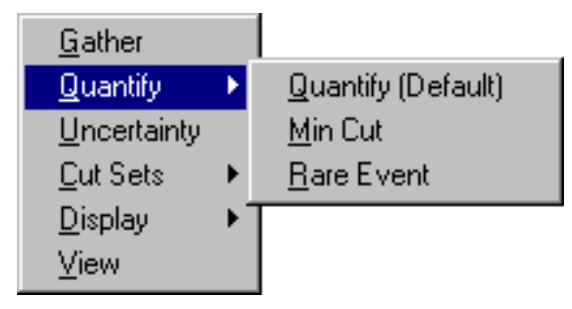

Quantify (Default) - $\quad$ Re-quantify using the quantification method for the specified end state. This is selected in the Modify | End State option.

Min Cut Re-quantify using the Minimal Cut Set Upper Bound Approximation. Rare Event Re-quantify by adding together the probabilities for the cut sets of a top event. 
If the selected quantification method differs from the end state's default, a warning dialog with the message, "The chosen quantification method was different from the End State default" is displayed. Choose the Ok button to continue processing.

During processing, the Quantification Results dialog is displayed. If an error occurs, the message, "Error quantifying cut sets" will be displayed in the message bar.

If a single end state was selected, upon completion of the quantification process, the results are displayed in the Quantification Results dialog. Choose Ok to close the dialog.

\subsubsection{Minimal Cut Set Upper Bound Approximation}

This calculation approximates the probability of the union of the minimal cut sets for the fault trees. The equation for the minimal cut set upper bound is

$$
S=1-\prod_{i=1}^{m}\left(1-C_{i}\right)
$$

where

$$
\begin{aligned}
& \mathrm{S}=\text { minimal cut set upper bound for the fault tree unavailability, } \\
& \mathrm{C}_{\mathrm{i}}=\text { probability of the } \mathrm{i}=\text { th cut set, and } \\
& \mathrm{m}=\text { the number of cut sets. }
\end{aligned}
$$

Example: If the cut sets for a fault tree are $\mathrm{X}=\mathrm{A} \cup \mathrm{B} \cup \mathrm{C}$ (i.e., the union of three events, $\mathrm{A}, \mathrm{B}$, and $\mathrm{C}$ ); then the cut sets can be written as $\mathrm{X}=\mathrm{A}+\mathrm{B}+\mathrm{C}$ with the plus symbol indicating union. The fault tree unavailability computed from the minimal cut set upper bound approximation is then $X=1-(1-A)(1$ B)(1 - C).

\section{SEE TECHNICAL REFERENCE}

\section{Minimal Cut Set Upper Bound}

\subsubsection{Rare Event Approximation}

The rare event approximation approach adds together the probabilities for the cut sets of a top event. This approximation is a good method when the cut set probabilities are small.

\section{SEE TECHNICAL REFERENCE}

Rare Event 


\subsection{Uncertainty Analysis}

\subsubsection{Uncertainty Analysis}

\section{PURPOSE}

This option can estimate the variability (due to the uncertainties in the basic event probabilities) of either a fault tree top event probability or an event tree sequence frequency. To do this, SAPHIRE provides two sampling techniques: Monte Carlo simulation and Latin Hypercube simulation. In addition, you may perform a seismic analysis to integrate the seismic fragility curve with the site hazard curve using the uncertainty analysis option.

In a traditional uncertainty analysis, SAPHIRE samples the user-specified distributions for each basic event in a group of cut sets, then quantifies these cut sets using the sample values. For a seismic uncertainty, however, SAPHIRE performs the analysis at each G-level in the site hazard curve for the current project. The seismic events are sampled at each new G-level and the quantification performed.

You are given the option of performing an uncertainty analysis on the current case cut sets for either a single, for a group, or for all of the fault trees (or sequences or end states) within the current project.

\section{STEPS}

1. From the SAPHIRE menu select Fault Tree/Sequence/End State. The Fault Trees List/Sequences/End State List dialog will be displayed.

2. Highlight the desired fault tree(s)/sequence(s)/end state(s) and right-click to invoke the pop-up menu.

3. Choose Uncertainty. The Uncertainty Calculation Values dialog will be displayed.

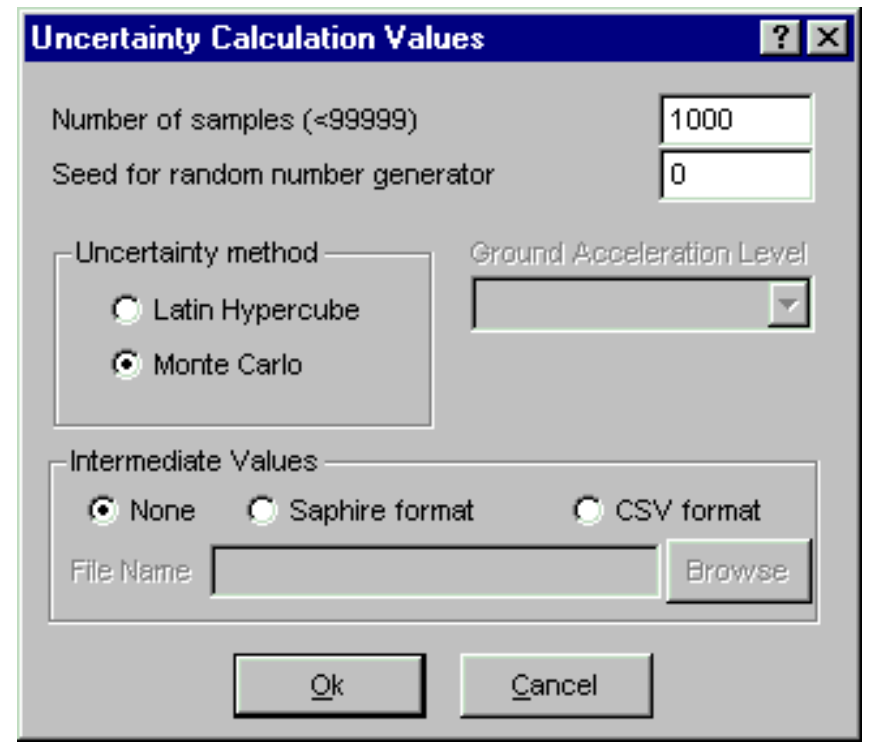

You may change any of the data fields on this dialog. The default values that appear on this dialog may be reset to new values by selecting the Utility $\mid$ Define Constants option. 
Number of samples $(<99999)$ -

Seed for random number generator -

Uncertainty method -

Ground Acceleration Level -

Intermediate Values None -

SAPHIRE format -

CSV format -

File Name -

Browse -
A default value is provided for the number of samples to use in simulation. You may use this value or enter another value. The number must be less than 99,999.

A default seed is provided for the random seed. You may use this value or enter a new value for the seed. To obtain a random seed from the system clock, you must enter a zero in this field.

Select the appropriate radio button for the desired uncertainty sampling technique.

If seismic uncertainty is selected, a ground level acceleration must be provided. This indicates the acceleration rate at which the component will always fail. The user can select to process all G-levels combined, all G-levels separately, or a specified G-level only.

Select this check box if you wish for the results and intermediate samples to be written to an ASCII file. Select this check box if you wish for the results and intermediate samples to be written to an ASCII file, formatted in the traditional SAPHIRE format.

Select this check box if you wish for the results and intermediate samples to be written to an ASCII file, formatted as a comma delimited file, for easy import into a spreadsheet program.

Specifies the name of the ASCII file where the intermediate values will be stored.

Opens the Choose File dialog, to select a directory and file name to store the intermediate results.

\subsubsection{Intermediate Values}

\section{PURPOSE}

This option allows you to output the results and intermediate samples to the ASCII file specified in the File Name field.

\section{STEPS}

1. From the SAPHIRE menu select Fault Tree/Sequences/End State. The Fault Trees List/Sequences/End State List dialog will be displayed.

2. Highlight the desired fault tree(s)/sequence(s)/end state(s) and right-click to invoke the pop-up menu.

3. Choose Uncertainty. The Uncertainty Calculation Values dialog will be displayed.

4. Check the Output Values check box and enter a name in the File Name field. 
The example below describes the layout of a SAPHIRE formatted ASCII file. The CSV (comma delimited file) layout is similar, but is comma delimited to be easily imported into a spreadsheet program.

Format for ASCII file containing intermediate samples:

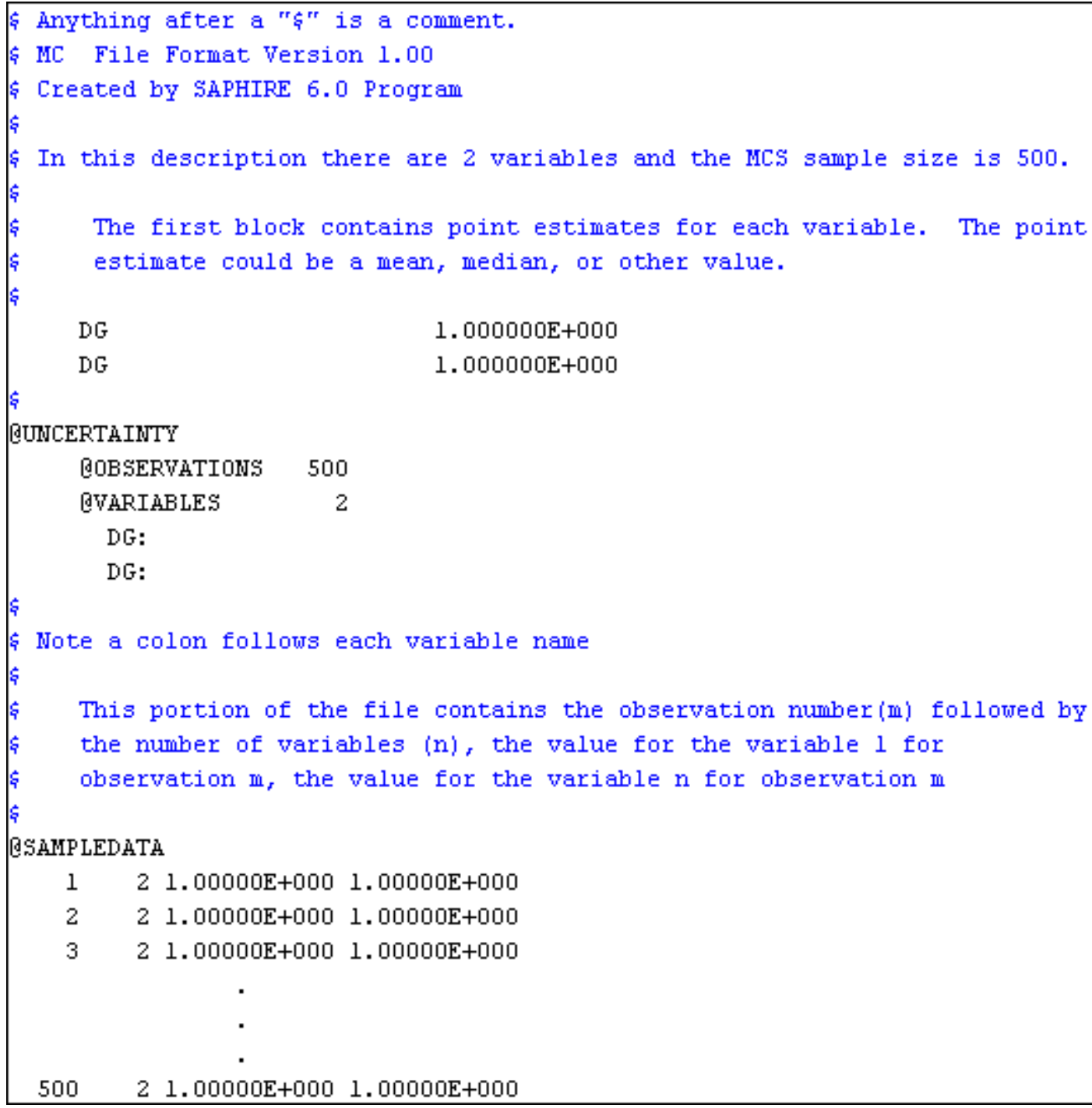

\subsubsection{Monte Carlo Sampling (MCS)}

Simple MCS is a fundamental uncertainty sampling approach. To perform the sampling, SAPHIRE makes repeated quantifications of the fault tree/sequence/end state cutsets using samples from the basic event uncertainty distributions. This type of sampling requires more samples than LHS for the same degree of accuracy.

When using this sampling technique, if the number of samples entered is less than ten, then the number of samples will be increased to ten before the uncertainty analysis process will continue. Any number of samples greater than or equal to ten will be allowed, but a number of at least 1000 is probably a better value for improving the reliability of the Monte Carlo results. 


\subsubsection{Latin Hypercube Sampling (LHS)}

LHS is a stratified sampling technique where the random variable distributions are divided into equal probability intervals. A probability is randomly selected from within each interval for each basic event. Generally, LHS will require fewer samples than simple MCS for similar accuracy. However, due to the stratification method, it may take longer to generate a value than for a MCS.

When using this technique, if the number of samples entered is less than twice the total number of unique events in the fault tree/sequence/end state, then the number of samples will be increased to two times the total number of unique events before the analysis will continue. The LHS technique gives its best results if the number of samples is at least twice the total number of unique events.

\subsubsection{The Uncertainty Analysis Process}

The following is a description of the uncertainty analysis process performed by SAPHIRE after values have been entered in the Uncertainty Calculation Values dialog.

Once the number of samples has been accepted and a seed obtained from the system clock (if necessary), checks will be run to ensure the events with the same correlation classes have consistent failure data, uncertainty data, and distribution types. If any events with inconsistencies exist, an error message will be displayed and the uncertainty analysis process will be terminated so that the inconsistent values may be corrected.

If an error occurs during the uncertainty analysis process, the process is terminated and a message box providing information about the specific error is displayed.

If all of the events successfully pass the correlation class checks, then the distribution parameters for the events will be checked to ensure that they are valid. If any of the parameters are invalid, error messages will be displayed and the process will be terminated so the distribution parameters may be corrected.

After both of these checks have been passed, a point estimate will be calculated for the selected fault tree (or sequence or end state). At this point the samples for each event will be generated using the selected sampling technique, either the Monte Carlo Sampling technique or the Latin Hypercube Sampling technique. The uncertainty analysis function provides you with 11 different distribution types for both sampling techniques. The distribution types include normal, lognormal, beta, gamma, chi-squared, exponential, uniform, Dirichlet, constrained non-informative, maximum entropy, and the user-defined histograms.

During processing, the Uncertainty Results dialog will be displayed and updated as the samples are generated. When the requested number of samples have been generated, statistical information will be calculated using the generated samples. A sample mean, median, and standard deviation will be calculated for the selected fault tree (or sequence or end state). Coefficients of skewness and kurtosis, and quantile values will also be calculated. This data will be saved in the database for the selected fault tree (or sequence or end state).

Upon completion of these calculations, the following values will be displayed on the Uncertainty Results dialog for viewing: the name, random seed used, the number of samples generated in this process, the total number of events and cut sets being processed, the point estimate, the mean, the median, the 5th and 95th percentile values, the minimum and maximum generated sample values, the standard deviation, the skewness and kurtosis, and the time involved to perform the analysis. 


\begin{tabular}{|c|c|c|}
\hline Uncertainty Results & \multicolumn{2}{|r|}{$\mathrm{x}$} \\
\hline \multicolumn{3}{|l|}{ Name } \\
\hline Random Seed 41877 & Events & 10 \\
\hline Sample Size $\quad 1000$ & Cut Sets & 15 \\
\hline Point estimate & \multicolumn{2}{|c|}{$2.120 \mathrm{E}-002$} \\
\hline Mean Value & \multicolumn{2}{|c|}{$2.047 \mathrm{E}-002$} \\
\hline 5th Percentile Value & \multicolumn{2}{|c|}{$1.524 \mathrm{E}-003$} \\
\hline Median Value & \multicolumn{2}{|c|}{$8.788 \mathrm{E}-003$} \\
\hline 95th Percentile Value & \multicolumn{2}{|c|}{$6.852 E-002$} \\
\hline Minimum Sample Value & \multicolumn{2}{|c|}{$2.150 \mathrm{E}-004$} \\
\hline Maximum Sample Value & \multicolumn{2}{|c|}{ 6.591E-001 } \\
\hline Standard Deviation & \multicolumn{2}{|c|}{$4.138 \mathrm{E}-002$} \\
\hline Skewness & \multicolumn{2}{|c|}{$7.312 \mathrm{E}+000$} \\
\hline Kurtosis & \multicolumn{2}{|c|}{$8.155 E+001$} \\
\hline Elapsed Time & \multicolumn{2}{|c|}{ 00:00:00.690 } \\
\hline \multicolumn{3}{|c|}{ Cancel } \\
\hline
\end{tabular}

If only one fault tree (or sequence or end state) was selected (highlighted) for the uncertainty analysis process, then you will need to close the Uncertainty Results dialog. However, if more than one is being processed, the Uncertainty Results dialog will be displayed for each, and when all of the selected fault trees (or sequences or end states) have been processed, the Uncertainty Results dialog will automatically be closed.

\subsubsection{Seismic Uncertainty}

\section{PURPOSE}

A seismic analysis integrates the seismic fragility curve with the site hazard curve using the uncertainty analysis option. If the user has chosen to perform a seismic analysis, then a G-level was also selected. This option allows the user to specify how SAPHIRE performs the analysis.

\section{STEPS}

1. From the SAPHIRE menu select Fault Tree/Sequence/End State. The Fault Trees List/Sequences/End State List dialog will be displayed.

2. Select the "SEISMIC" value from the Analysis Type drop-down list.

3. Highlight the desired fault tree(s)/sequence(s)/end state(s) and right-click to invoke the pop-up menu.

4. Choose Uncertainty. The Uncertainty Calculation Values dialog will be displayed.

5. Provide appropriate values for the data entry fields on this dialog and choose one of the Ground Acceleration Level values.

The G-levels are described here: 
ALL COMBINED - SAPHIRE performs an uncertainty analysis on all G-levels defined in the site hazard curve for this project. The combined results are then stored in the database.

ALL SEPARATE - SAPHIRE performs an uncertainty analysis on all G-levels, but does the analysis and stores the results separately for each G-level.

Specific G-level - $\quad$ SAPHIRE only performs the analysis for that specified G-level.

\subsubsection{Uncertainty Distributions for Basic Events}

Within the SAPHIRE code, eleven types of uncertainty distributions are supported. The table below lists the different distributions, their identifier within SAPHIRE, and the uncertainty parameter that is needed by the code.

Along with the uncertainty parameter, most distributions require a second parameter. SAPHIRE requires that the mean value be specified for all distributions. The mean value is put in the database as a mean failure probability or a mean failure rate, depending on which calculation type is used.

Basic event uncertainty distribution types supported by SAPHIRE.

\begin{tabular}{lll} 
Distribution & Identifier & Uncertainty Parameter \\
none & blank & none \\
lognormal & L & error factor \\
normal & N & standard deviation \\
beta & B & b in Beta $(\mathrm{a}, \mathrm{b})$ \\
gamma & $\mathrm{G}$ & $\mathrm{r}$ in $\Gamma(\mathrm{r})$ \\
chi-squared & $\mathrm{C}$ & degrees of freedom \\
exponential & $\mathrm{E}$ & none \\
uniform & $\mathrm{U}$ & upper end point \\
histograms & $\mathrm{H}$ & histogram number (i.e., identifier) \\
maximum entropy & $\mathrm{M}$ & lower and upper end point \\
constrained noninformative & $\mathrm{O}$ & none \\
Seismic & $\mathrm{S}$ & Beta $\mathrm{r}$, Beta $\mathrm{u}$ \\
\hline
\end{tabular}

If the event was assumed to be normally distributed, we would simply put the mean value in the probability data field and the standard deviation in the uncertainty parameter data field. To enter this distribution, the mean value of 0.5 would be entered, a normal distribution would be chosen (type N), and the standard deviation of 0.2 would be specified.

To review the process of specifying an uncertainty distribution in SAPHIRE, the required steps are to: 
1. Enter the mean value for the basic event.

2. Choose the distribution type (from those shown in the "Identifier" column in the table above).

3. Specify the appropriate uncertainty distribution parameter (shown in the "Uncertainty Parameter" column in the table above).

Additional information on the uncertainty distributions and their parameters can be found in the SAPHIRE Technical Reference Manual.

\subsection{End State Cut Set Analysis}

\subsubsection{End State Cut Set Analysis}

\section{PURPOSE}

This option provides the ability to perform a variety of analysis functions on the end state cut sets.

\section{STEPS}

1. From the SAPHIRE menu select End State. The End State List dialog will be displayed.

2. Highlight the desired end state(s) and right-click to invoke the pop-up menu.

3. Choose Quantify, then one of the sub-menu options.

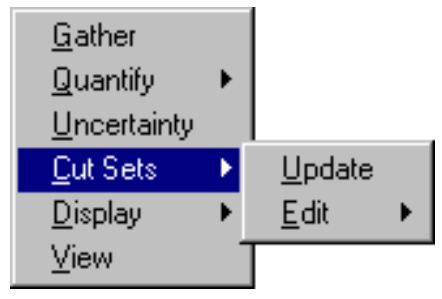

Update - $\quad$ Update the current cut sets for selected end state(s) based on cut set generation cutoff values.

Edit - $\quad$ Modify the base case/current end state cut sets.

\subsubsection{Updating Cut Sets}

\section{PURPOSE}

This option will update the current case cut sets for a selected fault tree (or sequence or end state) based on cut set generation cutoff values. You are given the opportunity to specify several cut set generation cutoff values that will be used to determine if a cut set is to be retained or discarded. You are also given the choice of using either the base case cut sets or the current case cut sets as the starting set of cut sets to be updated. The updated version of the cut sets will be saved as the new current case cut sets. 
Current case cut sets can be updated for a selected fault tree (or sequence or end state), a group of fault trees (or sequences or end states), or all of the fault trees (or sequences or end states) within the current project.

\section{STEPS}

1. From the SAPHIRE menu select Fault Tree/Sequence/End State. The Fault Trees List/Sequences/End State List dialog will be displayed.

2. Highlight the desired fault tree(s)/sequence(s)/end state(s) and right-click to invoke the pop-up menu.

3. Choose Cut Sets, then the Update sub-menu option.

4. The Cut Set Generation Truncation dialog will be displayed.

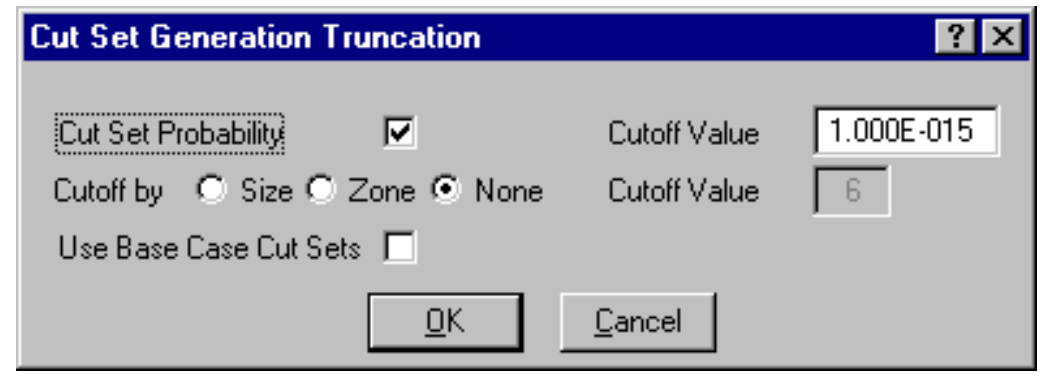

You may change any of the data fields on this dialog. The default values that appear on this dialog may be reset to new values by selecting Utility on the SAPHIRE menu and then invoking the Define Constants option.
Cut Set Probability -
Used for determining if the cut set should be retained or
Cutoff by Size -
discarded based on the value in the Cutoff Value field.
Used for determining if the cut set should be retained or discarded based on zoned flagged events or the value in the Cutoff Value field.
Use Base Case Cut Sets -
Used to specify base case cut sets or current case cut sets.

During processing, the Cut Set Generation Results dialog is displayed and updated as the calculations proceed. If a single fault tree (or sequence or end state) was selected, upon completion of the cut set generation, the results are displayed in this dialog. Choose the Ok button to close the dialog.

Once the cut sets are updated, they are automatically quantified.

\subsubsection{Cut Set Generation Truncation}

\subsubsection{Cut Set Probability}

If you select this check box, then only the cut sets whose product for all of its event probabilities is greater than or equal to the value in the Cutoff Value field will be kept. All other cut sets will be removed from current case cut sets for that fault tree.

If you uncheck this box, then the probability for the cut set will not be relevant for determining if the cut set should be retained or discarded. 


\subsubsection{Cutoff by Size}

Size - If you choose this radio button, then only the cut sets whose number of events is less than or equal to the value specified in the $>$ Cutoff Value field will be kept in the cut sets for that fault tree. All other cut sets will be removed.

Zone -If you choose this radio button, then only zone flagged events will be checked.

None -If you choose this radio button, then the number of events in a cut set will be irrelevant for determining if the cut set should be retained or discarded.

\subsubsection{Use Base Case Cut Sets}

If you select this check box, then base case cut sets will be used as the cut sets to be updated and then stored in the current case cut sets. However, if unchecked, the current cut sets will be used as the cut sets to be updated and then resaved in the current case cut sets.

\subsubsection{Editing End State Cut Sets}

\subsubsection{Editing Cut Sets}

\section{PURPOSE}

The Cut Set Editor provides you with the means to edit the fault tree (or sequence or end state) cut sets. SAPHIRE provides space for two sets of cut sets in the database: base case and current case. Whenever SAPHIRE generates cut sets, they are stored in the current case location. The user may save a set of cut sets to the base case location by performing a Base Case Update. The user can choose to edit either base case or current cut sets; however, the results of the editing are always stored in the current case location.

Using the cut set editor, you can insert new cut sets, delete cut sets, or add or modify basic events in the cut sets. Any event name entered during cut set editing may be preceded by a "/" to indicate that it is to be treated as a complemented event. The probability of a complemented event is one minus the failure probability.

\section{STEPS}

1. From the SAPHIRE menu select Fault Tree/Sequence/End State. The Fault Trees List/Sequences/End State List dialog will be displayed.

2. Highlight the desired record(s) and right-click to invoke the pop-up menu.

3. Choose Cut Sets, then the Edit sub-menu option.

4. Choose either Current or Base from the sub-menu. The Cut Set Editor dialog will be displayed. 


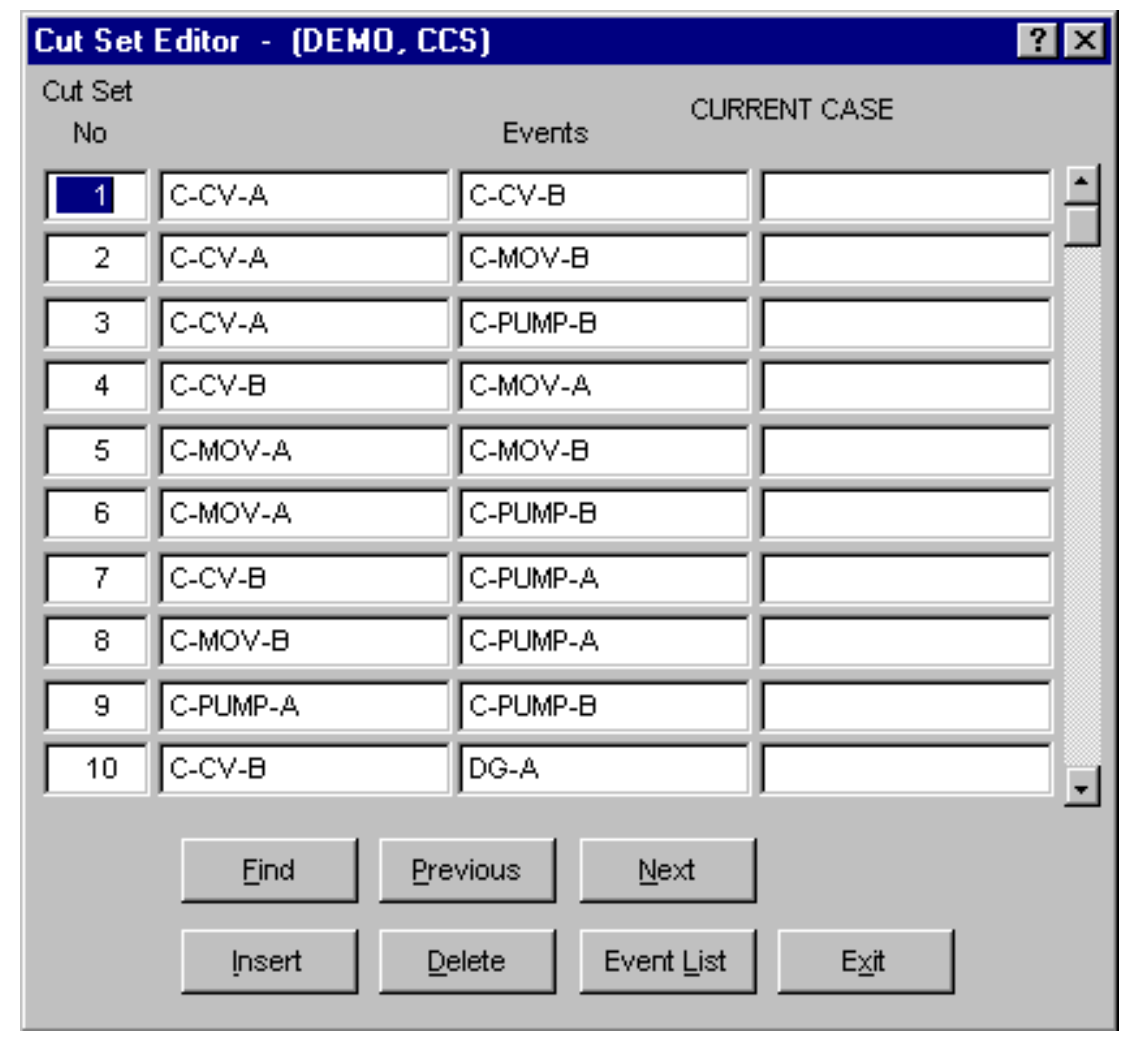

Find Locate the cut set(s) containing the marked basic event(s).

Previous - $\quad$ Locate the previous cut set containing the event(s) matching the criteria of most recent "Find" operation.

Next - $\quad$ Locate the next cut set containing the event(s) matching the criteria of most recent "Find" operation.

Insert - $\quad$ Add a new cut set or basic event.

Delete - Delete an existing cut set or basic event.

Event List - $\quad$ List the basic events in the data base.

Exit -

Close the Cut Set Editor dialog. The Cut Set Editor message box is displayed with three options:

Yes - Close the dialog and save changes to the cut sets.

No - Close the dialog without saving changes to the cut sets.

Cancel - Do not close the dialog. Changes are not saved.

\subsubsection{Find}

\section{PURPOSE}

This option locates the cut set(s) containing the selected criteria.

\section{STEPS}

1. From the SAPHIRE menu select Fault Tree/Sequence/End State. The Fault Trees List/Sequences/End State List dialog will be displayed. 
2. Highlight the desired record(s) and right-click to invoke the pop-up menu.

3. Choose Cut Sets, then the Edit sub-menu option.

4. Choose either Current or Base from the sub-menu. The Cut Set Editor dialog will be displayed.

5. Choose the Find button. The Event List dialog will be displayed.

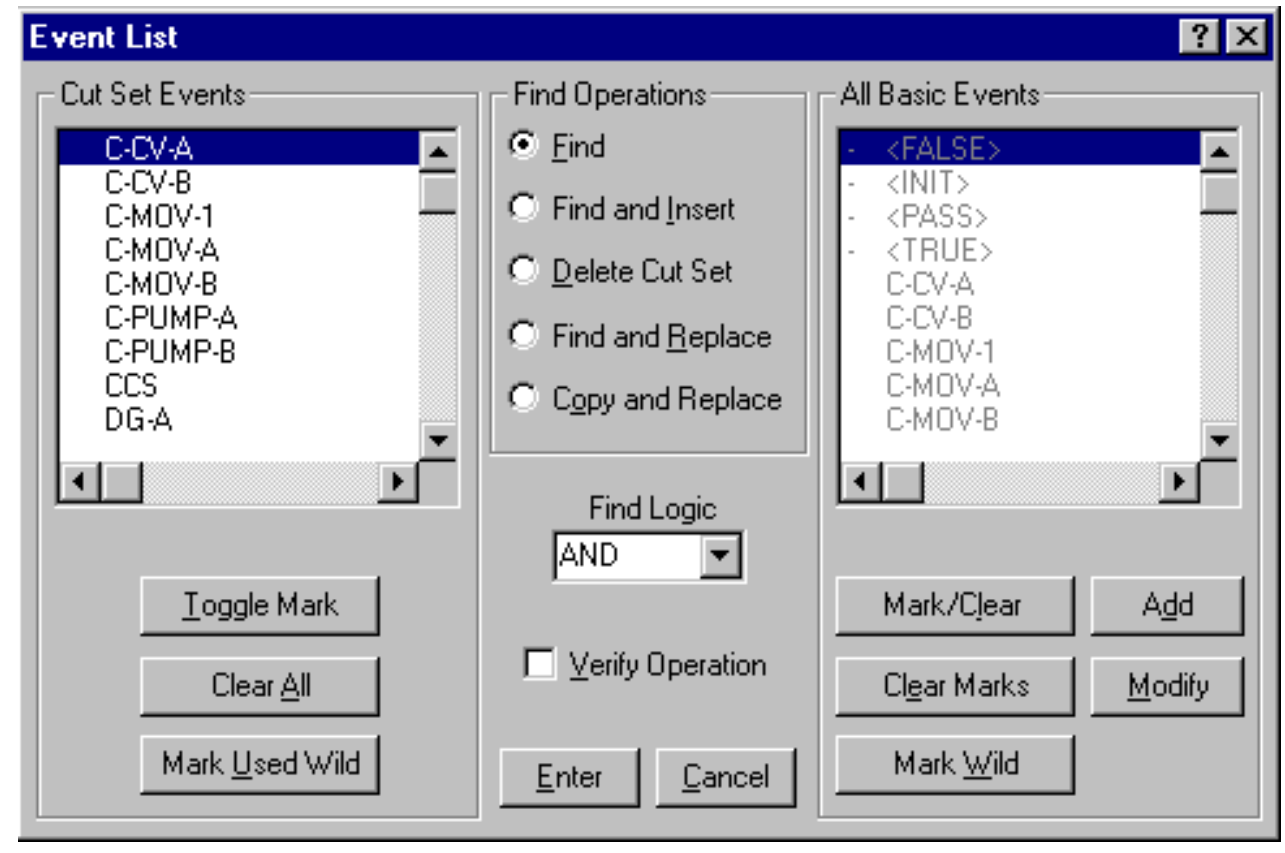

The Event List dialog consists of four sections. The functions that apply to each section are discussed briefly:

\section{Cut Set Events}

Basic Event List -

Toggle Mark -

Clear All -

Mark Used Wild -

Find Operations

Find -

Find and Insert -
Contains all basic events used in the cut sets being edited.

Marks or unmarks selected events in the Cut Set Events list. An asterisk

$\left(^{*}\right)$ next to the event name indicates that it is currently marked. A slash

(/) indicates that the event is marked as a complimented event.

Removes all marks from the events in the Cut Set Events list.

Marks events in the Cut Set Events list based on matching criteria. The Event Class Mask dialog is displayed.

Locate the cut set(s) containing the marked event(s) in the Cut Set Events list using the selected Find Logic.

Locate the cut sets(s) containing the marked event(s) in the Cut Set Events list using the selected Find Logic and insert the marked event(s) from the All Basic Events list. The new events are inserted into the cut set after the "find" event(s). 
Delete Cut Set - $\quad$ Remove the cut set(s) containing the marked event(s) in the Cut Set Events list using the selected Find Logic.

Find and Replace - $\quad$ Locate the cut sets(s) containing the marked event(s) in the Cut Set Events list using the selected Find Logic and replace them with the marked event(s) from the All Basic Events list.

Copy and Replace - Copy the cut sets(s) containing the marked event(s) in the Cut Set Events list using the selected Find Logic and replace the event(s) with the marked event(s) from the All Basic Events list. This creates a new cut set for each cut sets matching the "find" criteria.

All Basic Events - This section is enabled for use with the Find and Insert, Find and Replace, and Copy and Replace functions:

Basic Event List - $\quad$ Contains all basic events in the database.

Mark/Clear - $\quad$ Marks or unmarks selected events in the All Basic Events list.

Clear Marks - $\quad$ Remove all marks from the events in the All Basic Events list.

Mark Wild - $\quad$ Marks events in the All Basic Events list based on matching criteria. The Event Class Mask dialog is displayed.

Add Add a new basic event to the database. This new event will appear in the

Modify All Basic Events list. Modify the selected event in the All Basic Events list.

\section{Other options:}

Find Logic drop-down list - Allows selection between the logical AND and OR operations. The selected logical operation is applied to the events in the Cut Set Events.

Verify Option - $\quad$ If this check box is selected, you are prompted to confirm the operation for each successful find. If this check box is deselected, the operation will be performed immediately.

Enter - $\quad$ Perform the "find" operation using the currently selected criteria.

Cancel - Do not perform the "find" operation and close the Event List dialog.

\subsubsection{Insert}

\section{PURPOSE}

This option allows you to insert a new cut set or basic event. If the I-bar is in a Cut Set No cell (i.e., the first column of cells on the Cut Set Editor dialog), then a row for a cut set will be added at the end of the cut set list. If the I-bar is in one of the Events cells, and the last event row for the current cut set (i.e., all three cells in the current cut set row) is filled, then a row for the current cut set will be added.

\section{STEPS}

1. From the SAPHIRE menu select Fault Tree/Sequence/End State. The Fault Trees List/Sequences/End State List dialog will be displayed.

2. Highlight the desired record(s) and right-click to invoke the pop-up menu.

3. Choose Cut Sets, then the Edit sub-menu option. 
4. Choose either Current or Base from the sub-menu. The Cut Set Editor dialog will be displayed.

5. Place the cursor in the desired cell and choose the Insert button.

\subsubsection{Delete}

\section{PURPOSE}

This option allows you to delete an existing cut set or basic event. If the I-bar is in a Cut Set No cell (i.e., the first column of cells on the Cut Set Editor dialog), then the entire cut set will be deleted. If the I-bar is in one of the Events cells, then the event in that cell will be removed from the cut set.

\section{STEPS}

1. From the SAPHIRE menu select Fault Tree/Sequence/End State. The Fault Trees List/Sequences/End State List dialog will be displayed.

2. Highlight the desired record(s) and right-click to invoke the pop-up menu.

3. Choose Cut Sets, then the Edit sub-menu option.

4. Choose either Current or Base from the sub-menu. The Cut Set Editor dialog will be displayed.

5. Place the cursor in the desired cell and choose the Delete button.

If you are deleting a cut set, you will be prompted:

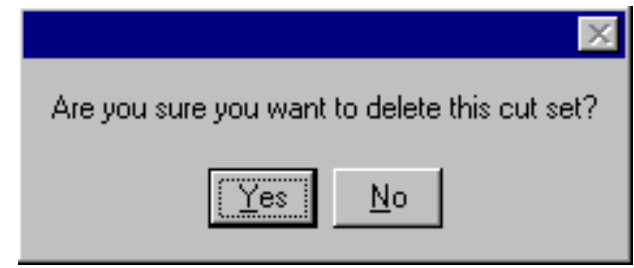

Yes - Continue the delete operation.

No - Cancel the delete operation.

\subsubsection{Event List}

\section{PURPOSE}

This option lists all the basic events in the database. From this dialog you can add a new event to the database, modify an existing event's data, or select the event to be included in the current cut set. The cursor must be positioned in one of the Events cells in order to activate this option. The selected event will replace an existing event in the current cell.

\section{STEPS}

1. From the SAPHIRE menu select Fault Tree/Sequence/End State. The Fault Trees List/Sequences/End State List dialog will be displayed.

2. Highlight the desired record(s) and right-click to invoke the pop-up menu.

3. Choose Cut Sets, then the Edit sub-menu option.

4. Choose either Current or Base from the sub-menu. The Cut Set Editor dialog will be displayed.

5. Place the cursor in the desired Event cell. 
6. Choose the Event List button. The Event List dialog will be displayed.

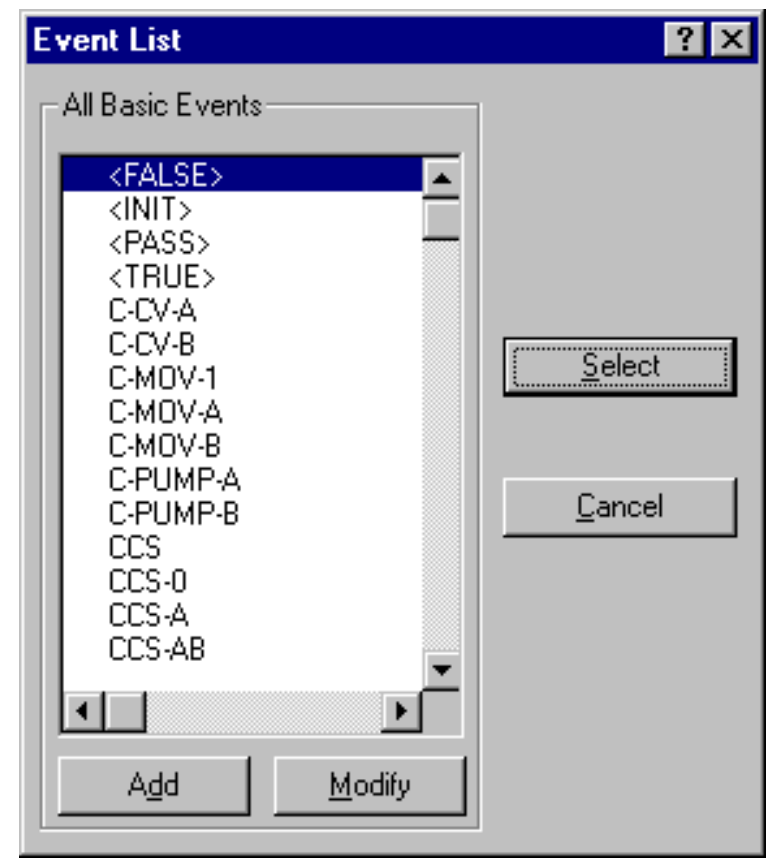

Add - $\quad$ Add basic event to the data base. The Basic Event data entry dialog will be displayed.

Modify - $\quad$ Modify the data of the highlighted basic event. The Basic Event data entry dialog will be displayed.

Select - $\quad$ Include the highlighted basic event in the cut set. The selected event will be placed in the cell currently occupied by the cursor.

Cancel - $\quad$ Close the Event List dialog and do not change the current cell.

\subsection{Displaying End State Results}

\subsubsection{Displaying End State Results}

\section{PURPOSE}

This option displays the results of end state analysis functions. You can view the current case cut sets and uncertainty analysis results, and perform importance measure analyses.

STEPS

1. From the SAPHIRE menu select End State. The End State List dialog will be displayed.

2. Highlight the desired end state(s) and right-click to invoke the pop-up menu.

3. Choose Display, then one of the sub-menu options. 


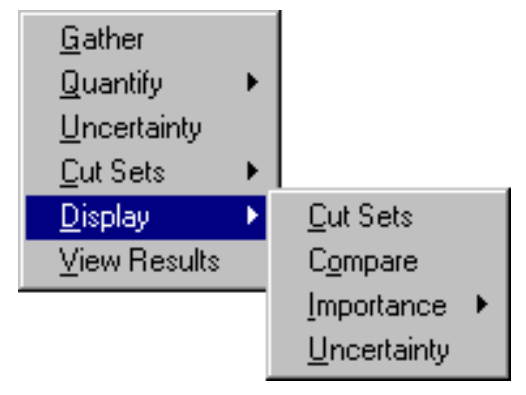

Cut Sets - $\quad$ Display the end states' cut sets, their percent of contribution to the end state, the frequency, and the event names that make up the cut sets.

Comparison - Display the end states' base and current cut sets, their percent of contribution to the end state, the frequency, and the event names that make up the cut sets.

Importance - Perform and display "reliability-worth" information about the basic events in the cut sets.

Uncertainty - Display uncertainty analysis results.

\subsubsection{Displaying Cut Sets}

\subsubsection{Displaying Cut Sets}

\section{PURPOSE}

This option displays the current case cut sets. Three tabbed pages provide different views of the cut sets. The first tab, labeled Full List, contains the complete list of cut sets for the selected fault tree (or sequences or end states). This page always contains the complete set of cut sets found for the selected fault tree/sequences/end state.

When the dialog is invoked, the fault tree (or sequence or end state) cut sets are shown. Displayed with each cut set is its associated percent of contribution to the minimal cut set upper bound, its probability (or, perhaps, frequency), and the event names that make up the cut sets. Also displayed are the minimal cut set upper bound for the total, the number of cut sets that make up the total, the current slice minimal cut set upper bound, the percentage that the slice contributes to the total, and the number of cut sets in the slice.

The Included In List and Excluded From List tabs will not contain data until a slice has been defined, via one of the Slice By options.

The second tab, labeled Included In Slice, contains those cut sets specified (qualified) by the user via either the Remove and/or Slice By options. The third tab, labeled Excluded From Slice, contains the inverse of the second tab; it contains those cut sets not included in the qualified cut set list. The summary information at the top of each page shows the summary information for just the cut sets shown on the current page, and also in relation to the full list of cut sets.

\section{STEPS}

1. From the SAPHIRE menu select Fault Tree/Sequence/End State. The Fault Trees List/Sequences/End State List dialog will be displayed.

2. Highlight the desired list item(s) and right-click to invoke the pop-up menu.

3. Choose Display, then the Cut Sets sub-menu option. The Selected Cut Sets dialog will be displayed. 
Full List | Included In Slice | Excluded From Slice |

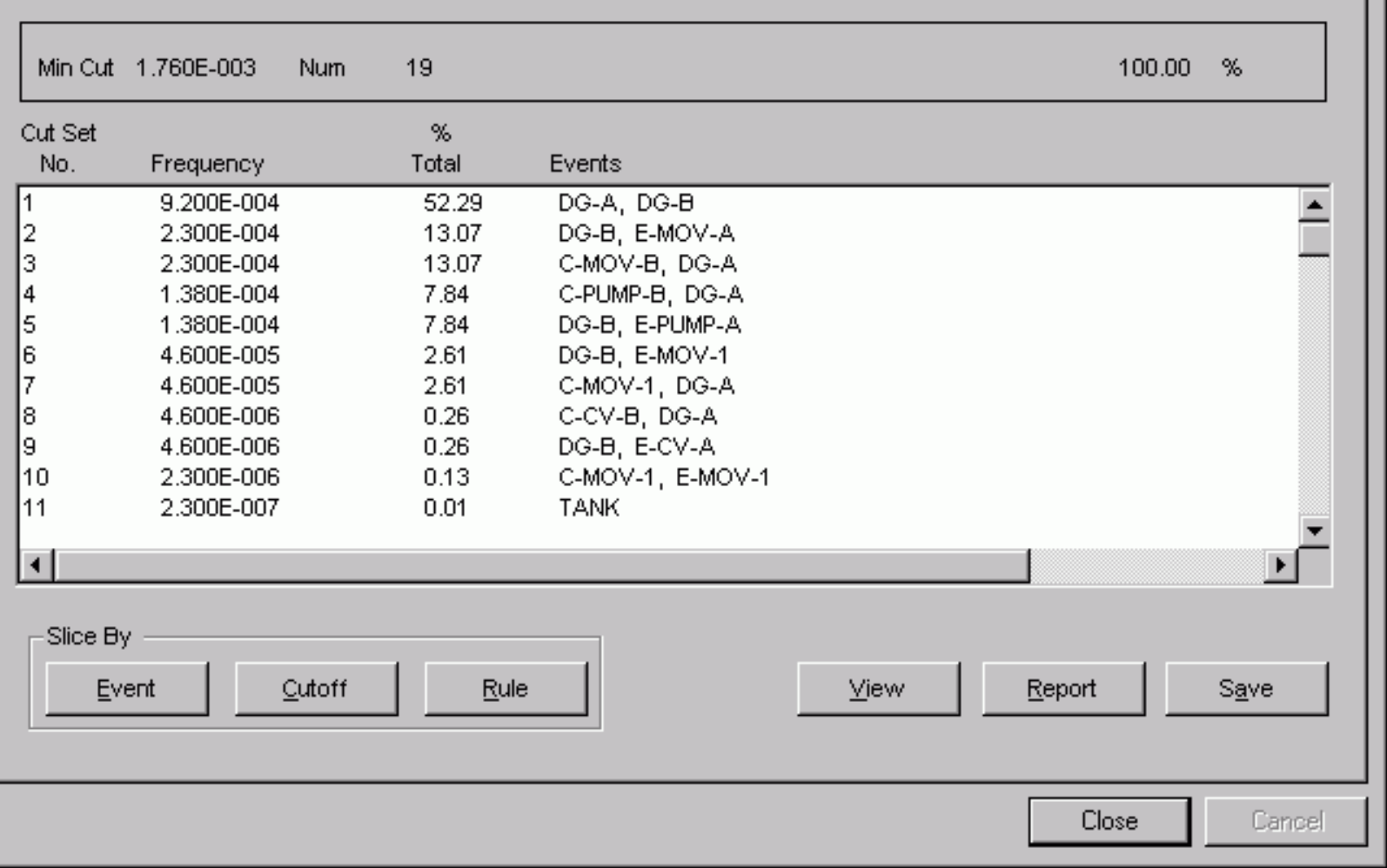

Tabbed pages:

Full List -

Included in Slice -

Display the complete (unsliced) list of cut sets.

Excluded From Slice -

Display all currently qualified cut sets.

Display all currently unqualified cut sets.

Pop-up menu (only) functions:

Path -

Remove -

Reset Explicit -
Display the logic that generated the highlighted cut set.

Remove the highlighted cut set(s) from the current list, and place them into the Excluded From Slice list.

Restore the cut set(s) that were deleted, via the Remove option, to the current list.

\section{Slice By:}

Event - Define the event criteria upon which cut sets will be qualified.

Cutoff - Define the cut off criteria upon which cut sets will be qualified.

Rule - Define the rule-based criteria upon which cut sets will be qualified.

View - Display information about the selected cut set including basic event data.

Report - Generate a report of the cut sets that are displayed in the currently selected tab.

Save - Copy the cut sets from the currently selected tab to an end state.

Close - Close the Selected Cut Sets dialog.

\subsubsection{Path Search}




\section{PURPOSE}

This option displays the representation of the logic that generated the selected cut set. The representation is in the form of a hierarchical tree, where each gate can be expanded or condensed by clicking on the gate. Only the failure path is traced here.

\section{STEPS}

1. From the SAPHIRE menu select Fault Tree/Sequence/End State. The Fault Tree List/Sequences/End State List dialog will be displayed.

2. Highlight the desired list item and right-click to invoke the pop-up menu.

3. Choose Display | Cut Sets. The Selected Cut Sets dialog will be displayed.

4. Highlight the desired cut set and right-click to invoke the pop-up menu.

5. Choose the Path option. The Selected Cut Set Events dialog will be displayed.

See the topic, "About Logic Dialogs," for a description of the icons presented on this dialog.

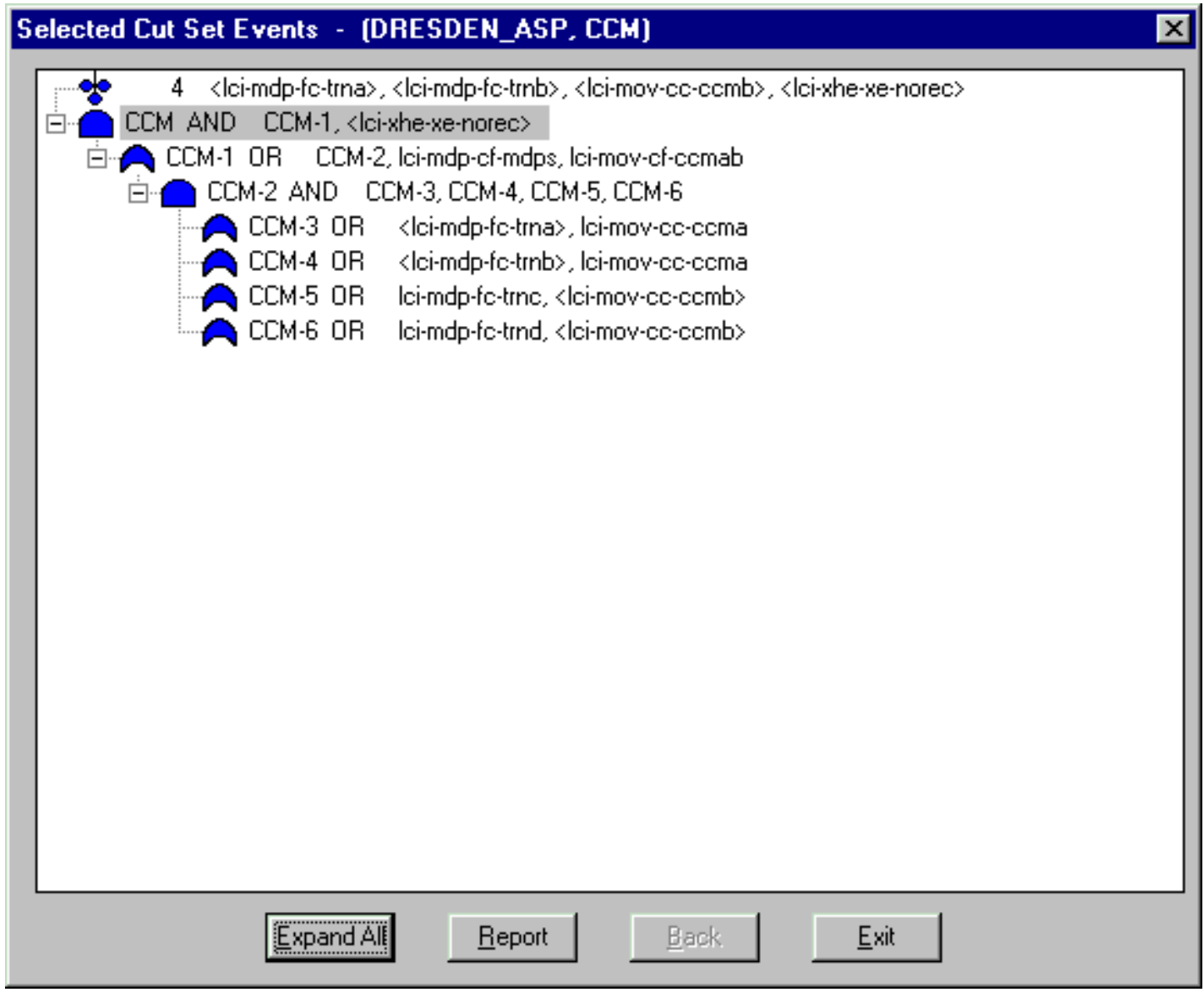

Expand All - $\quad$ Expand all gates.

Report - $\quad$ Generate a report representing the expanded hierarchical tree. The output destination for the report must be specified. 
Back - $\quad$ Return to the referenced gate. Active only after a referenced gate has been traced to the location where it was originally defined (i.e., the reference gate icon has been clicked).

Exit -

Close the Selected Cut Set Events dialog.

\subsubsection{Save}

\section{PURPOSE}

Save the results of the existing cut set slice (currently displayed on the Selected Cut Sets dialog) to an end state.

\section{STEPS}

1. From the SAPHIRE menu select Fault Tree/Sequence/End State. The Fault Tree List/Sequences/End State List dialog will be displayed.

2. Highlight the desired list item and right-click to invoke the pop-up menu.

3. Choose Display | Cut Sets. The Selected Cut Sets dialog will be displayed.

4. Slice, or qualify, the cut sets as desired. Select the tab containing the cut set list you want to save.

5. Choose the Save button. The Save To An End State dialog will be displayed.

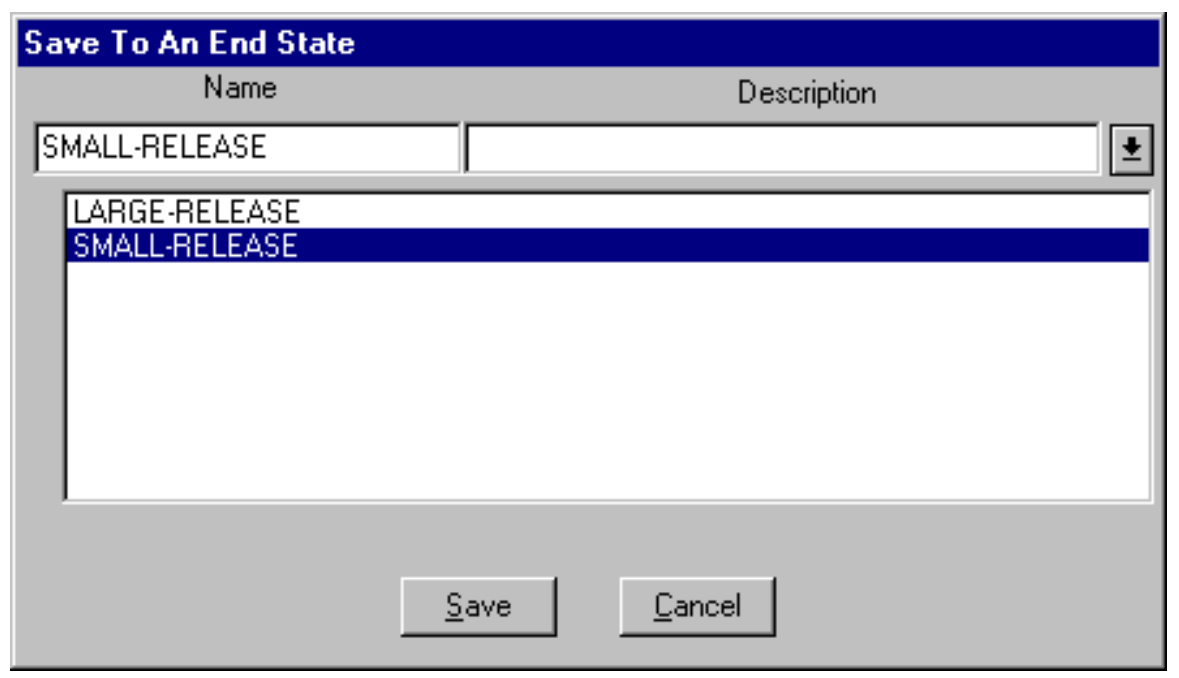

In the example here, the down arrow to the right of the Description field was selected to display the list of existing end states.

Name - $\quad$ Enter the name of the new end state. Up to 24 uppercase, alphanumeric characters are allowed.

Description - Enter the description of the new end state. Up to 60 upper- and lowercase, alphanumeric characters area allowed 
Save - $\quad$ Perform the save operation. A new end state record will be saved in the SAPHIRE data base with the name and description as entered here, and current slice for its cut sets. Selecting an existing end state will cause the existing cut sets for that end state to be overwritten.

Cancel - $\quad$ Close the Save To An End State dialog without saving the cut sets or creating a new end state record.

\subsubsection{Slice Option}

\subsection{Slice By Cutoff}

\section{PURPOSE}

This option provides the ability to divide the original cut set list into two subsets (slices) - qualified and unqualified cut sets. This is accomplished by defining a cut off value to determine whether or not a cut set is qualified. This qualification criteria can be used to help the analyst determine the affect of specified cut sets on the minimal cut set upper bound.

\section{STEPS}

1. From the SAPHIRE menu select Fault Tree/Sequence/End State. The Fault Tree List/Sequences/End State List dialog will be displayed.

2. Highlight the desired list item and right-click to invoke the pop-up menu.

3. Choose Display | Cut Sets. The Selected Cut Sets dialog will be displayed.

4. Choose the Slice By Cutoff button. The Slice By Value dialog will be displayed.

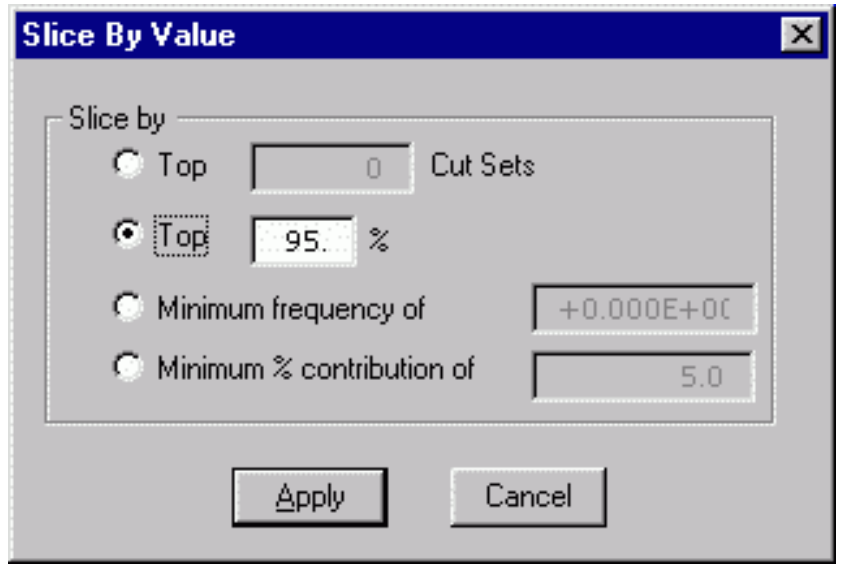

Select one of four types of cutoff value expressions:

Top X Cut Sets -

Top X\% -

Minimum frequency of $\mathbf{X}-$

Minimum \% contribution of $\mathrm{X}$ -

Apply -
Display only the top X cut sets in the qualified list.

Display only the cut sets which contribute to the top X\% of the min cut upper bound.

Display only the cut sets which contribute at least a value of $\mathrm{X}$ to the min cut upper bound.

- Display only the cut sets which contribute at least a X\% to the min cut upper bound.

Cut sets in the current slice will be evaluated. Those cut sets meeting the qualification criteria will be retained in 
Cancel -

\subsection{Slice By Event}

\subsection{Slice By Event}

\section{PURPOSE}

This option provides the ability to divide the original cut set list into two subsets (slices) - qualified and unqualified cut sets. This is accomplished by defining a set of events used to evaluate whether or not a cut set is qualified. This qualification criteria can be used to help the analyst determine the affect of specified events on the minimal cut set upper bound.

\section{STEPS}

1. From the SAPHIRE menu select Fault Tree/Sequence/End State. The Fault Tree List/Sequences/End State List dialog will be displayed.

2. Highlight the desired list item and right-click to invoke the pop-up menu.

3. Choose Display | Cut Sets. The Selected Cut Sets dialog will be displayed.

4. Choose the Slice By Event button. The Event List dialog will be displayed. 


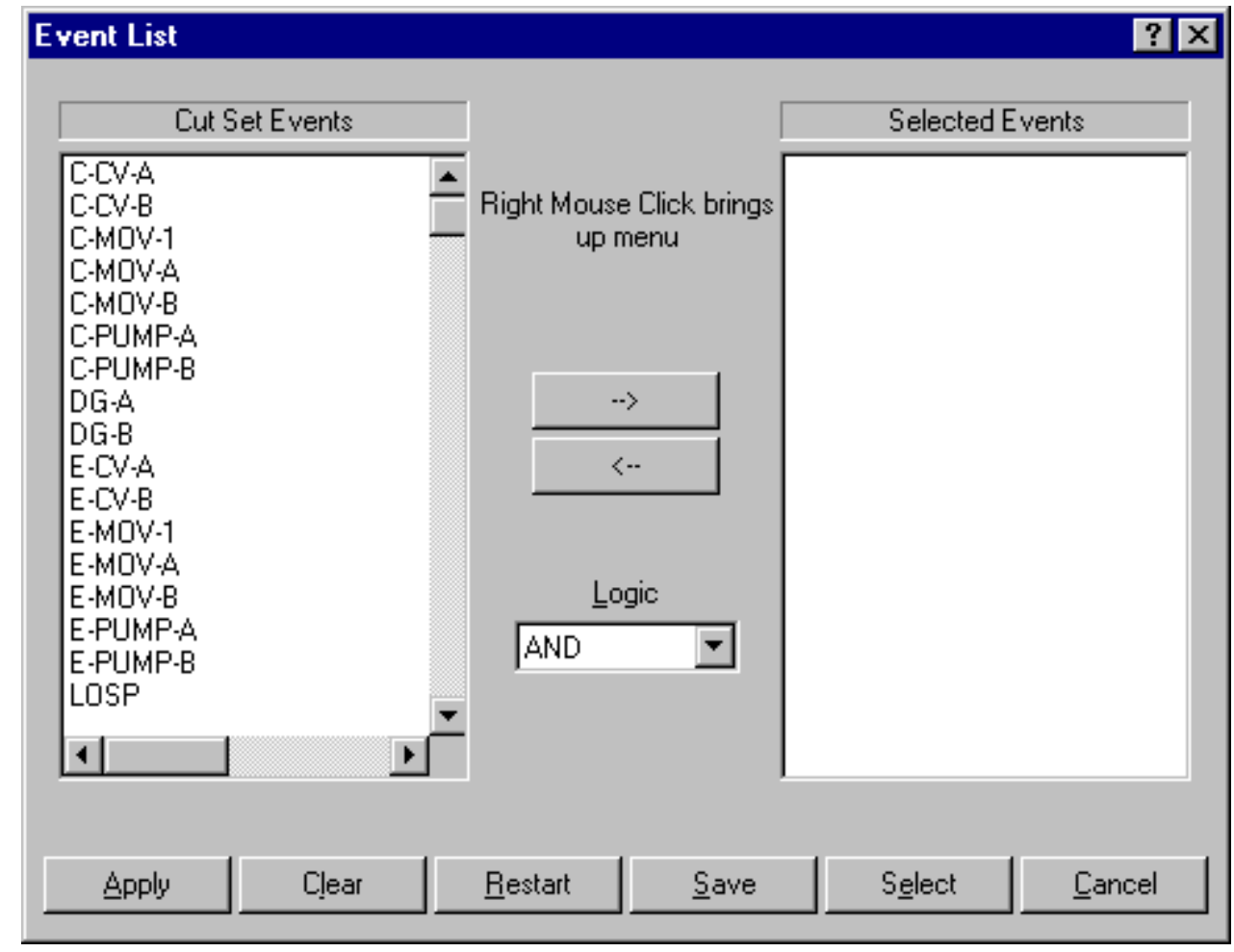

The Event List dialog is divided into three sections:

Cut Set Events list - $\quad$ Contains all the basic events included in the cut set(s) for the currently selected fault tree, sequence(s) or end state(s).

Selected Events list - Contains all basic events that define the qualification criteria. This list is initially empty.

Logic drop-down list - $\quad$ Allows selection between the logical "AND" and "OR" operations. The selected logical operation is applied to the events in the Selected Events list to determine which cut sets are qualified.

The various functions available from this dialog are accessed three different ways. When an event in the Cut Set Events list is highlighted, the following options are available from the pop-up menu. The highlighted event(s) will be added to the Selected Events list.

Wild Card Mark -

Add Event -

Add Failure Event-

Add Success EventAdd NOT Event In-
Use the event class mask to highlight events in the Cut Set Events list.

Cut sets containing this event, in any state (either failed or success), will be qualified.

Cut sets containing the failure $(+)$ of this event will be qualified. Cut sets containing the success (/) of this event will be qualified. Cut sets that do not contain $(\sim)$ this event, in any state (either failed or success), will be qualified. 
Apply Selected - $\quad$ Evaluate the qualification criteria. This is the same process as if the Apply button was chosen.

When an event in the Selected Events list is highlighted, the following options are available from the pop-up menu. The qualification criteria state will be changed for the highlighted event(s):
Remove Event - $\quad$ Delete this event from the Selected Events list. This event will no longer be used in the qualification criteria.
Event (either / or + ) - $\quad$ The state of the highlighted event, whether successful or failed, is irrelevant.
Failed Event -
Change the state of the highlighted event to failed $(+)$.
Success Event -
Change the state of the highlighted event to successful (/).
Event NOT In -
The highlighted event are is not contained $(\sim)$ in qualified cut sets.
Apply Selected - $\quad$ Evaluate the qualification criteria. This is the same process as if the Apply button was chosen.

The following functions are performed by choosing the appropriate button:
Apply - Cut sets in the current slice will be evaluated. Those cut sets meeting the qualification criteria will be retained in the current slice of qualified cut sets. Other cut sets not meeting the qualification criteria will be placed in the unqualified list.
Clear - $\quad$ Remove all events from the Selected Events list (i.e., clear the qualification criteria).
Restart - Restore the original cut sets and clear the qualification criteria. The Event List dialog will be closed and the Included In List and Excluded From List pages will be cleared.
Save - $\quad$ Save the selected events in a stored slice record.
Select - Choose a saved slice record and place the events in the Selected Events list.
Cancel - Close the Event List dialog without applying the qualification criteria.

\subsection{Wild Card Mark}

\section{PURPOSE}

This option uses the event class mask to highlight events in the Cut Set Events list. This selection is used with either the Cut Set Editor or the Cut Set | Slice option.

\section{STEPS}

1. From the SAPHIRE menu select Fault Tree/Sequence/End State. The Fault Tree List/Sequences/End State List dialog will be displayed.

2. Highlight the desired list item and right-click to invoke the pop-up menu.

To access from the Slice\#1

1. Choose Display | Cut Sets. The Selected Cut Sets dialog will be displayed.

2. Choose the Slice button. The Event List dialog will be displayed.

3. Right-click to invoke the pop-up menu and choose the Wild Card Mark option. 
To access from the Cut Set Editor\#1

1. Choose Cut Sets | Edit | Current or Base. The Cut Set Editor dialog will be displayed.

2. Choose the Find button. The Event List dialog will be displayed.

3. Choose the Mark Used Wild button.

4. The Event Class Mask dialog will be displayed.

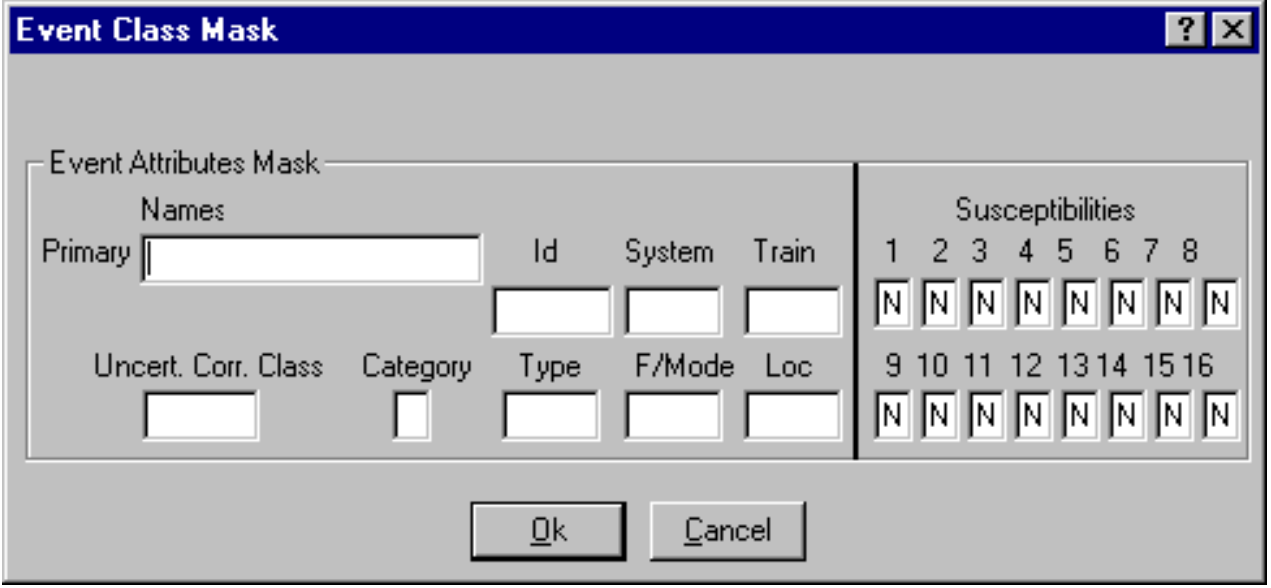

Primary -

Id -

System -

Train -

Uncert. Corr. Class -

Category -

Type -

F/Mode -

Location -

Susceptibilities -
Select all basic events with matching primary names (maximum 24 characters). Wildcard characters may be used.

Select all basic events with matching component identifiers.

Select all basic event with matching systems.

Select all basic event with matching trains

Select all basic event with matching uncertainty correlation classes.

Select all basic event with matching categories.

Select all basic event with matching types.

Select all basic event with matching failure modes.

Select all basic event with matching locations.

Select all basic event with matching susceptibility attributes set.

\subsection{Save Slice}

\section{PURPOSE}

This option allows you to save the basic events currently displayed in the Selected Events list in a stored slice record or a set.

\section{STEPS}

1. From the SAPHIRE menu select Fault Tree/Sequence/End State. The Fault Tree List/Sequences/End State List dialog will be displayed.

2. Highlight the desired list item and right-click to invoke the pop-up menu.

3. Choose Display | Cut Sets. The Selected Cut Sets dialog will be displayed.

4. Choose the Slice button. The Event List dialog will be displayed. 
5. Select the desired events to be saved in the slice.

6. Choose the Save button. The Save Slice dialog will be displayed.

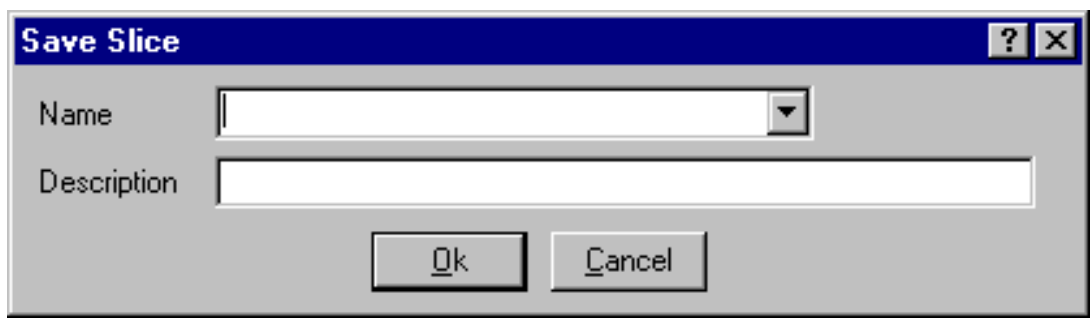

Ok - Save the new slice record and close the dialog.

Cancel - Close the dialog without saving.

\subsection{Select Slice}

\section{PURPOSE}

Choose a saved slice record and place the events in the Selected Events list. When a slice record is loaded, the basic events saved in the slice replace all basic events currently displayed in the Selected Events list. If a basic event that was saved in a slice does not appear in the Cut Set Events list, it will not be displayed in the Selected Events list, although it will remain in the slice record.

\section{STEPS}

1. From the SAPHIRE menu select Fault Tree/Sequence/End State. The Fault Tree List/Sequences/End State List dialog will be displayed.

2. Highlight the desired list item and right-click to invoke the pop-up menu.

3. Choose Display | Cut Sets. The Selected Cut Sets dialog will be displayed.

4. Choose the Slice button. The Event List dialog will be displayed.

5. Choose the Select button. The Select Slice dialog will be displayed. 


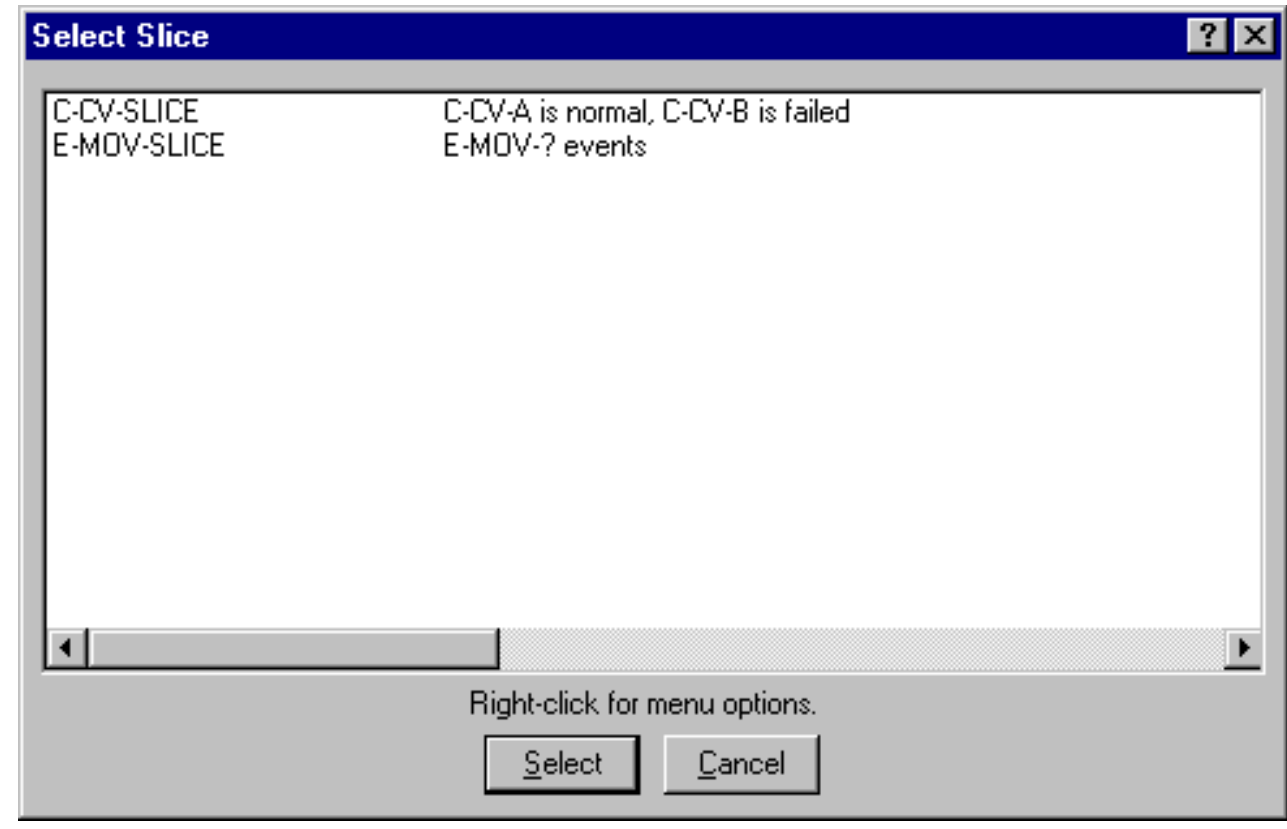

\section{Pop-up Menu Options}

Modify - Change the slice record name and/or description. See Save Slice .

Delete - Delete the slice record from the database.

Exit - $\quad$ Close the Select Slice dialog.

\section{Button Options}

Select - Choose the highlighted slice record and load the saved events.

Cancel - Close the Select Slice dialog.

\subsection{Slice By Rule}

\subsection{Slice By Rule}

\section{PURPOSE}

This option provides the ability to divide the original cut set list into two subsets (slices) - qualified and unqualified cut sets. This is accomplished by defining one or more rules to evaluate whether or not a cut set is qualified. The rules provide the ability to qualify cut sets using logical expressions, including mixed logic (combining "ands" and "ors") if desired. The rules are saved in the project for repeated use.

When the dialog is invoked, a list of slice rules is displayed. The highlighted rule is previewed in the bottom text area. To add/modify/delete a slice rule name and description, right click and select the appropriate menu option. To edit a rule, choose the Edit Rule button. To apply a rule to the current cut sets, choose the Apply button.

To clear the qualified and unqualified lists, choose the Reset option from the Slice By Event option, or, apply a different slice option to override the current slice.

\section{STEPS}

1. From the SAPHIRE menu select Fault Tree/Sequence/End State. The Fault Tree List/Sequences/End State List dialog will be displayed. 
2. Highlight the desired list item and right-click to invoke the pop-up menu.

3. Choose Display | Cut Sets. The Selected Cut Sets dialog will be displayed.

4. Choose the Slice By Rule button. The Slice Rules dialog will be displayed.

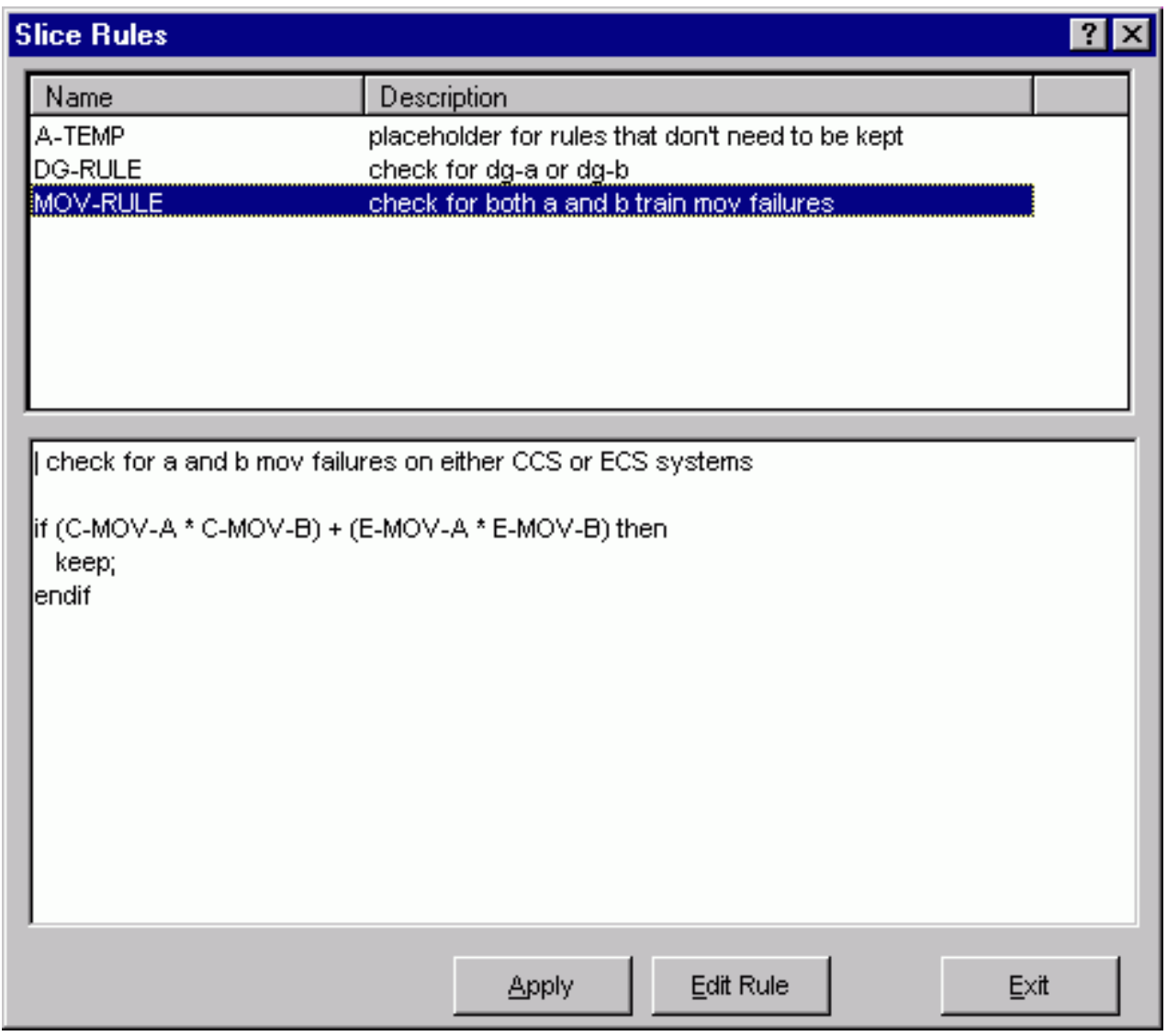

\section{Pop-up Menu Options}

Add - Create a new, empty rule.

Copy - Make a copy of an existing rule. You must supply a unique name before saving the rule.

Modify - Modify the name and/or description of the selected rule.

Delete - Remove the rule from the project.

Apply - The full list of current cut sets will be evaluated. Those cut sets meeting the qualification criteria will be retained in the current slice of qualified cut sets. Other cut sets not meeting the qualification criteria will be placed in the unqualified list.

Edit Rules-Edit the selected rule logic.

Exit - $\quad$ Close the Slice Rules dialog without applying a rule. 


\subsection{Slice Rules Editor}

\section{PURPOSE}

This option allows you to create or modify rules that divide cut sets into two subsets - qualified and unqualified. The Slice Rules editor provides a means to develop logic rules that locate and separate cut sets containing desired event combinations.

The slice rules are entered in a free-form text editor within SAPHIRE. The editor options and rule syntax are similar to that of SAPHIRE's recovery rules. However, unlike to recovery rules, slice rules are not tied to the fault tree, sequence(s) or end state(s) whose cut sets are currently displayed. (A slice rule is available to be applied to any type of displayed cut sets.)

Slice rules follow a format similar to the structure that is found in traditional programming languages (e.g., BASIC or PASCAL). As such, the ability exists to define "macros" and "if...then" type of structures. After creating or editing rules, SAPHIRE compiles the rules to check their validity.

Note: An alternative method of entering rules into the project is to export them from SAPHIRE and create or edit them in any word processor or text editor (that can output ASCII files) and then loaded directly into the SAPHIRE database.

\section{STEPS}

1. From the SAPHIRE menu select Fault Tree/Sequence/End State. The Fault Trees List/Sequences/End States dialog will be displayed.

2. Highlight the desired fault tree(s)/sequence(s)/end state(s) and right-click to invoke the pop-up menu.

3. Choose Display | Cut Sets. The Selected Cut Sets dialog will appear.

4. Choose the Slice By Rule button. The Slice Rules dialog will appear.

5. If necessary, add a new rule by right clicking and choosing the Add menu option.

6. Highlight the desired slice rule and choose Edit Rules. If rules have been previously defined they will be displayed in the edit window. 


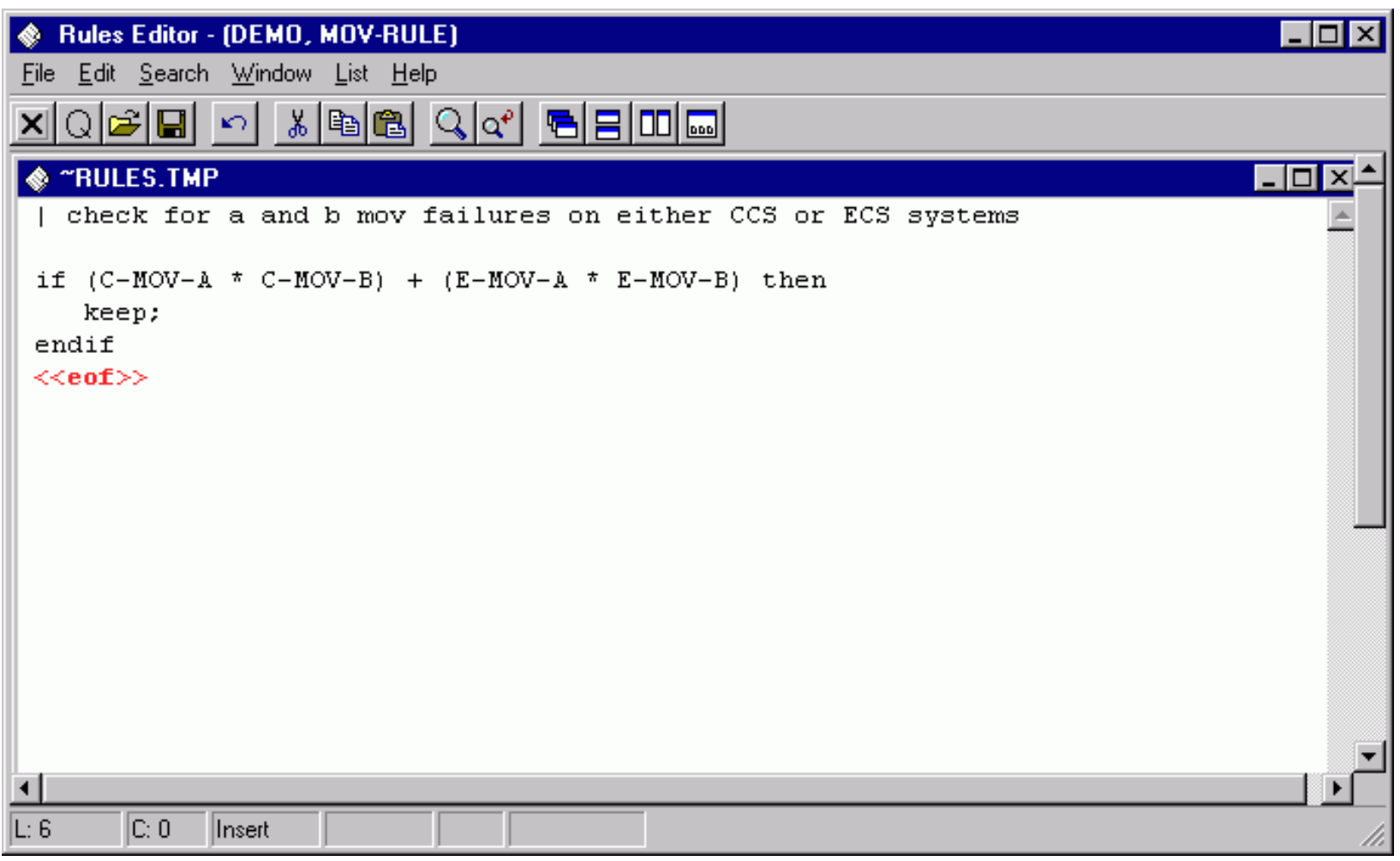

Type the rules in the editor. Note that the rules are not case sensitive.

File -

File functions such as open, save, print, preferences, etc.

Edit Editing functions such as copy and paste along with text format operations.

Search Search and cursor positioning operations such as find, replace, goto, etc.

Window - Window management functions such as cascade, tile horizontally, etc.

Lists - $\quad$ Display lists of macros and events for inserting into the rules. Three different event lists are available: initiators, a complete list of events, and only those events available in the currently displayed cut sets.

\subsection{Editor File Functions}

These options provide file management functions.

Open - $\quad$ Open a file into a new window. This feature is not usually used while editing SAPHIRE rules.

New - $\quad$ Create a new file in a new window. This feature is not usually used while editing SAPHIRE rules.

Close - $\quad$ Close the edit current window. If changes have been made you will be prompted to save the file.

Quit - $\quad$ Close the current edit window without saving.

Save - $\quad$ Save the file in the current window to disk.

Save As - $\quad$ Save the file in the current window with a new file name.

Save All - $\quad$ Save all the files in all edit windows currently open.

Save Block - Save the currently highlighted text into a new file. The Save As dialog will be invoked. 
Import File - Insert the contents of a file into the current edit window at the current cursor position. The Edit external file dialog will be invoked.

Print - $\quad$ Print the file in the current edit window.

Page setup - $\quad$ Set printer page layout options, .such as pages per sheet and line numbers.

Preferences - $\quad$ Select editing options such as tab width, undo levels, etc.

Exit -

Terminate the Editor session.

\subsection{Editor Edit Functions}

These options provide editing and text formatting functions.
Undo -
Undo the previous editing operation.
Cut -
Remove the highlighted text from the current window and place it in the
Copy - clipboard.
Paste -
Delete -
Copy the highlighted text from the current window and place it in the clipboard.
Copy the clipboard text into the current window at the cursor position.
Shift left indent - in the clipboard.
Shift right indent - Shift the text to the left by the Shift Size specified under Options.
Shift left space - Shift the text to the right by the Shift Size specified under Options. Shift the text to the left by a single space.
Shift right space - Shift the text to the right by a single space.
Delete to end of line -
Delete to the end of the current line beginning after the current cursor position.
Delete line - $\quad$ Delete the line on which the cursor is currently positioned.
Capitalize word - $\quad$ Convert the entire word under the current cursor position to upper case.
Downcase word - $\quad$ Convert the entire word under the current cursor position to lower case.

\subsection{Editor Search Functions}

These options provide search and cursor positioning operations.

Find -

Replace -

Find Procedure Find/Replace again Find Altered lines Find line number Match symbol -

Goto next error Goto previous error Goto Marker -

Set Marker -
Search the text in the current window for the specified string.

Search the text in the current window for a string and replace it with another string.

Search the text in the current window for the specified procedure. Repeat the previous search operation.

Find the lines of text that have changed since the last save operation.

Go to the specified text line number in the current window.

Find the matching symbol pair for the character under the cursor " ()$,\{\}$, [], $(* *) "$

Position the cursor at the next compilation error.

Position the cursor at the previous compilation error.

Position the cursor at the previously saved file position. Up to four marker positions can be retrieved.

Save the current file position for later retrieval.

\subsection{Editor Window Functions}


These options are used for managing the open edit windows within the application window, such as the Linkage Rules Editor or the Event Tree Logic Editor window. Along with the options listed below and segregated by a separation bar, the names of each open file is listed in the menu.

Cascade windows -

Tile windows horizontally -

Tile windows vertically -

Arrange Icons -
Resize and reposition the non-minimized windows in an overlapping fashion so that the title of each window is displayed in a cascade arrangement. The current edit window will be on top.

Resize and reposition the non-minimized windows in a horizontally tiled arrangement.

Resize and reposition the non-minimized windows in a vertically tiled arrangement.

Arrange the minimized window icons at the bottom of the application window.

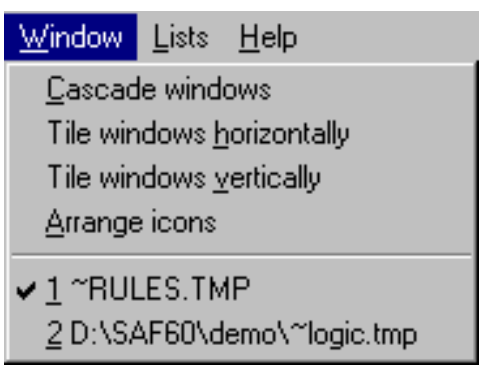

In the example above, the files " $\sim$ RULES.TMP" and " $\sim$ LOGIC.TMP" are open. $\sim$ RULES.TMP is the active window, as indicated by the check mark on the menu.

\subsection{7 $\quad$ Slice Rules Lists}

Selecting from the lists allows you to insert or replace items in the editor.

Macros - $\quad$ Allows you to insert a macro into the editor.

Events - $\quad$ Select any basic event instead of typing in the basic event name.

Cut Set Events - Select from a list of only those events found in the currently displayed cut sets, instead of typing in the event name.

Initiators - $\quad$ Select an initiating event instead of typing in the initiator name.

\subsection{MACRO}

(Basic Rule syntax)

A macro is a user-definable keyword that specifies a search criteria. The macro name must be all uppercase, must be 24 characters or less, and must not include any of the restricted characters (e.g., a space, $*, ?, \backslash, /)$. The macro line can wrap around to more than one line, but must end with a semicolon.

MACRO-NAME = SEARCH-CRITERIA; 
if MACRO-NAME “and optional other search criteria” then

perform some action on each cut set...;

endif

| Macros are only applicable in the particular rule they are entered into.

\subsubsection{Importance}

\subsection{Sets Importance}

\section{PURPOSE}

This option displays the Fussell-Vesely importance of the selected set(s) of cut sets including interactions among the events. The selected sets are previously saved slices.

\section{STEPS}

1. From the SAPHIRE menu select Fault Tree/Sequence/End State. The Fault Tree List/Sequences/End State List dialog will be displayed.

2. Highlight the desired list item and right-click to invoke the pop-up menu.

3. Choose Display | Cut Sets. The Selected Cut Sets dialog will be displayed.

4. Choose the Sets button. The Select Set(s) for Set Importance dialog will be displayed.

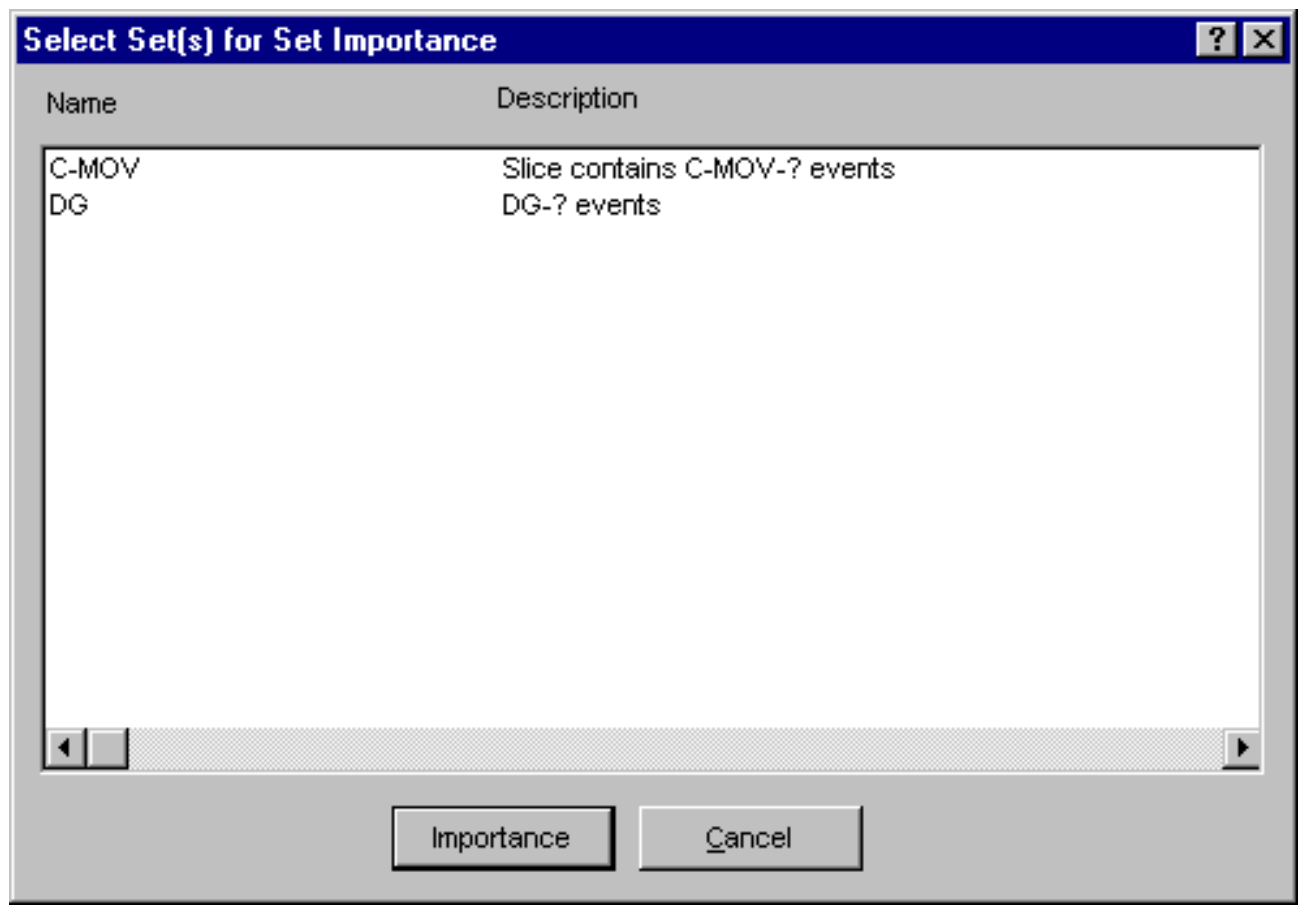

Select the desired set(s) and choose the Importance button. The Sets Importance dialog will be displayed containing the Fussell-Vesely importance, normalized Fussell-Vesely, name of the set, and its description, for each selected set. 


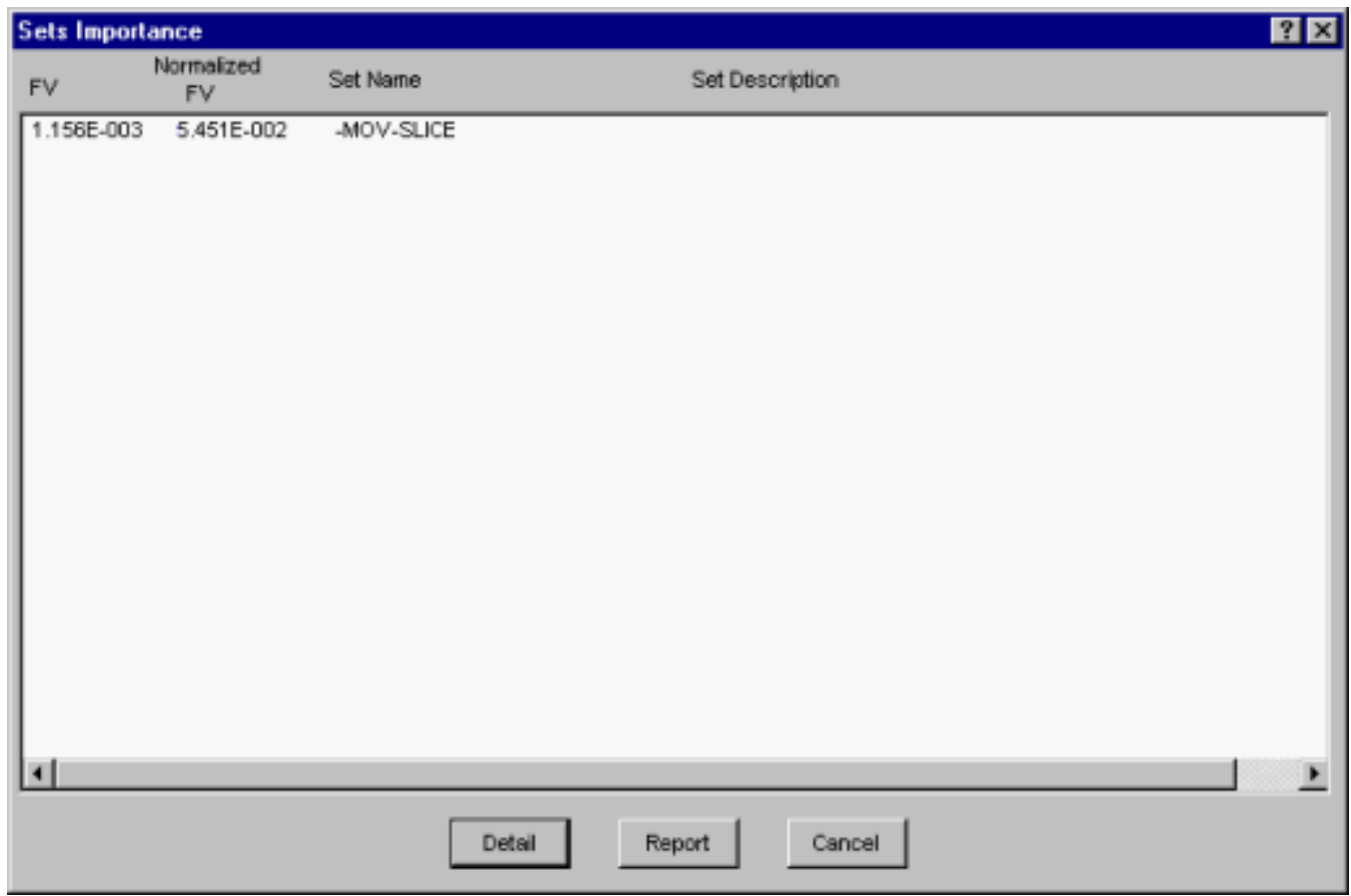

Detail - Displays the Cut Set Importance dialog after selecting one of the sets. Choose the Top or Bottom buttons for Sets importance details .

Report - Generates a report containing the Fussell-Vesely importance, normalized Fussell-Vesely, set name, and description of the selected set.

Cancel - Closes the Sets Importance dialog.

\subsubsection{View Option}

\subsection{View Cut Set}

\section{PURPOSE}

This option displays the basic events of the selected cut set with their failure probabilities and descriptions. At the bottom of the Selected Cut Set Events dialog, information about this cut set is displayed: percent of contribution to the total, the cut set frequency, and the number of events that make up this cut set.

\section{STEPS}

1. From the SAPHIRE menu select Fault Tree/Sequence/End State. The Fault Tree List/Sequences/End State List dialog will be displayed.

2. Highlight the desired list item and right-click to invoke the pop-up menu. 
3. Choose Display | Cut Sets. The Selected Cut Sets dialog will be displayed.

$O R$

4. Choose Display | Comparison. The Base \& Current Case Cut Sets dialog will be displayed.

5. Highlight the desired cut set.

6. Choose the View button. The Selected Cut Set Events dialog will be displayed.

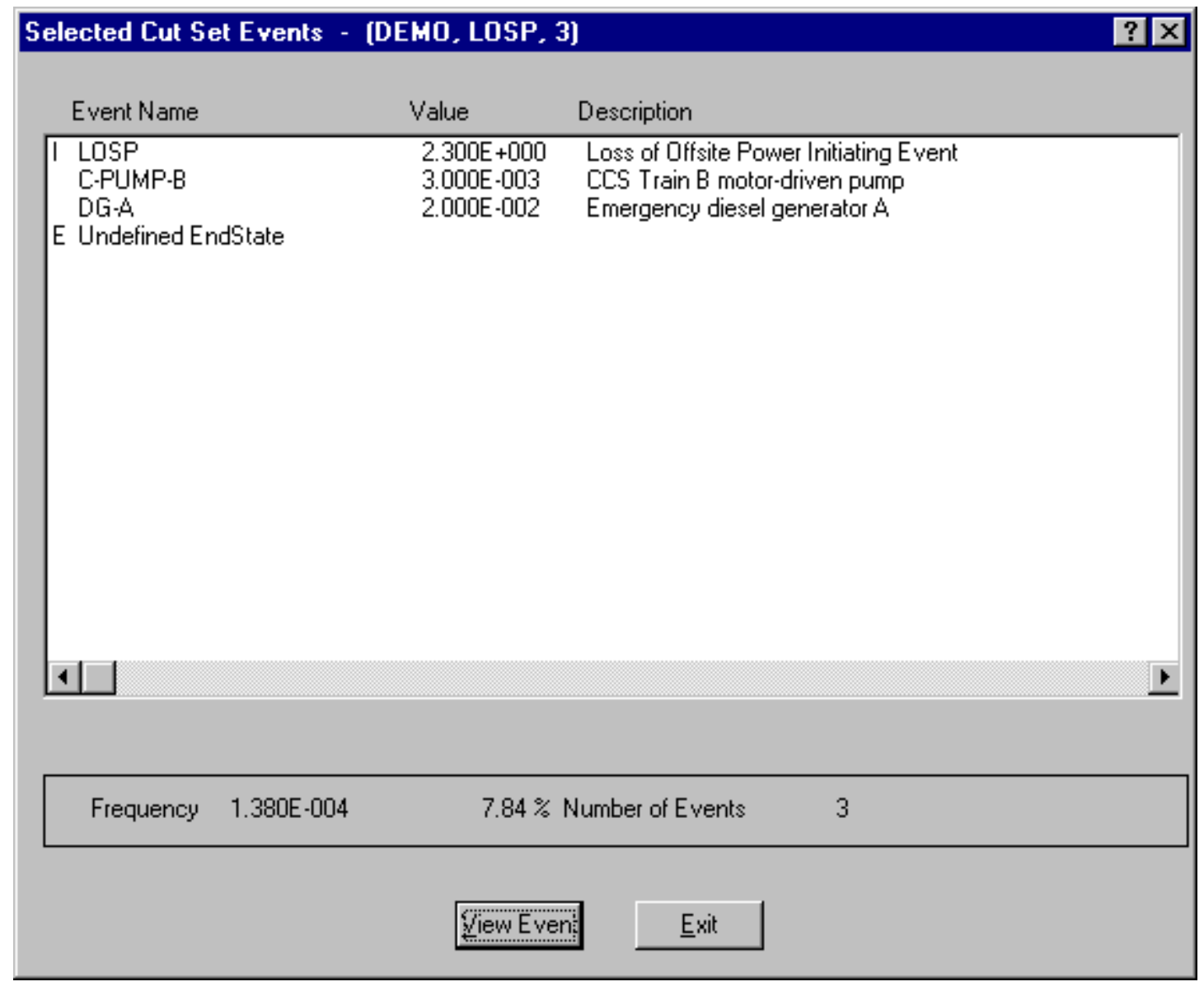

When viewing fault tree cut sets, the end state, if any, is designated with the letter "E" to the left of the Event Name column. It will be the last item listed.

When viewing sequence cut sets, the initiating event is designated with the letter "I" to the left of the Event Name column. It will be the first item listed. The end state will be designated with the letter "E" to the left of the Event Name column and will be the last item listed.

When viewing end state cut sets, the initiating event is designated with the letter "I" to the left of the Event Name column. It will be the first item listed. The originating sequence is designated by the letter "S" to the left of the Event Name column and will be the last item listed.

View Event - Display individual basic event information.

\subsection{View Event}




\section{PURPOSE}

This option provides the following detailed information about the basic events that make up a cut set: event names and description, event attributes, random failure data, uncertainty data, susceptibility flags, and transformation data.

\section{STEPS}

1. From the SAPHIRE menu select Fault Tree/Sequence/End State. The Fault Tree List/Sequences/End State List dialog will be displayed.

2. Highlight the desired list item and right-click to invoke the pop-up menu.

3. Choose Display | Cut Sets. The Selected Cut Sets dialog will be displayed. $O R$

4. Choose Display | Comparison. The Base \& Current Case Cut Sets dialog will be displayed.

5. Highlight the desired cut set.

6. Choose the View Cut Set button. The Selected Cut Set Events dialog will be displayed.

7. Highlight the desired basic event.

8. Choose the View Event button. The View Event dialog will be displayed. 


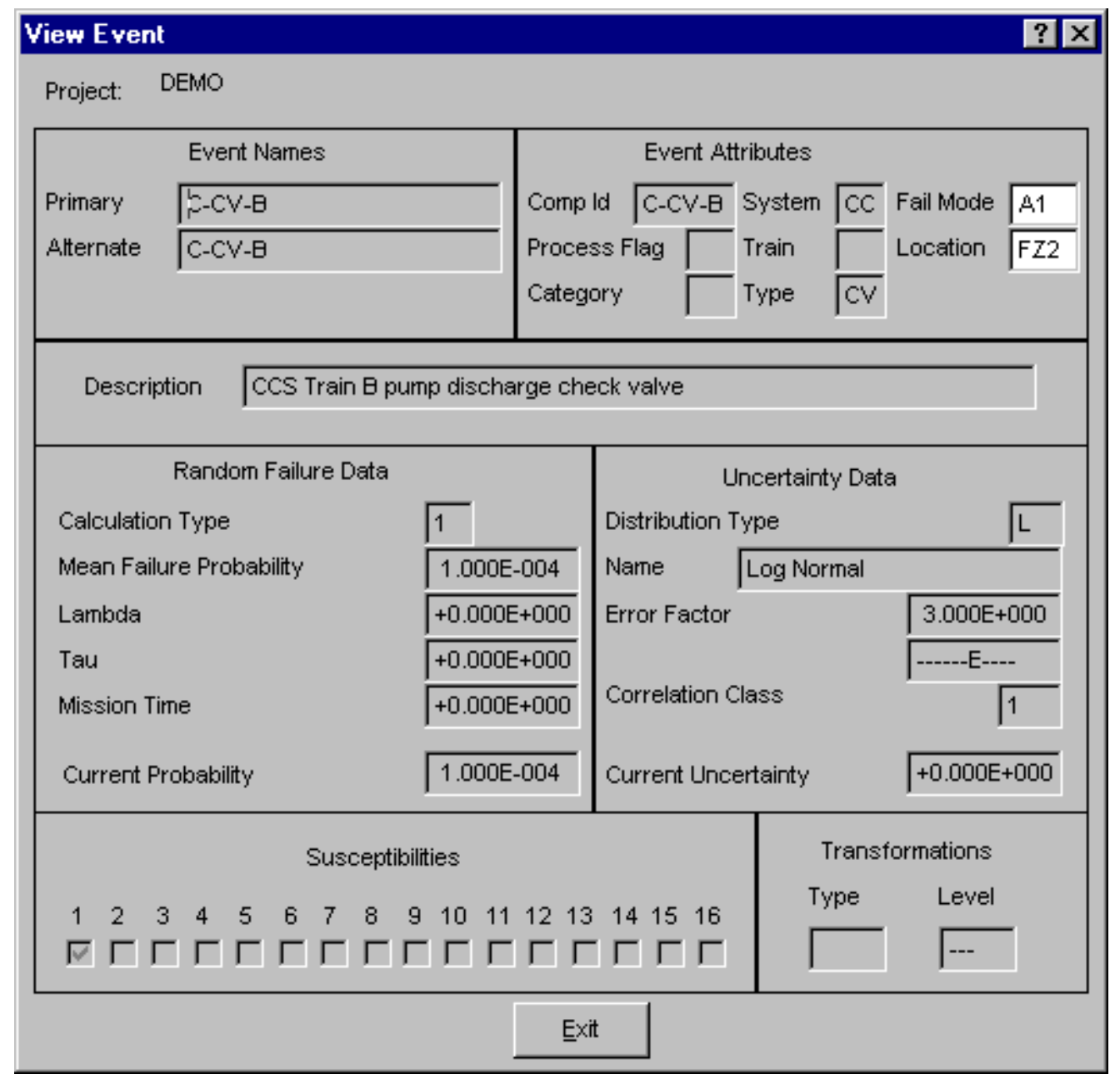

Information on this dialog is for display only and cannot be modified.

\subsubsection{Report Dialog}

\subsection{Cut Set Report}

\section{PURPOSE}

This option allows you to generate a report of the data that is currently displayed on the dialog. The report may be displayed in the Report Viewer, sent to a printer, or saved to a file for later processing.

\section{STEPS}

1. From the SAPHIRE menu select Fault Tree/Sequence/End State. The Fault Tree List/Sequences/End State List dialog will be displayed.

2. Highlight the desired list item and right-click to invoke the pop-up menu.

3. Choose Display | Cut Sets. The Selected Cut Sets dialog will be displayed.

4. Choose the Report button. The Report dialog will be displayed.

5. Select the desired report options and choose $\mathbf{O K}$. The Report Viewer will display the report. 


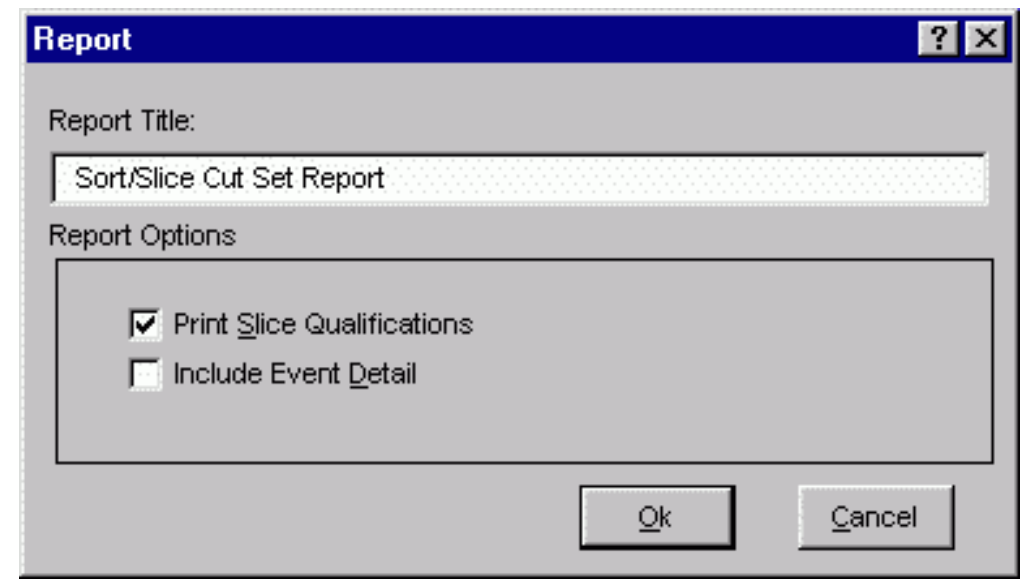

Report Title -

Print Slice Qualifications -

Include Event Detail -
A default report title is provided. Edit this value to customize the title. When checked, the criteria used to qualify the cut sets is included in the report.

When checked, the report will report one event per line, and including event probability and description information. When this option is not checked, the events will appear as a comma delimited list, with no supplementary event information included.

\subsubsection{Comparing Cut Sets}

\subsubsection{Cut Set Comparison}

\section{PURPOSE}

This option displays both the base case and current case cut sets for the selected fault tree (or sequences or end states). The source (either base or current) of the cut sets is indicated both by color and a symbol. The color red and the "-" symbol in the first column indicate a base case cut set. The color black and a blank (" ") in the first column indicate that the cut set is both a current case and base case cut set. The color green and the "+" symbol in the first column indicate a current case cut set.

\section{STEPS}

1. From the SAPHIRE menu select Fault Tree/Sequence/End State. The Fault Trees List/Sequences/End State List dialog will be displayed.

2. Highlight the desired list item(s) and right-click to invoke the pop-up menu.

3. Choose Display, then the Comparison sub-menu option. The Base \& Current Case Cut Sets dialog will be displayed. 


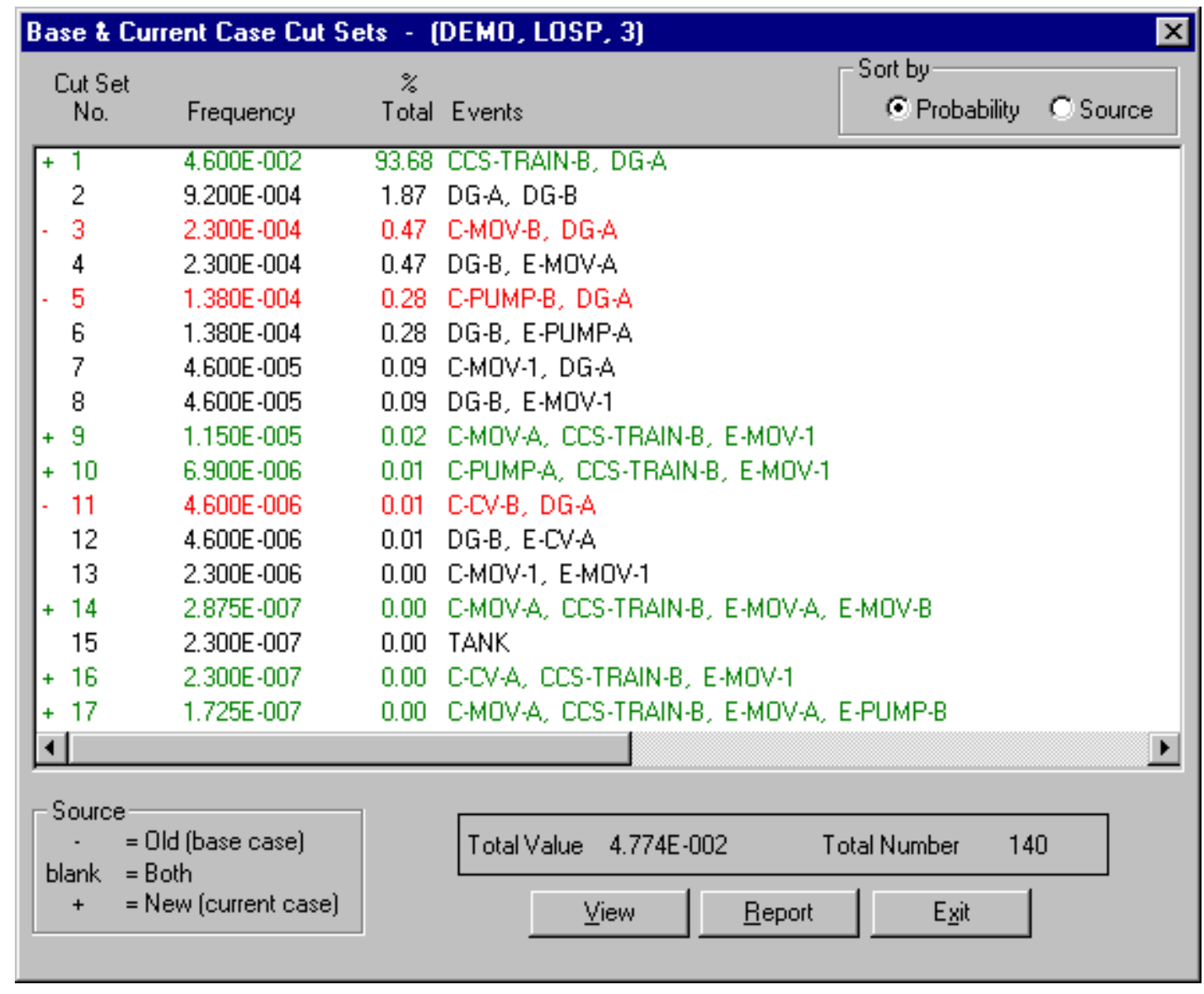

\section{Sort by}

Probability - $\quad$ List the cut sets by frequency in descending order.

Source - $\quad$ List the cut sets by source, listing first base case cut sets, then cut sets existing in both, followed by current case cut sets.

View - Display information about the selected cut set including basic event data.

Report - Generate a report of the data that is displayed on the dialog.

Exit - $\quad$ Close the Base \& Current Case Cut Sets dialog.

\subsubsection{View Cut Set}

\section{PURPOSE}

This option displays the basic events of the selected cut set with their failure probabilities and descriptions. At the bottom of the Selected Cut Set Events dialog, information about this cut set is displayed: percent of contribution to the total, the cut set frequency, and the number of events that make up this cut set. 


\section{STEPS}

1. From the SAPHIRE menu select Fault Tree/Sequence/End State. The Fault Tree List/Sequences/End State List dialog will be displayed.

2. Highlight the desired list item and right-click to invoke the pop-up menu.

3. Choose Display | Cut Sets. The Selected Cut Sets dialog will be displayed. OR

4. Choose Display | Comparison. The Base \& Current Case Cut Sets dialog will be displayed.

5. Highlight the desired cut set.

6. Choose the View button. The Selected Cut Set Events dialog will be displayed.

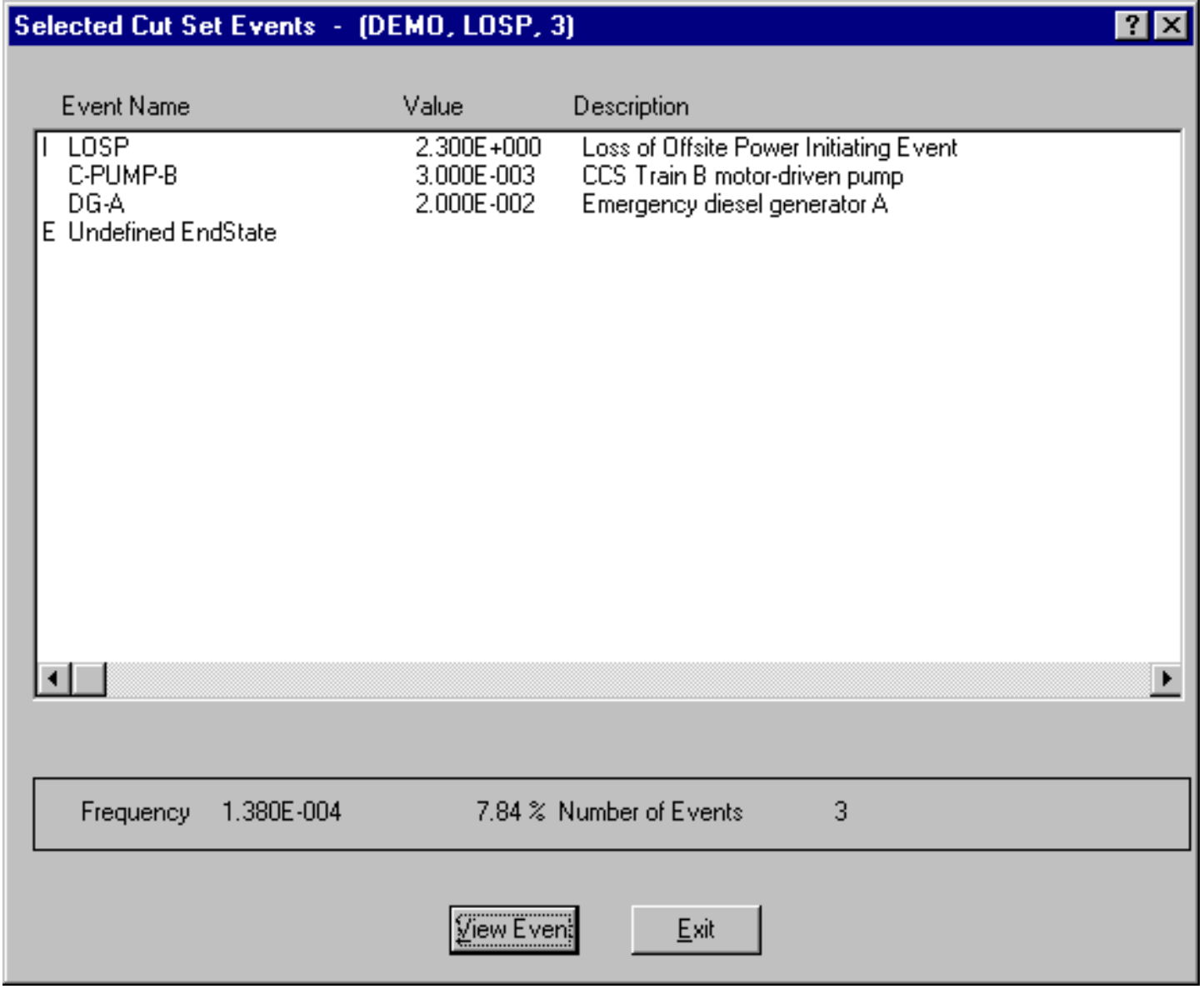

When viewing fault tree cut sets, the end state, if any, is designated with the letter "E" to the left of the Event Name column. It will be the last item listed.

When viewing sequence cut sets, the initiating event is designated with the letter "I" to the left of the Event Name column. It will be the first item listed. The end state will be designated with the letter "E" to the left of the Event Name column and will be the last item listed. 
When viewing end state cut sets, the initiating event is designated with the letter "I" to the left of the Event Name column. It will be the first item listed. The originating sequence is designated by the letter "S" to the left of the Event Name column and will be the last item listed.

View Event - Display individual basic event information.

\subsubsection{Cut Set Report}

\section{PURPOSE}

This option allows you to generate a report of the data that is currently displayed on the dialog. The report may be displayed in the Report Viewer, sent to a printer, or saved to a file for later processing.

\section{STEPS}

1. From the SAPHIRE menu select Fault Tree/Sequence/End State. The Fault Tree List/Sequences/End State List dialog will be displayed.

2. Highlight the desired list item and right-click to invoke the pop-up menu.

3. Choose Display | Cut Sets. The Selected Cut Sets dialog will be displayed.

4. Choose the Report button. The Report dialog will be displayed.

5. Select the desired report options and choose OK. The Report Viewer will display the report.

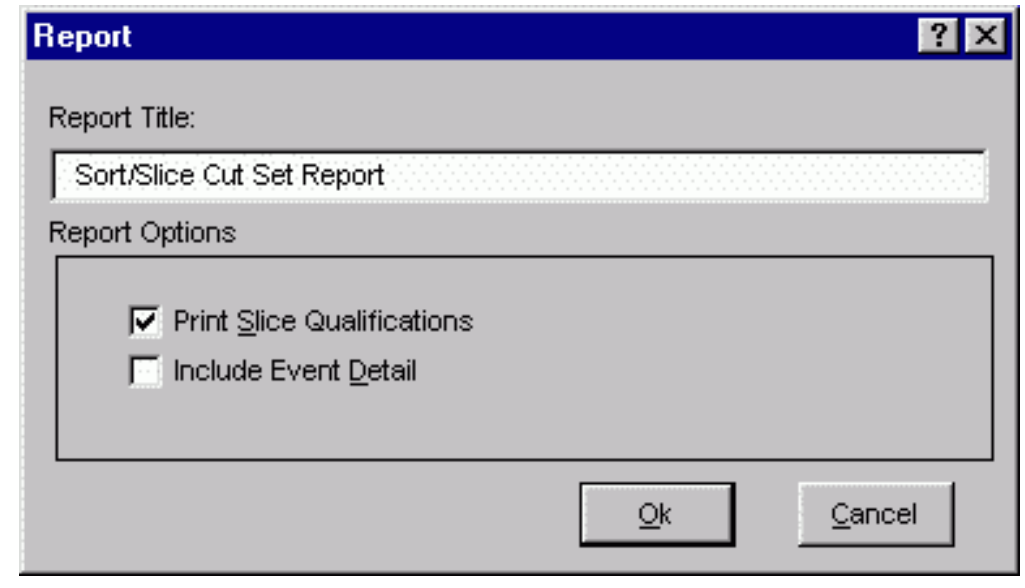

\section{Report Title - \\ Print Slice Qualifications -}

Include Event Detail -
A default report title is provided. Edit this value to customize the title. When checked, the criteria used to qualify the cut sets is included in the report.

When checked, the report will report one event per line, and including event probability and description information. When this option is not checked, the events will appear as a comma delimited list, with no supplementary event information included.

\subsubsection{Displaying Importance Results}

\subsubsection{Importance Measures}




\section{PURPOSE}

Importance measures provide "reliability-worth" information about basic events appearing in the cut sets for a fault tree or accident sequence. The "importance" for a basic event is essentially the event's contribution to the overall top event probability (for fault trees) or sequence frequency (for event trees). The measure of contribution can take on many forms depending on what concern the analyst has for a particular basic event. Consequently, SAPHIRE calculates seven different basic event importance measures. These are the Fussell-Vesely importance, risk reduction ratio, risk increase ratio, Birnbaum (or first derivative) importance, risk reduction difference, risk increase difference, and the uncertainty importance.

The ratio importance measures are dimensionless and consider only relative changes. The difference definitions account for the actual risk levels that exist and are more appropriate when actual risk levels are of concern, such as comparisons or prioritizations across different plants. For purely relative evaluations, such as prioritizations within a plant, the ratios sometimes give more graphic results.

These importance measures are calculated for each basic event for the respective fault tree or accident sequence. Once the results have been calculated, they can be displayed and then partitioned as a subset of the original cut sets.

\section{STEPS}

1. From the SAPHIRE menu select Fault Tree/Sequence/End State. The Fault Tree List/Sequences/End State List dialog will be displayed.

2. Highlight the desired list item and right-click to invoke the pop-up menu.

3. Choose Display, then the Importance sub-menu option.

4. Choose from one of the following sub-menu options:

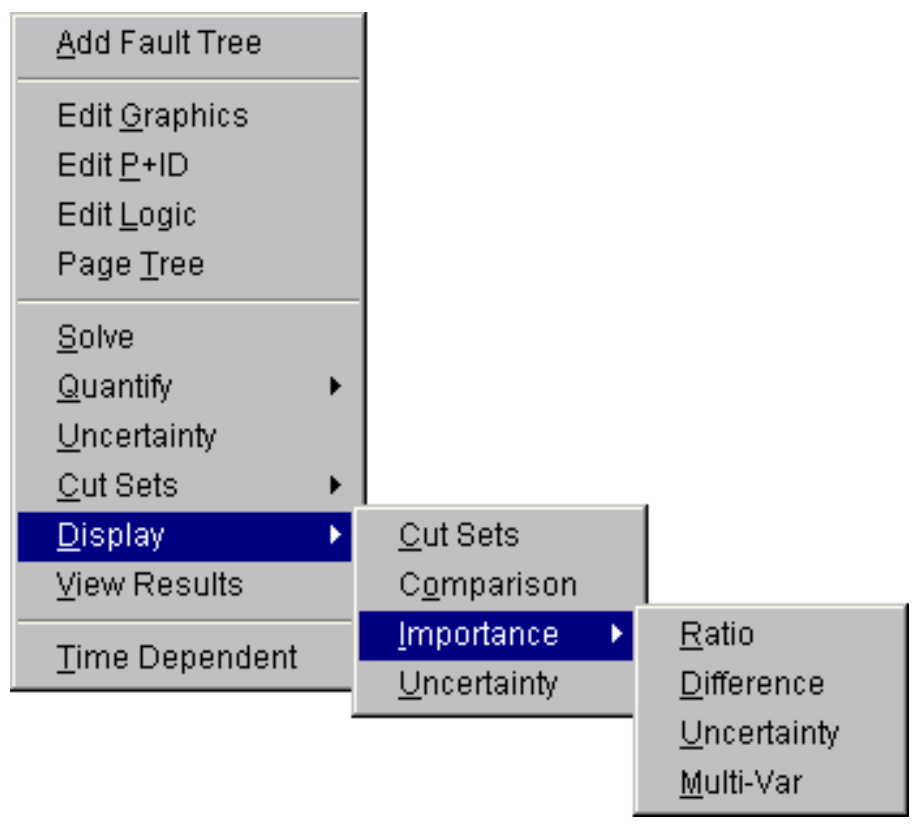

Ratio - $\quad$ Fussell-Vesely importance, Risk Reduction Ratio, and Risk Increase Ratio will be calculated and displayed. 
Difference - $\quad$ Birnbaum importance, Risk Reduction Interval, and Risk Increase Interval will be calculated and displayed.

Uncertainty - Quantification of the contribution of each individual basic event's uncertainty to the total output uncertainty.

Multi-Var - Importance for a group of events will be calculated and displayed.

\subsubsection{Fussell-Vesely Importance (FV)}

The FV measure is an indication of the percentage of the minimal cut set upper bound contributed by the cut sets containing the basic event. The equation for FV importance is

$$
\mathbf{F V}=\mathbf{F}(\mathbf{i}) / \mathbf{F}(\mathbf{x})
$$

where

$\mathrm{F}(\mathrm{i})$ is the minimal cut set upper bound for the group of cut sets containing the event

$\mathrm{F}(\mathrm{x})$ is the original minimal cut set upper bound

Prior to SAPHIRE version 7.27, the FV equation used and approximate expression

$$
\mathbf{F V}=\mathbf{1}-\mathbf{F}(\mathbf{0}) / \mathbf{F}(\mathbf{x})
$$

where

$F(0)$ is the minimal cut set upper bound with the event probability set equal to 0.0 .

$\mathrm{F}(\mathrm{x})$ is the original minimal cut set upper bound.

\subsubsection{Birnbaum Importance (B)}

This indicates the sensitivity of the minimal cut set upper bound with respect to a change in the basic event probability.

$$
\mathbf{B}=\mathbf{F}(\mathbf{1})-\mathbf{F}(\mathbf{0})
$$

where

$\mathrm{F}(1)$ is the minimal cut set upper bound with the event probability set equal to 1.0.

$\mathrm{F}(0)$ is the minimal cut set upper bound with the event probability set equal to 0.0 .

\subsubsection{Risk Reduction Ratio (RRR) or Risk Reduction Interval (RRI)}

These are an indication of how much the minimal cut set upper bound would decrease if the basic event was reduced to a probability or 0.0 (typically if the corresponding component never failed).

$$
\begin{aligned}
& R R R=F(x) / F(0) \\
& R R I=F(x)-F(0)
\end{aligned}
$$

Note the similarity between RRI and FV; the relative importance ranking of basic events will be the same for the two importance measures. 


\subsubsection{Risk Increase Ratio (RIR) or Risk Increase Interval (RII)}

These are an indication of how much the minimal cut set upper bound would increase if the basic event was increased to 1.0 (typically if the corresponding component always failed). Note: If the event probability is close to 1.0, this importance measure may yield a small RIR or RII.

$$
\begin{aligned}
& \text { RIR }=\mathbf{F}(1) / F(x) \\
& \text { RII }=\mathbf{F}(1)-F(x)
\end{aligned}
$$

where

$\mathrm{F}(\mathrm{x})$ is the original minimal cut set upper bound

$\mathrm{F}(1)$ is the minimal cut set upper bound with the event probability set equal to 1.0 .

\subsubsection{Uncertainty Importance}

The uncertainty in each input parameter, as expressed through its probability distribution, contributes to the uncertainty in the output parameter of interest (e.g., core damage frequency). The uncertainty importance measure in SAPHIRE attempts to quantify the contribution of each individual basic event $=\mathrm{s}$ uncertainty to this total output uncertainty. The measure used in SAPHIRE is based on a Taylor series expansion of the variance of the output of interest. The equation used by SAPHIRE is

$$
\operatorname{Var}(R) \approx \sum_{i=1}^{n}\left(\frac{\partial R}{\partial p_{i}}\right)^{2} \sigma_{i}^{2}
$$

where $\mathrm{R}$ is the output of interest, $\mathrm{p}_{\mathrm{i}}$ is the probability of the ith basic event, and $\sigma_{\mathrm{i}}{ }^{2}$ is the variance of the uncertainty distribution for the ith event. This approximation, which hinges upon the basic events being mutually statistically independent, says that the variance of the output is approximately the sum of $\mathrm{n}$ separate contributions, one from each basic event. The magnitude of each contribution (each contribution is positive) measures how much of the output variance is contributed by each basic event. Because it is more convenient, SAPHIRE uses the square root of each individual contribution as the uncertainty importance:

$$
I_{u n c}=\frac{\partial R}{\partial p_{i}} \sigma_{i}
$$

where $\sigma_{\mathrm{i}}$ is the standard deviation of the uncertainty distribution of the ith basic event. Note that the partial derivative in the above equation for the uncertainty importance is, by definition, the Birnbaum importance of that event. Therefore, no new calculations are needed; the uncertainty importance is the Birnbaum importance multiplied by the standard deviation of the input probability distribution.

\subsubsection{Multi-Var Importance}

This option allows the user to calculate an importance for a group of events.

For instance, the user can select all motor operated valves in a set of cut sets and get a combined importance measure for these valves together. All of the valves are treated as a single event in the importance calculations and the result is displayed with the name "Multi-variable." 


\subsubsection{Importance Measures Dialog}

\subsection{Importance Measures Results}

\section{PURPOSE}

This option displays the results of the importance measure analysis.

\section{STEPS}

1. From the SAPHIRE menu select Fault Tree/Sequence/End State. The Fault Tree List/Sequences/End State List dialog will be displayed.

2. Highlight the desired list item and right-click to invoke the pop-up menu.

3. Choose Display, then the Importance submenu option.

4. Choose from one of the submenu options: Ratio, Difference, Uncertainty, Mult-Var. The Importance Measures dialog will be displayed.

The results shown here are Fussell-Vesely importance measures. If the analysis type is seismic, the program will prompt you to select a ground acceleration level defined in the project hazard curve.

\begin{tabular}{|c|c|c|c|c|c|c|}
\hline \multicolumn{7}{|c|}{ Importance Heasures - [DEMO, CCS] } \\
\hline \multirow[b]{3}{*}{ Event Name } & \multirow[b]{3}{*}{$\begin{array}{l}\# \text { of } \\
\text { Occur }\end{array}$} & \multirow[b]{3}{*}{ Probability } & \multirow[b]{3}{*}{$F \cdot V$} & \multicolumn{3}{|c|}{ Sort } \\
\hline & & & & \multicolumn{2}{|l|}{$F \cdot V$} & $\nabla$ \\
\hline & & & & $\begin{array}{l}\text { Risk Reduc. } \\
\text { Ratio }\end{array}$ & $\begin{array}{l}\text { Risk Incre. } \\
\text { Ratio }\end{array}$ & \\
\hline \multirow[t]{2}{*}{$\begin{array}{l}\text { DG-B } \\
\text { C-MOV-1 } \\
\text { DG-A } \\
\text { C-MOV-B } \\
\text { C-PUMP-B } \\
\text { C-MOV-A } \\
\text { C-PUMP-A } \\
\text { C-CV-A } \\
\text { C-CV-B }\end{array}$} & $\begin{array}{l}1 \\
1 \\
3 \\
4 \\
4 \\
3 \\
3 \\
3 \\
4\end{array}$ & $\begin{array}{l}2.000 \mathrm{E}-002 \\
1.000 \mathrm{E}-003 \\
2.000 \mathrm{E}-002 \\
5.000 \mathrm{E}-003 \\
3.000 \mathrm{E}-003 \\
5.000 \mathrm{E}-003 \\
3.000 \mathrm{E}-003 \\
1.000 \mathrm{E}-003 \\
1.000 \mathrm{E}-004\end{array}$ & $\begin{array}{l}9.418 \mathrm{E}-001 \\
4.619 \mathrm{E}-002 \\
7.477 \mathrm{E}-003 \\
6.692 \mathrm{E}-003 \\
4.015 \mathrm{E}-003 \\
1.869 \mathrm{E}-003 \\
1.121 \mathrm{E}-003 \\
3.738 \mathrm{E}-004 \\
1.338 \mathrm{E}-004\end{array}$ & $\begin{array}{l}1.718 \mathrm{E}+001 \\
1.048 \mathrm{E}+000 \\
1.008 \mathrm{E}+000 \\
1.007 \mathrm{E}+000 \\
1.004 \mathrm{E}+000 \\
1.002 \mathrm{E}+000 \\
1.001 \mathrm{E}+000 \\
1.000 \mathrm{E}+000 \\
1.000 \mathrm{E}+000\end{array}$ & \multicolumn{2}{|l|}{$\begin{array}{l}4.715 E+001 \\
4.715 E+001 \\
1.366 E+000 \\
2.322 E+000 \\
2.325 E+000 \\
1.371 E+000 \\
1.372 E+000 \\
1.373 E+000 \\
2.329 E+000\end{array}$} \\
\hline & Description & \multicolumn{2}{|c|}{ Partition } & Report & \multicolumn{2}{|l|}{ Exxit } \\
\hline
\end{tabular}

Sort -

Select from the drop-down list to sort results by Occurrence, Probability, F-V / Birnbaum / Uncertainty, Reduction, or Increase. By default, when the importance data are first displayed it is sorted, high to low, by F-V / Birnbaum / Uncertainty importance value.

Description - Display the full description for the highlighted event in the message bar of the SAPHIRE main window.

Partition - $\quad$ Limit which events are displayed/reported.

Report - Generate a report of the data that is displayed on the dialog.

Exit - Close the Importance Measures dialog. 


\subsection{Importance Results Partition}

\section{PURPOSE}

This option allows you to redefine a fault tree as a subset of the original cut sets. This is accomplished by defining a set of events to be used to determine whether a cut set belongs to a partition.

\section{STEPS}

1. From the SAPHIRE menu select Fault Tree/Sequence/End State. The Fault Tree

List/Sequences/End State List dialog will be displayed.

2. Highlight the desired list item and right-click to invoke the pop-up menu.

3. Choose Display | Importance.

4. Choose from one of the submenu options: Ratio, Difference, Uncertainty, Mult-Var. The Importance Measures dialog will be displayed.

5. Choose the Partition button. The Partition dialog will be displayed.

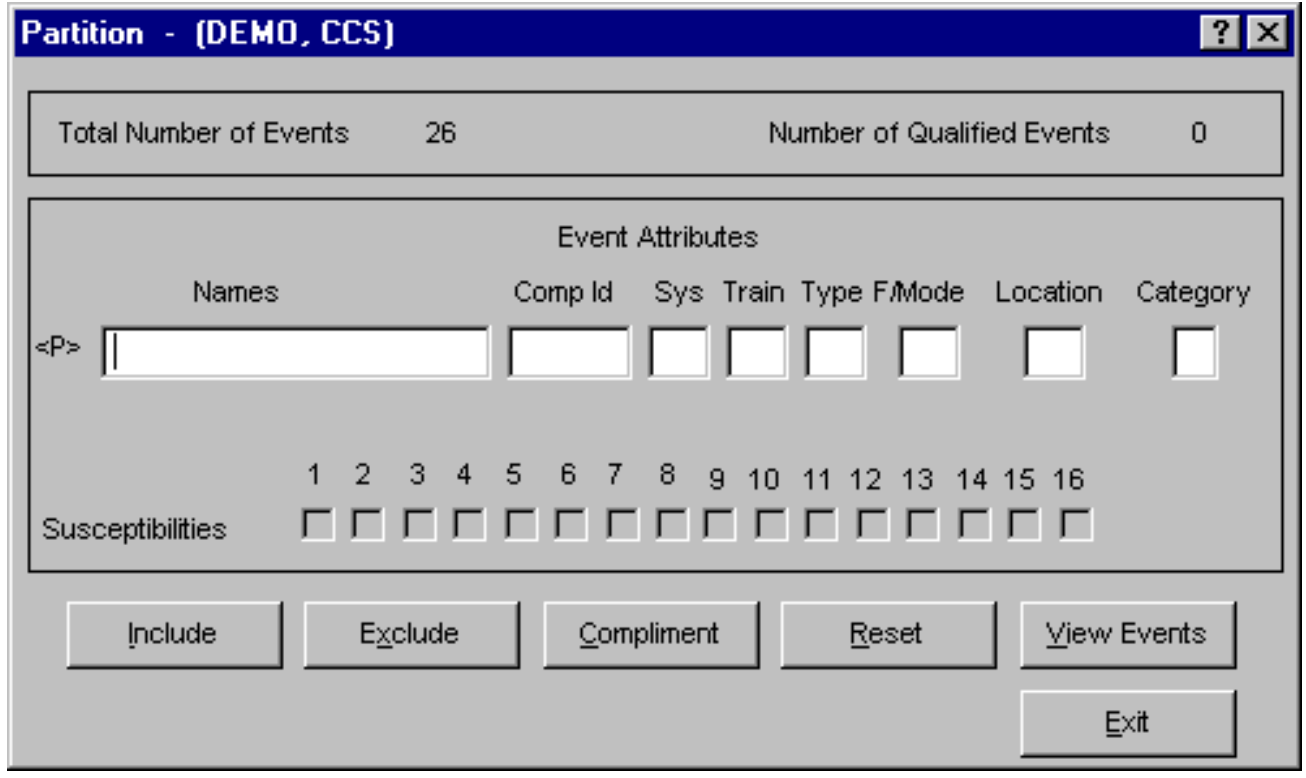

Include - $\quad$ Fill in the entry fields that are to be used to qualify the events that may be used in the new partition. Choose the Include button. The events in the cut set are qualified and when complete, will update the Number of Qualified Events field that appears in the upper right of the Partition dialog.

Exclude - $\quad$ Fill in the entry fields that are to be used to remove events from the list of qualified events. Choose the Exclude button. The events in the cut set are qualified and when complete, will update the Number of Qualified Events field that appears in the upper right of the Partition dialog.

Compliment - Cause all currently qualified events to be disqualified, and all unqualified events to become the set of qualified events.

Reset - $\quad$ Set all events in the database to be qualified. This removes all partitioning from the current cut sets. 
View Events - Display the list of all events in the project. Mark those events that are to be considered qualified.

Exit - $\quad$ Close the Partition dialog. Only those cut sets that are made up of qualified events are displayed on the Importance Measures dialog.

\subsection{View Events}

\section{PURPOSE}

This option allows you to display the list of all events in the project and mark those events that are to be used to determine whether a cut set belongs to a partition.

\section{STEPS}

1. From the SAPHIRE menu select Fault Tree/Sequence/End State. The Fault Tree List/Sequences/End State List dialog will be displayed.

2. Highlight the desired list item and right-click to invoke the pop-up menu.

3. Choose Display | Importance.

4. Choose from one of the sub-menu options: Ratio, Difference, Uncertainty, Mult-Var.

5. The Importance Measures dialog will be displayed.

6. Choose the Partition button. The Partition dialog will be displayed.

7. Choose the View Events button. The Events dialog will be displayed.

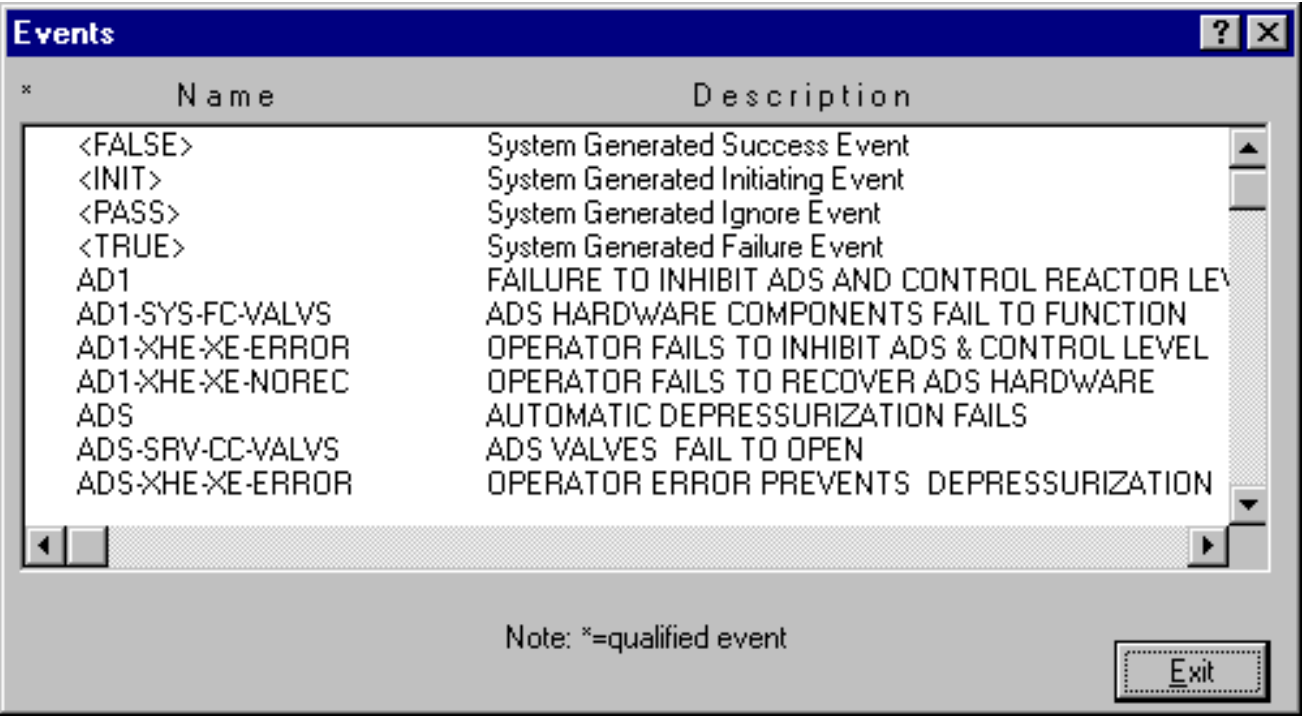

Double-click - Mark the selected event. An asterisk (*) will appear to the left of the event name. 
Exit - $\quad$ Close the Events dialog. Choose the Include or Exclude button from the Partition dialog..

\subsubsection{Displaying Uncertainty}

\subsubsection{Displaying Uncertainty Results}

\section{PURPOSE}

This option displays the results of the uncertainty analysis previously performed. The results displayed are the distribution and probability bounds for both base and current results. These values were calculated using either the Latin Hypercube or the Monte Carlo simulation technique.

\section{STEPS}

1. From the SAPHIRE menu select Fault Tree/Sequence/End State. The Fault Tree List/Sequences/End State List dialog will be displayed.

2. Highlight the desired list item and right-click to invoke the pop-up menu.

3. Choose Display, then the Uncertainty sub-menu option. The Fault Tree/Sequence/End State Uncertainty dialog will be displayed.

If the analysis type is seismic, you will be prompted to select a ground acceleration level defined in the project hazard curve.

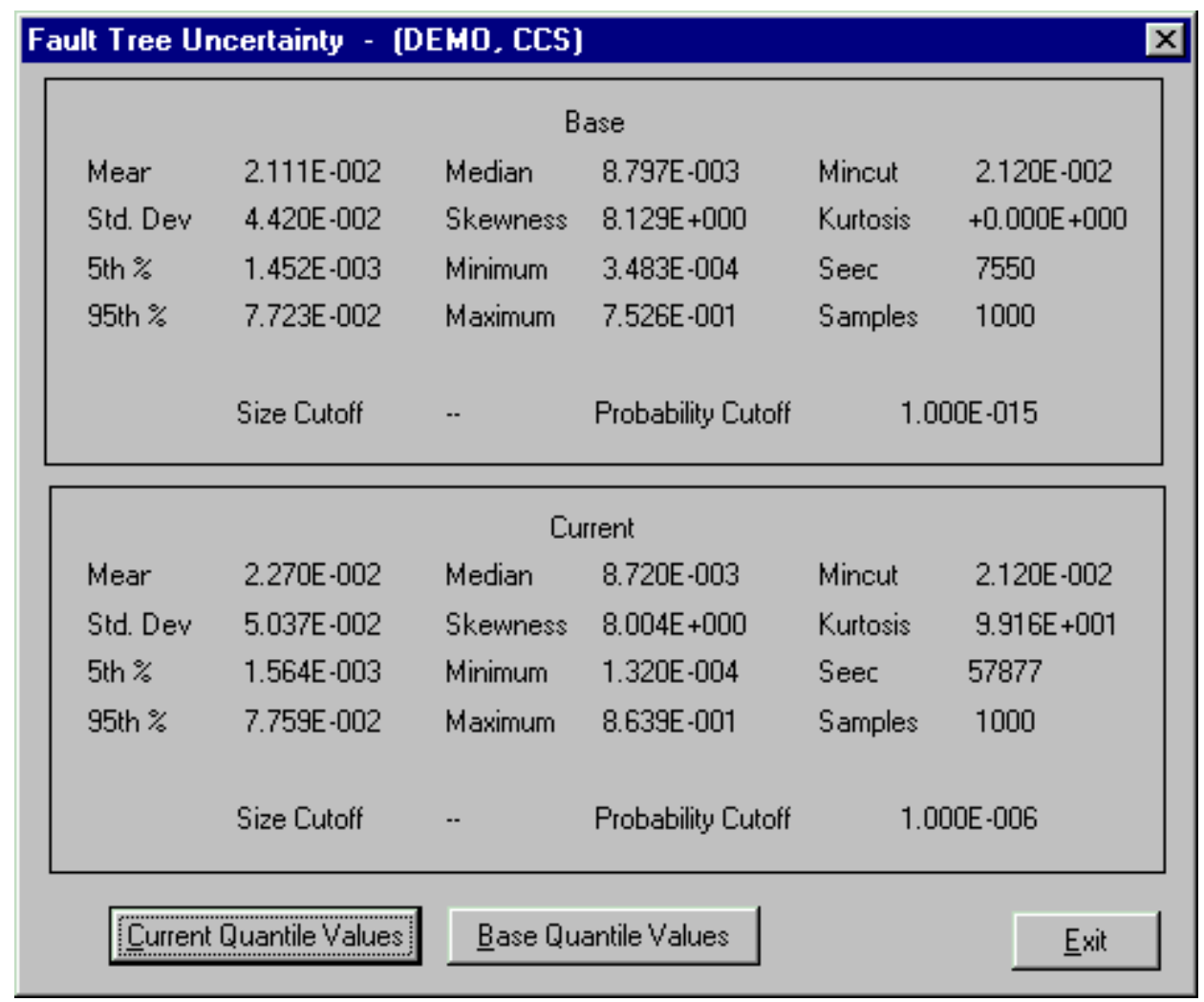


Current Quantile Values Base Quantile Values-

Exit dialog.
View the quantile values associated with the current case data. View the quantile values associated with the base case data.

Close the Fault Tree/Sequence/End State Uncertainty

\subsubsection{Uncertainty Quantile Values}

\section{PURPOSE}

Display the uncertainty quantile (or probability) values associated with either the current or base case data. These quantile values can be used to construct a cumulative distribution plot of the analysis uncertainty results.

\section{STEPS}

1. From the SAPHIRE menu select Fault Tree/Sequence/End State. The Fault Tree List/Sequences/End State List dialog will be displayed.

2. Highlight the desired fault tree and right-click to invoke the pop-up menu.

3. Choose Display, then the Uncertainty sub-menu option. The Fault Tree/Sequence/End State Uncertainty dialog will be displayed.

4. Choose the Current Quantile Values or Base Quantile Values button.

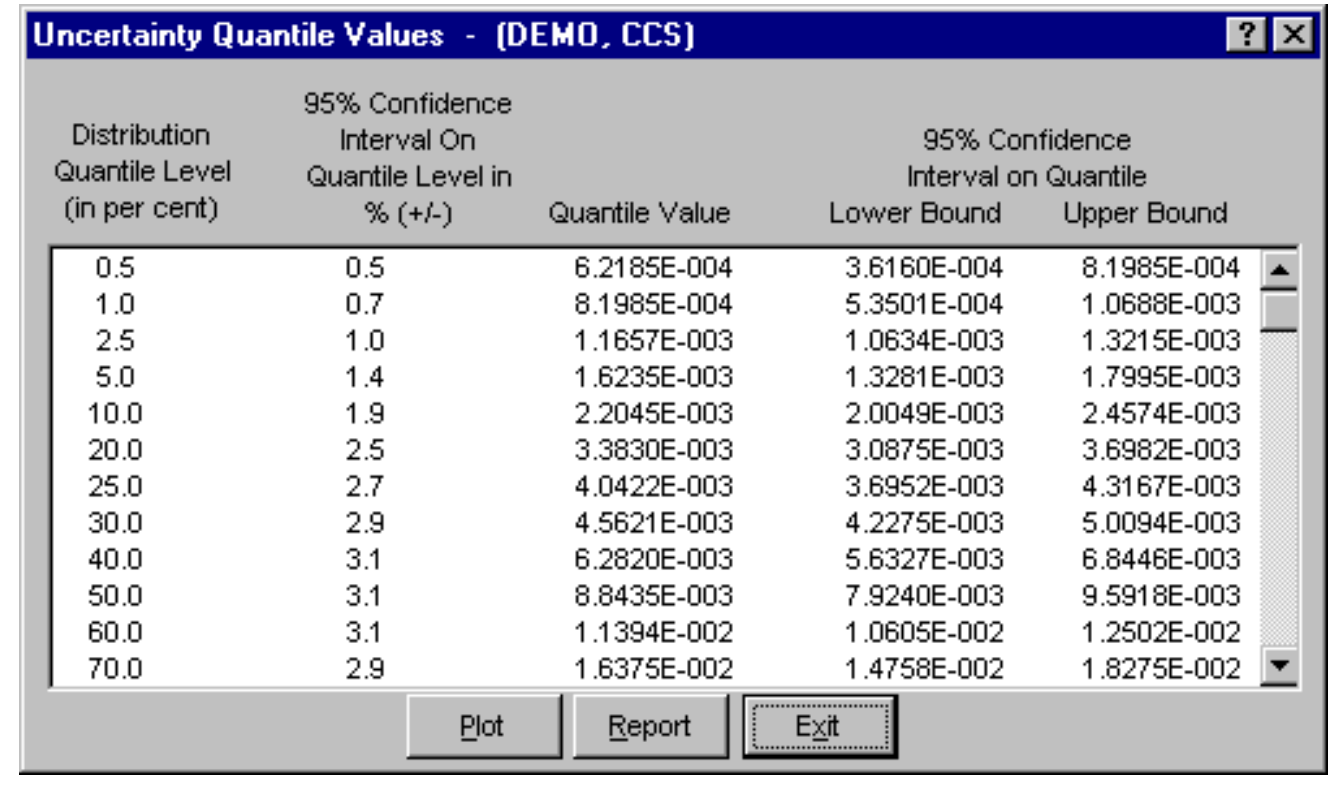

Distribution Quantile Level (in percent) - The probability level, which ranges from $0.5 \%$ to $99.5 \%$.

95\% Confidence Interval On Quantile Level in \% (+/-) - The 95\% confidence level on the quantile level (e.g., $5 \%, 50 \%, 90 \%$ ) expressed in terms of the percent of the quantile level. Note that this is the confidence on the quantile level, not the quantile value.

Quantile Value - The value obtained from the uncertainty analysis at the i'th \% quantile (or probability) level. The units on the value varies depending on the type of analysis (e.g., failure probability, core damage frequency, etc.). 
95\% Lower Bound Confidence Level On Quantile Value - The lower bound confidence level on the quantile value.

95\% Upper Bound Confidence Level On Quantile Value - The upper bound confidence level on the quantile value.

Plot - Show a graphical representation of the uncertainty distribution defined by the uncertainty quantile values.

Report - Generate a report containing the quantile values as displayed here.

Exit - Close the Uncertainty Quantile Values dialog.

\subsubsection{Plot Uncertainty Quantile Values}

\section{PURPOSE}

Show a graphical representation of the distribution defined by the uncertainty quantile (or probability) values associated with either the current or base case data.

\section{STEPS}

1. From the SAPHIRE menu select Fault Tree/Sequence/End State. The Fault Tree List/Sequences/End State List dialog will be displayed.

2. Highlight the desired fault tree and right-click to invoke the pop-up menu.

3. Choose Display, then the Uncertainty sub-menu option. The Fault Tree/Sequence/End State Uncertainty dialog will be displayed.

4. Choose the Current Quantile Values or Base Quantile Values button.

5. Choose the Plot button. The Uncertainty plot dialog will be displayed. 


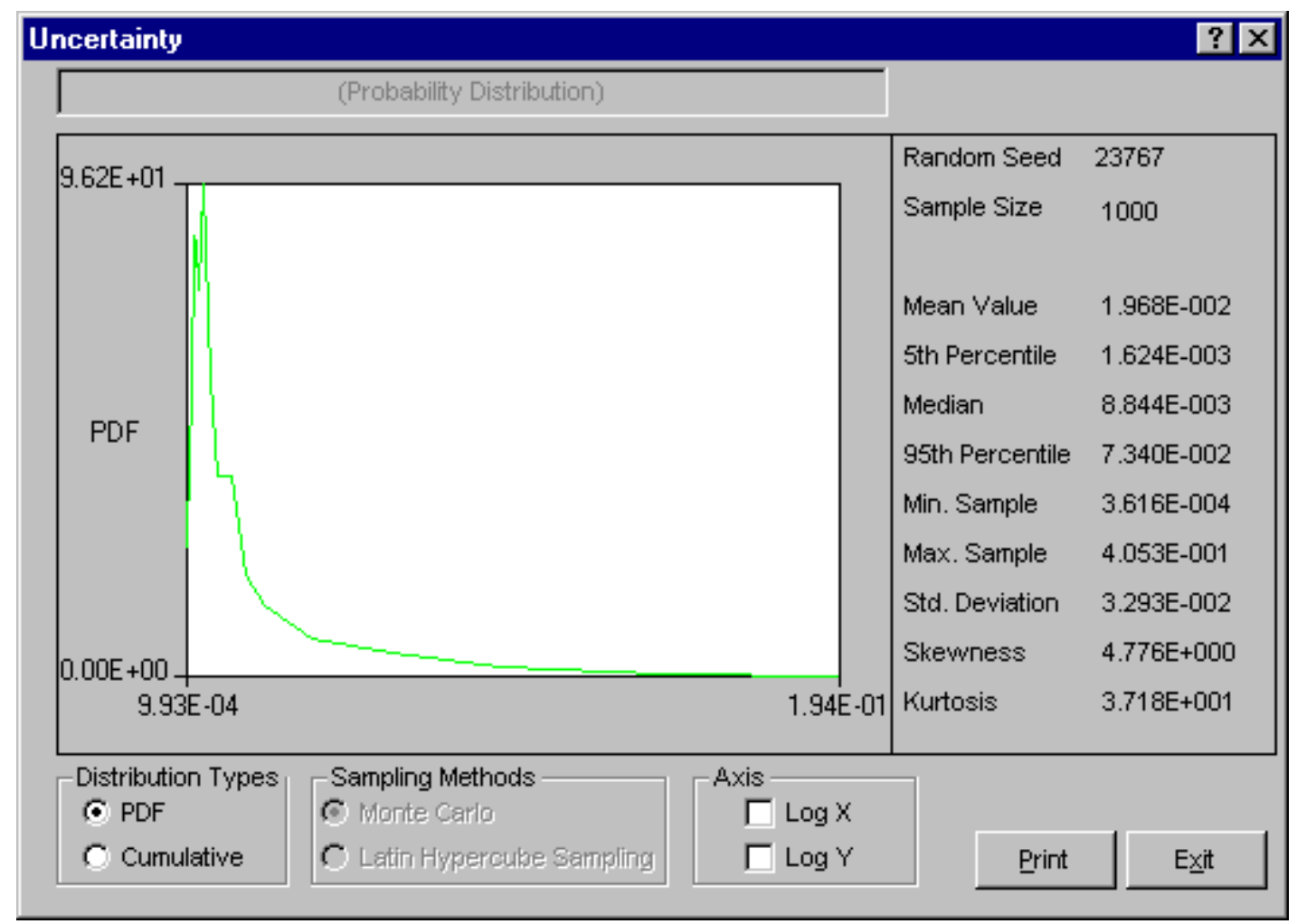

Print - Print the plot as displayed. The Print common dialog will be launched where you can select the desired printer.

Exit - Close the Uncertainty plot dialog.

\subsubsection{Distribution Types}

Select the type of graph to be displayed:

PDF - $\quad$ (Default) - Displays a graph showing the Probability Density Function.

Cumulative - Displays a graph showing the Cumulative Density Function.

\subsubsection{Sampling Methods}

The selected radio button indicates the sampling method used for the uncertainty calculation. Note the sampling method used cannot be changed here. In order to view results using a different sampling method, the uncertainty analysis must be re-run using the desired sampling method.
Monte Carlo -
Displays a graph that is based on Monte Carlo Sampling of the
Latin Hypercube Sampling - defined uncertainty distribution.
Displays a graph that is based on Latin Hypercube Sampling of the defined uncertainty distribution.

\subsubsection{Axis}


$\log \mathbf{X}$ - Produces a graph where the Log base 10 of the X-Values are used instead of just the XValues.

$\log$ Y - Produces a graph where the Log base 10 of the Y-Values are used instead of just the YValues.

\subsection{Viewing End States}

\subsubsection{Viewing End States}

\section{PURPOSE}

This option presents the end state analyses in various report forms. One or more end states can be viewed using this option.

\section{STEPS}

1. From the SAPHIRE menu select End State. The End State List dialog will be displayed.

2. Highlight the desired end state(s) and right-click to invoke the pop-up menu.

3. Choose View Results from the menu. The EndState Review dialog will be displayed.

\begin{tabular}{|c|c|c|c|c|c|}
\hline \multicolumn{5}{|c|}{ EndState Review - [DEMO] } & \multirow{2}{*}{$\begin{array}{l}x \\
\end{array}$} \\
\hline Sort Order: Name & & $\nabla$ Analysis Type & RANDOM & & \\
\hline End State & Curr Freq & Base Freq & Difference & Ratio & $\Delta$ \\
\hline LARGE-RELEASE & $1.760 \mathrm{E}-03$ & $1.760 \mathrm{E}-03$ & $0.000 \mathrm{E}+00$ & $1.000 E+00$ & \\
\hline SMALL-RELEASE & $0.000 \mathrm{E}+00$ & $0.000 \mathrm{E}+00$ & $0.000 \mathrm{E}+00$ & $0.000 \mathrm{E}+00$ & \\
\hline TOTALS $=$ & $1.760 \mathrm{E}-03$ & $1.760 \mathrm{E}-03$ & $0.000 \mathrm{E}+00$ & $1.000 \mathrm{E}+00$ & \\
\hline & & & & & \\
\hline & & & & & \\
\hline & & & & & \\
\hline & & & & & \\
\hline & & & & & \\
\hline & & & & & \\
\hline & & & & & \\
\hline & & & & & \\
\hline & & & & & \\
\hline & & & & & \\
\hline & & & & & $\nabla$ \\
\hline \begin{tabular}{|l|l|}
1 & \\
\end{tabular} & & & & D & \\
\hline Views & & & & & \\
\hline C Description & C Base Case & $C$ Base Case & Unc. & Report & \\
\hline (c) Current Vs. Base & C Current Case & $\mathrm{C}$ Current $\mathrm{C} \varepsilon$ & Se Unc. & Exit & \\
\hline
\end{tabular}

The selected end states are displayed in this dialog. The End State column contains the end states' primary names. Other columns' titles and data vary depending on the selected "View". 
The last row in the grid show the summation totals of applicable columns.

Report - Produce a report of the data currently displayed. The output can go to the Report Viewer, a printer, or a file.

Exit - $\quad$ Close the EndState Review dialog.

\subsubsection{View Options}

Description -

Current Vs. Base -

Base Case -

Current Case -
Includes the full description. (60 character maximum)

Includes the current case cut set probability, base case cut set probability, the probability difference (current - base), ratio, current case cut set count, base case cut set count, the cut set count difference (current base).

Includes base case values for cut set probability, mean, $5^{\text {th }}$ percentile, median, $95^{\text {th }}$ percentile, probability truncation, and size truncation used when solving.

Includes current case values for cut set probability, mean, $5^{\text {th }}$ percentile, median, $95^{\text {th }}$ percentile, probability truncation, and size truncation used when solving.

Base Case Uncertainty - Includes base case values for cut set probability, mean, $5^{\text {th }}$ percentile, median, $95^{\text {th }}$ percentile, minimum, maximum, standard deviation, skewness, kurtosis, sample size, and random number seed.

Current Case Uncertainty-Includes current case values for cut set probability, mean, $5^{\text {th }}$ percentile, median, $95^{\text {th }}$ percentile, minimum, maximum, standard deviation, skewness, kurtosis, sample size, and random number seed. 


\section{MODIFYING THE DATA BASE}

\subsection{Modifying the Data Base}

\section{PURPOSE}

This option allows you to modify SAPHIRE database files.

\section{STEPS}

1. From the SAPHIRE menu select Modify. The drop-down menu with available items will be displayed.

After selecting an option from the menu, a dialog containing a list of all records for the selected data type appears. In general, the functions: add, copy, modify, and delete are then available from a pop-up menu. Some of the dialogs have additional options accessible by choosing the appropriate button.

\begin{tabular}{|l|}
\hline Modify Report Utili \\
\hline Project \\
Event Trees \\
Fault Trees \\
End States \\
Easic Events \\
Attributes \\
Analysis Iypes \\
Gates \\
Histograms \\
P \& IDs \\
Change Sets \\
Finag Sets \\
\hline
\end{tabular}

\subsection{Modifying Project Data}

\subsubsection{Project Data Entry Dialog}

\section{PURPOSE}

The Project dialog allows you to modify project data. 


\section{STEPS}

1. From the menu select Modify.

2. Choose Project from the menu. The Project dialog is displayed.

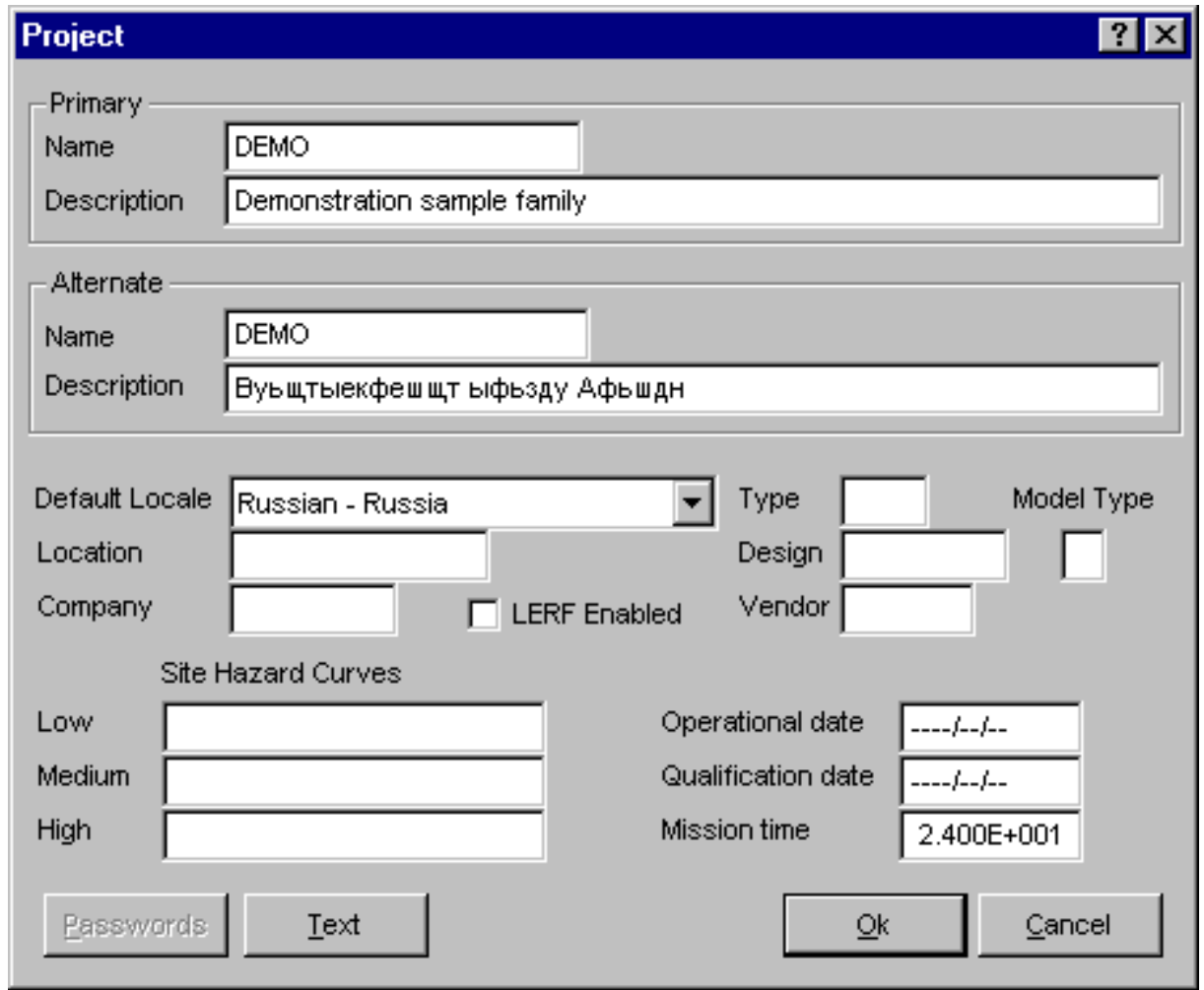

Ok - Save the modified project record and close the dialog.

Cancel - Close the dialog without saving.

\subsubsection{Name}

This is a required field. A maximum of 24 uppercase, alphanumeric characters may be entered. Embedded blanks are not allowed. The name should be descriptive so it can be readily identified.

\subsubsection{Type}

This is a user-defined field used to classify the facility under study. A maximum of 3 characters (uppercase, alphanumeric) may be entered.

\subsubsection{Location}

This is a user-defined field used to specify the geographical location of the facility under study. A maximum of 10 characters (uppercase, alphanumeric) may be entered. 


\subsubsection{Design}

This is a user-defined field used to identify the facility's design type. A maximum of 10 characters (uppercase, alphanumeric) may be entered. This field may contain embedded blanks.

\subsubsection{Model Type}

This field is used to identify the model solution type for this facility. An " $\mathrm{N}$ " indicates that this is a normal model. An "A" indicates that this is an ASP model. You may specify you own model designation or leave the field blank.

\subsubsection{Company}

This is user-defined field used to identify the company responsible for the facility. A maximum of 10 characters (alphanumeric) may be entered. This field may contain embedded blanks.

\subsubsection{Vendor}

This is a five-character, uppercase, alphanumeric field used to identify the company that built the facility.

\subsubsection{LERF Enabled}

Indicates that the model has been developed for use in Large Early Release Frequency (LERF) study. The "LERF" option in GEM allows a user to select an existing initiating event assessment or condition assessment (or make a new one), but evaluate the assessment using a LERF end state gather instead of the core damage sequences. GEM has been designed to look specifically for sequences labeled "PDS-" (either on the event tree graphic or created via event tree partition rules). As such, the plant model must be designed such that only LERF sequences are assigned to end states beginning with "PDS-" for the correct LERF results to be generated.

\subsubsection{Description}

This is a 120-character, uppercase or lowercase, alphanumeric field that provides brief, descriptive information.

\subsubsection{Default Locale}

Set the default or primary locale for this project. The locales listed are those that are loaded into memory when your computer is started. To add locales, see International SAPHIRE .

\subsubsection{Site Hazard Curves}

This is a 24-character field that identifies histograms to be used as hazard curves. Low, Medium and High histograms represent the differing views about susceptibility to earthquakes.

\subsubsection{Operational Date}

This is the date the facility became operational. The format for this field is YYYY/MM/DD.

\subsubsection{Qualification Date}

This is the qualification date of the facility. The format for this field is YYYY/MM/DD. 


\subsubsection{Mission Time}

The default mission time for this project.

\subsection{Modifying Event Tree Data}

\subsubsection{Event Trees}

\section{PURPOSE}

This option allows you to add, modify, copy, or delete event tree data records, or associated text. Additionally, you can update selected event tree base case data or clear selected event tree current case data. You can add, modify, copy or delete sequence data records associated with the selected event tree.

\section{STEPS}

1. From the SAPHIRE menu select Modify.

2. Choose Event Trees from the menu. The Edit Event Trees dialog is displayed, listing all of the event trees belonging to the current project.

3. Highlight the desired event tree.

4. Right-click to invoke the pop-up menu or choose the desired button.

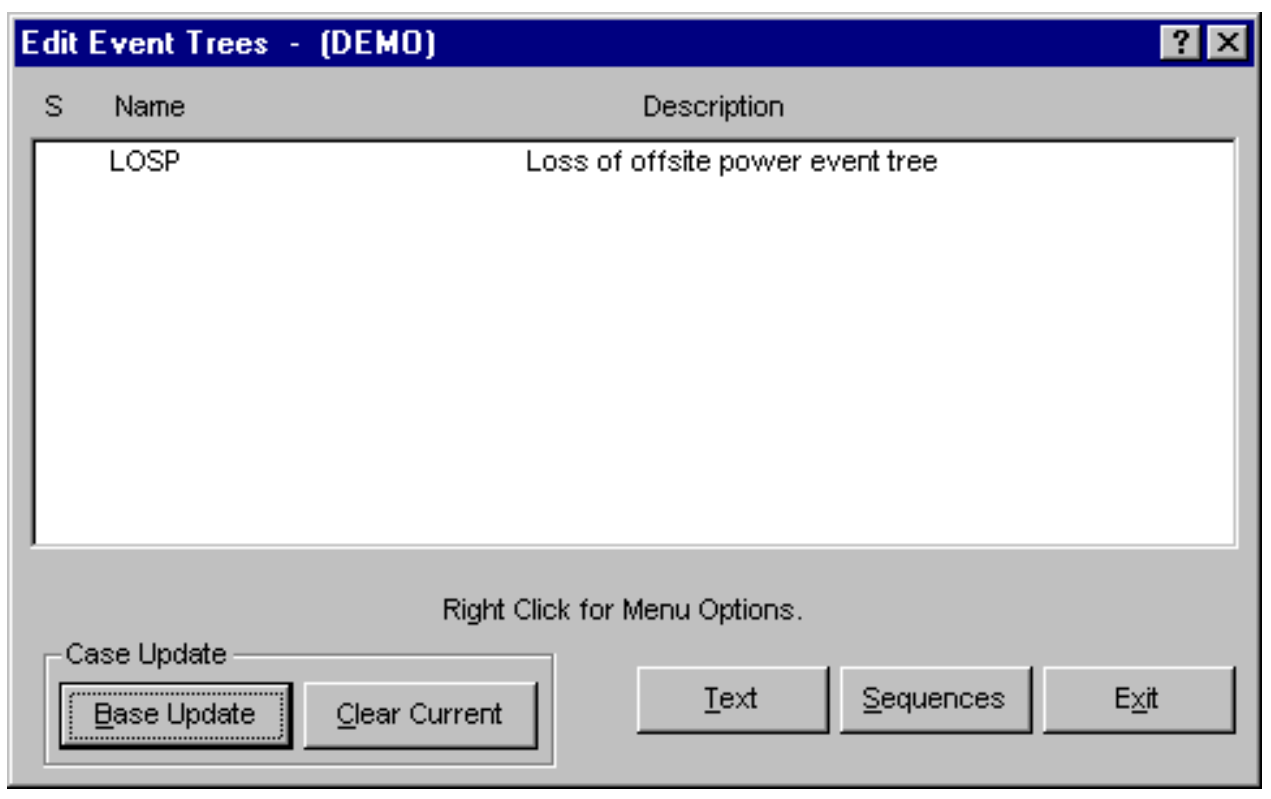

\section{Pop-up Menu Options}

Add -

Copy -

Modify -

Delete -

Graphics-

Reset -
Add a new event tree record to the database.

Create a new event tree record by copying an existing one.

Edit the selected event tree record.

Delete the selected event tree record(s).

Edit the graphical picture of the event tree.

Delete all the sequences that belong to the selected event tree. 


\section{Button Options}

Base Update - $\quad$ Update selected event tree base case data. The Base Case Update dialog will have slightly different wording than the dialog in this example.

Clear Current - Clear selected event tree current case data. The Clear Current Case dialog will have slightly different wording than the dialog in this example.

Text - $\quad$ View and edit the descriptive text associated with the selected event tree. Sequences - $\quad$ Add, modify, copy or delete sequence data records.

Exit Close the Edit Event Trees dialog.

\subsubsection{Delete An Event Tree}

\section{PURPOSE}

This option allows you to delete an event tree record and associated sequence records from the database.

\section{STEPS}

1. From the SAPHIRE menu select Modify.

2. Choose Event Trees from the menu. The Edit Event Trees dialog is displayed.

3. Highlight the event tree you wish to delete.

4. Right-click to invoke the pop-up menu and choose Delete. A warning dialog will appear, allowing you to cancel the deletion at this point.

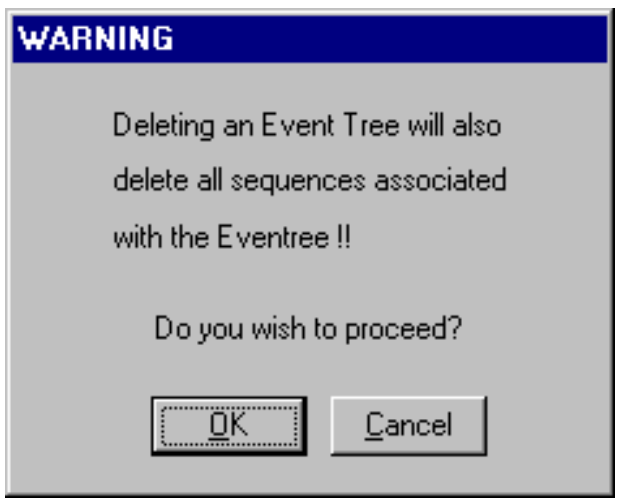

OK - Continue the delete operation. Once you choose $\mathbf{O K}$ at the warning screen to continue with the deletion process, another warning dialog is shown. You will be prompted to choose Yes or No. Upon choosing the Yes button, the selected event tree record and all sequence records associated with the event tree will be deleted from the database.

Cancel - Terminate the delete operation.

NOTE: You cannot delete a sub-tree (if it is used by another event tree) using this option. 


\subsubsection{Base Case Update}

\section{PURPOSE}

This option allows you to overwrite all base case (original) data with the current case data for the selected analysis type. If you choose to update the base data, four operations will occur. (1) The base case cut sets will be overwritten with the current case cut sets. (2) The base case uncertainty results will be overwritten with the current case uncertainty results. (3) The base case uncertainty quantile values will be overwritten with the quantile values from the current case. (4) The base case minimum cut set upper bound will be overwritten with the current case minimum cut set upper bound. As a note of caution, the original base case results will be overwritten if this option is executed!

\section{STEPS}

1. From the SAPHIRE menu select Modify.

2. Choose Event Trees. The Edit Event Trees dialog is displayed.

OR

3. Choose Event Trees | Sequences. The Edit Sequences dialog is displayed.

OR

4. Choose Fault Trees. The Edit Fault Trees dialog is displayed.

$O R$

5. Choose End States. The Edit End State dialog is displayed.

6. Highlight the desired list item.

7. Choose the Base Update button. The Base Case Update dialog will be displayed.

You will be prompted for confirmation before performing the update. In addition, you must select the analysis type from the drop-down list.

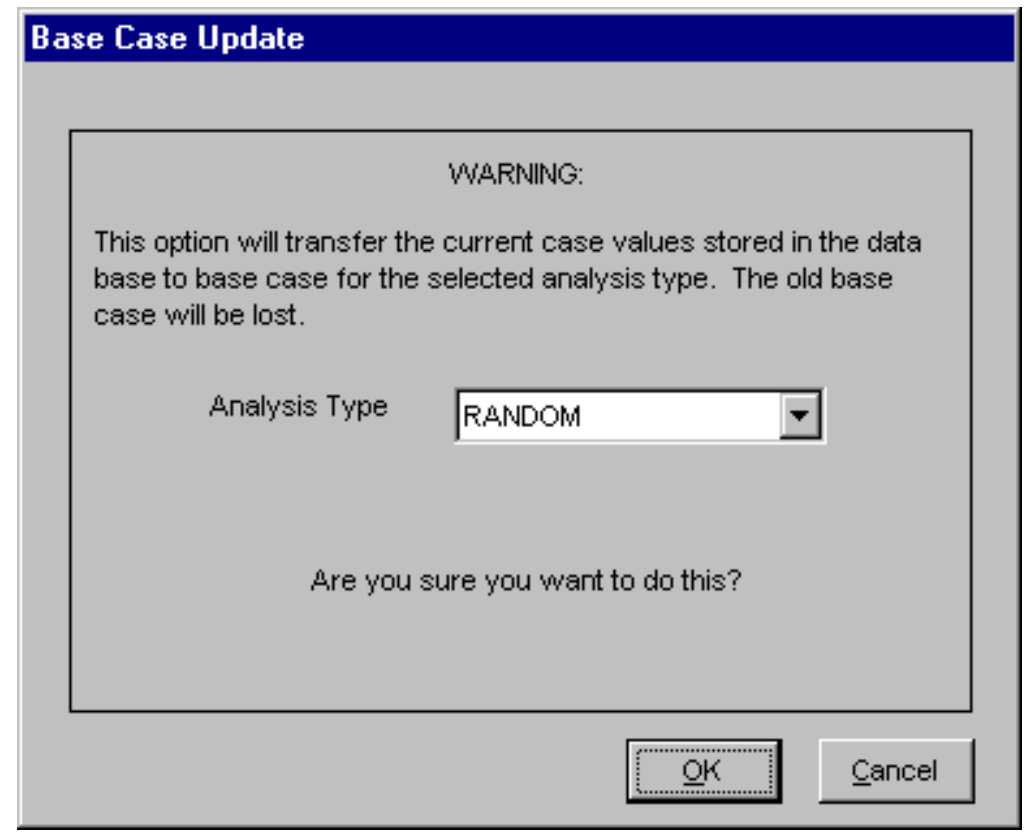


OK - Cause the current case data to overwrite the base case data. Upon completion of this process, a message, "Base case update complete" will be displayed in the message bar of the SAPHIRE window.

Cancel - Terminate the update operation.

\subsubsection{Clear Current Case}

\section{PURPOSE}

This option deletes all current case information for the specified analysis type. All SAPHIRE calculations use data stored in the current case for sensitivity and event analysis, and cut set generation results are stored in the current case.

\section{STEPS}

1. From the SAPHIRE menu select Modify.

2. Choose Event Trees. The Edit Event Trees dialog is displayed. OR

3. Choose Event Trees $\mid$ Sequences. The Edit Sequences dialog is displayed. OR

4. Choose Fault Trees. The Edit Fault Trees dialog is displayed. OR

5. Choose End States. The Edit End State dialog is displayed.

6. Highlight the desired list item.

7. Choose the Clear Current button. The Clear Current Case dialog will be displayed.

You will be prompted for confirmation before performing the clear operation. In addition, you must select the analysis type from the drop-down list.

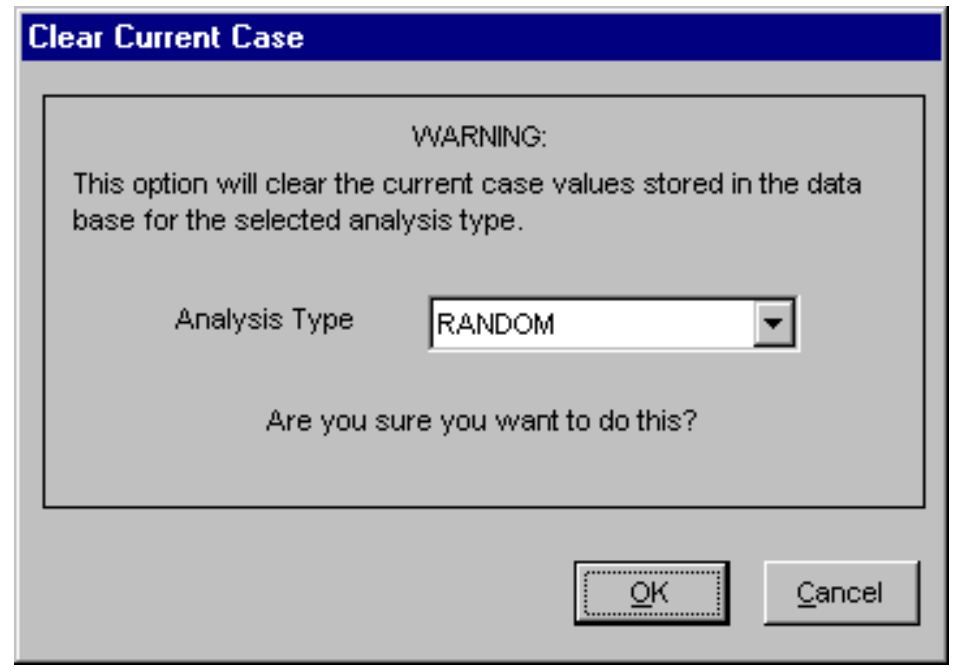

OK - Cause the current case data to be deleted. Upon completion of this procsss, a message, "Current Case Cleared..." will be displayed in the message bar of the SAPHIRE window.

Cancel - Terminate the clear current case process. 


\subsubsection{Database Text}

\section{PURPOSE}

This option allows you to view and edit the descriptive text associated with a specific record. The Primary text option is generally used for text in the desired primary language. The Alternate text option is generally used for information entered in the secondary language using a different alphabet (locale ).

\section{STEPS}

1. From the SAPHIRE menu select Modify.

2. Choose the desired option from the menu.

3. Highlight the record whose text you wish to modify.

4. Choose the Text button. The Select Text Type dialog is displayed.

5. Choose Primary or Alternate. The Text dialog is displayed.

Initially, the first 18 lines of the text block are displayed. If there are more lines of text, the vertical scroll bar will be available. In the example here, the Primary text dialog is displayed.

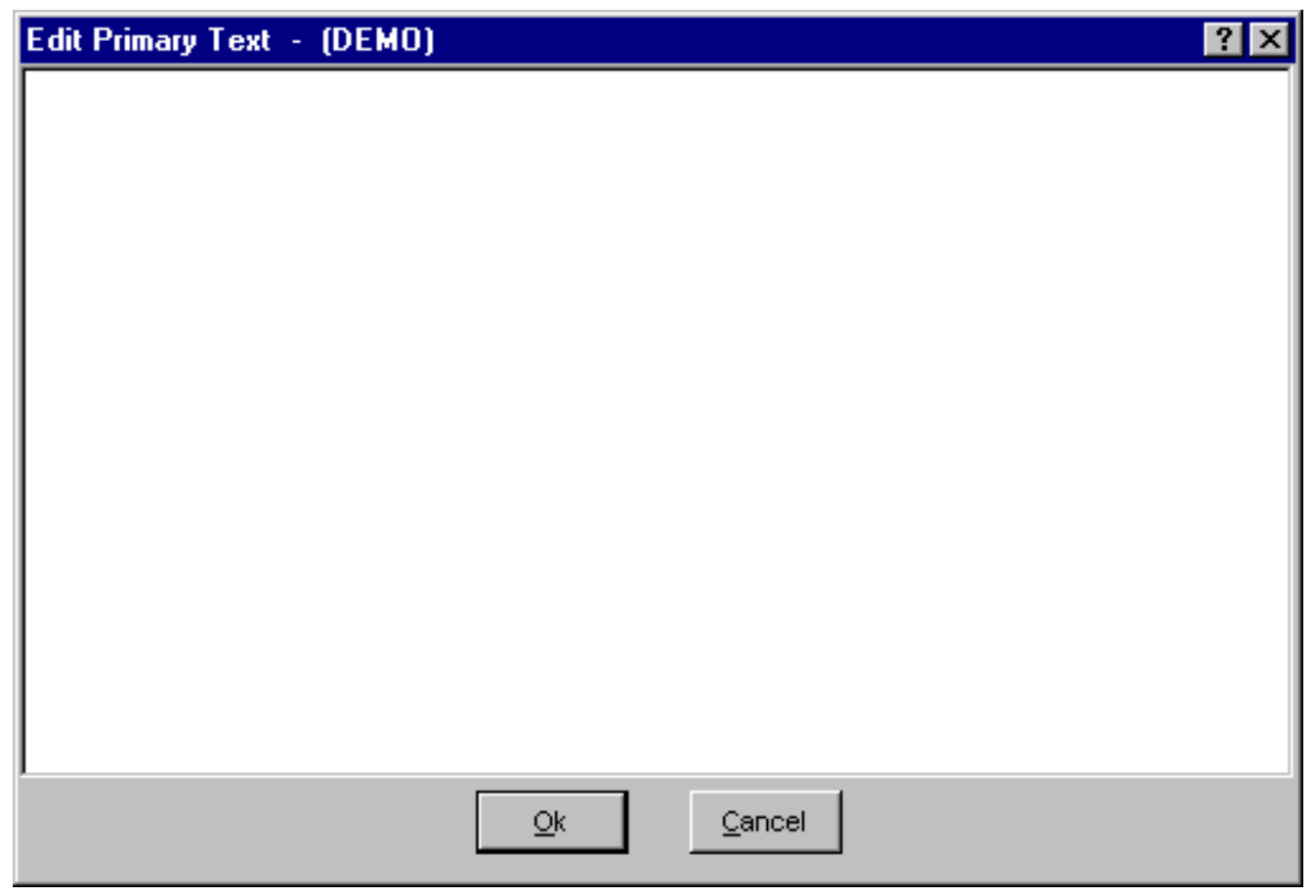

Page Down - $\quad$ Presents the next 18 lines of text.

Page Up - Presents the previous 18 lines of text.

Ctrl Page Down-Places the cursor at the end of the last line of text currently visible.

Ctrl Page Up - Places the cursor at the beginning of the first line of text currently visible.

Ctrl- : Moves the cursor left to the beginning of the word on the current line.

Ctrl- $\rightarrow$ - $\quad$ Moves the cursor right to the beginning of the word on the current line.

Home - Places the cursor in front of the first character of the current line.

Ctrl-Home - Places the cursor at the beginning of the text block. 

End -
Places the cursor behind the last character of the current line.
Ctrl-End - Places the cursor at the end of the text block.
Insert - Works as a toggle. Initially, the editor is in "insert" mode - existing characters are pushed to the right as you type. Pressing the Insert key toggles to "overwrite" mode - existing characters are over written as you type.
Delete - Deletes the character to the right of the current cursor position; or deletes the currently highlighted text.
Ctrl-Delete - Deletes the characters from the current cursor position to the beginning of the following word.
Backspace - $\quad$ Deletes the character to the left of the current cursor position; or deletes the currently highlighted text.
Ctrl-C - $\quad$ Copies highlighted text to the clipboard.
Ctrl-V - Pastes clipboard text at the current cursor position.
Ctrl-X - $\quad$ Cuts highlighted text to the clipboard.

The editor does not automatically line wrap; therefore, you must use $<$ Enter $>$ to establish each new line of text.

Ok - $\quad$ Save the new or modified text and close the Text dialog.

Cancel - Close the Text dialog without saving changes. You will be prompted with the dialog shown below.

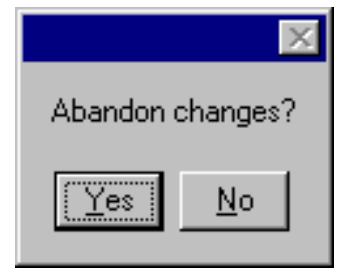

Yes - $\quad$ Close the Text dialog without saving changes.

No - $\quad$ Save the new or modified text and close the Text dialog.

\subsubsection{Event Tree Data Entry Dialog}

\section{PURPOSE}

The Add/Modify Event Tree dialog allows you to enter or modify event tree data.

\section{STEPS}

1. From the SAPHIRE menu select Modify.

2. Choose Event Trees from the menu. The Edit Event Trees dialog is displayed.

3. Highlight the event tree you wish to edit or copy. (Skip this step if adding a new event tree record.)

4. Right-click to invoke the pop-up menu and choose Add, Copy, or Modify.

$O R$

5. From the SAPHIRE menu select Event Tree. The Event Tree List dialog will be displayed.

6. Right-click to invoke the pop-up menu and select Add Event Tree. 


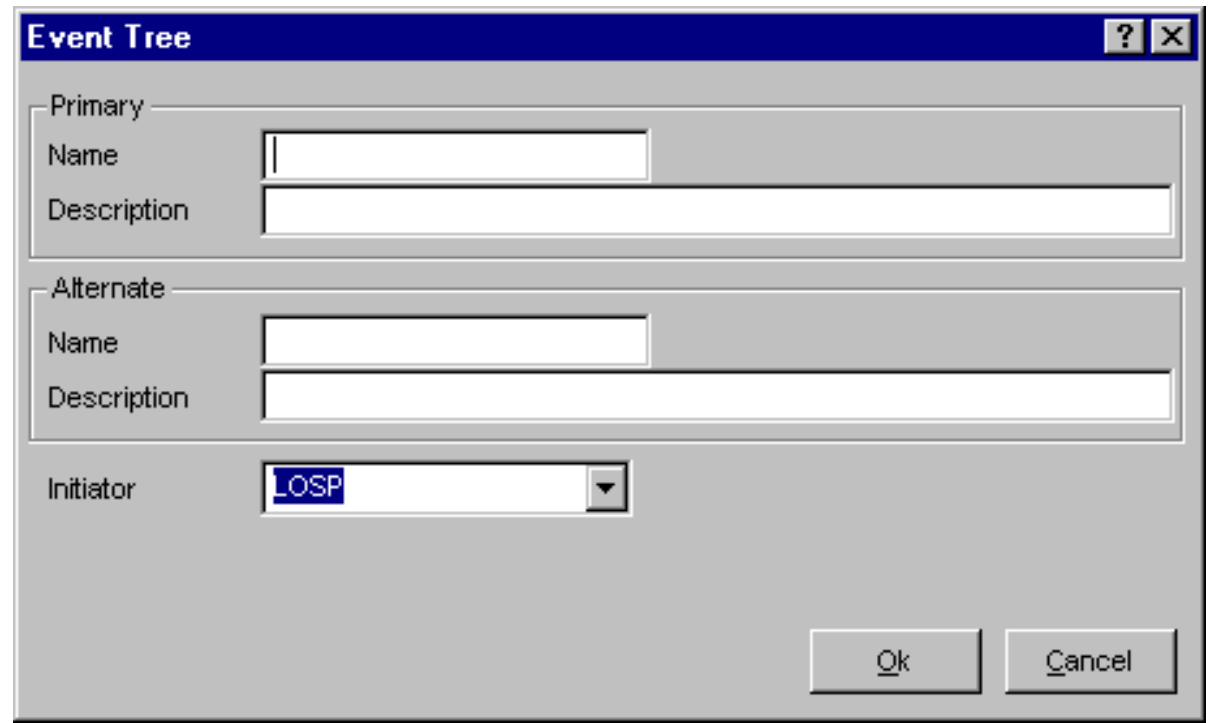

Ok - $\quad$ Save the new or modified event tree record and close the dialog.

Cancel - Close the dialog without saving.

\subsubsection{Event Tree Data Entry Dialog}

\subsubsection{Name}

This is a required field. A maximum of 24 uppercase, alphanumeric characters may be entered. Embedded blanks are not allowed. The name should be descriptive so it can be readily identified.

\subsubsection{Description}

This is a 120-character, uppercase or lowercase, alphanumeric field that provides brief, descriptive information.

\subsubsection{Initiating Event Name}

This is the current event tree's initiating event. The drop-down list contains all initiating events in the current project. Choose an initiating event from the drop-down list or enter a new one.

\subsubsection{Edit Sequences Dialog}

\subsubsection{Sequences}

\section{PURPOSE}

This option allows you to add, modify, copy, or delete data records, or text for the sequences associated with an event tree. Additionally, you can update selected sequence base case data or clear selected sequence current case data. 


\section{STEPS}

1. From the SAPHIRE menu select Modify.

2. Choose Event Trees from the menu. The Edit Event Trees dialog is displayed.

3. Highlight the desired event tree and choose the Sequences button.

4. The Edit Sequences dialog will be displayed listing all of the sequences associated with the selected event tree.

5. Highlight the desired sequence.

6. Right-click to invoke the pop-up menu or choose the desired button.

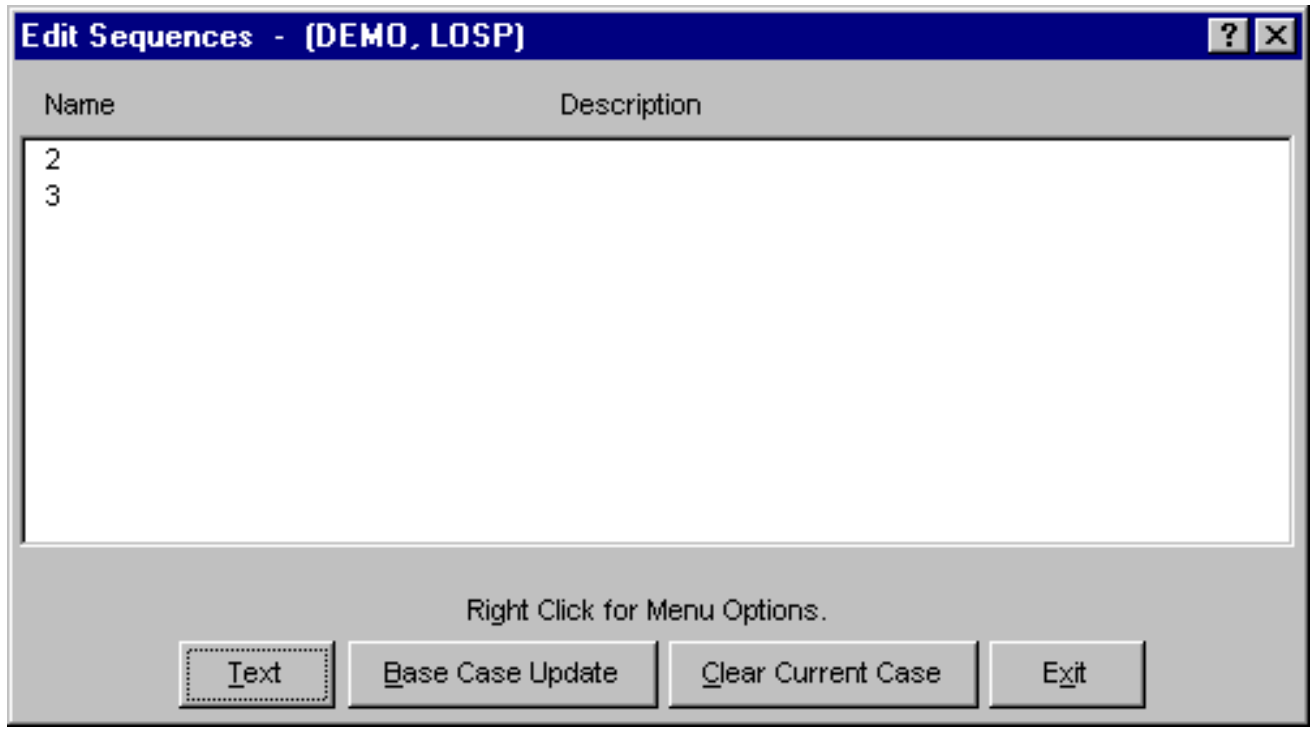

Pop-up Menu Options

Add -

Copy -

Modify -

Delete -

\section{Button Options}

Text -

Base Case Update Clear Current Case Exit -
Add a new sequence record to the database.

Create a new sequence record by copying an existing one.

Edit the selected sequence record.

Delete the selected sequence record(s).

\subsubsection{Delete Record}

\section{PURPOSE}

This option allows you to delete the highlighted record from the database.

\section{STEPS}

View and edit the descriptive text associated with the selected sequence.

Update selected sequence base case data.

Clear selected sequence current case data.

Close the Edit Sequences dialog.

1. From the SAPHIRE menu select Modify. 
2. Choose the desired option from the menu.

3. Highlight the record you wish to delete.

4. Right-click to invoke the pop-up menu and choose Delete. A warning dialog will be displayed, allowing you to cancel the deletion at this point.

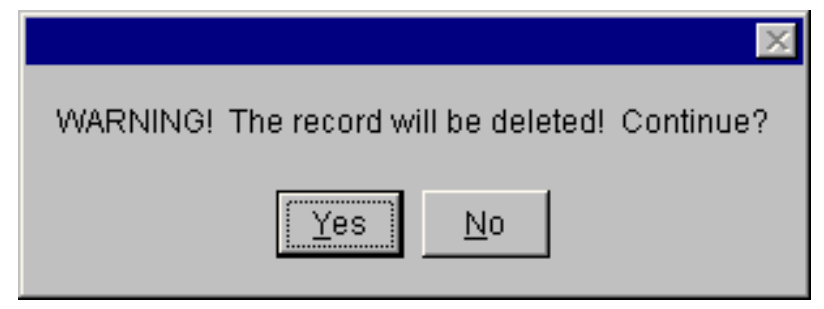

Yes - Continue the delete operation. The selected record will be deleted from the database. The message, "Record deleted," is displayed in the message bar of the SAPHIRE window.

No - Cancel the delete operation. The selected record will NOT be deleted from the database.

\subsubsection{Database Text}

\section{PURPOSE}

This option allows you to view and edit the descriptive text associated with a specific record. The Primary text option is generally used for text in the desired primary language. The Alternate text option is generally used for information entered in the secondary language using a different alphabet (locale).

\section{STEPS}

1. From the SAPHIRE menu select Modify.

2. Choose the desired option from the menu.

3. Highlight the record whose text you wish to modify.

4. Choose the Text button. The Select Text Type dialog is displayed.

5. Choose Primary or Alternate. The Text dialog is displayed.

Initially, the first 18 lines of the text block are displayed. If there are more lines of text, the vertical scroll bar will be available. In the example here, the Primary text dialog is displayed. 


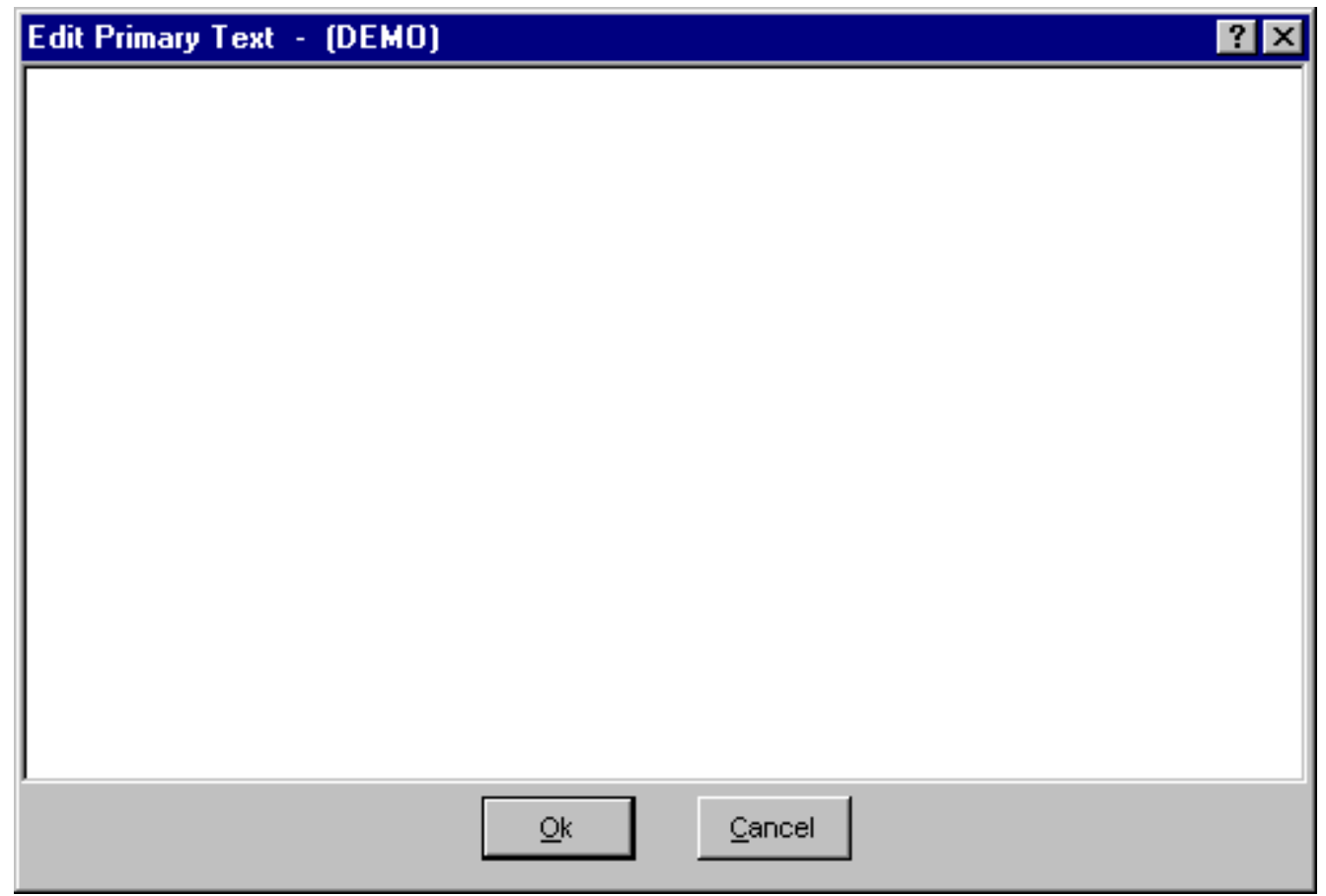

Page Down - $\quad$ Presents the next 18 lines of text.

Page Up - $\quad$ Presents the previous 18 lines of text.

Ctrl Page Down-Places the cursor at the end of the last line of text currently visible.

Ctrl Page Up Places the cursor at the beginning of the first line of text currently visible.

Ctrl- - - $\quad$ Moves the cursor left to the beginning of the word on the current line.

Ctrl- $\rightarrow$ - $\quad$ Moves the cursor right to the beginning of the word on the current line.

Home - $\quad$ Places the cursor in front of the first character of the current line.

Ctrl-Home - Places the cursor at the beginning of the text block.

End -

Ctrl-End - $\quad$ Places the cursor at the end of the text block.

Insert - $\quad$ Works as a toggle. Initially, the editor is in "insert" mode - existing characters are pushed to the right as you type. Pressing the Insert key toggles to "overwrite" mode - existing characters are over written as you type.

Delete - $\quad$ Deletes the character to the right of the current cursor position; or deletes the currently highlighted text.

Ctrl-Delete - Deletes the characters from the current cursor position to the beginning of the following word.

Backspace - Deletes the character to the left of the current cursor position; or deletes the currently highlighted text.

Ctrl-C - $\quad$ Copies highlighted text to the clipboard.

Ctrl-V - $\quad$ Pastes clipboard text at the current cursor position.

Ctrl-X - Cuts highlighted text to the clipboard.

The editor does not automatically line wrap; therefore, you must use $<$ Enter $>$ to establish each new line of text.

Ok - Save the new or modified text and close the Text dialog. 
Cancel - Close the Text dialog without saving changes. You will be prompted with the dialog shown below.

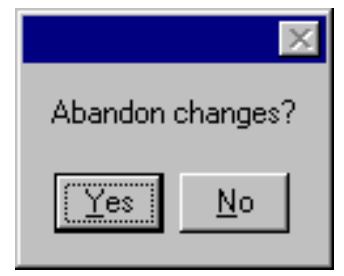

Yes - $\quad$ Close the Text dialog without saving changes.

No - $\quad$ Save the new or modified text and close the Text dialog.

\subsubsection{Base Case Update}

\section{PURPOSE}

This option allows you to overwrite all base case (original) data with the current case data for the selected analysis type. If you choose to update the base data, four operations will occur. (1) The base case cut sets will be overwritten with the current case cut sets. (2) The base case uncertainty results will be overwritten with the current case uncertainty results. (3) The base case uncertainty quantile values will be overwritten with the quantile values from the current case. (4) The base case minimum cut set upper bound will be overwritten with the current case minimum cut set upper bound. As a note of caution, the original base case results will be overwritten if this option is executed!

\section{STEPS}

1. From the SAPHIRE menu select Modify.

2. Choose Event Trees. The Edit Event Trees dialog is displayed. $O R$

3. Choose Event Trees | Sequences. The Edit Sequences dialog is displayed. OR

4. Choose Fault Trees. The Edit Fault Trees dialog is displayed. $O R$

5. Choose End States. The Edit End State dialog is displayed.

6. Highlight the desired list item.

7. Choose the Base Update button. The Base Case Update dialog will be displayed.

You will be prompted for confirmation before performing the update. In addition, you must select the analysis type from the drop-down list. 


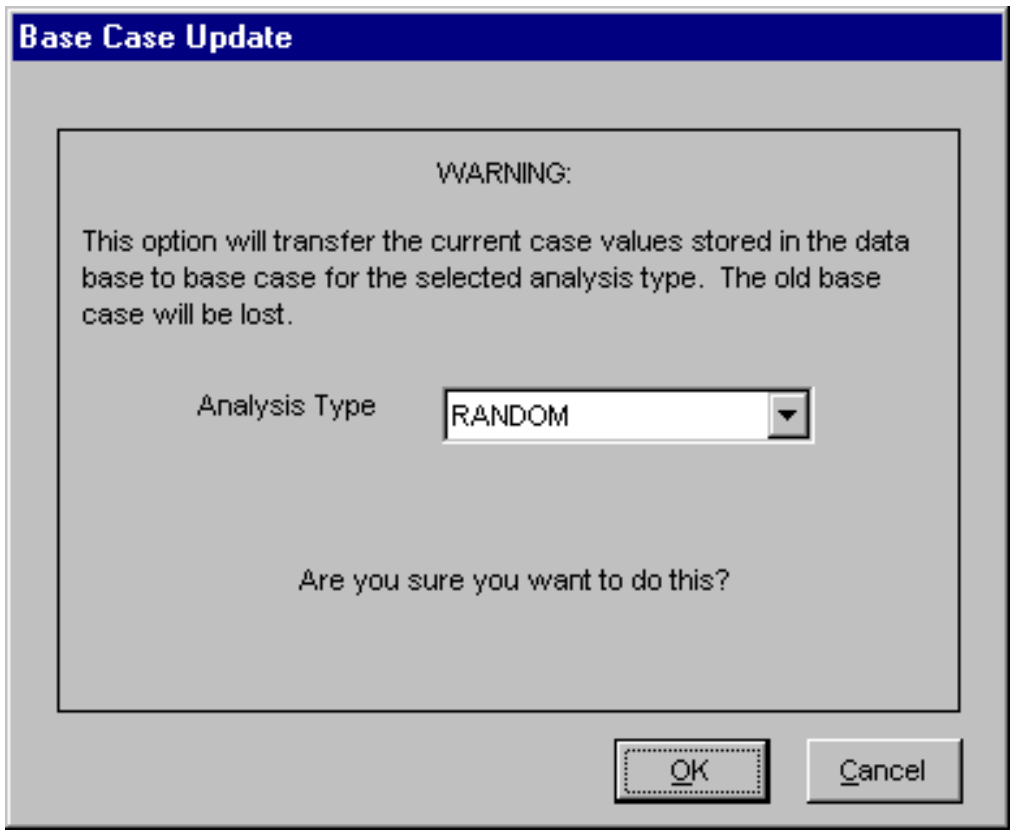

OK - Cause the current case data to overwrite the base case data. Upon completion of this process, a message, "Base case update complete" will be displayed in the message bar of the SAPHIRE window.

Cancel - Terminate the update operation.

\subsubsection{Clear Current Case}

\section{PURPOSE}

This option deletes all current case information for the specified analysis type. All SAPHIRE calculations use data stored in the current case for sensitivity and event analysis, and cut set generation results are stored in the current case.

\section{STEPS}

1. From the SAPHIRE menu select Modify.

2. Choose Event Trees. The Edit Event Trees dialog is displayed.

OR

3. Choose Event Trees | Sequences. The Edit Sequences dialog is displayed. $O R$

4. Choose Fault Trees. The Edit Fault Trees dialog is displayed. OR

5. Choose End States. The Edit End State dialog is displayed.

6. Highlight the desired list item.

7. Choose the Clear Current button. The Clear Current Case dialog will be displayed.

You will be prompted for confirmation before performing the clear operation. In addition, you must select the analysis type from the drop-down list. 


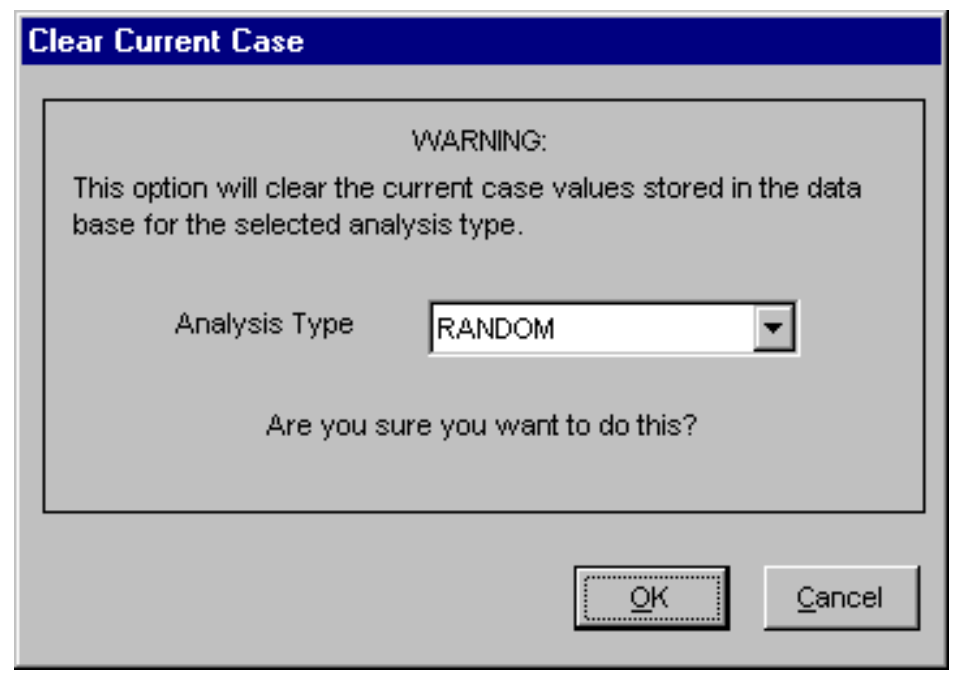

OK - Cause the current case data to be deleted. Upon completion of this procsss, a message, "Current Case Cleared..." will be displayed in the message bar of the SAPHIRE window. Cancel - Terminate the clear current case process.

\subsubsection{Sequence Data Entry Dialog}

\section{PURPOSE}

The Add/Modify Sequence dialog allows you to enter or modify sequence data.

\section{STEPS}

1. From the SAPHIRE menu select Modify.

2. Choose Event Trees from the menu. The Edit Event Trees dialog is displayed.

3. Highlight the desired event tree and

4. Choose the Sequences button. The Edit Sequences dialog will be displayed.

5. Highlight the sequence you wish to edit or copy. (Skip this step if adding a new sequence record.)

6. Right-click to invoke the pop-up menu and choose Add, Copy, or Modify. 


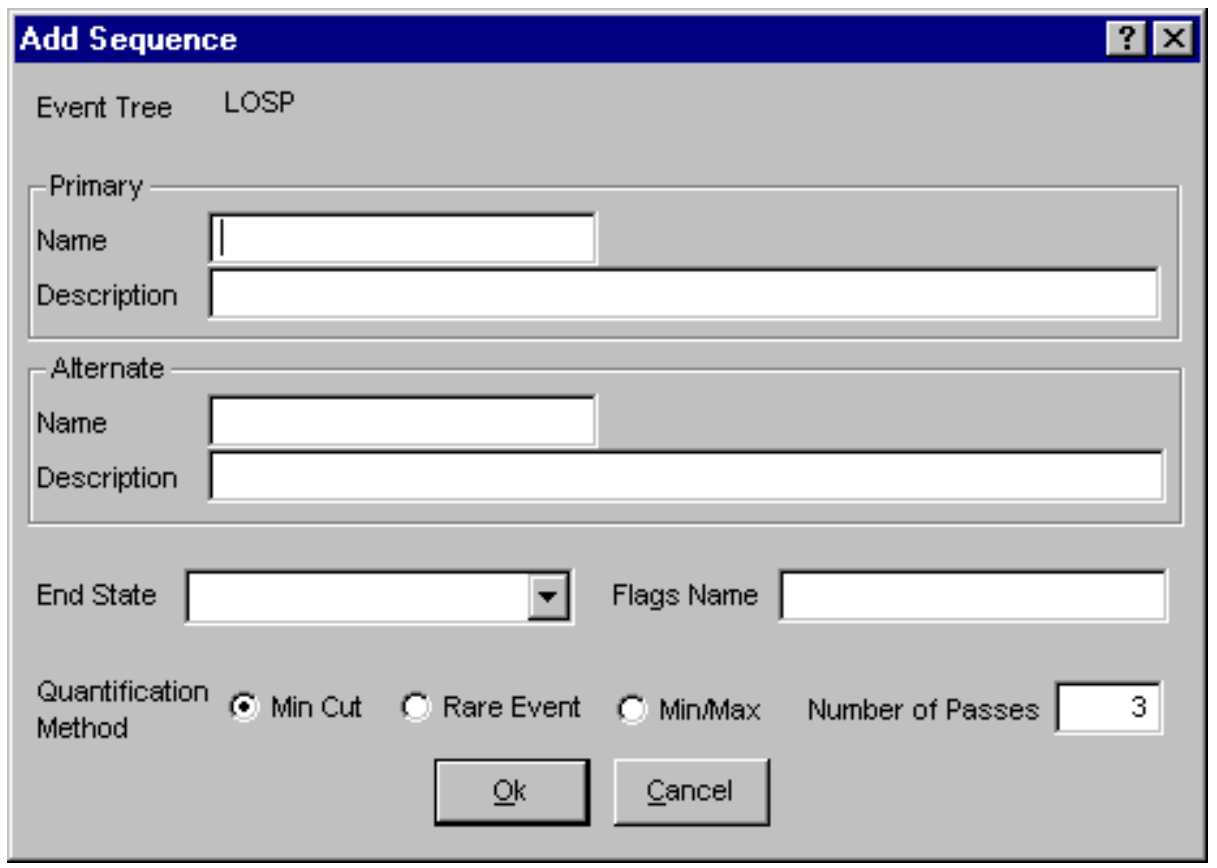

Ok - Save the new or modified sequence record and close the dialog.

Cancel - Close the dialog without saving.

\subsubsection{Sequence Data Entry Dialog}

\subsection{Name}

This is a required field. A maximum of 24 uppercase, alphanumeric characters may be entered. Embedded blanks are not allowed. The name should be descriptive so it can be readily identified.

\subsection{End State}

This is a 24-character uppercase, alphanumeric field used to identify the end state. Select an end state from the drop-down list.

\subsection{Description}

This is a 120-character, uppercase or lowercase, alphanumeric field that provides brief, descriptive information.

\subsection{Flags Name}

This is a 24-character uppercase, alphanumeric field used to identify a flag set. Embedded blanks are not allowed. The flag set contains flags to be used when generating cut sets for this sequence. SAPHIRE uses this default flag set name to modify or prune the fault tree logic for this sequence before it is solved (see Sequence Analysis). Enter a flag name or leave blank. 


\subsection{Quantification Method}

Choose the default quantification method for this sequence/fault tree/end state. If the Min/Max radio button is chosen, you must also enter the Number of Passes (from 1 to 10).

\subsection{Modifying Fault Tree Data}

\subsubsection{Fault Trees}

\section{PURPOSE}

This option allows you to add, modify or delete fault tree data records. When modifying an existing fault tree record, you can also enter current case mincut upper bound values for each of the 16 analysis types.

\section{STEPS}

1. From the SAPHIRE menu select Modify.

2. Choose Fault Trees from the menu. The Edit Fault Trees dialog is displayed, listing all of the fault trees belonging to the current project.

3. Highlight the desired fault tree.

4. Right-click to invoke the pop-up menu or choose the desired button.

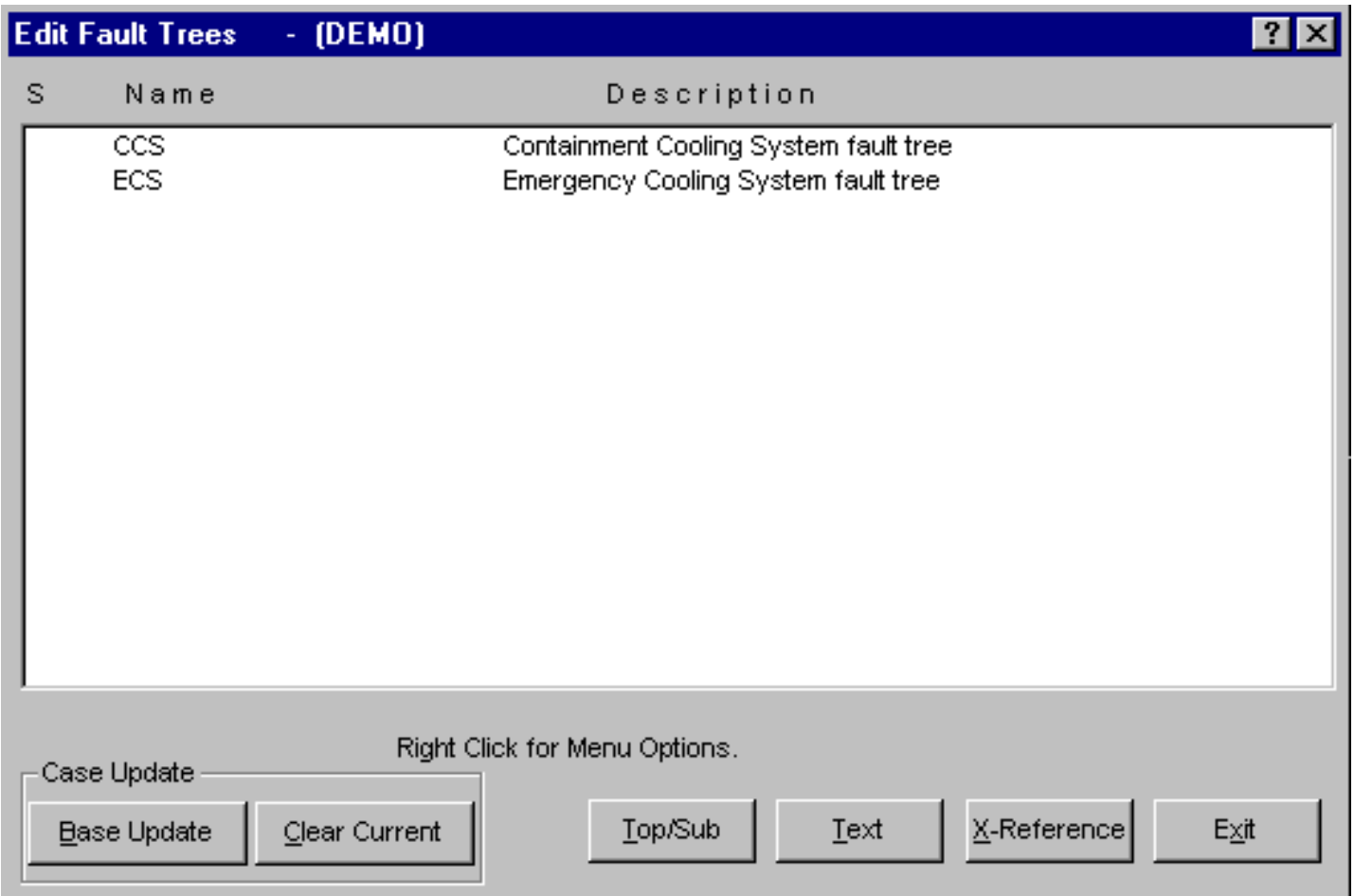


Pop-up Menu Options

Add -

Copy -

Modify -

Delete -

Graphics -

Logic -

Button Options

Base Update -

Clear Current -

Top/Sub -

Text -

X-Reference -

Exit -
Add a new fault tree record to the current project database.

Create a new fault tree record by copying an existing one.

Edit the selected fault tree record.

Delete the selected fault tree record(s).

Edit the graphical picture associated with the selected fault tree record.

Edit the logic associated with the selected fault tree record.
Update selected fault tree base case data.

Clear selected fault tree current case data.

If the fault tree is a top, change it to a sub-tree or alternately, if the fault tree is a sub-tree, change it to a top.

View and edit the descriptive text associated with the selected fault tree.

Display the cross-reference map of the highlighted fault tree.

Close the Edit Fault Trees dialog.

\subsubsection{Delete Fault Tree}

\section{PURPOSE}

This option allows you to delete the highlighted fault tree from the database.

\section{STEPS}

1. From the SAPHIRE menu select Modify.

2. Choose Fault Trees from the menu. The Edit Fault Trees dialog is displayed.

3. Highlight the fault tree you wish to delete.

4. Right-click to invoke the pop-up menu and choose Delete. A warning dialog will be displayed, allowing you to cancel the deletion at this point.

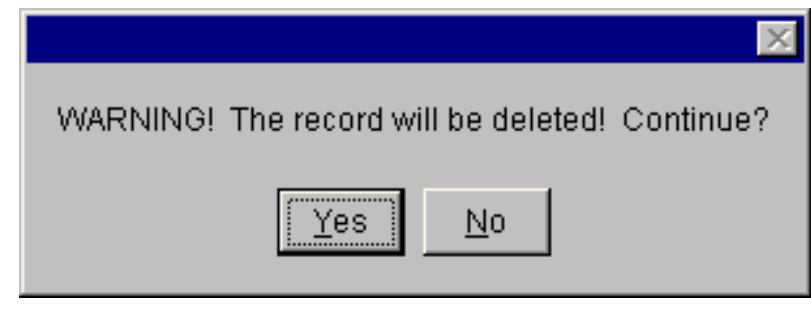

Yes - Continue the delete operation. The selected fault tree will be deleted from the database. The message, "Record deleted," will be displayed in the message bar of the SAPHIRE window.

No - Cancel the delete operation.

NOTE: SAPHIRE will not allow you to delete a fault tree that is used by another fault tree. 


\subsubsection{Base Case Update}

\section{PURPOSE}

This option allows you to overwrite all base case (original) data with the current case data for the selected analysis type. If you choose to update the base data, four operations will occur. (1) The base case cut sets will be overwritten with the current case cut sets. (2) The base case uncertainty results will be overwritten with the current case uncertainty results. (3) The base case uncertainty quantile values will be overwritten with the quantile values from the current case. (4) The base case minimum cut set upper bound will be overwritten with the current case minimum cut set upper bound. As a note of caution, the original base case results will be overwritten if this option is executed!

\section{STEPS}

1. From the SAPHIRE menu select Modify.

2. Choose Event Trees. The Edit Event Trees dialog is displayed.

OR

3. Choose Event Trees | Sequences. The Edit Sequences dialog is displayed.

$O R$

4. Choose Fault Trees. The Edit Fault Trees dialog is displayed.

$O R$

5. Choose End States. The Edit End State dialog is displayed.

6. Highlight the desired list item.

7. Choose the Base Update button. The Base Case Update dialog will be displayed.

You will be prompted for confirmation before performing the update. In addition, you must select the analysis type from the drop-down list.

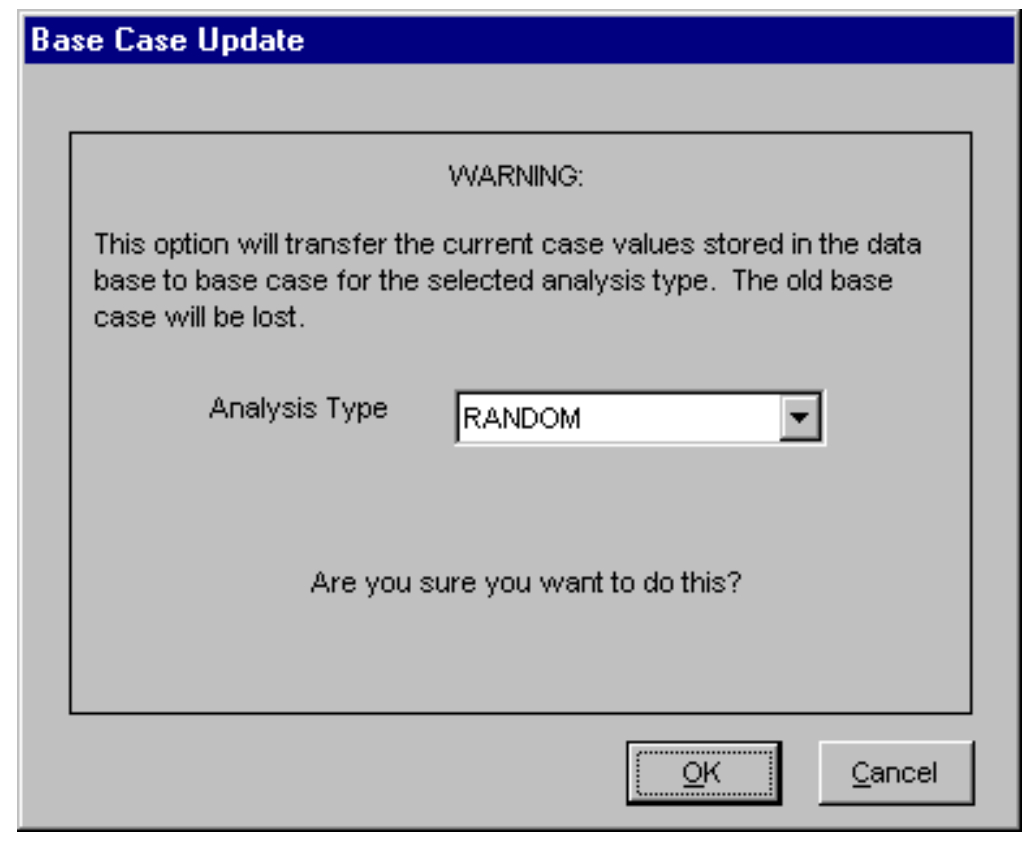


OK - Cause the current case data to overwrite the base case data. Upon completion of this process, a message, "Base case update complete" will be displayed in the message bar of the SAPHIRE window.

Cancel - Terminate the update operation.

\subsubsection{Clear Current Case}

\section{PURPOSE}

This option deletes all current case information for the specified analysis type. All SAPHIRE calculations use data stored in the current case for sensitivity and event analysis, and cut set generation results are stored in the current case.

\section{STEPS}

1. From the SAPHIRE menu select Modify.

2. Choose Event Trees. The Edit Event Trees dialog is displayed. OR

3. Choose Event Trees $\mid$ Sequences. The Edit Sequences dialog is displayed. OR

4. Choose Fault Trees. The Edit Fault Trees dialog is displayed. OR

5. Choose End States. The Edit End State dialog is displayed.

6. Highlight the desired list item.

7. Choose the Clear Current button. The Clear Current Case dialog will be displayed.

You will be prompted for confirmation before performing the clear operation. In addition, you must select the analysis type from the drop-down list.

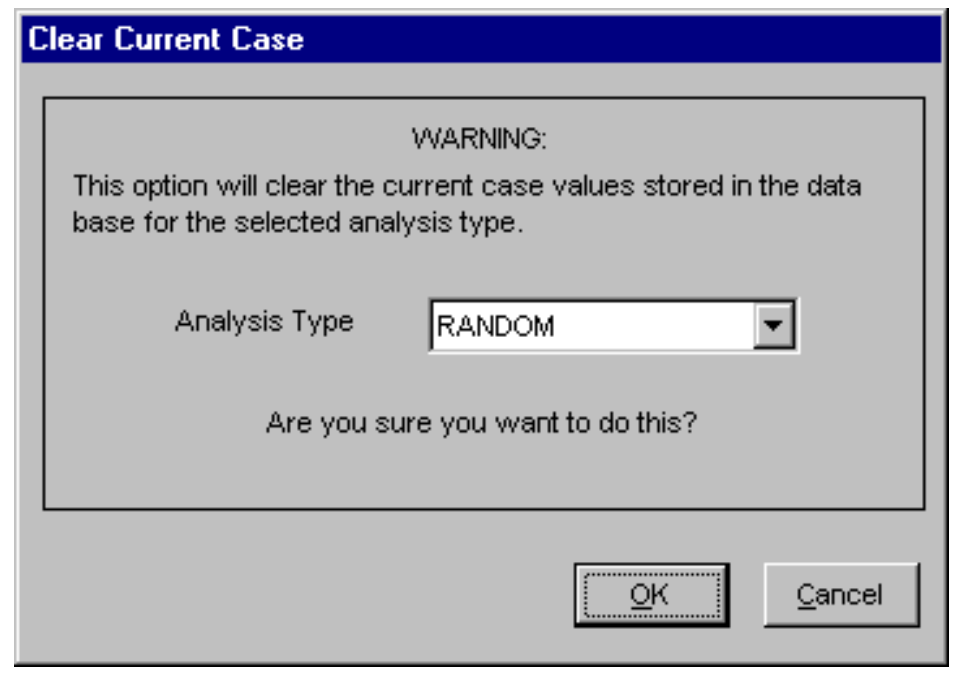

OK - Cause the current case data to be deleted. Upon completion of this procsss, a message, "Current Case Cleared..." will be displayed in the message bar of the SAPHIRE window.

Cancel - Terminate the clear current case process. 


\subsubsection{Top/Sub-tree Convert}

\section{PURPOSE}

This option allows you to convert the highlighted fault tree from a top to a sub-tree, or a sub-tree to a top. This option acts as a toggle. If the selected fault tree is being used by a sequence, it cannot be converted to a sub-tree.

\section{STEPS}

1. From the SAPHIRE menu select Modify.

2. Choose Fault Trees from the menu. The Edit Fault Trees dialog is displayed.

3. Highlight the fault tree you wish to convert.

4. Choose the Top/Sub button. A warning dialog will be displayed, allowing you to cancel the conversion at this point.

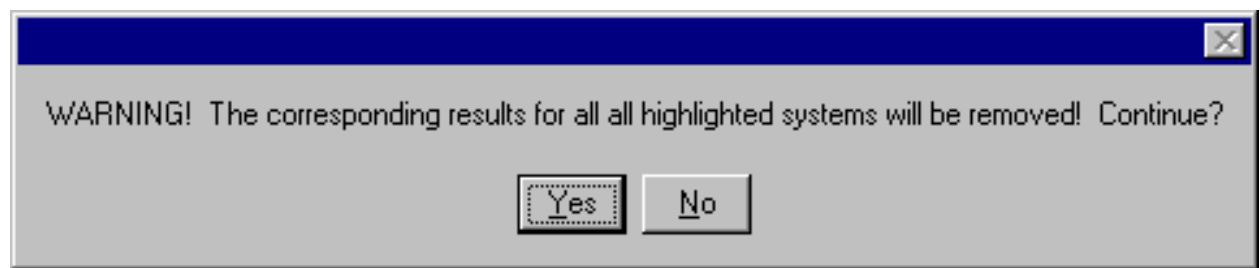

Yes - Continue the conversion operation. The selected fault tree will be converted from its current type.

No - Cancel the conversion operation.

NOTE: When a top fault tree is converted to a sub-tree, the analysis results will be deleted.

\subsubsection{Database Text}

\section{PURPOSE}

This option allows you to view and edit the descriptive text associated with a specific record. The Primary text option is generally used for text in the desired primary language. The Alternate text option is generally used for information entered in the secondary language using a different alphabet (locale ).

\section{STEPS}

1. From the SAPHIRE menu select Modify.

2. Choose the desired option from the menu.

3. Highlight the record whose text you wish to modify.

4. Choose the Text button. The Select Text Type dialog is displayed.

5. Choose Primary or Alternate. The Text dialog is displayed.

Initially, the first 18 lines of the text block are displayed. If there are more lines of text, the vertical scroll bar will be available. In the example here, the Primary text dialog is displayed. 


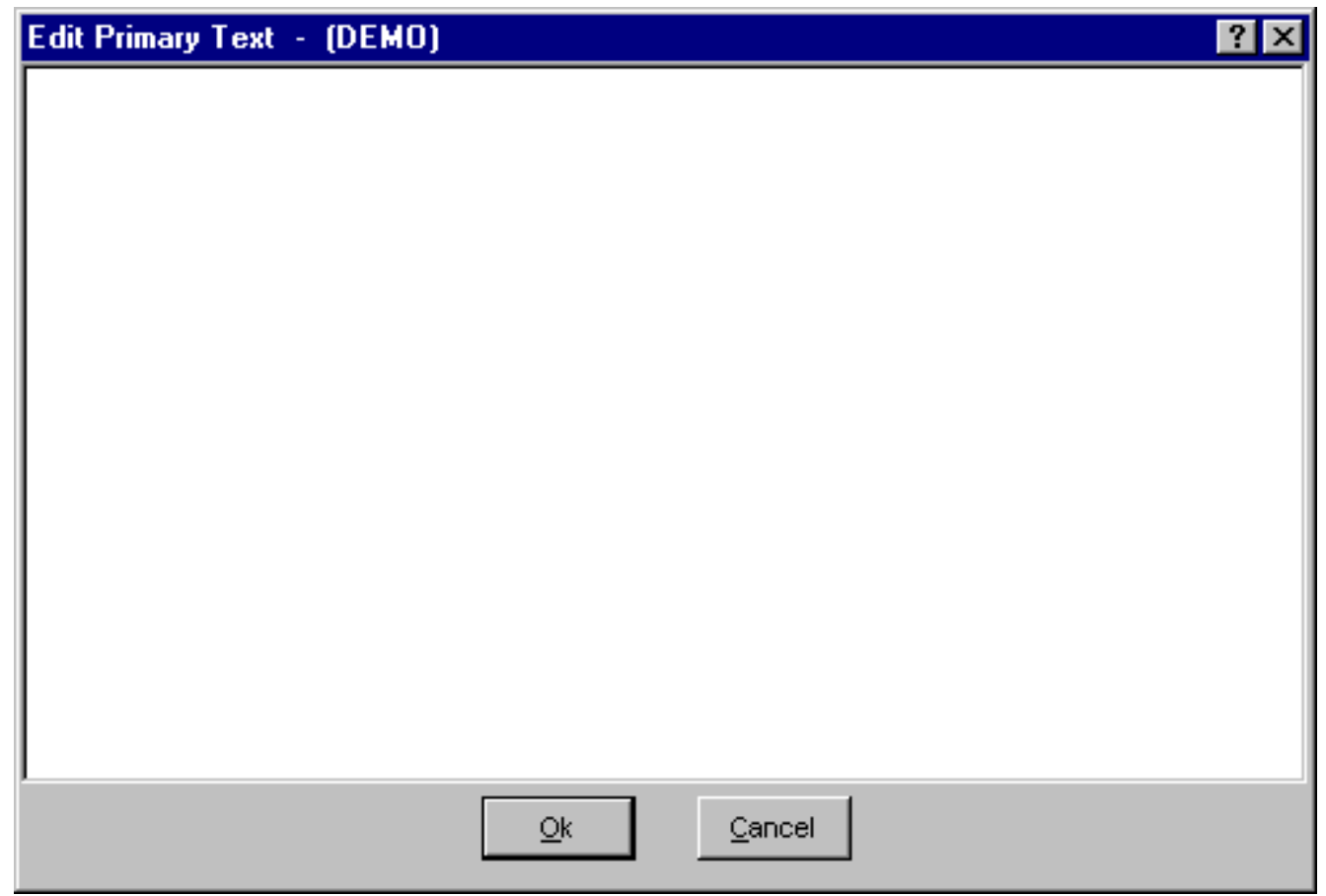

Page Down - $\quad$ Presents the next 18 lines of text.

Page Up-Presents the previous 18 lines of text.

Ctrl Page Down-Places the cursor at the end of the last line of text currently visible.

Ctrl Page Up - Places the cursor at the beginning of the first line of text currently visible.

Ctrl- - - $\quad$ Moves the cursor left to the beginning of the word on the current line.

Ctrl- $\rightarrow$ - $\quad$ Moves the cursor right to the beginning of the word on the current line.

Home - $\quad$ Places the cursor in front of the first character of the current line.

Ctrl-Home - Places the cursor at the beginning of the text block.

End -

Ctrl-End - $\quad$ Places the cursor at the end of the text block.

Insert - $\quad$ Works as a toggle. Initially, the editor is in "insert" mode - existing characters are pushed to the right as you type. Pressing the Insert key toggles to "overwrite" mode - existing characters are over written as you type.

Delete - $\quad$ Deletes the character to the right of the current cursor position; or deletes the currently highlighted text.

Ctrl-Delete - Deletes the characters from the current cursor position to the beginning of the following word.

Backspace - Deletes the character to the left of the current cursor position; or deletes the currently highlighted text.

Ctrl-C - $\quad$ Copies highlighted text to the clipboard.

Ctrl-V - $\quad$ Pastes clipboard text at the current cursor position.

Ctrl-X - Cuts highlighted text to the clipboard.

The editor does not automatically line wrap; therefore, you must use $<$ Enter $>$ to establish each new line of text.

Ok - Save the new or modified text and close the Text dialog. 
Cancel - Close the Text dialog without saving changes. You will be prompted with the dialog shown below.

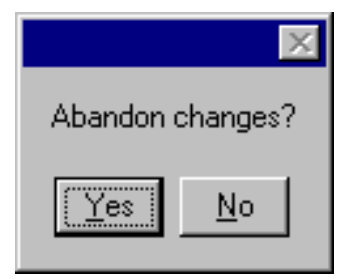

Yes - Close the Text dialog without saving changes.

No - Save the new or modified text and close the Text dialog.

\subsubsection{Fault Tree Cross-reference}

\section{PURPOSE}

This option displays a cross-reference map of the highlighted fault tree. This cross-reference map displays the sequence logic and event tree logic in which the fault tree is used.

\section{STEPS}

1. From the SAPHIRE menu select Modify.

2. Choose Fault Trees from the menu. The Edit Fault Trees dialog is displayed.

3. Highlight the fault tree you wish to convert.

4. Choose the $\mathbf{X}$-Reference button. The System Cross-reference dialog will be displayed. 


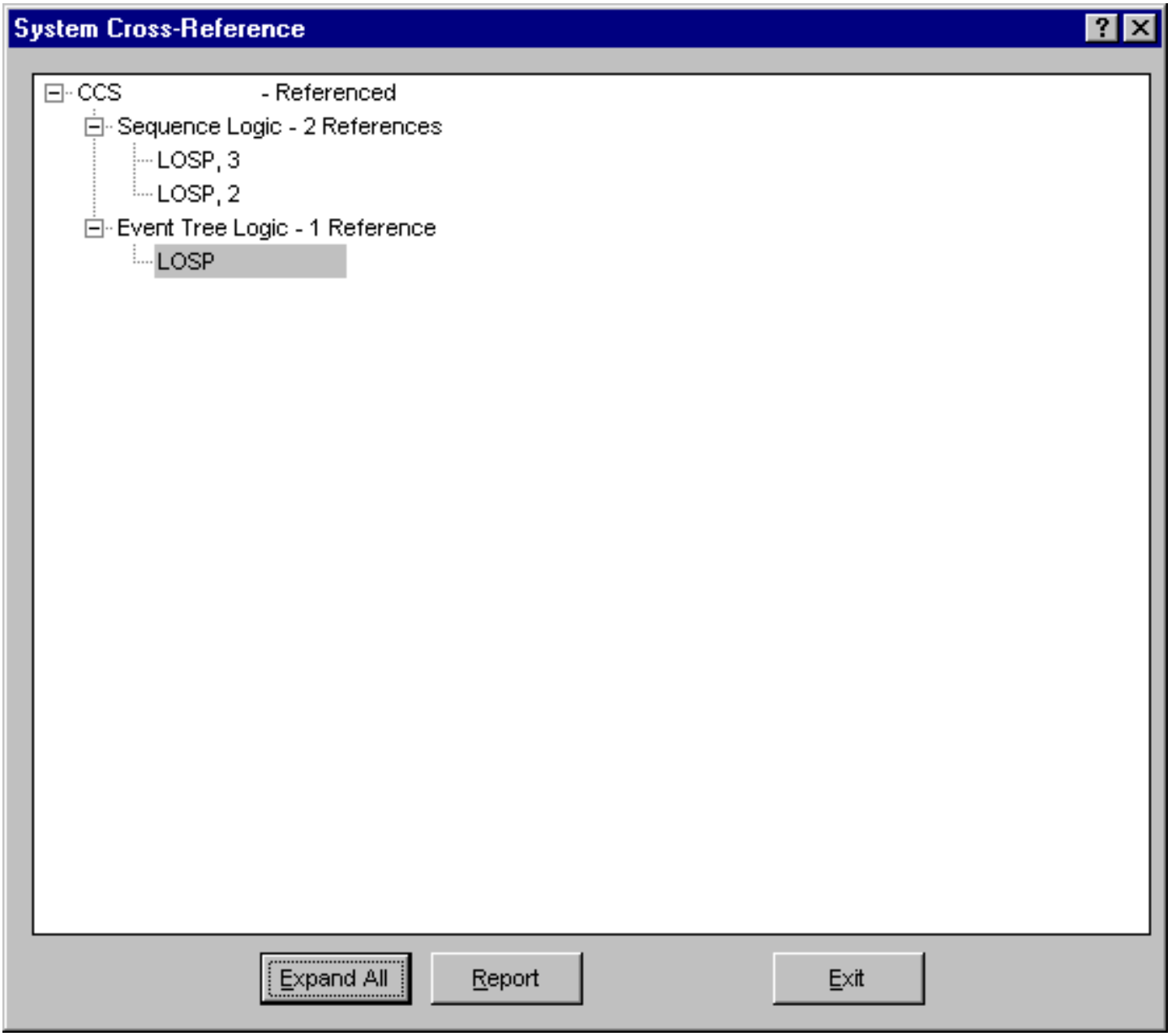

\#- Indicates that the item can be expanded to display more information. Click on this symbol to expand the item.

$\square$ - $\quad$ Indicates that the item is fully expanded. Click on this symbol to collapse the item.

Expand All - Expand all items in the cross-reference map.

Report - Generate the cross-reference report for the fault tree.

Exit - Close the System Cross-reference dialog.

\subsubsection{Add Fault Tree Dialog}

\subsubsection{Add Fault Tree}

\section{PURPOSE}

The Add Fault Tree dialog allows you to enter data for a new fault tree record. 


\section{STEPS}

1. From the SAPHIRE menu select Modify.

2. Choose Fault Trees from the menu. The Edit Fault Trees dialog is displayed.

3. Right-click to invoke the pop-up menu and choose Add.

OR

4. From the SAPHIRE menu select Fault Tree. The Fault Trees List dialog will be displayed.

5. Right-click to invoke the pop-up menu and choose Add Fault Tree.

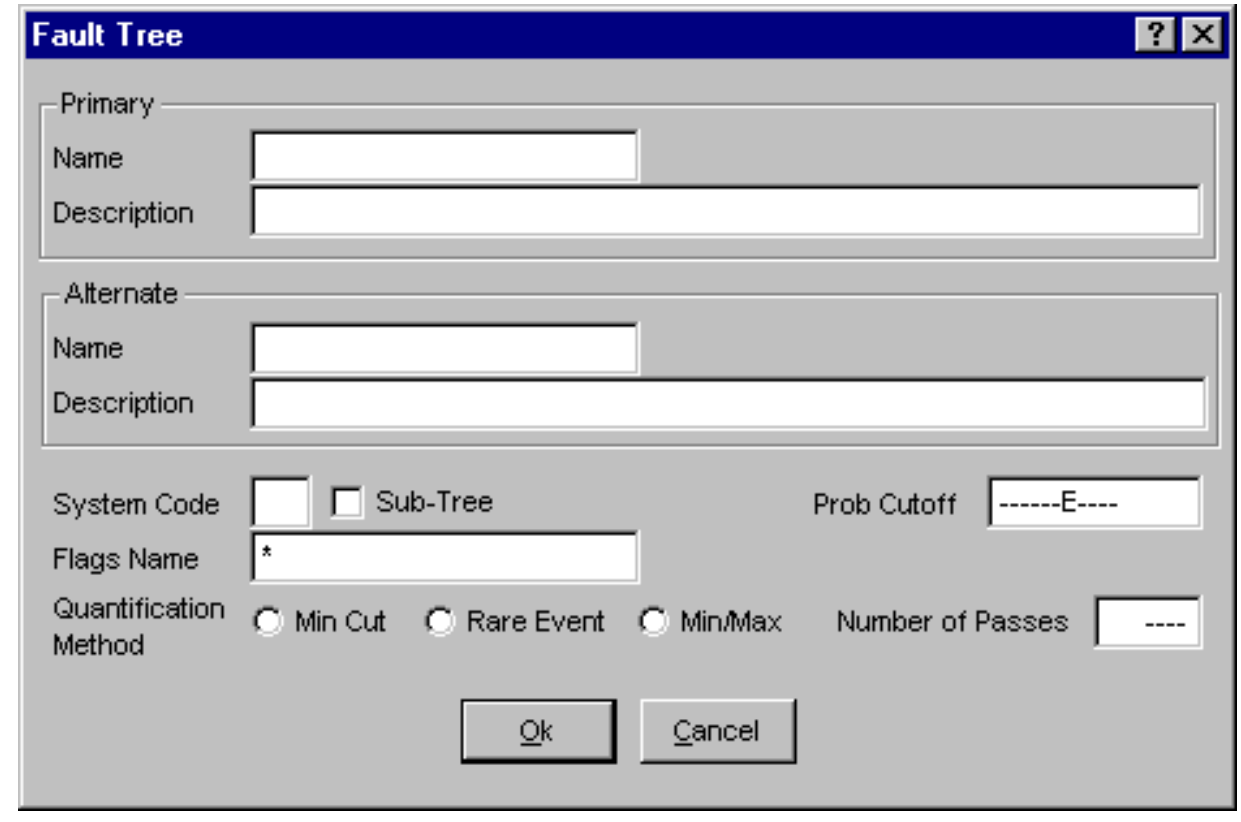

Ok - $\quad$ Add the new fault tree record to the database. When complete, the Add Fault Tree dialog will be closed and the message, "Record added," will be displayed in the message bar of the SAPHIRE dialog.

Cancel - The fault tree record is not saved or added to the database.

\subsubsection{Name}

This is a required field. A maximum of 24 uppercase, alphanumeric characters may be entered. Embedded blanks are not allowed. The name should be descriptive so it can be readily identified.

\subsubsection{Description}

This is a 120-character, uppercase or lowercase, alphanumeric field that provides brief, descriptive information. 


\subsubsection{Fault Tree Code}

This is a three-character, uppercase, alphanumeric field used to identify the graphic diagram display of the fault tree.

\subsubsection{Sub-tree}

Check this box if this is a sub-tree. Leave unchecked if not.

\subsubsection{Prob Cutoff}

The default probability cutoff value for the fault tree. Use scientific notation. When solving fault trees, this value is used when the Cutoff by Cut Set Probability box is checked in the Cut Set Generation dialog. If no value is entered here and the Cutoff by Cut Set Probability box is checked, then the value entered in the Global Cutoff Value field of that dialog will be used.

\subsubsection{Flags Name}

This is a 24-character uppercase, alphanumeric field used to identify a flag set. Embedded blanks are not allowed. The flag set contains flags to be used when generating cut sets for the fault tree. SAPHIRE uses this default flag set name to modify or prune the logic for the fault tree before it is solved (see Fault Tree Analysis). Enter a flag name or leave blank.

\subsubsection{Quantification Method}

Choose the default quantification method for this sequence/fault tree/end state. If the Min/Max radio button is chosen, you must also enter the Number of Passes (from 1 to 10).

\subsubsection{Modify Fault Tree Dialog}

\subsubsection{Modify Fault Tree}

\section{PURPOSE}

The Modify Fault Tree dialog allows you to modify fault tree record data.

\section{STEPS}

1. From the SAPHIRE menu select Modify.

2. Choose Fault Trees from the menu. The Edit Fault Trees dialog is displayed.

3. Highlight the fault tree you wish to edit.

4. Right-click to invoke the pop-up menu and choose Modify. 


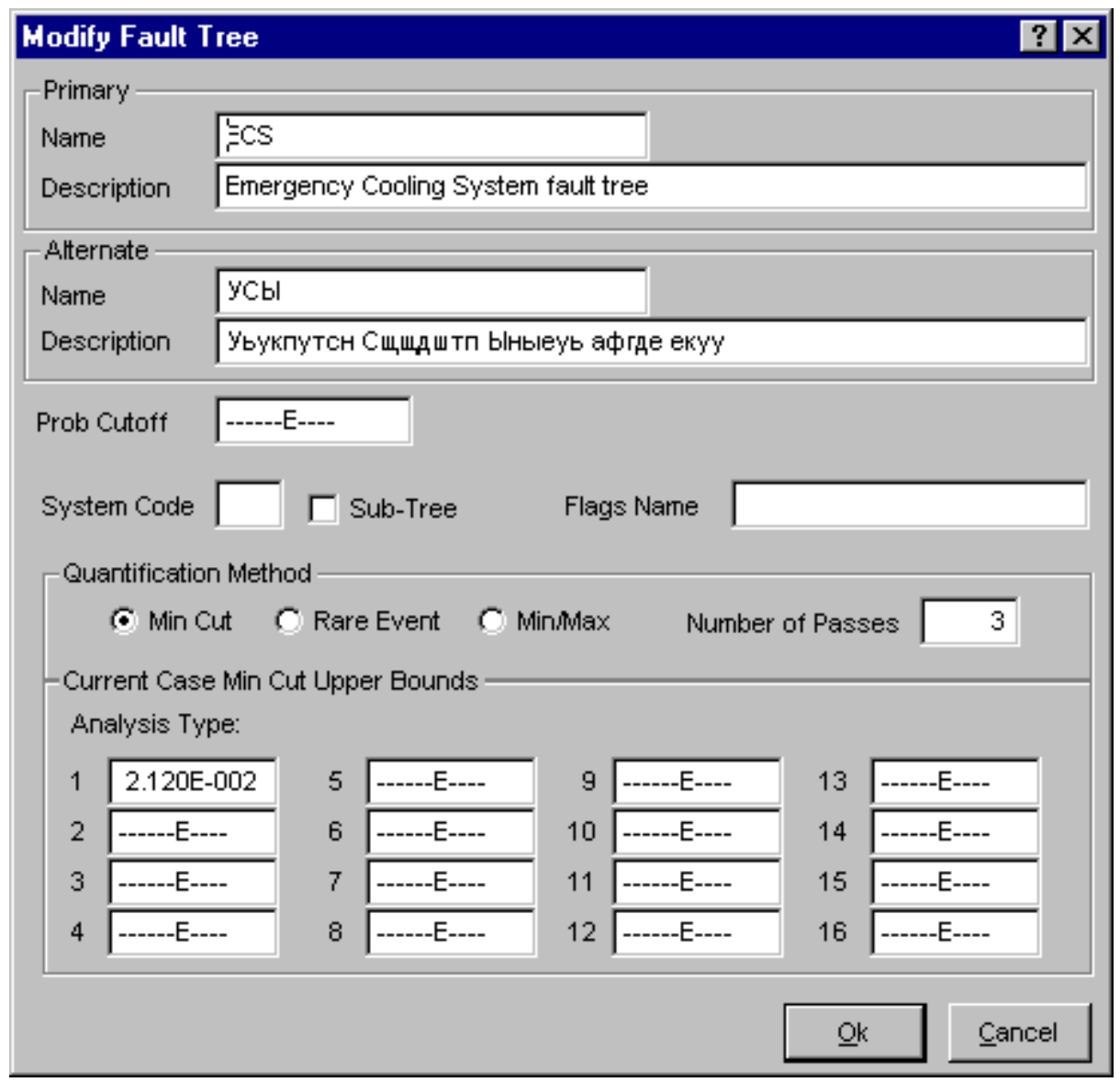

Ok - Save the changes made to the fault tree record. When complete, the Modify Fault Tree dialog will be closed and the message, "Record modified," will be displayed in the message bar of the SAPHIRE dialog.

Cancel - The changes to the fault tree record are not saved.

\subsubsection{Name}

This is a required field. A maximum of 24 uppercase, alphanumeric characters may be entered. Embedded blanks are not allowed. The name should be descriptive so it can be readily identified.

\subsubsection{Prob Cutoff}

The default probability cutoff value for the fault tree. Use scientific notation. When solving fault trees, this value is used when the Cutoff by Cut Set Probability box is checked in the Cut Set Generation dialog. If no value is entered here and the Cutoff by Cut Set Probability box is checked, then the value entered in the Global Cutoff Value field of that dialog will be used.

\subsubsection{Description}

This is a 120-character, uppercase or lowercase, alphanumeric field that provides brief, descriptive information. 


\subsubsection{Fault Tree Code}

This is a three-character, uppercase, alphanumeric field used to identify the graphic diagram display of the fault tree.

\subsubsection{Sub-tree}

Check this box if this is a sub-tree. Leave unchecked if not.

\subsubsection{Flags Name}

This is a 24-character uppercase, alphanumeric field used to identify a flag set. Embedded blanks are not allowed. The flag set contains flags to be used when generating cut sets for the fault tree. SAPHIRE uses this default flag set name to modify or prune the logic for the fault tree before it is solved (see Fault Tree Analysis). Enter a flag name or leave blank.

\subsubsection{Quantification Method}

Choose the default quantification method for this sequence/fault tree/end state. If the Min/Max radio button is chosen, you must also enter the Number of Passes (from 1 to 10).

\subsubsection{Current Case Min Cut Upper Bounds}

Optionally specify the minimal cut set upper bound for each analysis type.

\subsection{Modifying End State Data}

\subsubsection{End States}

\section{PURPOSE}

This option allows you to add, modify, or delete the end state data records, or associated text. Additionally, you can update selected end state base case data or clear selected end state current case data. You can also delete all unused end state records from the database.

\section{STEPS}

1. From the SAPHIRE menu select Modify.

2 Choose End States from the menu. The Edit End State dialog is displayed, listing all of the end states belonging to the current project.

3. Highlight the desired end state.

4. Right-click to invoke the pop-up menu or choose the desired button. 


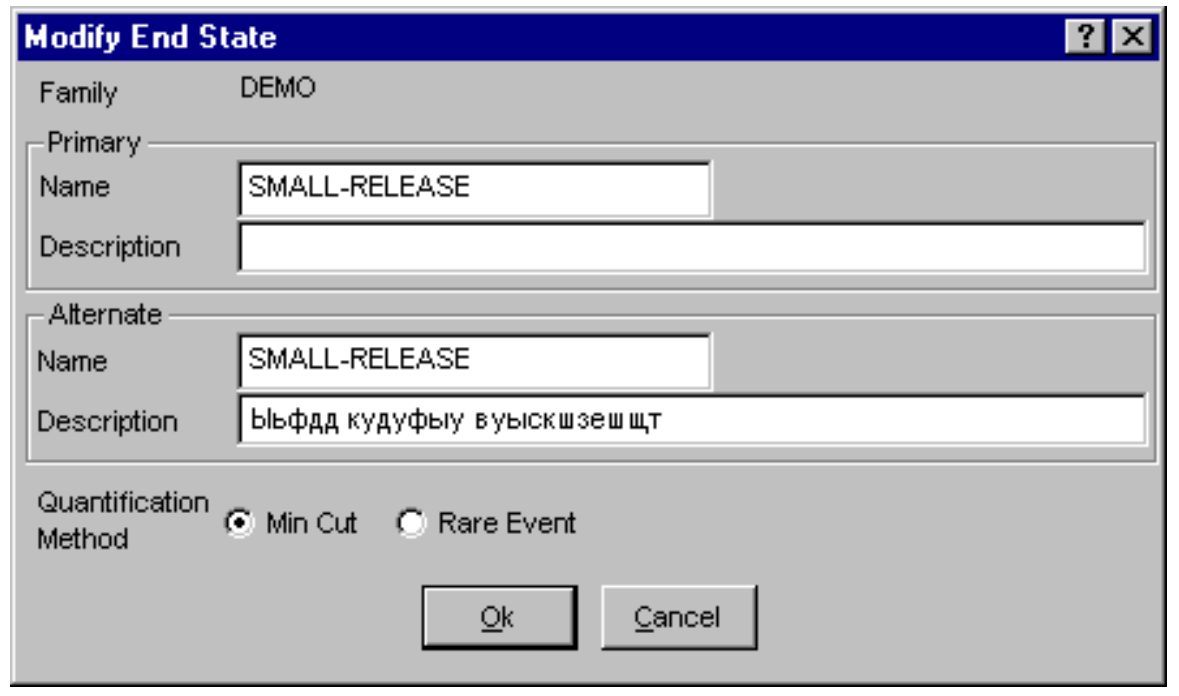

\section{Pop-up Menu Options}

Add -

Modify -

Delete -
Add a new end state record to the current project database. When complete, the message, "Record added," is displayed in the message bar of the SAPHIRE window.

Create a new end state record by copying an existing one.

Edit the selected end state record. When complete, the message, "Record modified," is displayed in the message bar of the SAPHIRE window.

Delete the selected end state record(s).

\section{Button Options}

Base Update - Update selected end state base case data.

Clear Current - Clear selected end state current case data.

Edit Text - $\quad$ View and edit the descriptive text associated with the selected end state.

X-Ref - $\quad$ Display the cross-reference map of the highlighted end state.

Remove Unused - Delete all unused end state records.

Show Unused - Show all unused end state records. The unused end states are indicated by a dash (-) to the left of the end state name.

\section{Exit -}

Close the Edit End State dialog.

\subsubsection{Delete Record}

\section{PURPOSE}

This option allows you to delete the highlighted record from the database.

\section{STEPS}

1. From the SAPHIRE menu select Modify.

2. Choose the desired option from the menu.

3. Highlight the record you wish to delete.

4. Right-click to invoke the pop-up menu and choose Delete. A warning dialog will be displayed, allowing you to cancel the deletion at this point. 


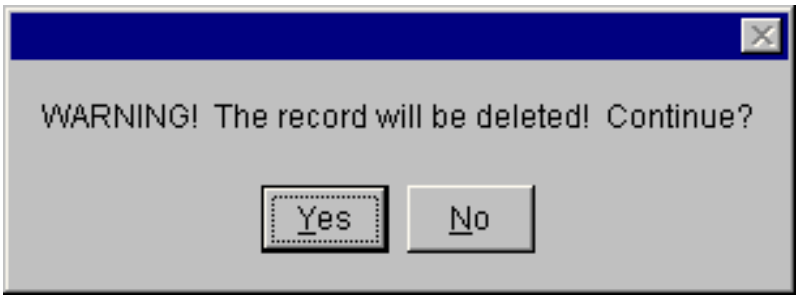

Yes - Continue the delete operation. The selected record will be deleted from the database. The message, "Record deleted," is displayed in the message bar of the SAPHIRE window.

No - Cancel the delete operation. The selected record will NOT be deleted from the database.

\subsubsection{Base Case Update}

\section{PURPOSE}

This option allows you to overwrite all base case (original) data with the current case data for the selected analysis type. If you choose to update the base data, four operations will occur. (1) The base case cut sets will be overwritten with the current case cut sets. (2) The base case uncertainty results will be overwritten with the current case uncertainty results. (3) The base case uncertainty quantile values will be overwritten with the quantile values from the current case. (4) The base case minimum cut set upper bound will be overwritten with the current case minimum cut set upper bound. As a note of caution, the original base case results will be overwritten if this option is executed!

\section{STEPS}

1. From the SAPHIRE menu select Modify.

2. Choose Event Trees. The Edit Event Trees dialog is displayed. OR

3. Choose Event Trees | Sequences. The Edit Sequences dialog is displayed. $O R$

4. Choose Fault Trees. The Edit Fault Trees dialog is displayed. OR

5. Choose End States. The Edit End State dialog is displayed.

6. Highlight the desired list item.

7. Choose the Base Update button. The Base Case Update dialog will be displayed.

You will be prompted for confirmation before performing the update. In addition, you must select the analysis type from the drop-down list. 


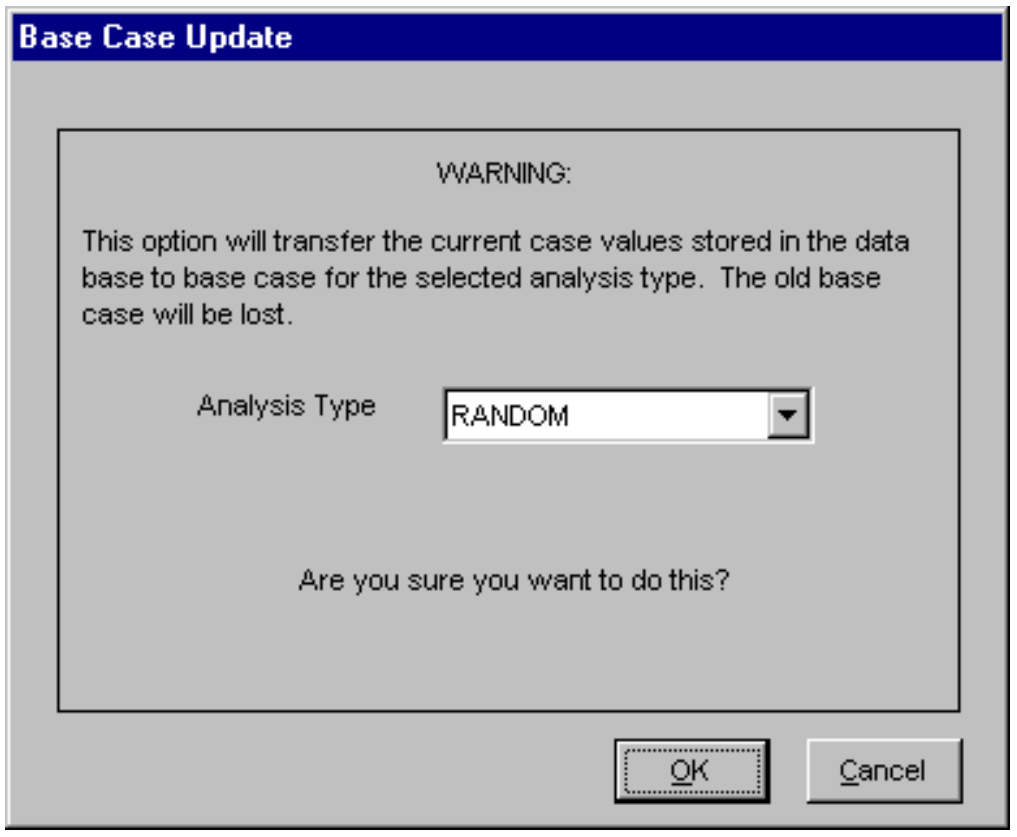

OK - Cause the current case data to overwrite the base case data. Upon completion of this process, a message, "Base case update complete" will be displayed in the message bar of the SAPHIRE window.

Cancel - Terminate the update operation.

\subsubsection{Clear Current Case}

\section{PURPOSE}

This option deletes all current case information for the specified analysis type. All SAPHIRE calculations use data stored in the current case for sensitivity and event analysis, and cut set generation results are stored in the current case.

\section{STEPS}

1. From the SAPHIRE menu select Modify.

2. Choose Event Trees. The Edit Event Trees dialog is displayed. $O R$

3. Choose Event Trees $\mid$ Sequences. The Edit Sequences dialog is displayed. OR

4. Choose Fault Trees. The Edit Fault Trees dialog is displayed. $O R$

5. Choose End States. The Edit End State dialog is displayed.

6. Highlight the desired list item.

7. Choose the Clear Current button. The Clear Current Case dialog will be displayed.

You will be prompted for confirmation before performing the clear operation. In addition, you must select the analysis type from the drop-down list. 


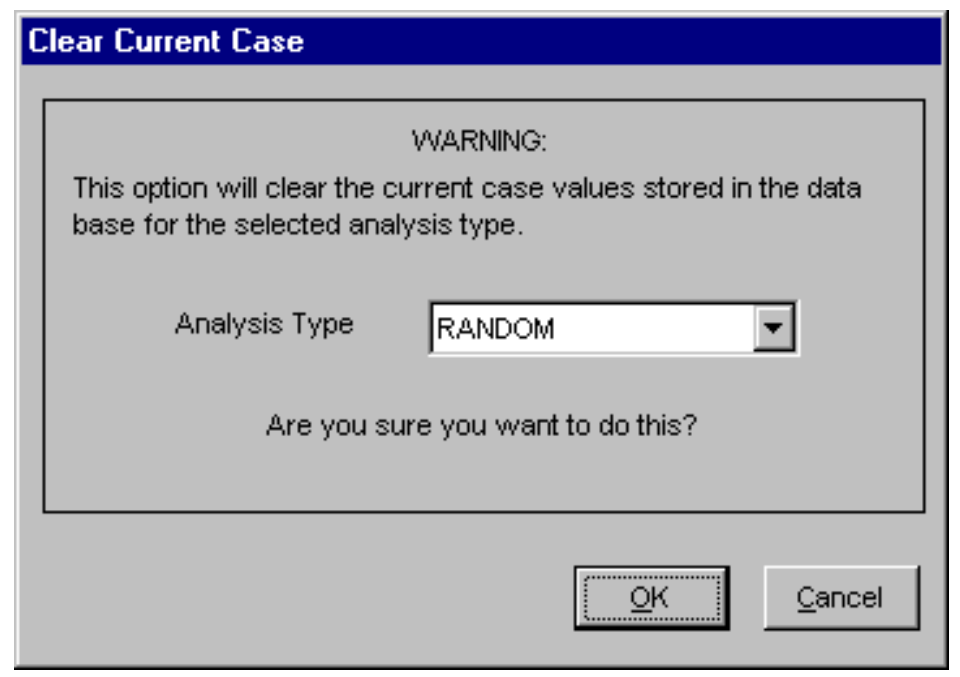

OK - Cause the current case data to be deleted. Upon completion of this procsss, a message, "Current Case Cleared..." will be displayed in the message bar of the SAPHIRE window. Cancel - Terminate the clear current case process.

\subsubsection{Database Text}

\section{PURPOSE}

This option allows you to view and edit the descriptive text associated with a specific record. The Primary text option is generally used for text in the desired primary language. The Alternate text option is generally used for information entered in the secondary language using a different alphabet (locale ).

\section{STEPS}

1. From the SAPHIRE menu select Modify.

2. Choose the desired option from the menu.

3. Highlight the record whose text you wish to modify.

4. Choose the Text button. The Select Text Type dialog is displayed.

5. Choose Primary or Alternate. The Text dialog is displayed.

Initially, the first 18 lines of the text block are displayed. If there are more lines of text, the vertical scroll bar will be available. In the example here, the Primary text dialog is displayed. 


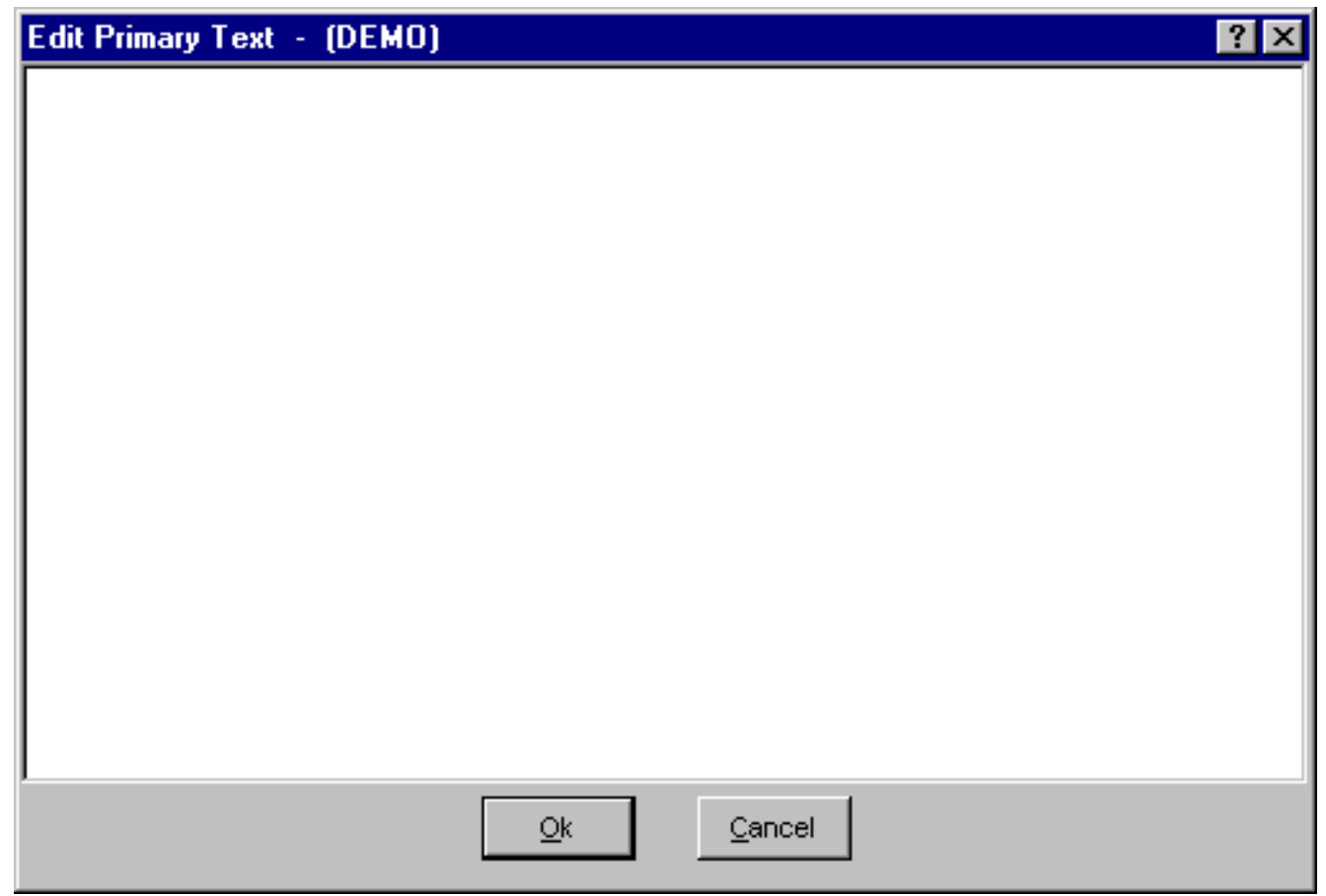

Page Down- Presents the next 18 lines of text.

Page Up-Presents the previous 18 lines of text.

Ctrl Page Down-Places the cursor at the end of the last line of text currently visible.

Ctrl Page Up- Places the cursor at the beginning of the first line of text currently visible.

Ctrl- - - $\quad$ Moves the cursor left to the beginning of the word on the current line.

Ctrl- $\rightarrow$ - $\quad$ Moves the cursor right to the beginning of the word on the current line.

Home - $\quad$ Places the cursor in front of the first character of the current line.

Ctrl-Home - Places the cursor at the beginning of the text block.

End -

Ctrl-End - $\quad$ Places the cursor at the end of the text block.

Insert - $\quad$ Works as a toggle. Initially, the editor is in "insert" mode - existing characters are pushed to the right as you type. Pressing the Insert key toggles to "overwrite" mode - existing characters are over written as you type.

Delete - $\quad$ Deletes the character to the right of the current cursor position; or deletes the currently highlighted text.

Ctrl-Delete - Deletes the characters from the current cursor position to the beginning of the following word.

Backspace - Deletes the character to the left of the current cursor position; or deletes the currently highlighted text.

Ctrl-C - $\quad$ Copies highlighted text to the clipboard.

Ctrl-V - $\quad$ Pastes clipboard text at the current cursor position.

Ctrl-X - Cuts highlighted text to the clipboard.

The editor does not automatically line wrap; therefore, you must use $<$ Enter $>$ to establish each new line of text.

Ok - $\quad$ Save the new or modified text and close the Text dialog. 
Cancel - Close the Text dialog without saving changes. You will be prompted with the dialog shown below.

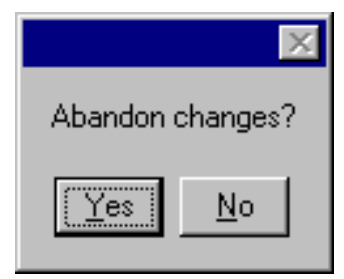

Yes - Close the Text dialog without saving changes.

No - Save the new or modified text and close the Text dialog.

\subsubsection{Cross Reference}

\section{PURPOSE}

This option displays a cross-reference map of the highlighted end state. This cross-reference map will show the sequence logic, and cut sets in which the end state is used. For each sequence in which the end state is used, base and/or current case cut sets will be listed for each analysis type.

\section{STEPS}

1. From the SAPHIRE menu select Modify.

2. Choose End States from the menu. The Edit End States dialog is displayed.

3. Highlight the desired end state.

4. Choose the Cross-reference button. The End State Cross-reference dialog is displayed. 


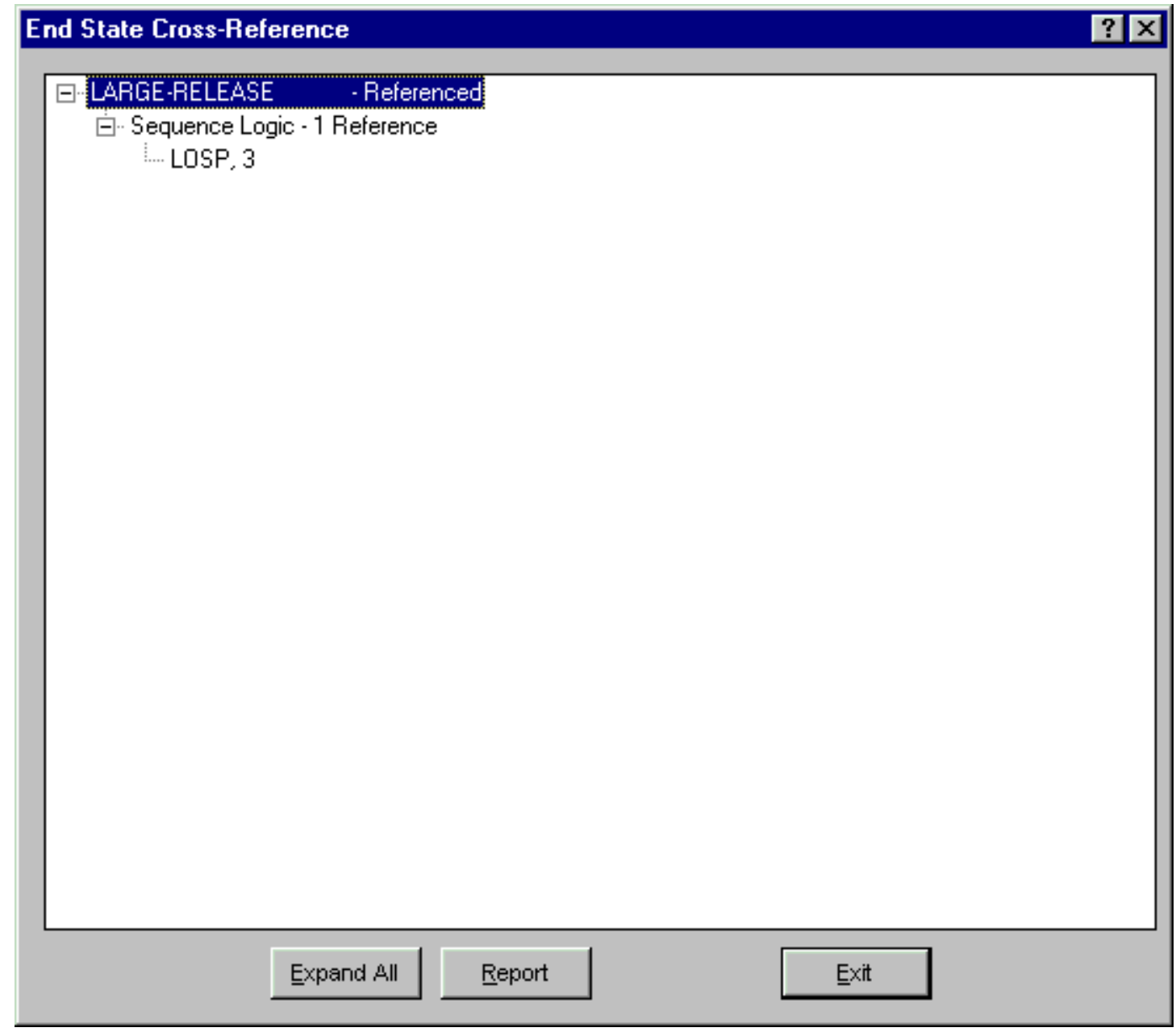

\#- Indicates that the item can be expanded to display more information. Click on this symbol to expand the item.

$\square$ - $\quad$ Indicates that the item is fully expanded. Click on this symbol to collapse the item.

Expand All - Expand all items in the cross-reference map.

Report - Generate the cross-reference report for the end state.

Exit - $\quad$ Close the End State Cross-reference dialog.

\subsubsection{Remove Unused End States}

\section{PURPOSE}

This option allows you to delete all unused end state records from the data base.

\section{STEPS}

1. From the SAPHIRE menu select Modify.

2. Choose End States from the menu. The Edit End State dialog is displayed.

3. Choose the Remove Unused button. A warning dialog will appear, allowing you to cancel the operation at this point. 


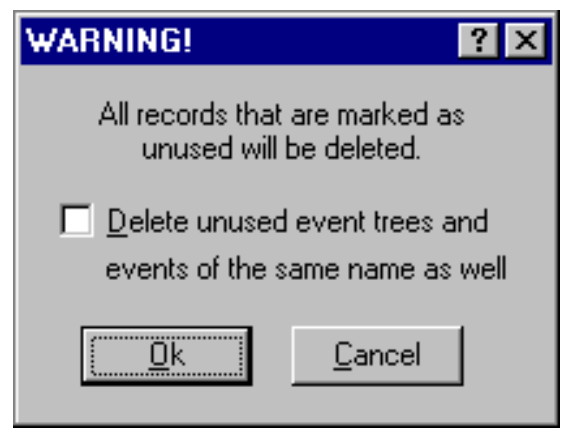

Choose the check box if you wish to delete unused event trees and basic events of the same name when you perform this operation.

OK - Continue the remove operation. The unused end state records will be deleted from the database.

Cancel - Terminate the delete operation.

\subsubsection{End State Data Entry Dialog}

\subsubsection{End State Data Entry}

\section{PURPOSE}

The Add/Modify End State dialog allows you to enter or modify end state data.

\section{STEPS}

1. From the SAPHIRE menu select Modify.

2. Choose End States from the menu. The Edit End State dialog is displayed.

3. Highlight the end state you wish to edit or copy. (Skip this step if adding a new end state record.)

4. Right-click to invoke the pop-up menu and choose Add, Copy or Modify.

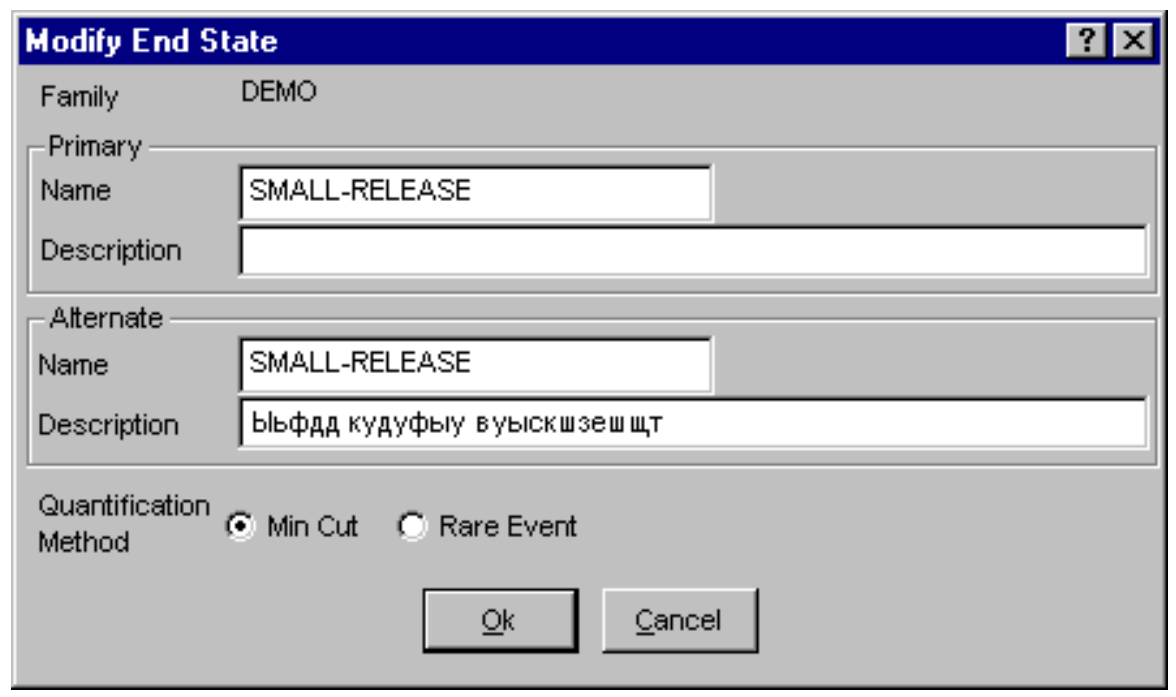


Ok - $\quad$ Save the new or modified end state and close the dialog.

Cancel - Close the dialog without saving.

\subsubsection{Name}

This is a required field. A maximum of 24 uppercase, alphanumeric characters may be entered. Embedded blanks are not allowed. The name should be descriptive so it can be readily identified.

\subsubsection{Description}

This is a 120-character, uppercase or lowercase, alphanumeric field that provides brief, descriptive information.

\subsubsection{Quantification Method}

Choose the default quantification method for this sequence/fault tree/end state. If the Min/Max radio button is chosen, you must also enter the Number of Passes (from 1 to 10).

\subsection{Modifying Basic Event Data}

\subsubsection{Basic Events}

\section{PURPOSE}

This option allows you to add, modify, or delete the basic event data records. Deletion of all unused basic event records is also provided.

\section{STEPS}

1. From the SAPHIRE menu select Modify.

2. Choose Basic Events from the menu. The Edit Events dialog is displayed, listing all of the basic events belonging to the current project.

3. Highlight the desired event.

4. Right-click to invoke the pop-up menu or choose the desired button. 


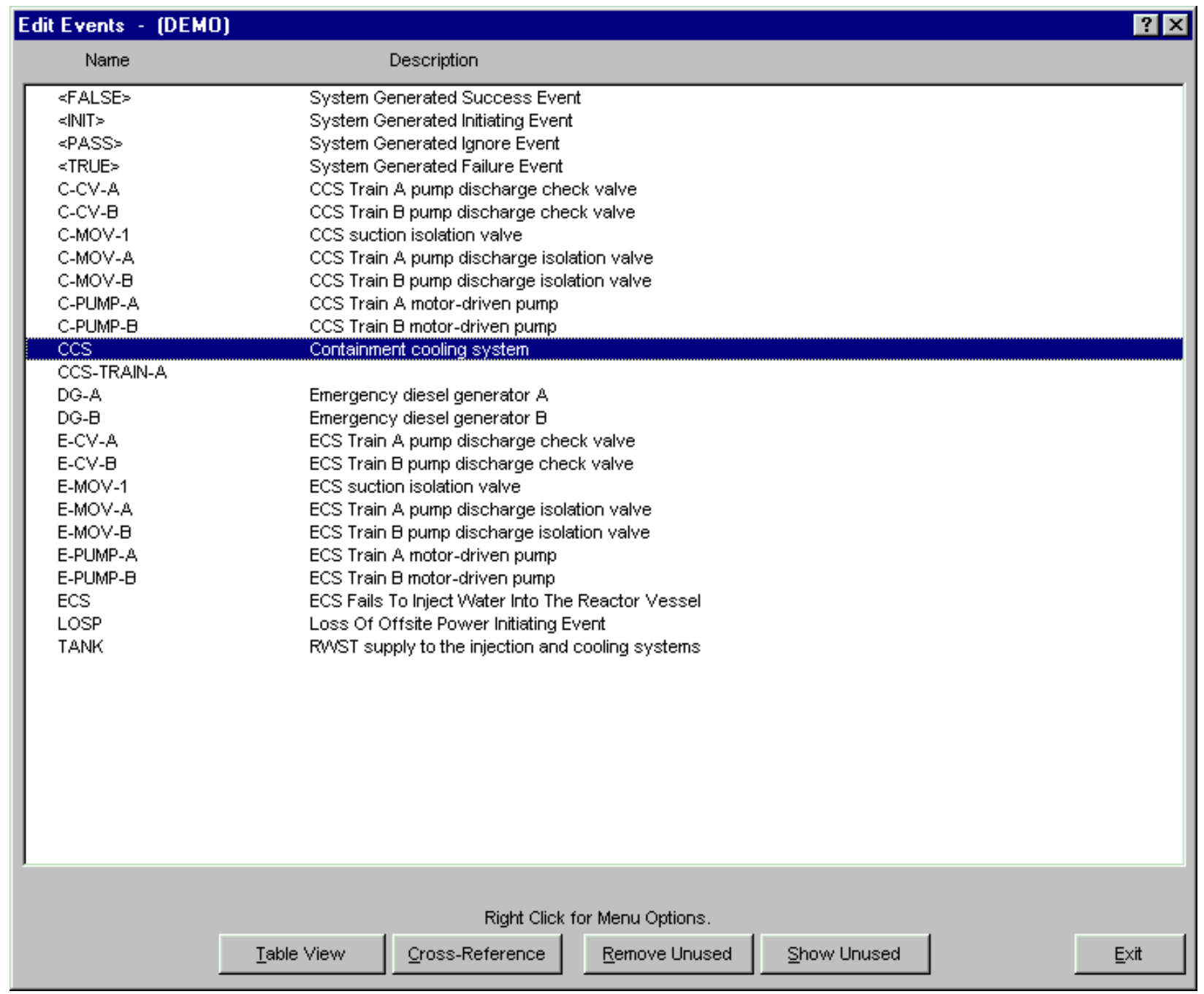

\section{Pop-up Menu Options}

Add -

Copy -

Modify -

Delete -
Add a new basic event record to the current project database. When complete, the message, "Record added," is displayed in the message bar of the SAPHIRE window.

Create a new basic event record by copying an existing one.

Edit the selected basic event record. When complete, the message, "Record modified," is displayed in the message bar of the SAPHIRE window.

Delete the selected basic event record(s). Only unused events will be deleted.

\section{Button Options}

Table View - Display fields of the selected event records in a table format.

Cross-reference - Display the cross-reference map of the highlighted event.

Remove Unused - Delete all unused basic event records.

Show Unused - Show all unused basic event records. The unused basic events are

Exit indicated by a dash (-) to the left of the basic event name.

Close the Edit Events dialog. 


\subsubsection{Delete Record}

\section{PURPOSE}

This option allows you to delete the highlighted record from the database.

\section{STEPS}

1. From the SAPHIRE menu select Modify.

2. Choose the desired option from the menu.

3. Highlight the record you wish to delete.

4. Right-click to invoke the pop-up menu and choose Delete. A warning dialog will be displayed, allowing you to cancel the deletion at this point.

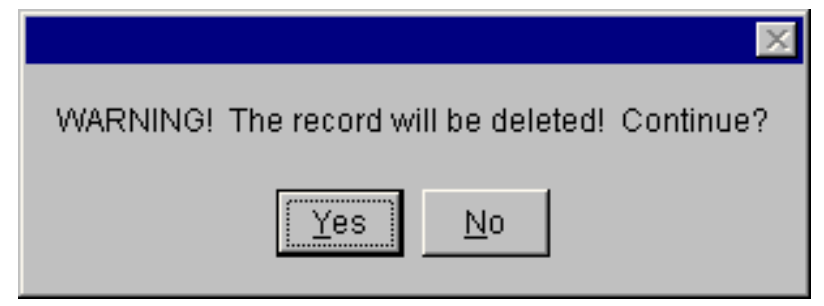

Yes - Continue the delete operation. The selected record will be deleted from the database. The message, "Record deleted," is displayed in the message bar of the SAPHIRE window.

No - Cancel the delete operation. The selected record will NOT be deleted from the database.

\subsubsection{Basic Event Cross-reference}

\section{PURPOSE}

This option displays a cross-reference map of the highlighted basic event. This cross-reference map will show the fault tree logic, change sets, and cut sets in which the basic event is used. For each fault tree, sequence and/or end state in which the basic event is used, base and/or current case cut sets will be listed for each analysis type.

\section{STEPS}

1. From the SAPHIRE menu select Modify.

2. Choose Basic Events from the menu. The Edit Events dialog is displayed.

3. Highlight the desired event.

4. Choose the Cross-reference button. The Event Cross-reference dialog is displayed. 


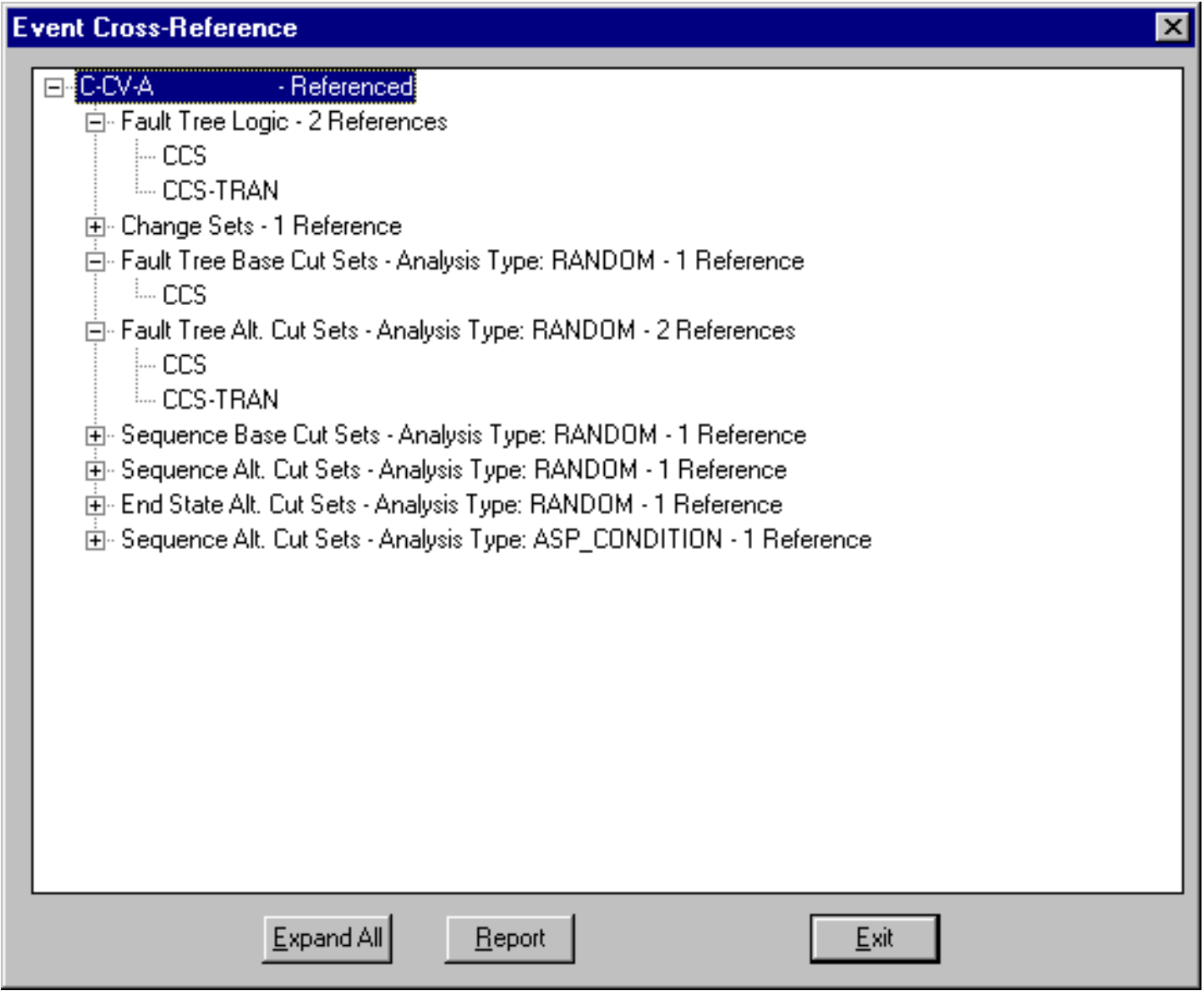

H- $\quad$ Indicates that the item can be expanded to display more information. Click on this symbol to expand the item.

$\square$ - Indicates that the item is fully expanded. Click on this symbol to collapse the item.

Expand All - Expand all items in the cross-reference map.

Report - $\quad$ Generate the cross-reference report for the basic event.

Exit - $\quad$ Close the Event Cross-reference dialog.

\subsubsection{Remove Unused Event}

\section{PURPOSE}

This option looks at all references to an event in the current project and deletes any events that are not referenced by anything.

\section{STEPS}

1. From the SAPHIRE menu select Modify.

2. Choose Basic Events from the menu. The Edit Events dialog is displayed.

3. Choose the Remove Unused button. 
A warning dialog will be displayed informing you that all records that are marked as unused will be deleted.

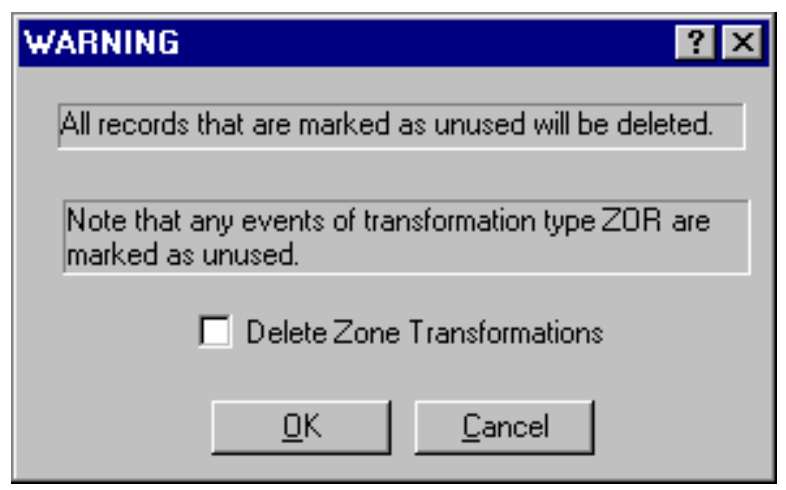

Delete Zone Transformations - By checking this box, events of transformation type ZOR will also be deleted.

OK -

Cancel -
Continue the deletion process.

Terminate without deleting the unused events.

\subsubsection{Table View}

\subsubsection{Table View}

\section{PURPOSE}

This option allows you to view selected basic event details in a table format.

\section{STEPS}

1. From the SAPHIRE menu select Modify.

2. Choose Basic Events from the menu. The Edit Events dialog is displayed.

3. Highlight the desired events to include in the table.

4. Choose the Table View button. 


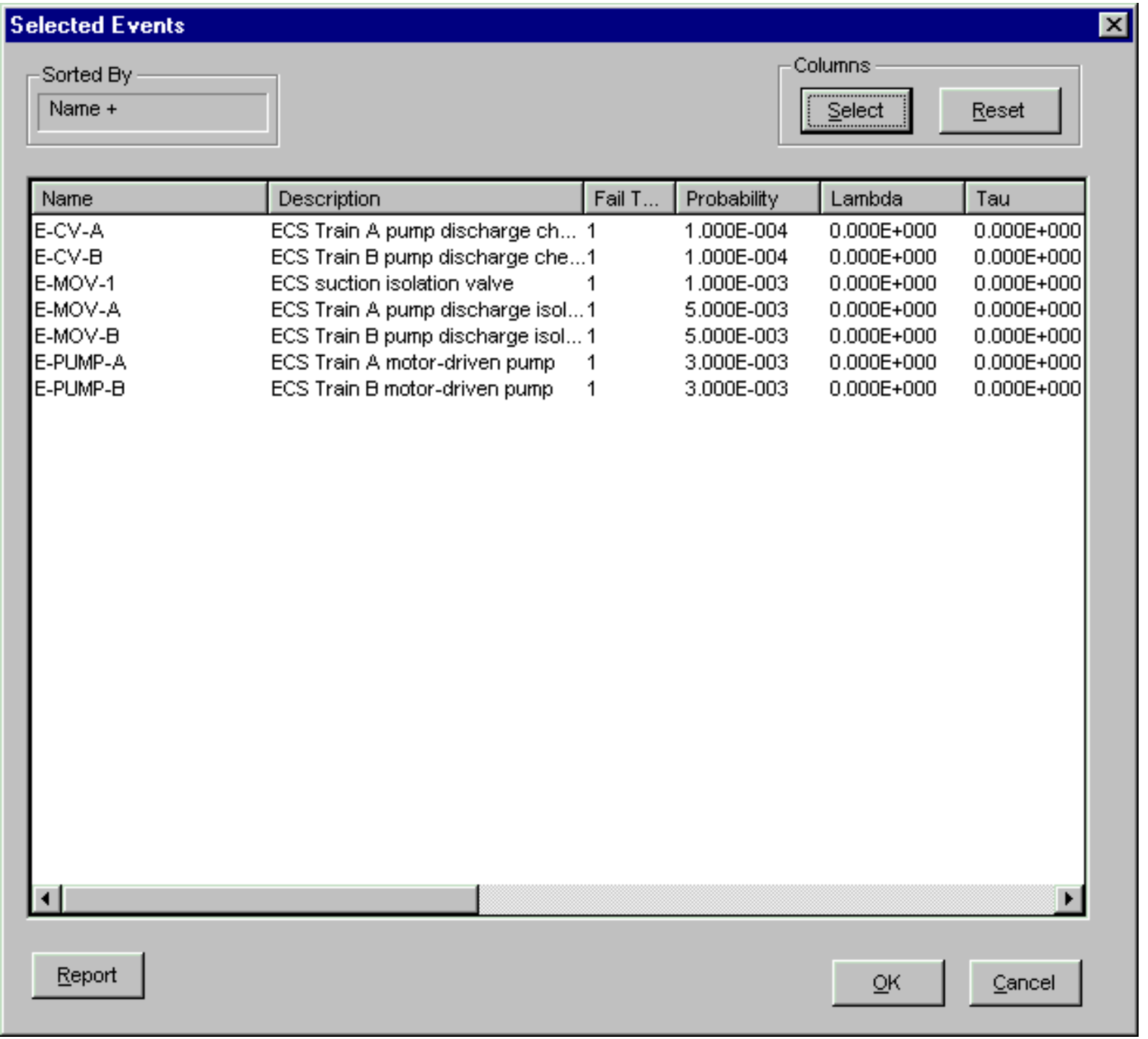

Sorted By - $\quad$ Indicates the name of the column the data is currently sorted by. A plus $(+)$ indicates ascending order. A minus (-) indicates descending order.

Click Column - Sort the table by the selected column. Additional clicks on the same column toggle the sort between ascending and descending order.

Resize Column - Move the mouse cursor to a column divider. The cursor will change to the Move Column cursor right to resize the column. Release the mouse when the desired width is obtained.

\section{Columns}

Select - Display the Select Columns to Display dialog.

Reset - $\quad$ Reset the column display to the SAPHIRE default.

\section{Pop-up Menu Option}

Modify - Edit the selected event record. When complete, the message, "Record modified," is displayed in the message bar of the SAPHIRE window. 


\section{Button Options}

Report - Create a report of the current table. The selected fields, order, and width will match the currently selected options for display. The report will be a table in RTF format. If you select a report preview, whatever program the operating system has associated with RTF files (such as Word, WordPerfect, Wordpad, etc), will be invoked to display the report.

OK - Close the Selected Events dialog. Retain the current column settings until SAPHIRE is closed.

Cancel - Close the Selected Events dialog. Do not retain the current column settings.

\subsubsection{Select Columns}

\section{PURPOSE}

This option allows you to select the columns to display in a table format. It also reports the current width of each column in inches so a report of desirable width can be created.

\section{STEP}

1. From the Selected Events dialog, press the Column Select button.

2. A list of basic event field names appears in the order they are to be displayed in the Selected Events dialog (and report option).

3. Field can be included or excluded from display by clicking on the first column of each field. Fields can be reordered by selecting the Up and Down Position buttons.

4. The current width of each column is displayed along with a total displayed width. This information is provided to help you create a custom view that can be attractively printed to a report. (Each field width can be modified from the Selected Events dialog by using the mouse to resize the column width.) 


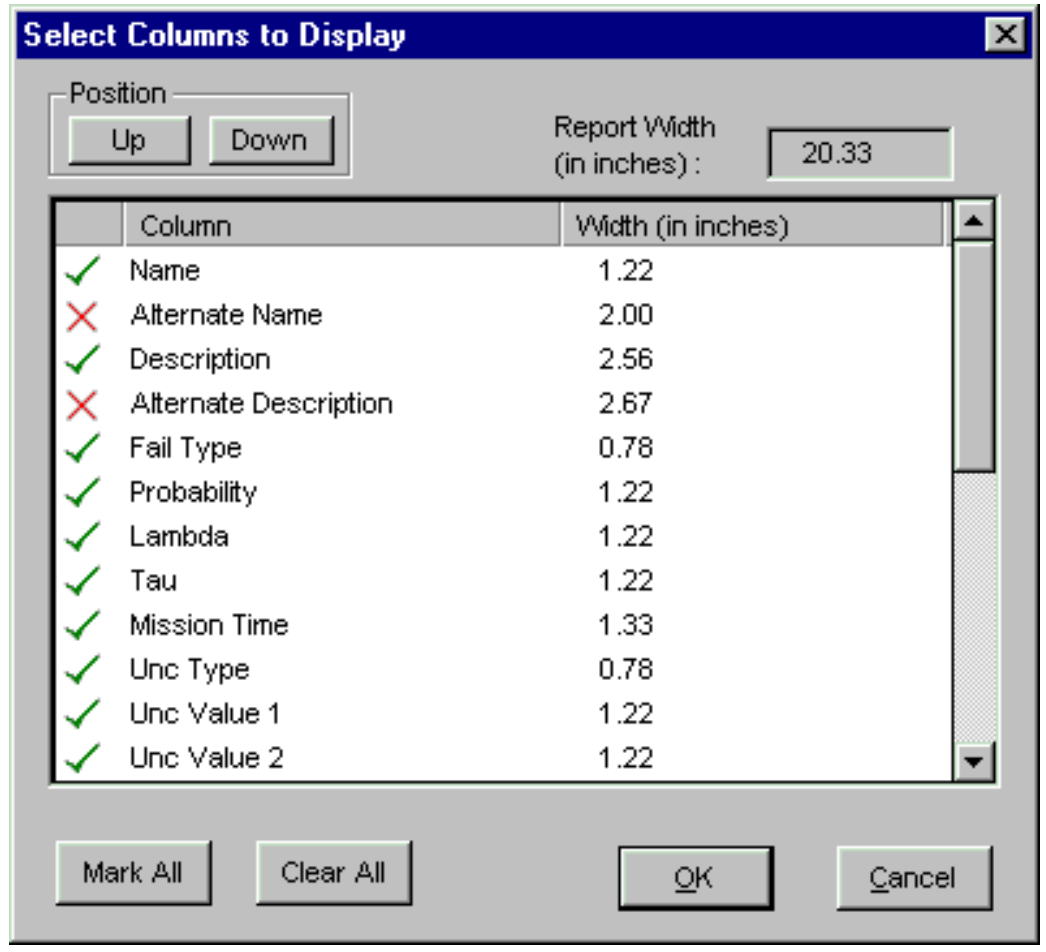

\section{Position}

Up-

Down-

\section{Button Options}

Mark All -

Clear All -

OK -

Cancel -
Indicates that the field will be included in the table view. Click on this symbol to toggle the status.

Indicates that the field will be excluded from the table view. Click on this symbol to toggle the status.

Each time you press the Up button, the selected field will move up one position in the list. (The field column in the table view will be moved left.)

Each time you press the Down button, the selected field will move down one position in the list. (The field column in the table view will be moved right.)

Include all available fields in the table view.

Exclude all available fields in the table view.

Close the Select Columns to Display dialog. Return to the Selected Events dialog using the selected column settings to display the events. Close the Select Columns to Display dialog. Do not use the selected column settings; instead, use the previously selected column settings. 
Note : Some of the fields available for reporting in SAPHIRE are multi-purpose fields, and thus may have a somewhat generic name. For example, the meaning of Unc Value 1 \#1Unc Type field. If the Unc Type field is "L" for Log Normal, then Unc Value 1 will be an Error Factor, and Unc Value 2 will be unused. Similarly, if the field Trans Typecontains an "A", "O", or "Z", it indicates the event is a transformation event with respective types "And", "Or", or "Zor"; but a "Y" value actually indicates a compound event; and a value " $\mathrm{T}$ " indicates a template event.

\subsubsection{Basic Event Property Sheet}

\subsubsection{Event Page}

\subsection{Basic Event Data Entry}

\section{PURPOSE}

The Add/Modify Event property sheet allows you to enter or modify basic event data. This property sheet contains six tabs containing information pertaining to basic events, grouped by related properties. To edit the desired properties click on the tab.

\section{STEPS}

1. From the SAPHIRE menu select Modify.

2. Choose Basic Events from the menu. The Edit Events dialog is displayed.

3. Highlight the event you wish to edit or copy. (Skip this step if adding a new basic event record.)

4. Right-click to invoke the pop-up menu and choose Add, Modify, or Copy. 


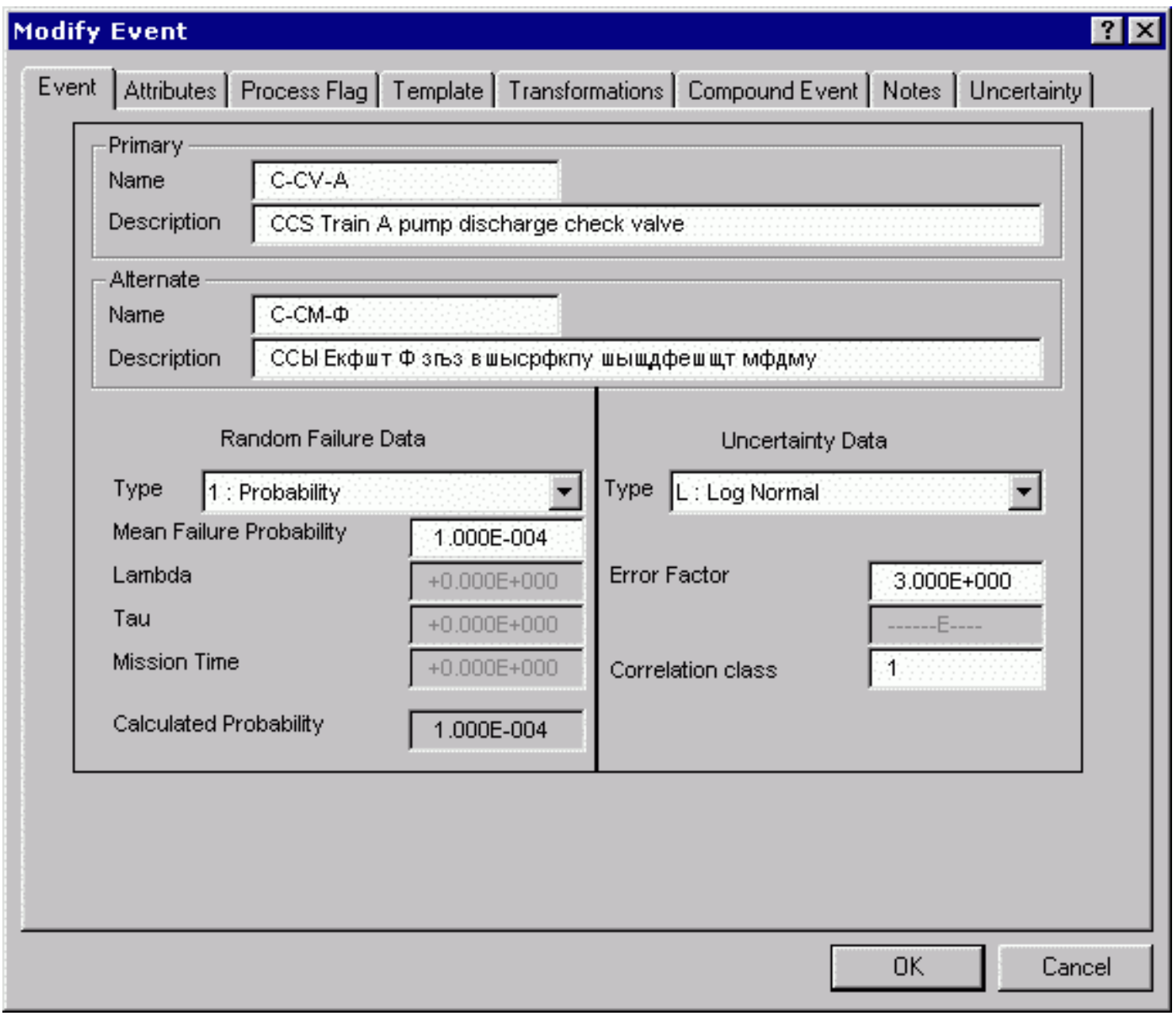

OK - Save the new or modified basic event and close the dialog.

Cancel - Close the dialog without saving.

\subsection{Primary Name}

The Primary name is the fundamental name used in the fault trees and event trees. A unique Primary name must be specified for every basic event in the logic models. A maximum of 24 uppercase, alphanumeric characters may be entered. Embedded blanks are not allowed. The name should be descriptive so it can be readily identified.

\subsection{Alternate Name}

The Alternate name, which can be different than the Primary name, can be used to report cut set results. If a name is not entered, the primary name will be copied to this field. This feature allows cut sets to be reported using a different naming scheme that is more descriptive or using another locale. For this name to be displayed in lists and on reports instead of the Primary name, choose Use alternate names for display in the Define Constants option. A maximum of 24 uppercase, alphanumeric characters may be entered in this field. Embedded blanks are not allowed. 


\subsection{Description}

This is a 120-character, uppercase or lowercase, alphanumeric field that provides brief, descriptive information.

\subsection{Alternate Description}

This is a 120-character, uppercase or lowercase, alphanumeric field that provides brief, descriptive information. This feature allows descriptions to be displayed and reported using a different naming scheme or another locale. For this description to be displayed in lists and on reports instead of the Primary description, choose Use alternate names for display in the Define Constants option.

\subsection{Calculation Types}

In the Failure Data section the calculation type is a numerical reference to the calculation method to be used. Choose the desired calculation type from the drop-down list.

An equation for each calculation type follows. See the symbol table for more details.

$1 \quad \mathrm{P}=\mathrm{p}$.

The value specified in the probability field is directly used as the basic event failure probability or initiating event frequency

$3 \quad \mathrm{P}=1-\operatorname{Exp}\left(-\mathrm{L} * \mathrm{t}_{\mathrm{m}}\right)$.

This calculation type is the full equation for the failure probability of an operating component without repair in a non-demand failure mode.

$5 \quad \mathrm{P}=([\mathrm{L} * \mathrm{~T}] /[1+\{\mathrm{L} * \mathrm{~T}\}]) *\left(1 \mathrm{EXP}\left[(\mathrm{L}+1 / \mathrm{T}) * \mathrm{t}_{\mathrm{m}}\right]\right)$

This calculation type is the full equation for the failure probability of an operating component with consideration given to the ability to repair the component.

$7 \quad \mathrm{P}=1+(\operatorname{EXP}[\mathrm{L} * \mathrm{~T}] 1) /(\mathrm{L} * \mathrm{~T})$.

This calculation type is the full equation for the failure probability of a standby component in a non-demand failure mode with consideration given to periodic testing.

$8 \quad \mathrm{P}=\mathrm{bp}+\mathrm{p}$.

This calculation type is available only in change sets. It indicates the value given in the change set probability field is to be added to the base case probability value.

$9 \quad \mathrm{P}=\mathrm{bp} * \mathrm{p}$.

This calculation type is available only in change sets. It indicates the value given in the change set probability field is to be multiplied with the base case probability value.

$\mathrm{T} \quad \mathrm{P}=1.0$ (House event - failed).

This calculation type indicates that the basic event is to be treated as a house event that is always failed. A house event never appears in the minimal cut sets. The model is modified to reflect the logic, given that the indicated basic event is always failed. To do this for an event that is guaranteed to occur (failure probability $=1.0$ ), the event is removed from the logic 
where it appears as an input to an AND gate. If the basic event is input to an OR gate, the entire gate and its inputs are removed from the logic. The resulting minimal cut sets show the failure combinations that must occur for top event or sequence failure given that the indicated basic event is always failed.

F $\quad \mathrm{P}=0.0$ (house event - successful).

This calculation type indicates that the basic event is to be treated as a house event that is never failed. A house event never appears in the minimal cut sets. The model is modified to reflect the logic given that the indicated basic event is never failed. To do this for an event that is guaranteed successful, the basic event is removed from the logic where it appears as an input to an OR gate. If the basic event is input to an AND gate, the entire gate and its inputs are removed from the logic. The resulting minimal cut sets show the failure combinations that must occur for top event or sequence failure given that the indicated basic event can never fail.

I $\quad \mathrm{P}=0.0$ (ignore event).

This calculation type indicates that the basic event is to be treated as if it did not exist in the logic for the fault tree. Before the tree is solved, the logic is edited to remove all references to the specified event from the fault tree.

$\mathrm{S} \quad \mathrm{P}=0.0$ (find a fault tree with the same name and use its current mincut upper bound as the probability)

This calculation type indicates that the basic event is to replace its matching fault tree. If no matching fault tree exists, the probability will be set to 0.0 .

E $\quad \mathrm{P}=0.0$ (find an end state tree with the same name and use its current mincut upper bound as the probability)

This calculation type indicates that the basic event is to use its matching end state value. If no matching end state exists, the probability will be set to 0.0. This type is useful in level two analysis.

G $\quad \mathrm{P}=\Phi[\ln (\mathrm{g} / \mathrm{a}) / \mathrm{Br}]$.

This calculation type indicates that the basic event is to be treated as a seismic event. The probability value for screening will be calculated using the ground acceleration, failure acceleration, and $\mathrm{Br}$ entered by the user.

$\mathrm{H} \quad \mathrm{P}=\Phi[\ln (\mathrm{g} / \mathrm{a}) / \mathrm{Br}]$.

This calculation type indicates that the basic event is to be treated as a seismic event. The probability for screening will be calculated from the failure acceleration, $\mathrm{Br}$, and ground acceleration. The ground acceleration will be the highest g-level specified in the medium project hazard curve.

$\mathrm{V} \quad \mathrm{P}=\mathrm{N} / \mathrm{A}$ (not applicable)

The event is a "value" event and will not appear directly in fault tree or sequence logic. The value specified in the Value field is not necessarily a probability or frequency, but is rather an input value to a compound event.

B $\quad \mathrm{P}=\mathrm{bp} /$

This option is only available for use in a change set. It indicates that the base case value is to 
be used. This is useful when multiple change sets are marked and it is desired to override a previous change.

C $\quad \mathrm{P}=\mathrm{F}(\mathrm{x})$.

The event is a compound event whose probability/frequency value is defined as a function of other input values, formulas, and/or events (value events and regular basic events). This option is not currently available in change sets, since compound event data cannot be added via a change set (but it can be removed via a change set).

\subsection{Mean Failure Probability}

Enter the probability that a component will fail between $\mathrm{t}$ and $\mathrm{t}+\Delta \mathrm{t}$ given that no failure has occurred before time $\mathrm{t}$ (use scientific notation).

\subsection{Lambda}

Enter the event's failure rate per hour (use scientific notation).

\subsection{Tau}

Enter the average time to repair in hours (use scientific notation).

\subsection{Mission Time}

Enter the mission time in hours (use scientific notation). The mission time is the period of time that a component is required to operate in order to characterize the component operation as being successful.

An example would be for a pump that must run for 24 hours after a particular initiating event occurs. The mission time for this case would be 24 hours.

\subsection{Distribution Types}

For the Uncertainty Data section, there are nine predefined distribution types available. The predefined distribution types are:

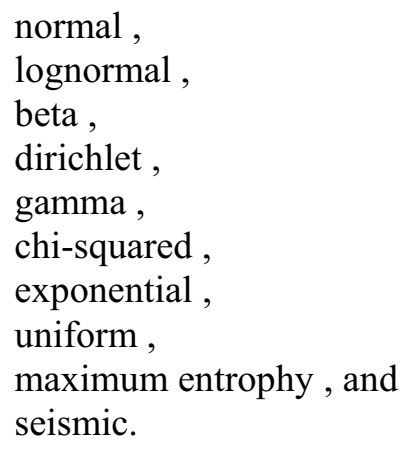

In addition to these predefined distribution types, user-defined histograms may be used. The default distribution type is no distribution. Choose the desired distribution type from the drop-down list.

\subsection{Value 1}


Enter the first parameter of the distribution, if one is required.

\subsection{Value 2}

Enter the second parameter of the distribution, if one is required.

\subsection{Correlation Class}

Used to account for data dependencies among like events in the database. Enter up to 24 uppercase, alphanumeric values. A blank correlation class indicates that there are no data dependencies. When running the uncertainty analyses, the same sample value will be used for all basic events with the same correlation class.

\subsubsection{Attributes Page}

\subsection{Basic Event Data - Attributes}

\section{PURPOSE}

Basic event attributes define the traits of the event. These traits can be used to group the events, for example, in change set processing.

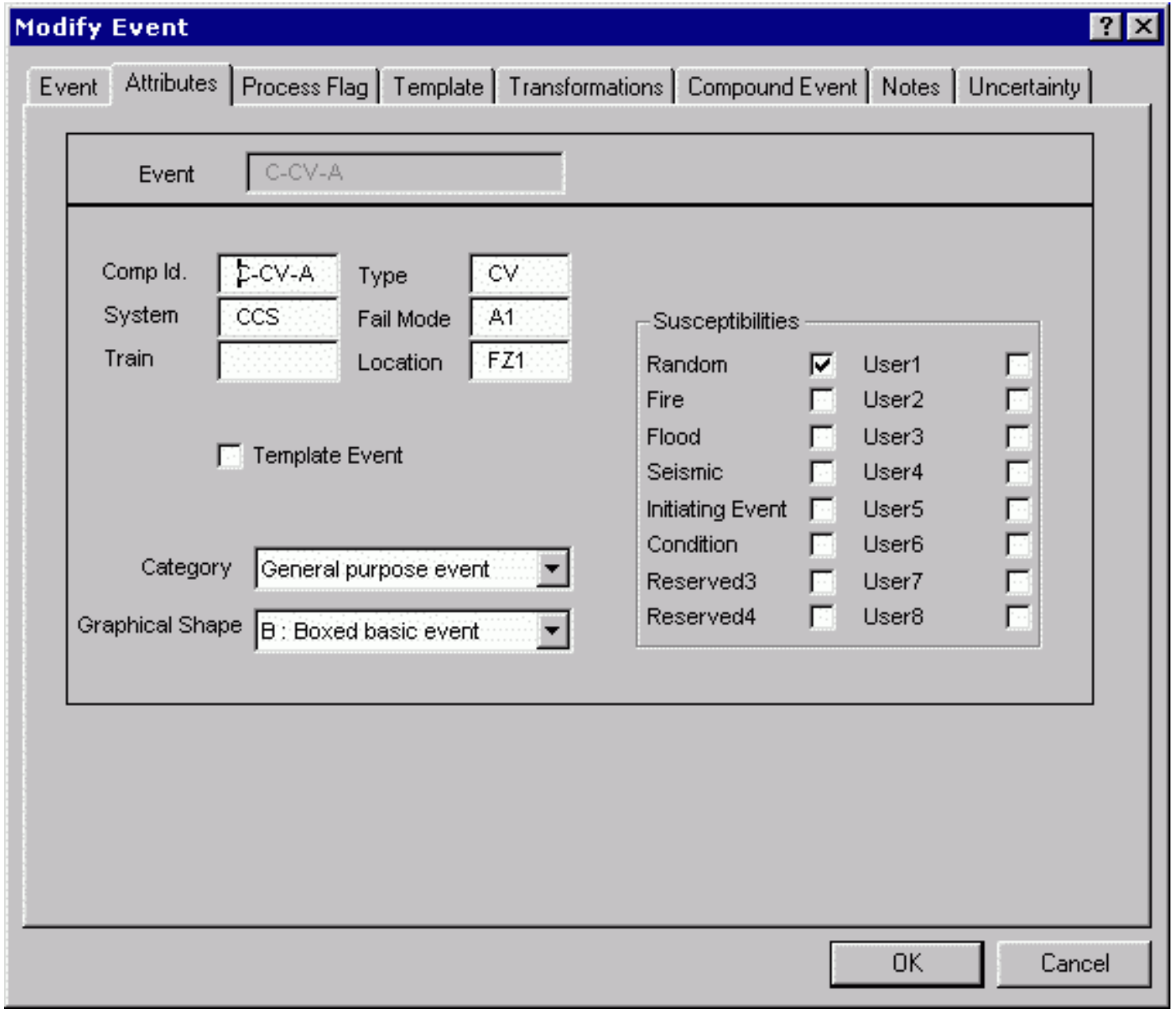


Ok - Save the new or modified basic event and close the dialog.

Cancel - Close the dialog without saving.

\subsection{Comp Id}

Component Identifier. Enter up to seven (7) alphanumeric characters to identify a component by a unique designator. This is usually part of the component label (e.g., DG01). No embedded blanks are allowed.

\subsection{System}

Enter up to three (3) alphanumeric characters to identify the system containing the component.

\subsection{Train}

Enter up to two (2) alphanumeric characters to identify the train containing the component.

\subsection{Type}

Enter the event type attribute.

\subsection{Fail Mode}

Enter up to two (2) alphanumeric characters to identify the failure mode for the component.

\subsection{Location}

Enter up to three (3) alphanumeric characters to identify the physical location for the component.

\subsection{Category}

Select the from the drop-down list to specify the category or use of the event.

General purpose event - This is the default and is appropriate for must basic events.

'I' - Initiating event - Any initiating events should be identified with this category designation. The event tree editor will automatically enter an 'I' when the user specifies that the first event is an initiating event.

'H' - Hazard event - This is a special calculation type assigned to histogram bins for hazard analysis.

' $\mathbf{R}$ ' - Recovery event - Events with this category designation will be listed in a list of recovery events used in the Recovery Rules editor

\subsection{Susceptibilities}

The susceptibility flags indicate whether or not the event is susceptible to a specific kind of failure. There are 16 susceptibilities as defined below:

Random (default)

Fire

Flood 


\author{
Seismic \\ Initiating Event \\ Condition \\ Reserved 3 (Reserved for internal use) \\ Reserved 4 (Reserved for internal use) \\ User1 - User 8 (User-defined)
}

Susceptibility flags must be checked for the event to be considered susceptible to a specific type of failure. All events are susceptible to random failure regardless of the random flag's value.

\title{
9.6.6.2.10 Template Event
}

Checking this box flags the event as a template event. A template event can used by other events to inherit the template event's field values. This box cannot be unchecked if any other events in the project reference it as a template.

\subsection{Graphical Shape}

The graphical shape selected here will determine what basic event shape will be used in the Alpha-toGraphics conversion. Select the graphical shape (e.g., basic event, boxed basic event, etc.) for this event from the drop-down list. The "Default" shape uses the shape selected at the time the conversion is done.

9.6.6.3 Process Flag Page

\subsection{Basic Event Data - Process Flag}

\section{PURPOSE}

The process flag specifies if certain processes should take special note of the selected event. Select one of the available values: 


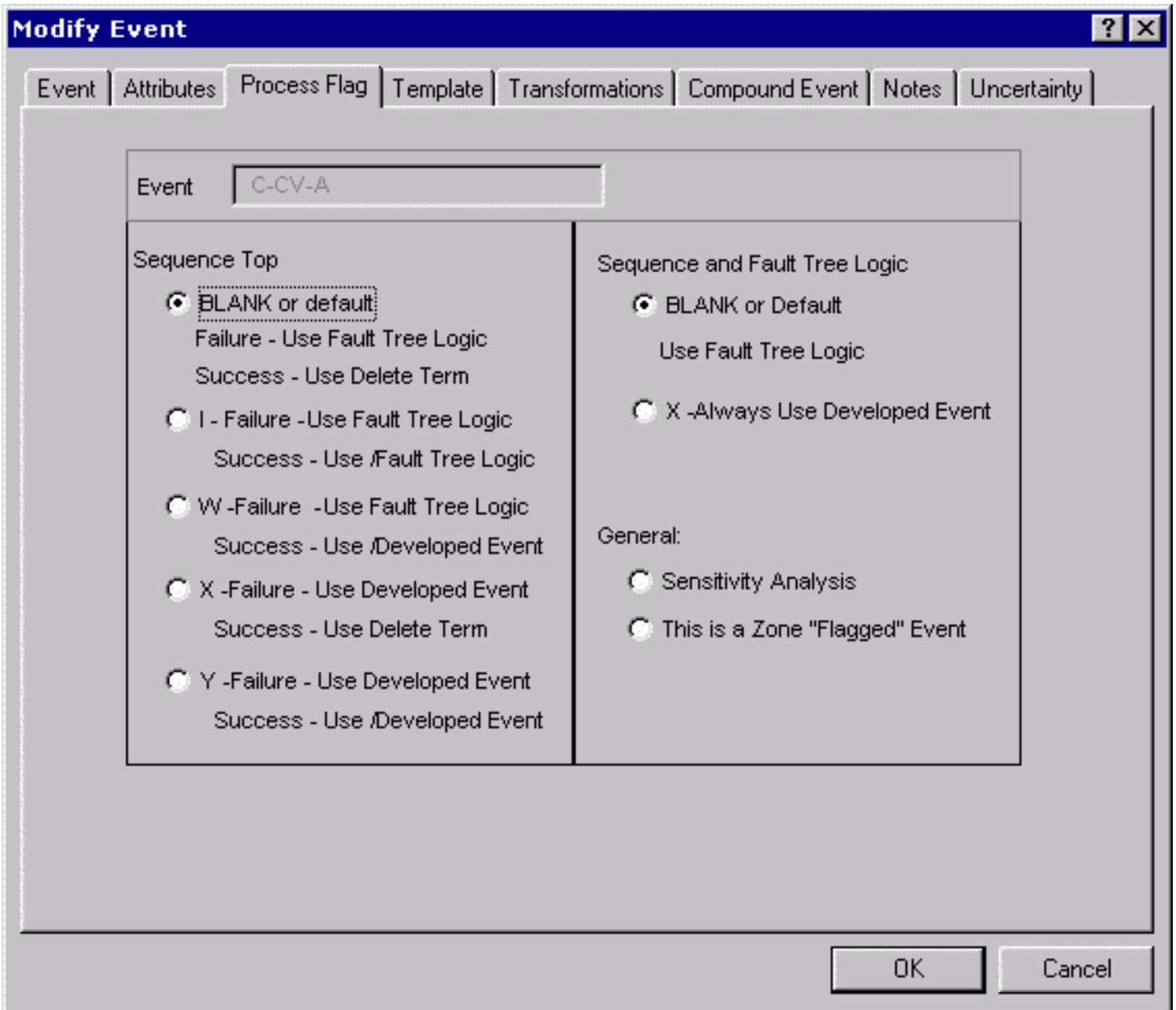

Ok - Save the new or modified basic event and close the dialog.

Cancel - Close the dialog without saving.

\subsection{Blank or Default}

When the Process Flag field is blank, the transfer logic associated with this event is expanded for failure references. For success references, the transfer is also expanded; however, any "impossible" cut sets (i.e., those both failing and succeeding) are removed from the resulting failure cut sets using cut set matching (also known as the "delete term" process).

\subsection{Process Flag ' $I$ '}

Use fault tree logic (if top event fails), use the complement of the fault tree logic (if top event succeeds). That is, if the top event is a failure, SAPHIRE will expand the fault tree and solve; if the event succeeds, SAPHIRE will complement the fault tree logic and solve it. An "I" causes SAPHIRE to treat the transfer as independent. Logic below this transfer is expanded for failure references, and for success references the complement of the logic is used.

\subsection{Process Flag ' $W$ '}


Use fault tree logic (if top event fails), use complement of the developed event (if top event succeeds). That is, if the event fails SAPHIRE will expand the fault tree and solve; if the event succeeds, SAPHIRE will use the complement of the developed event for the fault tree.

\subsection{Process Flag ' $X{ }^{\prime}$}

Use developed event (if event fails), use cut set matching to eliminate cut sets (if event succeeds). That is, an "X" tells SAPHIRE that the top event is to be used for failure references, but success references are to be treated the same as if the flag was blank.

\subsection{Process Flag ' $\mathrm{Y}^{\prime}$}

Use developed event (if event fails), use complement of developed event (if event succeeds). That is, a "Y" indicates that a transfer is to be replaced with its basic event for failed references and the complement of the event is to be used for success references.

\subsection{Sensitivity Analysis}

If an event is marked for sensitivity analysis, SAPHIRE will map a results frequency or probability plot. A sensitivity analysis allows you to see how sensitive the frequency or probability is in relation to an event.

\subsection{Zoned Flagged Event}

A zone flagged event is an event that has been marked as representing a zone (i.e., location or area). An example of a zone is a fire zone or a flood zone. When SAPHIRE encounters a zone flagged event, it performs a transformation. A transformation is an event or set of events that replace a zone flagged event.

\subsubsection{Template Page}

\subsection{Basic Event Data - Template}

\section{PURPOSE}

A template event is a basic event that can be used to define all or part of other basic events. Using templates can simplify the process of adding or updating a series of events which have common values.

When an event is marked as a template (from the Attributes page), the check boxes on the Template tab are enabled. All fields that are checked will be available to the events that use the template.

When a template event is selected from the template combo box, the check boxes below will be enabled, allowing you to mark the attributes, failure data, and uncertainty data that will be copied from the template event. By default all of the template's values will be copied to the basic event. A check mark indicates that the template event's value will be used. 


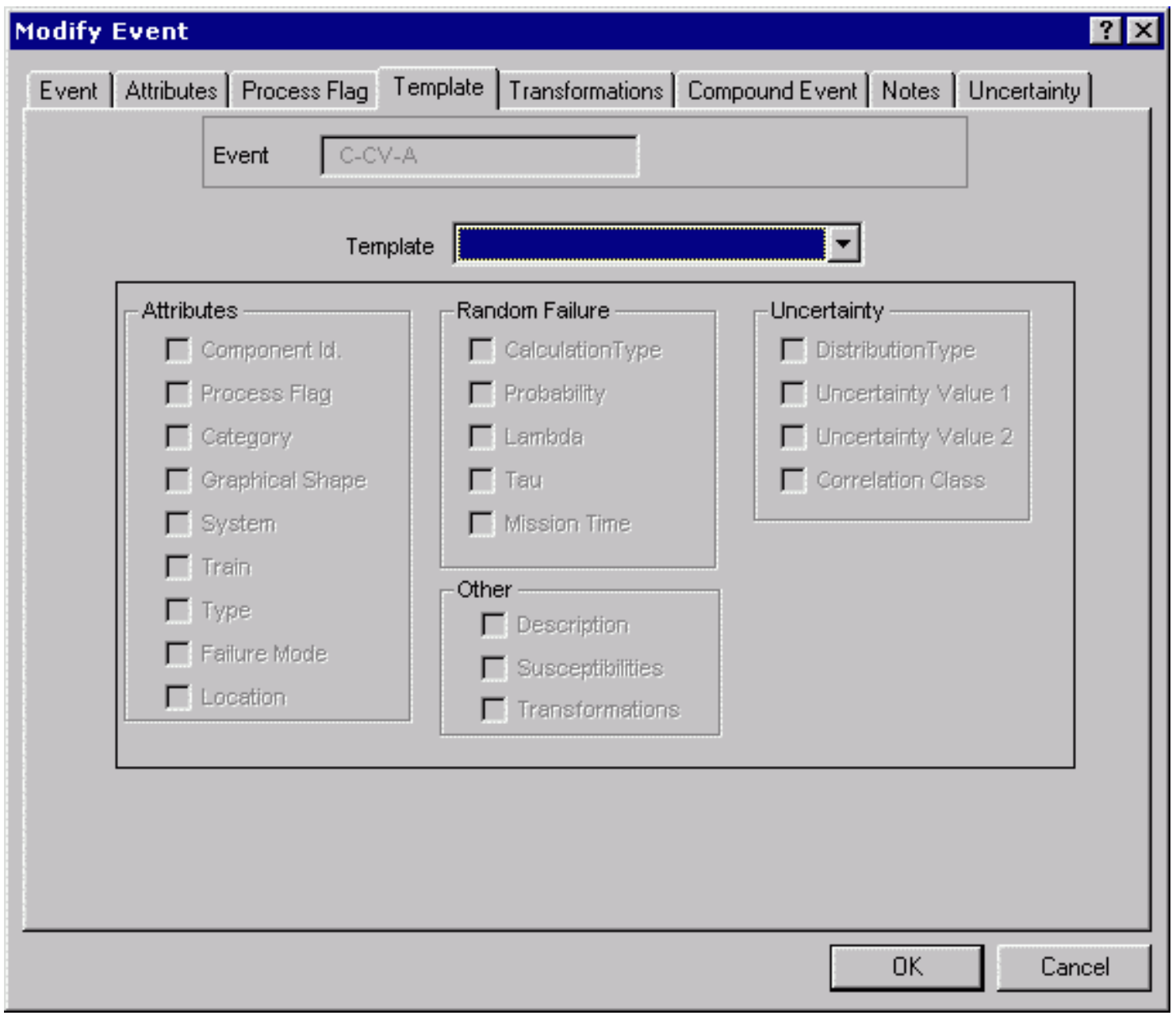

Ok - $\quad$ Save the new or modified basic event and close the dialog.

Cancel - Close the dialog without saving.

\subsection{Template Name}

If you wish to use another event's information as a template for this basic event, select the name of the event from the drop-down list. Then choose the Template tab to select the desired characteristics.

\subsection{Template Example}

Once a template basic event is defined, SAPHIRE has the capability to apply the template's values to all the basic events that reference it. This is accomplished by allowing you to specify a basic event as a template for other basic events in the database. Template events cannot reference other template events. Any basic event (except one designated as a template event) can be set up to use a template event. If a template event is chosen, the basic event can use all or part of the template's values. Whenever the template event's values are changed, all of the events that reference that template event will be modified to reflect that change.

The generation of current event data works with template events as well. Change sets are first applied to all template events and then to all non-template events. The change sets will be applied to the nontemplate events after all the template values have been loaded into them. 
Event ONE is defined as a template event. All values of Event One are available to be copied to events that references this one, except the Description field, which was unchecked for this example to demonstrate how to remove a field from a template.

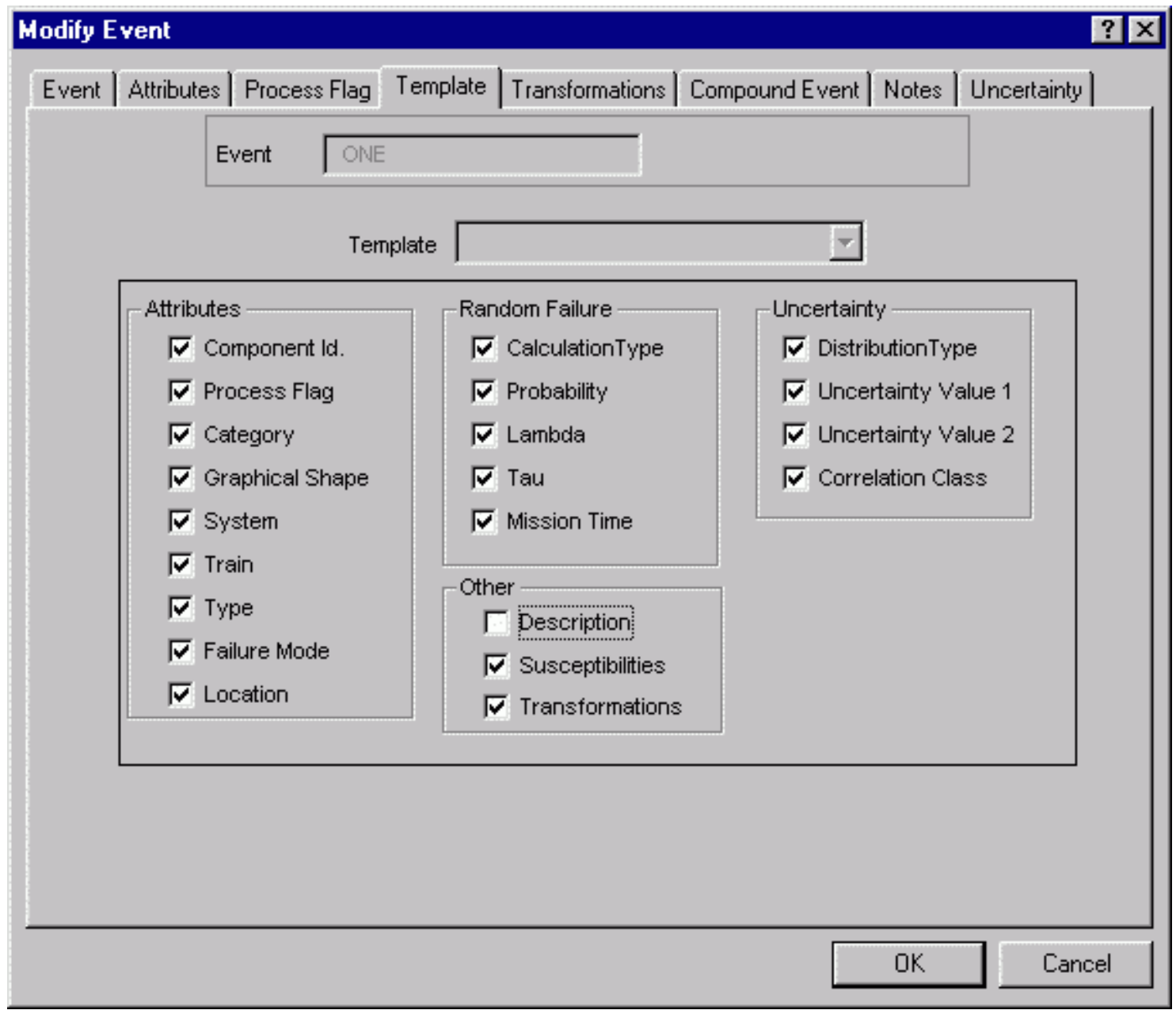

Event TWO references the template event ONE. All of the available values (the Description field is disabled because the template did not "share" this field) from the template event are copied into event TWO, with the exception of the Uncertainty Value 1 field, which was unchecked for this example to demonstrate how to override a template value. 


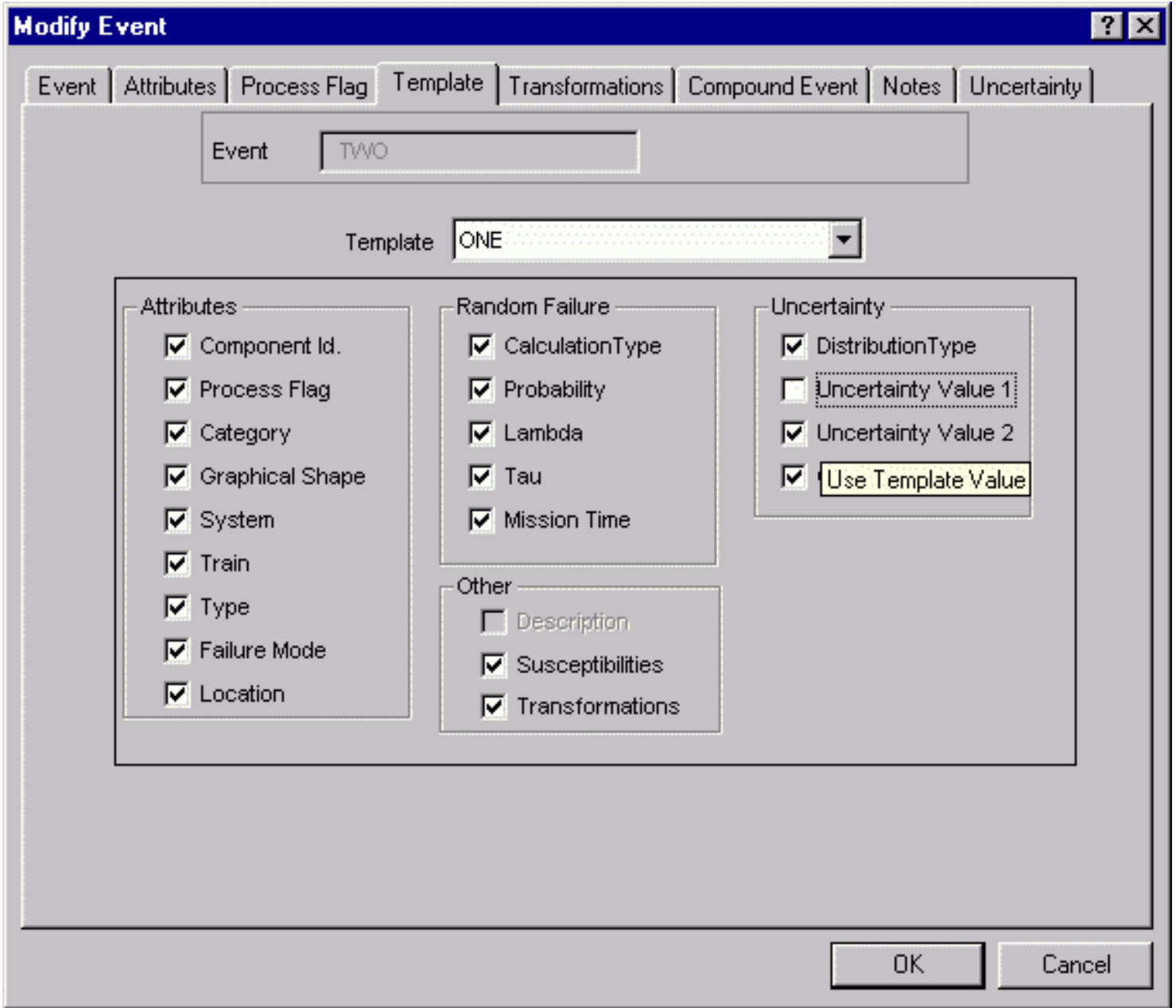

On the Event tab of the TWO event, the fields derived from the template are disabled and labeled in red to indicate that they cannot be edited. Because the Uncertainty Value 1 field was unchecked on the Template page, the Error Factor label is displayed in green, indicating that its value, (even though it is available from the template) can be overridden. The Description field is displayed normally, because the template event did not make that field available for use. 


\section{Modify Event}

\section{Event $\mid$ Attributes $\mid$ Process Flag $\mid$ Template $\mid$ Transformations $/$ Compound Event $\mid$ Notes $/$ Uncertainty|}

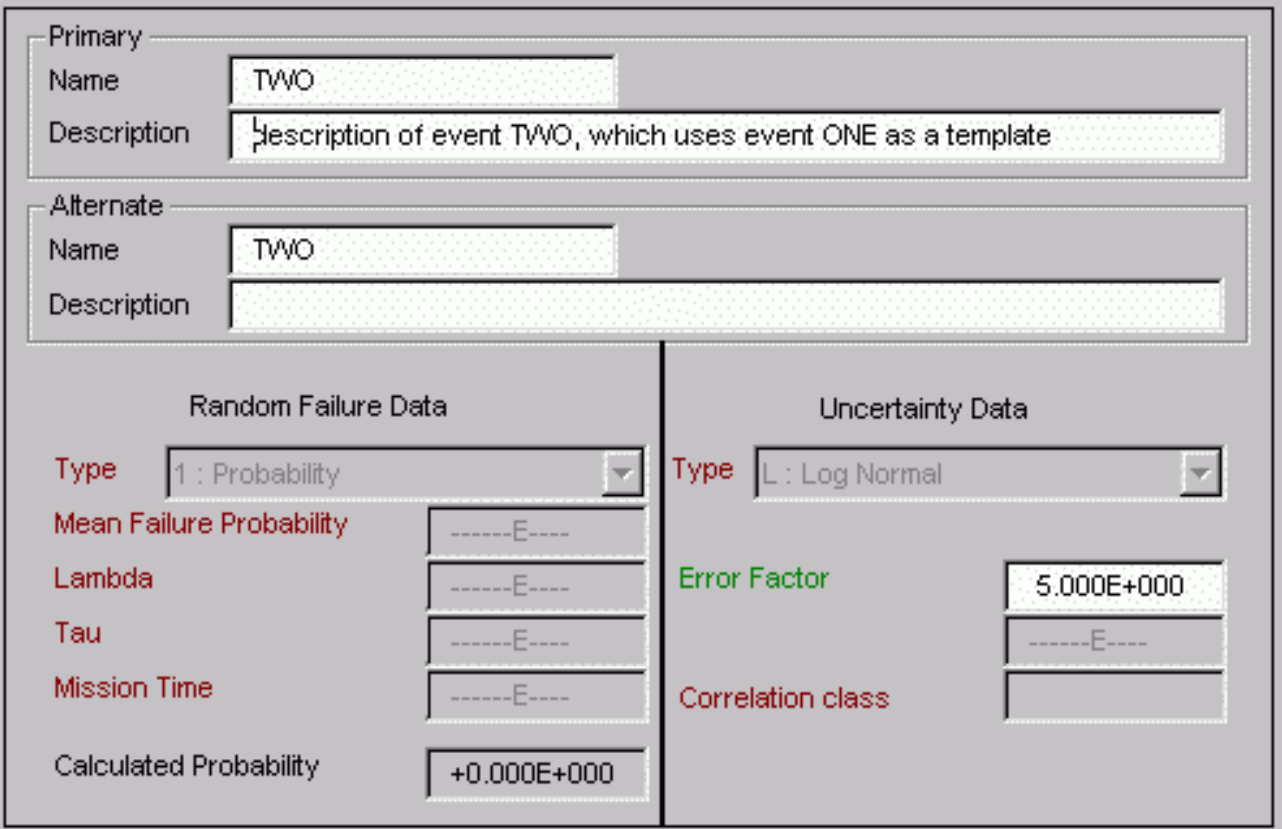

\subsubsection{Transformations Page}

\subsection{Basic Event Transformations}

\section{PURPOSE}

A transformation is a replacement or addition of basic event(s) inside the fault tree logic. During the transformation process, an event is replaced by a series of events; or, if any event exists then another set of events is added to the logic. Transformations occur when a zone flagged event is encountered.

The list on the left of the dialog displays all basic events defined for the current project. The list on the right displays the events that have been marked as replacements for the current event. 


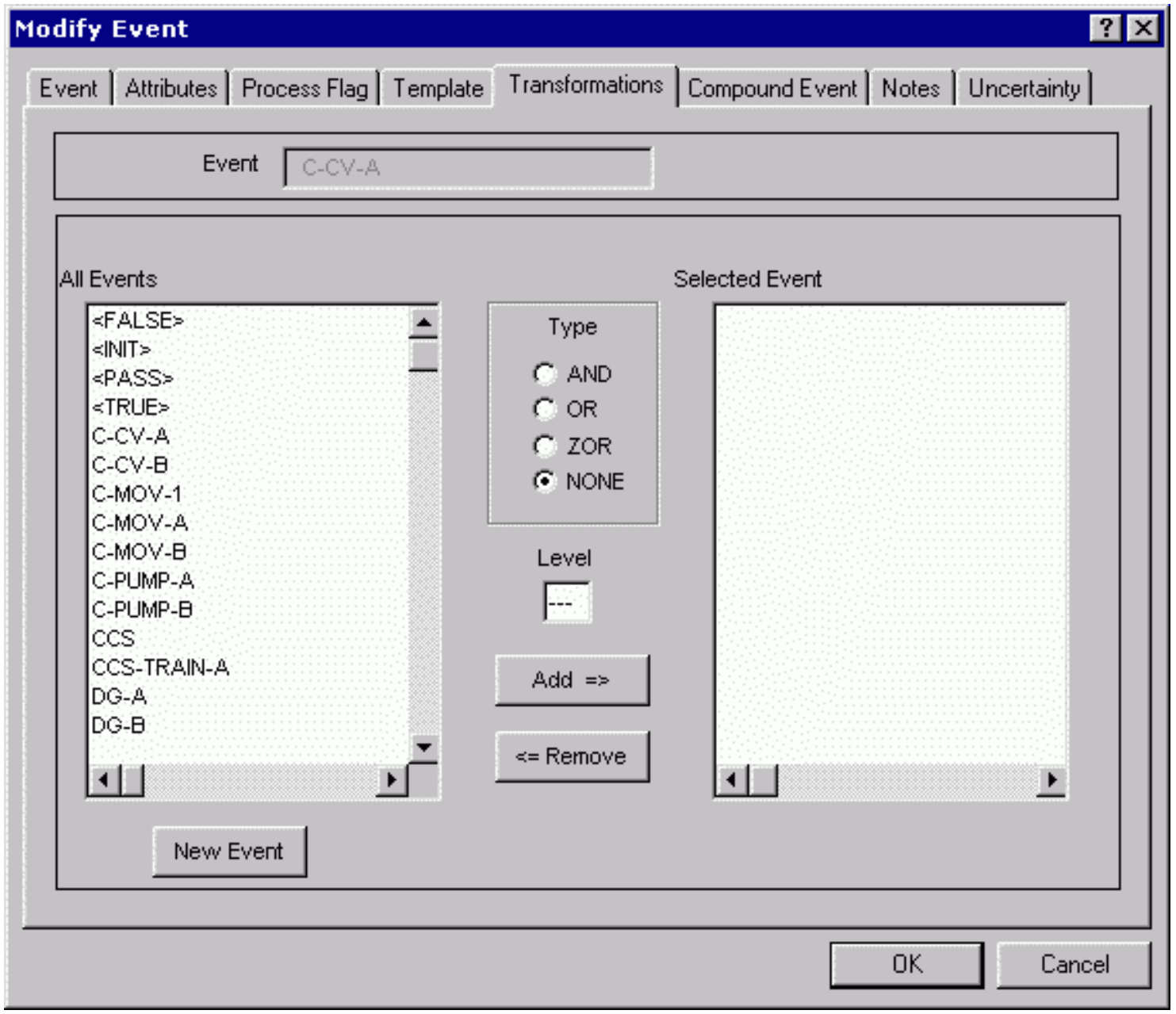

Add Add the highlighted event in the All Events list to the Selected Events list.

Remove - $\quad$ Remove the highlighted event from the Selected Events list.

Ok Save the new or modified basic event and close the dialog.

Cancel - $\quad$ Close the dialog without saving.

\subsection{Transformation - Type}

This field indicates the required behavior of the collective events. Enter one of the following:

$\mathrm{AND}=$ All included events must fail. Event is replaced with an AND gate, with all marked events as inputs.

$\mathrm{OR}=\quad$ Any included events must fail. Event is replaced with an OR gate with all marked events as inputs.

$Z O R=$ Events make up the zone. If any events in the list fail, all events fail.

\subsection{Transformation - Level}

Enter an integer between 0 and 255 indicating the level of substitution for the transformation. 


\subsection{Transformation Example}

Once transformations are defined, SAPHIRE has the capability to automatically perform event transformations during fault tree analysis. This is accomplished by allowing you to specify a transformation for each basic event in the database. This transformation can be one of three types, AND, OR, or ZOR and can define an optional level number. You may also specify a list a basic events that make up the transformation. When a fault tree is solved, you can specify that the logic for the fault tree be transformed by replacing each event that has a transformation defined for it by a gate with the events in the transformation as inputs. A simple example fault tree is as follows:

\begin{tabular}{|l|l|l|}
\hline TOP & AND & GATE1, GATE2 \\
\hline GATE1 & OR & EVENT1, EVENT2 \\
\hline GATE2 & AND & EVENT1, EVENT3 \\
\hline
\end{tabular}

You may then specify the following event transformations:

\begin{tabular}{|l|l|l|}
\hline EVENT1 & OR & LOC1, LOC2, LOC3, CABLE1 \\
\hline EVENT2 & OR & LOC2, LOC4, CABLE2 \\
\hline CABLE1 & OR & LOC4, LOC5 \\
\hline CABLE2 & OR & LOC5, LOC6, LOC7 \\
\hline
\end{tabular}

The CABLE events may represent the locations a cable passes through. The OR transformation type indicates that the specified event is to be replaced by an OR gate with the locations as inputs. You can then choose to expand the transformations resulting in the following logic:

\begin{tabular}{|l|l|l|}
\hline TOP & AND & GATE1, GATE2 \\
\hline GATE1 & OR & TRAN1, TRAN2 \\
\hline GATE2 & AND & TRAN1, TRAN3 \\
\hline TRAN1 & OR & LOC1, LOC2, LOC3, CABLE1, EVENT1 \\
\hline TRAN2 & OR & LOC2, LOC4, CABLE2, EVENT2 \\
\hline CABLE1 & OR & LOC4, LOC5 \\
\hline CABLE2 & OR & LOC5, LOC6, LOC7 \\
\hline
\end{tabular}

This logic is then reduced by combining like gate types to the following:

\begin{tabular}{|l|l|l|}
\hline TOP & AND & GATE1, GATE2 \\
\hline GATE1 & OR & TRAN1, TRAN2 \\
\hline GATE2 & AND & TRAN1, TRAN3 \\
\hline TRAN1 & OR & LOC1, LOC2, LOC3, LOC4, LOC5, EVENT1 \\
\hline TRAN2 & OR & LOC2, LOC4, LOC5, LOC6, LOC7, EVENT2 \\
\hline
\end{tabular}

When this fault tree is solved, the result is a list of cut sets in terms of independent failure events and locations. You can also choose to do a zone transformation by defining the locations that map to a particular zone. A zone or ZOR transformation as it is defined in the following example, effectively takes groups of locations and maps them into a single zone. Assuming you specified the following zone transformations: 


\begin{tabular}{|l|l|l|}
\hline ZONE1 & ZOR & LOC1, LOC2 \\
\hline ZONE2 & ZOR & LOC3, LOC4, LOC5 \\
\hline ZONE3 & ZOR & LOC6, LOC7 \\
\hline
\end{tabular}

Then the previous fault tree would be transformed into the following logic.

\begin{tabular}{|l|l|l|}
\hline TOP & AND & GATE1, GATE2 \\
\hline GATE1 & OR & TRAN1, TRAN2 \\
\hline GATE2 & AND & TRAN1, TRAN3 \\
\hline TRAN1 & OR & ZONE1, ZONE2, EVENT1 \\
\hline TRAN2 & OR & ZONE1, ZONE2, ZONE3, EVENT2 \\
\hline
\end{tabular}

This logic is obtained by replacing any occurrence of an input to a ZOR transformation by the name representing the transformation. Thus, you can map logic in terms of locations to logic in terms of zones, where a zone represents a collection of locations.

The susceptibility flags for each event are used to control which transformation are to be applied for a particular analysis type. For instance, if you want an event transformation to apply to a Fire analysis, then you must specify that the particular event is susceptible to Fire by checking fire susceptibility attribute.

\subsubsection{Basic Event Data - Notes}

\section{PURPOSE}

This option allows you to view and edit the descriptive text associated with a specific record. The Primary text option is generally used for text in the desired primary language. The Alternate text option is generally used for information entered in the secondary language using a different alphabet (locale). 


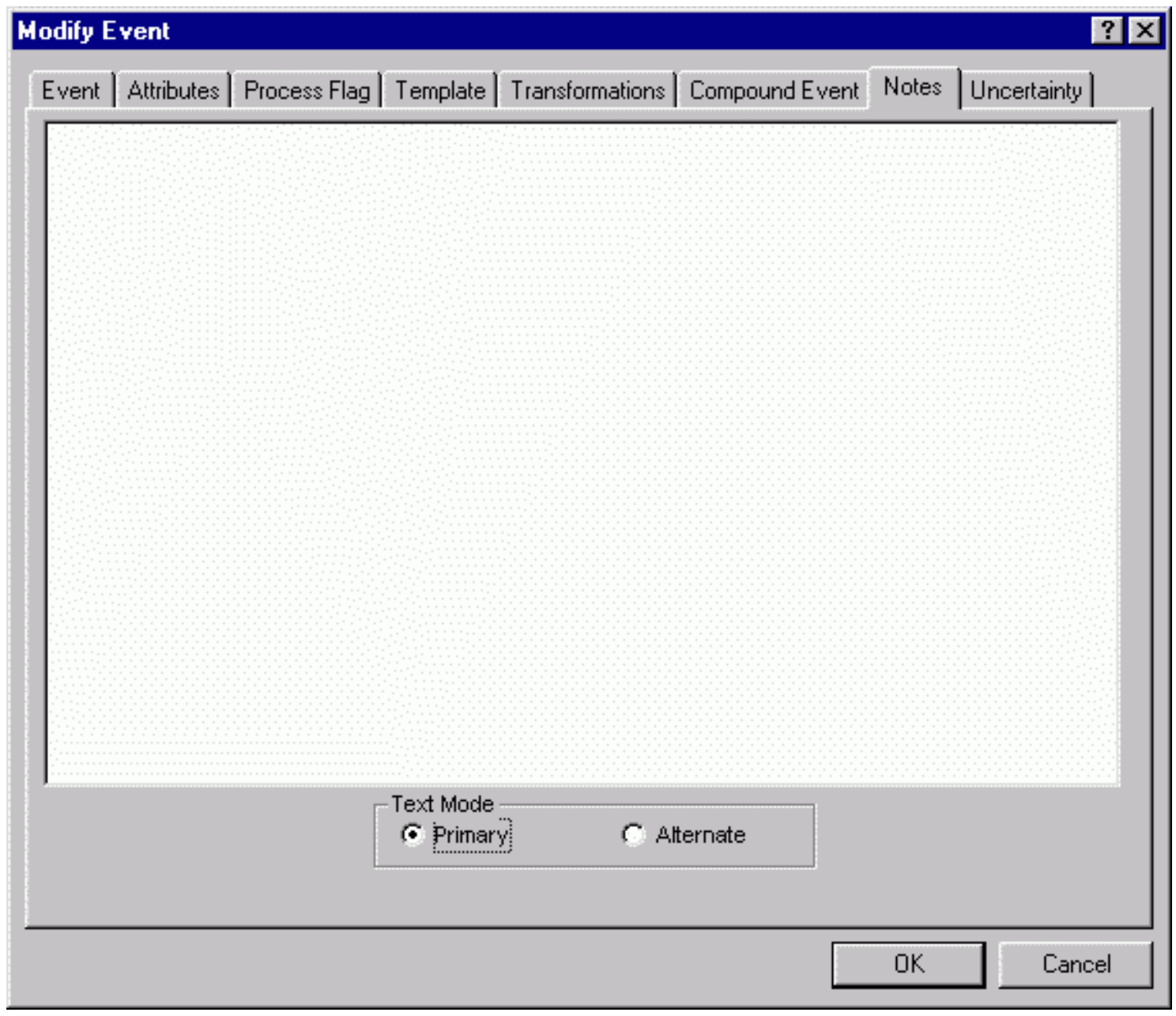

Ok - Save the new or modified basic event and close the dialog.

Cancel - Close the dialog without saving.

\subsubsection{Compound Event Page}

\subsection{Basic Event Data-Compound Event}

\section{PURPOSE}

A compound event's probability is defined by some combination of inputs, formulas, and other basic events.

\section{STEPS}

1. From the SAPHIRE menu select Modify | Basic Events. The Edit Events dialog is displayed. 
2. Highlight the event you wish to edit or copy. (Skip this step if adding a new basic event record.)

3. Right-click to invoke the pop-up menu and choose Add, Modify, or Copy. The Modify Event dialog will be displayed.

4. Choose the Attributes tab.

5. Select the Compound Event radio button in the Special Use Flags group. The Compound Event tab will appear.

6. Choose the Compound Event tab.

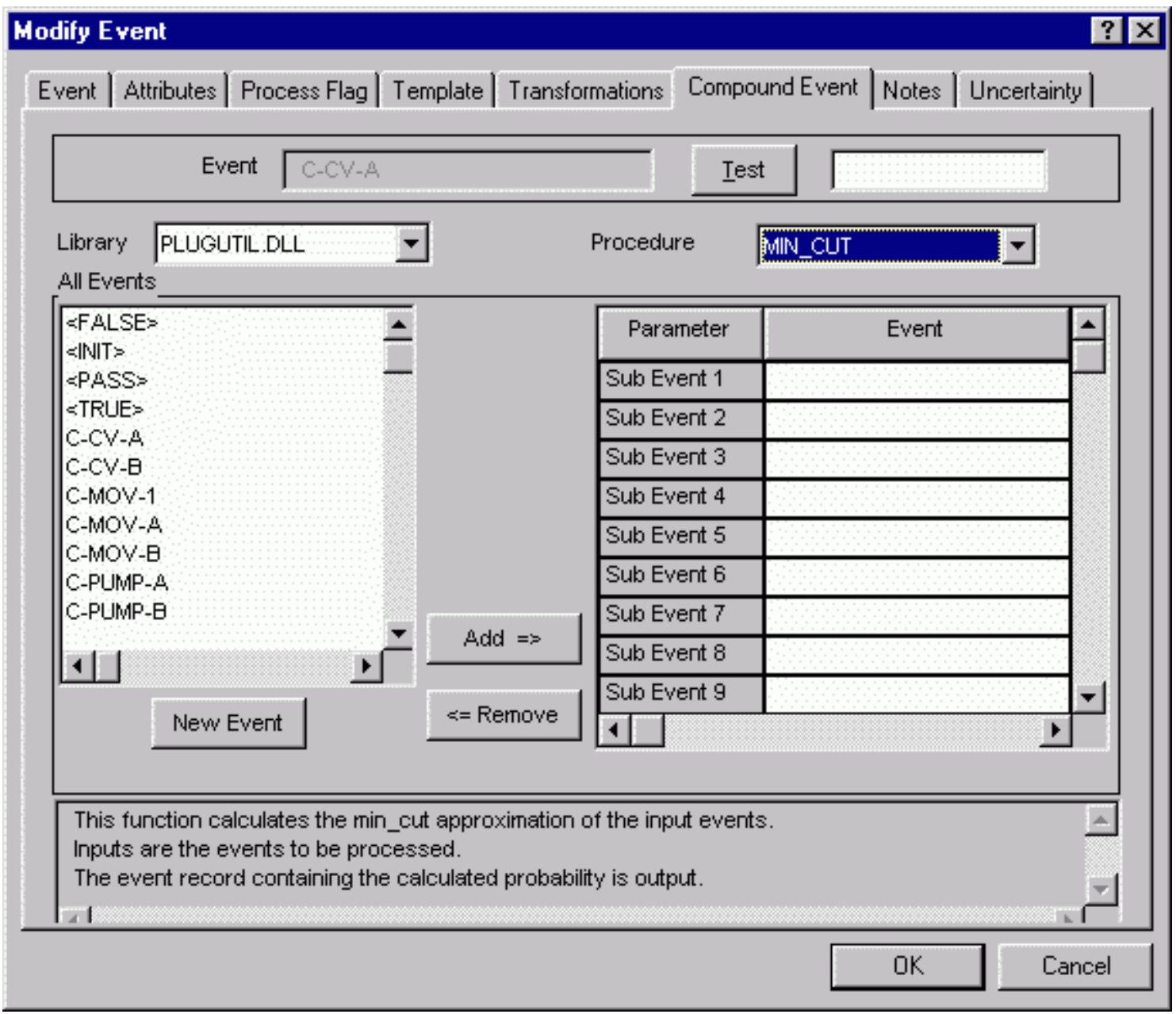

To calculate the compound event's probability, you must first select a SAPHIRE Dynamic Link Library (DLL) file from the Library drop-down list. Once the DLL is selected, choose a procedure from the Procedure drop-down list. After the desired procedure is selected, a list of appropriate parameters will be displayed in the Parameter column of Selected Event list. Choose an event from the All Events list for each parameter in the Selected Event list.

The All Events list on the left of the dialog displays all basic events defined for the current project. The Selected Event list on the right displays the events that have been selected as parameters for the selected DLL library procedure. 
Test - Calculate the compound event's probability based on the selected procedure and parameters. The basic event's probability values are applied at the end of the generate change set process.

Add - $\quad$ The highlighted event in the All Events list will be associated with the highlighted parameter in the Selected Event list.

Remove-The highlighted event in the Selected Event list will be removed from the list.

Ok - $\quad$ Save the new or modified basic event and close the dialog.

Cancel - Close the dialog without saving.

\subsection{Library}

The SAPHIRE Dynamic Link Libraries (DLLs) are provided during installation of SAPHIRE code. They contain one or more procedures used for calculating a compound event's probability.

\subsection{Procedure}

Contains the formula and defines the parameters used for calculating a compound event's probability. Found in a selected SAPHIRE DLL.

\subsection{Parameters}

The input values to a procedure used for calculating a compound event's probability. Select the parameter in the Selected Event list, then the desired event from the All Events list. The selected event's base probability value is used as the input parameter value.

\subsubsection{Uncertainty Page}

\subsection{Basic Event Data - Uncertainty}

\section{PURPOSE}

This property sheet page shows a graphical representation of the uncertainty distribution defined by the failure and uncertainty values. 


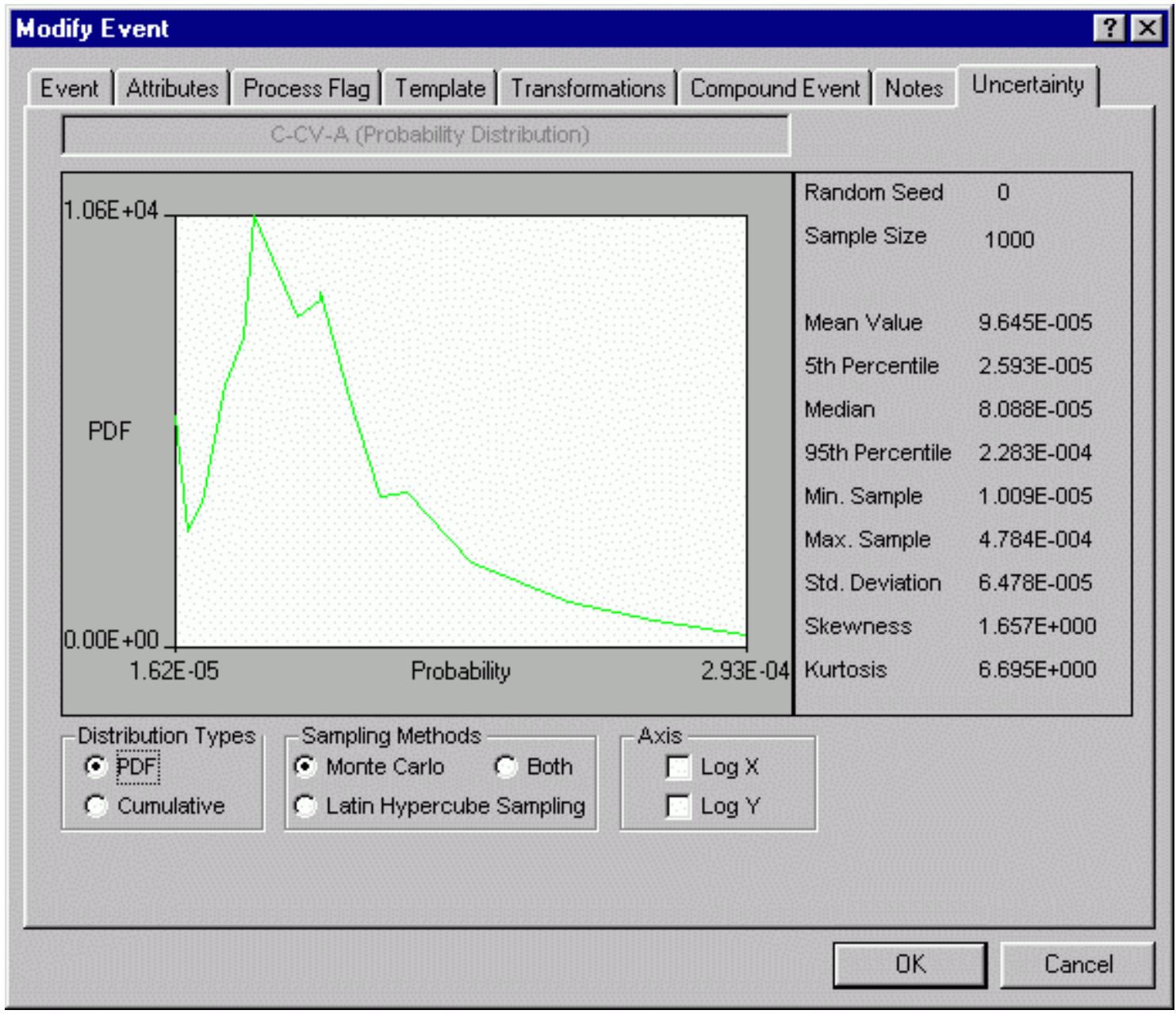

Ok - Save the new or modified basic event and close the dialog.

Cancel - Close the dialog without saving.

\subsection{Distribution Types}

Select the type of graph to be displayed:

PDF - $\quad$ (Default) - Displays a graph showing the Probability Density Function.

Cumulative - Displays a graph showing the Cumulative Density Function. 
Select the desired sampling method for uncertainty calculation:

Monte Carlo -

Latin Hypercube Sampling -

Both -
(Default) - Displays a graph that is based on Monte Carlo Sampling of the defined uncertainty distribution.

Displays a graph that is based on Latin Hypercube Sampling of the defined uncertainty distribution.

Displays a graph that will have two lines. One line is based on Monte Carlo Sampling of the defined uncertainty distribution and the other line is based on Latin Hypercube Sampling of the defined uncertainty distribution.

9.6.6.8.4 Axis

$\log \mathbf{X}$ - Produces a graph where the Log base 10 of the X-Values are used instead of just the XValues.

$\log$ Y - Produces a graph where the Log base 10 of the Y-Values are used instead of just the YValues.

\subsection{Modifying Attribute Data}

\subsubsection{Attributes}

\section{PURPOSE}

This option allows you to edit records of the five attribute types in the current project: Systems, Locations, Failure Modes, Basic Event Types, and Trains. The dialogs and operations are consistent for each of the five attribute categories, therefore, a generic discussion is provided. The Locations category has been used in the example graphics.

\section{STEPS}

1. From the SAPHIRE menu select Modify.

2. Choose Attributes from the menu.

3. Select the desired sub-menu option. The Edit Attributes dialog will be displayed listing all of the records of the selected attribute type. The selected attribute category is displayed in the Type field. 


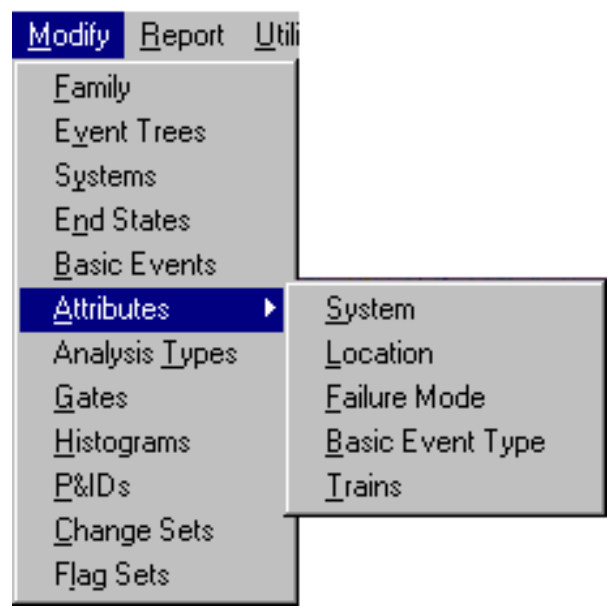

4. Highlight the desired list item.

5. Right-click to invoke the pop-up menu or choose the Exit button.

The example here shows the Location attribute records.

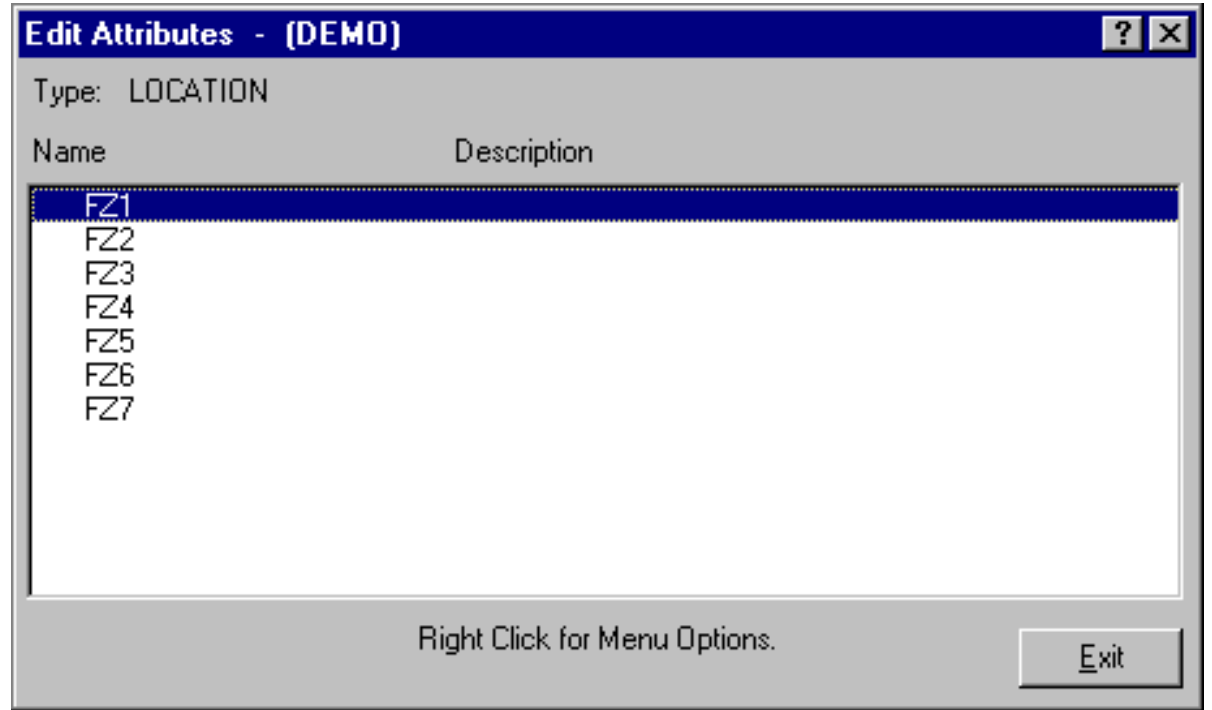

\section{Pop-up Menu Options}

Add - $\quad$ Add a new attribute record to the current project database. When complete, the message, "Record added," is displayed in the message bar of the SAPHIRE window.

Copy - Create a new attribute record by copying an existing one.

Modify - Edit the selected attribute record. When complete, the message, "Record modified," is displayed in the message bar of the SAPHIRE window.

Delete - Delete the selected attribute record(s). 


\section{Button Option}

Exit -

Close the Edit Attributes dialog.

\subsubsection{Delete Record}

\section{PURPOSE}

This option allows you to delete the highlighted record from the database.

\section{STEPS}

1. From the SAPHIRE menu select Modify.

2. Choose the desired option from the menu.

3. Highlight the record you wish to delete.

4. Right-click to invoke the pop-up menu and choose Delete. A warning dialog will be displayed, allowing you to cancel the deletion at this point.

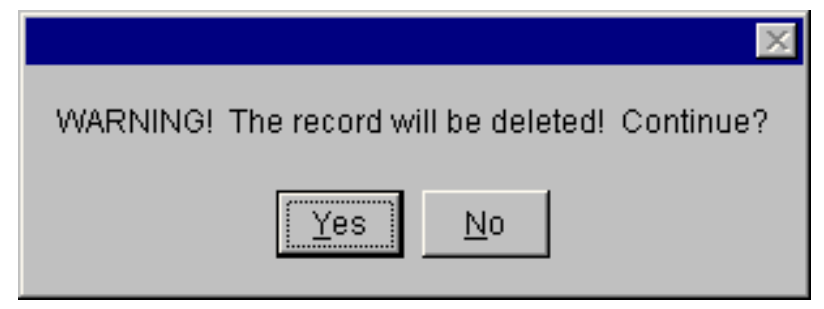

Yes - Continue the delete operation. The selected record will be deleted from the database. The message, "Record deleted," is displayed in the message bar of the SAPHIRE window.

No - Cancel the delete operation. The selected record will NOT be deleted from the database.

\subsubsection{Attribute Data Entry Dialog}

\subsubsection{Attribute Data Entry}

\section{PURPOSE}

The Add/Modify Attribute dialog allows you to enter or modify attribute data. The selected attribute category is displayed in the Type field.

\section{STEPS}

1. From the SAPHIRE menu select Modify.

2. Choose Attributes from the menu.

3. Select the desired sub-menu option. The Modify Attributes dialog will be displayed. The selected attribute category is displayed in the Type field. 


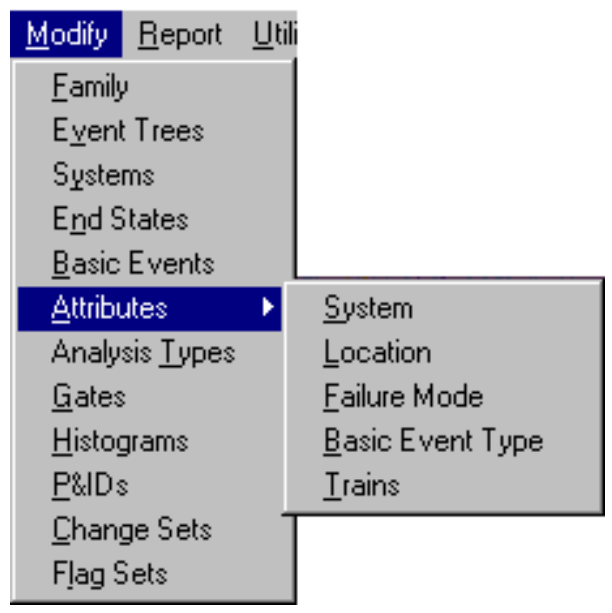

4. Highlight the list item you wish to edit or copy. (Skip this step if adding a new record.)

5. Right-click to invoke the pop-up menu and choose Add, Copy, or Modify.

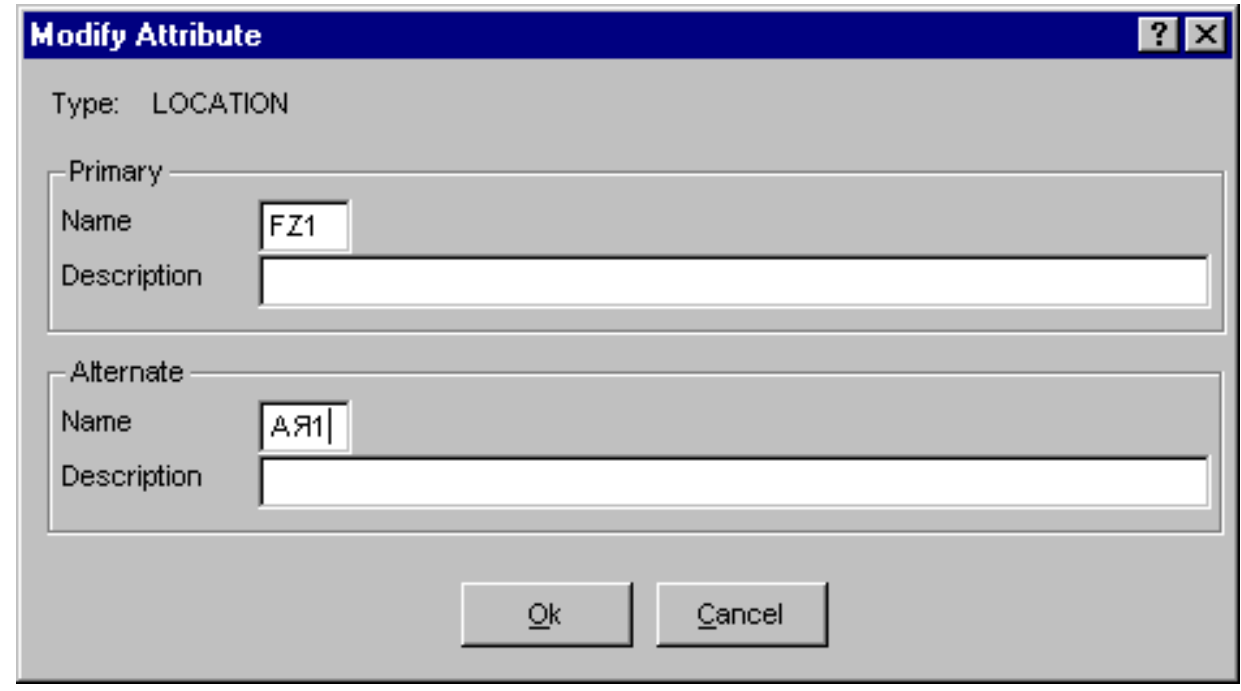

Ok - $\quad$ Save the new or modified attribute and close the dialog.

Cancel - Close the dialog without saving.

\subsubsection{Attribute Name}

This is a required field. A maximum of 5 uppercase, alphanumeric characters may be entered (embedded blanks are not allowed). The name should be descriptive so it can be readily identified.

\subsubsection{Description}

This is a 120-character, uppercase or lowercase, alphanumeric field that provides brief, descriptive information. 


\subsection{Modifying Analysis Type Data}

\subsubsection{Analysis Types}

\section{PURPOSE}

This option allows you to modify analysis types. SAPHIRE offers eight predefined analysis types and eight user-defined analysis types. You cannot edit the name of the eight predefined analysis types, but you can edit the description. You can modify both the name and description of the eight user-defined analysis types.

\section{STEPS}

1. From the SAPHIRE menu select Modify.

2. Choose Analysis Types from the menu. The Edit Analysis Types dialog is displayed, listing the 16 analysis types belonging to the current project.

3. Highlight the desired analysis type.

4. Choose the Modify button.

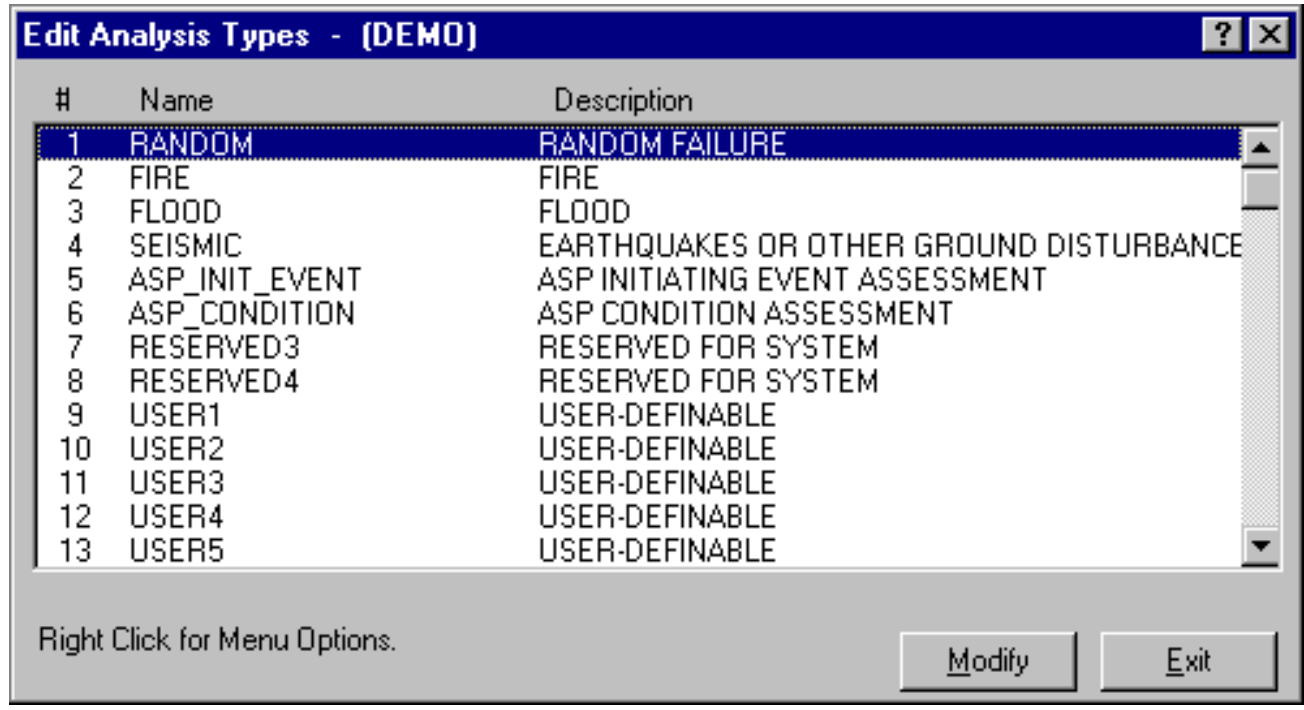

Modify - Edit the selected analysis type record. When complete, the message, "Record modified," is displayed in the message bar of the SAPHIRE window.

Exit - $\quad$ Close the Edit Analysis Types dialog.

\subsubsection{Analysis Type Data Entry Dialog}

\subsubsection{Analysis Type Data Entry}

\section{PURPOSE}

The Modify Analysis Type dialog allows you to edit analysis type data. 


\section{STEPS}

1. From the SAPHIRE menu select Modify.

2. Choose Analysis Types from the menu. The Edit Analysis Types dialog is displayed, listing the 16 analysis types belonging to the current project.

3. Highlight the desired analysis type.

4. Choose the Modify button.

You can edit only the description of the eight predefined analysis types. As shown here, the Name field is not accessible for modification:

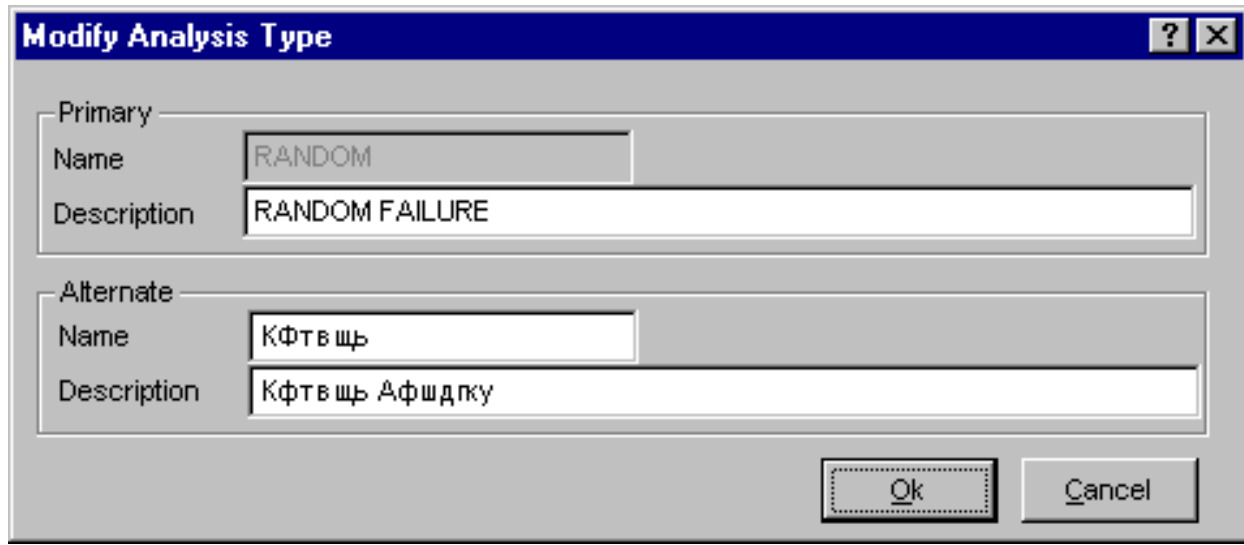

You can modify both the name and descriptions of the eight user-defined analysis types. As shown below, both fields are accessible for modification:

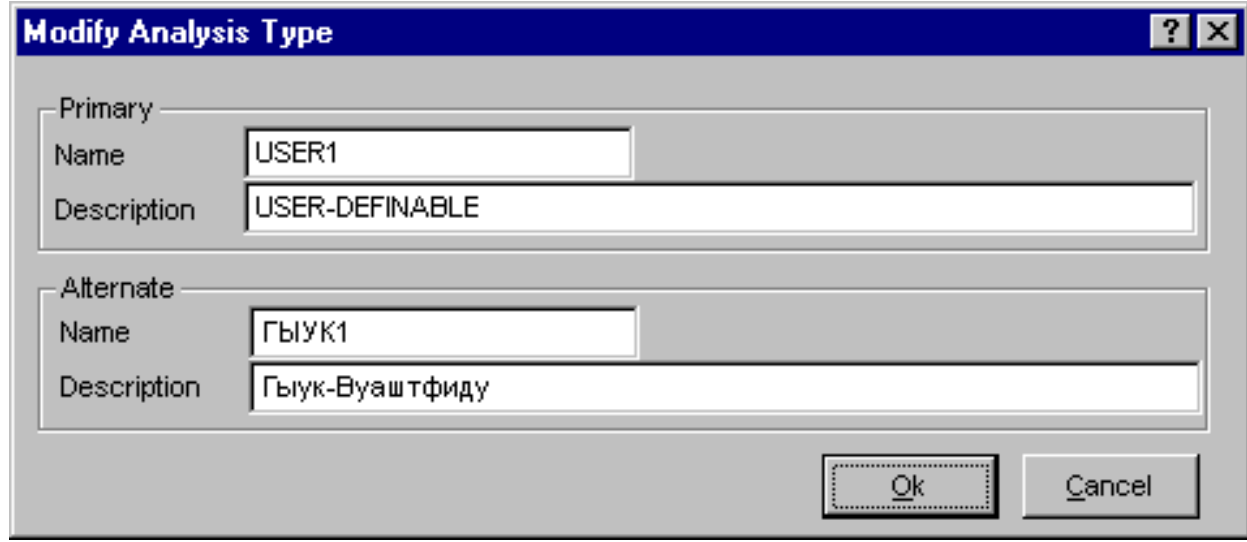

Ok - $\quad$ Save the modified analysis type and close the dialog.

Cancel - Close the dialog without saving.

\subsubsection{Name}

This is a required field. A maximum of 24 uppercase, alphanumeric characters may be entered. Embedded blanks are not allowed. The name should be descriptive so it can be readily identified. 


\subsubsection{Description}

This is a 120-character, uppercase or lowercase, alphanumeric field that provides brief, descriptive information.

\subsection{Modifying Gate Data}

\subsubsection{Gates}

\section{PURPOSE}

This option allows you to add, modify, or delete the gate data records.

\section{STEPS}

1. From the SAPHIRE menu select Modify.

2. Choose Gates from the menu. The Edit Gates dialog is displayed, listing all of the gates belonging to the current project.

3. Highlight the desired gate.

4. Right-click to invoke the pop-up menu or choose the desired button.

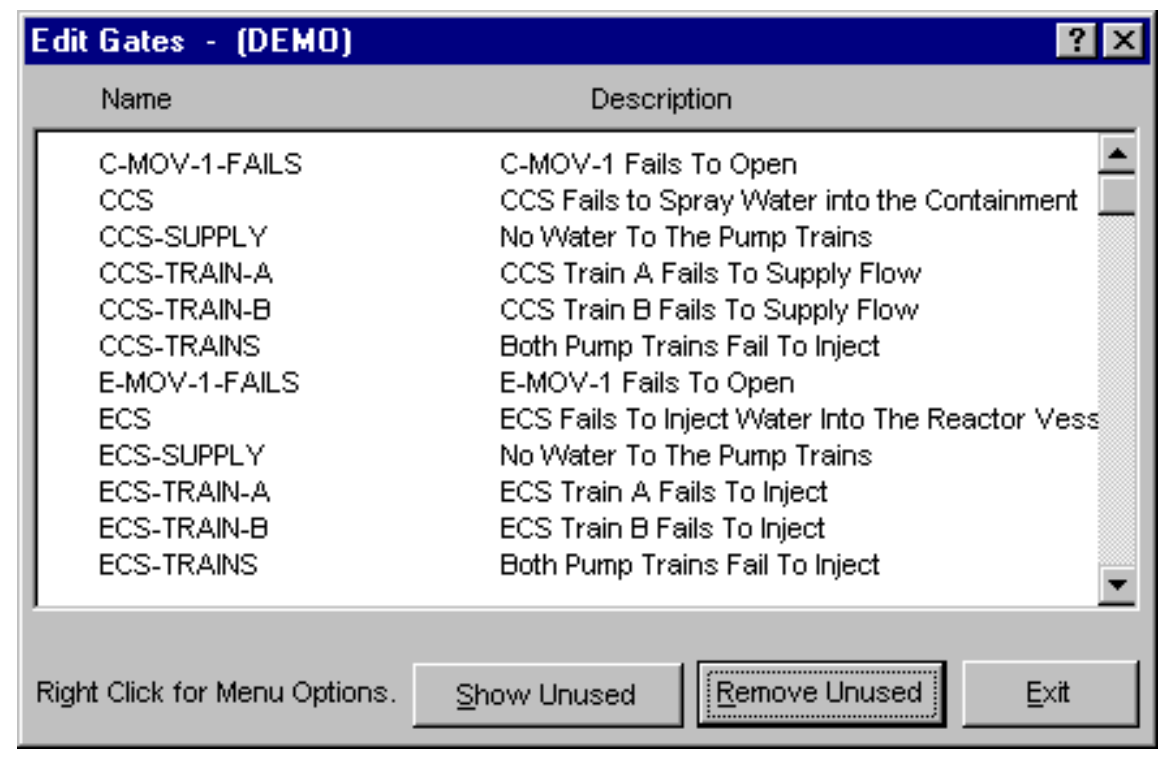

\section{Pop-up Menu Options}

Add - $\quad$ Add a new gate record to the current project database. When complete, the message, "Record added," is displayed in the message bar of the SAPHIRE window.

Copy - Create a new gate record by copying an existing one.

Modify - Edit the selected gate record. When complete, the message, "Record modified," is displayed in the message bar of the SAPHIRE window.

Delete - Delete the selected gate record(s). 


\section{Button Options}

Show Unused -

Show all unused gates. The unused gates are indicated by a dash $(-)$ to the left of the gate name.

Remove Unused Gates - Immediately delete all unused gate records.

Exit -

Close the Edit Gates dialog.

\subsubsection{Delete Record}

\section{PURPOSE}

This option allows you to delete the highlighted record from the database.

\section{STEPS}

1. From the SAPHIRE menu select Modify.

2. Choose the desired option from the menu.

3. Highlight the record you wish to delete.

4. Right-click to invoke the pop-up menu and choose Delete. A warning dialog will be displayed, allowing you to cancel the deletion at this point.

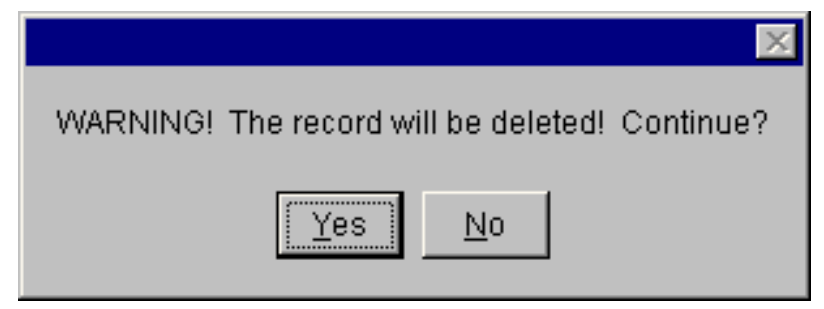

Yes - Continue the delete operation. The selected record will be deleted from the database. The message, "Record deleted," is displayed in the message bar of the SAPHIRE window.

No - Cancel the delete operation. The selected record will NOT be deleted from the database.

\subsubsection{Gate Data Entry Dialog}

\subsubsection{Gate Data Entry}

\section{PURPOSE}

The Add/Modify Gate dialog allows you to enter or modify gate data.

\section{STEPS}

1. From the SAPHIRE menu select Modify.

2. Choose Gates from the menu. The Edit Gates dialog is displayed.

3. Highlight the gate you wish to edit or copy. (Skip this step if adding a new gate.)

4. Right-click to invoke the pop-up menu and choose Add, Copy, or Modify. 


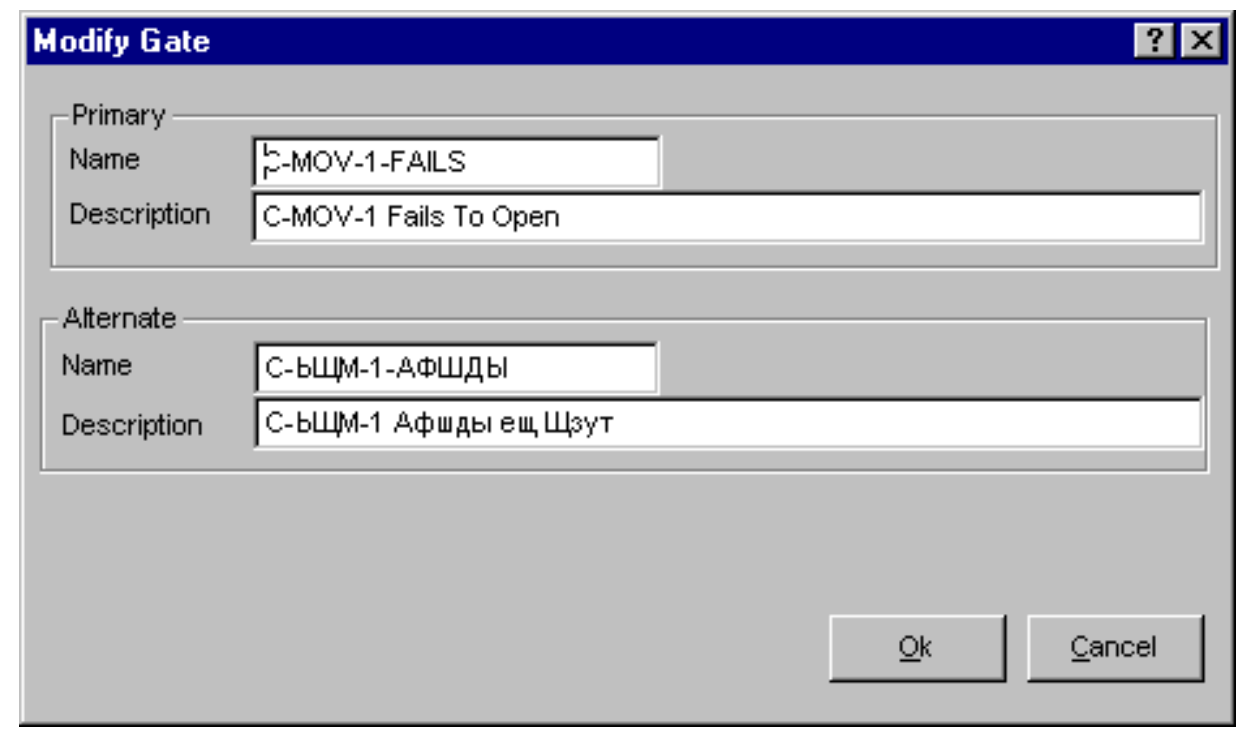

Ok - $\quad$ Save the new or modified gate and close the dialog.

Cancel - Close the dialog without saving.

\subsubsection{Name}

This is a required field. A maximum of 24 uppercase, alphanumeric characters may be entered. Embedded blanks are not allowed. The name should be descriptive so it can be readily identified.

\subsubsection{Description}

This is a 120-character, uppercase or lowercase, alphanumeric field that provides brief, descriptive information.

\subsubsection{Type}

Select the gate type from the drop-down list or enter your own of the type N/M.

AND - The output occurs if all inputs occur.

OR - The output occurs if any of the inputs occur.

TRAN - Transfer gate. This is a link to other logic structures.

NAND - Negation of an AND gate. The output occurs if any one of the inputs does not occur.

NOR - Negation of an OR gate. The output occurs if none of the inputs occur.

$\mathbf{N}$ of $\mathbf{M}$ - N/M gate. $\mathrm{N}$ of $\mathrm{M}$ input events must occur. Enter the $\mathrm{N}$ range from 2 to 9 and $\mathrm{M}$ range $\mathrm{N}<\mathrm{M}$. 


\subsection{Modifying Histogram Data}

\subsubsection{Histograms}

\section{PURPOSE}

This option allows you to add, modify, or delete user-defined histogram records. This is a useful option for allowing you to input your own distribution for a variable that cannot be expressed with one of the predefined uncertainty distribution types.

\section{STEPS}

1. From the SAPHIRE menu select Modify.

2. Choose Histograms from the menu. The Edit Histograms dialog is displayed, listing all of the currently existing histograms with their associated format type.

3. Highlight the desired histogram.

4. Right-click to invoke the pop-up menu or choose the Exit button.

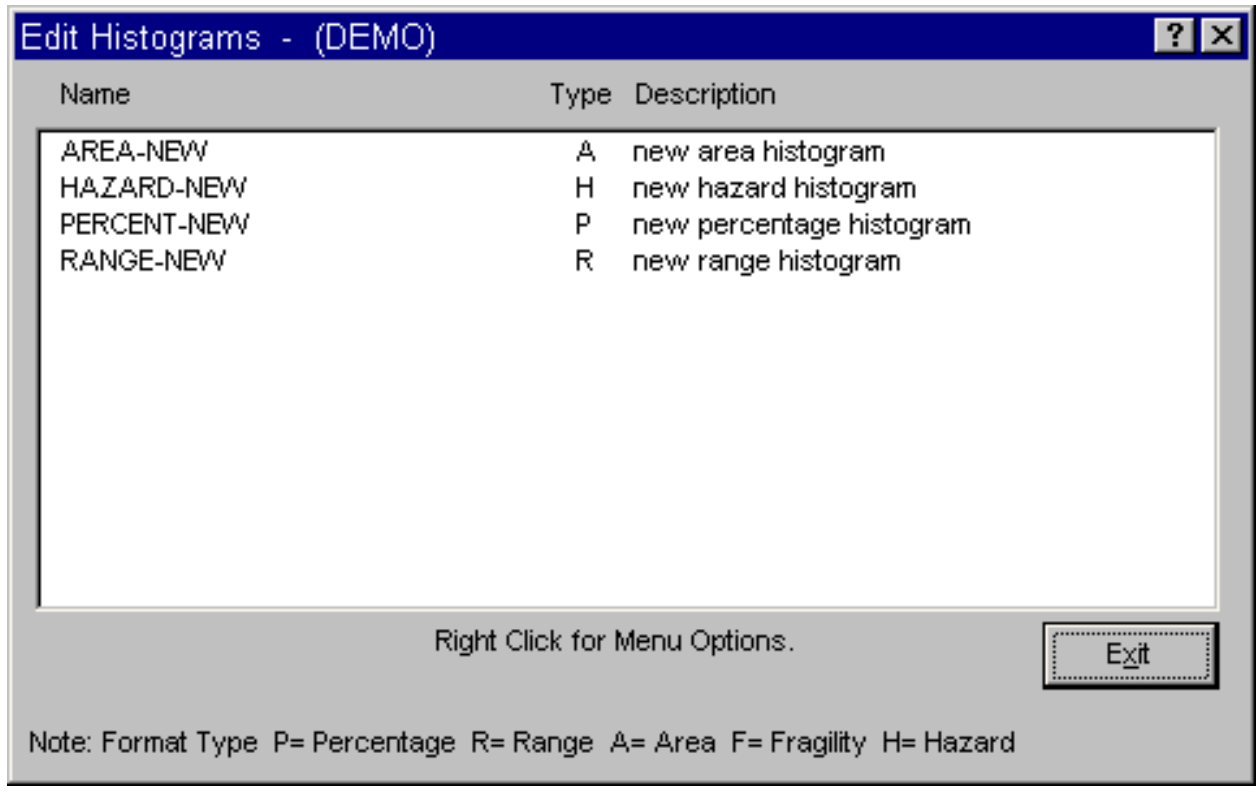




\section{Pop-up Menu Options}

Add - Add a new histogram record to the current project database. When complete, the message, "Record added," is displayed in the message bar of the SAPHIRE window.

Copy - Create a new histogram record by copying an existing one. The data entry dialog is dependent on the histogram's format.

Modify - Edit the selected histogram record. The data entry dialog is dependent on the histogram's format. When complete, the message, "Record modified," is displayed in the message bar of the SAPHIRE window.

Delete - Delete the selected histogram record(s).

\section{Button Option}

Exit -

Close the Edit Histograms dialog.

\subsubsection{Delete Record}

\section{PURPOSE}

This option allows you to delete the highlighted record from the database.

\section{STEPS}

1. From the SAPHIRE menu select Modify.

2. Choose the desired option from the menu.

3. Highlight the record you wish to delete.

4. Right-click to invoke the pop-up menu and choose Delete. A warning dialog will be displayed, allowing you to cancel the deletion at this point.

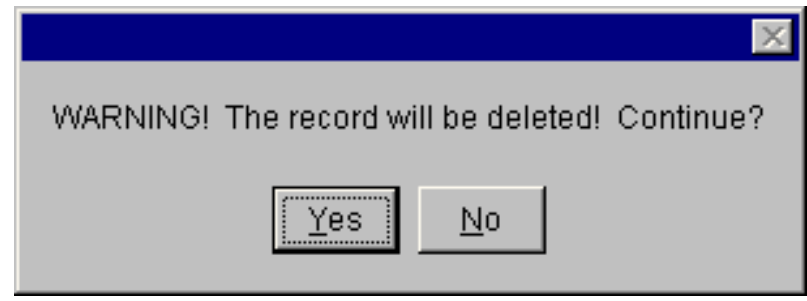

Yes - Continue the delete operation. The selected record will be deleted from the database. The message, "Record deleted," is displayed in the message bar of the SAPHIRE window.

No - Cancel the delete operation. The selected record will NOT be deleted from the database.

\subsubsection{Add Histograms}

\section{PURPOSE}

This option allows you to create a user-defined distribution type. 


\section{STEPS}

1. From the SAPHIRE menu select Modify.

2. Choose Histograms from the menu. The Edit Histograms dialog is.

3. Right-click to invoke the pop-up menu and choose the Add option. The Format dialog is displayed.

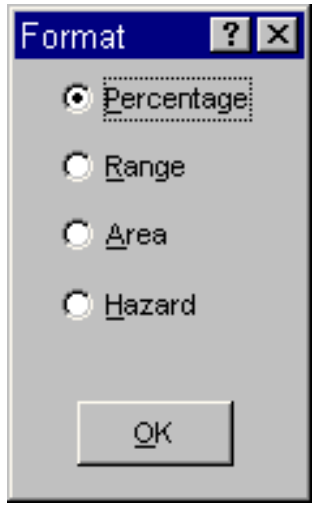

From the Format dialog, you are given the choice of adding the histogram data in either a percentage, range, area, or hazard format.

\subsubsection{Percentage Format Dialog}

\subsubsection{Percentage Histogram}

\section{PURPOSE}

The Add/Modify Histogram dialog allows you to enter or modify percentage histogram data. On this dialog, type in a name and a description for the percentage histogram. Enter the percentages for the histogram along with the corresponding probabilities.

\section{STEPS}

1. From the SAPHIRE menu select Modify.

2. Choose Histograms from the menu. The Edit Histograms dialog is displayed.

3. Highlighted the histogram, designated by Type "P", that you wish to edit or copy. (Skip this step if adding a new histogram.)

4. Right-click to invoke the pop-up menu and choose Add, Copy, or Modify. The Percentage Format dialog is displayed. 


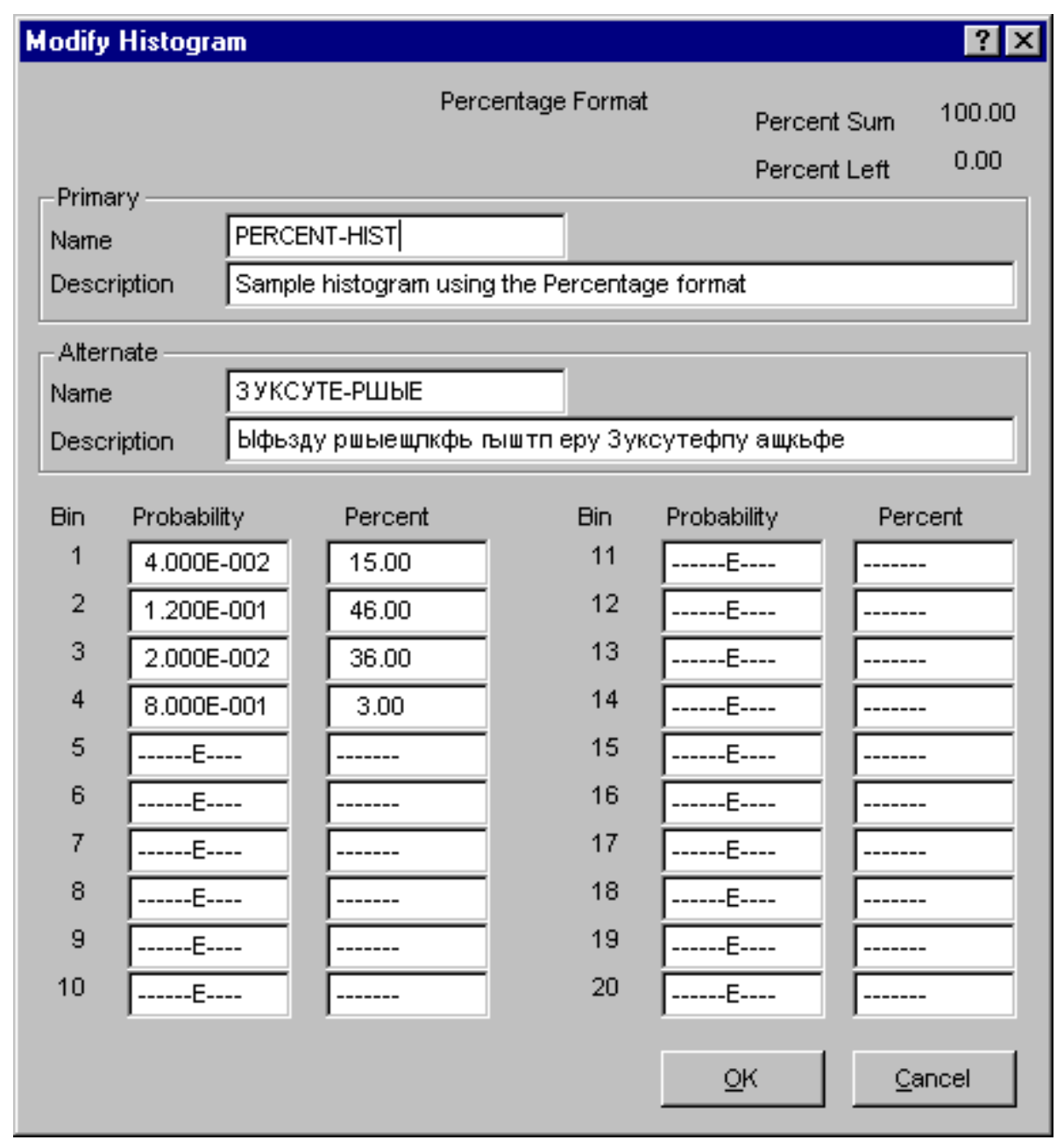

The example here demonstrates how to enter a percentage histogram, given that $15 \%$ of the data points have a probability of $0.04,46 \%$ of the data points have a probability of $0.12,36 \%$ of the data points have a probability of 0.02 , and the remaining $3 \%$ of the data points have a probability of 0.8 .

The sum of the percentages entered must total $100 \%$, in order for the histogram to be accepted as a valid percentage histogram. In the upper right-hand area of the dialog is a box that shows the current sum of the percentages that have been input and the remaining percentage needed to reach the $100 \%$ total.

NOTE: This format is a discrete distribution on the values entered. The percents give the degree of belief of each value.

Ok - Save the new or modified histogram and close the dialog.

Cancel - Close the dialog without saving the histogram.

\subsubsection{Name}

This is a required field. A maximum of 24 uppercase, alphanumeric characters may be entered. Embedded blanks are not allowed. The name should be descriptive so it can be readily identified.

\subsubsection{Description}


This is a 120-character, uppercase or lowercase, alphanumeric field that provides brief, descriptive information.

\subsubsection{Percent}

The percentage of data points that will have the failure probability value that is entered in this bin. The sum of the percentages in the bins must total $100 \%$.

\subsubsection{Probability}

The failure probability value.

\subsubsection{Range Format Dialog}

\subsubsection{Range Histogram}

\section{PURPOSE}

On this dialog, type in a name and description for the range histogram. Then, enter the starting probability point, the ending probability point, and the height associated with the first bin of the histogram. Next, for each successive bin of the histogram, an ending probability point and a height should be entered. There is a maximum of 20 bins allowed for each range histogram.

\section{STEPS}

1. From the SAPHIRE menu select Modify.

2. Choose Histograms from the menu. The Edit Histograms dialog is displayed.

3. Highlighted the histogram, designated by Type "R", that you wish to edit or copy. (Skip this step if adding a new histogram.)

4. Right-click to invoke the pop-up menu and choose Add, Copy, or Modify. The Range Format dialog is displayed. 


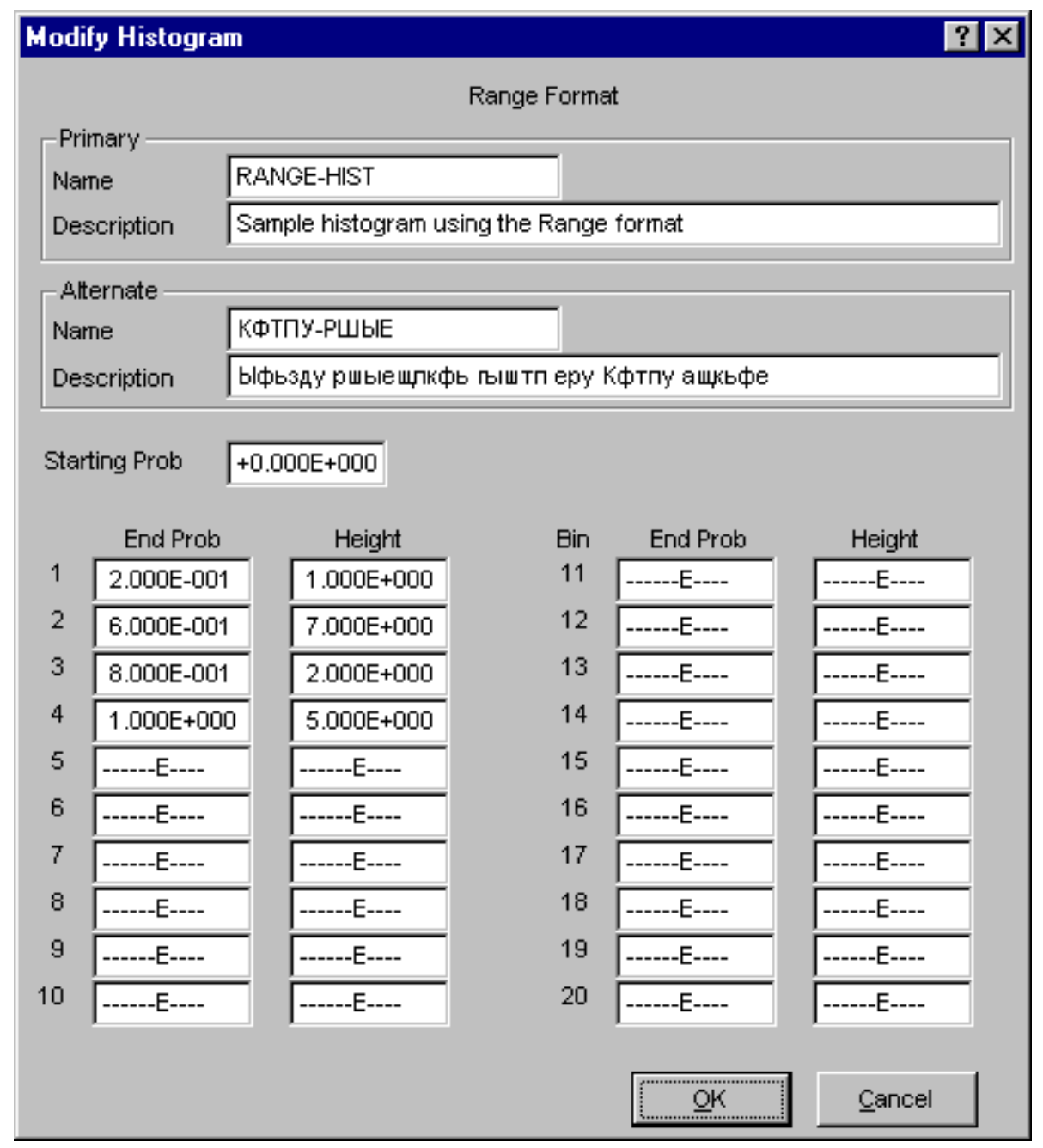

This is an example of inputting a range histogram whose data points lie on the closed interval of 0.0 and 1.0. The height associated with the data points on the subinterval of 0.0 to 0.2 is 10.0 (Bin 1), the height for the subinterval of 0.2 to 0.6 is 70.0 (Bin 2), the height for the subinterval of 0.6 to 0.8 is 20.0 (Bin 3 ), and the height for the last subinterval of 0.8 to 1.0 is 5.0 (Bin 4 ).

SAPHIRE calculates the midpoint of each bin, finds the area of each bin, and normalizes each area so the sum of the areas equals 1.0. The midpoint is the probability for each bin and the normalized area corresponds to the percent in the Percent Histogram format. The basic event mean probability should correspond to the mean of the histogram.

Ok - $\quad$ Save the new or modified histogram and close the dialog.

Cancel - Close the dialog without saving the histogram.

\subsubsection{Name}

This is a required field. A maximum of 24 uppercase, alphanumeric characters may be entered. Embedded blanks are not allowed. The name should be descriptive so it can be readily identified. 


\subsubsection{Description}

This is a 120-character, uppercase or lowercase, alphanumeric field that provides brief, descriptive information.

\subsubsection{Starting Probability}

The starting probability for the area or range. This value must $<=1.0$.

\subsubsection{End Probability}

The end probability value defining the subinterval between the starting probabilty (if this is Bin 1) or the previous bin's end probability.

\subsubsection{Height}

The height associated with the data points on the subinterval for this bin.

\subsubsection{Area Format Dialog}

\subsubsection{Area Histogram}

\section{PURPOSE}

On this dialog, type in a name and description for the area histogram. Then enter the starting probability point, the ending probability point, and the area associated with the first bin of the histogram. Next, for each successive bin of the histogram, an ending probability point and an area should be entered. There is a maximum of 20 bins allowed for each area histogram.

\section{STEPS}

1. From the SAPHIRE menu select Modify.

2. Choose Histograms from the menu. The Edit Histograms dialog is displayed.

3. Highlighted the histogram, designated by Type "A", that you wish to edit or copy. (Skip this step if adding a new histogram.)

4. Right-click to invoke the pop-up menu and choose Add, Copy, or Modify. The Area Format dialog is displayed. 


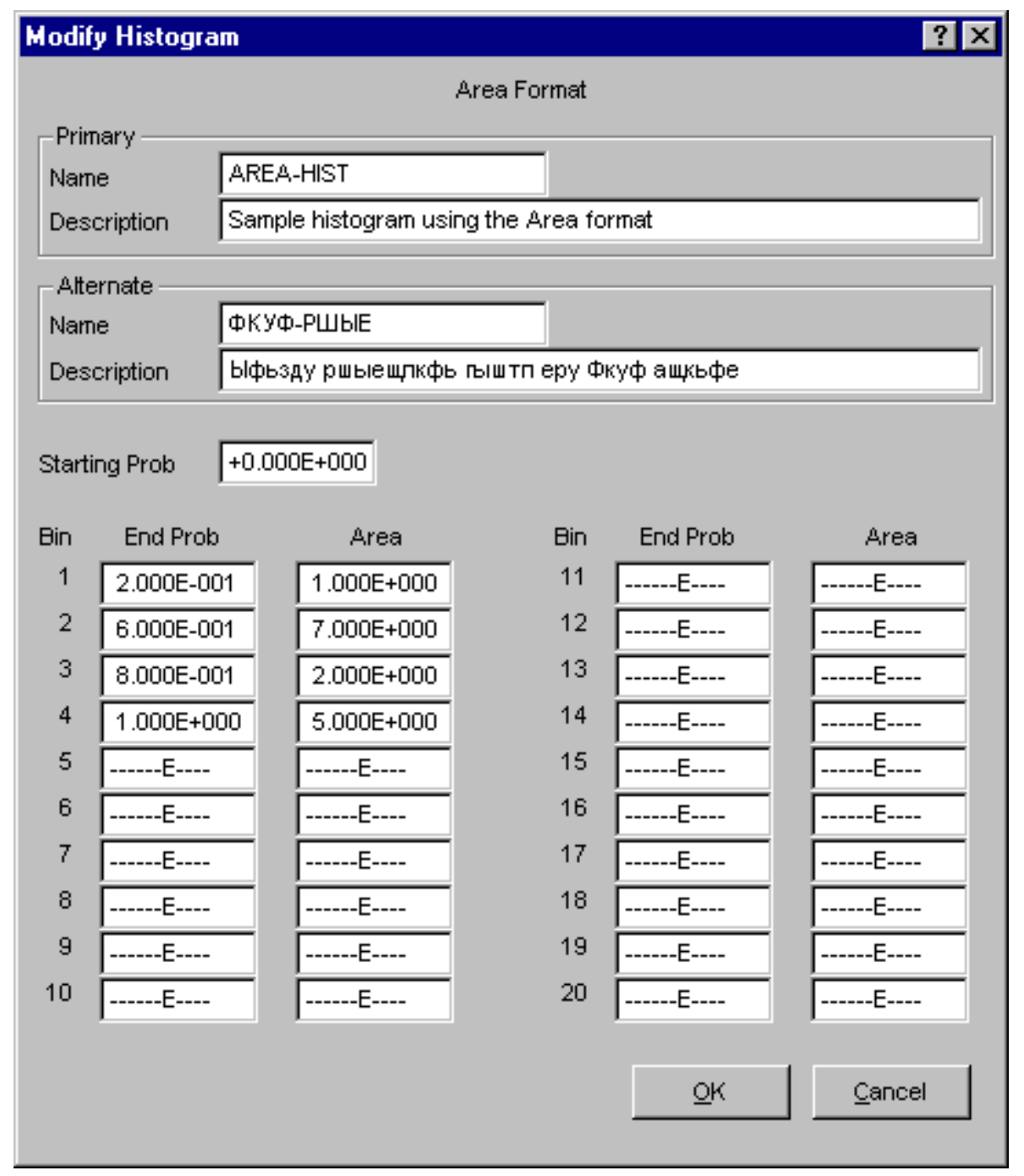

This is an example of inputting an area histogram whose data points lie on the closed interval of 0.0 and 1.0. The area associated with the data points on the subinterval of 0.0 to 0.2 is 10.0 (Bin 1), the area for the subinterval of 0.2 to 0.6 is 70.0 (Bin 2), the area for the subinterval of 0.6 to 0.8 is 20.0 (Bin 3), and the area for the last subinterval of 0.8 to 1.0 is 5.0 (Bin 4). SAPHIRE normalizes the area of each bin so the sum of the areas equals 1.0.

Ok - $\quad$ Save the new or modified histogram and close the dialog

Cancel - Close the dialog without saving the histogram.

\subsubsection{Name}

This is a required field. A maximum of 24 uppercase, alphanumeric characters may be entered. Embedded blanks are not allowed. The name should be descriptive so it can be readily identified.

\subsubsection{Description}

This is a 120-character, uppercase or lowercase, alphanumeric field that provides brief, descriptive information.

\subsubsection{Starting Probability}


The starting probability for the area or range. This value must $<=1.0$.

\subsubsection{End Probability}

The end probability value defining the subinterval between the starting probabilty (if this is Bin 1) or the previous bin's end probability.

\subsubsection{Area}

The area associated with the data points on the subinterval for this bin.

\subsubsection{Fragility Format Dialog}

\subsubsection{Name}

This is a required field. A maximum of 24 uppercase, alphanumeric characters may be entered. Embedded blanks are not allowed. The name should be descriptive so it can be readily identified.

\subsubsection{Description}

This is a 120-character, uppercase or lowercase, alphanumeric field that provides brief, descriptive information.

\subsubsection{Acceleration}

The ground acceleration level value for this bin.

\subsubsection{Frequency}

The frequency of occurrence (i.e., likelihood per year) for this bin.

\subsubsection{Hazard Format Dialog}

\subsubsection{Hazard Histogram}

\section{PURPOSE}

The Hazard histogram option is used to enter the site seismic hazard curve data for seismic analysis. This data consists of a table of values representing the G-level (ground acceleration level) and associated frequency of occurrence (i.e., likelihood per year). Note, the hazard histogram consists of discrete values.

\section{STEPS}

1. From the SAPHIRE menu select Modify.

2. Choose Histograms from the menu. The Edit Histograms dialog is displayed. 
3. Highlighted the histogram, designated by Type "H", that you wish to edit or copy. (Skip this step if adding a new histogram.)

4. Right-click to invoke the pop-up menu and choose Add, Copy, or Modify. The Hazard Format dialog is displayed.

On this dialog, type in a name and description for the hazard histogram. Then enter the acceleration Glevel and the frequency associated with the first bin of the histogram. Next, for each successive bin of the histogram, a G-level and a frequency value can be entered. There is a maximum of 20 bins allowed for each hazard histogram.

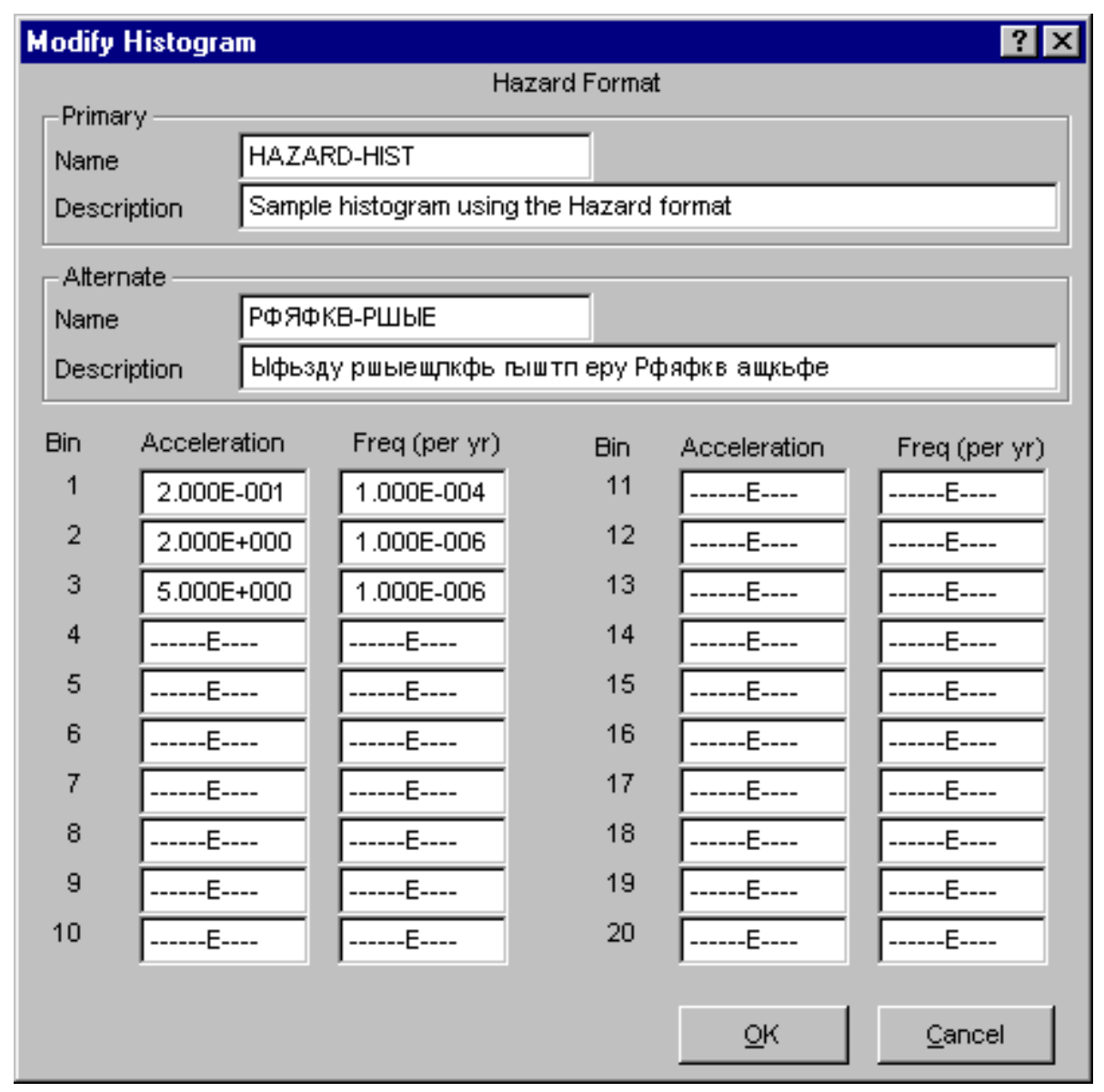

This is an example of entering a hazard histogram. The frequency associated with each G-level is G-level 0.2 , frequency 1.0E-4, G-level 2.0, frequency 1.0E-6, G-level 5.0, frequency 1.0E-6.

Ok - $\quad$ Save the new or modified histogram and close the dialog

Cancel - Close the dialog without saving the histogram.

SAPHIRE will use each acceleration bin and its associated frequency to quantify the seismic models. Note that the seismic models can be quantified using a single acceleration bin, using all acceleration bins separately, or using all acceleration bins and combining the results.

Multiple Hazard histograms can be defined, but only one histogram can be used at a time during quantification. The histogram to be used is identified in the Modify | Project option. 


\subsubsection{Name}

This is a required field. A maximum of 24 uppercase, alphanumeric characters may be entered. Embedded blanks are not allowed. The name should be descriptive so it can be readily identified.

\subsubsection{Description}

This is a 120-character, uppercase or lowercase, alphanumeric field that provides brief, descriptive information.

\subsubsection{Acceleration}

The ground acceleration level value for this bin.

\subsubsection{Frequency}

The frequency of occurrence (i.e., likelihood per year) for this bin.

\subsection{Modifying P\&ID Data}

\subsubsection{P\&IDs}

\section{PURPOSE}

This option allows you to edit Piping \& Instrumentation Diagram (P\&ID) graphic records.

\section{STEPS}

1. From the SAPHIRE menu select Modify.

2. Choose P\&IDs from the menu. The Edit PID Graphics dialog is displayed listing all of the PID graphics belonging to the current project.

3. Highlight the desired P\&ID graphic.

4. Right-click to invoke the pop-up menu or choose the Exit button. 


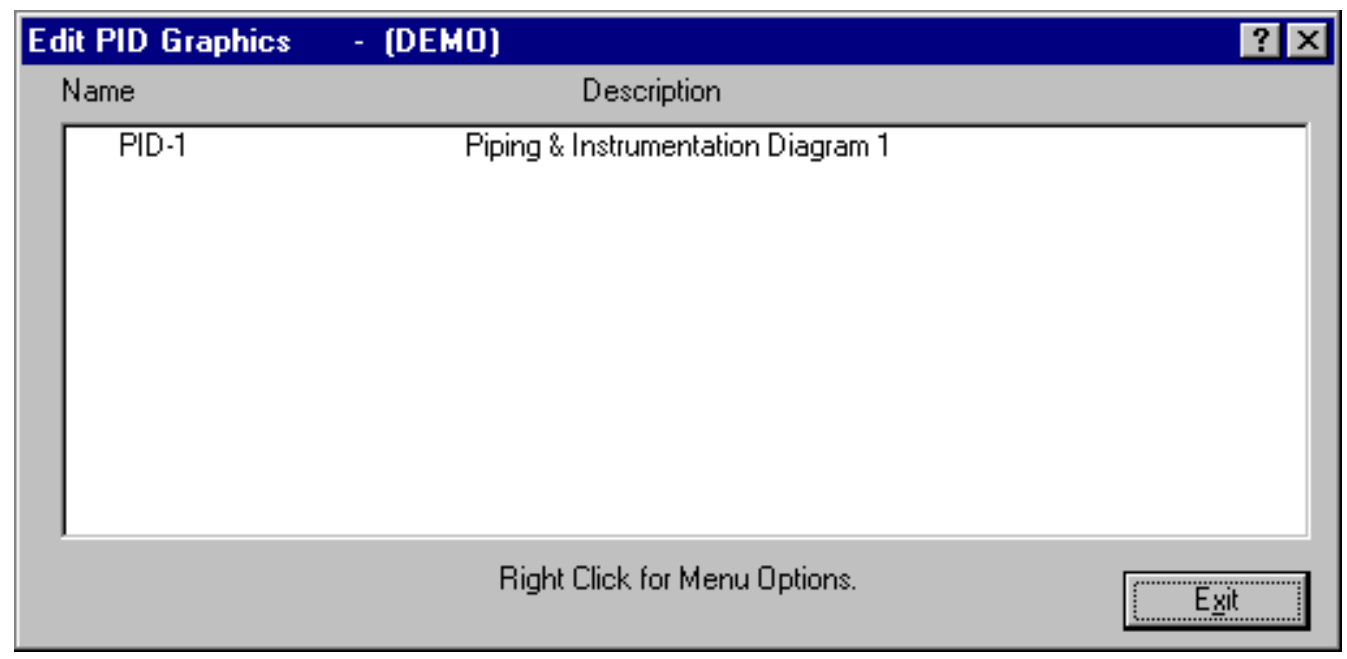

\section{Pop-up Menu Options}

Add - $\quad$ Add a new P\&ID record to the current project database. When complete, the message, "Record added," is displayed in the message bar of the SAPHIRE window.

Copy - Create a new P\&ID record by copying an existing one.

Modify - Edit the selected P\&ID record. When complete, the message, "Record modified," is displayed in the message bar of the SAPHIRE window.

Delete - Delete the selected P\&ID record(s).

\section{Button Option}

Exit - Close the Edit PID Graphics dialog.

\subsubsection{Delete Record}

\section{PURPOSE}

This option allows you to delete the highlighted record from the database.

\section{STEPS}

1. From the SAPHIRE menu select Modify.

2. Choose the desired option from the menu.

3. Highlight the record you wish to delete.

4. Right-click to invoke the pop-up menu and choose Delete. A warning dialog will be displayed, allowing you to cancel the deletion at this point.

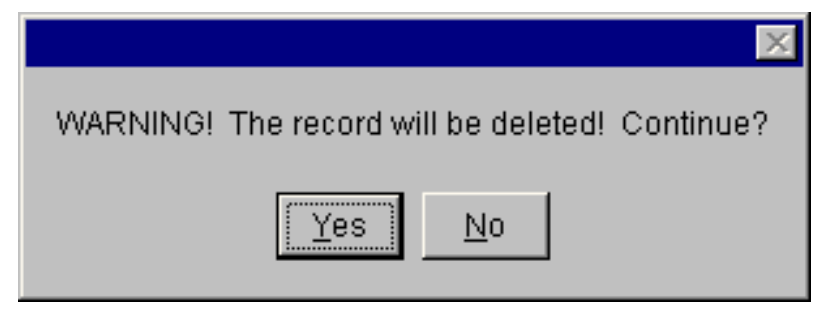


Yes - Continue the delete operation. The selected record will be deleted from the database. The message, "Record deleted," is displayed in the message bar of the SAPHIRE window.

No - Cancel the delete operation. The selected record will NOT be deleted from the database.

\subsubsection{P\&ID Data Entry Dialog}

\subsubsection{P\&ID Data Entry}

\section{PURPOSE}

The Add/Modify PID Graphic dialog allows you to enter or modify P\&ID data.

\section{STEPS}

1. From the SAPHIRE menu select Modify.

2. Choose P\&IDs from the menu. The Edit PID Graphics dialog is displayed.

3. Highlight the P\&ID graphic you wish to edit or copy. (Skip this step if adding a new record.)

4. Right-click to invoke the pop-up menu and choose Add, Copy, or Modify.

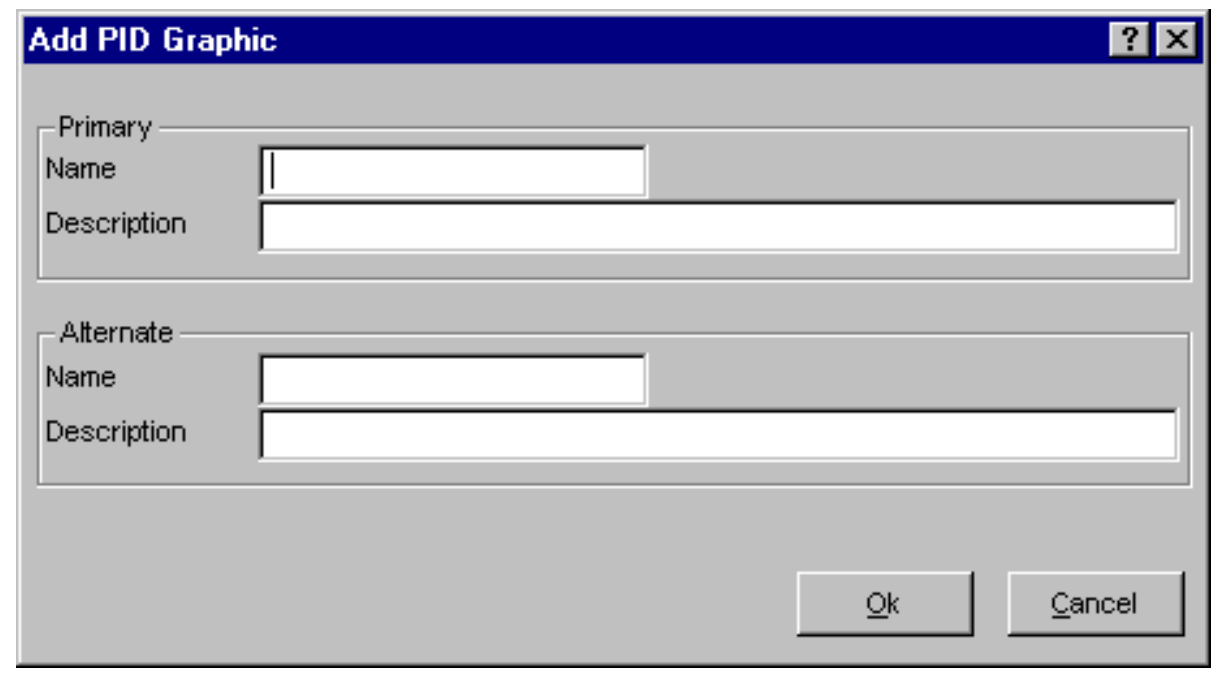

Ok - Save the newly created P\&ID record or changes made to the existing record, and close the dialog

Cancel - Close the dialog without saving the changes.

\subsubsection{Name}

This is a required field. A maximum of 24 uppercase, alphanumeric characters may be entered. Embedded blanks are not allowed. The name should be descriptive so it can be readily identified.

\subsubsection{Description}

This is a 120-character, uppercase or lowercase, alphanumeric field that provides brief, descriptive information. 


\subsection{Modifying Change Set Data}

\subsubsection{Change Sets}

\section{PURPOSE}

This option allows you to add, modify, delete, or copy change set records. You cannot edit the probability or class changes of event data in the change set using this option.

\section{STEPS}

1. From the SAPHIRE menu select Modify.

2. Choose Change Sets from the menu. The Change Sets dialog is displayed, listing all of the change sets belonging to the current project.

3. Highlight the desired change set.

4. Right-click to invoke the pop-up menu or choose the desired button.

The Change Sets dialog lists the change sets by marked order, then set type. First, all marked change sets are listed, then the condition assessments are listed alphabetically, followed by initiating event assessments (alphabetically), and finally, the change sets.

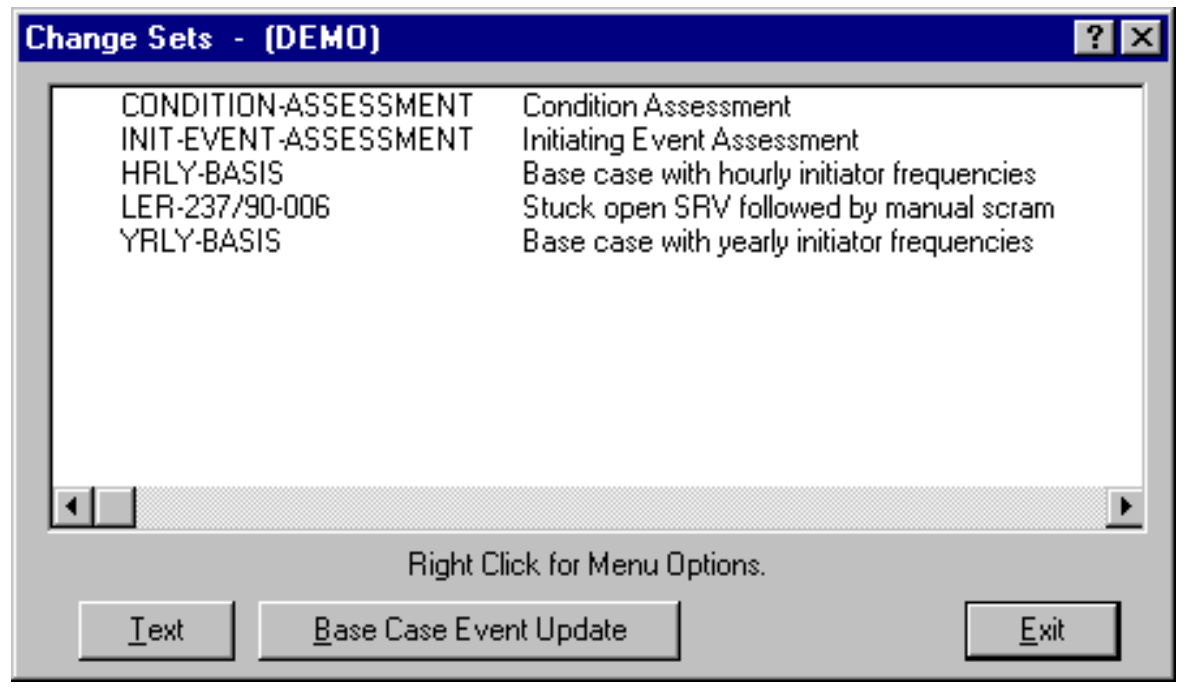

\section{Pop-up Menu Options}

Add - $\quad$ Add a new change set record to the current project database. When complete, the message, "Record added," is displayed in the message bar of the SAPHIRE window.

Copy - Create a new change set record by copying an existing one.

Modify - Edit the selected change set record. When complete, the message, "Record modified," is displayed in the message bar of the SAPHIRE window.

Delete - Delete the selected change set record(s).

\section{Button Options}

Text -
View and edit the descriptive text associated with the selected change set. 
Base Case Event Update - Overwrite the base case data values stored in the database with the current data values for each basic event.

Exit - $\quad$ Close the Change Sets dialog.

\subsubsection{Delete Record}

\section{PURPOSE}

This option allows you to delete the highlighted record from the database.

\section{STEPS}

1. From the SAPHIRE menu select Modify.

2. Choose the desired option from the menu.

3. Highlight the record you wish to delete.

4. Right-click to invoke the pop-up menu and choose Delete. A warning dialog will be displayed, allowing you to cancel the deletion at this point.

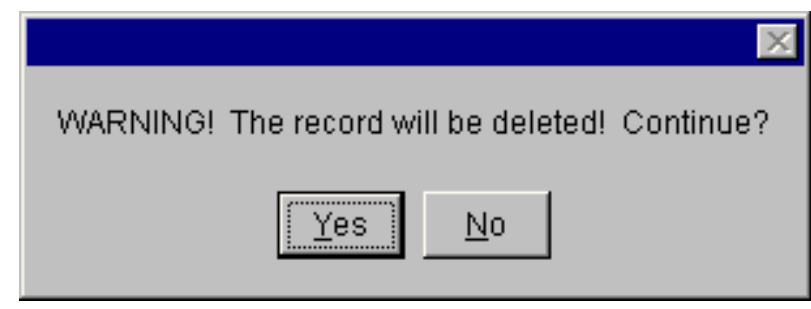

Yes - Continue the delete operation. The selected record will be deleted from the database. The message, "Record deleted," is displayed in the message bar of the SAPHIRE window.

No - Cancel the delete operation. The selected record will NOT be deleted from the database.

\subsubsection{Database Text}

\section{PURPOSE}

This option allows you to view and edit the descriptive text associated with a specific record. The Primary text option is generally used for text in the desired primary language. The Alternate text option is generally used for information entered in the secondary language using a different alphabet (locale ).

\section{STEPS}

1. From the SAPHIRE menu select Modify.

2. Choose the desired option from the menu. 
3. Highlight the record whose text you wish to modify.

4. Choose the Text button. The Select Text Type dialog is displayed.

5. Choose Primary or Alternate. The Text dialog is displayed.

Initially, the first 18 lines of the text block are displayed. If there are more lines of text, the vertical scroll bar will be available. In the example here, the Primary text dialog is displayed.

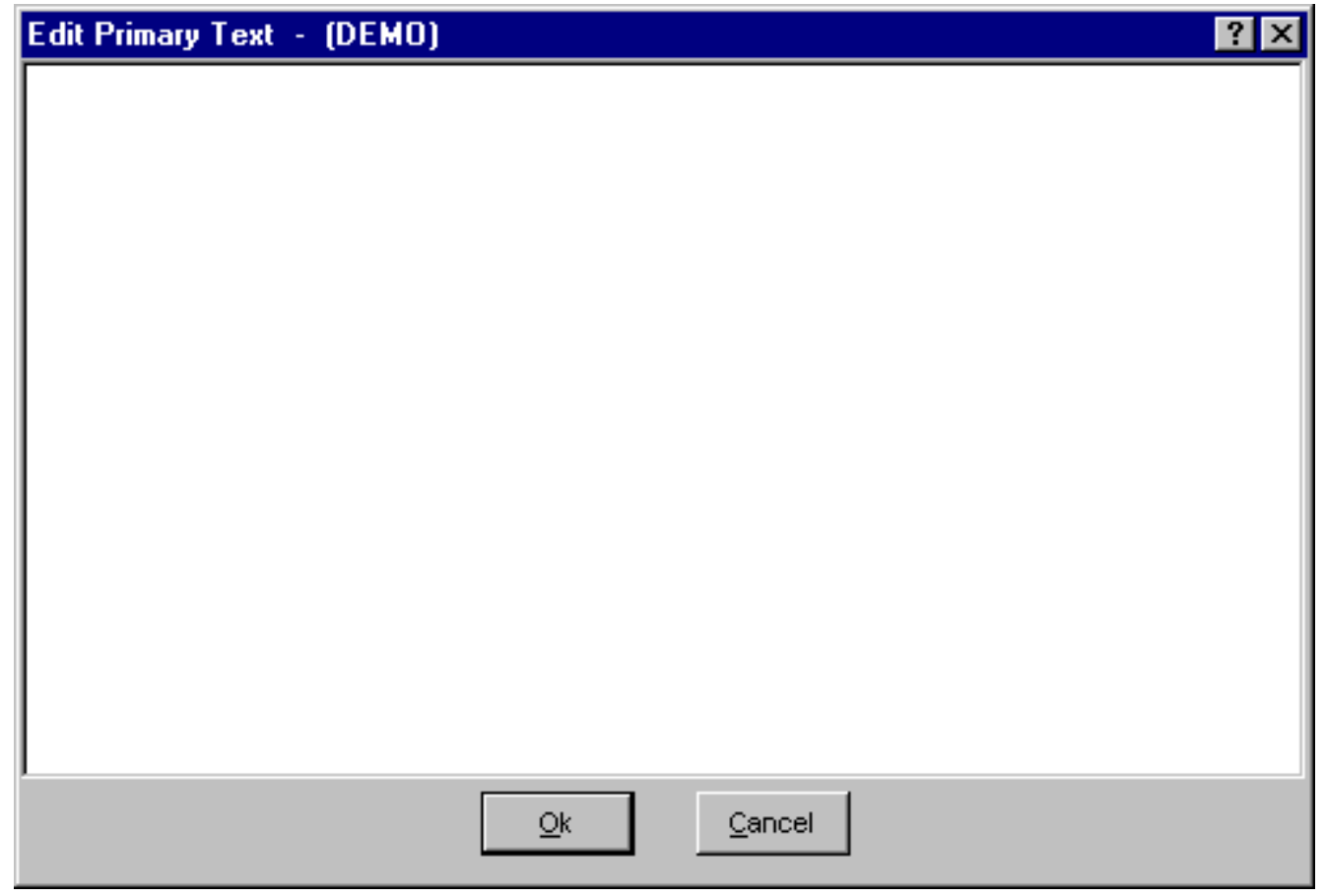

Page Down- Presents the next 18 lines of text.

Page Up- Presents the previous 18 lines of text.

Ctrl Page Down-Places the cursor at the end of the last line of text currently visible.

Ctrl Page Up- Places the cursor at the beginning of the first line of text currently visible.

Ctrl- - -

Moves the cursor left to the beginning of the word on the current line.

Ctrl- $\rightarrow$ - $\quad$ Moves the cursor right to the beginning of the word on the current line.

Home - $\quad$ Places the cursor in front of the first character of the current line.

Ctrl-Home - Places the cursor at the beginning of the text block.

End -

Ctrl-End - $\quad$ Places the cursor at the end of the text block.

Insert -

Works as a toggle. Initially, the editor is in "insert" mode - existing characters are pushed to the right as you type. Pressing the Insert key toggles to "overwrite" mode - existing characters are over written as you type.

Delete - Deletes the character to the right of the current cursor position; or deletes the currently highlighted text.

Ctrl-Delete - Deletes the characters from the current cursor position to the beginning of the following word.

Backspace - Deletes the character to the left of the current cursor position; or deletes the currently highlighted text.

Ctrl-C - Copies highlighted text to the clipboard. 
Ctrl-V - $\quad$ Pastes clipboard text at the current cursor position.

Ctrl-X - $\quad$ Cuts highlighted text to the clipboard.

The editor does not automatically line wrap; therefore, you must use $<$ Enter $>$ to establish each new line of text.

Ok - $\quad$ Save the new or modified text and close the Text dialog.

Cancel - Close the Text dialog without saving changes. You will be prompted with the dialog shown below.

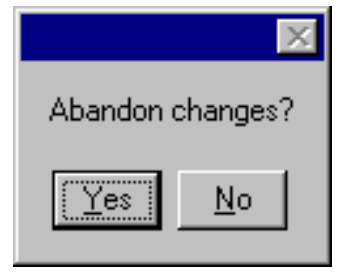

Yes - $\quad$ Close the Text dialog without saving changes.

No - $\quad$ Save the new or modified text and close the Text dialog.

\subsubsection{Change Set - Marking and Processing Order}

\section{PURPOSE}

Change sets are "marked" to determine which change sets will be used to generate current case data. Order of marking determines which event changes take precedence. This option acts in the manner of a toggle.

Marking Change Sets:

\section{STEPS}

1. From the SAPHIRE menu select Modify | Change Sets. The Change Sets dialog is displayed. OR

2. From the SAPHIRE menu select Generate. The Generate dialog is displayed.

3. Double-click on the desired change set to mark or unmark it.

If the selected change set was not previously marked, a numeric value (1 - 99) will appear to the left of the change set name, indicating the order of marking. The change sets in the list are rearranged by marked order, then alphabetically by type: first by condition assessment, then initiating event assessment type, finally by ordinary change set type.

If the change set was previously marked, it is unmarked (i.e., the numeric value is removed) and it is repositioned in the list.

If no change sets are marked when you select the Generate option, then the current case data will be initialized to the base case data. 


\section{Change Set Processing:}

During change set processing (i.e., generating event data or updating base case values), three criteria are evaluated: probability changes, class changes, and the order of marked change sets.

If an event in a marked change set has both a class change and a probability change associated with it, the probability change takes precedence over the class change if any discrepancies arise..

If more than one change set is marked, then the probability and class changes in the change sets marked with the highest number ( 2 is higher than 1, 3 is higher than 2 ) will take precedence over any changes from lower numbered change sets. For example, suppose that Event A has a probability change associated with it in Change Set 1, and a class change associated with it in Change Set 2, then the class change from Change Set 2 would overwrite the probability change from Change Set 1.

The following example illustrates the outcome of processing three marked change sets with four events:

Event A has both a probability change and a class change associated with it in Change Set 2.

Event B has a probability change in Change Set 1, and a class change in Change Set 3.

Event $\mathrm{C}$ has only a class change in Change Set 1 .

Event D has no probability changes or class changes associated with it in any of the three marked change sets.

The outcome of the Generate operation on these three marked change sets would be as follows:

The current case data for event A would be set to the values associated with the probability change in Change Set 2. (Probability changes take precedence over class changes when they occur for the same event within the same change set.)

The current case data for event B would be set equal to the values associated with the class change in Change Set 3. (The probability or class change in higher numbered change sets take precedence over changes in lower numbered change sets.)

The current case data for event $\mathrm{C}$ would be set to the values associated with the class change in Change Set 1 .

The current case data for event $\mathrm{D}$ would be set to the base case values for event $\mathrm{D}$. (There were no probability changes or class changes made to event $\mathrm{D}$ in any of the marked change sets.)

\subsubsection{Add Change Set Dialog}

\subsubsection{Adding a Change Set}




\section{PURPOSE}

This option allows you to create a new change set record. Enter the new change set's name, description and date.

\section{STEPS}

1. From the SAPHIRE menu select Modify $\mid$ Change Sets. The Change Sets dialog is displayed. $O R$

2. From the SAPHIRE menu select Generate. The Generate dialog is displayed.

3. Right-click to invoke the pop-up menu and choose Add. The Add Change Set dialog is displayed.

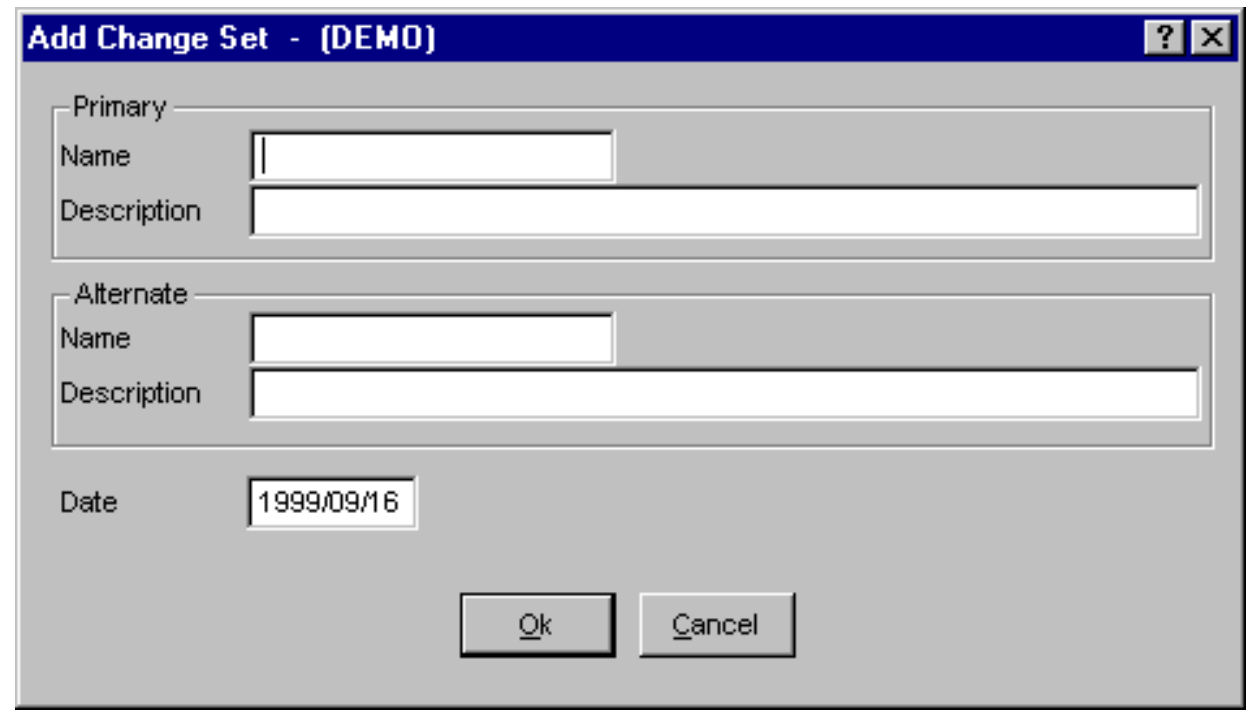

Ok - Save the new change set, and close the dialog. If the add operation is successful, the message, "Record added," is displayed in the message bar. If a change set record already exists with the same name, the message, "Duplicate record name - not added," is displayed in the message bar and the Add Change Set dialog remains open.

Cancel - Close the dialog without saving. A new change set record is not added to the database.

By default the set type is a Change Set and the Read-only attribute is not selected. These fields are not available on the Add Change Set dialog. If you wish to alter the set type or read-only attribute, you must Modify the change set.

\subsubsection{Name}

This is a required field. A maximum of 24 uppercase, alphanumeric characters may be entered. Embedded blanks are not allowed. The name should be descriptive so it can be readily identified.

\subsubsection{Description}

This is a 120-character, uppercase or lowercase, alphanumeric field that provides brief, descriptive information. 


\subsubsection{Date}

This is the creation date of the change set. It can be modified to reflect the modification date. The format for this field is YYYY/MM/DD.

\subsubsection{Copy Change Set Dialog}

\subsubsection{Copying a Change Set}

\section{PURPOSE}

This option allows you to create a new change set record based on the highlighted one. The original change set name is displayed in the Copy From: field. You MUST enter a unique name for the new change set. Along with the source change set's name, description, and date, probability and class changes are also duplicated.

\section{STEPS}

1. From the SAPHIRE menu select Modify | Change Sets. The Change Sets dialog is displayed. $O R$

2. From the SAPHIRE menu select Generate. The Generate dialog is displayed.

3. Highlight the change set you wish to copy.

4. Right-click to invoke the pop-up menu and choose Copy. The Copy Change Set dialog is displayed.

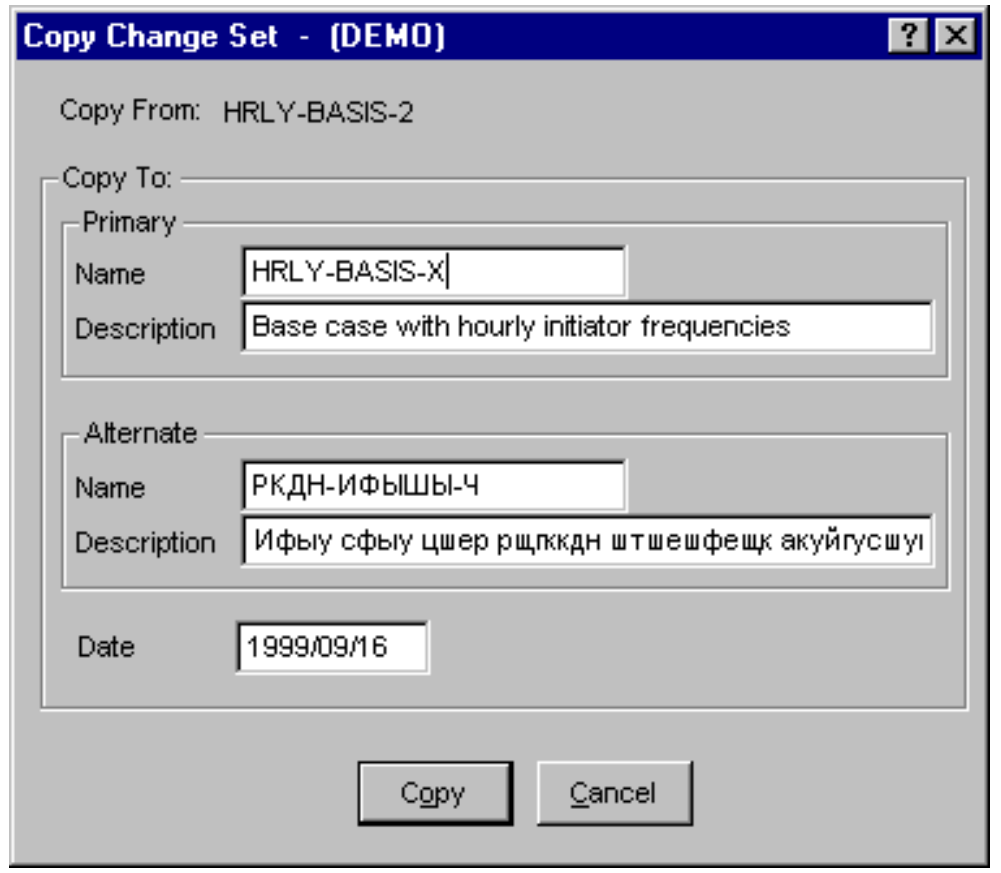

Copy - Save the newly created change set and close the dialog. If the copy operation is successful, the message, "Record added," is displayed in the message bar. If a change set 
record already exists with the same name, the message, "Duplicate record name - not added," is displayed in the message bar and the Copy Change Set dialog remains open.

Cancel - Close the dialog without saving the change set. A new change set record is not added to the database.

The set type and read-only flag of the source (original) change set will be duplicated in the destination (new) change set along with the basic event data changes. If you wish to alter the set type or read-only attribute, you must Modify the change set record. If you wish to alter the basic event data changes, you must choose the Class or Single buttons from the Generate dialog.

\subsubsection{Name}

This is a required field. A maximum of 24 uppercase, alphanumeric characters may be entered. Embedded blanks are not allowed. The name should be descriptive so it can be readily identified.

\subsubsection{Description}

This is a 120-character, uppercase or lowercase, alphanumeric field that provides brief, descriptive information.

\subsubsection{Date}

This is the creation date of the change set. It can be modified to reflect the modification date. The format for this field is YYYY/MM/DD.

\subsubsection{Modify Change Set Dialog}

\subsubsection{Modifying a Change Set}

\section{PURPOSE}

This option allows you to modify change set record data. In addition to editing the change set name, description, and date, you can also edit the set type and read-only attribute.

\section{STEPS}

1. From the SAPHIRE menu select Modify | Change Sets. The Change Sets dialog is displayed. OR

2. From the SAPHIRE menu select Generate. The Generate dialog is displayed.

3. Highlight the change set you wish to edit.

4. Right-click to invoke the pop-up menu and choose Modify. The Modify Change Set dialog is displayed. 


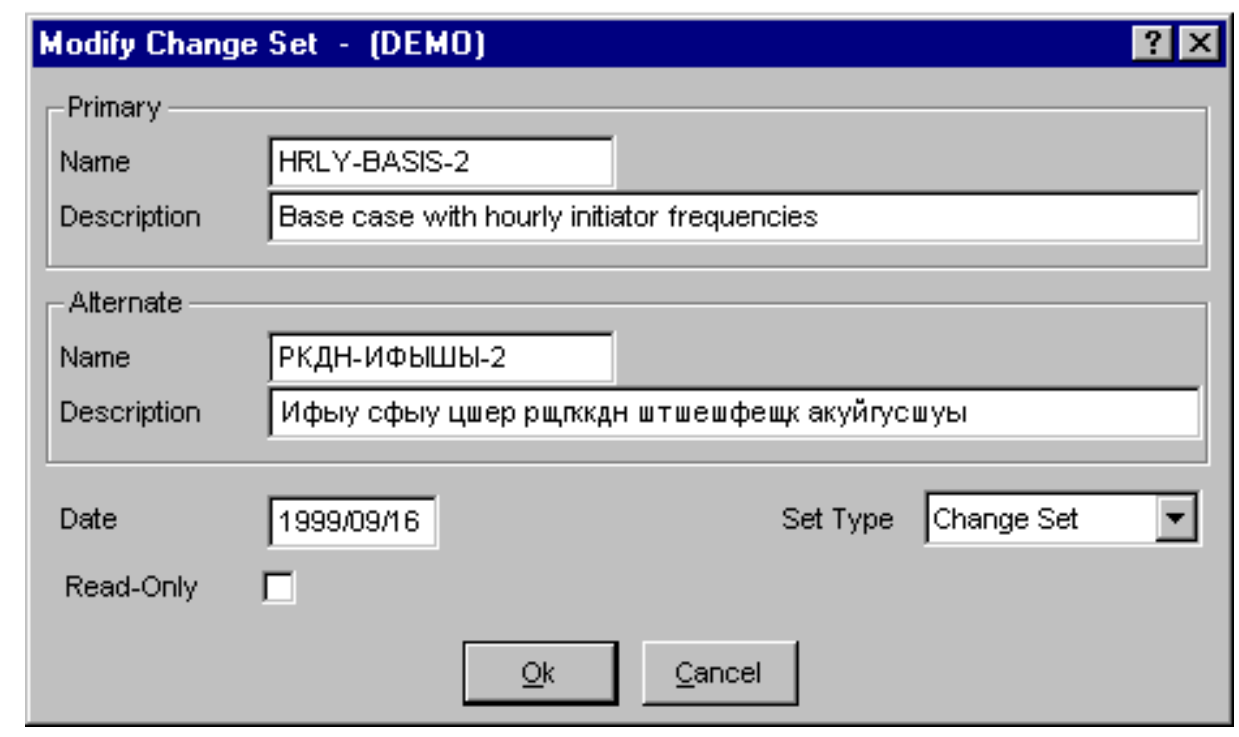

Ok - Save the modified change set and close the dialog. If the modify operation is successful, the message, "Record modified," is displayed in the message bar. If a change set record already exists with the same name, the message, "Revised name is a duplicate - not modified," is displayed in the message bar and the Modify Change Set dialog remains open.

Cancel - Close the dialog without saving the modifications.

\subsubsection{Name}

This is a required field. A maximum of 24 uppercase, alphanumeric characters may be entered. Embedded blanks are not allowed. The name should be descriptive so it can be readily identified.

\subsubsection{Set Type}

Select a change set type from the drop-down list.

Change Set - A user-defined set of changes that will be applied to the base case data when event data is transferred to the current case. Multiple change sets can be defined and applied singly or in combination.

Initiating Event- A special type of change set used specifically to evaluate an operational occurrence associated with an observed initiating event and other equipment failures, unavailabilities, or degradations.

Condition - $\quad$ A special type of change set used specifically to evaluate an operational occurrence in which no initiating event has taken place, but equipment failures, unavailabilities, or degradations have reduced reliability.

Flag Set - $\quad$ A special type of change set used to indicate modifications to particular events on a sequence-by-sequence basis. They are different from change sets in that they can only contain individually specified types of changes: either house flag changes to the calculation type or process type changes. NOTE: If a change set type is altered to a flag set, it will be displayed in the Flag Sets dialog after the Change Sets dialog is closed. 


\subsubsection{Description}

This is a 120-character, uppercase or lowercase, alphanumeric field that provides brief, descriptive information.

\subsubsection{Date}

This is the creation date of the change set. It can be modified to reflect the modification date. The format for this field is YYYY/MM/DD.

\subsubsection{Read-only}

This flag, if checked, indicates that the event data cannot be changed (in the Generate | Single/Class option). If you wish to make event data modifications, this box must be unchecked.

\subsubsection{Base Update Dialog}

\subsubsection{Base Case Event Update}

\section{PURPOSE}

This option allows you to overwrite the base case data values stored in the data base with the current case data values for each basic event. After executing this operation, the original base case data are no longer available.

\section{STEPS}

1. From the SAPHIRE menu select Modify.

2. Choose Change Sets from the menu.

3. Highlight the desired change set(s).

4. Choose the Base Case Event Update button. The Event Base Case Update dialog is displayed.

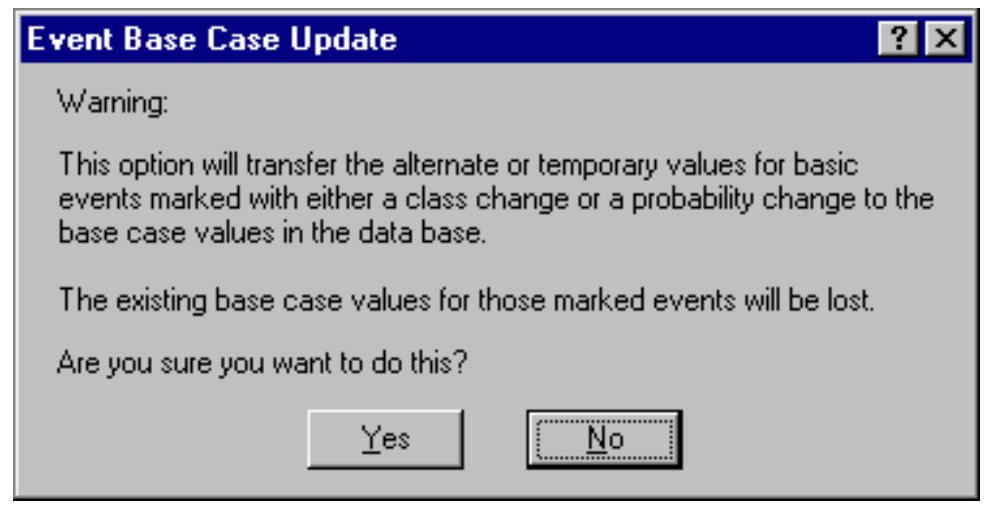

This dialog is to ensure that you wish to update the base case values with the marked change set(s). 
Yes - When you choose this button, the Set Mission Time dialog will be displayed. After entering the mission time, the base case values will be overwritten.

No - Terminate the process and close the Event Base Case Update dialog.

In order to perform a base case update, at least one change set must be marked and data generated. This option transfers the current values for the basic events marked either with a probability change or a class change in a change set to the base case values. The existing base case values will be overwritten. Any event in the change set that does not have either a class change or probability change associated with it will maintain its existing base case data.

\subsubsection{Mission Time}

Enter the mission time, using scientific notation, in hours.

\subsection{Modifying Flag Set Data}

\subsubsection{Flag Sets}

\section{PURPOSE}

This option allows you to add, modify, delete, or copy flag set records. Flag sets are a special type of change set used to indicate modifications to particular events on a sequence-by-sequence basis. They are different from change sets in that they can only contain individually-specified types of changes. No "Class Changes" are allowed in a flag set.

\section{STEPS}

1. From the SAPHIRE menu select Modify.

2. Choose Flag Sets from the menu. The Flag Sets dialog is displayed, listing all of the flag sets belonging to the current project.

3. Highlight the desired flag set.

4. Right-click to invoke the pop-up menu or choose the desired button.

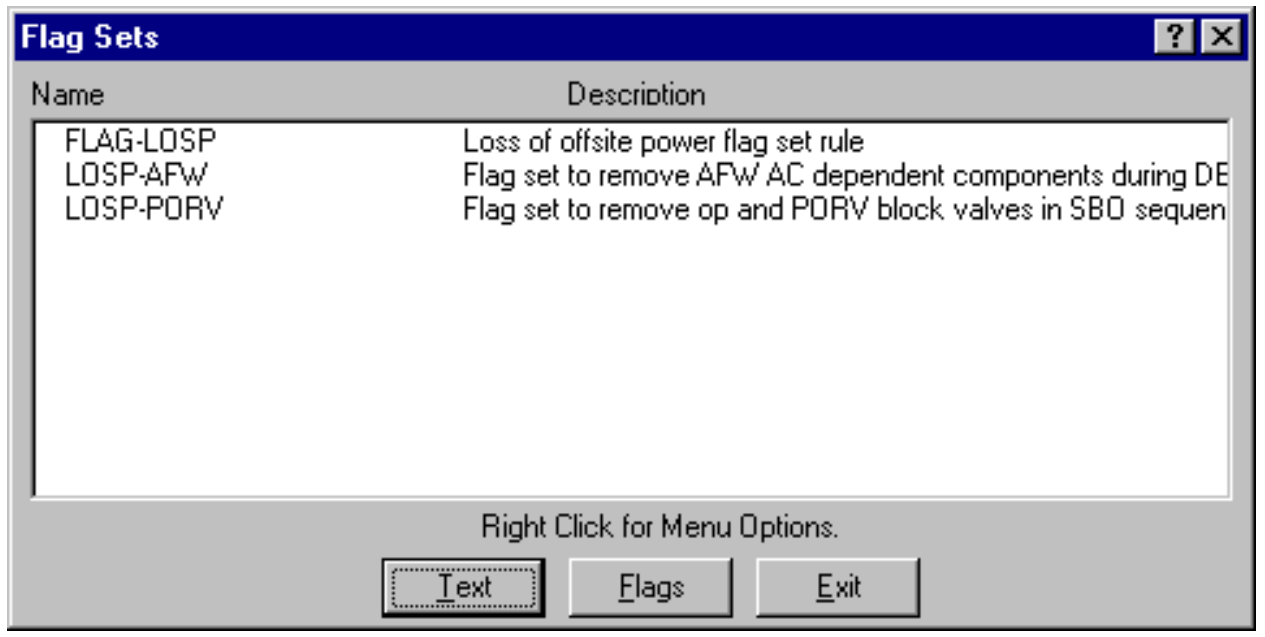




\section{Pop-up Menu Options}

Add - Create a new flag set record in the current project database. When complete, the message, "Record added," is displayed in the message bar of the SAPHIRE window.

Copy - Create a new flag set record by copying an existing one.

Modify - Edit the selected flag set record. When complete, the message, "Record modified," is displayed in the message bar of the SAPHIRE window.

Delete - Delete the selected flag set record(s).

\section{Button Options}

Text - View and edit the descriptive text associated with the selected flag set.

Flags - $\quad$ Add or modify basic event data in the flag set.

Exit - $\quad$ Close the Flag Sets dialog.

\subsubsection{Delete Record}

\section{PURPOSE}

This option allows you to delete the highlighted record from the database.

\section{STEPS}

1. From the SAPHIRE menu select Modify.

2. Choose the desired option from the menu.

3. Highlight the record you wish to delete.

4. Right-click to invoke the pop-up menu and choose Delete. A warning dialog will be displayed, allowing you to cancel the deletion at this point.

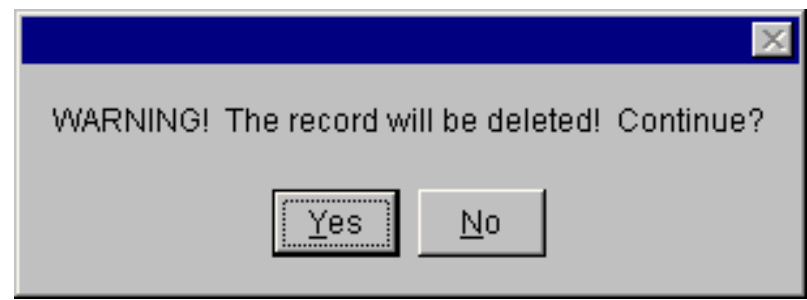

Yes - Continue the delete operation. The selected record will be deleted from the database. The message, "Record deleted," is displayed in the message bar of the SAPHIRE window.

No - Cancel the delete operation. The selected record will NOT be deleted from the database.

\subsubsection{Database Text}

\section{PURPOSE}

This option allows you to view and edit the descriptive text associated with a specific record. The Primary text option is generally used for text in the desired primary language. The Alternate text option is generally used for information entered in the secondary language using a different alphabet (locale ). 


\section{STEPS}

1. From the SAPHIRE menu select Modify.

2. Choose the desired option from the menu.

3. Highlight the record whose text you wish to modify.

4. Choose the Text button. The Select Text Type dialog is displayed.

5. Choose Primary or Alternate. The Text dialog is displayed.

Initially, the first 18 lines of the text block are displayed. If there are more lines of text, the vertical scroll bar will be available. In the example here, the Primary text dialog is displayed.

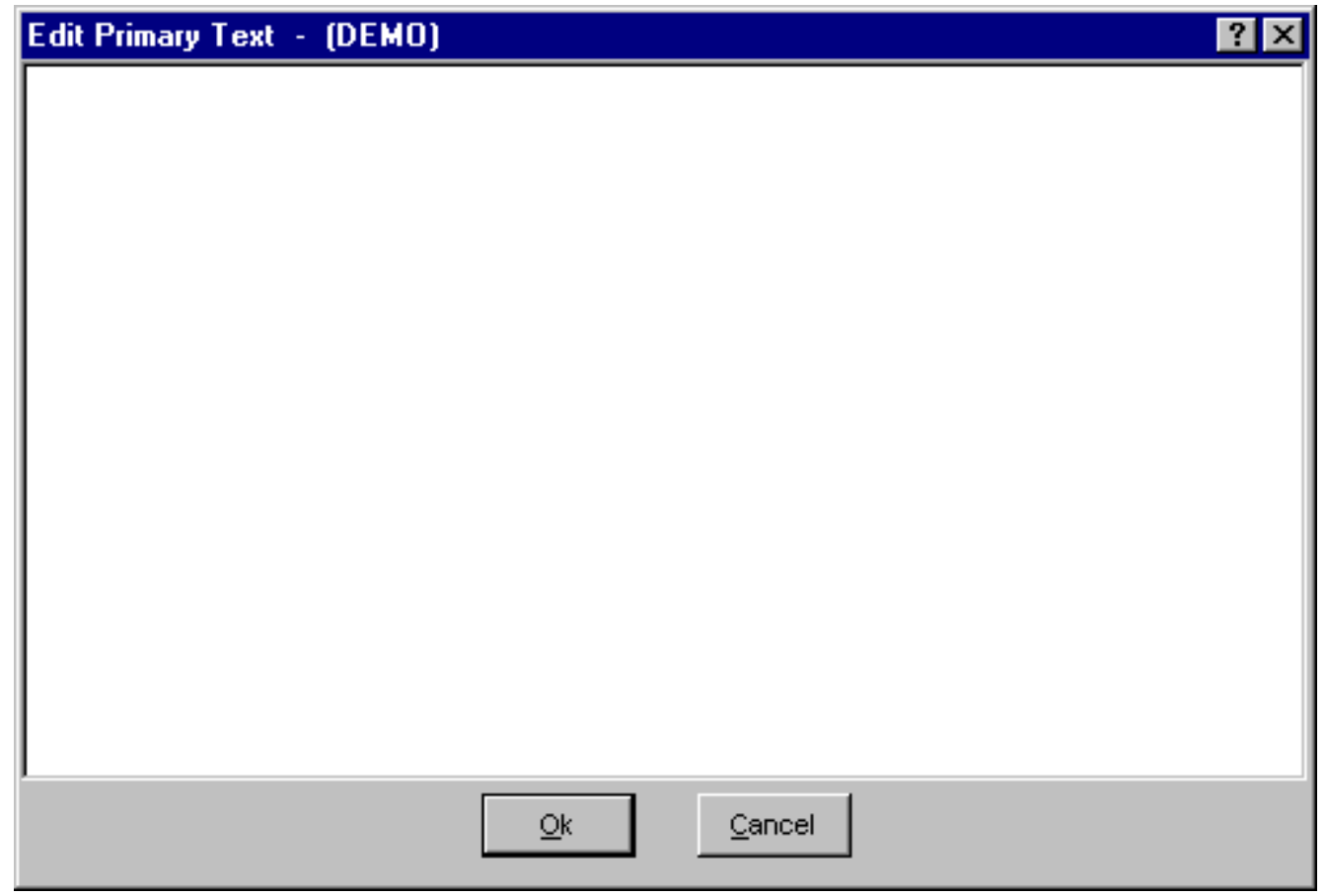

Page Down- Presents the next 18 lines of text.

Page Up- Presents the previous 18 lines of text.

Ctrl Page Down-Places the cursor at the end of the last line of text currently visible.

Ctrl Page Up- Places the cursor at the beginning of the first line of text currently visible.

Ctrl- : -

Moves the cursor left to the beginning of the word on the current line.

Ctrl- $\rightarrow \quad$ Moves the cursor right to the beginning of the word on the current line.

Home - $\quad$ Places the cursor in front of the first character of the current line.

Ctrl-Home - Places the cursor at the beginning of the text block.

End -

Places the cursor behind the last character of the current line.

Ctrl-End - $\quad$ Places the cursor at the end of the text block.

Insert - Works as a toggle. Initially, the editor is in "insert" mode - existing characters are pushed to the right as you type. Pressing the Insert key toggles to "overwrite" mode - existing characters are over written as you type.

Delete - $\quad$ Deletes the character to the right of the current cursor position; or deletes the currently highlighted text. 
Ctrl-Delete - Deletes the characters from the current cursor position to the beginning of the following word.

Backspace - Deletes the character to the left of the current cursor position; or deletes the currently highlighted text.

Ctrl-C - $\quad$ Copies highlighted text to the clipboard.

Ctrl-V - $\quad$ Pastes clipboard text at the current cursor position.

Ctrl-X - $\quad$ Cuts highlighted text to the clipboard.

The editor does not automatically line wrap; therefore, you must use $<$ Enter $>$ to establish each new line of text.

Ok - $\quad$ Save the new or modified text and close the Text dialog.

Cancel - $\quad$ Close the Text dialog without saving changes. You will be prompted with the dialog shown below.

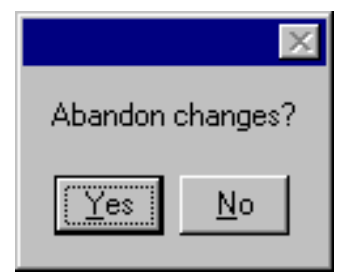

Yes - Close the Text dialog without saving changes.

No - Save the new or modified text and close the Text dialog.

\subsubsection{Add Flag Set Dialog}

\subsubsection{Adding a Flag Set}

\section{PURPOSE}

The Add Flag Set dialog allows you to create a new flag set record.

\section{STEPS}

1. From the SAPHIRE menu select Modify.

2. Choose Flag Sets from the menu. The Flag Sets dialog is displayed.

3. Right-click to invoke the pop-up menu and choose Add. 


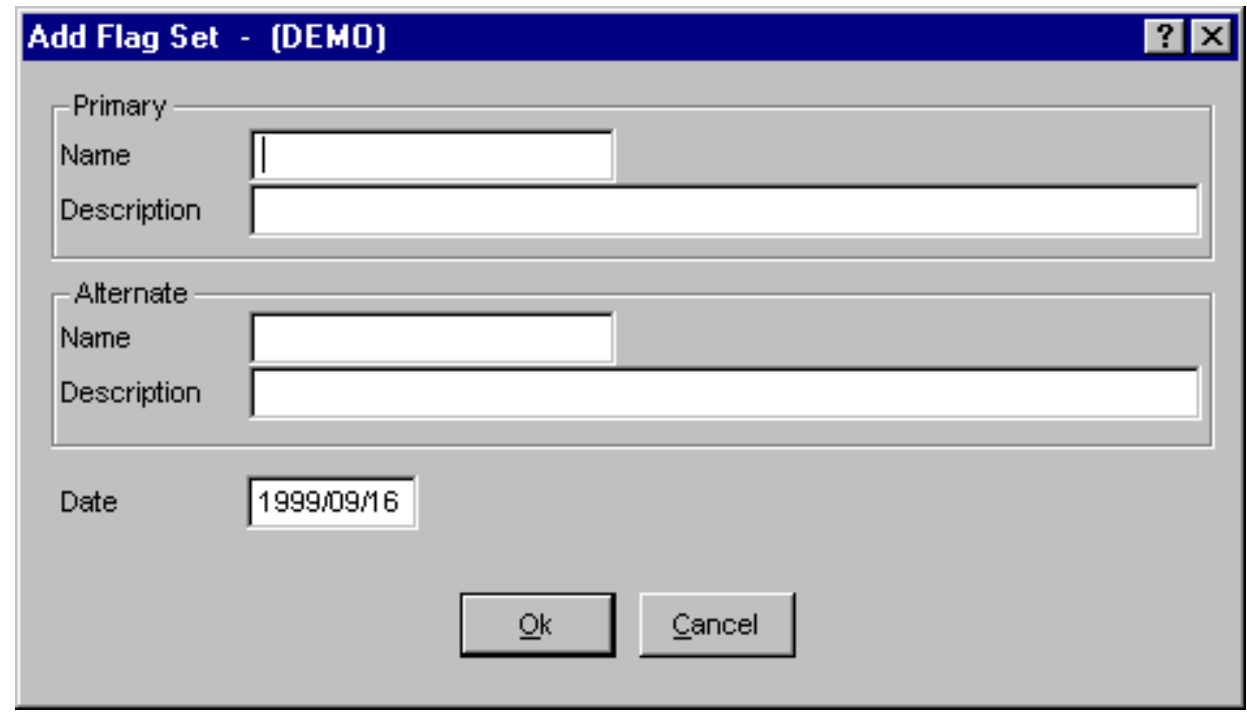

Ok - $\quad$ Save the new flag set, and close the dialog.

Cancel - Close the dialog without saving.

By default the set type is a Flag Set and the Read-only attribute is not selected. If you wish to alter the set type or read-only attribute, you must Modify the flag set.

\subsubsection{Name}

This is a required field. A maximum of 24 uppercase, alphanumeric characters may be entered. Embedded blanks are not allowed. The name should be descriptive so it can be readily identified.

\subsubsection{Description}

This is a 120-character, uppercase or lowercase, alphanumeric field that provides brief, descriptive information.

\subsubsection{Date}

This is the creation date of the change set. It can be modified to reflect the modification date. The format for this field is YYYY/MM/DD.

\subsubsection{Copy Flag Set Dialog}

\subsubsection{Copying A Flag Set}

\section{PURPOSE}

The Copy Flag Set dialog will be displayed, allowing you to create a new flag set record based on the highlighted one. The original flag set name is displayed in the Copy From: field. You MUST enter a unique name for the new flag set. 


\section{STEPS}

1. From the SAPHIRE menu select Modify.

2. Choose Flag Sets from the menu. The Flag Sets dialog is displayed.

3. Highlight the flag set you wish to copy.

4. Right-click to invoke the pop-up menu and choose Copy.

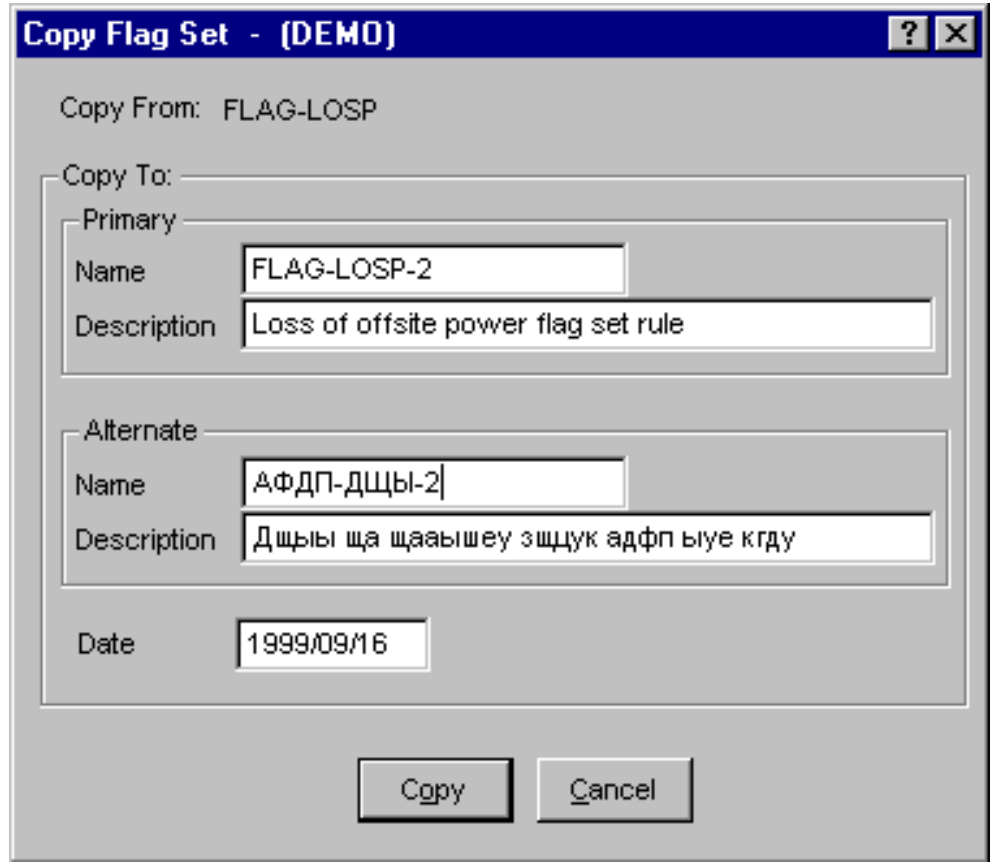

Copy - Save the new flag set, and close the dialog.

Cancel - Close the dialog without saving.

The set type and read-only flag of the source (original) flag set will be duplicated in the destination (new) flag set along with the basic event data changes.

\subsubsection{Name}

This is a required field. A maximum of 24 uppercase, alphanumeric characters may be entered. Embedded blanks are not allowed. The name should be descriptive so it can be readily identified.

\subsubsection{Description}

This is a 120-character, uppercase or lowercase, alphanumeric field that provides brief, descriptive information.

\subsubsection{Date}


This is the creation date of the change set. It can be modified to reflect the modification date. The format for this field is YYYY/MM/DD.

\subsubsection{Modify Flag Set Dialog}

\subsubsection{Modifying a Flag Set}

\section{PURPOSE}

The Modify Flag Set dialog allows you to edit flag set record data.

\section{STEPS}

1. From the SAPHIRE menu select Modify.

2. Choose Flag Sets from the menu. The Flag Sets dialog is displayed.

3. Highlight the flag set you wish to edit.

4. Right-click to invoke the pop-up menu and choose Modify.

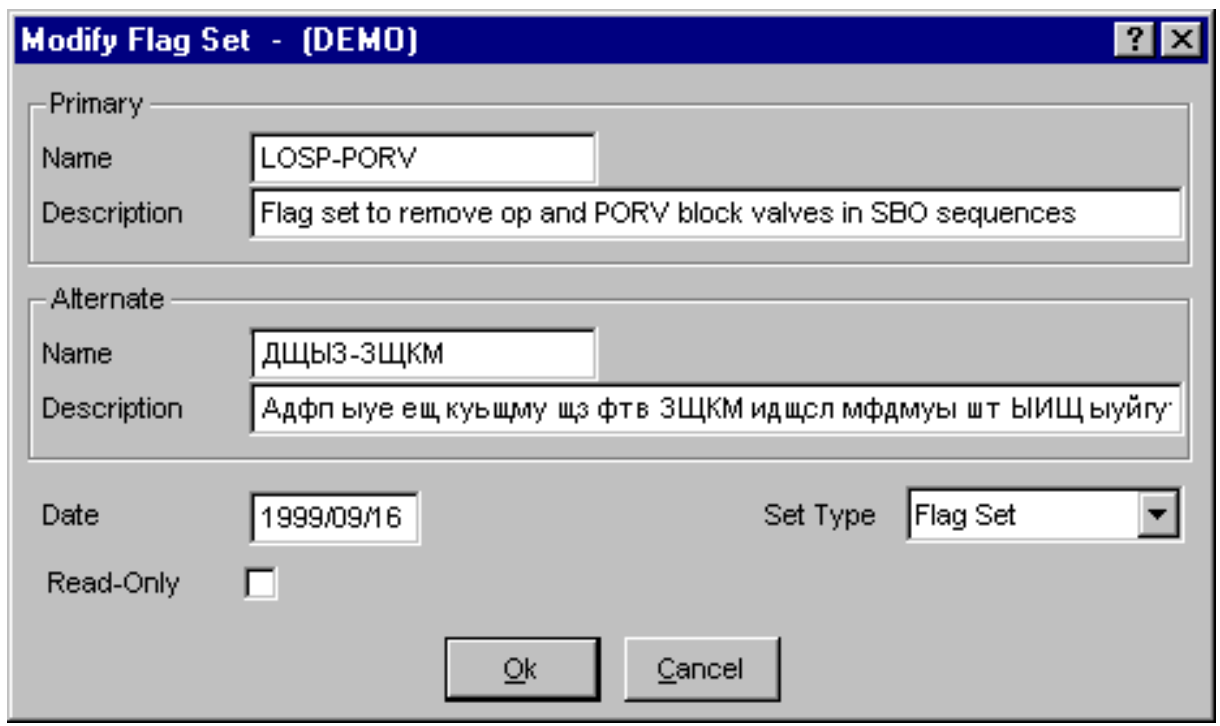

Ok - $\quad$ Save the modified flag set and close the dialog

Cancel - Close the dialog without saving the modifications.

\subsubsection{Name}

This is a required field. A maximum of 24 uppercase, alphanumeric characters may be entered. Embedded blanks are not allowed. The name should be descriptive so it can be readily identified.

\subsubsection{Set Type}


Select a change set type from the drop-down list.

Change Set - A user-defined set of changes that will be applied to the base case data when event data is transferred to the current case. Multiple change sets can be defined and applied singly or in combination.

Initiating Event-A special type of change set used specifically to evaluate an operational occurrence associated with an observed initiating event and other equipment failures, unavailabilities, or degradations.

Condition - $\quad$ A special type of change set used specifically to evaluate an operational occurrence in which no initiating event has taken place, but equipment failures, unavailabilities, or degradations have reduced reliability.

Flag Set - $\quad$ A special type of change set used to indicate modifications to particular events on a sequence-by-sequence basis. They are different from change sets in that they can only contain individually specified types of changes: either house flag changes to the calculation type or process type changes. NOTE: If a change set type is altered to a flag set, it will be displayed in the Flag Sets dialog after the Change Sets dialog is closed.

\subsubsection{Description}

This is a 120-character, uppercase or lowercase, alphanumeric field that provides brief, descriptive information.

\subsubsection{Date}

This is the creation date of the change set. It can be modified to reflect the modification date. The format for this field is YYYY/MM/DD.

\subsubsection{Read-only}

This flag, if checked, indicates that the event data cannot be changed (in the Generate | Single/Class option). If you wish to make event data modifications, this box must be unchecked.

\subsubsection{Flag Set Event Data}

\subsubsection{Flag Set Event Data}

\section{PURPOSE}

Edit or add house flag changes to the calculation type or process type.

Changes to the probability of failure (e.g., change the probability from 2E-3 to 1E-1) for events in a Flag Set cannot be made. Only the event changes of TRUE, FALSE, IGNORE, or process flags can be used in a SAPHIRE Flag Set. The changes that are made can then be applied to specified fault trees or sequences for use during fault tree or event tree analysis. The changes applied to basic event data may be for a single event or a group of events.

\section{STEPS}

1. From the SAPHIRE menu select Modify. 
2. Choose Flag Sets from the menu.

3. Highlight the desired flag set.

4. Choose the Flags button. The Flag Set Events dialog is displayed.

This dialog lists the event name and description of each event in the flag set. In addition, the dialog shows the event usage flags. The usage column (i.e., SLY) indicates whether the event has been marked or not used and whether the event is used in the base case, current, or both. The event usage flag is supplied for sequence cut sets (S), fault tree logic (L), or fault tree cut sets (Y).

The change set flag indicates if the event has been marked for a probability (single) change ("P"), a class change ("C") or both ("c"). Because only calculation or process type changes are allowed in flag sets, this flag will always be a "P." The flag set name is shown in the title bar of this dialog.

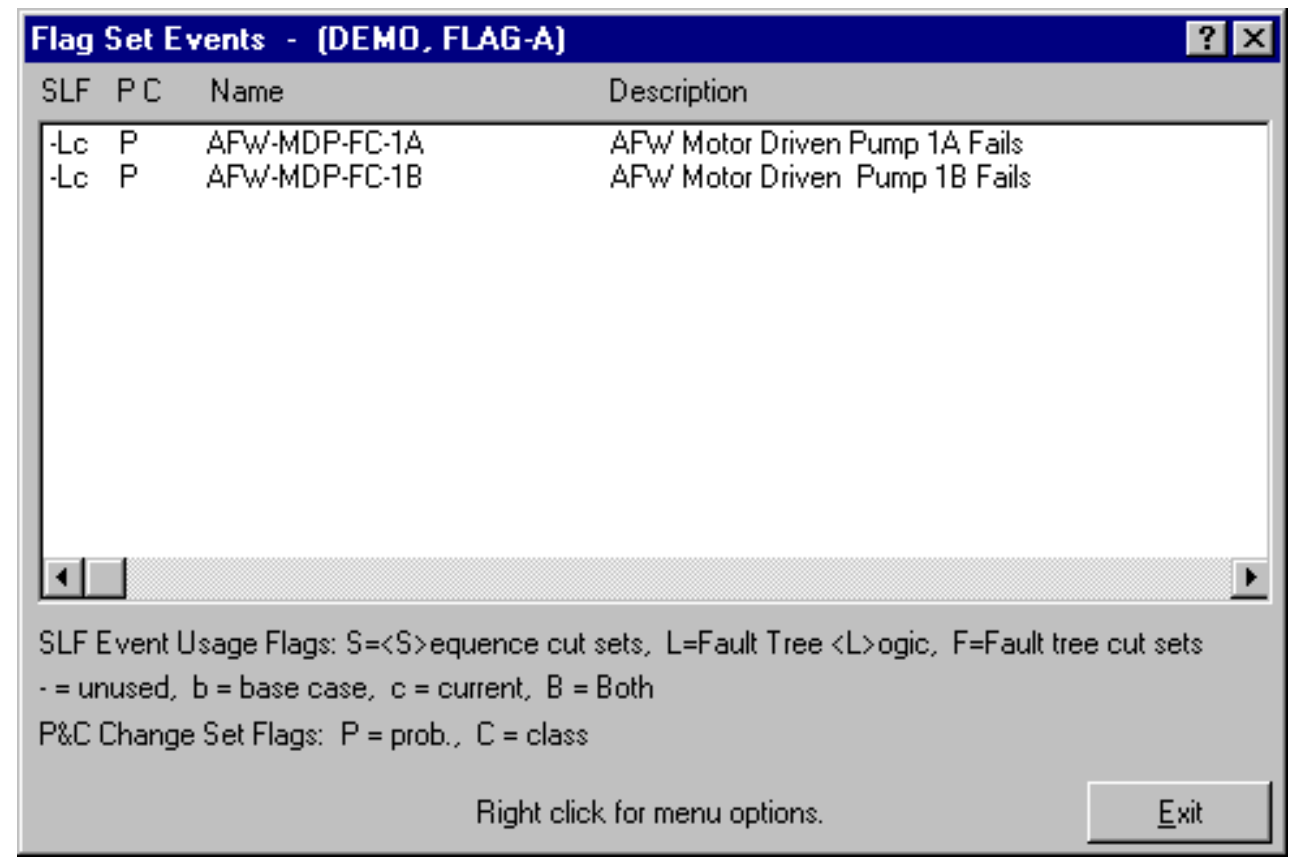

\section{Pop-up Menu Options}

Add - $\quad$ Add basic event(s) with flag changes to this flag set.

Modify - Modify basic event(s) flag changes in this flag set.

Reset - Reset changes for selected event(s) back to the base case values.

\section{Button Option}

Exit -
Closes the Flag Set Events dialog.

\subsubsection{Reset Event Data}




\section{PURPOSE}

This function allows you to reset changes for selected event(s) back to the base case values.

\section{STEPS}

1. From the SAPHIRE menu select Modify.

2. Choose Flag Sets from the menu.

3. From the Flag Sets dialog, highlight the desired flag set.

4. Choose the Flags button.

5. From the Flag Set Events dialog, highlight the desired basic event(s).

6. Right click to invoke the pop-up menu and choose the Reset option.

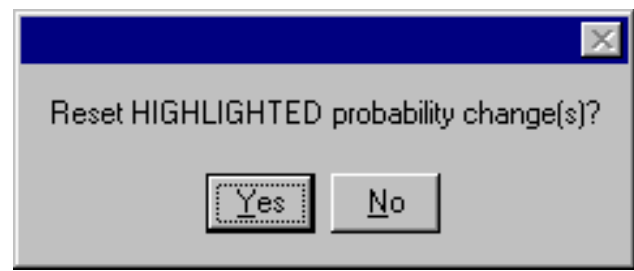

Yes - Set the changed events back to the base case values.

No - Cancel the reset operation. The changes to the basic events will remain.

\subsubsection{Add/Modify Flag Set Event Data}

\section{PURPOSE}

This function allows you to modify the event data house flag changes to the calculation type or process type. This can then be applied to specified fault trees or sequences for use during fault tree or event tree analysis. The changes applied to basic event data may be for a single event or a group of events.

\section{STEPS}

1. From the SAPHIRE menu select Modify.

2. Choose Flag Sets from the menu.

3. From the Flag Sets dialog, highlight the desired flag set.

4. Choose the Flags button.

\section{STEPS}

\section{To add event change:}

1. From the Flag Set Events dialog, right-click to invoke the pop-up menu.

2. Choose the Add option. The Select Flag Event dialog will be displayed. 


\begin{tabular}{|c|c|c|}
\hline Select Flag Event & it click for options] & $x$ \\
\hline$\langle$ FALSE $\rangle$ & System Generated Success Event & $\Delta$ \\
\hline$\langle\mathbb{N} \mid \mathrm{T}\rangle$ & System Generated Initiating Event & \\
\hline $\begin{array}{l}\langle\mathrm{PASS}\rangle \\
\langle\mathrm{TRUE}\rangle\end{array}$ & $\begin{array}{l}\text { System Generated Ignore Event } \\
\text { Sustem Generated Failure Event }\end{array}$ & - \\
\hline$A F W$ & No or Insufficient AFW' Flow & \\
\hline AFW-CST-TANK & CST system fails & \\
\hline$A F W-M D P-A-F$ & MDP train-A supercomponent & \\
\hline AFW-MDP-B-F & MDP train-B supercomponent & \\
\hline$A F W-M D P-C F-A B$ & Common Cause Failure of AFW Motor Driven Pumps & \\
\hline AFW-MDP-FC-1A & AFW Motor Driven Pump 1A Fails & \\
\hline AFW-MDP-FC-1B & AFW Motor Driven Pump 1B Fails & \\
\hline $\begin{array}{l}\text { AFW-PMP-CF-ALL } \\
\text { AFW-SUP }\end{array}$ & $\begin{array}{l}\text { Common Cause Failure of AFW Pumps (All Types] } \\
\text { AFW sustem fails }\end{array}$ & \\
\hline AFW-TDP-C-F & TDP train-A supercomponent & 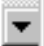 \\
\hline 11 & & 11 \\
\hline
\end{tabular}

3. Highlight the event(s) to be added to the flag set.

4. Right-click to invoke the pop-up menu and choose the Add option.

\section{STEPS}

\section{To modify event change:}

1. From the Flag Set Events dialog, highlight the desired basic event(s).

2. Right-click to invoke the pop-up menu and choose the Modify option.

If only a single event has been selected, then the Event Probability Flags dialog for single event changes will be displayed. If a group of events were selected, then the Event Probability Flags dialog for a group of events will be displayed.

The probability changes made are reflected on the Flag Set Events dialog by showing a "P" to the left of the event name.

\subsubsection{Event Probability Flags - Single}

\section{PURPOSE}

This function allows you to modify the current random failure data for the selected basic event.

\section{STEPS}

1. From the SAPHIRE menu select Modify.

2. Choose Flag Sets from the menu.

3. From the Flag Sets dialog, highlight the desired flag set. 
4. Choose the Flags button.

5. From the Flag Set Events dialog, highlight the basic event, if modifying

6. Right-click to invoke the pop-up menu and choose either Add or Modify. The Event Probability Flags dialog for a single event is displayed.

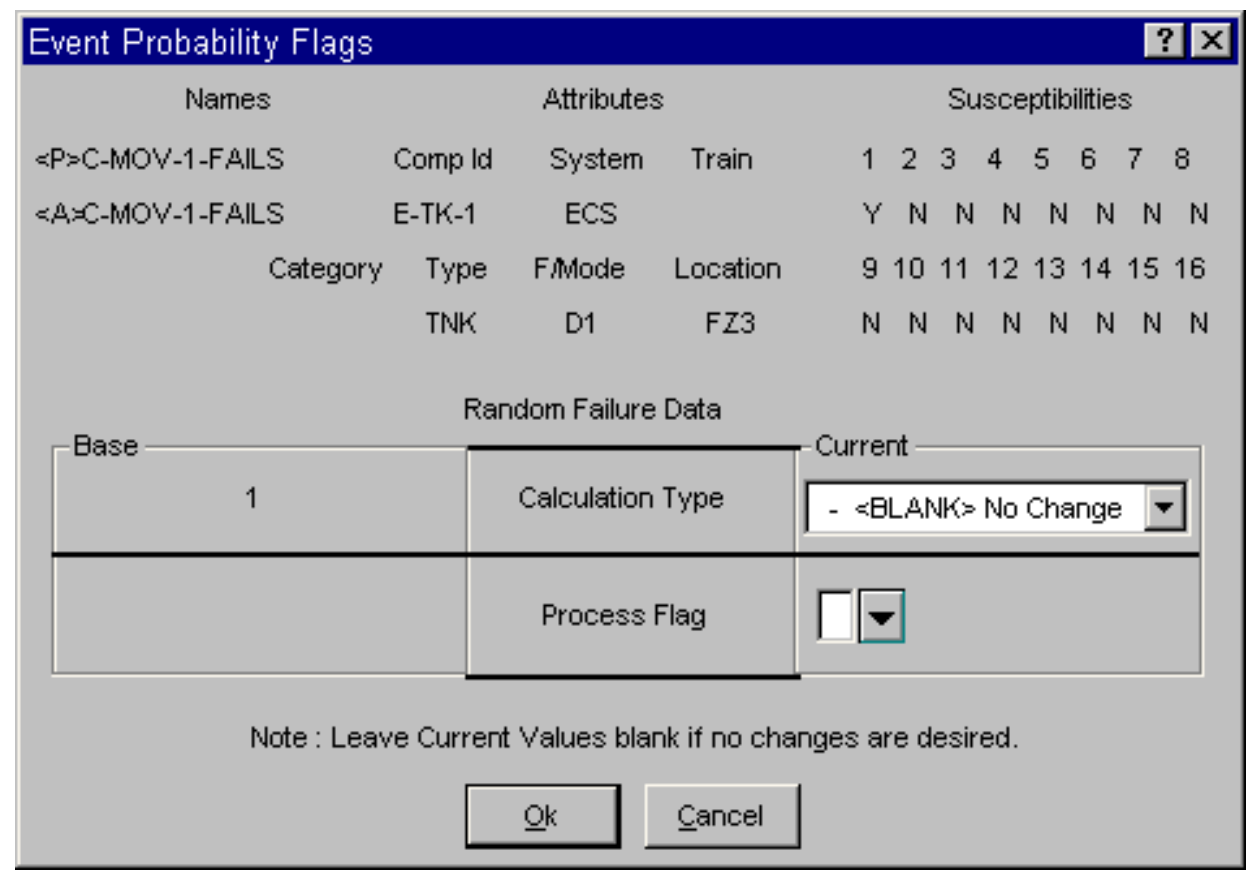

Calculation Type-Select from the drop-down list:

Blank (No Change)

$\mathrm{T}$ - House Event (failed, probability $=1.0$ )

$\mathrm{F}$ - House Event (successful, probability $=0.0$ )

I - Ignore this event (remove it from the logic)

Process Flag - Click on the down arrow button to the right of the field. The Process Flag dialog will be invoked.

Ok - $\quad$ Apply the change and close the Event Probability Flags dialog.

Cancel - $\quad$ Close the Event Probability Flags dialog without applying the change.

\subsubsection{Event Probability Flags - Group}

\section{PURPOSE}

This function allows you to modify the current random failure data for a group of selected basic events. 


\section{STEPS}

1. From the SAPHIRE menu select Modify.

2. Choose Flag Sets from the menu.

3. From the Flag Sets dialog, highlight the desired flag set.

4. Choose the Flags button.

5. From the Flag Set Events dialog, highlight the basic events, if modifying

6. Choose either the Add or Modify option. The Event Probability Flags dialog for a group of events is displayed.

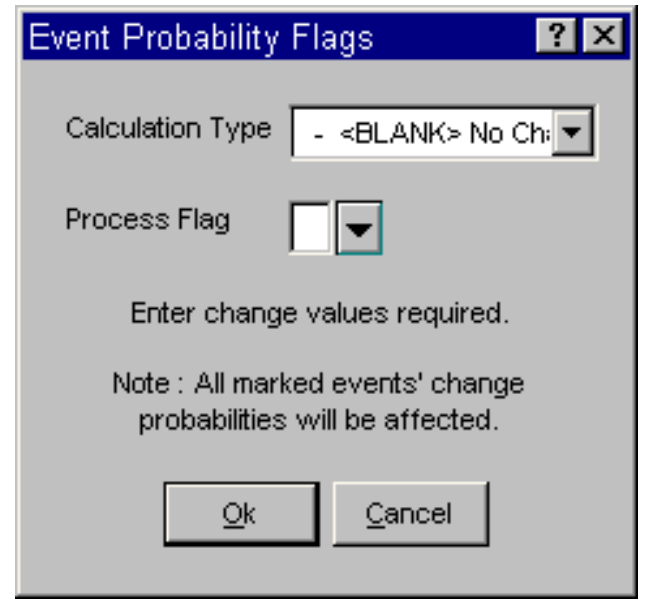

The changes entered on this dialog will be applied to all selected events.

Calculation Type-Select from the drop-down list:

Blank (No Change)

$\mathrm{T}$ - House Event (failed, probability $=1.0$ )

F - House Event (successful, probability $=0.0$ )

I - Ignore this event (remove it from the logic)

Process Flag - Click on the down arrow button to the right of the field. The Process Flag dialog will be invoked.

Ok - $\quad$ Apply the change and close the Event Probability Flags dialog.

Cancel - $\quad$ Close the Event Probability Flags dialog without applying the change. 


\section{REPORTING ON THE DATABASE}

\subsection{Reporting on the Data Base}

\section{PURPOSE}

This option allows you to obtain information about the current project. Various reports can be generated for the following data types: project, attributes, basic events, fault trees, event trees, end states, sequences, gates, histograms, and user information. The reports include options such as summary, cross-reference, and text information. The dialogs and operations are similar for each of the report data types, therefore, a generic discussion is provided here.

\section{STEPS}

1. From the SAPHIRE menu select Report. The Reports Menu dialog will be displayed.

2. From this dialog, select a specific report by choosing a radio button from each group (Data Type, Report Type, and Sub Type, if necessary).

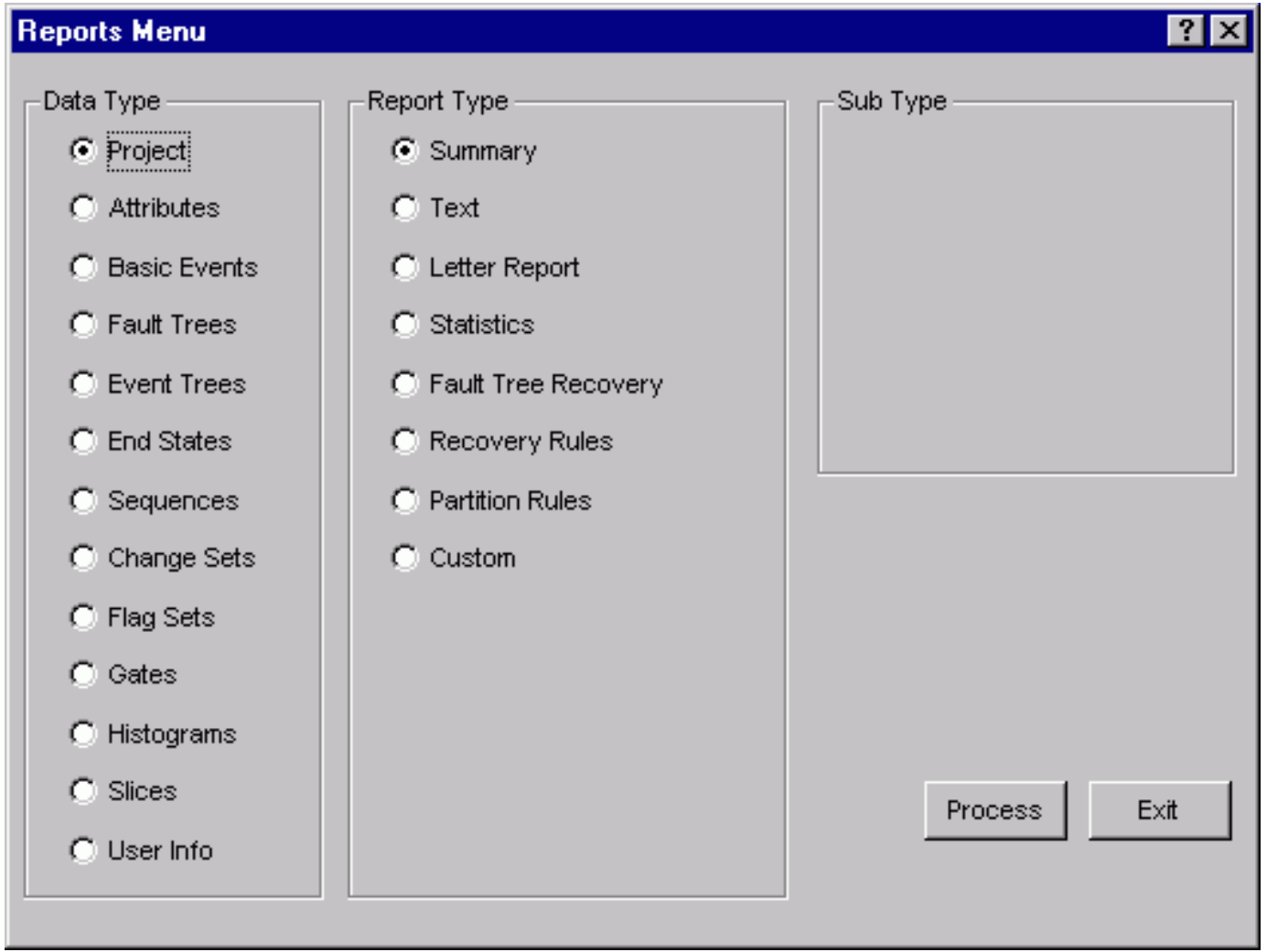

3. After a specific report has been selected, choose the Process button. A dialog containing the list of records associated with the selected report type will be displayed.

4. Select the desired records or process all records.

5. Choose the button on the dialog to generate the report. Often times this button is labeled Continue or it may be for a specific type of report, such as, Summary. The Report Viewer will display the report 
In addition to report type-specific information, each report will contain the report title and the date and time generated. Where applicable it will also contain the currently selected project name, selected case (base or current), and page numbers.

\subsection{Process All Entries}

Most of the report options supply a dialog with a list box for selecting specific records to be included in the report. If you do not highlight any of the records in the list box, and choose one of the options (with the exception of the Exit option), you will be prompted with the following dialog:

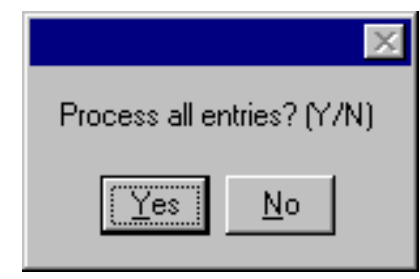

Yes - Generate the report and include all records in the current project database.

No - Do not generate the report. You will have the opportunity to select specific records from the list.

\subsection{Report Dialog}

\subsubsection{Report Output}

\section{PURPOSE}

Select a format and output destination for a previewed report.

This option allows you send a copy of the currently previewed report to an alternate destination, including a word processor (choose the Rich Text Format), browser (choose the HTML Format), or text editor (choose the Ascii Delimited format).

You may open the formatted report using the program associated with selected format option (Print Preview destination), send the formatted report directly to your default Windows printer (Print (Default Printer) destination), or send it directly to a file (File destination).

\section{NOTE}

SAPHIRE will use the default application associated with the selected format $(R T F, H T M L$, or Ascii/TXT) to preview or print the report. The Print Preview option will behave the same way as if you selected the File destination, and then double-clicked the resulting file from Windows Explorer.

To successfully use the RTF option, the word processor associated with the RTF extension must support RTF tables. Word processors such as Word and WordPerfect support both RTF and RTF tables, while WordPad may support basic RTF, but not RTF tables. If you do not have a word processor that supports RTF tables, you should select a different format. If you have a word processor that you know supports RTF tables, but SAPHIRE is not using that application to preview the RTF report, see your Windows 
documentation or system adminstrator for information on how to associate that word processor with the RTF file format.

Similarly, HTML formatted reports will be previewed using your system specified browser (usually Internet Explorer or Netscape); and Ascii delimited reports will be previewed using the default application associated with TXT files.

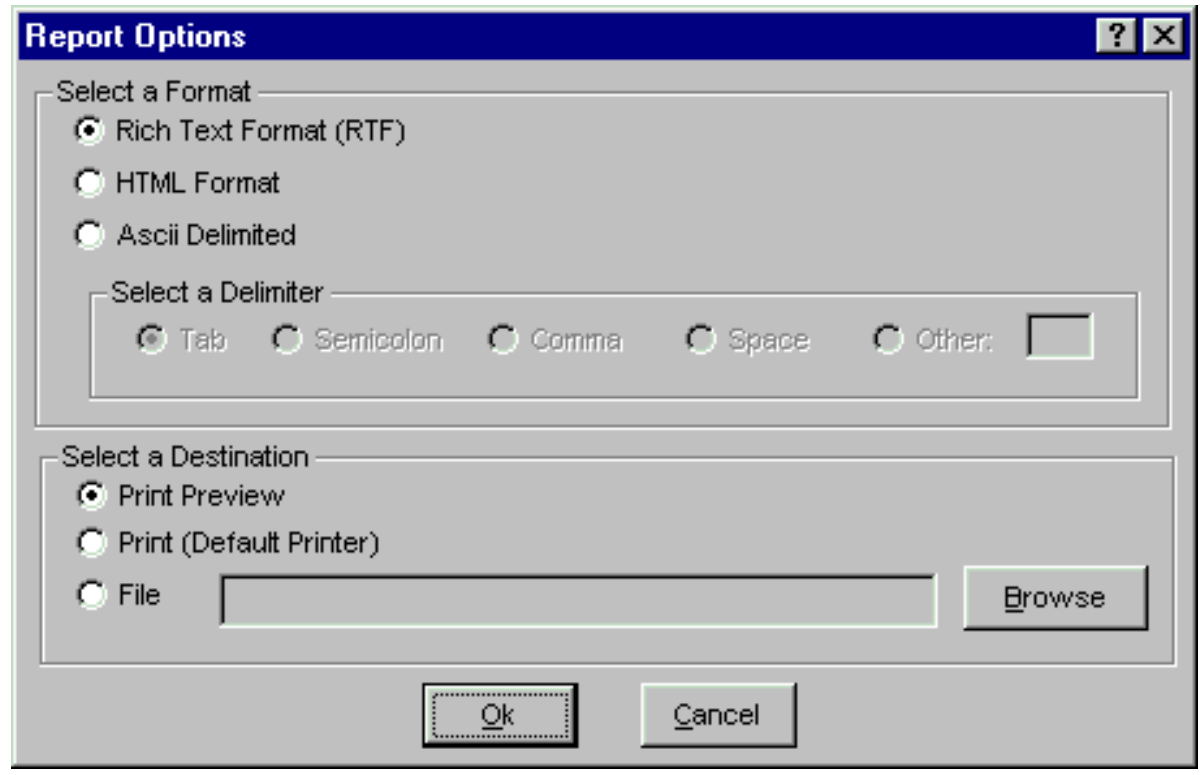

Select a Format

Rich Text Format (RTF) -

HTML Format Ascii Delimited -

Select a Delimiter -

Select a Destination

Print Preview -

Printer (Default Printer) -

File -

Browse -
Format the report data as RTF. This option is selected by default.

Format the report data as HTML.

Format the report data as Ascii text. Each column will be separated by the character indicated by the Select $a$ Delimiter option.

For the Ascii Delimited format, select Tab, Semicolon, Space, or Other as the column separator character. If Other is selected, indicate the character to use in the adjacent text box.

Show the report, using the default viewer for the selected format. This option is selected by default.

Print the report in the selected format directly to your Windows system default printer.

Save the report to a file. If the path is not included with the file name, the report file will be saved in the current project directory.

Open the Select Directory dialog to choose a directory.

OK -

Generate the report. 
Cancel -

Close the Report dialog without generating a report.

\subsubsection{Using the Report Viewer}

\subsubsection{Report Viewer}

\section{PURPOSE}

The Standard Report window allows you to scroll through data generated by selected report options.

\section{STEPS}

1. From one of the Report Menu options, choose the desired data type, report type, and, if available, sub type.

2. A list of the desired data type items (i.e., Basic Events, Fault Trees, etc), will appear in a list dialog. Select the items you want to appear in the report and choose the Continue button. The Standard Report window will be invoked.

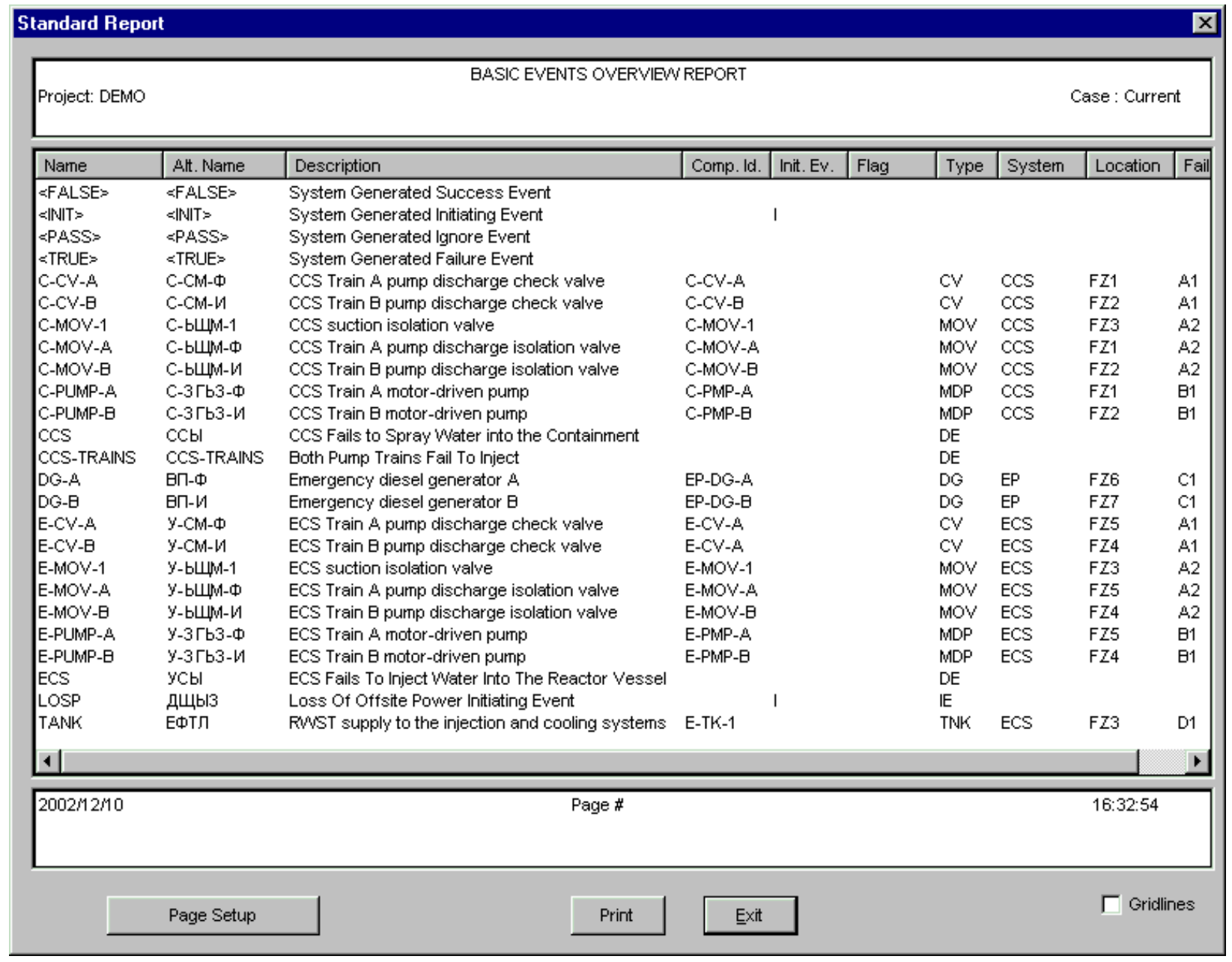

Columns may be resized by positioning the mouse cursor over the border between two column headings, clicking and dragging the cursor left or right to expand or contract the display width of the report. 
Page Setup - $\quad$ Open the Report Settings dialog to specify the desired report settings, such as fonts and margins.

Print - $\quad$ Open the Report Options dialog to send a copy of the report to a printer, file, or other application.

Exit Close the Standard Report window

Gridlines - $\quad$ Checked and unchecked, this option displays grid lines between each cell in the report.

\subsubsection{Print Dialog}

\section{PURPOSE}

Set printing options, such as, printer, page range, number of copies, etc. before printing the current file.

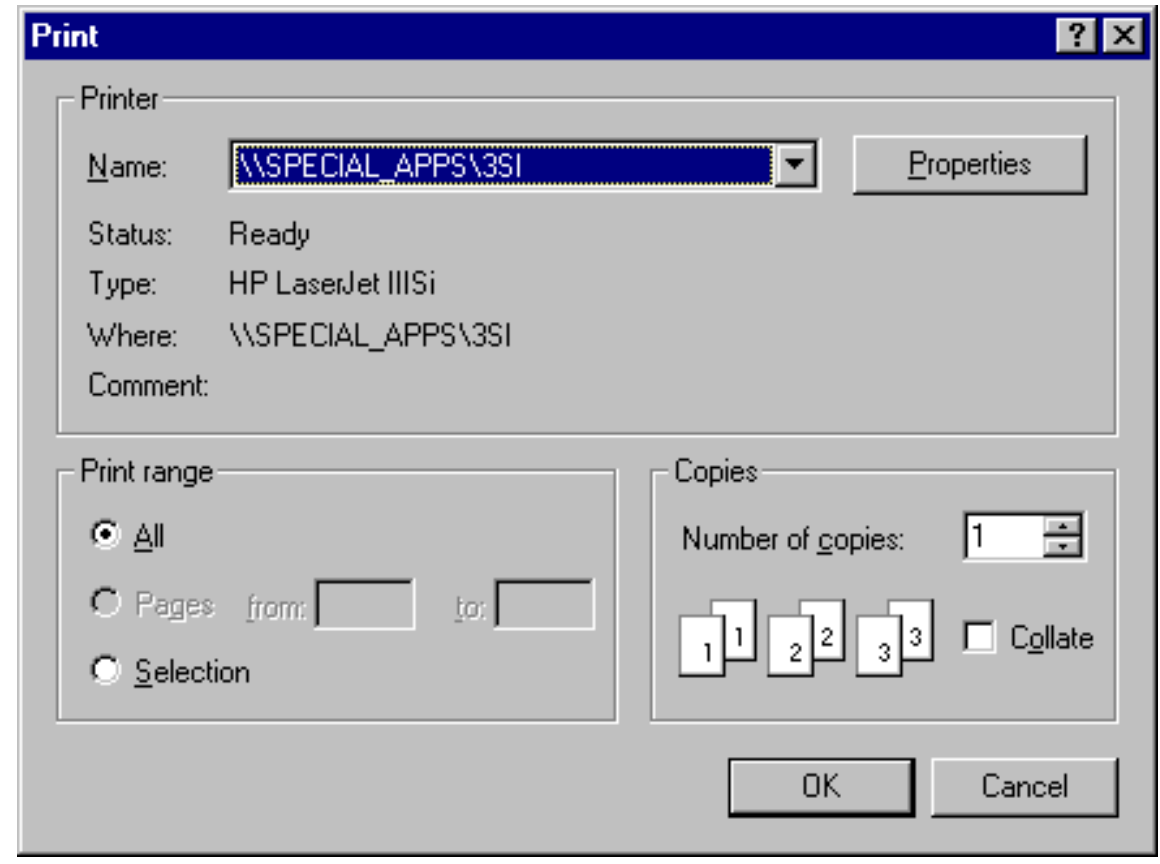

\footnotetext{
Name - $\quad$ List the printers that are set up for this computer.

Properties - $\quad$ Set up options for the selected printer. Available options depend on the features of the printer.

Status - $\quad$ Condition of the selected printer.

Type - $\quad$ Kind of selected printer.

Where - $\quad$ Location of the selected printer.

Comment - $\quad$ Miscellaneous information about the selected printer.

Print Range - Print the entire document, specified range, or highlighted selection.

Copies - $\quad$ Enter the number of copies you wish to print.

Collate - $\quad$ For more than one copy, specify whether you want the copies collated.
}

\subsubsection{Report Settings}




\section{PURPOSE}

This option allows you to modify general characteristics of a report.

The Report Viewer allows you to inspect the contents of the report, but does not reflect the settings available here. When a report is printed, via the Report Options dialog, an output format is selected, and where applicable, the output format will honor the settings selected here. Note that not all settings are applicable to each of the report formats available. For example, HTML output will utilize font information, but not utilize paper or orientation settings. ASCII format will not utilize the paper, margin, orientation, or font information.

\section{STEPS}

1. From one of the Report Menu options, choose the desired data type, report type, and, if available, sub type.

2. A list of the desired data type items (i.e., Basic Events, Fault Trees, etc), will appear in a list dialog. Select the items you want to appear in the report and choose the Continue button.

3. The Standard Report window will be invoked.

4. Press the Report Settings button. 


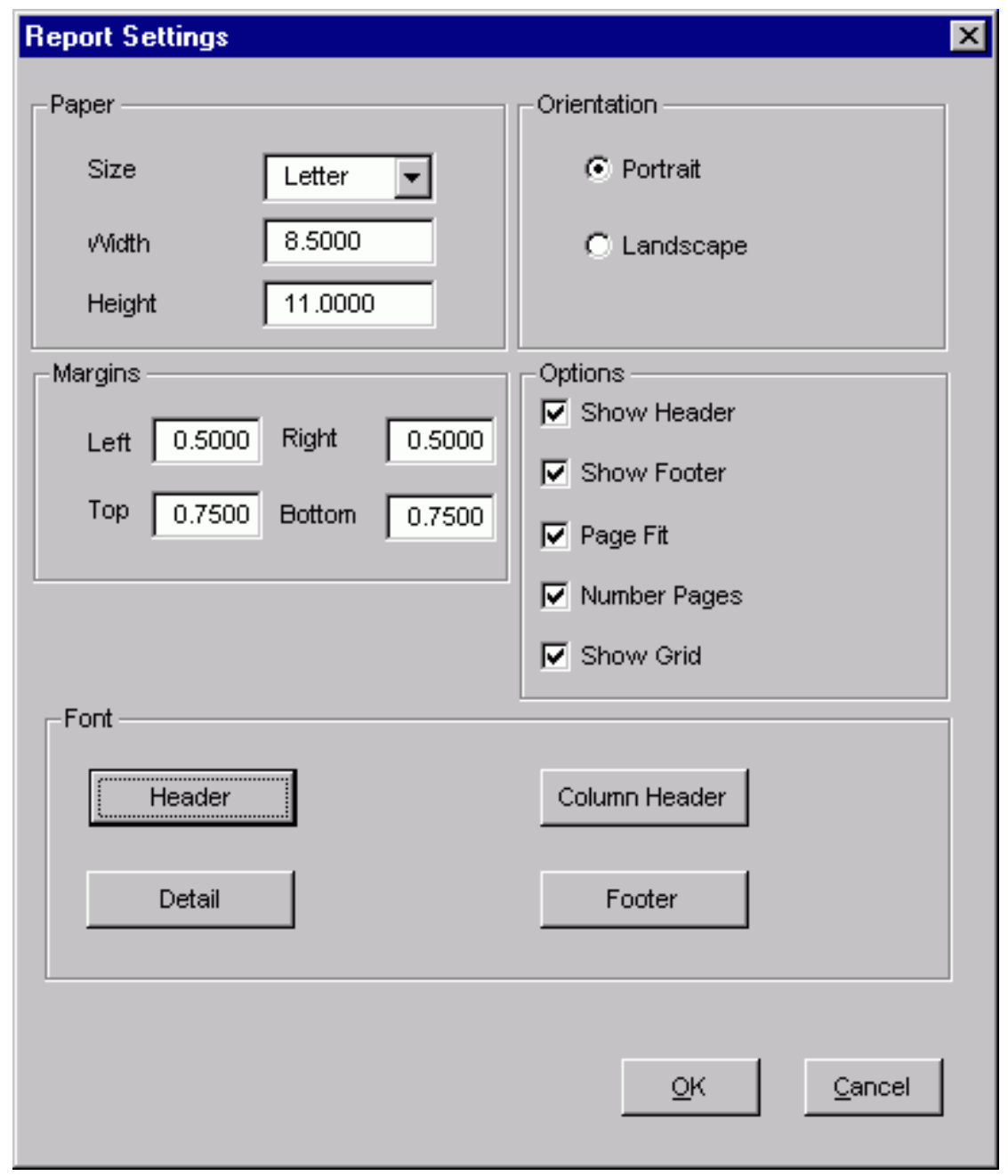

Paper - $\quad$ Set the desired paper size, height, and width.

Orientation - Select the desired paper orientation.

Options - $\quad$ Turn the header, footer, page numbering, page fit, and grid options on (checked) or off (unchecked).

Margins - $\quad$ Set the left, right, top, and bottom margins for the report.

Font - $\quad$ Select the font characteristics for the header, column header, detail, and footer areas of the report.

Ok -

Apply the selected settings to the report, and return to the report viewer.

Cancel - $\quad$ Return to the report viewer without changing any report settings.

\subsubsection{Report Options}

\section{PURPOSE}

This option sends a copy of the current report to a printer, file, or other application. The report can be sent in a variety of formats, including rich text format (RTF), HTML, and ASCII. 
The Print Preview option sends the report data to an external editor or viewer such as Microsoft Word or Internet Explorer, where you may then manipulate the report using the receiving application's native features. The Print (Default Printer) option uses the same application as the print preview option to print the report directly to your default printer. The File option sends the report data in the selected format directly to a file of your choosing. Any software application that supports that format can then be used to view or edit the report.

Note: In order to print or preview data in a particular format, you must have software installed on your computer that supports that format. To print or preview RTF data, you must have an editor or viewer that supports the RTF file format, such as Microsoft Word, WordPerfect, or WordPad. Likewise, to print or preview HTML data, you must have software such as Internet Explorer or Netscape. For ASCII data, you must have Notepad, WordPad, or another text editor available. The typical Windows operating system installation includes basic software to support each of the given file types.

If your system includes multiple software applications that support a particular format, SAPHIRE will invoke the program associated by default with that format's file extension. (The ASCII text format has a TXT file extension.) To access the report data via an application other than the default association, save the report data to a file destination, and then use the desired application to open the file. To change the default association of a file extension, see your Windows help system, or contact your help desk.

\section{STEPS}

1. From one of the Report Menu options, choose the desired data type, report type, and, if available, sub type.

2. A list of the desired data type items (i.e., Basic Events, Fault Trees, etc), will appear in a list dialog. Select the items you want to appear in the report and choose the Continue button.

3. The Standard Report window will be invoked.

4. Press the Print button.

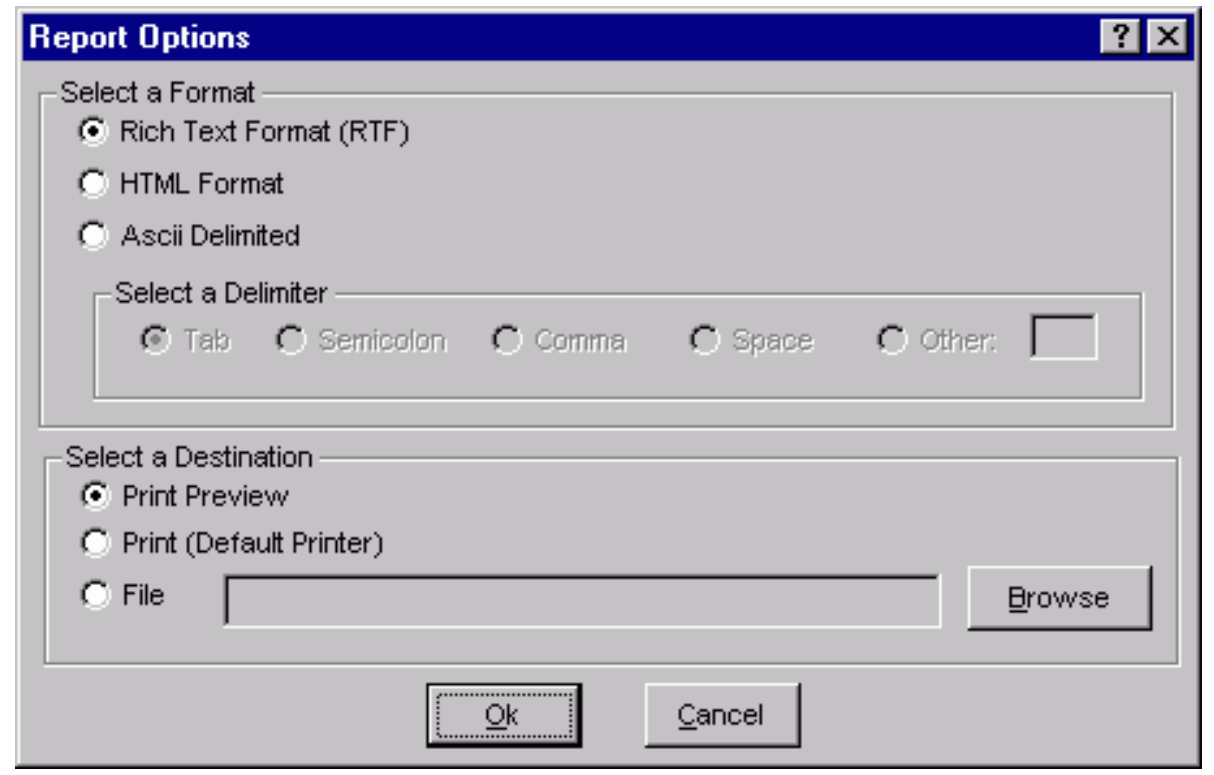




\begin{abstract}
Select a Format
Rich Text Format (RTF)- Output the report to the selected destination in RTF format. HTML Format - $\quad$ Output the report to the selected destination in HTML format. ASCII Delimited - $\quad$ Output the report to the selected destination in ASCII format. Within each line of data, items will be separated by the selected delimiter.
\end{abstract}

Select a Delimiter -

Select a Destination

Print Preview -

Print (default printer) -

File -

Browse -
When ASCII formatted output is selected, use this option to select the report cell delimiter character. Choose from commonly used delimiters such as tab, semicolon, comma, or space, or select Other and type the desired ASCII character delimiter into the adjacent field.

Preview the report in the selected output format. SAPHIRE will invoke whatever program your system has associated with the selected file type.

Send the report in the selected output format directly to your system's default printer. Hint: If you prefer to send the report to a printer that is not the default, choose the Print Preview option, and print from the associated application.

Send the report output in the selected format directly to the file indicated in the adjacent field.

Choose the folder and file name to send the report to when the File destination is selected.

\title{
10.4 Custom Reports Dialog
}

\subsubsection{Custom Reports}

\section{PURPOSE}

This option allows you to obtain information about the selected data type (e.g., project, fault tree, basic event, etc.) on an ad-hoc basis. Multiple ad-hoc reports can be created and saved in the SAPHIRE database.

The dialogs and operations are similar for each of the report data types, therefore, a generic discussion is provided here.

\section{STEPS}

1. From the SAPHIRE menu select Report. The Reports Menu dialog will be displayed.

2. Select the desired Data Type and Custom Report Type radio buttons.

3. Choose the Process button. The Custom Reports dialog will be displayed with a list of all existing custom reports for the selected data type. 


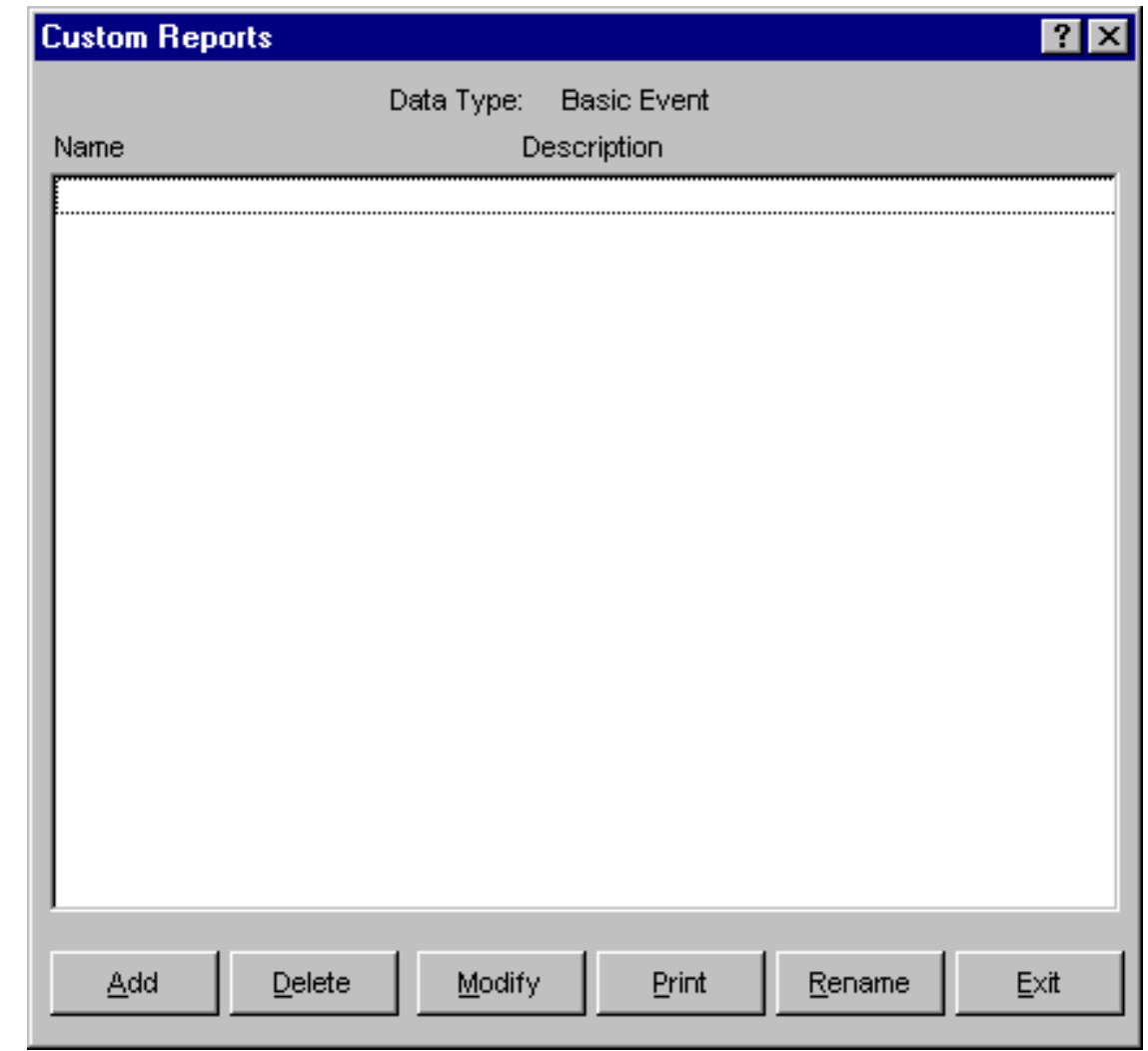

Add - Create a new custom report record.

Delete - Delete an existing custom report record.

Modify - $\quad$ Edit an existing custom report record. When you choose this button, the Custom Report Field Selection dialog will be displayed.

Print - View the selected custom report. When you choose this button, the Report Viewer is invoked.

Rename - $\quad$ Rename the existing custom report record.

Exit - $\quad$ Close the Custom Reports dialog.

\subsubsection{Add Custom Report}

\section{PURPOSE}

Create a new custom report record in the SAPHIRE database.

\section{STEPS}

1. From the SAPHIRE menu select Report. The Reports Menu dialog will be displayed.

2. Select the desired Data Type and Custom Report Type radio buttons.

3. Choose the Process button. The Custom Reports dialog will be displayed listing all existing custom reports for the selected data type.

4. Choose the Add button. The Add New Custom Report dialog will be displayed. 


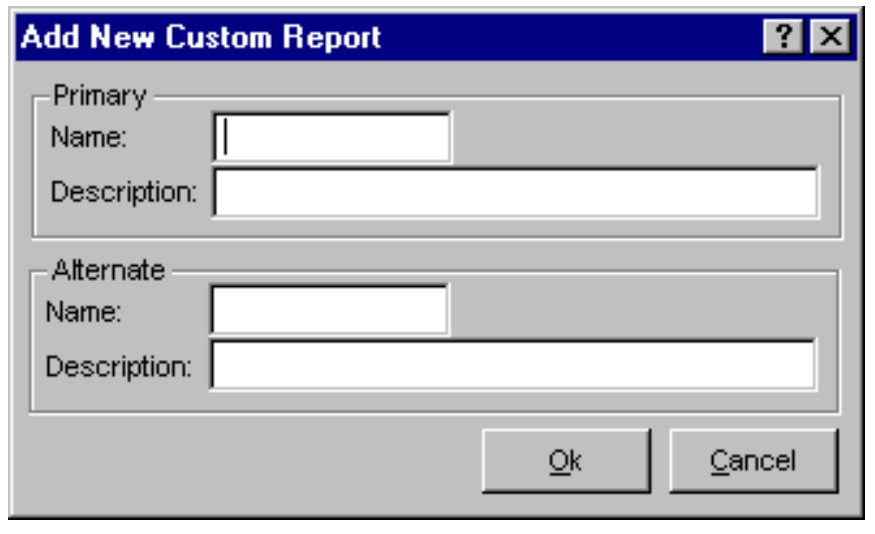

Primary Name - $\quad$ Required field. A maximum of 8 uppercase, alphanumeric may be entered to uniquely identify the report. The name should be descriptive so that the report can be readily identified. The name must be unique for the selected data type, but can be duplicated in other data types. For example you could have a report named "MYREPORT" for the Fault Tree data type and a report with the same name for the basic event data type.

Primary Description - This is a 30-character, uppercase or lowercase, alphanumeric field that provides brief descriptive information. Data entry in this field is optional.

Alternate Name -

Optional field. A maximum of 8 uppercase, alphanumeric characters may be entered. Embedded blanks are not allowed. If a name is not entered, the primary name will be copied to this field. This feature allows names to be displayed and reported using a different naming scheme or another locale. For this name to be displayed in lists and on reports instead of the Primary name, choose Use alternate names for display in the Define Constants option.

Alternate Description - This is a 30-character, uppercase or lowercase, alphanumeric field that provides brief, descriptive information. This feature allows descriptions to be displayed and reported using a different naming scheme or another locale. For this description to be displayed in lists and on reports instead of the Primary description, choose Use alternate names for display in the Define Constants option.

Ok -

Add the report record to the database. Upon pressing this button the Custom Report Field Selection dialog will be displayed. In order for this record to be added to the database, you must choose the Save button when complete.

Cancel - $\quad$ Close the Add New Custom Report dialog without adding a new record. 


\subsubsection{Delete Custom Report}

\section{PURPOSE}

Delete the selected custom report record from the SAPHIRE database.

\section{STEPS}

1. From the SAPHIRE menu select Report. The Reports Menu dialog will be displayed.

2. Choose the desired Data Type and Custom Report Type radio buttons.

3. Choose the Process button. The Custom Reports dialog will be displayed listing all existing custom reports for the selected data type.

4. Highlight the desired report.

5. Choose the Delete button. The Confirm Report Delete dialog will be displayed.

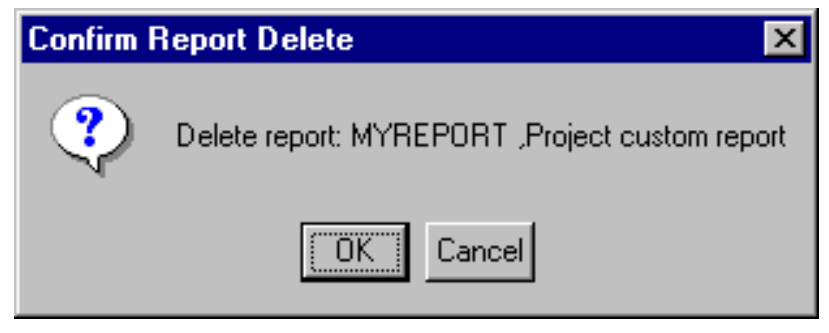

Ok - Delete the report record and close the Confirm Report Delete dialog.

Cancel - Close the Confirm Report Delete dialog without deleting the report.

\subsubsection{Rename Custom Report}

\section{PURPOSE}

Change the name or description of the selected custom report.

\section{STEPS}

1. From the SAPHIRE menu select Report. The Reports Menu dialog will be displayed.

2. Choose the desired Data Type and Custom Report Type radio buttons.

3. Choose the Process button. The Custom Reports dialog will be displayed listing all existing custom reports for the selected data type.

4. Highlight the desired report.

5. Choose the Rename button. The Rename Custom Report dialog will be displayed. 


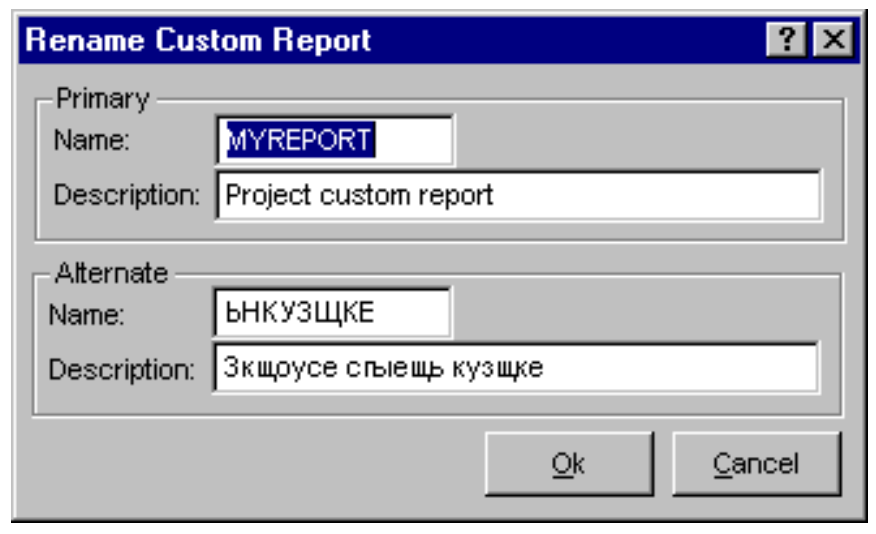

Primary Name - $\quad$ Required field. A maximum of 8 uppercase, alphanumeric may be entered to uniquely identify the report. The name should be descriptive so that the report can be readily identified. The name must be unique for the selected data type, but can be duplicated in other data types. For example you could have a report named "MYREPORT" for the Fault Tree data type and a report with the same name for the basic event data type.

Primary Description - This is a 30-character, uppercase or lowercase, alphanumeric field that provides brief descriptive information. Data entry in this field is optional.

Alternate Name -

Optional field. A maximum of 8 uppercase, alphanumeric characters may be entered. Embedded blanks are not allowed. If a name is not entered, the primary name will be copied to this field. This feature allows names to be displayed and reported using a different naming scheme or another locale. For this name to be displayed in lists and on reports instead of the Primary name, choose Use alternate names for display in the Define Constants option.

Alternate Description - This is a 30-character, uppercase or lowercase, alphanumeric field that provides brief, descriptive information. This feature allows descriptions to be displayed and reported using a different naming scheme or another locale. For this description to be displayed in lists and on reports instead of the Primary description, choose Use alternate names for display in the Define Constants option.

Ok -

Save the changes made to the report name and/or description.

Cancel Close the Rename Custom Report dialog without changing the record. 


\subsubsection{Custom Report Field Selection Dialog}

\subsubsection{Custom Report Field Selection Dialog}

\section{PURPOSE}

This option allows you to add or remove fields in the custom report. The field header names may also be modified.

\section{STEPS}

1. From the SAPHIRE menu select Report. The Reports Menu dialog will be displayed.

2. Choose the desired Data Type and Custom Report Type radio buttons.

3. Choose the Process button. The Custom Reports dialog will be displayed listing all existing custom reports for the selected data type.

4. Choose the Add or Modify button. The Custom Report Field Selection dialog will be displayed.

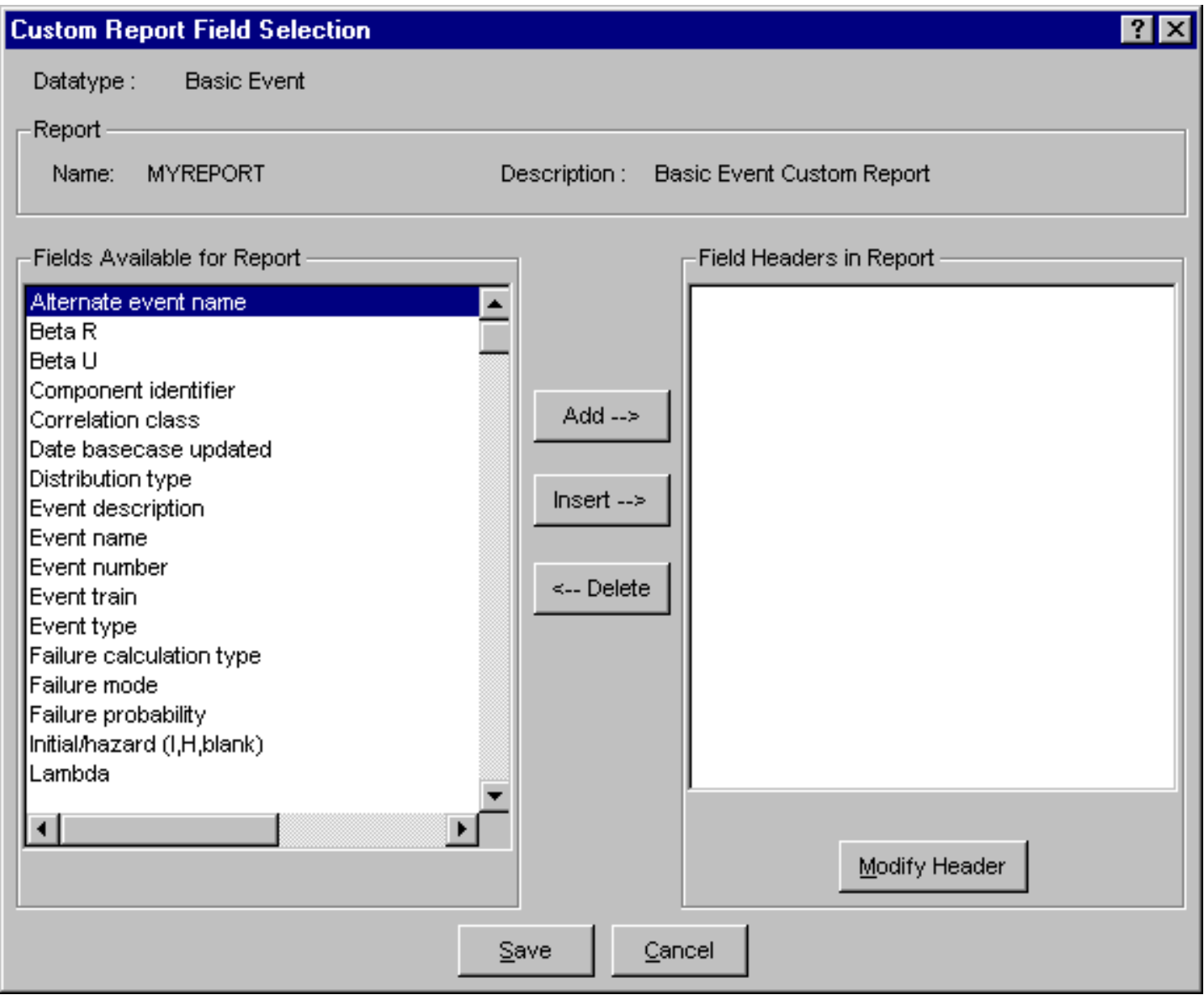

Fields Available for Report - $\quad$ List of all fields available for inclusion in the report, based on the selected data type. 
Field Headers in Report -

Add -

Insert -

Delete -

Modify Header -

Save -

Cancel -
List of fields currently included in the report. The order of the headers and the header name displayed in this list will be used for generating the report.

The highlighted field in the Fields Available list will be included in the report. The selected field's report header will be displayed at the bottom of the Field Headers list.

The highlighted field in the Fields Available list will be inserted in front of the currently highlighted header in the Field Headers list.

Remove the highlighted header from the Field Headers list. Change the name of the highlighted header.

Save the changes made and close the Custom Report Field Selection dialog.

Close the Custom Report Field Selection dialog without saving changes. When adding a new report, if you choose this button, the report record will not be saved in the database.

\subsubsection{Change Field Header Dialog}

\section{PURPOSE}

This option allows you to modify the selected field header name.

\section{STEPS}

1. From the SAPHIRE menu select Report. The Reports Menu dialog will be displayed.

2. Choose the desired Data Type and Custom Report Type radio buttons.

3. Choose the Process button. The Custom Reports dialog will be displayed listing all existing custom reports for the selected data type.

4. Choose the Add or Modify button. The Custom Report Field Selection dialog will be displayed.

5. Highlight an item in the Field Headers in Report list.

6. Choose the Modify Header button.

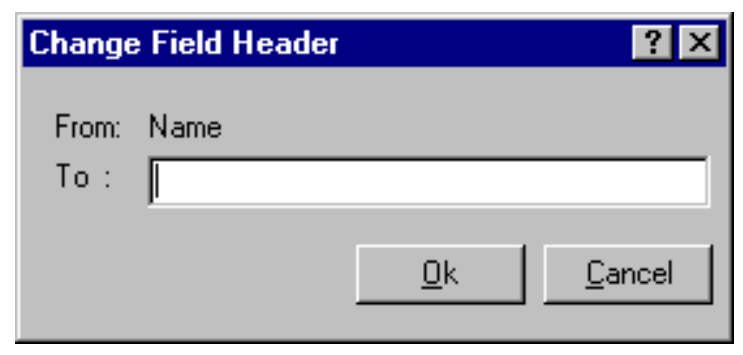

From - The current field header.

To - $\quad$ Enter the new field header.

Ok - $\quad$ Save the modification and close the Change Field Header dialog.

Cancel - Close the Change Field Header without saving the modification. 


\subsection{Project Reports Dialog}

\subsubsection{Project Reports}

\section{PURPOSE}

This option allows you to generate a variety of project reports.

\section{STEPS}

1. From the SAPHIRE menu select Report. The Reports Menu dialog will be displayed.

2. Select the Project Data Type and the desired Report Type radio buttons.

3. Choose the Process button. The Report Viewer will display the report.

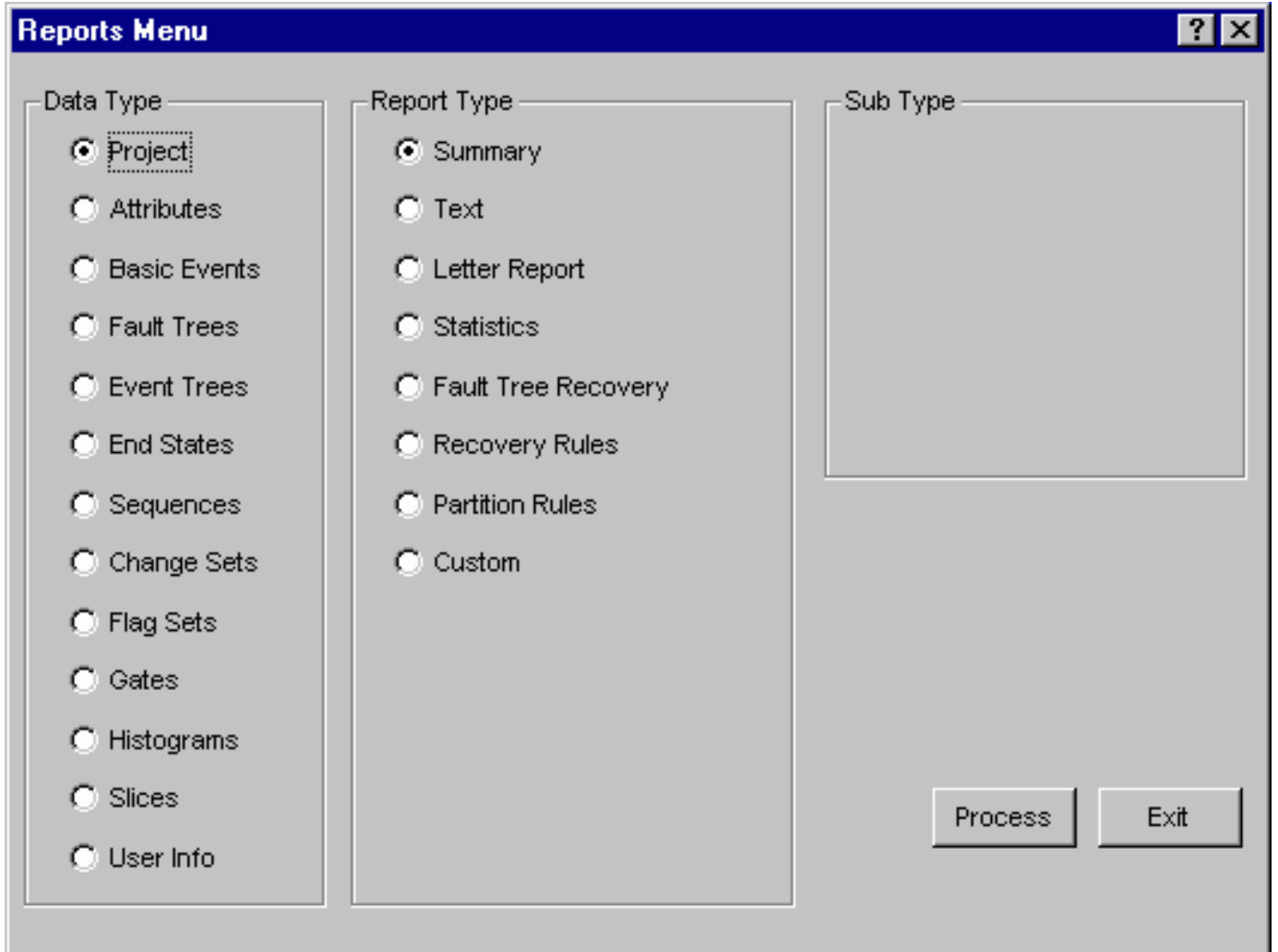

Report Type

Summary -

Text -

Letter Report -

Statistics -
Generate a summary report listing the current project name and its associated description.

Generate a report containing the descriptive text associated with the selected project.

Generate a report containing the contents of the README.DOC file in the current project's directory.

This report contains the total number of fault trees, subtrees, basic events, gates, attributes, event trees, sequences, end states, histograms, P\&IDs, and change sets defined for the current project. 
Fault Tree Recovery - This report outputs a listing of the recovery rule logic that applies to all fault trees, project-wide. Choose the Basic or Advanced radio button to indicate the desired rule type.

Recovery Rules - $\quad$ This report outputs a listing of the recovery rule logic that applies to all sequences, project-wide. Choose the Basic or Advanced radio button to indicate the desired rule type.

Partition Rules - $\quad$ This report outputs a listing of the partition rule logic that applies to all sequences, project-wide. Choose the Basic or Advanced radio button to indicate the desired rule type.

Custom - $\quad$ Create or select a user-defined report relating to project level descriptive information.

Process - $\quad$ Display the selected report.

Exit - $\quad$ This option closes the Reports Menu dialog.

\subsection{Attribute Reports}

\subsubsection{Attributes}

\section{PURPOSE}

This option allows you to generate a variety of attribute summary reports. These include reports based on system, location, failure mode, basic event type, and train attributes. Each report contains the name and description for all records of the specified attribute type in the current project.

\section{STEPS}

1. From the SAPHIRE menu select Report. The Reports Menu dialog will be displayed.

2. Select the Attributes Data Type and one of the Report Type radio buttons.

3. Choose the Process button. The Report Viewer will display the report. 


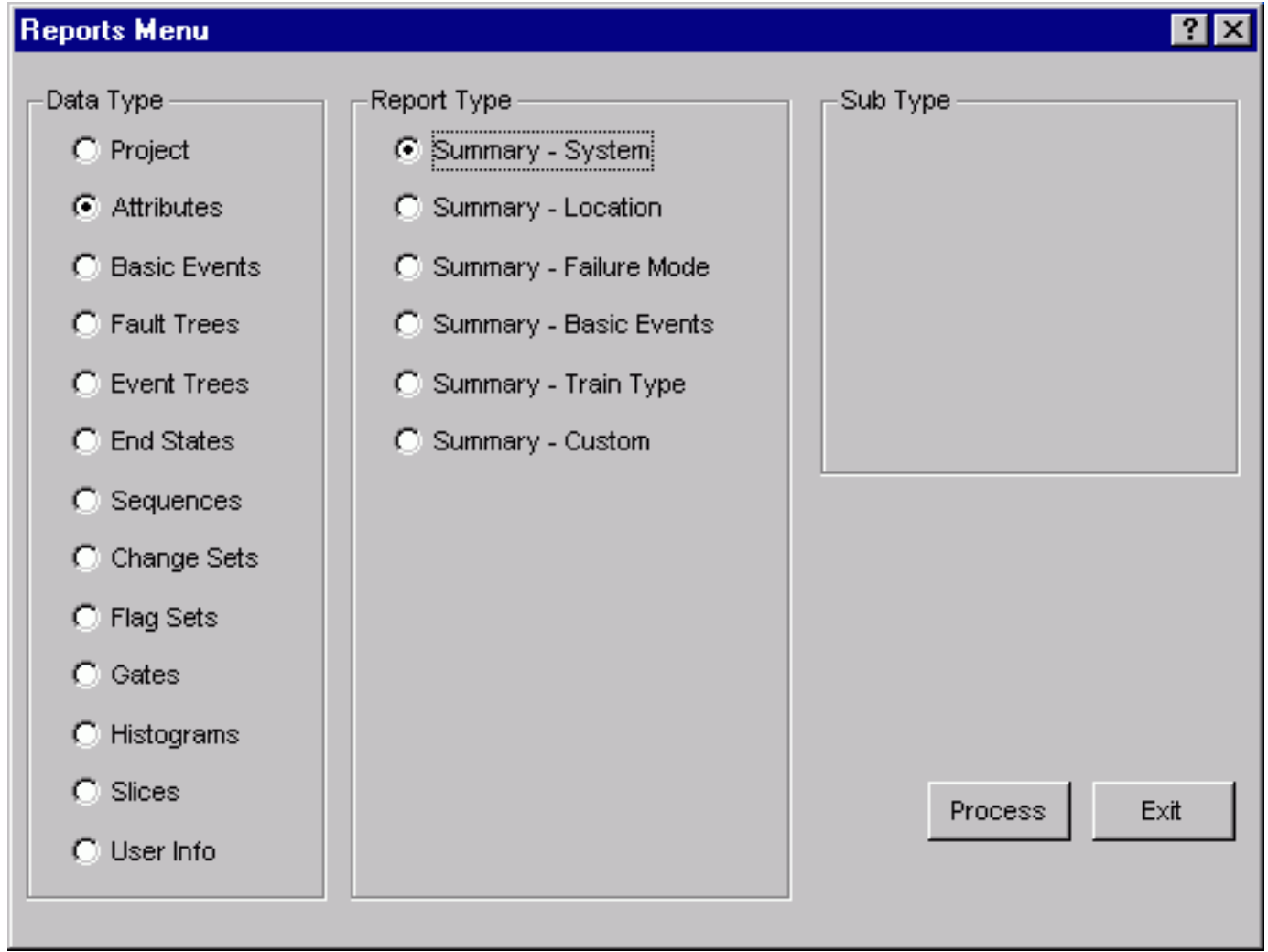

Process - Display the selected report.

Exit - $\quad$ This option closes the Reports Menu dialog.

\subsection{Basic Event Reports}

\subsubsection{Basic Events}

\section{PURPOSE}

The basic event report option allows you to generate overview, probabilities, uncertainty data, seismic data, transformation data, compound event data, template information, text, and cross reference reports.

\section{STEPS}

1. From the SAPHIRE menu select Report. The Reports Menu dialog will be displayed.

2. Select the Basic Events Data Type and one of the Report Type radio buttons.

3. Choose the Process button. A list of the basic events in the project will be displayed.

4. Highlight the desired basic event(s).

5. Choose the appropriate buttons to generate the report. The Report Viewer will display the report. 


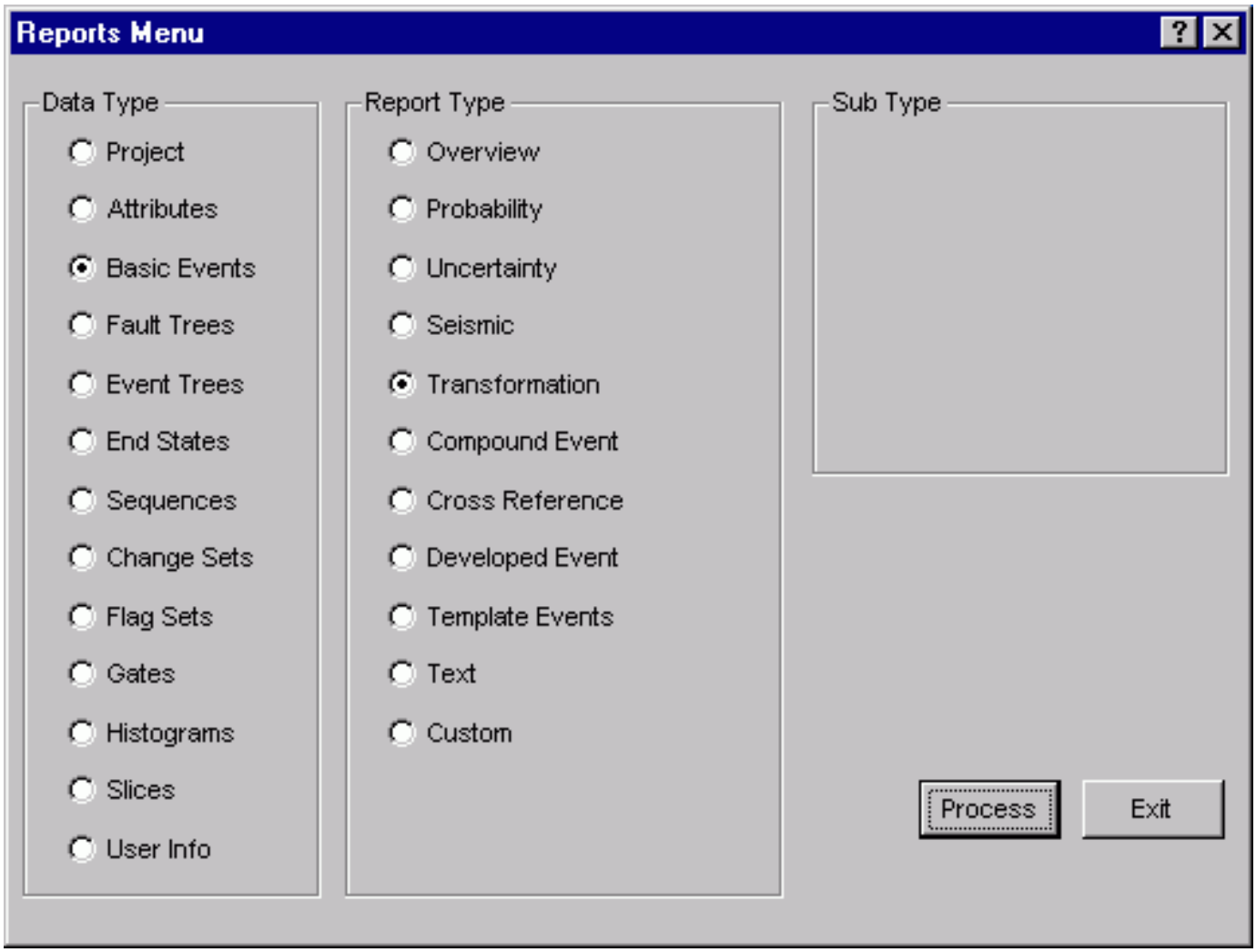

\subsubsection{Basic Event Overview}

\section{PURPOSE}

This option generates a basic event summary report. The overview report includes the basic event number, primary and secondary name, component type and ID, system location, and attribute fail mode.

\section{STEPS}

1. From the SAPHIRE menu select Report. The Reports Menu dialog will be displayed.

2. Select the Basic Events Data Type and the Overview Report Type radio buttons.

3. Choose the Process button. The Event Overview dialog will be displayed.

4. Highlight the desired basic event(s) or process all records.

5. Choose the Continue button. The Report Viewer will display the report. 


\begin{tabular}{|c|c|c|c|}
\hline Event Overvi & & & $x$ \\
\hline Name & Description & & \\
\hline $\begin{array}{l}\langle\text { FALSE } \\
\langle I N I T\rangle \\
\langle\text { PASS } \\
\text { 〈TRUE> } \\
\text { C-CV-A } \\
\text { C-CV-B } \\
\text { C-MOV-1 } \\
\text { C-MOV-A } \\
\text { C-MOV-B } \\
\text { C-PUMP-A } \\
\text { C-PUMP-B } \\
\text { CCS } \\
\text { DG-A }\end{array}$ & $\begin{array}{l}\text { System Generated Success Event } \\
\text { System Generated Initiating Event } \\
\text { System Generated lgnore Event } \\
\text { System Generated Failure Event } \\
\text { CCS Train A pump discharge check valve } \\
\text { CCS Train B pump discharge check valve } \\
\text { CCS suction isolation valve } \\
\text { CCS Train A pump discharge isolation valve } \\
\text { CCS Train B pump discharge isolation valve } \\
\text { CCS Train A motor-driven pump } \\
\text { CCS Train B motor-driven pump } \\
\text { Developed Event } \\
\text { Emergency diesel generator A }\end{array}$ & & $\bar{E}$ \\
\hline & Continue & Exit & \\
\hline
\end{tabular}

Continue - $\quad$ Generate the basic event summary report. The Report Viewer will be displayed. Exit Close the Event Overview dialog without generating a report.

\subsubsection{Basic Event Probability Report}

\section{PURPOSE}

This option allows you to generate a basic event probability report. The probability report shows the event number, primary name, failure calculation type, mean probability, and event lambda and tau values for selected events.

\section{STEPS}

1. From the SAPHIRE menu select Report. The Reports Menu dialog will be displayed.

2. Select the Basic Events Data Type and the Probability Report Type radio buttons.

3. Choose the Process button. The Event Probability dialog will be displayed.

4. Highlight the desired basic event(s) or process all records.

5. Select the desired Case radio button.

6. Choose Probability or Description button. The Report Viewer will display the report. 


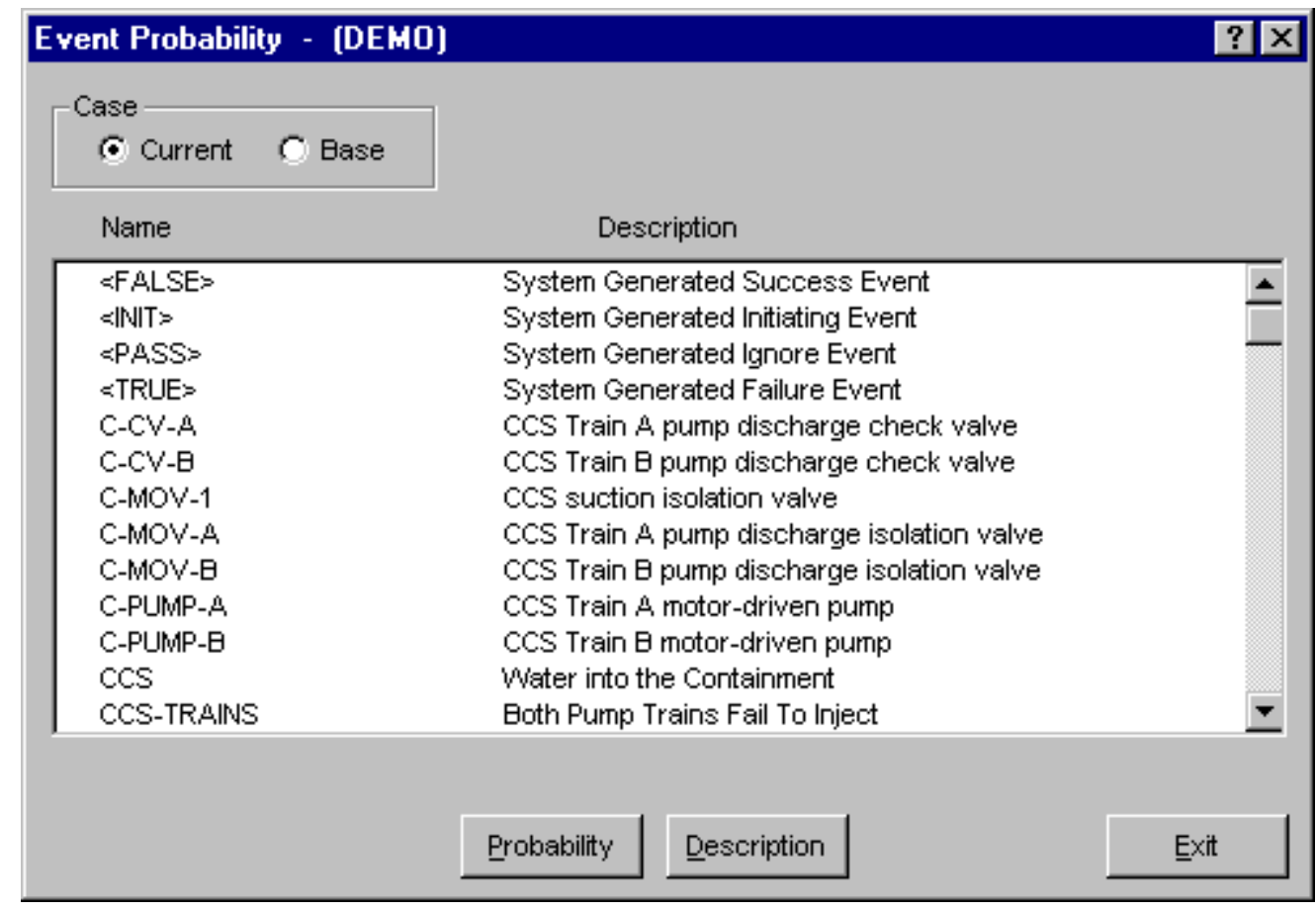

Case

Current - Use current case values for all calculations. This is the default.

Base - Use base case values for all calculations.

Probability - Generate the report based on the event selections. This report shows the event number, primary name, calculation type, mean probability, and lambda and tau values. The Report Viewer will display the report.

Description - Generate the Probability report, but also include the description for each event selected. The Report Viewer will display the report.

Exit - $\quad$ Close the Event Probability dialog without generating a report.

\subsubsection{Basic Event Uncertainty Report}

\section{PURPOSE}

This option allows you to generate a basic event uncertainty report. The report shows the event number, primary name, distribution type, mean probability, uncertainty value, and correlation class.

\section{STEPS}

1. From the SAPHIRE menu select Report. The Reports Menu dialog will be displayed.

2. Select the Basic Events Data Type and the Uncertainty Report Type radio buttons.

3. Choose the Process button. The Event Uncertainty dialog will be displayed.

4. Highlight the desired basic event(s) or process all records. 
5. Select the desired Case radio button.

6. Choose the Continue button. The Report Viewer will display the report.

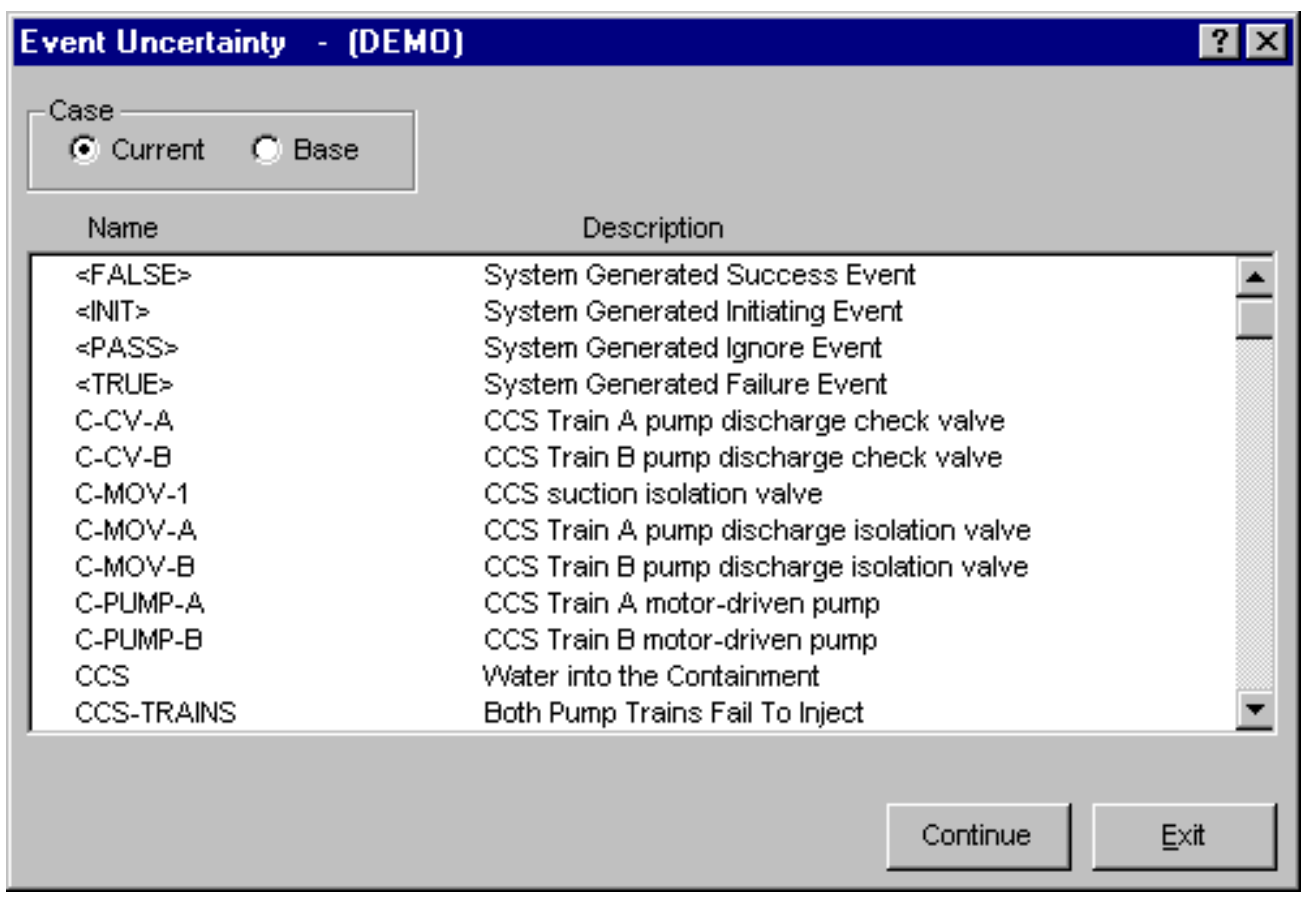

Case

Current - Use current case values for all calculations. This is the default.

Base - Use base case values for all calculations.

Continue - $\quad$ Generate the report based on event selections. The Report Viewer will display

Exit - $\quad$ Close the Event Uncertainty dialog without generating a report.

\subsubsection{Transformations Report}

\section{PURPOSE}

This option allows you to generate the event transformation reference report. The report shows the event number and name, event type, level, susceptibilities, and any referenced events.

\section{STEPS}

1. From the SAPHIRE menu select Report. The Reports Menu dialog will be displayed.

2. Select the Basic Events Data Type and the Transformation Report Type radio buttons.

3. Choose the Process button. The Transformations dialog will be displayed. A "T" to the left of the event name indicates that the event contains transformations.

4. Highlight the desired basic event(s) or process all listed records.

5. Choose the Continue button. The Report Viewer will display the report. 


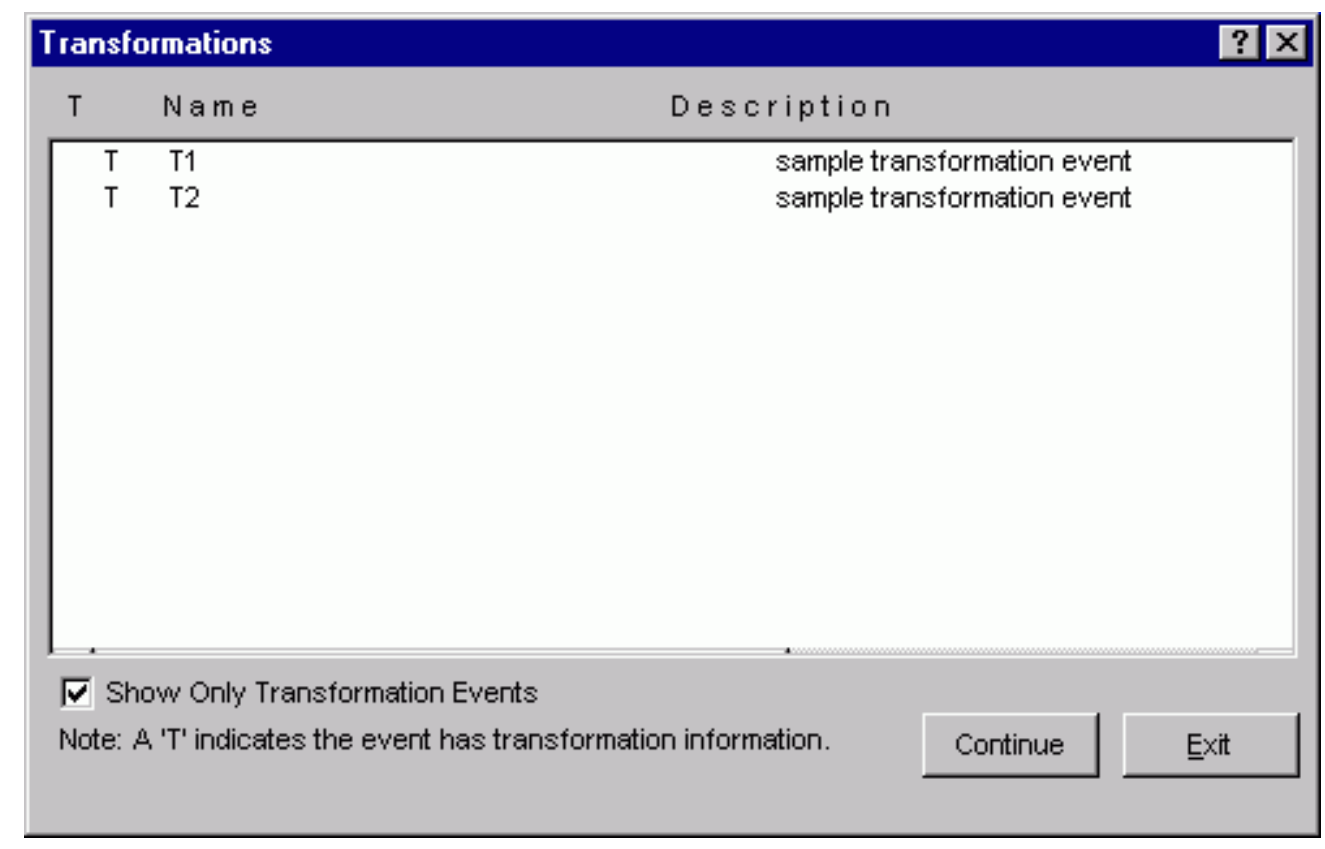

Show Only Transformation Events - The check box toggles the event list to display either all events in the project, or only those events that have transformations associated with them.

Continue Generate the basic event transformation report. The Report Viewer will display the report.

Exit Close the Transformations dialog without generating a report.

\subsubsection{Compound Event Report}

\section{PURPOSE}

This option allows you to generate the compound event reference report. The report shows the event name, plug-in library, function, and event inputs.

\section{STEPS}

1. From the SAPHIRE menu select Report. The Reports Menu dialog will be displayed.

2. Select the Basic Events Data Type and the Compound Event Report Type radio buttons.

3. Choose the Process button. The Compound Events dialog will be displayed. A " $\mathrm{C}$ " to the left of the event name indicates that the event contains compound event information.

4. Highlight the desired basic event(s) or process all listed records.

5. Choose the Continue button. The Report Viewer will display the report. 


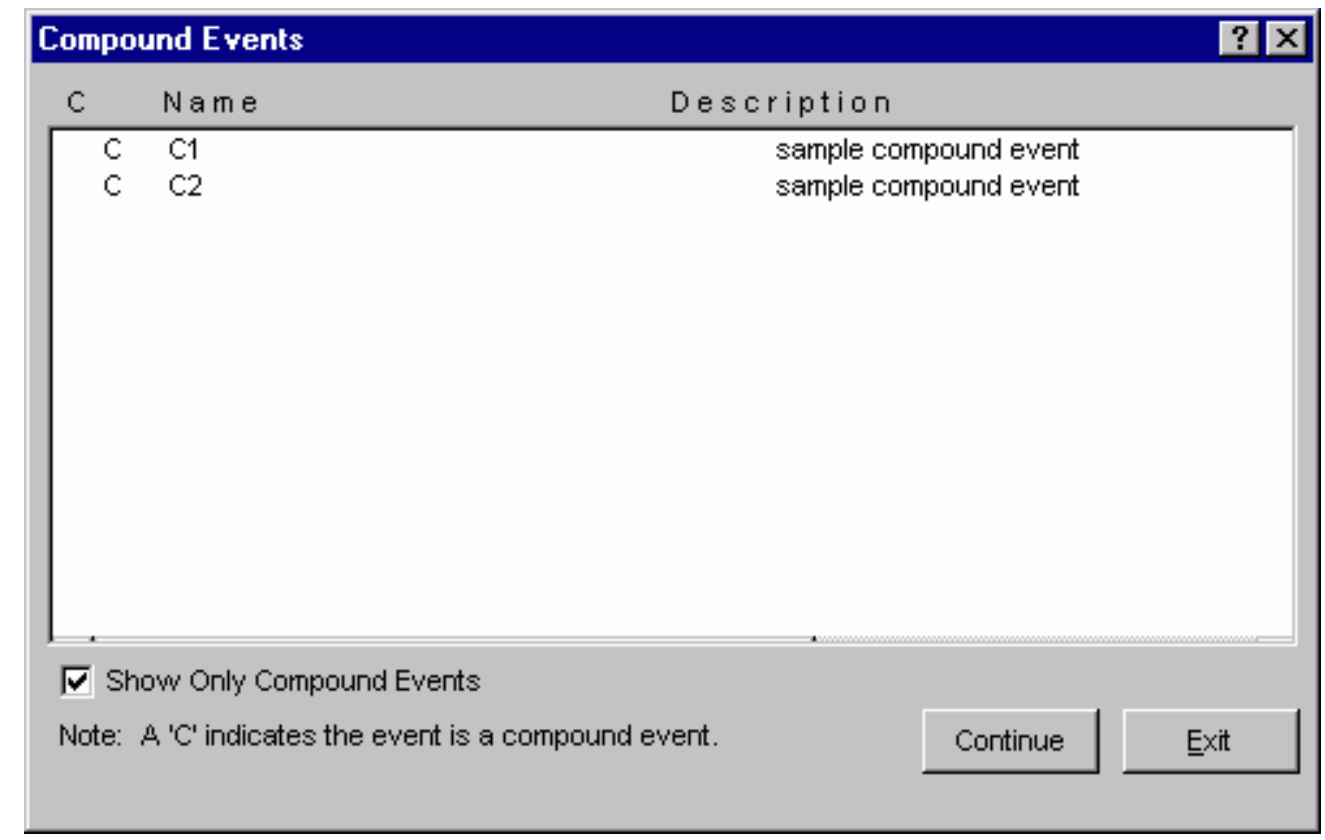

Show Only Compound Events -

Continue -

Exit -
The check box toggles the event list to display either all events in the project, or only those events that contain compound event information.

Generate the compound event report. The Report Viewer will display the report.

Close the Compound Events dialog without generating a report.

\subsubsection{Developed Event Report}

\section{PURPOSE}

This option allows you to generate the developed event report. The report shows the event name, base and current case calculation type, probability, and flag information. It also lists the fault trees that reference the developed event.

\section{STEPS}

1. From the SAPHIRE menu select Report. The Reports Menu dialog will be displayed.

2. Select the Basic Events Data Type and the Developed Events Report Type radio buttons.

3. Choose the Process button. The Transformations dialog will be displayed. A "DE" to the left of the event name indicates that the event is classified as a developed event.

4. Highlight the desired basic event(s) or process all listed records.

5. Choose the Continue button. The Report Viewer will display the report. 


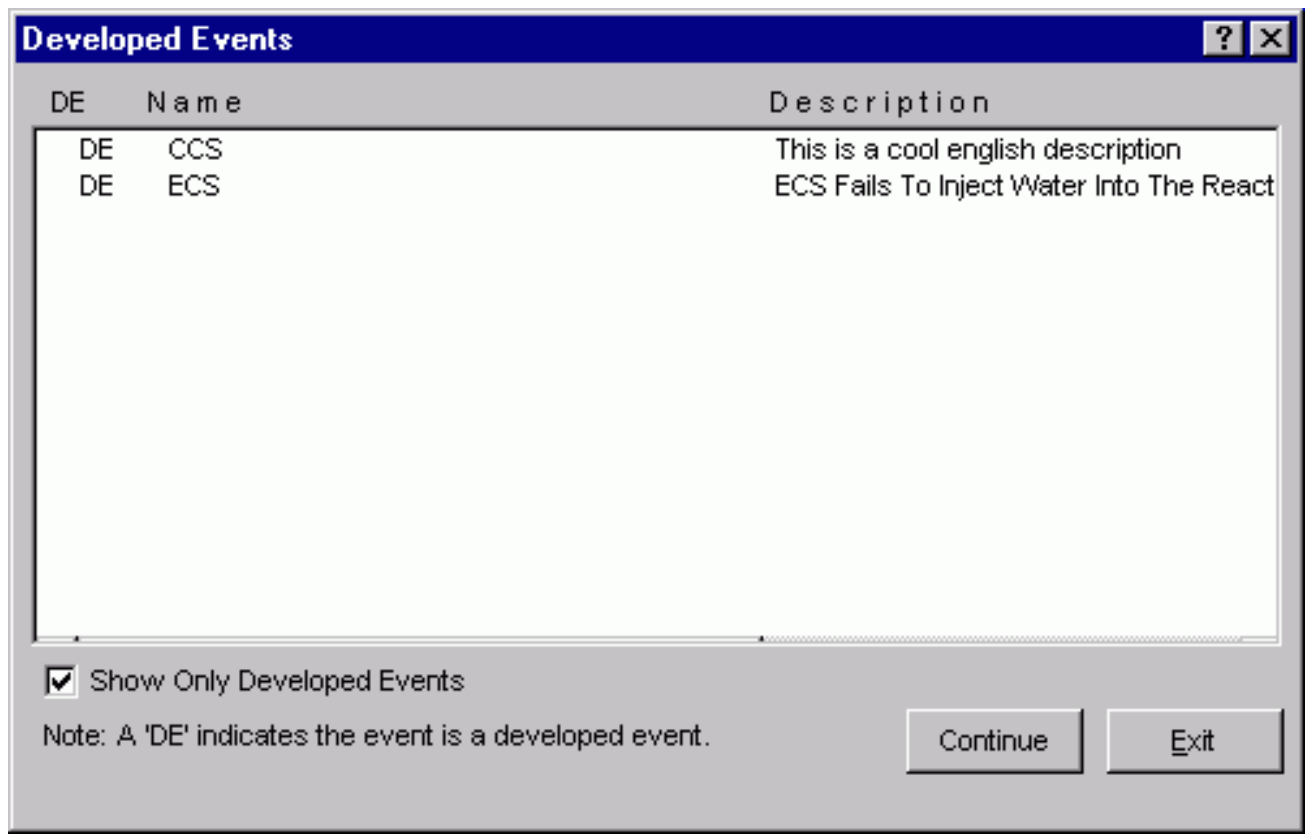

Show Only Developed Events - The check box toggles the event list to display either all events in the project, or only those events that are developed events.

Continue -

Exit report. Generate the developed event report. The Report Viewer will display the report.

Close the Developed Events dialog without generating a

\subsubsection{Template Event Report}

\section{PURPOSE}

This option allows you to generate the template event reference report. The report shows the template event name, and a list of all basic events that reference the template.

\section{STEPS}

1. From the SAPHIRE menu select Report. The Reports Menu dialog will be displayed.

2. Select the Basic Events Data Type and the Template Events Report Type radio buttons.

3. Choose the Process button. The Template Events dialog will be displayed. A "T" to the left of the event name indicates that the event contains template event information.

4. Highlight the desired basic event(s) or process all listed records.

5. Choose the Continue button. The Report Viewer will display the report. 


\begin{tabular}{|c|c|c|c|}
\hline \multicolumn{4}{|c|}{ Template Events } \\
\hline $\mathrm{T}$ & Name & \multicolumn{2}{|l|}{ Description } \\
\hline $\mathrm{T}$ & $\mathrm{AOV}-\mathrm{CC}$ & \multicolumn{2}{|c|}{ AIR OPERATED VALVE FAILS TO OPEI ^ } \\
\hline $\mathrm{T}$ & AOV-OO & \multicolumn{2}{|c|}{ AIR OPERATED FAILS TO CLOSE TEMF- } \\
\hline $\mathrm{T}$ & BAC-LP & \multicolumn{2}{|l|}{ AC BUS FAILS TEMPLATE } \\
\hline $\mathrm{T}$ & BAT-LP & \multicolumn{2}{|c|}{ BATTERY FAILS TEMPLATE } \\
\hline $\mathrm{T}$ & $\mathrm{BCH}-\mathrm{FC}$ & \multicolumn{2}{|c|}{ BATTERY CHARGER TEMPLATE } \\
\hline $\mathrm{T}$ & BDC-LP & \multicolumn{2}{|c|}{ DC BUS FAILS TEMPLATE } \\
\hline $\mathrm{T}$ & $\mathrm{CK} V-\mathrm{CC}$ & \multicolumn{2}{|c|}{ CHECK VAL VE FAILS TO OPEN TEMPL } \\
\hline $\mathrm{T}$ & CKV-LK & \multicolumn{2}{|c|}{ CHECK VALVE INTERNAL RUPTURE TE } \\
\hline $\mathrm{T}$ & $\mathrm{CK}-\mathrm{OO}$ & \multicolumn{2}{|c|}{ CHECK VALVE FAILS TO CLOSE TEMP } \\
\hline $\mathrm{T}$ & DGN-FS & \multicolumn{2}{|c|}{ DIESEL GENERATOR FAILS TO START } \\
\hline $\mathrm{T}$ & DGN-TM & \multicolumn{2}{|c|}{ DIESEL GENERATOR TEST AND MAINT } \\
\hline $\mathrm{T}$ & $\mathrm{EPV}-\mathrm{CC}$ & \multicolumn{2}{|c|}{ EXPLOSIVE VALVE FAILS TO OPEN TE } \\
\hline $\mathrm{T}$ & $\mathrm{HTX}-\mathrm{PG}$ & \multicolumn{2}{|c|}{ HEAT EXCHANGER PLUGGNG TEMPL,} \\
\hline \multicolumn{4}{|c|}{ 『 Show Only Template Events } \\
\hline \multicolumn{2}{|c|}{ Note: A ' $T$ ' indicates the event is a template event. } & Continue & Exit \\
\hline
\end{tabular}

Show Only Template Events - The check box toggles the event list to display either all events in the project, or only those events that are designated as template events.

Continue Generate the template event report. The Report Viewer will

Exit display the report.

report.

Close the Template Events dialog without generating a

\subsubsection{Basic Event Cross Reference Report}

\section{PURPOSE}

This option generates a basic event cross reference report.

\section{STEPS}

1. From the SAPHIRE menu select Report. The Reports Menu dialog will be displayed.

2. Select the Basic Events Data Type and the Cross Reference Report Type radio buttons.

3. Choose the Process button. The Event X-Reference dialog will be displayed.

4. Highlight the desired basic event(s) or process all records.

5. Select the desired Case radio button.

6. Select the desired analysis type from the drop-down list.

7. Choose the desired report type button. The Report Viewer will display the report. 


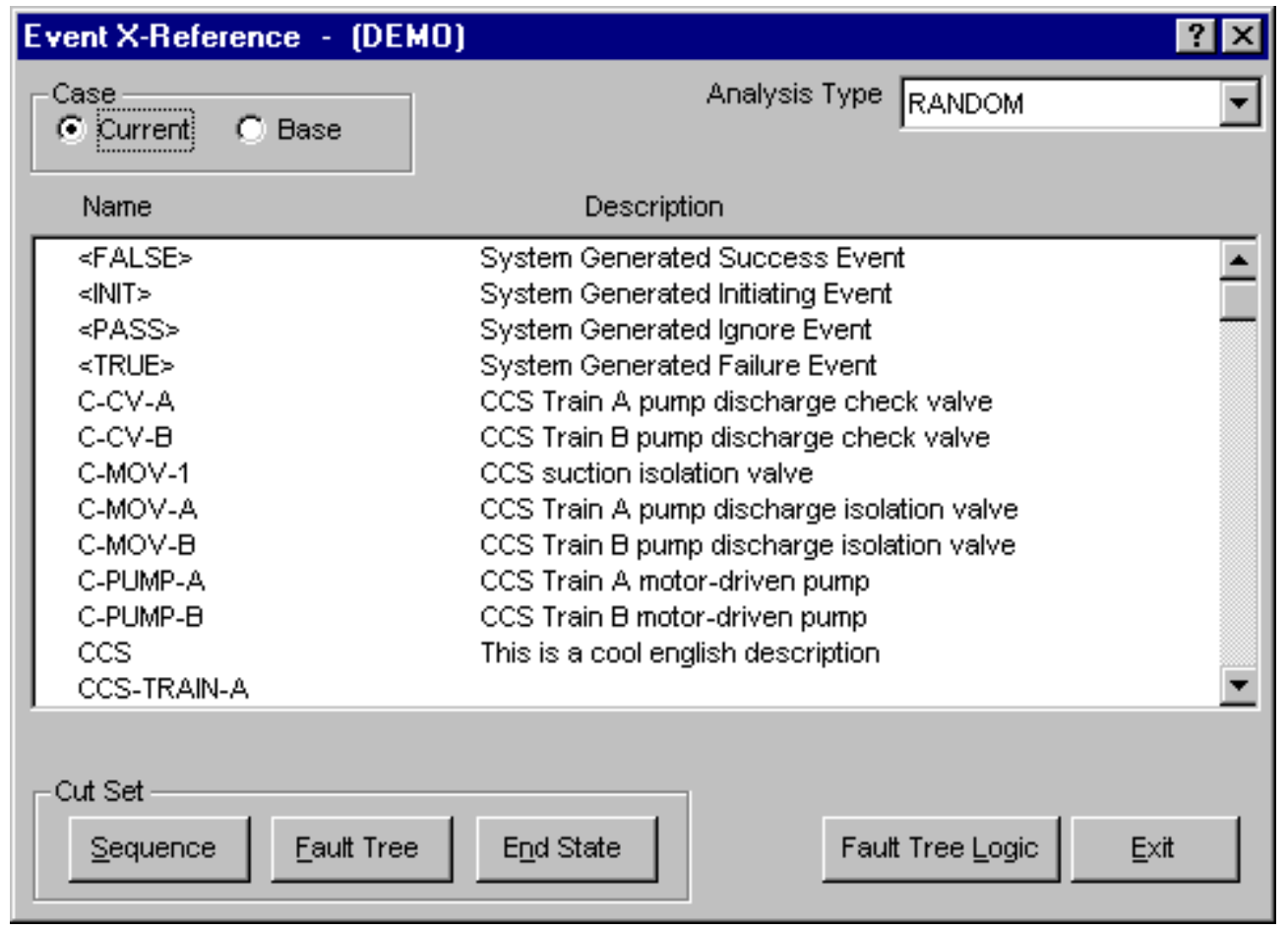

Case

Current - Use current case values for all calculations. This is the default.

Base - Use base case values for all calculations.

Sequence - $\quad$ Produce an event to sequence cut set cross-reference report. The report will include the event number, event name and associated event tree and sequence names.

Fault Tree - $\quad$ Produce an event to fault tree cut set cross-reference report. The report includes the event number, the event name and the corresponding fault tree name.

End State - $\quad$ Produce an event to end state cut set cross-reference report. The report includes the event number, the event name and the corresponding end state cut set name(s).

Fault Tree Logic-Produce an event to fault tree logic cross-reference report. The report includes the event number, the event name and the corresponding fault tree names.

Exit -

Close the Event $X$-Reference dialog without generating a report.

After report options have been selected and the button chosen, the Report dialog will be displayed and the output destination for the report must be specified.

\subsubsection{Seismic Events}

\subsubsection{Seismic}

\section{PURPOSE}

This option allows you to generate a seismic event report. The report shows the primary event name, fragility, beta R, beta $\mathrm{U}$, and HCLPF values for all seismic events. 


\section{STEPS}

1. From the SAPHIRE menu select Report. The Reports Menu dialog will be displayed.

2. Select the Basic Events Data Type and the Seismic Report Type radio buttons.

3. Choose the Process button. The Seismic Events dialog will be displayed. An "S" to the left of the event name indicates that the event is susceptible to seismic activity.

4. Highlight the desired basic event(s) or process all records.

5. Select the desired Case radio button.

6. Choose the Continue button.

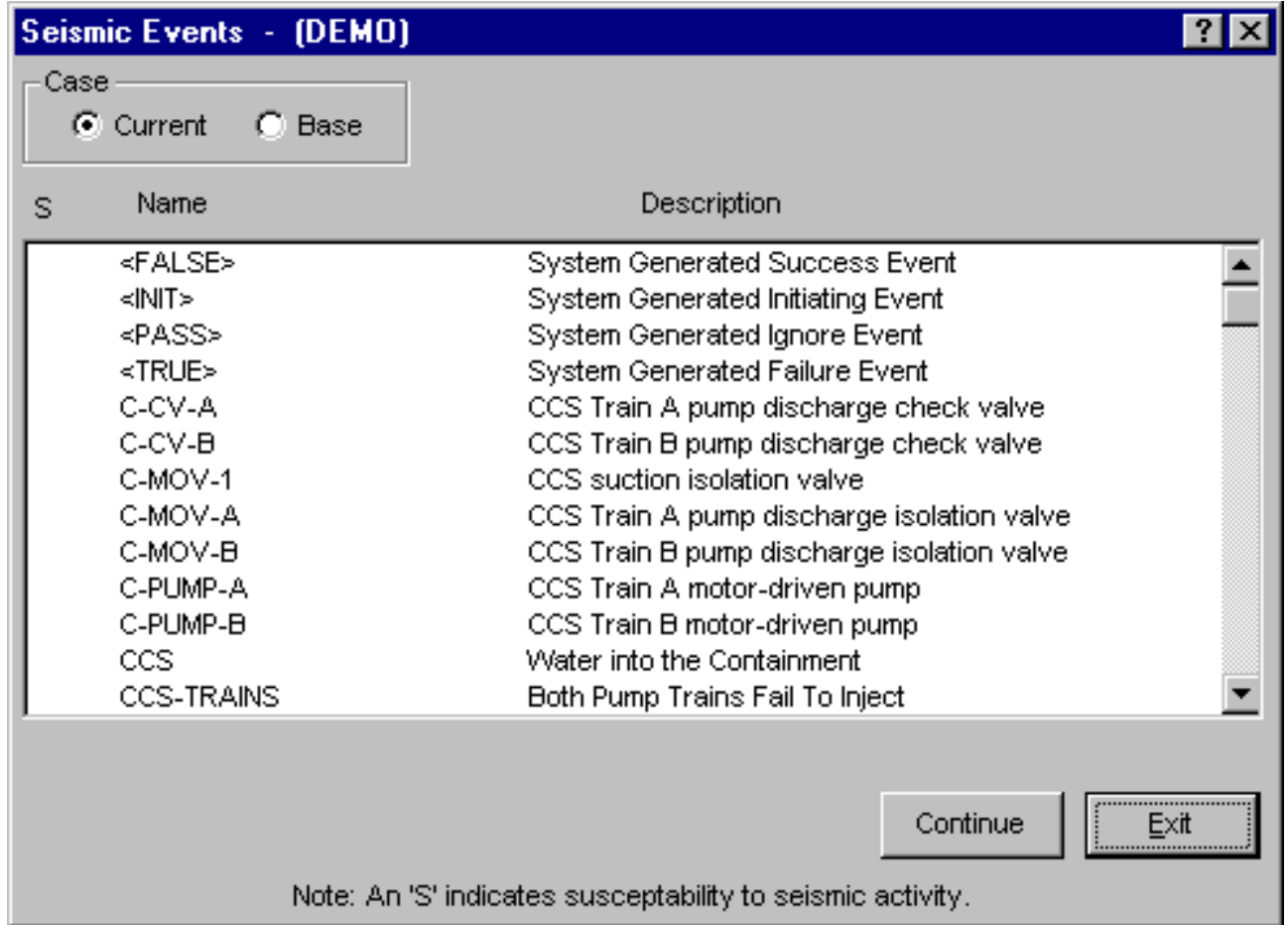

Case

Current - Use current case values for all calculations. This is the default.

Base - Use base case values for all calculations.

Continue - $\quad$ Generate the report based on event selections. The Report dialog will be displayed and output destination for the report must be specified.

Exit -

Close the Seismic Events dialog without generating a report.

\subsubsection{Seismic Event - Definition}

A seismic event has a calculation type of $\mathrm{G}$ (ground acceleration for screening G-levels) or $\mathrm{H}$ (hazard curve for screening G-levels), a distribution type of S (seismic log normal, Beta r, Beta u), and Susceptibility flag 4 set to "Y". 


\subsection{Fault Tree Reports}

\subsubsection{Fault Tree}

\section{PURPOSE}

This option allows you to generate a variety of fault tree reports. These include summary, logic, cut sets, importance, and cross reference reports.

\section{STEPS}

1. From the SAPHIRE menu select Report. The Reports Menu dialog will be displayed.

2. Select the Fault Tree Data Type and one of the Report Type radio buttons. Depending on the Report Type selected, you may be required to select an associated Sub Type as well.

3. Choose the Process button.

4. Highlight the desired fault tree(s) or process all records.

5. Choose the appropriate buttons to generate the report. The Report Viewer will display the report.

\begin{tabular}{|c|c|c|c|}
\hline Reports Menu & & & \\
\hline Data Type & Report Type & -Sub Type & \\
\hline C Project & c Summary & c Summary & \\
\hline C Attributes & C Logic & C Combination & \\
\hline C Basic Events & C Graphic & C Uncertainty & \\
\hline c Fault Trees & $\mathrm{C}$ Cut Sets & C Base Vs. Curr & \\
\hline C Event Trees & C Importance & C overview & \\
\hline C End States & $\mathrm{C} \times$ Reference Fault Tree & & \\
\hline C Sequences & C X Reference Sub-tree & & \\
\hline$C$ Change Sets & C Recovery Rules & & \\
\hline C Flag Sets & C Text & & \\
\hline C Gates & C Custom & & \\
\hline C Histograms & & & \\
\hline S Slices & & Process & Exit \\
\hline C User Into & & & \\
\hline
\end{tabular}

\section{Report Type}




\section{Summary -}

Logic -

Graphic -

Cut Sets -

Importance -

X-Reference Fault Tree -

X-Reference Sub-tree -

Recovery Rules -

Text -

Custom -
This option allows you to generate various fault tree summary reports.

Generate a report showing the fault tree logic information associated with each selected fault tree.

Generate graphical logic reports either to a printer or word processing compatible files.

Generate a report displaying cut set information for each selected fault tree.

Generate a report listing associated importance measures information for each selected fault tree.

This option allows you to generate logic cross reference reports for fault trees.

This option allows you to generate logic cross reference reports for sub-trees.

This report outputs a listing of the recovery rule logic that applies to each selected fault tree. Choose the Basic or

Advanced radio button to indicate the desired rule type.

Generate a report containing the descriptive text associated with the selected fault tree(s).

Create or select a user-defined report relating to fault tree level information.

\subsubsection{Fault Tree Summary Report}

\section{PURPOSE}

This option allows you to generate fault tree summary reports that are based on current or base case values for a specified analysis type.

\section{STEPS}

1. From the SAPHIRE menu select Report. The Reports Menu dialog will be displayed.

2. Select the Fault Tree Data Type and the Summary Report Type radio buttons.

3. Select the desired Sub Type radio button then choose the Process button.

4. Select the desired Case radio button.

5. Select the desired analysis type from the drop-down list.

6. Highlight the desired fault tree(s) or process all records.

7. Choose the Continue button to generate the report. The Report Viewer will display the report. 


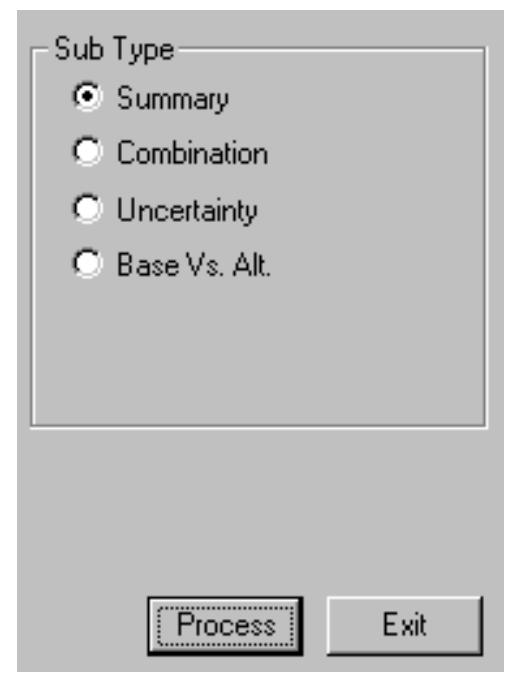

Case

Current - Use current case values for all calculations. This is the default.

Base - Use base case values for all calculations.

Summary - Generate the Fault Tree Brief Summary Report. This report contains the name, the associated minimum cut set upper bound, and the description of the selected fault trees.

Combination - Generate the Fault Tree Combination Report. This report contains the name, minimum cut set upper bound, mean, and number of cut sets in the selected fault trees.

Uncertainty - Generate the Fault Tree Uncertainty Value Report. This report contains the number, name, mean and median values, standard deviation, the 5th and 95th percentile, the minimum and maximum values, and the seed size of the selected fault trees.

BaseVs.Current-Generate the Fault Tree Base/Current Report. For each selected fault tree, this report contains the name, the minimal cut set upper bound for the base case and current case cut sets, the difference (base - current), and the number of base case and current case cut sets.

\subsubsection{Fault Tree Logic Report}

\section{PURPOSE}

This option generates fault tree logic reports. 


\section{STEPS}

1. From the SAPHIRE menu select Report. The Reports Menu dialog will be displayed.

2. Select the Fault Tree Data Type and the Logic Report Type radio buttons.

3. Choose the Process button. The Fault Tree Logic Reports dialog will be displayed, showing all of the fault trees in the currently selected project.

4. Highlight the desired fault tree(s) or process all records.

5. Choose the desired button to generate the report. The Report Viewer will display the report.

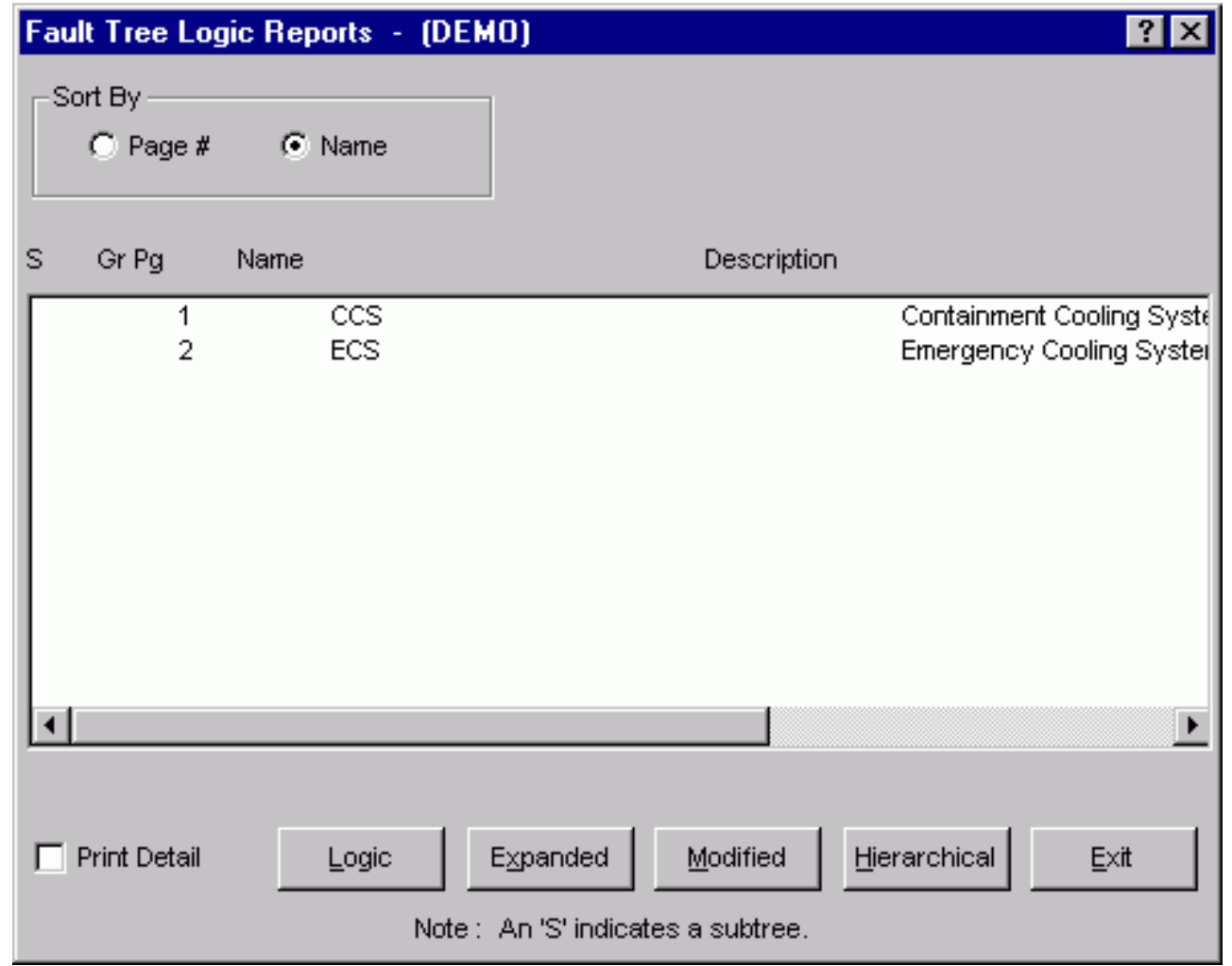

\section{Sort By}

Page \# - The fault list (and selected report items) will appear according to the page numbers assigned to each fault tree (see Fault Tree Page Numbering utility for information on how to assign page numbering.)

Name - The fault list (and selected report items) will appear in alphabetic order.

Print Detail - When checked, report will include event and gate descriptive information. This information is omitted from the reports when the option is unchecked.

Logic - $\quad$ Generate the Fault Tree Logic Report consisting of the logic as it is stored in the SAPHIRE database, including gate names, types, and inputs for the specified fault trees(s) or sub-tree(s). Transfer gates will not be expanded. 
Expanded - $\quad$ Generate the Fault Tree Expanded Logic Report consisting of the gate names, types, and inputs for the specified fault tree(s), including the logic of the fault trees represented by any transfer gates. When you choose this button, you will be prompted to enter a starting gate for the report. You may specify a gate or leave the field blank to include all gates.

Modified - $\quad$ Generate the Fault Tree Modified Logic Report consisting of the logic after it is loaded and restructured to the point just prior to cut set generation. When you choose this button, you will be prompted to enter a starting gate for the report. You may specify a gate or leave the field blank to include all gates.

Hierarchical - Generate the Hierarchical Logic Report. This option presents the logic in a format similar to that displayed in the Logic Editor. Gate and Event information is indented according to their position in the logic. (The Print Detail option does not affect this report - event and gate descriptions are always included.)

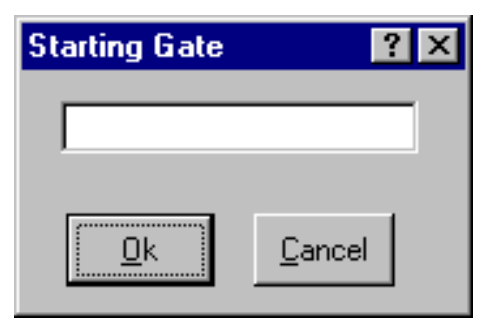

Exit - $\quad$ Close the Fault Tree Logic Reports dialog without generating a report.

\subsubsection{Fault Tree Graphics Report}

\section{PURPOSE}

This option allows you to generate a printout the selected fault tree(s).

\section{STEPS}

1. From the SAPHIRE menu select Report. The Reports Menu dialog will be displayed.

2. Select the Fault Tree Data Type and the Graphic Report Type radio buttons.

3. Choose the Process button. The Graphics Reports dialog will be displayed, showing all of the fault trees in the currently selected project. A " $G$ " next to the name indicates a graphic (.DLS) file is available.

4. Highlight the desired fault tree(s) or process all records.

5. Choose the Print button to generate the report. 


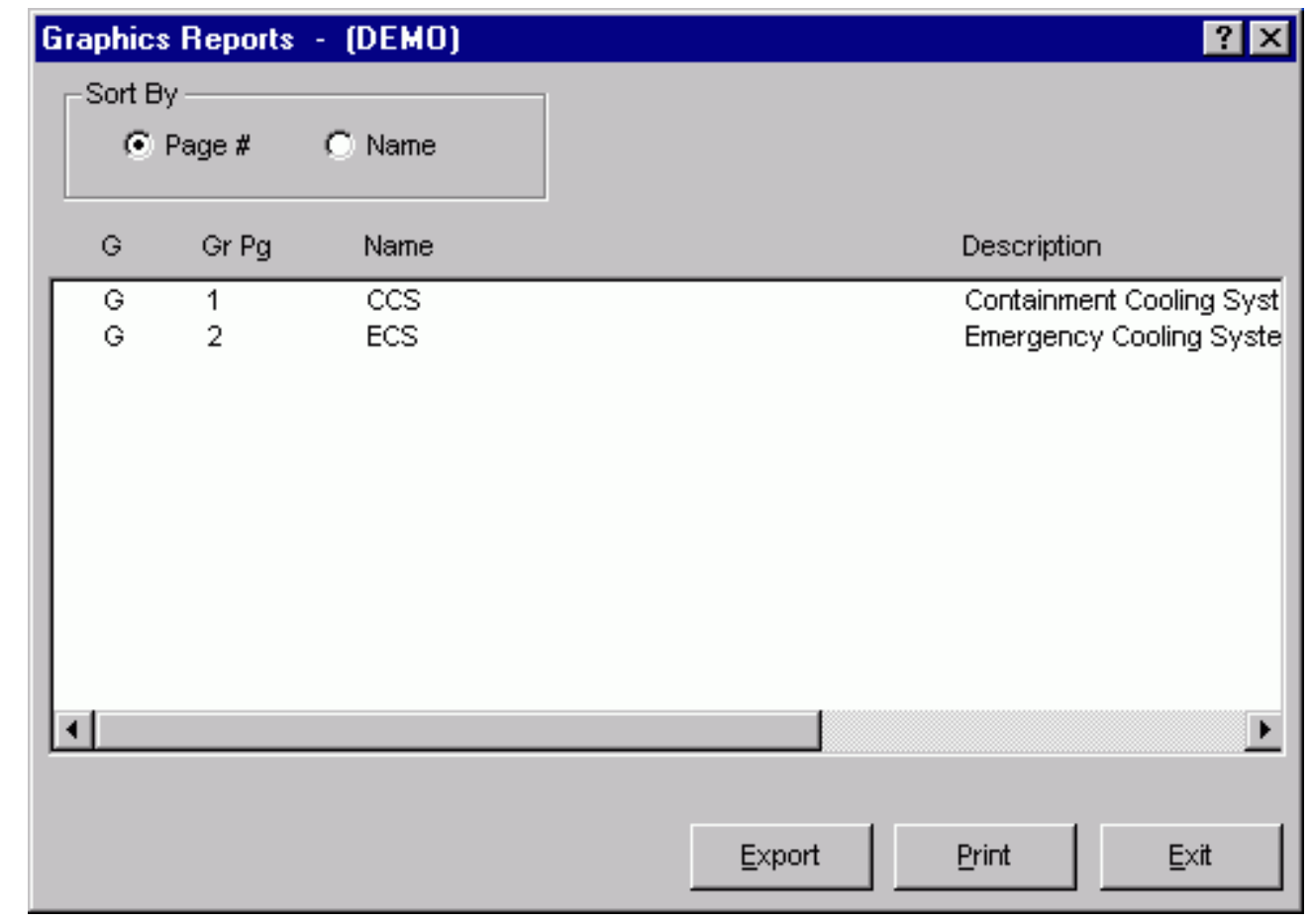

Sort By

Page \# - The fault list (and selected report items) will appear according to the page numbers assigned to each fault tree (see Fault Tree Page Numbering utility for information on how to assign page numbering.)

Name - The fault list (and selected report items) will appear in alphabetic order.

Export - $\quad$ Convert the graphic file(s) to either a file in Enhanced Metafile (EMF) or

Print - $\quad$ Print the graphic file(s) to the designated printer.

Exit - $\quad$ Close the Graphics Reports dialog without generating a report.

\subsubsection{Graphics Export}

\section{PURPOSE}

This option allows you to export the selected fault tree or event tree diagram(s) in WMF or EMF format.

\section{STEPS}

1. From the SAPHIRE menu select Report. The Reports Menu dialog will be displayed.

2. Select the Fault Tree/Event Tree Data Type and the Graphic Report Type radio buttons.

3. Choose the Process button. The Graphics Reports dialog will be displayed, showing all of the fault trees/event trees in the currently selected project. A " $G$ " next to the name indicates a graphic (.DLS/.ETG) file is available.

4. Highlight the desired fault tree(s)/event tree(s) or process all records.

5. Choose the Export button. The Select Export Type dialog will be displayed. 


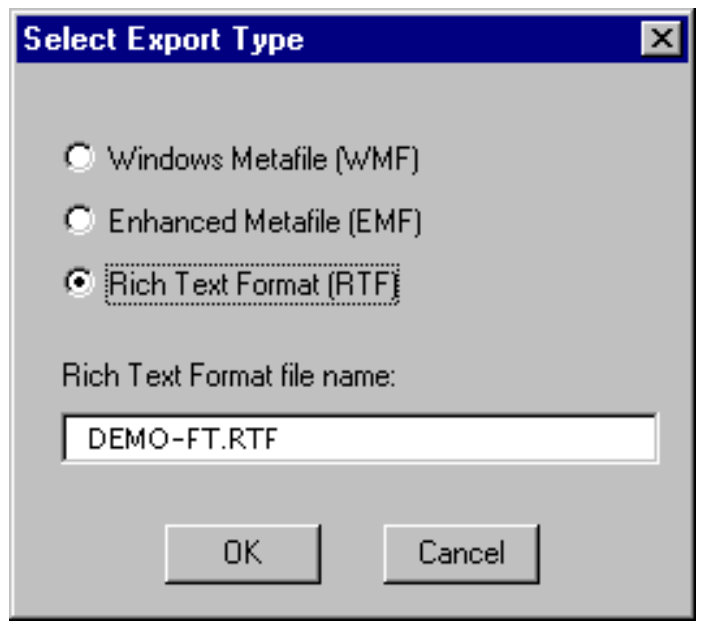

WMF - Export the diagram to Windows Metafile format.

EMF - Export the diagram to Enhanced Metafile format.

RTF - Export the diagram to Rich Text Format.

Cancel - Close the Select Export Type dialog without exporting the file(s).

\subsubsection{Cut Sets Report}

\section{PURPOSE}

This option allows you to generate cut set reports based the selected analysis type and on current or base case values.

\section{STEPS}

1. From the SAPHIRE menu select Report. The Reports Menu dialog will be displayed.

2. Select the Fault Tree Data Type and the Cut Sets Report Type radio buttons.

3. Choose the Process button. The Fault Tree Cut Sets dialog will be displayed, listing all of the fault trees residing in the current project.

4. Select the desired Case radio button.

5. Select the desired analysis type from the drop-down list.

6. Highlight the desired fault tree(s) or process all records

7. Choose the desired button to generate the report. The Report Viewer will display the report. 


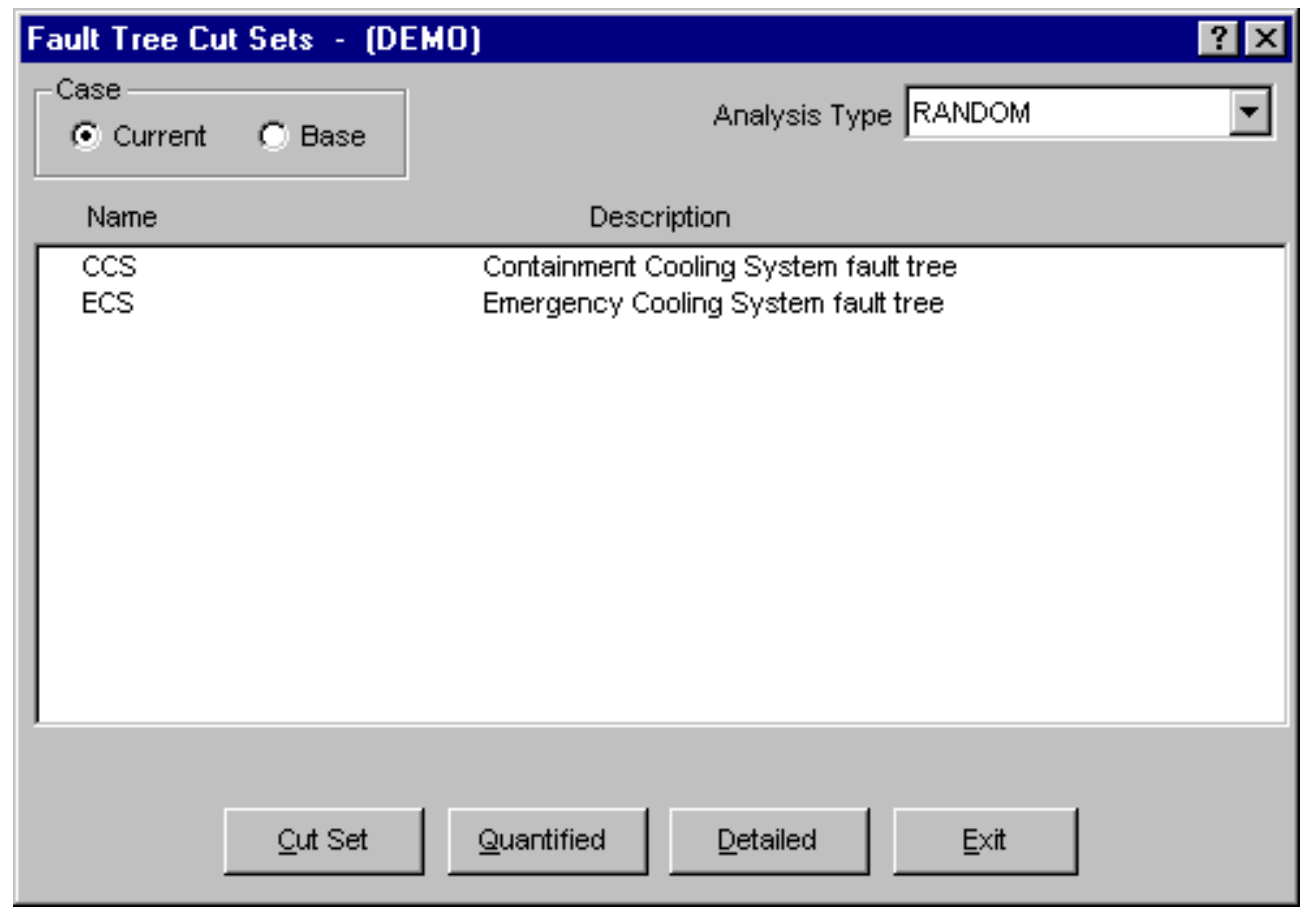

Case

Current - Use current case values for all calculations. This is the default.

Base - Use base case values for all calculations.

Cut Set - Generate the Fault Tree Cut Sets Report which consists of the cut set number, size, and the basic event names that are included in the corresponding cut sets (current or base) for the selected fault tree(s). When you choose this button, the Cut Set Report Options dialog will be displayed.

Quantified - Generate the Fault Tree Cut Set (Quantification) Report for quantified cut sets only. The report consists of the cut set number, percent of total, percent of the cut set, probability/frequency and the basic event names that are included in the associated cut sets (current or base) for the selected fault tree(s). When you choose this button, the Cut Set Report Options dialog will be displayed.

Detailed - $\quad$ Generate the Fault Tree Cut Set (Detailed) Report which consists of the cut set number, percent of the cut set, probability/frequency, and the basic event names and descriptions included in associated cut sets (current or base) for the selected fault tree(s). When you choose this button, the Cut Set Report Options dialog will be displayed.

Exit - $\quad$ Close the Fault Tree Cut Sets dialog without generating a report.

\subsubsection{Cut Set Report Options}

\section{PURPOSE}

This option allows you to specify several cut set cutoff values that will be used to determine if a cut set is to be included in the report. 
You can modify any of the default values provided by typing over the existing data.

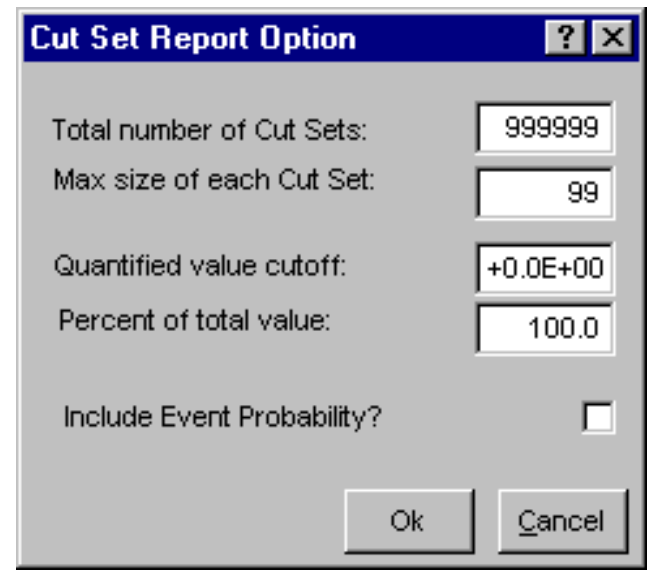

Total number of Cut Sets -

Max size of each Cut Set -

Quantified value cutoff -

Percent of total value -

Include Event Probability? -

Ok -

Cancel -
The number of cut sets included in the report will not exceed this number. Cut sets are considered beginning with the cut that contributes the highest percent frequency.

Include only those cut sets that contain no more than the number of events entered here.

Include only those cut sets whose frequency is greater than or equal to the value entered here. Applies only to the Quantified and Detailed cut set reports.

Include only those cut sets whose sum of the percent contribution is less than this value, beginning with the cut set that contributes the greatest percent. By entering 100.0 (the default) here, all cut sets will be included.

Preface the report with a list of each of the basic events referenced in the cut sets along with the basic event probabilities.

Generate the report based on the specified cutoff values. The Report Viewer will display the report.

Close the Cut Set Report Option dialog without generating a report.

\subsubsection{Fault Tree Importance Report}

\section{PURPOSE}

This option allows you to generate importance reports based the selected analysis type and on current or base case values.

\section{STEPS}

1. From the SAPHIRE menu select Report. The Reports Menu dialog will be displayed. 
2. Select the Fault Tree Data Type and Importance Report Type radio buttons.

3. Choose the Process button. The Fault Tree Importance dialog will be displayed, listing all of the fault trees residing in the current project.

4. Select the desired Case radio button.

5. Select the desired analysis type from the drop-down list.

6. Highlight the desired fault tree(s) or process all records.

7. Choose the desired button to generate the report. The Report Viewer will display the report.

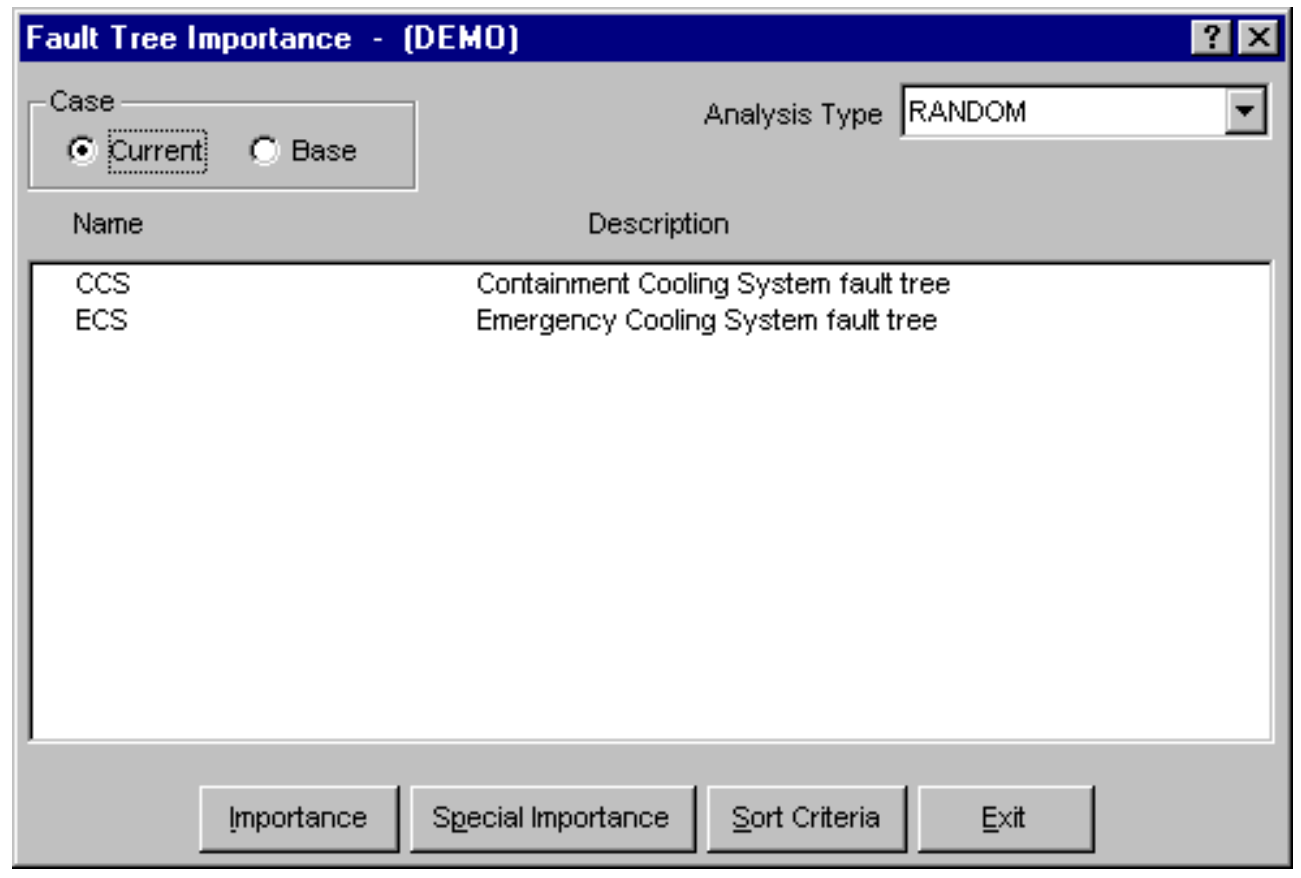

Case

Current - Use current case values for all calculations. This is the default.

Base - Use base case values for all calculations.

Importance -

Generate the Fault Tree Importance Measures report consisting of event names, number of times the event occurs, probability of failure, uncertainty importance value, risk reduction difference, and risk increase difference for the selected fault tree(s).

Special Importance - Generate the Fault Tree Importance Measures report consisting of event names, probability of failure, Fussell-Vesely value, risk reduction ratio, risk increase ratio, Birnbaum importance value, and event descriptions for the selected fault tree(s).

Sort Criteria -

Select the sort order in which to display the Fault Tree Importance Measures Report. When you choose this button, the Select Sort Criteria dialog will be displayed.

Exit -

Close the Fault Tree Importance dialog without generating a report. 


\subsubsection{Select Sort Criteria}

\section{PURPOSE}

This option allows you to select the sort order in which to display the Fault Tree/Sequence/End State Importance Measures Report.

\section{STEPS}

1. From the SAPHIRE menu select Report. The Reports Menu dialog will be displayed.

2. Select the Fault Tree/Sequence Data Type and Importance Report Type radio buttons.

3. Choose the Process button. The Fault Tree/Sequence Importance dialog will be displayed, listing all of the fault trees residing in the current project.

4. Select the desired Case radio button.

5. Select the desired analysis type from the drop-down list.

6. Highlight the desired fault tree(s) or process all records.

7. Choose the Sort Criteria button to select the sort order and choose OK.

8. Choose the desired report button from the Fault Tree/Sequence Importance dialog. The Report Viewer will display the report in the specified order.

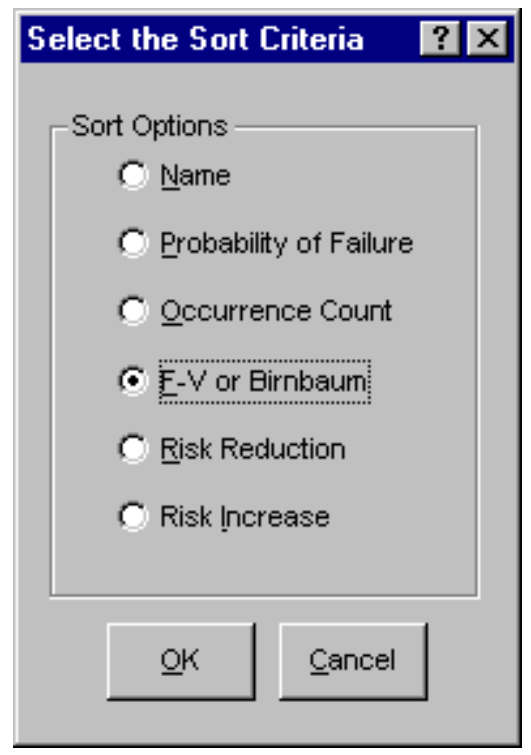

Name -

Sort by event name

Probability of Failure - Sort by the probability failures

Occurrence Count - Sort by the number of occurrences (most to fewest)

F-V or Birnbaum - $\quad$ Sort in Fussell-Vesely or Birnbaum order

Risk Reduction - $\quad$ Sort in risk reduction ratio or difference order

Risk Increase - $\quad$ Sort in risk increase ratio or difference order. 
OK -

Cancel -
Closes the Select the Sort Criteria dialog and saves the sort option.

Closes the Select the Sort Criteria dialog without saving the sort option.

\subsubsection{X-Reference Fault Tree}

\section{PURPOSE}

This option allows you to generate logic cross reference reports for fault trees.

\section{STEPS}

1. From the SAPHIRE menu select Report. The Reports Menu dialog will be displayed.

2. Select the Fault Tree Data Type and the X Reference Fault Tree Report Type radio buttons.

3. Select the desired Sub Type radio button.

4. Choose the Process button. From the subsequent Reports dialog, highlight the desired fault tree(s) or process all records. Notice that no sub-trees are included in this list.

5. Choose the Continue button to generate the report. The Report Viewer will display the report.

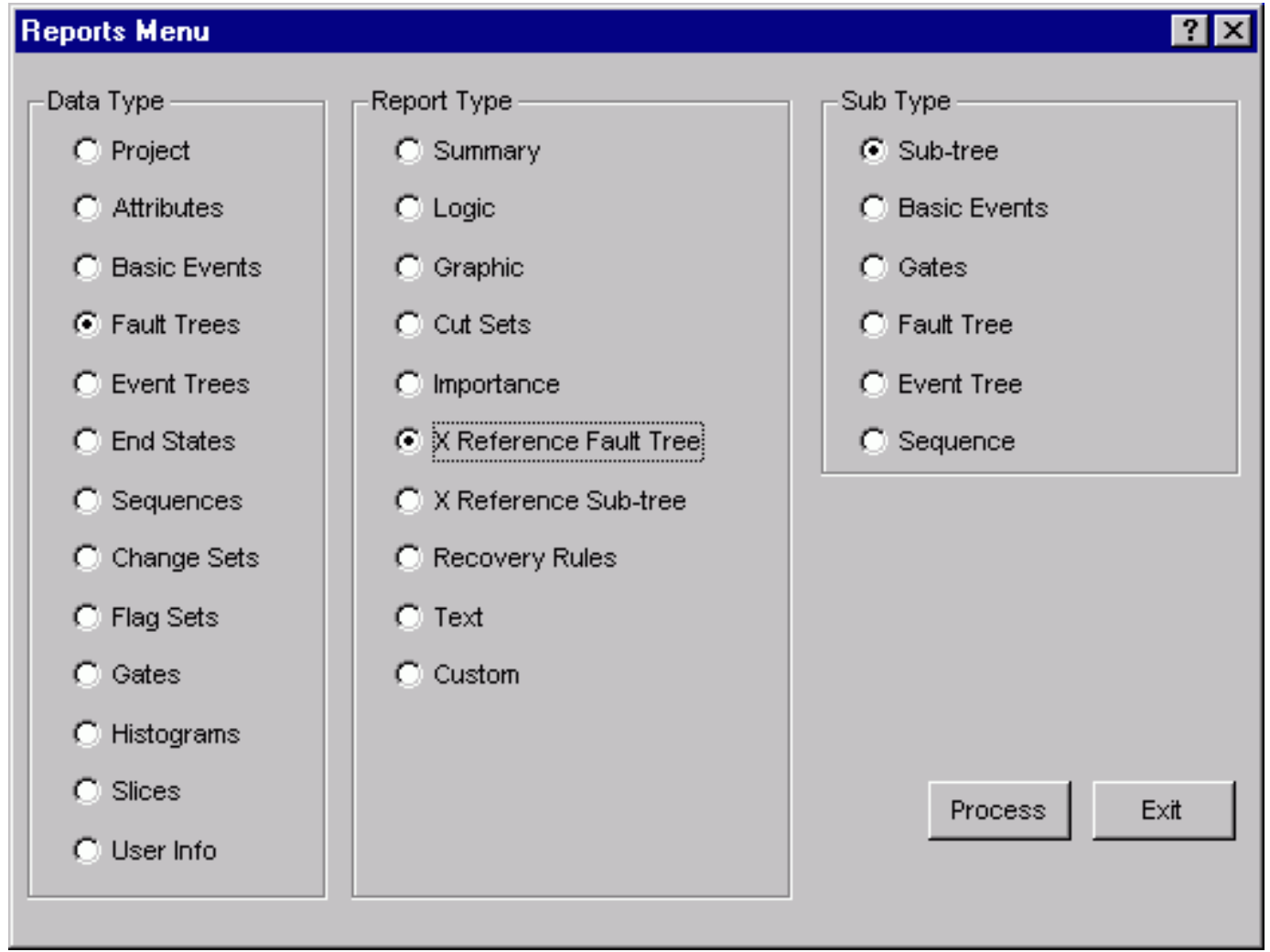

Sub-tree - $\quad$ Generate the Fault Tree Hierarchy Report. Each selected fault tree will be placed on a separate page with the selected fault tree name and a list of all sub-trees it uses.

Basic Events - Generate the Fault Tree/Basic Event Reference Report consisting of the selected fault tree(s) and a corresponding list of all basic events used by that fault tree. 
Gates - $\quad$ Generates the Fault Tree/Gate Reference Report consisting of the selected fault tree(s) and a corresponding list of gates used by that fault tree.

Fault Tree - Generate the Sub-tree Cross Reference Report consisting of the selected fault tree(s) listed as the Sub-tree Name with the corresponding trees using that subtree.

Event Tree - Generate the Fault Tree/Event Tree Reference Report consisting of the selected fault tree(s) and the corresponding event tree logic or rules using that fault tree.

Sequence - $\quad$ Generate the Fault Tree/Sequence Reference Report consisting of the selected fault tree(s) and the corresponding event trees and sequences using that fault tree.

\subsubsection{X-Reference Sub-tree}

\section{PURPOSE}

This option allows you to generate logic cross reference reports for sub-trees.

\section{STEPS}

1. From the SAPHIRE menu select Report. The Reports Menu dialog will be displayed.

2. Select the Fault Tree Data Type and the $\mathbf{X}$ Reference Sub-tree Report Type radio buttons.

3. Select the desired Sub Type radio button.

4. Choose the Process button.

5. From the subsequent Reports dialog, highlight the desired sub-tree(s) or process all records. Notice that both fault trees and sub-trees are included in this list

6. Choose the Continue button to generate the report. The Report Viewer will display the report. 


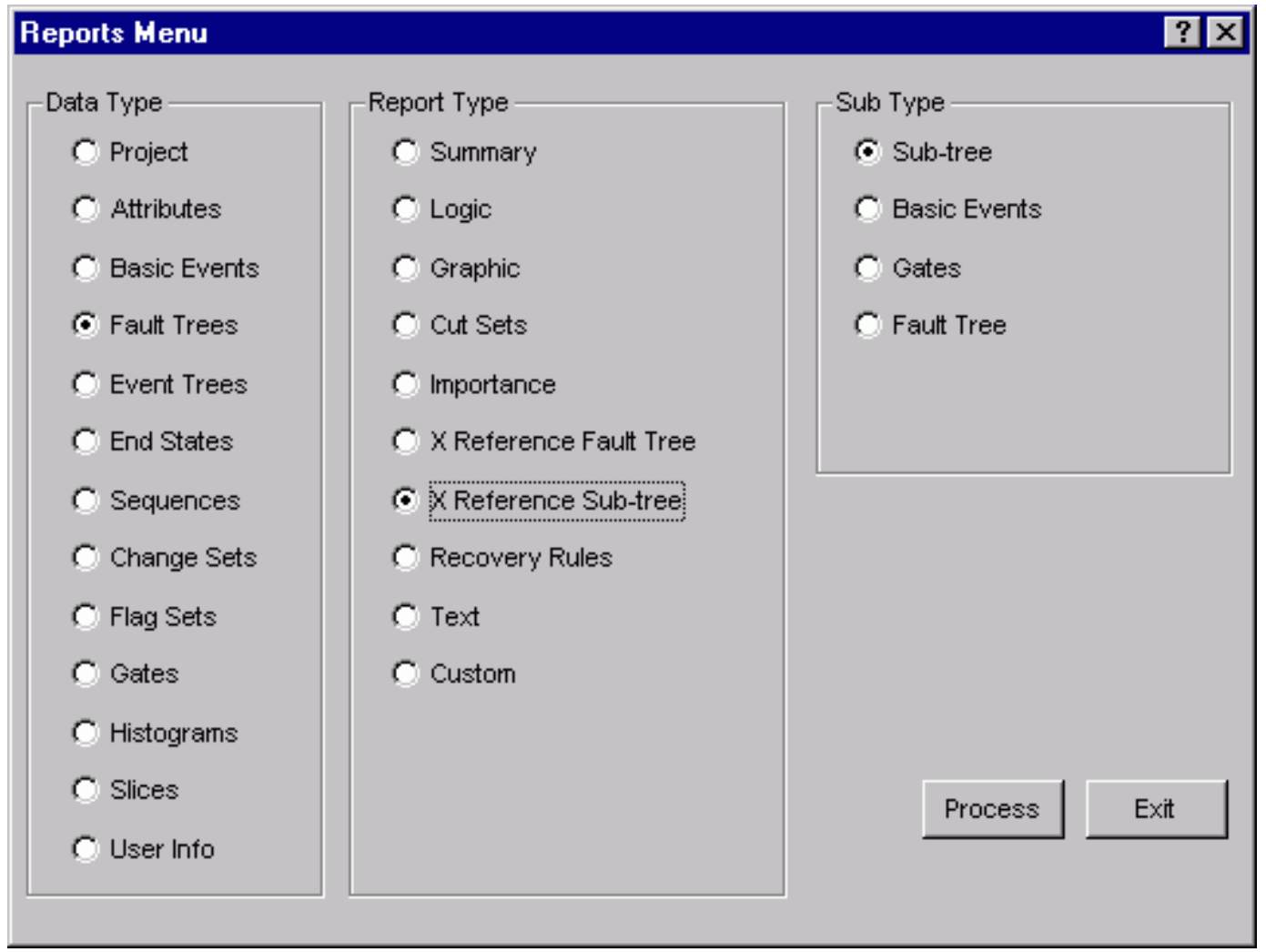

Sub-tree - $\quad$ Generate the Fault Tree Hierarchy Report. Each selected fault tree or sub-tree will be placed on a separate page with the selected fault tree name and of a list of all sub-trees it uses.

Basic Events - Generate the Fault Tree/Basic Event Reference Report consisting of the selected fault tree(s) and/or sub-tree(s) and a corresponding list of all basic events used by that fault tree.

Gates - $\quad$ Generates the Fault Tree/Gate Reference Report consisting of the selected fault tree(s) and/or sub-tree(s) and a corresponding list of gates used by that fault tree.

Fault Tree - $\quad$ This option generates the Sub-tree Cross Reference Report consisting of a subtree and a list of all fault trees/sub-trees that reference or use it.

\subsection{Event Tree Reports}

\subsubsection{Event Tree}

\section{PURPOSE}

This option generates a variety of event tree reports. The following reports may be generated: Logic/Rules, Graphics, Initiating Events, and Cross Reference. 


\section{STEPS}

1. From the SAPHIRE menu select Report. The Reports Menu dialog will be displayed.

2. Select the Event Tree Data Type and one of the Report Type radio buttons.

3. Choose the Process button.

4. Highlight the desired event tree(s) or process all records.

5. Choose the appropriate buttons to generate the report. The Report Viewer will display the report.

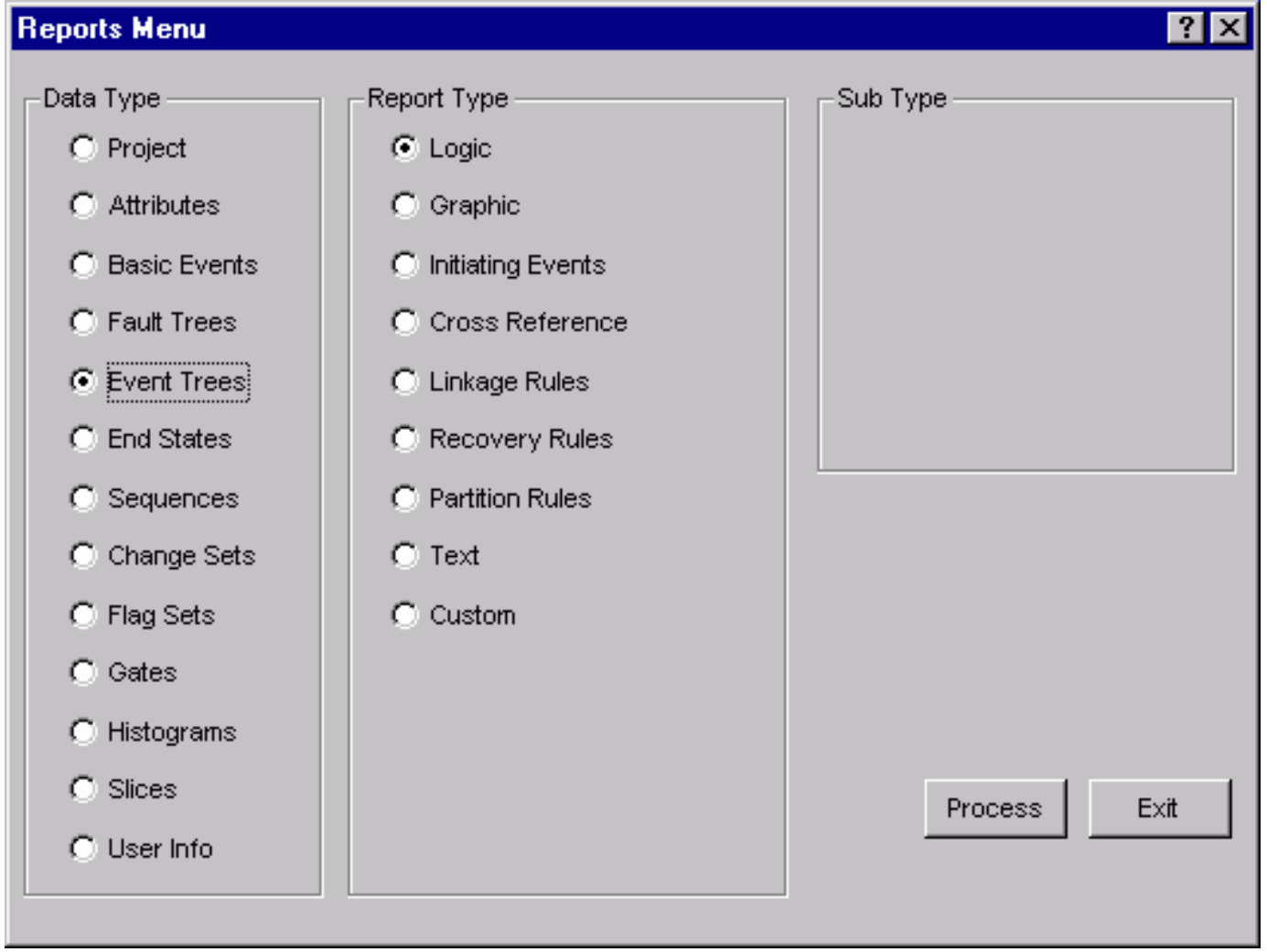

Report Type

Logic -

Graphic -

Initiating Events -

Linkage Rules -

Recovery Rules -
Generate a report showing the sequence logic information associated with each selected event tree.

Generate graphical logic reports either to a printer or word processing compatible files.

Generate a report summarizing the initiating event and description and top/subtree status of the selected event tree(s).

This report outputs a listing of the linkage rule logic defined for each event tree. Choose the Basic or Advanced radio button to indicate the desired rule type.

This report outputs a listing of the recovery rule logic that applies to all sequence cut sets within each selected event tree. Choose the Basic or Advanced radio button to indicate the desired rule type. 
Partition Rules -

Text -

Custom -
This report outputs a listing of the partition rule logic that applies to all sequence cut sets within each selected event tree. Choose the Basic or Advanced radio button to indicate the desired rule type.

Generate a report containing the descriptive text associated with the selected event tree(s).

Create or select a user-defined report relating to event tree level information.

\subsubsection{Event Tree Logic}

\section{PURPOSE}

This option allows you to generate the Event Tree Logic Report and the Event Tree Rules report.

\section{STEPS}

1. From the SAPHIRE menu select Report. The Reports Menu dialog will be displayed.

2. Select the Event Tree Data Type and the Logic Report Type radio buttons.

3. Choose the Process button. The Event Tree Logic dialog will be displayed, listing all of the event trees contained in the current project. An "S" to the left of the event tree name indicates a subtree.

4. Highlight the desired event tree(s) or process all records.

5. Choose the desired button to generate the report. The Report Viewer will display the report.

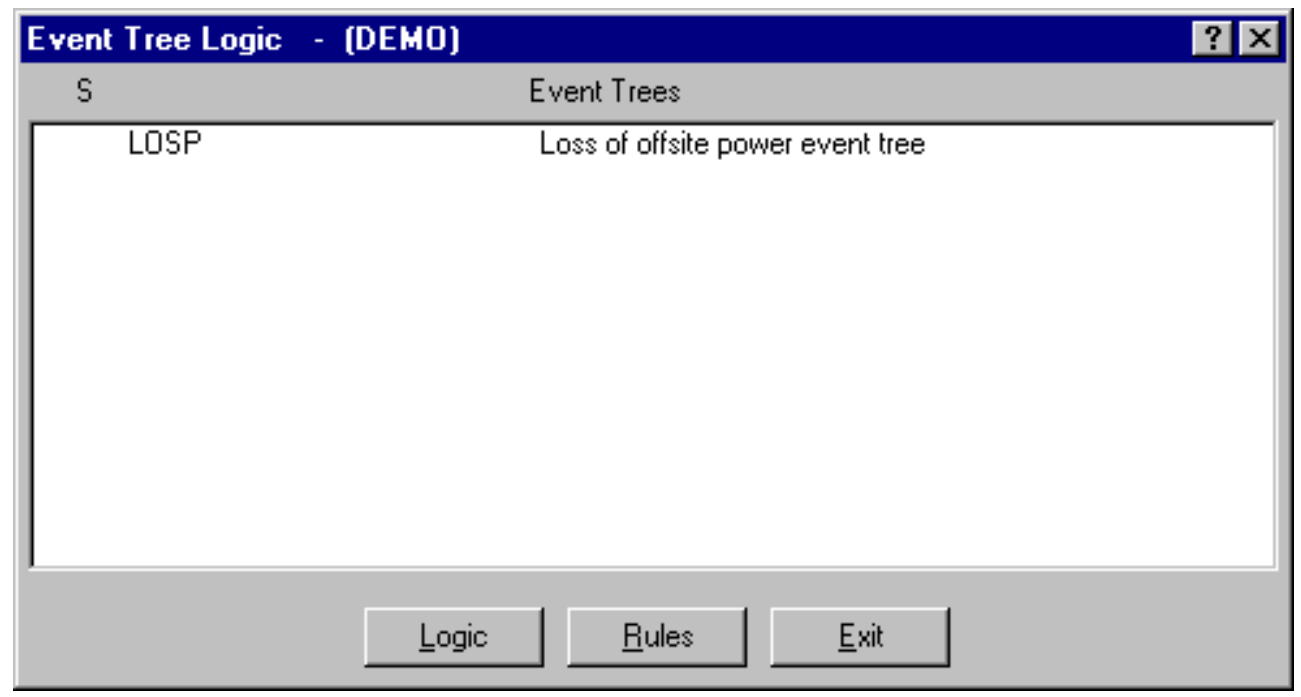

Logic - Produce the Event Tree Logic Report. This report lists all sequence names and the associated logic (pass/fail) associated with each selected event tree.

Rules - Produce the Event Tree Linkage Rules Report. This report lists all linkage rules (exceptions) associated with each selected event tree.

Exit - $\quad$ Close the Event Tree Logic dialog without generating a report.

After report options have been selected and the button chosen, the Report dialog will be displayed at which point the output destination for the report must be specified. 


\subsubsection{Event Tree Graphics}

\section{PURPOSE}

This option allows you to generate a printout the selected event tree(s).

\section{STEPS}

1. From the SAPHIRE menu select Report. The Reports Menu dialog will be displayed.

2. Select the Event Tree Data Type and the Graphic Report Type radio buttons.

3. Choose the Process button. The Event Tree Graphics dialog will be displayed, showing all of the event trees in the currently selected project. A " $G$ " next to the name indicates a graphic (.ETG) file is available.

4. Highlight the desired event tree(s) or process all records.

5. Choose the Print button to generate the report.

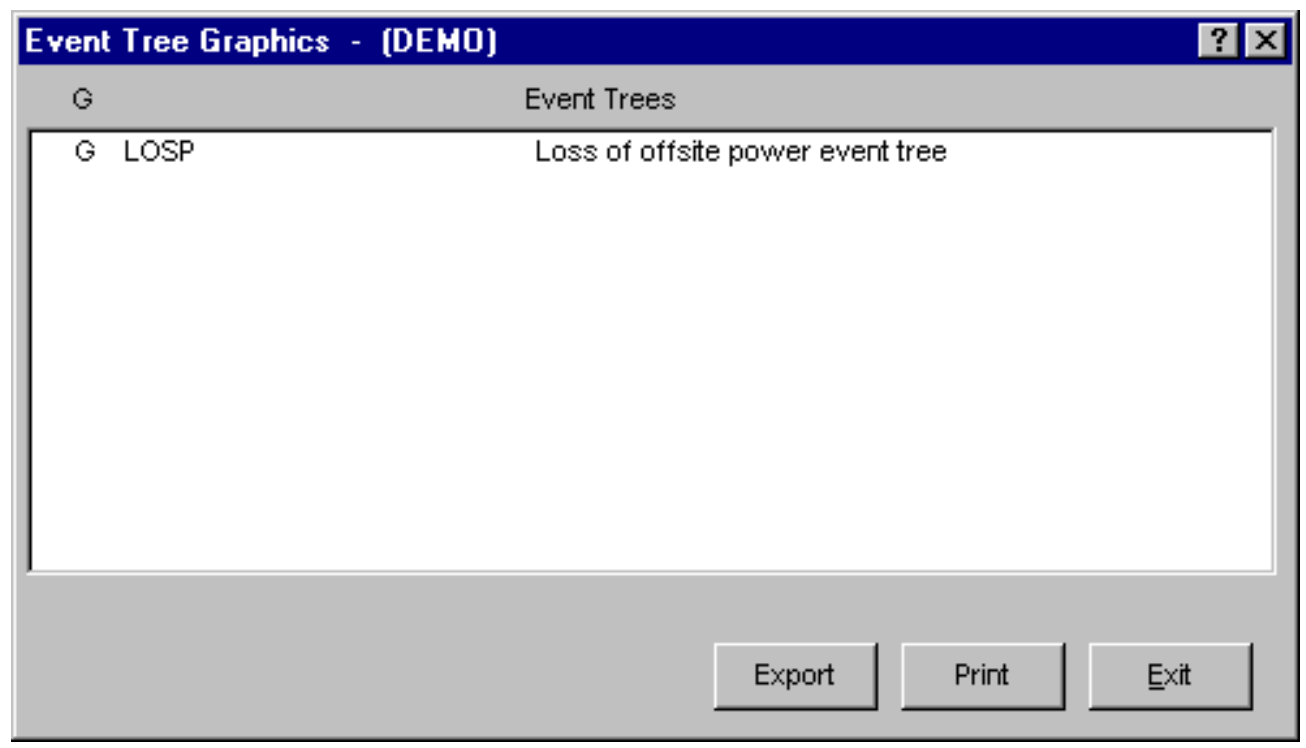

Export - Convert the graphic file(s) to either a file in Enhanced Metafile (EMF) or Windows Metafile (WMF) format.

Print - Print the graphic file(s) to the designated printer.

Exit - $\quad$ Close the Graphics Reports dialog without generating a report.

\subsubsection{Initiating Events Report}

\section{PURPOSE}

This option allows you to generate the Event Tree Initiating Events Report. This report contains the event tree name and the corresponding event tree description and initiating event for all event trees in the current project. 


\section{STEPS}

1. From the SAPHIRE menu select Report. The Reports Menu dialog will be displayed.

2. Select the Event Tree Data Type and the Initiating Events Report Type radio buttons.

3. Choose the Process button.

4. Highlight the desired event tree(s) or process all records.

5. The Report Viewer will display the report.

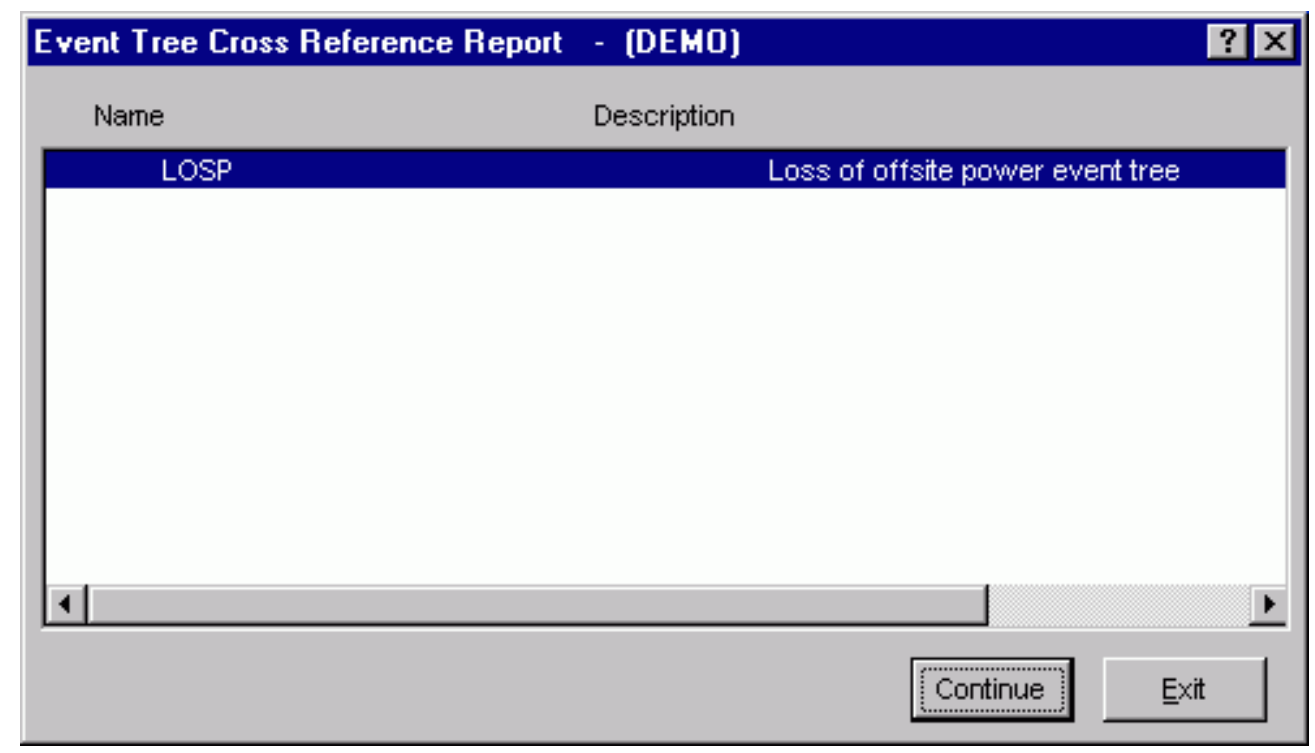

\subsubsection{Cross Reference}

\section{PURPOSE}

This option provides the means for generating a cross reference report for event trees or sub-trees.

\section{STEPS}

1. From the SAPHIRE menu select Report. The Reports Menu dialog will be displayed.

2. Select the Event Tree Data Type and the Cross Reference Report Type radio buttons.

3. Select the desired Sub Type radio button and the Show Sub-Trees check box, if desired.

4. Choose the Process button.

5. From the subsequent dialog, highlight the desired event tree(s) or process all records.

6. Choose the Continue button to generate the report. The Report Viewer will display the report. 


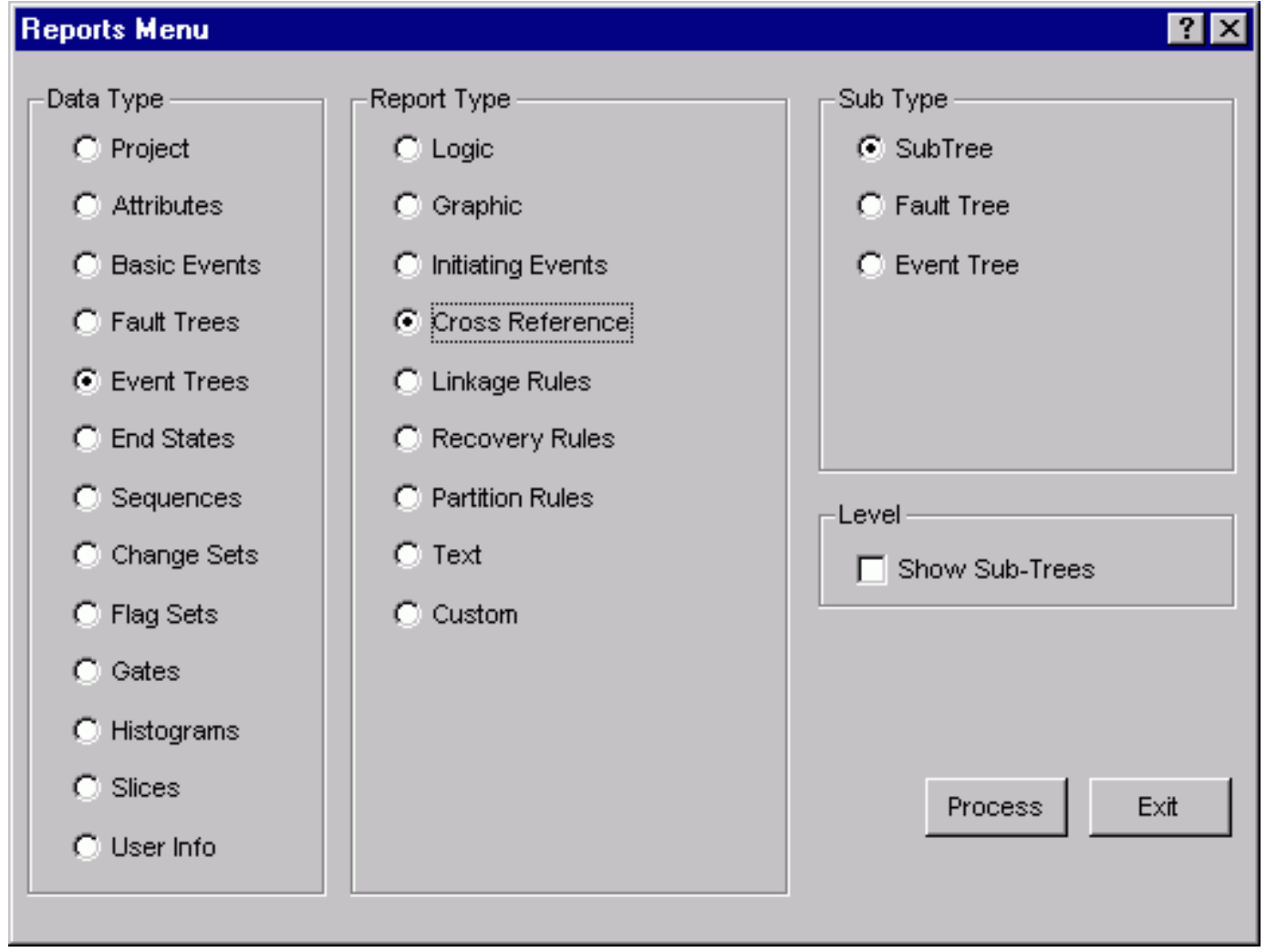

Level

Show Sub-trees - Include the event trees marked as sub-trees in the list of event trees.

SubType

Sub-tree - $\quad$ Generate the Sub Event Tree Hierarchy Report. Each selected event tree or sub-tree will be placed on a separate page with the selected event tree name and of a hierarchical list of all sub-trees it uses.

Fault Tree - $\quad$ Generate the Event Tree / Fault Tree Reference Report consisting of the selected event tree(s) and/or sub-tree(s) name with the corresponding list of fault trees that it uses.

Event Tree - $\quad$ Generate the Sub-tree Cross Reference Report consisting of the selected event tree(s) and/or sub-tree(s) name and the corresponding trees that use it.

\subsection{End State Reports}

\subsubsection{End State}

\section{PURPOSE}

This option provides the means to generate end state reports. These include summary, combination, uncertainty, and base vs. current case reports. 


\section{STEPS}

1. From the SAPHIRE menu select Report. The Reports Menu dialog will be displayed.

2. Select the End State Data Type and one of the Report Type radio buttons.

3. Choose the Process button.

4. From the subsequent dialog, highlight the desired end state(s) or process all records.

5. Choose the appropriate buttons to generate the report. The Report Viewer will display the report.

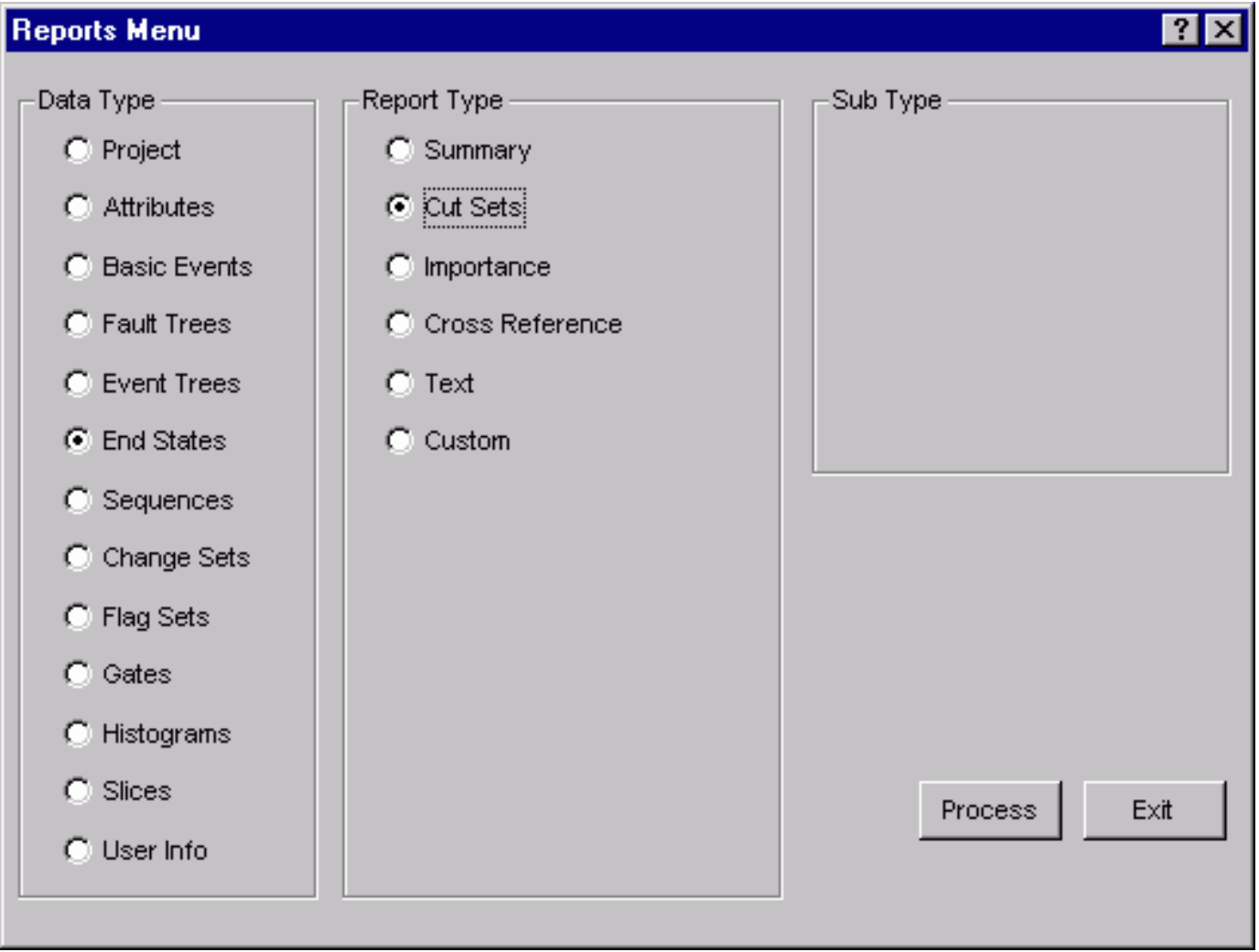

\subsubsection{End State Summary Report}

\section{PURPOSE}

This option allows you to generate an end state summary report based on current or base case values for a specified analysis type. 


\section{STEPS}

1. From the SAPHIRE menu select Report The Reports Menu dialog will be displayed.

2. Select the End State Data Type and the Summary Report Type radio buttons.

3. Choose the desired Sub Type radio button then

4. Choose the Process button.

5. From the subsequent dialog, select the desired Case radio button and the desired analysis type from the drop-down list.

6. Highlight the desired end state(s) or process all records

7. Choose the Continue button to generate the report. The Report Viewer will display the report.

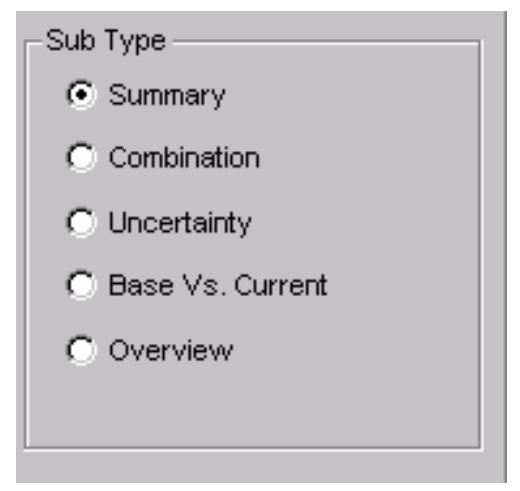

\section{Case}

Current - $\quad$ Use current case values for all calculations. This is the default.

Base -

Use base case values for all calculations.

Summary -

Combination -

Uncertainty -

Base Vs. Current -

Overview -
Generate the End State Brief Summary Report. This report contains the name, the associated minimum cut set upper bound, and the description of the selected fault trees.

Generate the End State Combination Report. This report contains the name, minimum cut set upper bound, mean, and number of cut sets in the selected fault trees.

Generate the End State Uncertainty Value Report. This report contains the number, name, mean and median values, standard deviation, the 5th and 95th percentile, the minimum and maximum values, and the seed size of the selected end states

Generate the End State Base/Current Report. For each selected fault tree, this report contains the name, the minimal cut set upper bound for the base case and current case cut sets, the difference (base - current), and the number of base case and current case cut sets.

This option is not yet implemented. 


\subsubsection{End State Cut Sets Report}

\section{PURPOSE}

This option allows you to generate cut set reports based on current and base case values for a specified analysis type.

\section{STEPS}

1. From the SAPHIRE menu select Report. The Reports Menu dialog will be displayed.

2. Select the End State Data Type and the Cut Sets Report Type radio buttons.

3. Choose the Process button. The End State Cut Sets dialog will be displayed, listing all of the end states residing in the current project.

4. Select the desired Case radio button.

5. Select the desired analysis type from the drop-down list.

6. Highlight the desired end state(s) or process all records

7. Choose the desired button to generate the report. The Report Viewer will display the report.

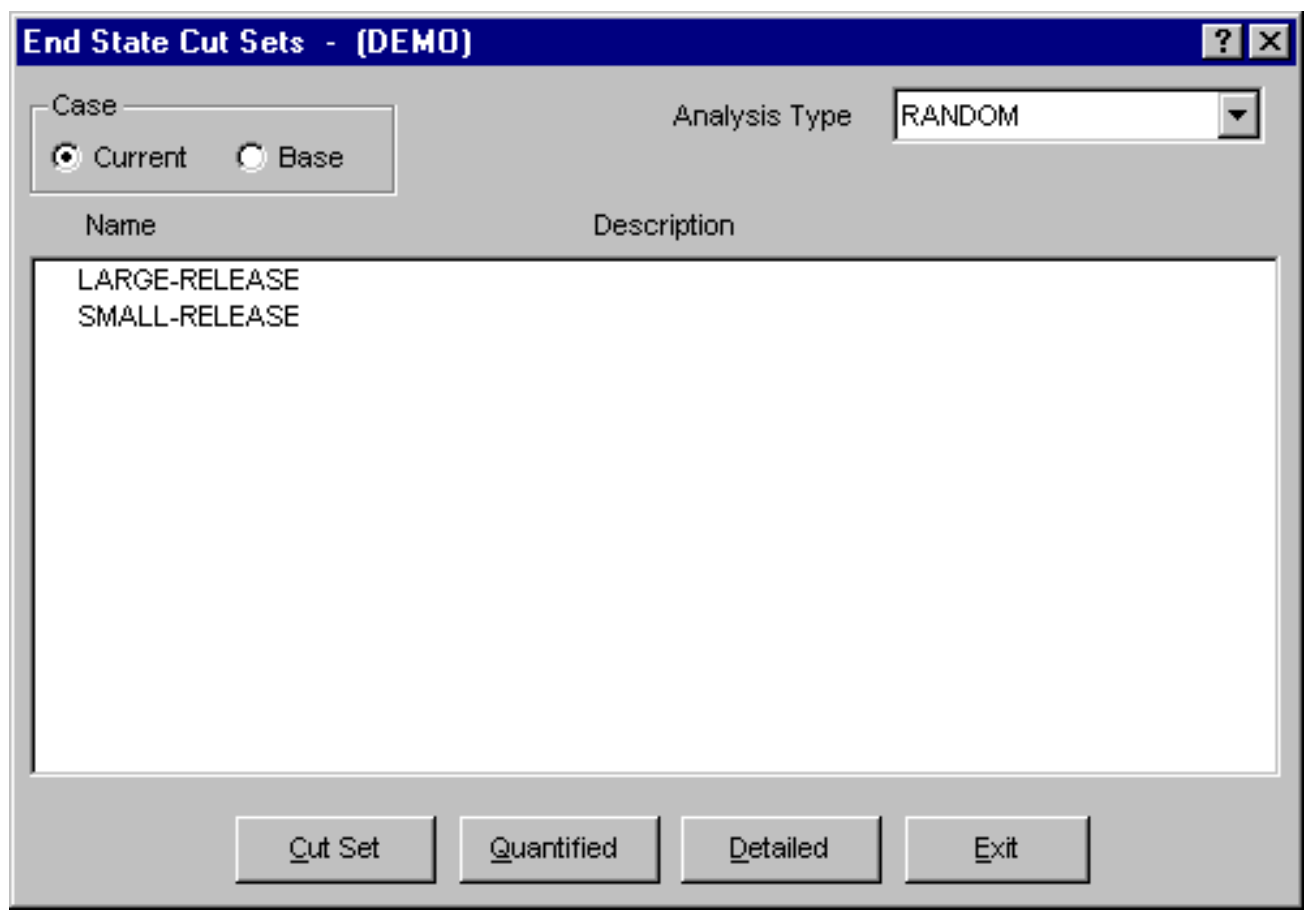

Case

Current - $\quad$ Use current case values for all calculations. This is the default. Base - $\quad$ Use base case values for all calculations. 
Cut Set -

Quantified -

Detailed -
Generate the End State Cut Sets Report which consists of the cut set number, size, and the basic event names that are included in the corresponding cut sets (current or base) for the selected end state(s). When you choose this button, the Cut Set Report Options dialog will be displayed.

Generate the End State Cut Sets (Quantification) Report for quantified cut sets only. The report consists of the cut set number, percent of total, percent of the cut set, probability/frequency and the basic event names that are included in the associated cut sets (current or base) for the selected end state(s). When you choose this button, the Cut Set Report Options dialog will be displayed.

Generate the End State Cut Sets (Detailed) Report which consists of the cut set number, percent of the cut set, probability/frequency, and the basic event names and descriptions included in associated cut sets (current or base) for the selected end state(s). When you choose this button, the Cut Set Report Options dialog will be displayed.

Exit - Close the End State Cut Sets dialog without generating a report.

\subsubsection{Cut Set Report Options}

\section{PURPOSE}

This option allows you to specify several cut set cutoff values that will be used to determine if a cut set is to be included in the report.

You can modify any of the default values provided by typing over the existing data.

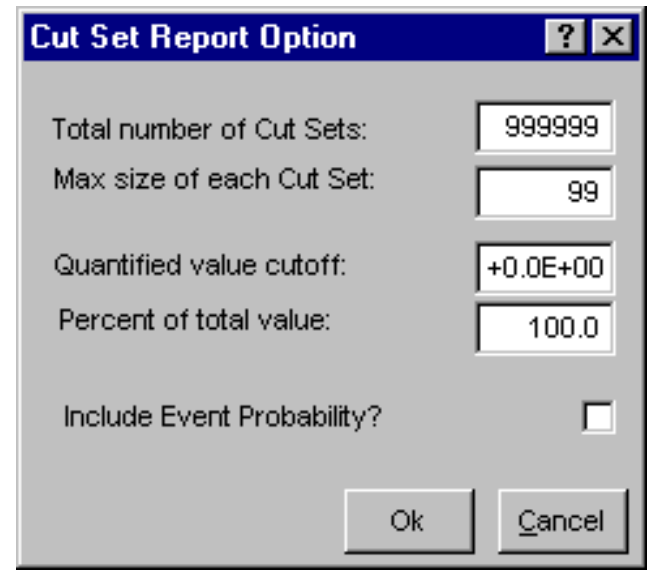


Total number of Cut Sets -

Max size of each Cut Set -

Quantified value cutoff -

Percent of total value -

Include Event Probability? -

Ok -

Cancel -
The number of cut sets included in the report will not exceed this number. Cut sets are considered beginning with the cut that contributes the highest percent frequency.

Include only those cut sets that contain no more than the number of events entered here.

Include only those cut sets whose frequency is greater than or equal to the value entered here. Applies only to the Quantified and Detailed cut set reports.

Include only those cut sets whose sum of the percent contribution is less than this value, beginning with the cut set that contributes the greatest percent. By entering 100.0 (the default) here, all cut sets will be included.

Preface the report with a list of each of the basic events referenced in the cut sets along with the basic event probabilities. Generate the report based on the specified cutoff values. The Report Viewer will display the report.

Close the Cut Set Report Option dialog without generating a report.

\subsubsection{End State Importance Reports}

\section{PURPOSE}

This option allows you to generate end state importance reports based the selected analysis type and on current or base case values.

\section{STEPS}

1. From the SAPHIRE menu select Report. The Reports Menu dialog will be displayed.

2. Select the End State Data Type and the Importance Report Type radio buttons.

3. Choose the Process button. The End State Importance dialog will be displayed, listing all of the end states residing in the current project.

4. Select the desired Case radio button.

5. Select the desired analysis type from the drop-down list.

6. Highlight the desired end state(s) or process all records.

7. Choose the desired button to generate the report. The Report Viewer will display the report. 


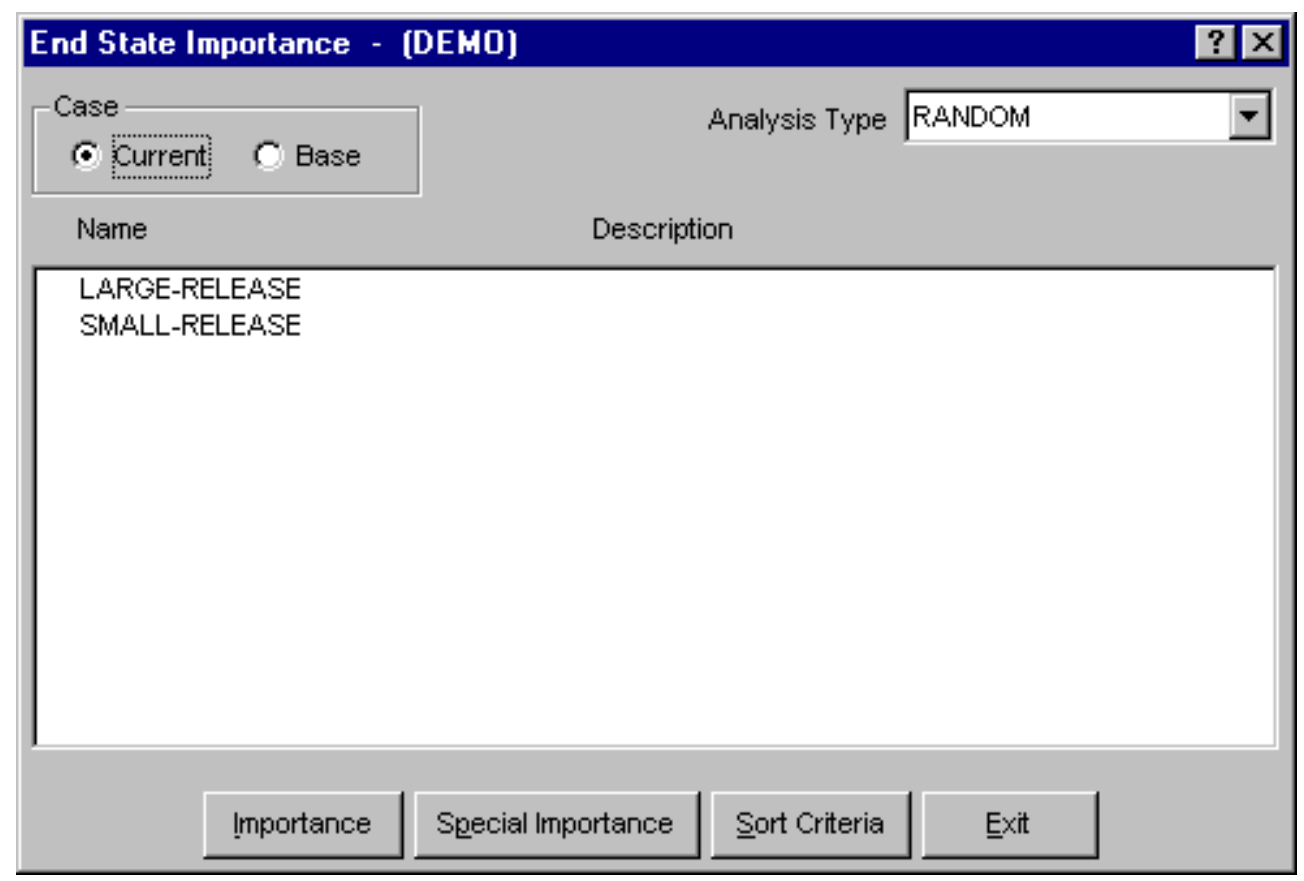

Case

Current - $\quad$ Use current case values for all calculations. This is the default.

Base -

Use base case values for all calculations.

Importance -

Generate the End State Importance Measures report consisting of event names, number of times the event occurs, probability of failure, uncertainty importance value, risk reduction difference, and risk increase difference for the selected end state(s).

Special Importance - Generate the End State Importance Measures report consisting of event names, probability of failure, Fussell-Vesely value, risk reduction ratio, risk increase ratio, Birnbaum importance value, and event descriptions for the selected end state(s).

Sort Criteria - $\quad$ Select the sort order in which to display the End State Importance Measures Report. When you choose this button, the Select Sort Criteria dialog will be displayed.

Exit report.

Close the End State Importance dialog without generating a

\subsubsection{Select Sort Criteria}

\section{PURPOSE}

This option allows you to select the sort order in which to display the Fault Tree/Sequence/End State Importance Measures Report. 


\section{STEPS}

1. From the SAPHIRE menu select Report. The Reports Menu dialog will be displayed.

2. Select the Fault Tree/Sequence Data Type and Importance Report Type radio buttons.

3. Choose the Process button. The Fault Tree/Sequence Importance dialog will be displayed, listing all of the fault trees residing in the current project.

4. Select the desired Case radio button.

5. Select the desired analysis type from the drop-down list.

6. Highlight the desired fault tree(s) or process all records.

7. Choose the Sort Criteria button to select the sort order and choose OK.

8. Choose the desired report button from the Fault Tree/Sequence Importance dialog. The Report Viewer will display the report in the specified order.

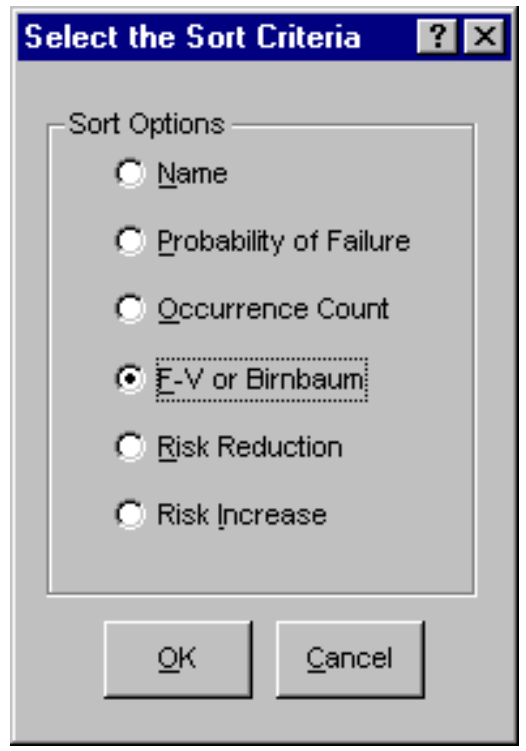

Name - $\quad$ Sort by event name

Probability of Failure - Sort by the probability failures

Occurrence Count - Sort by the number of occurrences (most to fewest)

F-V or Birnbaum - $\quad$ Sort in Fussell-Vesely or Birnbaum order

Risk Reduction - $\quad$ Sort in risk reduction ratio or difference order

Risk Increase - $\quad$ Sort in risk increase ratio or difference order.

OK -

Cancel - Closes the Select the Sort Criteria dialog without saving the sort option.

\subsubsection{End State Cross Reference Report}

\section{PURPOSE}

This option allows you to generate a cross reference report consisting of the end state number, end state name, and a list of event trees and sequences for each selected end state. 


\section{STEPS}

1. From the SAPHIRE menu select Report. The Reports Menu dialog will be displayed.

2. Select the End State Data Type and the Cross Reference Report Type radio buttons.

3. Choose the Process button. The End State X-Reference dialog will be displayed, listing all of the end states residing in the current project.

4. Select the desired Case radio button.

5. Select the desired analysis type from the drop-down list.

6. Highlight the desired end state(s) or process all records.

7. Choose the Continue button to generate the report. The Report Viewer will display the report.

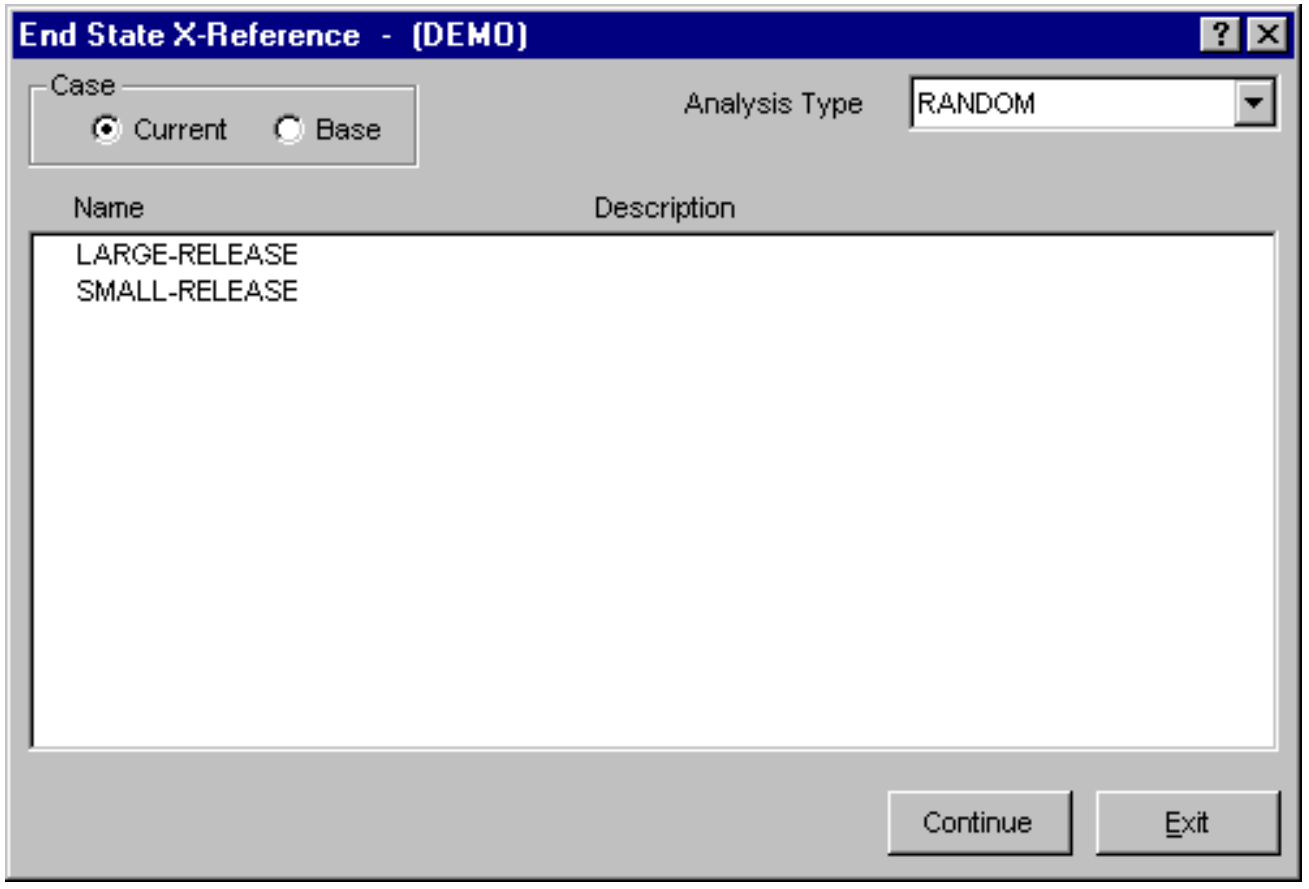

Case

Current - Use current case values for all calculations. This is the default.

Base - Use base case values for all calculations.

Continue - $\quad$ Generate the End State - Sequence Cut Set Reference Report. After choosing this button, the Report Viewer will display the report

Exit - $\quad$ Close the End State X-Reference dialog without generating a report. 


\subsection{Sequence Reports}

\subsubsection{Sequence}

\section{PURPOSE}

This option provides the means to generate a variety of sequence reports. These include summary, logic, cut sets, and importance reports.

\section{STEPS}

1. From the SAPHIRE menu select Report. The Reports Menu dialog will be displayed.

2. Select the Sequence Data Type and one of the Report Type radio buttons.

3. Choose the Process button.

4. From the subsequent dialog, highlight the desired sequence(s)

5. Choose the appropriate buttons to generate the report. The Report Viewer will display the report.

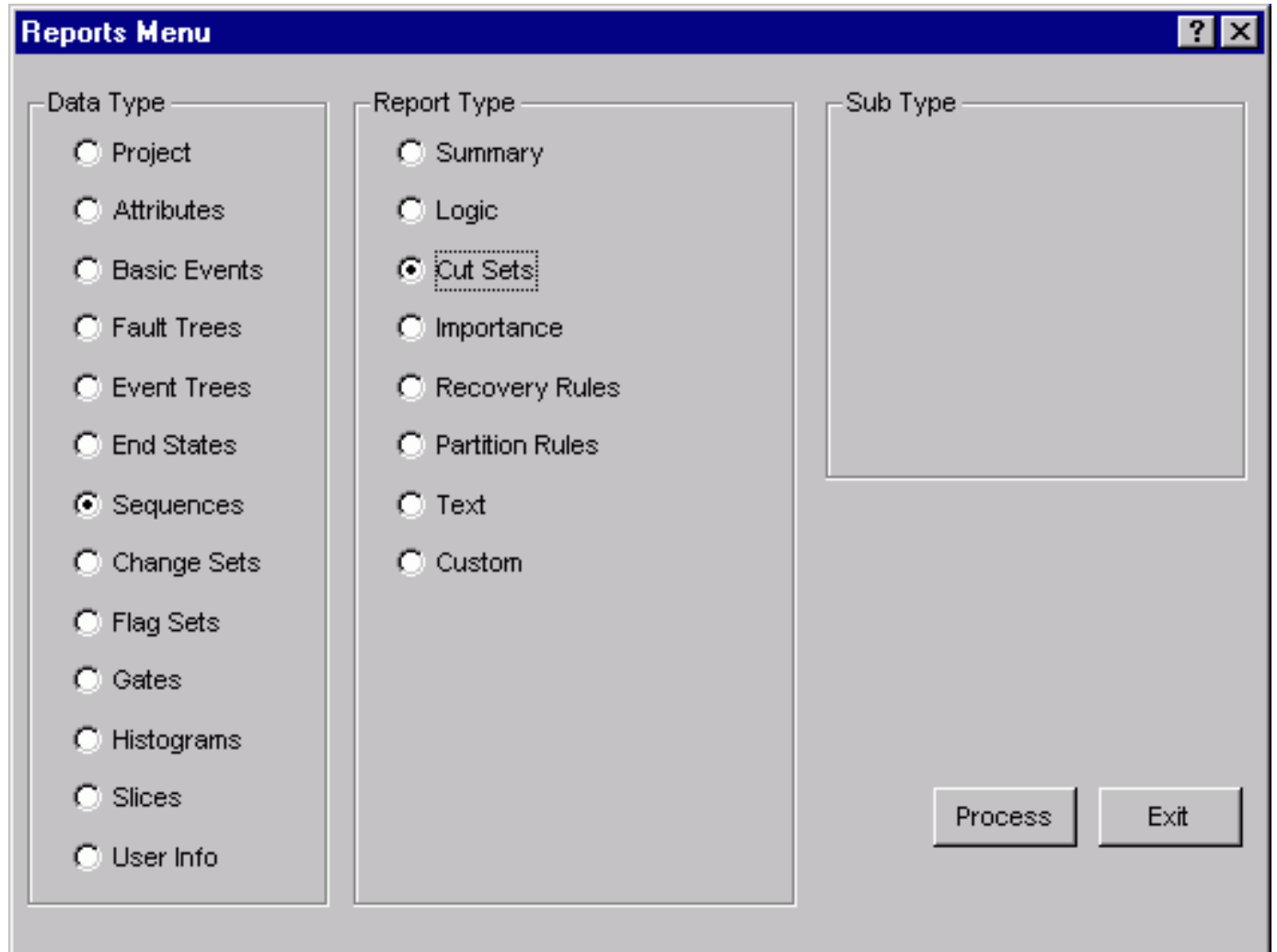




\section{Report Type}

Summary - $\quad$ This option allows you to generate various fault tree summary reports.

Logic - $\quad$ Generate a report showing the sequence logic information associated with each selected event tree.

Cut Sets - $\quad$ Generate a report displaying cut set information for each selected sequence.

Importance - Generate a report listing associated importance measures information for each selected sequence.

Recovery Rules -This report outputs a listing of the recovery rule logic that applies to each selected sequence. Choose the Basic or Advanced radio button to indicate the desired rule type.

Partition Rules - This report outputs a listing of the partition rule logic that applies to each selected sequence. Choose the Basic or Advanced radio button to indicate the desired rule type.

Text - $\quad$ Generate a report containing the descriptive text associated with the selected sequence(s).

Custom - $\quad$ Create or select a user-defined report relating to sequence level information.

\subsubsection{Sequence Summary Reports}

\section{PURPOSE}

This option allows you to generate sequence summary reports that are based on current or base case values for a specified analysis type.

\section{STEPS}

1. From the SAPHIRE menu select Report. The Reports Menu dialog will be displayed.

2. Select the Sequence Data Type and the Summary Report Type radio buttons.

3. Select the desired Sub Type radio button

4. Choose the Process button.

5. From the subsequent dialog, select the desired Case radio button and the desired analysis type from the drop-down list.

6. Highlight the desired sequence(s) or process all records.

7. Choose the Continue button to generate the report. The Report Viewer will display the report. 


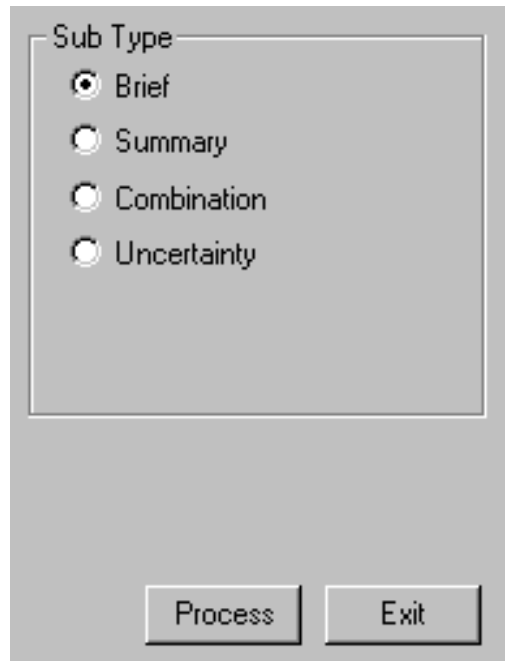

Case

Current - Use current case values for all calculations. This is the default.

Base - Use base case values for all calculations.

Brief Summary -Generate the Sequence Brief Summary Report. This report contains the event tree name, sequence name, end state name, and the minimum cut set upper bound for the selected sequence(s). The minimum cut set upper bound values for the selected sequences are totaled at the end or the report.

Summary - Generate the Sequence Summary Report. This report contains the event tree name, sequence name, the associated minimum cut set upper bound, and the description of the selected sequence(s). The minimum cut set upper bound values for the selected sequences are totaled at the end or the report.

Combination - Generate the Sequence Combination Report. This report contains the event tree name, sequence name, minimum cut set upper bound, mean, and number of cut sets in the selected sequence(s). Totals for the minimum cut set upper bound, mean and number of cut sets are provided at the end of the report.

Uncertainty - Generate the Sequence Uncertainty Values Report. This report contains the number, event tree name, sequence name, mean and median values, standard deviation, the 5th and 95th percentile, the minimum and maximum values, and the seed size of the selected sequence(s).

\subsubsection{Sequence Logic Report}

\section{PURPOSE}

This option allows you to generate the Sequence Logic Report. This report contains the sequence number, event tree name, sequence name, initiating event name, flag set name, and the logic paths (pass/fail) for the selected sequence(s).

\section{STEPS}

1. From the SAPHIRE menu select Report. The Reports Menu dialog will be displayed. 
2. Select the Sequence Data Type and the Logic Report Type radio buttons.

3. Choose the Process button. The Sequence Logic dialog will be displayed, listing all of the sequences in the currently selected project.

4. Highlight the desired sequence(s) or process all records.

5. Choose the Continue button to generate the report. The Report Viewer will display the report.

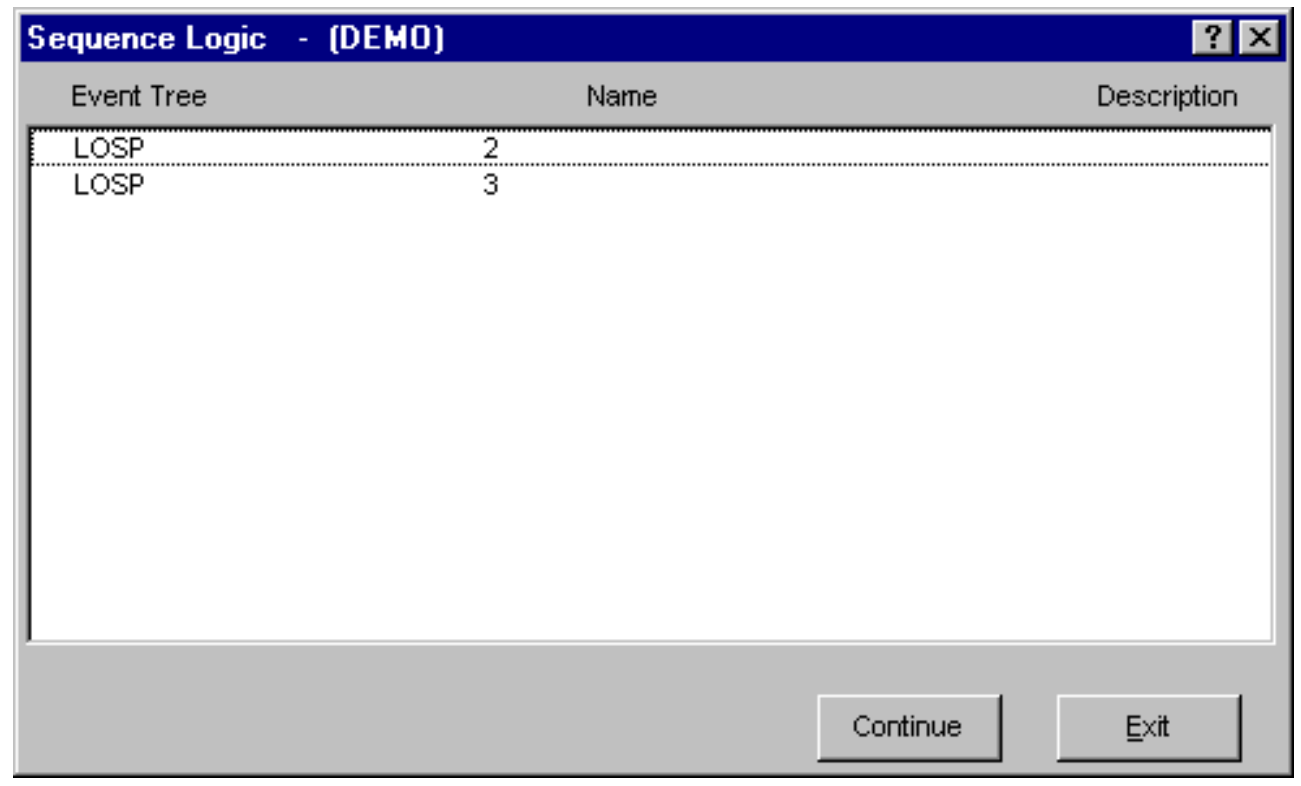

Continue - Generate the report. After this button has been selected, the Report Viewer will display the report.

Exit - $\quad$ Close the Sequence Logic dialog without generating a report.

\subsubsection{Sequence Cut Sets Reports}

\section{PURPOSE}

This option allows you to generate three types of sequence cut set reports based the selected analysis type and on current or base case values.

\section{STEPS}

1. From the SAPHIRE menu select Report. The Reports Menu dialog will be displayed.

2. Select the Sequence Data Type and the Cut Sets Report Type radio buttons.

3. Choose the Process button. The Sequence Cut Sets dialog will be displayed, listing all of the sequences residing in the current project. 
4. Select the desired Case radio button.

5. Select the desired analysis type from the drop-down list.

6. Highlight the desired sequence(s) or process all records.

7. Choose the desired button to generate the report. The Report Viewer will display the report.

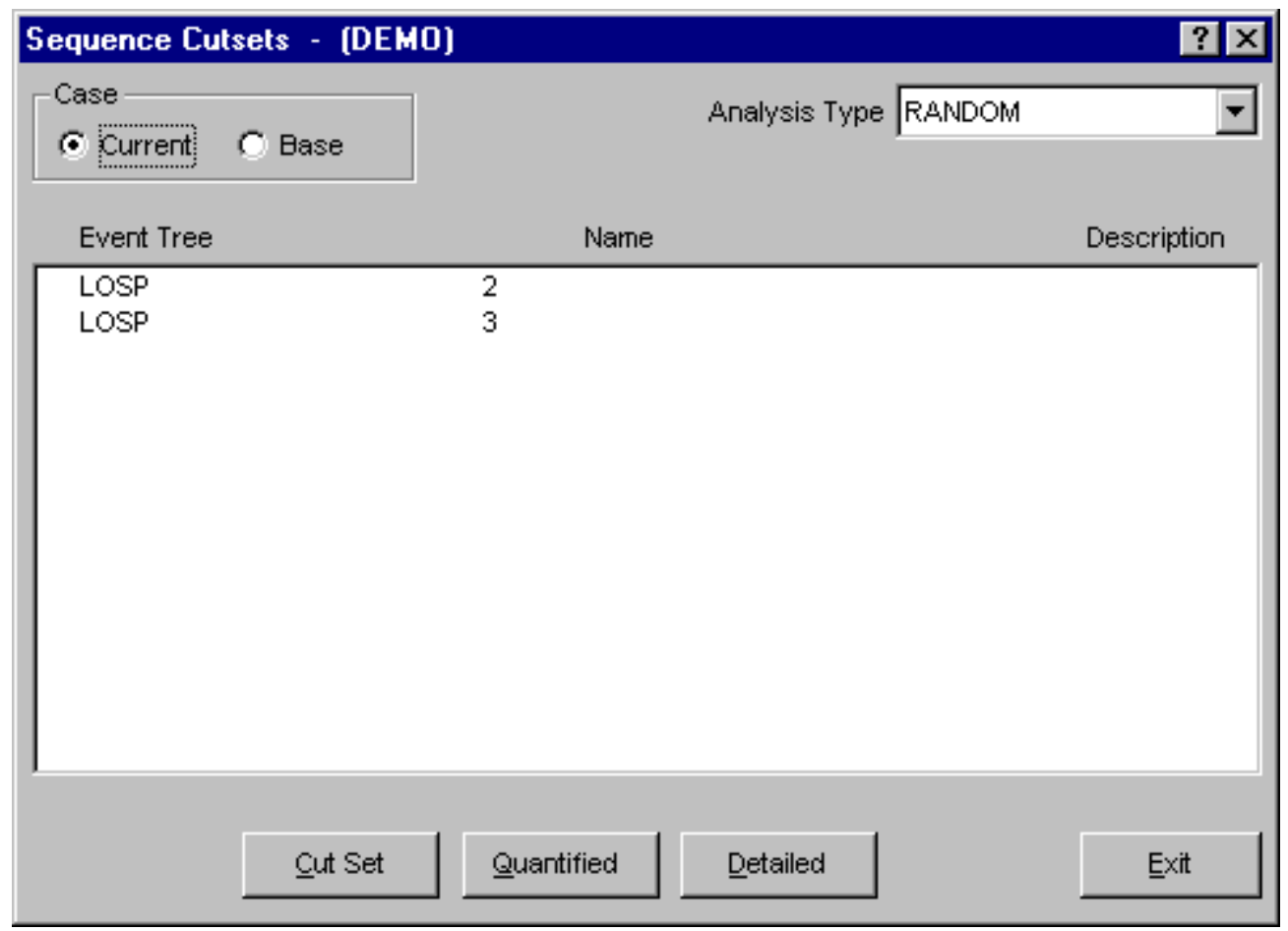

Case

Current - Use current case values for all calculations. This is the default.

Base - Use base case values for all calculations.

Cut Set - $\quad$ Generate the Sequence Cut Sets Report which consists of the cut set number, size, and the basic event names that are included in the corresponding cut sets (current or base) for the selected sequence(s). When you choose this button, the Cut Set Report Options dialog will be displayed followed by the Report dialog.

Quantified - Generate the Sequence Cut Sets (Quantification) Report for quantified cut sets only. The report consists of the cut set number, percent of total, percent of the cut set, probability/frequency and the basic event names that are included in the associated cut sets (current or base) for the selected sequence(s). When you choose this button, the Cut Set Report Options dialog will be displayed followed by the Report dialog.

Detailed - Generate the Sequence Cut Sets (Detailed) Report which consists of the cut set number, percent of the cut set, probability/frequency, and the basic event names and descriptions included in associated cut sets (current or base) for the selected 
sequence(s). When you choose this button, the Cut Set Report Options dialog will be displayed followed by the Report dialog.

Exit - $\quad$ Close the Sequence Cut Sets dialog without generating a report.

\subsubsection{Cut Set Report Options}

\section{PURPOSE}

This option allows you to specify several cut set cutoff values that will be used to determine if a cut set is to be included in the report.

You can modify any of the default values provided by typing over the existing data.

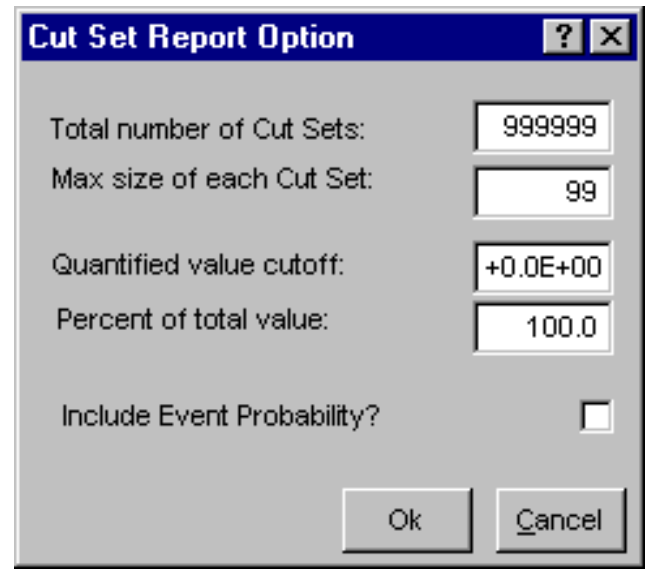

Total number of Cut Sets -

Max size of each Cut Set -

Quantified value cutoff -

Percent of total value -

Include Event Probability? -

Ok -

Cancel -
The number of cut sets included in the report will not exceed this number. Cut sets are considered beginning with the cut that contributes the highest percent frequency.

Include only those cut sets that contain no more than the number of events entered here.

Include only those cut sets whose frequency is greater than or equal to the value entered here. Applies only to the Quantified and Detailed cut set reports.

Include only those cut sets whose sum of the percent contribution is less than this value, beginning with the cut set that contributes the greatest percent. By entering 100.0 (the default) here, all cut sets will be included.

Preface the report with a list of each of the basic events referenced in the cut sets along with the basic event probabilities. Generate the report based on the specified cutoff values. The Report Viewer will display the report.

Close the Cut Set Report Option dialog without generating a report. 


\subsubsection{Sequence Importance Reports}

\section{PURPOSE}

This option allows you to generate importance reports based on the selected analysis type and on current or base case values. The header for each page contains the currently selected project name, analysis type, case (base or current), the sequence name, event tree name, and initiating event name. Also included, in parenthesis, is the selected sort order.

\section{STEPS}

1. From the SAPHIRE menu select Report. The Reports Menu dialog will be displayed.

2. Select the Sequence Data Type and the Importance Report Type radio buttons.

3. Choose the Process button. The Sequence Importance dialog will be displayed, listing all of the sequences residing in the current project.

4. Select the desired Case radio button.

5. Select the desired analysis type from the drop-down list.

6. Highlight the desired sequence(s) or process all records.

7. Choose the desired button to generate the report. The Report Viewer will display the report.

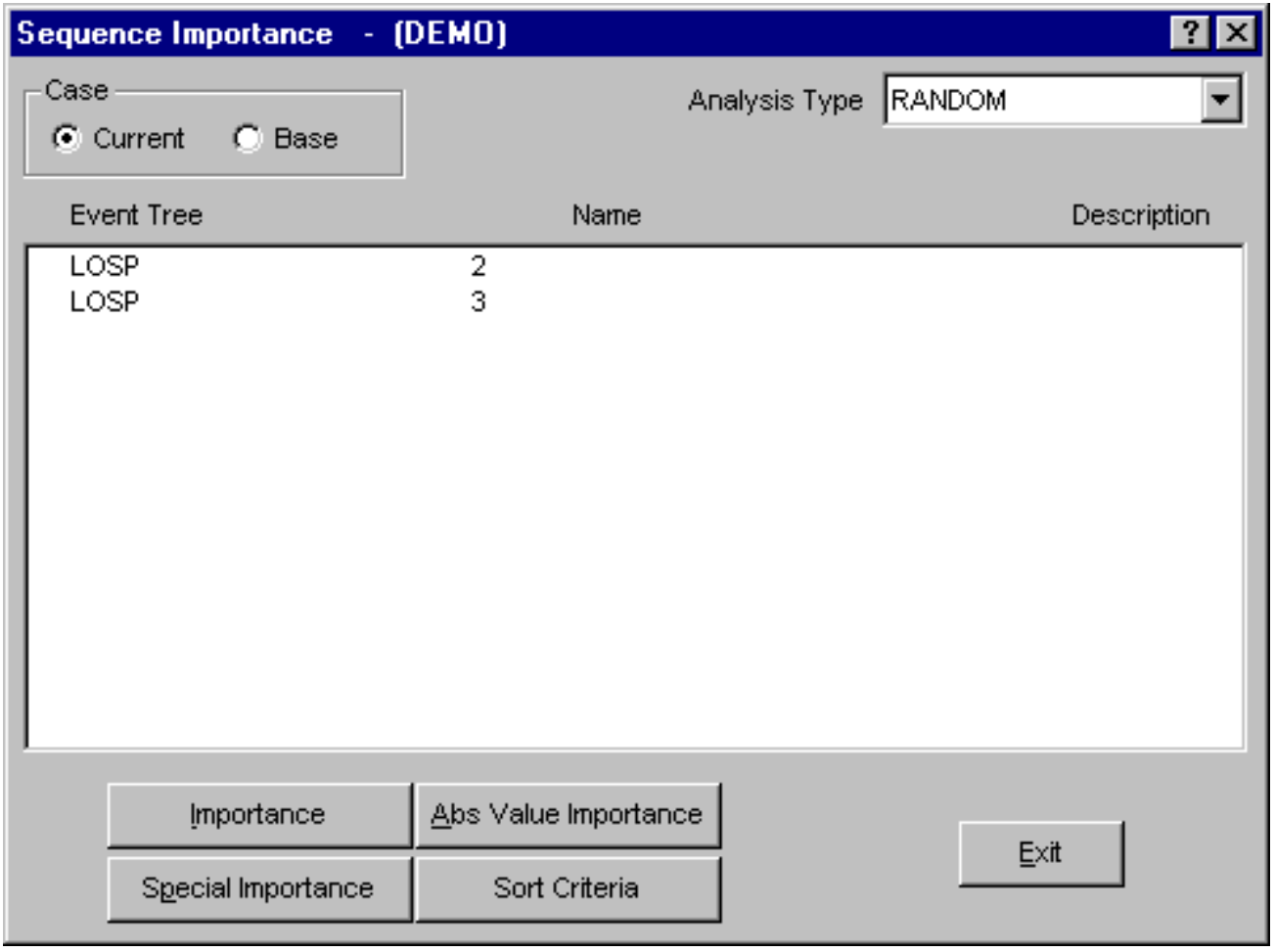

Case

Current - Use current case values for all calculations. This is the default. Base - Use base case values for all calculations. 
Importance -

Special Importance -

Sort Criteria -

Exit report.
Generate the Sequence Importance Measures report consisting of event names, number of times the event occurs, probability of failure, uncertainty importance value, risk reduction difference, and risk increase difference for the selected sequence(s).

Generate the Sequence Importance Measures report consisting of event names, probability of failure, Fussell-Vesely value, risk reduction ratio, risk increase ratio, Birnbaum importance value, and event descriptions for the selected sequence(s).

Abs. Value Importance -Generate the Sequence Absolute Value Importance report consisting of event names, number of times the event occurs, probability of failure, uncertainty importance value, risk reduction difference, and risk increase difference for the selected sequence(s).

Select the sort order in which to display the Sequence Importance Measures Report. When you choose this button, the Select Sort Criteria dialog will be displayed.

Close the Sequence Importance dialog without generating a

\subsubsection{Select Sort Criteria}

\section{PURPOSE}

This option allows you to select the sort order in which to display the Fault Tree/Sequence/End State Importance Measures Report.

\section{STEPS}

1. From the SAPHIRE menu select Report. The Reports Menu dialog will be displayed.

2. Select the Fault Tree/Sequence Data Type and Importance Report Type radio buttons.

3. Choose the Process button. The Fault Tree/Sequence Importance dialog will be displayed, listing all of the fault trees residing in the current project.

4. Select the desired Case radio button.

5. Select the desired analysis type from the drop-down list.

6. Highlight the desired fault tree(s) or process all records.

7. Choose the Sort Criteria button to select the sort order and choose OK.

8. Choose the desired report button from the Fault Tree/Sequence Importance dialog. The Report Viewer will display the report in the specified order. 


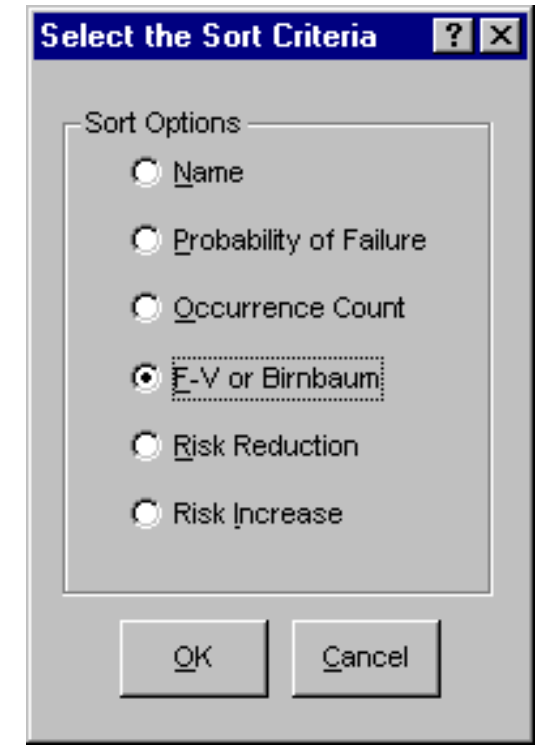

Name -

Sort by event name

Probability of Failure - Sort by the probability failures

Occurrence Count - $\quad$ Sort by the number of occurrences (most to fewest)

F-V or Birnbaum - $\quad$ Sort in Fussell-Vesely or Birnbaum order

Risk Reduction - $\quad$ Sort in risk reduction ratio or difference order

Risk Increase - $\quad$ Sort in risk increase ratio or difference order.

OK -

Cancel -

Closes the Select the Sort Criteria dialog and saves the sort option.

Closes the Select the Sort Criteria dialog without saving the sort option.

\subsection{Change Set Reports}

\subsubsection{Change Sets}

\section{PURPOSE}

This option provides the means to generate change set reports. These include separate reports for summary, single, and class change information.

\section{STEPS}

1. From the SAPHIRE menu select Report. The Reports Menu dialog will be displayed.

2. Select the Change Sets Type and one of the Report Type radio buttons.

3. Choose the Process button.

4. From the subsequent dialog, highlight the desired change set(s)

5. Choose the appropriate buttons to generate the report. The Report Viewer will display the report. 


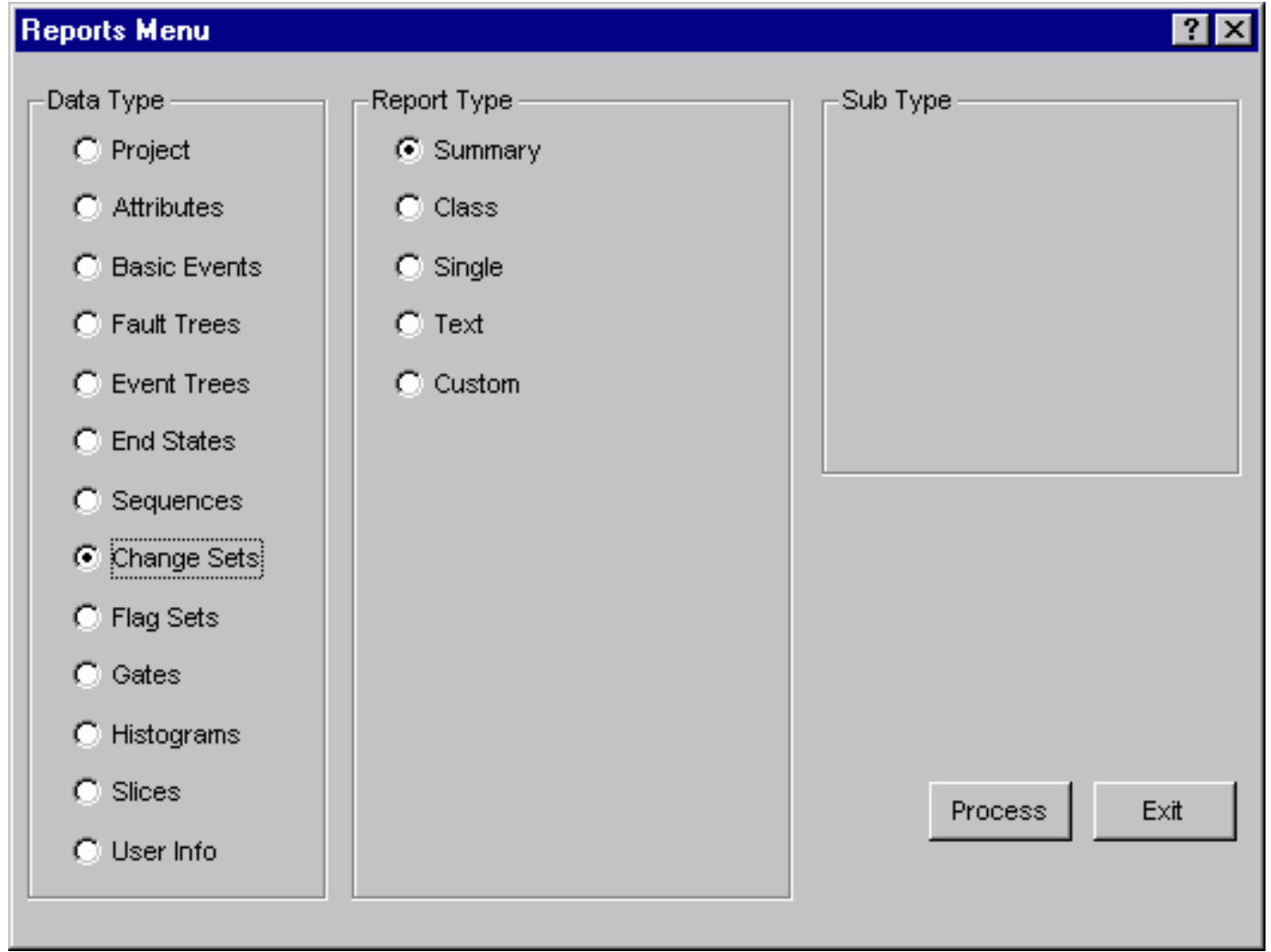

\subsubsection{Change Set/Flag Set Summary Reports}

\section{PURPOSE}

This option allows you to generate change set/flag set summary reports listing the name, description, and, for change sets, the current mark status.

\section{STEPS}

1. From the SAPHIRE menu select Report. The Reports Menu dialog will be displayed.

2. Select the Change Set Data Type and the Summary Report Type radio buttons.

3. Choose the Process button.

4. Highlight the desired change sets/flag sets or process all records.

5. Choose the Continue button to generate the report. The Report Viewer will display the report. 


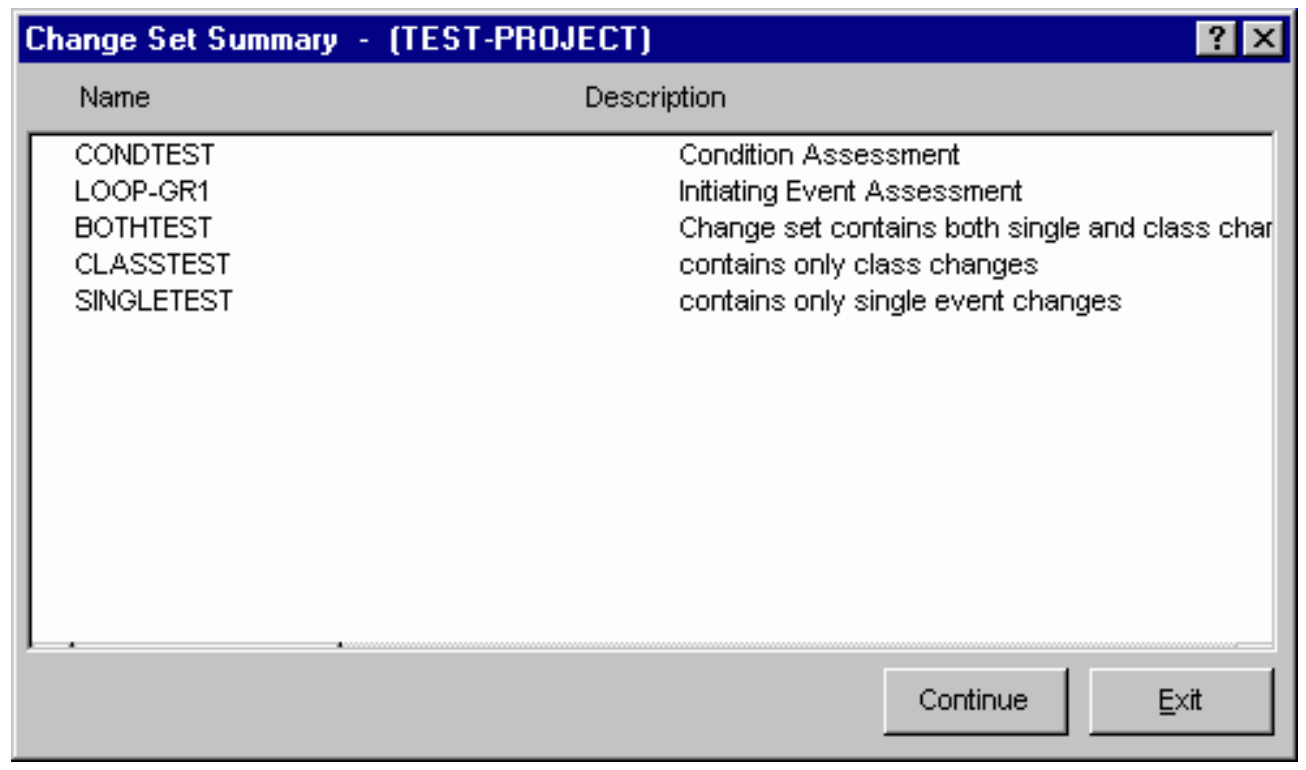

Continue - Generate the summary event report. The Report Viewer will display the report. Exit - $\quad$ Close the summary dialog without generating a report.

\subsubsection{Change Set Class Reports}

\section{PURPOSE}

This option allows you to generate a detailed change set report. The report will list the mark status and name of each selected change set, as well as a list of all events in the project that would be affected by the class change. (Single changes will not be included in this report.) For each included event the affected field change information is displayed. (Unaffected field information will be blank.)

\section{STEPS}

1. From the SAPHIRE menu select Report. The Reports Menu dialog will be displayed.

2. Select the Change Set Data Type and the Class Report Type radio buttons.

3. Choose the Process button.

4. Highlight the desired change sets or process all records.

5. Choose the Continue button to generate the report. The Report Viewer will display the report. 


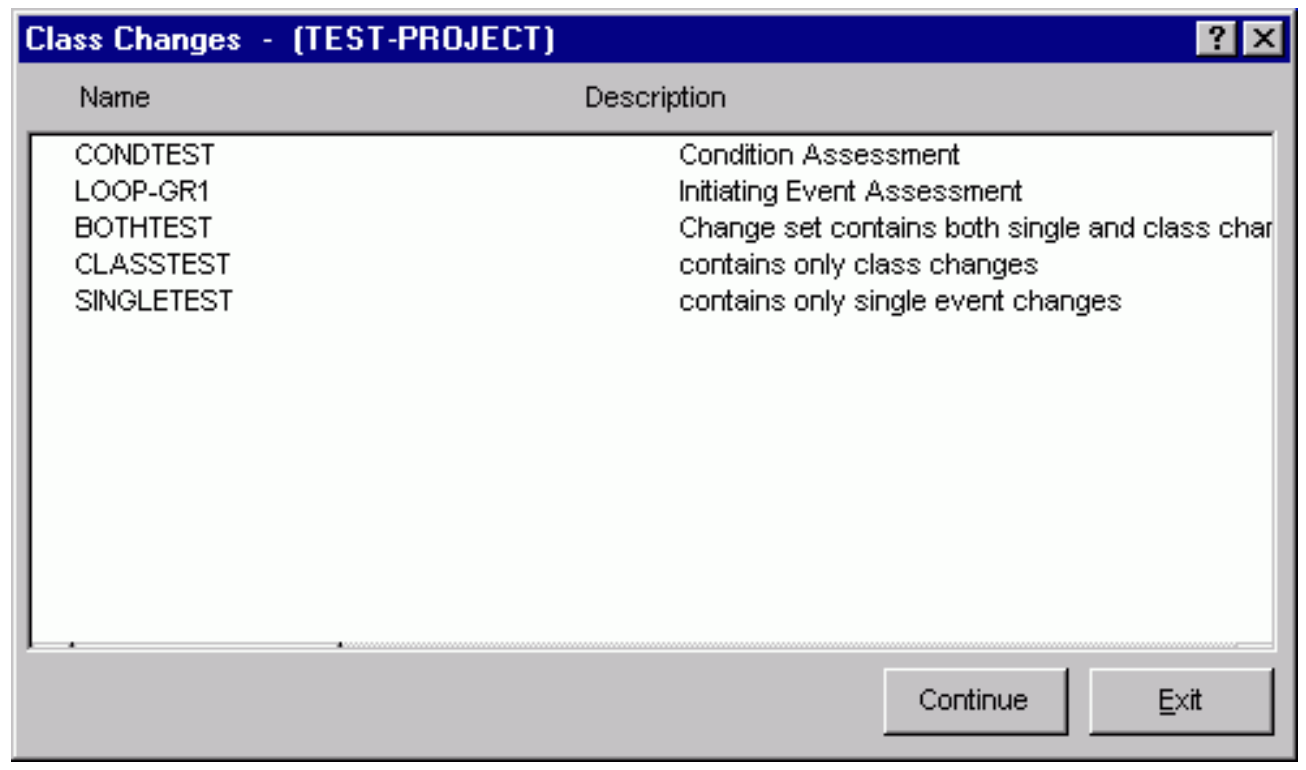

Continue - Generate the class change report. The Report Viewer will display the report. Exit - $\quad$ Close the dialog without generating a report.

\subsubsection{Single Changes Report}

\section{PURPOSE}

This option allows you to generate a detailed change set report. The report will list the mark status and name of each selected change set, as well as a list of all events explicitly included in the change set via the Single Change option. (Class changes will not be included in this report.) For each included event the affected field change information is displayed. (Unaffected field information will be blank.)

\section{STEPS}

1. From the SAPHIRE menu select Report. The Reports Menu dialog will be displayed.

2. Select the Change Set Data Type and the Class Report Type radio buttons.

3. Choose the Process button.

4. Highlight the desired change sets or process all records.

5. Choose the Continue button to generate the report. The Report Viewer will display the report. 


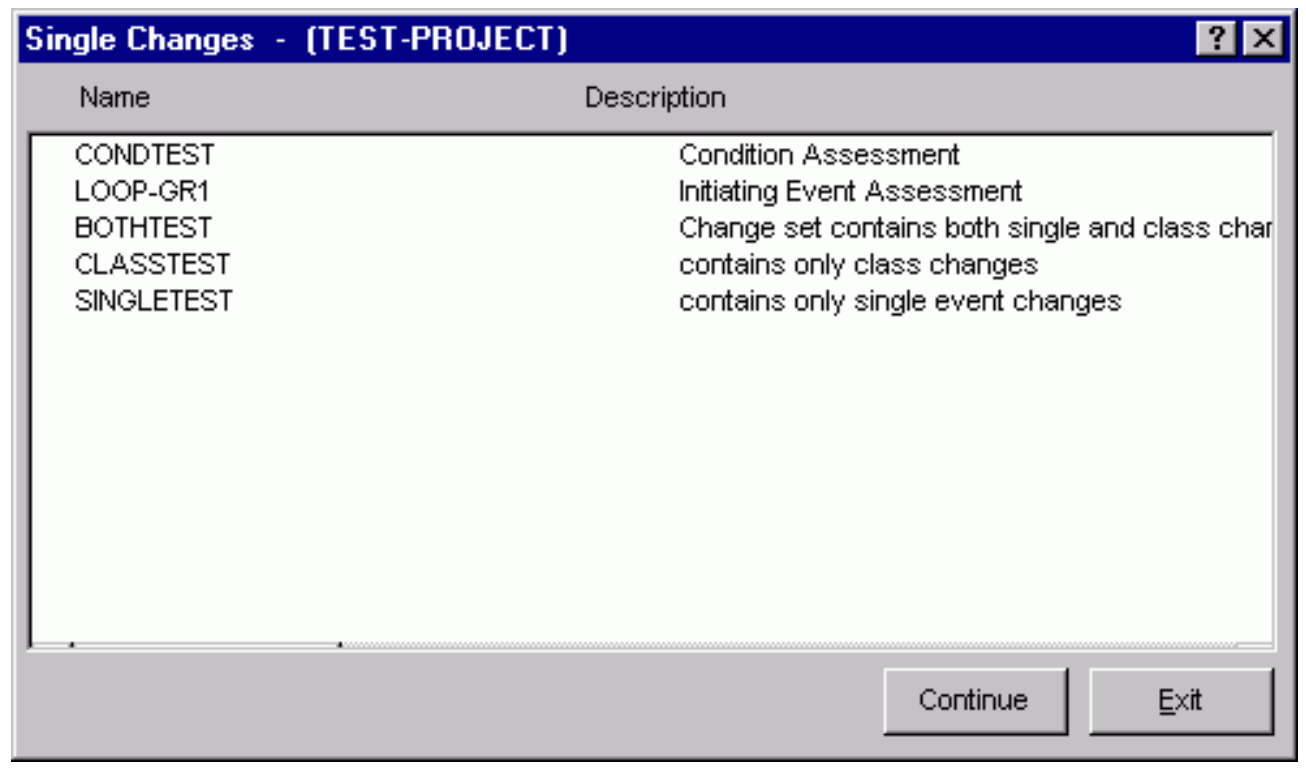

Continue - Generate the class change report. The Report Viewer will display the report. Exit - $\quad$ Close the dialog without generating a report.

\subsection{Flag Set Reports}

\subsubsection{Flag Sets}

\section{PURPOSE}

This option provides the means to generate flag set reports. These include separate reports for summary, detail (flag set events), and cross references.

\section{STEPS}

1. From the SAPHIRE menu select Report. The Reports Menu dialog will be displayed.

2. Select the Flag Sets Type and one of the Report Type radio buttons.

3. Choose the Process button.

4. From the subsequent dialog, highlight the desired change set(s)

5. Choose the appropriate buttons to generate the report. The Report Viewer will display the report. 


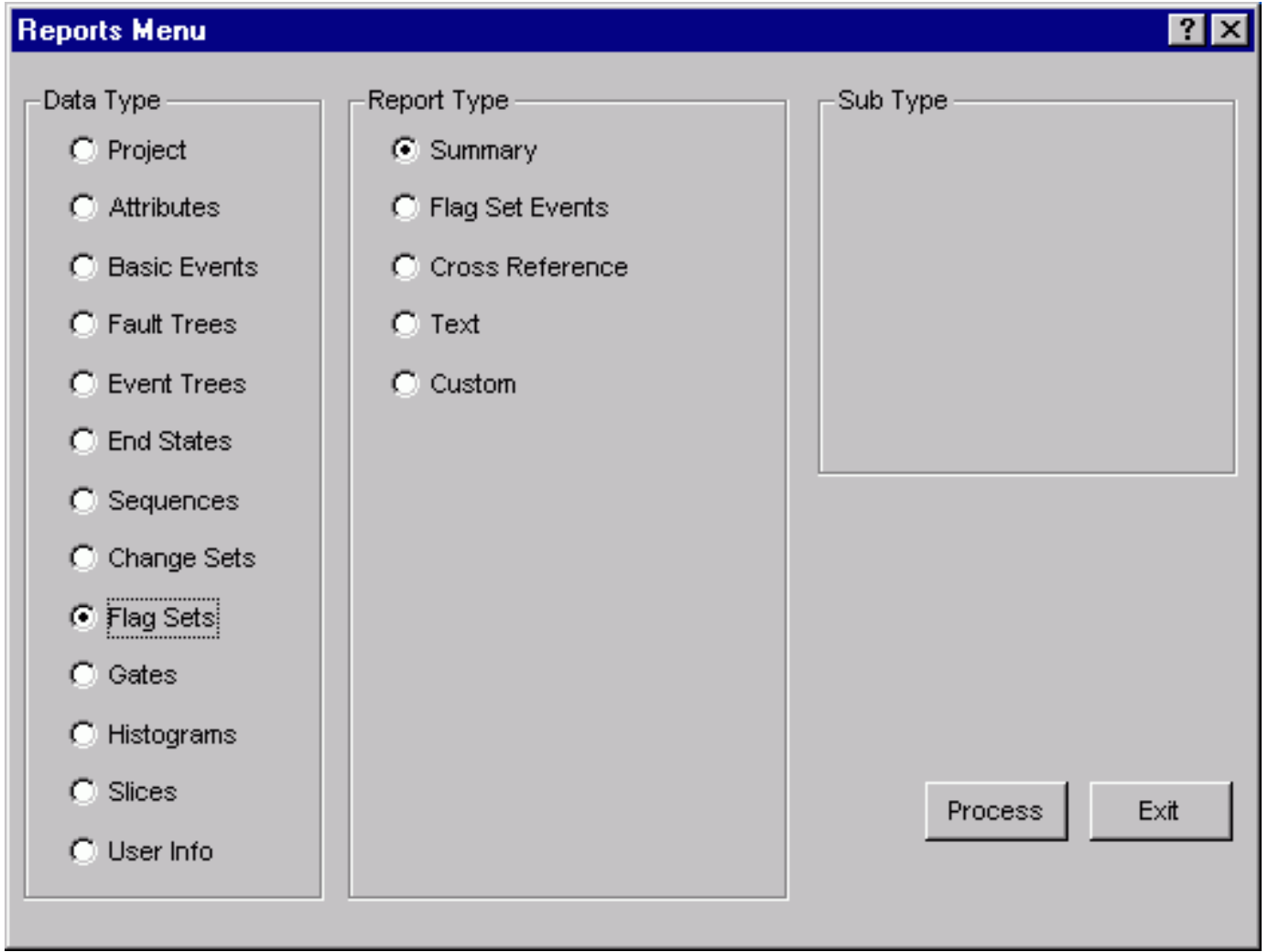

\subsubsection{Change Set/Flag Set Summary Reports}

\section{PURPOSE}

This option allows you to generate change set/flag set summary reports listing the name, description, and, for change sets, the current mark status.

\section{STEPS}

1. From the SAPHIRE menu select Report. The Reports Menu dialog will be displayed.

2. Select the Change Set Data Type and the Summary Report Type radio buttons.

3. Choose the Process button.

4. Highlight the desired change sets/flag sets or process all records.

5. Choose the Continue button to generate the report. The Report Viewer will display the report. 


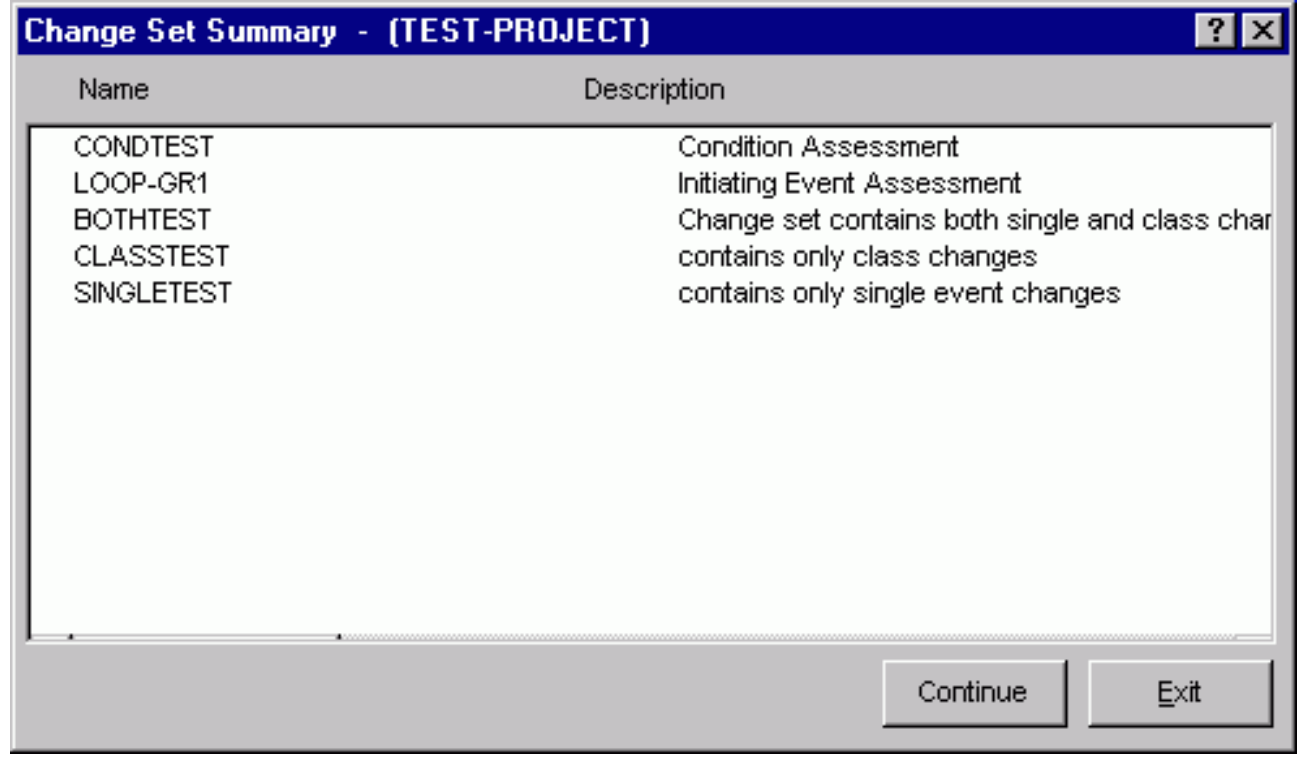

Continue - Generate the summary event report. The Report Viewer will display the report. Exit - Close the summary dialog without generating a report.

\subsubsection{Single Changes Report}

\section{PURPOSE}

This option allows you to generate a detailed flag set report. The report will list the name of each selected flag set, as well as a list of all events included in the flag set. For each included event the affected field change information is displayed. (Unaffected field information will be blank.)

\section{STEPS}

1. From the SAPHIRE menu select Report. The Reports Menu dialog will be displayed.

2. Select the Flag Set Data Type and the Flag Set Events Report Type radio buttons.

3. Choose the Process button.

4. Highlight the desired change sets or process all records.

5. Choose the Continue button to generate the report. The Report Viewer will display the report. 


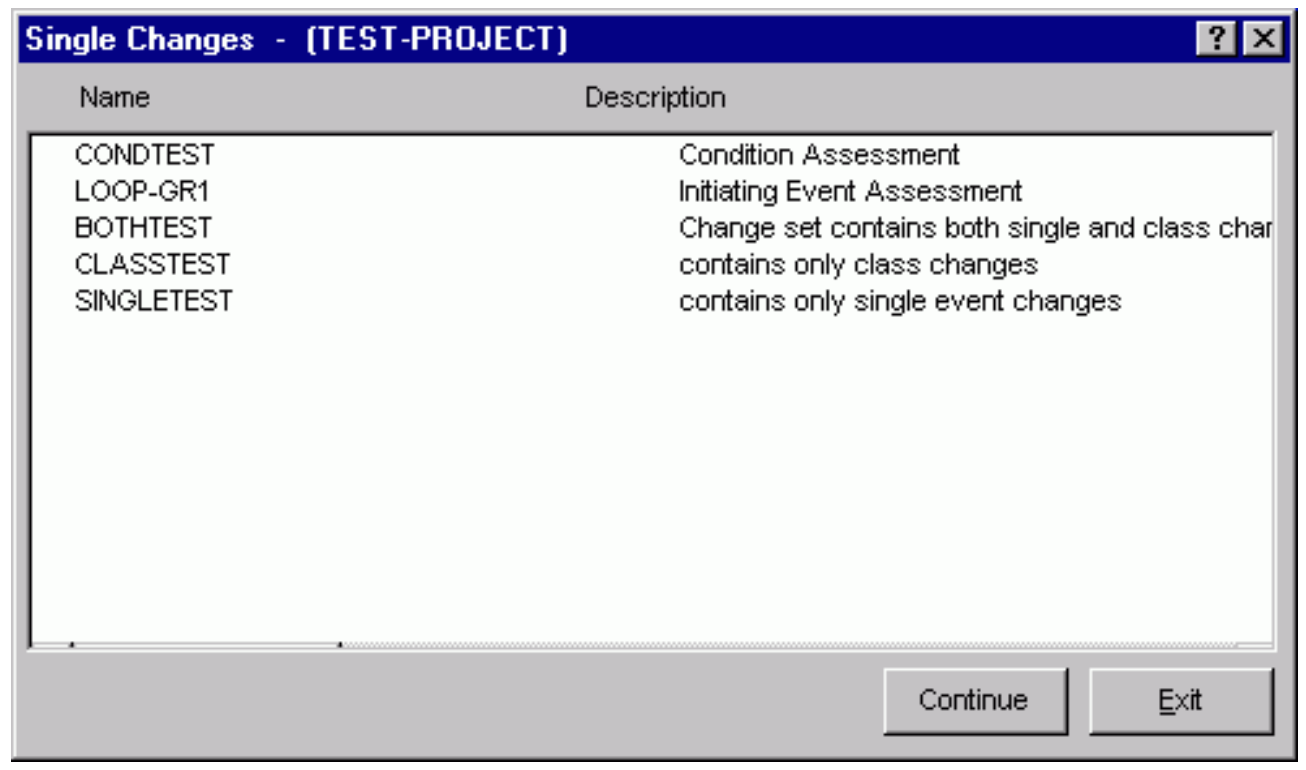

Continue - Generate the class change report. The Report Viewer will display the report. Exit - $\quad$ Close the dialog without generating a report.

\subsubsection{Flag Set Cross Reference Report}

\section{PURPOSE}

This option allows you to generate the Flag Set Cross Reference Report. Reports

\section{STEPS}

1. From the SAPHIRE menu select Report. The Reports Menu dialog will be displayed.

2. $\quad$ Select the Flag Set Data Type and Cross Reference Report Type radio buttons.

3. Choose the Process button. The Flag Set Cross Reference dialog will be displayed, listing all of the flag sets in the currently selected project .

4. Highlight the desired flag set(s) or process all records.

5. Choose the Continue button. The Report Viewer will display the report. 


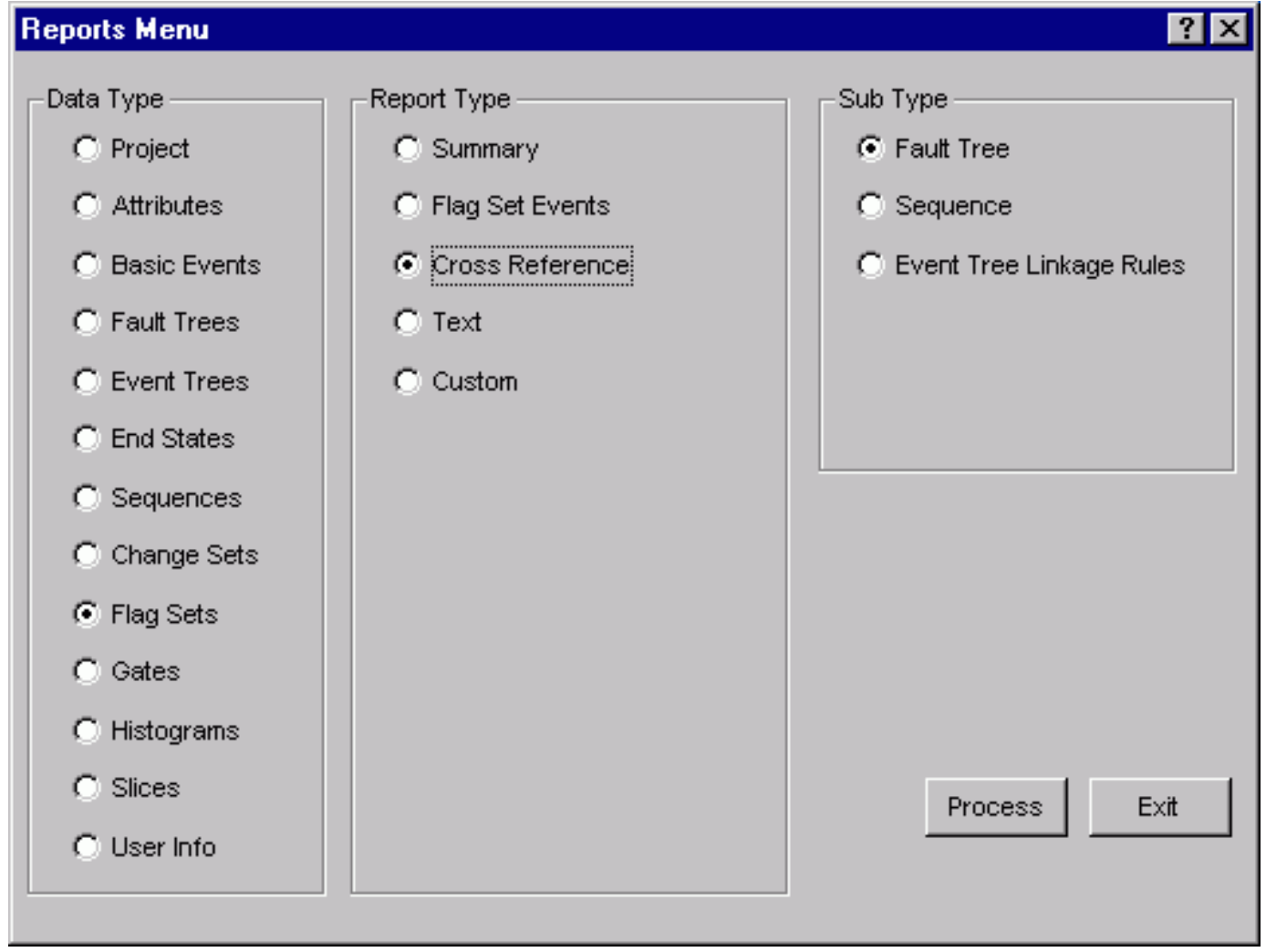

Fault Tree -

Sequence -

Event Tree Linkage Rules -

Exit -
Produce a flag set to fault tree cross-reference report. The report will include the flag set names and all fault trees that reference them.

Produce a flag set to sequence cross-reference report. The report will include the flag set names and all event tree/sequences that reference them.

Produce a flag set to event tree link rules cross-reference report. The report will include the flag set names and all event trees having either basic or advanced rules that reference them.

Close the dialog without generating a report.

\subsection{Gate Report}

\subsubsection{Gates}

\section{PURPOSE}

This option provides the means to generate the Gate-Fault Tree Logic Reference Report. This report lists the selected gate's name and the fault tree(s) that reference the gate.

\section{STEPS}

1. From the SAPHIRE menu select Report. The Reports Menu dialog will be displayed. 
2. Select the Gate Data Type and Cross Reference Report Type radio buttons.

3. Choose Process button. The Gate X-Reference dialog will be displayed listing all of the gates for the currently selected project.

4. Highlight the desired gate(s) or process all records.

5. Choose the desired button.

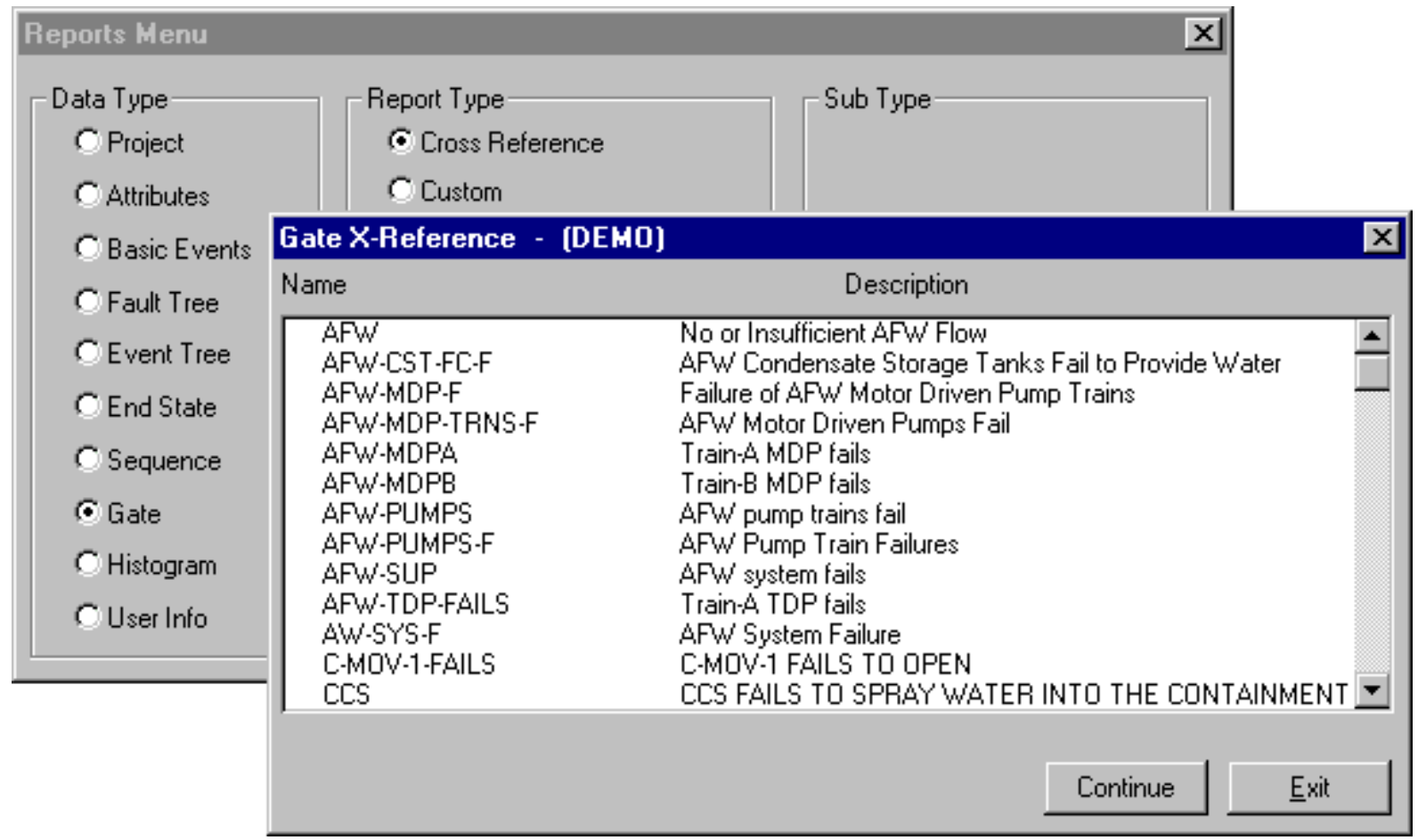

Continue - $\quad$ Generate the report. When this button is selected, the Report Viewer will display the report.

Exit Close the Gate X-Reference dialog without generating a report.

\subsection{Histogram Reports}

\subsubsection{Histogram}

\section{PURPOSE}

This option provides the means to generate histogram reports. Two histogram reports are available: Summary and Detail.

\section{STEPS}

1. From the SAPHIRE menu select Report. The Reports Menu dialog will be displayed.

2. Select the Histogram Data Type and one of the Report Type radio buttons. 
3. Choose the Process button.

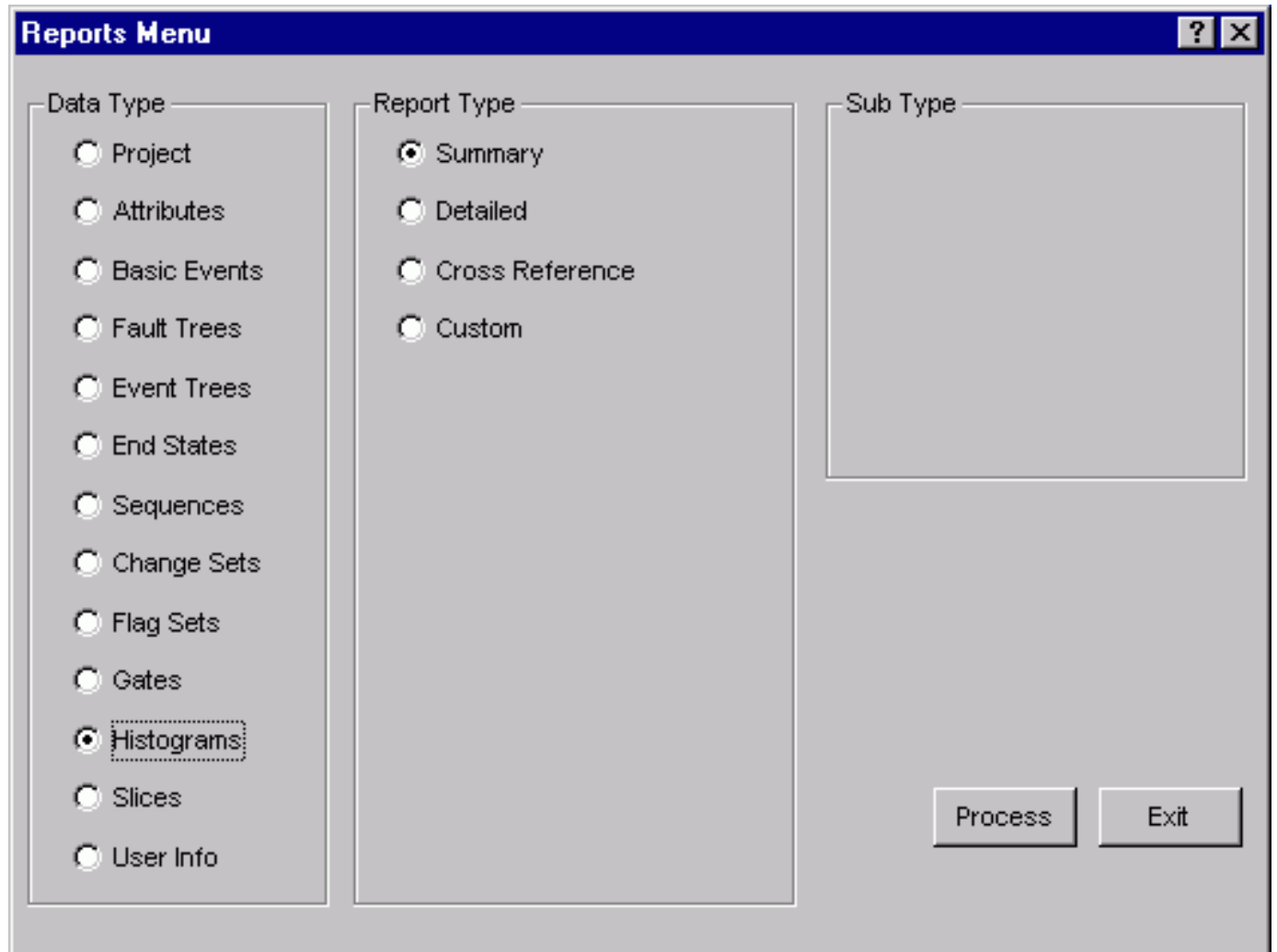

\subsubsection{Histogram Summary Report}

\section{PURPOSE}

This option allows you to generate the Histogram Summary Report. This report contains the histogram type and subtype, its name and description for each histogram defined in the current project.

\section{STEPS}

1. From the SAPHIRE menu select Report. The Reports Menu dialog will be displayed.

2. Select the Histogram Data Type and the Summary Report Type radio buttons.

3. Choose the Process button. The Report Viewer will display the report.

\subsubsection{Histogram Detail Report}

\section{PURPOSE}

This option allows you to generate the Histogram Detail Report. This report consists of the histogram name, format and where applicable, the starting value, ending probability and area or height values, or acceleration and frequency values. 


\section{STEPS}

1. From the SAPHIRE menu select Report. The Reports Menu dialog will be displayed.

2. Select the Histogram Data Type and Detail Report Type radio buttons.

3. Choose the Process button. The Histogram Detail dialog will be displayed, listing all of the histograms in the currently selected project along with the histogram type and description.

4. Highlight the desired histogram(s) or process all records.

5. Choose the Continue button. The Report Viewer will display the report.

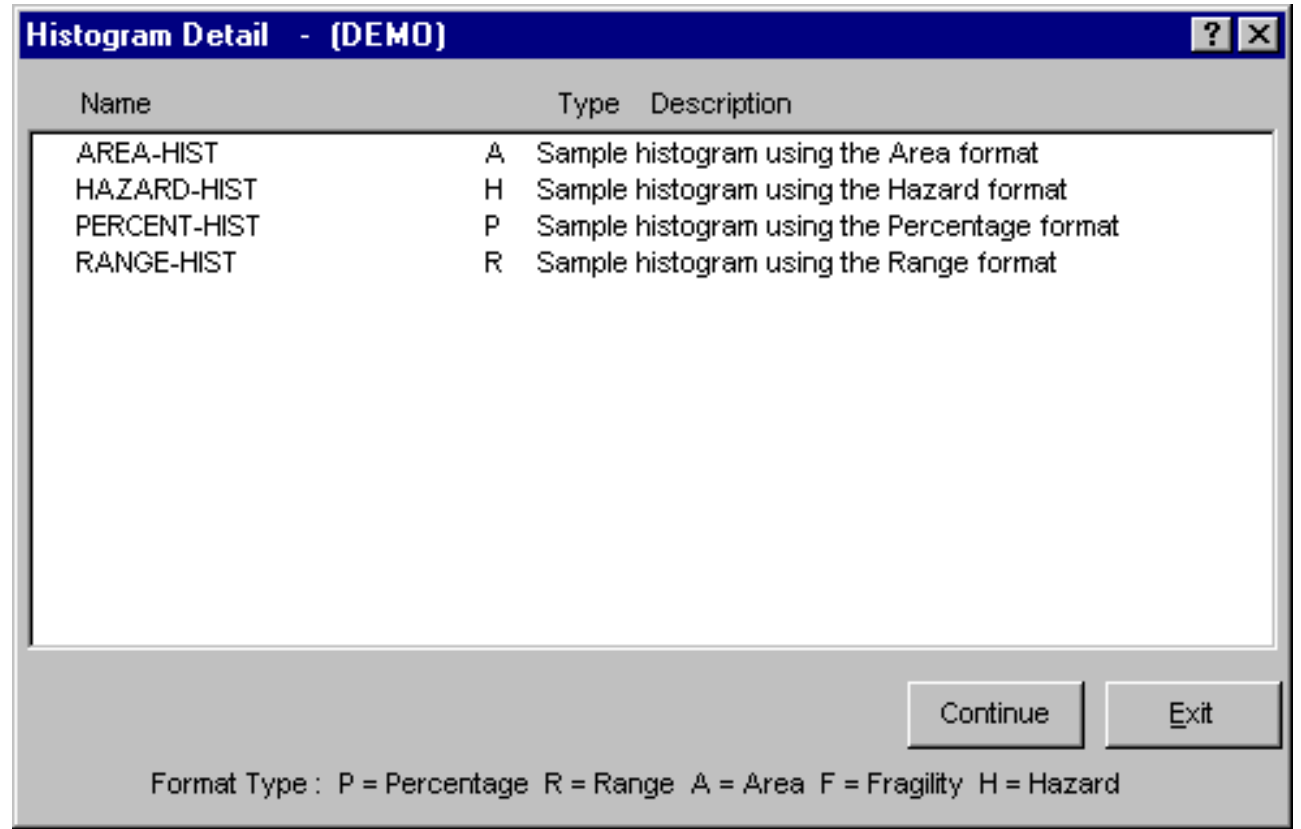

Continue - $\quad$ Generate the report. When this button is selected, the Report Viewer will display the report.

Exit -

Close the Histogram Detail dialog without generating a report.

\subsubsection{Histogram Cross Reference Report}

\section{PURPOSE}

This option allows you to generate the Histogram Cross Reference Report. This report consists of the histogram name, format and where applicable, the starting value, ending probability and area or height values, or acceleration and frequency values.

\section{STEPS}

1. From the SAPHIRE menu select Report. The Reports Menu dialog will be displayed.

2. Select the Histogram Data Type and Cross Reference Report Type radio buttons.

3. Choose the Process button. The Histogram Cross Reference dialog will be displayed, listing all of the histograms in the currently selected project along with the histogram type and description.

4. Highlight the desired histogram(s) or process all records. 
5. Choose the desired report button. The Report Viewer will display the report.

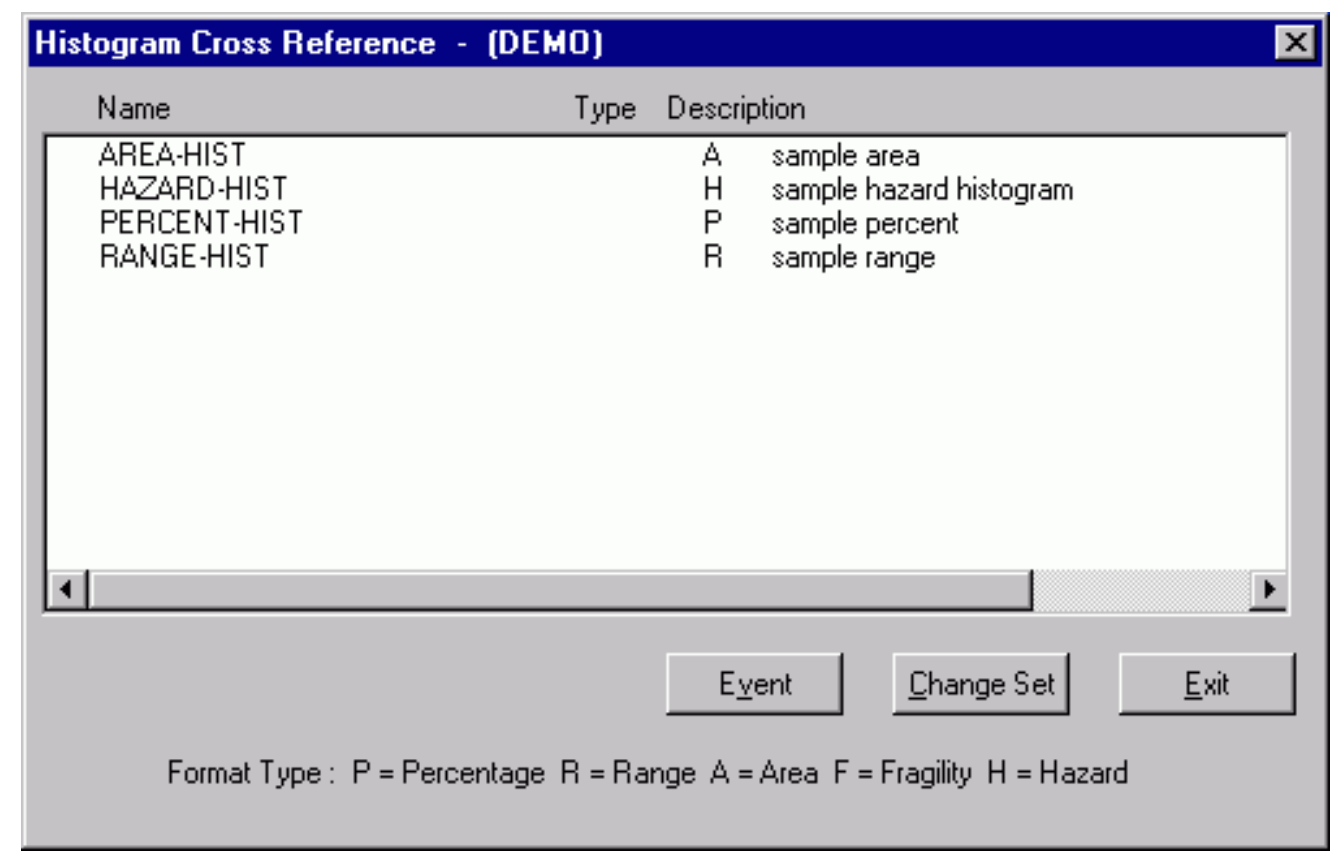

Event - $\quad$ Produce a histogram to event cut set cross-reference report. The report will include the histogram names and all events that reference them.

Change Set - Produce a histogram to change set cut set cross-reference report. The report will include the histogram names and all change sets that reference them.

Exit - $\quad$ Close the dialog without generating a report.

\subsection{Slices Report}

\subsubsection{Slices}

\section{PURPOSE}

This option provides the means to generate slice reports. There are two kinds of slices that can be stored in SAPHIRE, slice lists (referred to as simply a "slice"), and slice rules. The two are reported separately. To view slice list information, choose the Summary, Slice Events, or Custom option. To view slice rule information, choose Rule Summary, Slice Rule, Custom Slice Rule.

\section{STEPS}

1. From the SAPHIRE menu select Report. The Reports Menu dialog will be displayed.

2. Select the Slice Data Type and one of the Report Type radio buttons.

3. Choose the Process button.

4. Highlight the desired slice(s) or process all records. 
5. Choose the appropriate buttons to generate the report. The Report Viewer will display the report.

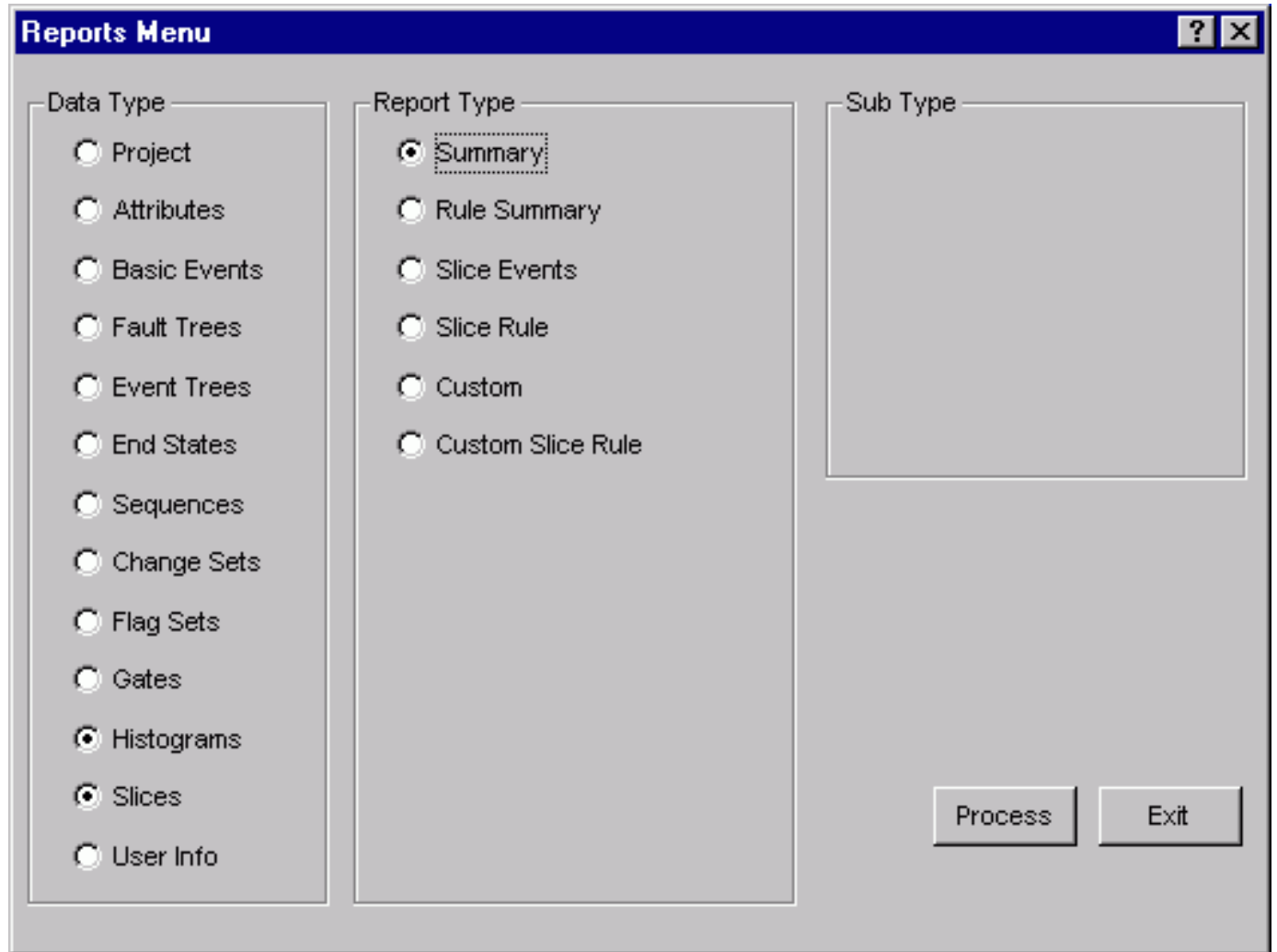

\section{Report Type}

Summary - Generate a summary report listing the selected slice(s) name and associated description.

Rule Summary - Generate a report containing the selected slice rule(s) name and associated description.

Slice Events - Generate a report containing the list of events included in the slice rule.

Slice Rule - Generate a report containing the rule logic for each selected slice rule.

Custom - $\quad$ Create or select a user-defined report relating to slice(s).

Custom Rules - Create or select a user-defined report relating to slice rule(s).

Process - $\quad$ Display the selected report in the Report Viewer.

Exit - $\quad$ This option closes the Reports Menu dialog. 


\subsection{User Information Report}

\subsubsection{User Information}

\section{PURPOSE}

This option provides the means to generate the User Defined Constants Report. This report lists the user's name, hardware configuration, default file locations, archive information, default values for cut set generation and uncertainty calculations, and default flag settings.

\section{STEPS}

1. From the SAPHIRE menu select Report. The Reports Menu dialog will be displayed.

2. Select the User Info Data Type and Defaults Report Type radio buttons.

3. Choose the Process button. The Report Viewer will display the report.

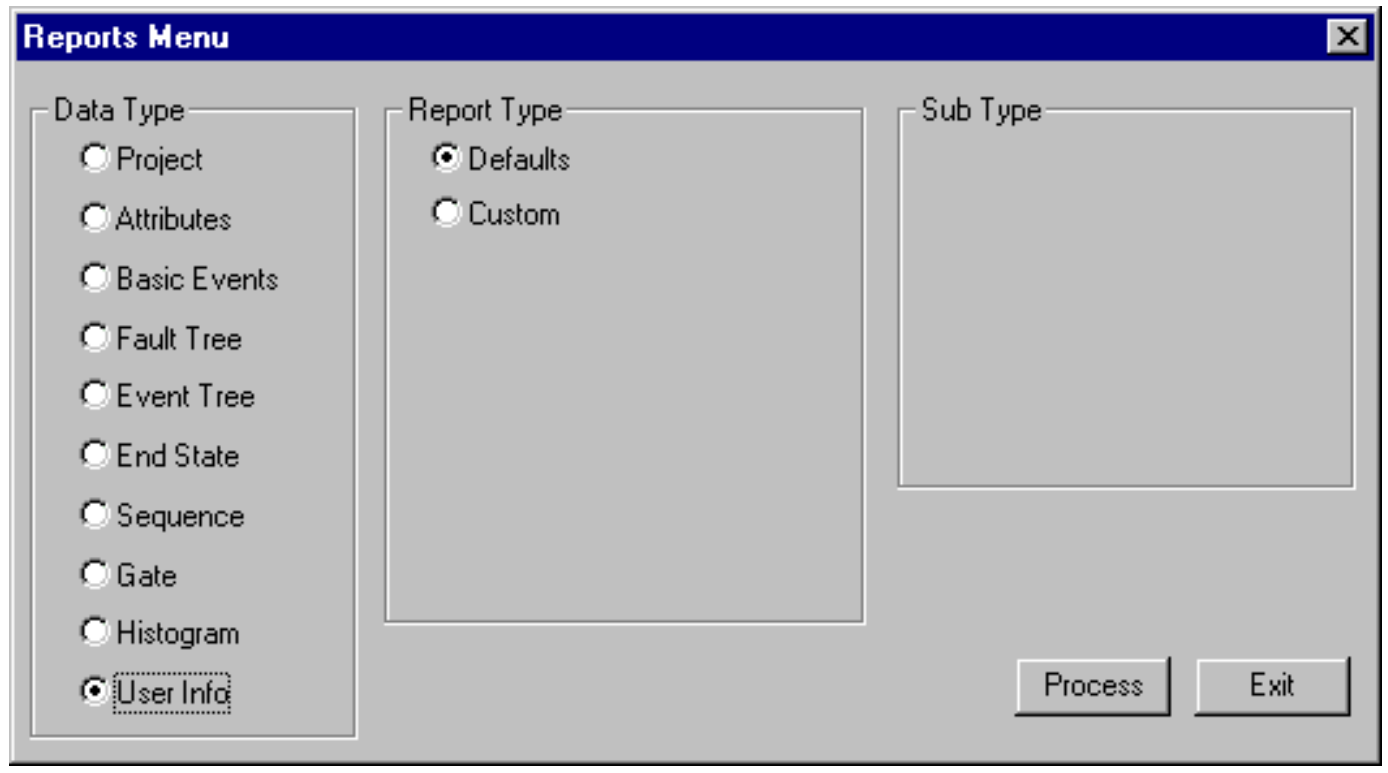




\begin{tabular}{|c|c|}
\hline 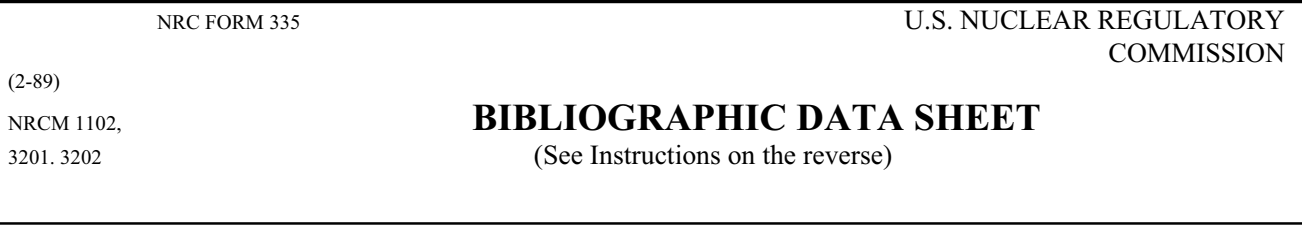 & $\begin{array}{l}\text { 1. REPORT NUMBER } \\
\text { (Assigned by NRC, Add Vol., } \\
\text { Supp., Rev., and Addendum } \\
\text { Numbers, if any.) } \\
\text { NUREG/CR-6952 } \\
\text { INL/EXT-05-00644 }\end{array}$ \\
\hline \multirow{4}{*}{$\begin{array}{l}\text { 2. TITLE AND SUBTITLE } \\
\text { Systems Analysis Programs for Hands-on Integrated Reliability Evaluations } \\
\text { (SAPHIRE) Vol. } 3 \text { Code Reference Manual - Part A }\end{array}$} & 3. DATE REPORT PUBLISHED \\
\hline & \begin{tabular}{l|l} 
MONTH & YEAR \\
\end{tabular} \\
\hline & September \\
\hline & $\begin{array}{l}\text { 4. FIN OR GRANT NUMBER } \\
\text { N6203 }\end{array}$ \\
\hline 5. $\operatorname{AUTHOR}(\mathrm{S})$ & $\begin{array}{c}\text { 6. TYPE OF REPORT } \\
\text { Technical }\end{array}$ \\
\hline K. J. Kvarfordt, C. L. Smith, S. T. Wood & 7. PERIOD COVERED (Inclusive Dates) \\
\hline \multicolumn{2}{|c|}{$\begin{array}{l}\text { 8. PERFORMING ORGANIZATION - NAME AND ADDRESS (If NRC, provide Division, Office or Region, U.S. Nuclear Regulatory Commission, and } \\
\text { mailing address; if contractor, provide name and mailing address.) } \\
\text { Idaho National Laboratory } \\
\text { Battelle Energy Alliance } \\
\text { P.O. Box } 1625 \\
\text { Idaho Falls, ID } 83415-3850\end{array}$} \\
\hline \multicolumn{2}{|l|}{$\begin{array}{l}\text { 9. SPONSORING ORGANIZATION - NAME AND } \\
\text { U.S. Nuclear Regulatory Commission, and mailing ad } \\
\text { Division of Risk Analysis } \\
\text { Office of Nuclear Regulatory Research } \\
\text { U.S. Nuclear Regulatory Commission } \\
\text { Washington, DC 20555-0001 }\end{array}$} \\
\hline \multicolumn{2}{|l|}{$\begin{array}{l}\text { 10. SUPPLEMENTARY NOTES } \\
\text { D. O’Neal, NRC Project Manager }\end{array}$} \\
\hline \multicolumn{2}{|c|}{$\begin{array}{l}\text { 11. ABSTRACT ( } 200 \text { words or less) } \\
\text { The Systems Analysis Programs for Hands-on Integrated Reliability Evaluations (SAPHIRE) is a software application } \\
\text { developed for performing a complete probabilistic risk assessment (PRA) using a personal computer (PC) running the } \\
\text { Microsoft Windows operating system. SAPHIRE is primarily funded by the U.S. Nuclear Regulatory Commission (NRC) } \\
\text { and developed by the Idaho National Laboratory (INL). This reference guide will introduce the SAPHIRE Version } 7.0 \\
\text { software. A brief discussion of the purpose and history of the software is included along with general information such as } \\
\text { installation instructions, starting and stopping the program, and some pointers on how to get around inside the program. } \\
\text { Next, database concepts and structure are discussed. Following that discussion are nine sections, one for each of the menu } \\
\text { options on the SAPHIRE main menu, wherein the purpose and general capabilities for each option are furnished. Next, the } \\
\text { capabilities and limitations of the software are provided. Finally, a series of appendices are provided that detail information } \\
\text { about some of the more complex operations of the software. }\end{array}$} \\
\hline \multirow[t]{4}{*}{ 12. KEY WORDS/DESCRIPTORS (List words or phrases that will assist researchers in locating the report.) } & $\begin{array}{l}\text { 13. AVAILABILITY STATEMENT } \\
\text { Unlimited }\end{array}$ \\
\hline & $\begin{array}{l}\text { 14. SECURITY CLASSIFICATION } \\
\text { (This page) } \\
\text { Unclassified } \\
\text { (This report) } \\
\text { Unclassified }\end{array}$ \\
\hline & 15. NUMBER OF PAGES \\
\hline & 16. PRICE \\
\hline
\end{tabular}


INL/EXT-05-00644

Rev. 1

\title{
Systems Analysis Programs for Hands-On Integrated Reliability Evaluations (SAPHIRE) Code Reference Manual
}

\author{
Part B
}

\author{
K. J. Kvarfordt \\ S. T. Wood \\ C. L. Smith
}

August 2008

\section{Idaho National Laboratory \\ Idaho Falls, Idaho 83415}

http://www.inl.gov

Prepared for the Division of Risk Analysis

Office of Nuclear Regulatory Research

U.S. Nuclear Regulatory Commission

Washington D.C. 20555

Job Code $\mathrm{N} 6203$ 


\title{
AVAILABILITY NOTICE
}

\author{
Availability of Reference Materials Cited in NRC Publications
}

Most documents cited in NRC publications will be available from one of the following sources:

1. The NRC Public Document Room, 11555 Rockville Pike, Rockville, MD 20852 (pdr@nrc.gov)

2. The Superintendent of Documents, U. S. Government Printing Office (GPO), Mail Stop SSOP, Washington, DC 20402-9328

3. The National Technical Information Service, Springfield, VA 22161

Although the listing that follows represents the majority of documents cited in NRC publications, it is not intended to be exhaustive.

Referenced documents available for inspection and copying for a fee from the NRC Public Document Room include NRC correspondence and internal NRC memoranda; NRC bulletins, circulars, information notices, inspection and investigative notices; licensee event reports; vendor reports and correspondence; Commission papers; and applicant and licensee documents and correspondence.

The following documents in the NUREG series are available for purchase from the GPO Sales Program: formal NRC staff and contractor reports, NRC-sponsored conference proceedings, international agreement reports, grant publications, and NRC booklets and brochures. Also available are regulatory guides, NRC regulations in the Code of Federal Regulations, and Nuclear Regulatory Commission Issuances.

Documents available from the National Technical Information Service include NUREG-series reports and technical reports prepared by other Federal agencies and reports prepared by the Atomic Energy Commission, forerunner agency to the Nuclear Regulatory Commission.

Documents available from public and special technical libraries include all open literature items, such as books, journal articles, and transactions. Federal Register notices, Federal and State legislation, and congressional reports can usually be obtained from these libraries.

Documents such as theses, dissertations, foreign reports and translations, and non-NRC conference proceedings are available for purchase from the organization sponsoring the publication cited.

Single copies of NRC draft reports are available free, to the extent of supply, upon written request to the Office of Administration, Distribution and Mail Services Section U. S. Nuclear Regulatory Commission, Washington, DC 20555-0001.

The public maintains copies of industry codes and standards used in a substantive manner in the NRC regulatory process at the NRC Library, Two White Flint North, 11545 Rockville Pike, Rockville, MD, 20852, for use. Codes and standards are usually copyrighted and may be purchased from the originating organization or, if they are American National Standards, from the American National Standards Institute, 1430 Broadway, New York, NY 10018. 


\section{DISCLAIMER NOTICE}

This report was prepared as an account of work sponsored by an agency of the United States Government. Neither the United States Government nor any agency thereof, or any of their employees, makes any warranty, expressed or implied, or assumes any legal liability of responsibility for any third party's use, or the results of such use, or any information, apparatus, product or process disclosed in this report, or represents that its use by such third party would not infringe privately owned rights. 



\section{PREVIOUS REPORTS}

Smith, C. L., et al., Testing, Verifying, and Validating SAPHIRE Versions 6.0 and 7.0, NUREG/CR6688, October 2000.

K. D. Russell, et al. Systems Analysis Programs for Hands-on Reliability Evaluations (SAPHIRE) Version 6.0 - System Overview Manual, NUREG/CR-6532, May 1999.

K. D. Russell et al., Integrated Reliability and Risk Analysis System (IRRAS) Version 5.0, Volume 2 Reference Manual, NUREG/CR-6116, EGG-2716, July 1994.

K. D. Russell et al., Verification and Validation (V\&V), Volume 9 - Reference Manual, NUREG/CR6116, EGG-2716, July 1994.

K. D. Russell et al., Integrated Reliability and Risk Analysis System (IRRAS) Version 4.0, Volume 1 Reference Manual, NUREG/CR-5813, EGG-2664, January 1992.

K. D. Russell et al., Integrated Reliability and Risk Analysis System (IRRAS) Version 2.5 Reference Manual, NUREG/CR-5300, EGG-2613, March 1991.

K. D. Russell, M. B. Sattison, D. M. Rasmuson, Integrated Reliability and Risk Analysis System (IRRAS) - Version 2.0 User's Guide, NUREG/CR-5111, EGG-2535, manuscript completed March 1989, published June 1990.

K. D. Russell, D. M. Snider, M. B. Sattison, H. D. Stewart, S.D. Matthews, K. L. Wagner, Integrated Reliability and Risk Analysis System (IRRAS) User's Guide - Version 1.0 (DRAFT), NUREG/CR-4844, EGG-2495, June 1987. 


\section{ABSTRACT}

The Systems Analysis Programs for Hands-on Integrated Reliability Evaluations (SAPHIRE) is a software application developed for performing a complete probabilistic risk assessment (PRA) using a personal computer. SAPHIRE is funded by the U.S. Nuclear Regulatory Commission (NRC) and developed by the Idaho National Laboratory (INL). The INL's primary role in this project is that of software developer. However, the INL also plays an important role in technology transfer by interfacing and supporting SAPHIRE users comprised of a wide range of PRA practitioners from the NRC, national laboratories, the private sector, and foreign countries.

SAPHIRE can be used to model a complex system's response to initiating events, quantify associated damage outcome frequencies, and identify important contributors to this damage (Level 1 PRA) and to analyze containment performance during a severe accident and quantify radioactive releases (Level 2 PRA). It can be used for a PRA evaluating a variety of operating conditions, for example, for a nuclear reactor at full power, low power, or at shutdown conditions. Furthermore, SAPHIRE can be used to analyze both internal and external initiating events and has special features for transforming models built for internal event analysis to models for external event analysis. It can also be used in a limited manner to quantify risk in terms of release consequences to both the public and the environment (Level 3 PRA).

SAPHIRE includes a separate module called the Graphical Evaluation Module (GEM). GEM provides a highly specialized user interface with SAPHIRE that automates SAPHIRE process steps for evaluating operational events at commercial nuclear power plants. Using GEM, an analyst can estimate the risk associated with operational events in a very efficient and expeditious manner.

This reference guide will introduce the SAPHIRE Version 7.0 software. A brief discussion of the purpose and history of the software is included along with general information such as installation instructions, starting and stopping the program, and some pointers on how to get around inside the program. Next, database concepts and structure are discussed. Following that discussion are nine sections, one for each of the menu options on the SAPHIRE main menu, wherein the purpose and general capabilities for each option are furnished. Next, the capabilities and limitations of the software are provided. 


\title{
FOREWORD
}

The U.S. Nuclear Regulatory Commission has developed the Systems Analysis Programs for Hands-on Integrated Reliability Evaluations (SAPHIRE) software used to perform probabilistic risk assessments (PRAs) on a personal computer. SAPHIRE enables users to supply basic event data, create and solve fault and event trees, perform uncertainty analyses, and generate reports. In that way, analysts can perform PRAs for any complex system, facility, or process.

SAPHIRE can be used to model a plant's response to initiating events, quantify core damage frequencies, and identify important contributors to core damage (Level 1 PRA). The program can also be used to evaluate containment failure and release models for severe accident conditions, given that core damage has occurred (Level 2 PRA). In so doing, the analyst could build the PRA model assuming that the reactor is initially at full power, low power, or shutdown. In addition, SAPHIRE can be used to analyze both internal and external events, and it includes special features for transforming models built for internal event analysis to models for external event analysis. It can also be used in a limited manner to quantify the frequency of release consequences (Level 3 PRA). Because this software is a very detailed technical tool, users should be familiar with PRA concepts and methods used to perform such analyses.

SAPHIRE has evolved with advances in computer technology. The versions currently in use (6 and 7) run in the Microsoft Windows ${ }^{\circledR}$ environment. A user-friendly interface, Graphical Evaluation Module (GEM), streamlines and automates selected SAPHIRE inputs and processes for performing event assessments.

SAPHIRE has also evolved with users' needs, and Versions 6 and 7 include new features and capabilities for developing and using larger, more complex models. For example, Version 7 can solve up to 2 million sequences and includes enhancements for cut set slicing, event tree rule linkage, and reporting options.

This NUREG-series report comprises seven volumes, which address SAPHIRE/GEM Versions 6 and 7. Volume 1, "Overview/Summary," gives an overview of the functions available in SAPHIRE and presents general instructions for using the software. Volume 2, "Technical Reference," discusses the theoretical background behind the SAPHIRE functions. Volume 3, "SAPHIRE Users' Manual," provides installation instructions and a step-by-step approach to using the program's features. Volume 4, "SAPHIRE Tutorial Manual," provides an example of the overall process of constructing a PRA database. Volume 5, "GEM/GEMDATA Reference Manual," discusses the use of GEM. Volume 6, "SAPHIRE Quality Assurance (QA) Manual," discusses QA methods and tests. Lastly, Volume 7, "SAPHIRE Data Loading Manual," assists the user in entering PRA data into SAPHIRE using the built-in MAR-D ASCII-text file data transfer process.

\author{
Christiana H. Lui, Director \\ Division of Risk Analysis \\ Office of Nuclear Regulatory Research
}




\section{CONTENTS}

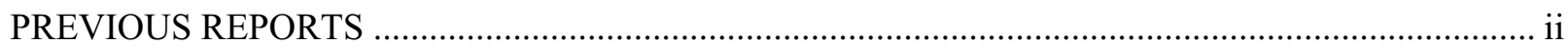

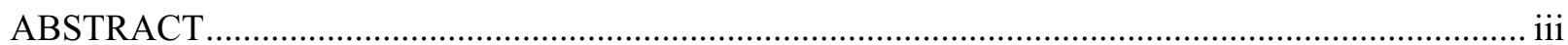

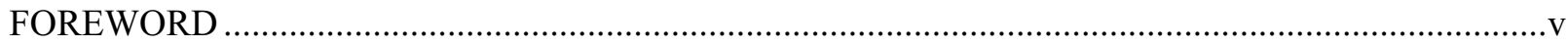

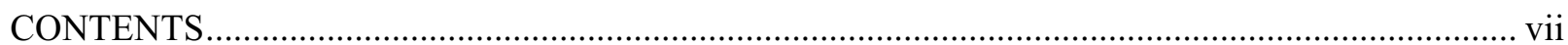

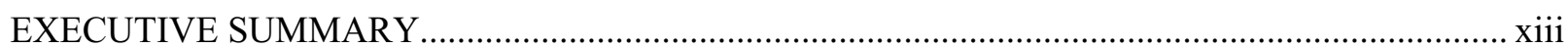

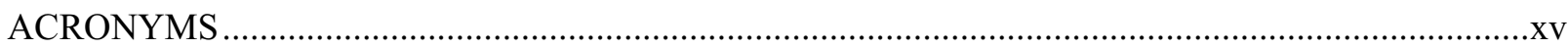

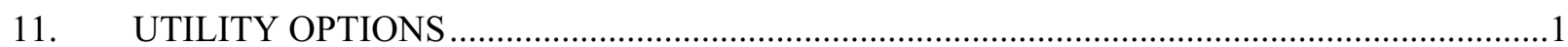

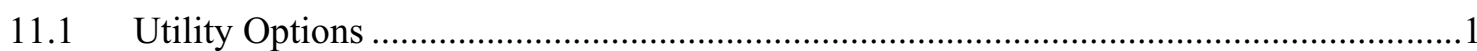

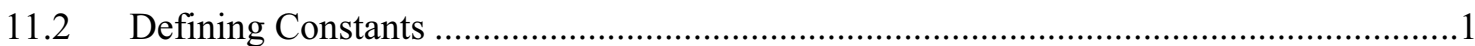

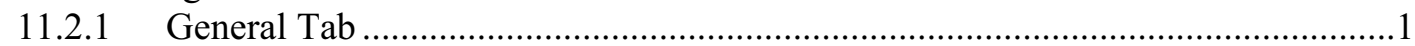

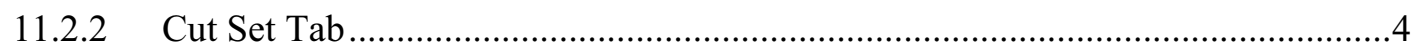

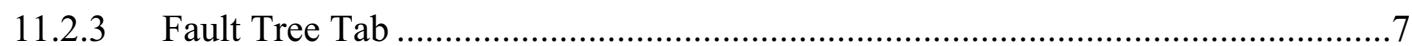

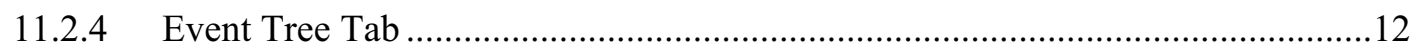

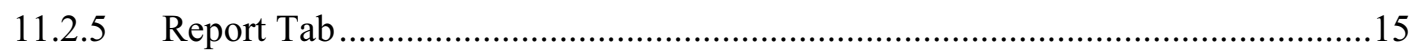

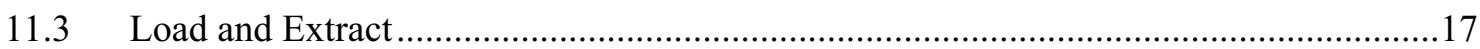

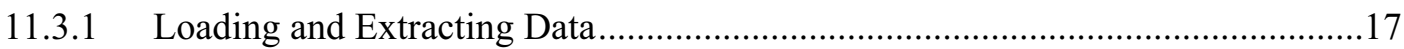

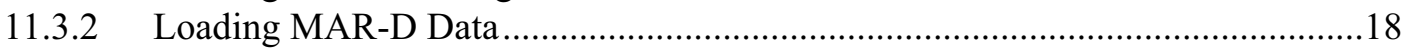

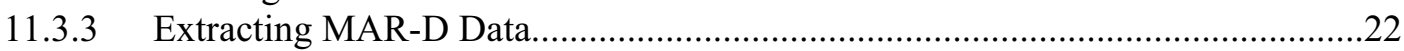

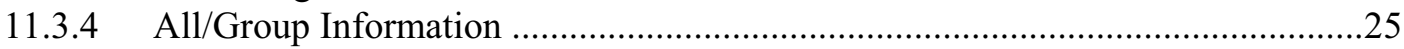

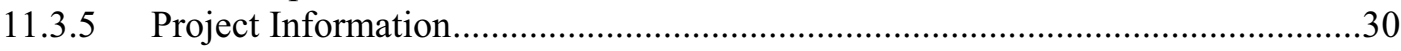

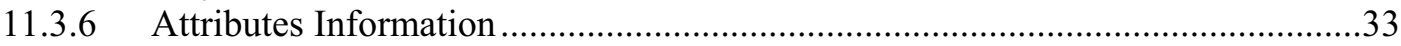

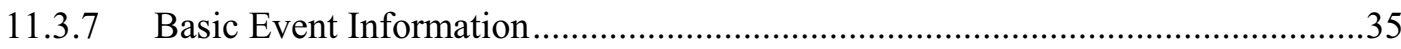

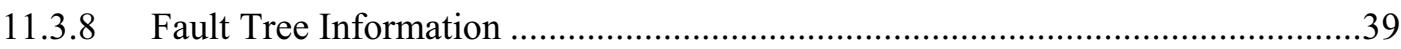

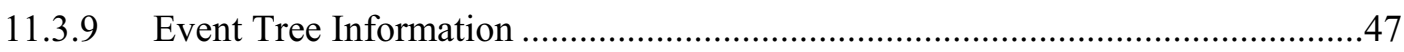

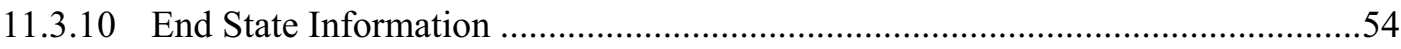

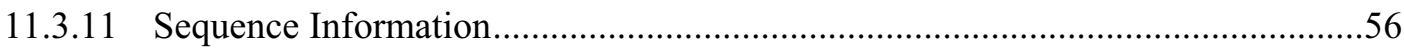

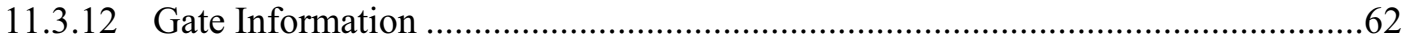

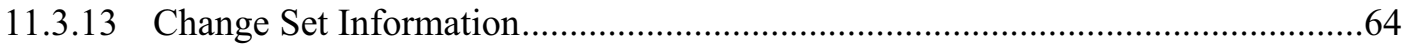

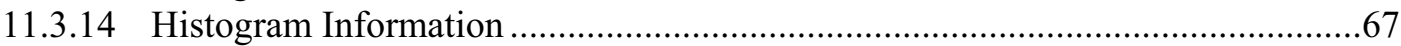

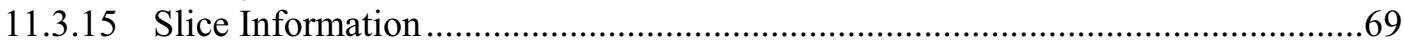

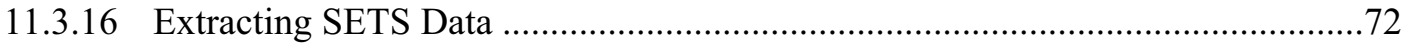

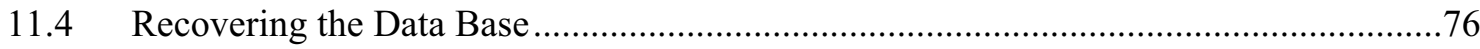

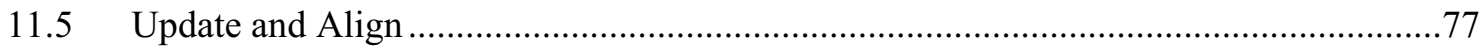

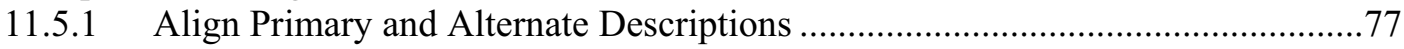

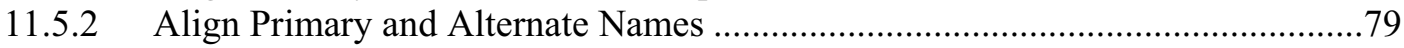

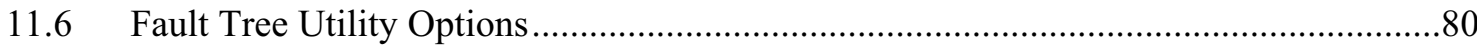

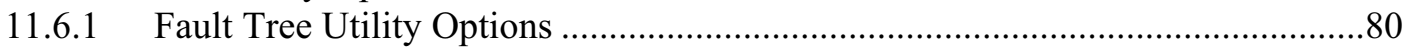

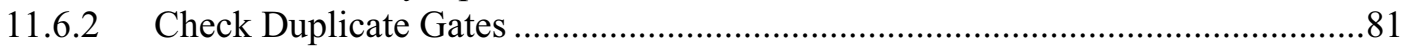

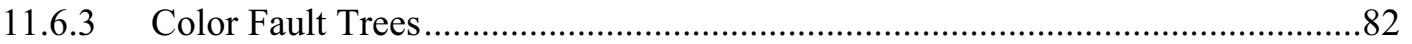




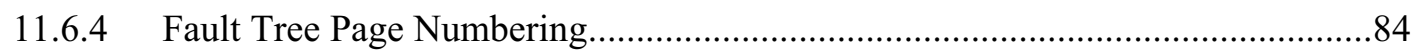

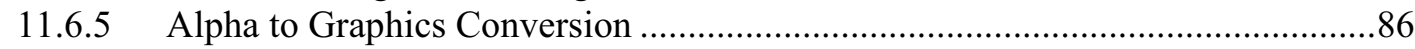

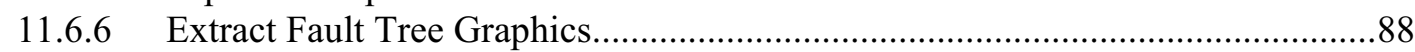

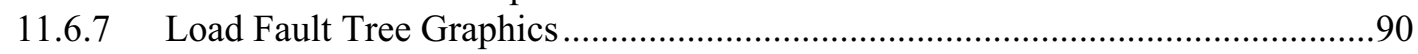

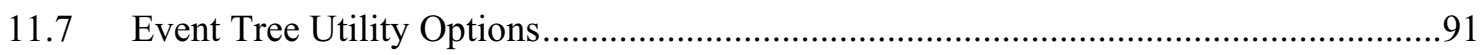

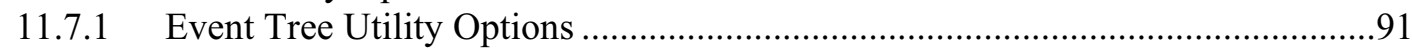

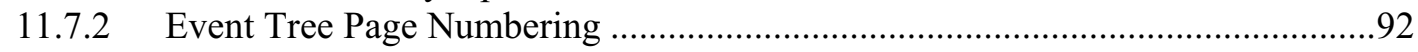

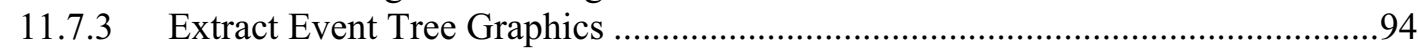

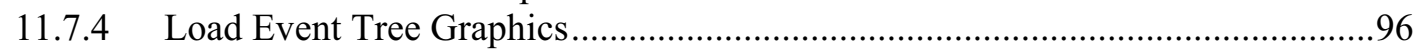

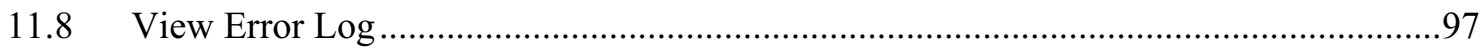

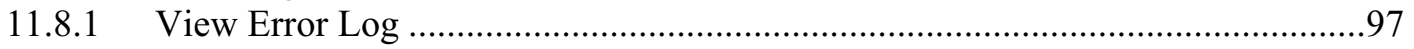

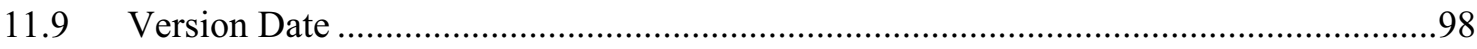

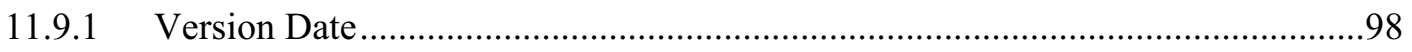

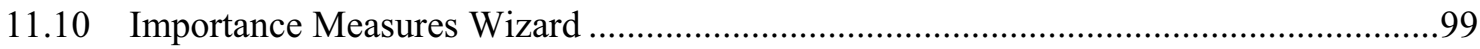

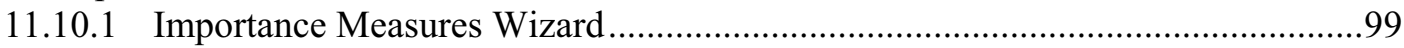

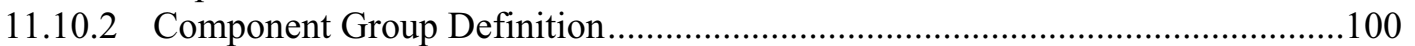

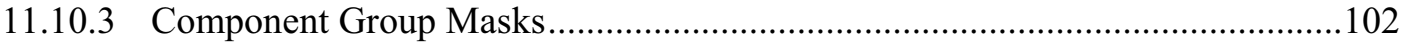

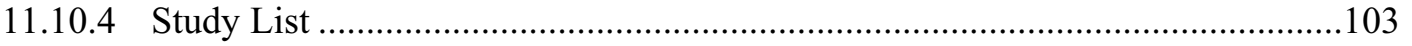

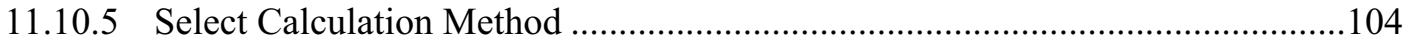

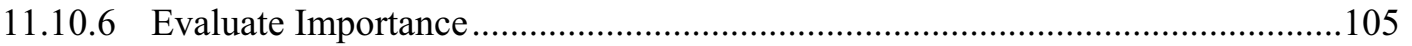

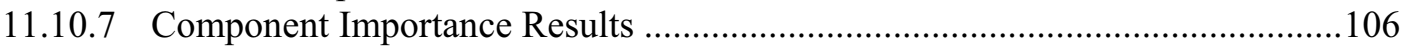

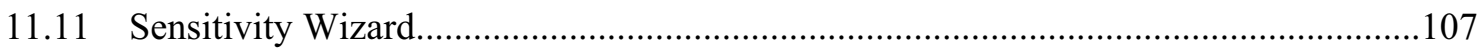

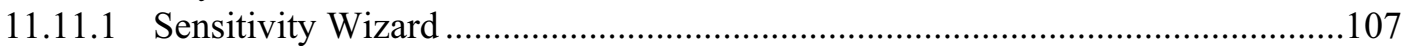

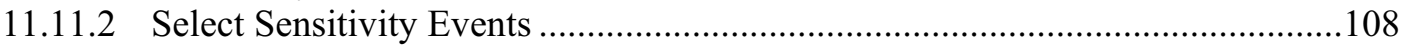

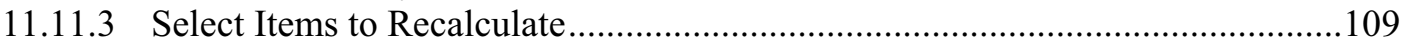

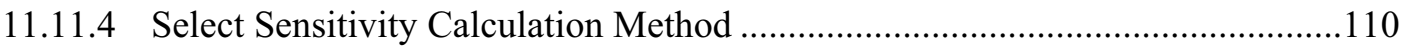

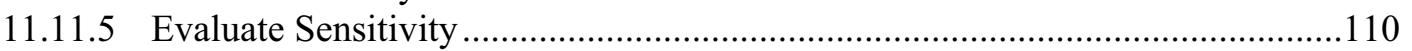

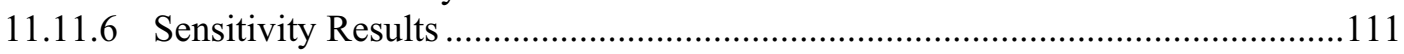

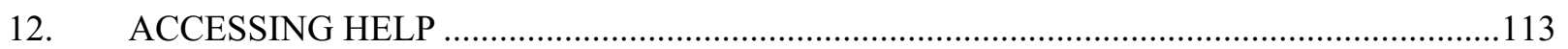

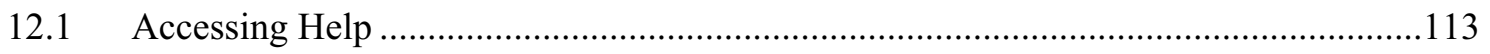

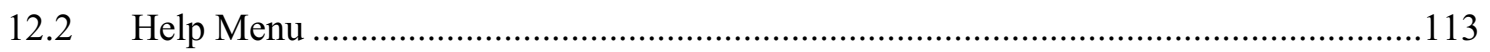

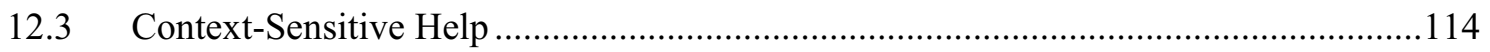

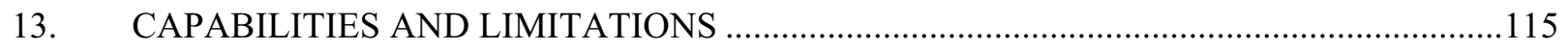

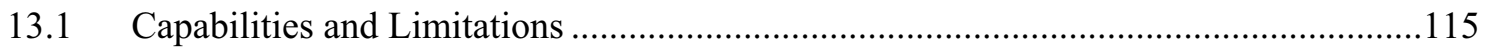

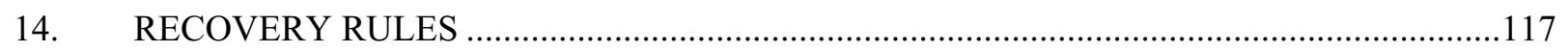

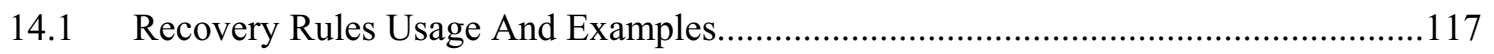

14.2 Search Criteria Examples - Basic Recovery …....................................................... 118

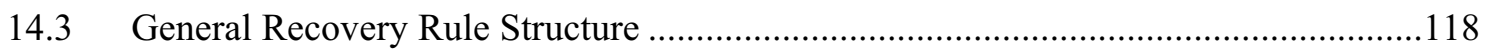




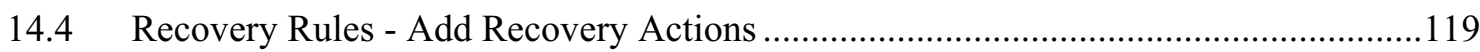

14.5 Recovery Rules - Mutually Exclusive Event Removal.............................................119

14.6 Recovery Rules - Common-Cause Failure Modeling .................................................120

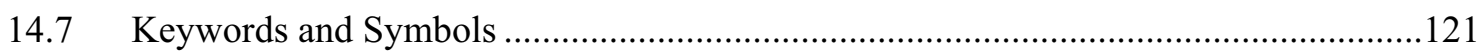

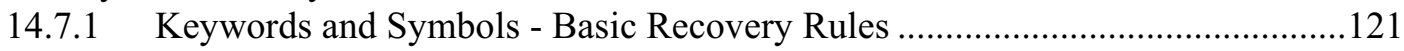

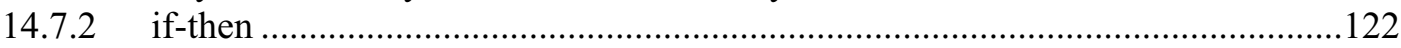

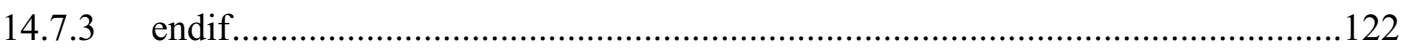

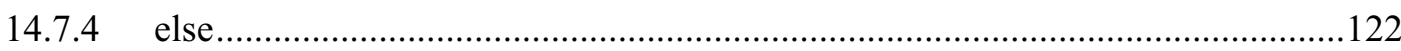

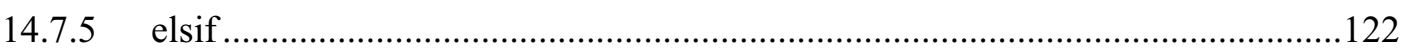

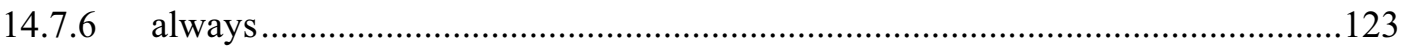

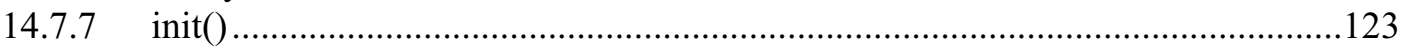

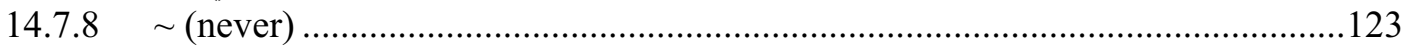

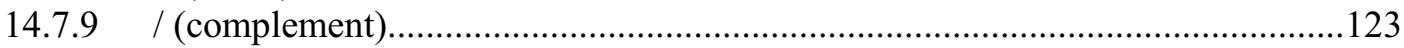

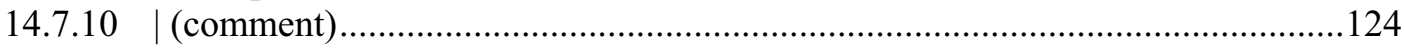

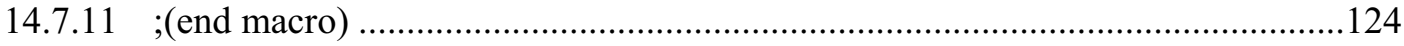

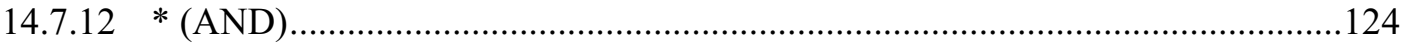

$14.7 .13+(\mathrm{OR})$

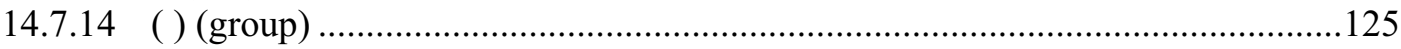

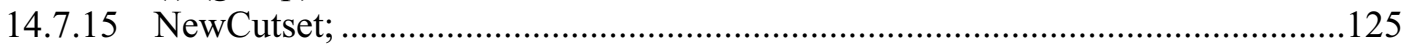

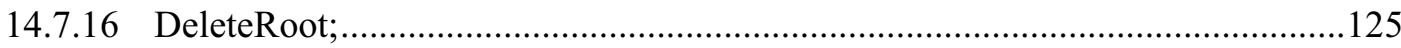

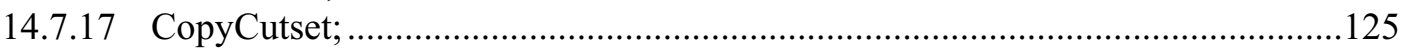

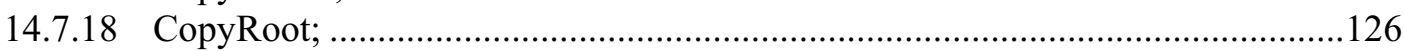

14.7.19 Recovery Rules - Keyword Examples.......................................................126

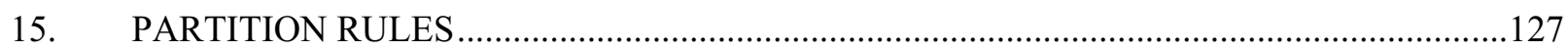

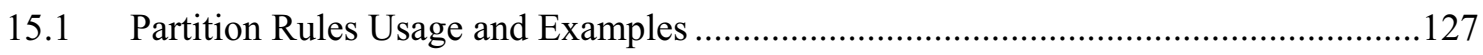

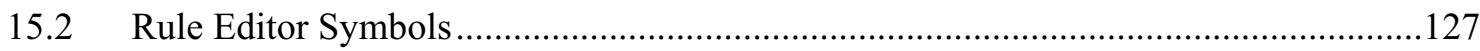

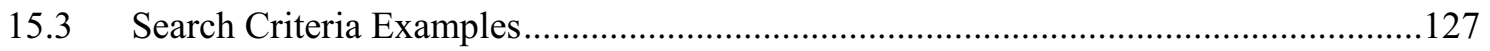

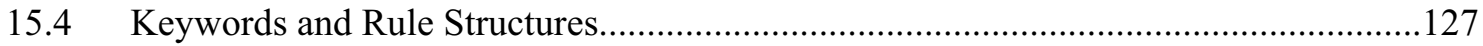

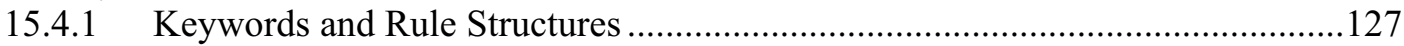

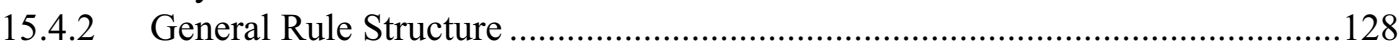

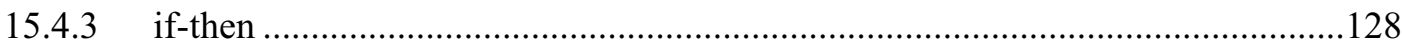

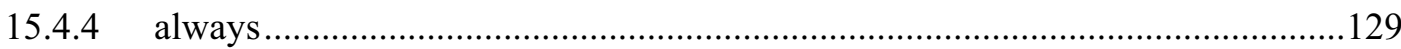

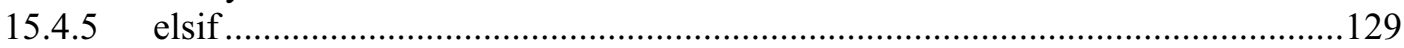

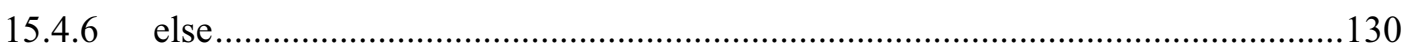

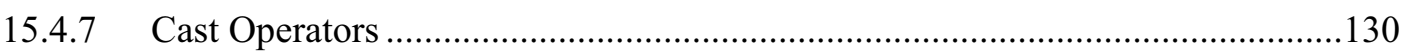

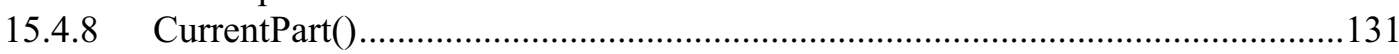

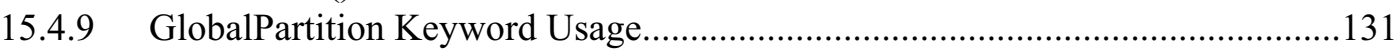

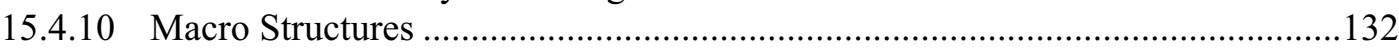

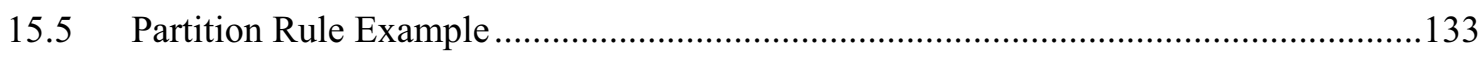

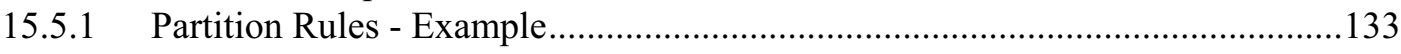

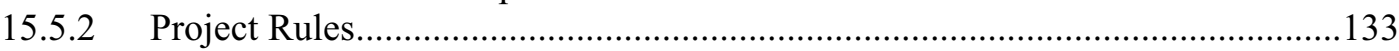

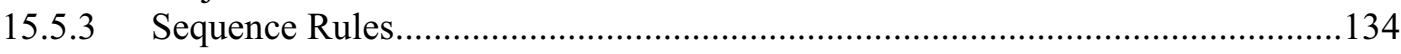

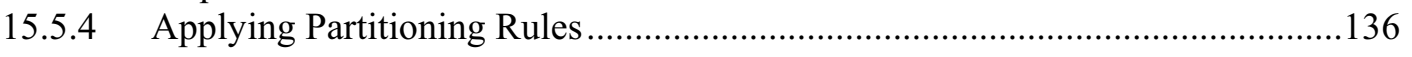




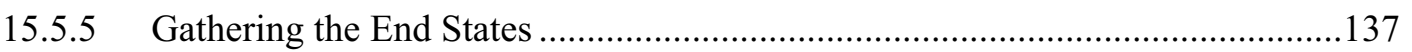

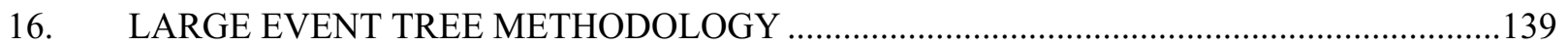

16.1 Application of the Large Event Tree Methodology .................................................139

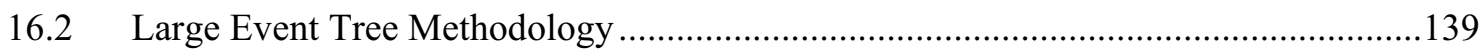

16.3 Connecting Support System Event Trees to Plant Response Event Trees....................140

16.4 Using "Link Event Tree" Rules to Assign Split-Fractions .........................................141

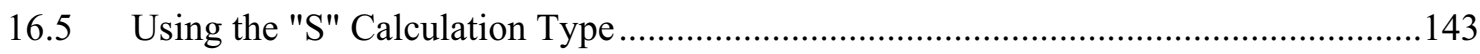

16.6 Truncating Sequences During Event Tree Linking ...................................................144

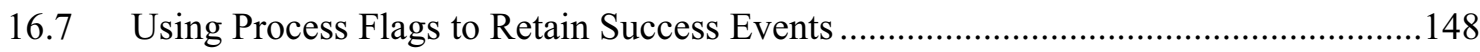

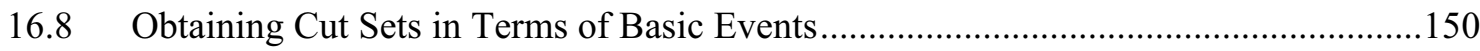

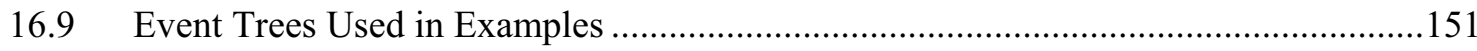

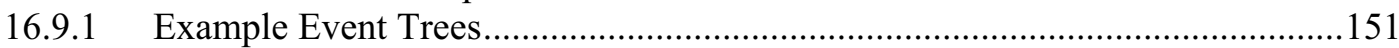

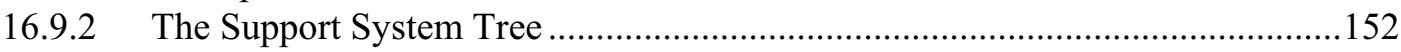

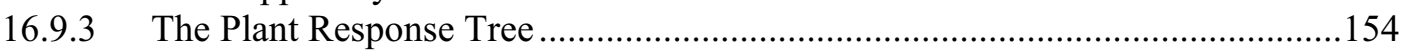

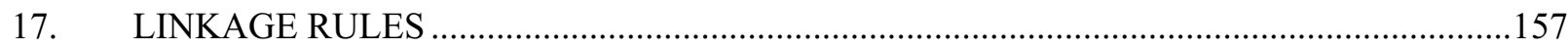

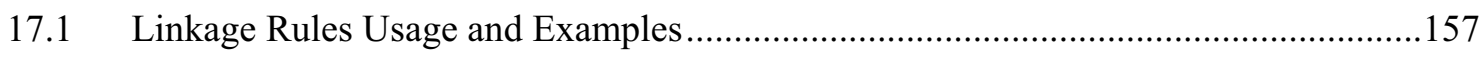

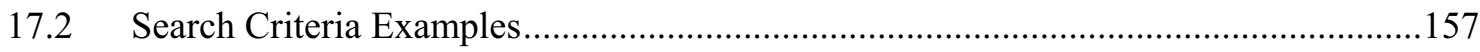

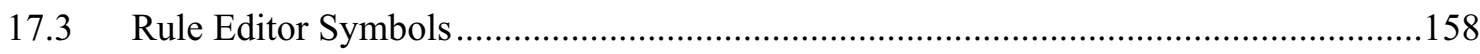

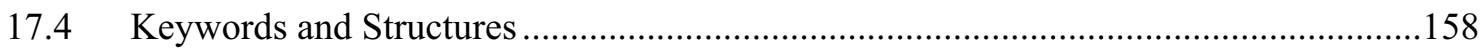

17.4.1 Linkage Rules Keywords and Structure ........................................................ 158

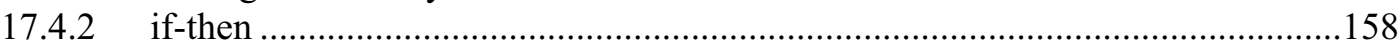

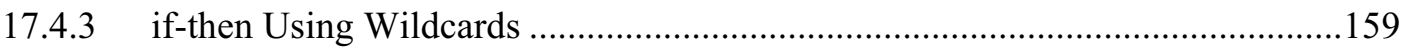

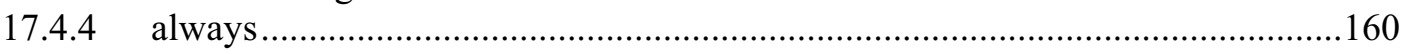

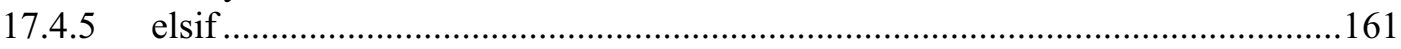

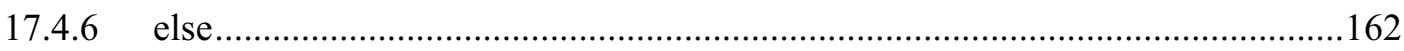

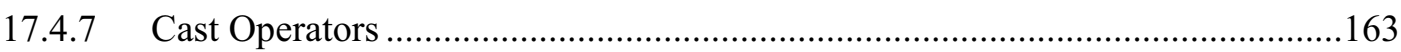

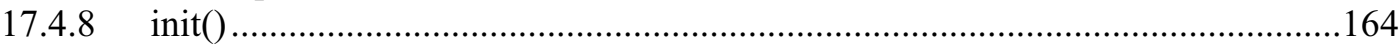

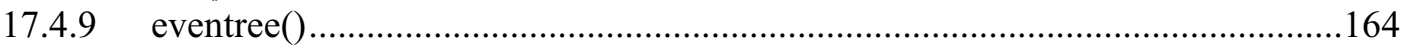

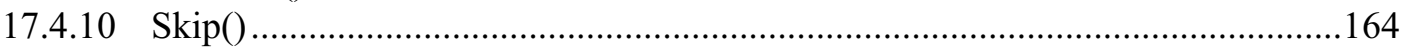

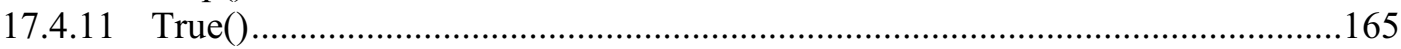

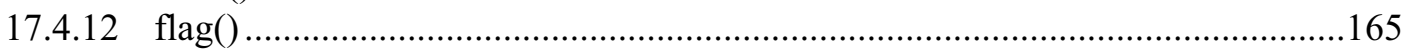

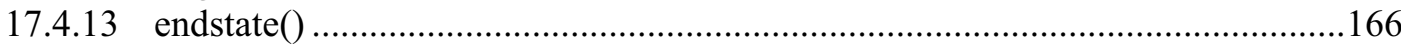

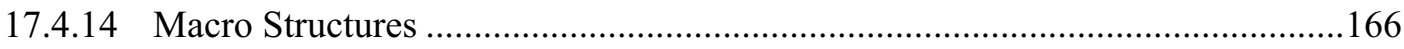

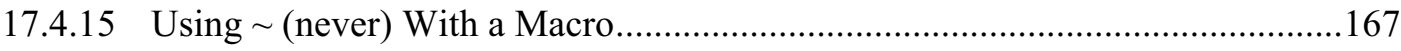

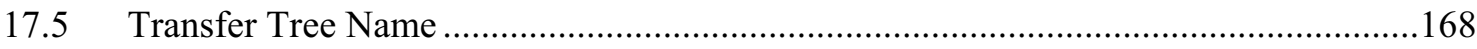

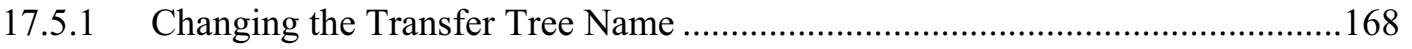




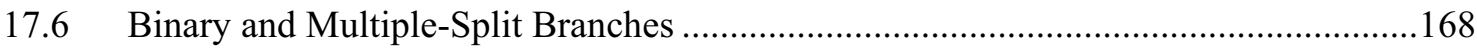

17.6.1 Rules for Binary and Multiple-Split Branches ................................................168

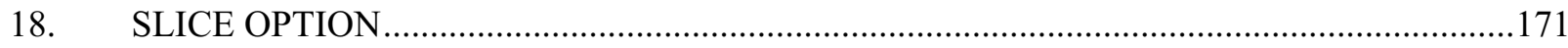

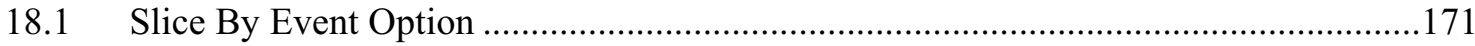

18.1.1 Displaying Cut Sets Using The Slice By Event Option ..................................171

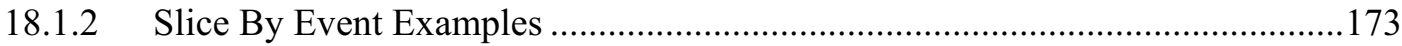

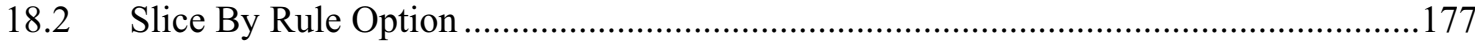

18.2.1 Displaying Cut Sets Using The Slice By Rule Option .....................................177

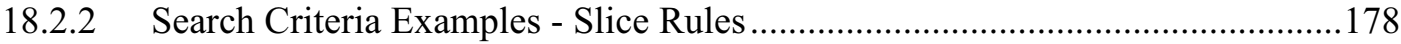

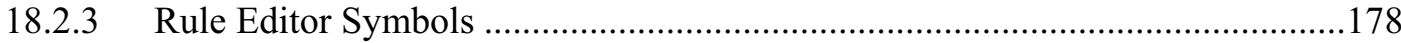

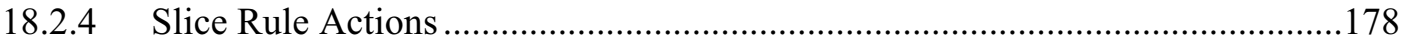

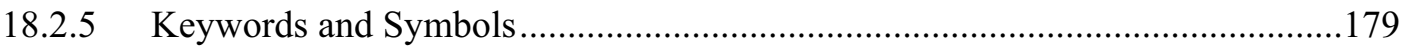

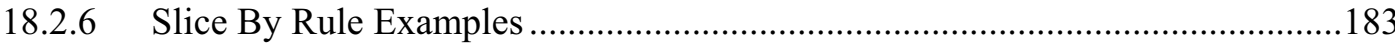

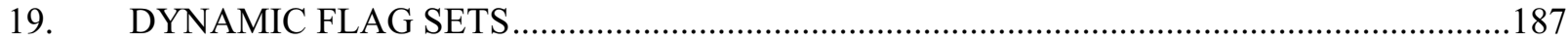

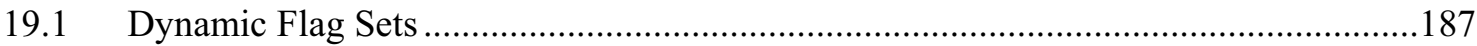

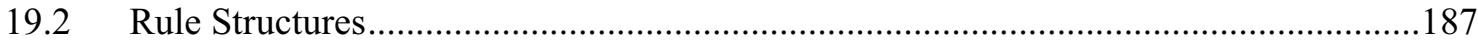

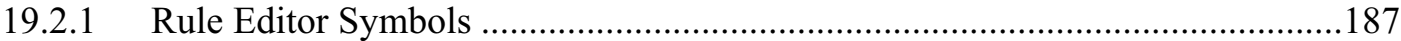

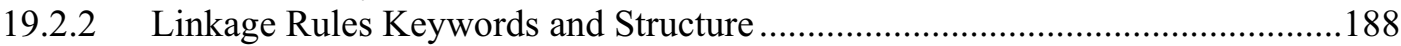

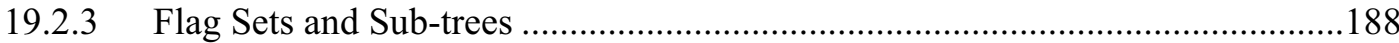

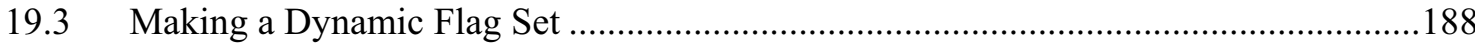

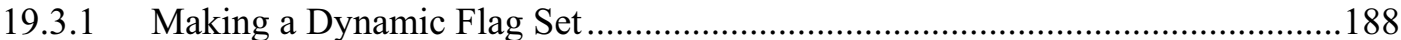

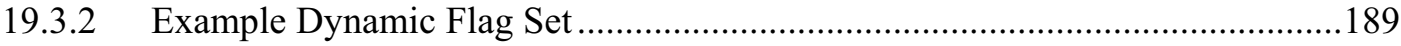

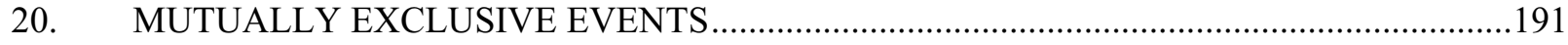

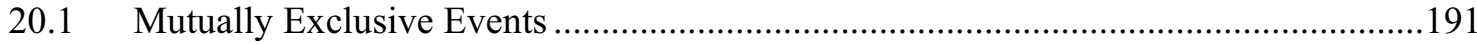

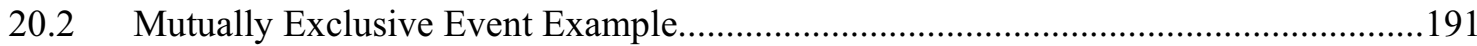

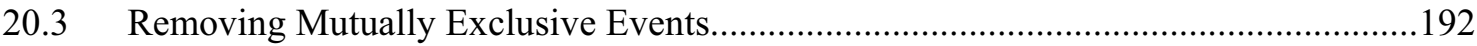

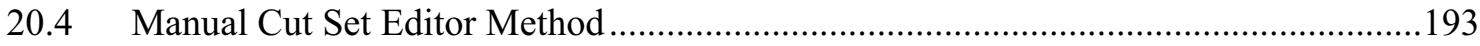

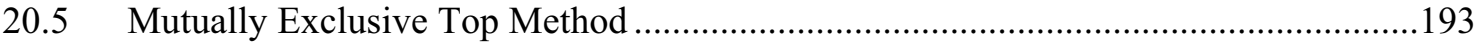

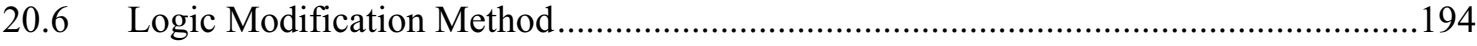

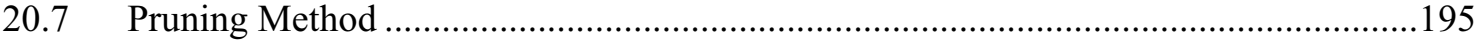

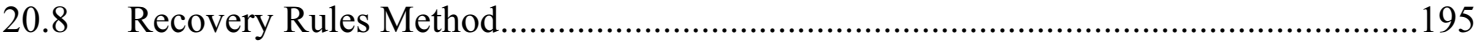

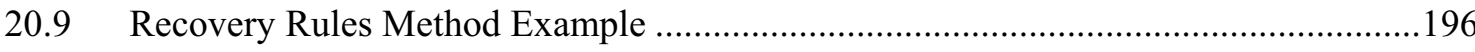

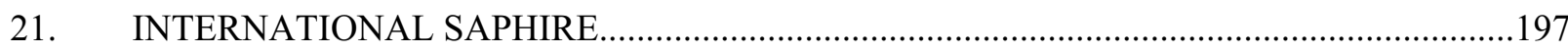

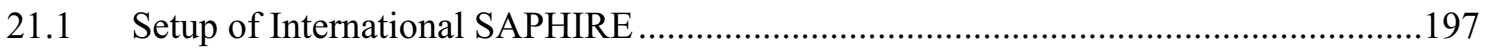




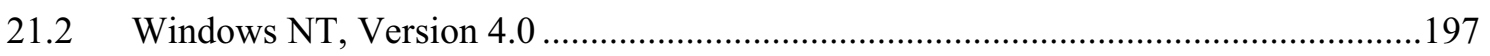

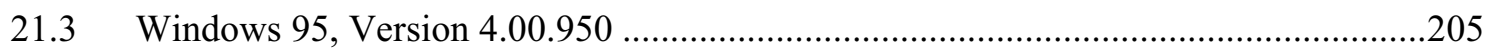

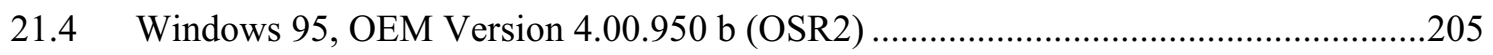

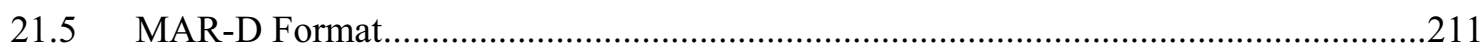

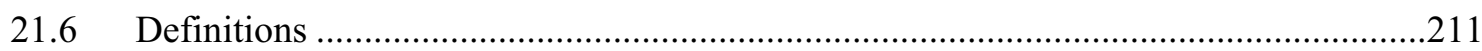

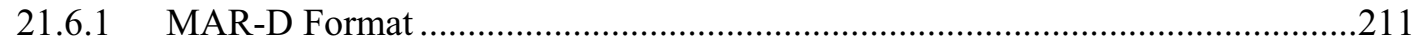

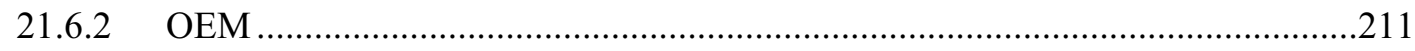

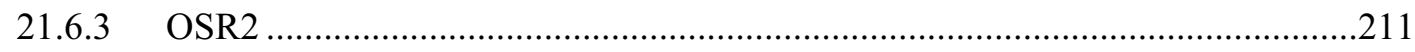

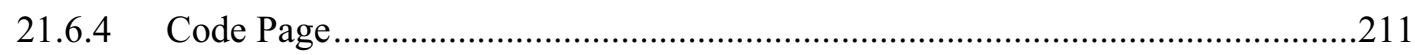

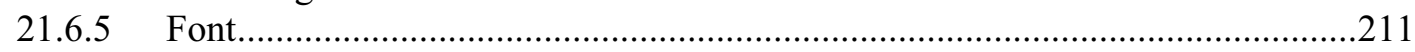

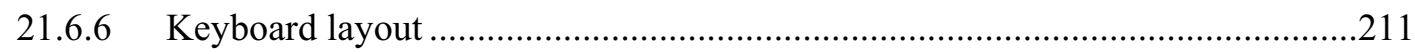

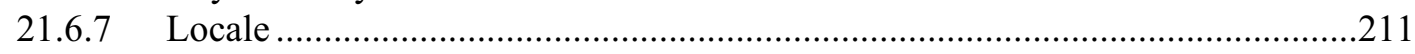

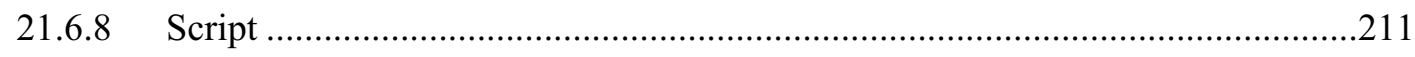

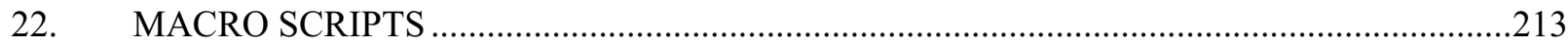

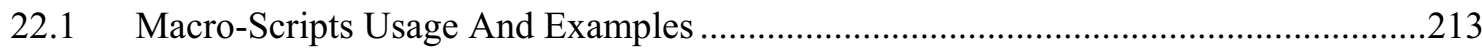

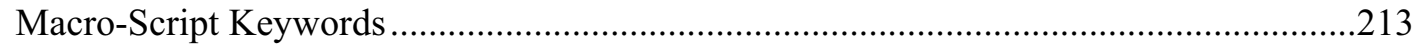

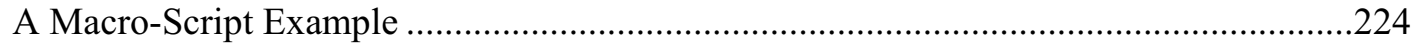

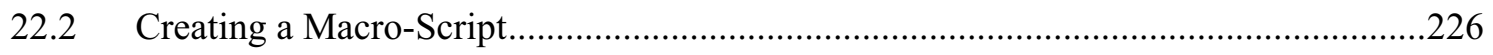

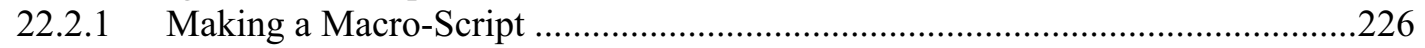

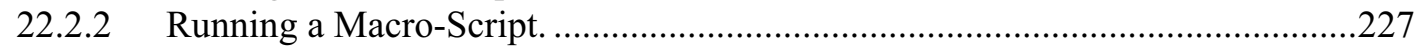




\section{EXECUTIVE SUMMARY}

The U.S. Nuclear Regulatory Commission (NRC) has developed a powerful personal computer (PC) software application for performing probabilistic risk assessments (PRAs), called Systems Analysis Programs for Hands-on Integrated Reliability Evaluations (SAPHIRE). SAPHIRE capabilities for performing a PRA are summarized in this volume, and a more detailed description can be found in the individual sections of the documentation.

With the release of SAPHIRE versions 5 and 6, INL included a separate module called the Graphical Evaluation Module (GEM). GEM provides a highly specialized user interface with SAPHIRE, automating SAPHIRE process steps for evaluating operational events at commercial nuclear power plants. In particular, GEM implements many of the accident sequence precursor (ASP) program analysis methods. Using GEM, an analyst can estimate the risk associated with operational events very efficiently and expeditiously.

This SAPHIRE manual is designed to be an on-line reference guide to the program. Divided into two parts (A and B), this manual is a step-by-step approach to using the features of SAPHIRE. Introductions and installation instructions for the SAPHIRE are first presented, along with some information about the basic features of SAPHIRE. The next sections contain information about SAPHIRE data base concepts so that you can begin learning how SAPHIRE works. The sections following present a step-by-step approach to using the features of SAPHIRE. Later sections provide information about the capabilities and limitations of SAPHIRE, including some of the advanced features of SAPHIRE.

For readers who are familiar with previous versions of SAPHIRE, following are a list of some of the features that have been enhanced or are new to SAPHIRE version 7.

- $\quad$ Improved Graphical Editing

- $\quad$ Improved Fault Tree Logic Editing

- $\quad$ Event Tree Linking Rules

- $\quad$ Sequence Recovery Rules

- $\quad$ Fault Tree Recovery Rules

- Partition Rules

SAPHIRE automatically detects when the database schema for a project is different from the current version of the code. When SAPHIRE attempts to open a project created with an older version of the database schema, the Version Conflict dialog will appear. Once the data has been converted to version 7, this data cannot be used with older versions of SAPHIRE (IRRAS, SARA, GEM, or FEP).

Users will find that SAPHIRE has evolved with advances in computers. Previously, it was run in the DOS operating system. It consisted of a suite of modules as described in the NUREG/CR-6116, "System Analysis Programs for Hands-on Integrated Reliability Evaluations (SAPHIRE) Version 5.0," series of volumes $1-8$. The current versions in use, 6 and 7, run under the Windows environment. Certain modules have been integrated in the SAPHIRE environment; however, the Graphical Evaluation Module (GEM) remains a separate interface with the SAPHIRE code. GEM is a user-friendly interface that streamlines and automates select SAPHIRE inputs and processes for performing events assessments.

SAPHIRE has also evolved with users' needs. New features and capabilities have been added in Versions 6 and 7 for developing and using larger, more complex models. For example, Version 7 can solve up to 2 million sequences. In addition, enhancements have also been made in Version 7 for cut set slicing, event tree rule linkage rules, and reporting options. 
To help assure the quality of new releases, SAPHIRE Versions 6 and 7 were used extensively with models created in earlier versions of SAPHIRE. The models were loaded into the current version of the software and results of the analyses were compared against SAPHIRE Version 5.0. Any discrepancies found were corrected in subsequent minor versions of the code. SAPHIRE has been validated in several ways. 


\section{ACRONYMS}

$\begin{array}{ll}\text { DOE } & \text { Department of Energy } \\ \text { GEM } & \text { Graphical Evaluation Module } \\ \text { INL } & \text { Idaho National Laboratory } \\ \text { IRRAS } & \text { Integrated Reliability and Risk Analysis System } \\ \text { NRC } & \text { Nuclear Regulatory Commission } \\ \text { PRA } & \text { probabilistic risk analysis } \\ \text { SAPHIRE } & \text { Systems Analysis Programs for Hands-on Integrated Reliability Evaluations }\end{array}$ 


\section{Systems Analysis Programs for Hands-on Integrated Reliability Evaluations (SAPHIRE) Vol. 3 Code Reference Manual - Part B}

\section{UTILITY OPTIONS}

\subsection{Utility Options}

\section{PURPOSE}

This option allows you to perform routine functions that are required by SAPHIRE such as defining constants, recovering the database, and MAR D data exchange.

\section{STEPS}

1. From the SAPHIRE menu select Utility.

2. The drop-down menu with available options will be displayed.

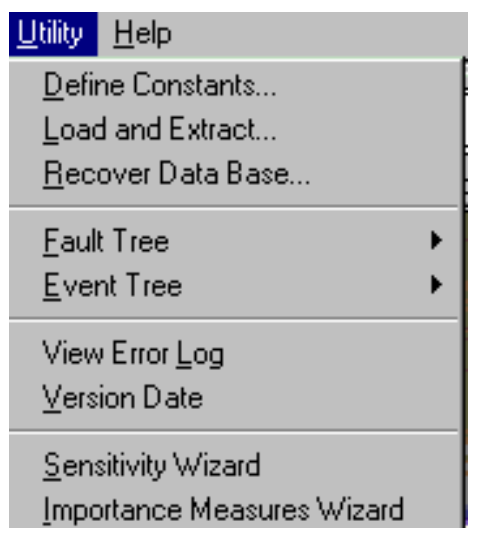

\subsection{Defining Constants}

\subsubsection{General Tab}

\subsubsection{Defining Constants}

\section{PURPOSE}

This option provides the means to specify general information, cut set option defaults, and graphical editor defaults. The define constants dialog consists of four property sheets or "pages," each containing information pertaining to program defaults, grouped by related properties. Select the desired page by clicking on the tab. 


\section{STEPS}

1. From the menu select Utility.

2. Choose Define Constants from the menu. The Define Constants dialog will be displayed.

Initially, the General constants are available when this option is selected.

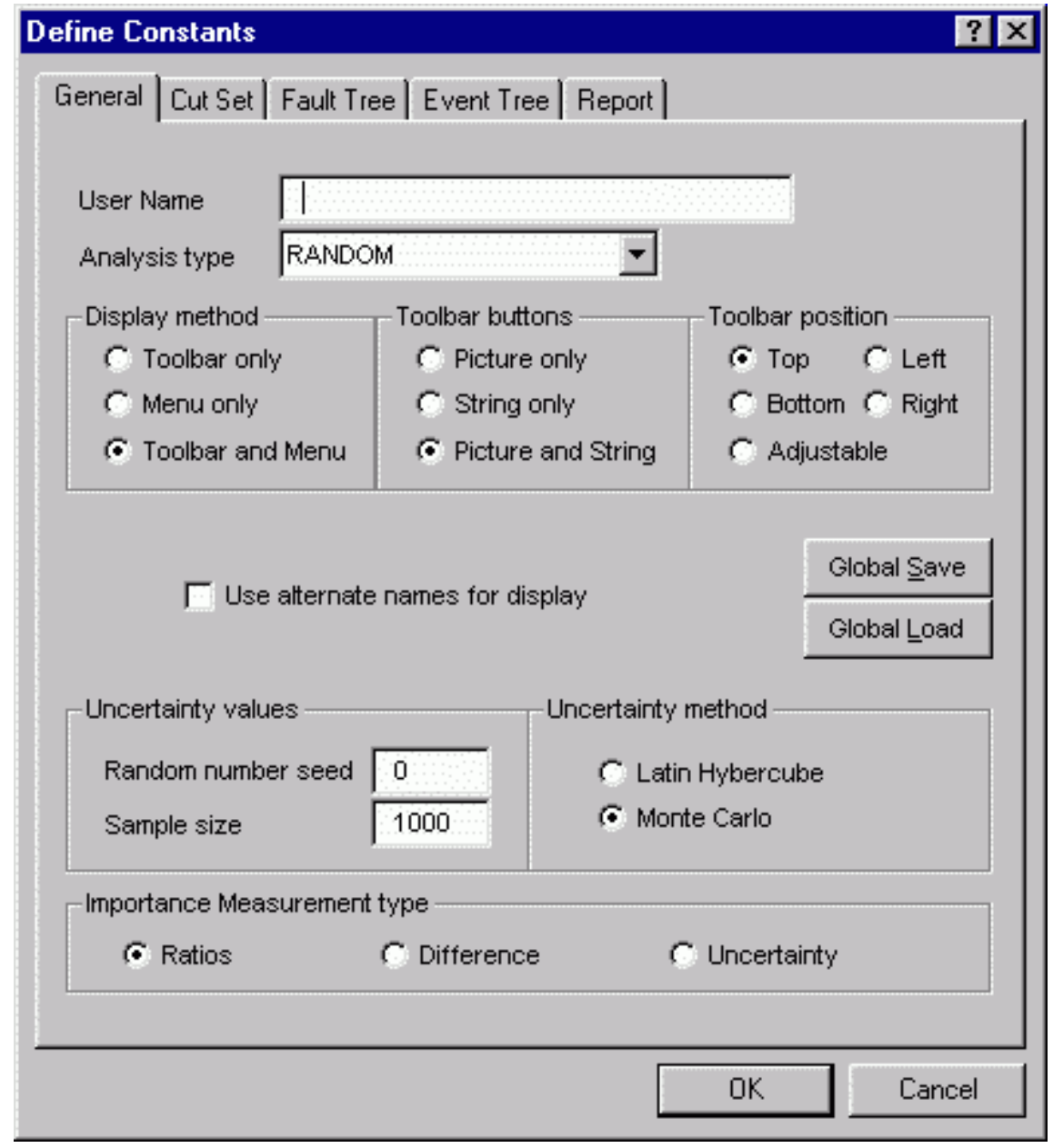

After setting the general information you may choose one of the other pages: Cut Set, Fault Tree, Event Tree, or Report to change other default values.

Global Save - $\quad$ Save the current values as the SAPHIRE global defaults. Global defaults will be used as the initial constants each time a new project is created.

Global Load - Load the SAPHIRE global default values.

OK - $\quad$ Save the changes made and close the Define Constants dialog.

Cancel - $\quad$ Close the Define Constants dialog without saving changes.

\subsubsection{User Name}

This 36-character, alphanumeric field identifies the program user. This information is optional. 


\subsubsection{Analysis type}

The default type of analysis to perform. Initially, the RANDOM analysis type is selected.

\subsubsection{Display Method}

Toolbar only -

Menu only -

Toolbar and Menu -
Shows only the toolbar in the main window. Shows only the menu in the main window.

Shows both the toolbar and menu on the main window.

\subsubsection{Toolbar Buttons}

These options are applicable if the toolbar is displayed in the main window.

Picture only -

String only -

Picture and String -
Shows only the bitmap (picture) on the toolbar buttons.

Shows only the text (string) on the toolbar buttons.

Shows both the bitmap and text on the toolbar buttons.

\subsubsection{Toolbar Position}

These options are applicable if the toolbar is displayed in the main window.

Top - $\quad$ Positions the toolbar horizontally at the top of the main window.

Bottom - $\quad$ Positions the toolbar horizontally at the bottom of the main window.

Left - Positions the toolbar vertically on the left side of the main window.

Right - Positions the toolbar vertically on the right side of the main window.

Adjustable - Allows the toolbar to be resized.

\subsubsection{Use alternate names}

Choose the primary or alternate name field to display throughout SAPHIRE. If this check box is selected, the alternate names will be used throughout the system; otherwise the primary names will be used. This is useful when a project has been developed for dual languages.

\subsubsection{Random number seed}

The default 5-digit numeric field indicating the first random number in the seed to be used in the uncertainty calculation. The initial value, 0 , indicates that the random number will be the current value of the real system clock. This value can be temporarily changed when performing uncertainty calculations.

\subsubsection{Sample size}

The default 6-digit numeric field indicating the number of uncertainty samples to be run. The sample size may range from 1 to 999999 . The initial value is 1000 . This value can be temporarily changed when performing uncertainty calculations.

\subsubsection{Uncertainty method}

Choose the default method for uncertainty analysis. The initial selection is Monte Carlo. This can be temporarily changed when performing uncertainty calculations.

\subsubsection{Importance Measurement type}


Choose the default importance measurement type.

Ratio - $\quad$ Fussell-Vessely importance, risk reduction ratio, and risk increase ratio .

Difference - Birnbaum importance, risk reduction interval, and risk increase interval .

Uncertainty - Quantification of the contribution of each individual basic event's uncertainty to the total output uncertainty.

\subsubsection{Cut Set Tab}

\subsubsection{Cut Set Constants}

\section{PURPOSE}

This option allows you to specify default values for cut set generation.

\section{STEPS}

1. From the menu select Utility.

2. Choose Define Constants from the menu. The Define Constants dialog will be displayed.

3. Select the Cut Set tab. 


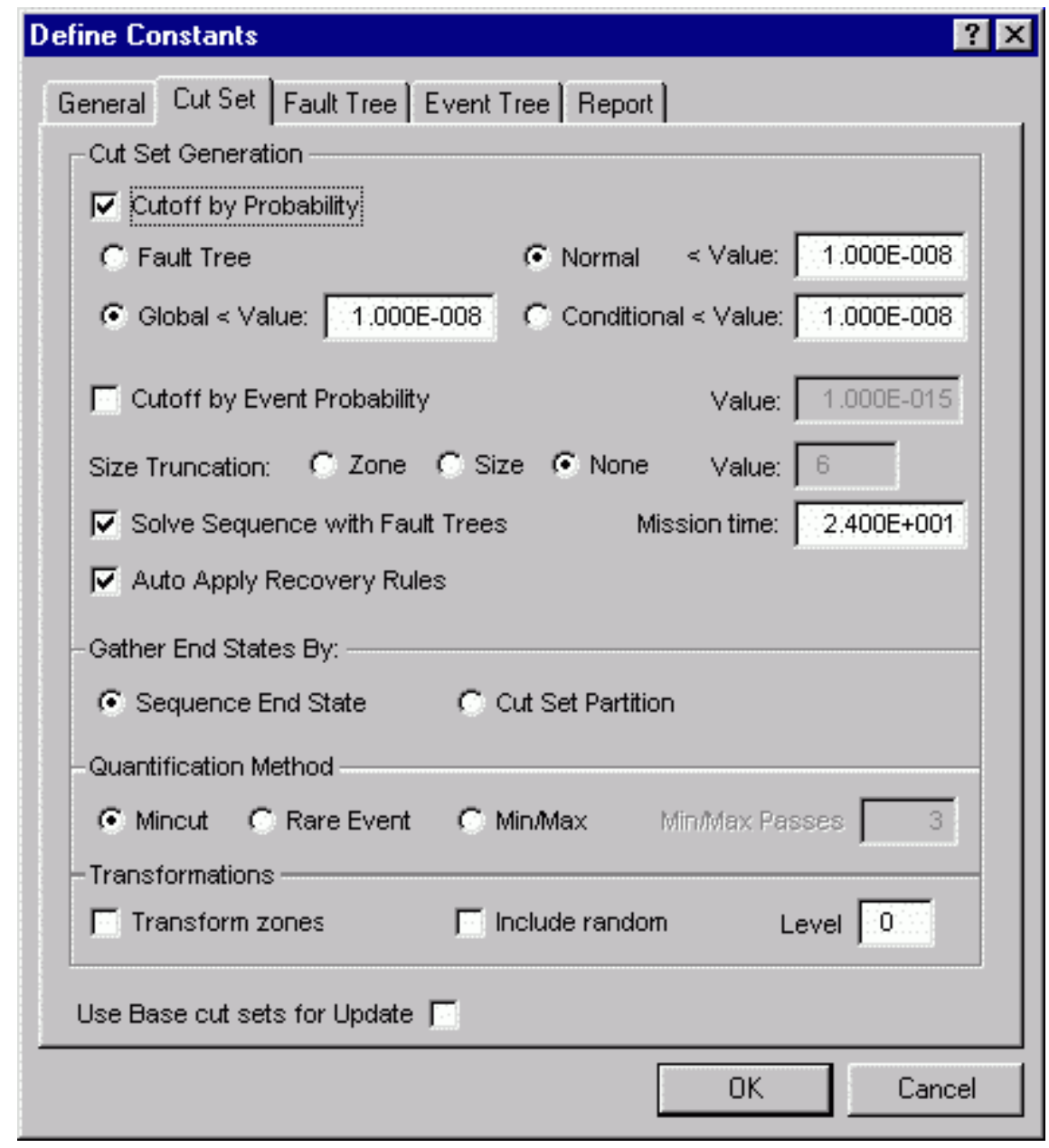

After setting the cut set defaults, you may choose one of the other pages: General, Fault Tree, Event Tree, or Report to change other default values.

Save - Save the changes made and close the Define Constants dialog.

Cancel - Close the Define Constants dialog without saving changes.

\subsubsection{Fault Tree Probability Cutoff}

If the Cutoff by Probability checkbox is selected, cut sets will be retained or discarded during fault tree cut set generation based on the cut set probability value. The default value is initially set to $1.0 \mathrm{E}-8$. These settings can be temporarily changed when you solve.

If you select Cutoff by Probability checkbox and choose:

Fault Tree - $\quad$ Only those cut sets whose product for all of its event probabilities is greater than or equal to the fault tree's cutoff value will be kept. This value is entered in the Modify | Fault Trees option.

Global - $\quad$ Only those cut sets whose product for all of its event probabilities is greater than or equal to the value in the $<$ Global Cutoff Value field will be kept.

All other cut sets will be removed. 
If you deselect this check box, then the probability for the cut set will be irrelevant for determining if the cut set should be retained or discarded.

\subsubsection{Sequence Probability Cutoff}

If the Cutoff by Probability checkbox is selected, cut sets will be retained or discarded during sequence cut set generation based on the cut set probability value. The default value is initially set to $1.0 \mathrm{E}-8$. These settings can be temporarily changed when you solve.

If you select Cutoff by Probability checkbox and choose:

Normal - $\quad$ Only those cut sets whose product for all of its event probabilities is greater than or equal to the value in the $<$ Cutoff Value field will be kept.

Conditional - $\quad$ Only those cut sets whose product for all of its event probabilities, excluding the initiating event probability, is greater than or equal to the value in the $<$ Cutoff Value field will be kept.

All other cut sets will be removed.

If you deselect this check box, then the probability for the cut set will be irrelevant for determining if the cut set should be retained or discarded.

\subsubsection{Cutoff by Event Probability}

The default event probability cutoff value. This option will check all cut sets that are below the probability cutoff and remove them only if they contain an event whose probability is below this value.

If you select this check box, then you must also choose the Cutoff by Probability check box and provide a value.

\subsubsection{Size Truncation}

The default size truncation method and value. When generating fault tree, sequence, or end state cut sets, this field is used for determining if cut sets should be retained or discarded based on the number of basic events in the cut sets. These settings can be changed temporarily when you solve or gather.

Size - The default maximum number of basic events allowed in cut set generation. The default value is initially set to six.

Zone - Only zone flagged events will be checked.

None - The number of events in a cut set is irrelevant for determining if the cut set will be retained.

\subsubsection{Solve Sequences with Fault Trees}

The default method for solving sequence cut sets. If checked then by default, the fault tree logic will be used to solve the sequence cut sets. If unchecked, previously generated fault tree cut sets will be used. This value can be temporarily changed in when solving sequence cut sets. 


\subsubsection{Mission Time}

The default mission time to be used in the calculation of basic event probabilities, in hours. Initially, this value is $2.4 \mathrm{E}+01$.

\subsubsection{Auto Apply Recovery Rules}

If checked, automatically apply recovery rules after cut set generation.

\subsubsection{Gather End States by}

The default method for gathering end state cut sets. This can be temporarily changed when gathering end state cut sets.

\section{Seq End State - $\quad$ Gather cut sets by the end state assigned to each sequence.}

Cut Set Partition - $\quad$ The rule-based (via the partition rules) end state of each cut set will be used for gathering.

\subsubsection{Quantification Method}

Choose the default quantification method to be used for quantifying fault tree, sequence, or end state cut sets.

Mincut - $\quad$ Use the Minimal Cut Set Upper Bound Approximation method.

Rare Event - $\quad$ Add together the probabilities for the cut sets of a top event.

Min/Max - Use the "exact" probability quantification algorithm.

\subsubsection{Transform Zones}

If checked, perform zone transformation during cut set generation.

\subsubsection{Include Random}

If checked, when performing location transformations, include random failures of the event in the transformations.

\subsubsection{Level}

An integer $0-255$ which indicates the default level of substitution for the transformations to be performed.

\subsubsection{Use Base Case}

If checked, use base case cut sets for fault tree, sequence, and end state update. Otherwise use current case cut sets.

\subsubsection{Fault Tree Tab}

\subsubsection{Fault Tree Constants}




\section{PURPOSE}

This option allows you to specify default values for the graphical Fault Tree Editor.

\section{STEPS}

1. From the menu select Utility.

2. Choose Define Constants from the menu. The Define Constants dialog will be displayed.

3. Select the Fault Tree tab.

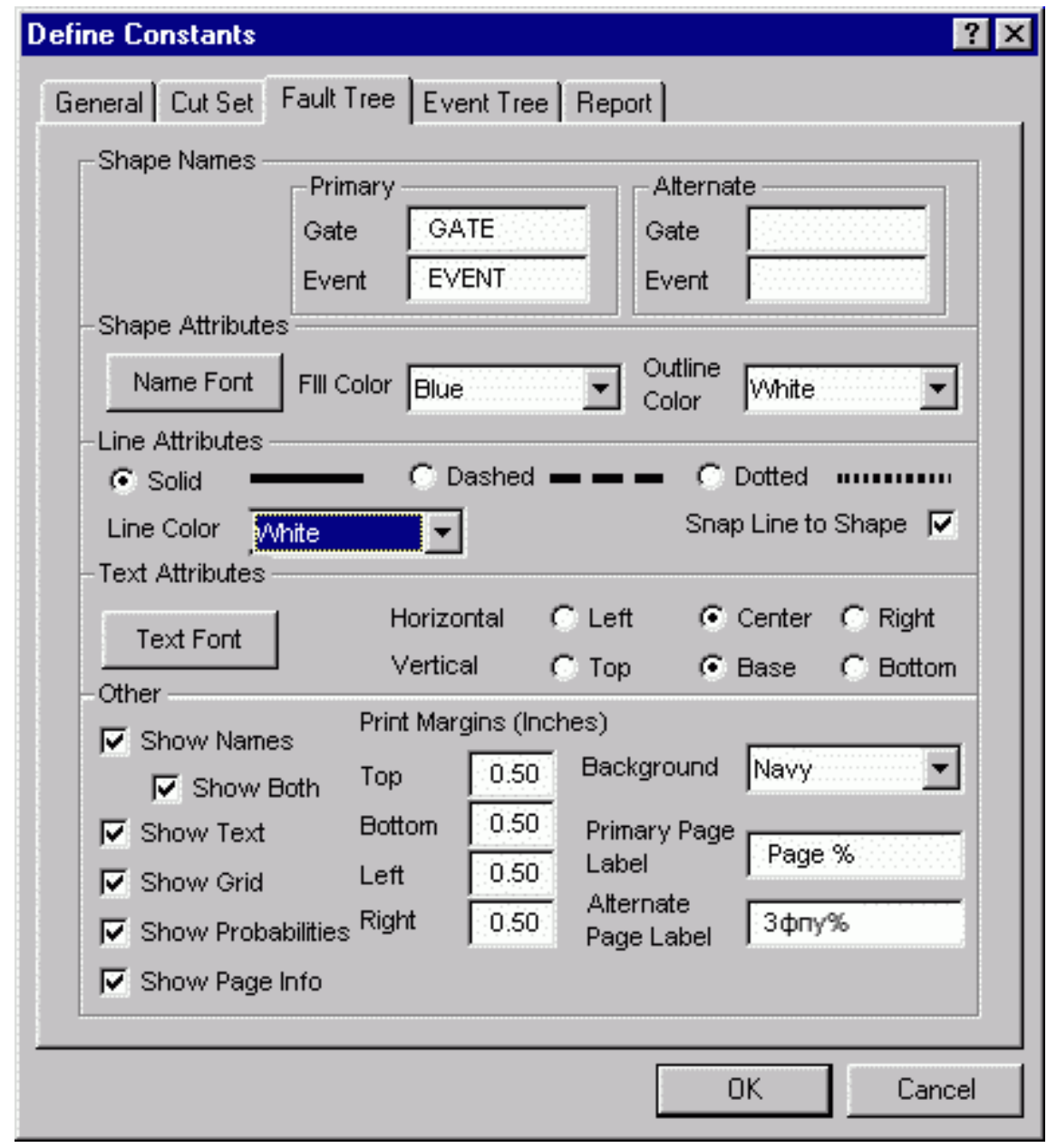

After setting the fault tree defaults, you may choose one of the other pages: General, Cut Set, or Event Tree, or Report to change other default values.

Save - Save the changes made and close the Define Constants dialog.

Cancel - Close the Define Constants dialog without saving changes.

\subsubsection{Gate Name}

10-character field for the fault tree default gate name. Each gate created in the fault tree editor will be given this name followed by a sequential number for each separate gate, e.g., GATE1, GATE2, etc. Both a default primary name and a default alternate name may be specified. 


\subsubsection{Alternate Gate Name}

10-character field for the fault tree default alternate gate name. This feature allows names to be displayed and reported using a different naming scheme or another locale. For this name to be displayed in lists and on reports instead of the Primary gate name, choose Use alternate names for display in the Define Constants option. Each gate created in the fault tree editor will be given this alternate name followed by a sequential number for each separate gate, e.g., ALTGATE1, ALTGATE2, etc.

\subsubsection{Event Name}

10-character field for the fault tree default basic event name. Each event created in the fault tree editor will be given this name followed by a sequential number for each separate event, e.g., EVENT1, EVENT2, etc.

\subsubsection{Alternate Event Name}

10-character field for the fault tree default alternate basic event name. This feature allows names to be displayed and reported using a different naming scheme or another locale. For this name to be displayed in lists and on reports instead of the Primary event name, choose Use alternate names for display in the Define Constants option. Each event created in the fault tree editor will be given thisalternate name followed by a sequential number for each separate event, e.g., ALTEVNT1, ALTEVNT2, etc.

\subsubsection{Name Font}

The default font for displaying names. Change the name font attributes by choosing this button.

\section{SEE ALSO}

Font Selection

\subsubsection{Font Selection}

This option allows you to change default attributes for a text object font or shape name font. This option is accessible from either the Fault Tree page or the Event Tree page on the Define Constants dialog.

\section{STEPS}

1. From the menu select Utility.

2. Choose Define Constants from the menu. The Define Constants dialog will be displayed.

3. Select the Fault Tree or Event Tree tab.

4. Choose the desired Font button. The Font dialog will be displayed.

5. Select the desired font from the list and choose or change the style, size, color, etc. 


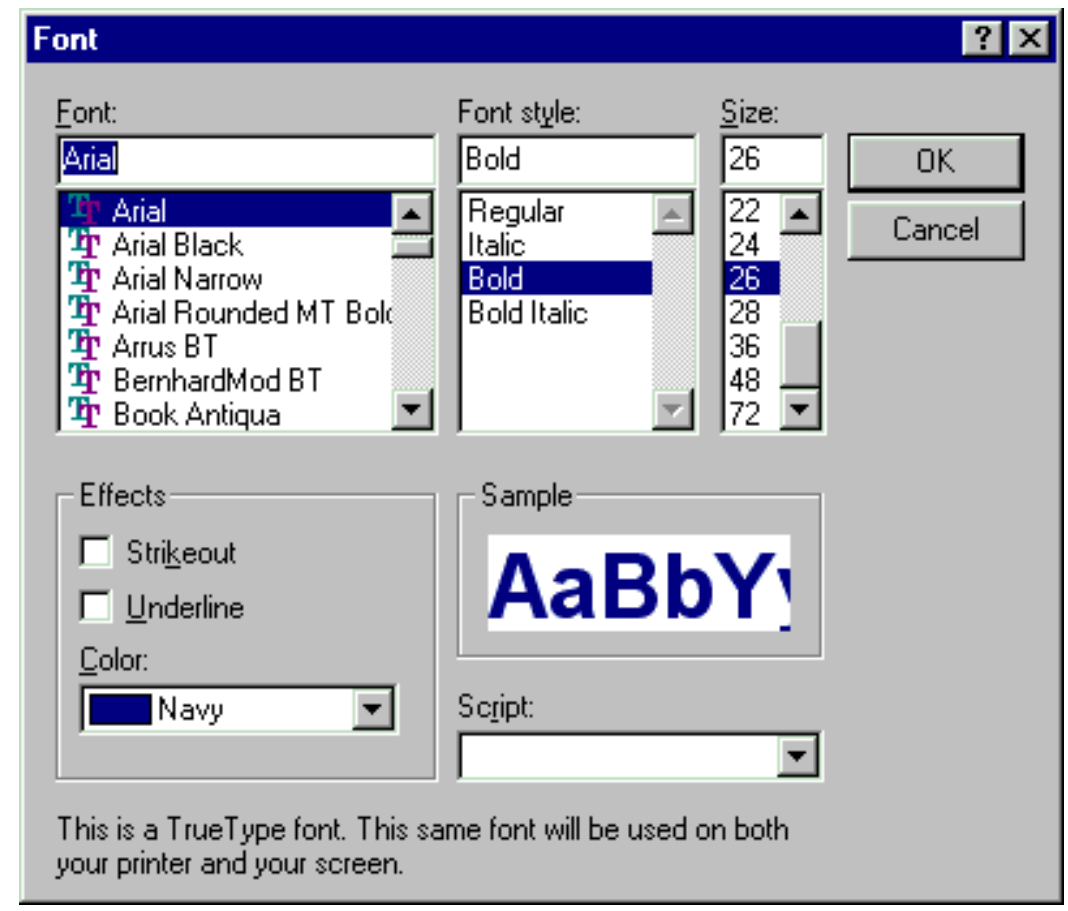

Font - $\quad$ List of available typeface names.

Font Style - $\quad$ Bold, italic, bold italic, or regular styles.

Size - $\quad$ Point size of the selected typeface.

Effects - $\quad$ Underline, strikeout text color.

Sample - $\quad$ Example of the appearance of the highlighted font.

Script - Character set.

OK - Close the Select Font dialog and change the font.

Cancel - $\quad$ Close the Select Font dialog without changing the font.

\subsubsection{Fill Color}

The default color for filling in shapes (i.e., the interior color of the shape). Change the fill color by selecting from the drop-down list.

\subsubsection{Outline Color}

The default color for outlining shapes (i.e., the exterior color of the shape). Change the outline color by selecting from the drop-down list.

\subsubsection{Line Style}

Select the default line type. Connecting lines will be drawn using this style.

\subsubsection{Line Color}

The default color for drawing connecting lines. Change the line color by selecting from the drop-down list.

\subsubsection{Text Font}


The default font for writing text. Change the text font attributes by choosing this button.

\subsubsection{Horizontal Justification}

Select the default horizontal justification for text objects:

Left - The text is aligned along the left margin.

Center - The text is centered on the page. This is the initial value.

Right - The text is aligned along the right margin.

\subsubsection{Vertical Justification}

Select the default vertical justification for text objects:

Base - The text is aligned slightly above the bottom of the text box. This is the initial value. Bottom - The text is aligned at the bottom of the text box.

Top - The text is aligned at the top of the text box.

\subsubsection{Show Names}

If selected, turns on the display of event and gate names. By default, this option is selected.

\subsubsection{Show Both}

If selected, turns on the display of both primary and alternate names for events and gates.

\subsubsection{Show Text}

If selected, turns on the display of descriptive text. By default, this option is selected.

\subsubsection{Show Grid}

If selected, turns the reference grid on. By default, this option is not selected.

\subsubsection{Show Probabilities}

If selected, turns on the display of basic event probabilites. By default, this option is not selected.

\subsubsection{Show Page Information}

If selected, turns on the display of page information. Information displayed includes the name and description of the fault tree or event tree, the current date, and the page number. By default, this option is selected.

\subsubsection{Print Margins}

Set the top, bottom, left, and right margins, in inches, for printing. Enter the values in real numbers. By default the margins are set to $0.50(1 / 2$ inch).

\subsubsection{Background Color}


The default background color for the graphical editor's window. Change the background color by selecting from the drop-down list.

\subsubsection{Primary Page Label}

If selected, turns on the page label display. The label entered is inserted in front of the page number. The symbol "\%" adds a space between the label and the page number. By default, the label "Page\%" is provided. If the label is left blank, then the page number will appear without any label. To omit both the label and the page number, use the carat $\left(^{\wedge}\right)$ symbol.

\subsubsection{Alternate Page Label}

If selected, turns on the alternate page label display. The label entered is inserted in front of the page number. This label is used when alternate names are displayed and printed. The alternate name display feature is set in the Define Constants option.

\subsubsection{Event Tree Tab}

\subsubsection{Event Tree Constants}

\section{PURPOSE}

This option allows you to specify default values for the event tree graphical editor.

\section{STEPS}

1. From the menu select Utility.

2. Choose Define Constants from the menu. The Define Constants dialog will be displayed.

3. Select the Event Tree tab. 


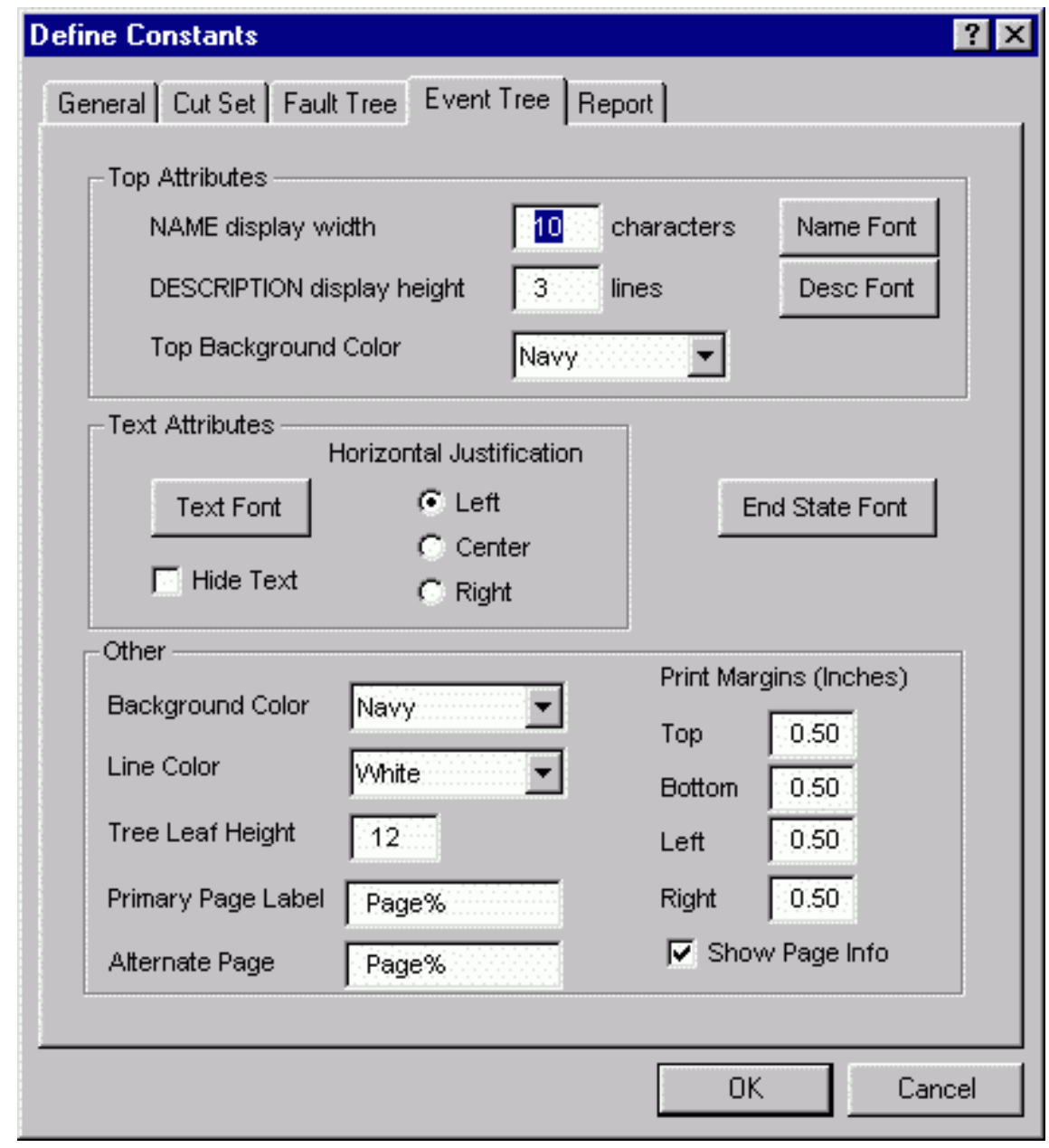

After setting the event tree defaults, you may choose one of the other constants pages: General, Cut Set, Fault Tree, or Report to change other default values.

OK - Save the changes made and close the Define Constants dialog.

Cancel - Close the Define Constants dialog without saving changes.

\subsubsection{Name Display Width}

The default number of characters in the name which will be displayed.

\subsubsection{Name Font}

The default font for displaying names. Change the name font attributes by choosing this button.

\subsubsection{Description Display Height}

The default number of lines of the description which will be displayed.

\subsubsection{Description Font}


The default font for displaying descriptions. Change the description font attributes by choosing this button .

\subsubsection{Top Background Color}

The default background color for the area where the top names are displayed in the graphical editor's window. Change the top background color by selecting from the drop-down list.

\subsubsection{Text Font}

The default font for writing text. Change the text font attributes by choosing this button.

\subsubsection{Hide Text}

If selected, the text will be hidden when displaying the tree.

\subsubsection{Horizontal Justification}

Select the default horizontal justification for text objects:

Left - The text is aligned along the left margin.

Center - The text is centered on the page. This is the initial value.

Right - The text is aligned along the right margin.

\subsubsection{End State Font}

The default font for displaying end state names. Change the end state name font attributes by choosing this button.

\subsubsection{Background Color}

The default background color for the graphical editor's window. Change the background color by selecting from the drop-down list.

\subsubsection{Line Color}

The default color of lines. Change the line color by selecting from the drop-down list.

\subsubsection{Tree Leaf Height}

The spacing, in relative units, between the branches.

\subsubsection{Primary Page Label}

If selected, turns on the page label display. The label entered is inserted in front of the page number. The symbol "\%" adds a space between the label and the page number. By default, the label "Page\%" is provided. If the label is left blank, then the page number will appear without any label. To omit both the label and the page number, use the carat $\left({ }^{\wedge}\right)$ symbol.

\subsubsection{Alternate Page Label}


If selected, turns on the alternate page label display. The label entered is inserted in front of the page number. This label is used when alternate names are displayed and printed. The alternate name display feature is set in the Define Constants option.

\subsubsection{Print Margins}

Set the top, bottom, left, and right margins, in inches, for printing. Enter the values in real numbers. By default the margins are set to 0.50 (1/2 inch).

\subsubsection{Show Page Information}

If selected, turns on the display of page information. Information displayed includes the name and description of the fault tree or event tree, the current date, and the page number. By default, this option is selected.

\subsubsection{Report Tab}

\subsubsection{Report Constants}

\section{PURPOSE}

This option allows you to specify default values for report output, where applicable.

\section{STEPS}

1. From the menu select Utility.

2. Choose Define Constants from the menu. The Define Constants dialog will be displayed.

3. Select the Report tab. 


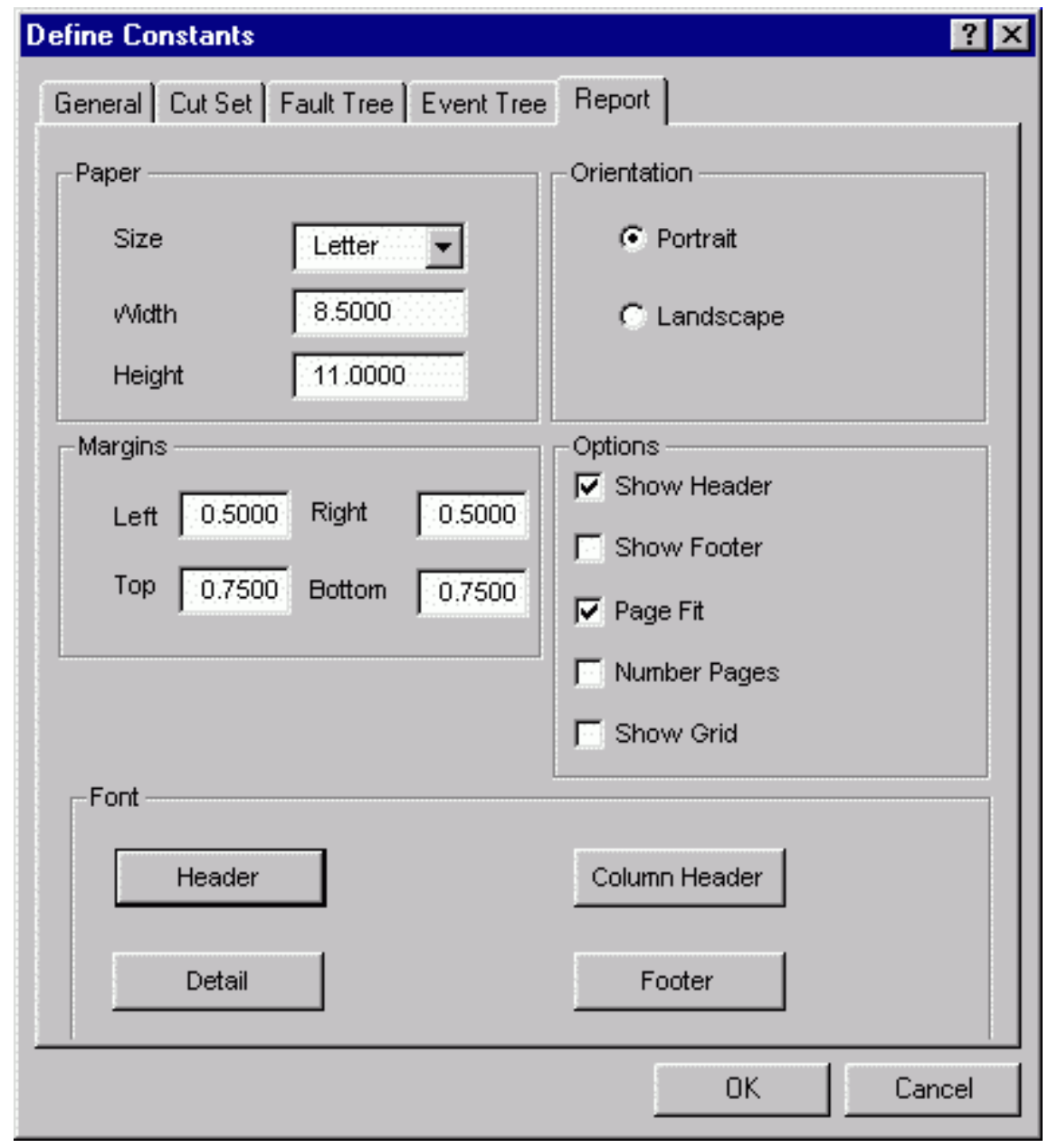

After setting the event tree defaults, you may choose one of the other constants pages: General, Cut Set, or Fault Tree to change other default values.

Paper - $\quad$ Set the desired paper size, height, and width.

Orientation - Select the desired paper orientation.

Options - $\quad$ Turn the header, footer, page numbering, page fit, and grid options on (checked) or off (unchecked).

Margins - $\quad$ Set the left, right, top, and bottom margins for the report.

Font - Select the font characteristics for the header, column header, detail, and footer areas of the report.

OK - $\quad$ Save the changes made and close the Define Constants dialog.

Cancel - $\quad$ Close the Define Constants dialog without saving changes. 


\subsection{Load and Extract}

\subsubsection{Loading and Extracting Data}

\section{PURPOSE}

This option allows you to load probabilistic risk assessment data from the Models and Results Data Base (MAR D) or Set Equation Transformation System (SETS) database into SAPHIRE. Additionally, you can output SAPHIRE formatted data to the generic MAR-D format or SETS format.

\section{STEPS}

1. From the SAPHIRE menu select Utility.

2. Choose Load and Extract from the menu. The Load and Extract Data dialog will be displayed.

3. Select the desired Data Action, Data Format, Data Type, then File Type.

4. Choose the Process button to continue.

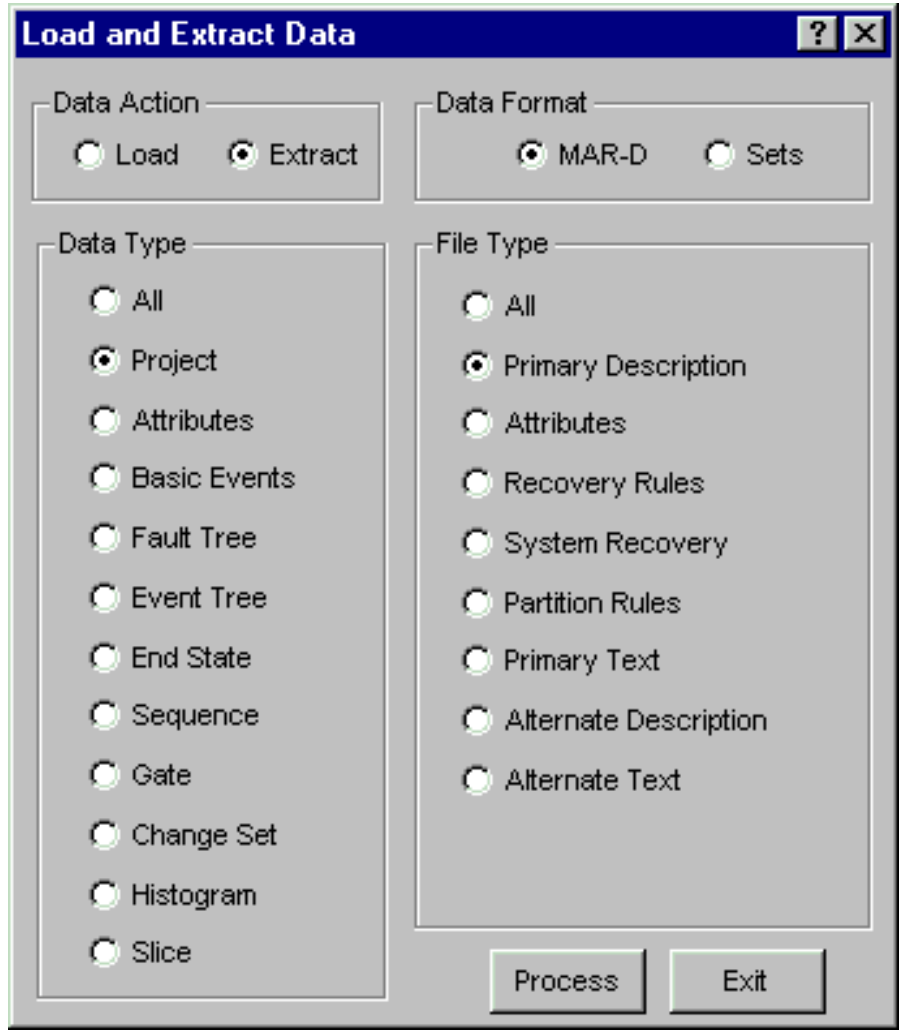

Data Action

Load - Load PRA data into the currently selected SAPHIRE project. 
Extract - Output SAPHIRE formatted data of the currently selected project to a file in the current project directory.

Data Format

MAR-D - Use the Models and Results Data Base format.

SETS - Use the Set Equation Transformation System file format.

Data Type - $\quad$ The type of data to be loaded or extracted, e.g., Project, Basic Event, etc.

File Type - $\quad$ The subset data of the selected Data Type.

Process - $\quad$ Proceed with the loading or extracting operation.

Exit - $\quad$ Close the Load and Extract Data dialog.

\subsubsection{Loading MAR-D Data}

\subsubsection{Loading MAR-D Data}

\section{PURPOSE}

This option facilitates loading of probabilistic risk assessment data from the Models and Results Data Base (MAR D). The process converts information from the generic format found in the MAR D database to the SAPHIRE database format. New data loaded into the database overwrites old data of the same name.

In general, the dialogs and operations are similar for each of the Data Types, therefore, a generic discussion is provided here.

\section{STEPS}

1. Before loading any data, a project must be created through the Modify | Project option.

2. Copy the files you wish to load into that project's folder (directory).

3. Select the project and verify that the project name displayed in the title bar of the SAPHIRE window is where the data should be loaded. If it is not correct, use the File | Open Project option again to choose the desired project.

4. Select the Utility | Load and Extract option. The Load and Extract Data dialog will be displayed. 


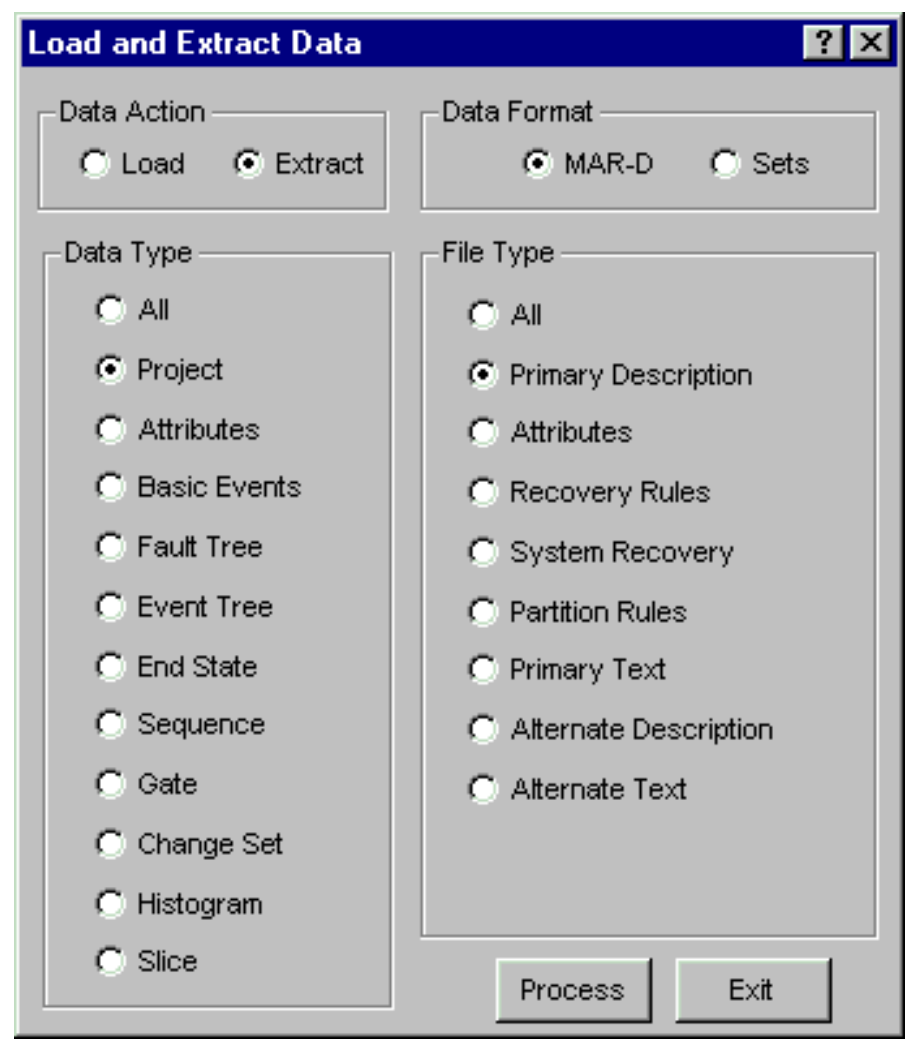

As you click the different Data Type radio buttons, corresponding File Type options will appear to the right, listing the types of data that can be loaded.

After selecting the data to load, choose the Process button. The Load dialog will appear listing the files that contain the specified data and have the proper file extension.

5. ĐClick on the Data Type in the graphic, above, for additional information on loading various types of data.

\subsubsection{Load Dialog}

\section{PURPOSE}

Converts information from the generic format found in the MAR D database to the SAPHIRE database format.

\section{STEPS}

1. Choose the desired Data Type and File Type radio buttons along with the Load Data Action radio button.

2. Select the Process button. The Select an input file dialog will be displayed.

3. Select the desired folder, if necessary.

4. Highlight the desired file and choose the Open button.

OR

5. Double click the desired file. 
This dialog lists the existing files of the appropriate file type (depending on the File Type radio button previously selected) that can be loaded.

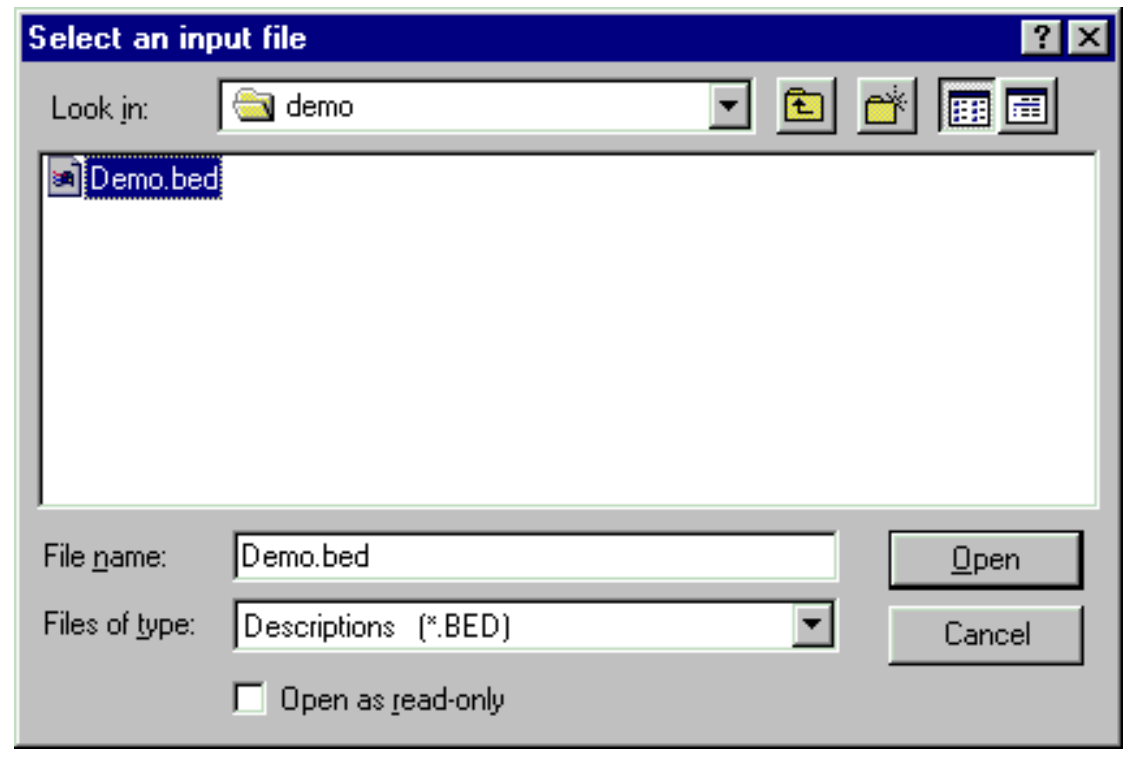

Open - Load the data in the selected file into the current project data base. If successful, the message, "Load complete," will be displayed in the status bar of the SAPHIRE window.

Cancel - Close the Select an input file dialog without loading data.

\subsubsection{General Format Rules}

The following general rules apply to the PRA Models and Results Data Base (MAR-D) data interchange format. These rules apply to all files unless specifically stated otherwise.

All name references (project names, event names, etc.) must be upper case alphanumeric. All lower case characters will be converted to upper case. Any alpha fields that are longer than the format specified will be truncated. No spaces are allowed in the middle of names.

Descriptions can have both upper-case and lower-case characters. No character checking will be done. No commas are allowed in the description.

Commas are used as field delimiters in most formats, and can be used as placeholders for unknown fields. Any number of leading and trailing field spaces can be inserted. Exceptions to this format are detailed as needed.

Text rules:

File is standard ASCII text, single spaced, upper and lower case.

First line of paragraph is indented 5 spaces, with a blank line between paragraphs.

${ }^{\wedge}$ EOS signals the End of Section so that multiple names in the same project can be collected in one file. 


\subsubsection{Load Verification}

\section{PURPOSE}

This provides consistency checking for names contained in multiple files. Data files that have no prerequisite loaded files (*.CTD, *.FTD, *.BED, etc.) are assumed to be correct and are loaded directly into the database without any field checking taking place. Data files having prerequisite loaded files contain dependent fields referenced in those data files (e.g., the *.BEI file contains event names also contained in the *.BED file).

MAR-D files load order dependencies.

\begin{tabular}{|c|c|c|c|}
\hline \multicolumn{2}{|c|}{ Group } & Prerequisite Files & Dependent Fields \\
\hline \multirow[t]{6}{*}{ Project $^{1}$} &. $\mathrm{FAD}$ & & \\
\hline & .FAT & & \\
\hline & .FAA & & \\
\hline & .FAY & & \\
\hline & .FAP & & \\
\hline & .FTT & & \\
\hline \multirow[t]{5}{*}{ Attributes } &. TTD & & \\
\hline & .CTD & & \\
\hline & .FMD & & \\
\hline &. $\mathrm{LCD}$ & & \\
\hline & .STD & & \\
\hline \multirow[t]{8}{*}{ Basic Events } &. $\mathrm{BED}$ & & \\
\hline & .BEI & .BED & Event Name \\
\hline & .BEA & .BED & Event Name \\
\hline & & .CAD & Class Attr \\
\hline & & .CTD & Comp Type \\
\hline & & FMD & Fail Mode \\
\hline & &. $\mathrm{LCD}$ & Location \\
\hline & & .STD & System Type \\
\hline \multirow[t]{9}{*}{ Fault Trees ${ }^{2}$} & .FTD & & \\
\hline & .DLS & & \\
\hline & .FTL & BED & Event Name \\
\hline &. FTC &. FTD & Fault Tree Name \\
\hline & & .BED & Event Name \\
\hline &. FTA &. FTD & Fault Tree Name \\
\hline & .FTT &. FTD & Fault Tree Name \\
\hline & .FTY & & \\
\hline & .PID & & \\
\hline \multirow[t]{6}{*}{ Event Trees ${ }^{3}$} & ETD & & \\
\hline & .ETA & ETD & Event Tree Name \\
\hline & & .BEI & Init Event \\
\hline & .ETG & .ETD & Event Tree Name \\
\hline & ETL & ETD & Event Tree Name \\
\hline & & .BEI & Init Event: unless Top \\
\hline
\end{tabular}




\begin{tabular}{|c|c|c|c|}
\hline \multirow{4}{*}{ End States } & .ETT & .ETD & Event Tree Name \\
\hline & .ESD & & \\
\hline & .ESI & $? ? ? ?$ & ???? \\
\hline & .EST & .ESD & End State Name \\
\hline \multirow[t]{11}{*}{ Sequences } &.$S Q D$ & ETD & Event Tree Name \\
\hline & .SQC & .ETD & Event Tree Name \\
\hline & & .SQD & Sequence Name \\
\hline & .SQA & .BED & Event Name \\
\hline & & .SQD & Sequence Name \\
\hline & & .ESD & End State Name \\
\hline & .SQL & & \\
\hline & .SQY & & \\
\hline &.$S Q P$ & & \\
\hline & .SQT & ETD & Event Tree Name \\
\hline & &.$S Q D$ & Sequence Name \\
\hline
\end{tabular}

\section{NOTES:}

Consistency checking for project names is active for all files.

.DLS and .FTL files are put into graphic relation instead of system relation. No cross relational cross checking available for graphics name.

.ETG is currently loaded as text. .ETL should look exactly like .ETG file.

If a dependent field does not exist in the database at load time, that field will be displayed in a confirmation/add menu. Thus, if an event name contained in a *.BEI file does not match any event name previously loaded by the *.BED file, the new name will be displayed with the option to add the event name or simply abort the load process. Note that the dependent field name cannot be edited at this point. If the name is not correct then exit, fix the mistake, and reload.

However, if you suspect your data files have multiple cross reference errors, load the files and allow the load procedure to find the errors for you. As an error is displayed on the dialog, write down the line number and file name and continue with the load. When the load is finished, make the necessary corrections to the data files, delete the database files for that project (i.e., the *.DAT, *.IDX, and *.BLK files), and restart the load procedure.

\subsubsection{Extracting MAR-D Data}

\subsubsection{Extracting MAR-D Data}




\section{PURPOSE}

This option allows you to output data in MAR D file format. The extracted file is created in the current project folder (directory). The default output file name for description, information, and attribute data is the project name plus the appropriate extension. For MAR D cut set, logic, graphic, and textual information, the name of the file is generated based on the selected output option and some internal numbers.

NOTE: If a file with this name already exists it will be overwritten.

In general, the dialogs and operations are similar for each of the Data Types, therefore, a generic discussion is provided here.

\section{STEPS}

1. From the SAPHIRE menu select Utility.

2. Choose the Load and Extract option. The Load and Extract Data dialog will be displayed.

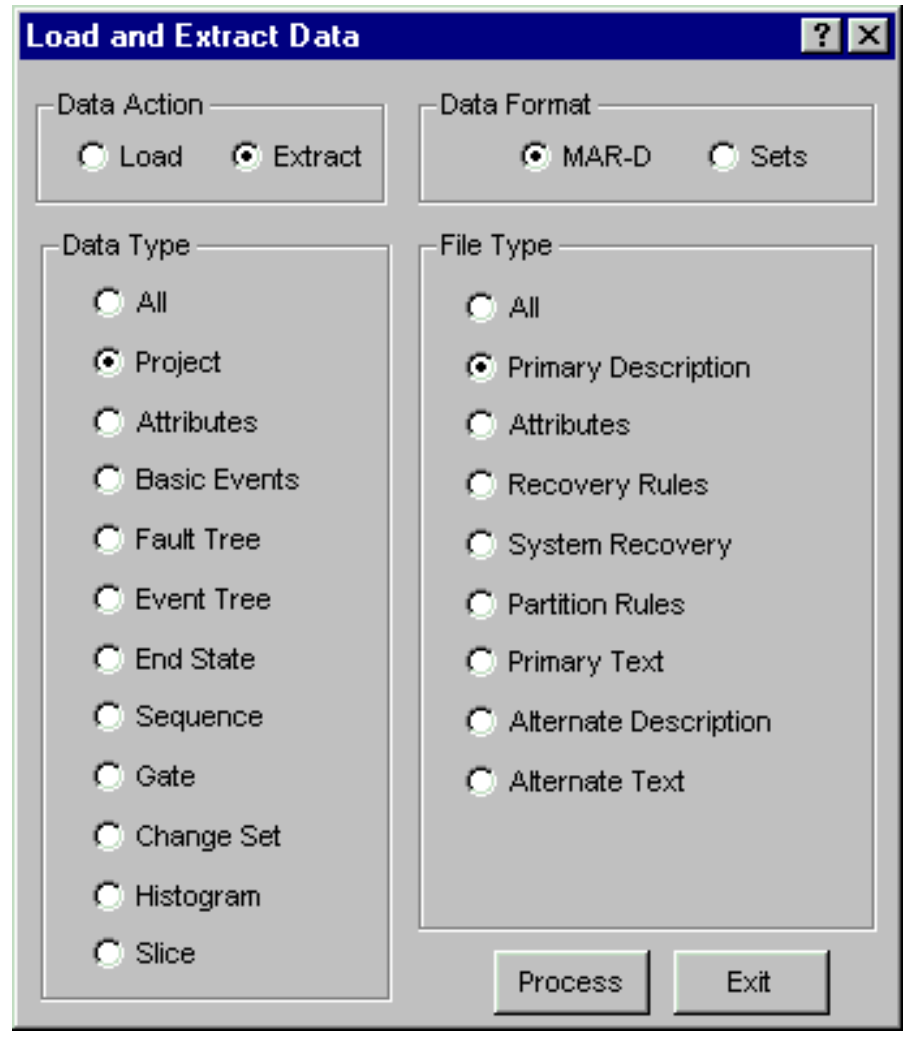

As you click the different Data Type radio buttons, corresponding File Type options will appear to the right, listing the types of data that can be loaded. Choose the data type and file type to output.

Usually, the Extract dialog will appear listing the records available for extraction. On occasion, when it is not necessary to select records, the Output Destination dialog will be displayed. 
3. Click on the Data Type in the graphic, above, for additional information on various types of data.

\subsubsection{Extract Dialog}

\section{PURPOSE}

Outputs information from the SAPHIRE database format to the generic format found in the MAR D database.

\section{STEPS}

1. Choose the desired Data Type and File Type radio buttons along with the Extract Data Action radio button.

2. Select the Process button. The Extract dialog will be displayed.

3. Highlight the desired record(s) and choose the Extract button.

This dialog lists the existing records in the current project database of the appropriate data type (depending on the File Type radio button previously selected) that can be extracted.

\begin{tabular}{|c|c|c|c|}
\hline Basic Erents - [DEMO] & & \begin{tabular}{|l|l}
$? \sqrt{2}$ \\
\end{tabular} & $x$ \\
\hline Name & Description & & \\
\hline $\begin{array}{l}\langle\text { FALSE } \\
\langle I N I T\rangle \\
\langle\text { PASS } \\
\langle\text { TRUE> } \\
\text { A-C-CV-A-SEISMIC } \\
\text { C-CV-A } \\
\text { C-CV-B } \\
\text { C-MOV-1 } \\
\text { C-MOV-A } \\
\text { C-MOV-B } \\
\text { C-PUMP-A } \\
\text { C-PUMP-B } \\
\text { CCS }\end{array}$ & $\begin{array}{l}\text { System Generated Success Event } \\
\text { System Generated Initiating Event } \\
\text { System Generated Ignore Event } \\
\text { System Generated Failure Event } \\
\text { CCS Train A pump discharge check valve } \\
\text { CCS Train A pump discharge check valve } \\
\text { CCS Train B pump discharge check valve } \\
\text { CCS suction isolation valve } \\
\text { CCS Train A pump discharge isolation valve } \\
\text { CCS Train B pump discharge isolation valve } \\
\text { CCS Train A motor-driven pump } \\
\text { CCS Train B motor-driven pump } \\
\text { Developed Event }\end{array}$ & $\Delta$ & $\underline{\underline{\underline{ }}}$ \\
\hline & Extract & Exit & \\
\hline
\end{tabular}

Extract - $\quad$ Output the data of the selected record(s) to a file in the current project subdirectory. After choosing this button, the Output Destination dialog will be displayed.

Exit - $\quad$ Close the Extract dialog without extracting data.

\subsubsection{Output Destination Dialog}

\section{PURPOSE}

Specify the output destination (file name) for extracted SAPHIRE data. 


\section{STEPS}

1. Once the Get Output Destination dialog is displayed, enter the name of the file to which the data will be output.

2. Choose the Ok button.

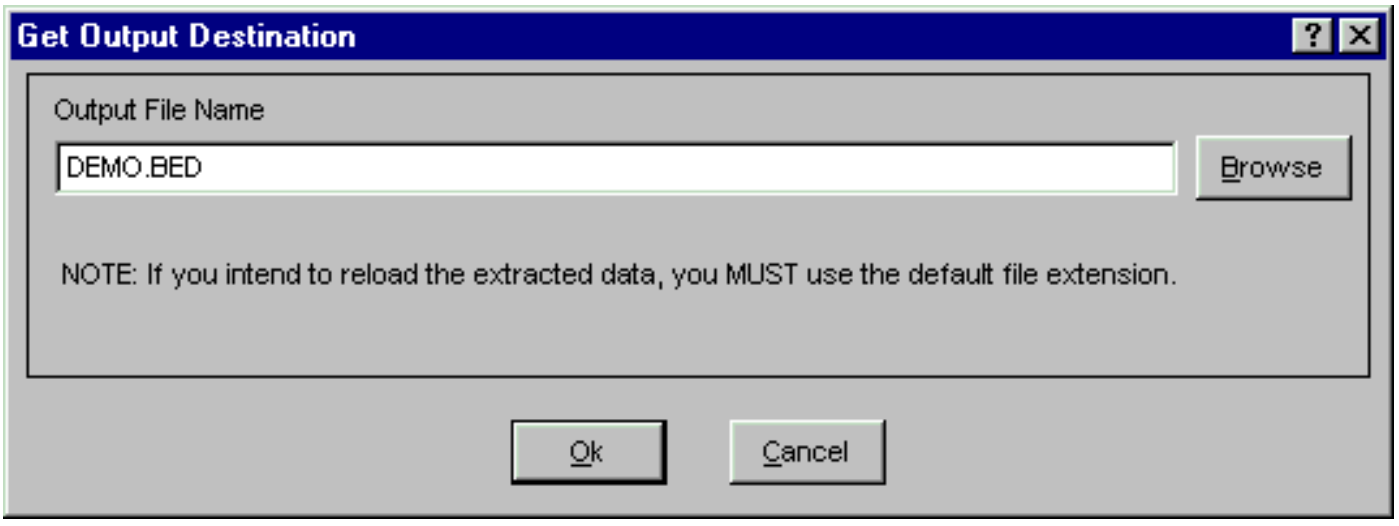

Output File Name - $\quad$ The name of the file that will contain the extracted data. Each combination of data type and file type has a unique file extension. A default file name will be suggested. The file name can be changed; however, the default file extension should be used if the data is to be reloaded by SAPHIRE. If the output file name does not include a directory path, the output directory will be the same as the current project folder.

Browse - $\quad$ Select a folder and file name for the output.

Ok - $\quad$ Extract the data to the specified file. The file will be created in the current project directory. If successful, the message "Output complete", will be displayed in the status bar of the SAPHIRE window.

Cancel Close the Get Output Destination dialog without extracting data.

Hint 1: By default, files will be extracted to the current project folder. However, it is recommended to extract files to a folder dedicated to load/extract files (a different dedicated folder for each project). In the Browse option, you can create a new folder by right clicking the file list and choosing New | Folder.

Hint 2: Once you have selected a load/extract folder that is different than the current project, that folder will be the initial directory selected each time you choose the Browse button. The most recently chosen folder will be remembered until SAPHIRE is closed.

\subsubsection{All/Group Information}

\subsubsection{All/Group Data Type}

\subsection{Extract All Data Type}




\section{PURPOSE}

This option allows you to output all of the data contained in the current project into MAR D file format. This option is equivalent to selecting each combination of Data Type and File Type. The desired folder (directory) may be selected. The default output file names will be used. In addition, a master file will be created. This file will contain the names of all the files created. You may select the name of this file, but the default extension (.MARD) is expected if the data is to be reloaded into SAPHIRE.

NOTE 1: If the selected master file or any other files with default names already exist, they will be overwritten.

NOTE 2: Do not confuse this option with the Project Data Type option. The All option extracts every piece of data contained in the current project, whereas the Project option extracts only data directly related to the Project record, such as name, description, and project-wide rules.

\section{STEPS}

1. From the SAPHIRE menu select Utility.

2. Choose the Load and Extract option. The Load and Extract Data dialog will be displayed.

3. Select the Extract Data Action.

4. Select the MAR-D Data Format.

5. Select the All Data Type.

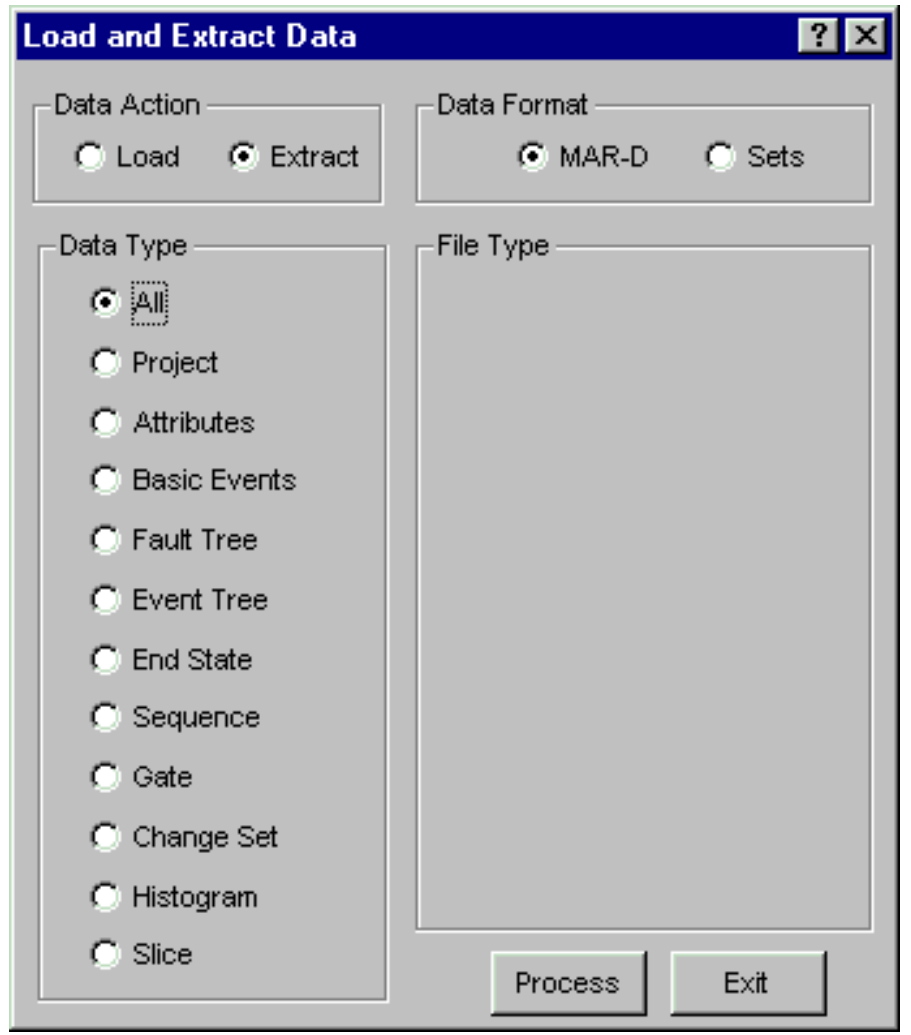


Process - Extract the current project data. The Output Destination dialog will be displayed. Exit - Close the Load and Extract Data dialog.

\subsection{Extract All File Type}

\section{PURPOSE}

This option allows you to output all of the data for a particular Data Type into MAR D file format. This option is equivalent to selecting each File Type option for the chosen Data Type. The desired folder (directory) may be selected. The default output file names will be used. In addition, a master file will be created. This file will contain the names of all the files created. You may choose the name of this file, but the default extension is expected if the data is to be reloaded into SAPHIRE.

NOTE: If the selected master file or any other files with default names already exist, they will be overwritten.

\section{STEPS}

1. From the SAPHIRE menu select Utility.

2. Choose the Load and Extract option. The Load and Extract Data dialog will be displayed.

3. Select the Extract Data Action.

4. Select the MAR-D Data Format.

5. $\quad$ Select the desired Data Type.

6. Select the All File Type.

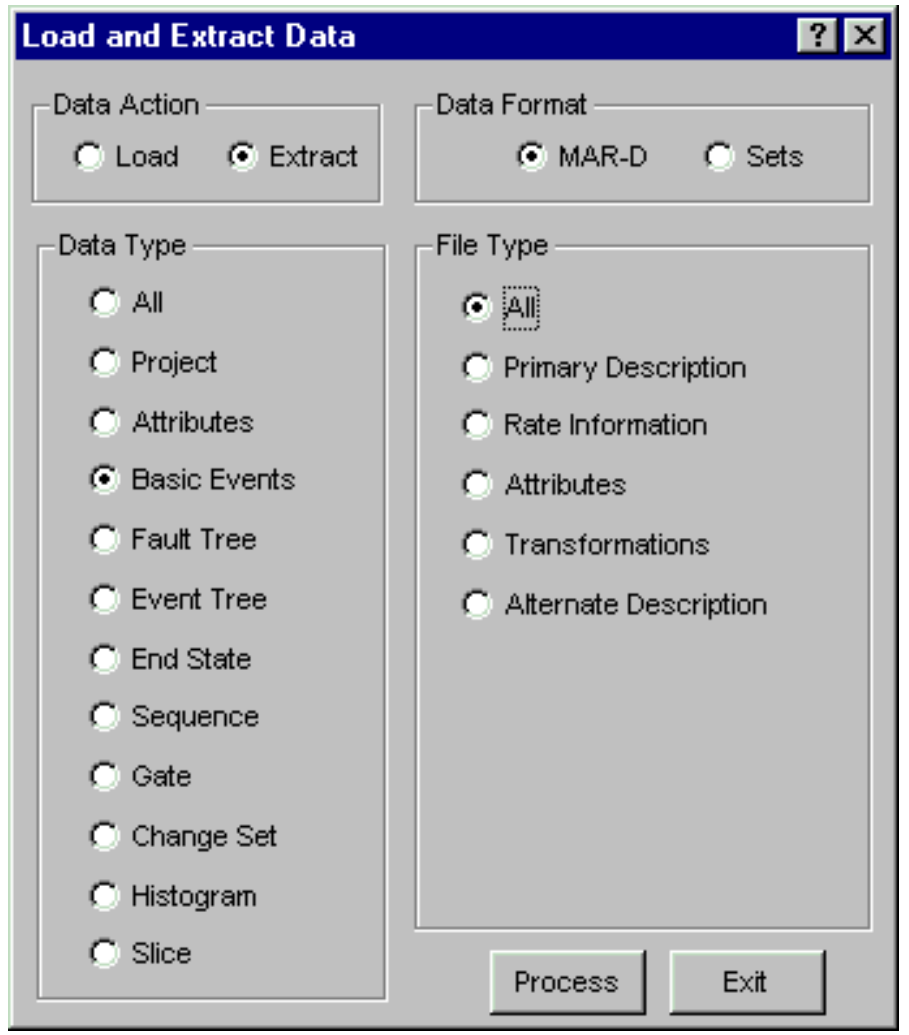


Process - Extract the data. The Output Destination dialog will be displayed.

Exit - Close the Load and Extract Data dialog.

\subsection{Load All/Group}

\section{PURPOSE}

This option facilitates loading of probabilistic risk assessment data from the Models and Results Data Base (MAR D). The process converts information from the generic format found in the MAR D database to the SAPHIRE database format. Use this option to load data that has been extracting using the corresponding Extract All Data Type option. You may also use this option to load data that was extracted using the Extract All File Type option, as well as for data extracted using the Related Information check box available in the Fault Tree Logic option.

NOTE: New data loaded into the database overwrites old data of the same name.

\section{STEPS}

1. From the SAPHIRE menu select Utility.

2. Choose the Load and Extract option. The Load and Extract Data dialog will be displayed.

3. Select the Load Data Action.

4. Select the MAR-D Data Format.

5. Select the All/Group Data Type.

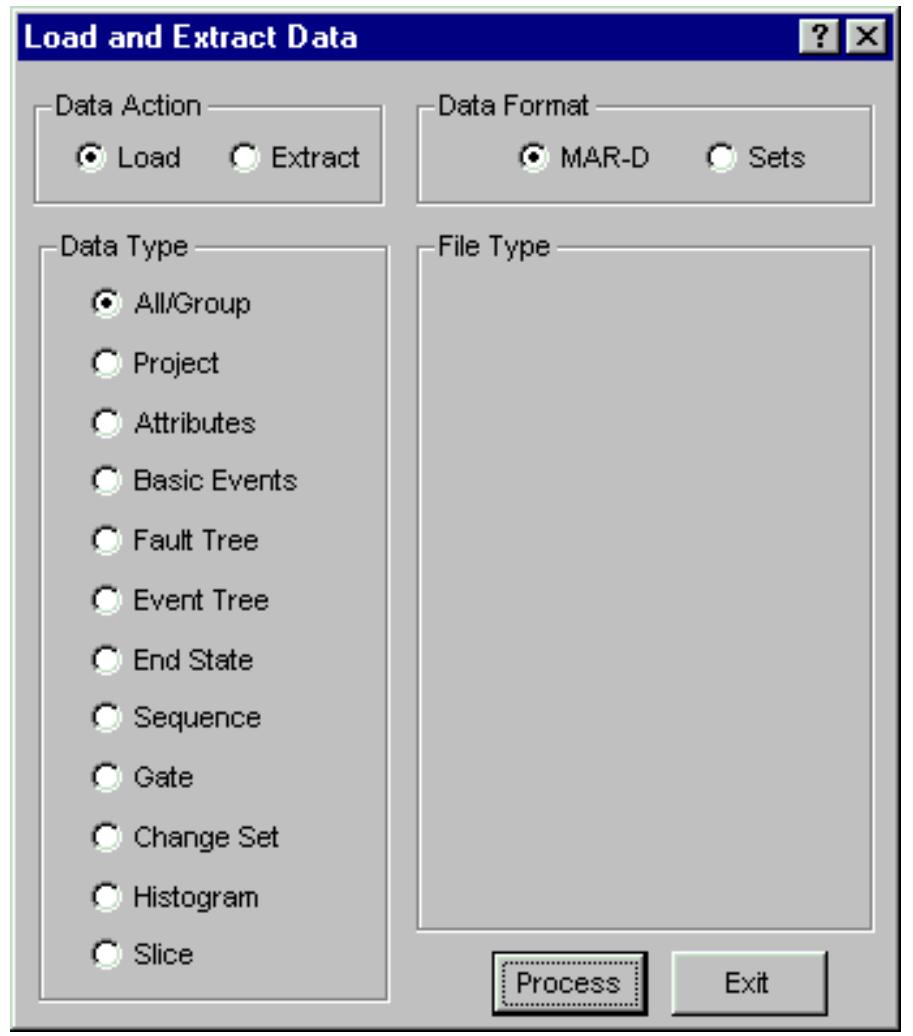


Process - Load data into the current project. The Load_dialog will be displayed.

Exit - Close the Load and Extract Data dialog.

\subsection{Load All/Group Dialog}

\section{PURPOSE}

Converts information from the generic format found in the MAR D database to the SAPHIRE database format. The All/Group option allows you to select a "master" file that contains a list of other MAR-D file formatted files to be loaded. SAPHIRE will load each of these files into the current project. If a file in the master list is not found, it will be ignored.

\section{STEPS}

1. Choose the All/Group Data Type and File Type radio buttons along with the Load Data Action radio button.

OR

2. Choose the desired Data Type radio button and the All File Type radio button.

3. Select the Process button. The Select an input file dialog will be displayed.

4. Select the desired folder, if necessary.

5. Select the desired file type from the Files of Type drop down list, if necessary.

6. Highlight the desired file and choose the Open button.

OR

7. Double click the desired file.

If you chose the All/Group Data Type option, the Files of Type drop down list will allow you to select any of the "master" list files. If you chose the All File Type option, only "master" list files for the selected Data Type will be available. 


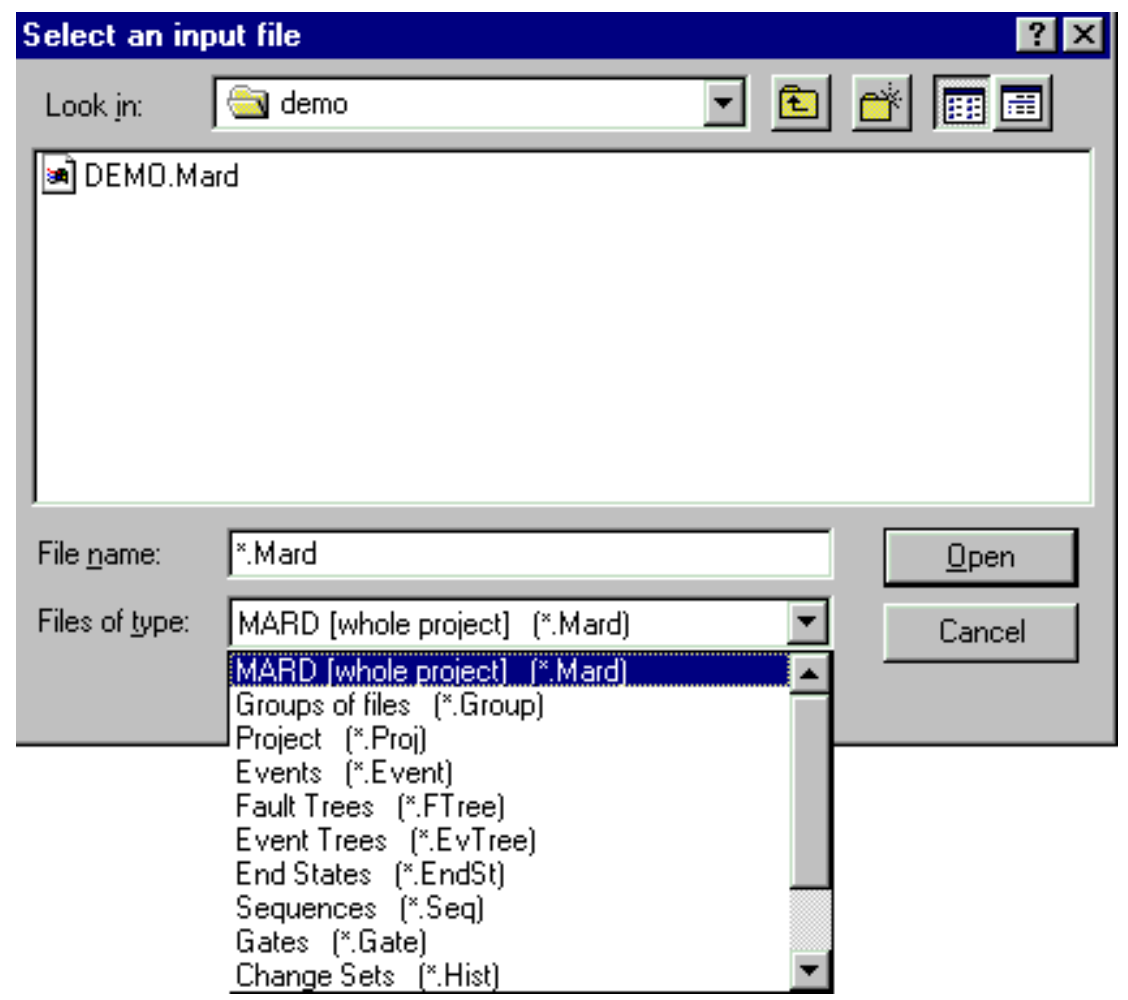

Open - Load the data from the selected ""master" file into the current project database. If successful, the message, "Load complete," will be displayed in the status bar of the SAPHIRE window.

Cancel - Close the Select an input file dialog without loading data.

\subsubsection{Project Information}

\subsubsection{Project Information}

\section{PURPOSE}

Project descriptions, attributes, and text can be loaded into the database or output from it. This information is currently stored in only the MAR D format. Note that each file contains data for only one project, and that a change in the data file project name will not change the database project name. When extracting, each file will contain information for the current project.

\section{STEPS}

1. From the SAPHIRE menu select Utility.

2. Choose Load and Extract from the menu. The Load and Extract Data dialog will be displayed.

3. Select the desired Data Action, MAR-D Data Format, Project Data Type, then the desired File Type. 


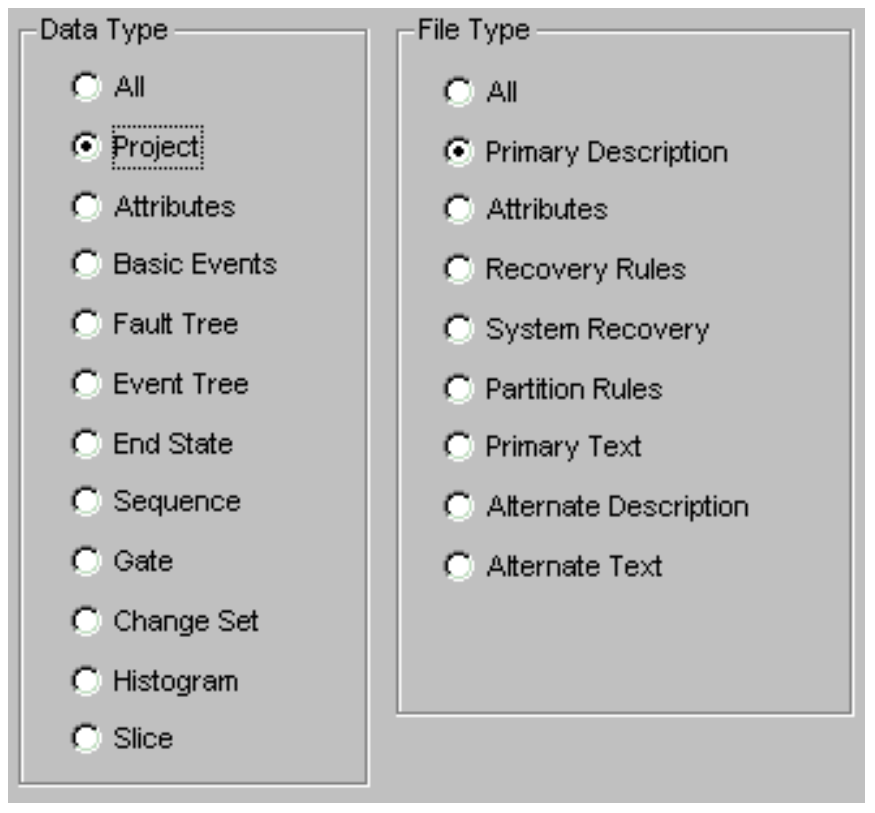

Process - $\quad$ Continue the load or extract operation for the selected Data Type and File Type. Cancel - $\quad$ Close the Load and Extract Data dialog.

\subsubsection{Project Names and Descriptions}

File Name:

xxxxxx.FAD

File Format:

name,description[,A]

where

name 24 character Project name (first 8 characters must be unique).

description $\quad 60$ character Project description

A 1 character If included indicates alternate description

\subsubsection{Project Attribute File}

File Name:

xxxxxx.FAA

File Format:

project $=$

name,mission,newSum,co,loc,type,design,vendor,AE,OpDate,QualDate

where

$\begin{array}{lll}\text { name } & 24 \text { character } & \text { Project name } \\ \text { mission } & \text { Floating point } & \text { Default mission time in hours } \\ \text { newSum } & \text { Floating point } & \text { New sequence frequency sum } \\ \text { co } & 10 \text { character } & \text { Company name } \\ \text { loc } & 16 \text { character } & \text { Location name } \\ \text { type } & 3 \text { character } & \text { Facility type }\end{array}$




$\begin{array}{lll}\text { design } & 10 \text { character } & \text { Facility design } \\ \text { vendor } & 5 \text { character } & \text { Vendor name } \\ \mathrm{AE} & 10 \text { character } & \text { Architectural Engineer } \\ \text { OpDate } & (\text { yyyy } / \mathrm{mm} / \mathrm{dd}) & \text { Operational date } \\ \text { QualDate } & (\text { yyyy } / \mathrm{mm} / \mathrm{dd}) & \text { Qualification date }\end{array}$

\subsubsection{Project Recovery Rules}

File Name:

xxxxxxxx.FAY

File Format:

project $=$

-- recovery rule text --

where

project 24 character Project name

\subsubsection{System Recovery Rules}

File Name:

$\operatorname{xxxxxxxx.FAS}$

File Format:

project $=$

-- recovery rule text --

where

project

24 character

Project name

\subsubsection{Project Partition Rules}

File Name:

xxxxxxxx.FAP

File Format:

project $=$

-- partition rule text --

where

project 24 character Project name

\subsubsection{Project Textual Information}

File Name:

xxxxxx.FAT

File Format:

$$
\begin{aligned}
& \text { Project }[, \mathrm{A}]= \\
& \text {-- text -- }
\end{aligned}
$$

where 
project

A
24 character

1 character
Project name

If included indicates alternate description

\subsubsection{Attributes Information}

\subsubsection{Event Attributes}

\section{PURPOSE}

Basic event attributes descriptions (locations, failure modes, class attributes, system types and component types) can be loaded or extracted using the MAR D Attributes option. Event attributes will be output for the entire project.

\section{STEPS}

1. From the SAPHIRE menu select Utility.

2. Choose Load and Extract from the menu. The Load and Extract Data dialog will be displayed.

3. Select the desired Data Action, MAR-D Data Format, Attributes Data Type, then the desired File Type.

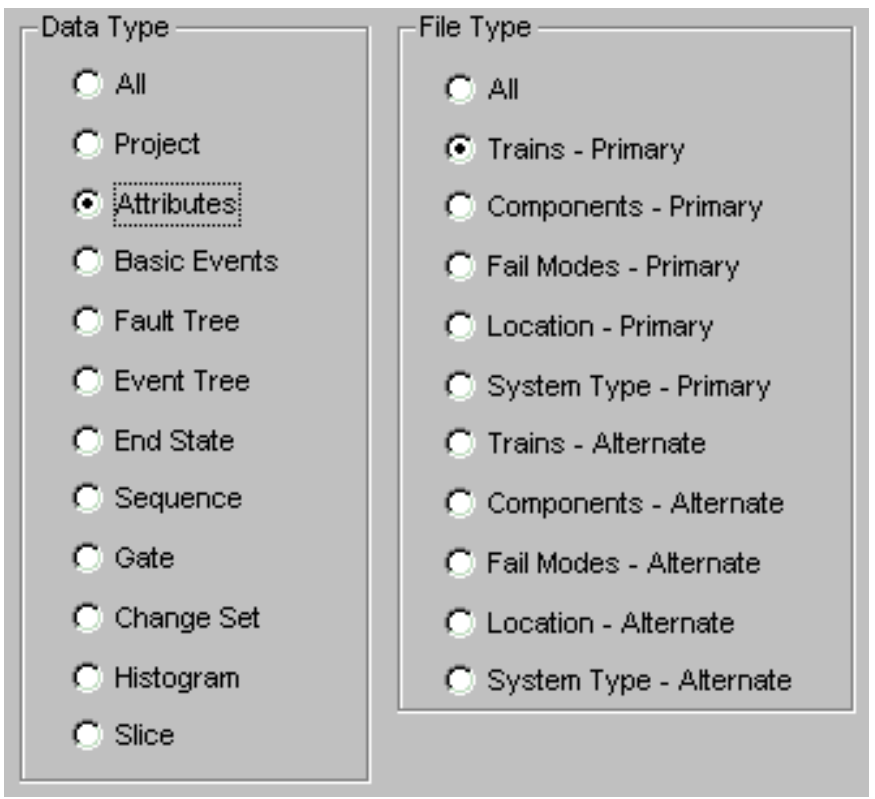

Process - $\quad$ Continue the load or extract operation for the selected Attributes File Type.

Cancel - $\quad$ Close the Load and Extract Data dialog.

\subsubsection{Class Attribute Descriptions}

File Name:

xxxxxx.TTD

File Format:

project $=$ 
attr,altAttr,description[,A]

$\ldots, \ldots$

where

$\begin{array}{lll}\text { project } & 24 \text { character } & \text { Project name } \\ \text { attr } & 5 \text { character } & \text { Class attribute primary name } \\ \text { altAttr } & 5 \text { character } & \text { Class attribute alternate name } \\ \text { description } & 60 \text { character } & \text { Class attribute description } \\ \text { A } & 1 \text { character } & \text { If included indicates alternate description }\end{array}$

\subsubsection{Component Type Descriptions}

File Name:

xxxxxx.CTD

File Format:

project $=$

comp, altComp, description [,A]

$\ldots, \ldots$

where

$\begin{array}{lll}\text { project } & 24 \text { character } & \text { Project name } \\ \text { comp } & 5 \text { character } & \text { Component type primary identifier } \\ \text { altComp } & 5 \text { character } & \text { Component type alternate identifier } \\ \text { description } & 60 \text { character } & \text { Component type description } \\ \text { A } & 1 \text { character } & \text { If included indicates alternate description }\end{array}$

\subsubsection{Failure Mode Descriptions}

File Name:

xxxxxx.FMD

File Format:

project $=$

fail,altFail,description[,A]

where

$\begin{array}{lll}\text { project } & 24 \text { character } & \text { Project name } \\ \text { fail } & 5 \text { character } & \text { Failure mode primary identifier } \\ \text { altFail } & 5 \text { character } & \text { Failure mode alternate identifier } \\ \text { description } & 60 \text { character } & \text { Failure mode description } \\ \text { A } & 1 \text { character } & \text { If included indicates alternate description }\end{array}$

\subsubsection{Location Descriptions}

File Name:

xxxxxx.LCD 
File Format:

project $=$

loc,altLoc,description[,A]

$\ldots, \ldots$

where

$\begin{array}{lll}\text { project } & 24 \text { character } & \text { Project name } \\ \text { loc } & 5 \text { character } & \text { Location primary identifier } \\ \text { altLoc } & 5 \text { character } & \text { Location alternate identifier } \\ \text { description } & 60 \text { character } & \text { Location description } \\ \text { A } & 1 \text { character } & \text { If included indicates alternate description }\end{array}$

\subsubsection{System Type Descriptions}

File Name:

xxxxxx.STD

File Format:

project $=$

sys,altSys, description[,A]

$\ldots, \ldots$

where

$\begin{array}{lll}\text { project } & 24 \text { character } & \text { Project name } \\ \text { sys } & 5 \text { character } & \text { System primary identifier } \\ \text { altSys } & 5 \text { character } & \text { System alternate identifier } \\ \text { description } & 60 \text { character } & \text { System description } \\ \text { A } & 1 \text { character } & \text { If included indicates alternate description }\end{array}$

\subsubsection{Basic Event Information}

\subsubsection{Basic Event Information}

\section{PURPOSE}

Event descriptions, failure rates, attributes, and transformations can be loaded into the database or extracted from it. When loading, the alternate name of the event defaults to the value of the primary name if an alternate name is not specified.

When extracting, if the Use Alternate Basic Event Names box is checked in the Utility | Define Constants | Analysis option, then the alternate name will be used instead of the primary name for descriptions and failure rates. Primary names will be output.

\section{STEPS}

1. From the SAPHIRE menu select Utility.

2. Choose Load and Extract from the menu. The Load and Extract Data dialog will be displayed. 
3. Select the desired Data Action, MAR-D Data Format, Basic Events Data Type, then the File Type.

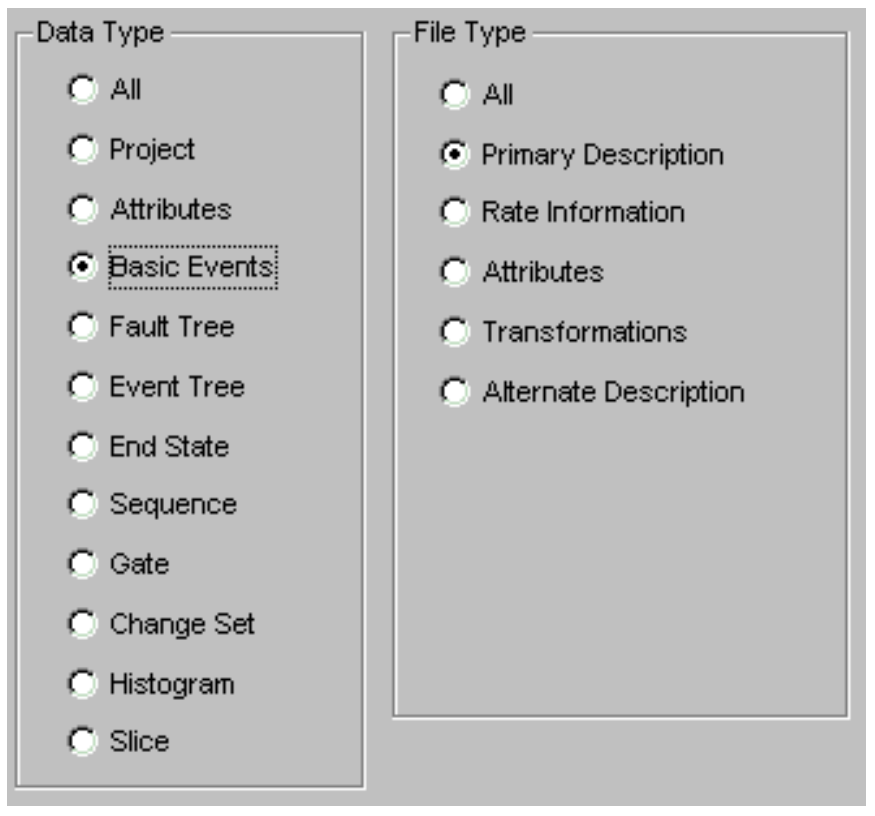

Process - $\quad$ Continue the load or extract operation for the selected Basic Events File Type. Cancel - $\quad$ Close the Load and Extract Data dialog.

\subsubsection{Basic Event Names and Descriptions}

File Name:

xxxxxx.BED

File Format:

project $=$

name,description[,A]

where

$\begin{array}{lll}\text { project } & 24 \text { character } & \text { Project name } \\ \text { name } & 24 \text { character } & \text { Event primary name } \\ \text { description } & 60 \text { character } & \text { Alphanumeric description } \\ \text { A } & 1 \text { character } & \text { If included indicates alternate description }\end{array}$

\subsubsection{Basic Event Failure Rate Information}

The basic event failure rates are stored in the Event relation.

File Name:

xxxxxx.BEI

File Format: 
project $=$

name, calc, udC, udT, udV, prob, lambda, tau, mission, init, Flag, udV2

where

\begin{tabular}{|c|c|c|}
\hline Project & 24 character & Project name \\
\hline Name & 24 character & Basic event name \\
\hline Calc & 1 character & Calculation type \\
\hline 1 & \multicolumn{2}{|c|}{ Probability } \\
\hline 2 & \multicolumn{2}{|c|}{ Lambda * Mission Time } \\
\hline 3 & \multicolumn{2}{|c|}{$1 \operatorname{Exp}($ Lambda $*$ Mission Time $)$} \\
\hline 4 & \multicolumn{2}{|c|}{ Lambda $*$ Min(Mission Time, Tau) } \\
\hline 5 & \multicolumn{2}{|c|}{ Operating component with full repair } \\
\hline 6 & \multicolumn{2}{|c|}{ Lambda * Tau / 2.0} \\
\hline 7 & \multicolumn{2}{|c|}{$1+(\operatorname{EXP}($ Lambda*Tau $) 1.0) /($ Lambda*Tau $)$} \\
\hline 8 & \multicolumn{2}{|c|}{ Base Probability $*$ Probability } \\
\hline 9 & \multicolumn{2}{|c|}{ Base Probability * Probability } \\
\hline $\mathrm{T}$ & \multicolumn{2}{|c|}{ Set to House Event (Failed, Prob=1.0) } \\
\hline $\mathrm{F}$ & \multicolumn{2}{|c|}{ Set to House Event (Successful,Prob=0.0) } \\
\hline I & \multicolumn{2}{|c|}{ Set to ignore } \\
\hline $\mathrm{S}$ & \multicolumn{2}{|c|}{ Use fault tree mincut upperbound } \\
\hline G & \multicolumn{2}{|c|}{ Seismic event - Enter g level for screening } \\
\hline M & \multicolumn{2}{|c|}{ Seismic event - Use medium site hazard curve for screening } \\
\hline $\mathrm{UdC}$ & 4 characters & $\begin{array}{l}\text { Uncertainty correlation class } \\
\text { Events in same class are } 100 \% \text { correlated. }\end{array}$ \\
\hline \multirow[t]{10}{*}{$\mathrm{UdT}$} & 1 character & Uncertainty distribution type \\
\hline & \multicolumn{2}{|c|}{ Log normal, error factor } \\
\hline & \multicolumn{2}{|c|}{ Normal, standard deviation } \\
\hline & \multicolumn{2}{|c|}{ Beta, $b$ of $\operatorname{Beta}(a, b)$} \\
\hline & \multicolumn{2}{|c|}{ Gamma, a Gamma(a) } \\
\hline & \multicolumn{2}{|c|}{ Chi-squared, degrees of freedom } \\
\hline & \multicolumn{2}{|c|}{ Exponential, none } \\
\hline & \multicolumn{2}{|c|}{ Uniform, Upper end pt. } \\
\hline & \multicolumn{2}{|c|}{ Histogram } \\
\hline & \multicolumn{2}{|c|}{ Maximum entropy } \\
\hline
\end{tabular}

$\begin{array}{lll}\text { UdV } & \text { Floating point } & \text { Uncertainty distribution value } \\ \text { Prob } & \text { Floating point } & \text { Probability value } \\ \text { Lambda } & \text { Floating point } & \text { Basic event failure rate per hr. } \\ \text { Tau } & \text { Floating point } & \text { Time to repair in hours } \\ \text { Mission } & \text { Floating point } & \text { Mission time } \\ \text { init } & \text { Boolean } & \text { Initiating event flag }(\mathrm{Y} / \mathrm{N}) \\ \text { Flag } & \text { 1-character } & \text { process flag }\end{array}$


udV2

Floating point

Uncertainty distribution value \#2

\section{General Rules:}

The name field is mandatory.

\subsubsection{Basic Event Attribute Codes}

Basic event attributes are stored in the Event relation.

File Name:

xxxxxx.BEA

File Format:

project $=$

name,Aname,type,sys,fail,loc,compID,Gname,train,att1,..,att16

where

$\begin{array}{lll}\text { project } & 24 \text { character } & \text { Project name } \\ \text { name } & 24 \text { character } & \text { Event name } \\ \text { Aname } & 24 \text { character } & \text { Alternate event name } \\ \text { type } & 3 \text { character } & \text { Event component type } \\ \text { sys } & 3 \text { character } & \text { Event component system } \\ \text { fail } & 3 \text { character } & \text { Failure mode } \\ \text { loc } & 3 \text { character } & \text { Component location } \\ \text { compID } & 7 \text { character } & \text { Component ID } \\ \text { Gname } & 24 \text { character } & \text { Event group identifier } \\ \text { train } & 3 \text { character } & \text { Train identifier } \\ \text { att1..att16 } & \text { Class attribute } & 16 \text { values of Y or N (yes or no) indicate whether } \\ & \text { flags } & \text { the attribute described in the class attribute file is } \\ & & \text { applicable. }\end{array}$

\section{General Rules:}

The name field is mandatory.

\subsubsection{Basic Event Transformations}

Basic event attributes are stored in the Event relation.

Site Name:

xxxxxx.BET

File Format:

project $=$

name1,level,type

bename1, bename2, ..., 
..., benameN

${ }^{\wedge} \mathrm{EOS}$

name2,level,type

bename1, bename $2, \ldots$,

$\ldots$, benameN

${ }^{\wedge} \mathrm{EOS}$

where

$\begin{array}{lll}\text { project } & 24 \text { character } & \text { Project name } \\ \text { name } & 24 \text { character } & \text { Event name } \\ \text { Type } & 4 \text { character } & \text { Transformation type } \\ \text { level } & 3 \text { character } & \text { Transformation level } \\ \text { bename1..N } & 24 \text { character } & \text { Event name }\end{array}$

\subsubsection{Fault Tree Information}

\subsubsection{Fault Tree Information}

\section{PURPOSE}

Fault tree descriptions, graphics, logic, cut sets, attributes, text and piping and instrumentation diagrams (P\&IDs) can be loaded into the database or output to files.

Fault tree cut sets and attributes data can be loaded into either the base case (permanent) or current (temporary) field areas. MAR-D database applications will normally use the base case load option because only permanent data should be loaded into the database. Analysis software such as SAPHIRE will use current case fields for comparisons of changed value results. Any basic event found within the fault tree logic or cut sets will be added to the Event relation.

For some of the File Types, if more than one of the fault trees are selected for extraction, the File Output dialog is displayed, allowing you to choose the manner in which files are created for output.

\section{STEPS}

1. From the SAPHIRE menu select Utility.

2. Choose Load and Extract from the menu. The Load and Extract Data dialog will be displayed.

3. Select the desired Data Action, MAR-D Data Format, Fault Tree Data Type, then the desired File Type. 


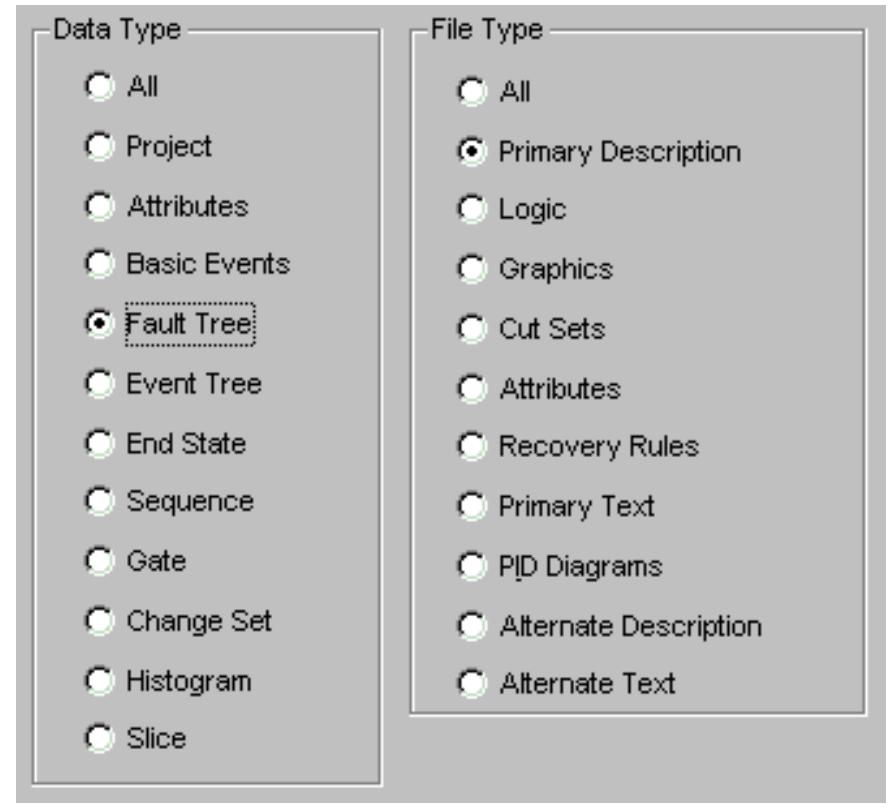

Process - $\quad$ Continue the load or extract operation for the selected Fault Tree File Type. Cancel Close the Load and Extract Data dialog. 


\subsubsection{File Output Dialog}

\section{PURPOSE}

This option provides you with the opportunity to choose the method for extracting multiple fault trees, event trees, or end states.

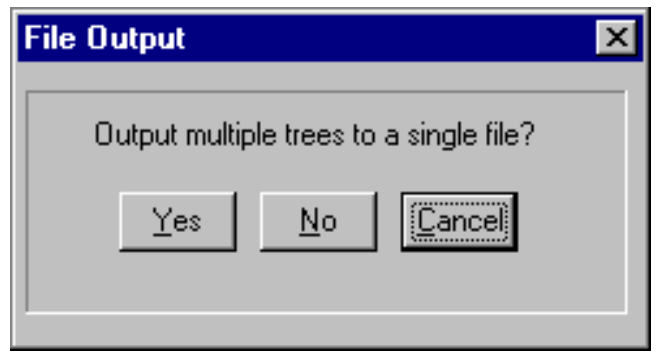

Yes - $\quad$ The output for all selected list items will be saved in one file, with the items separated by ${ }^{\wedge}$ EOS. The Get Output Destination dialog will be displayed.

No -

Cancel - Close the File Output dialog without extracting data.

\subsubsection{Fault Tree Logic Options}

\section{PURPOSE}

This option provides you with the opportunity to choose the way the logic will be extracted. 


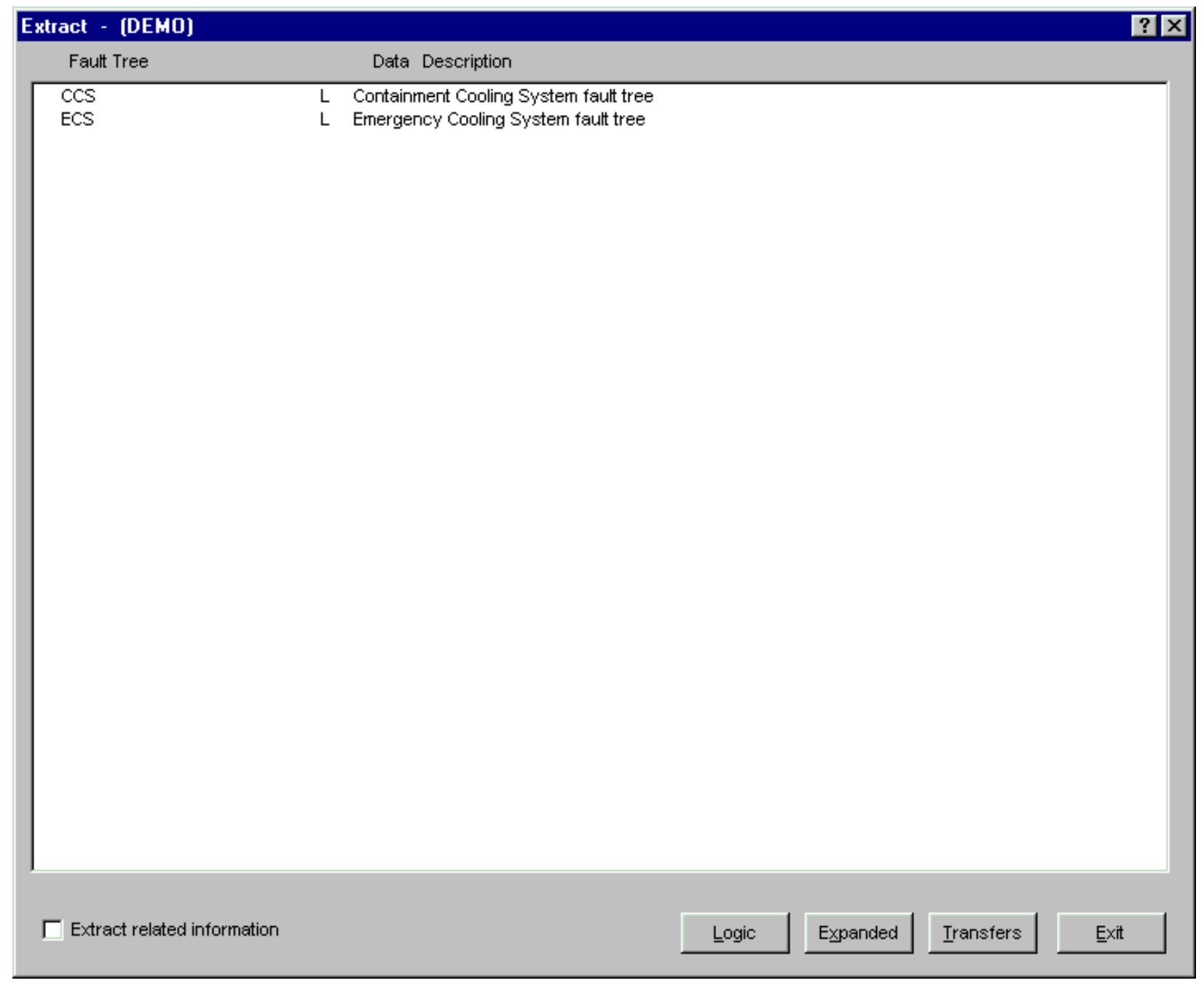

When you choose a Fault Tree Logic extraction method, either the Get Output Destination or the Select Directory dialog will be displayed. The File Output dialog may also be displayed.

Extract related information - When this is checked, related basic event and gate information for the selected fault trees are also extracted into their respective MAR-D file formats. A .Group master file is created, which contains a list of all files created. This .Group file can be used to

Logic -

Expanded -

Transfers load all of the related information back into SAPHIRE at once. Extract the logic "as is" for all selected items. Transfer gates will not be expanded.

Extract the expanded logic for all selected items. All transfer gates will be replaced with the complete transfer information. Paging information will be lost.

Extract the logic for all selected items, preserving transfer gates, but automatically extracting the transfer fault trees, whether they were explicitly marked or not. This option is a hybrid of the Logic and Expanded options. It both preserves paging information, and includes the transfer information. 
Exit data.
Close the Extract Fault Trees dialog without extracting 


\subsubsection{Fault Tree Names and Descriptions}

File Name:

xxxxxx.FTD

File Format:

project $=$

name,description[,s][,A]

$\ldots, \ldots$

where

$\begin{array}{lll}\text { project } & 24 \text { character } & \text { Project name } \\ \text { name } & 24 \text { character } & \text { Fault tree primary name } \\ \text { description } & 60 \text { character } & \text { Fault tree description } \\ \mathrm{s} & 1 \text { character } & \text { If included indicates fault tree is a sub-tree } \\ \mathrm{A} & 1 \text { character } & \text { If included indicates alternate description }\end{array}$

\subsubsection{Fault Tree Logic}

Fault tree logic is stored in the block data file of the System relation.

File Name:

xxxxxx.FTL

File Format:

project, fault tree $=$

* gatename1,description

gatename1 gatetype input 1 input $2 \ldots$ inputn

$\ldots \ldots \ldots \ldots$

* gatenamen,description

gatenamen gatetype input 1 input $2 \ldots$ inputn

where

\begin{tabular}{|c|c|c|c|}
\hline \multirow{4}{*}{\multicolumn{2}{|c|}{$\begin{array}{l}\text { project } \\
\text { fault tree } \\
\text { gatename } \\
\text { gatetype }\end{array}$}} & 24 character & Project name \\
\hline & & 24 character & Fault tree name \\
\hline & & 24 character & Gate name \\
\hline & & 4 character & Gate type \\
\hline & AND & & logical AND \\
\hline & OR & & logical OR \\
\hline & TBL & & table of events \\
\hline & TRAN & & transfer followed by a 24 -character fault tree name \\
\hline & NAND & & logical NOT AND \\
\hline & NOR & & logic NOT OR \\
\hline & $\mathrm{N} / \mathrm{M}$ & & $\mathrm{N}$ out of $\mathrm{M}$ logic gate \\
\hline
\end{tabular}


CONT

input

description
24 character

60 character continuation of inputs to the previous gate inputs to the gate (event or gate names) gate name descriptions included as comment

\section{General Rules:}

A gate definition cannot exceed 255 characters.

A line beginning with an asterisk $(*)$ is a comment.

For each gate name a comment should be included giving the gate description.

\subsubsection{Fault Tree Graphics}

Fault tree graphics are stored in the block data file of the System relation. The MAR D file (.DLS) is a display list sequence for the graphics in a binary format. It is loaded and output as is with no conversion performed.

File Name:

xxxxxx.DLS

File Format:

IRRAS 2.5/4.0/5.0, SAPHIRE 6.0 and 7.0 Fault Tree Graphics file (DLS format)

\subsubsection{Fault Tree Cut Sets}

The fault tree cut sets are stored in the System relation in the block data file.

File Name:

xxxxx.FTC

File Format:

project, fault tree, analysis $=$

eventname $*$ eventname +

eventname $*$ eventname $*$ eventname *

eventname +

eventname * eventname.

${ }^{\wedge} \mathrm{EOS}$

project, fault tree $2=$

where

\begin{tabular}{|c|c|c|c|}
\hline project & 24 character & Project name & \\
\hline fault tree & 24 character & Fault tree name & \\
\hline analysis & 1 character & Analysis type & \\
\hline 1 & & & Random \\
\hline 2 & & & Fire \\
\hline 3 & & & Flood \\
\hline 4 & & & Seismic \\
\hline
\end{tabular}


5 through 8

9 through 16

eventname
Reserved

user-defined

\section{General Rules:}

An asterisk (*) separates cut set events. Spaces are ignored.

A plus sign (+) separates cut sets.

A period (.) denotes the end of a sequence.

A slash (/) precedes complemented events.

Event names are a maximum of 4 characters including the "/".

A line beginning with an asterisk $(*)$ is a comment.

\subsubsection{Fault Tree Attributes}

File Name:

xxxxx.FTA

File Format:

project, analysis $=$

name,level,mission,mincut,proCut,sample,seed,sizCut,sys, cuts, events, value 1,..,value9

where

\begin{tabular}{|c|c|c|c|}
\hline project & 24 character & Project name & \\
\hline analysis & 1 character & Analysis type & \\
\hline 1 & & & Random \\
\hline 2 & & & Fire \\
\hline 3 & & & Flood \\
\hline 4 & & & Seismic \\
\hline 5 through 8 & & & Reserved \\
\hline 9 through 16 & & & user-defined \\
\hline name & 24 character & Fault tree name & \\
\hline level & Integer 2 & $0=$ top level tree & \\
\hline mission & $\begin{array}{l}\text { Floating } \\
\text { point }\end{array}$ & Mission time & \\
\hline mincut & $\begin{array}{l}\text { Floating } \\
\text { point }\end{array}$ & Mincut upper bound & \\
\hline proCut & $\begin{array}{l}\text { Floating } \\
\text { point }\end{array}$ & Probability cut off value & \\
\hline sample & Integer 4 & Sample size & \\
\hline seed & Integer 8 & Random number seed & \\
\hline sizecut & Integer 2 & Size cut off value & \\
\hline
\end{tabular}




$\begin{array}{lll}\text { sys } & 3 \text { character } & \text { System identifier } \\ \text { cuts } & \text { Integer } 5 & \text { Base number of cut sets } \\ \text { events } & \text { Integer } 5 & \text { Base number of events } \\ \text { value } & \begin{array}{l}\text { Floating } \\ \text { point }\end{array} & \text { Base uncertainty values } \\ & \text { r } & \end{array}$

\subsubsection{Fault Tree Recovery Rules}

File Name:

$\operatorname{xxxxxxxx.FTY~}$

File Format:

$$
\begin{aligned}
& \text { project }= \\
& \text {-- recovery rule text -- }
\end{aligned}
$$

where

$$
\text { project 24 character Project name }
$$

\subsubsection{Fault Tree Textual Information}

File Name:

xxxxxx.FTT

File Format:

project, fault tree $[, \mathrm{A}]=$

-- text --

${ }^{\wedge} \mathrm{EOS}$

project, fault tree $2=$

where

project

fault tree

A
24 character

24 character

1 character
Project name

Fault tree name

If included indicates alternate text

\subsubsection{Fault Tree Graphical P\&ID}

File Name:

$\operatorname{xxxxxxxx.PID}$

File Format:

IRRAS 4.0/5.0, SAPHIRE 6.0 P\&ID Graphics file (PID Format)

\subsubsection{Event Tree Information}

\subsubsection{Event Tree Information}




\section{PURPOSE}

Event tree descriptions, graphics, logic, attributes, rules, and text can be loaded into the database or output from it.

For some of the File Types, if more than one of the event trees are selected for extraction, the File Output dialog is displayed, allowing you to choose the manner in which files are created for output.

\section{STEPS}

1. From the SAPHIRE menu select Utility.

2. Choose Load and Extract from the menu. The Load and Extract Data dialog will be displayed.

3. Select the desired Data Action, MAR-D Data Format, Event Tree Data Type, then the desired File Type.

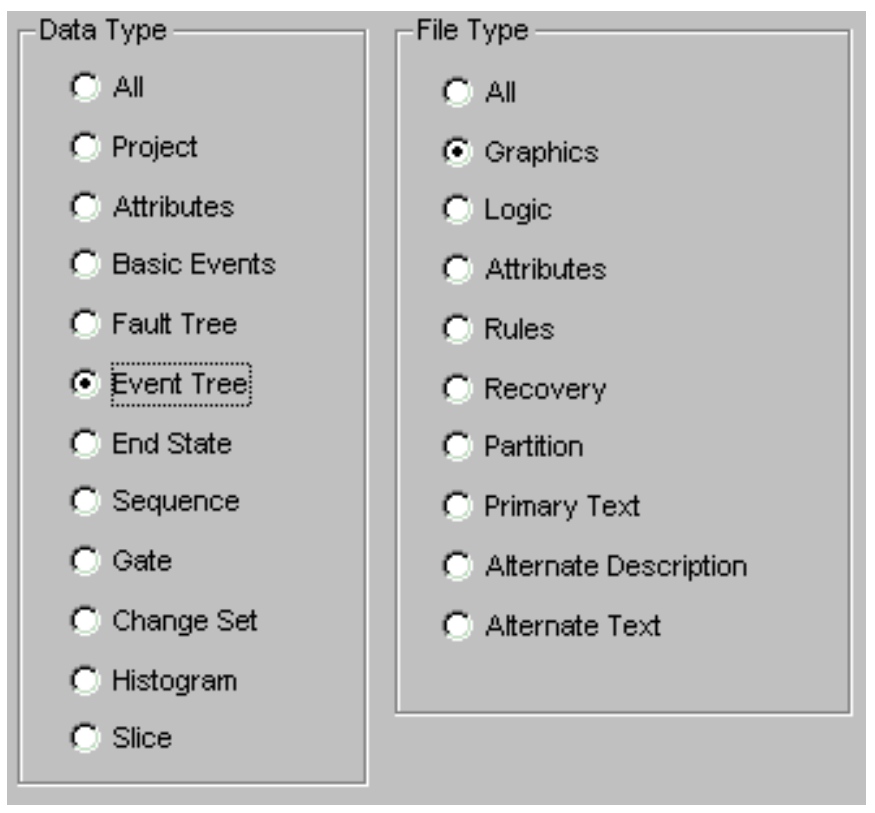

Process - Continue the load or extract operation for the selected Event Tree File Type.

Cancel - Close the Load and Extract Data dialog.

\subsubsection{File Output Dialog}

\section{PURPOSE}

This option provides you with the opportunity to choose the method for extracting multiple fault trees, event trees, or end states. 


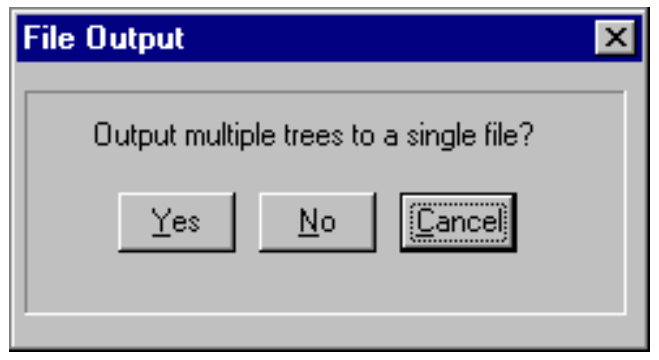

Yes - $\quad$ The output for all selected list items will be saved in one file, with the items separated by ${ }^{\wedge}$ EOS. The Get Output Destination dialog will be displayed.

No -

Cancel - Close the File Output dialog without extracting data.

\subsubsection{Event Tree Names and Descriptions}

File Name:

xxxxxx.ETD

File Format:

project $=$

name,description[,s][,A]

$\ldots, \ldots$

where

$\begin{array}{lll}\text { Project } & 24 \text { character } & \text { Project name } \\ \text { Name } & 24 \text { character } & \text { Event tree name } \\ \text { Description } & 60 \text { character } & \text { Event tree description } \\ \mathrm{S} & 1 \text { character } & \text { If included indicates event tree is a fault tree } \\ \mathrm{A} & 1 \text { character } & \text { If included indicates alternate description }\end{array}$

\subsubsection{Event Tree Graphics}

The SAPHIRE Event Tree Graphics file (*.ETG) is a display list sequence for the graphics. Its format and contents are the same as the Event Tree Logic File.

File Name:

xxxxxx.ETG

File Format:

See file format for the Event Tree Logic

\subsubsection{Event Tree Logic}

File Name:

xxxxxx.ETL

File Format:

project, event tree, init event $[, \mathrm{T}]=$

$\wedge^{\wedge}$ TOPS 
$* 1|2| 3|4| 5 \mid$ this is a comment

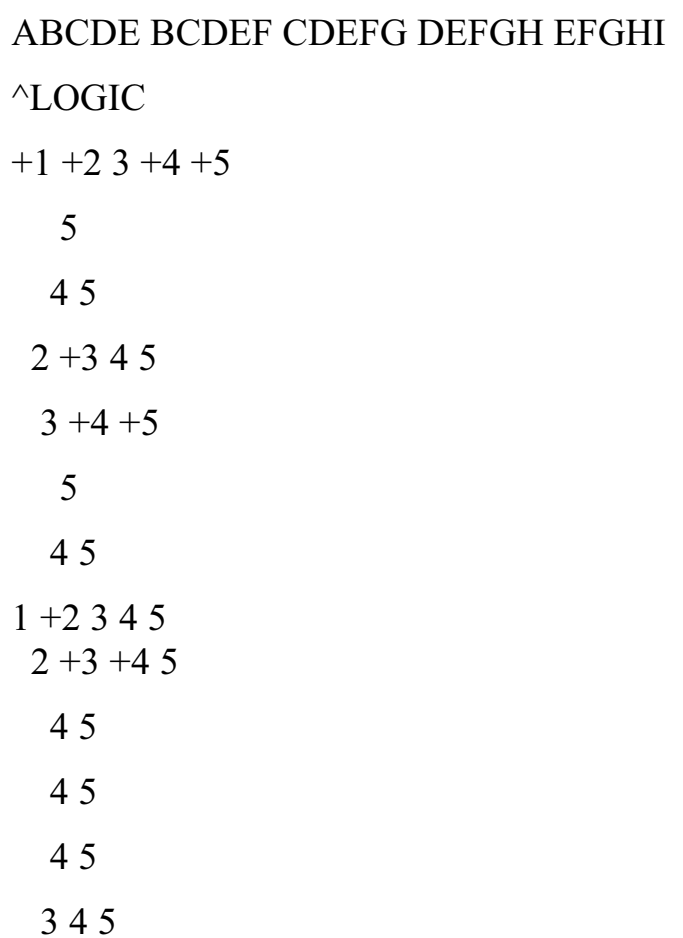

\section{${ }^{\wedge}$ SEQUENCES}

$\mathrm{Y} / \mathrm{N}$, header\#1,

$\mathrm{Y} / \mathrm{N}$, sequence\#1,

$\mathrm{Y} / \mathrm{N}$, header $\# 2$,

$\mathrm{Y} / \mathrm{N}$, end state\#1,

$\mathrm{Y} / \mathrm{N}$, sequence\#2,

$\mathrm{Y} / \mathrm{N}$, sequence\#3,

$\mathrm{Y} / \mathrm{N}$, sequence\#4,

$\mathrm{Y} / \mathrm{N}$, sequence\#5,

$\mathrm{Y} / \mathrm{N}$, sequence\#6,

$\mathrm{Y} / \mathrm{N}$, sequence\#7,

$\mathrm{Y} / \mathrm{N}$, sequence\#8,

$\mathrm{Y} / \mathrm{N}$, sequence\#9,

$\mathrm{Y} / \mathrm{N}$, sequence\#10,

$\mathrm{Y} / \mathrm{N}$, sequence\#11,

$\mathrm{Y} / \mathrm{N}$, sequence\#12,

$\mathrm{Y} / \mathrm{N}$, sequence\#13,

${ }^{\wedge}$ TEXT

SIZE $s$

JUST $\mathrm{j}$

COLOR $\mathrm{j}$

$\mathrm{XY}$ xvalue,yvalue

"60 character line of text"
$\mathrm{Y} / \mathrm{N}$, end state\#2,

$\mathrm{Y} / \mathrm{N}$, end state\#3, $\mathrm{Y} / \mathrm{N}$, end state\#4,

$\mathrm{Y} / \mathrm{N}$, end state $\# 5$, $\mathrm{Y} / \mathrm{N}$, end state\#6, $\mathrm{Y} / \mathrm{N}$, end state\#7, $\mathrm{Y} / \mathrm{N}$, end state\#8, $\mathrm{Y} / \mathrm{N}$, tran file\#9, $\mathrm{Y} / \mathrm{N}$, end state\#10, $\mathrm{Y} / \mathrm{N}$, end state\#11, $\mathrm{Y} / \mathrm{N}$, end state\#12, $\mathrm{Y} / \mathrm{N}$, end state\#13,
Y/N, header\#3, $\quad \mathrm{Y} / \mathrm{N}$, header\#4 $\mathrm{Y} / \mathrm{N}, \mathrm{xdata} 1 \# 1, \quad \mathrm{Y} / \mathrm{N}, \mathrm{xdata} \# 1$ $\mathrm{Y} / \mathrm{N}, \mathrm{xdata} 1 \# 2, \quad \mathrm{Y} / \mathrm{N}, \mathrm{xdata} 2 \# 2$ $\mathrm{Y} / \mathrm{N}, \mathrm{xdata} 1 \# 3, \quad \mathrm{Y} / \mathrm{N}, \mathrm{xdata} 2 \# 3$ $\mathrm{Y} / \mathrm{N}$, xdata1\#4, $\mathrm{Y} / \mathrm{N}, \mathrm{xdata} 2 \# 4$ $\mathrm{Y} / \mathrm{N}, \mathrm{xdata} 1 \# 5, \quad \mathrm{Y} / \mathrm{N}, \mathrm{xdata} 2 \# 5$ $\mathrm{Y} / \mathrm{N}, \mathrm{xdata} 1 \# 6, \quad \mathrm{Y} / \mathrm{N}, \mathrm{xdata} 2 \# 6$ $\mathrm{Y} / \mathrm{N}, \mathrm{xdata} 1 \# 7, \quad \mathrm{Y} / \mathrm{N}, \mathrm{xdata} 2 \# 7$ $\mathrm{Y} / \mathrm{N}, \mathrm{xdata} \# 8, \quad \mathrm{Y} / \mathrm{N}, \mathrm{xdata} 2 \# 8$ $\mathrm{Y} / \mathrm{N}, \mathrm{xdata} 1 \# 9, \quad \mathrm{Y} / \mathrm{N}, \mathrm{xdata} 2 \# 9, \mathrm{~T}$ $\mathrm{Y} / \mathrm{N}, \mathrm{xdata} 1 \# 10, \quad \mathrm{Y} / \mathrm{N}, \mathrm{xdata} 2 \# 10$ $\mathrm{Y} / \mathrm{N}$, xdata1\#11, $\mathrm{Y} / \mathrm{N}, \mathrm{xdata}$ 2\#11 $\mathrm{Y} / \mathrm{N}, \mathrm{xdata}$ \#12, $\mathrm{Y} / \mathrm{N}, \mathrm{xdata} 2 \# 12$ $\mathrm{Y} / \mathrm{N}, \mathrm{xdata}$ \#13, $\mathrm{Y} / \mathrm{N}, \mathrm{xdata}$ 2\#13 
XY xvalue, yvalue

"60 character line of text"

"60 character line of text"

$\wedge$ PARMS

START yvalue

WINDOW x1,y1,x2,y2

HEADER $\mathrm{x} 1, \mathrm{x} 2, \mathrm{x} 3, \mathrm{x} 4$

${ }^{\wedge} \mathrm{EOS}$

project, event tree $2=$

(additional event trees)

where

\begin{tabular}{|c|c|c|}
\hline project & 24 character & Project name \\
\hline name & 24 character & Event tree name \\
\hline init event & 24 character & Initiating Event \\
\hline$[, \mathrm{T}]$ & 1 character & $\begin{array}{l}\text { Optional flag indicating init event name is a Top } \\
\text { event fault tree }\end{array}$ \\
\hline TOPS & 24 character & Top event/fault tree names \\
\hline $\mathrm{Y} / \mathrm{N}$ & Boolean & End state text displayed? \\
\hline header & 24 character & Sequence header \\
\hline sequence & 24 character & Sequence name \\
\hline endstate & 24 character & End state name \\
\hline tran file & 24 character & Name of transfer file \\
\hline xdata1 & 24 character & Information (optional) \\
\hline xdata2 & 24 character & Information (optional) \\
\hline
\end{tabular}

\section{General Rules:}

A line beginning with an asterisk $(*)$ is a comment.

Literal "^TOPS", "^LOGIC", "^SEQUENCES" labels must be present.

Logic is built according to the position of the top event in the definition.

Plus sign (+)---the specified top event succeeded.

Minus sign ( )---the specified top event failed.

Blank ( )---the response of the indicated top event did not matter.

Header, Sequence name, End State name, Xdata1, Xdata fields associated with each sequence. "Y/N" indicates whether the specified field is visible. A "T" at the end indicates the sequence transfers to another tree.

User text is input following the ${ }^{\wedge}$ TEXT command. Parameters include the size, justification, color, and location of the text block.

The ^PARMS command allows input of program control parameters. 


\subsubsection{Sample Graphical Event Tree}

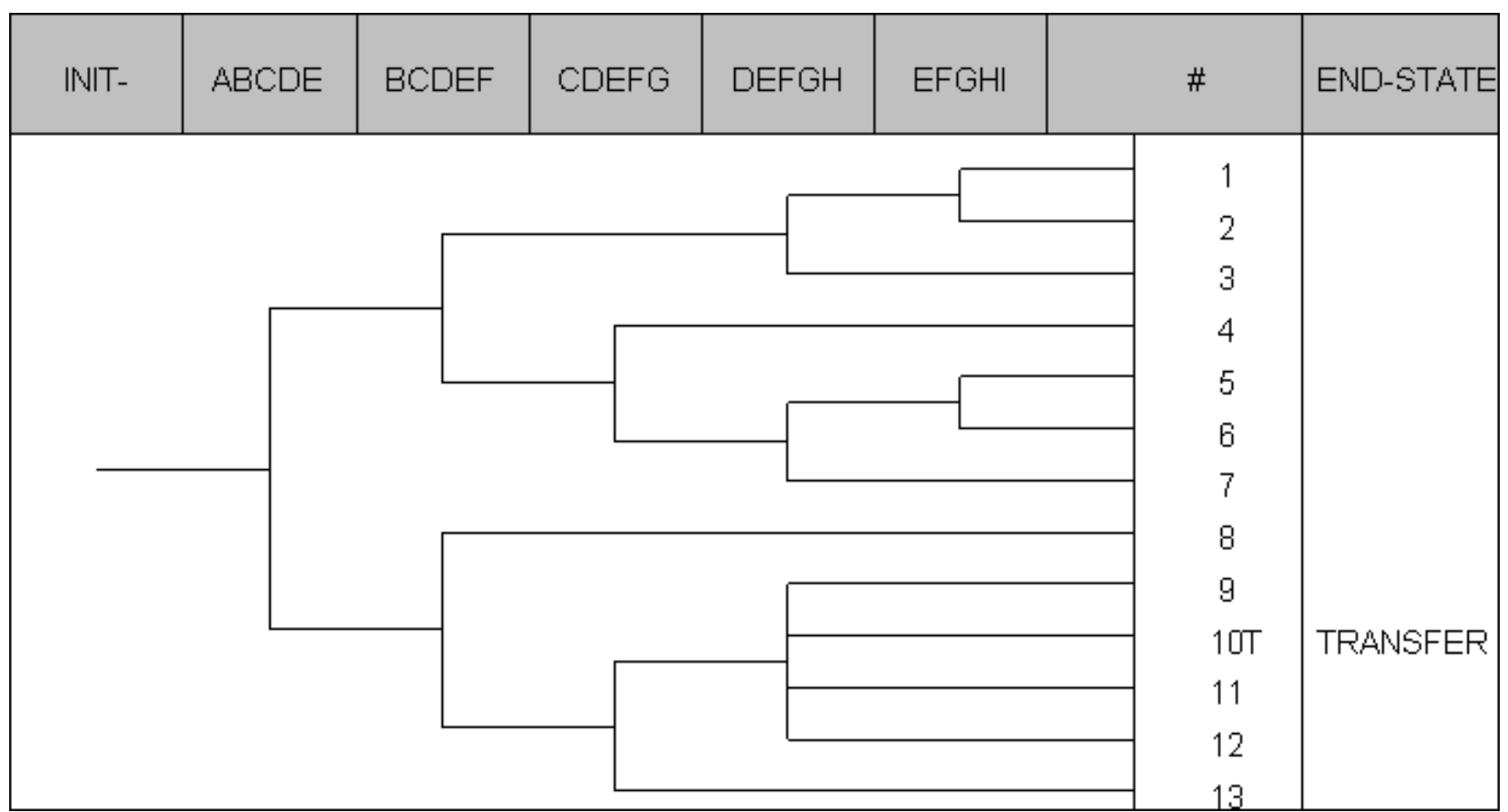

\subsubsection{Event Tree Attributes}

File Name:

xxxxxx.ETA

File Format:

project $=$

name,init

where

$\begin{array}{lll}\text { project } & 24 \text { character } & \text { Project name } \\ \text { name } & 24 \text { character } & \text { Event tree name } \\ \text { init event } & 24 \text { character } & \text { Initiating Event }\end{array}$

\subsubsection{Event Tree Rules}

File Name:

xxxxxxxx.ETR

File Format:

project, event tree $=$

-- event tree rule text 


\section{${ }^{\wedge} \mathrm{EOS}$}

project, event tree 2

where:

$\begin{array}{lll}\text { project } & 24 \text { character } & \text { Project name } \\ \text { name } & 24 \text { character } & \text { Event tree name } \\ \text { tops } & 24 \text { character } & \text { Top event/fault tree names }\end{array}$

\subsubsection{Event Tree Recovery Rules}

File Name:

xxxxxxxx.ETY

File Format:

project, event tree $=$

-- recovery rule text --

$\wedge^{\wedge} \mathrm{EOS}$

project, event tree $2=$

where
project
24 character
Project name
event tree
24 character
Event tree name

\subsubsection{Event Tree Partition Rules}

File Name:

xxxxxxxx.ETP

File Format:

project, event tree $=$

-- partition rule text --

${ }^{\wedge} \mathrm{EOS}$

project, event tree $2=$

where

$\begin{array}{lll}\text { project } & 24 \text { character } & \text { Project name } \\ \text { event tree } & 24 \text { character } & \text { Event tree name }\end{array}$

\subsubsection{Event Tree Textual Information}

File Name:

xxxxxx.ETT

File Format:

project, event tree [,A] $=$

-- text --

${ }^{\wedge} \mathrm{EOS}$

project, event tree $2=$ 
where
project
24 character
Project name
event tree
24 character
Event tree name
A
1 character
If included indicates alternate description

\subsubsection{End State Information}

\subsubsection{End State}

\section{PURPOSE}

End state names, descriptions, cut sets, and text can be loaded into the database or output from it. Each sequence can be tied to a single plant damage state. The cut sets for a sequence can be partitioned to map to separate end state. The name and description data are loaded with the *.PDS file.

For some of the File Types, if more than one of the end states are selected for extraction, the File Output dialog is displayed, allowing you to choose the manner in which files are created for output.

\section{STEPS}

1. From the SAPHIRE menu select Utility.

2. Choose Load and Extract from the menu. The Load and Extract Data dialog will be displayed.

3. Select the desired Data Action, MAR-D Data Format, End State Data Type, then the desired File Type.

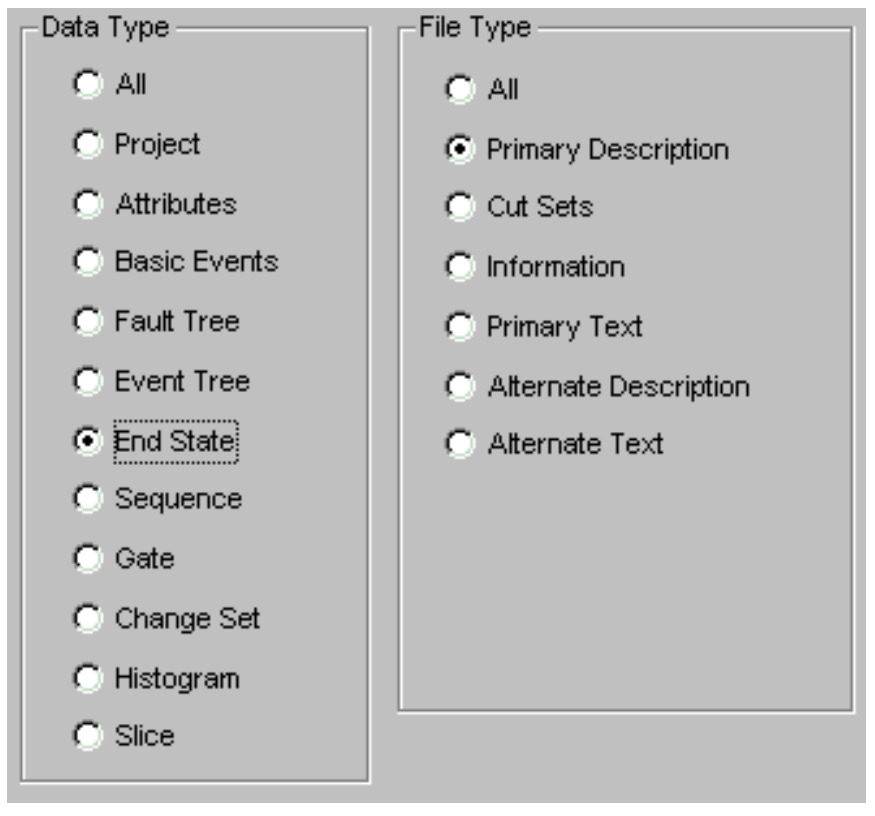

Process - Continue the load or extract operation for the selected End State File Type.

Cancel - Close the Load and Extract Data dialog. 


\subsubsection{End State Names and Descriptions}

File Name:

xxxxxx.ESD

File Format:

project $=$

name,description[,A]

$\ldots, \ldots$

where

$\begin{array}{lll}\text { project } & 24 \text { character } & \text { Project primary name } \\ \text { name } & 24 \text { character } & \text { End state primary name } \\ \text { description } & 60 \text { character } & \text { End state description } \\ \text { A } & 1 \text { character } & \text { If included indicates alternate description }\end{array}$

\subsubsection{End State Cut Sets}

The end state cut sets are the minimal cut sets for end state logic as derived from the fault tree logic. The cut sets are stored in the block data file of the Endstate relation.

The MAR D end state cut sets are in a format similar to that of the fault tree cut sets.

File Name:

xxxxxx.ENC

File Format:

project, event tree, end state $=$

eventname * eventname +

eventname * eventname * eventname *

eventname +

eventname $*$ eventname.

$\wedge^{\wedge} \mathrm{EOS}$

project, event tree 2 , end state $=$

where

$\begin{array}{lll}\text { project } & 24 \text { character } & \text { Project name } \\ \text { event tree } & 24 \text { character } & \text { Event tree name } \\ \text { end state } & 24 \text { character } & \text { End state name } \\ \text { eventname } & 24 \text { character } & \text { Event names in the cut set }\end{array}$

\section{General Rules:}

An asterisk $(*)$ separates events in a cut set. Spaces are ignored.

A plus sign $(+)$ separates cut sets.

A period (.) denotes the end of the sequence. 
A slash (/) precedes complemented events.

Event names have a maximum of 16 characters including the "/" character for complemented events.

A line beginning with an asterisk (*) is a comment.

\subsubsection{End State Information}

File Name:

xxxxxx.ESI

File Format:

project $=$

project $=$

Name, E-QMethod, E-QPasses, R-QMethod, R-QPasses,

where

$\begin{array}{lll}\text { project } & 24 \text { character } & \text { Project name } \\ \text { name } & 24 \text { character } & \text { End state name } \\ \text { e-Qmethod } & 1 \text { character } & \text { End state default quantification method } \\ \text { e-Qpasses } & \text { Integer } 3 & \text { End state default min/max quantification passes } \\ \text { r-QMethod } & 1 \text { character } & \text { Quantification method used for current results } \\ \text { r-Qpasses } & \text { Integer } 3 & \begin{array}{l}\text { Min/max quantification passes used for current } \\ \text { results }\end{array}\end{array}$

\subsubsection{End State Textual Information}

A separate file is created for each end state selected.

File Name:

end-state.EST

where

end-state 24 character $\quad$ End state name

File Format:

project, end state[, $\mathrm{A}]=$

-- text --

where

project

end state

A
24 character

24 character

1 character
Project name

End state name

If included indicates alternate description

\subsubsection{Sequence Information}

\subsubsection{Sequence}




\section{PURPOSE}

Sequence cut sets, descriptions, attributes, text, and logic for MAR D file formats can be loaded or output.

With cut sets and attributes, data can be loaded into either the base case or current field areas. The event tree initiating event must have been entered into the database prior to loading sequence cut sets and, therefore, does not need to be included; any found will be removed from the cut set data.

When extracting, if more than one of the sequences are selected, the Output dialog is displayed, allowing you to choose the manner in which files are created for output.

\section{STEPS}

1. From the SAPHIRE menu select Utility.

2. Choose Load and Extract from the menu. The Load and Extract Data dialog will be displayed.

3. Select the desired Data Action, MAR-D Data Format, Sequence Data Type, then the desired File Type.

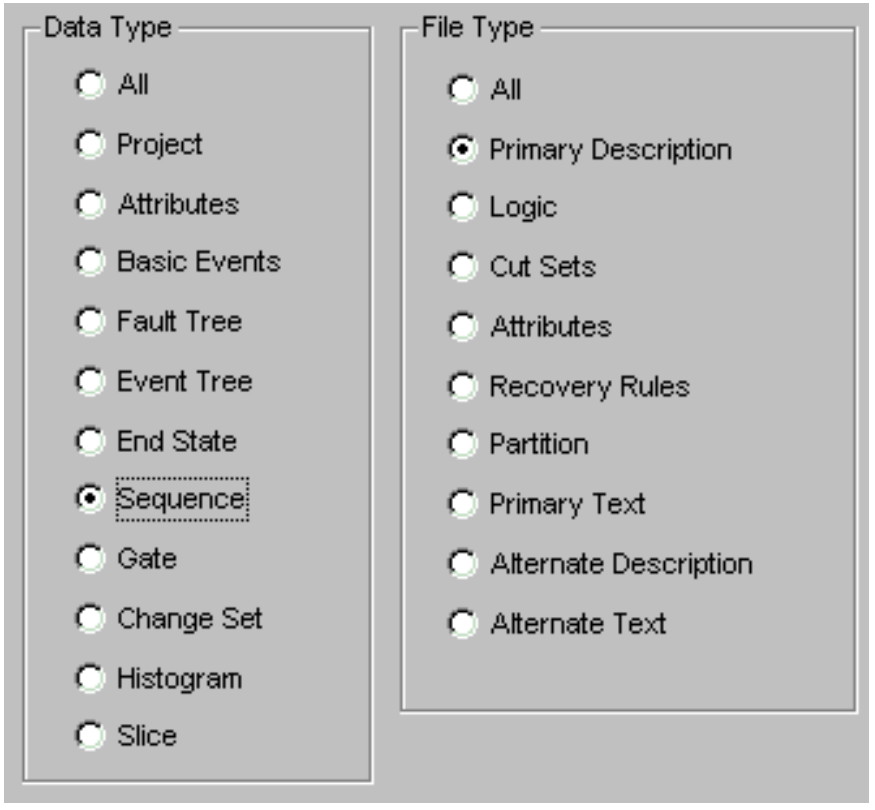

Process - $\quad$ Continue the load or extract operation for the selected Sequence File Type.

Cancel - $\quad$ Close the Load and Extract Data dialog.

\subsubsection{Output Dialog}

\section{PURPOSE}

This option provides you with the opportunity to choose the method for extracting multiple sequences. When extracting descriptions, attributes, or text, the Multiple Files by Sequence option is not available. 


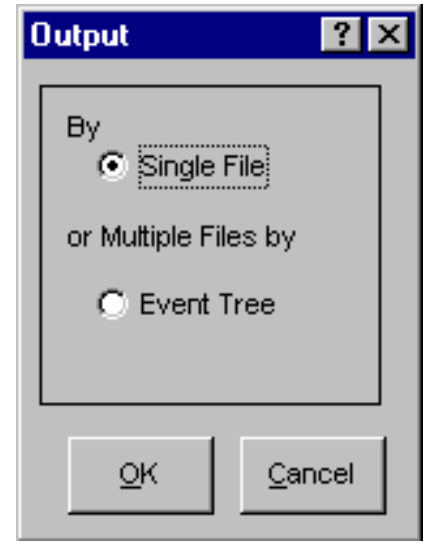

Single File - $\quad$ The output for the selected sequences will be saved in one file. The file name will be the first eight letters of the project name with the appropriate file extension.

Event Tree - $\quad$ The selected sequences will be grouped by event tree and output to individual event tree files. The names of the event tree files will be based on the event tree names and the appropriate file extension.

OK - $\quad$ Extract the data to the specified file(s). The file will be created in the current project directory. If successful, the message, "Output complete," will be displayed in the menu of the SAPHIRE window.

Cancel - $\quad$ Close the Output dialog without extracting data.

\subsubsection{Sequence Names and Descriptions}

File Name:

$\operatorname{xxxxxx.SQD}$

File Format:

project, eventree $=$

name,description[,A]

$\ldots, \ldots$

${ }^{\wedge} \mathrm{EOS}$

where

project

event tree

name

description

A
24 character

24 character

24 character

60 character

1 character
Project name

Event tree name

Sequence name

Sequence description

If included indicates alternate description

\subsubsection{Sequence Logic}

File Name:

$\operatorname{xxxxxxxx.SQL}$

File Format:

project, event tree, sequence $=$ 
sys 1 sys 2 /sys 3 sys 4

- .

${ }^{\wedge} \mathrm{EOS}$

project, event tree 2 , sequence $2=$

where

$\begin{array}{lll}\text { project } & 24 \text { character } & \text { Project name } \\ \text { event tree } & 24 \text { character } & \text { Event tree name } \\ \text { sequence } & 24 \text { character } & \text { Sequence name } \\ \text { sys } & 24 \text { character } & \text { Fault tree name }\end{array}$

\section{General Rules:}

Complemented fault trees are prefixed with "/".

\subsubsection{Sequence Cut Sets}

The sequence cut sets are the minimal cut sets for sequence logic as derived from the fault tree logic. The cut sets are stored in the block data file of the Sequence relation.

The MAR D sequence cut sets (.SQC) are in a format similar to that of the fault tree cut sets.

File Name:

$\operatorname{xxxxxx.SQC}$

File Format:

project, event tree, sequence, analysis $=$

eventname $*$ eventname +

eventname $*$ eventname $*$ eventname *

eventname +

eventname * eventname.

${ }^{\wedge} \mathrm{EOS}$

project, event tree 2 , sequence $2=$

where

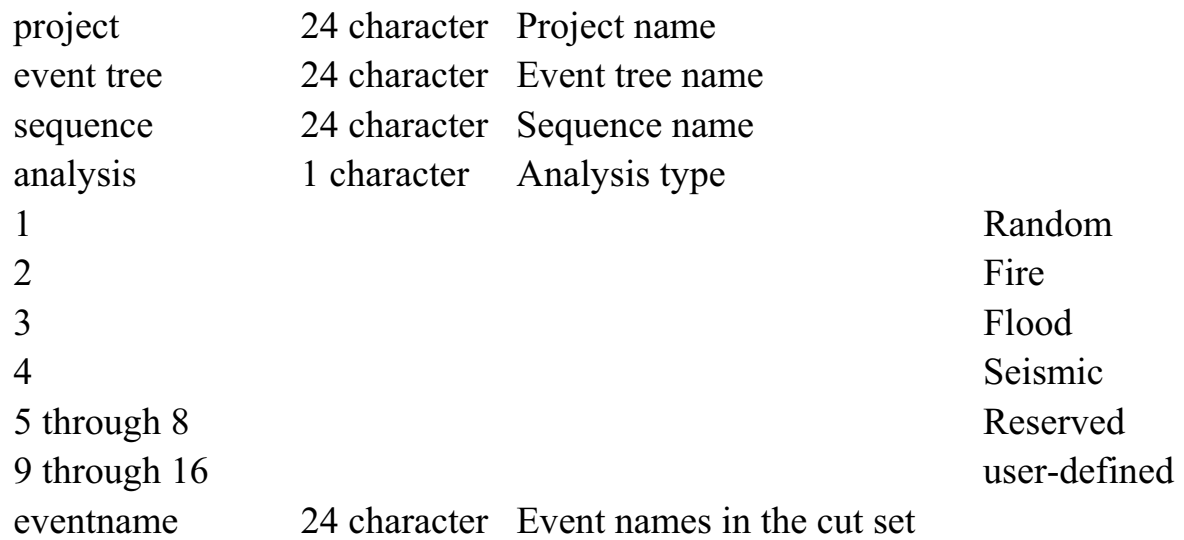




\section{General Rules:}

An asterisk (*) separates events in a cut set. Spaces are ignored.

A plus sign (+) separates cut sets.

A period (.) denotes the end of the sequence.

A slash (/) precedes complemented events.

Event names have a maximum of 24 characters including the "/" character for complemented events.

A line beginning with an asterisk $(*)$ is a comment.

\subsubsection{Sequence Attributes}

File Name:

$\operatorname{xxxxxx.SQA~}$

File Format:

project, event tree, analysis $=$

name,endstate,mincut,mission,procut,sample,seed,size,cuts,

events,value1, . . , value9, default flags, used flags

$\ldots, \ldots, \ldots, \ldots, \ldots, \ldots, \ldots, \ldots$

${ }^{\wedge} \mathrm{EOS}$

project, event tree $2=$

where

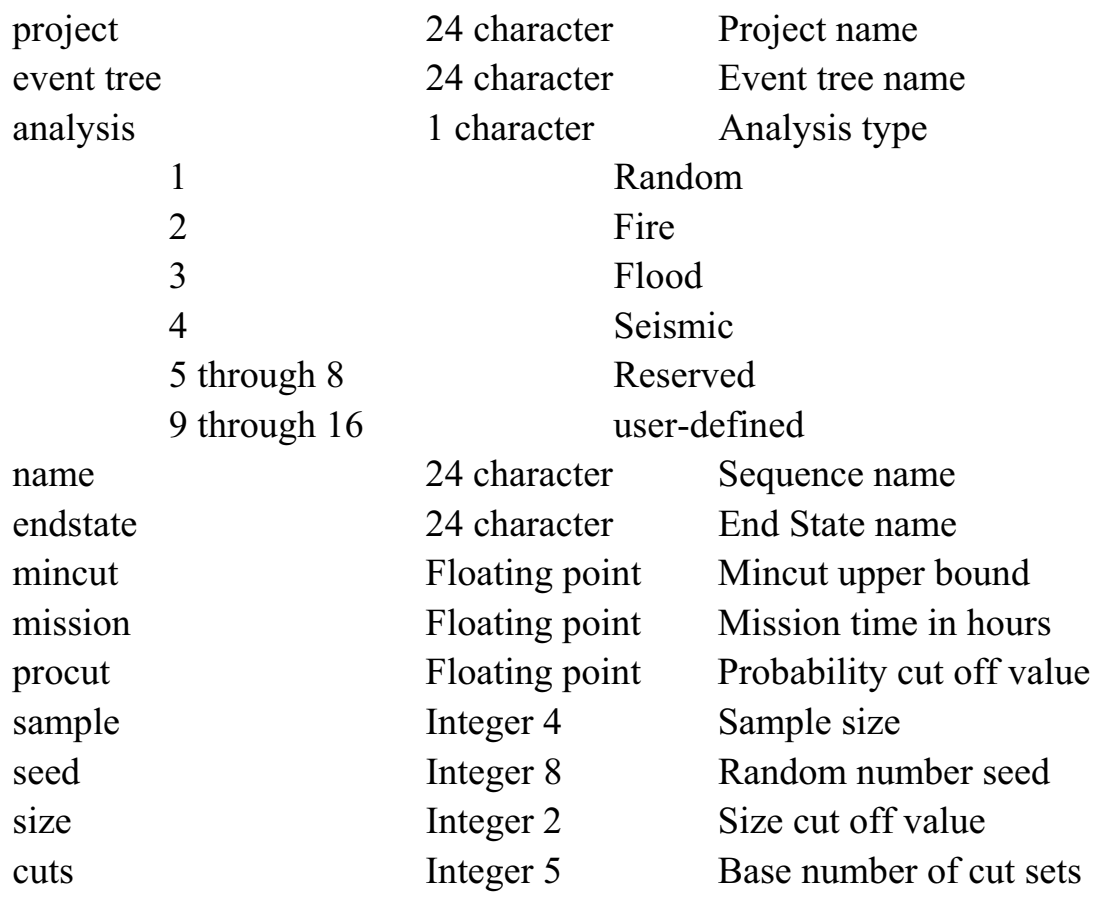




$\begin{array}{lll}\begin{array}{l}\text { events } \\ \text { value }\end{array} & \begin{array}{l}\text { Integer } 5 \\ \text { Floating point }\end{array} & \begin{array}{l}\text { Base number of events } \\ \text { Base uncertainty values } \\ \text { value1 }\end{array} \\ \text { value2 } & & \text { 5th percentile } \\ \text { value3 } & & \text { Median } \\ \text { value4 } & & \text { Mean } \\ \text { value5 } & & \text { 95th percentile } \\ \text { value6 } & & \text { Minimum sample } \\ \text { value7 } & & \text { Maximum sample } \\ \text { value8 } & & \text { Standard deviation } \\ \text { value9 } & & \text { Skewness } \\ \text { Default flags } & & \text { Kurtosis } \\ \text { Used flags } & 24 \text { character } & \text { Default flag set for this sequence } \\ \end{array}$

\subsubsection{Sequence Recovery Rules}

File Name:

$\operatorname{xxxxxxxx.SQY}$

File Format:

project, event tree, sequence $=$

-- recovery rule text --

$\wedge$ EOS

project, event tree, sequence $2=$

where

$\begin{array}{lll}\text { project } & 24 \text { character } & \text { Project name } \\ \text { event tree } & 24 \text { character } & \text { Event tree name } \\ \text { sequence } & 24 \text { character } & \text { Sequence name }\end{array}$

\subsubsection{Sequence Partition Rules}

File Name:

$\operatorname{xxxxxxxx.SQP}$

File Format:

project, event tree, sequence $=$

-- partition rule text --

${ }^{\wedge} \mathrm{EOS}$

project, event tree, sequence $2=$

where

$\begin{array}{lll}\text { project } & 24 \text { character } & \text { Project name } \\ \text { event tree } & 24 \text { character } & \text { Event tree name } \\ \text { sequence } & 24 \text { character } & \text { Sequence name }\end{array}$

\subsubsection{Sequence Textual Information}


File Name:

$\operatorname{xxxxxx.SQT}$

File Format:

project, event tree, sequence $[, \mathrm{A}]=$

--- text ---

${ }^{\wedge} \mathrm{EOS}$

project, event tree 2 , sequence $2=$

--- text ---

where

$\begin{array}{lll}\text { project } & 24 \text { character } & \text { Project name } \\ \text { sequence } & 24 \text { character } & \text { Sequence name } \\ \text { event tree } & 24 \text { character } & \text { Event tree name } \\ \text { A } & 1 \text { character } & \text { If included indicates alternate description }\end{array}$

\subsubsection{Gate Information}

\subsubsection{Gate}

\section{PURPOSE}

Gate names and descriptions and types can be loaded for use in graphics conversion or output from the database.

\section{STEPS}

1. From the SAPHIRE menu select Utility.

2. Choose Load and Extract from the menu. The Load and Extract Data dialog will be displayed.

3. Select the desired Data Action, MAR-D Data Format, Gate Data Type, then the desired File Type. 


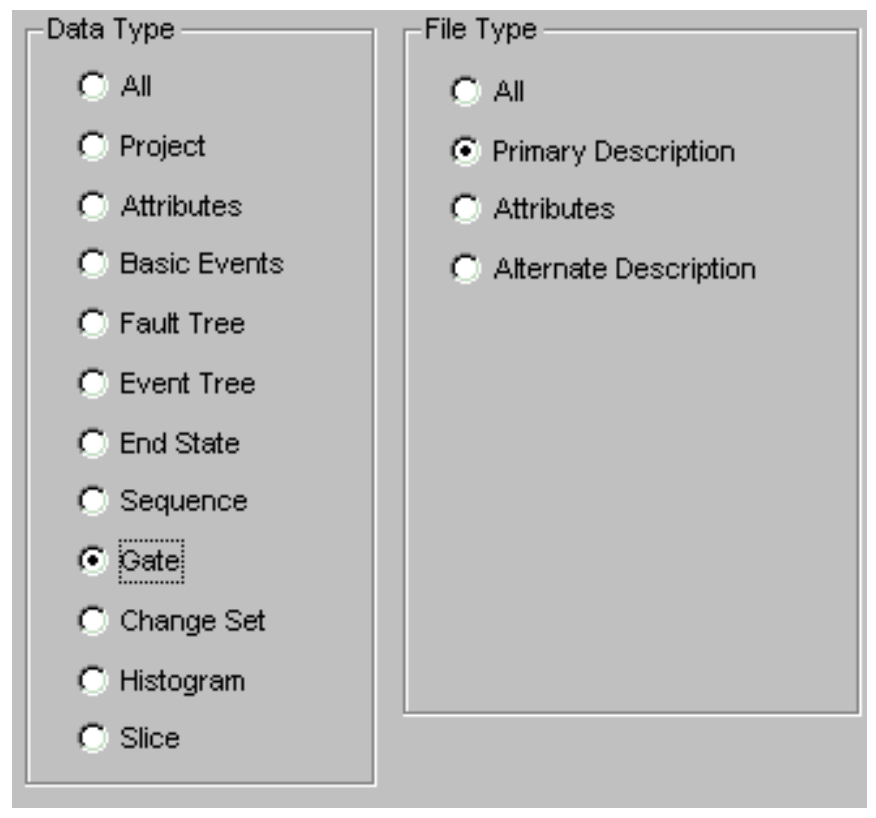

Process - $\quad$ Continue the load or extract operation for the selected Gate File Type.

Cancel - $\quad$ Close the Load and Extract Data dialog.

\subsubsection{Gate Description}

File Name:

xxxxxx.GTD

File Format:

project $=$

name,description[,A]

where

project

name

description

A

\subsubsection{Gate Attributes}

File Name:

xxxxxx.GTA

File Format

project $=$

name, attribute

where

project

name

attribute
24 character Project name

24 character Gate name

60 character Gate description

1 character 


\subsubsection{Change Set Information}

\subsubsection{Change Sets}

\section{PURPOSE}

All change set information used in the Generate option can be loaded via this file.

\section{STEPS}

1. From the SAPHIRE menu select Utility.

2. Choose Load and Extract from the menu. The Load and Extract Data dialog will be displayed.

3. Select the desired Data Action, MAR-D Data Format, Change Set Data Type, then the desired File Type.

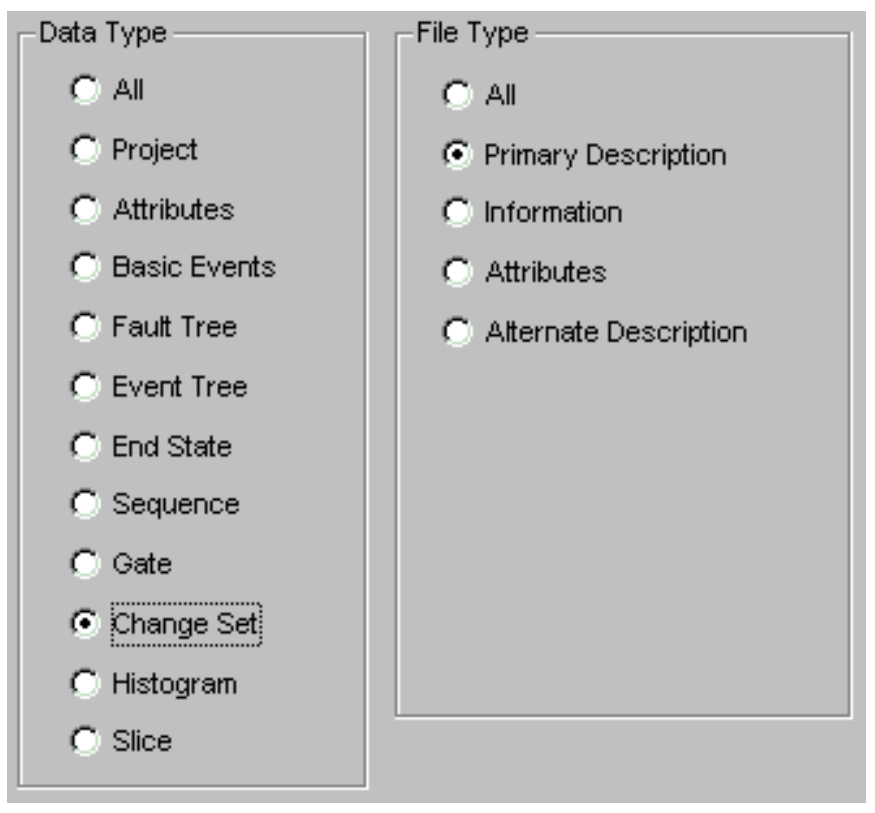

Process - Continue the load or extract operation for the selected Change Set File Type.

Cancel - Close the Load and Extract Data dialog.

\subsubsection{Change Set Description}

File Name:

$\operatorname{xxxxxx.CSD}$

File Format:

project $=$

name,description[,A]

where 


$\begin{array}{lll}\text { project } & 24 \text { character } & \text { Project name } \\ \text { name } & 24 \text { character } & \text { Change set name } \\ \text { description } & 60 \text { character } & \text { Change set description } \\ \text { A } & 1 \text { character } & \text { If included indicates alternate description }\end{array}$

\subsubsection{Change Set Information}

File Name:

XXXXxx.CSI

File Format:

project, change $=$

^PROBABILITY

eventname,calc,udT,prob,lambda,tau,udV,udC,mission,init

${ }^{\wedge}$ CLASS

eventname,group,compType,compId,system,location,failMode,train,init,att1,..att16

calcType,udT,prob,lambda,tau,udV,udC,mission, init

${ }^{\wedge} \mathrm{EOS}$

project,change $2=$

where

$\begin{array}{lll}\begin{array}{l}\text { change } \\ \text { eventname }\end{array} & 24 \text { character } & \begin{array}{l}\text { change set name } \\ \text { group }\end{array} \\ \text { compType } & 24 \text { character } & \text { name mask } \\ \text { event group mask } \\ \text { compId } & 7 \text { characters } & \text { component type mask } \\ \text { system } & 3 \text { characters } & \text { component ID mask } \\ \text { location } & 3 \text { characters } & \text { system mask } \\ \text { failMode } & 3 \text { characters } & \text { location mask } \\ \text { train } & 2 \text { characters } & \text { failure mode mask } \\ \text { init } & 2 \text { characters } & \text { train mask } \\ \text { att1..att16 } & 1 \text { character } & \text { initiating event }(\mathrm{Y} / \mathrm{N}) \\ & \text { Class attribute } & 16 \text { values of Y or } \mathrm{N} \text { (yes or no) indicate whether } \\ & \text { flags } & \text { the attribute described in the class attribute file is } \\ \text { calc } & 1 \text { character } & \text { applicable. } \\ & & \text { Calculation type }\end{array}$

Probability

Lambda* Mission Time

1 Exp(-Lambda * Mission Time)

Lambda * Min(Mission Time, Tau)

Operating component with full repair

Lambda * Tau / 2.0

$1+(\operatorname{EXP}($ Lambda*Tau $) 1.0) /($ Lambda*Tau $)$

Base Probability * Probability

Base Probability * Probability

Set to House Event (Failed, Prob=1.0) 
prob

lambda

tau

udV

udC

mission

init

Set to House Event (Successful,Prob=0.0)

Set to ignore

Use fault tree mincut upperbound

Seismic event - Enter g level for screening

Use low site hazard curve

Seismic event - Use medium site hazard curve for screening

Use high site hazard curve

1 character Uncertainty distribution type

Log normal, error factor

Normal, standard deviation

Beta, $b$ of $\operatorname{Beta}(a, b)$

Gamma, a Gamma(a)

Chi-squared, degrees of freedom

Exponential, none

Uniform, Upper end pt.

Histogram

Maximum entropy

Floating point

Floating point

Floating point

Floating point

4 characters

Floating point

Boolean (T/F)

\section{Probability value}

Basic event failure rate per hr.

Time to repair in hours

Uncertainty distribution value

Uncertainty correlation class. Events in same class are $100 \%$ correlated.

Mission time

Initiating event

\subsubsection{Change Set Attributes}

File Name:

xxxxxx.CSA

File Format:

project $=$

name,altName

where

$\begin{array}{lll}\text { project } & 24 \text { character } & \text { Project name } \\ \text { name } & 24 \text { character } & \text { Change set primary name } \\ \text { altName } & 24 \text { character } & \text { Change set alternate name }\end{array}$




\subsubsection{Histogram Information}

\subsubsection{Histograms}

\section{PURPOSE}

Histogram descriptions and information can be loaded into or extracted from the database.

\section{STEPS}

1. From the SAPHIRE menu select Utility.

2. Choose Load and Extract from the menu. The Load and Extract Data dialog will be displayed.

3. Select the desired Data Action, MAR-D Data Format, Histogram Data Type, then the desired File Type.

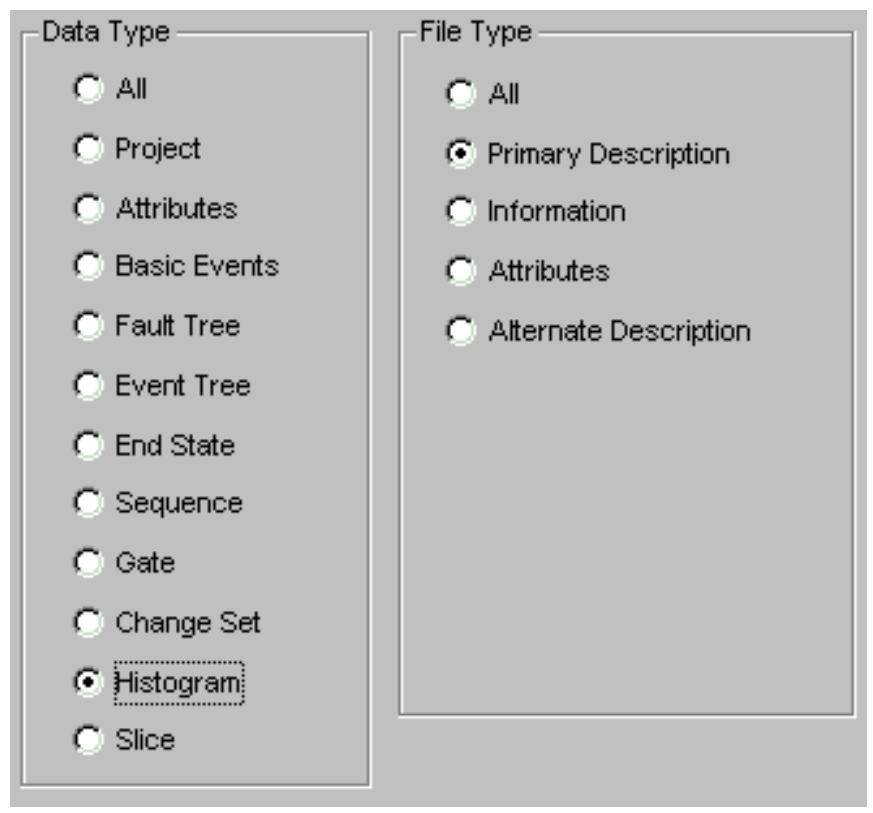

Process - Continue the load or extract operation for the selected Histogram File Type.

Cancel - Close the Load and Extract Data dialog.

\subsubsection{Histogram Description}

File Name:

xxxxxxxx.HID

File Format:

project $=$

name, type, subtype, description[, A]

where

project 24 character Project name 


\begin{tabular}{|c|c|c|c|}
\hline name & 24 character & Histogram primary name & \\
\hline type & 1 character & Histogram type & \\
\hline $\mathrm{H}$ & & & Hazard \\
\hline $\mathrm{U}$ & & & Uncertainty \\
\hline $\mathrm{F}$ & & & Fragility \\
\hline subtype & 1 character & Histogram subtype & \\
\hline $\mathrm{P}$ & & & Percent \\
\hline A & & & Area \\
\hline $\mathrm{R}$ & & & Range \\
\hline $\mathrm{H}$ & & & Hazard \\
\hline Description & 60 character & Histogram description & \\
\hline A & 1 character & $\begin{array}{l}\text { If included indicates } \\
\text { alternate description }\end{array}$ & \\
\hline
\end{tabular}

\subsubsection{Histogram Information}

File Name:

xxxxxxxx.HII

File Format:

project, name1=
type, subtype
bin1 value1, bin1 value2
bin2 value1, bin2 value2
..
bin20 value1, bin20 value2

${ }^{\wedge} \mathrm{EOS}$

project, name $2=$

where

$\begin{array}{llll}\text { Project } & \text { 24 character } & \text { Project name } & \\ \text { NameN } & \text { 24 character } & \text { Histogram primary name } & \\ \text { Type } & 1 \text { character } & \text { Histogram type } & \\ \mathrm{H} & & & \text { Hazard } \\ \mathrm{U} & & & \text { Uncertainty } \\ \mathrm{F} & & & \text { Fragility } \\ \text { Subtype } & 1 \text { character } & \text { Histogram subtype } & \\ \mathrm{P} & & & \text { Percent } \\ \mathrm{A} & & & \text { Area } \\ \mathrm{R} & & & \text { Range } \\ \mathrm{H} & & & \text { Hazard } \\ \text { bin value1 } & \text { Exponential } & \text { first value for bin } & \\ \text { bin value2 } & \text { Exponential } & \text { second value for bin } & \end{array}$

\subsubsection{Histogram Attributes}


File Name:

$\operatorname{xxxxxxxx.HII~}$

File Format:

project $=$

name, type, subtype, altName

where

\begin{tabular}{|c|c|c|c|}
\hline project & 24 character & Project name & \\
\hline name & 24 character & Histogram primary name & \\
\hline type & 1 character & Histogram type & \\
\hline $\mathrm{H}$ & & & Hazard \\
\hline $\mathrm{U}$ & & & Uncertainty \\
\hline $\mathrm{F}$ & & & Fragility \\
\hline subtype & 1 character & Histogram subtype & \\
\hline $\mathrm{P}$ & & & Percent \\
\hline A & & & Area \\
\hline $\mathrm{R}$ & & & Range \\
\hline $\mathrm{H}$ & & & Hazard \\
\hline altName & 24 character & Histogram alternate name & \\
\hline
\end{tabular}

\subsubsection{Slice Information}

\subsubsection{Slice}

\section{PURPOSE}

Slice descriptions and basic event information can be loaded into or extracted from the database.

\section{STEPS}

1. From the SAPHIRE menu select Utility.

2. Choose Load and Extract from the menu. The Load and Extract Data dialog will be displayed.

3. Select the desired Data Action, MAR-D Data Format, Slice Data Type, then the desired File Type. 


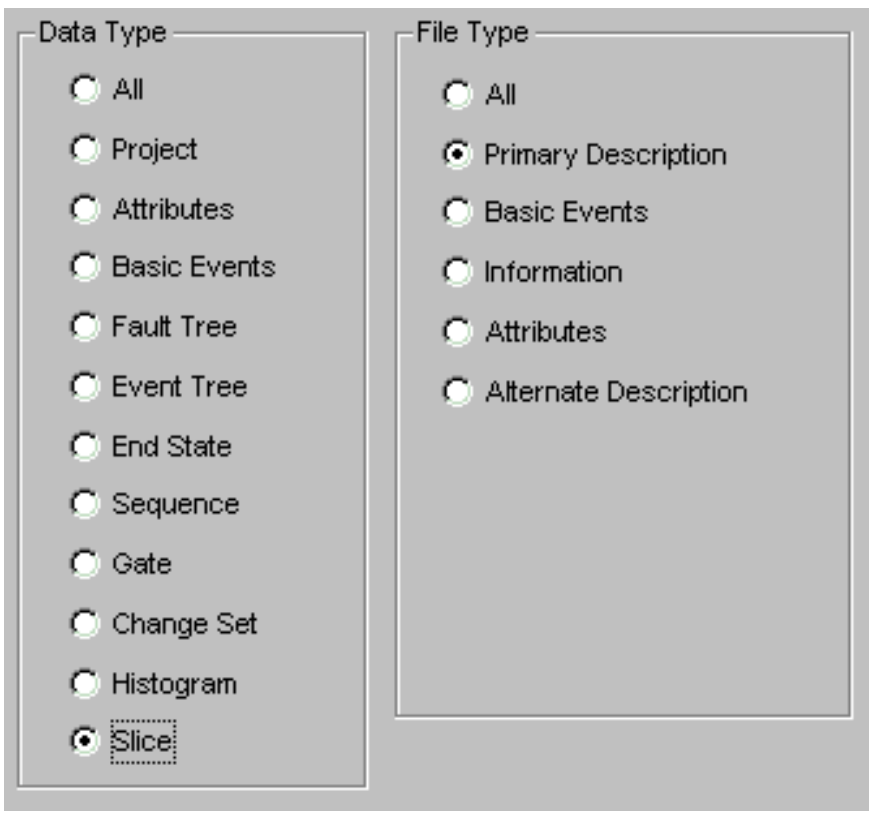

Process - $\quad$ Continue the load or extract operation for the selected Slice File Type.

Cancel - $\quad$ Close the Load and Extract Data dialog.

\subsubsection{Slice Description}

File Name:

$\operatorname{xxxxxxxx.SLD}$

File Format:

project $=$

name, description[, A]

where

project

name

description

A
24 character

24 character

60 character

1 character
Project name

Slice name

Slice description

If included indicates alternate description

\subsubsection{Slice Basic Events}

File Name:

$\operatorname{xxxxxxxx.SLB}$

File Format:

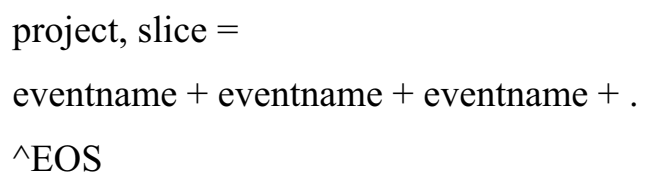

project, slice $2=$ where 


$\begin{array}{lll}\text { project } & 24 \text { character } & \text { Project name } \\ \text { slice } & 24 \text { character } & \text { Slice name } \\ \text { eventname } & 24 \text { character } & \text { Event names in the slice } \\ + \text { or } * & 1 \text { character } & \text { Slice logic: }+=\text { or }, *=\text { and }\end{array}$

\section{General Rules:}

A plus symbol $(+)$ or asterisk $(*)$ between event names represent the logic in a slice. Spaces are ignored. All logic must be the same in a slice.

A period (.) denotes the end of the slice.

A slash (/) precedes complemented events.

Event names have a maximum of 24 characters including the "/" character for complemented events.

A line beginning with an asterisk $(*)$ is a comment.

\subsubsection{Slice Information}

File Name:

$\operatorname{xxxxxxxx.SLI}$

File Format:

project, slice $=$

eventname, delta, factor

$\ldots, .$.

${ }^{\wedge} \mathrm{EOS}$

project, slice $2=$

where

$\begin{array}{lll}\text { project } & 24 \text { character } & \text { Project name } \\ \text { slice } & 24 \text { character } & \text { Slice name } \\ \text { eventname } & 24 \text { character } & \text { Event names in the slice } \\ \text { delta } & \text { Floating point } & \text { Delta value that is factored } \\ \text { factor } & 1 \text { character } & \text { Factor flag: } \mathrm{F}=\text { multiply, Blank=add }\end{array}$

\subsubsection{Slice Attributes}

File Name:

xxxxxx.SLA

File Format:

project $=$

name, altName

where 


$\begin{array}{lll}\text { project } & 24 \text { character } & \text { Project name } \\ \text { name } & 24 \text { character } & \text { Slice primary name } \\ \text { altName } & 24 \text { character } & \text { Slice alternate name }\end{array}$

\subsubsection{Extracting SETS Data}

\subsubsection{Basic Events Data Format}

\subsection{SETS Basic Event Descriptions}

File Name:

xxxxxxx.DES.

File Format:

name $\$$ description $\$$

name $\$$ description $\$$

where
name
event name
name list
description of event

\subsection{SETS Basic Event Failure Rates}

File Name:

xxxxxxx.VBK.

File Format:

VALUE BLOCK\$ value-block-name prob \$ name-list\$

where prob \$ name-list\$

prob point value probability estimate

name list list of event names separated by commas

\subsection{SETS Basic Events Data Format}

\section{PURPOSE}

Event descriptions, and failure rates for the Set Equation Transformation System (SETS) data format can be loaded into the database or extracted from it.

\section{STEPS}

1. From the SAPHIRE menu select Utility.

2. Choose Load and Extract from the menu. The Load and Extract Data dialog will be displayed.

3. Select the desired Data Action, SETS Data Format, Basic Events Data Type, then the desired File Type. 


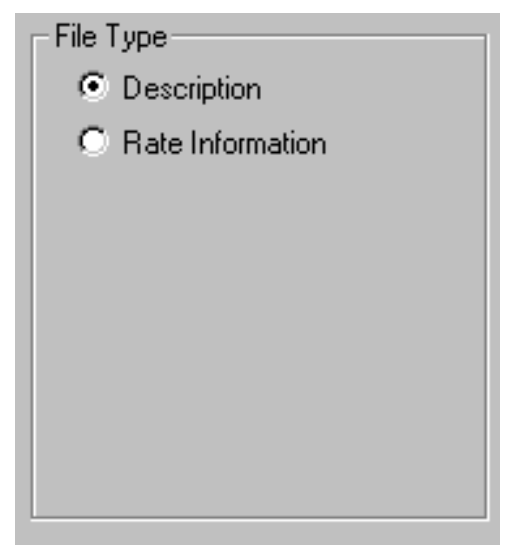

Process - Continue the load or extract operation for the selected Basic Events File Type.

Exit - Close the Load and Extract Data dialog.

\subsubsection{Fault Tree Data Format}

\subsection{SETS Fault Tree Data Format}

\section{PURPOSE}

Fault tree cut sets and logic for the Set Equation Transformation System (SETS) data format can be loaded into the database or output to files.

\section{STEPS}

1. From the SAPHIRE menu select Utility.

2. Choose Load and Extract from the menu. The Load and Extract Data dialog will be displayed.

3. Select the desired Data Action, SETS Data Format, Fault Tree Data Type, then the desired File Type.

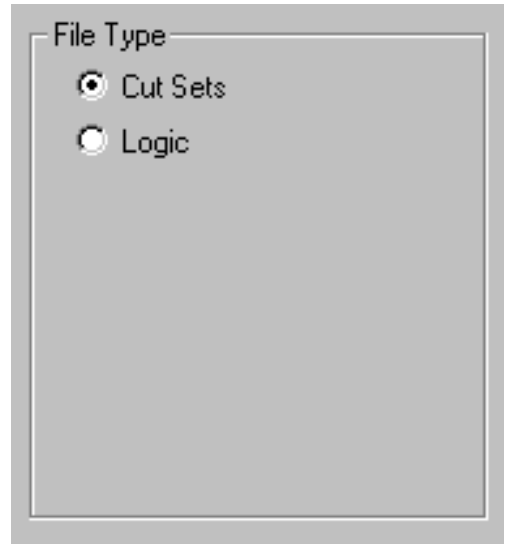

Process - $\quad$ Continue the load or extract operation for the selected Fault Tree File Type.

Cancel - $\quad$ Close the Load and Extract Data dialog. 


\subsection{SETS Fault Tree Cut Sets}

The fault tree cut sets are stored in the System relation in the block data file. The format of the cut set file (.DNF) is given in the SETS Sequence Cut Sets.

\subsection{SETS Fault Tree Logic}

File Name:

xxxxxx.SET.

File Format:

FAULT TREE\$ fault tree name.

COMMENT\$ descriptive material \$

gate type $\$$ gate name. IN $\$$ input 1 , input $2, \ldots$, input $n$.

OUT $\$$ output 1 , output $2, \ldots$, output $n$.

event type \$event name. OUT\$ output $1, \ldots$, output $n$.

where

fault tree name

gate type

AG

OG

EOR

EAG

$\mathrm{SG}$

gate name

output $n$

event type

$\mathrm{BE}$

CE

$\mathrm{UE}$

DE

$\mathrm{EE}$

COMMENT\$
The name of the fault tree.

The type of gate being defined.

$=$ AND gate

$=$ OR gate

$=$ Exclusive OR gate (converted to $\mathrm{SG}$ )

$=$ Exclusive AND gate (converted to $\mathrm{SG}$ )

$=$ Special Gate

The name of the gate being defined (16 characters) input $n$ The names of the gates or primary events that are the immediate inputs to the gate being defined (16 characters)

The names of the gates that are the immediate outputs of the gate or primary event being defined (16 characters).

The type of primary event being defined.

$=$ Basic Event

$=$ Conditional Event

$=$ Undeveloped Event

$=$ Developed Event

$=$ External Event

Defines a comment. Must follow a "." delimiter.

\subsubsection{Sequence Data Format}




\subsection{SETS Sequence Cut Sets}

File Name:

xxxxxx.DNF.

The format of the SETS output cut sets file (.DNF) is dependent upon the command issued within SETS. The factored form is

$$
\mathrm{A} *(\mathrm{~B}+\mathrm{C})
$$

The disjunctive normal form is

$$
\mathrm{A} * \mathrm{~B}+\mathrm{A} * \mathrm{C}
$$

ONLY the disjunctive normal form is accepted by the MAR D at this time.

File Format:

sequence-name $=$

eventName * eventName +

eventName * eventName.

where

\section{General Rules:}

An asterisk $\left(^{*}\right)$ separates event names. Spaces are ignored.

A plus sign $(+)$ separates cut sets.

A period (.) denotes the end of a sequence.

An asterisk (*) in the first column denotes a comment.

\subsection{SETS Sequence Data Format}

\section{PURPOSE}

Sequence cut sets, and list cut sets for the Set Equation Transformation System (SETS) data format can be loaded or output.

The event tree initiating event will be included in each cut set term for SETS *.DNF format output.

\section{STEPS}

1. From the SAPHIRE menu select Utility.

2. Choose Load and Extract from the menu. The Load and Extract Data dialog will be displayed. 
3. Select the desired Data Action, SETS Data Format, Sequence Data Type, then the desired File Type.

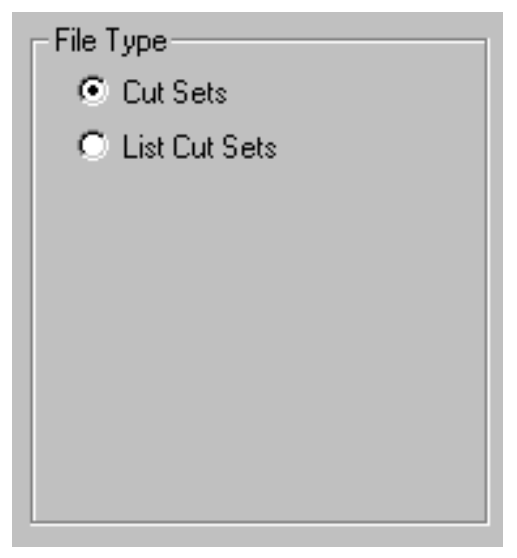

Process - $\quad$ Continue the load or extract operation for the selected Sequence File Type.

Cancel - $\quad$ Close the Load and Extract Data dialog.

\subsection{Recovering the Data Base}

\section{PURPOSE}

This option allows you to restructure the database and re index the data.

NOTE

Before recovering the database, it is suggested that you backup the *.DFL files and corresponding data files (*.IDX , *.DAT, and *.BLK) in the directory of the project to be rebuilt.

\section{STEPS}

1. From the menu select Utility.

2. Choose Recover Data Base from the menu. The Recover Everything warning dialog will be displayed.

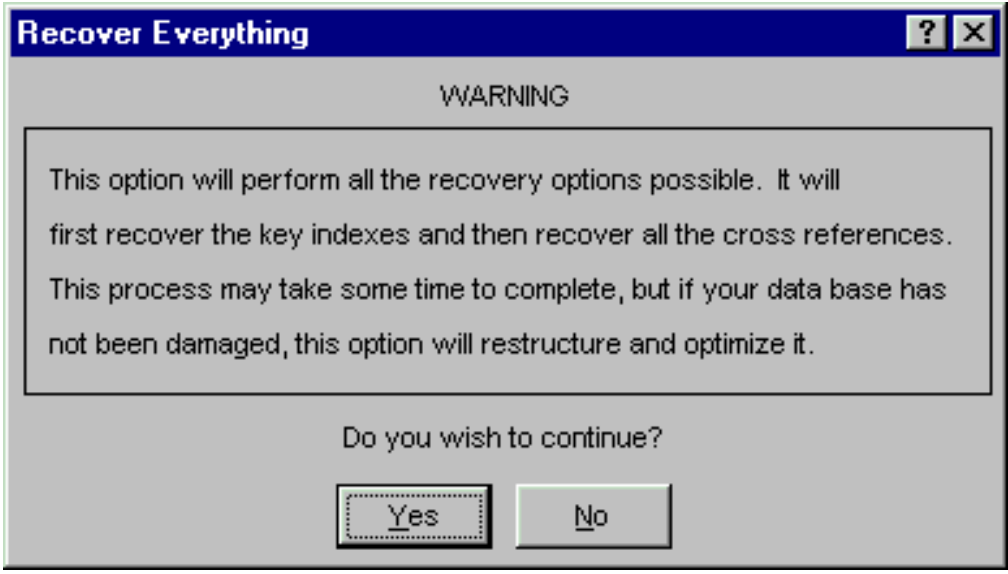


Yes - Continue with the recovery. Status messages will appear in the message bar of the SAPHIRE window as the recover process proceeds. "Successful Completion," will be the last message displayed in the message bar.

No - Close the Recover Everything dialog without recovering the database.

Some indications that a database rebuild is necessary include:

- Data elements such as events/fault trees have been deleted and seem to reappear

- $\quad$ During cut set generation or update, the min cut upper bound seems surprisingly high

- Cross Reference reports show/don't show events being used properly

- $\quad$ Events/fault trees that don't appear to be referenced cannot be deleted, and

- $\quad$ After a software version update

- $\quad$ SAPHIRE displays a message stating a database recovery is required.

You may rebuild the database anytime because the rebuild process compacts the data and generally helps the software run faster. This option will recover all key indexes and then recover the cross-references. This process may take several minutes to complete. If your database has not been damaged, this option will just restructure and optimize your database.

\subsection{Update and Align}

\subsubsection{Align Primary and Alternate Descriptions}

\section{PURPOSE}

This option allows you to synchronize the primary and alternate descriptions for all data items (fault trees, gates, events, etc) in the current project. When an alternate naming scheme is not desired, this utility can be used to ensure that all primary and alternate descriptions match.

SAPHIRE supports a dual naming scheme. Each record in a project has two names and two descriptions, referred to as primary and alternate. The purpose of the alternate name and description is to allow an alternate view of the project. The alternate name and description could be expressed in another language (see International SAPHIRE), or in layman's terms, or whatever the project developer wishes. The primary name and descriptions are displayed by default through SAPHIRE, in lists, graphics, and reports. The default display can be changed to show alternate names and descriptions (see Defining Constants).

Not all projects need to use this feature, particularly in the model development phase of the project. However SAPHIRE still requires that the alternate names be specified. As new items are added to the project, SAPHIRE defaults the alternate name and description to match the primary name and description, unless otherwise specified. When these items are later modified by the user, SAPHIRE does not automatically update the alternate name and description information; it assumes the name and description are different by design. This can cause confusion, since the item can now be referred to in the project by either name. When an alternate naming scheme is not desired, this utility can be used to ensure that all primary and alternate descriptions match. 


\section{STEPS}

1. From the menu select Utility.

2. Choose Update Descriptions from the menu. The Align Primary and Alternate Descriptions warning dialog will be displayed.

3. Select Primary, Alternate, or as the name field to retain.

4. Choose $\mathbf{O K}$ to perform the alignment.

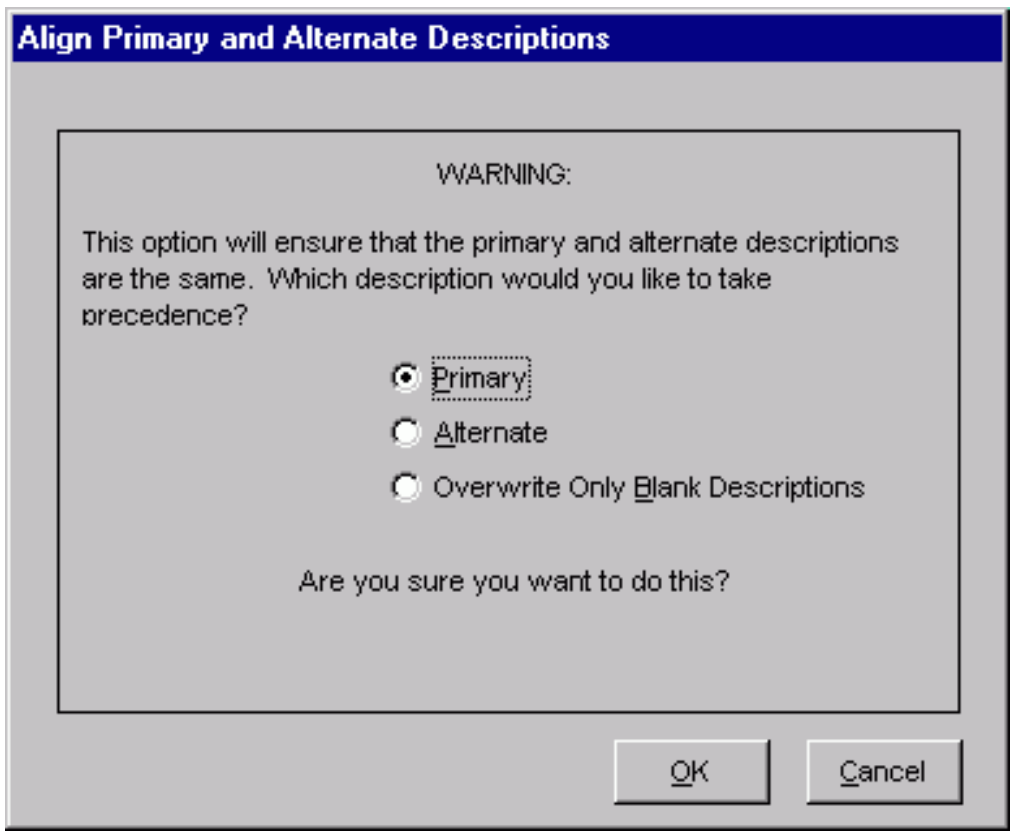

Primary -

Alternate -

Overwrite Only Blank Descriptions -

OK -

Cancel -
Overwrite the alternate description with the primary description.

Overwrite the primary description with the alternate description.

Overwrite the alternate description with the primary description only when the alternate description is blank. Align all project item descriptions to match the selected option.

Close the dialog without performing the alignment. 


\subsubsection{Align Primary and Alternate Names}

\section{PURPOSE}

This option allows you to synchronize the primary and alternate names for all data items (fault trees, gates, events, etc) in the current project. When an alternate naming scheme is not desired, this utility can be used to ensure that all primary and alternate names match each other.

SAPHIRE supports a dual naming scheme. Each record in a project has two names and two descriptions, referred to as primary and alternate. The purpose of the alternate name and description is to allow an alternate view of the project. The alternate name and description could be expressed in another language (see International SAPHIRE), or in layman's terms, or whatever the project developer wishes. The primary name and descriptions are displayed by default through SAPHIRE, in lists, graphics, and reports. The default display can be changed to show alternate names and descriptions (see Defining Constants).

Not all projects need to use this feature, particularly in the model development phase of the project. However SAPHIRE still requires that the alternate names be specified. As new items are added to the project, SAPHIRE defaults the alternate name and description to match the primary name and description, unless otherwise specified. When these items are later modified by the user, SAPHIRE does not automatically update the alternate name and description information; it assumes the name and description are different by design. This can cause confusion, since the item can now be referred to in the project by either name. When an alternate naming scheme is not desired, this utility can be used to ensure that all primary and alternate names match.

\section{STEPS}

1. From the menu select Utility.

2. Choose Align Names from the menu. The Align Primary and Alternate Names warning dialog will be displayed.

3. Select Primary or Alternate as the name field to retain.

4. Choose $\mathbf{O K}$ to perform the alignment. 


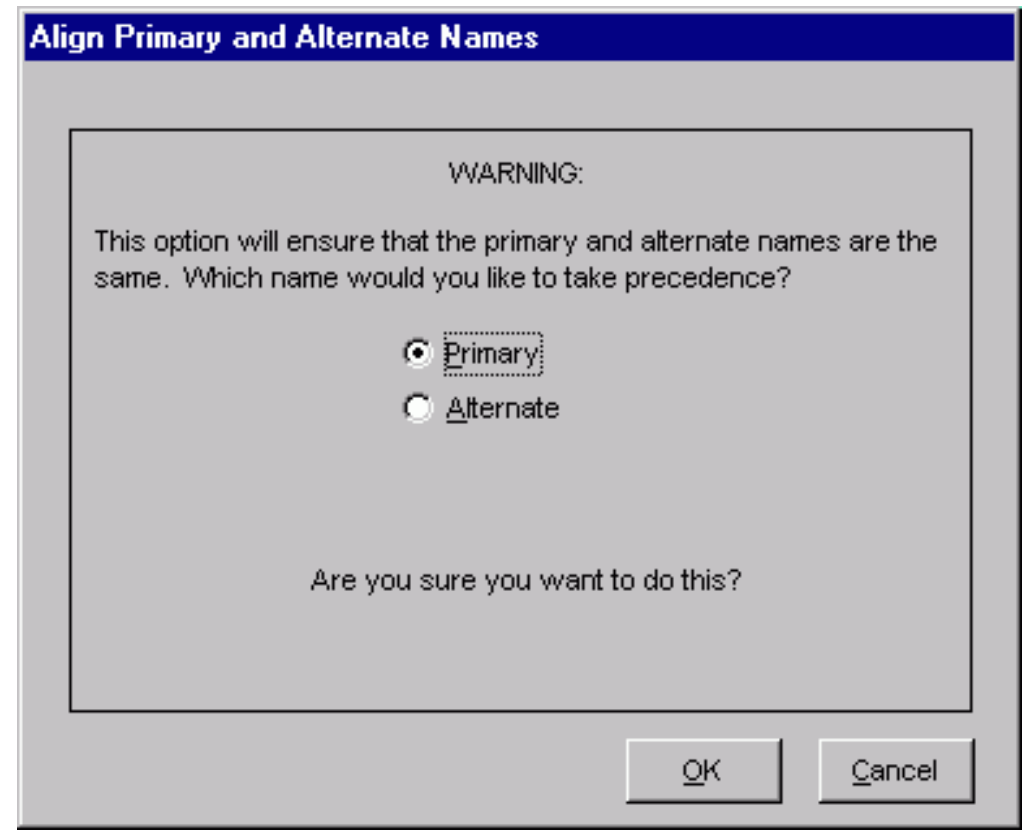

Primary - $\quad$ Overwrite the alternate name with the primary name.

Alternate - $\quad$ Overwrite the primary name with the alternate name.

OK - $\quad$ Align all project item names to match the selected option.

Cancel - $\quad$ Close the dialog without performing the alignment.

\section{SEE ALSO}

Align Primary and Alternate Descriptions

\subsection{Fault Tree Utility Options}

\subsubsection{Fault Tree Utility Options}

\section{PURPOSE}

This option provides you with the ability to manage fault tree graphics files. You can convert fault tree logic from alphanumeric format to graphical format, and load or extract fault tree graphics.

\section{STEPS}

1. From the SAPHIRE menu select Utility.

2. Choose Fault Tree from the menu. Select a sub-menu option. 


\section{나ility}

\section{Define Constants... \\ Load and Extract.. \\ Recover Data Base...}

\begin{tabular}{|l|l|}
\hline Eault Tree & Blpha to Graphics \\
\hline Event Tree & Check Duplicate Gates \\
\hline View Error Log & Extract Graphics \\
Version Date & Load Graphics \\
\hline
\end{tabular}

Sensitivity Wizard

Importance Measures Wizard
Alpha to Graphics -

Check Duplicate Gates Extract Graphics -

Load Graphics -

Fix Color -
Convert fault tree or sub-tree alphanumeric logic to a graphical format.

Finds and reports duplicate gates.

Extract fault trees from the database.

Load fault tree graphic files into the database.

Quickly map unique fault tree diagram display attributes (including color and font) to individual or groups of gates and events. The selected attributes will be automatically applied to all fault tree diagrams in the project.

\subsubsection{Check Duplicate Gates}

\section{PURPOSE}

This option searches the logic of all project fault trees for duplicate gates. A duplicate gate is a gate that appears in more than one fault tree. Although it is acceptable practice to have a gate in more than one fault tree, the gate with the same name in different fault trees should be of the same type (e.g., AND, OR, $\mathrm{N} / \mathrm{M}$, etc.) and should have the same inputs.

\section{STEPS}

1. From the SAPHIRE menu select Utility.

2. Choose Fault Tree from the menu.

3. Select the Check Duplicate Gates sub-menu option.

The software will search for duplicate gates. If no duplicate gates are found, a message on the status bar such as, "No Duplicate Gates were found" will be displayed. If duplicate gates were found the message, "Duplicate Gates were found. Check error.log file."

Sample error.log file: 


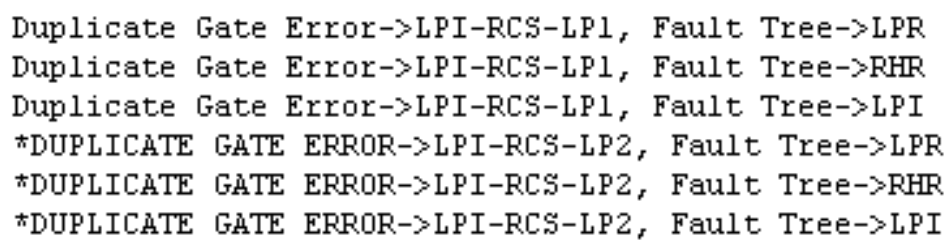

The example above shows part of the error log file that is generated after checking for duplicate gates. The first three lines indicate that the gate, LPI-RCS-LP1 is duplicated in three fault trees: LPR, RHR, and LPI. The next three lines indicate that the gate, LPI-RCS-LP2 is also duplicated in three fault trees, but because those lines begin with an asterisk, there is an error in the fault tree logic. There could be an error because the gate, LPI-RCS-LP2, is defined as a different type in one of the trees or because it has different inputs in one of the trees. Duplicate gates with an asterisk indicate conflicts that will need to be resolved before sequence cut set generation is performed.

\subsubsection{Color Fault Trees}

\section{PURPOSE}

This option allows you to quickly assign distinctive display attributes to one or more gates or events across all fault tree diagrams. You can change the name and description fonts, and the shape and outline colors.

\section{STEPS}

1. From the SAPHIRE menu select Utility.

2. Choose Fault Tree from the menu. Select the Fix Colors sub-menu option.

3. The Color Fault Tree dialog will be displayed listing either all of the events or all of the gates in the current project. 


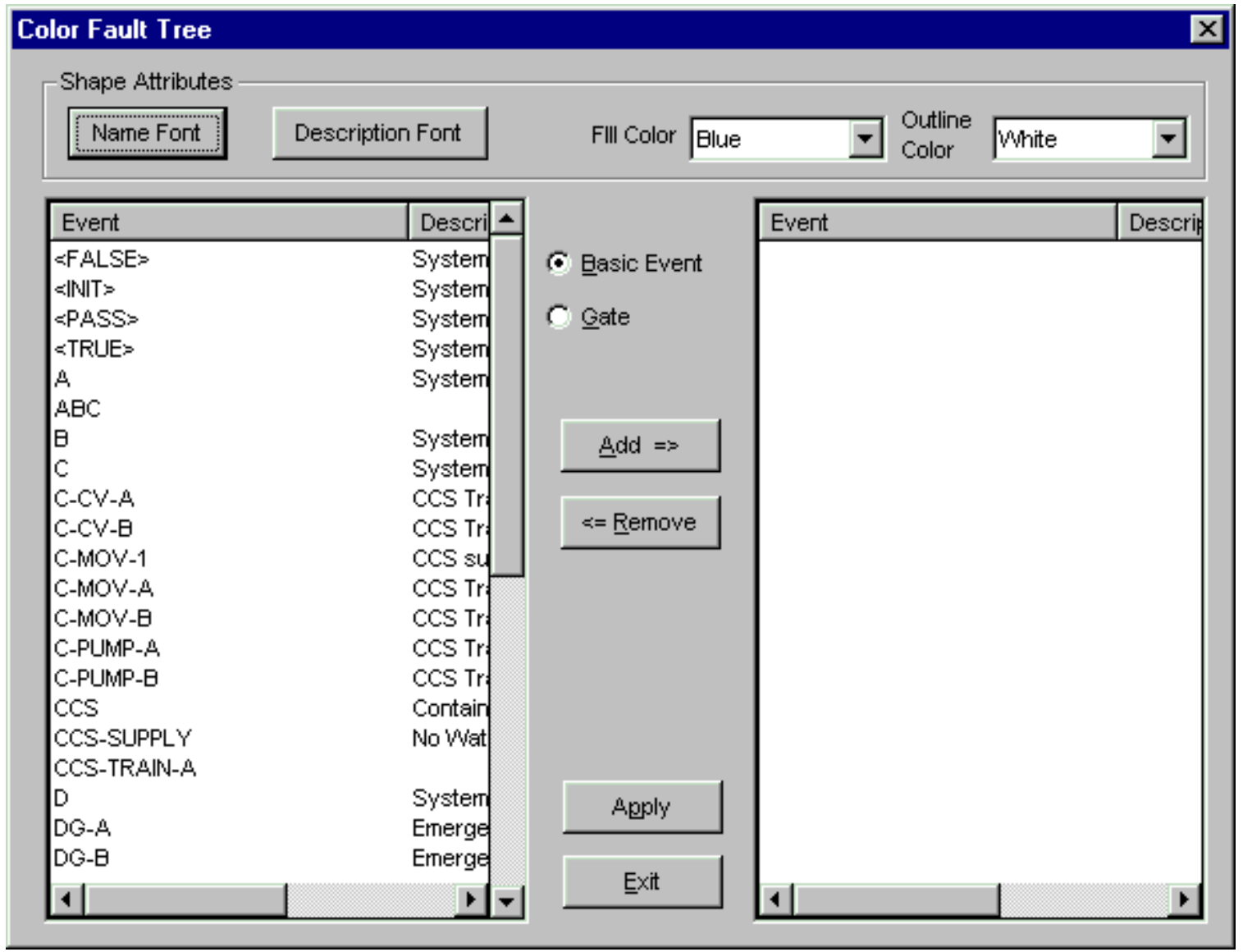

Set the desired shape display attributes. Use the Add and Remove buttons to create a list of selected gates or events from the current project. When the list and the attributes are satisfactory, press the Apply button to update the fault tree graphics.

\section{Shape Attributes}

Name Font - $\quad$ Select a font name, size, and color to apply to the selected event(s)/gate(s) names.

Description Font - Select a font name, size, and color to apply to the selected event(s)/gate(s) descriptions.

Fill Color - $\quad$ Select a color to apply to the selected event(s)/gate(s) shapes.

Outline Color - Select a color to apply to the selected event(s)/gate(s) shape outline.

\section{List Type}

Basic Event - When this option is selected, all of the basic events in the current project

Gate will be listed on the left.

When this option is selected, all of the gates in the current project will be listed on the left.

Add -

Add the selected event(s)/gate(s) from the list on the left, and add them $\mathrm{t}$ o the list on the right.

Remove Remove the selected event(s)/gate(s) from the list on the right. 
Apply - For each fault tree in the database, apply the currently selected shape attributes to each occurrence of the events/gates listed in the list on the right. If any fault trees have logic but no graphics, you will be prompted to optionally create the graphics.

Exit - $\quad$ Close the Color Fault Tree dialog.

Note: Choose your colors with care. For example, a white description font color will not show up on a white shape color, nor will blue name color show up on a blue background color.

\subsubsection{Fault Tree Page Numbering}

\subsubsection{Fault Tree Page Numbering}

\section{PURPOSE}

This option allows you to select a page numbering scheme to use for all fault trees in the current project. Three options are currently available: By Hierarchy, By Name, and By Level. The starting page number can also be set.

The By Name option orders fault trees strictly alphabetically. The By Level option orders top level fault trees first, followed by sub-trees. Within that order, fault trees are numbered alphabetically. The By Hierarchy option groups fault trees with their sub-trees. Top level trees appear in relative alphabetic order.

The dialog lists the name, description, number of transfers contained in the fault tree, top/sub tree status, and proposed page number assignment. Click any of the columns (except description) to view the list in order of that column. Click the column again to reverse the sort order.

Note: When a new fault tree is added to the project, an unused page number will be assigned to it. The page numbering utility must be run before the new fault tree's page number will fit into the selected page numbering scheme. Likewise, when a fault tree is deleted, there will be a hole in the page numbering scheme until the fault trees are renumbered using this utility.

\section{STEPS}

1. From the SAPHIRE menu select Utility.

2. Choose Fault Tree from the menu. Select the Number Pages sub-menu option.

3. The Fault Tree Page Numbering dialog will be displayed listing all of the fault trees in the current project.

4. Enter the desired Start Page number.

5. Choose the desired Re-Number button. The page numbers will be updated.

6. (Optional) Click on the Page Number column heading to view the list in the updated page number order.

7. Choose Save to update the page numbers, or Cancel to revert to the original numbers. 


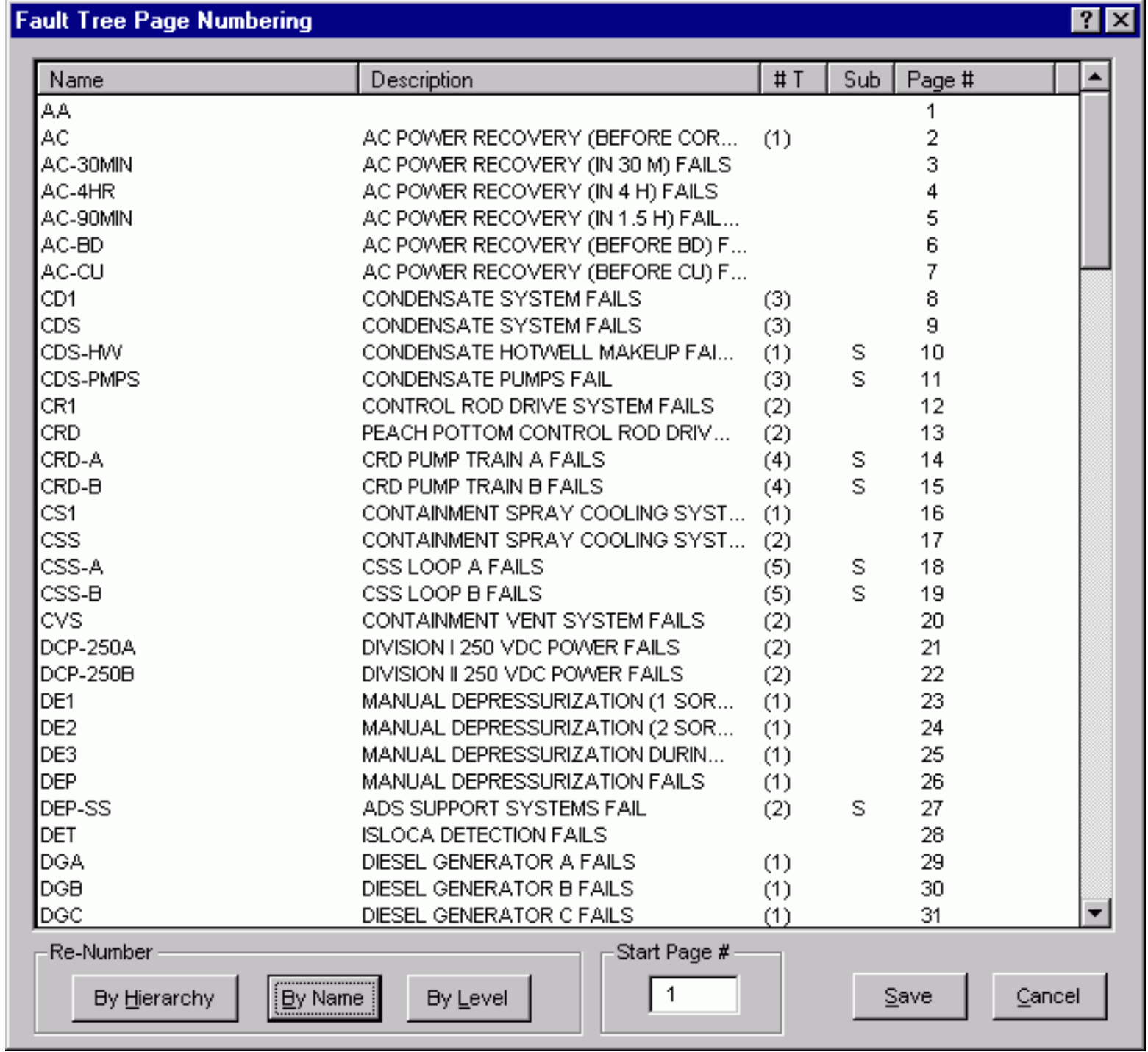

Click on the column headings to view the list in that column's order.

Re-Number

By Hierarchy - When this option is selected, fault trees and their referenced sub-trees will be grouped together. Top level fault trees appear in relative alphabetical order.

By Name - $\quad$ When this option is selected, fault trees will be numbered in alphabetical order.

By Level - $\quad$ When this option is selected, all top level trees will be numbered first, followed by all sub-trees. Within each level, the fault trees will be ordered alphabetically.

Start Page \# - $\quad$ Enter the desired page number of the first fault tree.

Save - Permanently assign the fault tree page numbers as currently shown in the fault tree list, and close the Page Numbering dialog.

Cancel - Close the Page Numbering dialog without changing the previously assigned page numbers. 


\subsubsection{Fault Tree Page Numbering Example}

Given a project containing the following fault trees,
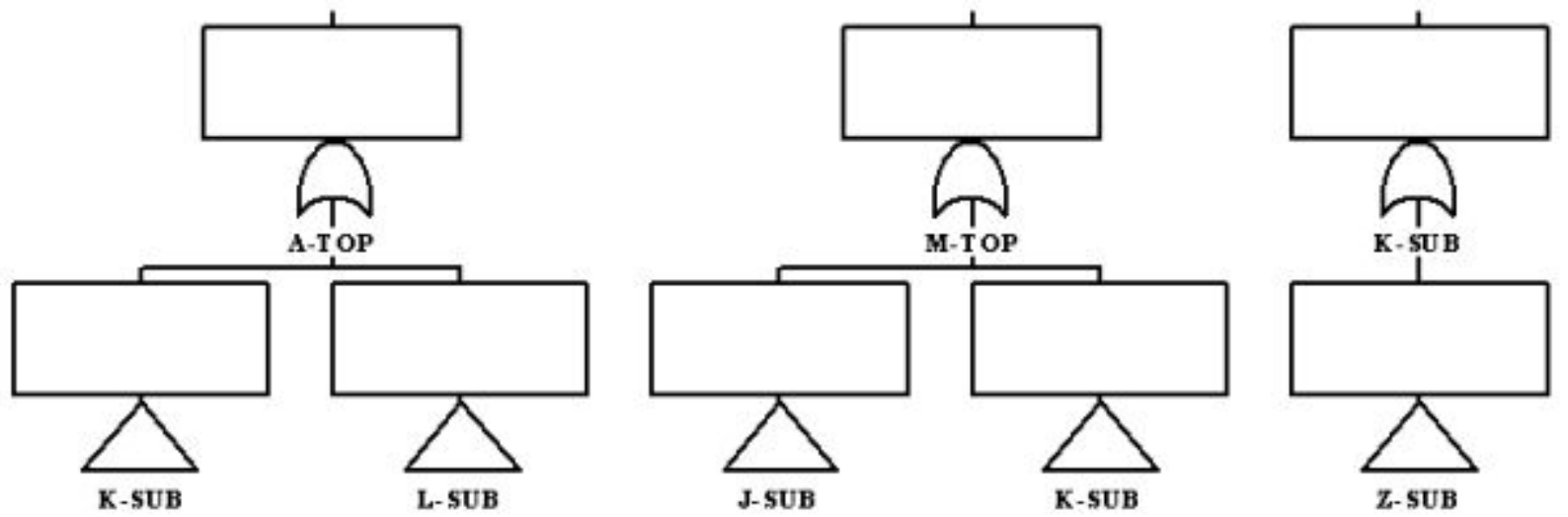

the table below shows the page numbers that would result from each ordering scheme.

\begin{tabular}{|l|l|l|l|}
\hline $\begin{array}{l}\text { Page } \\
\text { Number }\end{array}$ & $\begin{array}{l}\text { Order By } \\
\text { Hierarchy }\end{array}$ & $\begin{array}{l}\text { Order By } \\
\text { Name }\end{array}$ & $\begin{array}{l}\text { Order By } \\
\text { Level }\end{array}$ \\
\hline 1 & A-TOP & A-TOP & A-TOP \\
\hline 2 & K-SUB & J-SUB & M-TOP \\
\hline 3 & Z-SUB & K-SUB & J-SUB \\
\hline 4 & L-SUB & L-SUB & K-TOP \\
\hline 5 & M-TOP & M-TOP & L-SUB \\
\hline 6 & J-TOP & Z-SUB & Z-SUB \\
\hline
\end{tabular}

\subsubsection{Alpha to Graphics Conversion}

\subsubsection{Alpha to Graphics}

\section{PURPOSE}

This option allows you to convert the alphanumeric logic for a fault tree or sub-tree to a graphical format. Use this option when you define the fault tree logic with an ASCII file or use the logic editor to change the logic for a fault tree. The graphics and logic information for a fault tree are two separate entities, therefore, a conversion must be made if any changes are made to the alphanumeric logic. However, if changes are made the to the graphical representation, the changes are automatically reflected in the alphanumeric logic. 


\section{STEPS}

1. From the SAPHIRE menu select Utility.

2. Choose Fault Tree from the menu. Select the Alpha to Graphics sub-menu option.

3. The Alpha to Graphics dialog will be displayed listing all of the fault trees in the current project.

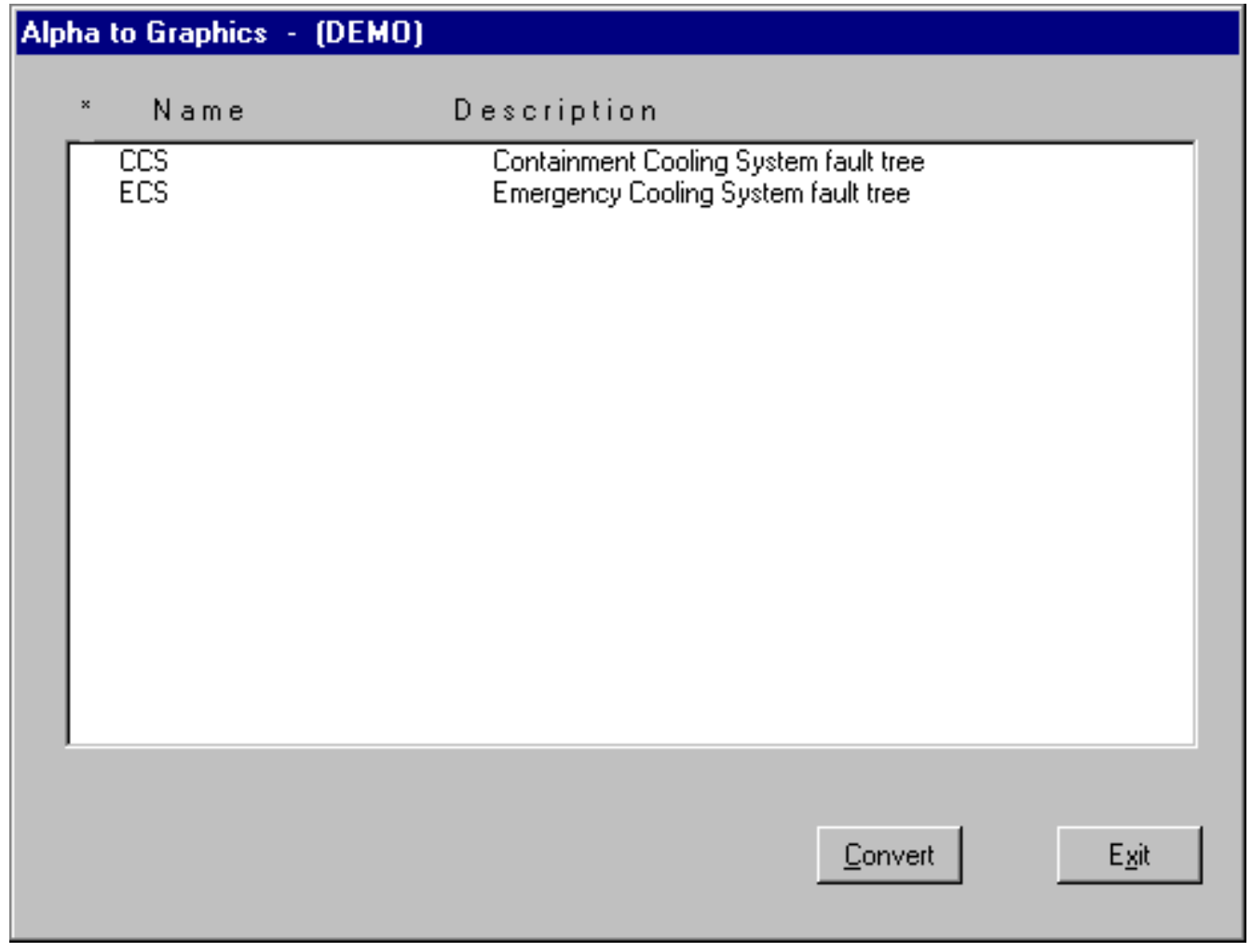

Convert - $\quad$ Continue with the conversion process.

Exit - Close the Alpha to Graphics dialog without performing conversions.

\subsubsection{Convert}

\section{PURPOSE}

Convert the selected alphanumeric format file(s) to a graphical format.

\section{STEPS}

1. From the SAPHIRE menu select Utility.

2. Choose Fault Tree from the menu.

3. Select the Alpha to Graphics sub-menu option. The Alpha to Graphics dialog will be displayed.

4. Highlighted the desired fault tree(s) and choose the Convert button.

5. A dialog, as shown below, will be displayed. Make the selections as desired and choose the OK button. 


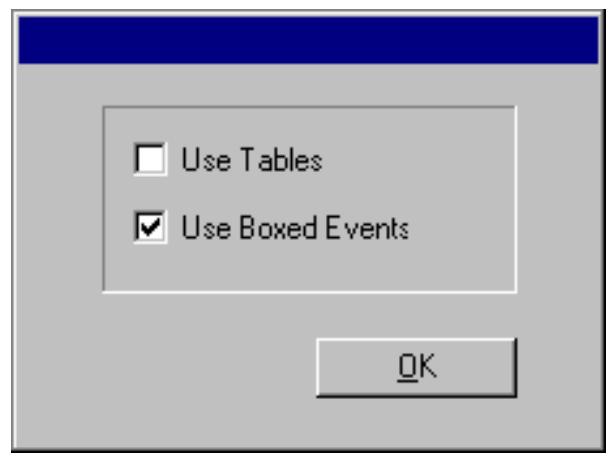

If neither check box is selected, normal basic events are used in the model.

Use Tables -

Use Boxed Events -

OK -
For each logic gate with many basic events as inputs, create a table of basic events, using the Primary name field. SAPHIRE processes the table of basic events as if they were separate symbols.

Create each basic events with a box for descriptive text. The text from the description field of the associated event will be placed in the box. This does not influence the logic of the fault tree, but adds clarity to the model for those using and reviewing it.

Complete the conversion process.

\subsubsection{Extract Fault Tree Graphics}

\subsubsection{Extract Fault Tree Graphics}

\section{PURPOSE}

This option allows you to extract fault trees from the database. When you create a fault tree diagram and save it, the .DLS file is saved in the database as well as in a temporary .DLS file in the current project directory. When you delete the .DLS file, the file still exists in the database. With this option, you can extract the stored .DLS file from the database.

\section{STEPS}

1. From the SAPHIRE menu select Utility.

2. Choose Fault Tree from the menu.

3. Select the Extract Graphics sub-menu option. The Extract Graphic Fault Trees dialog will be displayed listing all of the fault trees in the current project. 


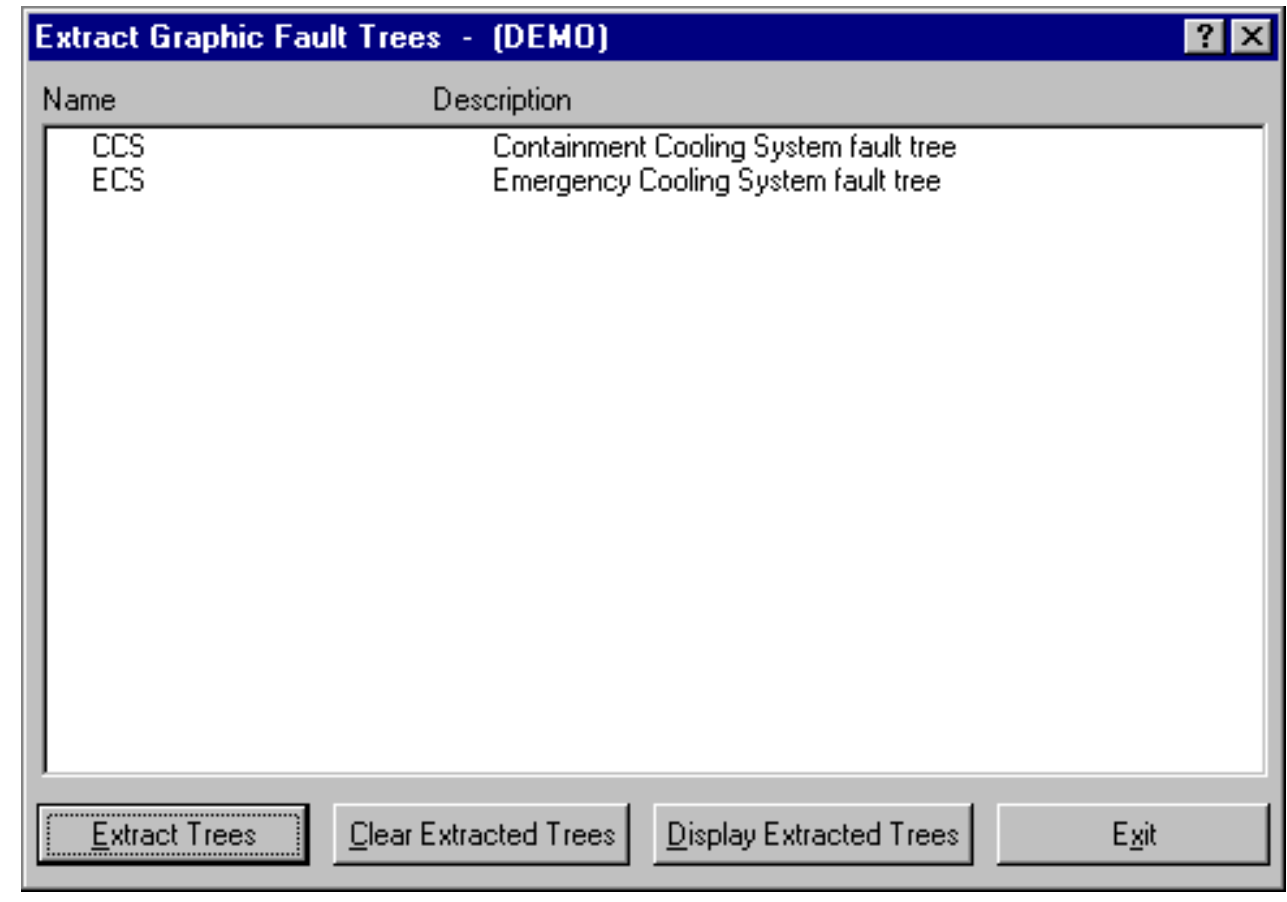

\section{Extract Trees -}

\section{Clear Extracted Trees -} Display Extracted Trees Exit -
Copy the specified trees out of the database.

Delete all extracted trees from the project directory.

Display all available extracted trees.

Close the Extract Graphic Fault Trees dialog.

\subsubsection{Extract Trees}

This option allows you to copy the highlighted trees from the database to a file in the directory of your choice. When complete, a message indicating that the tree was successfully extracted will be displayed in the message bar of the SAPHIRE window. The .DLS or .ETG file name of the extracted tree will be included in the message.

The extraction process does not delete the tree from the database.

\subsubsection{Clear Extracted Trees}

This option allows you to clear all extracted tree files from the current project directory. When you choose this button, a warning dialog, as shown below, will be displayed.

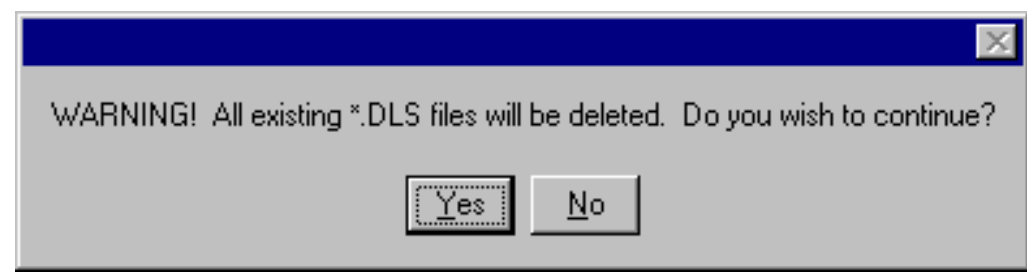

Yes - Delete all existing extracted tree files (.DLS or .ETG) from the current project directory. When complete the message, "All extracted files cleared..." will be displayed in the message bar of the SAPHIRE window. 
No - Terminate the delete process.

\subsubsection{Display Extracted Trees}

This option lists all the extracted trees, by file name, residing in the current project directory. When you choose this button, the Extracted Fault Trees or Extracted Event Trees dialog will be displayed.

The example here shows the dialog for fault trees.

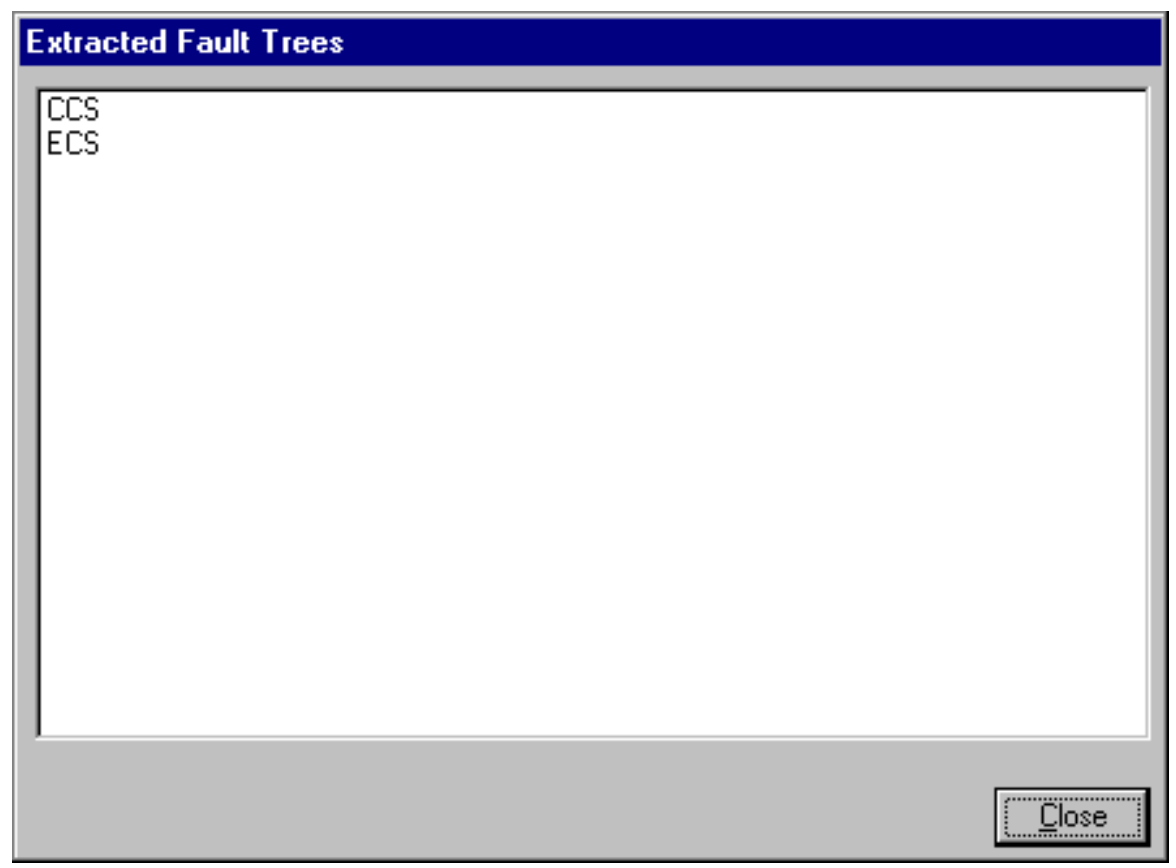

Close - Close the Extracted Fault Trees or Extracted Event Trees dialog.

\subsubsection{Load Fault Tree Graphics}

\subsubsection{Load Fault Tree Graphics}

\section{PURPOSE}

This option allows you to load graphic files into the database. When you perform this operation, the selected file will be converted and loaded into the existing fault tree record whose name matches that of the file. If no record exists, SAPHIRE will create a new fault tree record using the file name for the name of the fault tree in the database.

\section{STEPS}

1. From the SAPHIRE menu select Utility.

2. Choose Fault Tree from the menu. 
3. Select the Load Graphics sub-menu option. The Load Graphic Fault Trees dialog will be displayed listing all of the .DLS files in the current project directory.

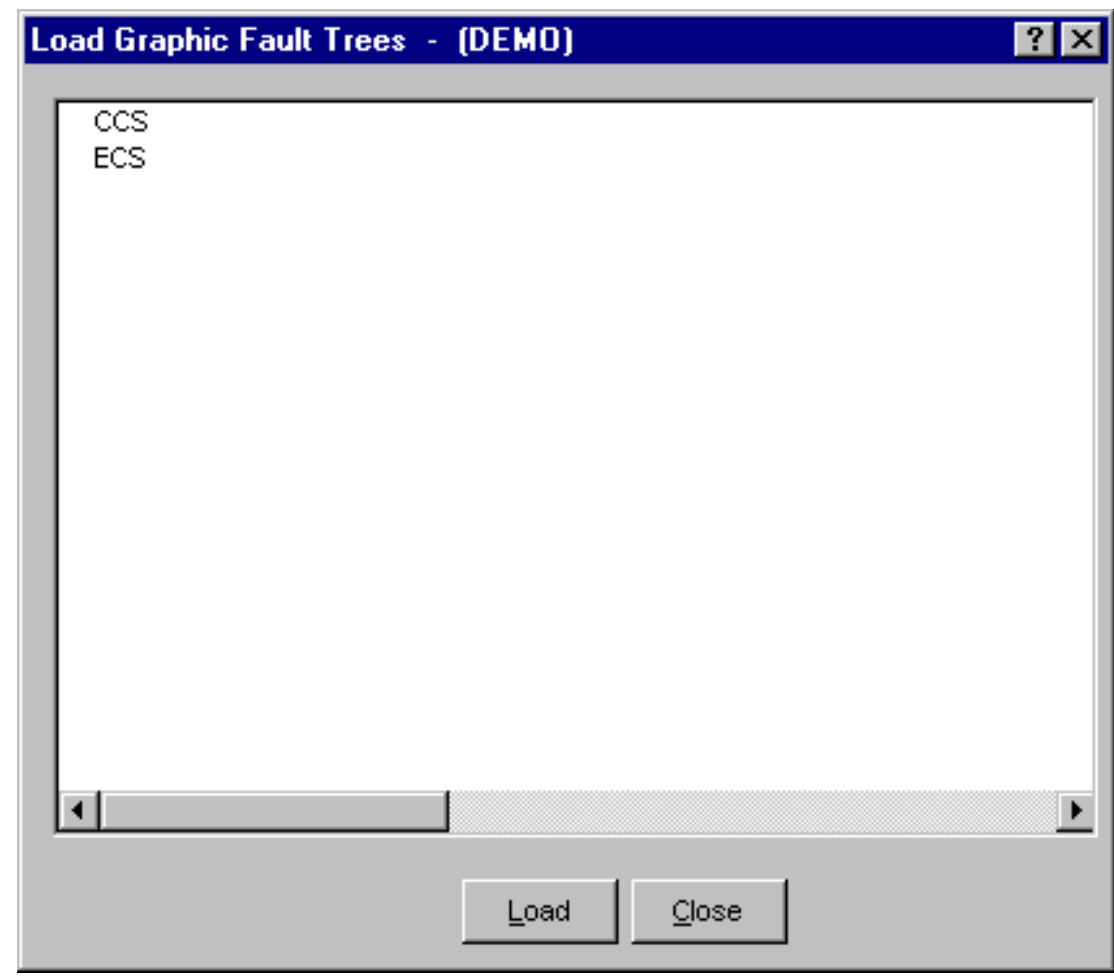

Load - Perform the load operation.

Close - Close the Load Graphic Fault Trees dialog.

\subsection{Event Tree Utility Options}

\subsubsection{Event Tree Utility Options}

\section{PURPOSE}

This option allows you to load or extract event tree graphics files.

\section{STEPS}

1. From the SAPHIRE menu select Utility.

2. Choose Event Tree from the menu. Select a sub-menu option. 


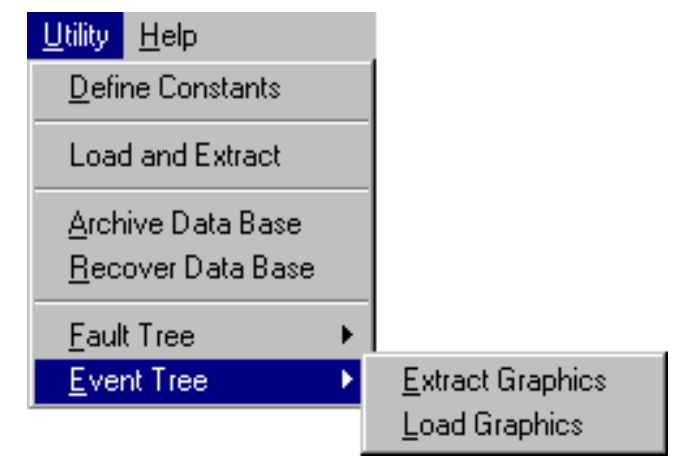

$\begin{array}{ll}\text { Extract Graphics - } & \text { Extract event trees from the database. } \\ \text { Load Graphics - } & \text { Load event tree graphic files into the database. }\end{array}$

\subsubsection{Event Tree Page Numbering}

\section{PURPOSE}

This option allows you to select a page numbering scheme to use for all event trees in the current project. Three options are currently available: By Hierarchy, By Name, and By Level. The starting page number can also be set.

The By Name option orders event trees strictly alphabetically. The By Level option orders top level event trees first, followed by sub-trees (or transfer trees). Within that order, event trees are numbered alphabetically. The By Hierarchy option groups event trees with their sub-trees. Top level trees appear in relative alphabetic order.

The dialog lists the name, description, number of transfers contained in the event tree, top/sub tree status, and proposed page number assignment. Click any of the columns (except description) to view the list in order of that column. Click the column again to reverse the sort order.

Note: When a new event tree is added to the project, an unused page number will be assigned to it. The page numbering utility must be run before the new event tree's page number will fit into the selected page numbering scheme. Likewise, when an event tree is deleted, there will be a hole in the page numbering scheme until the event trees are renumbered using this utility.

\section{STEPS}

1. From the SAPHIRE menu select Utility.

2. Choose Event Tree from the menu. Select the Number Pages sub-menu option.

3. The Event Tree Page Numbering dialog will be displayed listing all of the fault trees in the current project.

4. Enter the desired Start Page number.

5. Choose the desired Re-Number button. The page numbers will be updated.

6. (Optional) Click on the Page Number column heading to view the list in the updated page number order.

7. Choose Save to update the page numbers, or Cancel to revert to the original numbers. 


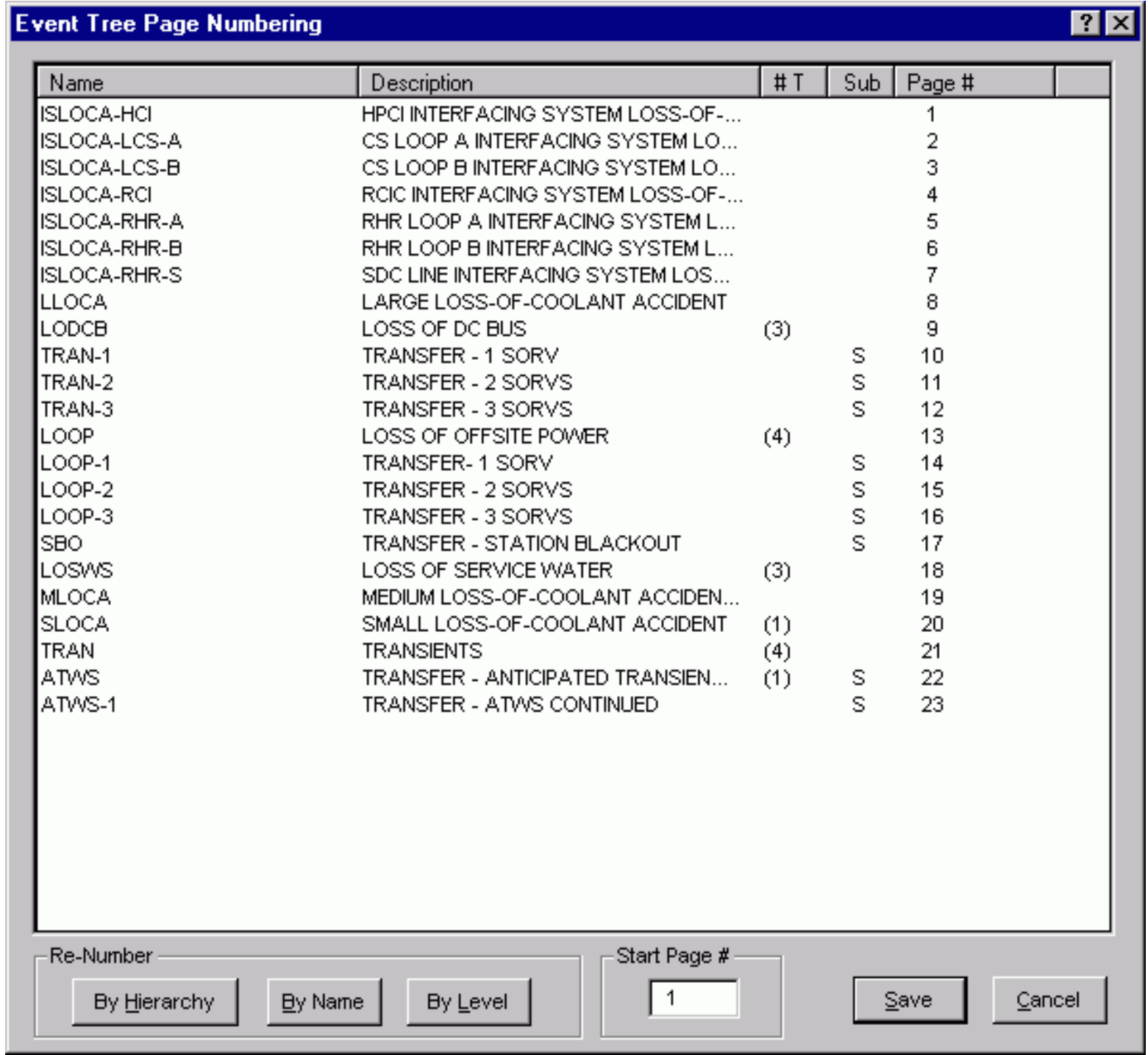

Click on the column headings to view the list in that column's order.

Re-Number

By Hierarchy - When this option is selected, event trees and their referenced transfer trees will be grouped together. Top level event trees appear in relative alphabetical order.

By Name - $\quad$ When this option is selected, event trees will be numbered in alphabetical order.

By Level - $\quad$ When this option is selected, all top level trees will be numbered first, followed by all transfer trees. Within each level, the event trees will be ordered alphabetically.

Start Page \# -

Enter the desired page number of the first event tree.

Save -

Permanently assign the event tree page numbers as currently shown in the event tree list, and close the Page Numbering dialog.

Cancel -

Close the Page Numbering dialog without changing the previously assigned page numbers. 


\subsubsection{Extract Event Tree Graphics}

\subsubsection{Extract Event Tree Graphics}

\section{PURPOSE}

This option allows you to extract event trees from the database. When you create an event tree diagram and save it, the .ETG file is saved in the database as well as in a temporary .ETG file in the current project directory. When you delete the .ETG file, the file still exists in the database. With this option, you can extract the stored .ETG file from the database.

\section{STEPS}

1. From the SAPHIRE menu select Utility.

2. Choose Event Tree from the menu.

Select the Extract Graphics sub-menu option. The Extract Graphic Event Trees dialog will be displayed listing all of the event trees in the current project.

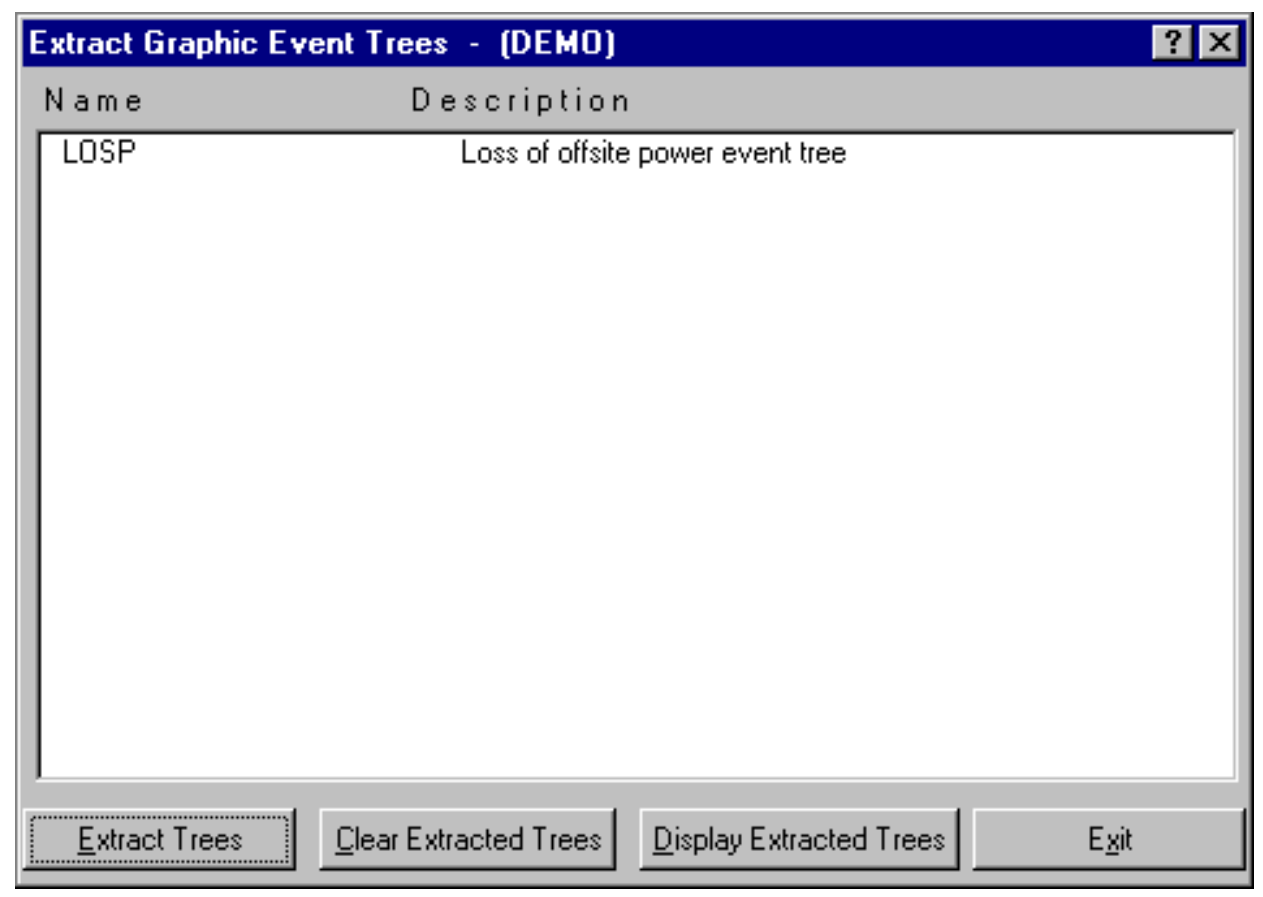

Extract Trees - $\quad$ Remove the specified trees from the database.

Clear Extracted Trees - Delete all extracted trees from the project directory.

Display Extracted Trees-Display all extracted trees to date.

Exit - Close the Extract Graphic Event Trees dialog. 


\subsubsection{Extract Trees}

This option allows you to copy the highlighted trees from the database to a file in the directory of your choice. When complete, a message indicating that the tree was successfully extracted will be displayed in the message bar of the SAPHIRE window. The .DLS or.ETG file name of the extracted tree will be included in the message.

The extraction process does not delete the tree from the database.

\subsubsection{Clear Extracted Trees}

This option allows you to clear all extracted tree files from the current project directory. When you choose this button, a warning dialog, as shown below, will be displayed.

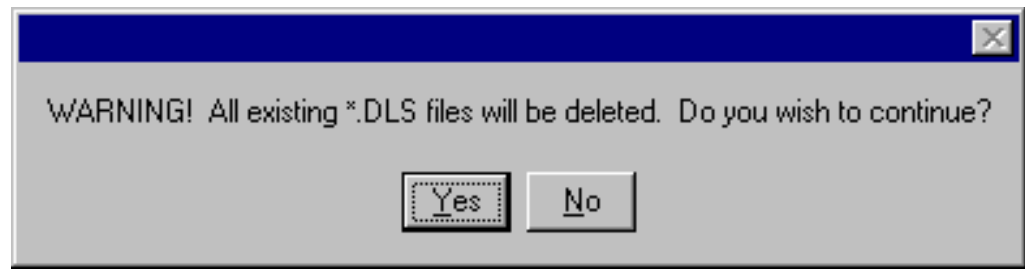

Yes - Delete all existing extracted tree files (.DLS or .ETG) from the current project directory. When complete the message, "All extracted files cleared..." will be displayed in the message bar of the SAPHIRE window.

No - Terminate the delete process.

\subsubsection{Display Extracted Trees}

This option lists all the extracted trees, by file name, residing in the current project directory. When you choose this button, the Extracted Fault Trees or Extracted Event Trees dialog will be displayed.

The example here shows the dialog for fault trees. 


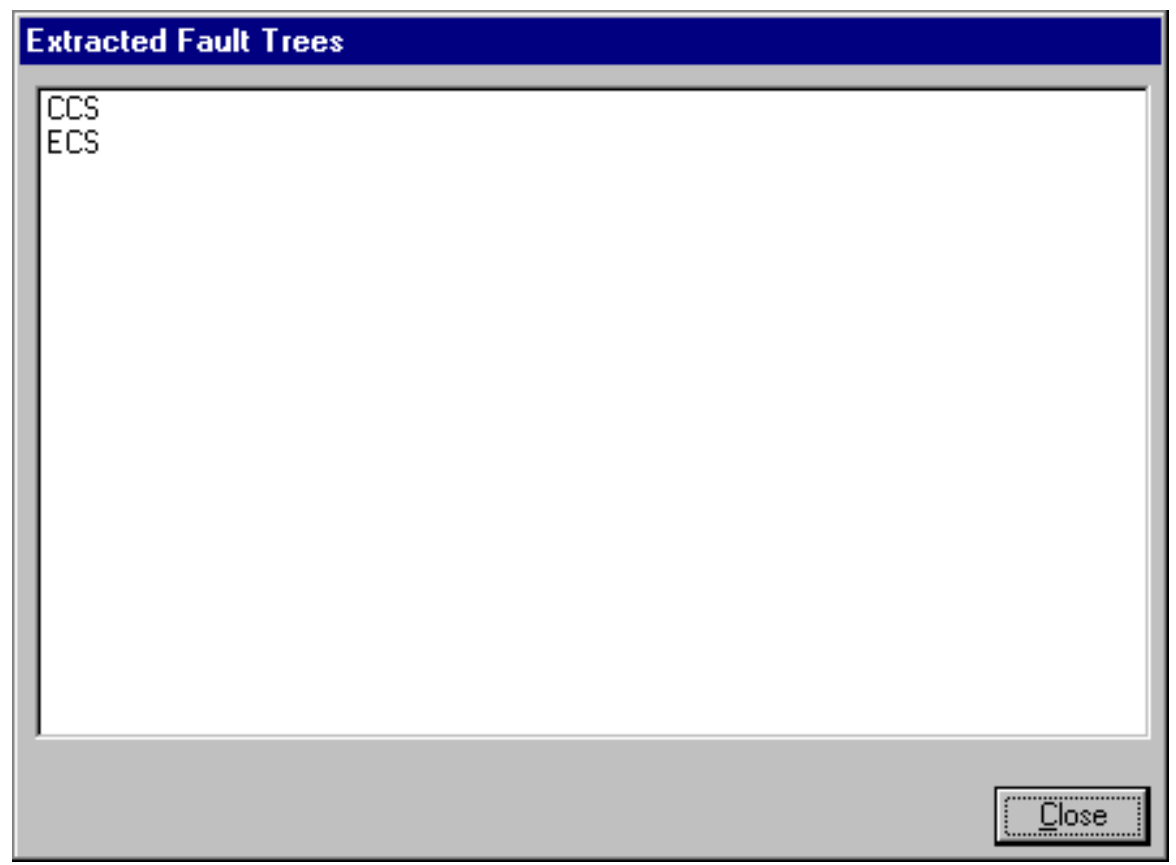

Close - Close the Extracted Fault Trees or Extracted Event Trees dialog.

\subsubsection{Load Event Tree Graphics}

\subsubsection{Load Event Tree Graphics}

\section{PURPOSE}

This option allows you to load event tree graphic files into the database. When you perform this operation, the selected file will be converted and loaded into the existing event tree record whose name matches that of the file. If no record exists, SAPHIRE will create a new event tree record using the file name for the name of the event tree in the database.

\section{STEPS}

1. From the SAPHIRE menu select Utility.

2. Choose Event Tree from the menu.

3. Select the Load Graphics sub-menu option. The Load Graphic Event Trees dialog will be displayed listing all of the .ETG files in the current project directory. 


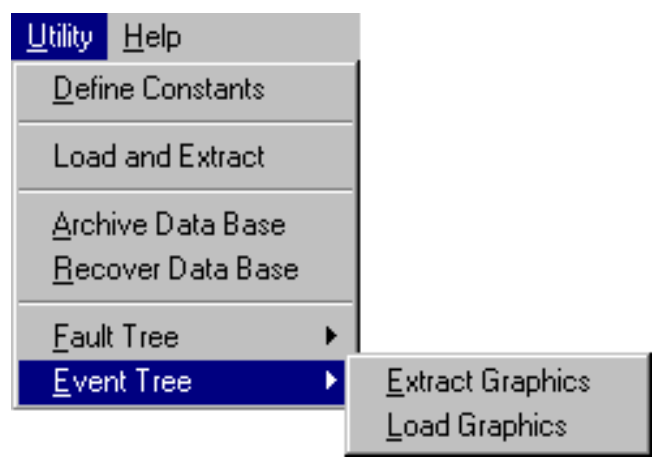

Load - Perform the load operation.

Close - Close the Load Graphic Event Trees dialog.

\subsection{View Error Log}

\subsubsection{View Error Log}

\section{PURPOSE}

This option allows you to view the results of the SAPHIRE error log. An error log is produced each time basic event data is generated. The log reports errors, warnings, and inconsistencies detected in the current event data. The error log can remain open while other SAPHIRE dialogs are accessed, allowing you to see the error list while making any desired corrections. The error log is automatically cleared upon exiting SAPHIRE.

\section{STEPS}

1. From the SAPHIRE menu select Utility.

2. Choose View Error Log from the menu. The View Error Log dialog will be displayed. 
Unc Var: (Type $L=2.44 E-003$ ) $>$ (Constr Noninfo $=8.01 E-004)->D G-A$

Unc Var: (Type $L=2.44 E-003$ ) $>$ (Constr Noninfo $=8.01 E-004)->D G-A$

Unc Var: (Type L=2.44E-003) > (Constr Noninfo=8.01E-004) $>$ DG-B

Unc Var: (Type L=2.44E-003) > (Constr Noninfo $=8.01 \mathrm{E}-004)$ - $>$ DG-B

Unc Var: (Type $L=3.22 \mathrm{E}+0011)>$ (Constr Noninfo $=1.06 \mathrm{E}+001)$ - $>\mathrm{L} 0 \mathrm{SP}$

Unc Var: (Type $L=3.22 E+001$ ) > (Constr Noninfo $=1.06 E+001)>>0 S P$

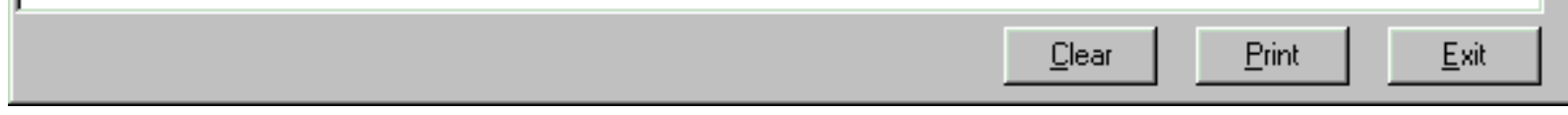

Print - Print the error log.

Clear - Clear the error log.

Exit - Close the View Error Log dialog.

\subsection{Version Date}

\subsubsection{Version Date}

\section{PURPOSE}

This option allows you to set the version date of the current project (i.e., model).

\section{STEPS}

1 From the SAPHIRE menu select Utility.

2. Choose Version Date from the menu. The Set Version Dates dialog will be displayed. 


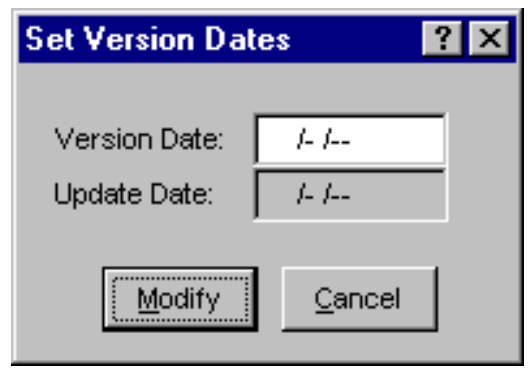

Version Date - Enter the new version date in YYYY/MM/DD format. The version date indicates the date the model development was complete.

Update Date - Displays the last date that the model was updated in YYYY/MM/DD format. The update date is changed by SAPHIRE whenever any of the following are changed: fault tree logic, fault tree graphics, event tree logic, or event tree graphics. The update date will also be changed if a basecase update is performed.

Modify - $\quad$ Close the Set Version Dates dialog and change the version date for the project. When you choose this button, the Warning dialog, below, will be displayed.

Cancel - $\quad$ Close the Set Version Dates without changing the project's version date.

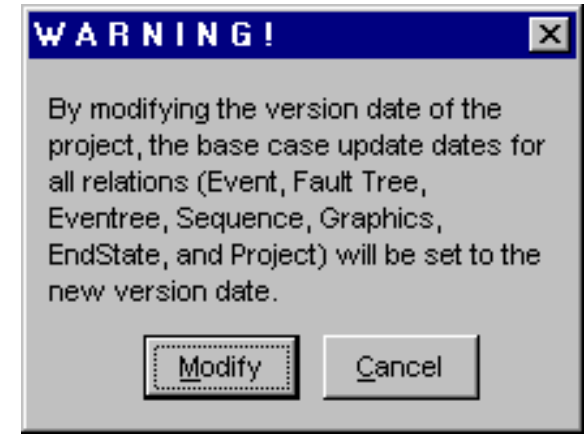

Modify - $\quad$ Close the Warning dialog and change the version date for the project.

Cancel - $\quad$ Close the Warning dialog without changing the project's version date.

\subsection{Importance Measures Wizard}

\subsubsection{Importance Measures Wizard}

\section{PURPOSE}

The Importance Measures Wizard is an extension of the basic Importance Measurescalculations originally provided in earlier versions of SAPHIRE. It provides a convenient interface for extending importance concepts for single events into groups of events (components). It also provides the option to readily recalculate rather than just requantify cut sets. This may result in more accurate answers since event interactions will be more fully accounted for.

\section{STEPS}

1. From the SAPHIRE menu select Utilities | Importance Measures Wizard. 
2. The Importance Measure Wizard dialog will be displayed.

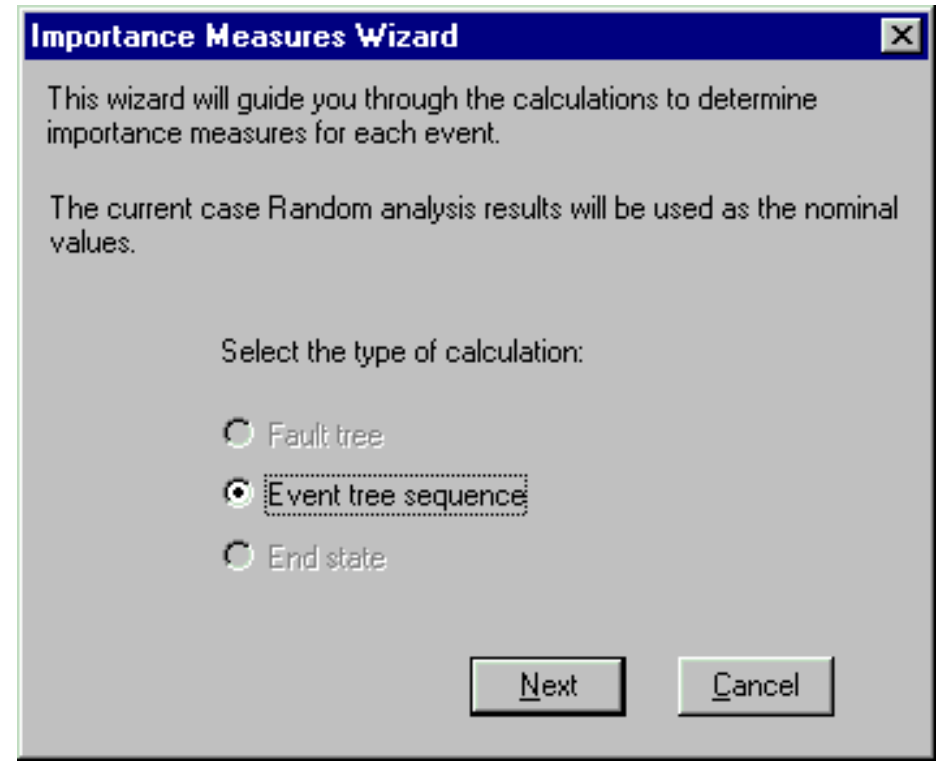

From this dialog, you can select the type of cut sets to analyze: Fault tree, Event tree sequence, or End state.

\section{Navigation Options}

Next - $\quad$ Proceed to the next step in the Importance Measures Wizard process.

Cancel - Close the Importance Measures Wizard dialog.

\subsubsection{Component Group Definition}

\section{PURPOSE}

The Component Groups dialog allows you to select and/or group basic events into component groups. These groups will be analyzed as single entities later in the Importance Measures wizard.

The Mask feature allows you to quickly group basic events together based on the schema of their names. Then, the Component and Event features can be used to refine the groupings as needed. Finally, the Study options allow you to save the component group definitions for later access.

\section{STEPS}

1. From the SAPHIRE menu select Utilities | Importance Measures Wizard.

2. The Importance Measures Wizard dialog will be displayed. Select the type of cut sets to analyze, and choose the Next button. The Component Groups dialog will appear. 


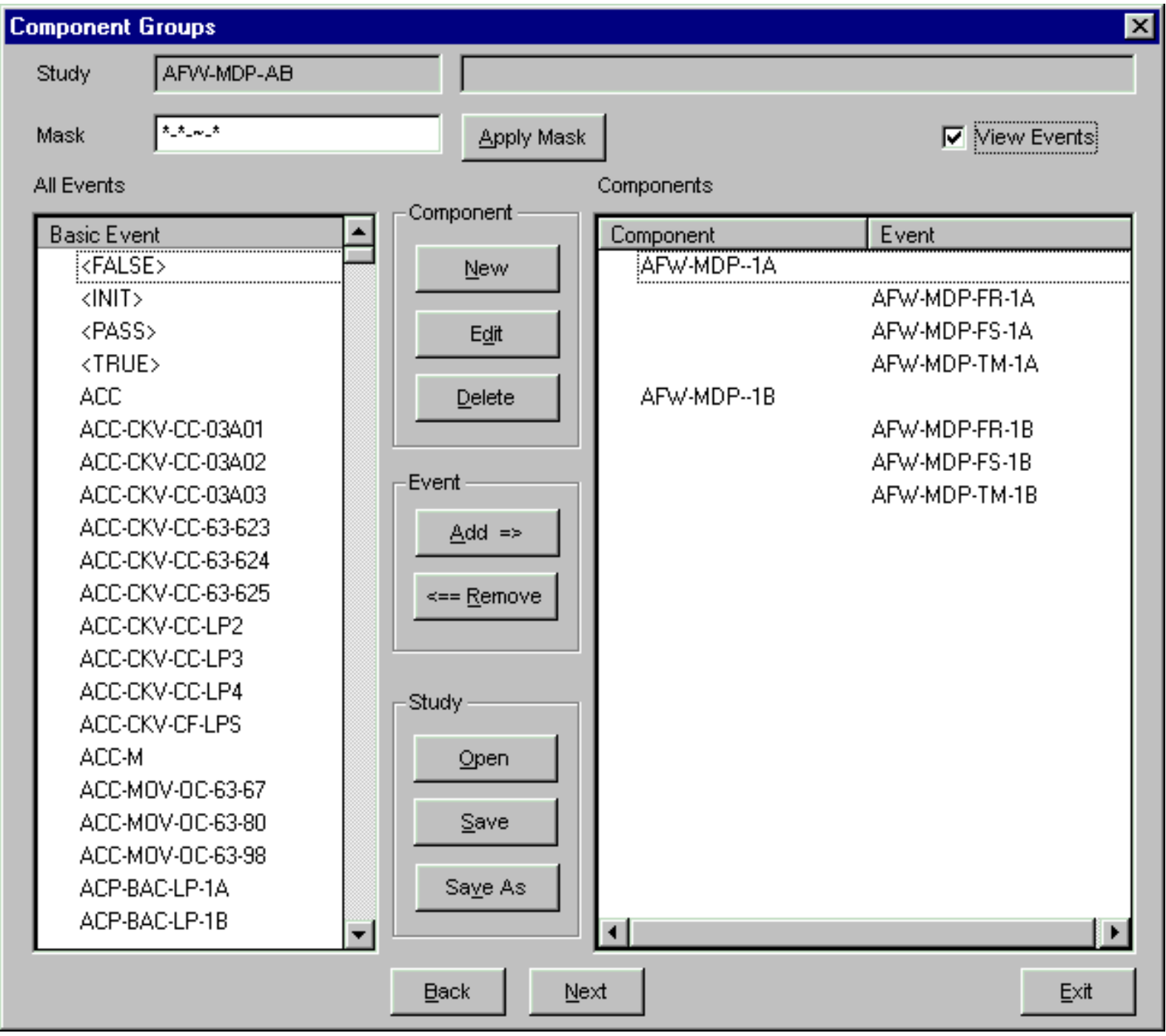

Study - $\quad$ The name and description of the currently selected study. If there are no studies defined in the project, the study name will read "New", until a study definition is saved.

Mask Options

Mask - Define a wild card mask to collectively assign basic events from the project to component groups based on the naming scheme of the basic events.

Apply Mask - $\quad$ Apply the Mask to create component groups. Warning: all existing component groups will be replaced when this option is selected.

View Events - When this option is selected, basic events appear in the Component list beneath the component group to which they are assigned. When this option is not selected, the Component list contains only component names.

\section{Component Options}

New - Create a component group name to include in the Component list.

Edit - Rename the selected component group.

Delete - Remove the component group and its events from the study.

\section{Event Options}


Add - $\quad$ Add the selected event(s) from the All Events list to the selected component group in the Component list. A check mark will appear beside each selected event in the All Events list, indicating it belongs to a component group.

Study Options

Open - Load a previously saved "study", or component groups definition.

Save - Save the current study.

Save As - Save the currently defined component groups definition as a study.

Navigation Options

Back - $\quad$ Return to the previous step in the Importance Measures Wizard process.

Next - $\quad$ Proceed to the next step in the Importance Measures Wizard process.

Exit - $\quad$ Close the Importance Measures Wizard dialog.

\subsubsection{Component Group Masks}

* (asterisk) - $\quad$ Designates a group of one or more characters to be used to define a group. Each unique combination of characters indicates a distinct component.

$\sim$ (twiddle) - $\quad$ Designates a group of one or more characters not to be used to define a group. That is, this part of a basic event name does not contribute to the distinctness of a component.

separator character(s) - Designates boundary marker(s) within the naming scheme.

\section{Example 1}

PRA models typically use a naming scheme for basic events that includes the system, component, failure mode, and possibly train, divided by a separator character, such as "_" or "_".

To combine events into groups of distinct train, system, and components, a mask will use a * (asterisk) in the character positions for train, system, and component, and a (twiddle) in the failure mode and train positions. The * (asterisk) and $\sim$ (twiddle) characters will be split up by a separator character(s), which indicate the boundaries. So, if the naming scheme follows the format SYS_COMP_FM_T, the desired mask would be ** ${ }^{*} *$

Example 2

Consider the following events:

M-CHILD-JOHN M-CHILD-JAKE

M-CHILD-BOB M-ADULT-JOHN

M-ADULT-JACK F-CHILD-SUSAN

F-CHILD-JANE F-ADULT-SHIRLEY

GEORGE

The mask $*_{-} *_{-} \sim$ would produce the following groups:

M-CHILD-

M-CHILD-JOHN M-CHILD-JAKE M-CHILD-BOB

M-ADULT-

M-ADULT-JOHN M-ADULT-JACK 
F-CHILD-

F-CHILD-SUSAN F-CHILD-JANE

F-ADULT-

F-ADULT-SHIRLEY

GEORGE

GEORGE

Note: George starts out as a possible match to the mask specifications, but does not conform to the entire mask, so it is placed into a single member group, "as is".

Alternatively, the mask $\sim_{-} *_{-}$would produce the following groups:

-CHILD-

M-CHILD-JOHN M-CHILD-JAKE M-CHILD-BOB

F-CHILD-SUSAN F-CHILD-JANE

-ADULT-

M-ADULT-JOHN M-ADULT-JACK F-ADULT-SHIRLEY

Note: GEORGE does not appear in any groups, because it never even begins to match the mask specification.

\subsubsection{Study List}

\section{PURPOSE}

The Study List dialog allows you select and manage studies created through the Importance Measures Wizard. Each study consists of a collection of events grouped into components. 


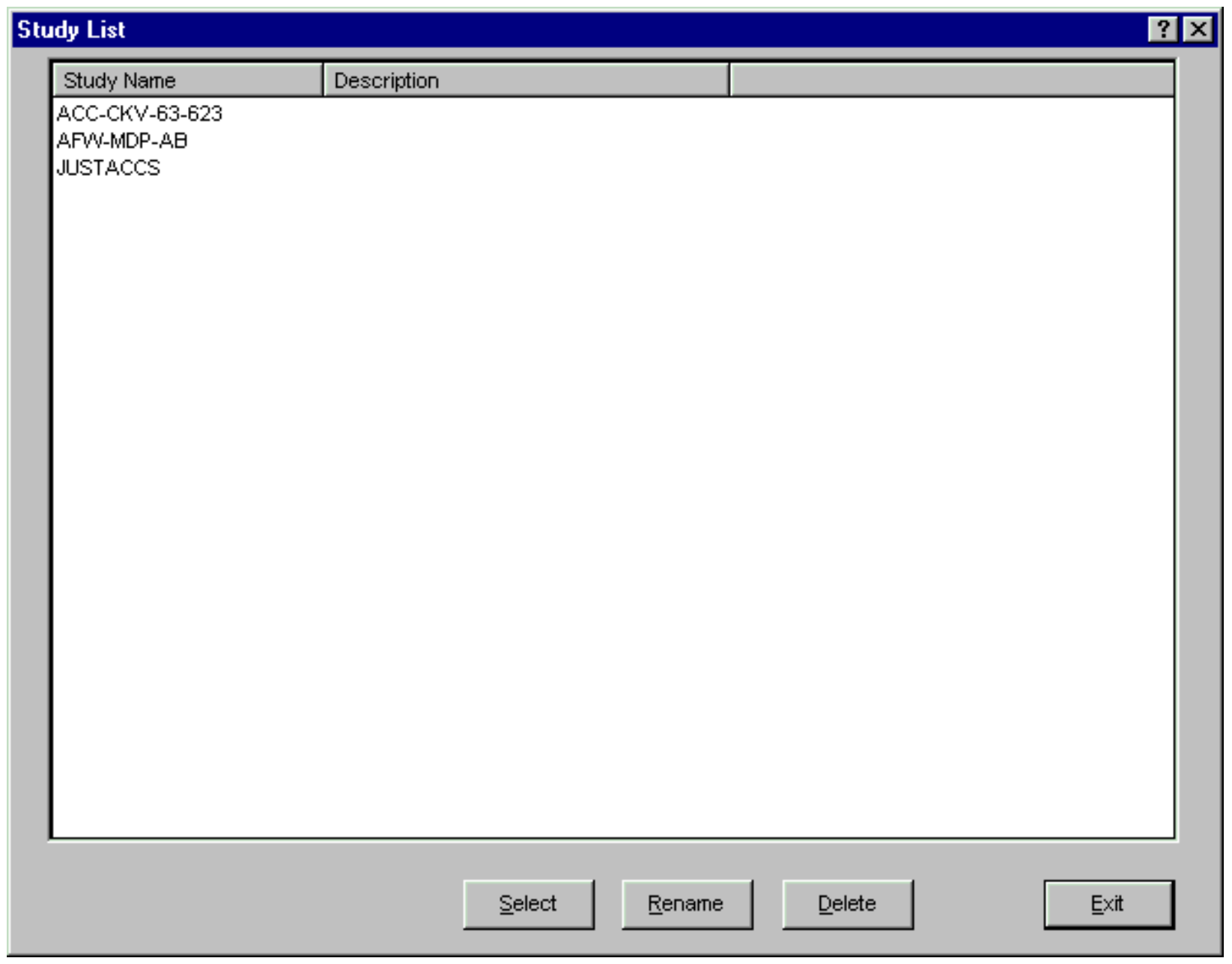

Select - $\quad$ Load the selected study into the Component Group dialog.

Rename - $\quad$ Rename the selected study.

Delete - $\quad$ Delete the selected study.

Exit - $\quad$ Return to the Component Group dialog without selecting a study.

\subsubsection{Select Calculation Method}

\section{PURPOSE}

From this dialog, you can choose to either requantify the existing cut sets, or generate new cut sets for each component group. The requantify option is generally much faster, but the regenerate option may be more accurate, since any interactions between events will be more fully taken into account. 


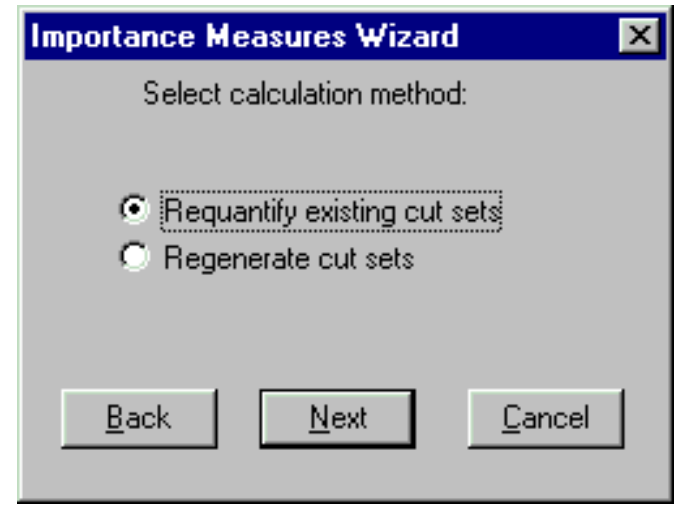

\section{Navigation Options \\ Back - $\quad$ Return to the previous step in Importance Measures Wizard process. \\ Next - $\quad$ Proceed to the next step in the Importance Measures Wizard. \\ Cancel - Cancel the Importance Measures Wizard calculations.}

\subsubsection{Evaluate Importance}

\section{PURPOSE}

The Importance Measures Wizard will perform the importance evaluations according to the inputs given in earlier steps of the wizard. If the option to regenerate the cut sets was selected from an earlier step in the Importance Measures Wizard, you will be prompted to enter the cut set probability. This value should match the one used to generate the nominal cut sets.

Depending upon the size of the cut sets and the number of component groups, this process could take some time to complete.
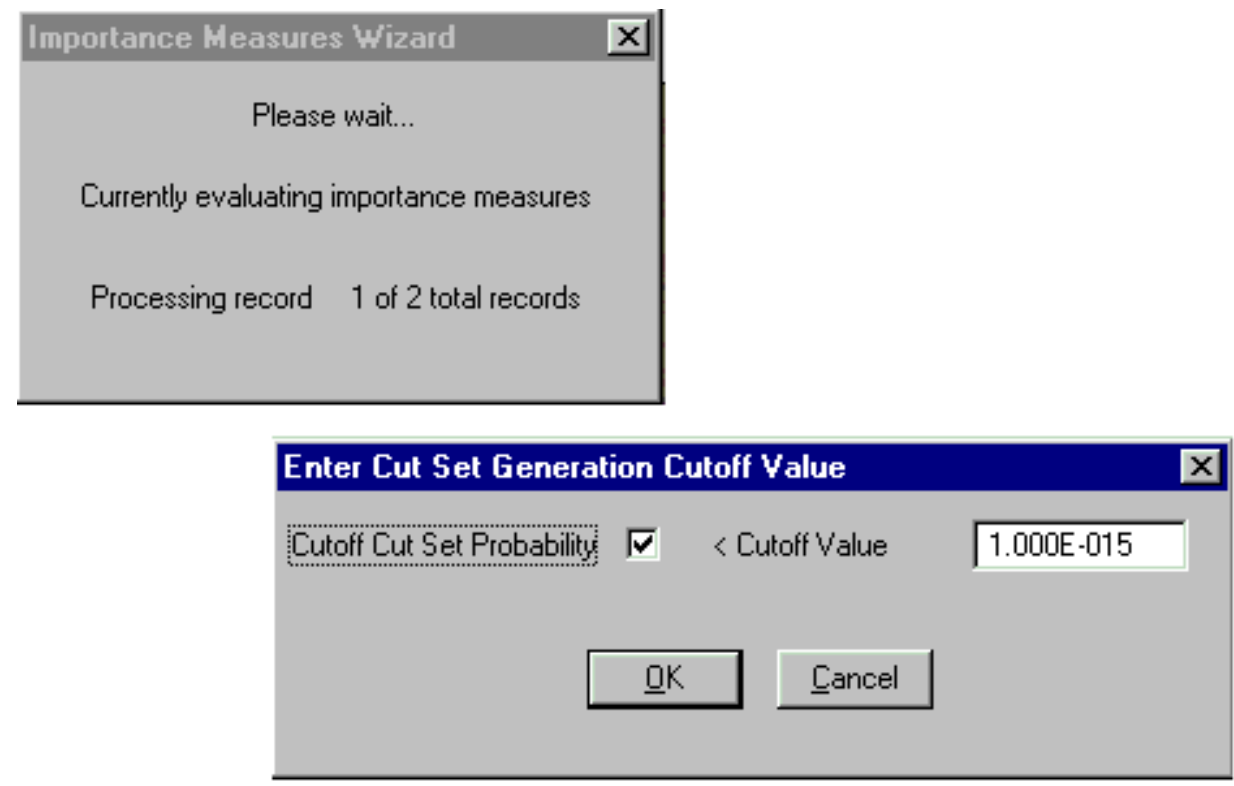


\section{Navigation Options}

OK - Perform the importance evaluation .

Cancel - Cancel the Importance Measures Wizard calculation.

\subsubsection{Component Importance Results}

\section{PURPOSE}

The Component Importance dialog displays the results of the importance measures evaluation.

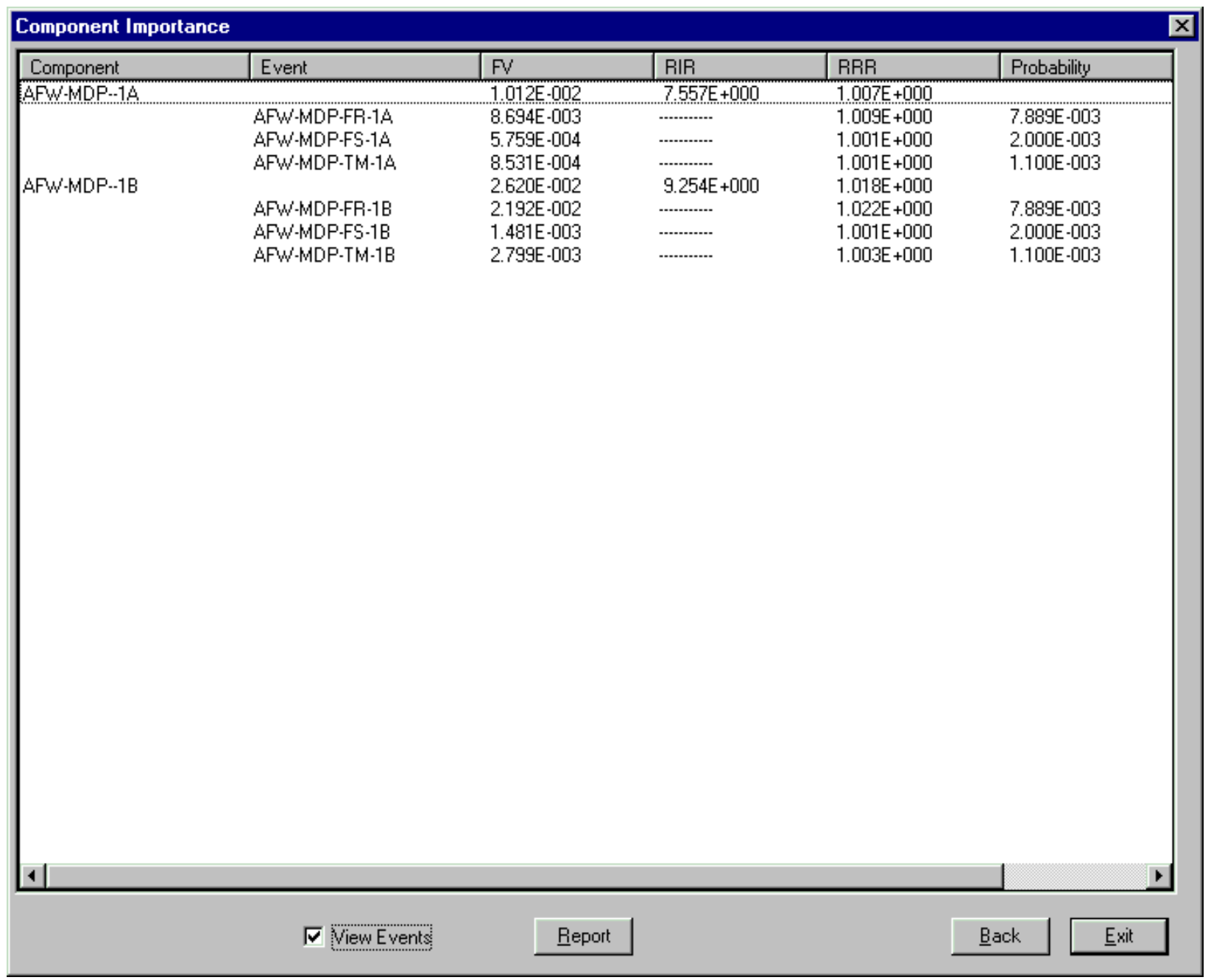

View Events - When this option is selected, basic events appear in the list beneath the component group to which they are assigned. When this option is not selected, the Component list contains only component names

Report - $\quad$ Report the results to a comma delimited file. The Choose file dialog (similar to the Save As dialog) will be displayed. 


\section{Navigation Options}

Back - Return to the previous step in the Importance Measures Wizard process.

Exit - Close the Importance Measures Wizard. The wizard will restore the database to the state it was in when the wizard was invoked, which may take a few moments.

\subsection{Sensitivity Wizard}

\subsubsection{Sensitivity Wizard}

\section{PURPOSE}

The Sensitivity Wizard provides a convenient interface for evaluating changes in overall risk and reliability resulting from changes in basic event probabilities. The wizard gives you the option of recalculating cut sets, rather than just requantifying them. This may result in more accurate answers since any event interactions will be accounted for.

\section{STEPS}

1. From the SAPHIRE menu select Utilities | Sensitivity Wizard.

2. The Sensitivity Wizard dialog will be displayed.

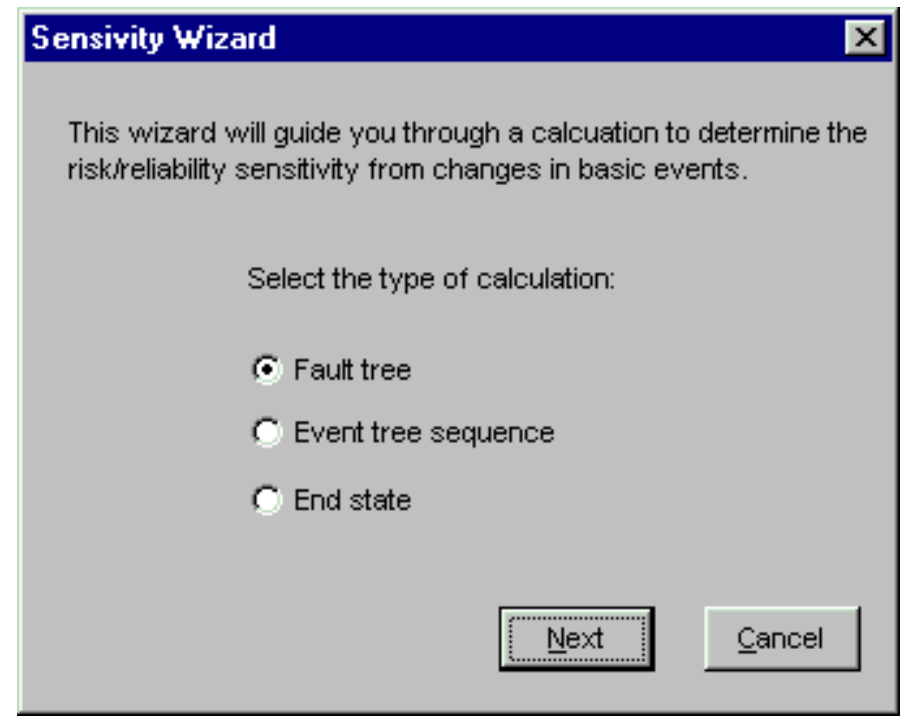

From this dialog, you can select the type of cut sets to analyze: Fault tree, Event tree sequence, or End state.

\section{Navigation Options}

Next - Continue to the next step in the Sensitivity Wizard process.

Cancel - Close the Sensitivity Wizard dialog. 


\subsubsection{Select Sensitivity Events}

\section{PURPOSE}

This option allows you to choose the basic events to study.

\section{Sensitivity Wizard}

Double click to select events to be modified for sensitivity analysis

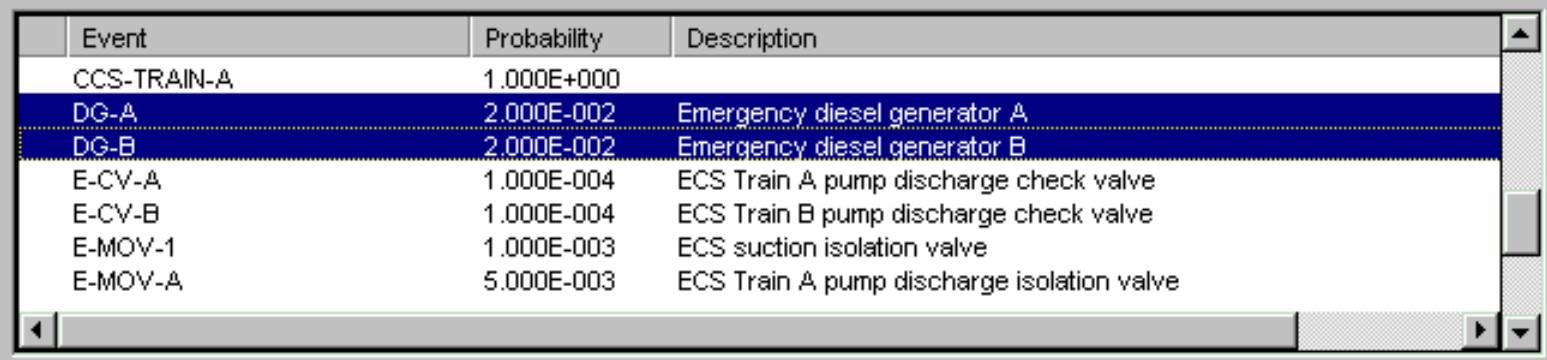

Click on a cell to enter the modified data values for each selected event, or right click to remove an event from the selection list.

\begin{tabular}{l|l|l|l|l|l|}
\hline Event & Start Value & Step Type & Step Change & TiF & Description \\
\hline DG-A & $2.000 E-002$ & & 10.000 & \\
\hline
\end{tabular}

The top of this dialog lists all the basic events in the project. From this list you choose the events to study by selecting one or more events, invoking the pop-up menu and choosing the Add option. The chosen events will appear in the bottom list, where you indicate the changes to make to those events.

\section{Popup Menu Options}

Add -

Delete -

\section{Event Change Columns}

Event -

Start Value -

Step Type -

Step Change -
Copy the selected event(s) in the top list to the bottom list.

Remove the selected events from the bottom list.

The name of the event chosen from the top list.

Type in the desired starting probability/frequency for the event. The successive columns indicate how to modify this value for sensitivity analysis.

Click the cell to toggle the increment type between $+($ add $)$ and *(multiply). A + will add the specified Step Change value to the Start Value. A * will multiply the Step Change to the Start Value.

Type in the desired increment value. This value will be initially added or multiplied to the Start Value, depending on the chosen Step Type. 
$\mathbf{T} / \mathbf{F}-$

Number of Steps -

Navigation Options

Back -

Next -

Cancel -
Click the cell to toggle between TRUE, FALSE, and BLANK values. A blank setting will not make the event a house event - it will use the Start and Step values from the other columns. A TRUE or FALSE setting will ignore the Start and Step values, and will perform the analysis as if the basic event were a house event.

The description of the basic event (for information purposes only).

Select the number of times to increment the selected events' probabilities, and recalculate the min cuts. The chosen Step Value will be used at step 1. Each additional step will add or multiply the Step Value to the previous step value, according to the chosen Step Type. Events designated as house events will remain house events for each step.

Return to the previous step in the Sensitivity Wizard process.

Proceed to the next step in the Sensitivity Wizard.

Cancel Sensitivity Wizard calculation.

\subsubsection{Select Items to Recalculate}

\section{PURPOSE}

This option allows you to choose the sequences, fault trees, sequences, or end states (depending on the type of analysis selected in an earlier step) to recalculate.

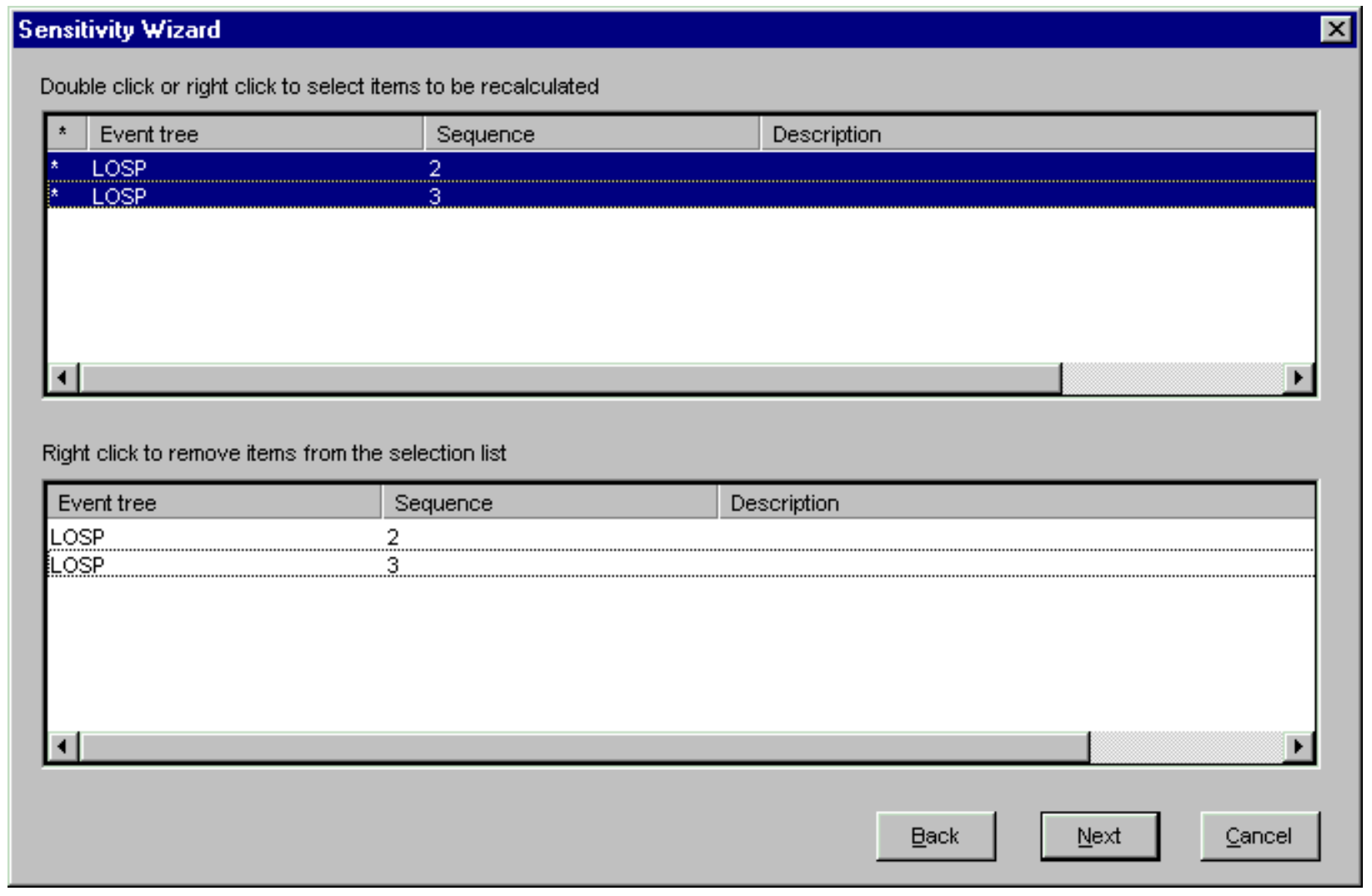


The top of this dialog lists all the fault trees/sequences/end states in the project. Each item that has a * (asterisk) in the first column has been detected as possibly affected by the events selected in the previous step. From this list you choose the fault trees/sequences/end states to study by selecting one or more, invoking the pop-up menu, and choosing the Add option. The chosen items will appear in the bottom list.

\section{Popup Menu Options}

Add - Copy the selected fault tree(s)/sequence(s)/end state(s) from the top list to the bottom list.

Delete - Remove the selected fault tree(s)/sequence(s)/end state(s) from the bottom list.

\section{Navigation Options}

Back - $\quad$ Return to the previous step in the Sensitivity Wizard process.

Next - $\quad$ Proceed to the next step in the Sensitivity Wizard.

Cancel - Cancel Sensitivity Wizard calculation.

\subsubsection{Select Sensitivity Calculation Method}

\section{PURPOSE}

This option allows you to choose whether to recalculate new cut sets or requantify existing cut sets. Requantification is generally faster, but recalculation is generally more accurate.

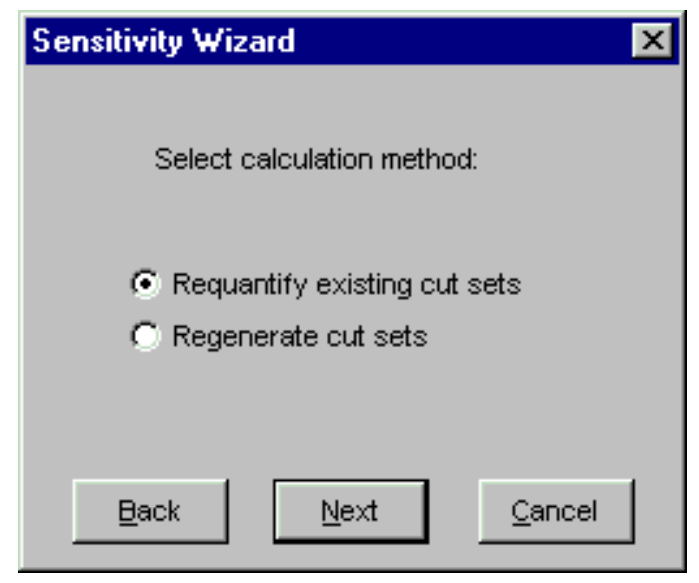

\section{Navigation Options}

Back - $\quad$ Return to the previous step in the Sensitivity Wizard process.

Next - $\quad$ Proceed to the next step in the Sensitivity Wizard.

Cancel - Cancel Sensitivity Wizard calculation.

\subsubsection{Evaluate Sensitivity}

\section{PURPOSE}

The Sensitivity Wizard will perform the sensitivity evaluations according to the inputs given in earlier steps of the wizard. If the option to regenerate the cut sets was selected in the Sensitivity Wizard, you will be prompted to enter the cut set generation options. 
Depending upon the size of the cut sets and the number of chosen steps, this process could take some time to complete.

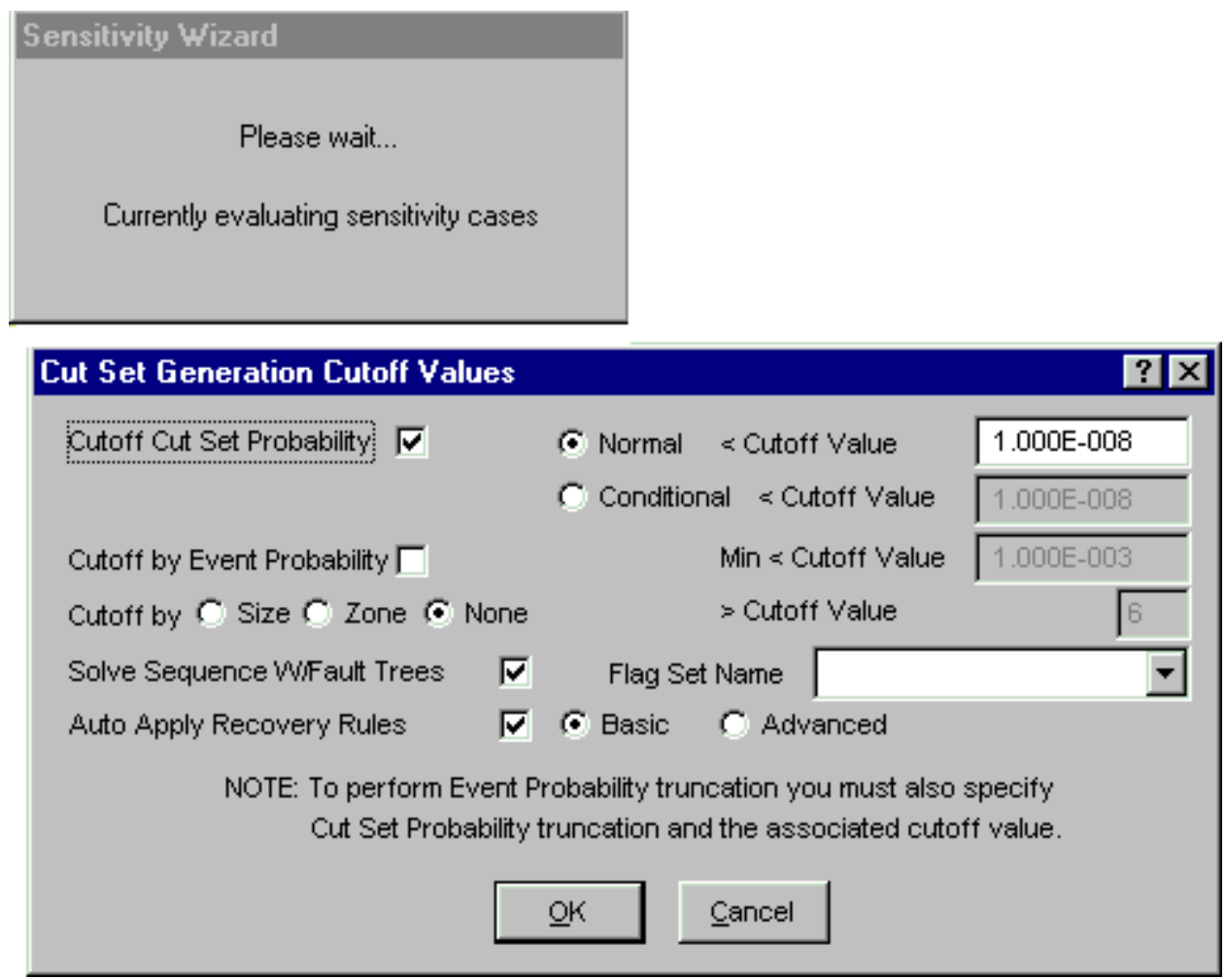

\section{Navigation Options}

OK - Perform the sensitivity analysis .

Cancel - Cancel Sensitivity Wizard calculation.

\subsubsection{Sensitivity Results}

\section{PURPOSE}

The Sensitivity Wizard Results dialog presents the calculated results. 


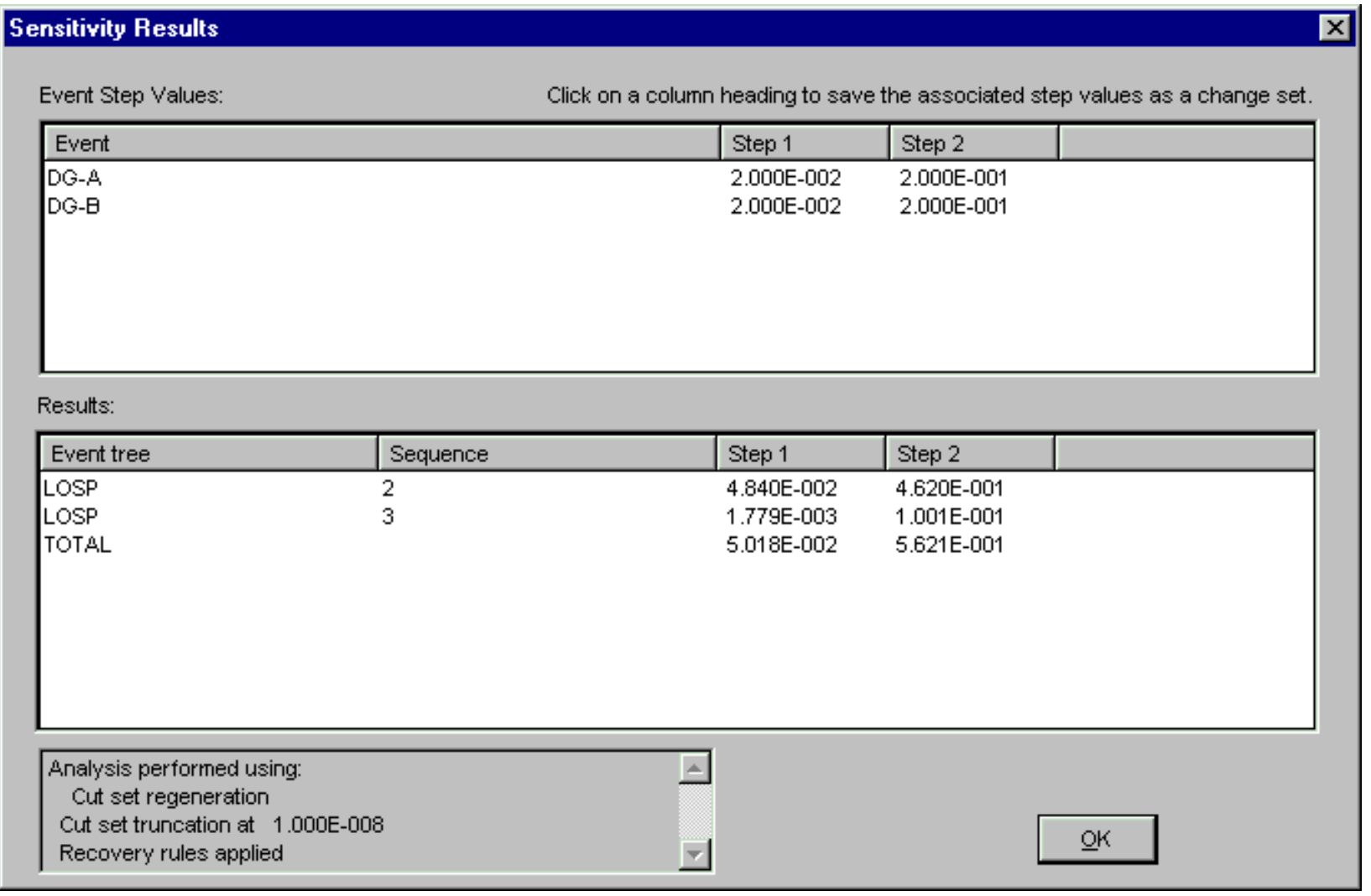

The top of this dialog lists the basic events selected for sensitivity analysis. The bottom list contains the selected fault trees/sequences/end states. Additional columns correspond to the number of steps selected in an earlier step. The step values for the basic events represent the probability/frequency used in each step. The step values for the fault trees/sequences/end states represents the min cut upper bound for each step calculated using the basic event values listed for the corresponding step.

Step Column Click -

Step Value Double Click -

\section{Navigation Options \\ OK -}

Create a change set containing the event probabilities used in that step. (Each calculation type will be set to 1). The Add Change Set dialog will appear.

Double click on a min cut upper bound result to view the cut sets associated with it. A View Step Detail dialog will appear, asking you to choose which step results to view.

Close the Sensitivity Wizard. You may be prompted to regenerate the basic event data. This is recommended in order to return the database to the state it was in prior to invoking the Sensitivity Wizard. 


\section{ACCESSING HELP}

\subsection{Accessing Help}

Included with the SAPHIRE installation is an on-line hypertext help system containing a complete reference to SAPHIRE.

The SAPHIRE help system was designed using the Windows 95 and NT 4.0 help features, based on "Help tabs". The Contents Tab is arranged like a table of contents containing a list of topics available in the help system. It is organized with books and pages. The pages are the link to the specified topic. The Index Tab provides access to the help system index of topic keywords. The Find Tab provides the functionality of a full-text search.

In general, the topic pages in the SAPHIRE system contain a "PURPOSE" section which provides a description of the selected topic and its primary function. This is followed by a "STEPS" section which provides step-by-step instructions for performing the function. Often, a picture of a dialog is included on the topic page. Brief explanations of the options on the dialog are provided below the graphic image and when necessary, links for additional details are presented. On occasion, the graphic image on the topic page will contain hot-spots which provide links to other topic pages or invoke a pop-up window containing a definition. When applicable, a "SEE ALSO" section is included for links to related topics.

Some of the topic pages are general and text-oriented. They provide background information or in-depth discussion about a specific concept and step-by-step directions are not applicable.

The help system is accessed via the Help option on the SAPHIRE menu or through the context-sensitive help on each dialog. The Help menu offers access to the on-line hypertext help system through the Contents Tab or Index Tab. Context-sensitive help offers a brief description of the selected feature on the current dialog.

\subsection{Help Menu}

\section{PURPOSE}

This option provides access to the SAPHIRE hypertext help system through a variety of options.

\section{STEPS}

1. From the SAPHIRE menu select Help.

2. The drop-down menu with available items will be displayed.

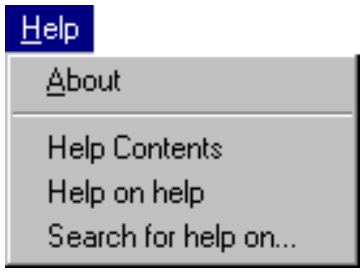




\begin{abstract}
About -
Provides product information about the version of SAPHIRE currently running.

Help Contents - $\quad$ Invokes the SAPHIRE help table of contents. From the table of contents, any "book" or "chapter" can be opened and the topic "page" of interest can be selected.

Search for help on... - Invokes the SAPHIRE detailed help index. Any keyword in this index can be selected to access information about the associated topic.

Help on help - $\quad$ Invokes the Windows help system table of contents. Information on how to use a hypertext help system is provided.
\end{abstract}

\title{
12.3 Context-Sensitive Help
}

\section{PURPOSE}

Context-sensitive help usually provides definition-style information about the selected control on a dialog box in a pop-up window. An entire topic page from the SAPHIRE help system may be invoked when the function of the selected control requires a detailed explanation.

\section{STEPS}

1. On the active dialog box, choose the Help button $(? \vec{?})$, located in the upper right-hand corner of the dialog. The cursor will now be the Help cursor (arrow with a question mark).

2. Click on the desired control (i.e., field, button, etc.) on the dialog.

When you are done viewing help topics:

If a pop-up window was invoked, simply click on the dialog to close the pop-up window and proceed with SAPHIRE operations.

If the SAPHIRE help system was invoked, you can close the help system or leave it open. In either case, because the help system runs independently of the SAPHIRE program, you can click on the dialog and proceed with SAPHIRE operations. 


\section{CAPABILITIES AND LIMITATIONS}

\subsection{Capabilities and Limitations}

\section{SAPHIRE Database Limits:}

(limits are per project unless otherwise indicated)

Number of projects limited by user's available disk space.

Number of cut sets limited by user's available disk space.

Number of basic events 64,000

Number of event trees 64,000

Number of sequences 2,000,000

Number of end states 64,000

Number of fault trees 64,000 - \# of basic events

Number of gates 64,000

Number of change sets 10,000

Number of analysis types 16

Size of block text fields limited by user's available disk space.

Lines of rules limited by user's available disk space.

Inputs per OR gate 5,000

Inputs per AND gate 256

Inputs per $\mathrm{N} / \mathrm{M}$ gate $\mathrm{N} / 99$, where $\mathrm{N}=2$ to 98 .

Gates/events per fault tree 10,000 (Limit when loading a fault tree through MAR-D).

Number of events per cut set 256

Number of uncertainty samples 99,999

Alpha to Graphics conversion 50 levels deep

Logic display 255 characters wide (dialog display and reporting only)

Path search display 255 characters wide (dialog display and reporting only)

\section{General Field Limits:}

Name references - Unless specified otherwise, limited to 24 uppercase characters. If lowercase characters are entered, they will be converted by SAPHIRE to uppercase. Embedded spaces (or blanks) are not allowed.

Descriptions - 120-character upper- or lowercase alphanumeric characters. Embedded spaces (or blanks) are allowed.

File names - Long file names are supported.

Floating point fields - Use scientific notation (e.g., 1.0E-15).

Information on specific fields can be found in the Utilities | Load and Extract section. 


\section{RECOVERY RULES}

\subsection{Recovery Rules Usage And Examples}

\section{PURPOSE}

The Recovery Rule editor allows you to create rules that affect existing cut sets in a "post-processing" fashion. The rule-based editor is available for both fault tree and sequence cut sets.

The recovery rules are "free-form" logic rules that allow for the alteration or deletion of fault tree or sequence cut sets. They follow a format similar to the structure that is found in traditional programming languages (e.g., BASIC or PASCAL). As such, the ability exists to define "macros" and "if...then" types of structures. The rules are entered in a free-form text editor within SAPHIRE. They can also be exported and imported through the SAPHIRE Utility | Load and Extract option.

The rules can be used for probabilistic risk assessment techniques such as the automated inclusion of sequence recovery events or common-cause failure cut sets, or elimination of mutually exclusive events (e.g., impossible combinations of events). They may be developed for a particular fault tree, all fault trees (project fault tree rules), a particular sequences, a single event tree, or all sequences (project sequence rules). Initiating events, basic events, and recovery events can all be added into the relational database directly from within the rule editor.

When the rules are applied, existing fault tree or sequence cut sets are scanned for cut sets matching the search criteria defined in the rule. The rule is used to modify the cut sets matching the search criteria. This searching process is a multi-step procedure:

If the first cut set in the list matches the search criteria in the first rule, the applicable modifications defined in the first rule are made to the cut set. Once a cut set qualifies for a search criterion it is not affected by any of the remaining rules.

If the first cut set in the list does not match the search criteria in the first rule, the second rule (SAPHIRE processes the rules from top to bottom) is evaluated.

After all the rules are evaluated for the first cut set, the second cut set in the list is evaluated, starting again with the first rule. This process is repeated until all cut sets are evaluated.

Since the recovery rules have the capability to remove basic events from individual cut sets, application of the rules could result in non-minimal cut sets. Consequently, it is recommended practice to re-minimize the cut sets after applying recovery rules. Thus, the typical steps you would take in performing an analysis using recovery rules are:

Finalize logic models and data changes and then Generate changes

Generate fault tree or sequence cut sets

Apply recovery rules to applicable fault trees or sequences

Perform a cut set update to fault tree or sequence cut sets

Perform uncertainty analysis

Display or report results. 


\subsection{Search Criteria Examples - Basic Recovery}

These examples are used based on basic events $\mathrm{X}, \mathrm{Y}$, and Z.

Search Criteria
$\mathrm{X}$
$/ \mathrm{X}$
$\sim \mathrm{X}$
$\mathrm{X}+\mathrm{Y}$
$\mathrm{X} * \mathrm{Y}$
$\sim \mathrm{X} * \mathrm{Y}$
$\mathrm{X} *(\mathrm{Y}+\mathrm{Z})$
always

Meaning of Search Criteria

Basic event $X$ appears in the cut set

Success of event $X$ appears in the cut set

Basic event $X$ never occurs in the cut set

Either event X or Y appear in the cut set

Both events $\mathrm{X}$ and $\mathrm{Y}$ appear in the cut set

$\mathrm{Y}$ does appear and $\mathrm{X}$ does not appear in the cut set

Either $\mathrm{X}$ and $\mathrm{Y}$ or $\mathrm{X}$ and $\mathrm{Z}$ appear in the cut set

This pre-defined macro name means the criteria is always met

\subsection{General Recovery Rule Structure}

Shown below are two examples of the actual basic rule structure and syntax.

The following example shows how the "if...then" rule structure can be used to check if a failure of an electric power bus event appears in a cut set. If the event does occur, the rule will muliply the cut set containing the event by an operator non-recovery probability named "BUSREC" via the "recovery" keyword.

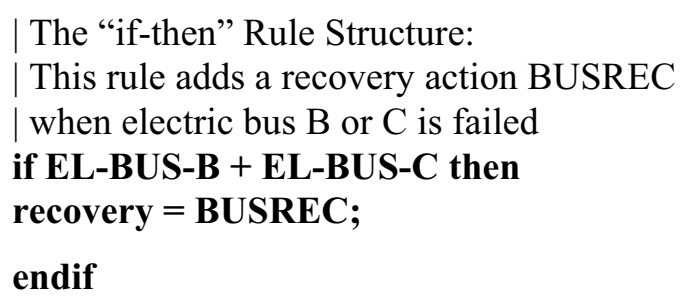

The following example demonstrates the use of the "elsif" keyword. For this second example, the rule first checks to see if two diesel generators (DG) maintenance events appear in the same cut set. If the do, the cut set is deleted since plant technical specifications do not permit two diesel generators to be out of service simultaneously for routine maintenance. Or, if the cut set does not qualify under the first search criteria, the rule checks to see if the random failure events, DG-1-RAND and DG-2-RAND appear in the same cut set. If they do, the rule will first copy the original cut set, then remove them and add the common-cause event, "DG-CCF-1AND2."

\footnotetext{
| The "if-then-elsif" Structure:

| This rule deletes the cut set if both diesel | generators are out for maintenance.

If the two DGs fail randomly, add a common-cause event.

if (DG-MAINT * DG-2-MAINT) then

DeleteRoot;

elsif (DG-1-RAND * DG-2-RAND) then

| Copy the original cut set, remove the

| two failure events, then add CC
} 


\author{
CopyRoot; \\ DeleteEvent $=$ DG-1-RAND; \\ DeleteEvent $=$ DG-2-RAND; \\ AddEvent = DG-CCF-1AND2; \\ Endif
}

14.4 Recovery Rules - Add Recovery Actions

In a PRA, cut sets for accident sequences are generated using fault tree and event tree logic. Since most PRAs are analyzed in a failure space, each cut sets represents the minimal set of components or fault trees that have to fail (given a particular initiating event) in order to result in an undesired condition (e.g., core damage). As such, operator actions that could prevent the accident sequence from progressing to the point of an accident may not be specifically included in the logic models. to model the PRA accident sequences as accurately as practical, the analyst will typically want to apply recovery events (such as appropriate) to the accident sequence cut sets.

The recovery events represent the probability that the operator or operators fail to successfully prevent the accident by restoring one or more of the failed components in the sequence cut sets. Consequently, the recovery events are frequently called the non-recovery probability events.

To demonstrate the potential uses of the recovery rules, the example here shows how the rules could be used to include recovery actions on specific cut sets in a particular sequence. As shown, the rule will add the NRAC-12HR operator recovery event (recovery of DGs within 12 hours) to those cut sets that contain LOSP as the sequence initiating event and failure of either DG A or DG B. This rule would probably be typed into the event tree sequence rule editor for the sequence of interest.

(Basic Rule syntax)

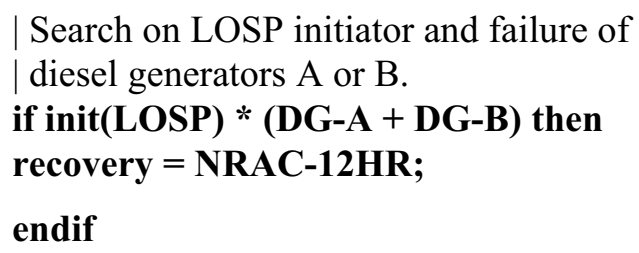

Once this rule is saved in the project database, SAPHIRE will evaluate all the sequence cut sets and then automatically include the recovery actions as specified in the recovery rule. The "if...then" line can be as complex as needed.

\title{
14.5 Recovery Rules - Mutually Exclusive Event Removal
}

To demonstrate the potential uses of the recovery rules, the example here shows how the rules could be used to remove a particular cut set from the cut set list.

NOTE: There may be instances where a cut set should be removed because of mutually exclusive events (i.e., the combination of basic events should not occur). 
(Basic Rule syntax)

| This rule could be placed in either (or both) the

| fault tree project rules or the event tree project rules.

| Define a macro to get those cut sets that have

combinations of two motor driven pumps out

for maintenance.

PUPMS-IN-MAINT = MDP-A-MAINT * MDP-B-MAINT;

| Search for the maintenance vents and then

delete the cut set.

if PUMPS-IN-MAINT then

| Delete the cut set

DeleteRoot;

endif

\subsection{Recovery Rules - Common-Cause Failure Modeling}

To demonstrate the potential uses of the recovery rules, the example here shows how the rules could be used to incorporate common cause modeling into the PRA.

Common cause failure modeling, one part of dependent failure analysis, is a standard PRA modeling technique. It attempts to model simultaneous failures of multiple components due to a single cause (i.e., common mode failure). Recovery rules can be created to search for cut sets containing specific groups of independent, random failure events and replace them with a single common-cause basic event in the cut sets.

Note these two important issues when using recovery rules for common-cause failure modeling:

The usefulness of the recovery rules for common-cause failure modeling is limited by the fact that groups of independent failures must be present in the cut sets in order for a rule to replace the independent failures with a single common cause failure event.

If a probability or size truncation is specified when generating fault tree or sequence cut sets, the independent failure cut sets may be omitted that would be above the truncation limit after being modified by the rule.

(Basic Rule syntax)

| The search criteria identifies the failure

| combination of two auxiliary feedwater pumps.

If these two basic events are found in a cut set

| then a new cut set will be created that replaces

| the independent failures of the two pumps with

a single common-cause basic event. This rule

| could be placed in either (or both) the fault tree

| project rules or the event tree project rules.

| Define a macro to only pick up those cut sets 
| that have combinations of AFW-PUMP-A

and AFW-PUMP-B.

CCF-AFW-PUMPS = AFW-PUMP-A * AFW-PUMP-B;

| Search for the AFW pump basic events and

I make a new cut set with the CCF event.

if CCF-AFW-PUMPS then

| First make a copy of the original cut set

CopyRoot;

| Now remove the two independent failure events

DeleteEvent $=$ AFW-PUMP-A;

DeleteEvent $=$ AFW-PUMP-B;

| Now add the CCF event

AddEvent $=$ AFW-PUMP-CCF;

endif

\subsection{Keywords and Symbols}

\subsubsection{Keywords and Symbols - Basic Recovery Rules}

Below is a list of keywords and symbols used in the SAPHIRE slice rules. Note the spelling of each keyword.

\begin{tabular}{|c|c|}
\hline n- & \\
\hline & es the end of a particular rule. \\
\hline se - & $\begin{array}{l}\text { Specifies some action to be taken if all the search criteria are not met. The "else" } \\
\text { should be the last condition in the recovery rule. }\end{array}$ \\
\hline 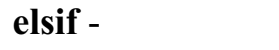 & Specifies an alternative search criteria. \\
\hline re & eing eval \\
\hline it $O$ & Irch criteria to indicate that a sequence cut set has a particular \\
\hline (never) - & $\begin{array}{l}\text { ch criteria to indicate that a particular event will not be in the cut } \\
\text { evaluated. }\end{array}$ \\
\hline \multicolumn{2}{|c|}{$\begin{array}{l}\text { / (complement) - Repr } \\
\text { | (comment) - Repr }\end{array}$} \\
\hline & Repr \\
\hline ; (end macro) - & line that modifies the cut set being \\
\hline JD) - & Indicates the logical AND co \\
\hline (OR) - & Indice \\
\hline & Indic \\
\hline & Indic \\
\hline Event $=$ - & nt will be added to the cut set being evaluated \\
\hline & es that an event will be deleted from the cut set being evaluated. \\
\hline & dde \\
\hline & hat the "found" cut will be deleted. \\
\hline
\end{tabular}


CopyCutset; - Indicates that the cut set being evaluated will be copied and added to the list of cut sets.

CopyRoot; - $\quad$ Indicates that the original cut set will be copied

MACRO - $\quad$ A user-definable keyword that specifies a search criteria.

\subsection{2 if-then}

(Basic Rule syntax)

This keyword indicates that a search criteria is being specified.

if "search criteria" then

perform some action on each cut set...;

endif

\subsection{3 endif}

(Basic Rule syntax)

This keyword indicates that the end of a particular rule.

if "search criteria" then

perform some action on each cut set...;

endif

\subsection{4 else}

(Basic Rule syntax)

This keyword specifies that some action to be taken if all the search criteria are not met. The "else" should be the last condition in the recovery rule.

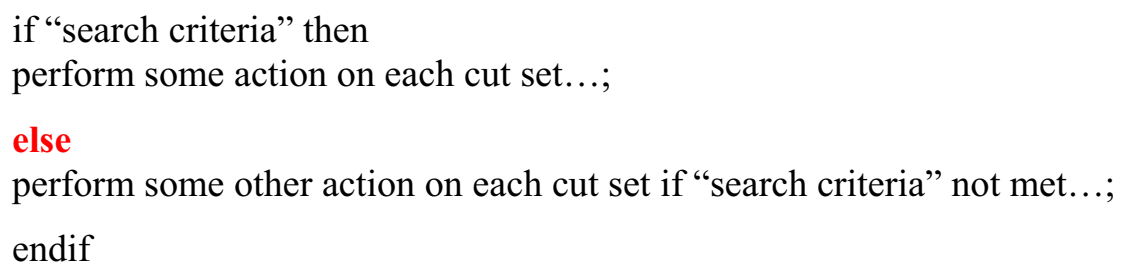

\subsection{5 elsif}

(Basic Rule syntax)

This keyword specifies an alternative search criteria. Any number of "elsifs" can be used within a recovery rule.

if "search criteria" then

perform some action on each cut set...; 


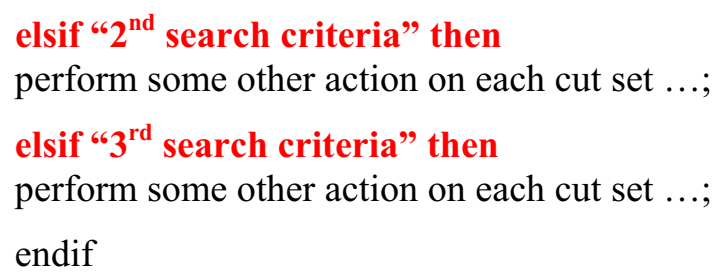

\subsection{6 always}

(Basic Rule syntax)

This keyword indicates that every cut set that is being evaluated satisfies the search criteria.

if always then

perform some action on each cut set...;

endif

\subsection{7 init()}

(Basic Rule syntax)

This keyword is used in the search criteria to indicate that a sequence cut set has a particular initiating event.

if init(INITIATOR-NAME) "and optional other search criteria" then

perform some action on each cut set...;

endif

\subsection{8 $\sim$ (never)}

(Basic Rule syntax)

This symbol is used in the search criteria to indicate that a particular event will not be in the cut set that is being evaluated.

if ( SEARCH-CRITERIA) "and optional other search criteria" then $\cdots$

The search criteria will be satisfied for cut sets that do not contain SEARCH-CRITERIA (and also contains the optional ("other search criteria"). SEARCH-CRITERIA may be either an initiating event, basic event, macro, or logic expression.

\subsection{9 / (complement)}

(Basic Rule syntax)

This symbol is used to represent a complemented event (i.e., the success of a failure basic event). 
if (/BASIC-EVENT) “and optional other search criteria” then

The search criteria will be satisfied for all cut sets that contain the complement of BASIC-EVENT (and also contains the optional "other search criteria").

\subsubsection{0 | (comment)}

(Basic Rule syntax)

This symbol is used to represent a comment contained in the rules. Everything on a line to the right of this symbol will be ignored by the rule compiler. Blank lines are encouraged in the editor to improve readability and comments can be entered following the "|" character.

| Place your comments here!

| Note that blank lines are also permissible.

\subsubsection{1 ;(end macro)}

(Basic Rule syntax)

This symbol is used to indicate the end of a macro line or a line that modifies the cut set being evaluated.

| usage for a macro command

MACRO-NAME = "search criteria";

| usage for a cut set modification line

recovery $=$ RECOVERY-EVENT;

\subsubsection{2*(AND)}

(Basic Rule syntax)

This symbol is used to indicate the logical AND command.

if SEARCH-CRITERIA1 * SEARCH-CRITERIA2 then

The search criteria will be satisfied for all cut sets that match SEARCH-CRITERIA1 and SEARCHCRITERIA2. The SEARCH-CRITERIA\# may be either an initiating event, macro, or logic expression.

\subsubsection{3 + (OR)}

(Basic Rule syntax)

This symbol is used to indicate the logical OR command.

if SEARCH-CRITERIA1 + SEARCH-CRITERIA2 then

The search criteria will be satisfied for all cut sets that match either SEARCH-CRITERIA1 or SEARCHCRITERIA2. The SEARCH-CRITERIA\# may be either an initiating event, macro, or logic expression. 


\subsubsection{4 ( ) (group)}

(Basic Rule syntax)

These symbols together indicate a specific grouping of items.

$$
\text { if }(\mathrm{A}+\mathrm{B}) *(\mathrm{C}+\mathrm{D}) \text { then }
$$

The search criteria above would return all cut sets that contain:

$[\mathrm{A} * \mathrm{C}],[\mathrm{A} * \mathrm{D}],[\mathrm{B} * \mathrm{C}]$, or $[\mathrm{B} * \mathrm{D}]$.

\subsubsection{NewCutset;}

(Basic Rule syntax)

This keyword indicates that a new, empty cut set will be added to the list of cut sets. The new cut set then becomes the cut set that is being evaluated. Note the particular capitalization of the keyword.

if "search criteria" then

NewCutset;

now make additions to the empty cut set...

endif

\subsubsection{DeleteRoot;}

(Basic Rule syntax)

This keyword indicates that the original cut set (i.e., that cut set which satisfies the search criteria) will be deleted. Note the particular capitalization of the keyword.

if "search criteria" then
DeleteRoot;
endif

\subsubsection{CopyCutset;}

(Basic Rule syntax)

This keyword indicates that the cut set being evaluated will be copied and added to the list of cut sets. This copied cut set then becomes the cut set that is being evaluated.

if "search criteria" then

CopyCutset;

now make modifications to a copy of the cut set...

endif 


\subsubsection{CopyRoot;}

(Basic Rule syntax)

This keyword indicates that the original cut set (i.e., that cut set which satisfied the search criteria) will be copied. The copied cut set will then become the cut set that is being evaluated.

if "search criteria" then

CopyRoot;

now make modifications to a copy of the original cut set...

endif

\subsubsection{Recovery Rules - Keyword Examples}

The example here illustrates how cut sets can be changed by the use of some of the recovery rule keywords occurring in the basic rule syntax.

For this example, assume that only a single cut set matches the search criteria. This cut set starts with a single basic event, A, and is called the Root cut set.

\begin{tabular}{|c|c|c|c|}
\hline Step & $\begin{array}{l}\text { Keyword that is } \\
\text { applied }\end{array}$ & $\begin{array}{l}\text { Resulting } \\
\text { cut set(s) }\end{array}$ & Comment \\
\hline 1 & AddEvent = B & (1) $A * B$ & $\begin{array}{l}\text { Event B is attached to the "currently evaluated" cut } \\
\text { set. }\end{array}$ \\
\hline 2 & NewCutset; & $\begin{array}{l}\text { (1) A*B } \\
\text { (2) blank }\end{array}$ & $\begin{array}{l}\text { A new blank cut set is included in the list of cut sets. } \\
\text { This new cut set now becomes the "currently } \\
\text { evaluated" cut set. }\end{array}$ \\
\hline 3 & AddEvent $=\mathrm{C}$ & $\begin{array}{l}\text { (1) } A * B \\
\text { (2) } C\end{array}$ & $\begin{array}{l}\text { Event C is attached to the "currently evaluated" cut } \\
\text { set. }\end{array}$ \\
\hline 4 & DeleteRoot; & (1) $\mathrm{C}$ & The Root cut set is removed. \\
\hline 5 & CopyCutset; & $\begin{array}{l}\text { (1) } \mathrm{C} \\
\text { (2) } \mathrm{C}\end{array}$ & $\begin{array}{l}\text { A new cut set is included in the list of cut sets that is } \\
\text { a duplicate of the old "currently evaluated" cut set. }\end{array}$ \\
\hline 6 & AddEvent = D; & $\begin{array}{l}\text { (1) } C \\
\text { (2) } C * D\end{array}$ & $\begin{array}{l}\text { Event D is attached to the "currently evaluated" cut } \\
\text { set }\end{array}$ \\
\hline
\end{tabular}




\section{PARTITION RULES}

\subsection{Partition Rules Usage and Examples}

\section{PURPOSE}

The partition rules test the existing sequence cut sets for the presence or absence of specific combinations of basic events or initiating events, and assigns characters in the end state name when the criteria are met. This allows end state names to be built as the rules are applied.

\section{TOPICS}

Symbols

Search Criteria Examples

Keywords and Rule Structures

Partitioning Rule Example

\subsection{Rule Editor Symbols}

$\begin{array}{cl}\mid \text { (comment) } & \text { Denotes a comment line } \\ * \text { (AND) } & \text { Logical AND operator } \\ +(\text { OR }) & \text { Logical OR operator } \\ \sim(\text { never }) & \text { Logical operator for "never" or "not present" } \\ / \text { (complement) } & \text { Complement } \\ ; \text { (end macro }) & \text { Indicates the end of a macro line or a line that } \\ & \text { modifies the criteria being evaluated } \\ () & \text { Parentheses for grouping terms }\end{array}$

\subsection{Search Criteria Examples}

(Basic Rule syntax)

Search Criteria

DG-A

Meaning of the Search Criteria

/DG-A

Basic event DG-A (failure)

$\sim$ DG-A

Complemented basic event DG-A (success)

$\operatorname{init(LOSP)}$

Failure of DG-A is not in the cut set

SYSTEM(ECS)

Initiating event with the name LOSP

Fault tree with the name ECS

\subsection{Keywords and Rule Structures}

\subsubsection{Keywords and Rule Structures}

Below is a list of keywords used in the SAPHIRE partition rules (Basic Rule syntax). Note the spelling of each keyword. The General Rule Structures describe how the keywords can be used. 
if-then - $\quad$ Indicates that a search criteria is being specified.

always - Indicates that the search criteria is always satisfied.

elsif - $\quad$ Specifies an alternative search criteria.

else - $\quad$ Specifies that some action to be taken if all the search criteria are not met.

Cast Operators - Define substitutions for macros, fault trees, end states, events, and initiating events.

CurrentPart() - Creates a different end state.

GlobalPartition - Partitions all cut sets in a sequence to an end state.

Macro - $\quad$ A user-definable keyword that specifies a search criteria.

\subsubsection{General Rule Structure}

(Basic Rule syntax)

The information between the "if-then " and the "endif" clause defines the end state for the cut set. The user can either specify an entire end state name as in the example or substitute only certain character positions in the name.

$$
\begin{aligned}
& \text { if always then } \\
& \text { partition = "NO-FLOW-"; } \\
& \text { endif }
\end{aligned}
$$

The following rules demonstrate the substitution process.

$$
\begin{aligned}
& \text { if NO-FLOW then } \\
& \text { partition = "????????END"; } \\
& \text { elsif } \sim \text { EVENT7 then } \\
& \text { partition = "????????START"; } \\
& \text { else } \\
& \text { partition = "????????NONE"; } \\
& \text { endif }
\end{aligned}
$$

This rule will result in one of three end states being assigned to a cut set. Either, "NO-FLOW-END", "NO-FLOW-START", or "NO-FLOW-NONE". A rule can contain some additional clauses as indicated above.

\subsection{3 if-then}

(Basic Rule syntax)

This keyword indicates that a search criteria is being specified. The information between the "if" and the "endif" clause defines the tops to be replaced. There is no limit on the number of replacements that can be defined between these clauses.

This is an example of the if-then rule structure along with the use of wildcard characters. 


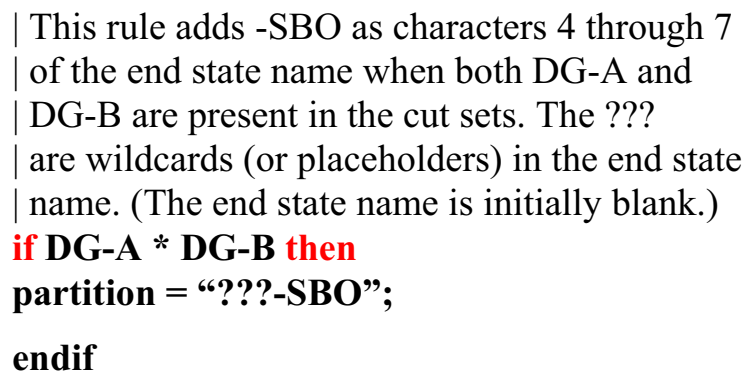

The partition statement must end with a semicolon. The end state name must be less than or equal to 24 characters in total length. The end state characters are enclosed in quotation marks.

\subsection{4 always}

(Basic Rule syntax)

This keyword indicates that the search criteria is always satisfied. The substitutions following this condition are always made.

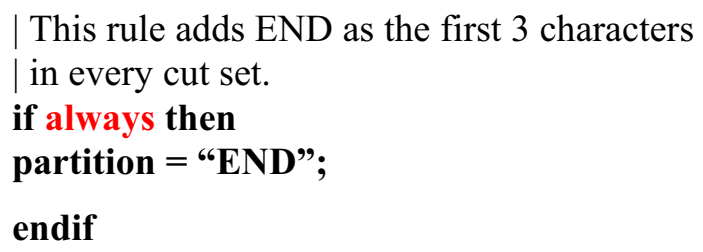

The partition statement must end with a semicolon. The end state name must be less than or equal to 24 characters in total length. The end state characters are enclosed in quotation marks.

\subsection{5 elsif}

(Basic Rule syntax)

This keyword specifies an alternative search criteria. Any number of "elsifs" can be used within a rule. The first condition that is met is used and the others are ignored.

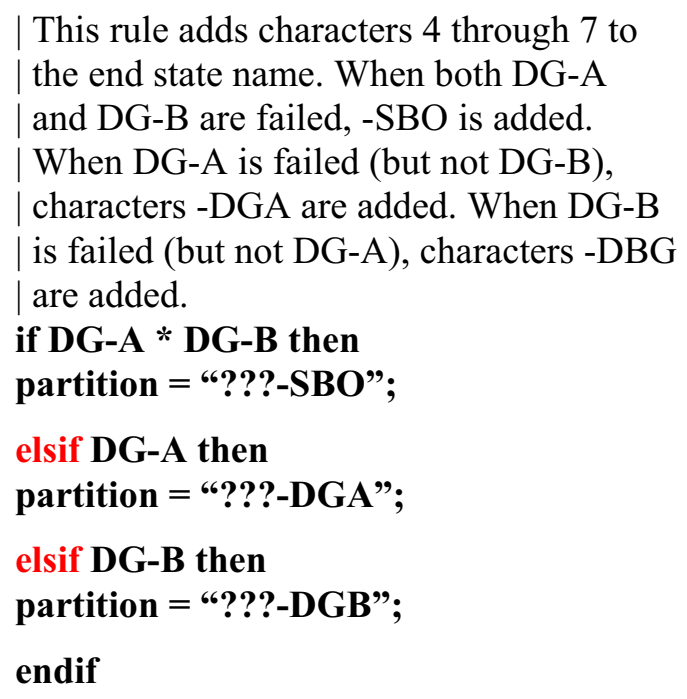


Note that the cut sets that do not contain DG-A or DG-B are not assigned to any end state in the rule above. See the "else" statement to resolve this.

\subsection{6 else}

\section{(Basic Rule syntax)}

This keyword specifies that some action to be taken if all the search criteria are not met. The "else" should be the last condition in the rule.

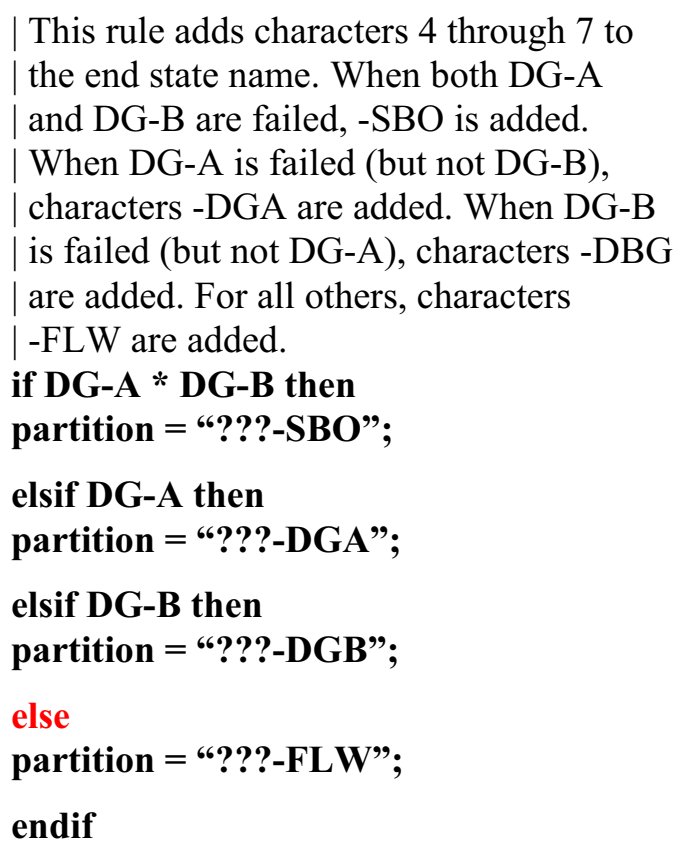

Note that the cut sets that do not contain DG-A or DG-B are assigned to the ???-FLW end state by the "else" statement.

\subsubsection{Cast Operators}

(Basic Rule syntax)

SAPHIRE provides type cast functions for macros, fault trees, end states, events, and initiating events. If you want to change the default assumptions, then you can "cast" the names to something else by enclosing the name in parenthesis with a cast name preceding it.

For instance, if the name "EVENT5" was really the name of a fault tree then you could change its default type with the following syntax; "SYSTEM(EVENT5)". The editor will then treat this name as a fault tree. 


\subsubsection{CurrentPart()}

(Basic Rule syntax)

The "current partition" rule structure uses the end state created by a partition to append on other information and create a different end state. This rule can use wildcards as part of its search criteria.

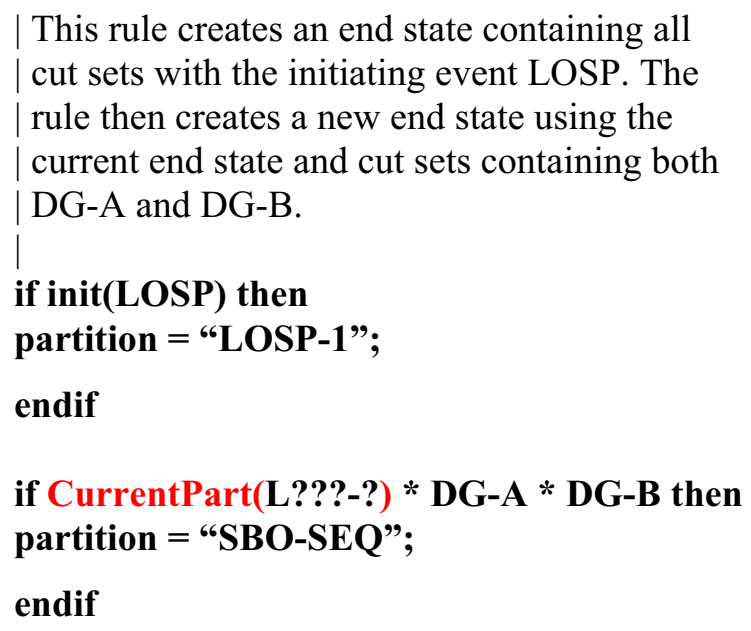

\subsubsection{GlobalPartition Keyword Usage}

\subsubsection{GlobalPartition - Transfer to Level 2 Tree}

(Basic Rule syntax)

Global partition rules are geared more for Level 2 studies since the end state that is created is also an event tree with the same name. The event tree that is created uses the end state frequency as its initiating event frequency and then transfers to a Level 2 event tree. The end state event tree can be looked at as an event tree which transfers Level 1 information to Level 2 trees.

This rule creates an end state event tree to be used by a Level 2 event tree already created.

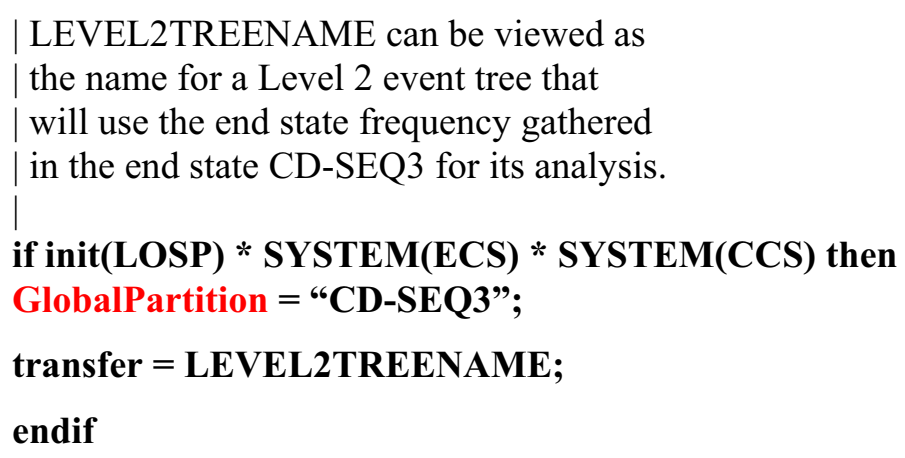

NOTE: Global partitioning is designed for partitioning cut sets into end states based upon sequence logic. 


\subsubsection{GlobalPartition - Wildcards}

(Basic Rule syntax)

The use of wildcards can also be used by the global partition rules. This adds the ability of partition rules to test on the initiating event name.

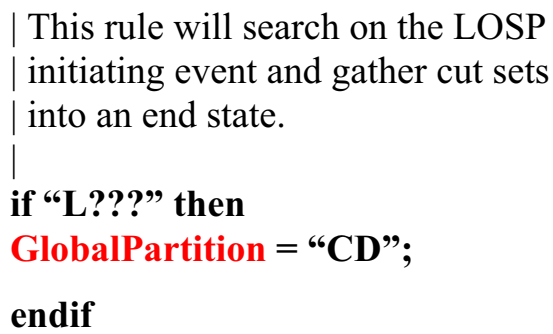

NOTE: Global partitioning is designed for partitioning cut sets into end states based upon sequence logic.

\subsubsection{Macro Structures}

\subsubsection{Macro Structures}

(Basic Rule syntax)

Macros can be used to streamline complex rules. A macro is simply a statement to define a search criteria (or variable) and assign a name to the search criteria. The macro name must be all uppercase, must be 24 characters or less, and must not include any of the restricted characters (e.g., a space, *, ?, ।, /). The macro line can wrap around to more than one line, but must end with a semicolon.

The macro defined by the following syntax,

$$
\text { NO-FLOW }=(\text { EVENT1 + EVENT2 }) *(\text { EVENT3 + /EVENT4 })
$$

indicates that the macro "NO-FLOW" is to be true if the following conditions are true. (1) EVENT1 or EVENT2 appear in the cut set and EVENT3 appears or EVENT4 appears as a complemented event. Once a macro is defined, it can be used in an "if" test. The following rule is an example of an "if" test using a macro.

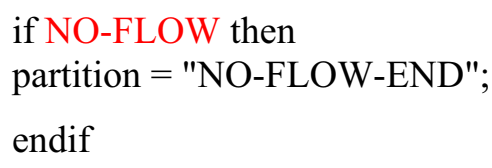

The following is an example using the "?" wildcard character for string substitution. This character is a place holder character and has no effect on the resulting string substitution. 


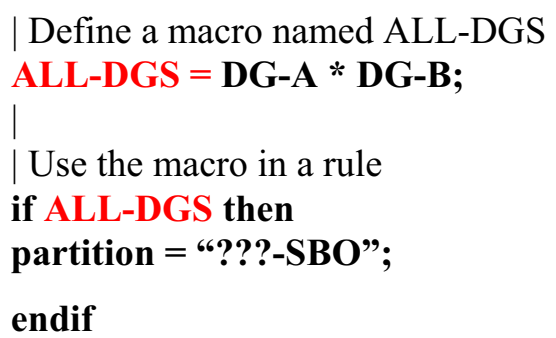

You can also use the $\sim$ (never) character to indicate that the item following the character is not in the current cut set.

\subsubsection{Using (never) With a Macro}

(Basic Rule syntax)

When creating a rule that applies when the events in the macro do not occur, use the $\sim$ (never) symbol. Do not "complement" a macro.

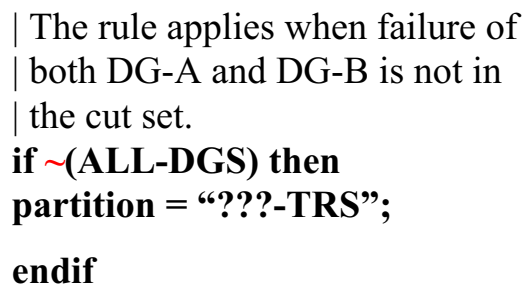

\subsection{Partition Rule Example}

\subsubsection{Partition Rules - Example}

\section{PURPOSE}

Provide an example, along with the steps for using partitioning rules.

\section{STEPS}

1. Create project rules

2. Create individual sequence rules

3. Apply the rules

4. Gather the end states

End state results can be displayed and/or reported from either the End State | View or Report | End State options.

\subsubsection{Project Rules}

\section{PURPOSE}

Create partition rules for the DEMO project. 


\section{STEPS}

1. Select Sequence from the SAPHIRE menu. The Sequences dialog will be displayed.

2. Right-click to invoke the pop-up menu and select Cut Sets | Partition | Edit Rules.

3. The Edit Partition Rules dialog will be displayed. Select Rule Level Project, Rule Type Basic, and choose the $\mathbf{O K}$ button.

4. The Partition Rules Editor window will be displayed. Because these are project rules, only the project name appears in the title bar.

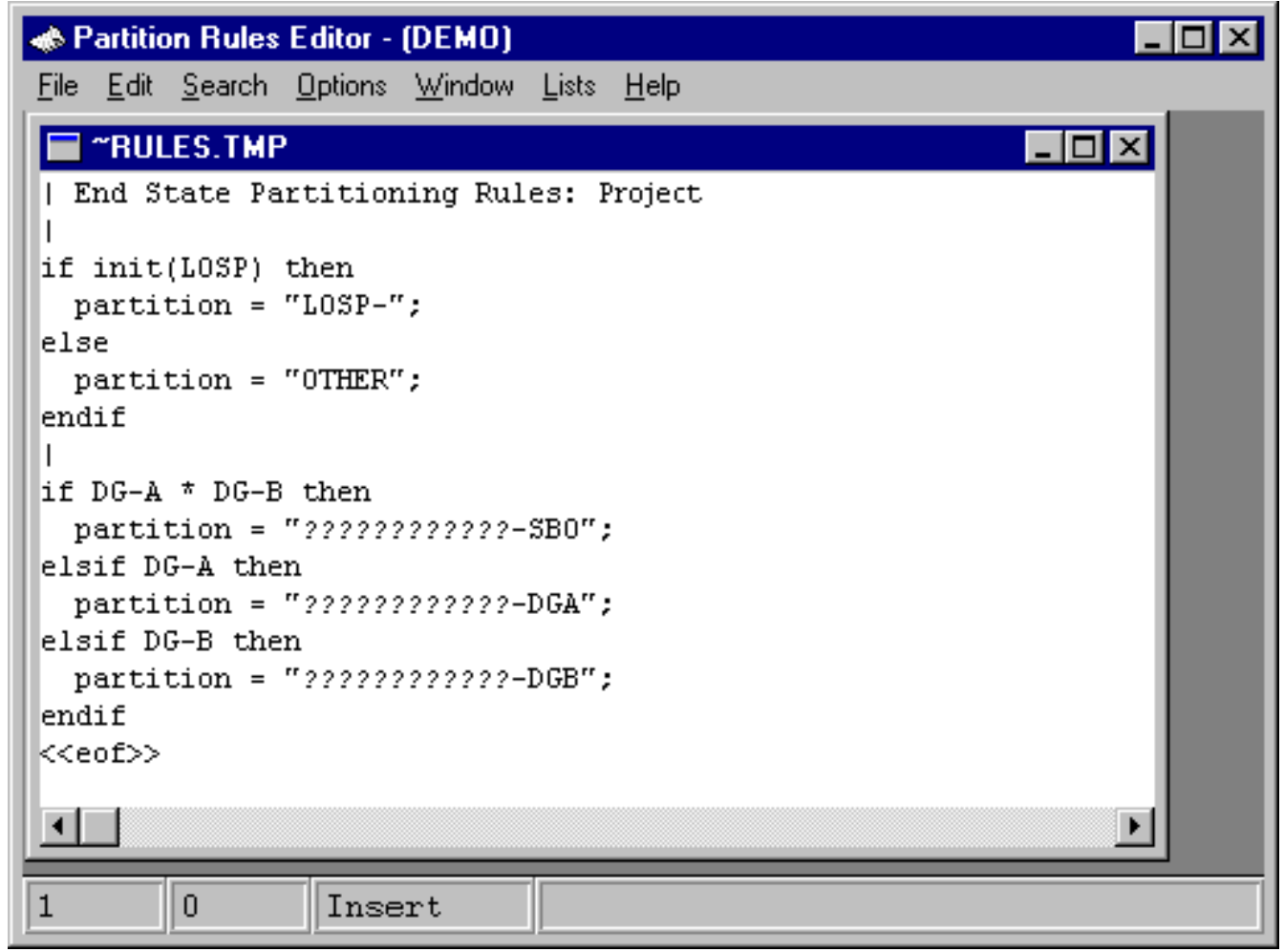

5. Enter the partition rules as displayed. Select File | Exit when complete.

The first project rule shown adds characters 1 through 5 as "LOSP-" when LOSP is the initiating event, and "OTHER" if LOSP is not the initiating event.

The next rule adds characters 13 through 16 as "-SBO" when both DG-A and DG-B are failed. When DG-A is failed, "DGA" is added, and when DG-B is failed, "DGB" is added (as characters in the end state name).

\subsubsection{Sequence Rules}

\section{PURPOSE}

Create sequence partition rules for each of the sequences in the DEMO project LOSP event tree.

\section{STEPS}

1. Select Sequence from the SAPHIRE menu. The Sequences dialog will be displayed. 
2. Highlight the LOSP 2 sequence.

3. Right-click to invoke the pop-up menu and select Cut Sets | Partition | Edit Rules.

4. The Edit Partition Rules dialog will be displayed. Select Rule Level Sequence, Rule Type Basic, and choose the $\mathbf{O K}$ button.

5. The Edit Partition Rules dialog will be displayed.

6. The Partition Rules Editor window will be displayed. The event tree and sequence name, along with the current project name will appear in the title bar when an individual sequence is selected.

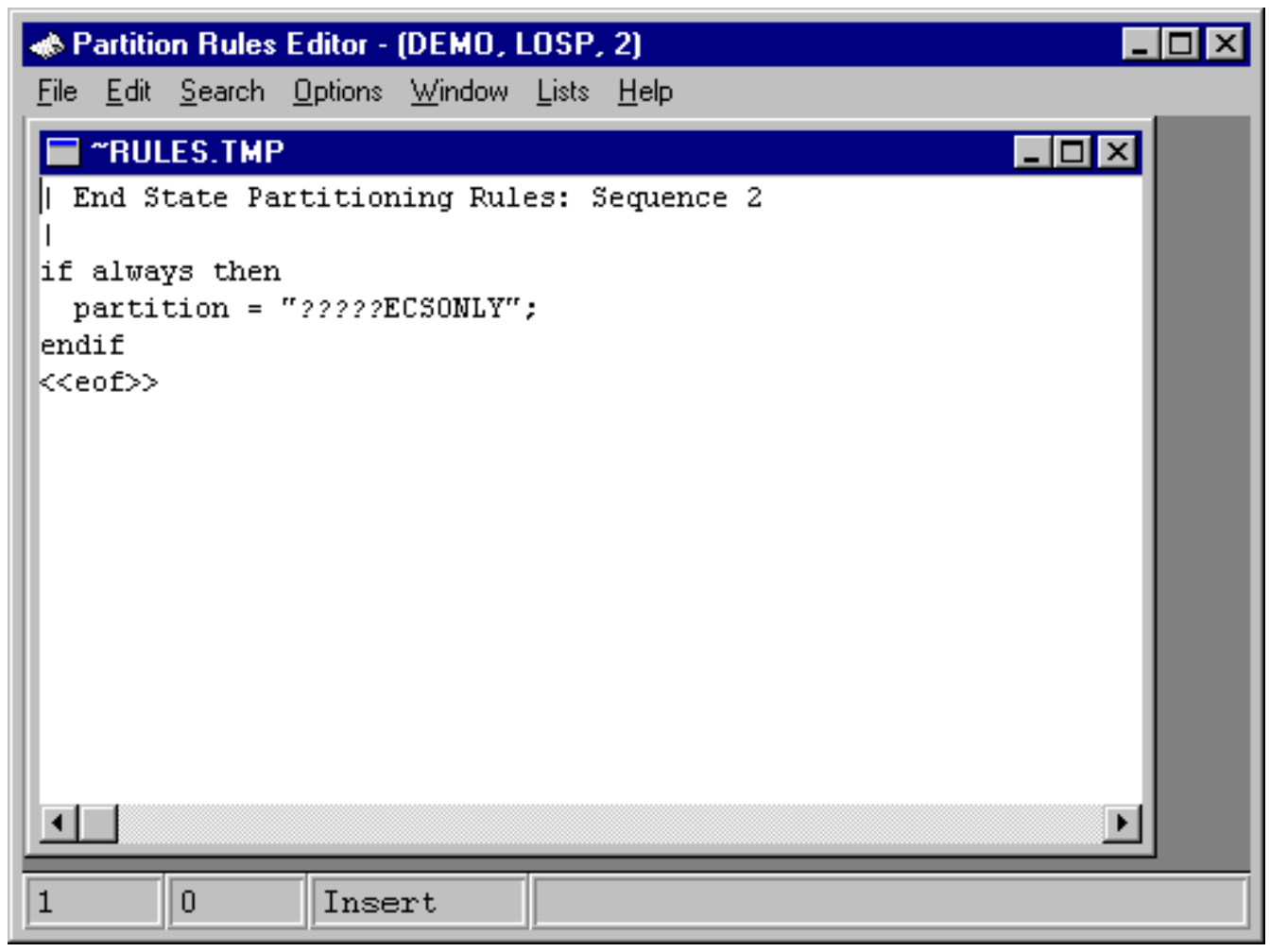

7. Enter the partition rules as displayed. Select File | Exit when complete.

The rule in sequence 2 adds characters 2 through 12 as "ECSONLY".

Repeat the process above for the LOSP 3 sequence, beginning with step 2. 


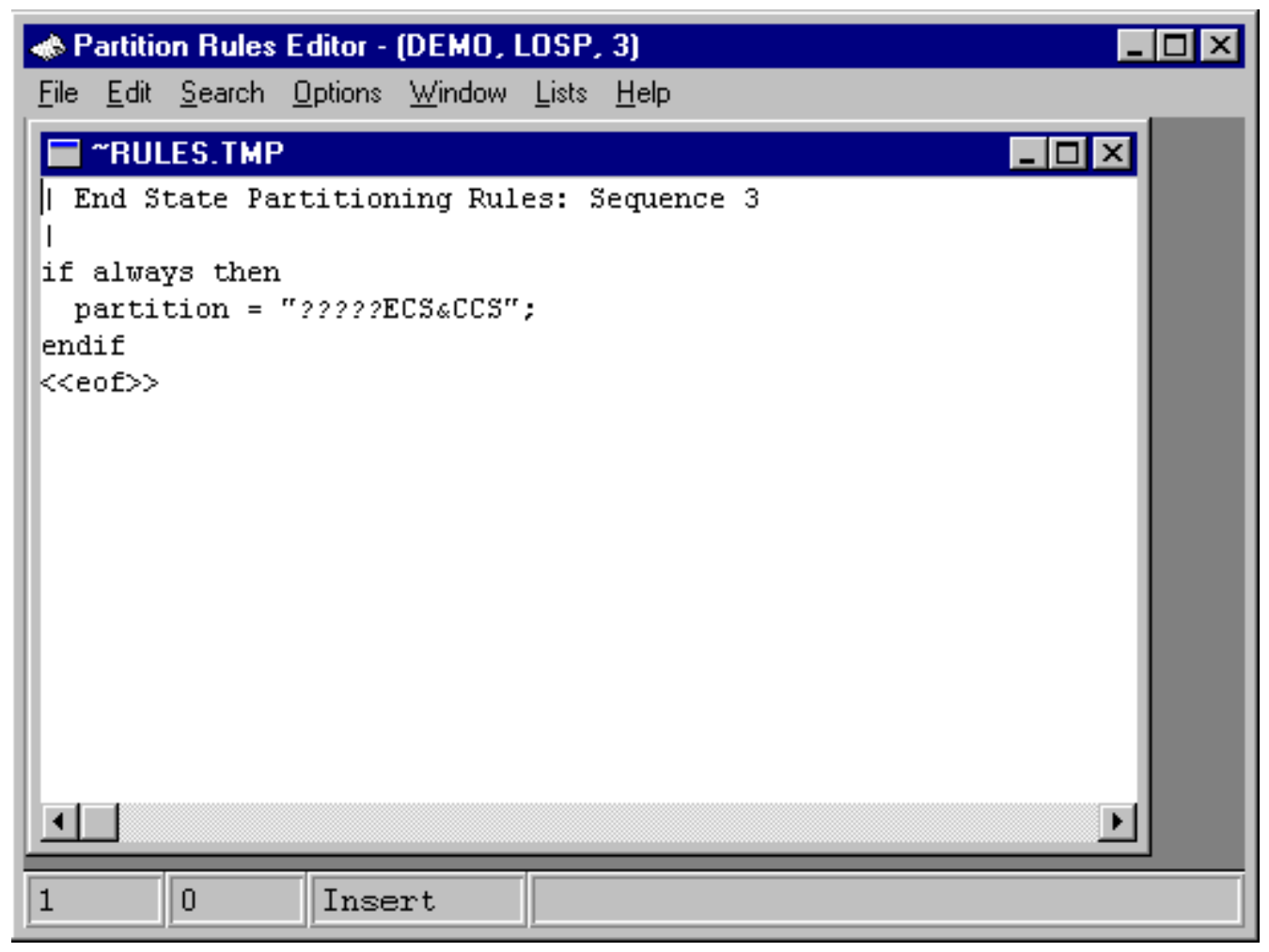

The rule in sequence 3 adds characters 6 through 12 as "ECS\&CCS".

\subsubsection{Applying Partitioning Rules}

\section{PURPOSE}

Apply the partition rules after entering them via the Partition Rules Editor. After creating the partition rules, they must now be applied.

\section{STEPS}

1. From the Sequences dialog, highlight the LOSP sequences.

2. Right-click to invoke the pop-up menu and select Cut Sets | Partition | Apply Batch. 


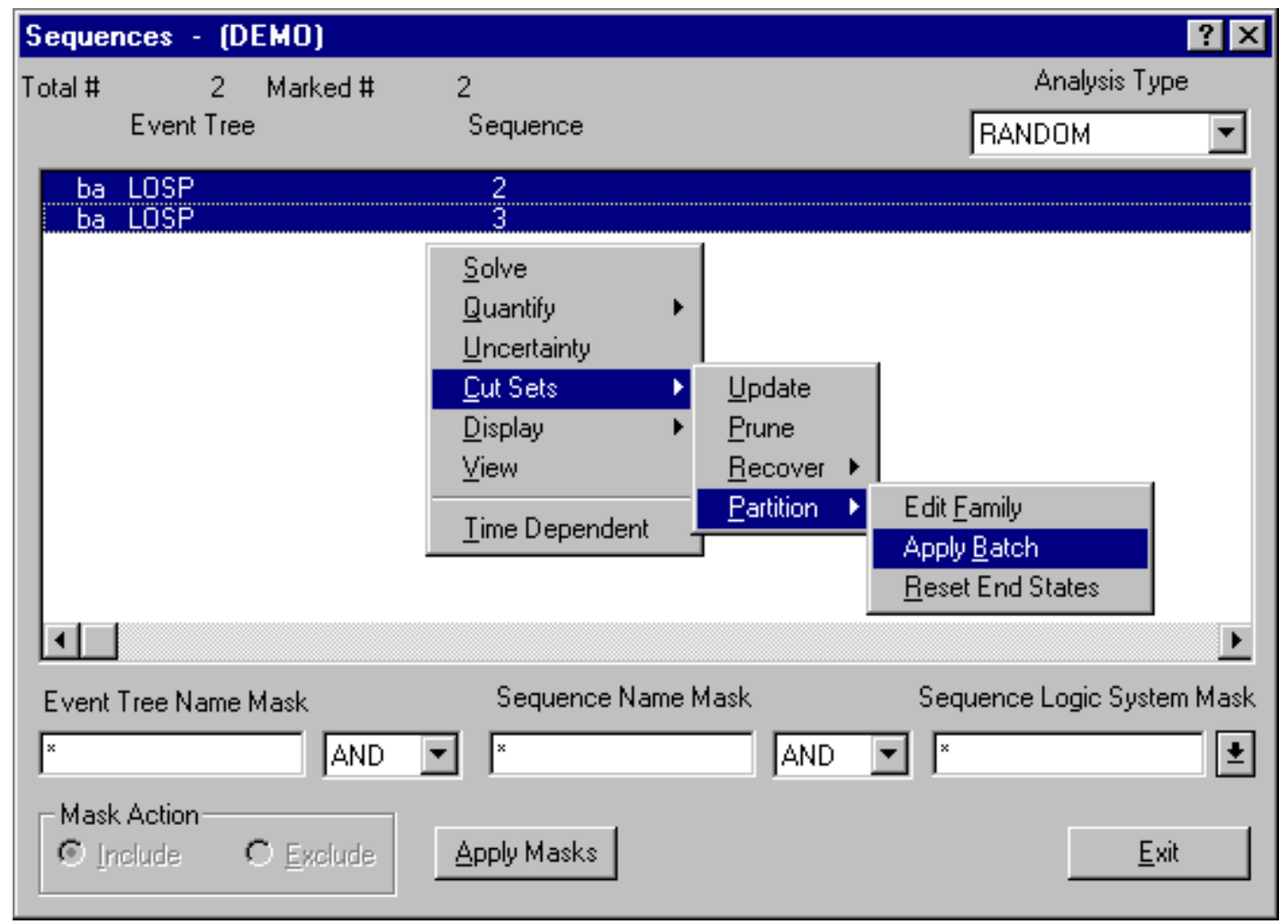

3. A warning dialog will be displayed. Choose Ok to continue.

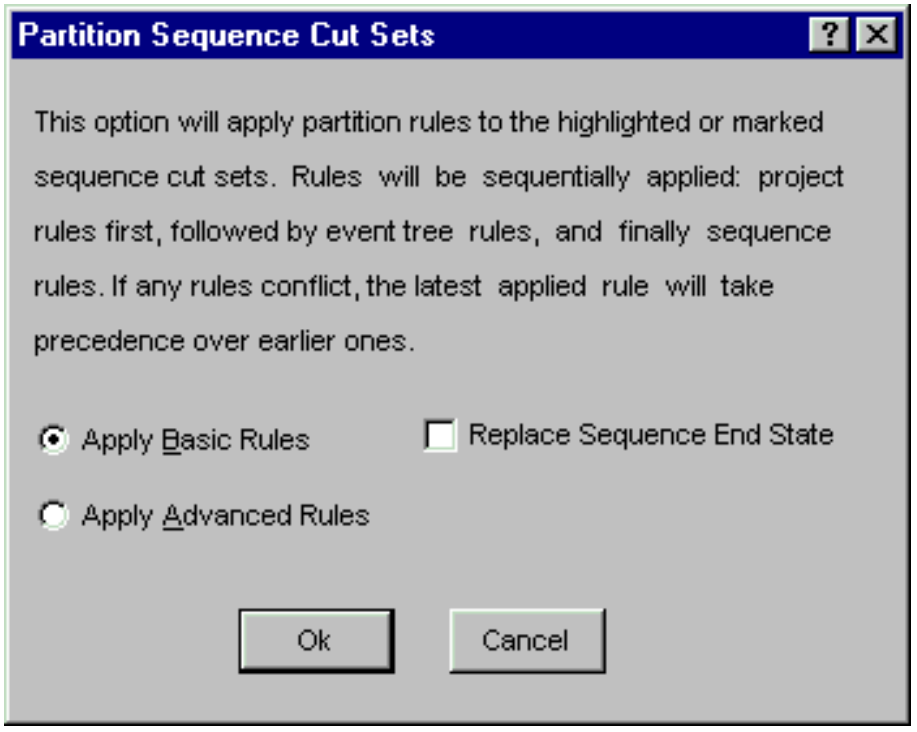

\subsubsection{Gathering the End States}

\section{PURPOSE}

Gather the cut sets for the end states created by the partition rules. After applying the partition rules the end states must now be "gathered". 


\section{STEPS}

1. From the SAPHIRE menu select End State. The End State List dialog will be displayed.

2. Highlight the end states created by the partition rules.

3. Right-click to invoke the pop-up menu and choose Gather. The Cut Set Generation dialog will be displayed.

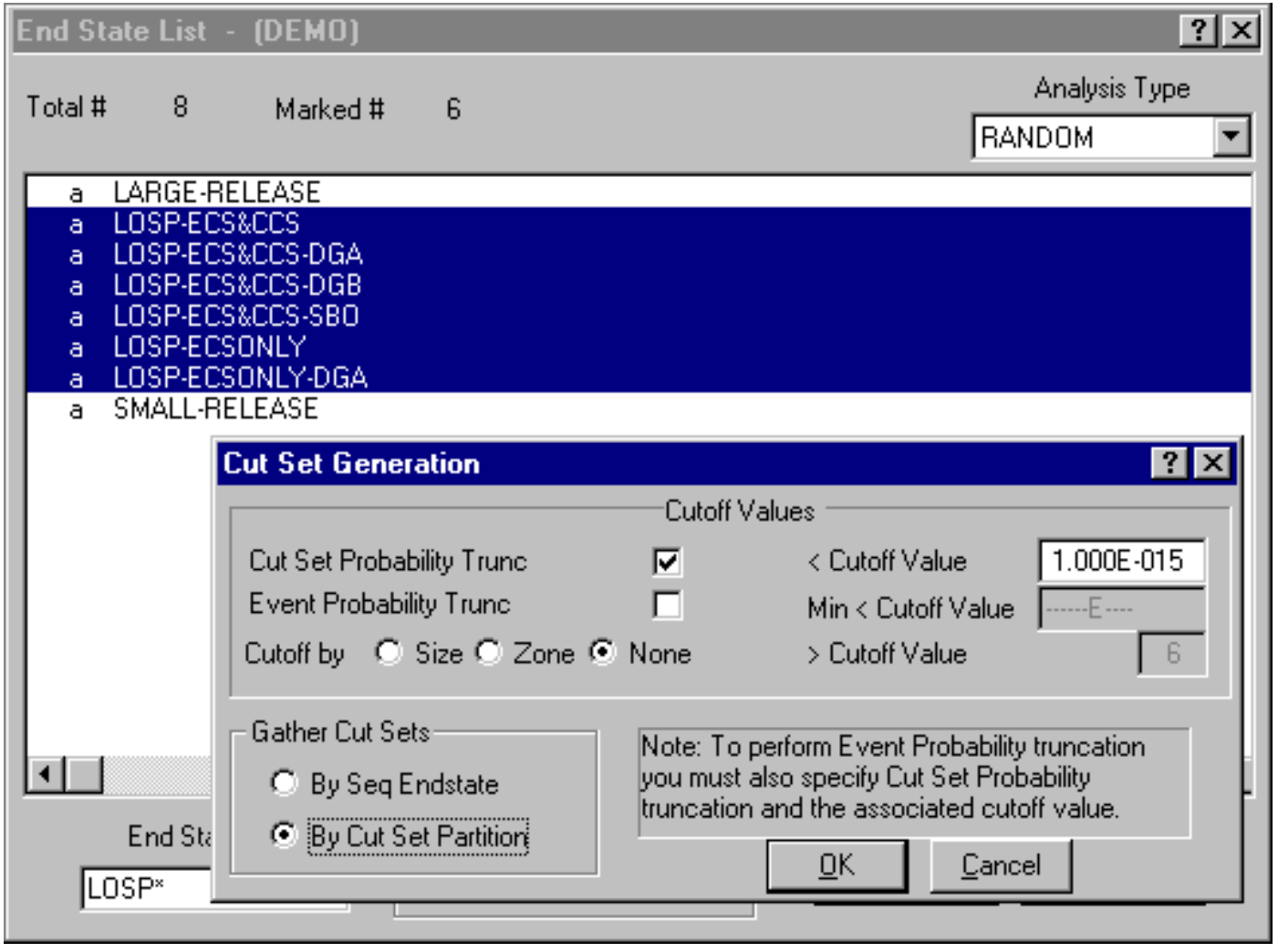

Hyou may change any of the data on this dialog. For more information about the fields in the graphic above, click on the desired field.

In order to gather the partitioned cut sets, from the Gather Cut Sets section you must select the By Cut Set Partition radio button. 


\section{LARGE EVENT TREE METHODOLOGY \\ 16.1 Application of the Large Event Tree Methodology}

\section{PURPOSE}

Describe the "large event tree" methodology and how SAPHIRE can be used to evaluate sequences using this approach. The options that allow truncation of sequences during the process of linking event tree sequences and other options related to analyzing large event trees are presented.

\section{TOPICS}

Large Event Tree Methodology

Connecting Support System to Plant Response Trees

Example Event Trees

Using Link Event Tree Rules to Assign Split-fractions

Using the "S" Calculation Type

Truncating Sequences During Linking

Using Process Flags

Obtaining Cut Sets in Terms of Basic Events

\subsection{Large Event Tree Methodology}

There are two basic approaches for accident sequence quantification:

1. Fault tree linking

2. Large event tree methodology (also called "event trees with boundary conditions")

Characteristics of the large event tree methodology include:

1. Important support systems are modeled as top events in the event trees rather than being contained in the "front line system" or "plant response system" fault trees.

This type of modeling removes shared dependencies from the plant response system fault trees.

2. The paths through the event tree (i.e., sequences) can be quantified by multiplying the splitfranctions along the path because the top events are independent.

This multiplication is in contrast to the fault tree linking approach, where simply multiplying the branch probabilities together would probably yield incorrect results because of the potential for double-counting component failures unless Boolean processes are applied.

3. The split-fraction for each branch point in the model is derived from a fault tree that applies to the branch point. The successes and failures on the path leading to that branch point (which define the 
"boundary conditions" for the fault tree) must be recognized when the fault tree is developed and solved. The resulting "split-fraction" is conditional upon the path through the event tree.

This is in contrast to the fault tree linking approach which usually has only one fault tree that corresponds to a particular top event.

The split-fractions underneath the top event are assigned by using the "Link Event Tree" rules to specify the particular fault tree that corresponds to the branch point.

4. Each path through the event tree (i.e., sequence) is characterized by the initiating event and by the combination of failed and successful fault trees in the path. Success branch probabilities are retained along with the failed branch probabilities for the sequence.

In SAPHIRE, the sequence is stored as a "cut set" even though the term "cut set" implies retaining only the failed branch probabilities.

There are important differences that arise between the fault tree linking approach and the large event tree approach. How the user must construct the model and choose analysis options for the large event tree approach in SAPHIRE are summarized below:

- The sharing of support system event trees with different plant response event trees, depending on the initiating event.

- $\quad$ The use of the Link Event Tree rules to assign split-fractions

- The use of multiple-split branching in the event tree, such as 3-split or 4-split branching.

- $\quad$ The need to use truncation when generating sequences because of the large number of sequences that could be generated. (Currently, a SAPHIRE database cannot retain more than 100,000 sequences.)

- $\quad$ The analysis options and use of Process Flags used to retain success events in the cut sets.

\subsection{Connecting Support System Event Trees to Plant Response Event Trees}

A "support system event tree" (that represents power systems, instrument air, etc.) may be used by several initiating events.

The "support system event tree" needs to transfer to the appropriate plant response event tree, depending on the initiating event.

To connect the event trees (and avoid having to duplicate event trees), the following approach is preferred:

1. Create an event tree that will contain the initiating event and will transfer to the appropriate support system event tree. (Note: SAPHIRE requires that at least two tops be present in each event tree; however, there does not need to be any branching.) 
2. The path through the support system event trees (which may contain many transfers to include all of the support system event trees) will ultimately result in the need to transfer to the appropriate plant response event tree.

3. Rules can be written in the "Link Event Tree" rule editor to enact this transfer.

Notice that more than one initiating event could call the same support system tree. And the support system tree could transfer to different plant response trees.

"Initiating Event" Trees
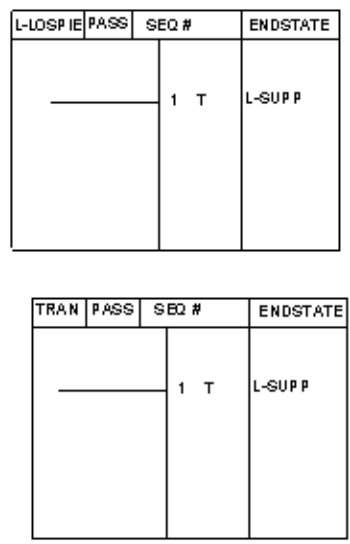

"Plant Response" Trees

The "Support System" Tree
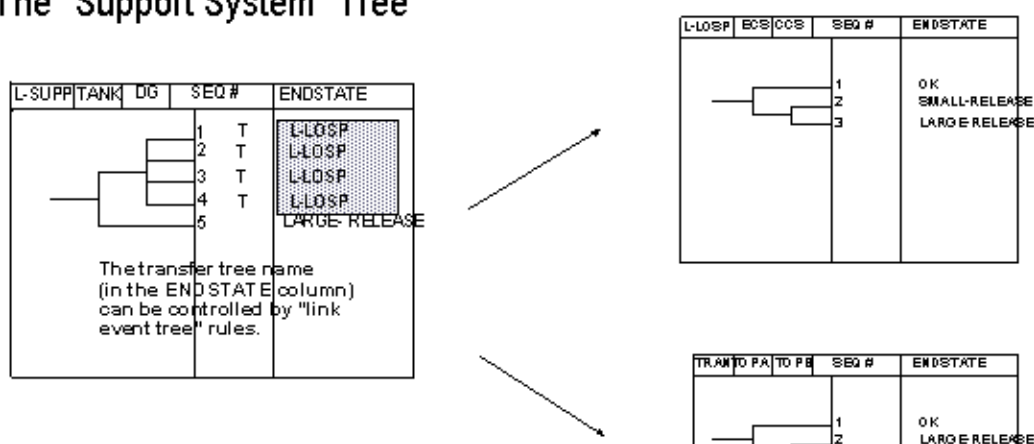

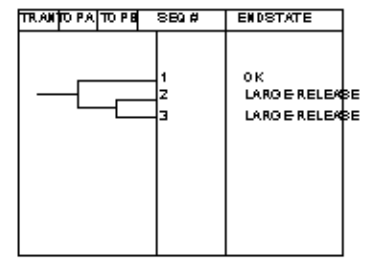

\subsection{Using "Link Event Tree" Rules to Assign Split-Fractions}

In the large event tree approach, the particular path through the event tree determines the status of support systems and therefore, determines the appropriate fault tree for plant response systems that have dependencies on the support systems. There may be several variations of the basic fault tree used underneath each event tree top event.

In contrast, with the fault tree linking approach, each top event usually corresponds to a single fault tree or single top event probability for the failed branch.

\section{Plant Response Tree Rules}

To assign the proper split-fractions, the rules shown here must be applied during sequence generation. (Additionally, the sample support system rules must also be applied.) 


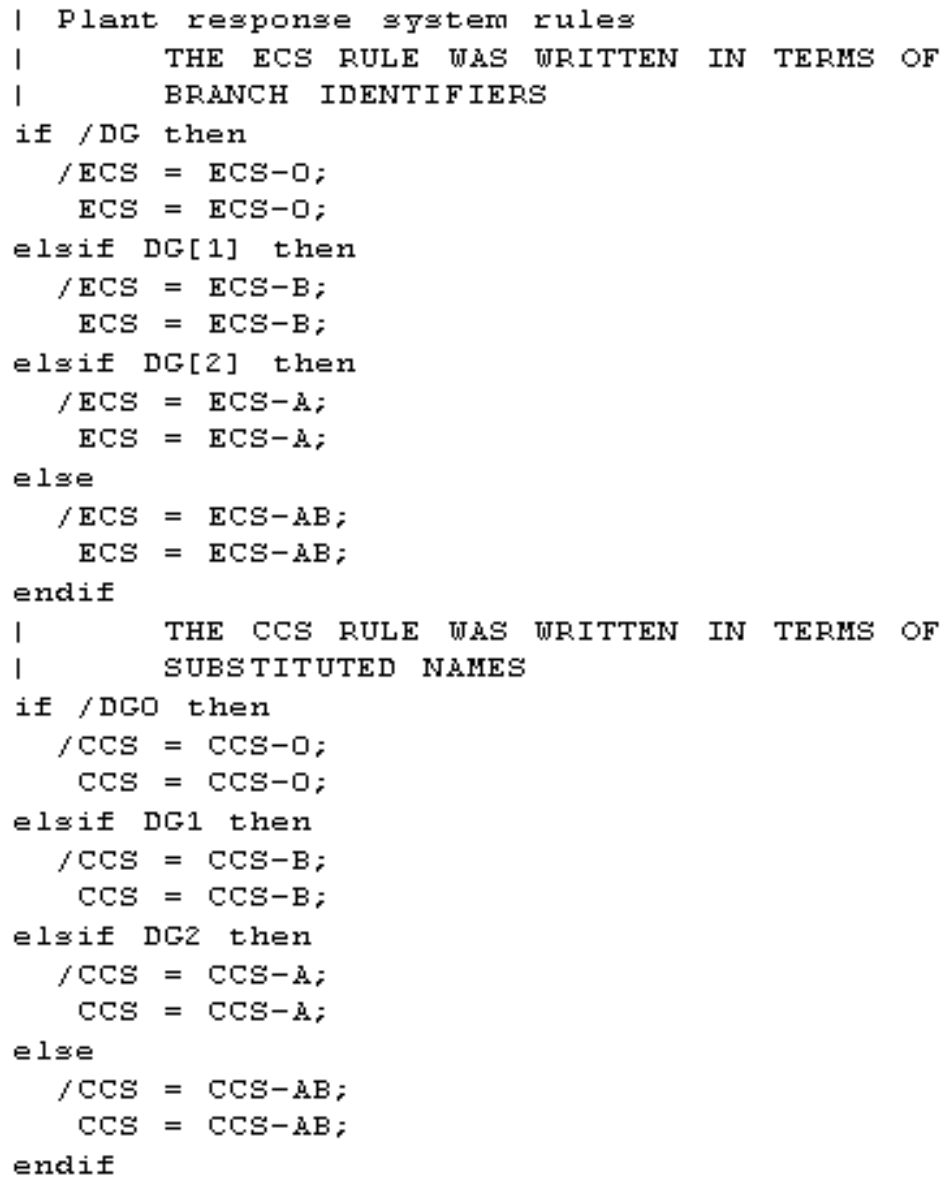

Notice that the rules can be written in terms of the branch identifier (e.g., DG[1]) or in terms of the fault tree name (e.g., DG1).

The fault tree name must have been assigned by earlier rules, usually, the support system rules.

\section{SEE ALSO}

Link Event Tree rule language and rule editor. 


\subsection{Using the "S" Calculation Type}

The "S" calculation type is specified in the Modify | Basic Events option.

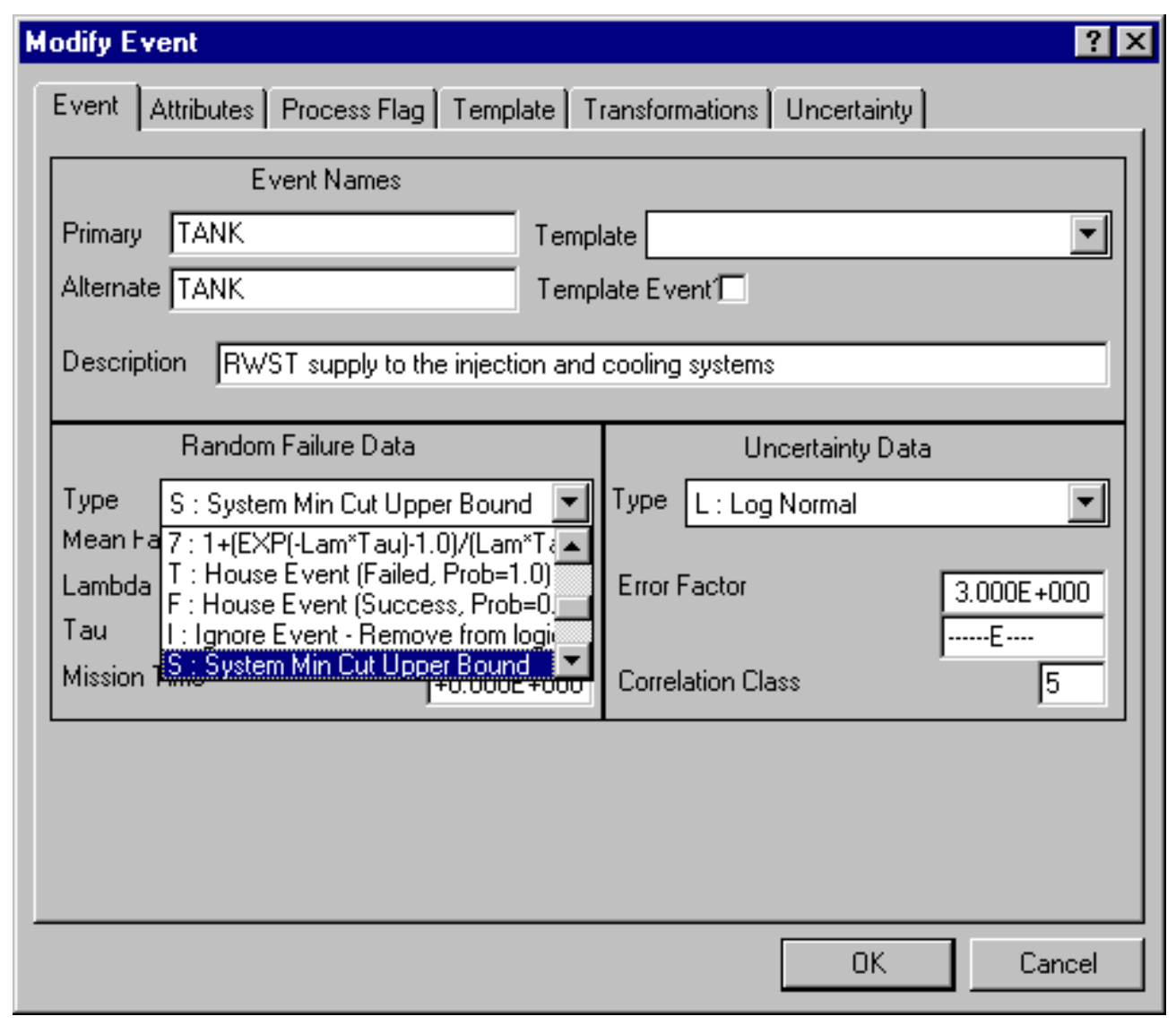

The "S" calculation type allows the split-fraction to be set to the previously calculated minimal cut set upper bound for the fault tree. Using this feature saves the user from having to manually type in the splitfraction probability.

When the "S" calculation type is used, the proper steps must be taken to load the minimal cut set upper bound value into the current database. The steps are as follows:

1. Enter the fault tree logic and basic event data associated with the fault tree.

2. Specify the S calculation type for the fault tree or "split-fraction" name which corresponds to the fault tree top gate name.

3. Perform the Generate function from the Generate menu option. This must be performed prior to generating the fault tree cut sets so that the new basic event data is in the "current" data base.

4. Analyze the fault tree using the Fault Tree option from the SAPHIRE menu. (For a new fault tree, you would begin with the Solve option.)

5. Repeat the Generate option, as in Step 3. This step must be performed prior to generating sequences with truncation. You can use the Report | Basic Event option to report current fault tree probabilities. 


\subsection{Truncating Sequences During Event Tree Linking}

\section{Prerequisites for Generating Sequences with Truncation}

The fault trees that do not have logic should have a failure probability specified prior to generating (and truncating) sequences. This is entered via the Modify | Basic Events option.

The fault trees that have logic should also have a failure probability specified prior to generating (and truncating) sequences. This is also entered in the Modify | Basic Events option either by directly specifying a probability or by using the "S" calculation type.

\section{Entering Fault tree Split-Fractions}

Fault trees, whether they have logic or not, are listed both as Fault Trees and as Basic Events in the SAPHIRE database. When a Fault Tree is entered into the database, it is automatically added to the Basic Event list.

The fault tree failure probability provides the split-fraction for the failed branches and its complement provides the split-fraction for the success branches.

In this example, the L-LOSPIE event tree will transfer to the L-SUPP tree, which transfers to the L-LOSP tree. To generate all of the desired sequences:

1. Select Event Tree from the SAPHIRE menu.

2. The Event Tree List dialog will be displayed.

3. Highlight only the L-LOSPIE tree. Selecting the L-SUPP or L-LOSP trees would generate unwanted sequences in this example case.

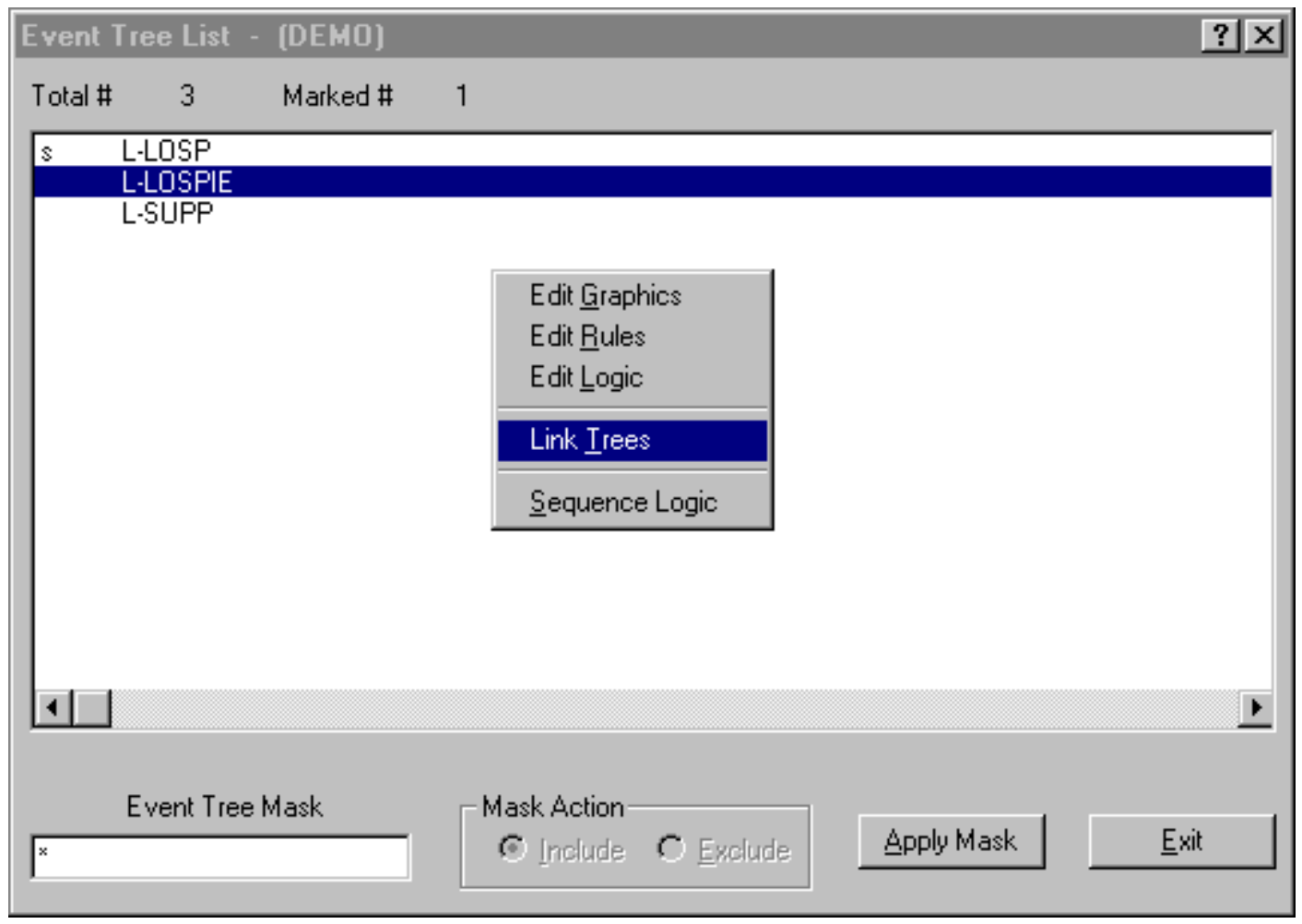


4. Right-click to invoke the pop-up menu and select the Link Trees option.

5. The Generate Options dialog will be displayed.

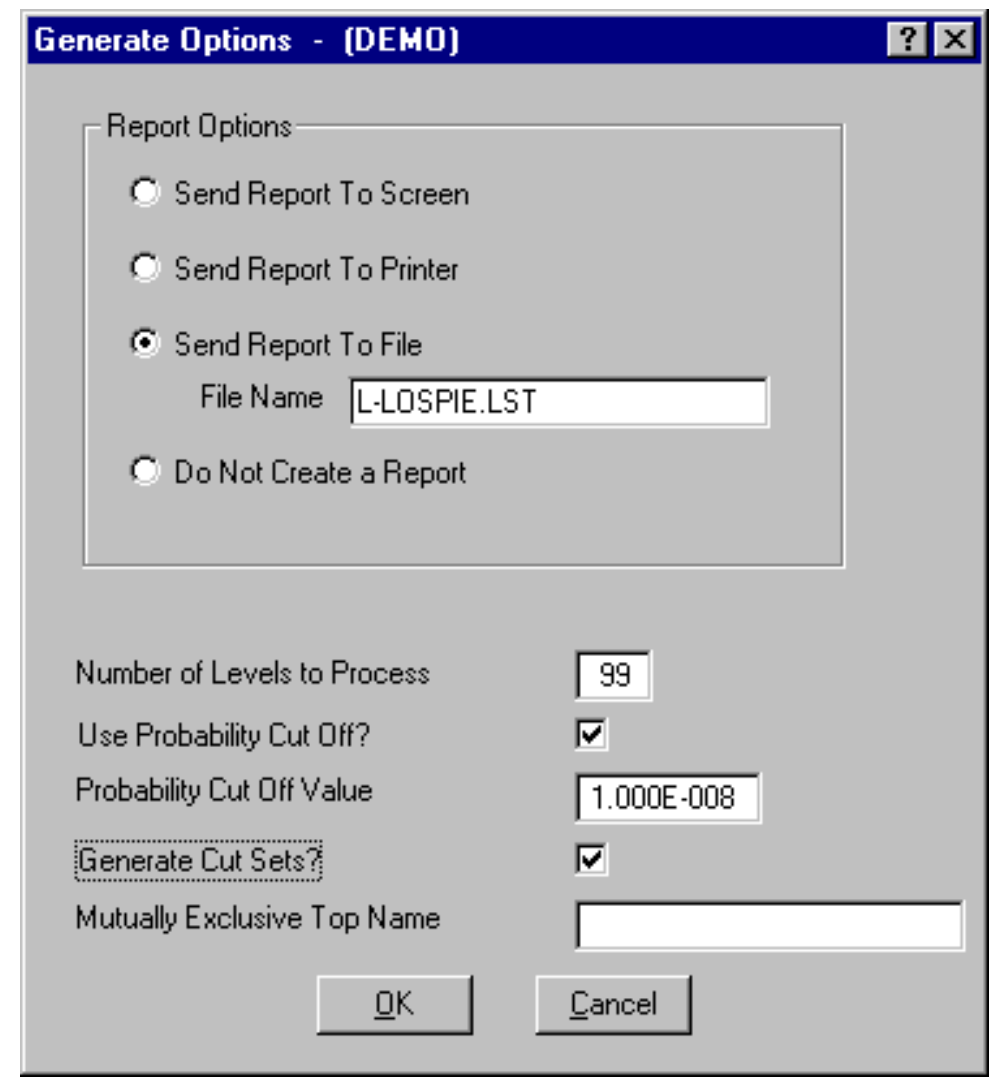

When using the large event tree approach, perform truncation when generating sequences by selecting the following options:

1. Select the Use Probability Cut Off? check box, and enter the cutoff value. 1.0E-08 was used here.

2. Select the Generate Cut Set? check box.

Using these options will eliminate any sequences that are below the specified truncation value. The calculated sequence frequencies are based on the fault tree failure probabilities entered in the Modify | Basic Events option or entered via a change set.

NOTE: During the sequence truncation process, fault trees are not evaluated to obtain the sequence frequency.

The sequences generated by this tree are shown in the table. 


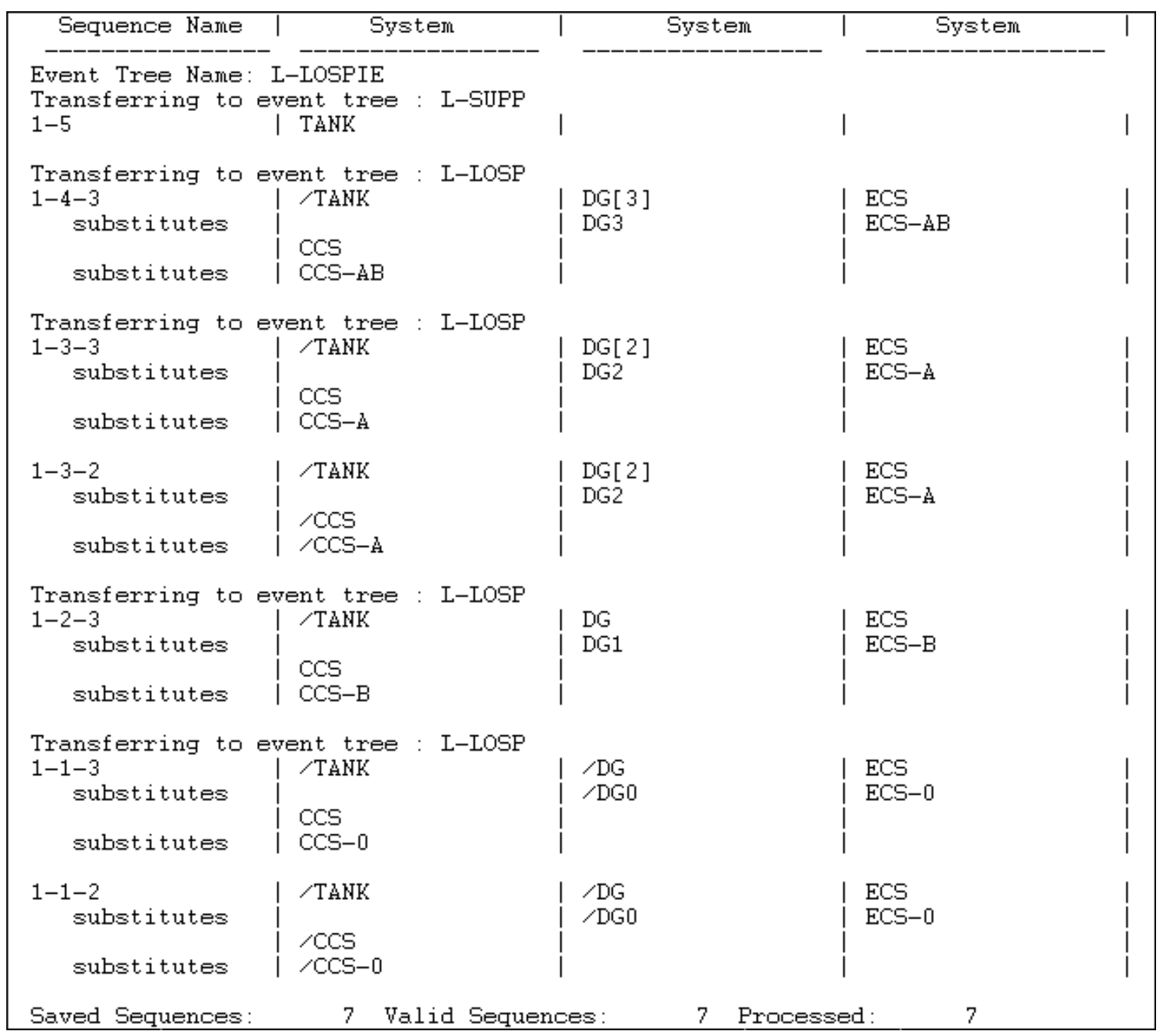

The file that the generated sequences are listed in can be named from the "File Name" field of the Generate Options dialog. If many sequences are generated, this file can become large - slowing down the sequence generation process and taking up disk space. It may be desirable to prevent this file from being created by selecting the Do Not Create a Report radio button.

3. After linking the event trees, you will need to quantify the sequences prior to reporting sequence results. Select Sequence from the SAPHIRE menu.

4. Highlight the L-LOSPIE sequences and right-click to invoke the pop-up menu. 


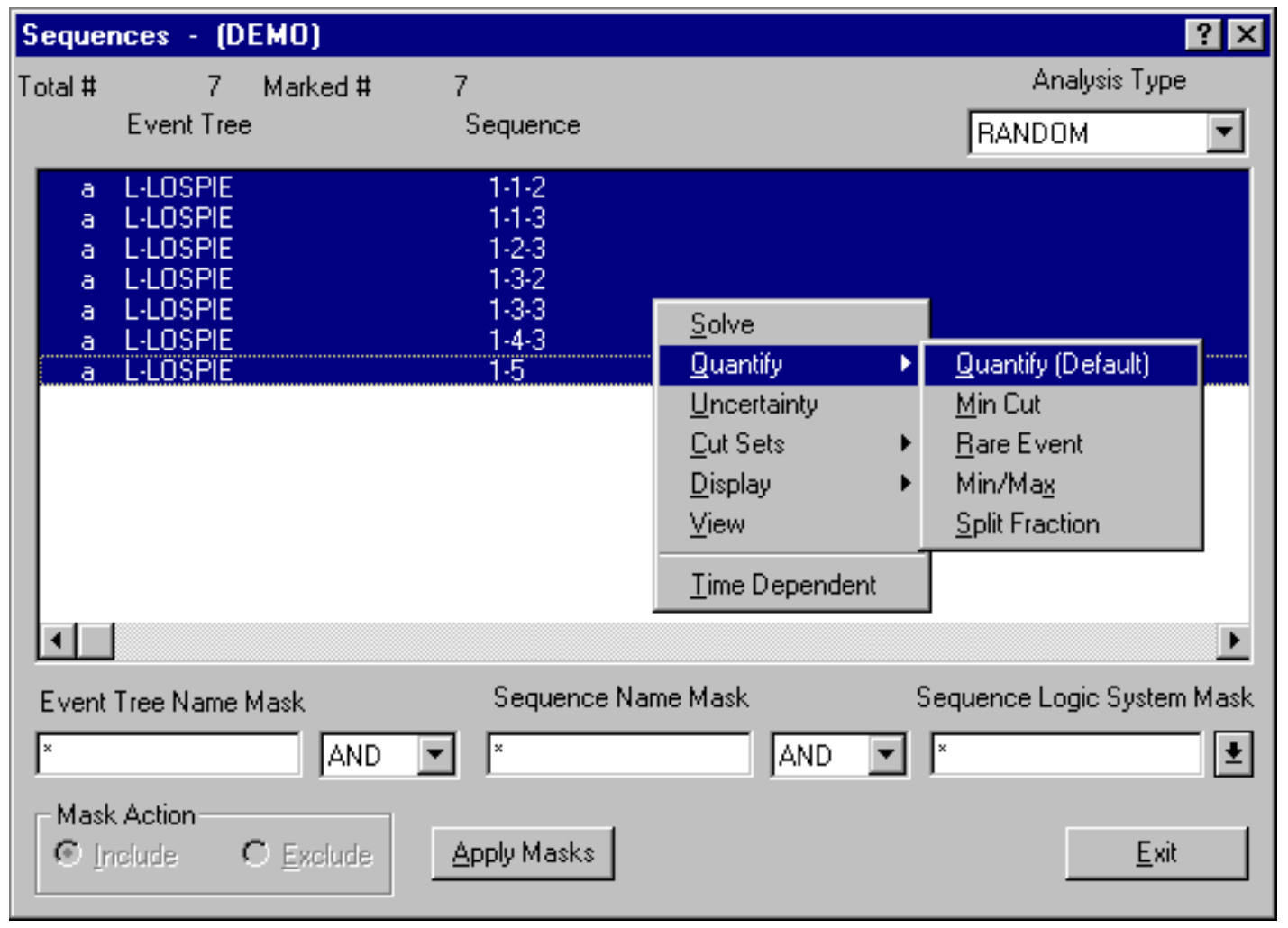

\section{Choose Quantify | Default.}

6. The sequence results can be viewed by highlighting the desired sequence(s) and the right-clicking and choosing Display | Cut Sets from the pop-up menu. The success and failure split-fractions are displayed in the Selected Cut Sets dialog. 


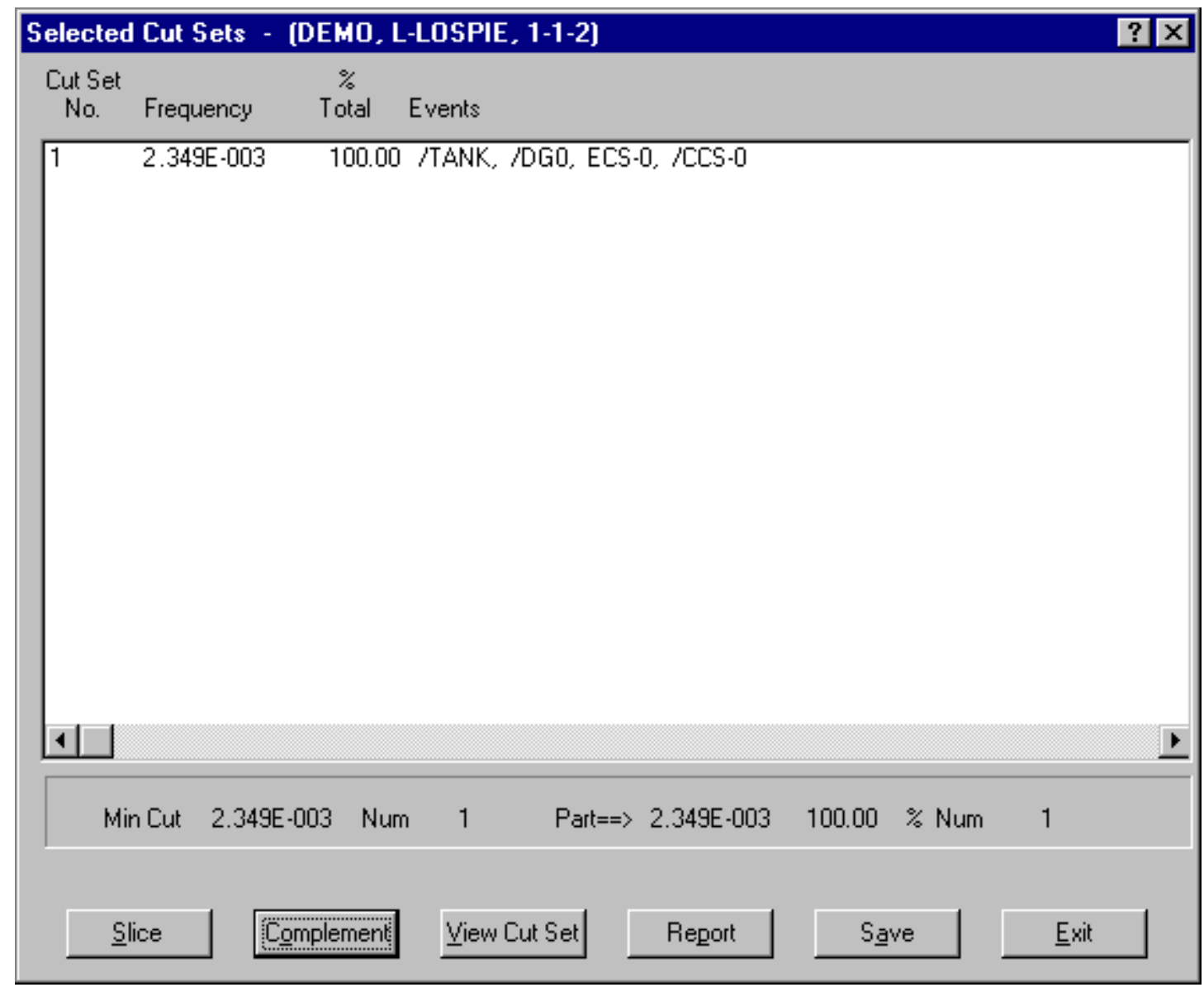

Notice that each sequence will contain only one cut set which represents the split-fraction along the event tree path.

\subsection{Using Process Flags to Retain Success Events}

Process Flags are needed to specify the proper treatment of success events if cut sets are to be generated in terms of basic events following sequence generation. They are specified in the Modify | Basic Events option. Choose the down arrow adjacent to the Process Flag field to invoke the Process Flag dialog. 


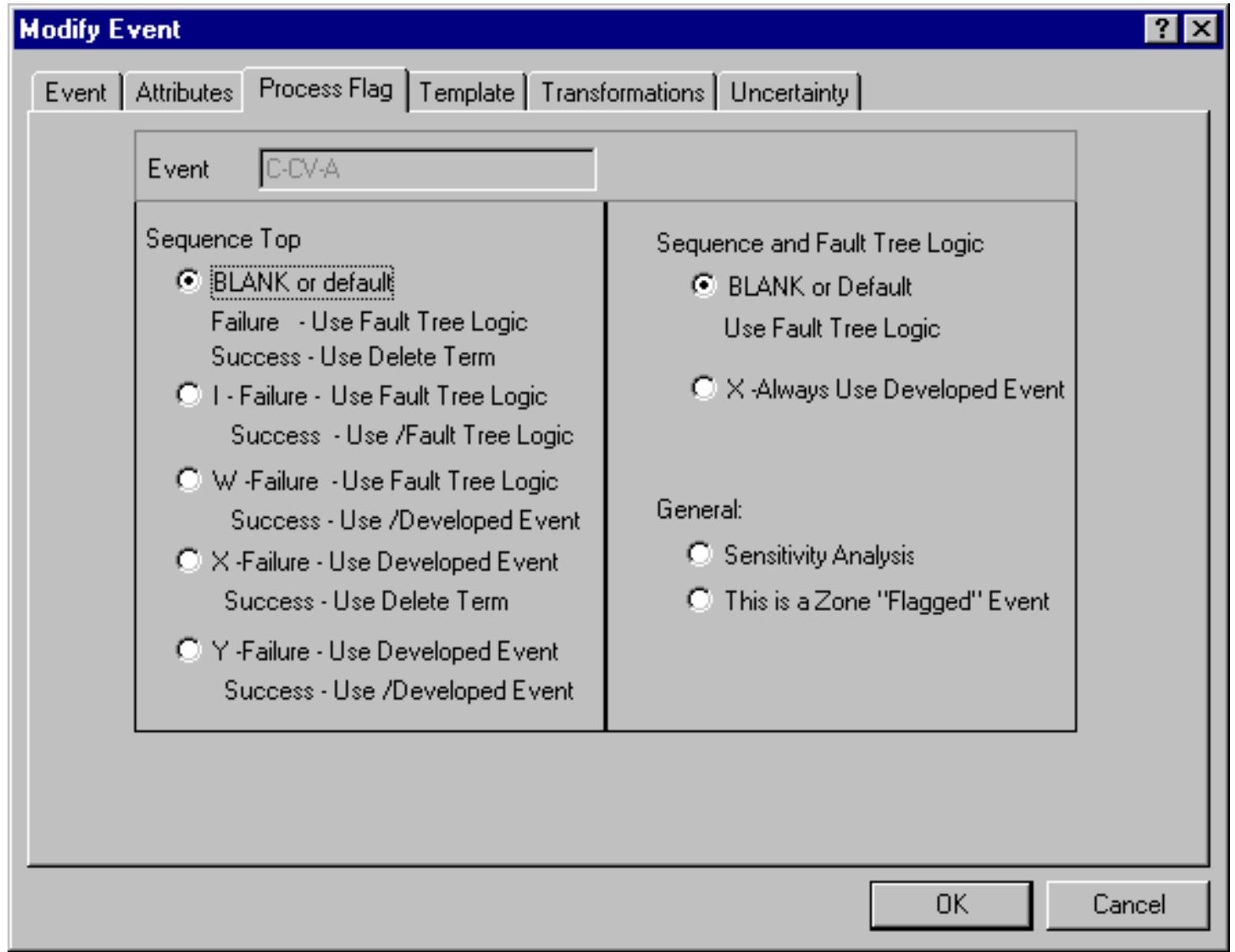

The Y Process Flag is used when the top event does not have fault tree logic (or you chose to ignore the fault tree logic).

The W Process Flag is used when the top event has fault tree logic that is to be used for the failed branch, but the split-fraction is to be used for the success branch.

\section{The Process Flags and Calculation Types specified in this example are shown below:}

\begin{tabular}{|c|c|c|c|}
\hline Name & $\begin{array}{l}\text { Calc } \\
\text { Type }\end{array}$ & $\begin{array}{c}\text { Process } \\
\text { Flag }\end{array}$ & Comment \\
\hline CCS-0 & S & $\mathrm{W}$ & System calls a fault tree \\
\hline CCS-A & S & W & System calls a fault tree \\
\hline CCS-AB & 1 & $\mathrm{Y}$ & System uses a probability \\
\hline CCS-B & 1 & $\mathrm{Y}$ & System uses a probability \\
\hline DG0 & $\mathrm{S}$ & Y & $\begin{array}{l}\text { System calls a fault tree, but only the success split-fraction is } \\
\text { needed }\end{array}$ \\
\hline DG1 & S & & $\begin{array}{l}\text { System calls a fault tree that is only used for failed branches, } \\
\text { not for success branches }\end{array}$ \\
\hline DG2 & S & & $\begin{array}{l}\text { System calls a fault tree that is only used for failed branches, } \\
\text { not for success branches }\end{array}$ \\
\hline DG3 & $\mathrm{S}$ & & $\begin{array}{l}\text { System calls a fault tree that is only used for failed branches, } \\
\text { not for success branches }\end{array}$ \\
\hline ECS-0 & $\mathrm{S}$ & $\mathrm{W}$ & System calls a fault tree \\
\hline
\end{tabular}




$\begin{array}{lccl}\text { ECS-A } & 1 & \text { Y } & \text { System uses a probability } \\ \text { ECS-AB } & 1 & \text { Y } & \text { System uses a probability } \\ \text { ECS-B } & \text { S } & \text { W } & \text { System calls a fault tree } \\ \text { TANK } & 1 & \text { Y } & \text { Top event uses a probabliity }\end{array}$

\subsection{Obtaining Cut Sets in Terms of Basic Events}

To obtain cut sets in terms of basic events from the fault tree rather than in terms of fault tree splitfractions, use the Sequence | Solve option. You need to have generated the sequences before you use this option.

The cut sets can be viewed from the Sequence | Display | Cut Sets option. In this example, the success and failures in terms of basic events are displayed.

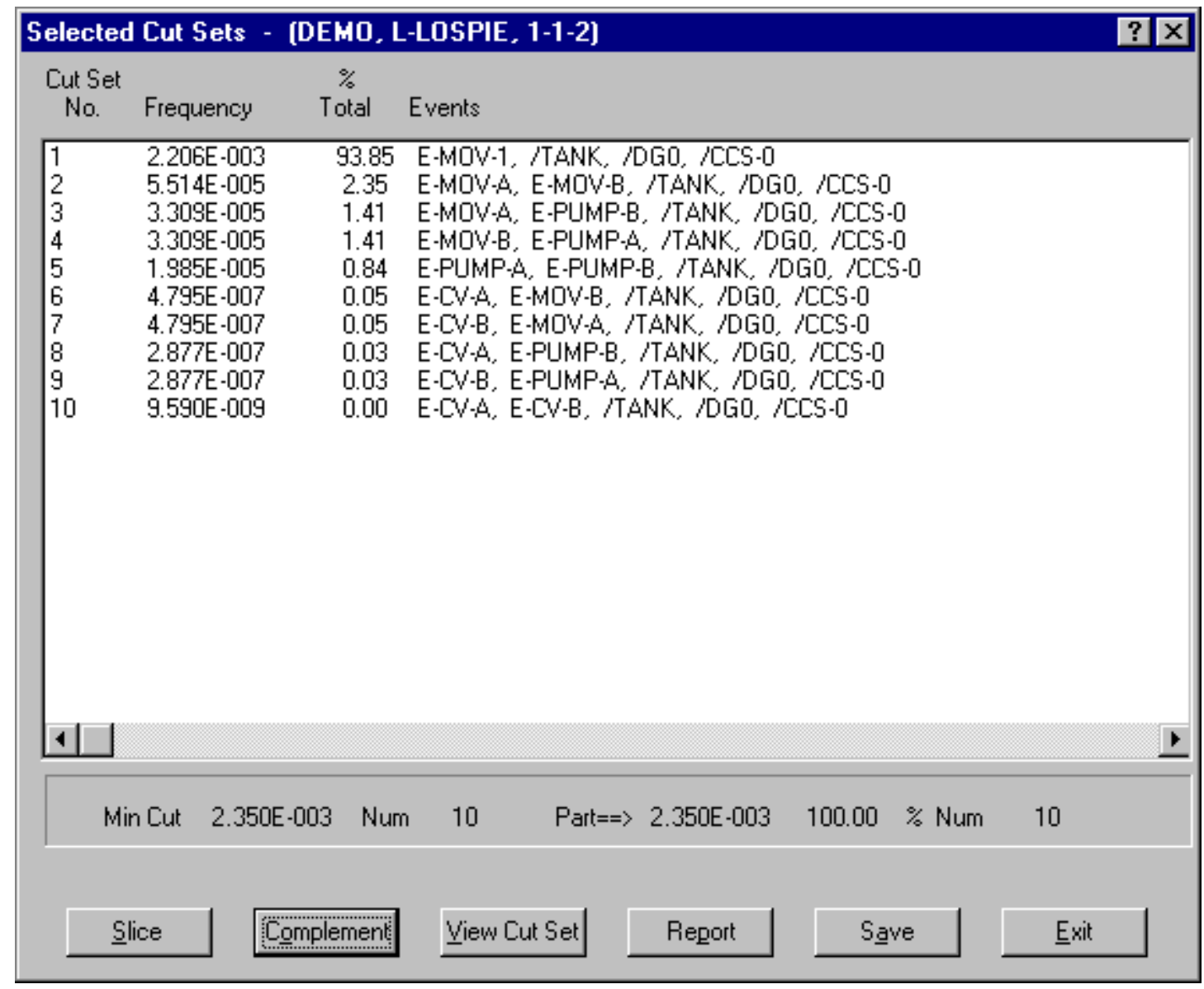

NOTE: To obtain cut sets in terms of basic events, the fault trees for every split-fraction that is affected by a basic event in a fault tree must be in the database. 


\subsection{Event Trees Used in Examples}

\subsubsection{Example Event Trees}

These three event trees will be used as examples in this section:

The "Initiating Event" Tree

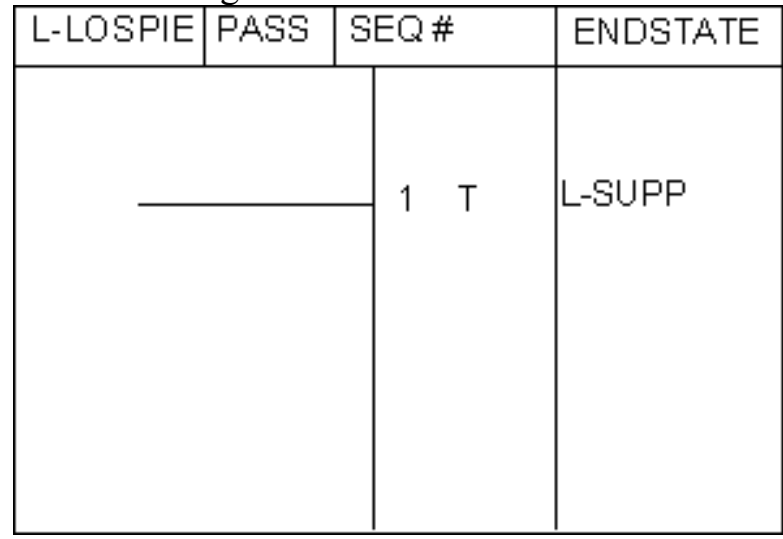

The "Support System" Tree

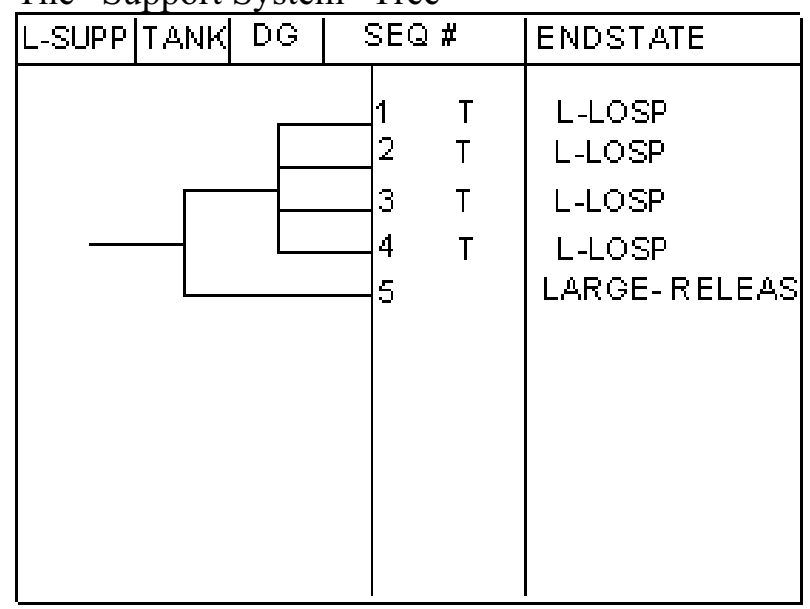

The "Plant Response" Tree

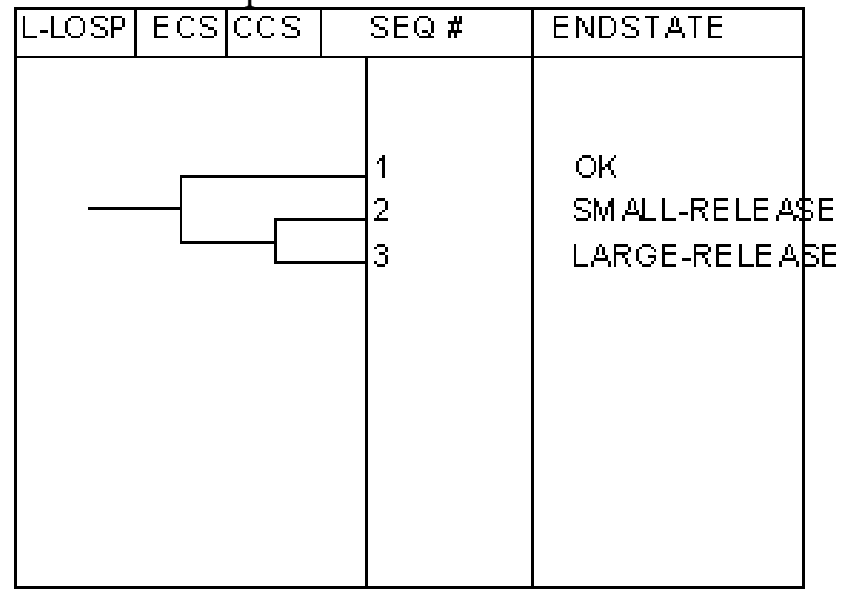




\subsubsection{The Support System Tree}

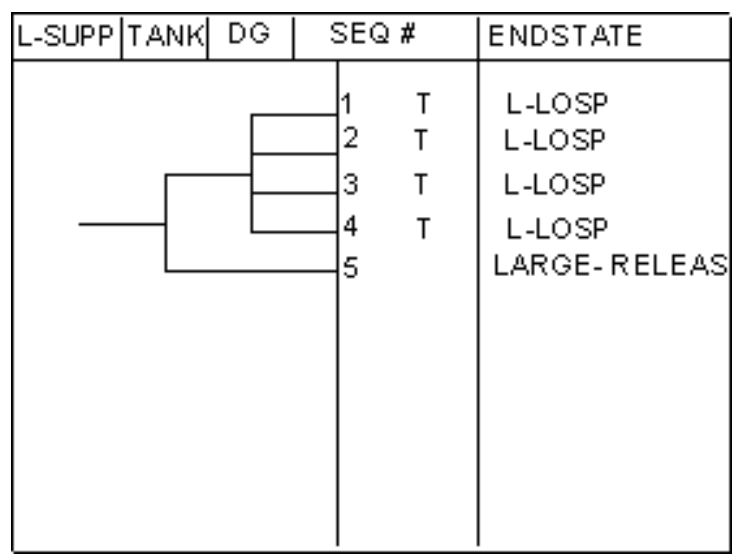

In this example, top event TANK questions the status of the tank, and if the tank is failed, both ECS and CCS fail.

The probability of failure is specified directly, without using a fault tree. The probability of failure is entered via the Modify | Basic Events menu. SAPHIRE determines the success probability as the complement of the failure probability.

$/ \mathrm{TANK}=1-\mathrm{TANK}$

Top event DG questions the status of two diesel generators that provide support to ECS and CCS.

The fault tree for each diesel generator is assumed to consist of a single basic event. Therefore, failure of diesel generator $\mathrm{A}$ is modeled by a fault tree consisting of the basic event DG-A, and failure of diesel generator B is modeled by a basic event DG-B.

The top branch (/DG) represents success of both diesel generators. The next branch down represents success of diesel generator A and failure of diesel generator B. Thus, DG1 is a fault tree containing DGB. The third branch represents success of diesel generator B and failure of diesel generator A. Thus, DG2 is a fault tree containing DG-A. The bottom branch represents both diesel generators being failed.

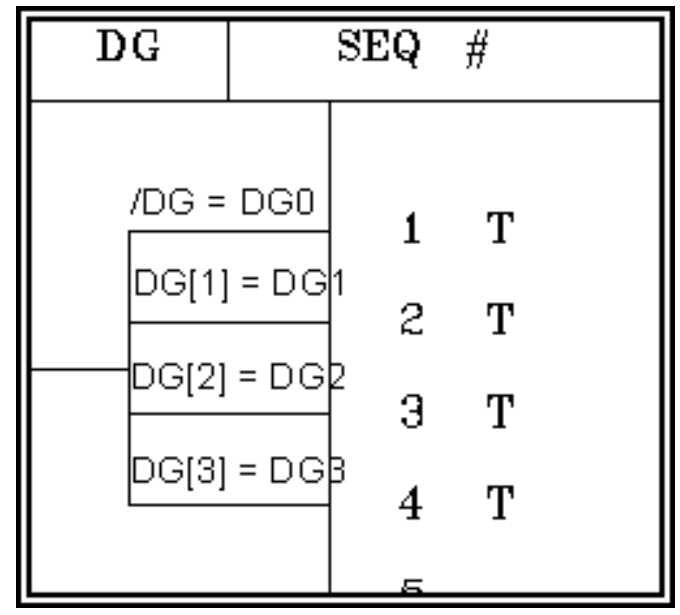


The split-fractions for each branch are determined from the corresponding fault tree. The splitfraction for /DG is taken as the complement of the DG0 fault tree.

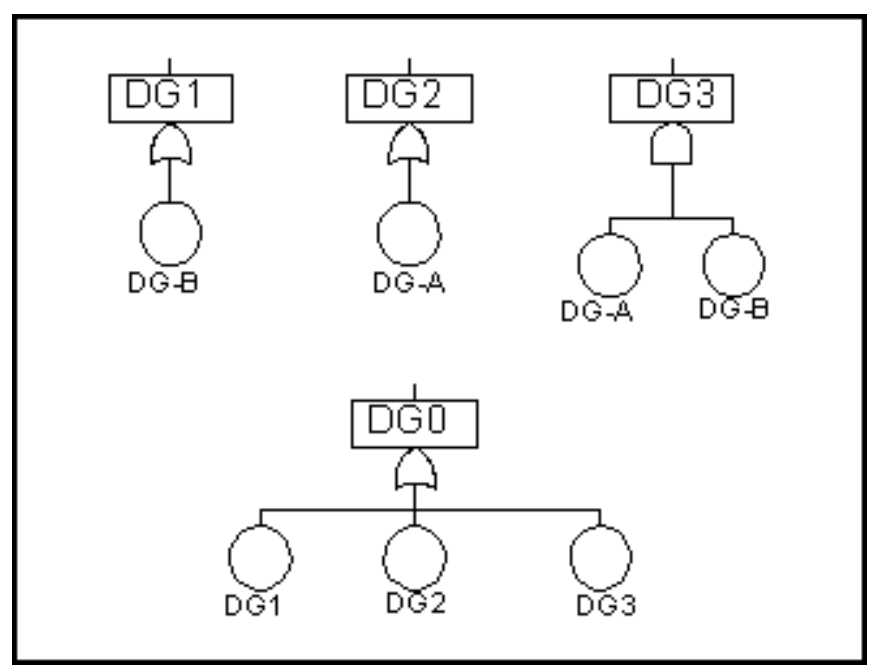

The "link event tree" rule editor is used to assign the appropriate "system" to each branch. The rules for the support system event tree are as shown:

I Support system rules
if always then
$/ \mathrm{DG}=\mathrm{DG} ;$
$\mathrm{DG}[1]=\mathrm{DG} ;$
$\mathrm{DG}[2]=\mathrm{DG} ;$
$\mathrm{DG}[3]=\mathrm{DG} ;$
endif




\subsubsection{The Plant Response Tree}

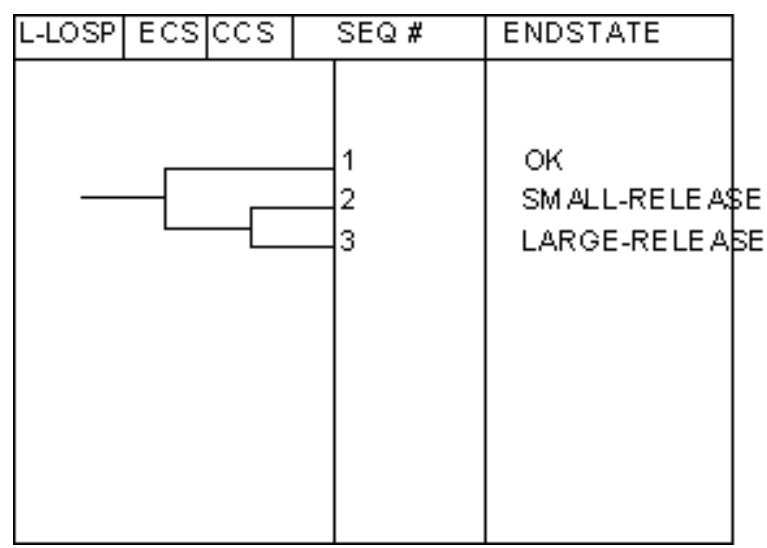

The ECS and CCS top events are similar to the fault tree linking example in the DEMO project, with the following exceptions:

- $\quad$ The TANK failure event is no longer contained in the fault trees for ECS and CCS.

- $\quad$ The status of the DGs depends on the path through the support system event tree.

When the top branch of the support system tree transfers to the plant response tree (PRT), both DGs are successful. When the second branch of the support system tree transfers to the PRT, DG A is successful but DG B is failed, and so on. As you can see, the probability of ECS (and CCS) failing depends on the path through the system support event tree. A special version of the ECS (and CCS) fault tree is created for each situation. The "Link Event Tree" rules are used to assign the fault tree name which corresponds to the proper fault tree based on the status of the DGs. The success or failure probability for each fault tree (the split-fractions) can be derived from each fault tree prior to generating sequences (via the "S" calculation type) or can be directly specified by the user as a probability.

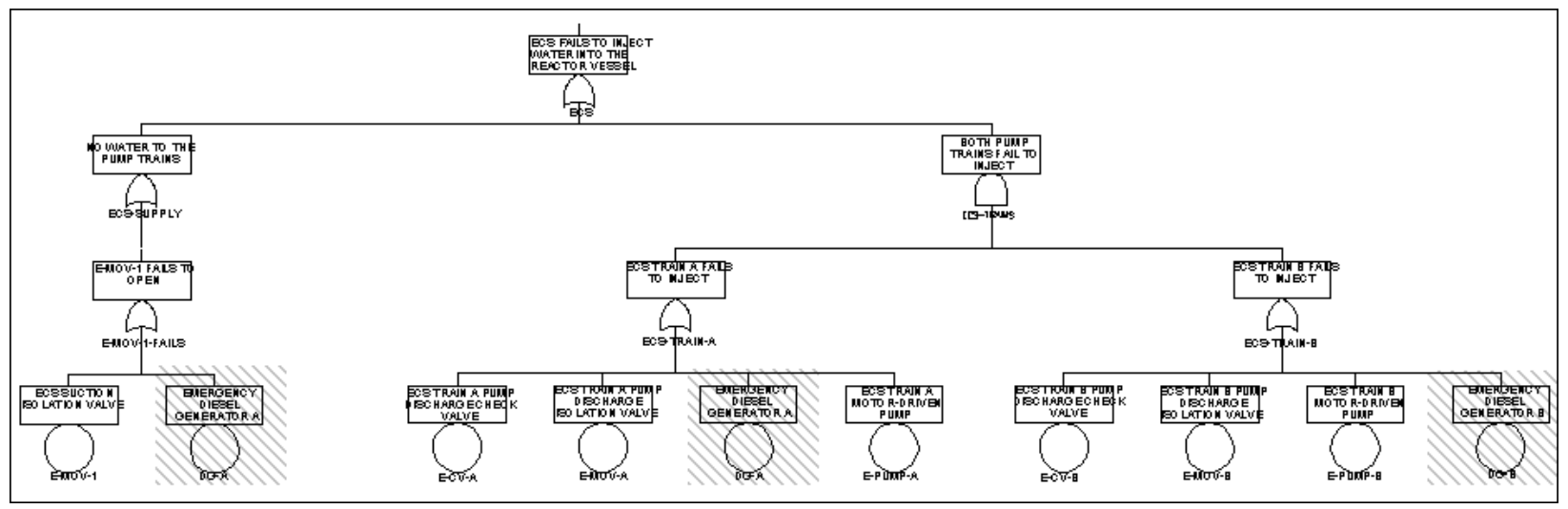




\begin{tabular}{lll}
\multicolumn{1}{c}{ DG Status } & \multicolumn{1}{c}{ ECS Fault Tree Name } & \multicolumn{1}{c}{ Split-Fraction } \\
No DGs failed & ECS-0 & $1.065 \mathrm{E}-3$ \\
Only DG-A failed & ECS-A (FAILED) & 1.0 \\
Only DG-B failed & ECS-B & $9.076 \mathrm{E}-3$ \\
Both DGs failed & ECS-AB (FAILED) & 1.0
\end{tabular}

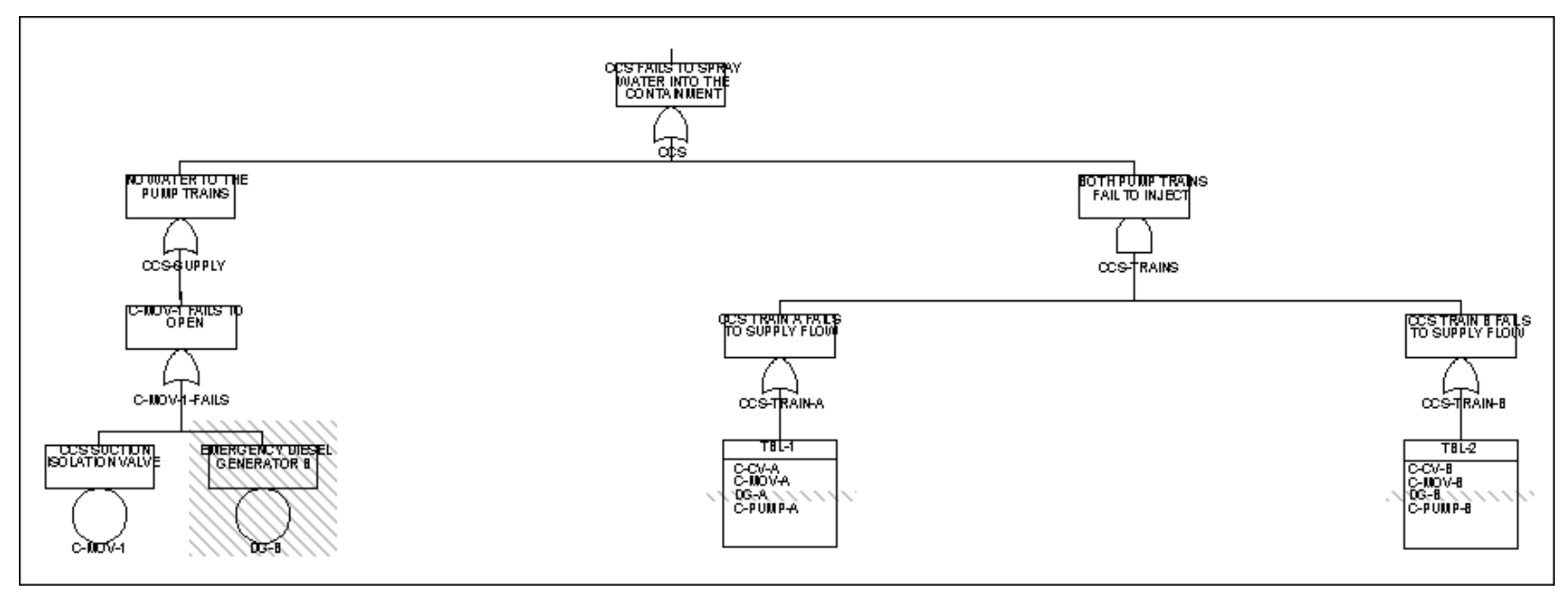

\begin{tabular}{lll}
\multicolumn{1}{c}{ DG Status } & \multicolumn{1}{c}{ CCS Fault Tree Name } & \multicolumn{1}{c}{ Split-Fraction } \\
No DGs failed & CCS-0 & $1.065 \mathrm{E}-3$ \\
Only DG-A failed & CCS-A & $9.076 \mathrm{E}-3$ \\
Only DG-B failed & CCS-B (FAILED) & 1.0 \\
Both DGs failed & CCS-AB (FAILED) & 1.0
\end{tabular}




\section{LINKAGE RULES}

\subsection{Linkage Rules Usage and Examples}

\section{PURPOSE}

The linkage rule editor allows you to create rules that affect sequence generation. Typically, these rules are used to replace default fault trees with substituted fault trees (or "split-fractions") based on logical conditions that are specified in the rules.

These rules are applied when sequence logic is generated. Without using rules, the sequence logic consists of the logical combinations of failed and successful top events (and the initiating event for the event tree). These rules allow the user to replace the top event (which is called a "fault tree" in SAPHIRE) with a substituted fault tree based on the logical conditions dictated by the rule.

\section{TOPICS}

Search Criteria Examples

Symbols

Keywords and Structures

Binary and Multiple-Split Branches

\subsection{Search Criteria Examples}

These examples are based upon an event tree with initiating event IE-1 and top events A, B, and C.

\begin{tabular}{|c|c|c|c|}
\hline $\begin{array}{c}\text { Search } \\
\text { Criteria }\end{array}$ & $\begin{array}{l}\text { Meaning of the Search } \\
\text { Criteria }\end{array}$ & $\begin{array}{c}\text { Search } \\
\text { Criteria }\end{array}$ & Meaning of the Search Criteria \\
\hline $\operatorname{init(IE-1)}$ & $\begin{array}{l}\text { Initiating event with the name } \\
\text { IE-1 }\end{array}$ & $\sim \mathrm{A}$ & Failure of A never occurs \\
\hline A & $\begin{array}{l}\text { Failure of top event or fault } \\
\text { tree A }\end{array}$ & $\sim(/ \mathrm{A}+\mathrm{A})$ & $\begin{array}{l}\text { Success of A and failure of A } \\
\text { never occurs (can be used to test } \\
\text { for a "pass" condition) }\end{array}$ \\
\hline /A & $\begin{array}{l}\text { Success of top event or fault } \\
\text { tree A }\end{array}$ & $A * B$ & Failure of A and of B occur \\
\hline$(\mathrm{A}+\mathrm{B}) * \mathrm{C}$ & $\begin{array}{l}\text { Failure of A or B occurs and } \\
\text { failure of } C \text { occurs }\end{array}$ & always & $\begin{array}{l}\text { This pre-defined macro name } \\
\text { means the criteria is always met. }\end{array}$ \\
\hline
\end{tabular}




\title{
17.3 Rule Editor Symbols
}

\author{
| (comment) Denotes a comment line \\ * (AND) Logical AND operator \\ $+(\mathrm{OR}) \quad$ Logical OR operator \\ $\sim$ (never) Logical operator for "never" or "not present" \\ / (complement) Complement \\ ; (end macro) Indicates the end of a macro line or a line that modifies the criteria being \\ evaluated. \\ ( ) Parentheses for grouping terms
}

\subsection{Keywords and Structures}

\subsubsection{Linkage Rules Keywords and Structure}

if-then - Indicates that a search criteria is being specified.

if-then using wildcards - A search criteria can be specified using wildcards.

always - Indicates that the search criteria is always satisfied.

elsif - Specifies an alternative search criteria.

else - Specifies some action to be taken if all the search criteria are not met.

Cast Operators - Define substitutions for the end state or flag set of a sequence, or define a new transfer event tree

Skip - Indicates that the specified item should be ignored.

Macro - A user-definable keyword that specifies a search criteria.

\subsection{2 if-then}

This keyword indicates that a search criteria is being specified. The information between the "if" and the "endif" clause defines the tops to be replaced. There is no limit on the number of replacements that can be defined between these clauses.

| The "if-then" rule structure:

| This rule replaces $\mathrm{C}$ with C-SYS when

$\mathrm{A}$ and $\mathrm{B}$ are both failed.

Only sequences 6 and 7 are affected by this rule.

if $\mathbf{A} * \mathbf{B}$ then

$/ \mathrm{C}=\mathrm{C}-\mathrm{SYS}$;

$\mathrm{C}=\mathrm{C}-\mathrm{SYS}$;

endif 


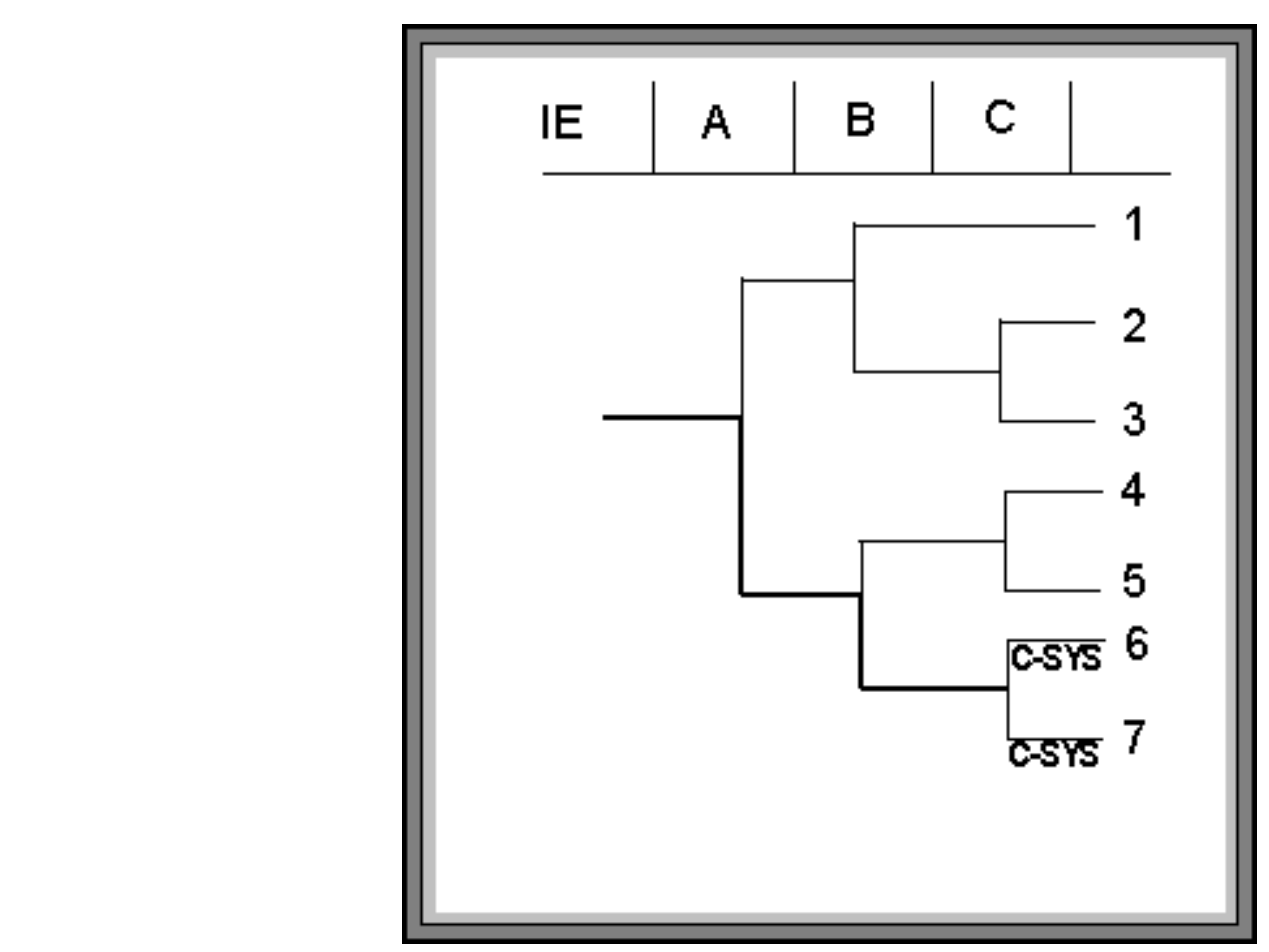

\section{Important Reminders:}

Each replacement line must end with a semicolon.

You can add as many replacement lines in the rule as you want.

The rules are sensitive to UPPER and lower case - the cOrReCtcASe mUSt bE uSeD!

\subsection{3 if-then Using Wildcards}

Wildcard characters can be used with the if-then structure to indicate a search for a group of criteria whose names have something in common. A wildcard is a character that can represent one or more characters in a search criteria. You can use one or more wildcards to specify groups of search criteria. The wildcard characters must be enclosed in quotation marks.

| The "if-then" rule structure using wildcards:

| This rule replaces $\mathrm{C}$ with C-SYS when

the initiating event occurs.

1

| (Sequence 3, 5 and 7 are affected)

if "??" then

$\mathrm{C}=\mathrm{C}-\mathrm{SYS}$;

endif 


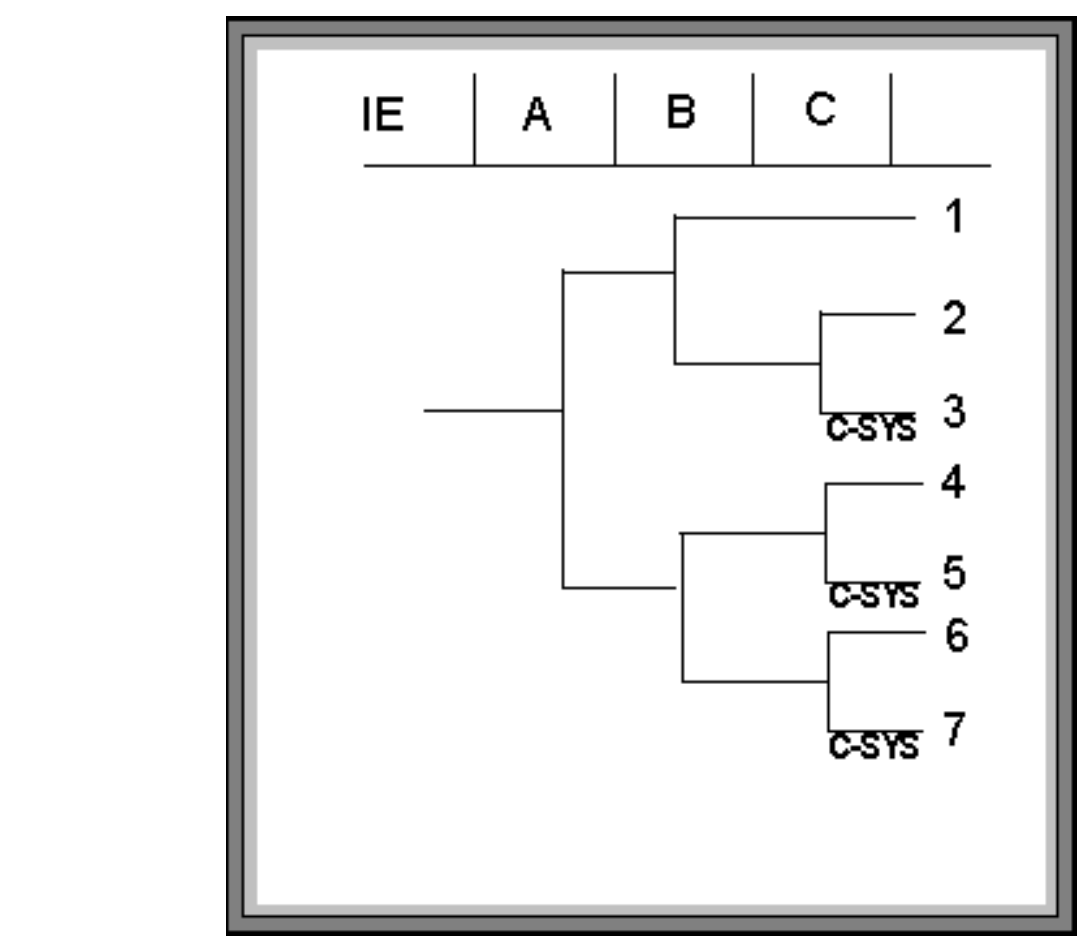

| The "??" refers to the initiating event, but will | key on any top of only two characters.

\subsection{4 always}

This keyword indicates that the search criteria is always satisfied. The substitutions following this condition are always made.

| The "if-always" rule structure:

| This rule replaces every occurrence

of C with C-SYS.

| (Sequences 2 through 7 are affected)

if always then

$/ \mathrm{C}=\mathrm{C}-\mathrm{SYS}$;

$\mathrm{C}=\mathrm{C}-\mathrm{SYS}$;

endif 


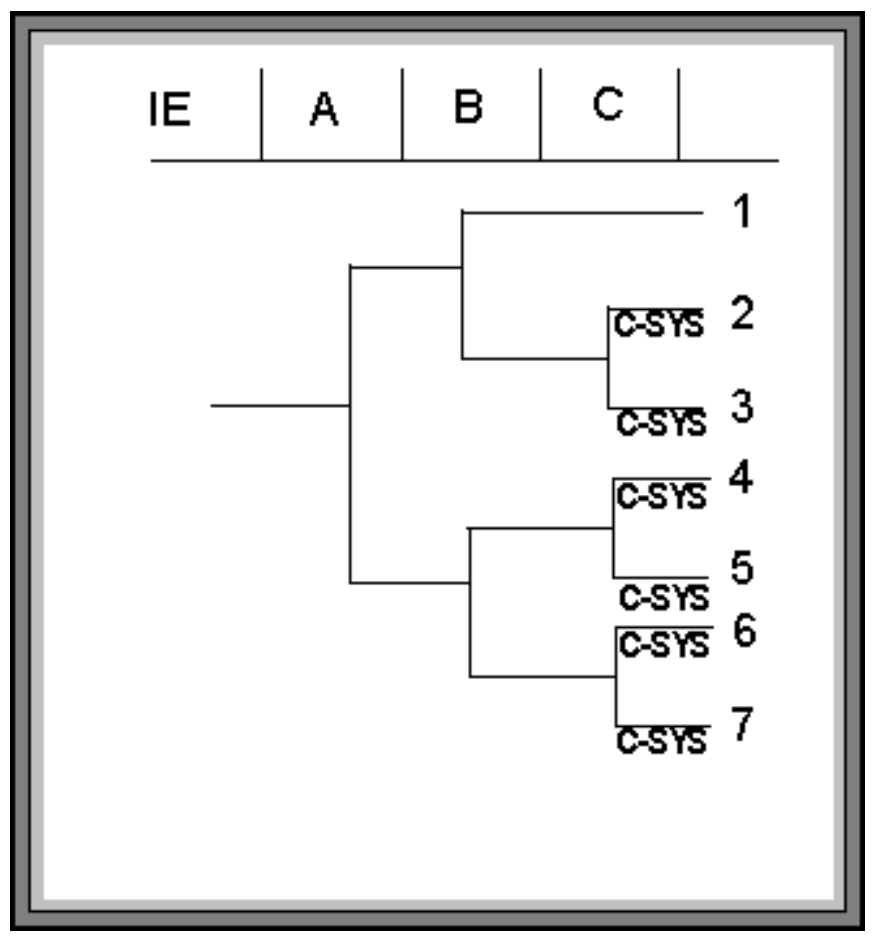

\subsection{5 elsif}

This keyword specifies an alternative search criteria. Any number of "elsifs" can be used within a rule. The first condition that is met is used and the others are ignored.

| The "if-then-elsif" structure:

| This rule replaces $\mathrm{C}$ with $\mathrm{C}-\mathrm{AB}$ if

$\mathrm{A}$ and $\mathrm{B}$ are failed, and replaces

C with $\mathrm{C}-\mathrm{B}$ if only $\mathrm{B}$ is failed.

if $\mathrm{A} * \mathbf{B}$ then

$/ \mathrm{C}=\mathrm{C}-\mathbf{A B}$;

$\mathbf{C}=\mathbf{C}-\mathbf{A B}$;

elsif $B$ then

$/ \mathbf{C}=\mathbf{C}-\mathbf{B}$;

$\mathrm{C}=\mathrm{C}-\mathrm{B}$;

endif 


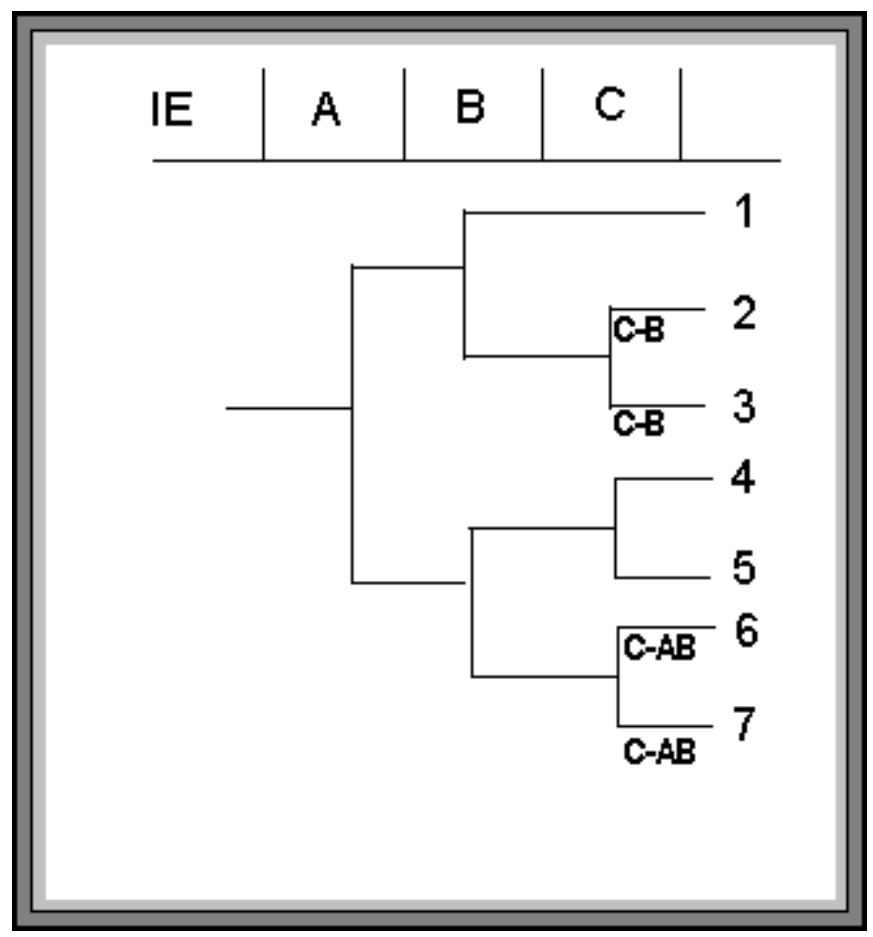

\section{Only One Substitution Per Branch:}

In the "elsif "rule structure, for every applicable branch, only the first substitution that applies is made. Subsequent substitutions are ignored.

In fact, the linkage rules as a whole work this way because only the first substitution for a branch is made. In other words, after a substitution has been assigned, no other rule will overwrite the substitution.

\subsection{6 else}

This keyword specifies that some action to be taken if all the search criteria are not met. The "else" should be the last condition in the rule.

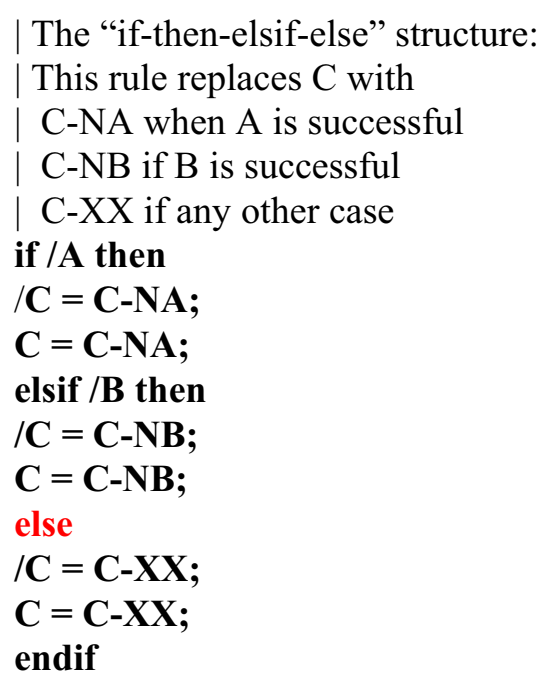




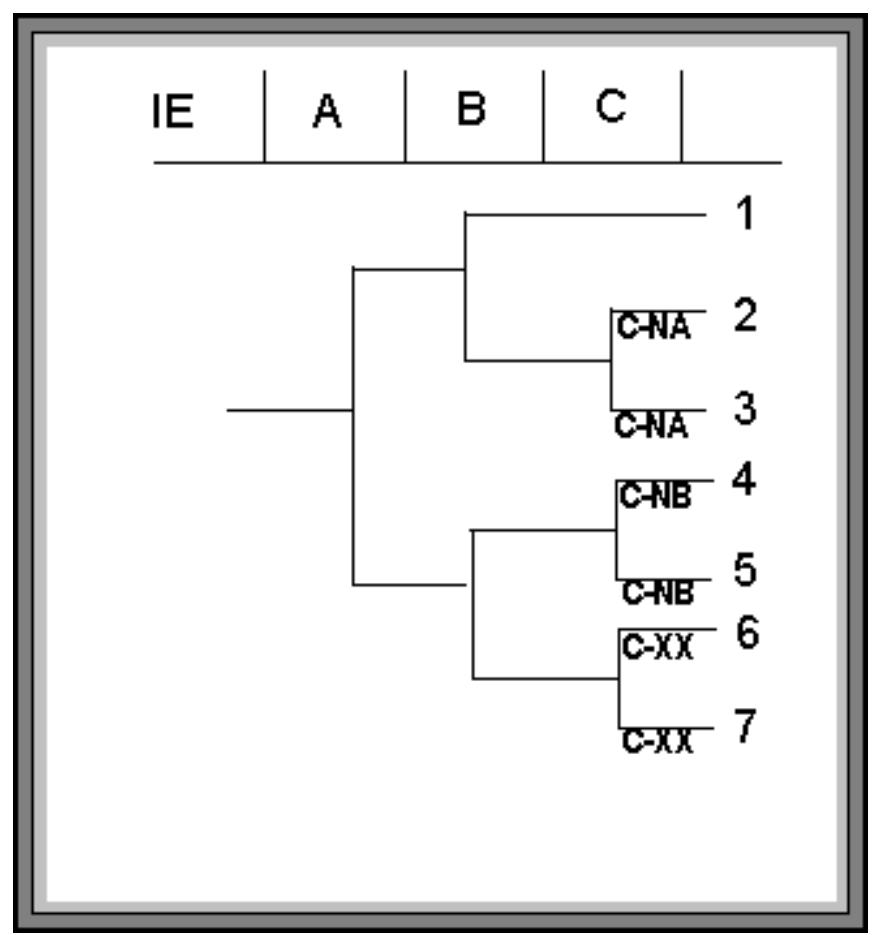

\subsubsection{Cast Operators}

SAPHIRE provides type cast functions for the following:

- $\quad$ event trees

- $\quad$ initiators

- $\quad$ fault trees

- $\quad$ flag sets

- $\quad$ end states

- $\quad$ macros

With these functions you can define substitutions for the end state or flag set of a sequence, or define a new transfer event tree for the sequence.

For instance, if the name "SYS5" was really the name of an initiating event then the user could change its default type with the following syntax; "init(SYS5)". The editor will then treat this name as an initiating event.

\section{OTHER CAST OPERATORS}

True ()- Cast operator that sets basic events to house event TRUE.

False - Cast operator that sets basic events to house event FALSE.

Ignore - Cast operator that sets basic events to house event IGNORE. 


\subsection{8 init()}

This keyword is used to indicate a particular initiating event.

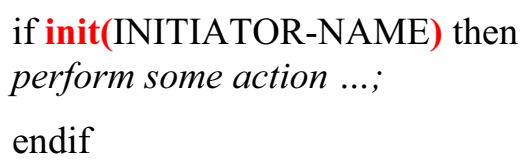

See Changing the Transfer Tree Name for a specific example of how to use this keyword.

\subsection{9 eventree 0}

This keyword is used to indicate a particular event tree. Make note of the spelling of this keyword.

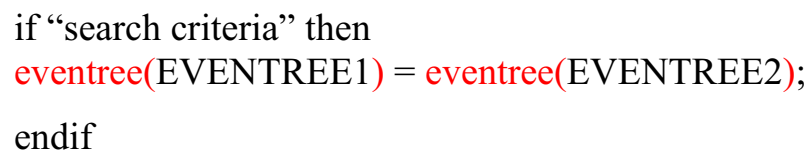

See Changing the Transfer Tree Name for a specific example of how to use this keyword.

\subsubsection{Skip ()}

This keyword indicates that the specified item should be ignored. Usually, this keyword is used to "skip" sequences in the event tree logic. No data will be generated for any "skipped" sequences. The sequences are not generated when the rule is applied, however, the sequence names (numbers) are left unchanged.

| This rule "skips" C given the failure of B.

if $B$ then

$/ \mathbf{C}=\operatorname{Skip}(\mathbf{C})$;

$\mathrm{C}=\operatorname{Skip}(\mathbf{C})$;

endif 


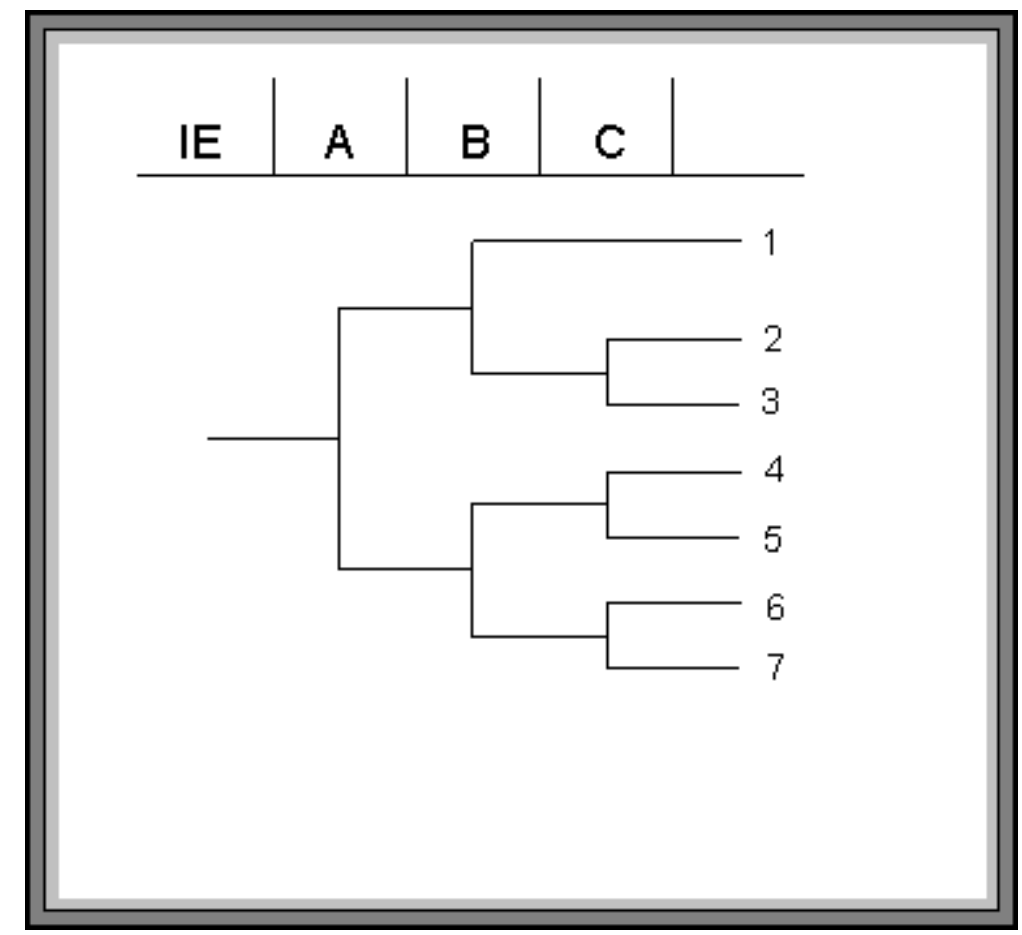

For this rule only sequences 1,4 , and 5 will be generated, since all sequences where B fails have been skipped.

\subsubsection{True()}

This rule structure is used to set the basic event(s) in parenthesis to house event TRUE.

In this example, the rule could alternatively set the basic events in parenthesis to house events FALSE or IGNORE by replacing the keyword "True" with either "False" or "Ignore".

| This rule sets E-MOV-A and E-PUMP-A

to TRUE given ECS fails in the LOSP event tree.

if ECS then

eventree $($ LOSP $)=$ True $($ E-MOV-A, E-PUMP-A $)$;

endif

\subsubsection{2 flag()}

This rule structure can be used to add an existing flag set to a sequence. The flag set needs to be created using the Modify | Flag Set option for the information to be applied to the sequence.

| This rule adds the flag set "FLAG-SET-1" to | the sequence that meets the criteria specified.

if ECS then eventree $($ LOSP $)=$ flag $($ FLAG-SET-1 $)$;

endif 


\subsubsection{3 endstate 0}

This rule structure can be used to add or create an end state for the sequence cut sets.

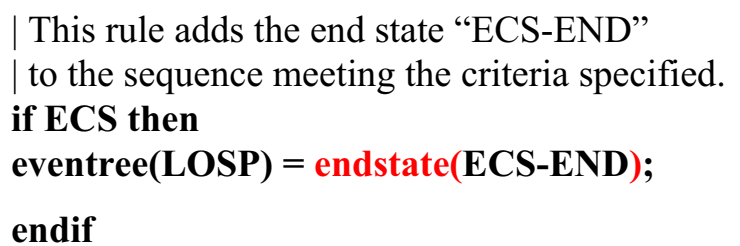

\subsubsection{Macro Structures}

Macros can streamline complex rules. A macro is simply a statement to define a search criteria (or variable) and assign a name to the search criteria. The macro name must be all uppercase, must be 24 characters or less, and must not include any of the restricted characters (e.g., a space, *, ?, l, /). The macro line can wrap around to more than one line, but must end with a semicolon.

Once a macro is defined, it can be used in an "if" test.
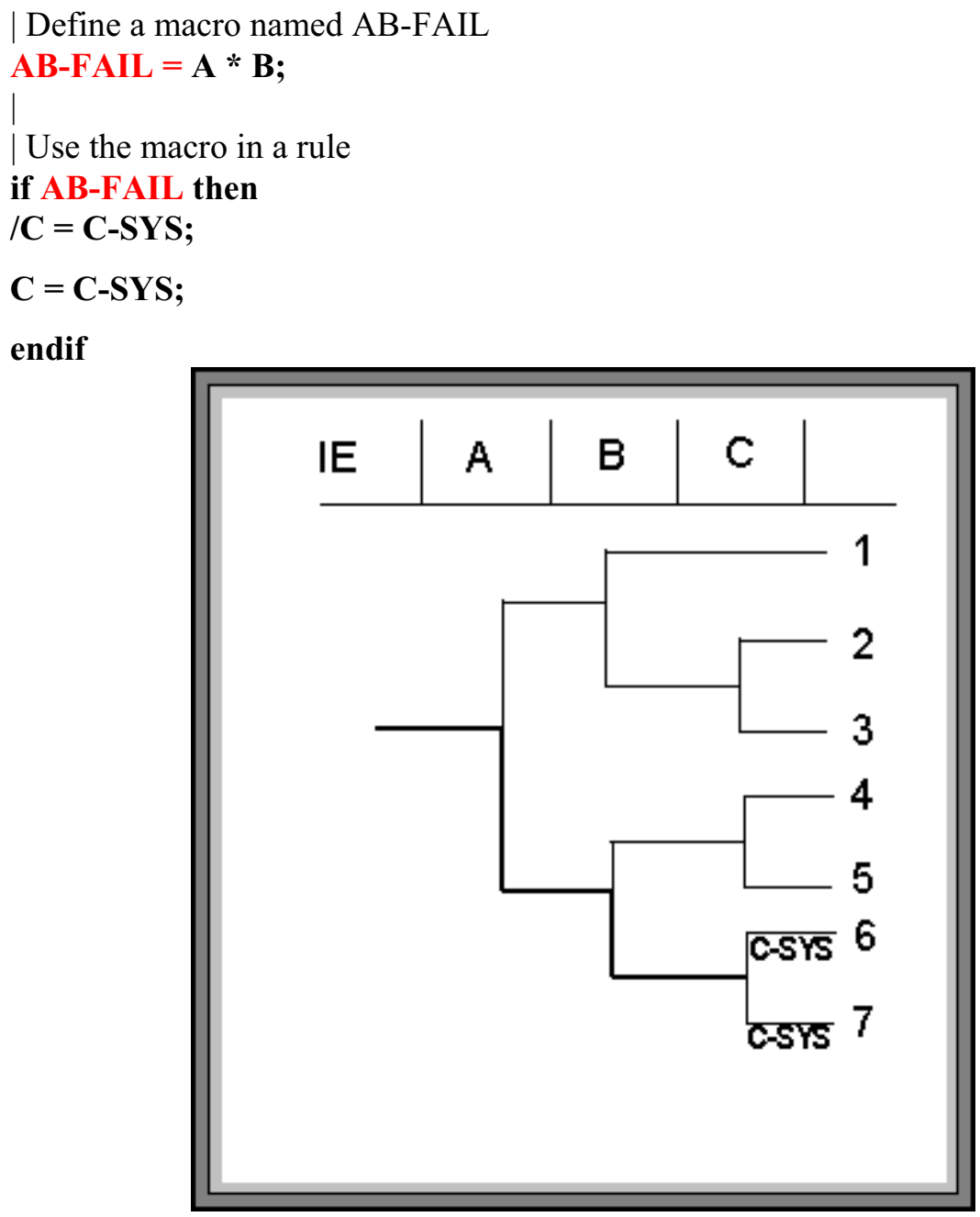


\section{SEE ALSO}

Using (never) with a Macro

\subsubsection{Using (never) With a Macro}

When creating a rule where the events in the macro do not occur, use the $\sim$ (i.e., never) symbol. Do not "complement" a macro.

| Using the $\sim$ macro as the search criteria:

| The rule applies when $\mathrm{A}$ and $\mathrm{B}$

| have not both failed.

| Define a macro named AB-FAIL

$\mathrm{AB}-\mathrm{FAIL}=\mathbf{A} * \mathbf{B}$;

Use the macro in a rule

if $\sim \mathrm{AB}-\mathrm{FAIL}$ then

$/ \mathrm{C}=\mathrm{C}-\mathrm{SYS}$;

C = C-SYS;

endif

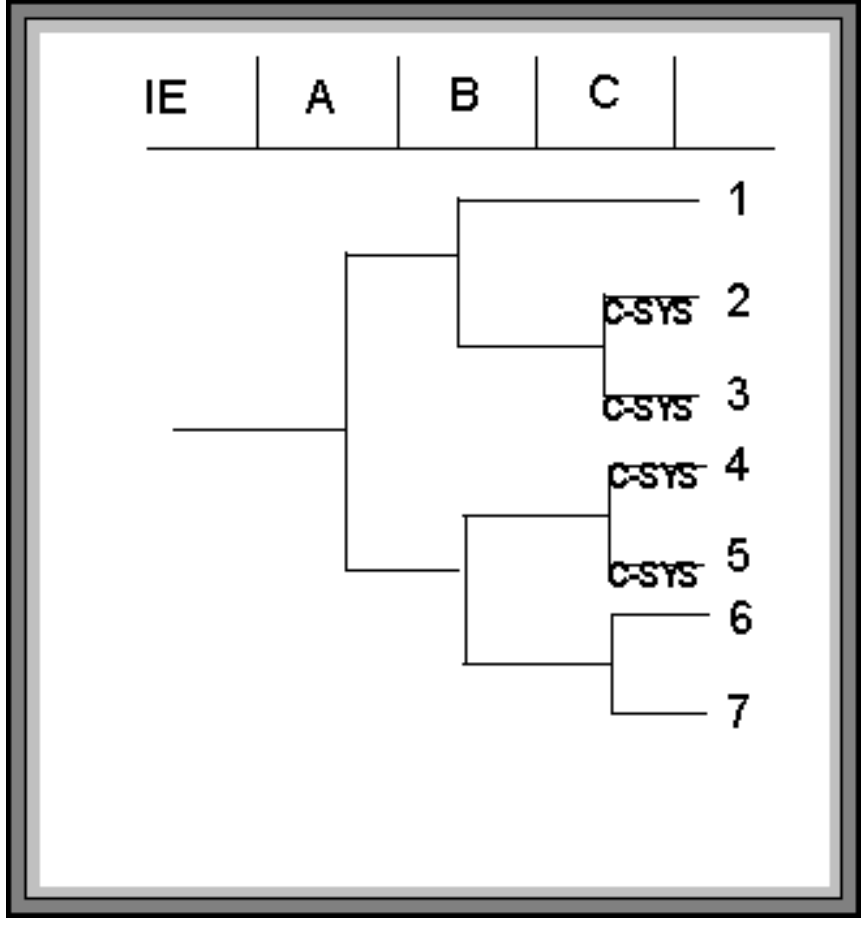




\subsection{Transfer Tree Name}

\subsubsection{Changing the Transfer Tree Name}

This is an example of how the transfer tree can be changed by using the init() and eventree() rules.

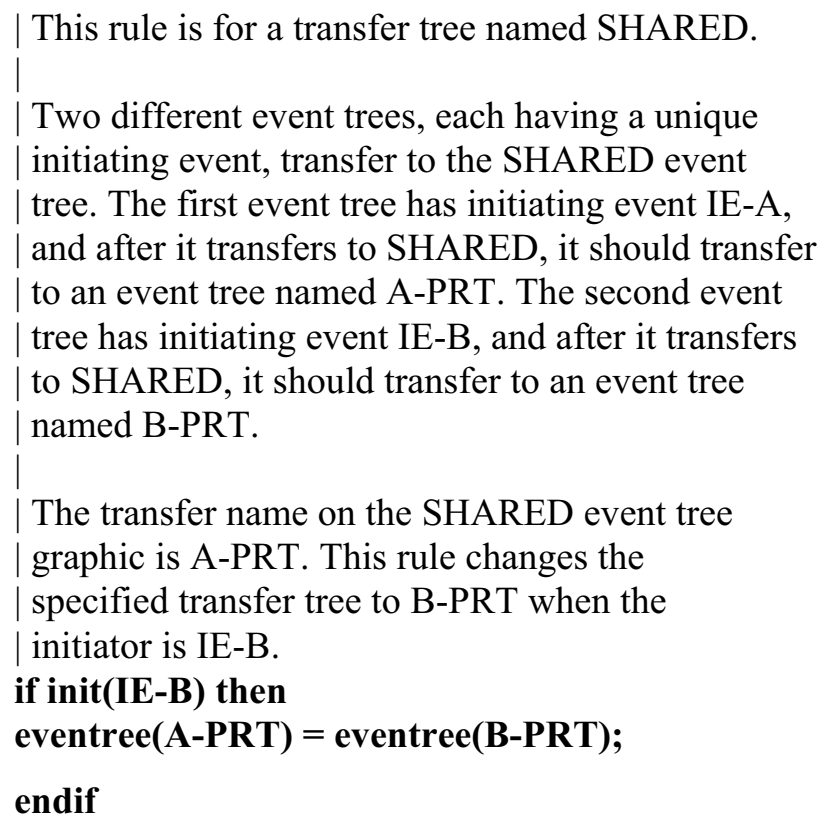

\subsection{Binary and Multiple-Split Branches}

\subsubsection{Rules for Binary and Multiple-Split Branches}

Several important modeling conventions are provided in the following example. The language for specifying a particular event tree branch is exemplified. The event tree in the figure here will be used for all rules in this discussion. 


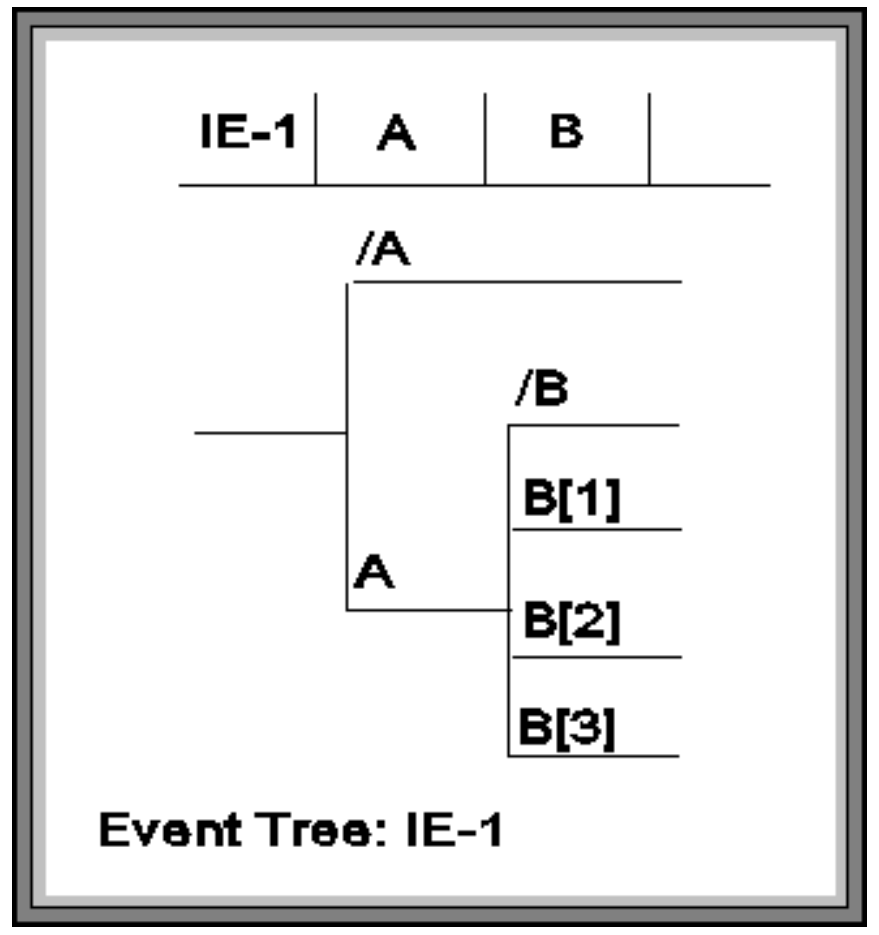

For binary branching, the success branch for a top is denoted with the complement symbol "/". SAPHIRE computes the probability for /A as (1-ATOP).

For multiple-split branching, the failed branches are designated with the top event name and the branch number in brackets.

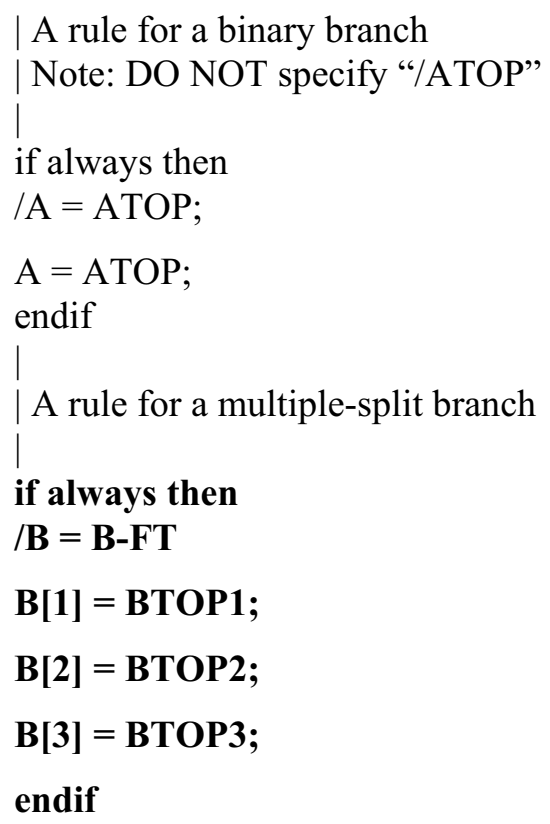

When you generate sequences using the event tree rules (from the Event Tree | Link Trees option), the sequences are reported as shown below. 


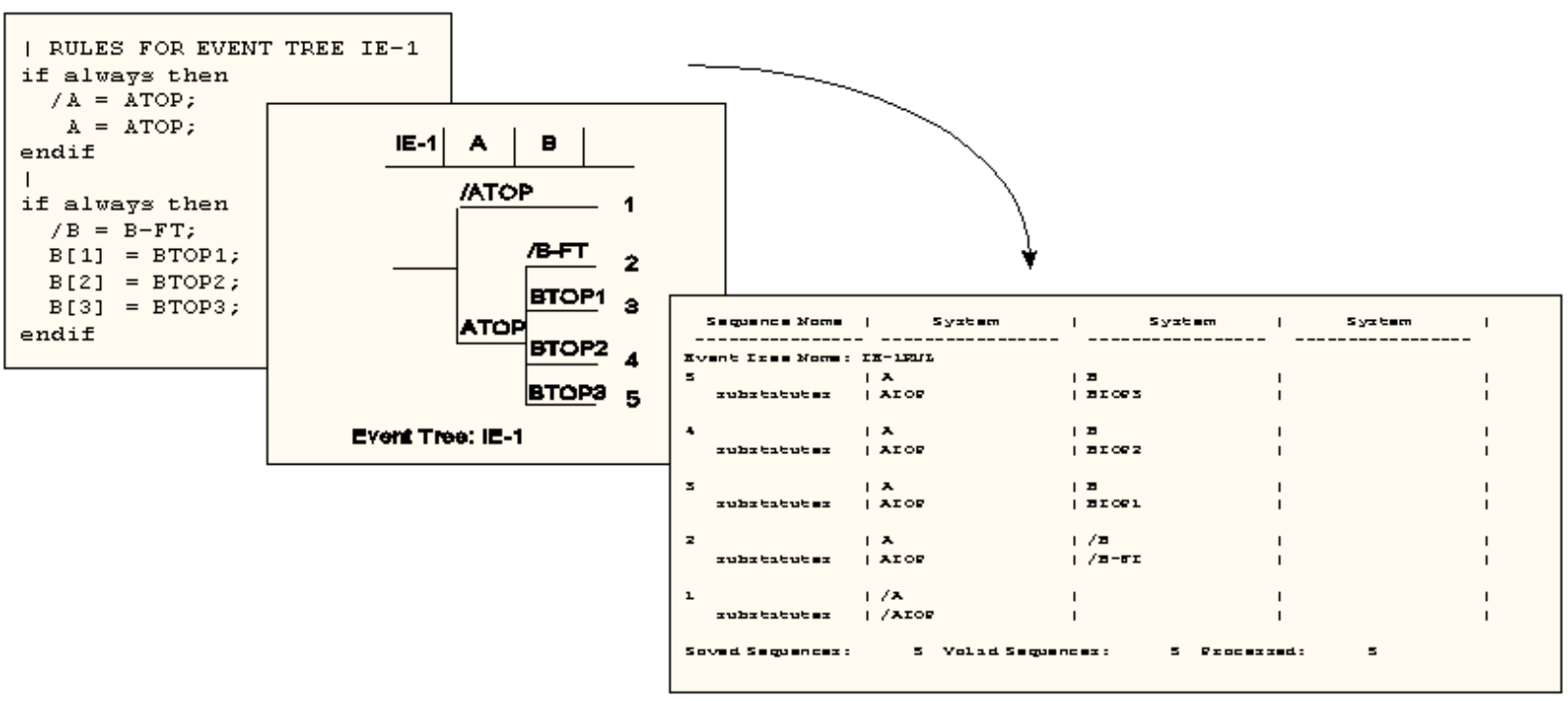

To include complemented events in sequence cut sets, you must specify the Y Process Flag (in the Modify | Basic Events option) for the applicable fault tree. In this example, you would set the Y Process Flag for ATOP and B-FT.

For multiple-split branches, you may want to construct a fault tree with the name that corresponds to the substituted success branch name. The fault tree would consist of the failed branch fault trees, BTOP1, BTOP2, and BTOP3 "ORed" together. SAPHIRE will automatically complement the fault tree result. You will need to solve the fault tree and use the S Calculation Type (in the Modify | Basic Events option). 


\section{SLICE OPTION \\ 18.1 Slice By Event Option}

\subsubsection{Displaying Cut Sets Using The Slice By Event Option}

\section{PURPOSE}

This section describes the "Slice By Event" cut set display feature that allows you to divide cut sets for viewing and reporting. The "Slice By Event" option is available for fault tree, sequence, and end state cut sets.

With this option, the user can quickly subdivide a list of cut sets into two lists based upon user-defined qualification criteria. The first list contains cut sets matching the current qualification criteria. Initially, this list contains all the generated cut sets. The second list, called the unqualified cut sets, contains all the cut sets not matching the qualification criteria.

The Slice By Event button is available from the Selected Cut Sets dialog. (Via the Display | Cut Sets pop-up menu from fault trees, sequences or end states lists.) Once the slice by event option is selected, the Event List dialog is displayed. This is the dialog used to define the criteria upon which cut sets will be qualified.

\section{STEPS}

To define qualification criteria:

1. Choose the slice type (Event, Cutoff, or Rule)

2. If Event, then

- Specify the logic option. Choose either "AND" or "OR” from the Logic drop-down list.

Choosing AND tells SAPHIRE that all of the selected events must appear in the cut set to meet the qualification criteria.

Choosing OR tells SAPHIRE that any of the selected events can appear in a cut set for the cut set to meet the qualification criteria.

- $\quad$ Specify the events on which the qualification criteria are based. Three ways of specifying basic events are provided:

Choose the Wild Card Mark option,

Highlight the desired event(s) in the Cut Set Events list and right-click to invoke the pop-up menu. Then choose from one of the four types: (Normal) Event, Failure Event, Success Event, or NOT Event.

Highlight the desired event(s) in the Cut Set Events list and choose the $\rightarrow$ button. This method adds (Normal) Event(s) to the Selected Events list. Conversely, choosing the $\leftarrow$ button removes the highlighted event(s) from the Selected Events list. 


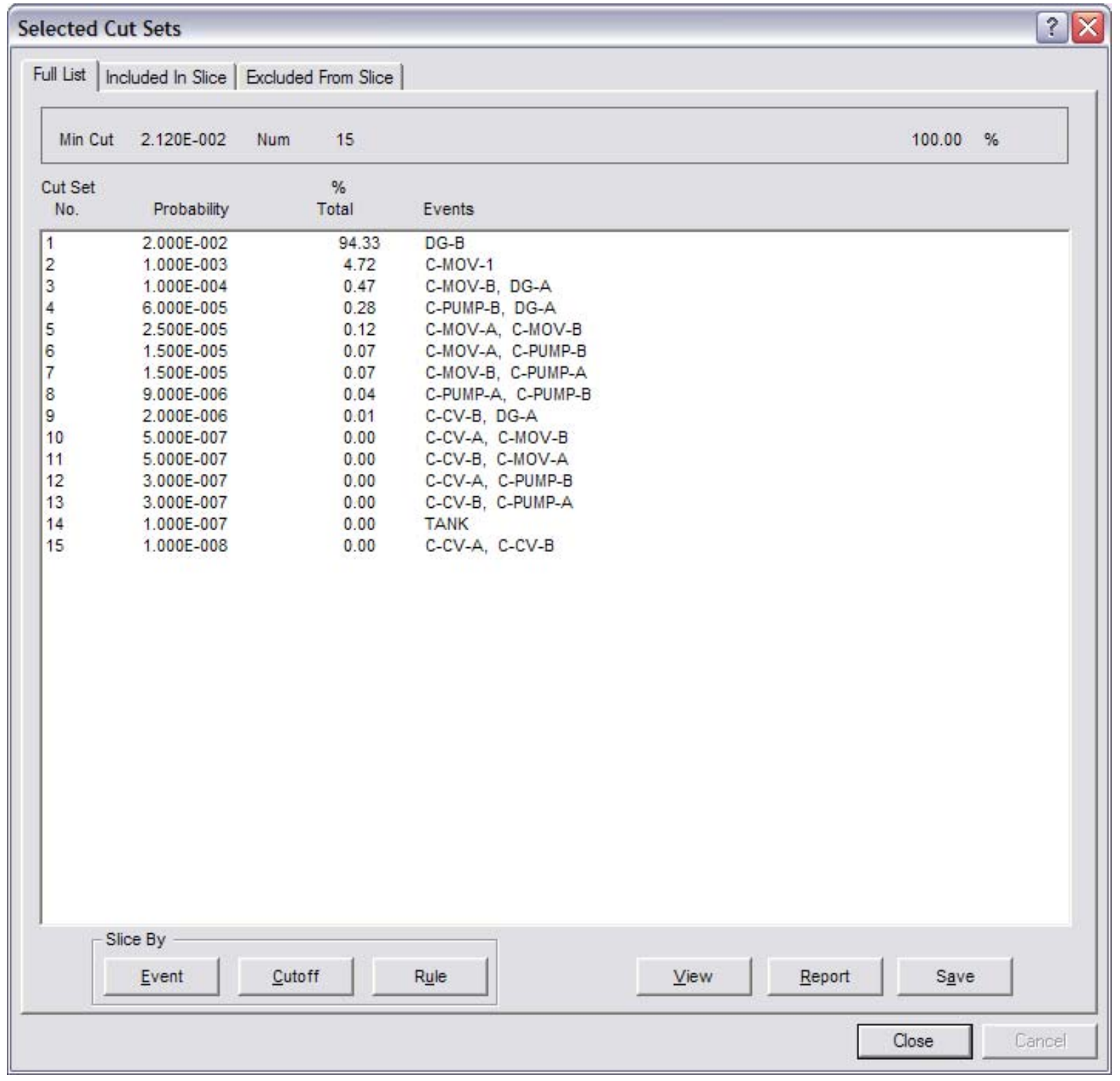

As an example, the qualification criteria events are the four events in the Selected Events list and the Logic to be performed is the "OR" operation. This tells SAPHIRE that any cut set:

containing the failure of event "C-CV-A" (+C-CV-A) or containing the success of event "C-MOV-1" (/C-MOV-1) or not containing the event "C-PUMP-A" ( C-PUMP-A) or containing the event "DG-A" (DG-A)

will meet the qualification criteria.

Thus, the evaluation can be defined in terms of successes, failures, exclusion (not contained in), or inclusion (contained in) for any of the basic events in the model. 
Choose the Apply button. SAPHIRE searches the cut sets for the qualified events (i.e., those matching the criteria). The Event List dialog will be closed and only the qualified cut sets (i.e., those containing events matching the criteria) will be displayed in the Selected Cut Sets dialog.

\subsubsection{Slice By Event Examples}

\subsubsection{Viewing Cut Sets Containing DG-A}

\section{PURPOSE}

This example demonstrates a simple use of the cut set slice option. A single event will be selected with the qualification criteria defined in terms of either failure or success for the event (i.e., the state of the event is irrelevant).

\section{STEPS}

1. Select Sequence from the SAPHIRE menu.

2. The Sequences dialog will be displayed. Highlight LOSP 3.

3. Right-click to invoke the pop-up menu and choose Display | Cut Sets.

4. The Selected Cut Sets dialog will be displayed with all of the cut sets generated for the LOSP 3 sequence. Choose the Slice By Event button.

5. The Event List dialog will be displayed. Highlight DG-A in the Cut Set Events list.

6. Right-click to invoke the pop-up menu and choose Add Event.

7. Choose the Apply button. The Event List dialog will be closed and the qualification criteria will be evaluated.

8. The Included In Slice tab will be displayed in the Selected Cut Sets dialog, showing only those cut sets meeting the qualification criteria. The Excluded From Slice tab will show the unqualified cut sets. 


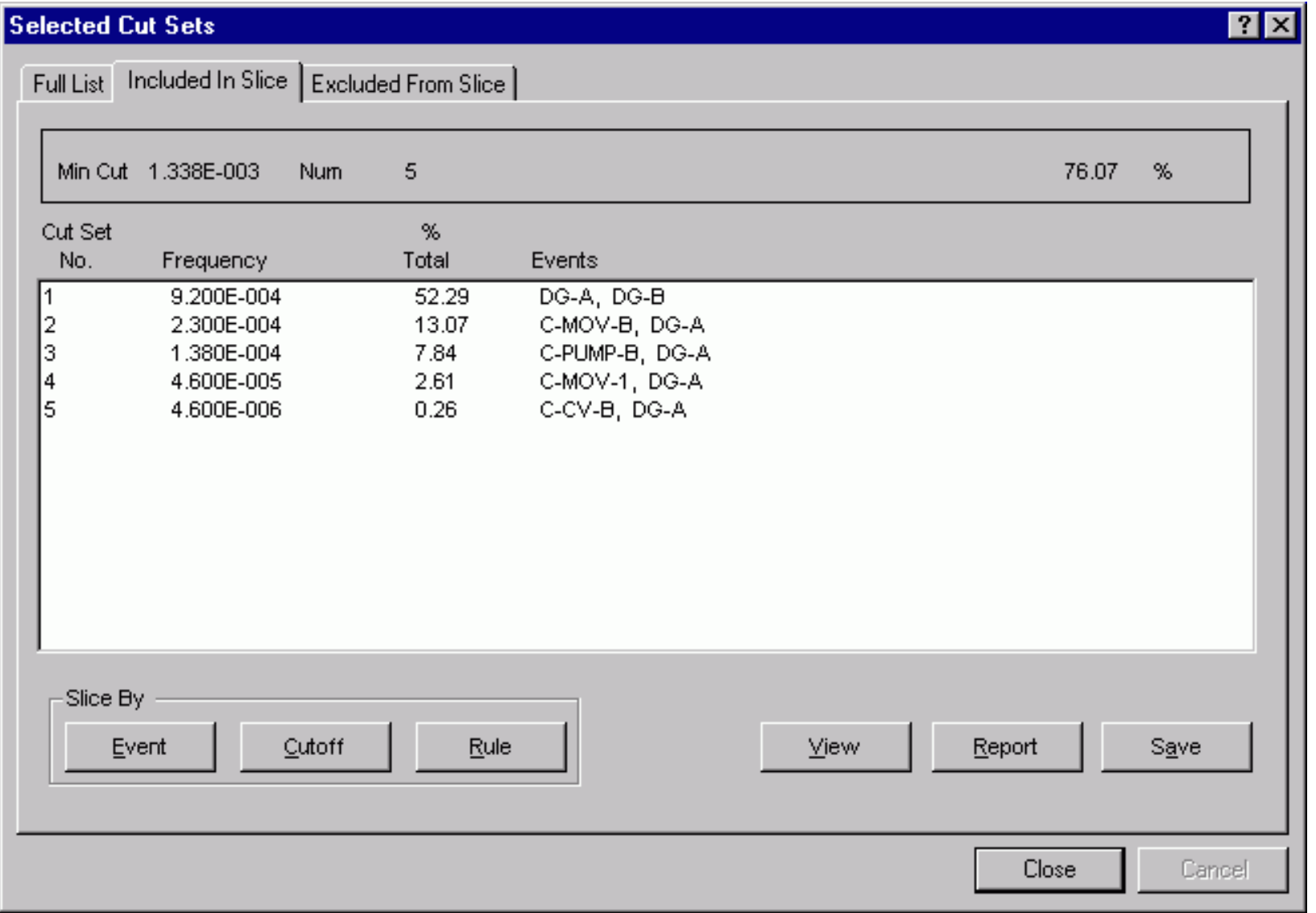

Notice that in this example, only five cut sets have been included that contain the basic event DG-A. The number of cut sets, their percent contribution, and subtotal are shown on the bottom right hand side of the dialog.

If you select the Excluded From List tab, the cut sets that do not contain DG-A will be displayed.

\subsubsection{Using the Slice Option to Select DG-A or DG-B}

\section{PURPOSE}

This example demonstrates how to use the Wild Card Mark feature to select multiple basic events.

Notice when using this option that the events matching the event class mask are highlighted in the Cut Set Events list, but are not automatically added to the Selected Events list. This provides the analyst with the opportunity to choose the state of the highlighted events (e.g., failed, success, not in), via the pop-up menu, before they are added to the Selected Events list. 


\section{STEPS}

1. To start a fresh slice session for LOSP 3, choose the Slice By Event button (from the Selected Cut Sets dialog).

2. On the Event List dialog, choose the Restart button. The original cut set list for LOSP 3 will be restored in the Selected Cut Sets dialog.

3. Choose the Slice By Event button again.

4. On the Event List dialog, select the "OR" Logic option.

5. Right-click to invoke the pop-up menu and choose the Wild Card Mark option.

6. The Event Class Mask dialog will be displayed. Enter "DG" in the Type field.

7. Choose the Ok button. The Event Class Mask dialog will be closed and the DG-A and DG-B events will be highlighted in the Cut Set Events list.

8. Choose the Add Event option from the pop-up menu. Both events will be included in the Selected Events list.

9. Choose the Apply button. The Event List dialog will be closed and the qualification criteria will be evaluated.

10. The Included In Slice tab will be displayed in the Selected Cut Sets dialog, showing only those cut sets meeting the qualification criteria. The Excluded From Slice tab will show the unqualified cut sets. 


\section{Full List Included In Slice | Excluded From Slice}

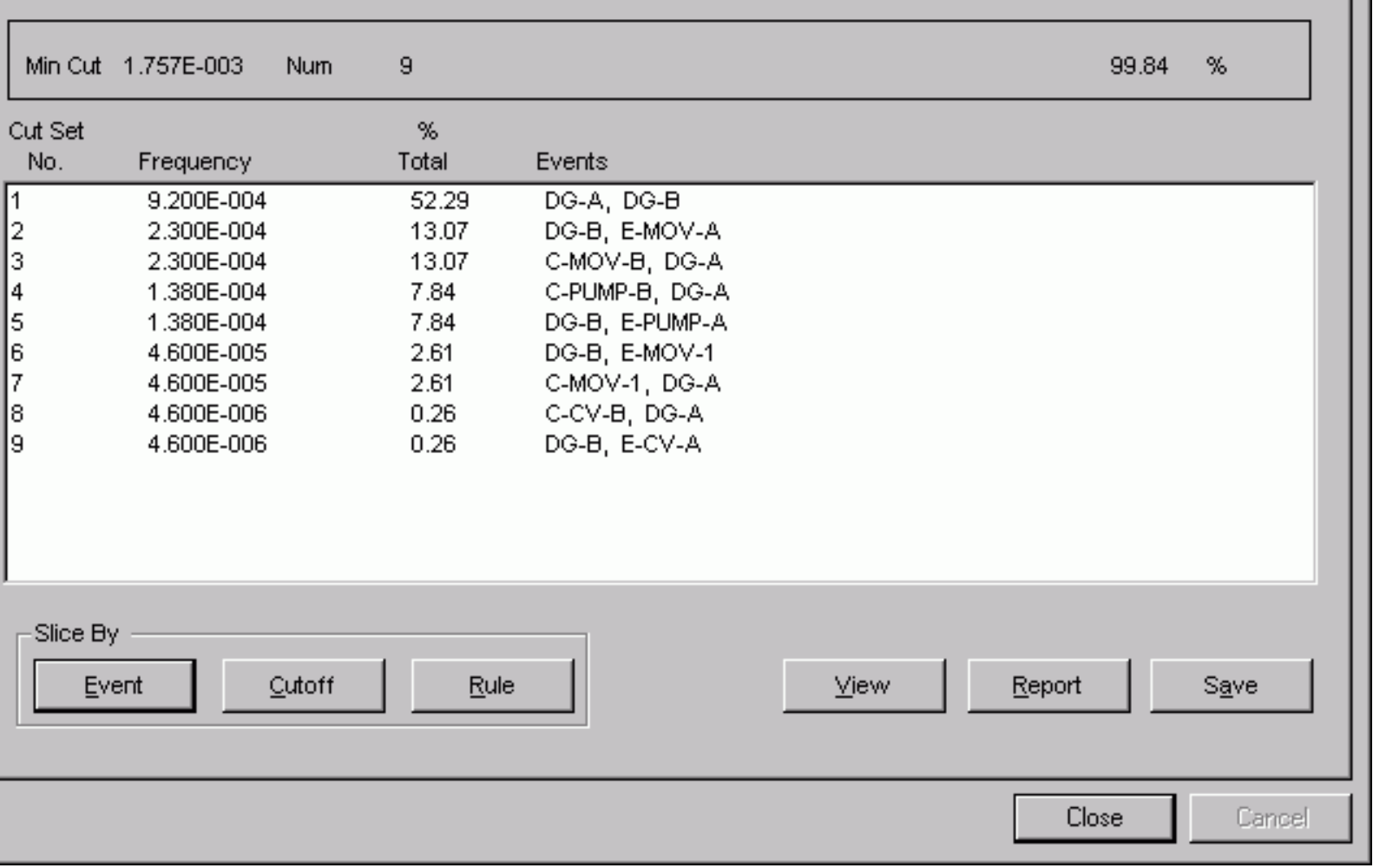

Now there are nine cut sets displayed. Each cut set contains either DG-A or DG-B (or both).

If you select the Excluded From List tab the cut sets that do not contain either DG-A or DG-B will be displayed.

If you leave the Logic option set to "AND" (the default), only one cut set will meet the qualification criteria. This cut set includes both DG-A and DG-B.

\section{Alternate ways to select both DGs:}

- Highlight the basic events in the Cut Set Events list on the Event List dialog.

- $\quad$ Enter "DG-?" in the Primary Name field on the Event Class Mask dialog. 


\subsection{Slice By Rule Option}

\subsubsection{Displaying Cut Sets Using The Slice By Rule Option}

\section{PURPOSE}

This section describes the "Slice By Rule" cut set display feature that allows you to divide cut sets for viewing and reporting. This "Slice By Rule" option is available for fault tree, sequence, and end state cut sets.

With this option, the user can quickly subdivide a list of cut sets into two lists based upon user-defined qualification criteria. The first list contains cut sets matching the current qualification criteria. Initially, this list contains all the generated cut sets. The second list, called the unqualified cut sets, contains all the cut sets not matching the qualification criteria.

The Slice By Rule button is available from the Selected Cut Sets dialog (via the Display | Cut Sets popup menu from fault trees, sequences or end states lists). Once the slice option is selected, the Slice Rules dialog is displayed. This is the dialog used to manage the list of slice rules upon which cut sets will be qualified. Right clicking in this dialog displays a menu to Add, Copy, Modify, and Delete slice rule name and descriptions. Once a slice name has been defined and highlighted, the Edit Rule button is used to display the Slice Rule Editor, where search criteria for the rule is specified.

The slice rules are "free-form" logic rules that allow for the inclusion or exclusion of cut sets from a "keep" list. They follow a format similar to the structure that is found in traditional programming languages (e.g., BASIC or PASCAL). As such, the ability exists to define "macros" and "if...then" types of structures. The rules are entered in a free-form text editor within SAPHIRE. They can also be exported and imported through the SAPHIRE Utility | Load and Extract option.

Upon saving and exiting the rule logic, SAPHIRE compiles the rules to check their validity. Errors will be reported to the user if found; otherwise the editor will exit normally. To apply a slice rule to the full list of displayed cut sets, highlight the desired rule and press the Apply Button. Cut sets will be divided into the Included in List tab and Excluded From List tab according to the rule logic.

To clear the Included and Excluded lists, choose the Reset option from the Slice By Event option, or, apply a different slice option to override the current option.

\section{EXAMPLES}

Viewing Cut Sets Containing DG-A

Using the Slice Option to Select DG-A and DG-B 


\subsubsection{Search Criteria Examples - Slice Rules}

These examples are used based on basic events X, Y, and Z.

\begin{tabular}{cl} 
Search Criteria & \multicolumn{1}{c}{ Meaning of Search Criteria } \\
$\mathrm{X}$ & Basic event $\mathrm{X}$ appears in the cut set \\
/X & Success of event $\mathrm{X}$ appears in the cut set \\
$\sim \mathrm{X}$ & Basic event $\mathrm{X}$ never occurs in the cut set \\
$\mathrm{X}+\mathrm{Y}$ & Either event $\mathrm{X}$ or $\mathrm{Y}$ appear in the cut set \\
$\mathrm{X} * \mathrm{Y}$ & Both events $\mathrm{X}$ and $\mathrm{Y}$ appear in the cut set \\
$\sim \mathrm{X} * \mathrm{Y}$ & $\mathrm{Y}$ does appear and $\mathrm{X}$ does not appear in the cut set \\
$\mathrm{X} *(\mathrm{Y}+\mathrm{Z})$ & Either $\mathrm{X}$ and $\mathrm{Y}$ or $\mathrm{X}$ and $\mathrm{Z}$ appear in the cut set \\
always & This pre-defined macro name means the criteria is always met
\end{tabular}

\subsubsection{Rule Editor Symbols}

$\begin{array}{cl}\mid \text { (comment) } & \text { Denotes a comment line } \\ * \text { (AND) } & \text { Logical AND operator } \\ +(\text { OR }) & \text { Logical OR operator } \\ \sim(\text { never }) & \text { Logical operator for "never" or "not present" } \\ /(\text { complement }) & \text { Complement } \\ ; \text { (end macro }) & \text { Indicates the end of a macro line or a line that } \\ & \text { modifies the criteria being evaluated. } \\ (\text { ) } & \text { Parentheses for grouping terms }\end{array}$

\subsubsection{Slice Rule Actions}

The slice utility provides the ability to divide a list of cut sets into two groups, or slices: the Included in Slice group, and the Excluded From Slice group. This

feature allows the analyst to locate and separate out cut sets containing a specified combination of events. As such, there are two actions a rule can perform. The "keep" action will include qualified cut set in the Included in Slice group, and the "discard" action will include unqualified cut sets in the Excluded From Slice group.

Note that when a cut set is evaluated, and does not meet the specified search criteria, the default action is to "discard". Therefore, if the slice action is "discard" (which may sometimes be a desirable way to frame the search logic), you must include a "keep" action in order to separate unqualified cut sets from those the qualification is discarding.

To demonstrate the potential uses of the slice rules, the example here shows how the rules could be used to remove cut sets relating to loss of off-site power and diesel generator failures. As shown, the rule will place any cut sets having the initiating event LOSP and either diesel generator A or B into the Excluded from Slice List. Those cut sets that do not meet this criteria will be placed into the Included In Slice list. 
| Search on LOSP initiator and failure of

| diesel generators $\mathrm{A}$ or $\mathrm{B}$ and remove them from display.

if init(LOSP) * (DG-A + DG-B) then

discard;

else

keep;

endif

When this rule is applied to the displayed cut sets, SAPHIRE will evaluate each cut set and then automatically separate each one into the appropriate list. The "if...then" line can be as complex as needed.

As written, the above rule would only discard cut sets for sequence cut sets, since fault tree cut sets do not contain initiating events.

\subsubsection{Keywords and Symbols}

\subsubsection{Keywords and Symbols - Slice Rules}

Below is a list of keywords and symbols used in the SAPHIRE slice rules. The search criteria and rule structure of the slice rules is parallel to that of recovery rules; only the recovery and slice actions differ.

Note the spelling of each keyword.

if-then - Indicates a search criteria is being specified.

endif - Indicates the end of a particular rule.

else - Specifies some action to be taken if all the search criteria are not met. The "else" should be the last condition in the slice rule.

elsif - Specifies an alternative search criteria.

always - Indicates that every cut set that is being evaluated satisfies the search criteria.

init()- Used in the search criteria to indicate that a sequence cut set has a particular initiating event.

$\sim$ (never) - Used in the search criteria to indicate that a particular event will not be in the cut set that is being evaluated.

/ (complement) - Represents a complemented event (i.e., the success of a failure basic event).

| (comment) - Represents a comment contained in the rules.

; (end macro) - Indicates the end of a macro line or a line that directs the destination of the cut set being evaluated.

* (AND) - Indicates the logical AND command.

+ (OR) - Indicates the logical OR command.

() - Indicate a specific grouping of items.

keep; - Indicates that the cut set being evaluated will be placed in the Included In Slice list.

discard; - Indicates that the cut set being evaluated will be placed in the Excluded From Slice list.

MACRO - A user-definable keyword that specifies a search criteria.

\subsubsection{2 if-then}


(Basic Rule syntax)

This keyword indicates that a search criteria is being specified.

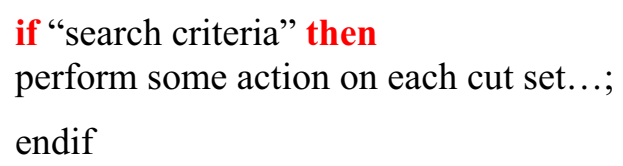

\subsubsection{3 endif}

(Basic Rule syntax)

This keyword indicates that the end of a particular rule.

if "search criteria" then

perform some action on each cut set...;

endif

\subsubsection{4 else}

(Basic Rule syntax)

This keyword specifies that some action to be taken if all the search criteria are not met. The "else" should be the last condition in the recovery rule.

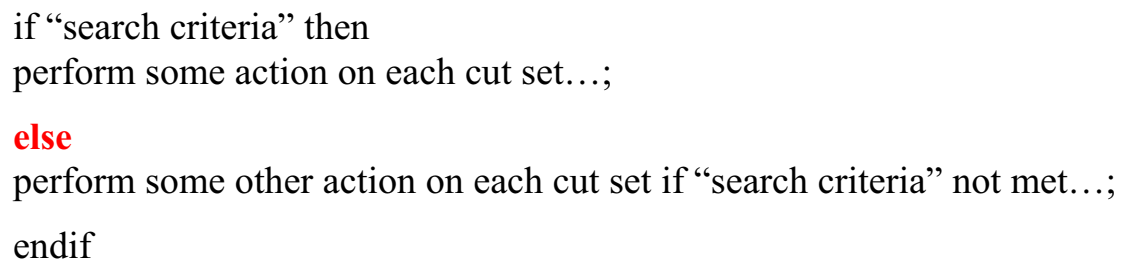

\subsubsection{5 elsif}

(Basic Rule syntax)

This keyword specifies an alternative search criteria. Any number of "elsifs" can be used within a recovery rule.

if "search criteria" then

perform some action on each cut set...;

elsif " 2 nd search criteria" then 
perform some other action on each cut set ...;

elsif " 3 rd search criteria" then

perform some other action on each cut set ...;

endif

\subsubsection{6 always}

(Basic Rule syntax)

This keyword indicates that every cut set that is being evaluated satisfies the search criteria.

if always then

perform some action on each cut set...;

endif

\subsubsection{7 init()}

(Basic Rule syntax)

This keyword is used in the search criteria to indicate that a sequence cut set has a particular initiating event.

if init(INITIATOR-NAME) "and optional other search criteria" then

perform some action on each cut set...;

endif

\subsubsection{8 $\sim$ (never)}

(Basic Rule syntax)

This symbol is used in the search criteria to indicate that a particular event will not be in the cut set that is being evaluated.

if ( SEARCH-CRITERIA) “and optional other search criteria” then $\ldots$

The search criteria will be satisfied for cut sets that do not contain SEARCH-CRITERIA (and also contains the optional ("other search criteria"). SEARCH-CRITERIA may be either an initiating event, basic event, macro, or logic expression.

\subsubsection{9 | (comment)}

(Basic Rule syntax) 
This symbol is used to represent a comment contained in the rules. Everything on a line to the right of this symbol will be ignored by the rule compiler. Blank lines are encouraged in the editor to improve readability and comments can be entered following the "|" character.

| Place your comments here!

| Note that blank lines are also permissible.

\subsubsection{0 /(complement)}

(Basic Rule syntax)

This symbol is used to represent a complemented event (i.e., the success of a failure basic event).

if (/BASIC-EVENT) "and optional other search criteria" then

The search criteria will be satisfied for all cut sets that contain the complement of BASIC-EVENT (and also contains the optional "other search criteria").

\subsubsection{1 ;(end macro)}

(Basic Rule syntax)

This symbol is used to indicate the end of a macro line or a line that modifies the cut set being evaluated.

$$
\begin{aligned}
& \text { | usage for a macro command } \\
& \text { MACRO-NAME = "search criteria"; } \\
& \text { | usage for a cut set modification line } \\
& \text { recovery = RECOVERY-EVENT; }
\end{aligned}
$$

\subsubsection{2 *(AND)}

(Basic Rule syntax)

This symbol is used to indicate the logical AND command.

$$
\text { if SEARCH-CRITERIA1 * SEARCH-CRITERIA2 then }
$$

The search criteria will be satisfied for all cut sets that match SEARCH-CRITERIA1 and SEARCHCRITERIA2. The SEARCH-CRITERIA\# may be either an initiating event, macro, or logic expression.

\subsubsection{3 +(OR)}

(Basic Rule syntax) 
This symbol is used to indicate the logical OR command.

$$
\text { if SEARCH-CRITERIA1 + SEARCH-CRITERIA2 then }
$$

The search criteria will be satisfied for all cut sets that match either SEARCH-CRITERIA1 or SEARCHCRITERIA2. The SEARCH-CRITERIA\# may be either an initiating event, macro, or logic expression.

\subsubsection{4 () (group)}

(Basic Rule syntax)

These symbols together indicate a specific grouping of items.

$$
\text { if }(\mathrm{A}+\mathrm{B}) *(\mathrm{C}+\mathrm{D}) \text { then }
$$

The search criteria above would return all cut sets that contain:

$$
[\mathrm{A} * \mathrm{C}],[\mathrm{A} * \mathrm{D}],[\mathrm{B} * \mathrm{C}] \text {, or }[\mathrm{B} * \mathrm{D}] \text {. }
$$

\subsubsection{Slice By Rule Examples}

\subsubsection{Slice Rule Example: View MOV pairs}

\section{PURPOSE}

This example demonstrates how to write a slice rule containing mixed logic to view only cut sets which have one or more groups of events in them. The ability to specify one or more groups of events is a feature available only through the Slice by Rule option.

The example also makes use of the "discard" keyword. Whenever the "discard" keyword is used, the "keep" keyword must also be used, because the default action for unqualified cut sets is to discard.

\section{STEPS}

1. From the Selected Cut Sets dialog, choose the Slice By Rule button. The Slice Rules dialog will appear.

2. Right click to invoke the pop-up menu and choose the Add menu option. 
3. Give the rule a name, such as "MOV-RULE", and, optionally, a description, such as "find MOV groups".

4. Choose the $\mathbf{O K}$ button to close the Add Rule dialog and save the new rule .

5. With the new rule still highlighted, choose the Edit Rules button. The Rules Editor window will appear.

6. Type the following rule:

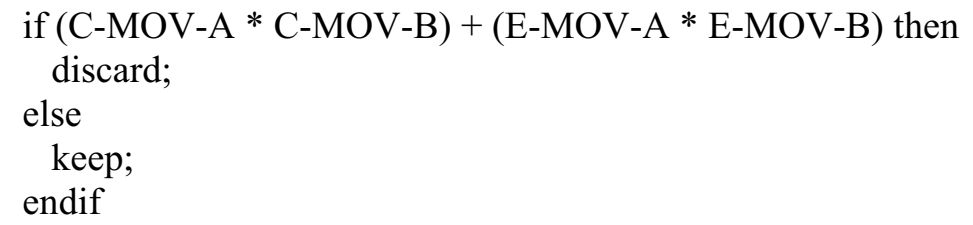

7. From the Edit Rules menu, choose File | Exit to close the Rules Editor. When prompted to save the file, choose Yes.

8. From the Slice Rules dialog (with the new rule still highlighted), choose the Apply button. The Slice Rules dialog will be closed and the cut sets will be evaluated against the rule.

9. The Included In Slice tab will be displayed in the Selected Cut Sets dialog, showing only those cut sets which did not meet the qualification criteria. Because the "discard" keyword was used, it is the Excluded From Slice tab that shows the qualified cut sets.

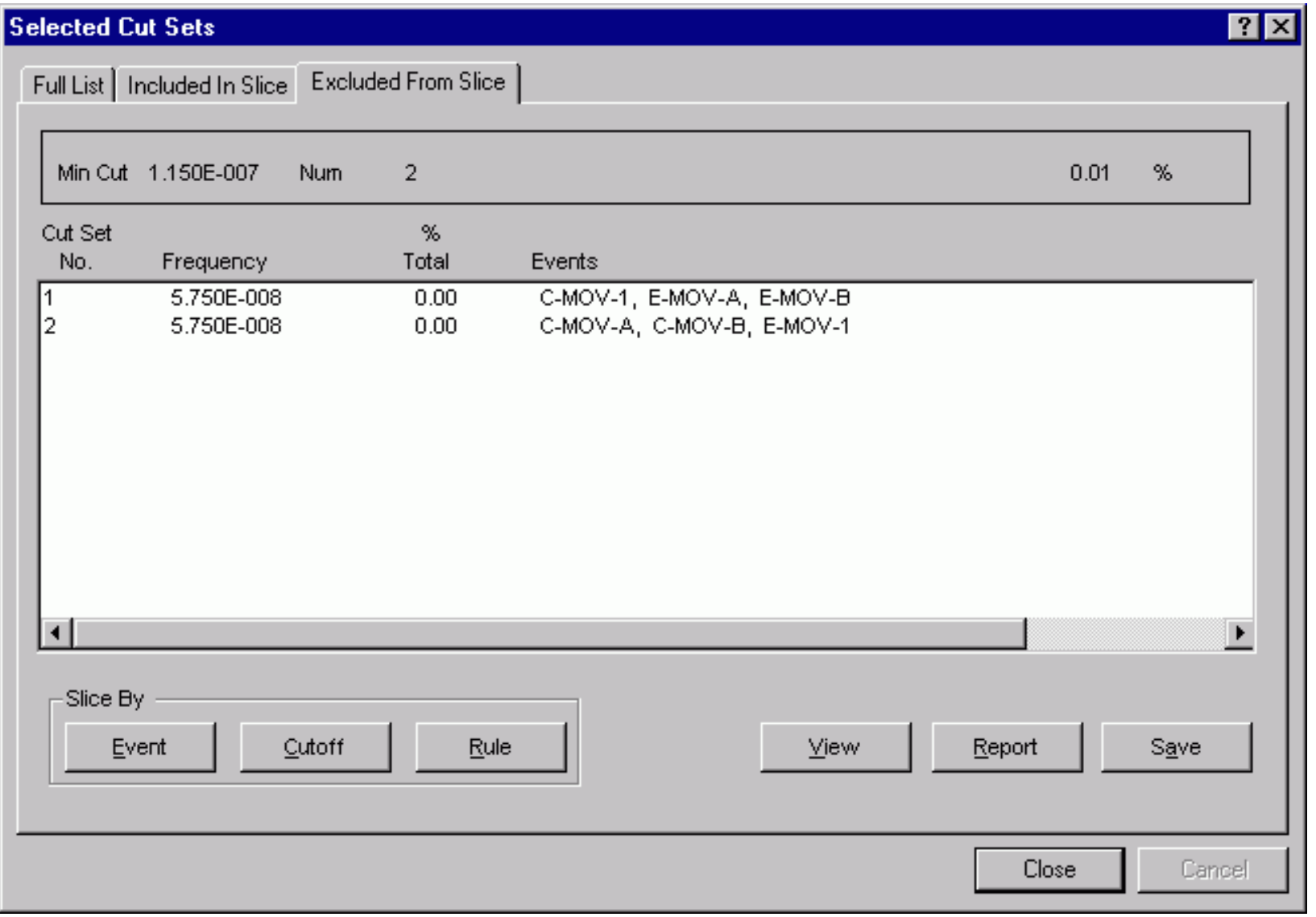


The alternate form of the slice rule that will reverse the contents of the Included In Slice and Excluded From Slice tabs is as follows:

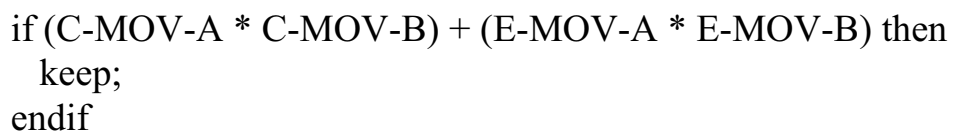

\subsubsection{Slice Rule Example: View DG-A or DG-B cut sets}

\section{PURPOSE}

This example demonstrates how to write a slice rule to view only cut sets which have one or more specified events in them.

In this example, the cut sets in the DEMO project will be "sliced" according to whether or not they contain DG-A or DG-B. See the Slice By Event example for an alternate method of performing the same task.

\section{STEPS}

1. From the Selected Cut Sets dialog, choose the Slice By Rule button. The Slice Rules dialog will appear.

2. Right click to invoke the pop-up menu and choose the Add menu option.

3. Give the rule a name, such as "DG-RULE", and, optionally, a description, such as "find any diesel generators".

4. Choose the $\mathbf{O K}$ button to close the Add Rule dialog and save the new rule .

5. With the new rule still highlighted, choose the Edit Rules button. The Rules Editor window will appear.

6. Type the following rule:

if DG-A + DG-B then

keep;

endif

7. From the Edit Rules menu, choose File | Exit to close the Rules Editor. When prompted to save the file, choose Yes.

8. From the Slice Rules dialog (with the new rule still highlighted), choose the Apply button. The Slice Rules dialog will be closed and the cut sets will be evaluated against the rule.

9. The Included In Slice tab will be displayed in the Selected Cut Sets dialog, showing only those cut sets meeting the qualification criteria. The Excluded From Slice tab will show the unqualified cut sets. 
Full List Included In Slice | Excluded From Slice |

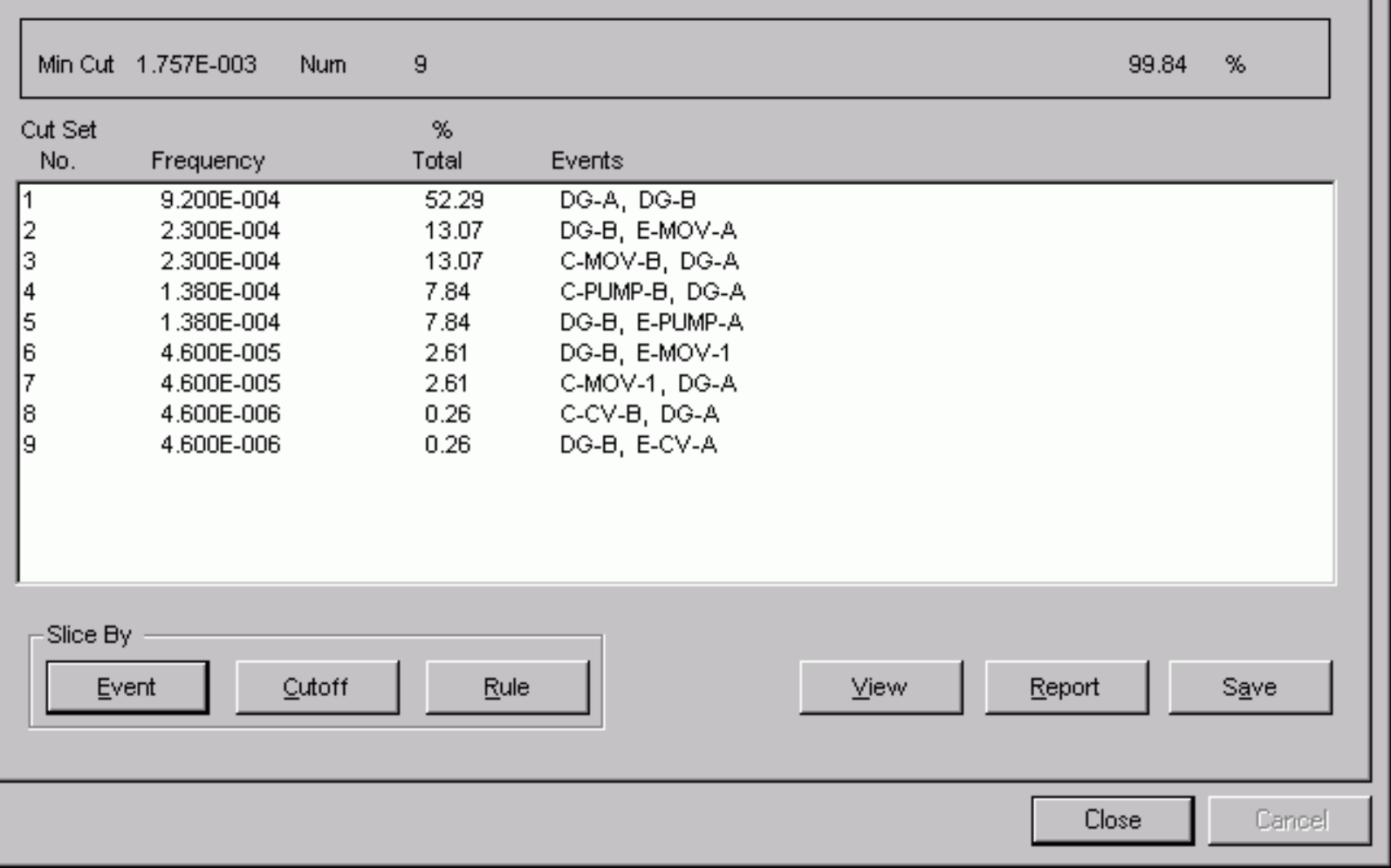

Now there are nine cut sets displayed. Each cut set contains either DG-A or DG-B (or both).

If you select the Excluded From List tab the cut sets that do not contain either DG-A or DG-B will be displayed.

The following variation of the above rule would reverse the results; The Excluded From List tab would contain the cut sets having either DG-A or DG-B (or both). The Included In List tab would contain the cut sets that do not contain either DG event. The "else keep" portion of the rule is necessary, because by default, any cut sets not meeting the rule criteria are placed in the Excluded From List, (i.e., discarded).

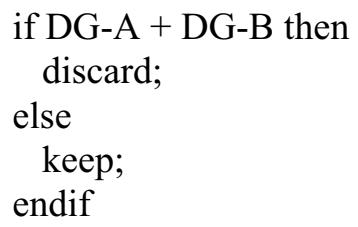




\section{DYNAMIC FLAG SETS}

\subsection{Dynamic Flag Sets}

Dynamic flag sets are a special type of flag set that are assigned to sequences by the use of event tree rules. The dynamic flag set is assigned to a sequence or sequences if they meet the criteria contained in the rule.

The advantage of using dynamic flag sets is evident when event tree logic changes are made which causes changes to the number of sequences. Since dynamic flag sets are assigned to sequences defined in the event tree rule, the dynamic flag set will automatically reassign itself to the correct sequence. Conversely, if regular flag sets were used, the analyst would have to modify each sequence individually to make sure the correct flag set name is assigned to the correct sequence. This makes the use of dynamic flag sets more efficient than regular flag sets if flag sets are assigned to specific sequences.

Dynamic flag sets can contain only individual basic event changes. No "Class Changes" are allowed.

Dynamic flag sets can contain only house event settings (calculation type T, F, or I changes) or top event process flag changes. No probability changes can be made.

Dynamic flag sets will appear in the same list as regular Flag Sets, however, the name given to a dynamic flag set (e.g., ET-000001-000001) is based upon the event tree, sequence name, and number of dynamic flag sets already created.

\section{TOPICS}

Search Criteria Examples

Symbols

Keywords and Structures

Making a Dynamic Flag Set

Example Dynamic Flag Set

Flag Sets and Subtrees

\subsection{Rule Structures}

\subsubsection{Rule Editor Symbols}

$\begin{array}{cl}\mid \text { (comment) } & \text { Denotes a comment line } \\ * \text { (AND) } & \text { Logical AND operator } \\ +(\text { OR }) & \text { Logical OR operator } \\ \sim(\text { never }) & \text { Logical operator for "never" or "not present" } \\ / \text { (complement) } & \text { Complement } \\ ; \text { (end macro }) & \text { Indicates the end of a macro line or a line that } \\ & \text { modifies the criteria being evaluated. } \\ (\text { ) } & \text { Parentheses for grouping terms }\end{array}$




\subsubsection{Linkage Rules Keywords and Structure}

\begin{tabular}{|c|c|}
\hline if-then & Indicates that a search criteria is being specified. \\
\hline if-then using wildcards - & A search criteria can be specified using wildcards. \\
\hline always - & Indicates that the search criteria is always satisfied. \\
\hline elsif - & Specifies an alternative search criteria. \\
\hline else - & $\begin{array}{l}\text { Specifies some action to be taken if all the search criteria are not } \\
\text { met. }\end{array}$ \\
\hline Cast Operators - & $\begin{array}{l}\text { Define substitutions for the end state or flag set of a sequence, or } \\
\text { define a new transfer event tree }\end{array}$ \\
\hline Skip - & Indicates that the specified item should be ignored. \\
\hline Macro - & A user-definable keyword that specifies a search criteria. \\
\hline
\end{tabular}

\subsubsection{Flag Sets and Sub-trees}

SAPHIRE has an enhanced feature that will append a flag set to the sequence meeting the search criteria even if the event tree stated in parenthesis transfers to a subtree. Therefore, either rule stated below will append a flag set to the same sequence.

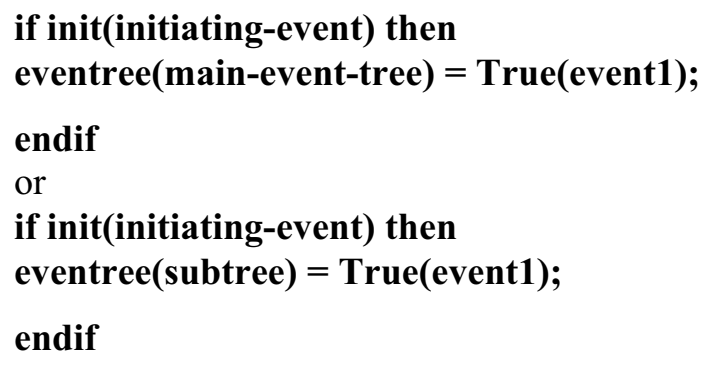

\subsection{Making a Dynamic Flag Set}

\subsubsection{Making a Dynamic Flag Set}

\section{PURPOSE}

Describe the steps for creating a dynamic flag set.

\section{STEPS}

1. Select Event Tree from the SAPHIRE menu.

2. In the Event Tree List, highlight the desired event tree.

3. Right-click to invoke the pop-up menu and select Edit Rules.

4. Using the rule structures discussed, construct a rule that will modify a basic event's calculation type and exit the editor.

5. Generate the event tree sequences by selecting Link Trees from the pop-up menu.

6. The flag set will automatically be assigned to the sequence without manually modifying the sequence.

7. The event tree sequences are ready to be analyzed from the Sequence menu option. 


\title{
19.3.2 Example Dynamic Flag Set
}

To illustrate the use of dynamic flag sets, the DEMO project will be used.

A rule was written to set DG-A and C-PUMP-B to a house event FALSE given CCS fails in the LOSP event tree.

\author{
if CCS then \\ eventree $($ LOSP $)=$ False $($ DG-A, C-PUMP-B $)$; \\ endif
}

The dynamic flag set will append itself to the sequences meeting the criteria specified in the rule. For this particular rule, Sequence 3 will have a flag set associated with it that will set DG-A and C-PUMP-B to a house event FALSE.

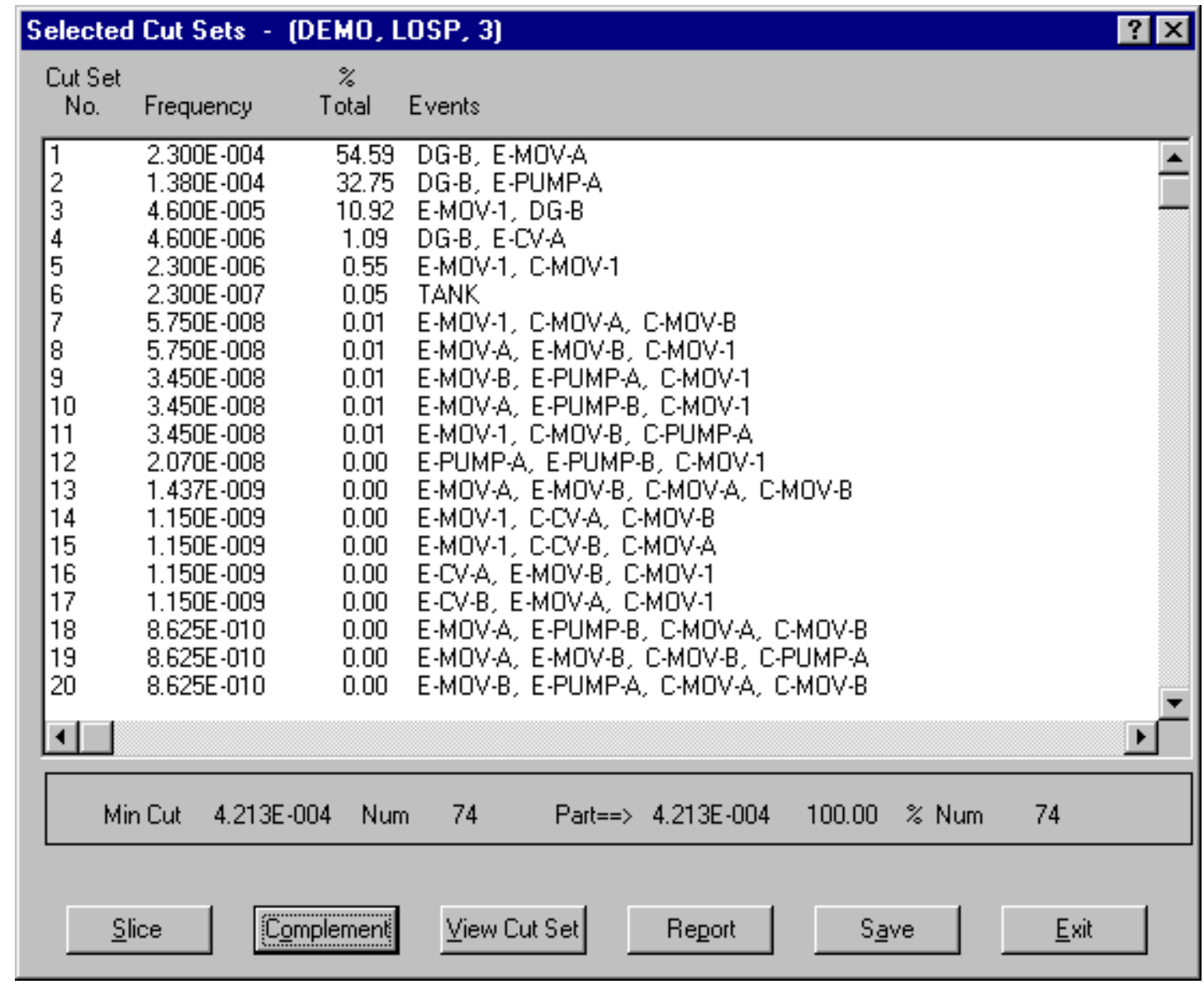

The resulting cut sets for Sequence 3 are shown above. Notice that DG-A and C-PUMP-B do not show up in the list of cut sets. 


\section{MUTUALLY EXCLUSIVE EVENTS}

\subsection{Mutually Exclusive Events}

\section{PURPOSE}

This section presents the topic of mutually exclusive events. The term is defined here, a fault tree logic example is provided, and the methods to remove these events from SAPHIRE PRA results are discussed.

The term "mutually exclusive events" refers to two or more basic events that appear in a single cut set (either for fault trees or sequences) which logically should not appear together. Generally, mutually exclusive events should not appear together in the list of cut sets for one of two reasons:

Plant technical specifications or other operating restrictions may prevent two components from being out of service (e.g., tested or maintained) at the same time. An example is not allowing two AFW pumps to be simultaneously out of service for testing and maintenance.

Other general logical modeling concerns may lead the analyst to remove specific combinations of events. An example of this involves the practice of including multiple initiating events in the fault tree logic. Given this case, sequence cut sets can e generated that include multiple initiating events.

Most mutually exclusive groups include only two or three components.

During the logic modeling phase, the analyst may recognize that certain combinations of mutually exclusive events will appear just by knowing how the fault tree and event tree logic modeling was performed. However, some unrecognized mutually exclusive events may not be evident until the analyst generates and evaluates the fault tree or sequence cut sets.

\section{TOPICS}

Mutually Exclusive Event Example

Removing Mutually Exclusive Events

\subsection{Mutually Exclusive Event Example}

As an example of how fault tree logic modeling can produce mutually exclusive events, the fault tree shown below will be used. 


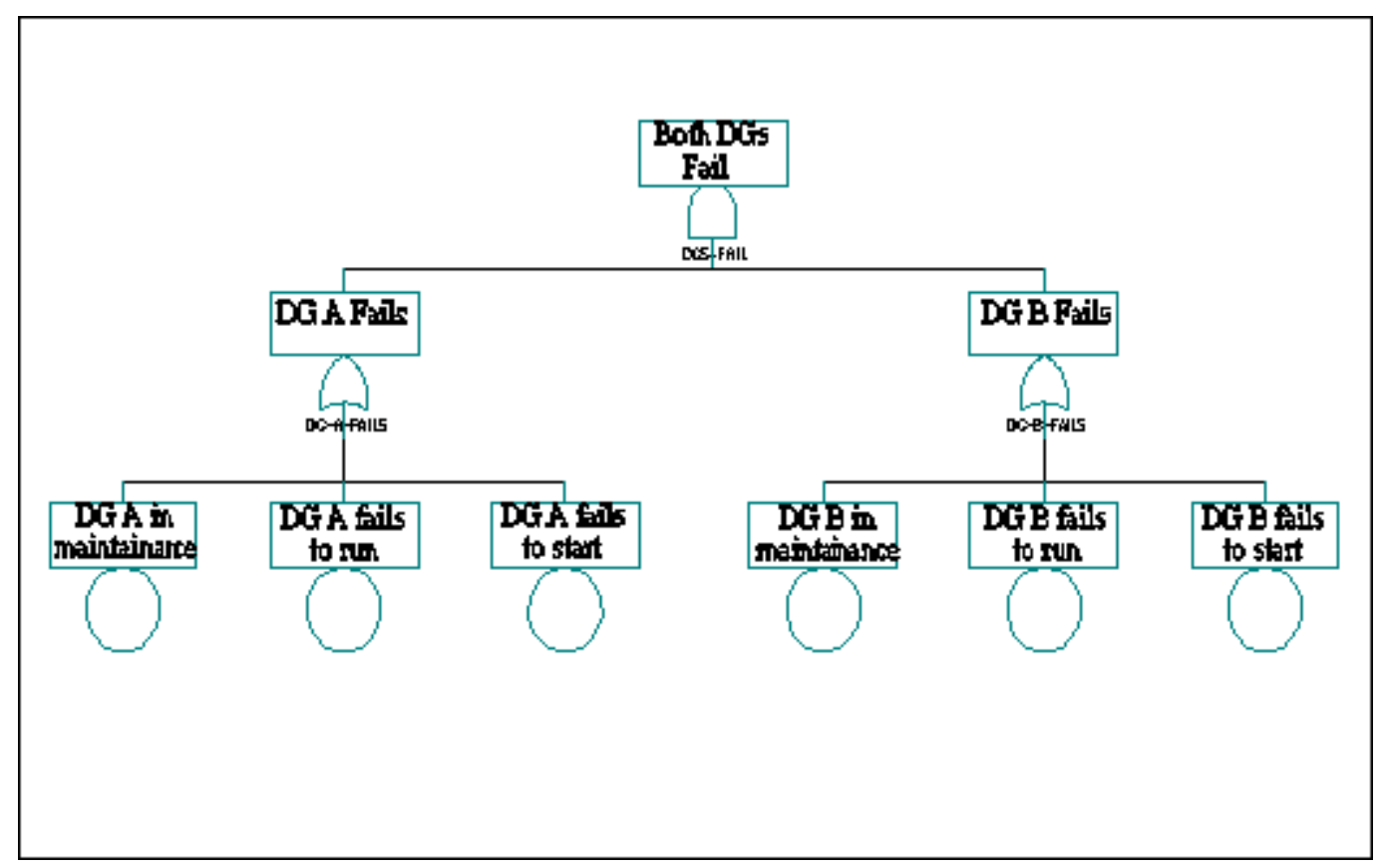

Generating cut sets for this fault tree will produce a cut set containing the two maintenance events:

\section{DG-A-MAINTENANCE * DG-B-MAINTENANCE}

Assuming that the plant technical specifications restrict both diesel generators from being in maintenance simultaneously while at power, this cut set is an example of mutually exclusive events. These mutually exclusive events would be handled by removing any cut sets that contain the mutually exclusive events (even if the cut set includes additional events).

Generally, mutually exclusive events fall under the "restriction case" (e.g., two AFW pumps out for testing at the same time) or under the "modeling concerns case" (e.g., initiating events in the fault trees lead to multiple initiating events in sequence cut sets).

\subsection{Removing Mutually Exclusive Events}

Mutually exclusive events are treated by removing any cut sets that contain the mutually exclusive events (even if the cut set includes additional events). In SAPHIRE, several methods exist that could perform this removal operation.

Editing the cut sets manually using the cut set editor to "weed-out' the mutually exclusive events.

Using the "mutually exclusive top event" feature when generating sequence logic so that impossible events can be defined at the sequence level.

Modify logic models (via NOT gates ore complemented events) to effectively remove impossible combinations of events.

Pruning cut sets to remove impossible events that are defined by a simple logic structure (i.e., fault tree).

Using recovery rules to define impossible combinations of events so that cut sets containing these events would be deleted. 


\subsection{Manual Cut Set Editor Method}

\section{PURPOSE}

Remove mutually exclusive events from a cut set using the cut set editor. This method can also be applied to fault tree cut sets in a similar manner.

\section{STEPS}

1. After sequences cut sets have been solved, select Sequence from SAPHIRE menu.

2. From the Sequences dialog, highlight the desired sequence. LOSP 3 was highlighted in this example.

3. Right-click to invoke the pop-up menu and select Cut Sets | Edit | Current.

4. Place the cursor in the Cut Set No. column of the desired cut set and choose the Delete button.

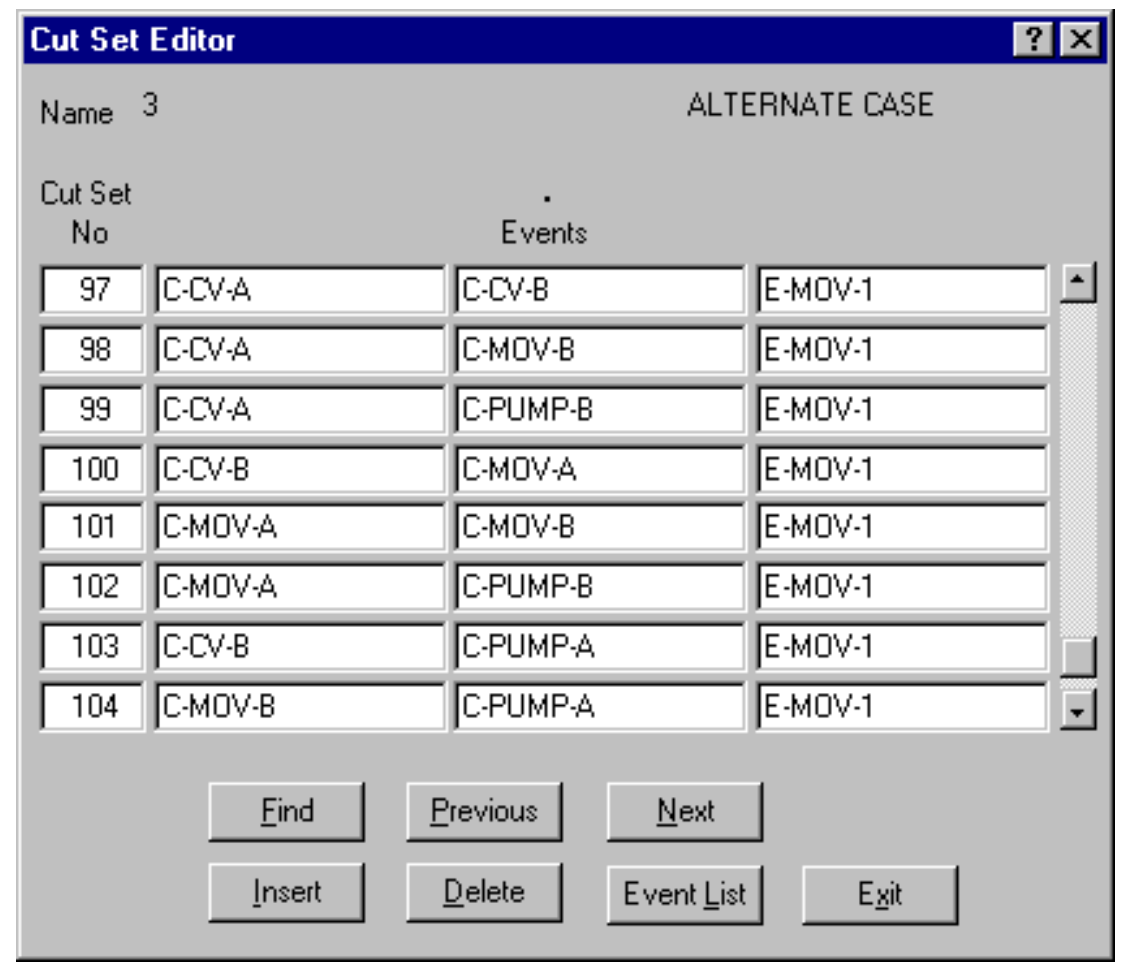

This process is not recommended since it is both error prone and time consuming.

\subsection{Mutually Exclusive Top Method}

\section{PURPOSE}

Remove mutually exclusive events from sequence cut sets using the "mutually exclusive top event" feature when generating sequence logic. Impossible events can be defined at the sequence level using this method. Using the "mutually exclusive top" method is only applicable to sequence cut sets. 


\section{STEPS}

1. Define fault tree logic that represents the combination of events that are mutually exclusive.

Using the example, the analyst would make a fault tree that had the two maintenance events "ANDed" together.

\section{ME-TOP AND DG-A-MAINTENANCE DG-B-MAINTENANCE}

2. The fault tree name for the fault tree containing the mutually exclusive combinations would then be typed in the space labeled "Mutually Exclusive Top Name" when generating sequence logic (via the Event Tree | Link Trees option).

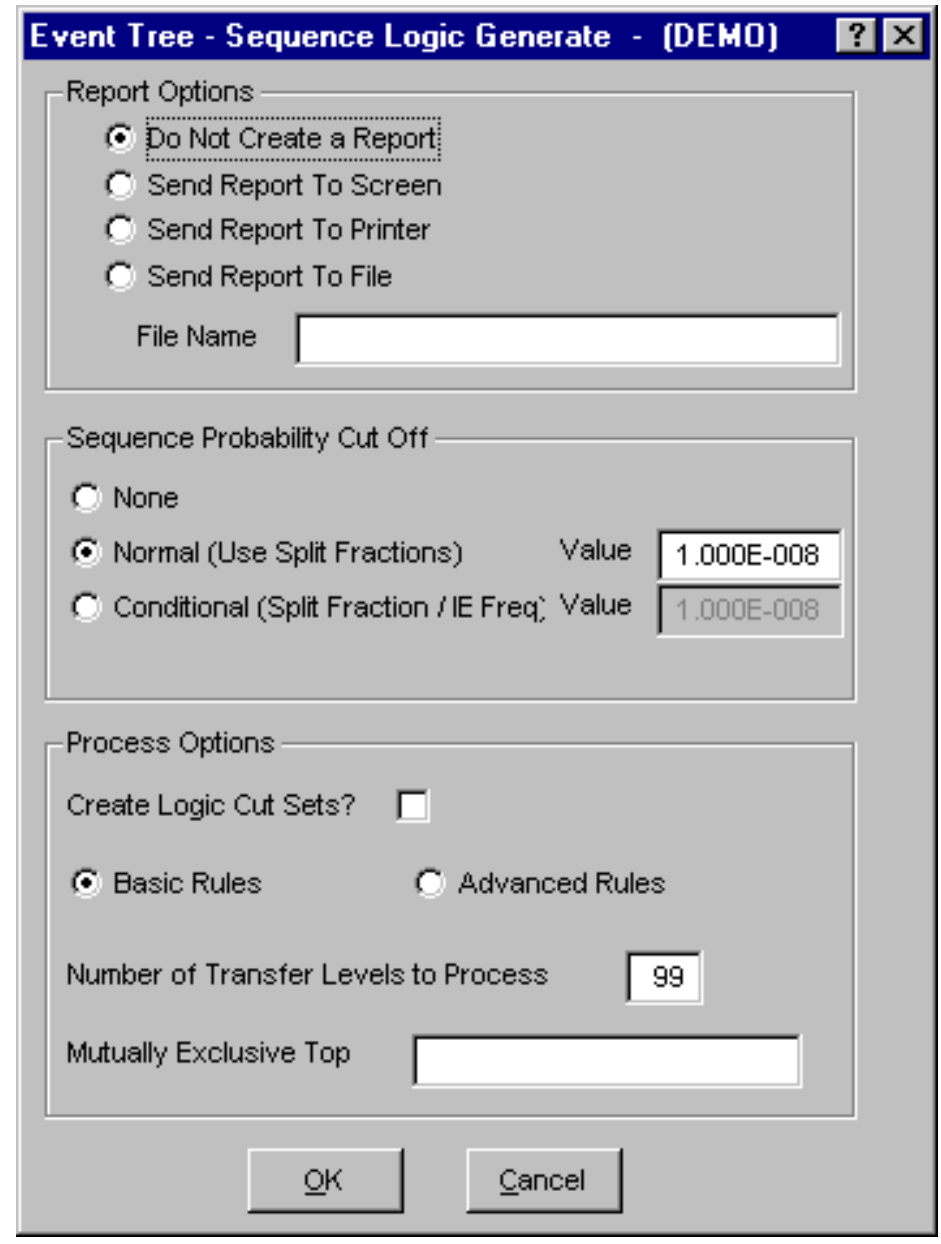

\subsection{Logic Modification Method}

\section{PURPOSE}

Remove mutually exclusive events by modifying logic models (via NOT gates or complemented events).

The illustration here demonstrates how the example problem could be modified so that combinations of diesel generator maintenance events will not appear in the list of cut sets. 


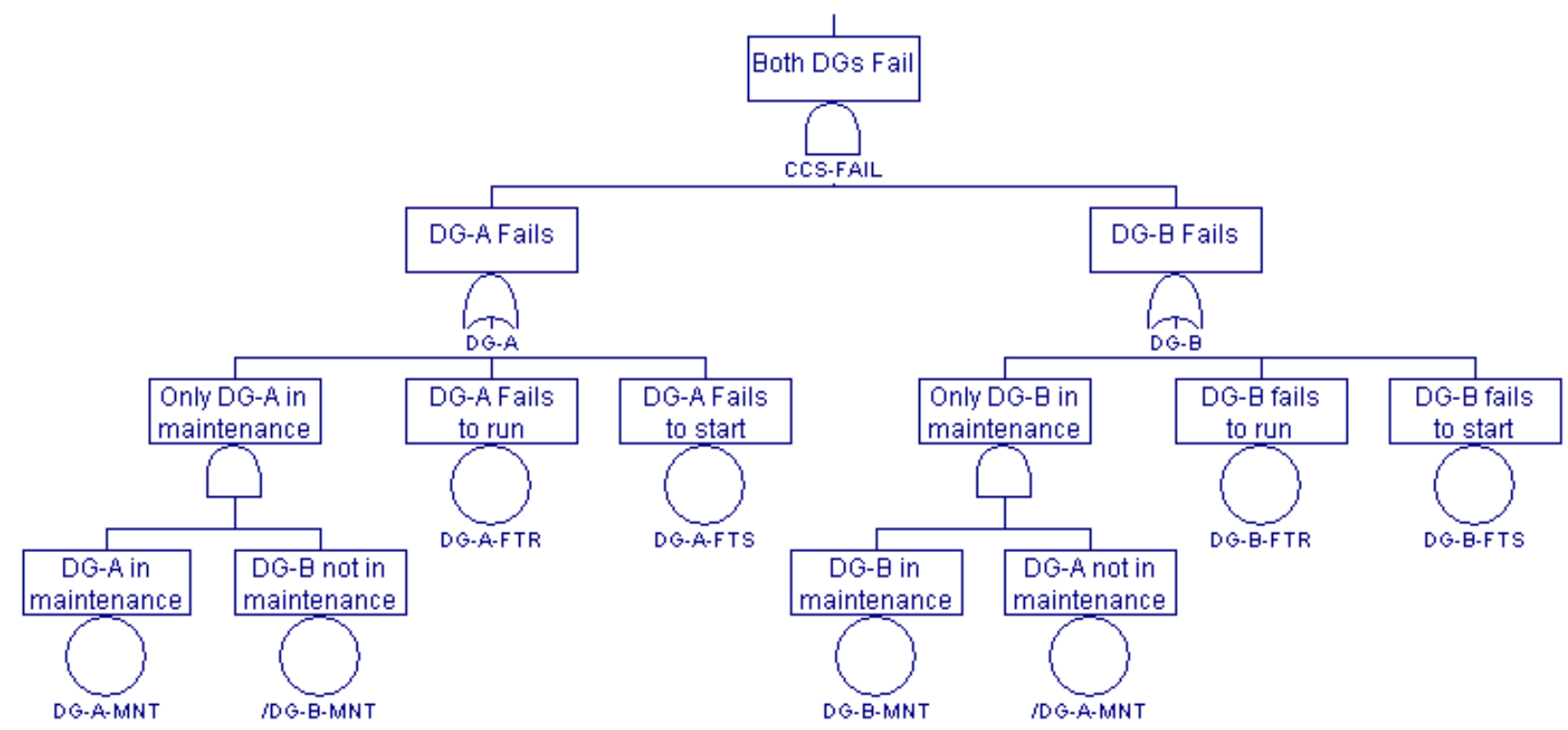

Using this method requires that the analyst modify the fault tree logic in order to directly remove impossible combinations of events.

Drawbacks to this method include the time needed to modify the fault tree logic and the realization that complemented basic events will not appear in the list of cut sets.

\subsection{Pruning Method}

\section{PURPOSE}

Remove mutually exclusive events by pruning cut sets using the Pruning feature.

To use this method, the analyst must first define fault tree logic that indicates (via existing cut sets) combinations of events that you want to keep. Thus, the tree

\section{ME-PRUNE OR DG-A-MAINTENANCE DG-B-MAINTENANCE}

would delete cut sets containing DG-A-MAINTENANCE * DG-B-MAINTENANCE, but would keep any single occurrence of the events DG-A-MAINTENANCE or DG-B-MAINTENANCE.

\subsection{Recovery Rules Method}

\section{PURPOSE}

Use Recovery Rules to define groups of events that, if appearing together, result in the deletion of the cut set. For most cases, this is the preferred method of removing cut sets for the following reasons:

The rules are automatically implemented on the cut sets when recovery rules are applied.

No changes to logic models are needed.

No manual manipulations to cut sets are required. 
The recovery rules for removing mutually exclusive events may be developed for a particular fault tree, all fault trees, a particular sequence, a single event tree, or all sequences.

Mutually exclusive event rules are generally typed into the fault tree project and sequence project rules.

\section{SEE ALSO}

Recovery Rules Method Example

\subsection{Recovery Rules Method Example}

To demonstrate the potential use of the recovery rules, the example here shows how the rules could be used to remove the cut set containing both diesel generators failing from the demo database.

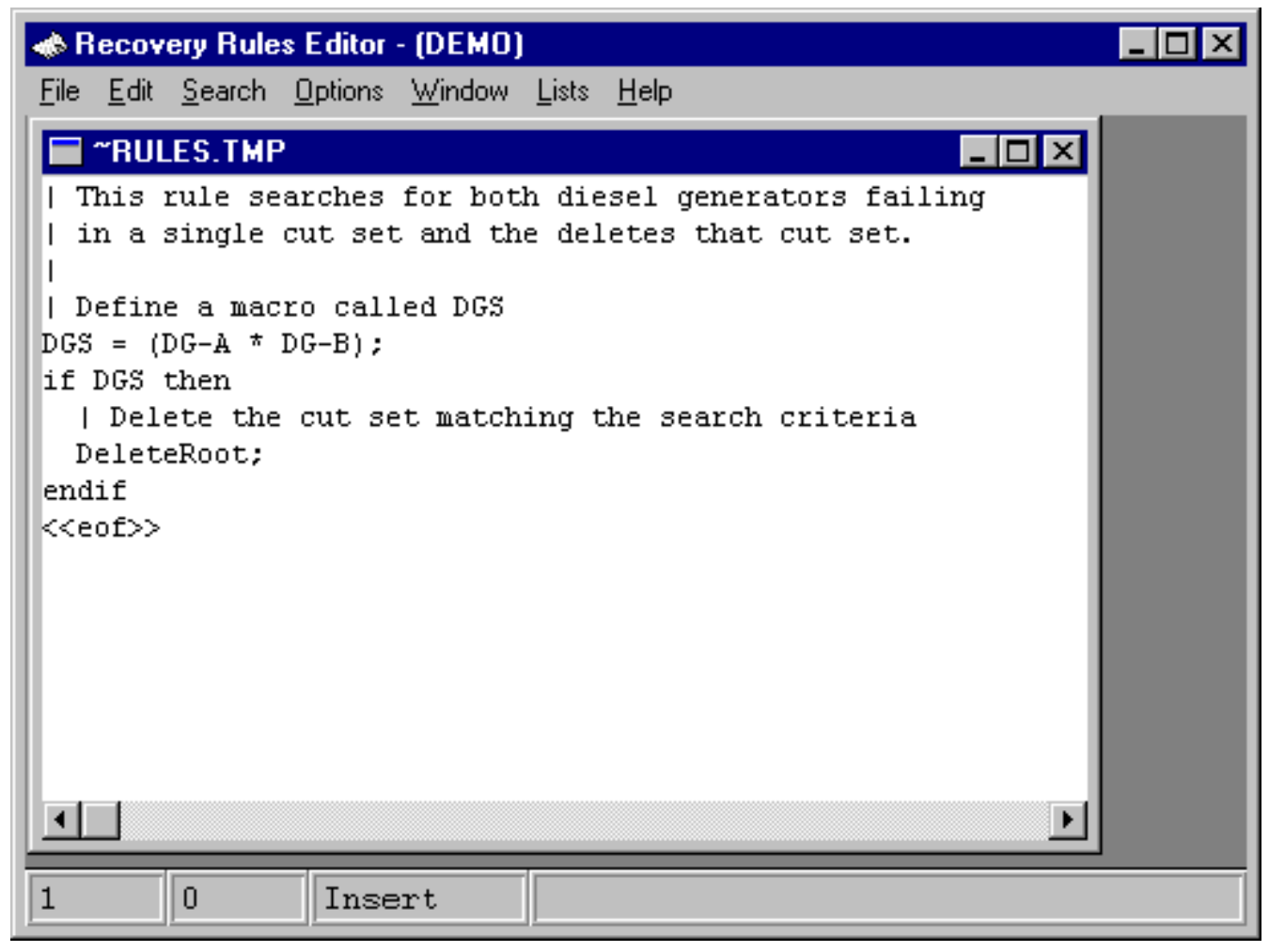

The rule shown above was entered in the sequence project rules.

The recovery rules were then applied to both sequences 2 and 3 (using no truncation).

Sequence 3 changed from an original value of $1.8 \mathrm{E}-3$ to a value of $8.4 \mathrm{E}-4$.

Only one cut set was removed from Sequence 3, but it happened to be the dominant cut set. 


\section{INTERNATIONAL SAPHIRE}

\subsection{Setup of International SAPHIRE}

This section applies to the English versions of the Windows operating systems. It is intended to guide you through the steps of enabling foreign language support while operating the SAPHIRE for Windows program. If your Windows operating system is the native version for the foreign language of your choice, it is not necessary for you to follow these instructions.

The following instructions are applicable only for the specified versions Windows 95 and Windows NT operating systems.

Because you will be required to restart your system, it is recommended that you close all other applications before beginning installation of the foreign language(s). You will also need to have your Windows operating system CD-ROM or floppy disks available.

\subsection{Windows NT, Version 4.0}

\section{PURPOSE}

This section guides you through the steps of enabling foreign language support for the Windows NT, Version 4.0 operating system.

SAPHIRE International Support is not available for earlier versions of Windows NT (e.g., Version 3.51 or earlier). SAPHIRE has been tested only with NT 4.0, Service Pack 3, but should run with prior versions of NT 4.0. It is strongly recommended that you run SAPHIRE with the most current NT Service Pack.

\section{STEPS}

1. Double-click on the My Computer in icon on your desktop. In the My Computer window double-click on the Control Panel and folder. In the Control Panel window double-click on the Display icon. The Display Properties dialog will be displayed. 


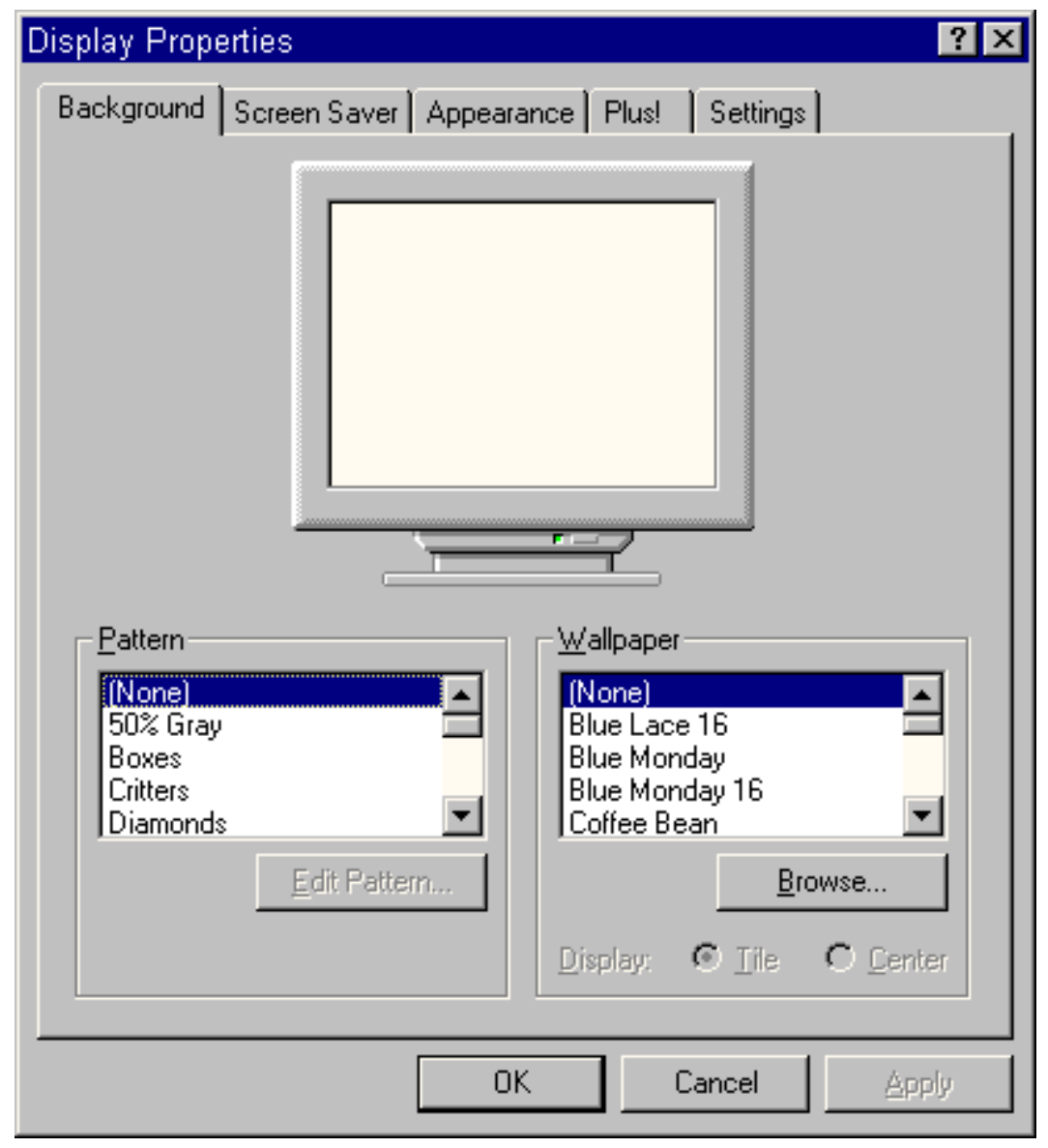

2. Select the Plus! tab. 


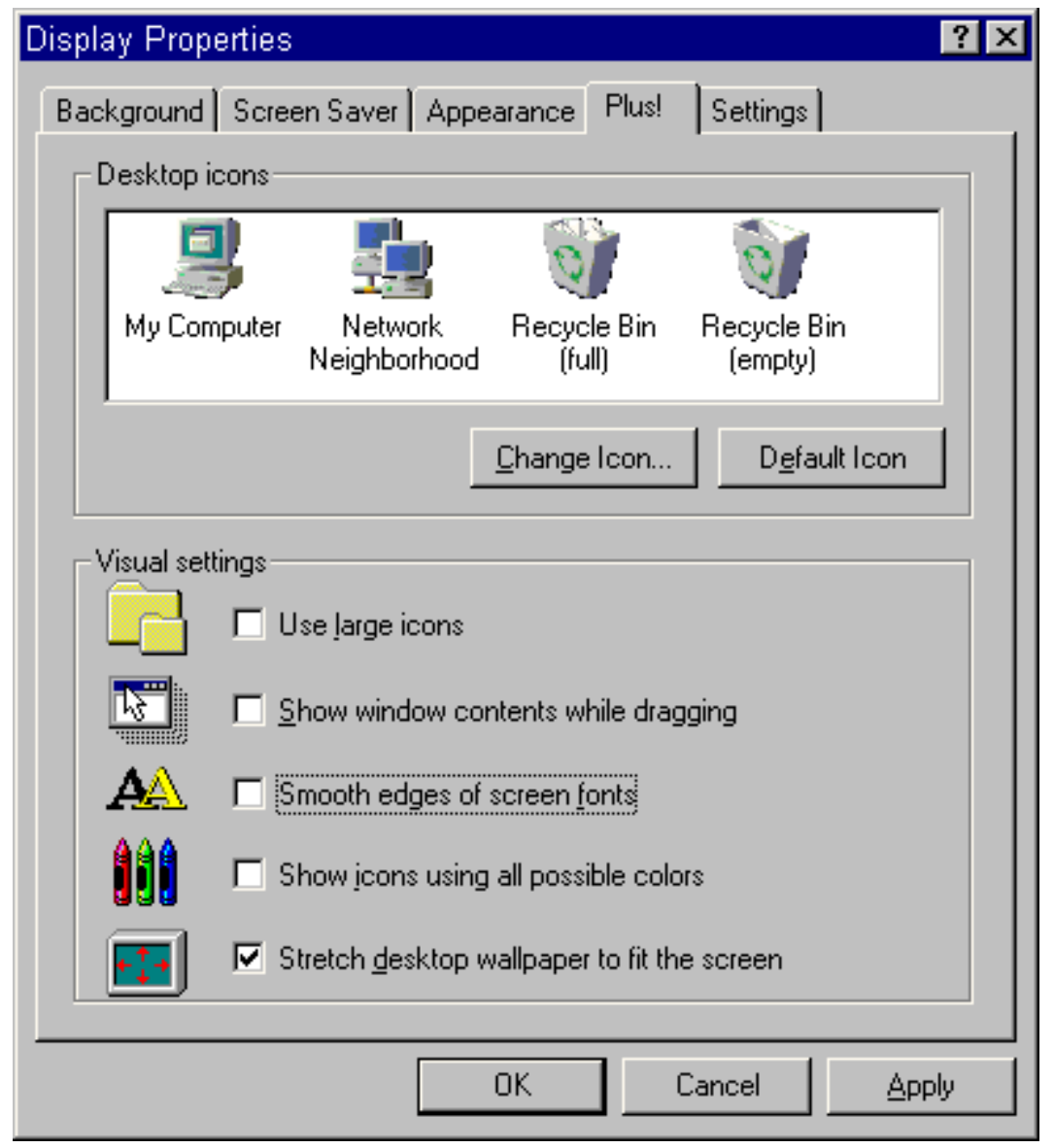

3. Ensure that the Smooth edges of screen fonts option is NOT checked. Choose the Apply button. Choose the OK button. The Display Properties dialog will be closed.

4. In the Control Panel window double-click on the Regional Settings Settings Properties dialog will be displayed. 


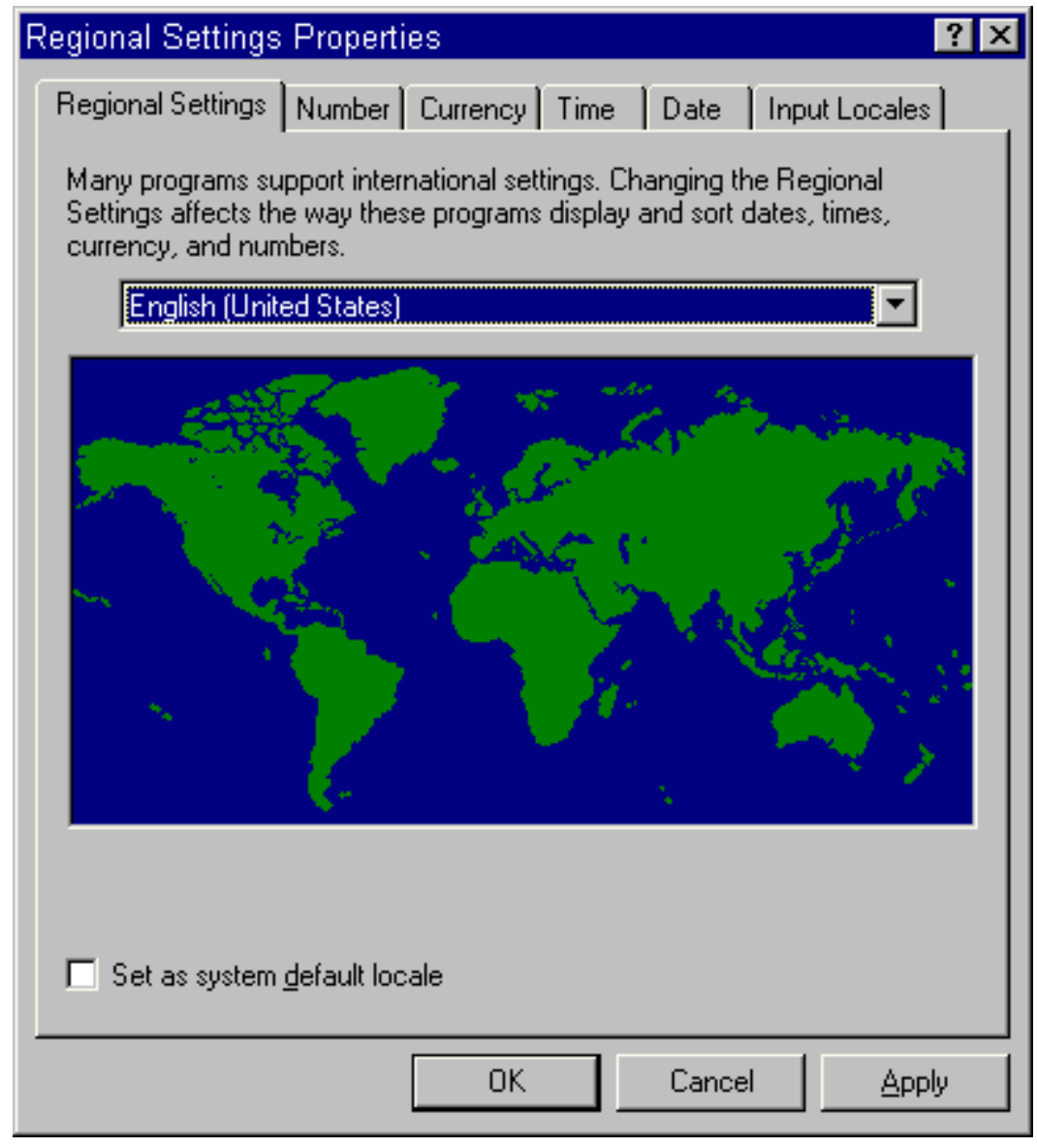

5. Choose the Input Locales tab. The currently installed input locales will be displayed in the list. If the language you wish to use is already in the list, skip to Step 7. If the language you wish to use is not in the list, choose the Add button. 


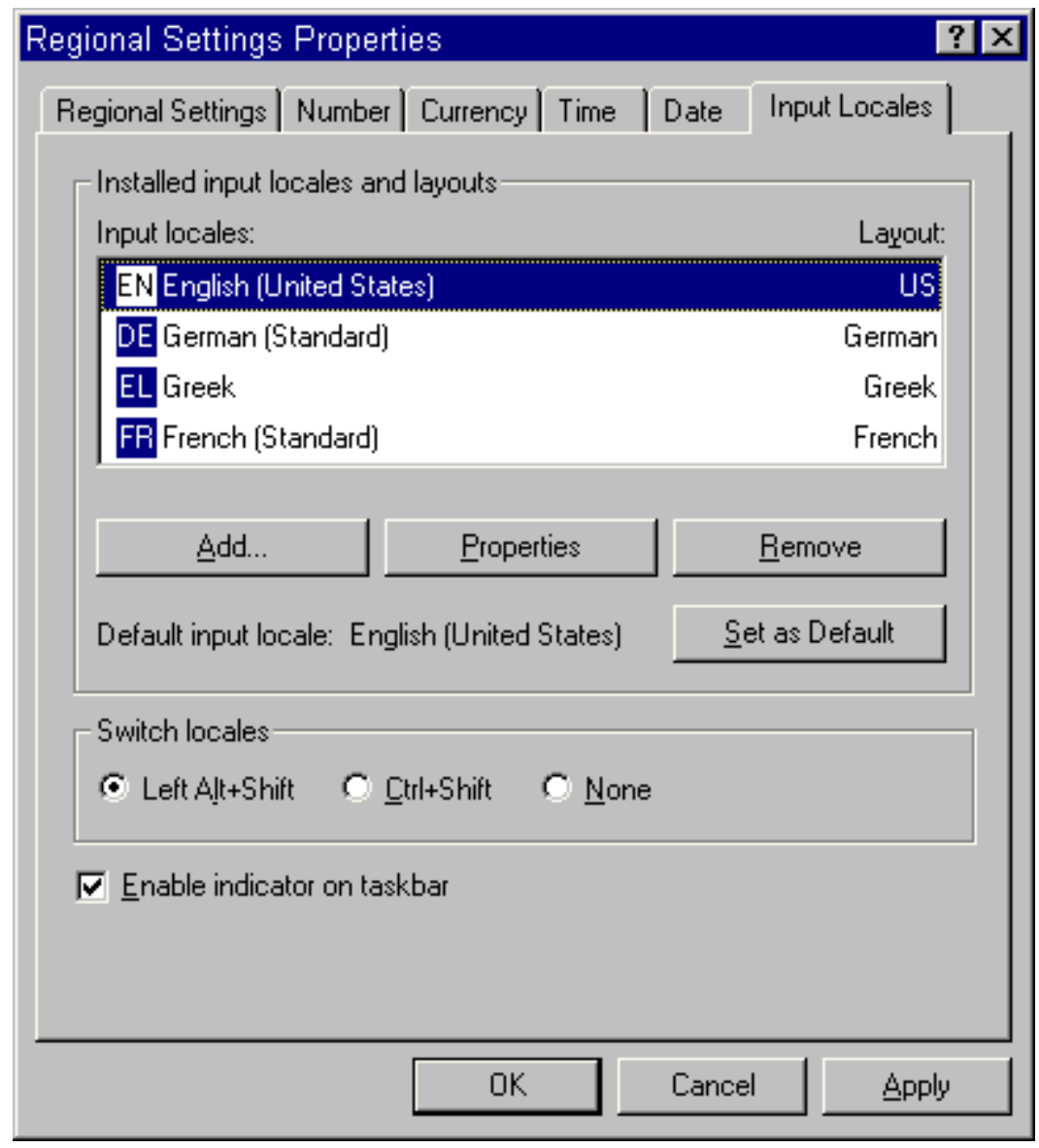

6. The Add Input Locale dialog will be displayed. Select the desired locale from the drop-down list and choose the $\mathbf{O K}$ button.

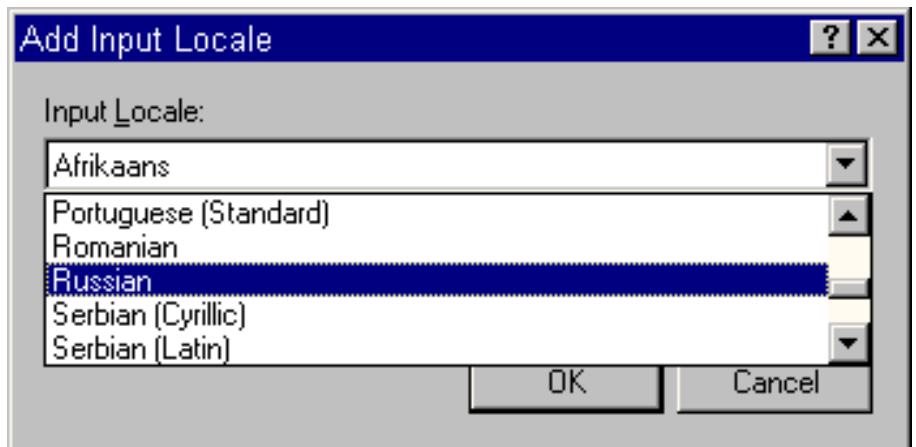

The Add Input Locale dialog will be closed and the selected locale will be displayed in the list of input locale choices on the Regional Settings Properties dialog, Input Locales tab.

7. To allow switching on-the-fly between installed input locales, choose either the Left Alt+Shift or $\mathbf{C t f l + S h i f t}$ radio buttons. Ensure that the Enable indicator on taskbar check box is checked.

Choose the Apply button. You may be instructed to install some required files on your computer. If necessary, follow the directions for copying the requisite files. 
8. On the Regional Settings Properties dialog choose the Regional Settings tab. Select the desired language from the drop-down list. Choose the Set as system default locale check box. Choose the $\mathbf{O K}$ button.

9. If you have not previously installed the required files, the Insert Disk dialog will be displayed. Follow the directions and choose the $\mathbf{O K}$ button.

\section{Insert Disk}

Please insert the Compact Disc labeled Windows NT Workstation CD-ROM' into your CD-ROM drive $[F$ : ) and then click OK. You can also click OK if you want files to be copied from an alternate location, such as a floppy disk or a network server.

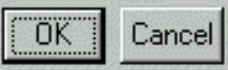

10. If you have previously installed the required language files, the Regional Settings dialog will be displayed. Choose the Yes button.

\section{Regional Settings}

(1) Required files are already installed on your hard disk. Setup can use these existing files, or Setup can recopy them from your original Windows NT CD-ROM or from a network share.

Would you like to skip file copying and use the existing files? (lf you click No, you will be prompted to insert your Windows NT CD-ROM or to supply an alternate location where the needed files may be found.)

\section{Yes No}

11. The Change Regional Settings dialog will be displayed. In order for the change to take place, you must restart your computer.

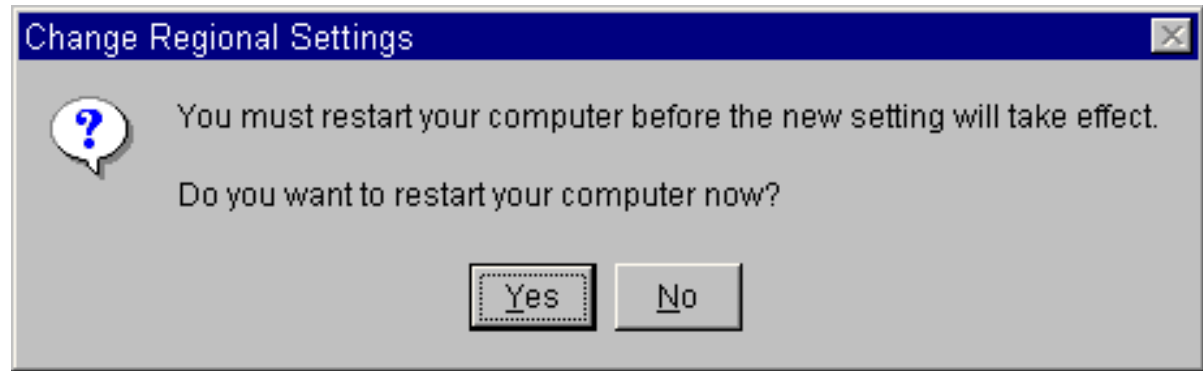

After your computer has restarted, you will need to change your system's "icon font." Open the Display Properties dialog. Select the Appearance tab. 


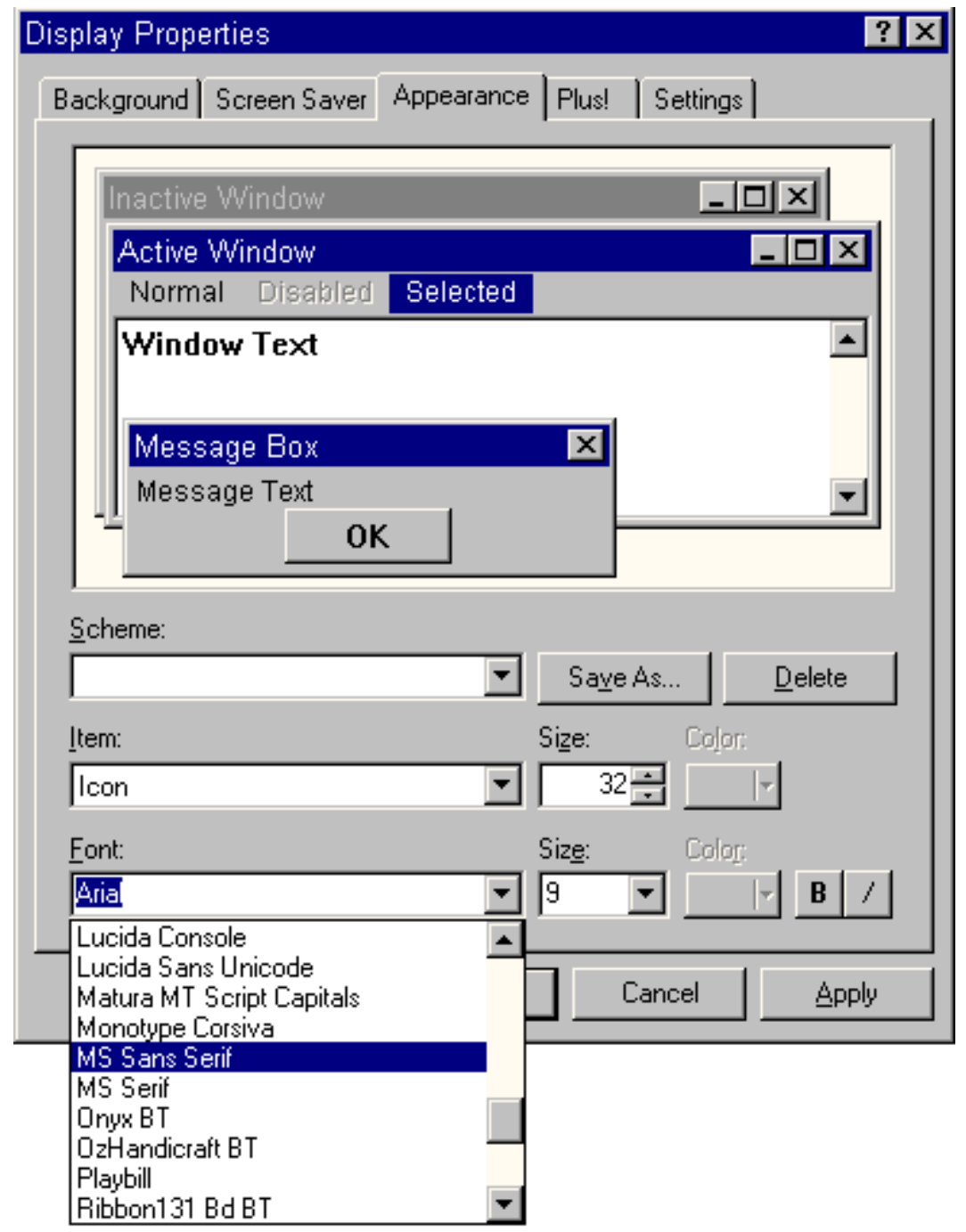

12. In the Item drop-down list, select the Icon choice. In the Font drop-down list, select the MS San Serif font. Choose the Apply button. Choose the OK button. The Display Properties dialog will be closed.

13. You may want to change the regional setting of your operating system's "current" locale to English, but leave the "default" locale set to Russian. On the Regional Settings Properties dialog, Regional Settings tab select English from the drop-down list. Choose the Apply button, then the OK button. By doing this you set the "current" locale to English which enables SAPHIRE to display certain controls using the English alphabet. DO NOT select the Set as system default locale check box since by doing this you will change the operating system's "default" locale from Russian back to English.

You can switch between languages (code pages ) anytime after installing more than one locale by using the shortcut keys selected in Step 7, above, or by clicking on the language indicator on the system taskbar and selecting the desired locale from the list. 


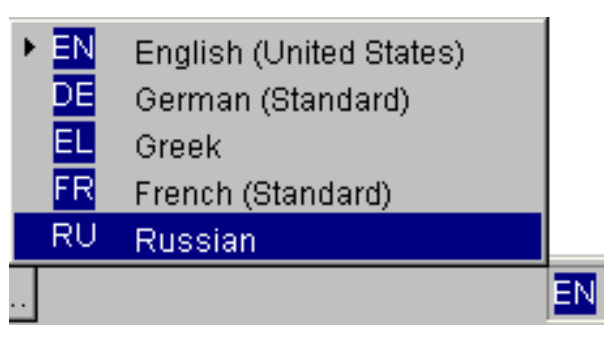

To enter data using the English character set, you must choose the English locale, to enter data using the Cyrillic character set, you must choose the Russian locale, etc.

This on-the-fly switching of code pages does not affect the way programs display dialog controls, times, dates, etc. It simply changes the way keyboard input is interpreted by the program, allowing entry using different character sets.

14. Start SAPHIRE and choose Modify | Project from the menu or choose the

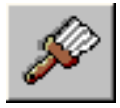
buttons on the toolbar. The Project dialog will be displayed.

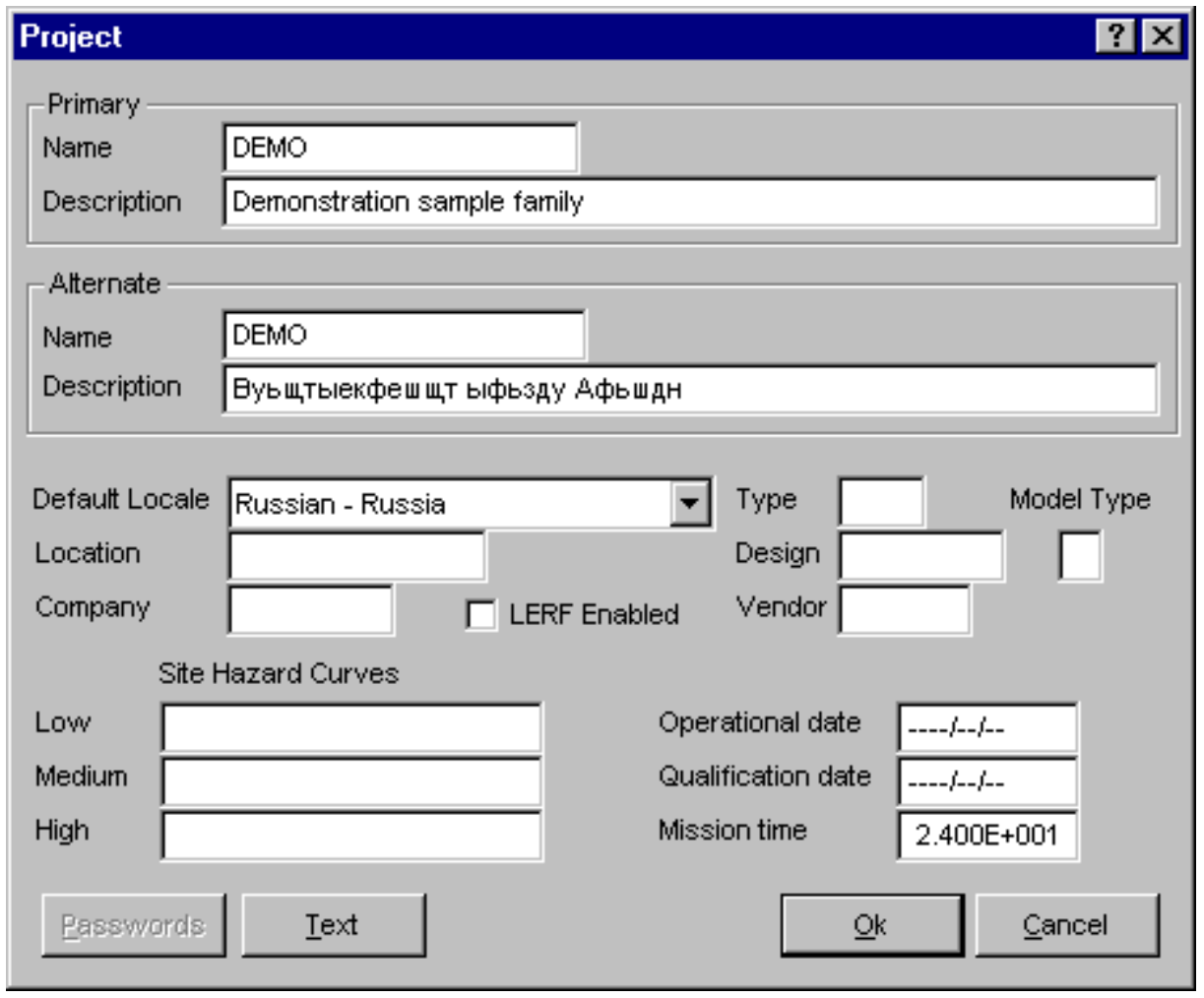

15. Choose the desired language from the Default Locale drop-down list. Choose the Ok button. You can now enter and view foreign language characters on SAPHIRE dialogs and reports. Switch between locales (described in Step 13) to enter data using the different character sets on subsequent SAPHIRE dialogs. 


\subsection{Windows 95, Version 4.00 .950}

\section{PURPOSE}

SAPHIRE does not run with this version of Windows 95. You MUST install Service Pack 1 for this version of Windows 95 in order to run SAPHIRE and use any language other than English.

After installing Service Pack 1, you will be running Windows 95, version 4.00.950a (Service Pack 1). Follow the instructions below for Windows 95 version $4.00 .950 \mathrm{~b}$.

\subsection{Windows 95, OEM Version 4.00.950 b (OSR2)}

\section{PURPOSE}

This section guides you through the steps of enabling foreign language support for the Windows 95, OEM Version $4.00950 \mathrm{~b}$ (OSR2 ) operating system.

\section{STEPS}

1. Double-click on the My Computer in icon on your desktop. In the My Computer window double-click on the Control Panel 7 folder. If you have previously installed Multilanguage support, skip to Step 6, otherwise, continue with Step 2.

2. In the Control Panel window double-click on the Add/Remove Programs icon. The Add/Remove Programs Properties dialog will be displayed. 


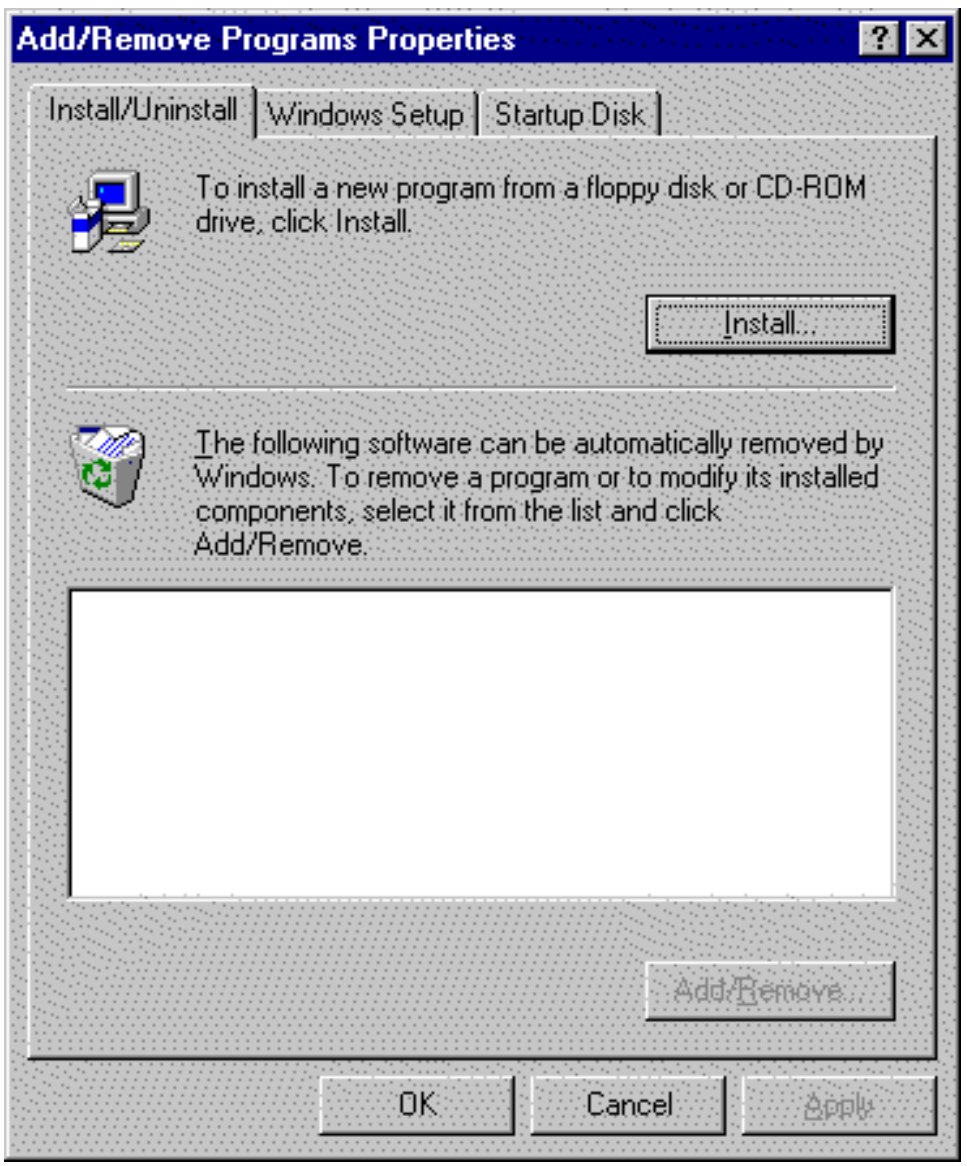

3. Select the Windows Setup tab. From the Components list check the Multilanguage Support option. Choose the Details button and ensure that a check mark appears next to the language(s) you want to use. Choose the Apply button. 


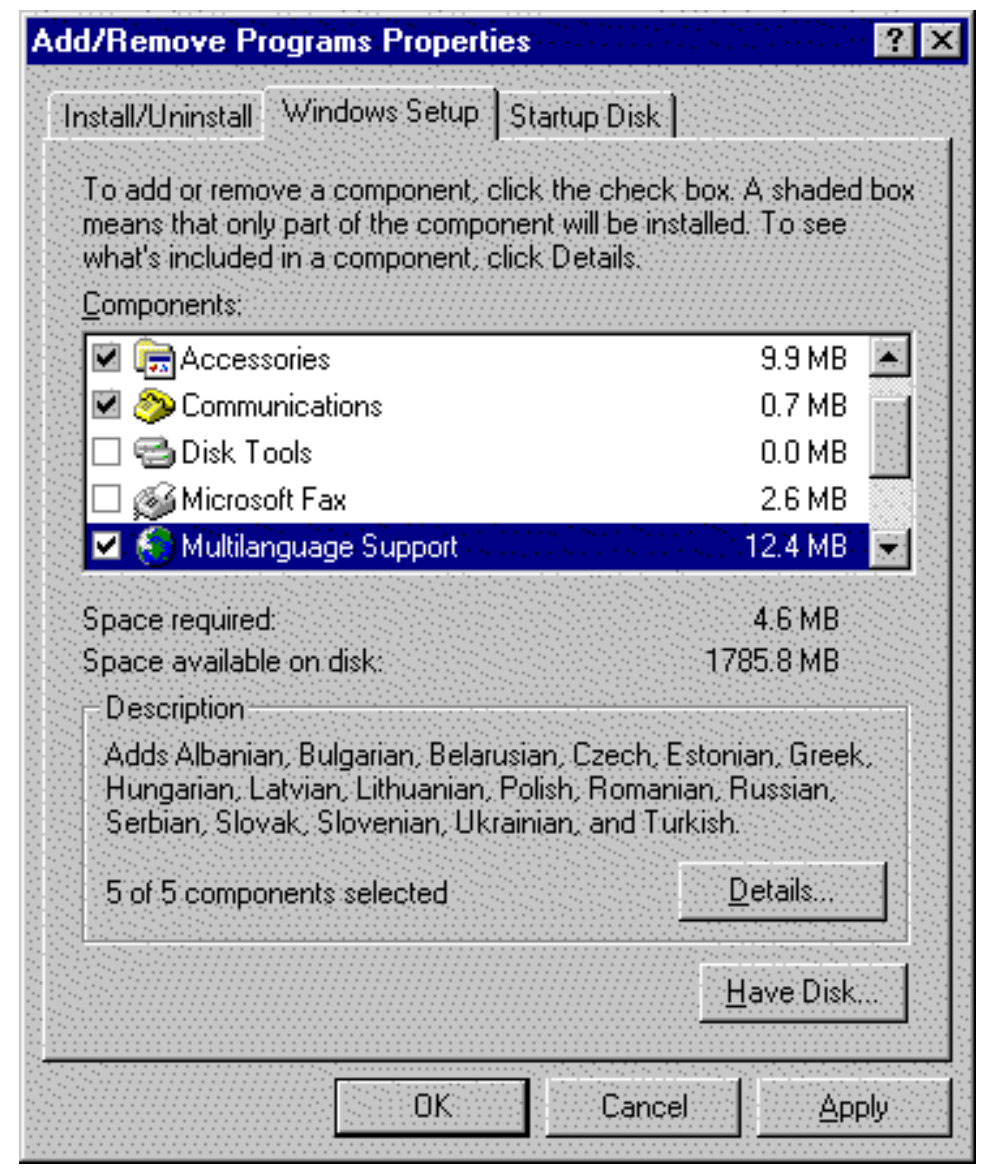

4. If the required files have not been previously installed, the Insert Disk dialog will be displayed. Follow the directions and choose the OK button.

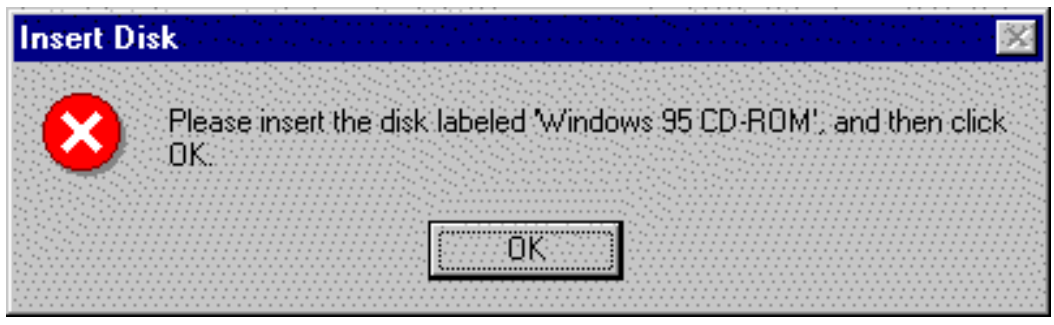

5. After the required files have been copied, the System Settings Change dialog will be displayed. Choose the Yes button. In order for the change to take place, you must restart your computer

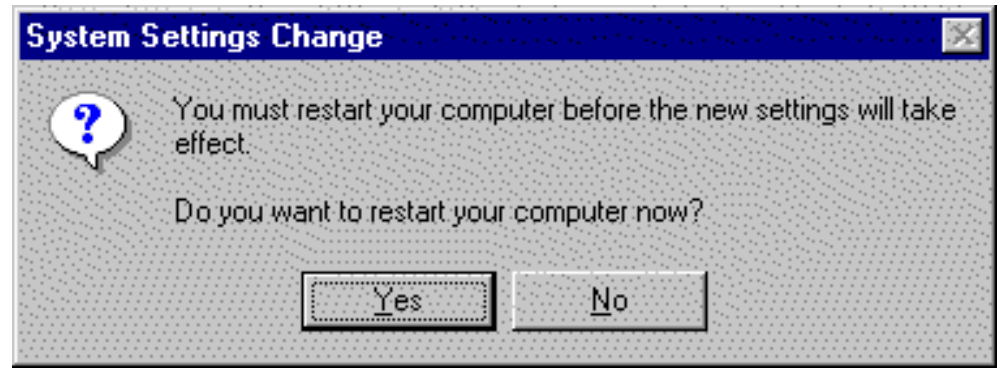


6. After your computer has restarted, you will need to change your system's keyboard properties. In the Control Panel window double-click on the Keyboard dialog will be displayed.

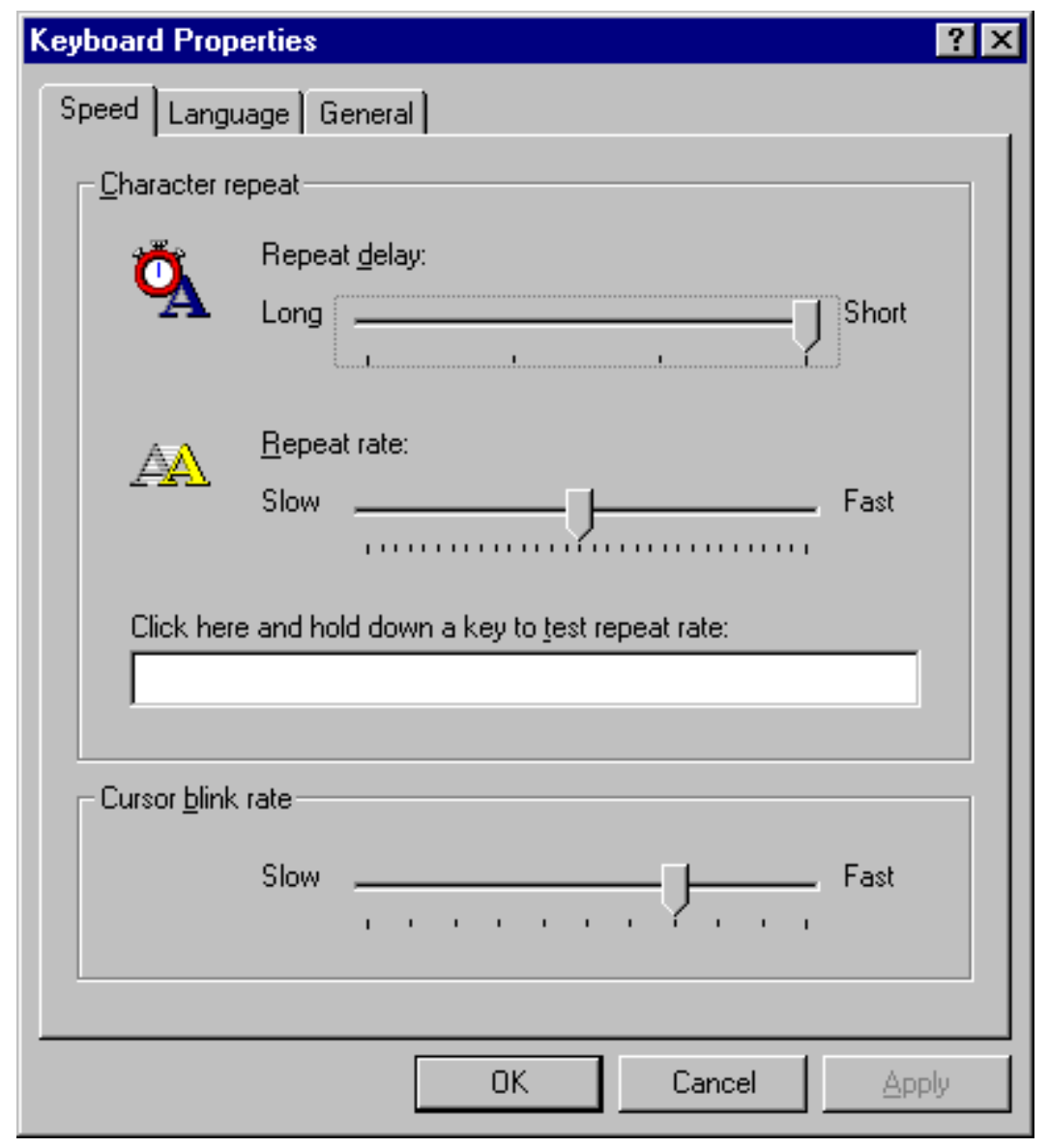

7. Choose the Language tab. The currently installed languages are displayed in the list. If the language you wish to use is already in the list, skip to Step 9. If the desired language is not in the list, choose the Add button. 


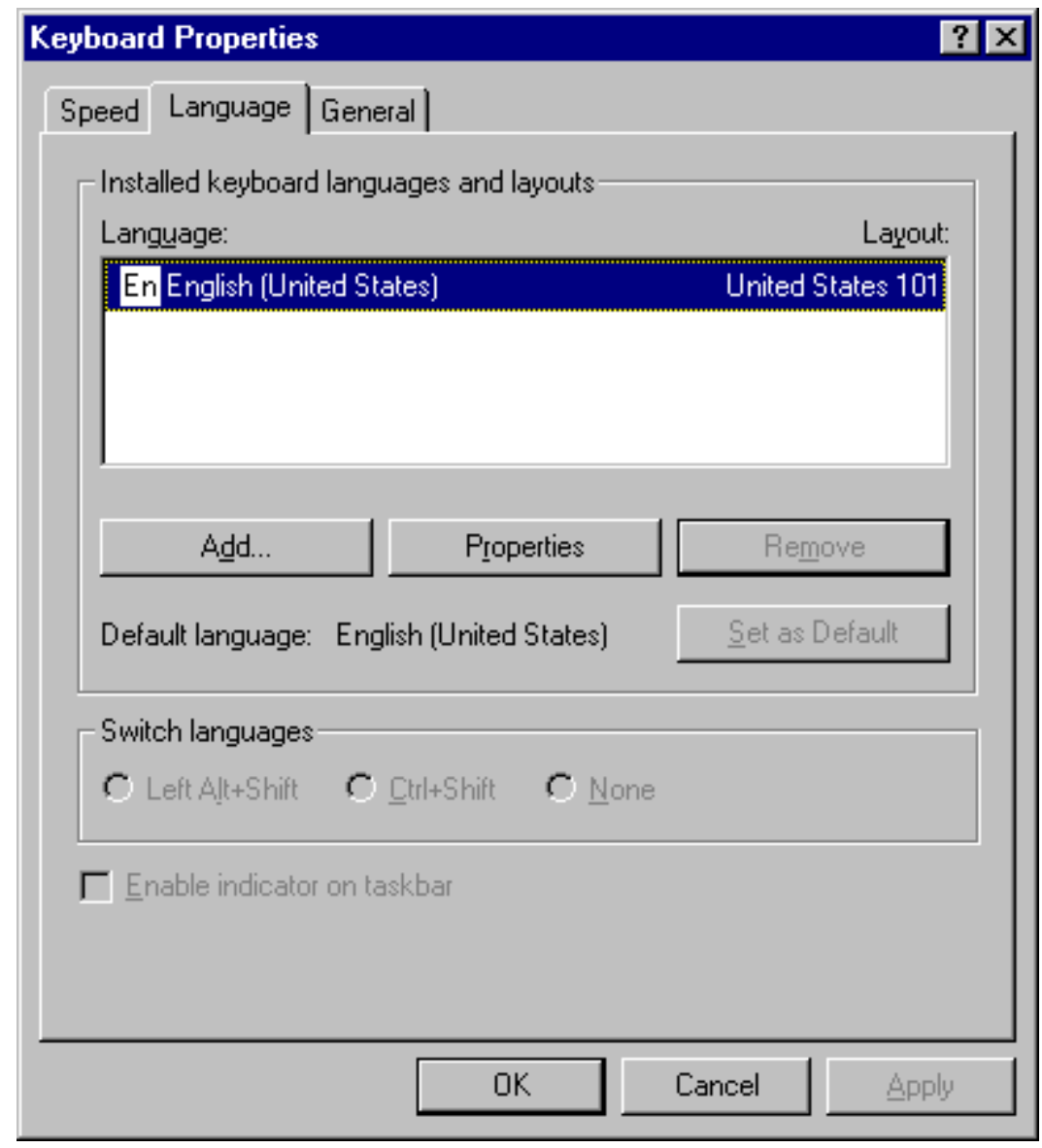

8. The Add Language dialog will be displayed. Select the desired language from the drop-down list and choose the OK button.

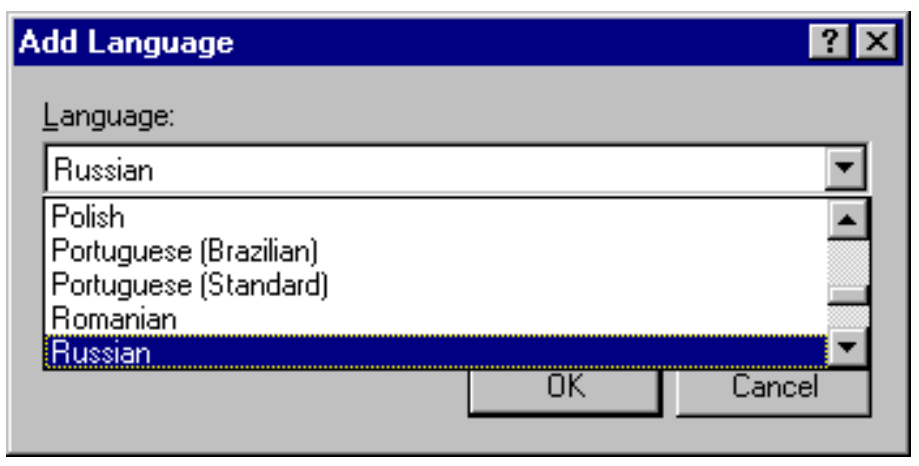

9. The Add Language dialog will be closed and the selected language will be displayed in the list of language choices on the Keyboard Properties dialog, Language tab (refer to Figure 17). To allow for switching on-the-fly between installed languages, choose either the Left Alt+Shift or

Ctrl+Shift radio buttons. Ensure that the Enable indicator on taskbar check box is checked. Choose the Apply button then choose the OK button. You may be instructed to install some required files on your computer. If necessary, follow the directions for copying the requisite files. 
10. In the Control Panel window double-click on the Regional Settings icon. The Regional Settings Properties dialog will be displayed.

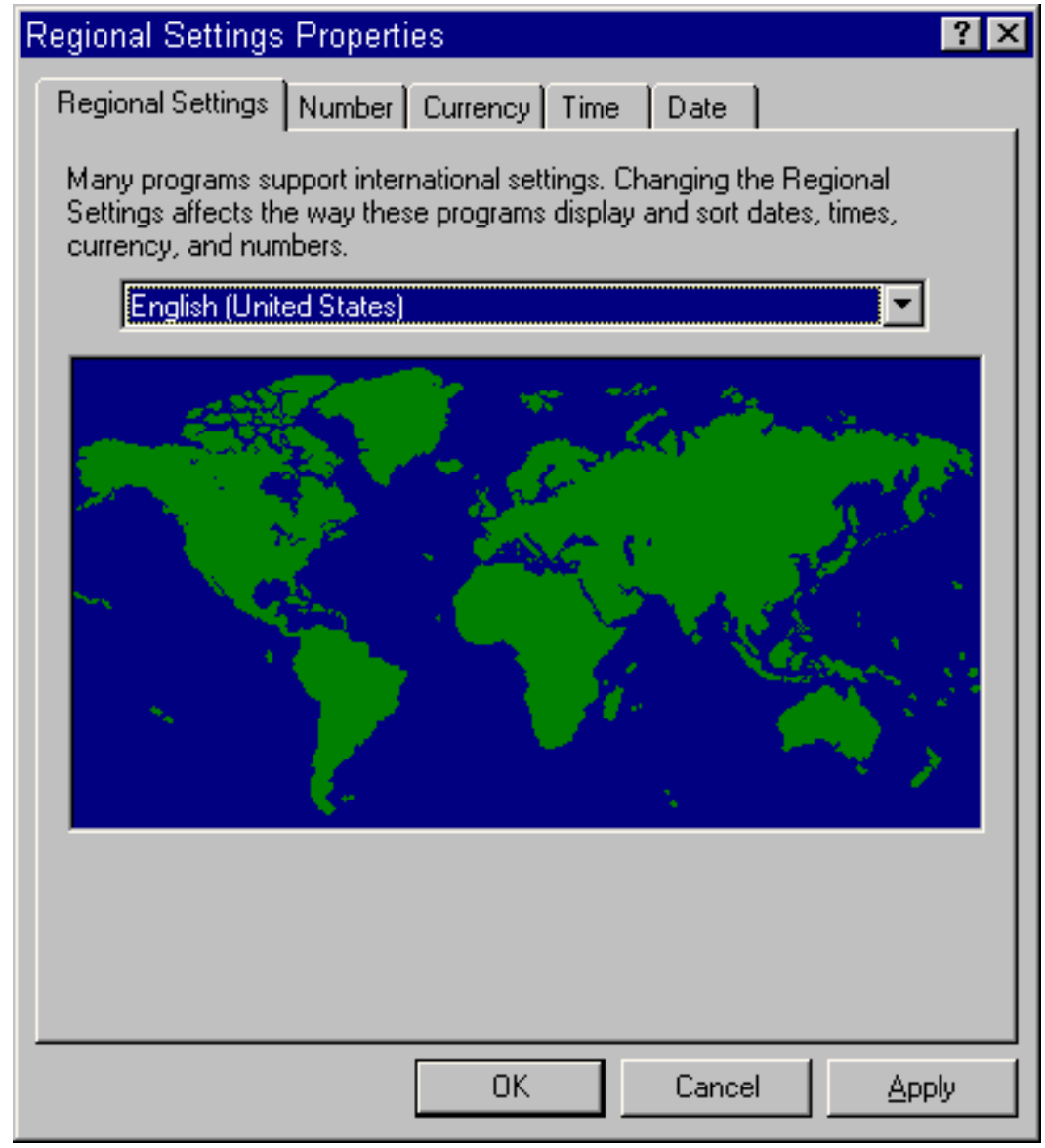

11. Select the desired language from the drop-down list. Choose the Apply button.

12. The Change Regional Settings dialog will be displayed. In order for the change to take place, you must restart your computer.

13. After your computer has restarted, you can switch between languages by using the shortcut keys selected in Step 9 above, or by clicking on the language indicator on the system taskbar and selecting the desired language from the list.

14. Go to Steps 14 and 15 under the Windows NT instructions. 


\subsection{MAR-D Format}

When editing MAR-D output files that contain data entered using other languages, you must use an editor that supports multiple languages (i.e., language scripts other than English) such as Multi-Edit for Windows and WordPad.

\subsection{Definitions}

\subsubsection{MAR-D Format}

When editing MAR-D output files that contain data entered using other languages, you must use an editor that supports multiple languages (i.e., language scripts other than English) such as Multi-Edit for Windows and WordPad.

\subsubsection{OEM}

Original Equipment Manufacturer

\subsubsection{OSR2}

Operating System Revision 2

\subsubsection{Code Page}

An internal table that the operating system uses to relate the keys on the keyboard to the characters displayed on the screen. Usually defined to support specific languages or groups of languages which share common writing systems. In Windows 95 and NT 4.0, code pages can be changed on-the-fly by the user without changing the default language system in use.

\subsubsection{Font}

A set of graphical representations of characters.

\subsubsection{Keyboard layout}

A standard arrangement of characters on a keyboard that defines which keys produce particular characters.

\subsubsection{Locale}

The features of the software environment that are dependent on language, country, and cultural conventions (e.g., sort order; keyboard layout; character sets; and time, date, and currency formats). In Windows, the locale information includes the name of the spoken language, the script used to write the language, and the cultural conventions.

\subsubsection{Script}

A system of characters used to write one or several languages. The characters of the script denote sounds, syllables, or word elements and are governed by a general set of rules for creating text, such as default writing direction. 


\section{MACRO SCRIPTS}

\subsection{Macro-Scripts Usage And Examples}

SAPHIRE (Version 7) is equipped with a feature that allows for the usage of Macro-scripts to run specified menu or analysis steps in a manner outside of SAPHIRE. This feature is useful for repetitive analysis procedures in databases or several databases. Macro-scripts in SAPHIRE are different than the Macros used in the linking, recovery and partition rule editors. Those Macros are used to define a combination of rule based operands, systems, or basic event for linking event trees or adding recovery actions or end state partitions. "Macro-scripts" are Keywords in text file format that SAPHIRE uses to automatically perform "analysis menu" functions. Though not directly designed to perform model modifications (i.e. modify basic event data, fault tree logic and event tree logic). Most modify functions can be used using macro-scripts using the MAR-D extract and load feature.

Macro-scripts are "programmed" using a standard text editor that SAPHIRE interprets to perform specified functions. The Macro-scripts are coded with Keywords that SAPHIRE recognizes as a specific menu or analysis functions.

The embedded macro code uses tags, similar to those in an "HTML" or "XML" type file to run the actual associated scripts. The tags are classified as verbs or "actions" which are the main actions or operations performed on a plant model; classes of "objects" which are acted upon by the verbs; and "parameters" which are passed to the actions. Each action, class, and parameter has opening and closing tags that mark the series of commands performed during a macro scenario. This code contains the declarations for all the tags utilized by the macrocode. The module also contains functions and procedures that parse the macro script into its actions and verbs, initializes the routines and parameters in the tool from the macro specified inputs, runs the code and outputs results into a text file. The macro scripts themselves can be run in normal or debug mode. All the commands executed can be submitted into an output ASCII text log file. Results are output into a separate ASCII text file. Time, date, and user identification information is logged with the test results. Key modules within the API and core functional areas have had macrocode hooks added to support this interface. Comment capability is available in the Macro-script program.

\section{TOPICS}

Keywords and Structures Example of a MACRO-SCRIPT
Making a MACRO-SCRIPT

Running a MACRO-SCRIPT

\section{Macro-Script Keywords}

The following table illustrates the Keywords and their usage for creating a macro-script in SAPHIRE. Each keyword having a opening tag and closing tag (i.e. $<$ tag $>$ being the opening and $</$ tag $>$ being the closing). The table also contains parameter setting commands that are used by the tags to perform specific functions. 


\begin{tabular}{|c|c|c|}
\hline Keyword & Use/Example & Notes \\
\hline$<$ add $>$ & $\begin{array}{l}<\text { add }> \\
\quad \cdots \\
</ \text { add }>\end{array}$ & $\begin{array}{l}\text { This keyword will add a new item to a list. Using this keyword, } \\
\text { a basic event could be added to a MAR-D cut set listing or a } \\
\text { change set added to a database. }\end{array}$ \\
\hline <analysis type> & $\begin{array}{l}<\text { analysis type }>4 \\
<\text { /analysis type }>\end{array}$ & $\begin{array}{l}\text { This keyword is used to specify analysis type that SAPHIRE is } \\
\text { to perform calculation in. Random }=1 \text {, Fire }=2 \text {, Flood }=3 \text {, } \\
\text { Seismic }=4 \text {, etc. For this example the analysis type would be } \\
\text { "seismic". }\end{array}$ \\
\hline <analysis> & $\begin{array}{l}<\text { analysis>random } \\
</ \text { analysis }>\end{array}$ & $\begin{array}{l}\text { This keyword is used to specify specific analysis type during a } \\
\text { base case update. For this example, during the base case } \\
\text { update, the analysis type would be "random." }\end{array}$ \\
\hline$<$ base case update $>$ & $\begin{array}{l}<\text { base case update }> \\
\text { (analysis type } \\
\text { specified) }<\text { /base } \\
\text { case update }>\end{array}$ & $\begin{array}{l}\text { This keyword is used to specify the start and end a base case } \\
\text { update action. }\end{array}$ \\
\hline <basic event> & $\begin{array}{l}<\text { basic event }> \\
\quad \cdots \\
<\text { < basic event }>\end{array}$ & $\begin{array}{l}\text { This keyword is used to specify the start and end of a basic } \\
\text { event action. Generally, basic event actions involves extracting } \\
\text { or loading MAR-D files or adding/deleting a basic event from a } \\
\text { MAR-D file. }\end{array}$ \\
\hline$<$ calc type $>$ & $\begin{array}{l}<\text { calc type }>1</ \text { calc } \\
\text { type }>\end{array}$ & $\begin{array}{l}\text { This keyword is used to specify the calculation type. For this } \\
\text { example, calculation type } 1 \text { would be use in the change set. }\end{array}$ \\
\hline$<$ case $>$ & $<$ case $>$ base </case $>$ & $\begin{array}{l}\text { This keyword is used to specify the base or current case. For } \\
\text { this example, the base case would be invoked. }\end{array}$ \\
\hline$<$ <hange set> & $\begin{array}{l}<\text { change set }> \\
\quad \cdots \\
</ \text { change set }>\end{array}$ & $\begin{array}{l}\text { This keyword is used to specify the start and end of change set } \\
\text { operations. For example, a change set can be added, deleted, } \\
\text { marked or unmarked. }\end{array}$ \\
\hline <class > & $\begin{array}{l}<\text { class }> \\
\cdots \\
</ \text { class }>\end{array}$ & $\begin{array}{l}\text { This keyword is used to specify the start and end of an event } \\
\text { class operation (i.e., class type basic event change in a change } \\
\text { set). Within this keyword function, the class of the basic events } \\
\text { to be changed and their desired changes is specified. }\end{array}$ \\
\hline <comment> & $\begin{array}{l}<\text { comment }> \\
\text { information } \\
</ \text { comment }>\end{array}$ & $\begin{array}{l}\text { This keyword is used to specify the start and end of a macro } \\
\text { comment block. Any text can be located within the comment } \\
\text { block. }\end{array}$ \\
\hline$<$ compare & $\begin{array}{l}<\text { compare file> } \\
\quad<\text { input } 1>\text { FILE1 } \\
\quad<\text { input1> } \\
\quad<\text { input 2>FILE2 } \\
\text { </input2> }\end{array}$ & $\begin{array}{l}\text { This keyword is used to specify the start and end of a file } \\
\text { comparison. Used with <input } 1>\text {, <input } 2>\text { tags to specify the } \\
\text { two files to compare (report files). The results of the } \\
\text { comparison can be viewed in a file using the } \\
\text { <output>filename</output }>\text { keyword. }\end{array}$ \\
\hline
\end{tabular}


Keyword Use/Example Notes

\begin{tabular}{|c|c|c|}
\hline & $</$ compare file $>$ & \\
\hline$<$ condition> & $\begin{array}{l}<\text { condition> } \\
\quad \cdots \\
<\text { <condition> }\end{array}$ & $\begin{array}{l}\text { This keyword is used to specify the start and end of a selected } \\
\text { GEM condition assessment process. Within the condition } \\
\text { block, a GEM condition assessment can be added, deleted, } \\
\text { marked or unmarked. }\end{array}$ \\
\hline <delete> & $\begin{array}{c}<\text { delete }> \\
\cdots \\
</ \text { delete }>\end{array}$ & $\begin{array}{l}\text { This keyword is used to specify the start and end of a list item } \\
\text { to be deleted. Using this keyword, a basic event could be } \\
\text { deleted to a MAR-D cut set listing and then reloaded or a } \\
\text { change set deleted from a database. }\end{array}$ \\
\hline <description> & $\begin{array}{l}<\text { description> } \\
\text { text description } \\
</ \text { description }>\end{array}$ & $\begin{array}{l}\text { This keyword is used to specify a text description to change } \\
\text { set, flag set, or scenario. For example if a change set is } \\
\text { created, <description }>\text { class change - All } \\
\text { events }<\text { /description>, the text between the description block } \\
\text { would be added as the change set's description. }\end{array}$ \\
\hline < duration> & $\begin{array}{l}<\text { duration>72 } \\
</ \text { duration }>\end{array}$ & $\begin{array}{l}\text { This keyword is used to specify a duration time for a GEM } \\
\text { condition assessment. For this example, the duration time } \\
\text { would be } 72 \text { hours. }\end{array}$ \\
\hline$<$ end state $>$ & $\begin{array}{l}<\text { end state }> \\
\quad \cdots \\
<\text { lend state }>\end{array}$ & $\begin{array}{l}\text { This keyword is used to specify the start and end of an end } \\
\text { state operation. Within the end state keyword blocks, end } \\
\text { states can be marked, gathered, solved, and an uncertainty } \\
\text { analysis performed. }\end{array}$ \\
\hline$<e n d>$ & $<$ end $></$ end $>$ & This keyword is used to specify the end of a scenario. \\
\hline$<$ event name> & $\begin{array}{l}<\text { event name> BE } \\
\text { NAME </event } \\
\text { name> }\end{array}$ & $\begin{array}{l}\text { This keyword is used to specify an event name to be adjusted } \\
\text { during a change set operation. For this example, the basic } \\
\text { event BE NAME, could be adjusted within a change set block. }\end{array}$ \\
\hline$<$ event tree $>$ & $\begin{array}{l}<\text { event tree> } \\
\quad \cdots \\
<\text { <event tree> }\end{array}$ & $\begin{array}{l}\text { This keyword is used to specify the start and end of an event } \\
\text { tree operation. Within the event tree keyword block, an event } \\
\text { tree can be marked, unmarked, and linked. }\end{array}$ \\
\hline <exclude> & $\begin{array}{l}\text { <exclude> FT-1 } \\
\text { </exclude> }\end{array}$ & $\begin{array}{l}\text { This keyword is used to specify the name of a event tree, fault } \\
\text { tree, or sequence to be excluded in an operation. For this } \\
\text { example, fault tree FT-1, would be excluded from any } \\
\text { operation. }\end{array}$ \\
\hline$<e x$ & $\begin{array}{l}<\text { extract }> \\
\cdots \\
<\text { extract }>\end{array}$ & $\begin{array}{l}\text { This keyword is used to specify the start and end of a MAR-D } \\
\text { extract operation. Within the extract keyword block, the MAR-D } \\
\text { file type and file name is specified. }\end{array}$ \\
\hline
\end{tabular}




\section{$\begin{array}{lll}\text { Keyword Use/Example Notes } & \end{array}$}

\begin{tabular}{|c|c|c|}
\hline$<$ fault tree $>$ & $\begin{array}{l}<\text { fault tree }> \\
\cdots \\
</ \text { fault tree }>\end{array}$ & $\begin{array}{l}\text { This keyword is used to specify the start and end of a fault tree } \\
\text { operation. Within the fault tree keyword block, cut sets can be } \\
\text { solved, flag sets applied, uncertainty analysis and importance } \\
\text { measures can be performed. }\end{array}$ \\
\hline$<$ file name $>$ & $\begin{array}{l}<\text { file name }> \\
\text { TEMP.BEI </file } \\
\text { name> }\end{array}$ & $\begin{array}{l}\text { This keyword is used to specify a file name for MAR-D extract } \\
\text { and load functions. For this example, the file name is MAR-D } \\
\text { file with a name "TEMP.BEI". This keyword is also used to } \\
\text { specify report output files names. }\end{array}$ \\
\hline$<$ flag set $>$ & $\begin{array}{l}<\text { flag set> } \\
\cdots \\
<\text { <flag set }>\end{array}$ & $\begin{array}{l}\text { This keyword is used to specify the start and end of flag set } \\
\text { operations. For example, a flag set can be added, deleted, and } \\
\text { applied to fault trees and sequences. }\end{array}$ \\
\hline <gather method> & $\begin{array}{l}<\text { gather method }>\text { by } \\
\text { sequence</gather } \\
\text { method }>\end{array}$ & $\begin{array}{l}\text { This keyword is used to specify the specific end state gather } \\
\text { operation. For this example, the cut sets would be gathered } \\
\text { "by sequence." }\end{array}$ \\
\hline$<$ generate $>$ & $\begin{array}{l}<\text { generate }> \\
</ \text { generate }\end{array}$ & $\begin{array}{l}\text { This keyword is used to specify the generate option. The } \\
\text { generate command implements the changes in a change set or } \\
\text { flag set and moves from the base case to the current case for } \\
\text { an analysis. }\end{array}$ \\
\hline$<$ group $>$ & $<$ group $>$ yes </group $>$ & $\begin{array}{l}\text { This keyword is used to specify the use of group importance } \\
\text { measures or not. For this example, the group importance is } \\
\text { "on". }\end{array}$ \\
\hline <ie name> & $\begin{array}{l}<\text { ie name>IE-LOSP } \\
<\text { <ie name> }\end{array}$ & $\begin{array}{l}\text { This keyword is used to specify the initiating event name. For } \\
\text { this example, the initiating event "IE-LOSP" would be selected } \\
\text { during a GEM initiating event assessment. }\end{array}$ \\
\hline <importance> & $\begin{array}{l}<\text { importance> } \\
\quad \cdots \\
\text { </importance> }\end{array}$ & $\begin{array}{l}\text { This keyword is used to specify the start and end of an } \\
\text { importance measure operation. }\end{array}$ \\
\hline <include> & $\begin{array}{l}<\text { include }>\text { FT-1 } \\
\text { </include }>\end{array}$ & $\begin{array}{l}\text { This keyword is used to specify the name of a event tree, fault } \\
\text { tree, or sequence to be included in an operation. For this } \\
\text { example, fault tree FT- } 1 \text {, would be included in the operation. }\end{array}$ \\
\hline <init event> & $\begin{array}{l}<\text { init event> } \\
\cdots \\
<\text { /init event> }\end{array}$ & $\begin{array}{l}\text { This keyword is used to specify the start and end of a GEM } \\
\text { initiating event assessment.. }\end{array}$ \\
\hline$<$ initial prompt> & $\begin{array}{l}<\text { initial prompt> yes } \\
<\text { /initial prompt> }\end{array}$ & $\begin{array}{l}\text { This keyword is not necessary to run a macro. However, if } \\
\text { "yes" is specified (as in this example) in the initial prompt block, } \\
\text { a confirmation box is displayed that ask the analyst do confirm } \\
\text { the operation of the macro-script. }\end{array}$ \\
\hline
\end{tabular}




\section{$\begin{array}{lll}\text { Keyword Use/Example Notes } & \end{array}$}

\begin{tabular}{|c|c|c|}
\hline$<$ input 1> & $\begin{array}{l}<\text { input } 1> \\
\text { FILENAME1</input } \\
1>\end{array}$ & $\begin{array}{l}\text { This keyword is used to designate the first file in a compare } \\
\text { operation. For this example, the first file is called FILENAME1. }\end{array}$ \\
\hline$<$ input 2> & $\begin{array}{l}<\text { input } 2> \\
\text { FILENAME2</input2> }\end{array}$ & $\begin{array}{l}\text { This keyword is used to designate the second file in a compare } \\
\text { operation. For this example, the second file is called } \\
\text { FILENAME2. }\end{array}$ \\
\hline$<$ lambda $>$ & $\begin{array}{l}<\text { lambda }>1.0 \mathrm{E}-2 \\
</ \text { lambda }>\end{array}$ & $\begin{array}{l}\text { This keyword is used to specify the value. For this example, } \\
\text { the lambda value would be } 1.0 \mathrm{E}-2 \text {. }\end{array}$ \\
\hline$<$ link $>$ & $<$ link $></$ link $>$ & This keyword is used to link event trees. \\
\hline$<$ load $>$ & $\begin{aligned}<\text { load }> \\
\quad \cdots \\
</ \text { load }>\end{aligned}$ & $\begin{array}{l}\text { This keyword is used to specify the start and end of a MAR-D } \\
\text { load operation. This operation can load in additional MAR-D } \\
\text { files or files that have been modified }\end{array}$ \\
\hline$<$ logic show save> & $\begin{array}{l}<\text { logic show save> } \\
</ \text { logic show save> }\end{array}$ & This keyword is used to show and save fault tree logic. \\
\hline$<$ loop class $>$ & $\begin{array}{l}<\text { loop class }>\text { grid } \\
<\text { /loop class }>\end{array}$ & $\begin{array}{l}\text { This keyword is used to specify the type of loop classification } \\
\text { being used during an analysis. For this example, the loop } \\
\text { class would be "Grid-related". }\end{array}$ \\
\hline $\begin{array}{l}<\text { mark event tree } \\
\text { mask> }\end{array}$ & $\begin{array}{l}<\text { mark event tree } \\
\text { mask }>\text { LOSP }</ \text { mark } \\
\text { event tree mask }>\end{array}$ & $\begin{array}{l}\text { This keyword is used to specify the specific event tree mask } \\
\text { and is used during sequence analysis. For this example, all } \\
\text { sequences generated with the event tree LOSP would be } \\
\text { marked. }\end{array}$ \\
\hline $\begin{array}{l}<\text { mark logic fault } \\
\text { tree> }\end{array}$ & $\begin{array}{l}<\text { mark logic fault } \\
\text { tree }>^{*}<\text { /mark logic } \\
\text { fault tree }>\end{array}$ & $\begin{array}{l}\text { This keyword is used to select a specific sequence having a } \\
\text { specific fault tree logic during sequence analysis. }\end{array}$ \\
\hline <mark mask> & $\begin{array}{l}<\text { mark mask }> \\
</ \text { mark mask }>\end{array}$ & $\begin{array}{l}\text { This keyword is used to specify the specific fault trees or event } \\
\text { trees to mark. For this example, a "*" would mark all fault trees } \\
\text { or event trees in the list (depending on if you were functioning } \\
\text { under a <fault tree> or <event tree> function block). }\end{array}$ \\
\hline$<$ mark name $>$ & $\begin{array}{l}<\text { mark name }> \\
\text { NAME1</mark } \\
\text { name> }\end{array}$ & $\begin{array}{l}\text { This keyword is used to specify the specific condition name in } \\
\text { GEM. For this example, the condition assessment NAME1 } \\
\text { would be marked. }\end{array}$ \\
\hline $\begin{array}{l}<\text { mark sequence } \\
\text { mask }>\end{array}$ & $\begin{array}{l}<\text { mark sequence } \\
\text { mask }>\text { ATWS }</ \text { mark } \\
\text { sequence mask }>\end{array}$ & $\begin{array}{l}\text { This keyword is used to specify the specific sequence mask. } \\
\text { For this example, all ATWS sequences would be selected for } \\
\text { analysis. }\end{array}$ \\
\hline
\end{tabular}




\section{$\begin{array}{lll}\text { Keyword Use/Example Notes } & \end{array}$}

\begin{tabular}{|c|c|c|}
\hline $\begin{array}{l}<\text { mark sequence } \\
\text { name }>\end{array}$ & $\begin{array}{l}<\text { mark sequence } \\
\text { name }>\text { LOSP2 } \\
</ \text { mark sequence } \\
\text { name }>\end{array}$ & $\begin{array}{l}\text { This keyword is used to mark a specific sequence for sequence } \\
\text { analysis. For this example, the sequence named LOSP2 would } \\
\text { be marked.. }\end{array}$ \\
\hline <mask operation> & $\begin{array}{l}<\text { mask operation>an } \\
<\text { /mask operation> }\end{array}$ & $\begin{array}{l}\text { This keyword is used to specify the specific mask operation for } \\
\text { masking/marking sequences. The logic mask operator can } \\
\text { either be "OR" or "AND". This function block allows more } \\
\text { flexibility in selecting event tree names, sequence names and } \\
\text { fault tree names for sequence analysis. }\end{array}$ \\
\hline$<$ mask $>$ & $<$ mask $><$ /mask $>$ & This keyword is used in conjunction with others. \\
\hline$<$ method $>$ & $\begin{array}{l}<\text { method }>\text { mcs } \\
</ \text { method }>\end{array}$ & $\begin{array}{l}\text { This keyword is used to specify the specific uncertainty } \\
\text { method. For this example, the uncertainty method would be } \\
\text { Monte Carlo. For Latin Hypercube, "Ihs" would be used. }\end{array}$ \\
\hline$<$ mission time $>$ & $\begin{array}{l}<\text { mission time }>10 \\
</ \text { mission time }>\end{array}$ & $\begin{array}{l}\text { This keyword is used to specify the specific mission time value. } \\
\text { Mission time can be in scientific notation i.e. }<\text { mission } \\
\text { time }>1.0 \mathrm{E}+1</ \text { mission time }>\text {. For this example the mission } \\
\text { time would be } 10 \text { hours. }\end{array}$ \\
\hline <name $>$ & $\begin{array}{l}<\text { name }>\text { test name } \\
<\text { /name }>\end{array}$ & $\begin{array}{l}\text { This keyword is used to specify the name of a specific test } \\
\text { passed in by a DOS script file, i.e. <name }>\% \mathrm{P}-11</ \text { name }>\text {. It } \\
\text { is also used to specify the name of a change set, flag set, fault } \\
\text { tree, event tree, etc to be added or deleted during an operation. }\end{array}$ \\
\hline <output> & $\begin{array}{l}<\text { output> file name } \\
<\text { /output> }\end{array}$ & $\begin{array}{l}\text { This keyword is used to specify the output to a specific file } \\
\text { name. For example, <output>compare.rpt</output>, would } \\
\text { output to a text file called "compare.rpt". }\end{array}$ \\
\hline$<$ page $>$ & $<$ page $></$ page $>$ & This keyword is used to turn on the MAR-D paging option. \\
\hline$<$ partition> & $\begin{array}{l}<\text { partition }> \\
</ \text { partition }>\end{array}$ & $\begin{array}{l}\text { This keyword is used to turn on the partition option for event } \\
\text { trees. }\end{array}$ \\
\hline$<$ probability > & $\begin{array}{l}<\text { probability> } 0.02 \\
</ \text { probability }>\end{array}$ & $\begin{array}{l}\text { This keyword is used to specify a specific probability. For this } \\
\text { example the probability of } 0.02 \text { would be used in a change set. }\end{array}$ \\
\hline$<$ process $>$ & $\begin{array}{l}<\text { process }> \\
</ \text { process }>\end{array}$ & $\begin{array}{l}\text { This keyword is used to specify the start and end of a condition } \\
\text { assessment timed test. }\end{array}$ \\
\hline <program exit> & $\begin{array}{l}<\text { program exit> } \\
</ \text { program exit> }\end{array}$ & This keyword is used to specify the end of the macro script. \\
\hline <project $>$ & $<$ project $><$ /project $>$ & This keyword is used to specify a "project level" uncertainty \\
\hline
\end{tabular}


operation.

This keyword is used to specify the destination of the report <report destination> <report destination>F output. There are three options: "F" is a file, "V" is a screen $</$ report destination> view, and " $P$ " is a default printer. It the report destination is not specified, the default is to a file.

\begin{tabular}{|c|c|}
\hline$<$ report format $>$ & $\begin{array}{l}<\text { report format }>A \\
<\text { /report format }>\end{array}$ \\
\hline$<$ report> & $\begin{array}{c}<\text { report> } \\
\ldots \\
<\text { </report> }\end{array}$ \\
\hline <sample> & $\begin{array}{l}\text { <sample> } 5000 \\
\text { </sample> }\end{array}$ \\
\hline <scenario> & $\begin{array}{c}<\text { scenario } \\
\ldots \\
<\text { <scenario }\end{array}$ \\
\hline
\end{tabular}

$<$ seed $>$

$<$ seismic bin>

<sequence>

set units>

$<$ setup $>$

<show save> </scenario $>$

$<$ seed $>1234<$ seed $>$

<seismic bin> 9

$</$ seismic bin $>$

<sequence>

$\cdots$

$</$ sequence $>$

$<$ set units> PPerYear $</$ set units $>$

$<$ setup $>$

$</$ setup $>$

<show save $><$ show save>
This keyword is used to specify the type of report format that is outputted. There are three options: " $A$ " is text file, " $R$ " is RTF format, and "H" is HTML format. The default is text format.

This keyword is used to specify a report creating operation.

This keyword is used to specify the number of samples in an uncertainty calculation. For this example, 5000 samples would be ran for the uncertainty operation.

This keyword is used to specify a specific scenario sequence of events. This keyword is used to help identify various macroscript functions in a file.

This keyword is used to specify the random number seed in an uncertainty measure calculation. If a " 0 " is used, then the clock number is implemented. For this example, the seed number would be "1234".

This keyword is used to specify the histogram bin number for a seismic analysis.

This keyword is used to specify the start and end of a sequence operation.

This keyword is used to specify the project units. For this example "per year" would be used. Other units include per month, per week, and per day.

This keyword is used to specify the test scenario state of operation. This keyword is used to during the validation and verification macro-script tests.

This keyword displays a fault tree graphic and saves it. 


\section{$\begin{array}{lll}\text { Keyword Use/Example Notes } & \end{array}$}

\begin{tabular}{|c|c|c|}
\hline$<$ single $>$ & $<$ single $></$ single $>$ & $\begin{array}{l}\text { This keyword is used to specify a single event operation in a } \\
\text { change set operation. To types of change sets are available, } \\
\text { single and class changes. }\end{array}$ \\
\hline <slice save> & $\begin{array}{c}\text { <slice save } \\
\quad \ldots \\
<\text { <lice save }>\end{array}$ & $\begin{array}{l}\text { This keyword is used to specify a cut set slice save operation. } \\
\text { This feature allow for specific cut sets to be saved in a file (or } \\
\text { end state). }\end{array}$ \\
\hline <solve $>$ & $\begin{array}{c}<\text { solve }> \\
\quad \cdots \\
</ \text { solve }>\end{array}$ & $\begin{array}{l}\text { This keyword is used to specify a fault tree/sequence solve } \\
\text { operation (i.e. solve for cut sets). }\end{array}$ \\
\hline <sort order> & $\begin{array}{l}<\text { sort order }>\text { name } \\
</ \text { sort order }>\end{array}$ & $\begin{array}{l}\text { This keyword is used to specify the type of sorted order of the } \\
\text { output. This is used for sorting using a specified importance } \\
\text { measure. This function sorts the basic events in alphabetical } \\
\text { order. }\end{array}$ \\
\hline$<$ start $>$ & $<$ start $></$ start $>$ & $\begin{array}{l}\text { This keyword is used to specify the start of a new validation } \\
\text { and verification test. }\end{array}$ \\
\hline <sub type> & $\begin{array}{l}\text { <sub type>base only } \\
<\text { /sub type> }\end{array}$ & $\begin{array}{l}\text { This keyword is used to specify a report sub-type. For this } \\
\text { example, the sub-type report is for base case results only. }\end{array}$ \\
\hline <suscept> & $\begin{array}{l}<\text { suscept> } 4 \\
</ \text { suscept }>\end{array}$ & $\begin{array}{l}\text { This keyword is used to specify an event susceptibility value. } \\
1=\text { random, } 2=\text { fire, } 3=\text { flood, } 4=\text { seismic, etc. }\end{array}$ \\
\hline$<$ tau $>$ & $<$ tau $>4<$ tau $>$ & $\begin{array}{l}\text { This keyword is used to specify a specific tau value in a change } \\
\text { set. }\end{array}$ \\
\hline <truncation> & $\begin{array}{l}<\text { truncation> } 1.0 \mathrm{E}-12 \\
</ \text { truncation> }\end{array}$ & $\begin{array}{l}\text { This keyword is used to specify a probability cut off level, a } \\
\text { truncation. This function block is used within the <solve }> \\
\text { block. }\end{array}$ \\
\hline <type> & $<$ type $>$ cut set $</$ type $>$ & $\begin{array}{l}\text { This keyword is used to specify the report type. For this } \\
\text { example, the report type would be for a cut set output report. }\end{array}$ \\
\hline <uncertainty> & $\begin{array}{l}\text { <uncertainty }> \\
\quad \cdots \\
\text { </uncertainty }>\end{array}$ & $\begin{array}{l}\text { This keyword is used to specify an uncertainty measure } \\
\text { operation. }\end{array}$ \\
\hline <unmark> & $<$ unmark $><$ /unmark $>$ & $\begin{array}{l}\text { This keyword is used to clear any marked change sets in a } \\
\text { plant model prior to additional analysis. }\end{array}$ \\
\hline <update> & $<$ update $><$ /update $>$ & $\begin{array}{l}\text { This keyword is used to specify an update operation for a cut } \\
\text { set. }\end{array}$ \\
\hline
\end{tabular}




\begin{tabular}{|c|c|c|}
\hline Keyword & Use/Example & Notes \\
\hline$<$ verbose $>$ & $\begin{array}{l}<\text { verbose }> \\
\quad \cdots \\
</ \text { verbose }>\end{array}$ & $\begin{array}{l}\text { This keyword is used to specify the verbose option on for the } \\
\text { verification and validation test procedure of SAPHIRE. This } \\
\text { keyword is used to setup the level of detail in the test results } \\
\text { output files. }\end{array}$ \\
\hline affected & $\begin{array}{l}<\text { sub type }>\text { affected } \\
</ \text { sub type }>\end{array}$ & $\begin{array}{l}\text { Parameter setting for a change set report that shows affected } \\
\text { events adjusted in the change set (i.e. shows current case } \\
\text { data) }\end{array}$ \\
\hline all & $\begin{array}{l}<\text { sub type }>\text { all }<\text { /sub } \\
\text { type }>\end{array}$ & $\begin{array}{l}\text { Parameter setting for a change set report that shows both } \\
\text { affected and unaffected basic events (i.e. shows both current } \\
\text { and base case). }\end{array}$ \\
\hline and & $\begin{array}{l}<\text { mask operation>anc } \\
<\text { /mask operation }>\end{array}$ & $\begin{array}{l}\text { dParameter setting for setting for filtering masking/marking } \\
\text { selections for fault trees and sequences. }\end{array}$ \\
\hline ascii & $\begin{array}{l}<\text { report format }>\text { ascii } \\
</ \text { report format }>\end{array}$ & $\begin{array}{l}\text { Parameter setting for setting the report file out format to } \\
\text { delimited ascii text. }\end{array}$ \\
\hline average & $\begin{array}{l}<\text { loop class }>\text { average } \\
<\text { /loop class }>\end{array}$ & $\begin{array}{l}\text { Parameter setting for use with GEM initiating event operations. } \\
\text { Set the loop class to be a combined average of Grid related, } \\
\text { Plant centered, severe weather, and extremely severe weather } \\
\text { classes. }\end{array}$ \\
\hline base & $<$ case $>$ base $<$ /case $>$ & Parameter setting to specify the base case is to be used. \\
\hline by partition & $\begin{array}{l}<\text { gather method }>\text { by } \\
\text { partition </gather } \\
\text { method }>\end{array}$ & $\begin{array}{l}\text { Parameter setting for selection of an end state gather } \\
\text { technique to be "gather cut sets by partition". }\end{array}$ \\
\hline by sequence & $\begin{array}{l}<\text { gather method }>\text { by } \\
\text { sequence</gather } \\
\text { method }>\end{array}$ & $\begin{array}{l}\text { Parameter setting for selection of an end state gather } \\
\text { technique to be "gather cut sets by sequence". }\end{array}$ \\
\hline combination & $\begin{array}{l}\text { <type> combination } \\
</ \text { type }>\end{array}$ & $\begin{array}{l}\text { Parameter setting to specify a combination report type } \\
\text { (summary report) for fault tree, sequences and end states } \\
\text { reports. }\end{array}$ \\
\hline current & $\begin{array}{l}<\text { case }>\text { current }</ \text { case } \\
>\end{array}$ & Parameter setting to specify the current case is to be used. \\
\hline current base & $\begin{array}{l}<\text { sub type }>\text { current } \\
\text { base }</ \text { sub type }>\end{array}$ & $\begin{array}{l}\text { Parameter setting for selection of a report sub type to have } \\
\text { output for both the current case and the base case. }\end{array}$ \\
\hline current only & $\begin{array}{l}<\text { sub type>current } \\
\text { only</sub type> }\end{array}$ & $\begin{array}{l}\text { Parameter setting for selection of a report sub type to be } \\
\text { current case only. }\end{array}$ \\
\hline
\end{tabular}




\begin{tabular}{|c|c|c|}
\hline cut set & $<$ type>cut set</type> & $\begin{array}{l}\text { Parameter setting for selection of a report type to be for "cut } \\
\text { sets." i.e. the output file will contain cut sets. }\end{array}$ \\
\hline debug & & $\begin{array}{l}\text { Parameter setting for selecting the mode of the log file output } \\
\text { used for validation and verification testing. }\end{array}$ \\
\hline detail & & $\begin{array}{l}\text { Parameter setting for selecting the mode of the log file output in } \\
\text { a high detail mode. }\end{array}$ \\
\hline esw & $\begin{array}{l}<\text { loop class }>\text { esw } \\
<\text { /loop class }>\end{array}$ & $\begin{array}{l}\text { Parameter setting for use with GEM initiating event operations. } \\
\text { Sets the loop class to "extreme severe weather." }\end{array}$ \\
\hline file & & Parameter setting for TBD \\
\hline grid & $\begin{array}{l}<\text { loop class }>\text { grid } \\
<\text { lloop class }>\end{array}$ & $\begin{array}{l}\text { Parameter setting for use with Initiating Event operations. Set } \\
\text { the loop class to "grid related." }\end{array}$ \\
\hline group & <type>group</type> & $\begin{array}{l}\text { Parameter setting for selection of an importance measure } \\
\text { calculation type "group." }\end{array}$ \\
\hline interval & $\begin{array}{l}<\text { type>interval } \\
</ \text { type }>\end{array}$ & $\begin{array}{l}\text { Setting for selection of an importance measure calculation, } \\
\text { "interval." The other option is "ratio". }\end{array}$ \\
\hline Ihs & $\begin{array}{l}<\text { method }>\text { lhs } \\
<\text { /method }>\end{array}$ & $\begin{array}{l}\text { Parameter setting for Latin Hypercube Simulation method in an } \\
\text { uncertainty calculation. }\end{array}$ \\
\hline mcs & $\begin{array}{l}<\text { method }>\text { mcs } \\
</ \text { method }>\end{array}$ & $\begin{array}{l}\text { Parameter setting for Monte Carlo Simulation method in an } \\
\text { uncertainty calculation. }\end{array}$ \\
\hline name & $\begin{array}{l}<\text { name }>\% \mathrm{P}-10 \\
<\text { /name }>\end{array}$ & $\begin{array}{l}\text { Parameter setting for assigning the name of the database (or } \\
\text { project) that the macro-script is being used on to the test } \\
\text { scenario. For this example, if the project name was "TEST", } \\
\text { then the name of the scenario would be "TEST-10". }\end{array}$ \\
\hline no & $\begin{array}{l}<\text { initial prompt>no } \\
<\text { /initial prompt }>\end{array}$ & $\begin{array}{l}\text { Parameter setting for turning off a feature. For this example, } \\
\text { the conformation prompt would not be implemented. }\end{array}$ \\
\hline none & & Parameter setting for TBD \\
\hline or & $\begin{array}{l}<\text { mask operation>or } \\
<\text { /mask operation> }\end{array}$ & $\begin{array}{l}\text { Parameter setting for setting for filtering masking/marking } \\
\text { selections for fault trees and sequences. }\end{array}$ \\
\hline PPerDay & $\begin{array}{l}<\text { set units> PPerDay } \\
</ \text { set units> }\end{array}$ & Parameter setting for <set units> to be "per day." \\
\hline PerDemand & $<$ set units> & Parameter setting for < set units> to be "per demand." \\
\hline
\end{tabular}




\begin{tabular}{|c|c|c|}
\hline & $\begin{array}{l}\text { PPerDemand }</ \text { set } \\
\text { units }>\end{array}$ & \\
\hline PPerHour & $\begin{array}{l}<\text { set units> PPerHour } \\
</ \text { set units }>\end{array}$ & Parameter setting for <set units> to be "per hour." \\
\hline PPerMinute & $\begin{array}{l}\text { <set units> } \\
\text { PPerMinute </set } \\
\text { units> }\end{array}$ & Parameter setting for <set units > to be "per minute." \\
\hline PPerMonth & $\begin{array}{l}\text { <set units> } \\
\text { PPerMonth </set } \\
\text { units> }\end{array}$ & Parameter setting for < set units> to be "per month." \\
\hline PPerWeek & $\begin{array}{l}<\text { set units> } \\
\text { PPerWeek </set } \\
\text { units> }\end{array}$ & Parameter setting for <set units > to be "per week." \\
\hline PPerYear & $\begin{array}{l}<\text { set units> PPerYear } \\
</ \text { set units }>\end{array}$ & Parameter setting for < set units > to be "per year." \\
\hline plant centered & $\begin{array}{l}<\text { loop class }>\text { plant } \\
\text { centered }</ \text { loop class }>\end{array}$ & $\begin{array}{l}\text { Parameter setting for use with an Initiating Event operation. } \\
\text { > Sets the loop class to "plant centered." }\end{array}$ \\
\hline print & $\begin{array}{l}<\text { report destination }> \\
\text { print }</ \text { report } \\
\text { destination> }\end{array}$ & $\begin{array}{l}\text { Parameter setting for selecting a report output option to be to a } \\
\text { system default printer. }\end{array}$ \\
\hline project & $<$ type $>$ project</type $>$ & $\begin{array}{l}\text { Parameter setting for selection of an uncertainty calculation } \\
\text { type to "project". }\end{array}$ \\
\hline random & $\begin{array}{l}<\text { analysis }>\text { random }</ a \\
\text { nalysis }>\end{array}$ & Parameter setting for an analysis type to random. \\
\hline ratio & $<$ type $>$ ratio</type > & $\begin{array}{l}\text { Parameter setting for selection of an importance measure } \\
\text { calculation to be "ratio". The other option is "interval" }\end{array}$ \\
\hline results & $<$ type $>$ results</type $>$ & $\begin{array}{l}\text { Parameter setting for selection of a report type for analysis } \\
\text { results. }\end{array}$ \\
\hline $\mathrm{rtf}$ & $\begin{array}{l}<\text { report format> rtf } \\
<\text { /report format }>\end{array}$ & $\begin{array}{l}\text { Parameter setting for setting the file output format of a report to } \\
\text { be RTF format. }\end{array}$ \\
\hline severe weather & $\begin{array}{l}<\text { loop class }>\text { severe } \\
\text { weather }</ \text { loop class }>\end{array}$ & $\begin{array}{l}\text { Parameter setting for use with initiating event operations. Set } \\
\text { loop class to severe weather." }\end{array}$ \\
\hline
\end{tabular}


Keyword

\begin{tabular}{|c|c|c|}
\hline single & <type>single</type> & $\begin{array}{l}\text { Parameter setting for selection of an importance measure } \\
\text { calculation to single. }\end{array}$ \\
\hline unaffected & $\begin{array}{l}<\text { sub type }> \\
\text { unaffected }</ \text { sub } \\
\text { type }>\end{array}$ & $\begin{array}{l}\text { Parameter setting for a change set report that shows } \\
\text { unaffected events not adjusted in the change set (i.e. shows } \\
\text { base case data) }\end{array}$ \\
\hline uncertainty & $\begin{array}{l}<\text { type>uncertainty } \\
</ \text { type }>\end{array}$ & $\begin{array}{l}\text { Parameter setting for selection of an importance measure } \\
\text { calculation to be by "uncertainty." }\end{array}$ \\
\hline view & $\begin{array}{l}<\text { report destination }> \\
\text { view }</ \text { report } \\
\text { destination }>\end{array}$ & $\begin{array}{l}\text { Parameter setting for selecting a report output option to be to } \\
\text { screen for viewing. }\end{array}$ \\
\hline yes & $<$ group $>$ yes </group $>$ & Parameter setting for turning on a feature. \\
\hline
\end{tabular}

\section{A Macro-Script Example}

The follow is a simple Macro-script example designed for the DEMO project that accompanies the SAPHIRE program. The Macro-script can be created using any standard text editor. The following Macro-script, when ran, creates a change set called "Example" and adds a description to it. The change set adjusts the probability of basic event C-PUMP-A to 1.0E-1. The macro-script then marks the change set and generates the new data. Next, all the sequences are marked and new cut sets are solved. Finally, the new cut set listing is displayed on the screen. 


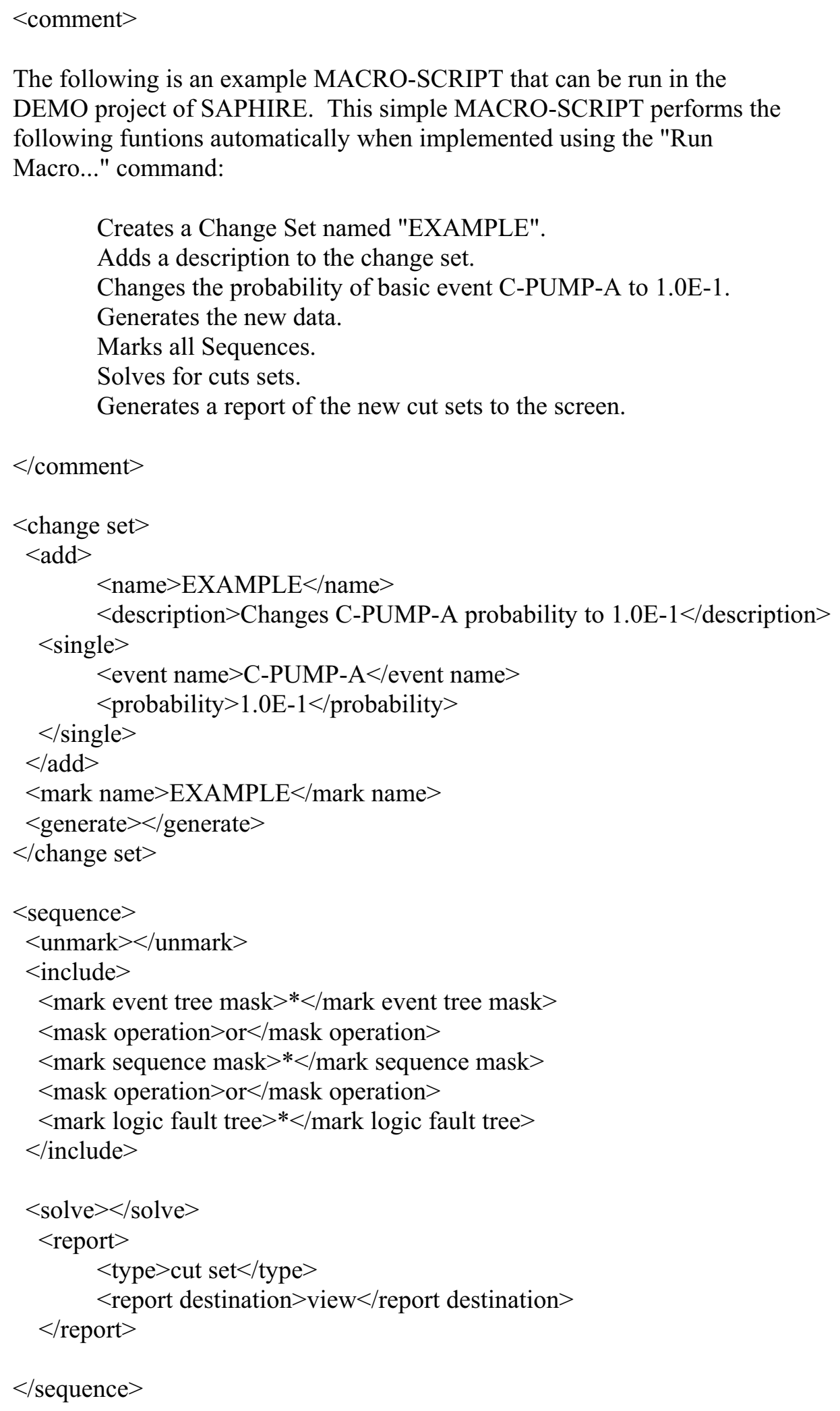




\subsection{Creating a Macro-Script}

\subsubsection{Making a Macro-Script}

\section{PURPOSE}

Describe the steps for creating a Macro-Script.

\section{STEPS}

1. Select any text editor or program that saves files in a standard ASCII file format. For this example, Microsoft ${ }^{\circledR}$ NotePad will be used.

2. In NotePad, type in the desired Macro-Script using the Keywords and commands shown in the table above.

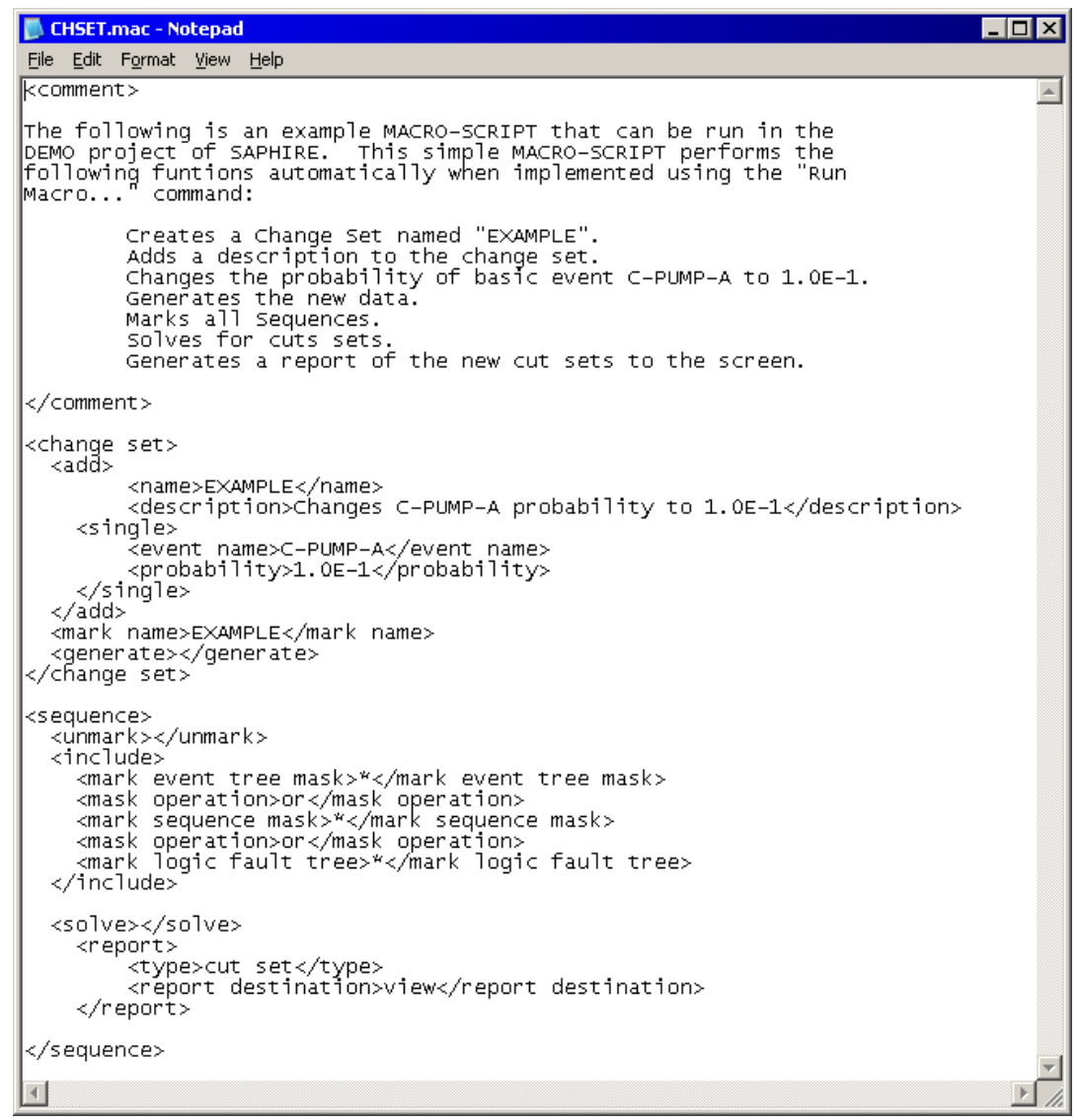

3. Save the file in NotePad by selecting File $\rightarrow$ Save As... and save the file in a specified directory or the Project directory. SAPHIRE reads the maco-script files with a ".MAC" file extention. For this example, the file was saved as CHSET.mac

The macro-script is ready to be accessed and implemented in SAPHIRE. 


\subsubsection{Running a Macro-Script.}

To illustrate the use of a macro-script, the DEMO project will be used.

1. To run a macro-script, select File from the menu bar and use the drop-down menu to select Run Maco.

\begin{tabular}{l} 
New Project... \\
Open Project... \\
Close Project \\
\hline Switch to GEM \\
\hline Run Macro \\
\hline Exit \\
\hline SEISMICDEMO \\
DEMO \\
SINGLE \\
STL1_3 \\
FLT1 \\
HYDROGEN
\end{tabular}

2. A "Select a macro file" pop-up window will be displayed. Use the "Look in:"drop box to select the location of the macro-script and select the desired file (macro-script files are read with a *.mac file extension). Click on the Open button to run the macro-script.

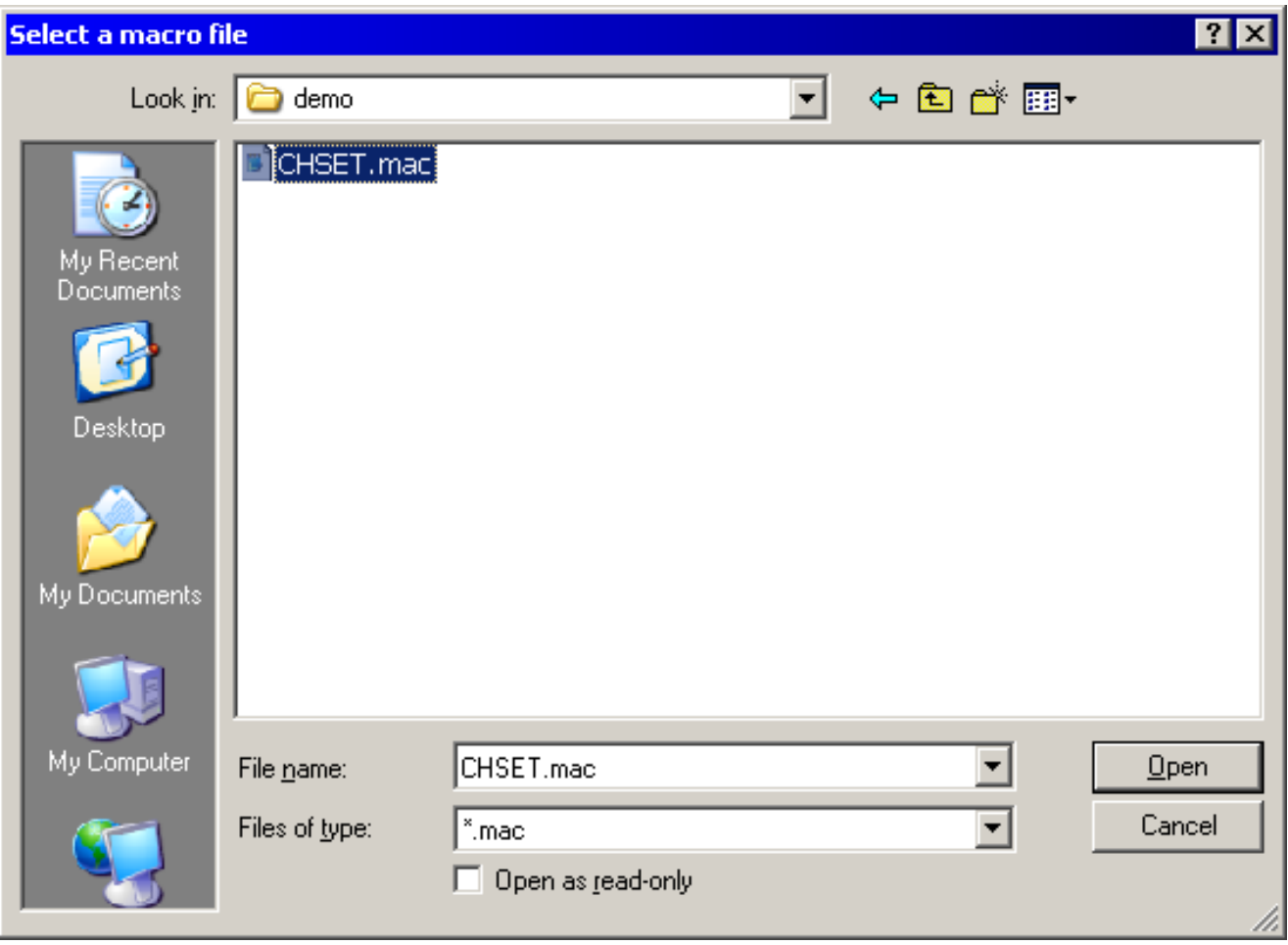




\begin{tabular}{|c|c|}
\hline 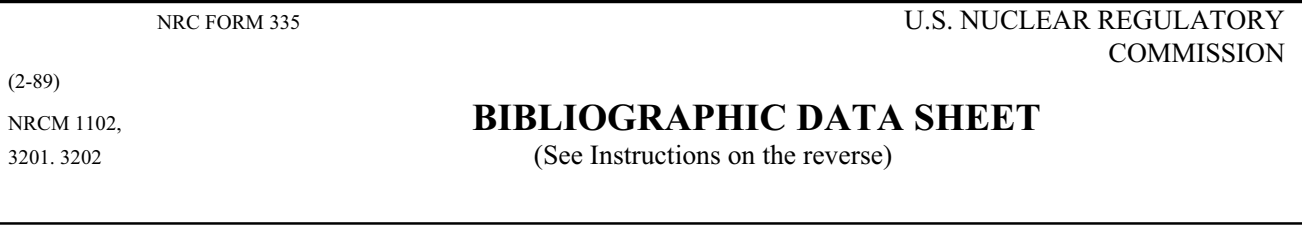 & $\begin{array}{l}\text { 1. REPORT NUMBER } \\
\text { (Assigned by NRC, Add Vol., } \\
\text { Supp., Rev., and Addendum } \\
\text { Numbers, if any.) } \\
\text { NUREG/CR-6952 } \\
\text { INL/EXT-05-00644 }\end{array}$ \\
\hline \multirow{4}{*}{$\begin{array}{l}\text { 2. TITLE AND SUBTITLE } \\
\text { Systems Analysis Programs for Hands-on Integrated Reliability Evaluations } \\
\text { (SAPHIRE) Vol. } 3 \text { Code Reference Manual - Part B }\end{array}$} & 3. DATE REPORT PUBLISHED \\
\hline & \begin{tabular}{l|l} 
MONTH & YEAR
\end{tabular} \\
\hline & September \\
\hline & $\begin{array}{l}\text { 4. FIN OR GRANT NUMBER } \\
\text { N6203 }\end{array}$ \\
\hline 5. $\operatorname{AUTHOR}(\mathrm{S})$ & $\begin{array}{c}\text { 6. TYPE OF REPORT } \\
\text { Technical }\end{array}$ \\
\hline K. J. Kvarfordt, C. L. Smith, S. T. Wood & 7. PERIOD COVERED (Inclusive Dates) \\
\hline \multicolumn{2}{|c|}{$\begin{array}{l}\text { 8. PERFORMING ORGANIZATION - NAME AND ADDRESS (If NRC, provide Division, Office or Region, U.S. Nuclear Regulatory Commission, and } \\
\text { mailing address; if contractor, provide name and mailing address.) } \\
\text { Idaho National Laboratory } \\
\text { Battelle Energy Alliance } \\
\text { P.O. Box } 1625 \\
\text { Idaho Falls, ID } 83415-3850\end{array}$} \\
\hline \multicolumn{2}{|l|}{$\begin{array}{l}\text { 9. SPONSORING ORGANIZATION - NAME AND } \\
\text { U.S. Nuclear Regulatory Commission, and mailing adc } \\
\text { Division of Risk Analysis } \\
\text { Office of Nuclear Regulatory Research } \\
\text { U.S. Nuclear Regulatory Commission } \\
\text { Washington, DC 20555-0001 }\end{array}$} \\
\hline \multicolumn{2}{|l|}{$\begin{array}{l}\text { 10. SUPPLEMENTARY NOTES } \\
\text { D. O’Neal, NRC Project Manager }\end{array}$} \\
\hline \multicolumn{2}{|c|}{$\begin{array}{l}\text { 11. ABSTRACT ( } 200 \text { words or less) } \\
\text { The Systems Analysis Programs for Hands-on Integrated Reliability Evaluations (SAPHIRE) is a software application } \\
\text { developed for performing a complete probabilistic risk assessment (PRA) using a personal computer (PC) running the } \\
\text { Microsoft Windows operating system. SAPHIRE is primarily funded by the U.S. Nuclear Regulatory Commission (NRC) } \\
\text { and developed by the Idaho National Laboratory (INL). This reference guide will introduce the SAPHIRE Version } 7.0 \\
\text { software. A brief discussion of the purpose and history of the software is included along with general information such as } \\
\text { installation instructions, starting and stopping the program, and some pointers on how to get around inside the program. } \\
\text { Next, database concepts and structure are discussed. Following that discussion are nine sections, one for each of the menu } \\
\text { options on the SAPHIRE main menu, wherein the purpose and general capabilities for each option are furnished. Next, the } \\
\text { capabilities and limitations of the software are provided. Finally, a series of appendices are provided that detail information } \\
\text { about some of the more complex operations of the software. }\end{array}$} \\
\hline \multirow[t]{4}{*}{ 12. KEY WORDS/DESCRIPTORS (List words or phrases that will assist researchers in locating the report.) } & $\begin{array}{l}\text { 13. AVAILABILITY STATEMENT } \\
\text { Unlimited }\end{array}$ \\
\hline & $\begin{array}{l}\text { 14. SECURITY CLASSIFICATION } \\
\text { (This page) } \\
\text { Unclassified } \\
\text { (This report) } \\
\text { Unclassified }\end{array}$ \\
\hline & 15. NUMBER OF PAGES \\
\hline & 16. PRICE \\
\hline
\end{tabular}

